\title{
Big Hole (41TV2161): Two Stratigraphically Isolated Middle Holocene Components in Travis County, Texas Volume I
}

\author{
J. Michael Quigg \\ Benjamin G. Bury \\ Robert A. Ricklis \\ Paul M. Matchen \\ Shannon Gray
}

See next page for additional authors

Follow this and additional works at: https://scholarworks.sfasu.edu/ita

Part of the American Material Culture Commons, Archaeological Anthropology Commons, Environmental Studies Commons, Other American Studies Commons, Other Arts and Humanities Commons, Other History of Art, Architecture, and Archaeology Commons, and the United States History Commons

Tell us how this article helped you.

This Article is brought to you for free and open access by the Center for Regional Heritage Research at SFA ScholarWorks. It has been accepted for inclusion in Index of Texas Archaeology: Open Access Gray Literature from the Lone Star State by an authorized editor of SFA ScholarWorks. For more information, please contact cdsscholarworks@sfasu.edu. 


\title{
Big Hole (41TV2161): Two Stratigraphically Isolated Middle Holocene Components in Travis County, Texas Volume I
}

\author{
Authors \\ J. Michael Quigg, Benjamin G. Bury, Robert A. Ricklis, Paul M. Matchen, Shannon Gray, Charles D. \\ Frederick, Tiffany Osburn, and Eric Shroeder

\section{Licensing Statement} \\ This is a work for hire produced for the Texas Department of Transportation (TxDOT), which owns all \\ rights, title, and interest in and to all data and other information developed for this project under its \\ contract with the report producer. The report may be cited and brief passages from this publication may \\ be reproduced without permission provided that credit is given to TxDOT and the firm that produced it. \\ Permission to reprint an entire chapter, section, figures or tables must be obtained in advance from the \\ Supervisor of the Archeological Studies Branch, Environmental Affairs Division, Texas Department of \\ Transportation, 125 East 11th Street, Austin, Texas, 78701
}




\section{Big Hole (41TV2161): Two Stratigraphically Isolated Middle Holocene Components in Travis County, Texas Volume I}

By:

J. Michael Quigg, Benjamin G. Bury, Robert A. Ricklis, Paul M. Matchen, Shannon Gray, Charles D. Frederick, Tiffany Osburn, and Eric Schroeder

with contributions by

Beta Analytic, Steven Bozarth, Henry and Mary Chigey, Hector Coronado, J. Phil Dering, Jeffrey R. Ferguson, Timothy Figol, Michael D. Glascock, Trisha-Ann Gonzales, Bruce Hardy, Mary Malainey, Linda Perry, Mary Schabel, Steven Shackley, Byron Sudbury, and Barbara Winsborough

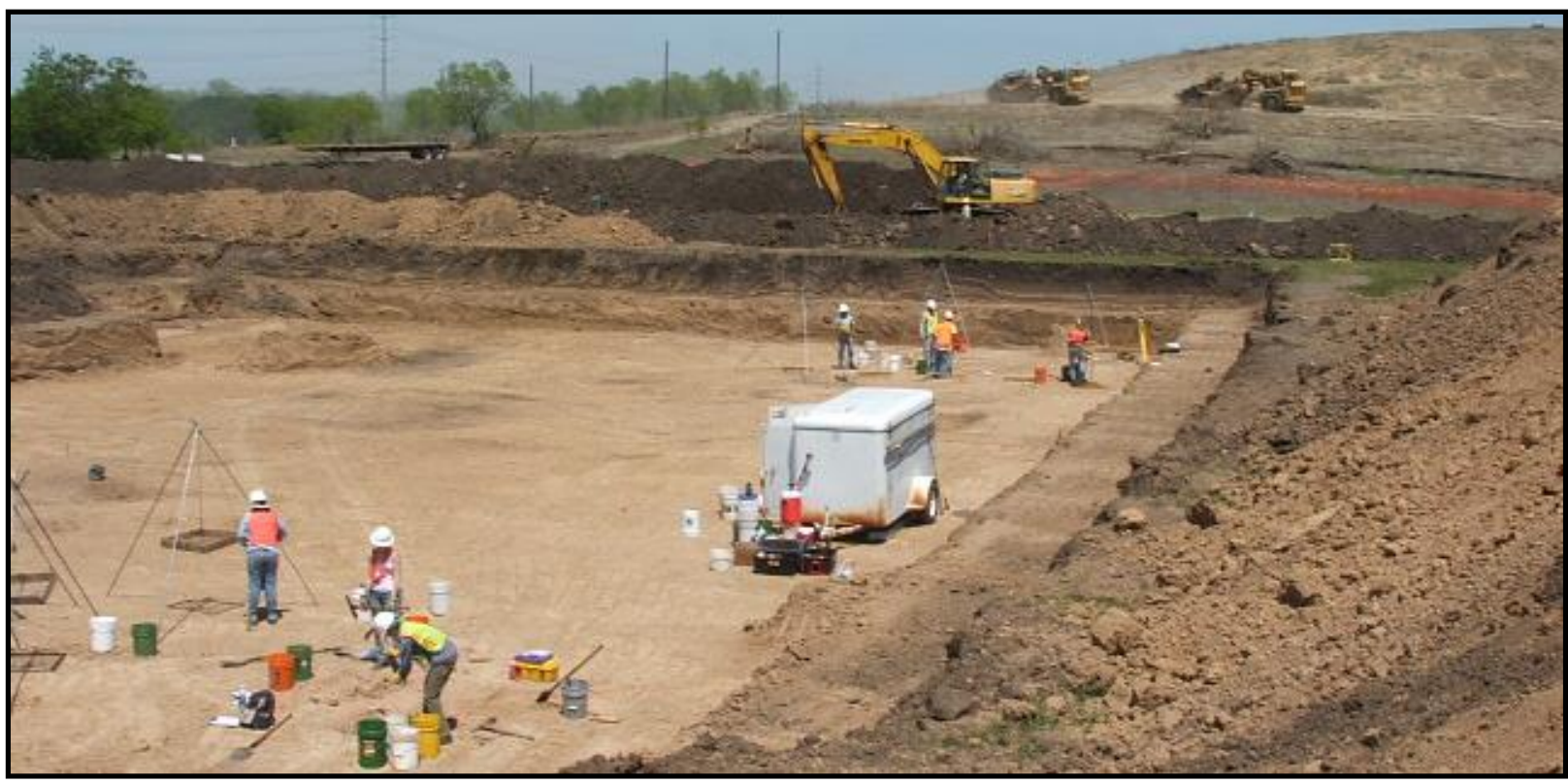

Prepared for:

Texas Department of Transportation Environmental Affairs Division Archeological Studies Program Report No. 144 (CSJ: 0440-06-006)

Austin, Texas
Prepared by:

TRC Environmental Corporation TRC Technical Report Nos. 51831 (112368), 181822, 208177, 228154 Austin, Texas

J. Michael Quigg, Principal Investigator Texas Antiquities Committee Permit No. 4064 
Copyright (C) 2016

Texas Department of Transportation (TxDOT)

This is a work for hire produced for the Texas Department of Transportation (TxDOT), which owns all rights, title, and interest in and to all data and other information developed for this project under Contracts 573XXSA006, 575XXSA008, 577XXSA003, 579XXSA003, and 573XXSA004. Brief passages from this publication may be reproduced without permission provided that credit is given to TxDOT and TRC Environmental Corporation. Permission to reprint an entire chapter, section, figures or tables must be obtained in advance from the Supervisor of the Archeological Studies Program, Environmental Affairs Division, Texas Department of Transportation, 125 East $11^{\text {th }}$ Street, Austin, Texas, 78701. Copies of this publication have been deposited with the Texas State Library in compliance with the State Depository Requirement.

Printed by:

Document Engine

Round Rock, Texas

July 2016

Jointly published by:

Texas Department of Transportation

Environmental Affairs Division

Archeological Studies Program

Scott Pletka, Ph.D., Supervisor

and

TRC Environmental Corporation

TRC Technical Report Nos. 181822, 184348, 208177, and 228154

Austin, Texas

ISBN \#978-1-935545-11-8 


\section{EXECUTIVE SUMMARY}

During April and May 2006, an archeological team from the Cultural Resources Section of the Planning, Permitting and Licensing Practice of TRC Environmental Corporation's (TRC) Austin office conducted geoarcheological documentation and data recovery excavations at prehistoric site 41TV2161 (CSJ: 0440-06-006). Investigations were restricted to a 70 centimeter $(\mathrm{cm})$ thick target zone between ca. 220 and $290 \mathrm{~cm}$ below surface (bs) on the western side of site 41TV2161 - the Big Hole site in eastern Travis County, Texas.

This cultural investigation was necessary under the requirements of Section 106 of the National Historic Preservation Act (NHPA), the implementing regulations of 36CRF Part 800 and the Antiquities Code of Texas (Texas Natural Resource Code, Title 9, Chapter 191 as amended) to recover a sample of the significant cultural materials prior to destruction by planned construction of State Highway 130 (SH 130). The latter by a private construction firm - Lone Star Infrastructure. This necessary data recovery was for Texas Department of Transportation (TxDOT), Environmental (ENV) Affairs Division under a Scientific Services Contract No. 577XXSA003 (Work Authorization No. 57701SA003). Over the years since the original award, multiple work authorizations between TxDOT and TRC were implemented and completed towards specific aspects of the analyses and reporting. The final analyses and report were conducted under contract 57-3XXSA004 (Work Authorization 57311SA004). All work was under Texas Antiquities Committee Permit No. 4064 issued by the Texas Historical Commission (THC) to J. Michael Quigg.

Initially, an archeological crew from Hicks \& Company encountered site 41TV2161 during an intensive cultural resource inventory conducted south of Pearce Lane along the planned construction zone of SH 130 in the fall of 2005. Following the initial site discovery, archeologists expanded their investigations to the west across the SH 130 right-of-way, and completed excavation of 10 backhoe trenches, 13 shovel tests, and 11 test units at site 41TV2161. The investigations encountered at least seven buried cultural features and 1,034 artifacts, some in relatively good context. The survey and testing report to TxDOT presented their findings and recommendations (Campbell et al. 2006). The ENV Affairs Division of TxDOT and the THC reviewed the initial findings and recommendations, and determined site 41TV2161 was eligible for listing on the National Register of Historic Places and as State Antiquities Landmark as the proposed roadway development was to directly impact this important site and further excavations were required.

Subsequently, TRC archeologists led by Paul Matchen (Project Archeologist) and J. Michael Quigg (Principal Investigator) initiated data recovery excavations through the mechanicalremoval of between 220 and $250 \mathrm{~cm}$ of sediment from a 30-by-40 meter $(\mathrm{m})$ block area (roughly $3,000 \mathrm{~m}^{3}$ ). This was conducted to allow handexcavations to start just above the deeply buried, roughly $70 \mathrm{~cm}$ thick targeted zone of cultural material. Mechanical stripping by Lone Star Infrastructure staff created a large hole with an irregular bottom that varied between 220 and 260 cmbs. To locate specific areas to initiate handexcavations within the mechanically stripped area, a geophysical survey that employed ground penetrating radar (GPR) was conducted by Tiffany Osburn then with Geo-Marine in Plano, Texas. Over a dozen electronic anomalies were detected through the GPR investigation. Following processing, data filtering, and assessment, Osburn identified and ranked the anomalies for investigation. The highest ranked anomalies (1 through 8) were thought to have the greatest potential to represent cultural features. Anomalies 1 through 6 were selected and targeted through 
hand-excavations of 1-by-1 m units that formed continuous excavation blocks of various sizes. Blocks were designated A, B, C, D, E, and F. The type, nature, quantity, and context of encountered cultural materials in each block led the direction and expansion of each excavation block as needed. In total, TRC archeologists hand-excavated $38.5 \mathrm{~m}^{3}$ $\left(150 \mathrm{~m}^{2}\right)$ from a vertically narrow target zone within this deep, multicomponent and stratified prehistoric site.

Hand-excavation in the two largest Blocks, B and D $\left(51 \mathrm{~m}^{2}\right.$ and $62 \mathrm{~m}^{2}$ respectively), revealed two vertically separate cultural components between roughly 220 and 290 cmbs. The younger component was restricted to Block B and yielded a Bell/Andice point and point base, plus a complete Big Sandy point. These points were associated with at least eight small burned rock features, one cluster of ground stone tools, limited quantities of lithic debitage, few formal chipped and ground stone tools, and a rare vertebrate faunal assemblage. Roughly 20 to $25 \mathrm{~cm}$ below the Bell/Andice component in Block B and across Block D was a component identified by a single corner-notched Martindale dart point. This point was associated with a scattered burned rocks, three charcoal stained hearth features, scattered animal, bird, and fish bones, mussel shells, and less than a dozen formal chipped and ground stone tools.

Both identified components contained cultural materials in good stratigraphic context with high spatial integrity. Significant, both were radiocarbon dated by multiple charcoal samples to a narrow 200-year period between 5250 and 5450 B.P. during the middle Holocene. With exception of the well-preserved faunal assemblages, perishable materials were poorly preserved in the moist silty clay loam. Charcoal lacked structure and was reduced to dark stains. Microfossils (e.g., phytoliths and starch gains) were present, although in very limited numbers and deteriorated conditions.

The four much smaller Blocks (A, C, E, and F) yielded various quantities of cultural material and features, but these blocks also lacked sufficient charcoal dates and diagnostic artifacts Those artifacts and samples were left unassigned and analyzed separately from the Bell/Andice and Martindale components. The two well-defined components in Blocks B and D are the focus of this technical report. The components provide very significant data towards understanding rare and poorly understood hunter-gatherer populations during late stages of the Altithermal climate period.

This final report builds upon the interim report submitted to TxDOT (Quigg et al. 2007) that briefly described the methods, excavations, preliminary findings, initial results from six feasibility studies, and proposed an initial research design for data analyses. Context and integrity of the cultural materials in the two identified components was excellent. This rare circumstance combined with detailed artifact analyses, solid documentation of their ages through multiple radiocarbon dates, and multidisciplinary approach to analyses, allowed significant insights and contributions concerning the two populations involved. Results provide a greater understanding of human behaviors during a rarely identified time in Texas Prehistory.

The cultural materials and various collected samples were temporarily curated at TRC's Austin laboratory. Following completion of analyses and acceptance of this final report, the artifacts, paper records, photographs, and electronic database were permanently curated at the Center for Archaeological Studies (CAS) at Texas State University in San Marcos. 


\section{TABLE OF CONTENTS}

EXECUTIVE SUMMARY iii

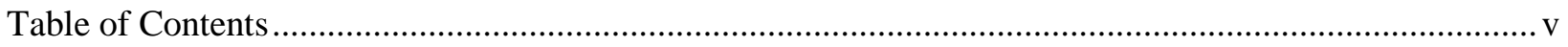

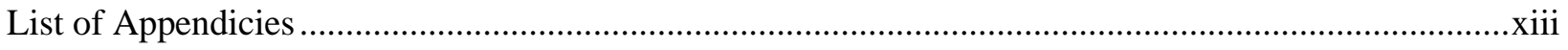

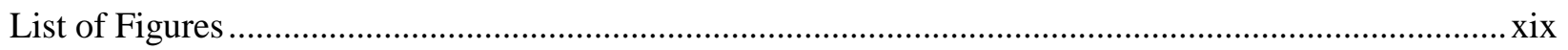

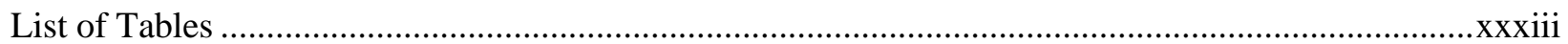

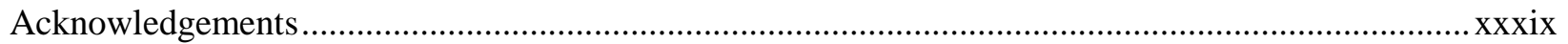

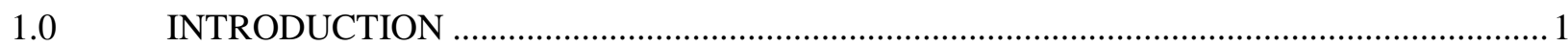

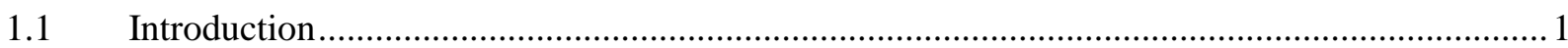

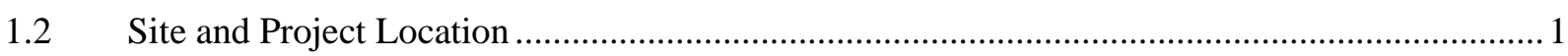

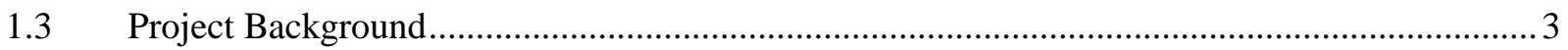

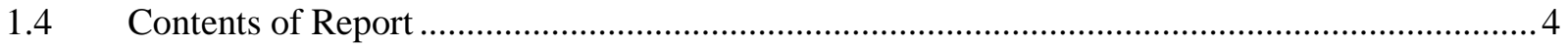

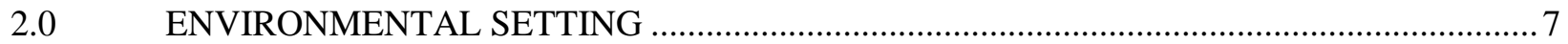

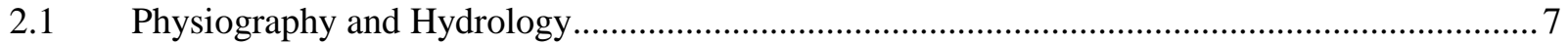

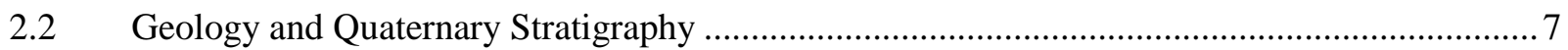

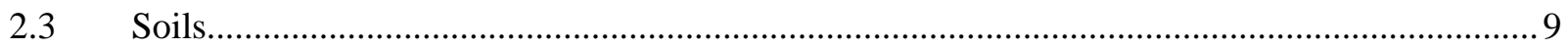

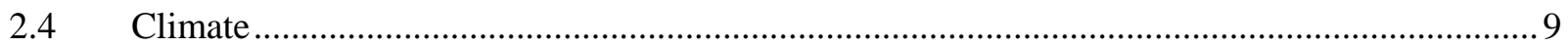

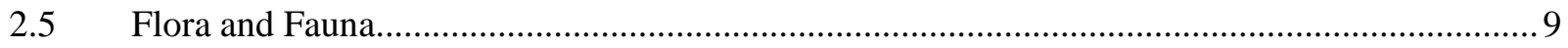

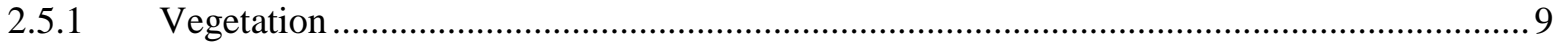

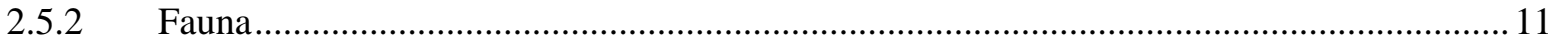

2.6 Paleoenvironment and Indications of Paleoclimate during the Middle Holocene ................... 12

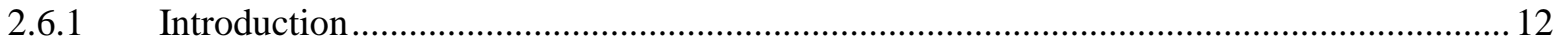

2.6.2 Paleoenvironmental Conductions in Central and Southeastern Texas .............................. 15

2.6.3 Paleoenvironmental Conditions in Northern Texas, Oklahoma, and Adjacent Areas ........22

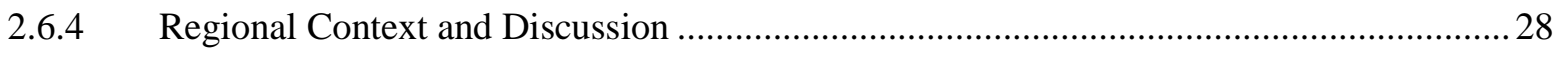

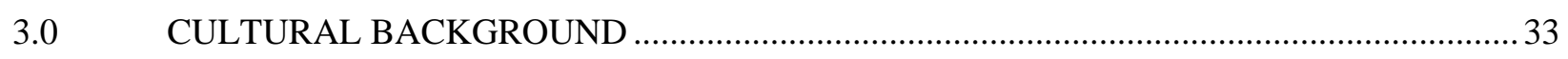

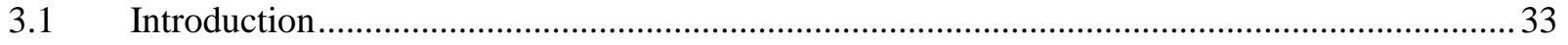

3.2 Bell/Andice/Calf Creek Horizon Background …...................................................................... 33

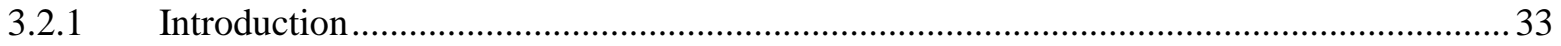

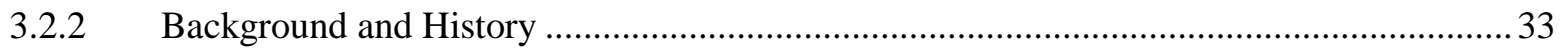

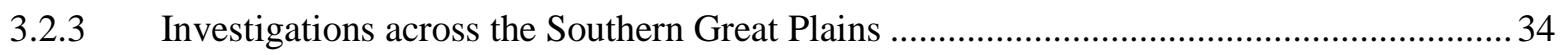

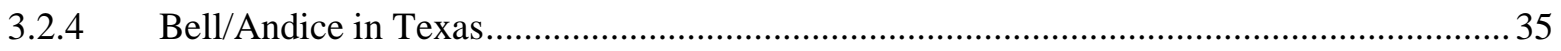




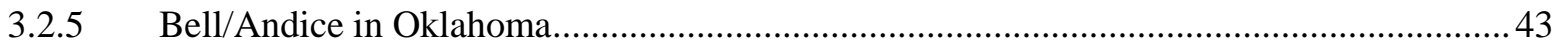

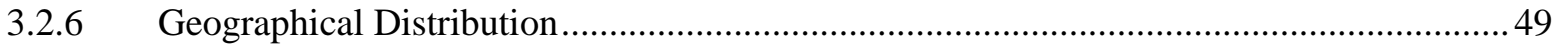

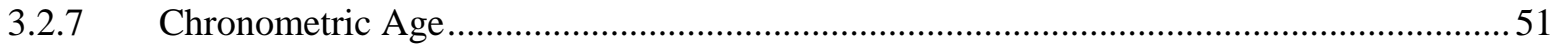

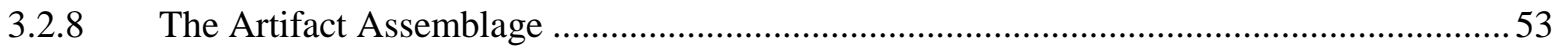

3.2.9 Lithic Procurement and Knapping Observations ..........................................................55

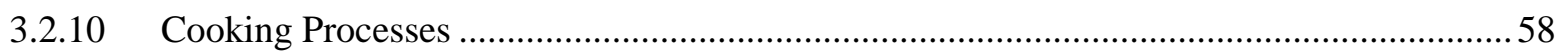

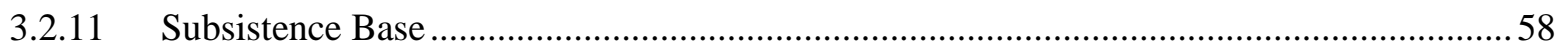

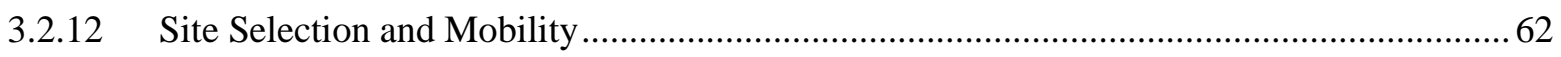

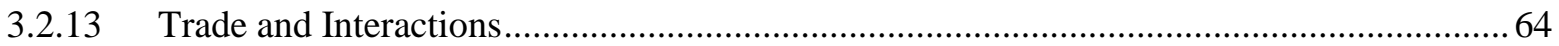

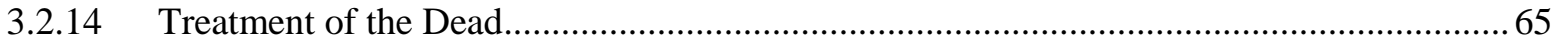

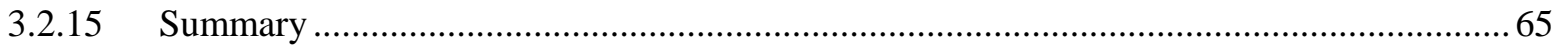

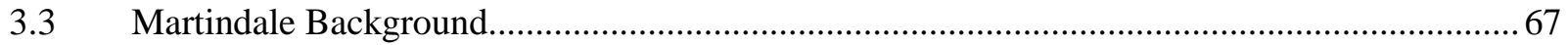

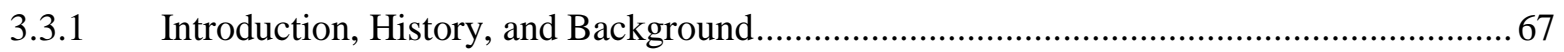

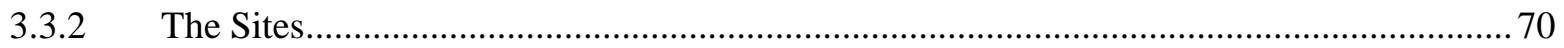

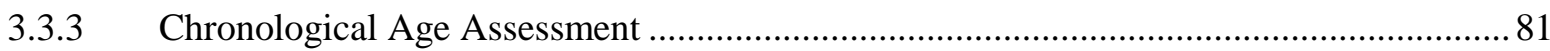

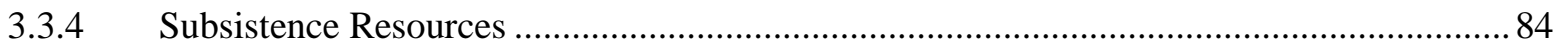

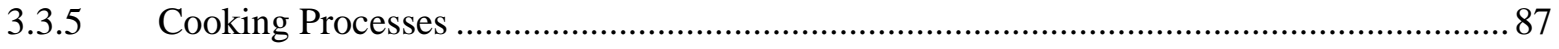

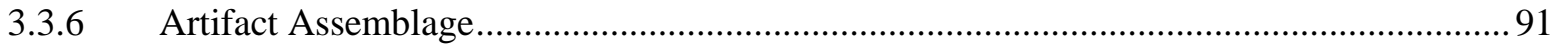

3.3.7 Settlement Patterns and Distribution.............................................................................. 91

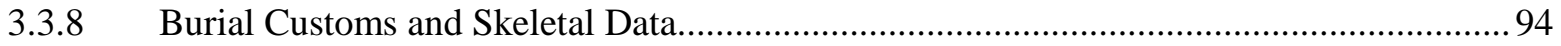

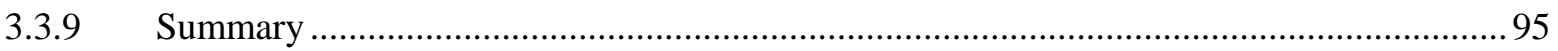

4.0 RESEARCH DESIGN FOR ANALYSIS AND REPORTING ..........................................97

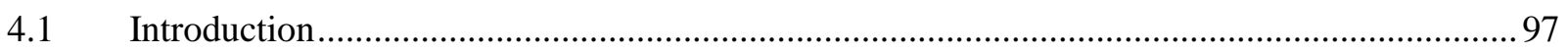

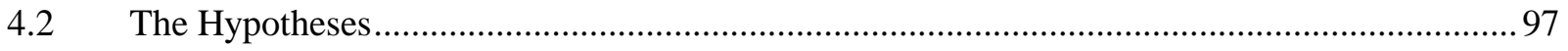

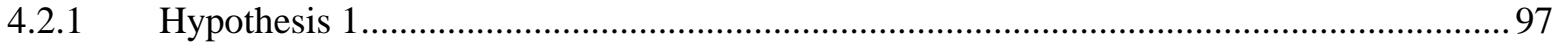

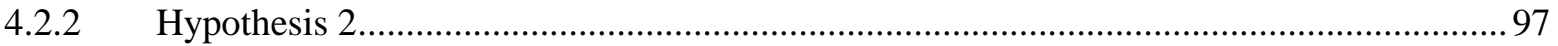

4.3 Prehistoric Cultural Components at 41TV2161 and their Chronological Placement ...............97

4.4 The Nature of the Pertinent Site Occupations as Inferable from features and Artifact

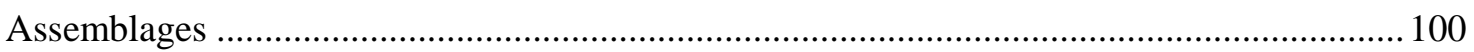

4.5 Regional Paleoclimate and Environment, ca. 5000 to 6000 B.P............................................ 101

4.6 Analysis for Testing the Two Hypotheses .......................................................................... 103

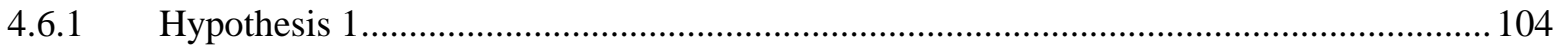

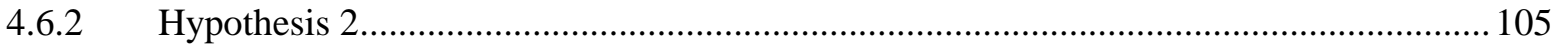




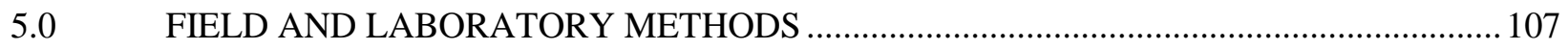

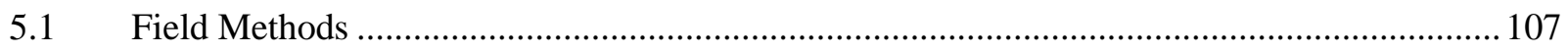

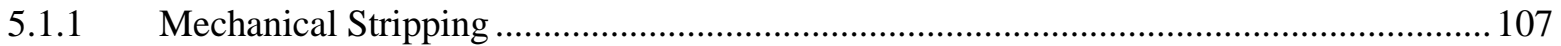

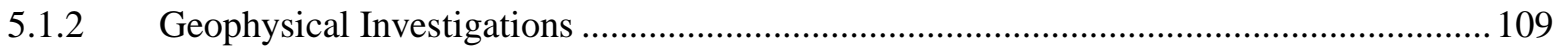

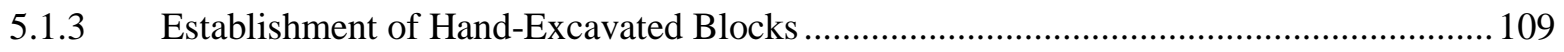

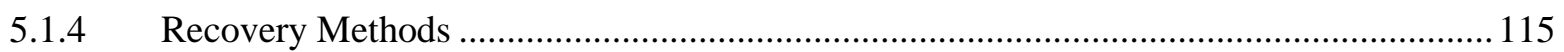

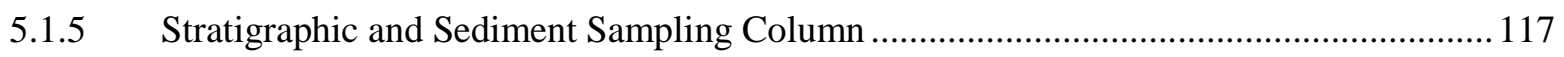

$5.2 \quad$ Laboratory Procedures and Technical Analyses ............................................................. 117

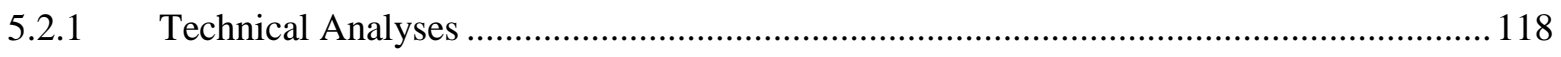

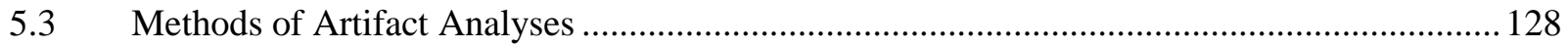

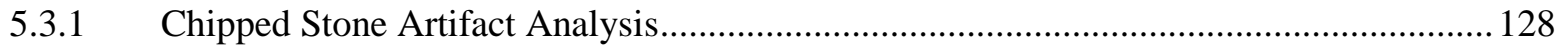

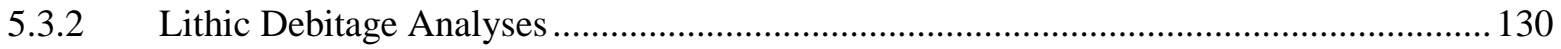

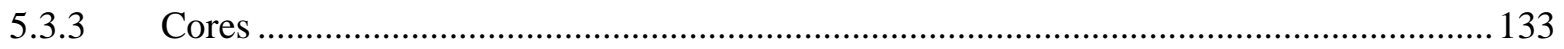

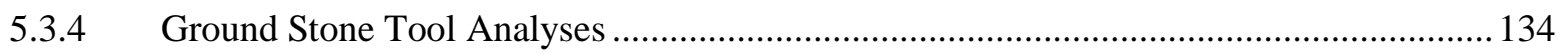

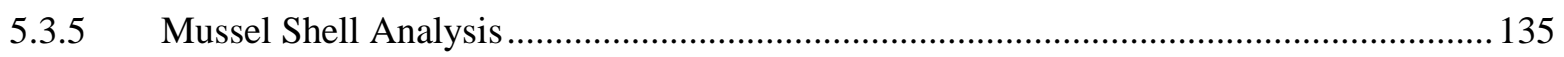

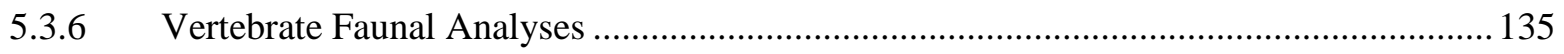

5.3.7 Burned Rock Analysis ........................................................................................... 139

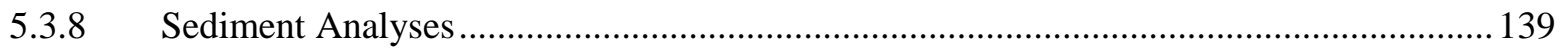

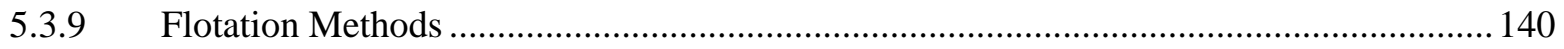

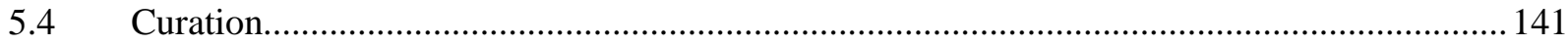

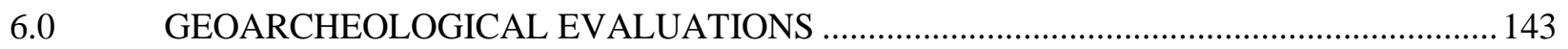

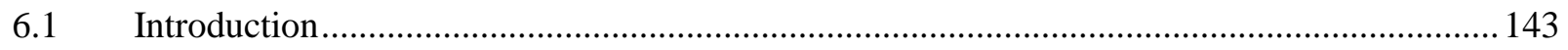

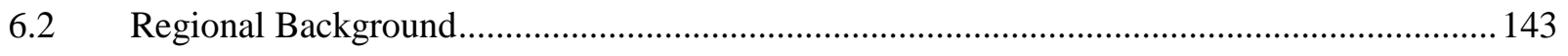

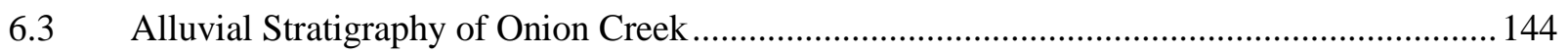

6.4 Late Quaternary Onion Creek Stratigraphy: A New Model .................................................. 145

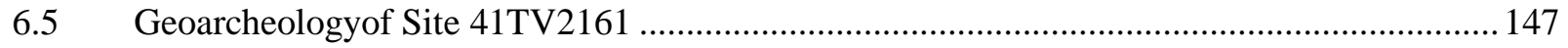

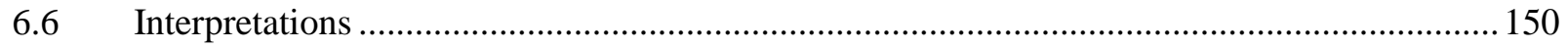

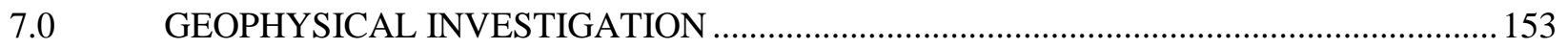

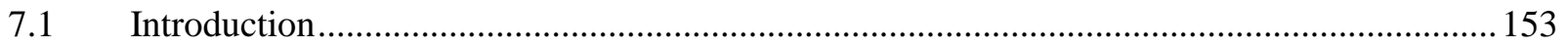

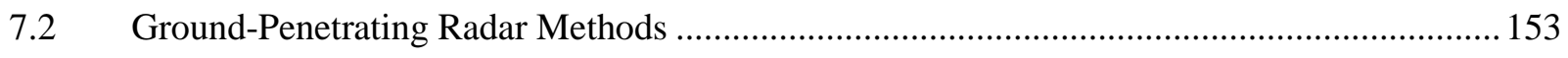

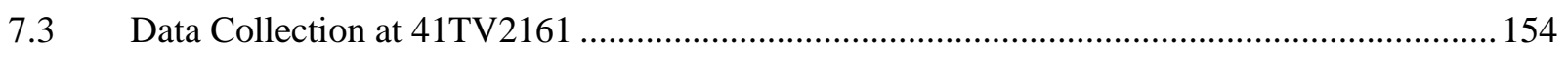

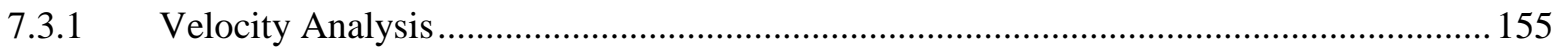

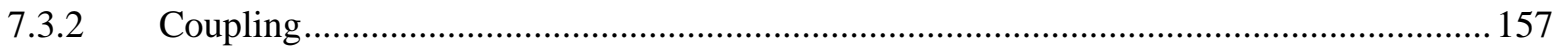




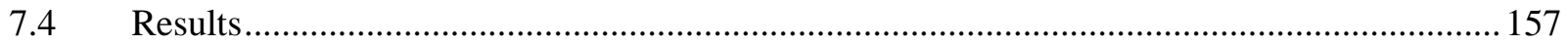

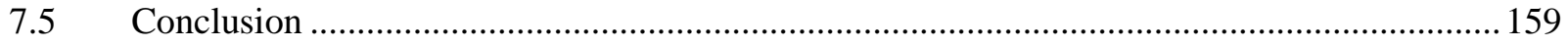

8.0 CULTURAL STRATIGRAPHY, INTEGRITY, AND COMPONENT ASSIGMENTS ....... 163

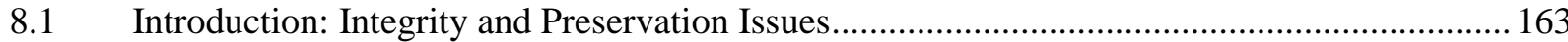

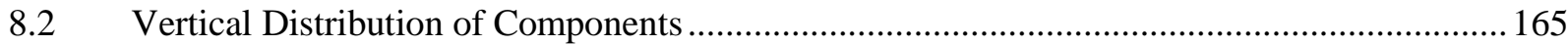

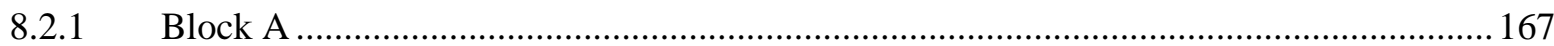

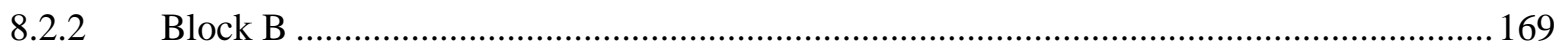

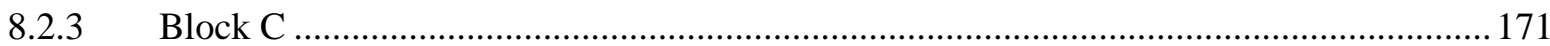

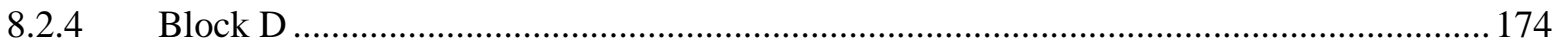

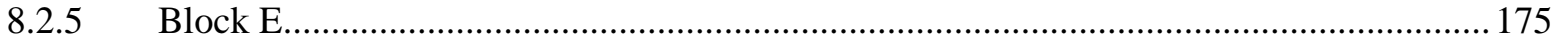

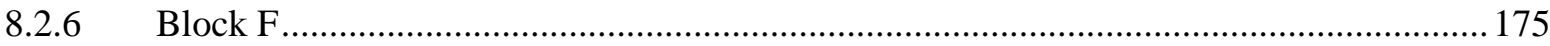

8.3 Site Stratigraphy Summary and Component Assignment................................................... 176

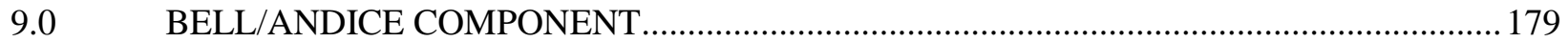

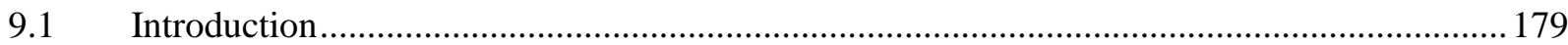

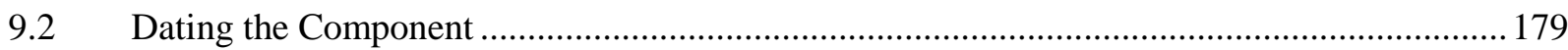

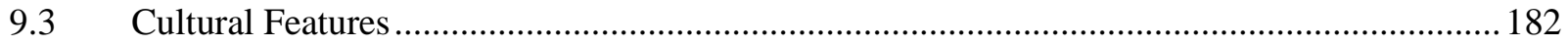

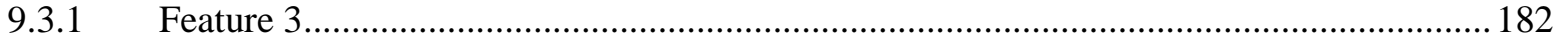

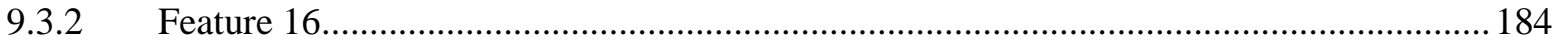

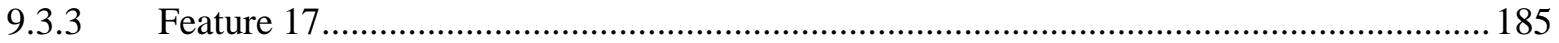

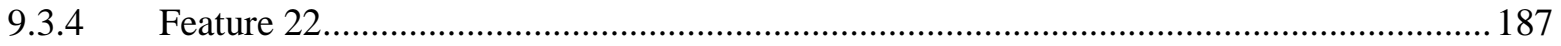

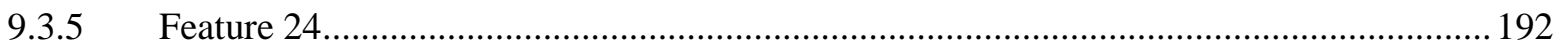

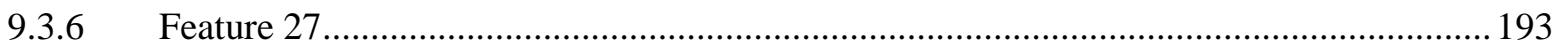

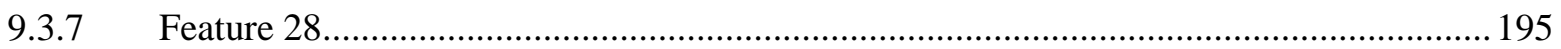

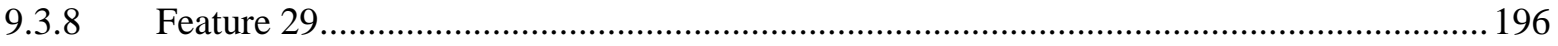

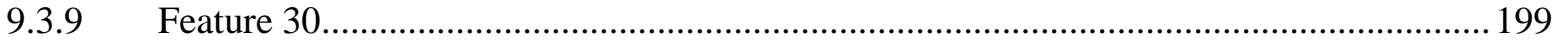

9.3.10 Feature 32

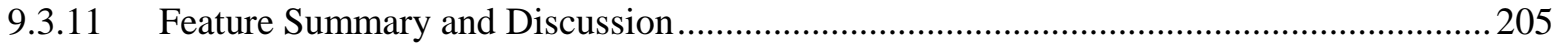

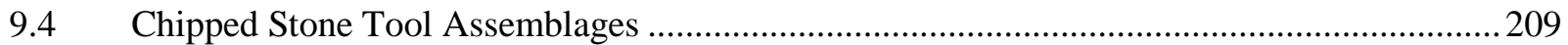

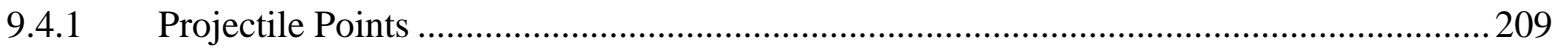

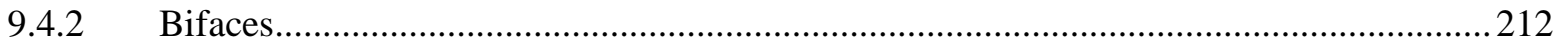

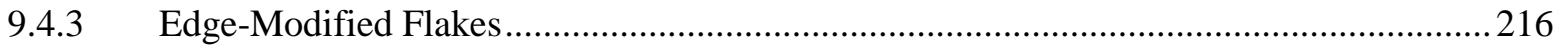

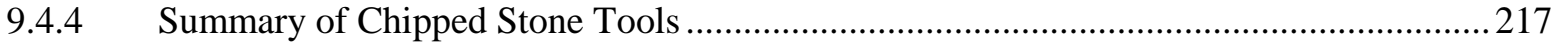

9.4.5 Horizontal Distribution of Chipped Stone Tools ..........................................................218 


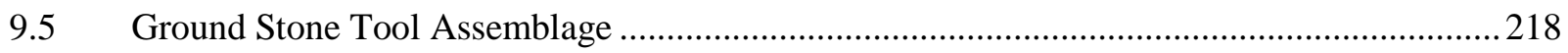

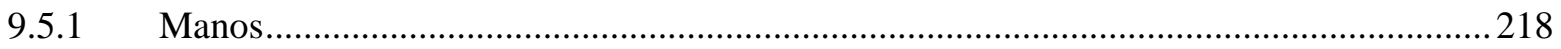

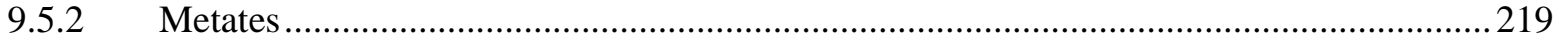

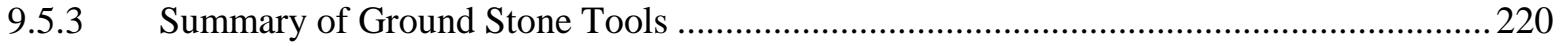

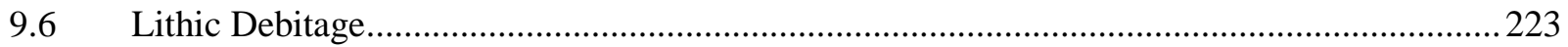

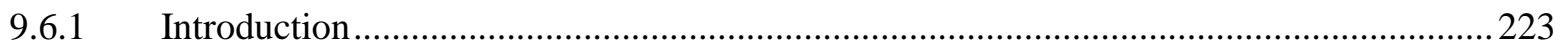

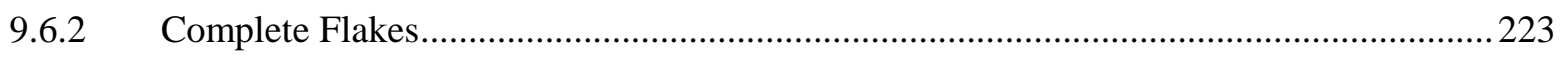

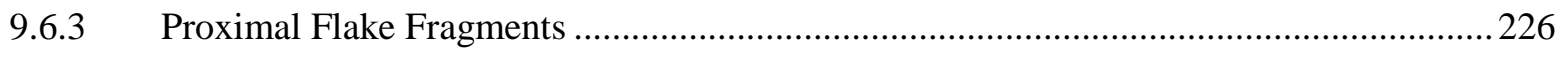

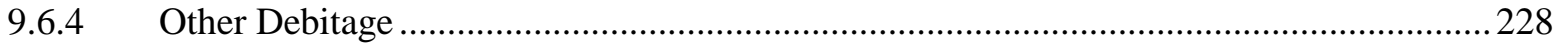

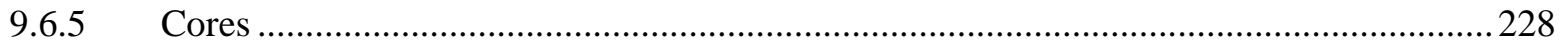

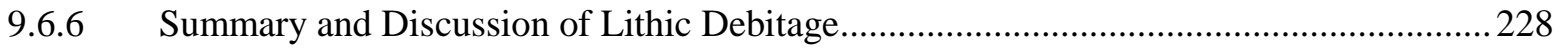

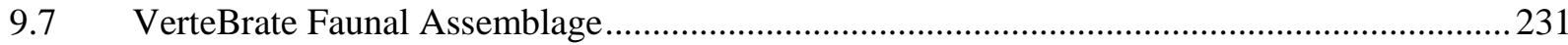

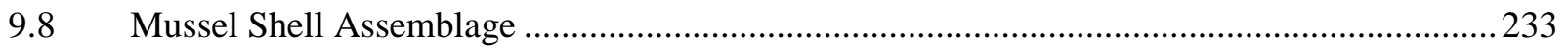

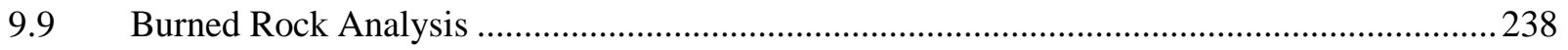

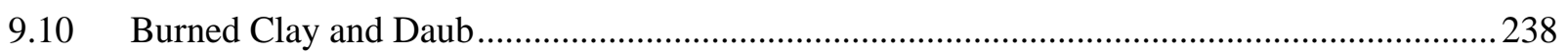

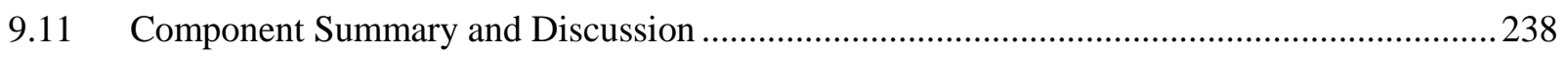

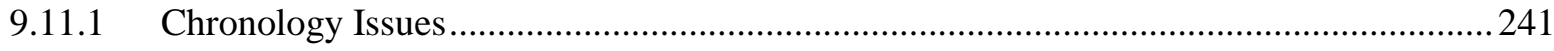

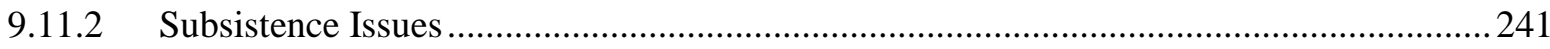

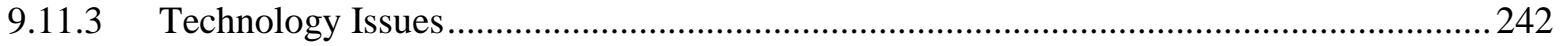

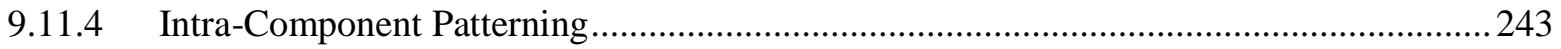

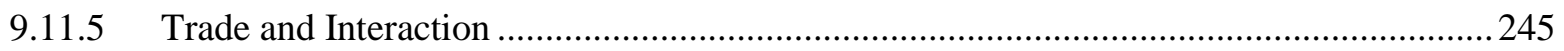

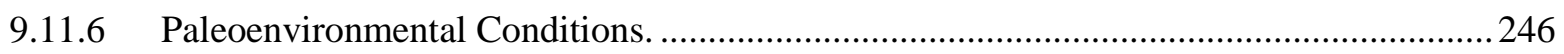

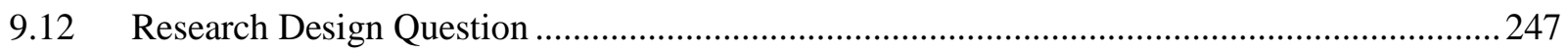

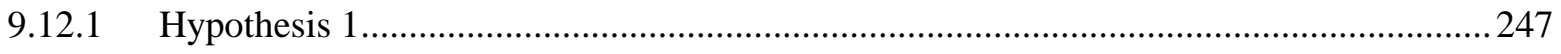

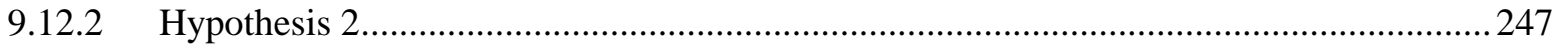

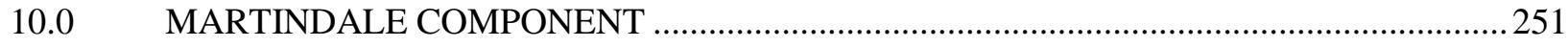

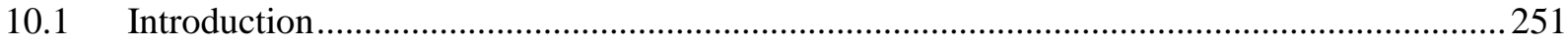

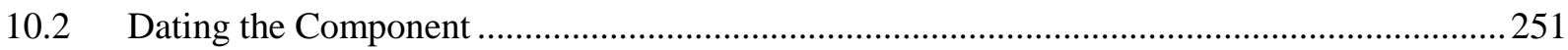

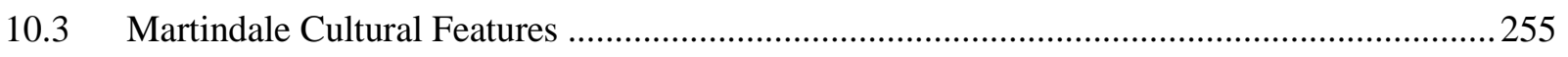

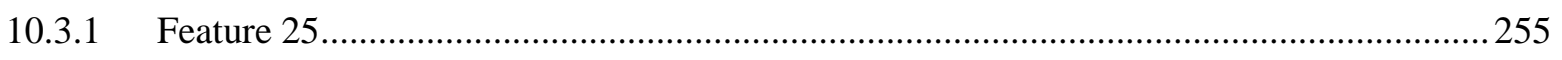

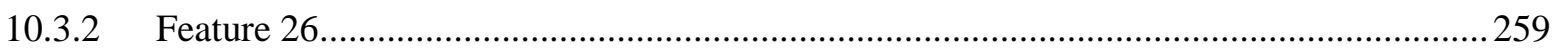

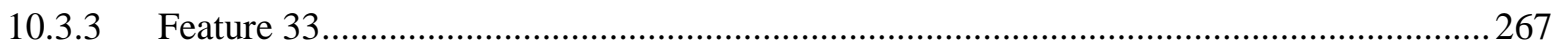

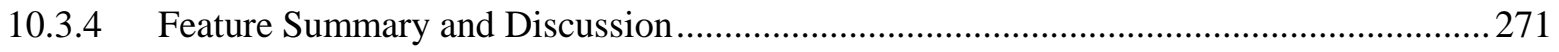




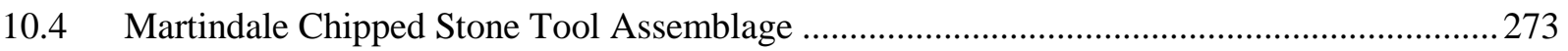

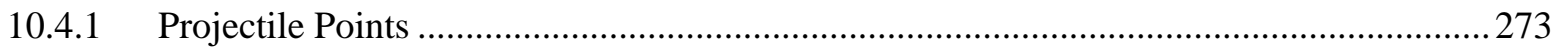

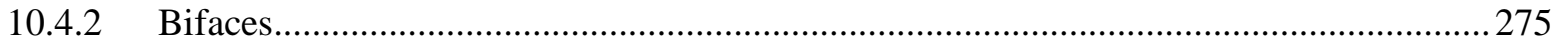

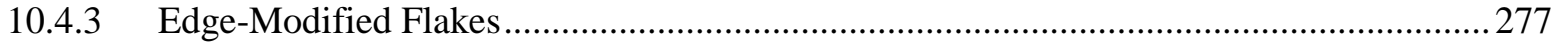

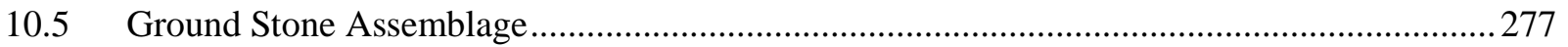

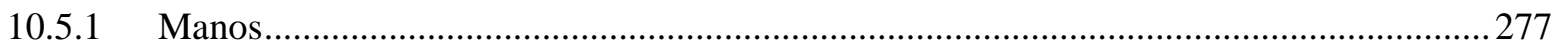

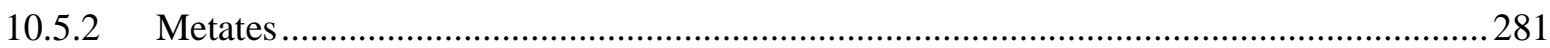

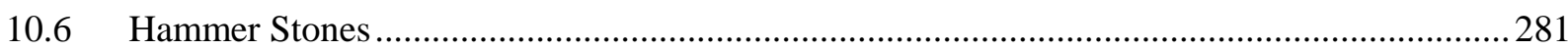

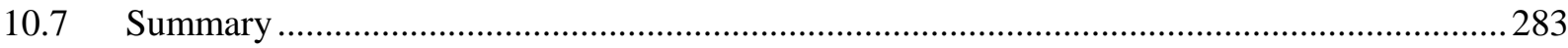

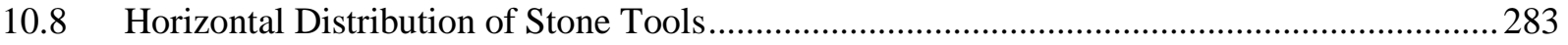

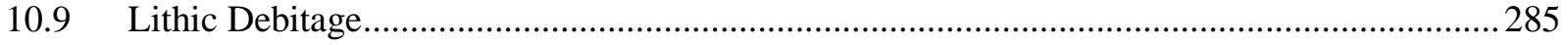

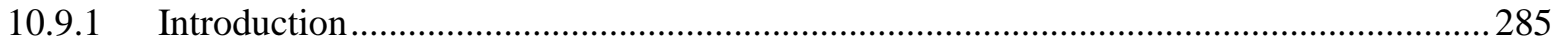

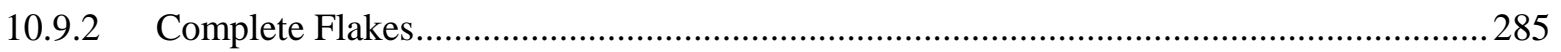

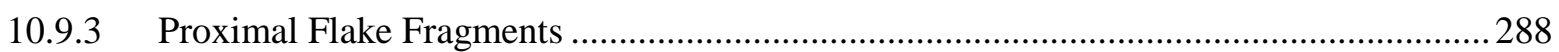

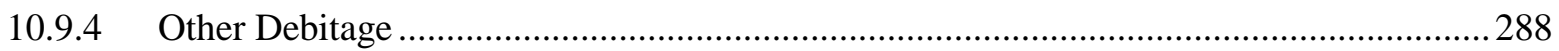

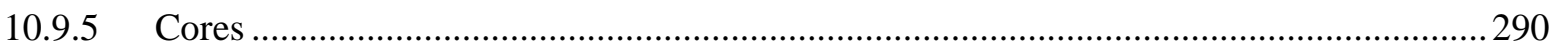

10.9.6 Summary of Martindale Lithic Debitage …...................................................................290

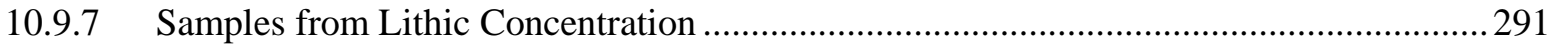

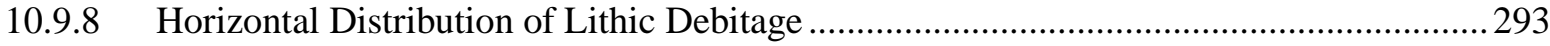

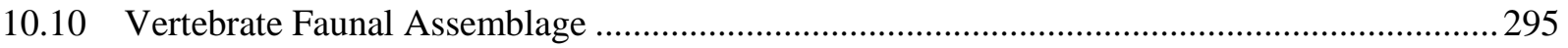

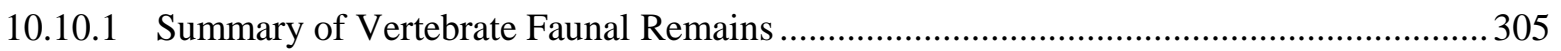

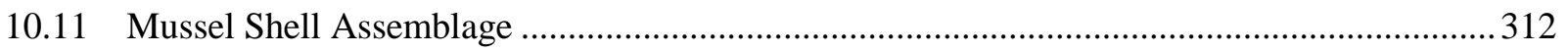

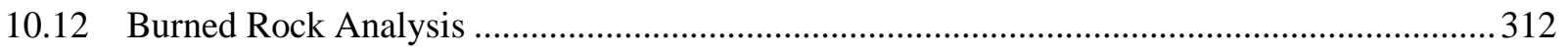

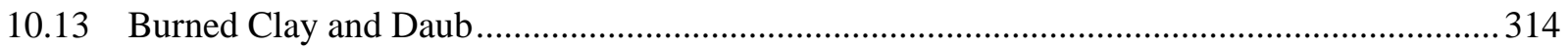

10.14 Martindale Component Summary and Discussion.................................................................. 314

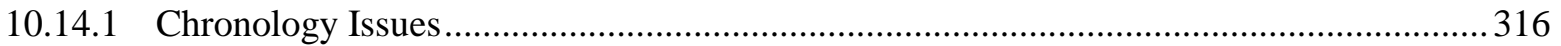

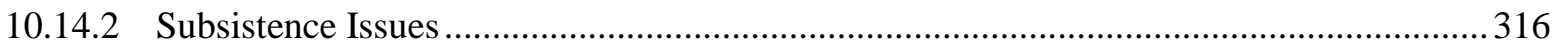

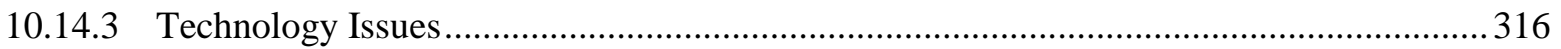

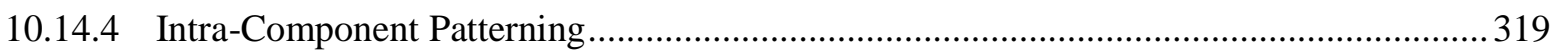

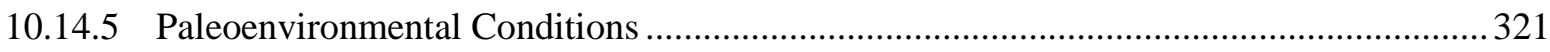

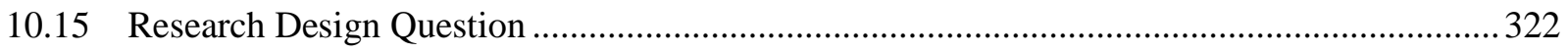

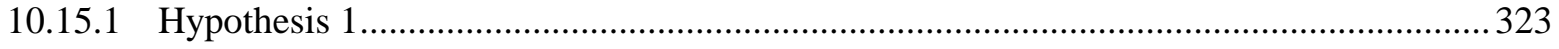

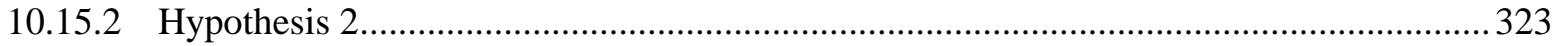




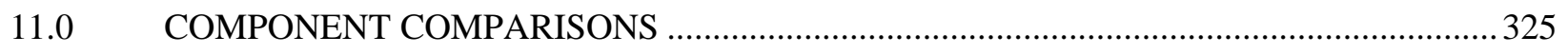

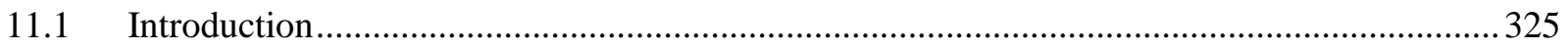

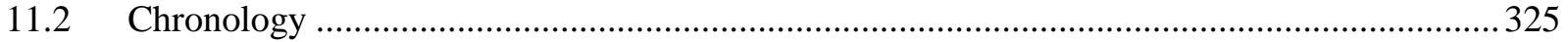

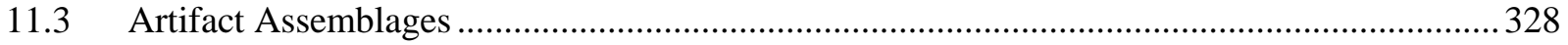

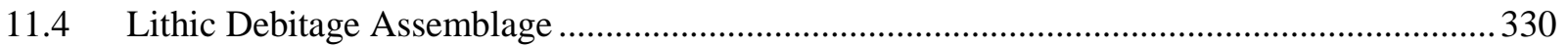

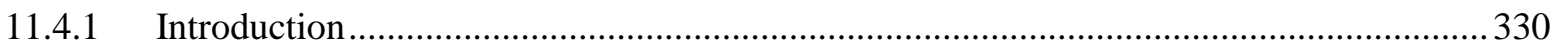

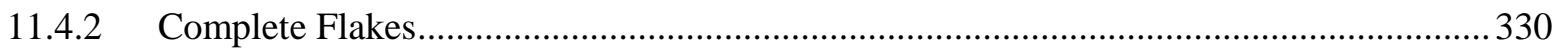

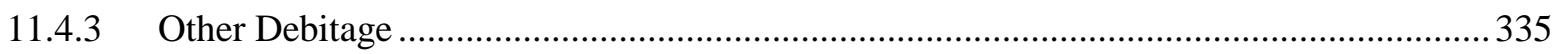

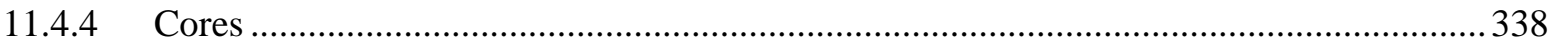

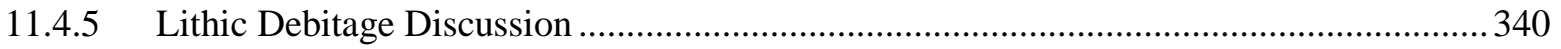

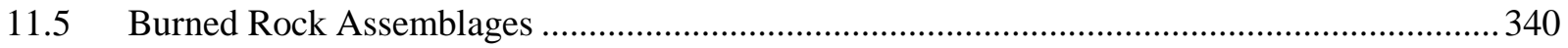

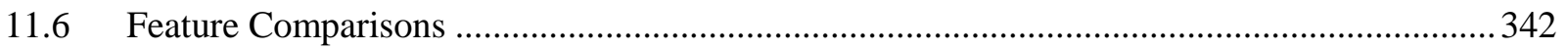

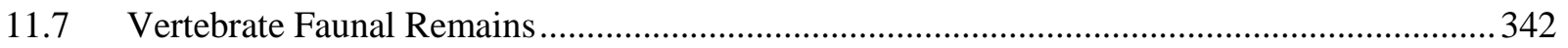

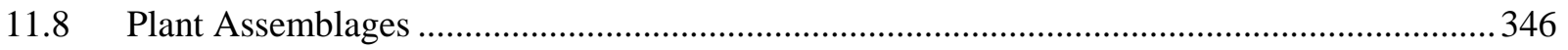

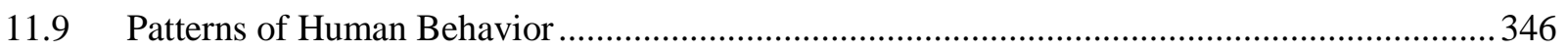

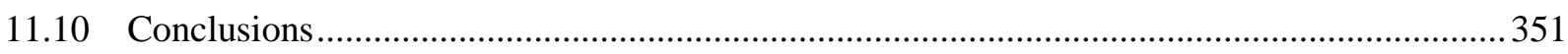

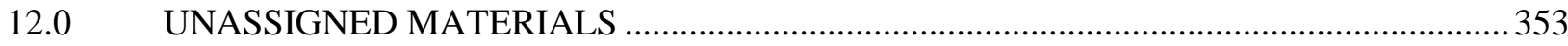

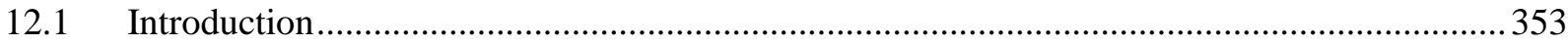

12.2 Dates Derived from Unassigned Features and Materials ....................................................... 353

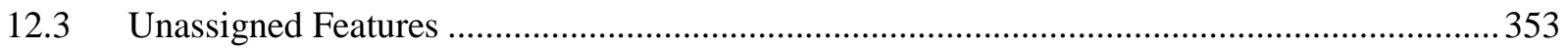

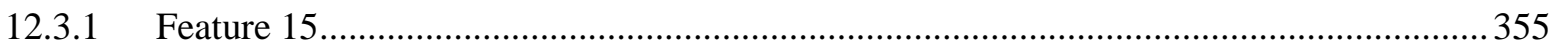

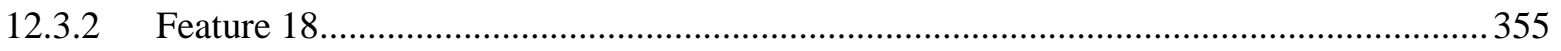

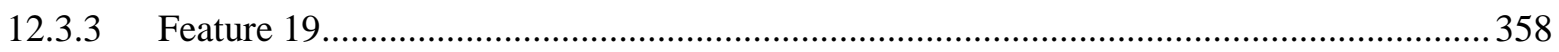

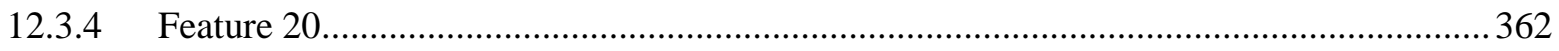

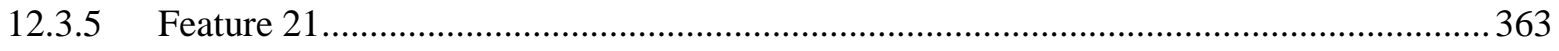

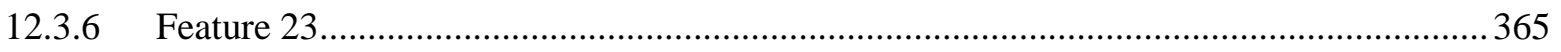

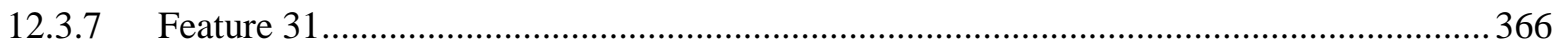

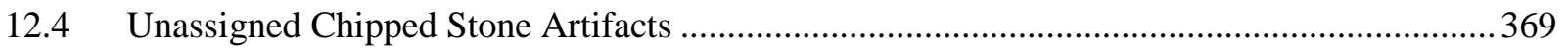

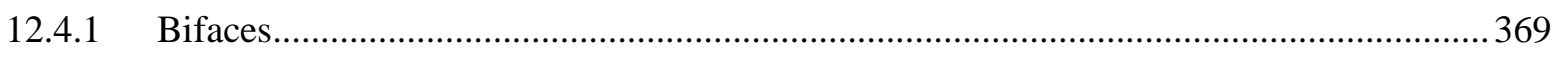

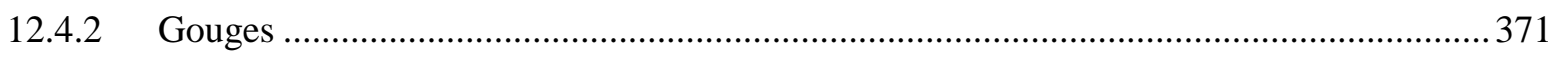

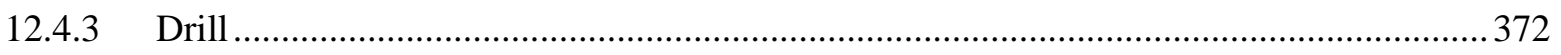

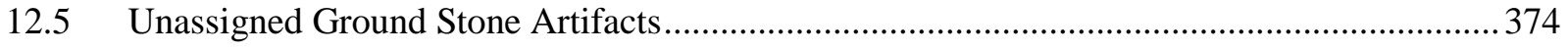

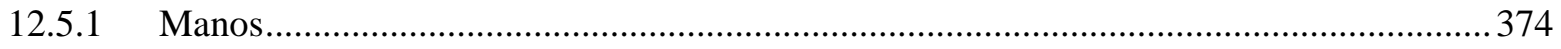




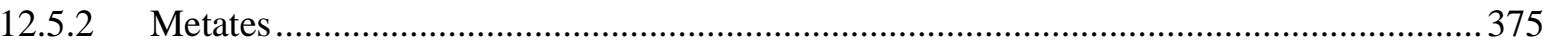

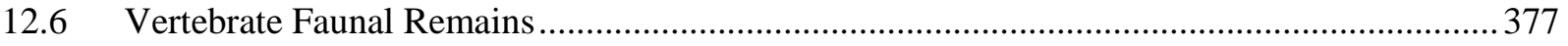

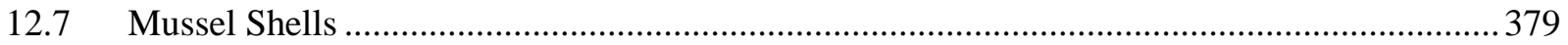

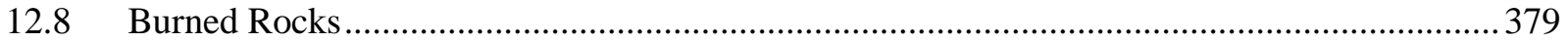

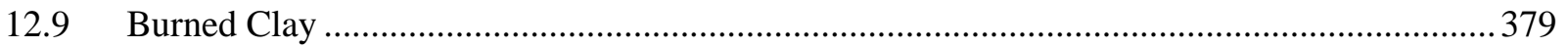

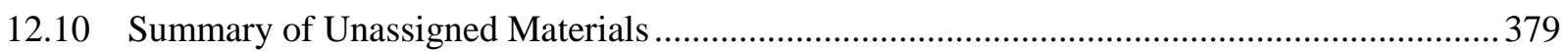

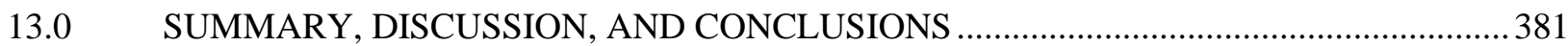

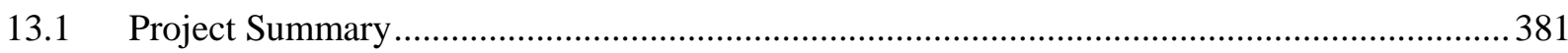

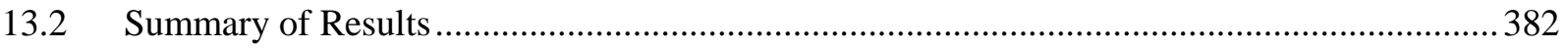

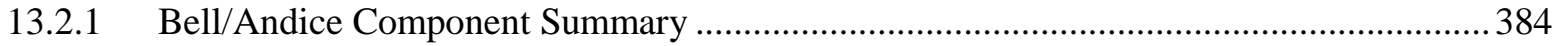

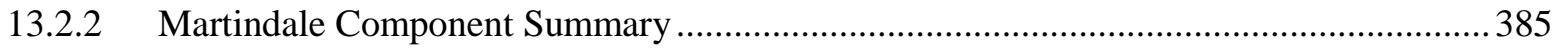

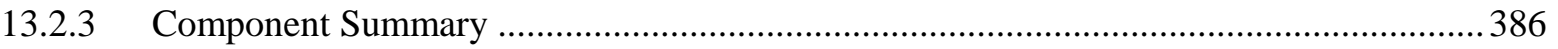

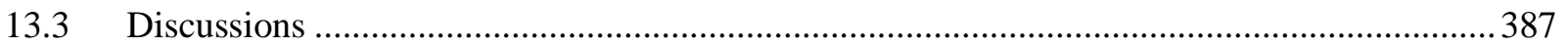

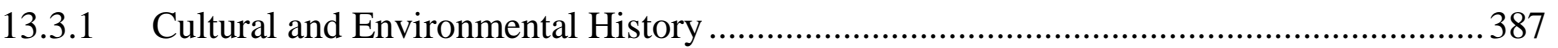

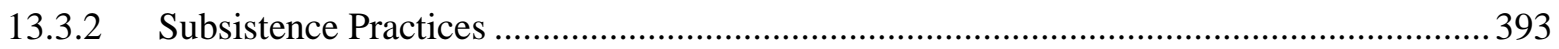

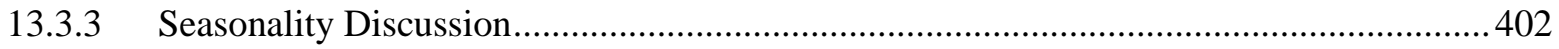

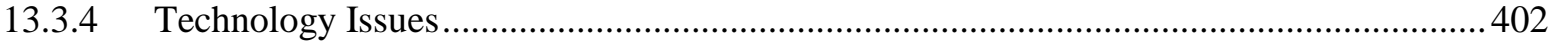

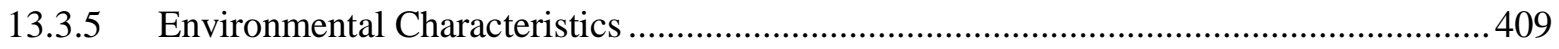

13.3.6 Settlement Patterns and Interaction ............................................................................. 412

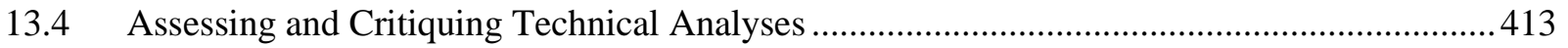

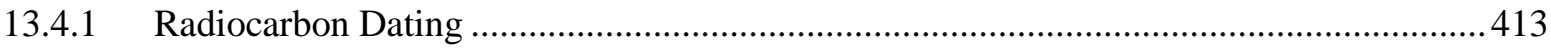

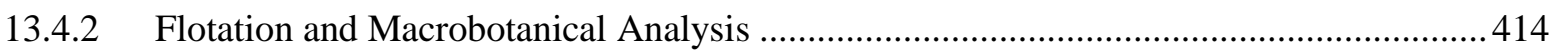

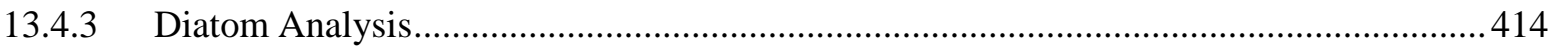

13.4.4 High-Powered Use-Wear Analysis ........................................................................... 415

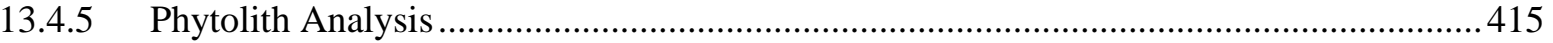

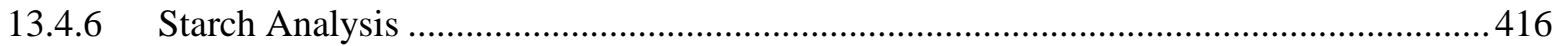

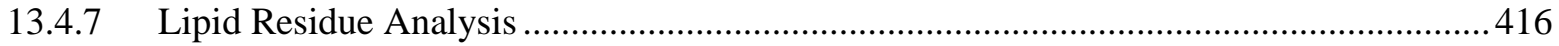

13.4.8 Instrumental Neutron Activation Analysis..................................................................... 417

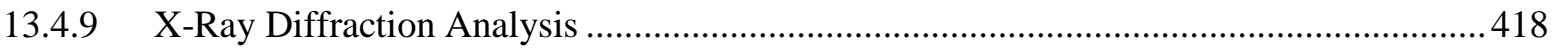

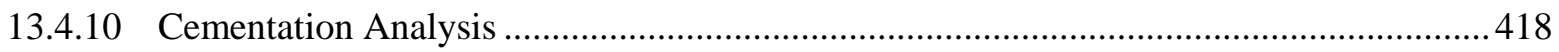

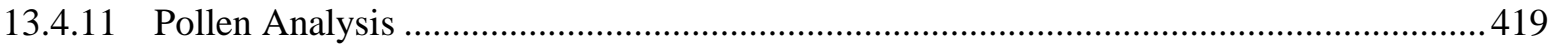

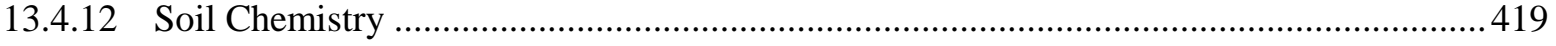

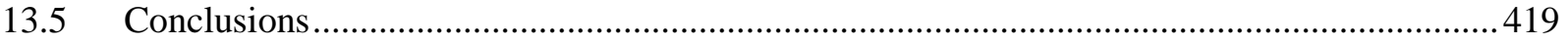


14.0 MANAGEMENT CONSIDERATIONS AND RECOMMENDATIONS ........................... 423

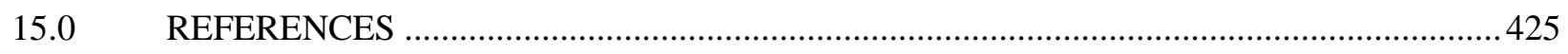

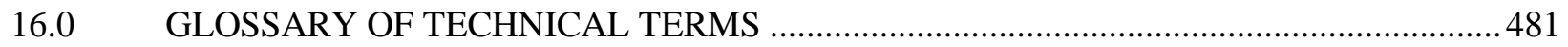

\section{LIST OF APPENDICIES}

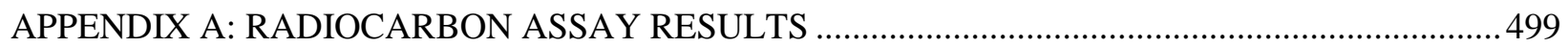

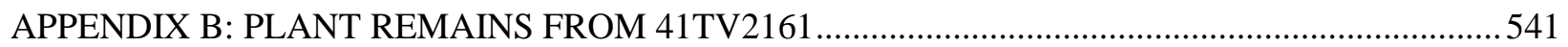

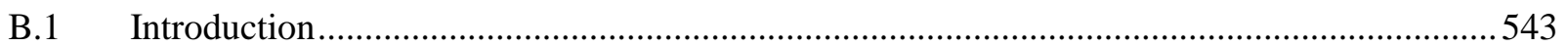

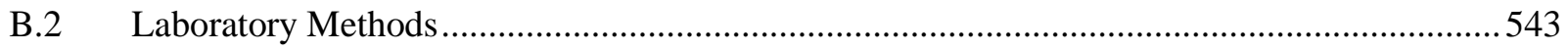

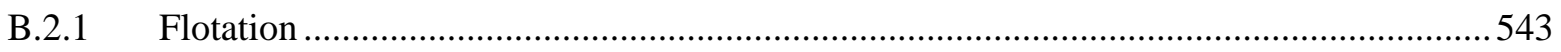

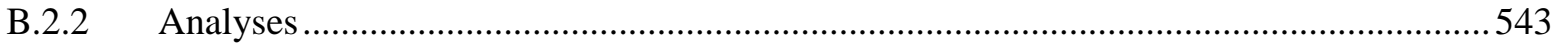

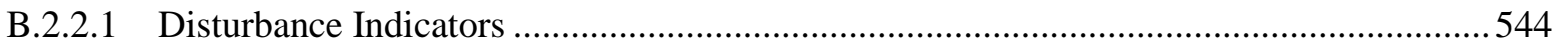

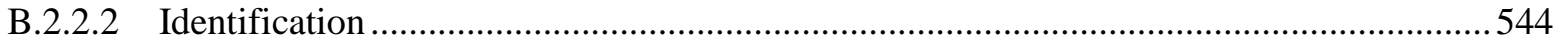

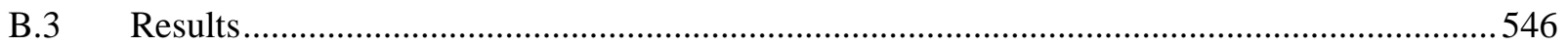

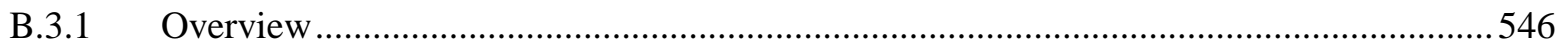

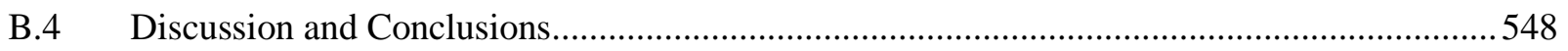

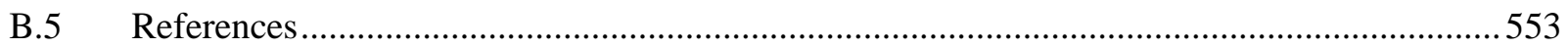

APPENDIX C: DIATOM PALEOENVIRONMENTAL ANALYSIS OF SEDIMENTS FROM ARCHEOLOGICAL SITE 41TV2161, TRAVIS COUNTY, TEXAS …............................................555

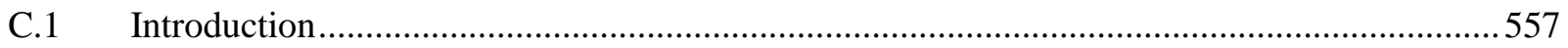

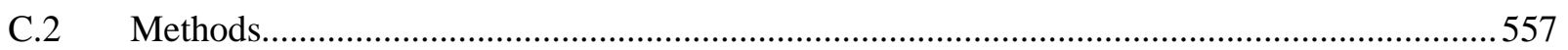

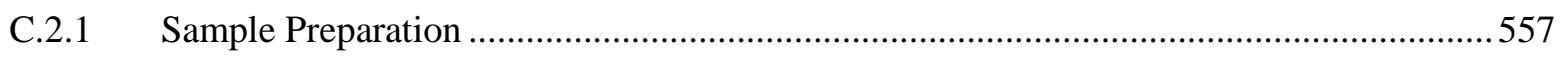

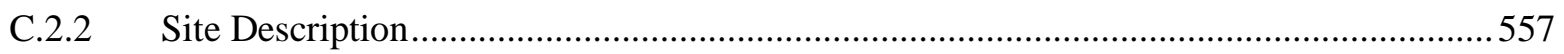

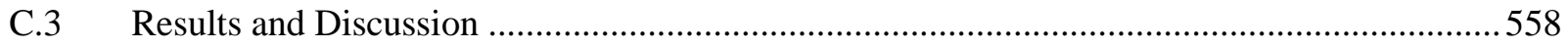

C.3.1 General Paleoenvironmental Description ................................................................558

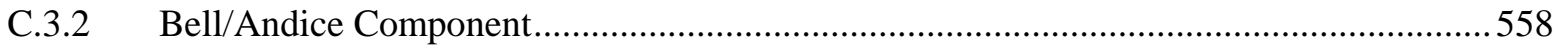

C.3.3 Corner-Notched or Martindale Component ....................................................................559

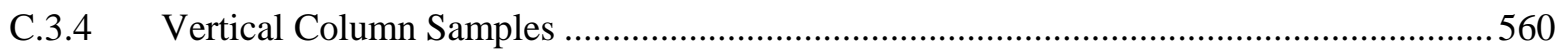

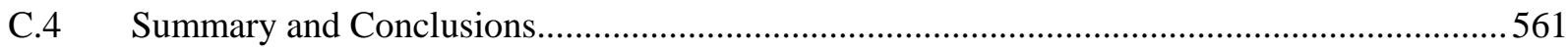

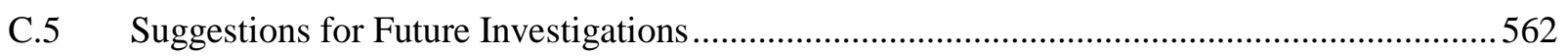

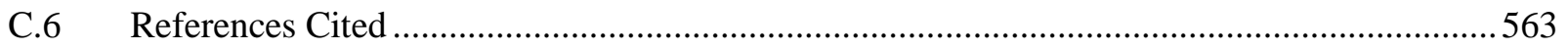

APPENDIX D: RESIDUE AND USE-WEAR ANALYSIS OF STONE ARTIFACTS FROM TRAVIS COUNTY, TEXAS, SITE 41TV2161 ….......................................................................... 581 


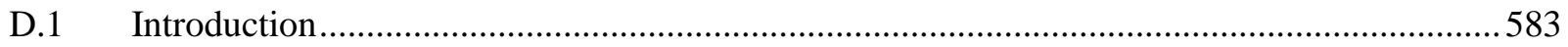

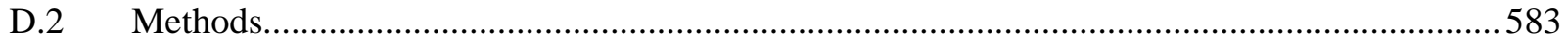

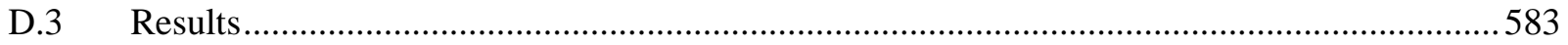

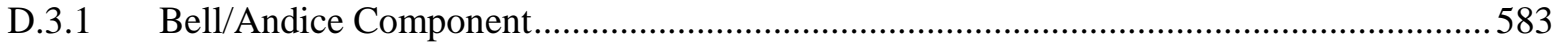

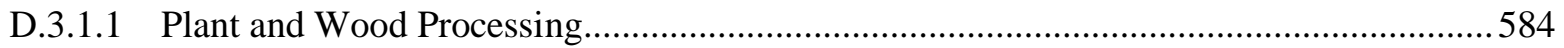

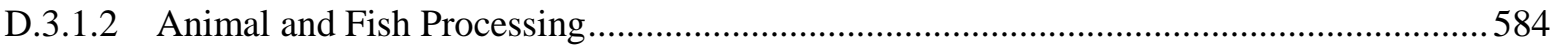

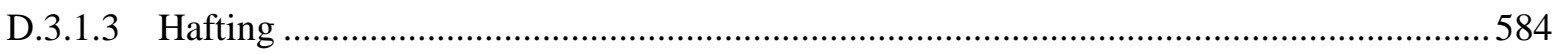

D.3.2 Martindale/Corner-Notched Component …...............................................................584

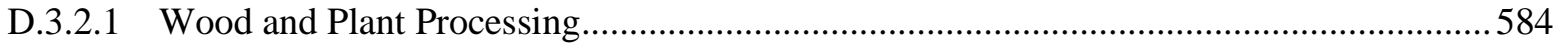

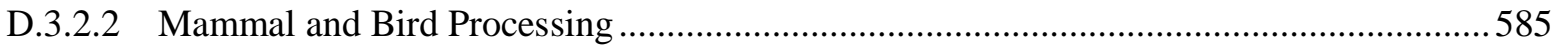

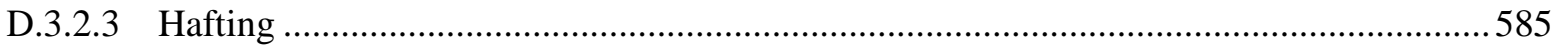

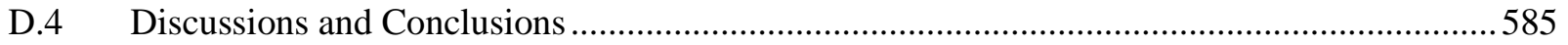

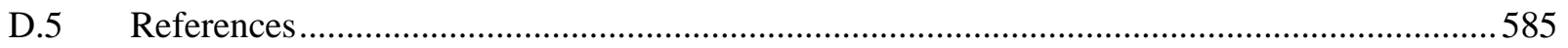

APPENDIX E: BIOGENIC SILICA ASSESSMENT OF SEDIMENT SAMPLES FROM THE SOIL PROFILE AND SELECT CULTURAL FEATURES AT 41TV2161 _......................................599

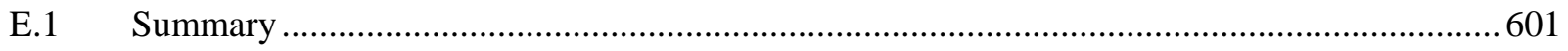

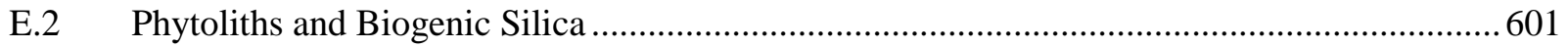

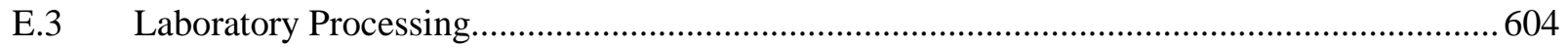

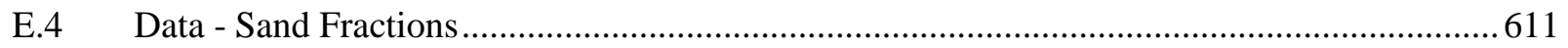

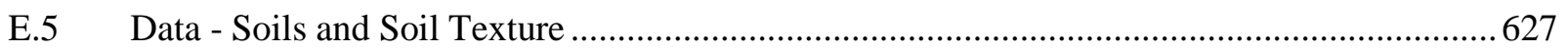

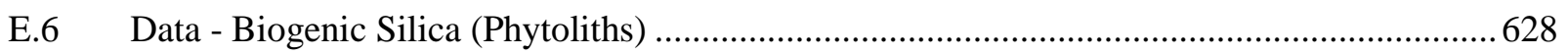

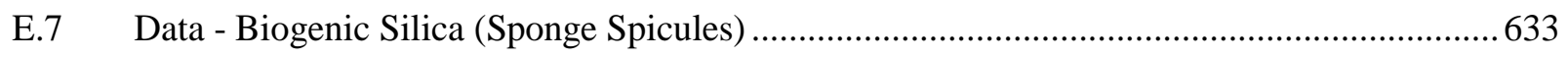

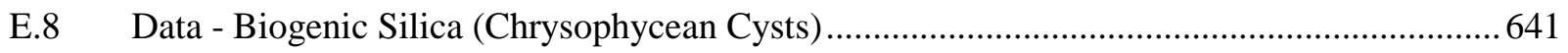

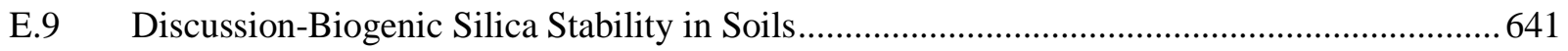

E.10 Data-Discussion-Surface Control Samples .........................................................................646

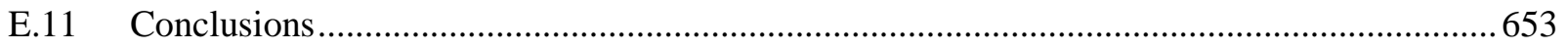

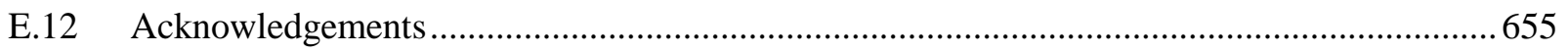

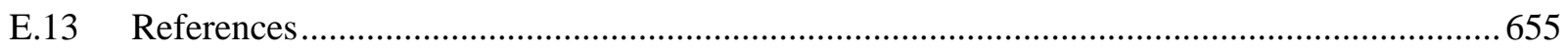

APPENDIX F: STARCH ANALYSIS OF 74 SAMPLES FROM SITE 41TV2161,TRAVIS

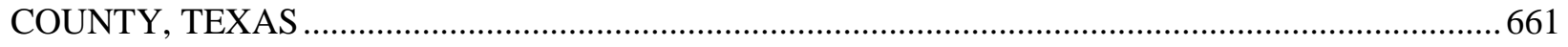

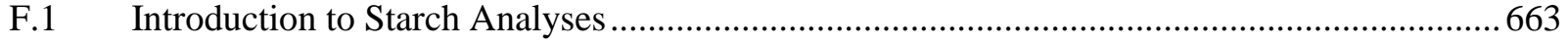

F.1.1 Understanding the Relationship between Residues and Artifacts..................................665

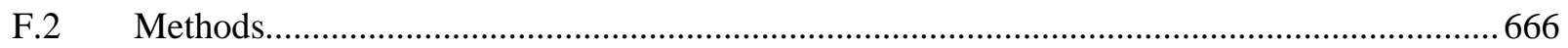

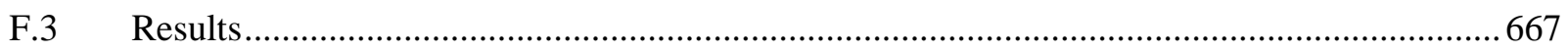




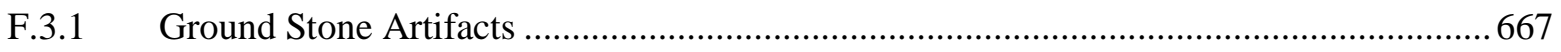

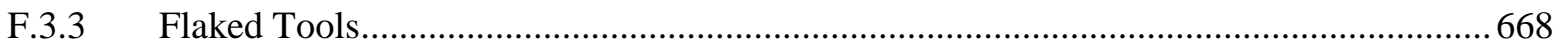

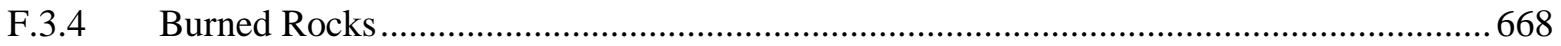

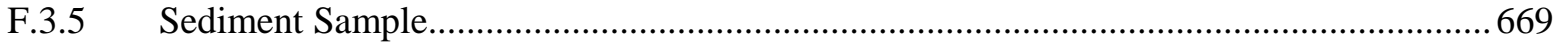

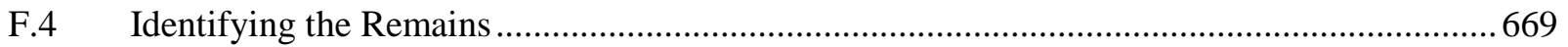

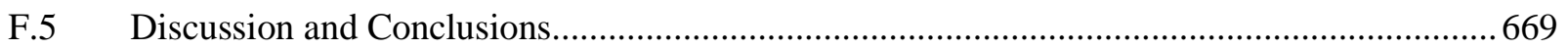

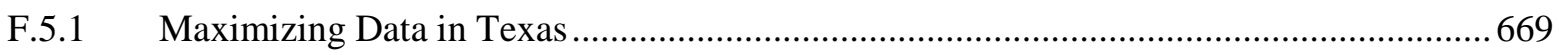

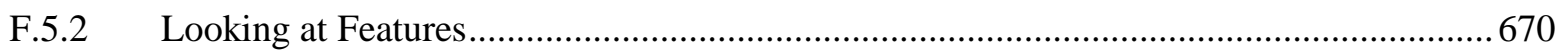

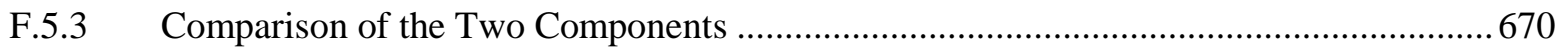

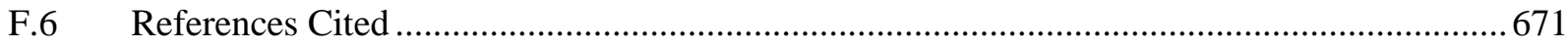

APPENDIX G: ANALYSIS OF THE LIPID COMPOSITIONS OF ARCHAEOLOGICAL BURNED ROCK AND TOOL RESIDUES FROM SITE 41TV2161 IN TRAVIS COUNTY, TEXAS

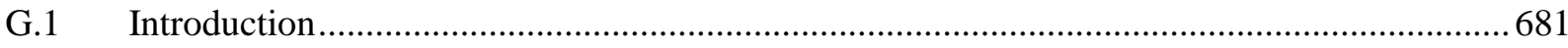

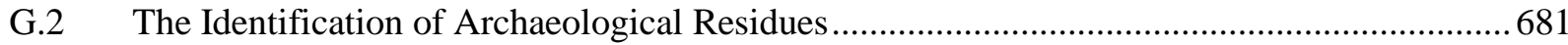

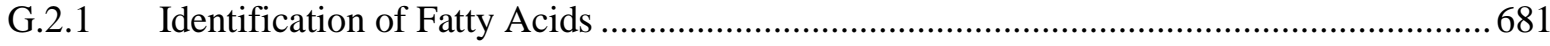

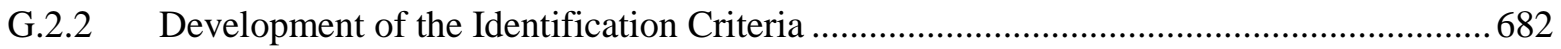

G.3 Using Lipid Distribution and Biomarkers to Indentify Archaeological Residues ..................683

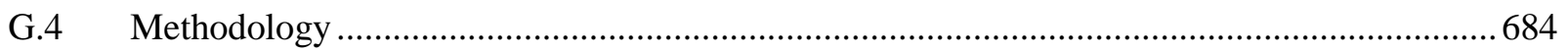

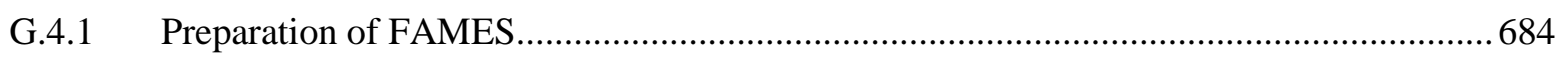

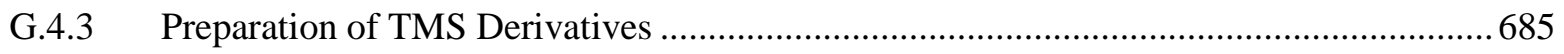

G.4.4 Gas Chromatography Analysis Parameters.................................................................685

G.4.5 High Temperature Gas Chromatography and Gas Chromatography with Mass

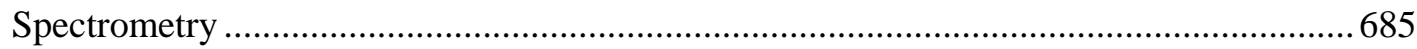

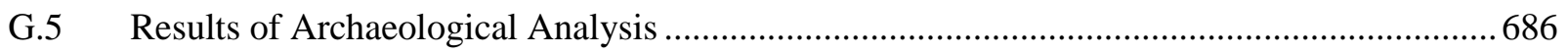

G.5.1 Very High Fat Content - Residues with Very High Levels of C18:1 Isomers .................686

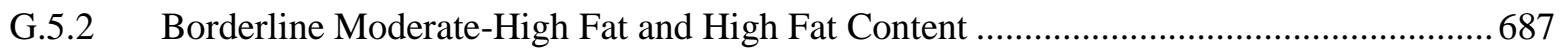

G.5.3 Low Fat Content Plant and Moderate-High Fat Content …...........................................687

G.5.4 Low Fat Content Plant and High Fat Content ................................................................. 688

G.5.5 Low Fat Content and High Fat Content ......................................................................68

G.5.6 Low Fat Plant and Borderline Medium and Moderate-High Fat Content.........................688

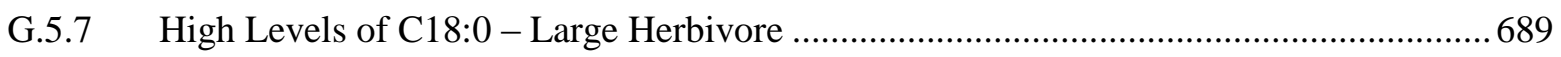

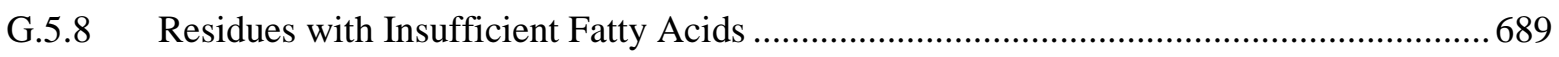

G.6 Comparison of the Bell/Andice and Martindale Components ............................................690 
G.7 References Cited . .690

APPENDIX H: CHERT SOURCING FOR THE BIG HOLE (41TV2161) PROJECT: COMPOSITIONAL ANALYSES OF CHERT GRAVELS AND ARTIFACTS CONDUCTED AS PART OF TRC PROJECT \#208177/WA0004/00002 ….......................................................... 708

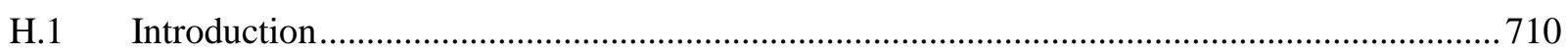

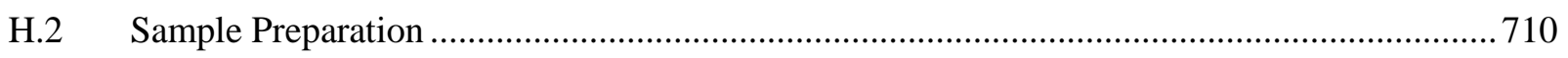

H.3 Irradiation and Gamma-Ray Spectroscopy …............................................................... 711

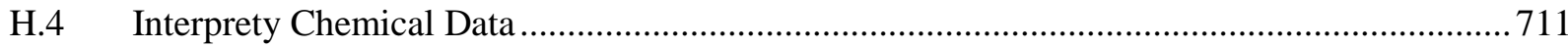

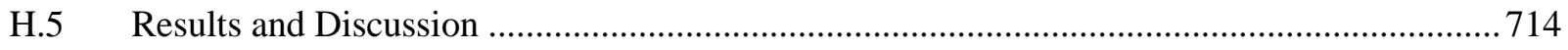

H.5.1 Comparison of Artifacts with Local Gravel Source...................................................... 714

H.5.2 Comparison with Extant Edwards Formation Database ............................................... 715

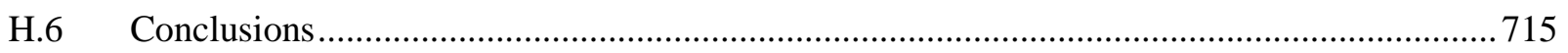

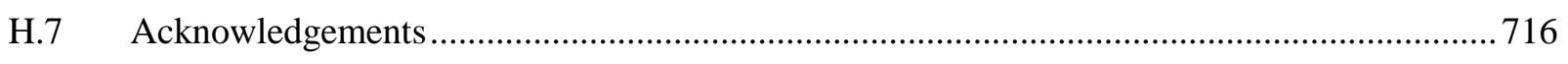

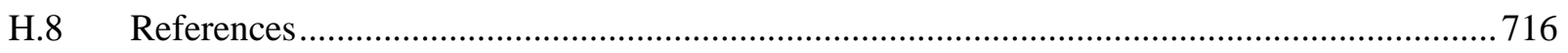

APPENDIX I: X-RAY FLUORESCENCE (XRF) ANALYSIS MAJOR AND MINOR OXIDE CONCENTRATIONS OF TWO ROCK SAMPLES FROM THE BIG HOLE SITE (41TV2161),

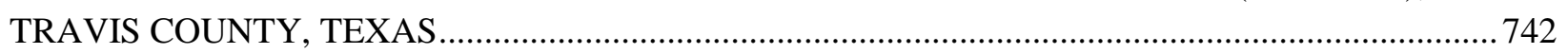

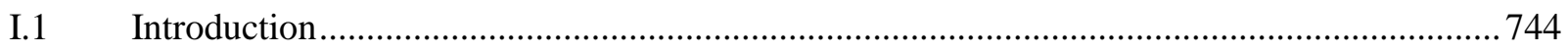

I.2 Laboratory Sampling, Analysis and Instrumentation..................................................... 744

I.2.1 Major and Minor Oxide Analysis ……...................................................................... 744

I.2.2 Conditions of Fundamental Parameter Analysis ......................................................... 744

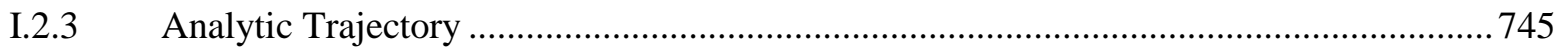

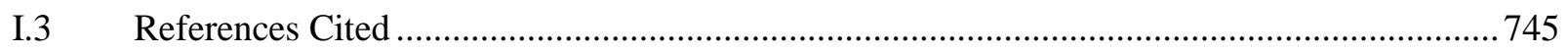

APPENDIX J: FORENSIC AGING OF TWO DEER INCISORS FROM 41TV2161 .........................752

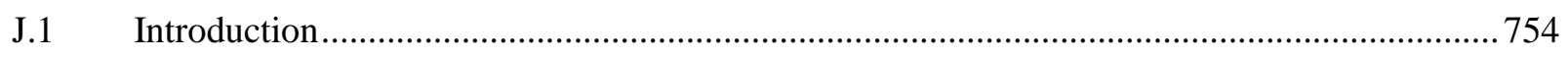

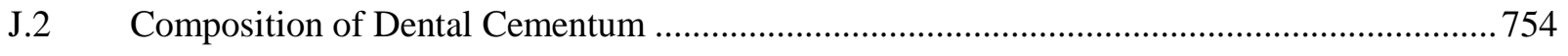

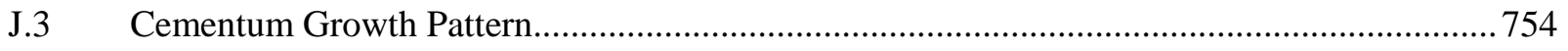

J.4 Methods - Stained, Histological Thin Section................................................................. 755

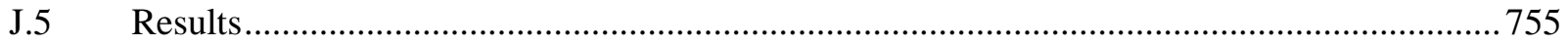

APPENDIX K: PRESENCE/ABSENCE ANALYSIS OF PHYTOLITHS AT 41TV2161_...................755

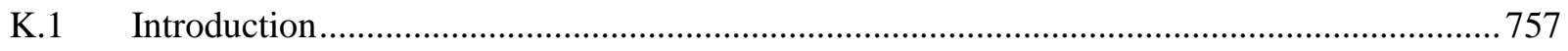

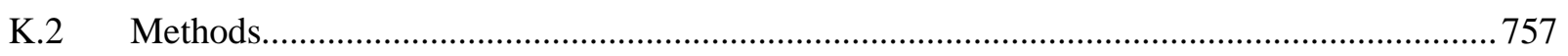

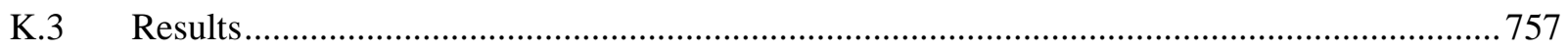

APPENDIX L: PRESENCE/ABSENCE ANALYSIS OF Pollen AT 41TV2161 ...............................759 


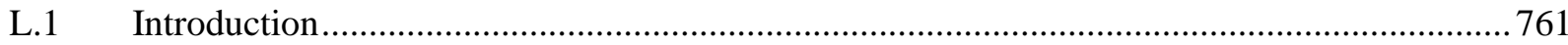

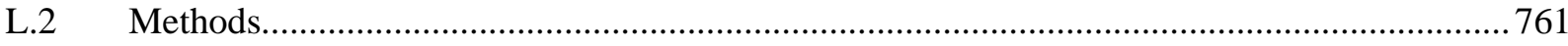

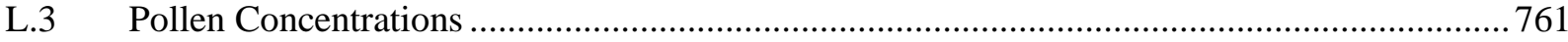

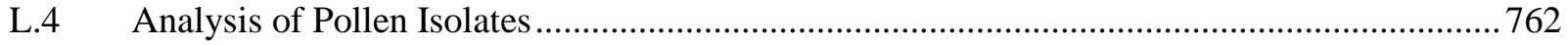

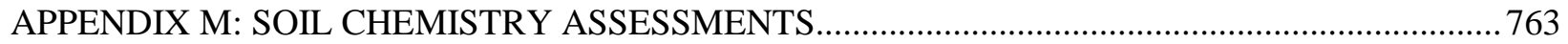

APPENDIX N: TXDOT CHIPPED STONE ANALYTICAL PROTOCOL, VERSION 2.1 ................ 779

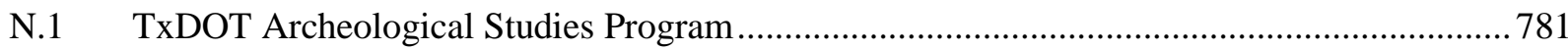

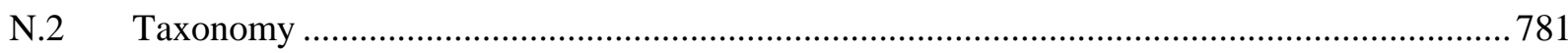

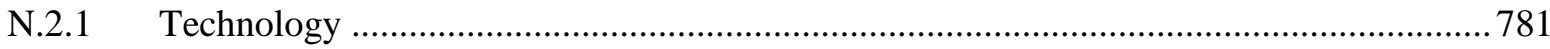

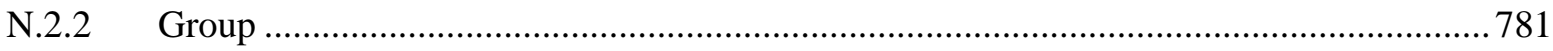

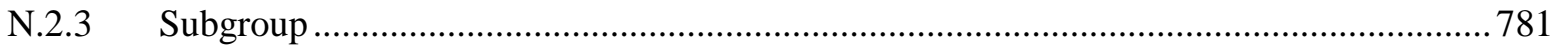

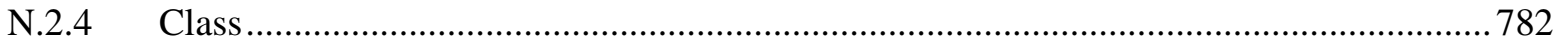

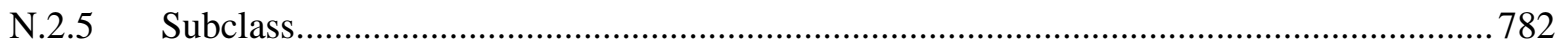

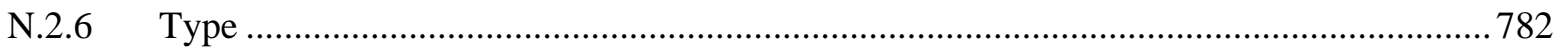

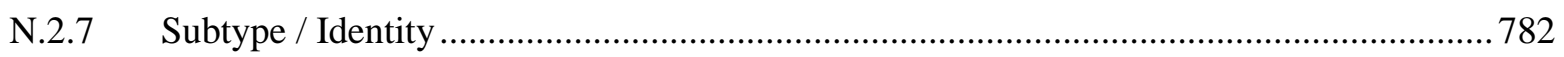

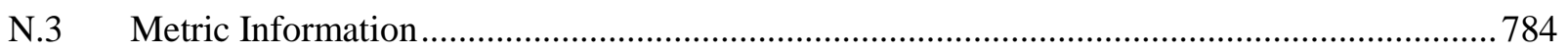

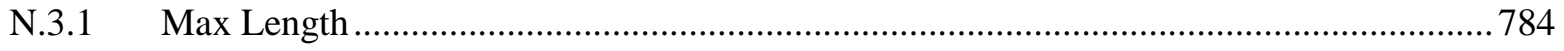

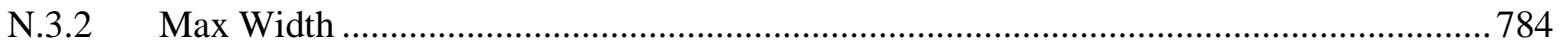

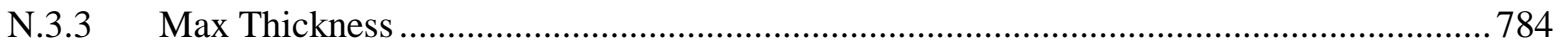

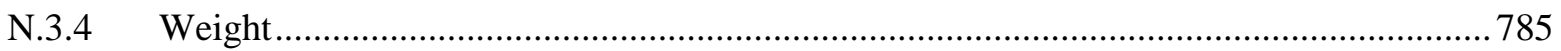

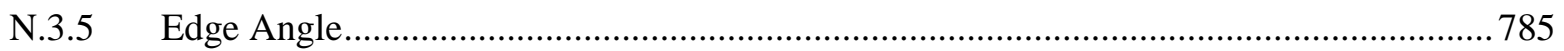

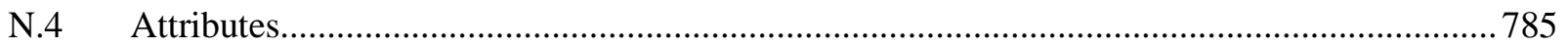

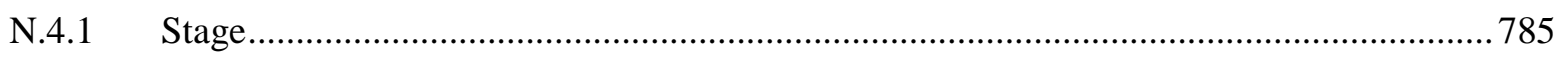

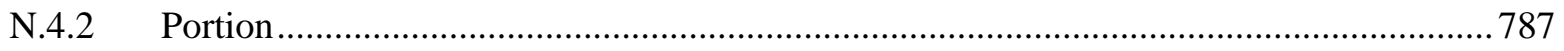

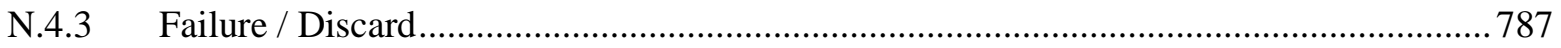

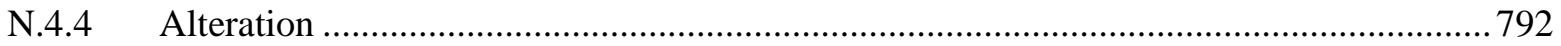

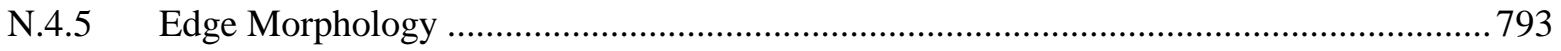

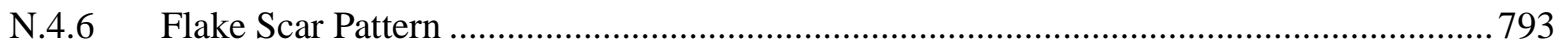

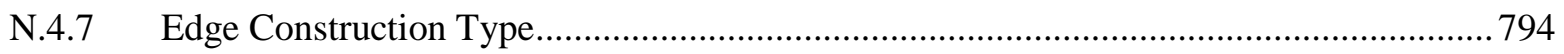

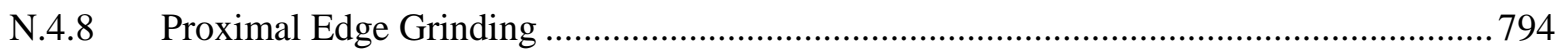

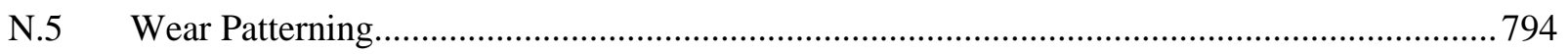

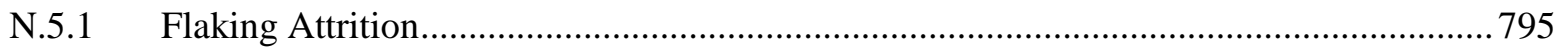

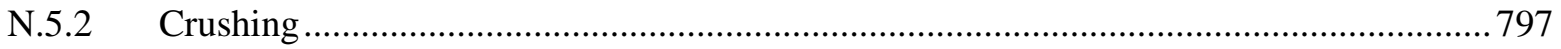




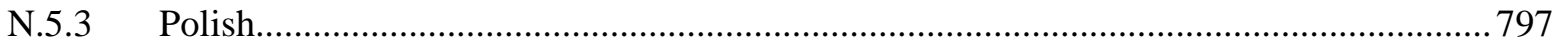

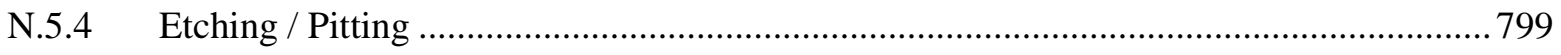

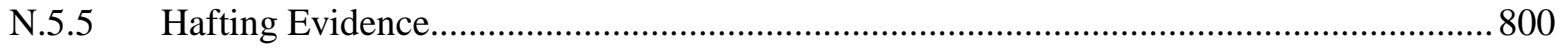

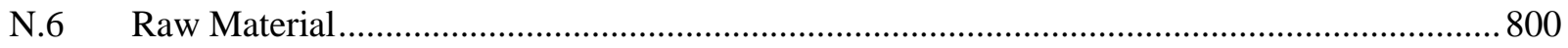

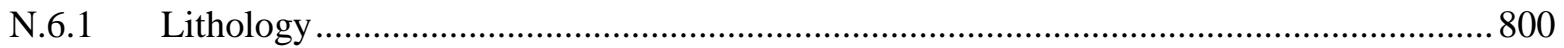

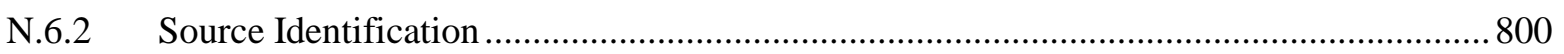

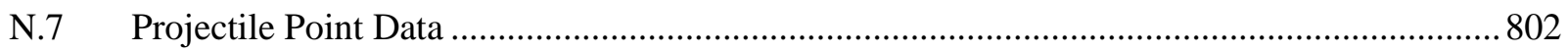

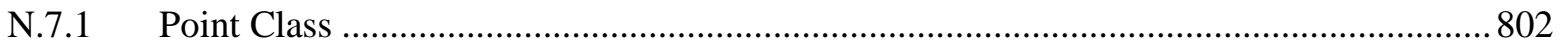

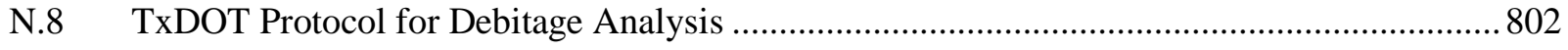

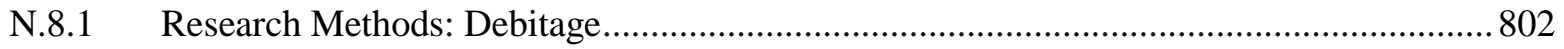

N.8.2 Metric Attributes (Number and Weight) ...................................................................... 802

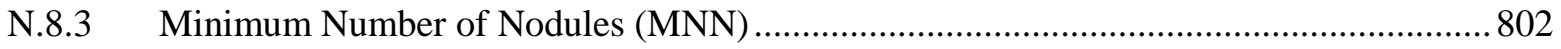

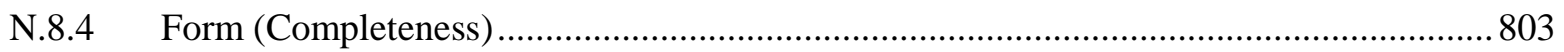

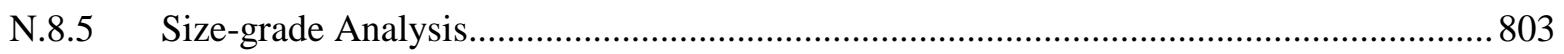

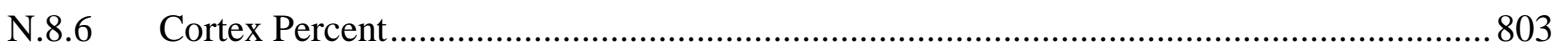

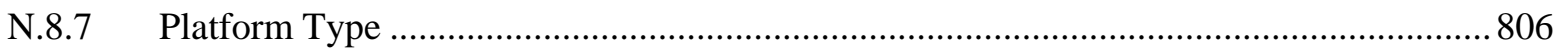

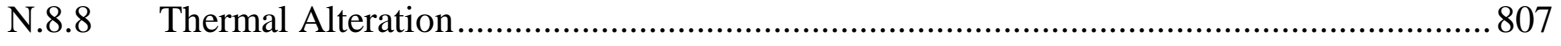

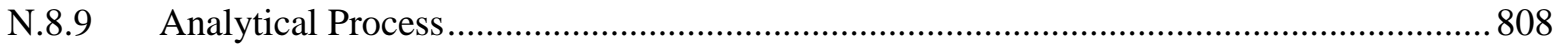

N.9 Questions for Middle-Level and High-Level Theory Using Debitage Data ..........................813

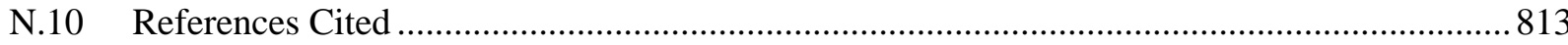

APPENDIX O: MARTINDALE EDGE-MODIFIED FLAKE DATA TABLES, TRAVIS

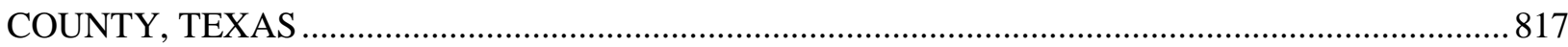




\section{LIST OF FIGURES}

Figure 1-1. General site location in Travis County, Texas......

Figure 1-2. General location of hand-excavations within the mechanically stripped block in relations to Hicks \& Company previous investigations. .5

Figure 2-1. General site environment across expansive, flat alluvial terrace of lower Onion Creek valley. Landscape has been altered in historic times. .8

Figure 2-2. Geology of the area surrounding the Big Hole site east of the Austin Bergstrom International Airport (ABIA) in eastern Travis County (after Barnes 1974, 1981).......................................8

Figure 2-3. Soils map (after Werchan et al. 1974) . .........................................................................

Figure 2-4. Area average temperature in degrees Fahrenheit (usclimatedata.com, after NOAA data)..............10

Figure 2-5. Average area precipitation in inches (usclimatedata.com, after NOAA data) ...............................10

Figure 2-6. Biotic regions across Texas with site location (modified from Blair 1950)................................11

Figure 2-7. Geophytes: wild onion (left) and winecup (right). Scale in centimeters, photographs by M. Quigg.

Figure 2-8. Geophytes: prairie pleatleaf (left photo, left side) and false onion (left photo, right side) and eastern camas (right photo). Scale in centimeters, photographs by M. Quigg (left), and A. Thoms (right)

Figure 2-9. Map depicting generalized interpretations of middle Holocene paleoenvironments and data collection localities.

Figure 2-10. Comparison of multiple interpretations of environmental variation in central Texas based on different kinds of proxy data

Figure 2-11. Carbon isotope values of soil organic carbon (SOC) in the sequence of buried soils at the Richard Beene site in Southern Texas (from Mandel et al. 2007, Figure 3.20).

Figure 2-12. Summary model of climatic and environmental changes projected from the Hall's Cave data in the Edwards Plateau (from Toomey et al. 1993:315, Fig.9).

Figure 2-13. Changes in the ratio of Notiosorex crawfordii to Notiosorex crawfordii + Cryptotis parva at the Hall Cave site and available radiocarbon dates (from Toomey et al. 1993:308, Fig.6).

Figure 2-14. Phytolith changes over time that demonstrate woodland to grassland ratio by depth and time (from Fredlund 1998:1643, Figure 41-2). Note log scale used for ratio.

Figure 2-15. Pedogenic carbon isotope data and inferred climate history (from Humphrey and Ferring 2001:67, Figure 4-6).

Figure 2-16. Short cell grass phytolith assemblages from Morgan Playa (from Fredlund et al. 1998:195, Figure 3).

Figure 3-1. Diagnostic Andice and Bell point examples from the Edwards Plateau in central Texas (from Quigg and Lintz et al. 2011b).

Figure 3-2. $\quad$ Projectile points from the Coffey site in Kansas (from Schmits 1976, 1978). Top two rows (a-h) from Horizon III-5, next row (i-k) from Horizon III-7, and bottom row (1-n) from Horizon III-8. 36

Figure 3-3. $\quad$ Munkers Creek points from Witty $(1982: 171)$...................................................................

Figure 3-4. Bell points from the Landslide site (from Sorrow et al. 1967: Fig.10).........................................38

Figure 3-5. Johnson's “Early Barbed” points from the Devil’s Mouth site (Johnson 1964, Figure 11;

E. Prewitt 1981a considers Bell/Andice varieties). 38 
Figure 3-6. Middle Holocene cultural constructs for the Southern Plains region. ...............................................40

Figure 3-7. The 1995 distribution of Andice (left) and Bell (right) points across Texas (after Prewitt 1995),....49

Figure 3-8. Location of key Bell/Andice/Calf Creek sites in the Southern Plains region...................................50

Figure 3-9. Martindale point forms from Suhm and Jelks 1962, Plate 107 .........................................................68

Figure 3-10. Martindale points forms from Weir (1976a, Figure 9), ...............................................................69

Figure 3-11. Group A Martindale points from the Wilson-Leonard site (from Dial et al. 1998:Figure 13-46).....70

Figure 3-12. Selected Early Archaic sites in and near the Edwards Plateau and central Texas. ............................71

Figure 3-13. Selected Martindale points from the Gatlin site (from Oksanen et al. 2008, Figure 3) ....................75

Figure 3-14. Stratified cultural components at the Cibolo Crossing site (from Kibler and Scott 2000,

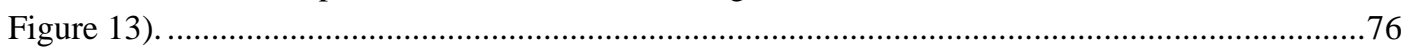

Figure 3-15. Martindale points from the Martindale component at the Cibolo Crossing site (Kibler and

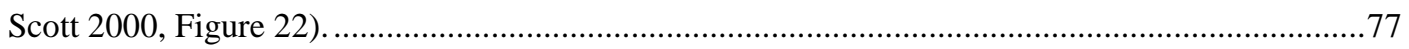

Figure 3-16. East-west profile along N190 at the Landslide site (from Sorrow et al. 1967: Figure 4). ................78

Figure 3-17. Martindale points from Stratum V at the Landslide site (41BL85) (from Sorrow et al.

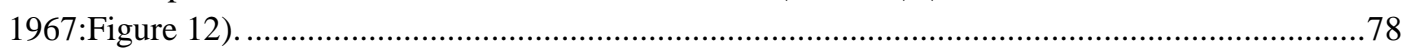

Figure 3-18. Martindale points from the Camp Pearl Wheat site (from Collins et al. 1990: Figure 19)................78

Figure 3-19. Plan map of south block depicting horizontal distribution of cultural features at the Camp

Pearl Wheat site (from Collins et al. 1990:Figure 19)....................................................................79

Figure 3-20. 'Martindale' point on bedrock next to fiber Feature 21 at Wroe Ranch shelter (from Turpin

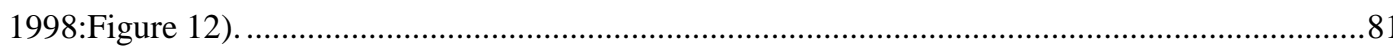

Figure 3-21. Photograph of earth oven profile of Feature 181 at the Wilson-Leonard site (from Collins et al. 1998b:237).

Figure 3-22. Photograph of Feature 22 from Martindale component at the Cibolo Crossing site (from Kibler and Scott 2000:Figure 24).

Figure 3-23. Profile photograph of Feature 26 from Martindale component at the Cibolo Crossing site (from Kibler and Scott 2000:Figure 26).

Figure 3-24. Martindale points from the Woodrow Heard site depicting impact fractures and beveled bases (Decker et al. 2000:Figure 184).

Figure 3-25. Martindale point distribution by county to 1995 (from Prewitt 1995:117, Black represents over 50 points, gray reveals 11 to 51 , hatched is 1 to 11 and white is no data). . .93

Figure 3-26. Early Corner-notched point from Seminole Sink (from Turpin 1988: Fig.12)...............................94

Figure 4-1. Profile of fine-grained alluvial sediments at the Big Hole site, 41TV2161, with the Bell/Andice and Martindale/Early Corner-notched components delineated by dashed lines (from Quigg et al. 2007).

Figure 4-2. Dart points from 41TV2161. $\mathrm{a}=$ basal stem fragment of a Bell point; $\mathrm{b}=$ Bell point; $\mathrm{c}=\mathrm{Big}$ Sandy point; $\mathrm{d}=$ Martindale point.

Figure 4-3. Plan (top) and profile (bottom) views of Feature 18, a burned rock cluster, showing the discrete and relatively intact condition typical of features at this site. 102

Figure 4-4. Non-scalar presentations of five basic patterns of Holocene climate fluctuations (relatively dry to relatively moist) based on pollen studies (Albert 2007; Bousman 1998, as summarized in Collins 2004) shifts in microfaunal species in cave deposits (Toomey et al. 1993), and fluctuations in percentages of $\mathrm{C}_{4}$ plants, based on carbon isotope values (Thoms 2007b). The Holocene climate 
history presented by Johnson and Goode (1995) draws upon various datasets, including sediment stratigraphy that reflect shifting hydrological conditions related to climate change.

Figure 5-1. Southern view across mechanically stripped excavation block with track hoe and other earth removal equipment in background. Note safety bench on left side and pedestalled burned rock feature in foreground.

Figure 5-2. Profile of the Big Hole site target block showing depth of mechanically stripped surface to roughly 2.5 meters deep. .108

Figure 5-3. Northern end of excavation block showing safety bench and water collection trench. Note Block $\mathrm{A}$ is on far side and Block D on right.

Figure 5-4. Tiffany Osburn employing the mounted ground penetrating radar.

Figure 5-5. Ground penetrating radar scans from the $900 \mathrm{MHZ}$ antenna showing anomalies from 6.7 to $13.3 \mathrm{~cm}$ and 13.3 to $20.0 \mathrm{~cm}$ below stripped surface of investigation block.

Figure 5-6. Examples of ground penetrating radar scans from the $400 \mathrm{MHZ}$ antenna showing anomalies from 13 to $26 \mathrm{~cm}$ and 26 to $39 \mathrm{~cm}$ below stripped surface of investigation block.

Figure 5-7. Plan map and distribution of completed hand-excavated blocks within mechanically stripped area.

Figure 5-8. Northern view of ongoing hand-excavations at Blocks B (left) and C (right) in the foreground, Block D (in center), and A in far corner.

Figure 5-9. Northern view of flooded excavation area...................................................................... 114

Figure 5-10. Western view of water filled trench along southern margin of excavation block.........................114

Figure 5-11. Example of profiled burned rock feature........................................................................... 116

Figure 5-12. Example of burned rock feature encountered and pedestalled during stripping........................116

Figure 5-13. View south of cleaned and sampled vertical stratigraphic column through the targeted deposits with the top collection hole at $250 \mathrm{cmbs}$. Note the visible safety bench at the boundary of the dark soil (above), whereas the smooth flat area (foreground) is the base of the emergency water collection trench (scale is 1 meter in $10 \mathrm{~cm}$ increments).

Figure 5-14. Examples of starch grains of wildrye grass recovered from burned rocks at an archeological site in Texas (photograph by Linda Perry).

Figure 5-15. Example of tiny charcoal pieces submitted for radiocarbon dating (scale in millimeters).... .123

Figure 5-16. Example of Rabdotus snail shells with apex removed and submitted for radiocarbon dating (scale in centimeters).

Figure 5-17. Example of piece plotted charcoal sent for identification.

Figure 5-18. Missouri University Research Reactor (MURR) laboratory assistant compiling data (photograph furnished by Dr. Glascock). .125

Figure 5-19. Rock with red pigment on surface (\#2396-10) subjected to XRF analysis. ................................125

Figure 5-20. $\quad$ Deer incisors (\#2246-2-10 and \#2246-2-11) subjected to cementation analysis..........................127

Figure 5-21. Chipped stone artifact analysis flowchart (from TxDOT 2010)..............................................128

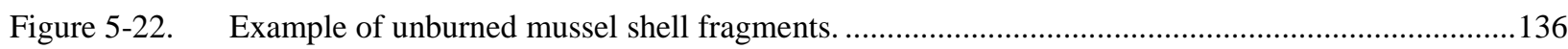

Figure 5-23. Example of small fragmented vertebrate bones..................................................................136

Figure 5-24. Examples of calcium carbonate adhering to a deer size long bone fragment (left \#2296)

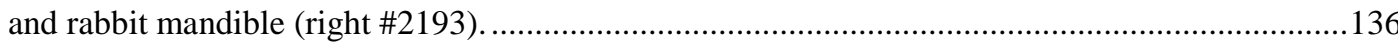

Figure 5-25. Examples of thin cut marks on bones (\#2292- left, \#2244-right; scale in millimeters).................137 
Figure 5-26. Examples of impact scar (middle top) on interior of deer size long bone (\#2250, scale in

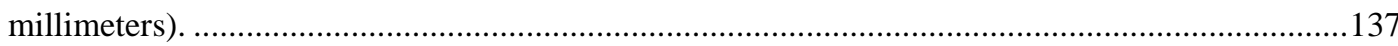

Figure 5-27. Examples of burned jackrabbit bones (\#2247, scale in centimeters).............................................138

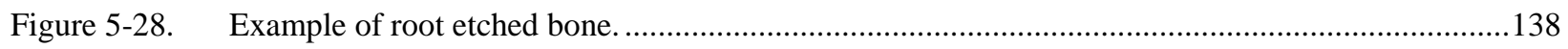

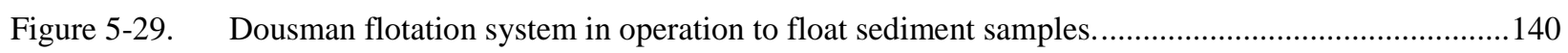

Figure 6-1. Schematic illustration showing the stratigraphic relationships observed in the lower Onion creek valley and selected radiocarbon ages. Horizontal is not to scale, and vertical scale is approximate (from Frederick 2011; Figure 8-10).

Figure 6-2. Soil column descriptions on south profile of mechanically stripped block at Big Hole.

Figure 6-3. View of sediment column described by geoarcheologist on south wall of deep pit showing sample locations (top hole is at boundary of Stratum II and III, flat base is bottom of drainage trench, base of upper dark zone is top of safety bench).

Figure 6-4. General view of upper Stratum II and III deposits above the targeted cultural deposits at bottom of photograph.

Figure 6-5. Very thin dispersed mottled greenish-gray clay lens that separated the Bell/Andice component from the lower Martindale component (bar scale in $10 \mathrm{~cm}$ increments)

Figure 6-6. Paleochannels of Onion Creek in vicinity of 41TV2161..........................................................151

Figure 7-1. Excavation grid placed within the mechanically-stripped excavation block. Surveyed surface is $230-250 \mathrm{~cm}$ below the modern ground surface.

Figure 7-2. Example of a GPR profile showing hyperbolic reflections resulting from discrete point sources, reflection signature of metal objects, and a sloping planar surface.

Figure 7-3. An attempted bar test to determine velocity. The metal bar should be visible at 2 meters along the profile.

Figure 7-4. Two reflection profiles showing areas (A) where coupling changes occurred, adversely affecting the resulting reflection amplitudes and obscuring archeological features.

Figure 7-5. GPR amplitude slice maps resulting from the $900 \mathrm{MHz}$ antenna. High amplitude reflections are seen as "warmer" colors, greens, yellows, and reds.

Figure 7-6. Amplitude slice maps from the $400 \mathrm{MHz}$ antenna. Each slice is 4 ns thick.

Figure 7-7. Indicates the location of 7 "anomalies" in the GPR data shown on slices 2 and 3 of the $400 \mathrm{MHz}$ data.

Figure 7-8. Indicates the location of 8 "anomalies" in the GPR data shown on slices 2 and 3 of the 900 $\mathrm{MHz}$ data

Figure 7-9. Sample of GPR transect profiles over recommended Anomalies 2, 3, and 5..............................161

Figure 8-1. Composite schematic site profile (data derived from Campbell et al. 2007).................................164

Figure 8-2. Profile of well-preserved, intact hearth Feature 3 (from Campbell et al. 2007:115)......................164

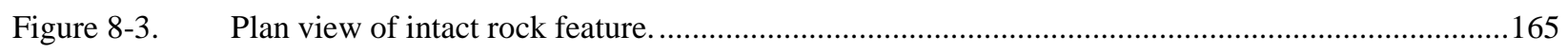

Figure 8-4. Profile of $50 \mathrm{~cm}$ thick silty clay deposits in north wall of N68/E96 in Block C. No obvious

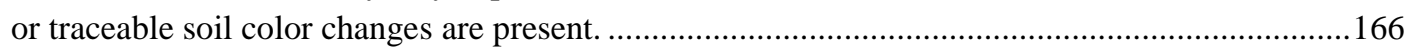

Figure 8-5. Profile of thin mottled greenish-gray lens that separates the two identified components.................166

Figure 8-6. Profile (left) and plan (right) distribution of encountered features (Features 15, 19, and 23)

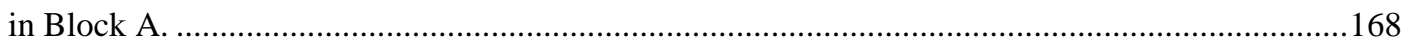

Figure 8-7. Overview of reddish and dark stained mottling of Feature 23 in Block A. ..................................168

Figure 8-8. Vertical distribution of cultural materials from three selected units in Block A. ..........................169 
Figure 8-9. Vertical distribution of encountered features (Features 16, 22, 24, 27, 28, 29, and 30 through 32) across Block B. Note Feature 26 was stratigraphically below other features.

Figure 8-10. Piece-plotted materials from two units in Block B (71N/82E and 71N/83E) that illustrate the vertical distribution of the Bell/Andice component stratigraphically above the light greenish-gray clay lens and the lower Feature 26 of the Martindale component.

Figure 8-11. Plan and profile distribution of piece-plotted materials and features (Features 18, 20 and 21) in Block C.

Figure 8-12. Oblique photograph of a portion of the mottled dark stained area of Feature 21.

Figure 8-13. Profile of Feature 18 in N66/E95 of Block C, documenting noncultural Rabdotus concentrations not directly associated with cultural feature.

Figure 8-14. Vertical distribution of cultural materials from three selected units in Block C. .173

Figure 8-15. Profile of north wall across Block D demonstrating vertical variability of the greenish-gray clay lens.

Figure 8-16. Profile of north wall of two units in Block D demonstrating vertical variability of the greenish-gray clay lens.

Figure 8-17. Vertical distribution of cultural materials in three selected units from Block D.

Figure 8-18. Vertical distribution Features 25 and 33 along with identified flake concentrations across Block D. 176

Figure 8-19. Vertical distribution of cultural materials within three selected units from Block F. 176

Figure 9-1.

Plot of radiocarbon assays from the Bell/Andice component. Samples are presented from top to bottom in the following order: charcoal (6), organic sediment (3), bone (2), and Rabdotus shells (1).....181

Figure 9-2. Plan view of Feature 3 (from Campbell et al. 2007, Figure 34)...................................................183

Figure 9-3. Profile of Feature 3 (from Campbell et al. 2007, Figure 35) ..........................................................183

Figure 9-4. Plan view drawing of Bell/Andice feature locations across Block B. ...........................................185

Figure 9-5. Plan view drawing of burned rock Feature 16........................................................................185

Figure 9-6. Drawing of tightly clustered ground stone cache in Feature 17.

Figure 9-7. Photograph of tapered sandstone pieces covered in calcium carbonate, possible metate fragment

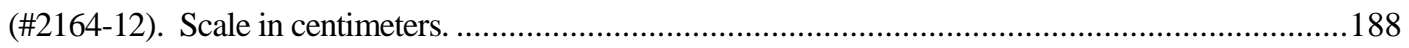

Figure 9-8. Photograph of complete mano (\#2164-10) from Feature 17. ..........................................................188

Figure 9-9. Photograph of complete mano (\#2164-11) from Feature 17. .........................................................188

Figure 9-10. Examples of spined freshwater sponge spicules (Appendix D) .....................................................188

Figure 9-11. Plan view drawing of Features 22, 24, 28, and 29, Block B........................................................189

Figure 9-12. Plan view drawing of Feature 24 with broken metate just west. ....................................................192

Figure 9-13. Example of true lily (Catesby's lily or pine lily, Lilium catesbaei) flower...................................193

Figure 9-14. Burned and unburned faunal remains (\#2179-4-g) in heavy fraction from Feature 24 (scale in millimeters).

Figure 9-15. Photograph of profile under Feature 27 burned rocks (north arrow is 10 centimeters)...................194

Figure 9-16. Overview photograph of burned rocks in Feature 28 in the northeastern corner of N74/E86 (north arrow is 20 centimeters).

Figure 9-17. Example of faunal fragments (\#2200-4-g) in heavy fraction from Feature 28 (scale in millimeters). 
Figure 9-18. Oblique photograph of top of Feature 29 that shows mottled, dark stained sediment in and around a circular cluster of burned rocks surrounded by scattered burned rocks (scales in 10 centimeters increments).

Figure 9-19. Oblique close-up of mottled, dark stained sediment in and around Feature 29 burned rocks (scales in 10 centimeters increments).

Figure 9-20. Oblique photograph of profile and excavated eastern half of shallow basin under Feature 29 (scale in 10 centimeter increments). 198

Figure 9-21. Oblique photograph of metate fragment (\#2223-10) recycled as a burned rock.

Figure 9-22. Close-up of burned and unburned bone fragments (\#2424-4-2g) from floated Feature 29 sediment (scale in millimeters).

Figure 9-23. Close-up of tiny lithic debitage (\#2024-4-g) from floated Feature 29 sediment (scale in millimeters).

Figure 9-24. Overview photograph of Feature 30 that shows dark stained sediment around five clustered burned rocks (scales in 10 centimeter increments)...

Figure 9-25. Profile photograph of Feature 30 that shows shallow basin with mottled dark stained sediment and no oxidation rim (scale in 1 centimeter increments).

Figure 9-26. Buffalo gourd fruit and leaves on vine (left), ripe fruit and seeds (right, from Texas Beyond

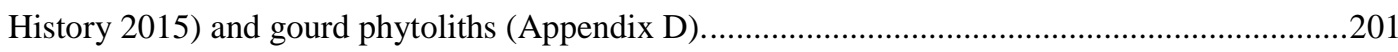

Figure 9-27. Plan view drawing of Feature 32 and its relation to Feature 30...................................................203

Figure 9-28. Profile drawing of Feature 30 and 32 ................................................................................203

Figure 9-29. Photograph of burned rocks and dark stained sediment in and around Feature 32 (scale in 10 centimeter increments)

Figure 9-30. Close-up of profile of burned rocks in of Feature 32 (scale in 10 centimeter increments).............204

Figure 9-31. Profile photograph of dark mottled stained sediment under Feature 32 burned rocks (scale in 10 centimeter increments).

Figure 9-32. Close-up of tiny burned and unburned bone fragments (\#2074-4-g) from floated Feature 32 sediment (scale in millimeters).

Figure 9-33. Photographs of Bell points (\#2139-11, left and \#2150-10, right).

Figure 9-34. Photographs of washed Big Sandy point (\#2224-10, left), use-wear results (middle), and

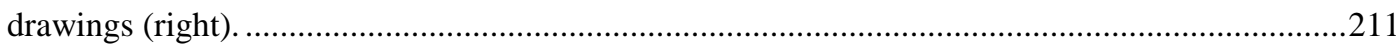

Figure 9-35. Photograph of unwashed, distal biface fragment (\#2105-10).

Figure 9-36. Photograph of unwashed, heat spalled, distal biface fragment (\#2146-10) adjacent to burned rock Feature 22 ......

Figure 9-37. Photograph of unwashed, lateral biface fragment (\#2175-12, left); three refit pieces (\#2175-10, \#2175-11, and \#2175-12) on right reconstructing biface outline (scale in centimeters).

Figure 9-38. Photograph of two examples of edge-modified flakes (\# 2041-10, left and \#2158-10 right).

Figure 9-39. Multiple microphotographs of edge-modified flake \#2149-10 showing soft polish (far left), two views of ctenoid (toothed) fish scale (far right), and hair (bottom left), (see Appendix D)...215

Figure 9-40. Dorsal and ventral views of an unwashed edge-modified flake (\#2114-10) depicting location of hard, high silica (HHS) polish in small concave notch area.

Figure 9-41. Horizontal distribution of Bell/Andice formal chipped stone tools, ground stone tools, and edge-modified flakes. 
Figure 9-42. Complete mano (\#2106-10)..................................................................................................218

Figure 9-43. General profiles of manos and metates from Bell/Andice component. ...........................................219

Figure 9-44. Complete mano (\#2164-10) from Feature 17. ............................................................................220

Figure 9-45. Complete mano (\#2164-11) from Feature 17 with use striations. ....................................................220

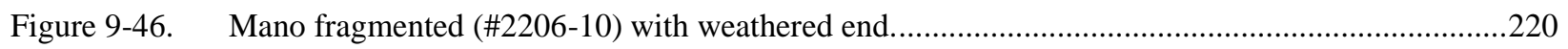

Figure 9-47. Oblique view of fragmented metate (\#2164-12) from Feature 17 (scale in centimeters)................222

Figure 9-48. Oblique view of fragmented metate (\#2175-13) from next to Feature 24 (scale in centimeters)....222

Figure 9-49. Oblique view of metate fragment (\#2223-10). ............................................................................223

Figure 9-50. Two examples of Bell/Andice cores (\#2146-1 and \#2197-1) ........................................................229

Figure 9-51. Horizontal distribution of the Bell/Andice lithic debitage across Block B...................................230

Figure 9-52. Example of small mammal remains (\#2202) between 230 and $240 \mathrm{cmbs}$ in N75/E83 .................231

Figure 9-53. Two adjoining deer size vertebrae (left, \#2158) from $247 \mathrm{cmbs}$ in N73/E81 and rabbit mandible (right, \#2193) from 220 to $230 \mathrm{cmbs}$ in N74/E85 covered in calcium carbonate.............232

Figure 9-54. Close-up of loose coyote $\mathrm{P}_{4}$ (\#2086) from 213 to $220 \mathrm{cmbs}$ in N71/E79 that shows no wear

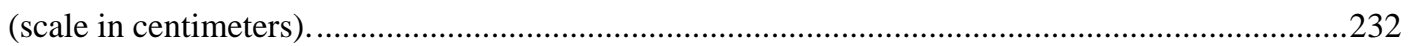

Figure 9-55. Example of bone fragments (\#2091) from 230 to $240 \mathrm{cmbs}$ in N71/E80...................................234

Figure 9-56. Close-up thin cut lines on tiny bone fragment (\#2202) from 230 to $240 \mathrm{cmbs}$ in N75/E83

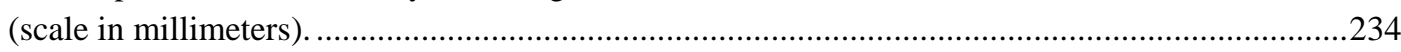

Figure 9-57. Vertebrate bone distribution across Block B for the Bell/Andice component................................235

Figure 9-58. Identified taxa distribution across Block B for the Bell/Andice component. .................................236

Figure 9-59. Bell/Andice burned rock distribution across Block B. ................................................................227

Figure 9-60. Example of wildrye (Elymus sp.) in natural setting (left) and close-up of ripe seed head (right,

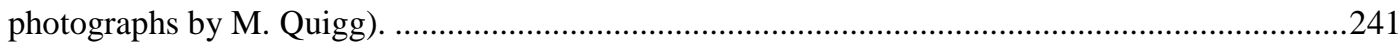

Figure 9-61. Distribution of Bell/Andice materials across Block B..............................................................24

Figure 10-1. Plot of radiocarbon assays from the Martindale component. Samples are presented from top to bottom in the following order: Rabdotus shells (2), organic sediment (5), bone (4), charcoal (2), and seeds (1)......

Figure 10-2. Charred cheno-am and grass seeds from Feature 26 radiocarbon dated to document the specific age of the Martindale component (scale in millimeters). ...................................................254

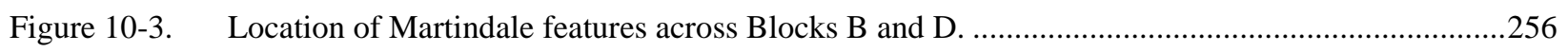

Figure 10-4. Overhead photograph of charcoal stained sediment of Feature 25 with burned rocks along the outer western margin.

Figure 10-5. Plan view drawing of Feature 25 and cultural materials in four units that surrounded the small

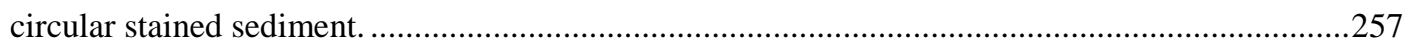

Figure 10-6. Plan view drawing of top portion of Feature 26 that depicts larger burned rocks in situ...............260

Figure 10-7. Oblique photograph of exposed top of Feature 26 depicting scattered burned rocks and dark stained sediments. Note complete oblong cobble is possible mano (\#2101-11). Scales in $10 \mathrm{~cm}$ intervals.

Figure 10-8. Oblique profile photograph of Feature 26 that depicts dark stained feature contents in shallow basin with burned rocks only along western margin. .261

Figure 10-9. Oblique profile photograph of Feature 26 that depicts shallow baked base with burned rocks concentrated on western margin of basin. 
Figure 10-10. Drawing of north-south profile through dark stained basin of Feature 26.

Figure 10-11. Burned rocks from inside Feature 26 with red hematite stained surfaces (\#2102-3-4 on left and \#2102-3-5 on right).

Figure 10-12. Possible mano (\#2101-11) from Feature 26.

Figure 10-13. Burned, calcined, and unburned animal bone fragments from floated Feature 26 sediment. Note small size pieces and small animal elements represented (scale in millimeters).

Figure 10-14. Close-up of burned and unburned gar fish scales from floated Feature 26 sediment (scale in millimeters).

Figure 10-15. Close-up of burned catfish spine (top) compared to modern unburned catfish spine (bottom).

Burned spine fragment from Feature 26 (scale in millimeters). .265

Figure 10-16. Micro debitage from floated Feature 26 sediment. Note consistency of material type (scale in millimeters).

Figure 10-17. Burned organic material (cheno-am and grass seeds, left) and burned fish bones and gar scales (right) from floated Feature 26 sediment (scale in millimeters). ...........................................266

Figure 10-18. Composite drawing of piece plotted cultural materials in and around hearth Feature $33 \ldots . . . . . . . . . .268$

Figure 10-19. Deer mandible (\#2275) fragment with two socketed molars and thin cut marks below M3 on outside edge

Figure 10-20. Metate fragment (\#2289-10) from inside Feature 33.

Figure 10-21. Lithic debitage (left) and burned clay chunks (right) from floated Feature 33 sediments (scale in centimeters)

Figure 10-22. Bone fragments from heavy fraction from Feature 33 sediments (Scale in millimeters). .............270

Figure 10-23. Photograph of burned clay nodules from Feature 33.

Figure 10-24. Unwashed Martindale point (\#2282-10, left) and possible corner-notched point medial fragment (\#2161-10, right). 275

Figure 10-25. Unwashed Martindale biface fragment (\#2257-10)..........................................................276

Figure 10-26. Unwashed Martindale biface fragment (\#2317-14) ..........................................................276

Figure 10-27. Unwashed Martindale biface (\#2326-10) ....................................................................276

Figure 10-28. Four examples of Martindale edge-modified flakes (left to right \#2338-10, \#2304-10, \#2325-10, and \#2318-10). 278

Figure 10-29. Six examples of small thin edge-modified flakes with pattern retouch (Top left to right \#2260-14, \#2260-13, \#2247-14, bottom left to right \#2246-13, 2254-10, and 2247-15). 278

Figure 10-30. Photograph of edge-modified flake (\#2325-10) with tiny downy feather barbule attached to distal end and possible starch grains documenting multiple uses (Appendix D). ...................278

Figure 10-31. General profiles of manos and metates in Martindale component...........................................279

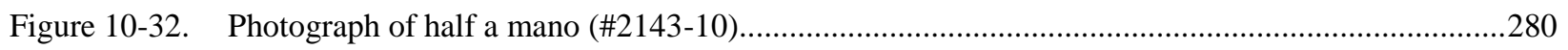

Figure 10-33. Photograph of complete mano (\#2102-11) from Feature 26...............................................280

Figure 10-34. Close-up photograph of pecked surface of metate fragment (\#2289-10) ....................................280

Figure 10-35. Photograph of hammer stones (\#2265-10 left and \#2226-10 right)........................................283

Figure 10-36. Horizontal distribution of Martindale features and formal stone tools......................................284

Figure 10-37. Photograph of unburned bone fragments (left) and lithic debitage (right) from heavy fraction (\#2248-4-g). 
Figure 10-38. Photograph of lithic debitage (left) and bone fragments (right) from heavy fraction (\#2261-4-g)

Figure 10-39. Horizontal distribution of Martindale lithic debitage across Blocks B and D.

Figure 10-40. Example of rabbit element fragments (\#2237). ..............................................................................297

Figure 10-41. Extensively butchered deer phalanges (\#2279) ..........................................................................298

Figure 10-42. Two views, exterior (left) and interior (right) of $\mathrm{M}_{2}$ and $\mathrm{M}_{3}$ socketed in deer mandible

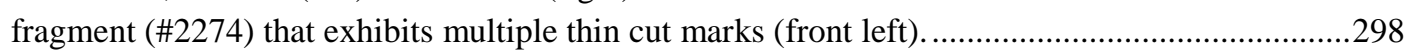

Figure 10-43. Well preserved right deer maxilla with socketed tooth row depicting tooth wear (\#2268)............298

Figure 10-44. Poorly preserved, weathered and worn left deer maxilla with individual teeth from same

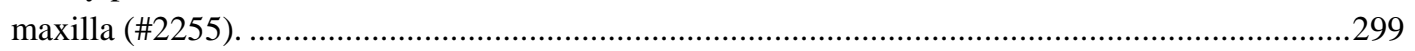

Figure 10-45. Two views of loose, right deer fawn $\mathrm{M}_{1}$ (\#2234) in eruption stage with no wear...........................299

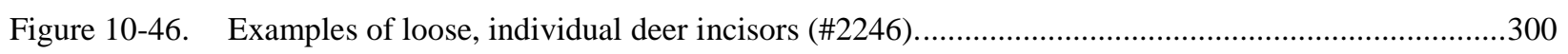

Figure 10-47. Left pronghorn left mandible section (\#2241) with socketed $\mathrm{P}_{3}$ and $\mathrm{P}_{4}$ that exhibits severe

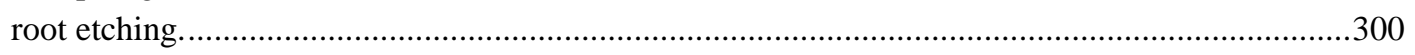

Figure 10-48. Right pronghorn mandible section with $\mathrm{P}_{2}, \mathrm{P}_{3}$ and $\mathrm{P}_{4}$ (\#2246, scale in millimeters)......................301

Figure 10-49. Socketed left pronghorn mandible $\mathrm{M}_{3}$ (\#2246) and socketed right pronghorn $\mathrm{M}_{3}$ (\#2281,

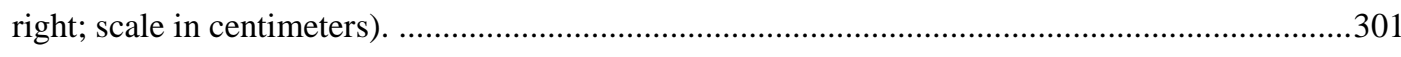

Figure 10-50. Two views of an unburned gar mandible (\#2233-2) fragment (scale in millimeters). .....................301

Figure 10-51. Examples of unburned gar scales (\#2238) (scale in millimeters). ..................................................302

Figure 10-52. Prairie chicken (Tympanuchus sp.) proximal humeral facet (upper left, \#2305) and distal sternal facet (lower right, \#2260) of coracoid element with complete modern grouse

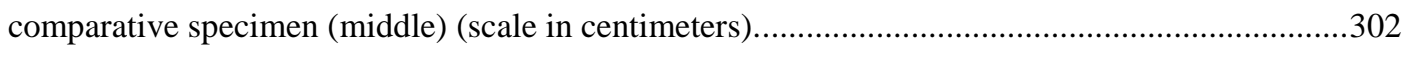

Figure 10-53. Male Attwater Prairie Chicken (Tympanuchus sp.)....................................................................303

Figure 10-54. Bird elements (\#2234, medial sacrum fragment left and scapula fragment right)...........................304

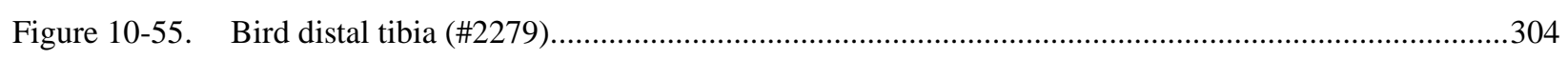

Figure 10-56. Large bird distal tibia (left, \#2279) compared to modern grouse distal tibia (right, scale in

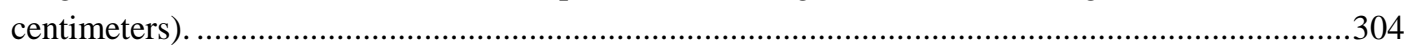

Figure 10-57. Martindale bone distribution across Blocks B and D. ................................................................

Figure 10-58. Martindale deer, pronghorn and deer size bone distribution across Blocks B and D.....................307

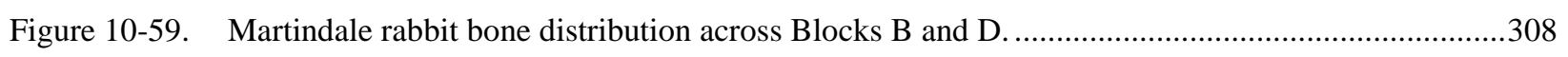

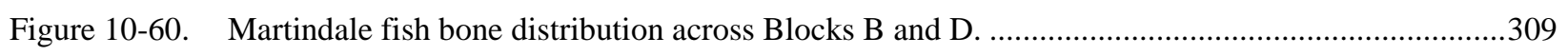

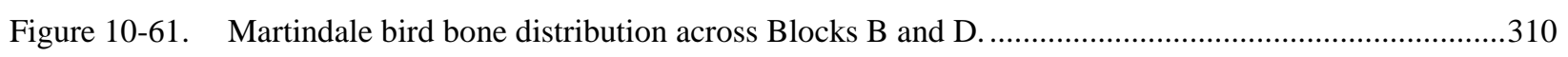

Figure 10-62. Martindale turtle bone distribution across Blocks B and D......................................................... 311

Figure 10-63. Martindale burned rock distribution across Blocks B and D. ....................................................313

Figure 10-64. Attwater prairie chicken in their natural coastal prairie habitat (courtesy of the Nature

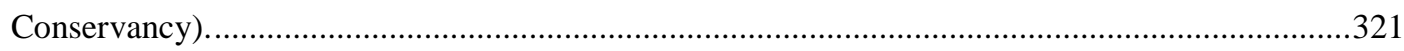

Figure 11-1. Plots of radiocarbon dates from Bell/Andice compared to Martindale dates. ...............................326

Figure 11-2. Calibrated radiocarbon assays from charcoal samples from the Bell/Andice and Martindale components.

Figure 11-3. Distribution of flakes by maximum dimension within the Martindale (MD) and Bell/Andice (BA) components. 
Figure 11-4. Distribution of flakes by maximum dimension within the Martindale (MD) and Bell/Andice

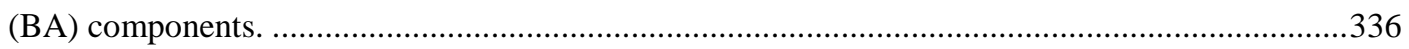

Figure 11-5. Comparison of materials classes of the two components. ............................................................341

Figure 11-6. Comparison of burned rock by count and weight between components. ........................................341

Figure 11-7. Composite of horizontal distribution of major materials classes in Bell/Andice component. ..........349

Figure 11-8. Composite of horizontal distribution of major materials classes in Martindale component.............350

Figure 12-1. Drawing depicting the location of nonassociated features in various blocks. .................................356

Figure 12-2. Overhead photograph of Rabdotus shell concentration, Feature 15 (scale in 10 centimeter

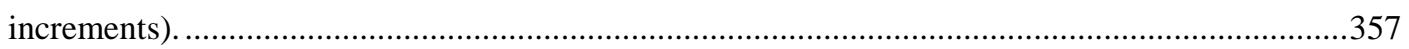

Figure 12-3. Overview photograph of exposed burned rock cluster of Feature 18 (left of arrow) and surround scattered cultural materials in Block C (scale in 10 centimeter increments). .....................357

Figure 12-4. Plan view drawing of Feature 18 in Block C.........................................................................358

Figure 12-5. Oblique view of partially excavated Feature 19 in Block A (scale in 10 centimeter increments)...362

Figure 12-6. Photograph of overhead view of clustered burned rocks in Feature 20 (scale in 10 centimeter

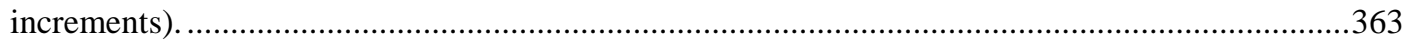

Figure 12-7. Top part of Feature 21 showing mottled dark, linear stained sediment in Block C (scale in 10

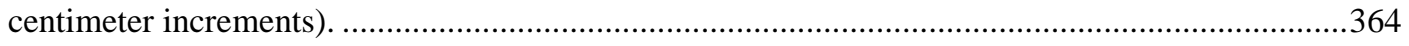

Figure 12-8. Overview of mottled dark stained sediment of Feature 21 across multiple units in Block C

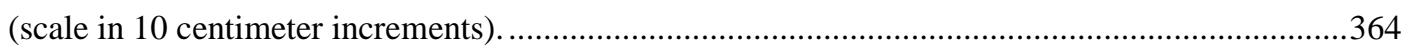

Figure 12-9. Close-up profile of part of Feature 21 in Block $\mathrm{C}$ (scale in 10 centimeter increments). .................364

Figure 12-10. Oblique view of mottled reddened and dark stained sediment in Feature 23 in Block C (scales

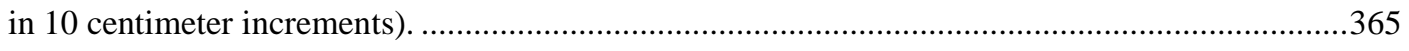

Figure 12-11. Plan view of exposed burned rocks in northern half of Feature 31 with metate fragment just

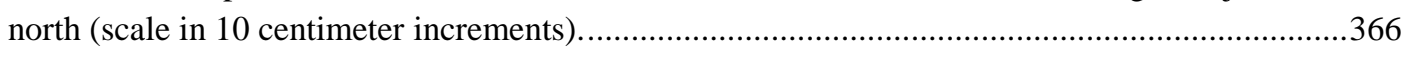

Figure 12-12. Profile of northern half of shallow dark stained basin of Feature 31 ............................................367

Figure 12-13. Plan view and profile drawing of northern half of Feature 31....................................................367

Figure 12-14. Metate fragment (\#2414-10) adjacent to burned rocks in Feature 31 (scale in centimeters)............368

Figure 12-15. Complete unwashed biface (\#2023-10)......................................................................................

Figure 12-16. Distal end of unwashed, early stage biface fragment (\#2416-10). .................................................371

Figure 12-17. Photographs of complete washed gouge (\#2417-10, left) and use-wear results (right)...................372

Figure 12-18. Complete unwashed drill stem (left, \#2162-11) depicting use-wear results (right) from just

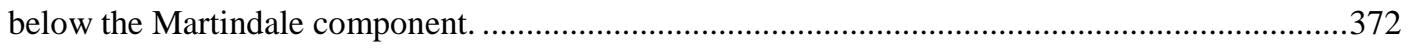

Figure 12-19. General profiles and plan views of manos and metates from unassigned context...........................374

Figure 12-20. Oblique view of mano fragment (\#2104-11) from below Feature 26............................................375

Figure 12-21. Plan view of possible mano fragment (\#2396-10) with red pigment staining from Block F

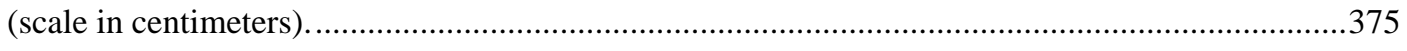

Figure 12-22. Two Plan views of fragmented metate (\#2396-11) with red (left) and black (right) pigment

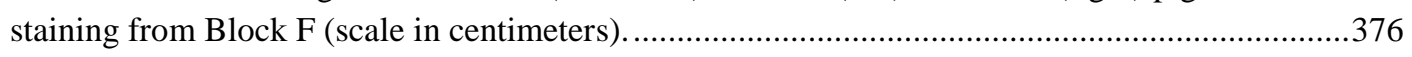

Figure 12-23. Loose pronghorn maxilla left $\mathrm{M}^{3}$ (\#2019, scale in centimeters) ....................................................377

Figure 13-1. As of the early 1990s, Big Sandy point distribution depicting counties with 1 to 11 points

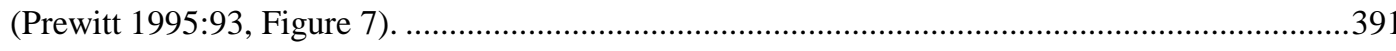

Figure 13-2. Close-up of wildrye (Elymus canadensis) grass seed heads (photograph by M. Quigg)................401 
Figure 13-3. Schematic drawing of the stone boiling process and burned rock patterning (drawing by and with permission of C. Lintz)......

Figure 13-4. Impressions of bone grease extraction process (left) and scooping bone grease floating on surface of water (right) (paintings by and with permission of Shayne Tolman).

Figure 13-5. Historic range for lesser prairie-chicken (LPC), Greater Prairie-Chicken (GPC) and Attwater's Prairie-Chicken (APC) (from Silvy et al. 2003:18).

Figure B-1. Silt/sand matrix mixed with decomposed and carbonized plant material. Scale is $1 \mathrm{~mm}$ per tic mark. (Sample \#2003-7: Magnification 7.5X).

Figure B-2. Silt/sand matrix coated in black, carbonized, and decomposed plant material. (Sample \#2246-7-2: Magnification 15X).

Figure D-1. Visual summary of results for Bell/Andice component...........................................................592

Figure D-2. Visual summary of results for Bell/Andice component.......................................................593

Figure D-3. Visual summary of results for Martindale component. ............................................................594

Figure D-4. Visual summary of results for Martindale component. ..............................................................595

Figure D-5. Visual summary of results for Martindale/Corner-Notched component. ........................................596

Figure D-6. $\quad$ Edge-modified flake 2419-10. A) possible fragment of ctenoid fish scale (orig. mag.; 100x); B) same scale at higher magnification (o.m. 500x); C) hair fragment (o.m. 500x); D) polish showing linearity (compare to fish polish Hogberg et al. 2009).

Figure D-7. Edge-modified flake 2325-10, A) fragment of downy feather barbule (o.m. 500x); B) fragment of feather barbule with starch grains (o.m. 500x, cross-polarized light); C) soft polish. .

Figure E-1. Representative short cell Poaceae phytoliths from floodplain surface A horizon soil control sample (4TV2161, PNUM 2451). A-L, N (lower), and Q (left) are various forms of Panicoideae short cell phytoliths. L, M (lower), P, Q (right), and rare Chloridoideae short cell phytoliths. N (upper) and S-AC are Pooideae short cell phytoliths ("crenate" morphology; the smaller Pooid forms were essentially absent in these samples). The Panicoid phytoliths in $\mathrm{G}$ and $\mathrm{H}$ have been burned - the discoloration is due to carbon embedded in the silica matrix. The white bar scales are 10 microns.

Figure E-2. Silt clay fraction solutions prior to first aspiration from $250 \mathrm{ml}$ sample bottles. Note incomplete settling visible in the middle two bottles in the top row. Also note the drastic difference in appearance of the two surface control samples (bottom row, last two bottles). The above samples are in the same order as listed in Table E-1....

Figure E-3. Close-up view of the lower portion of the twelve silt samples in the top row of Figure E-2. Samples F-H did not settle well — and at this point required acid pretreatment and a much longer time to settle. The other 9 samples show the tan/brown silica-based silt layer at the bottom of each sample jar below a whitish sediment layer. This thick white layer is not normally observed in the silt fraction at other sites, and was subsequently found to consist of a combination of carbonates and low density soil minerals. A fine intermediate grey layer(s) is also visible in some of these sample sediments. The white layer was significantly thinner in the hill top surface control sample, and nearly absent in the flood plain surface control sample.

Figure E-4. Sand fractions from Block C soil profile, Big Hole site (41TV2161). A: PNUM 2026; B: PNUM 2411; C: PNUM 2410; D: PNUM 2409; E: PNUM 2408; F: PNUM 2407; G: PNUM 2406; H: PNUM 2405; I: PNUM 2404; J: PNUM 2403; K: PNUM 2401; and L: PNUM 2400. Complete snails are readily visible in $\mathrm{E}, \mathrm{F}, \mathrm{H}, \mathrm{J}$ and $\mathrm{L}$. 
Figure E-5. $\quad$ Sand fractions from Block B and D samples, Big Hole site (41TV2161). A: PNUM 2143; B: PNUM 2261; C: PNUM 2257; D: PNUM 2110; E: PNUM 2112; F: PNUM 2197; G: PNUM 2200; H: PNUM 2424; I: PNUM 2078; J: PNUM 2092; K: PNUM 2224; and L: PNUM 2246. Complete snails are visible in A-C, E, G-H, J, and L.

Figure E-6. Sand fractions from miscellaneous Blocks A-D samples, and surface control samples, Big Hole site (41TV2161). A: PNUM 2367; B: PNUM 2146; C: PNUM 2149; D: PNUM 2176; E: PNUM 2060; F: PNUM 2179; G: PNUM 2239; H: PNUM 2280; I: PNUM 2044; J: PNUM 2275; K: PNUM 2450; and L: PNUM 2451. Complete snails are visible in A, E, and H-J.

Figure E-7. Representative sand fraction images 1 (PNUM 2406, 41TV2161). Larger particles present in sand fraction. A: Predominantly shell fragments and carbonates. B: Charcoal and snail in addition to other debris. C-F: Four specimens of egg-shaped ornamented particles which are calcareous oogonia of Charophytes (specimen in $\mathrm{F}$ is broken in half showing interior detail). The coiled white wire-like piece in Figure E-17H is a fragment of oogonia; the fragments were much more abundant than whole particles - possibly broken during laboratory shaking and particle size separations. Carbonate, fossil marine spicules, charcoal, snails, shell fragments, and miscellaneous items are present.

Figure E-8. Representative sand fraction images 2 (PNUM 2406, 41TV2161). A: Arrows highlight some of the many carbonate fragments some of which contain embedded quartz sand. B: Carbonate concretion highlighted. C: Carbonate sponge spicule in center (marine fossil). D: Possible echinoid spine in center (marine fossil). E: Arrow denotes a sheaf of carbonate that had encrusted a root. F: Arrows denote two foraminifera standing on edge ( $\# 2$ on the insert shows one of these forams in planar view).

Figure E-9. Significant bone fragments observed in the sand fractions. A: PNUM 2403; B, E: PNUM 2224; C, D: PNUM 2261; F, G: PNUM 31; H: PNUM 2146; and I: PNUM 2112. Scales at $3.5 \mathrm{x}, 10 \mathrm{x}$, and 25x. The specimens in $\mathrm{F}$ and $\mathrm{H}$ appear to be ends of long bones; the specimen in I may be the tip of a tool.

Figure E-10. Snails and fragments from Big Hole site (41TV2161) Block C Profile. PNUM 2026 specimens A-L

Figure E-11. Snails and fragments from Big Hole site (41TV2161) Block C Profile. PNUM 2026 specimens A-N.

Figure E-12. Snails and fragments from Big Hole site (41TV2161) Block C Profile. PNUM 2411 specimens A-M, and PNUM 2410 specimens N-O.

Figure E-13. Snails and fragments from Big Hole site (41TV2161) Block C Profile. PNUM 2409 specimens (A-G), and PNUM 2408 specimens (H-P).

Figure E-14. Snails and fragments from Big Hole site (41TV2161) Block C Profile. PNUM 2408 specimens A-L

Figure E-15. Snails and fragments from Big Hole site (41TV2161) Block C Profile. PNUM 2408 specimens A-F, and PNUM 2407 specimens G-K.

Figure E-16. Snails and fragments from Big Hole site (41TV2161) Block C Profile. PNUM 2407 specimens A-V. Specimen M is burned.

Figure E-17. Snails and fragments from Big Hole site (41TV2161) Block C Profile. PNUM 2406 specimens A-X.

Figure E-18. Snails and fragments from Big Hole site (41TV2161) Block C Profile. PNUM 2405 specimens A-X. Specimen L is burned. 
Figure E-19. Snails and fragments from Big Hole site (41TV2161) Block C Profile. PNUM 2404

specimens A-H, and PNUM 2403 specimens I-R.

Figure E-20. Snails and fragments from Big Hole site (41TV2161) Block C Profile. PNUM 2401 specimens A-R, and PNUM 2400 specimens S-AF. Specimens X and AC-AF are burned.

Figure E-21. Distinctive charcoal fragments from PNUM 2060. Specimens A-H all have the same distinctive circular "divots" in the surface. I-K also have distinctive traits. All specimens remain unidentified as to botanical origin(s). A piece of burned shell is on top of specimen in E.

Figure E-22. Soil texture values of the 4TV2161 sample suite. The laboratory sample numbers are on the $\mathrm{x}$-axis, and the PNUM values are in Table E-1. Samples 1-12 - showing the greatest variability - are the Block $C$ soil profile column samples. [Sample 34 omitted]

Figure E-23. Block C soil profile and soil texture plot [PNUM 2400-2411] shows a relatively stable clay content and significant variations in the sand and silt fractions over time.

Figure E-24. Representative weathered bulliform specimens from various samples (the specimen in E is likely an elongate or a tree type phytolith; $\mathrm{R}$ is amorphous silica, but may not be a bulliform cell). A, B: PNUM 2407; C-D: PNUM 2406; E-N: PNUM 2110; O-Q: PNUM 2176; R-T: PNUM 2060; U, W-X: PNUM 2239; V: PNUM 2179; and Y: PNUM 2280. The white bar scales are 10 microns.

Figure E-25. Cucurbita phytoliths. A-B: PNUM 2092; C: PNUM 2179; D-F: PNUM 2239; G-J: PNUM 2280; K: PNUM 2044; and L (left specimen): PNUM 2275. Several specimens may be burned (A, B, H, J, and L (left). Specimen J is atypically large for the period. White bar scales are 10 microns

Figure E-26. Smooth surfaced freshwater sponge spicules (megascleres). A \& C: PNUM 2280; B: PNUM 2411; D: PNUM 2149; E: PNUM 2143; F \& H: PNUM 2367; and G: PNUM 2110. All specimens are complete spicules except $\mathrm{C}$ and $\mathrm{F}$ (C shows a bending break on the right end). In the center of specimens B, C, and E the enlarged area is where the sclerocyte lived that synthesized the spicule.

Figure E-27. Spined freshwater sponge spicules. A: PNUM 2112; B, D-F: PNUM 2110, and C: PNUM 2406.

Figure E-28. Freshwater sponge spicules with thin-walls and large diameter axial canals. A and L: PNUM 2149; B: PNUM 2110; C, F-G, M: PNUM 2367; D: PNUM 2179; E: PNUM 2406; G: PNUM 2367; H: PNUM 2424; I and J: PNUM 2110; and K: PNUM 2143.

Figure E-29. Examples of weathered (physically abraded) spicules. A-B, E: PNUM 2060; C and I: PNUM 2146; D and J: PNUM 2110; F: PNUM 2112; G: PNUM 2239: and H: PNUM 2450.

Specimen I demonstrates signs of both physical abrasion and chemical weathering. The arrows in $\mathrm{J}$ indicate localized points of abrasion.

Figure E-30. Examples of chemically weathered spicules. Note that the thinner walls near the tip show more evidence of dissolution than the thicker areas (c.f., left end of F, H, and I; right end of B and D). A: PNUM 2408; B-C, E-F, and H-I: PNUM 2110; D: PNUM 2146; and G: PNUM 2112. Specimen A shows evidence of both physical abrasion and chemical weathering...........................638

Figure E-31. Gemmoscleres. A: PNUM 2112; and B: PNUM 2424 (broken).

Figure E-32. Chrysophycean Cysts. A-D: PNUM 2450; and E-J: PNUM 2451. Additional images from PNUM 2451: K (unidentified particle), and L (air bubble for comparison). The bar scales are 10 microns.

Figure E-33. Four plots of amorphous silica solubility vs. $\mathrm{pH}$ in aqueous systems (Iler 1979:42, Figure 1.6)...6642 
Figure E-34. Chloridoid ("saddle") phytolith plot of dimensional ratio vs. concentration with the 41TV2161 surface control data soil point added (PNUM 2451). The three squares denote upland prairie control surface samples located in Oklahoma. The red diamonds are dates for buried soils at the Bull Creek site (34BV176) (Bement et al. 2007), the downward pointing diamonds are from buried soils at the Burnham site (34WO69), and the upward pointing triangles are from the Opossum Creek site (34NW132) (Sudbury 2011c). The two large color ovals highlight two riparian settings in Oklahoma with more recent buried soils (black circles) and surface A horizons (black flower) data plotted [Carnegie Canyon site (34CD76), Lizard site (34WN107)]. The surface data point for 41TV2161 is in burnt orange.

Figure E-35. Unidentified large platy phytoliths (PNUM 2451). Scale bars 50 microns (A-E) and 25 microns (F-I).

Figure E-36. Miscellaneous phytoliths and particles (1). A-B: PNUM 2407; C: PNUM 2406; D-F: PNUM 2176; G, O: PNUM 2060; H-I, P: PNUM 2179; J, Q: PNUM 2239; K: PNUM 2044; L: PNUM 2092; M-N: PNUM 2146; R-S: PNUM 2280; T-V: PNUM 2275; W-X: PNUM 2450; Y-EE: PNUM 2451. Bar scales are 25 microns.

Figure E-37. Miscellaneous phytoliths and particles (2). A-B: PNUM 2110; C: PNUM 2424; D: PNUM 2092; E: PNUM 2246; F-G: PNUM 2367; H-I: PNUM 2146; J: PNUM 2176; K-M: PNUM 2060; N-P: PNUM 2179; Q-S: PNUM 2280; T: PNUM 2044; U: PNUM 2275; V-Z: PNUM 2450; AA-CC: PNUM 2451. Bar scales are 10 and 25 microns.

Figure E-38. Calcium carbonate nodule fragment (PNUM 2407). Four images of the same specimen. A: Specimen illuminated with plane polarized light at 500x; B: same as A with crossed polars; C. crossed polars with a full wave plate in place; and D. same as $\mathrm{C}$ with stage rotated 90 degrees to evaluate the change in birefringence.

Figure F-1. Starch remains recovered from site 41TV2161. The scale bar is equivalent to 20 microns, and all photomicrographs appear at identical magnification for comparative purposes. $\mathrm{A}=\mathrm{A}$ lenticular starch grain from flaked tool \#2021-10 from Martindale component. B = A starch grain from the lily family showing the typical feathered fissuring and soft lamellae. Note the large size. From flaked tool \#2103-10 in Martindale component. C = A gelatinized starch grain from burned rock \#2078-3-4, Feature 32. D = An "eroded" starch grain from burned rock \#2026-3-4, Feature 18

Figure H-1. Locations from which archaeological and geological samples were obtained for this study. Basemap: USGS 7.5' 1988 Montopolis, TX quadrangle.

Figure H-2. Dendrogram presenting a hierarchical cluster analysis of chert specimens. Cluster analysis performed using average linkage of mean Euclidean distance.

Figure H-3. Principal component biplot showing artifacts from 41TV2161 and source gravels from nearby hillside. Artifact specimens are labeled. Ellipse surrounding source gravel specimens is drawn at the $90 \%$ confidence interval.

Figure H-4. Plot of canonical discriminant functions one and two for quarry-related source groups within the Edwards Formation of central Texas. Ellipses are drawn at the $90 \%$ confidence interval around previously defined source groups. Newly generated data are projected against these groups.

Figure H-5. Plot of canonical discriminant (CD) functions one and two for quarry-related source groups within the Edwards Formation of central Texas. Source groups from the Fort Hood area have been removed from the $\mathrm{CD}$ analysis. Ellipses are drawn at the $90 \%$ confidence interval, and newly generated data are projected against these groups. 
Figure H-6. Plot of canonical discriminant (CD) functions one and two for quarry-related source groups within the Edwards Formation of central Texas. In this plot, specimens of source gravels analyzed in this study are treated as a discrete group. Ellipses are drawn at the $90 \%$ confidence interval, and newly generated data are projected against these groups. 728

Figure I-1. Analysis of reddish side of sample 2396-10.............................................................................748

Figure I-2. Analysis of non-reddish side of sample 2396-10 ....................................................................

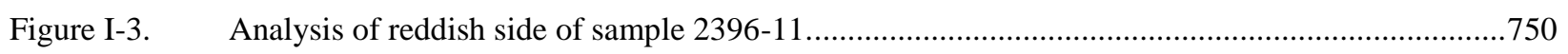

Figure N-1. Artifact taxonomy for chipped stone tools based on technological attributes and reduction

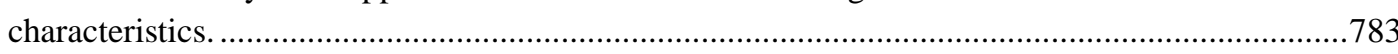

Figure N-2. Artifact taxonomy for chipped stone objects with primarily non-utilitarian, symbolic purpose. ....783

Figure N-3. Taxonomy for chipped stone cores. These are not tools, but rather the objective piece from

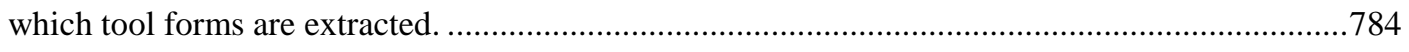

Figure N-4. Metric measurements recorded directly from tool....................................................................784

Figure N-5. Edge angle can be recorded with the use of a goniometer............................................................785

Figure N-6. In the illustration above, "retouched" and "fractured segments" are generally represented by

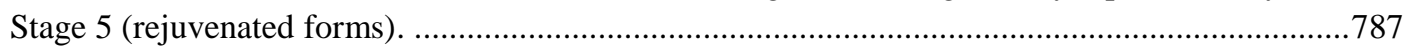

Figure N-7. Give stages of Chipped Stone Analytical Protocol.......................................................................789

Figure N-8. Chipped stone tools are more often discovered in a broken state.................................................790

Figure N-9. These terminations are often observed on bifacial blanks and preforms that were discarded

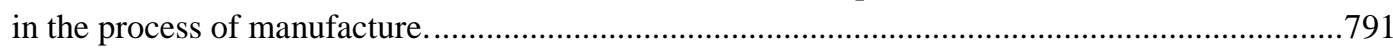

Figure N-10. These terminations illustrate additional failures that may render the objective piece unusable

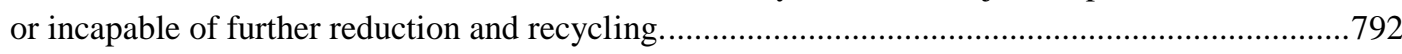

Figure N-11. Edge morphology has its greatest utility in characterizing projectile points, darts, and knives. .....793

Figure N-12. Patterns of flake removal in edge construction, potentially related to flaking technique, tool

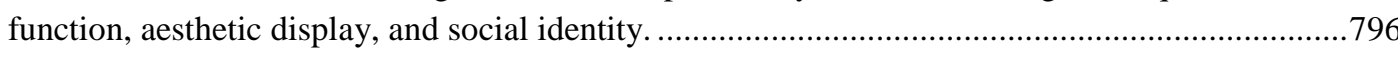

Figure N-13. Examples of lateral edge flaking attrition. (A) bifacial-unilateral; (B) unifacial-unilateral; (C)

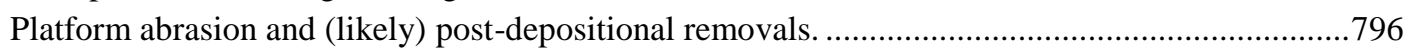

Figure N-14. Patterns of polish formation and distribution related to use-wear. ..................................................798

Figure N-15. Development of lateral scars (or striations) developed on the working edge of the tool derived from abrasive and repeated contact between the tool form and contact material.

Figure N-16. The graphic above illustrates both a quantifiable and a qualitative means by which to measure or estimate the amount of remnant dorsal cortex on a lithic flake.

Figure N-17. 10-20x magnification (hand lens or loop) is recommended for viewing platforms on debitage in the $1 / 4-1 / 2$ inch size grades. 805

\section{LIST OF TABLES}

Table 1-1. Summary of Trench and Unit Data from Site Discovery by Hicks \& Company (from Campbell

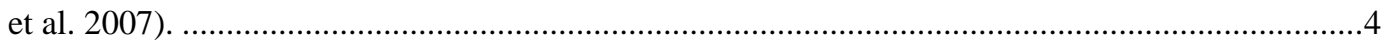

Table 3-1. Radiocarbon Dates for Sites Associated with Bell/Andice/Calf Creek Points..................................44

Table 3-2. $\quad$ Excavated Bell/Andice/Calf Creek Feature Data............................................................................59

Table 3-3. Martindale Site and Component Integrity Data. ..............................................................................72 
Table 3-4. $\quad$ Radiocarbon Dates Generally Associated with Martindale Points. ................................................82

Table 3-5. $\quad$ Early Archaic and Martindale Subsistence Data.......................................................................85

Table 3-6. $\quad$ Early Archaic and Martindale-Related Feature Frequency and Types. ......................................88

Table 5-1. $\quad$ Sediment Samples Sent to Milwaukee Soil Laboratory, LLC for Analyses. ................................119

Table 5-2. $\quad$ INAA Sample Numbers, Component Associations, and Descriptions. ......................................126

Table 9-1. $\quad$ Radiocarbon Dates from the Bell/Andice Component...............................................................180

Table 9-2. $\quad$ Burned Rock Quantities and Weights from Bell/Andice Features.............................................186

Table 9-3. Flotation Results with Counts and Weights by Material Class for Bell/Andice Component..........190

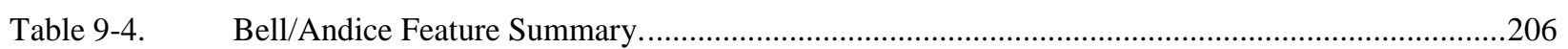

Table 9-5. Summary of Analytical Results from Bell/Andice Features..................................................207

Table 9-6. Bell/Andice Formal Chipped Stone Tool Data. ....................................................................210

Table 9-7. Bell/Andice Edge-Modified Flake Data. ............................................................................214

Table 9-8. Summary of Bell/Andice High-Powered Use-Wear Analyses. ...............................................216

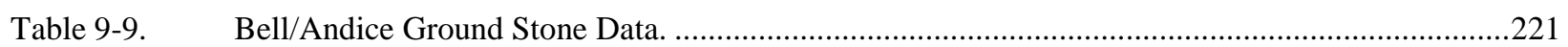

Table 9-10. Dorsal Cortex and Scar Counts for Complete Flakes. ........................................................224

Table 9-11. Bulb Size and Eraillure Scar Frequency for Complete Flakes. ................................................224

Table 9-12. Lipping and Longitudinal Curvature for Complete Flakes.....................................................224

Table 9-13. Platform Class and Preparation for Complete Flakes.......................................................224

Table 9-14. Size Class and Termination Types for Complete Flakes...........................................................226

Table 9-15. Metric Dimensions for Complete Flakes...........................................................................226

Table 9-16. Nonmetric Attributes Recorded on Proximal Flake Fragments..............................................227

Table 9-17. Metric Dimensions for Proximal Flake Fragments...........................................................227

Table 9-18. Metric Dimensions for Cores (measurements in $\mathrm{mm}$ ) .....................................................229

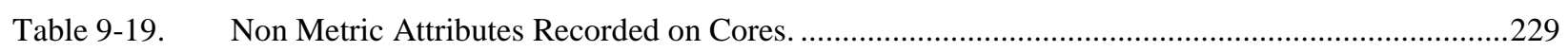

Table 9-20. Bell/Andice Bone Data.................................................................................................231

Table 9-21. Bell/Andice Burned Rock Frequency, Size, and Weight Categories..........................................238

Table 9-22. Summary of Bell/Andice Component Materials. .....................................................................239

Table 9-23. Relevant Excavated Hunter-Gatherer Occupations in the Central Texas Region.......................248

Table 10-1. Radiocarbon Dates from the Martindale Component. ...........................................................252

Table 10-2. Burned Rock Counts and Weights for Each Martindale Feature...............................................258

Table 10-3. Flotation Results with Counts and Weights by Material Class for Martindale Component. ...........264

Table 10-4. Martindale Feature Summary. ....................................................................................272

Table 10-5. Summary of Analytical Results from Martindale Features. ................................................272

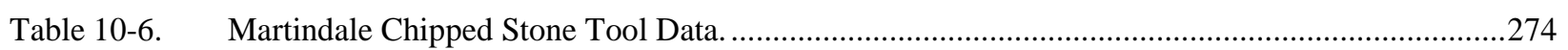

Table 10-7. Martindale Ground Stone Tool Data. .............................................................................279

Table 10-8. Martindale Hammer Stone Data.....................................................................................282

Table 10-9. High-Powered Use-wear Results from Martindale Artifacts................................................282

Table 10-10. Dorsal Cortex and Scar Counts for Complete Flakes. ….................................................286

Table 10-11. Bulb Size and Eraillure Scar Frequency for Complete Flakes. ...................................................286

Table 10-12. Lipping and Longitudinal Curvature for Complete Flakes.....................................................286 
Table 10-13. Platform Class and Preparation for Complete Flakes ...................................................................286

Table 10-14. Size Class and Termination Types for Complete Flakes................................................................28

Table 10-15. Metric Dimensions for Complete Flakes (measurements in mm). ...............................................288

Table 10-16. Nonmetric Attributes Recorded on Proximal Flake Fragments.......................................................289

Table 10-17. Descriptive Statistics of Metric Dimensions for Proximal Flake Fragments (measurements

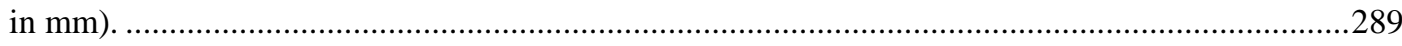

Table 10-18. Metric Dimensions for Cores (measurements in mm) …………..............................................290

Table 10-19. Nonmetric Attributes Recorded on Cores................................................................................290

Table 10-20. Float Results from Three Martindale Lithic Concentrations. ..........................................................292

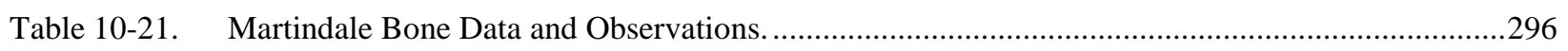

Table 10-22. Martindale Burned Rock Frequency, Size, and Weight Categories. ..............................................312

Table10-23. Summary of Materials Remains from the Martindale Component.................................................315

Table 11-1. Comparison of Material Remains from the Two Identified Components. ........................................328

Table 11-2. Summary of Tool Function from Use-Wear Analysis...................................................................329

Table 11-3. Dominant Attribute Classes in the Samples of Complete Flakes. .....................................................332

Table 11-4. Dorsal Cortex and Scar Counts for Complete Flakes..................................................................332

Table 11-5. Bulb Size and Eraillure Scar Frequency for Complete Flakes. ........................................................333

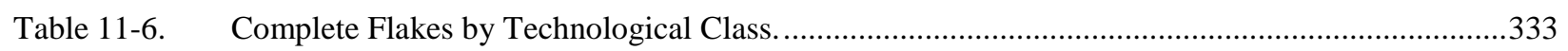

Table 11-7. Lipping and Longitudinal Curvature for Complete Flakes...........................................................333

Table 11-8. Platform Class and Preparation for Complete Flakes..................................................................334

Table 11-9. Size Class and Termination Types for Complete Flakes................................................................334

Table 11-10. Metric Dimensions for Complete Flakes (in millimeters). .............................................................335

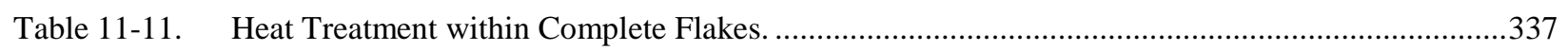

Table 11-12. Heat Treatment within Proximal Flake Fragments....................................................................337

Table 11-13. Metric Dimensions for Proximal Flake Fragments (in millimeters) ...............................................337

Table 11-14. Metric Dimensions for Cores (in millimeters).......................................................................

Table 11-15. Nonmetric Attributes Recorded on Cores.................................................................................339

Table 11-16. Metric Dimensions Recorded on Edge-Modified Flakes (in millimeters).......................................339

Table 11-17. Comparison of Feature Characteristics...................................................................................343

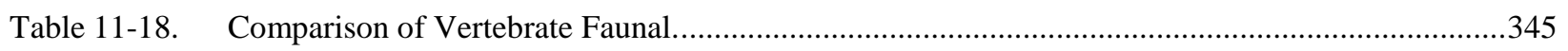

Table 11-19. Comparison of Plant Indicators from Technical Analyses. ...........................................................347

Table 11-20. Comparison of Chert Debitage and Bone Fragments less than $6.4 \mathrm{~mm}$ in Size Recovered in

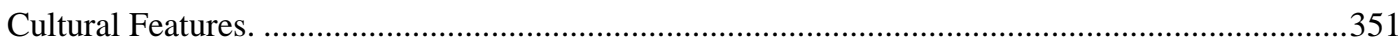

Table 12-1. Radiocarbon Dates from Multiple Blocks and Unassigned Features. ..............................................354

Table 12-2. Burned Rock Counts and Weights for Unassigned Features...........................................................359

Table 12-3. Flotation Results from Unassigned Features. ..................................................................................

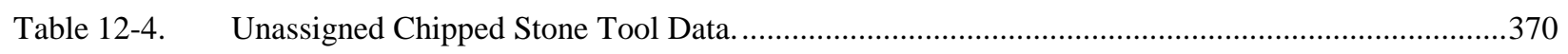

Table 12-5. Unassigned Ground Stone Data............................................................................................... 373

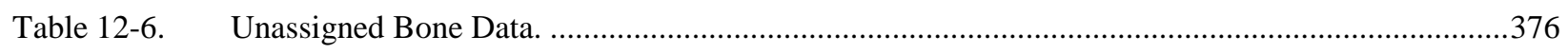

Table 12-7. Burned Rock Frequencies, Sizes and Weights from Unassigned Proveniences...............................378 
Table 12-8. Summary of Unassigned Materials from Data Recovery. .............................................................378

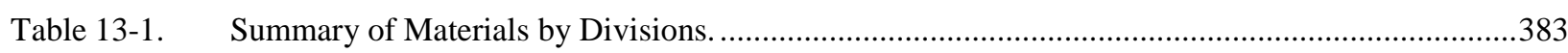

Table 13-2. Component Integrity, Point Types, and Radiocarbon Dates from Selected Excavated Sites in Texas for the General Martindale and Bell/Andice Middle Holocene Period...............................388

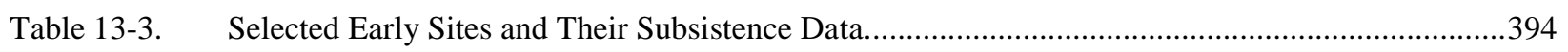

Table B-1. Flotation Sample Summaries for 41TV2161 (includes only floated samples)..............................546

Table B-2. Plant Materials Identified in the Flotation Samples.................................................................547

Table B-3. Plants Identified in the Macrobotanical Samples.......................................................................549

Table C-1. $\quad$ List and Provenience of Diatom Samples Analyzed from Site 41TV2161 …................................566

Table C-2. Diatoms Found in Sediment and Carbonate Samples from Site 41TV2161 ..................................569

Table C-3. Autecological Characteristics of Diatoms Found in 41TV2161 ...................................................572

Table C-4. Glossary of Diatom Attributes and Water Quality Terms.............................................................577

Table D-1. Results for the Bell/Andice (B/A) Component. ...........................................................................589

Table D-2. Results for the Martindale/Corner-Notched (M/CN) Component and Unassigned Materials. .......590

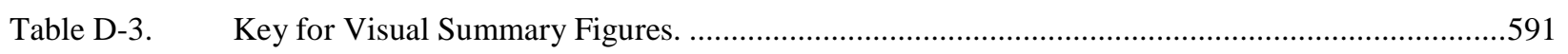

Table E-1. Big Hole Site (41TV2161) Sediment Samples. ...........................................................................602

Table E-2. Bone Fragments Observed in the Sand Fractions. ....................................................................612

Table E-3. Flood Plain Control Sample Phytolith Signature (Normalized \%). ................................................629

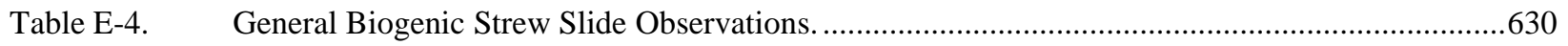

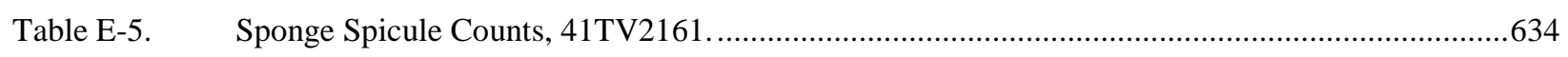

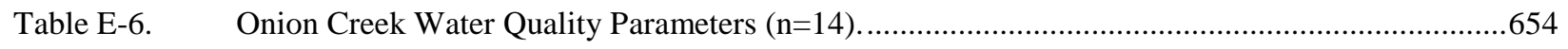

Table F-1. Starch Remains Recovered from Site 41TV2161 ...................................................................675

Table G-1. Summary of Average Fatty Acid Compositions of Modern Food Groups Generated by Hierarchical Cluster Analysis.

Table G-2. Criteria for the Identification of Archaeological Residues Based on the Decomposition Patterns of Experimental Cooking Residues Prepared in Pottery Vessels...................................................695

Table G-3. Known Food Sources for Different Types of Decomposed Residues............................................695

Table G-4. List of Ground Stone Tool and Burned Rock Samples Analyzed in 2007....................................696

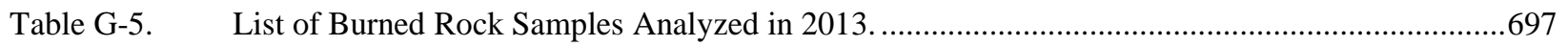

Table G-6. Lipid Compositions and Identifications of Residues from Site 41TV2161 .................................698

Table G-7. Biomarker and TAG Distributions of Lipid Residues with Insufficient Fatty Acids......................704

Table G-8. Residues from the Bell/Andice (Upper) Component. .....................................................................705

Table G-9. Residues from the Martindale (Lower) Component. .......................................................................706

Table H-1. Prior Studies of Texas Cherts Conducted by NAA at MURR. ...................................................720

Table H- 2. Recovery Contexts for Specimens in the Present Sample of Chert from Central Texas. .................720

Table H-3. RQ-Mode Principal Component Analysis of Chert Specimens from Central Texas. The First 10 Principal Components are Shown, Accounting for Greater than $90 \%$ of the Cumulative Variance in the Dataset. Bold Values Indicate Significant Loading on a Component...

Table H-4. Mahalanobis-Distance-Based Group-Membership Probabilities for Artifact Specimens from 41TV2161. Mahalanobis Distances Calculated Using Seven Discriminant Functions Calculated for all Source Groups in the Edwards Formation Database (excepting those 
from Fort Hood) and Including the Newly Analyzed Gravel Specimens as an Unique Source. Highest Probabilities of Membership are shown in Bold. Note that most specimens have a High Probability of Belonging to the Source Gravel Group.

Table I-1. Elemental Concentrations for the Artifacts and USGS RGM-1 by Site. ......................................747

Table K-1. Results of Presence/Absence Analysis of Phytoliths at 41TV2161 .............................................757

Table L-1. Preservation and Concentration of Pollen in Samples from 41TV2161 ......................................762

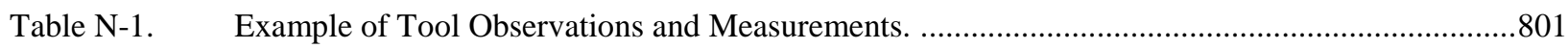


This page intentionally left blank. 


\section{ACKNOWLEDGEMENTS}

Many individuals and institutions assisted the authors in completing this project. I thank all participants for their diverse contributions, no matter how large or small it may appear to others, as each and every contribution was necessary to complete these analyses and the writing of this technical report.

This data recovery project was under the overall direction of the Environmental (ENV) Affairs Division of the Texas Department of Transportation (TxDOT) and many TxDOT individuals helped guide and direct this effort over the past 10 years. At the project initiation in 2006, Dr. Nancy A. Kenmotsu was the Director of the Cultural Resource Management Section, with Dr. G. Lain Ellis Supervisor of the Archeological Studies Branch, followed by the existing Supervisor Dr. Scott Pletka, who guided this program to its completion. Dr. Kenmotsu initiated and conducted contract talks and negotiations with TRC Environmental Corporation (TRC) archeological staff. Dr. Ellis secured the appropriate construction maps from Lone Star Infrastructure, corresponded with the Texas Historical Commission (THC), and was a conduit for passing information to TRC.

Mr. Jon Budd, District Archeologist, was responsible for this project and facilitated communications between agencies and TRC, prepared the initial and many subsequent Work Authorizations, and supplementals that followed. He also contributed to many meetings held during this long multiple step process that lead to the analyses and production of this final report. Dr. James Abbott, TxDOT geoarcheologist, visited the site on multiple occasions during the 2006 fieldwork, commented on the 2007 interim report, reviewed and commented on multiple requests for dating radiocarbon samples in 2006, 2007 and again in 2014, approved the samples, facilitated the delivering the samples to the radiocarbon laboratory, and commented on the final draft of this report. Multiple TxDOT staff archeologists visited the site during fieldwork on one or more occasions.

Jason Buntz of Lone Star Infrastructure facilitated an initial meeting between the parties involved, and provided maps and location data of previous trenches. Lone Star Infrastructure hosted a prefield meeting and safety session attended by the various parties. The company, under Dan Young's direction, also provided the massive excavation and hauling equipment, plus operators to dig more than $2 \mathrm{~m}$ to our selected target zone, which created the big hole from which our hand-excavations proceeded.

Geo-Marine Inc. of Plano was kind enough to allow then staff archeologist and geophysical specialist Tiffany Osburn to conduct the 2006 ground penetrating radar work across the stripped surface and provide her expert opinion and direction as to which anomalies most likely would yield cultural features. Thank you Tiffany for your dedication to the fieldwork and providing your technical expertise concerning the best potential anomalies. Subsequently, in 2015 Tiffany wrote the chapter concerning her earlier geophysical work.

TRC field archeologists are thanked for their hard work and dedication to the various tasks in retrieving and recording the necessary data in a timely and precise manner, and surviving the very muddy conditions following our "mini flood" in April 2006. The crew included Charles Bludau, Josh Haefner, Michael Kirby, Lisa Kraus, Kendra DuBois, David Peyton, Matthew Senn, Greg Sundborg, Sarah Terry, Jennifer Williamson, under the field direction of Mike Quigg and Paul Matchen.

Eric Schroeder of Applied Archeological Sciences, Inc. in Cedar Creek served as the geoarcheologist and conducted the field inspection, soil 
descriptions, and contributed the geoarcheological chapter. Subsequently, Dr. Charles Frederick, an independent geoarcheologist from Dublin, Texas provided insights into the soils and stratigraphy from his previous work in the immediate area, and was the principal author for the geoarcheological chapter.

In the TRC Austin laboratory, former Laboratory Director Kendra DuBois initially processed the artifacts, created and maintained the electronic database, and managed the paper records through the end of the interim report. She also produced a number of the figures used in the interim report. Her diverse skills and organization aided in the successful completion of the interim report. Two excellent laboratory assistants, David Yelacic and Shelly Fischbeck, performed multiple tasks in sorting, assessing, crossing checking, and organizing the artifacts and paper records. Subsequently, during the final stages of data analyses and reporting phase, Trisha-Ann Gonzales also conducted numerous and diverse laboratory tasks. She helped in sample preparation, documentation and packaging of the materials sent for technical analyses, maintained and constantly updated the database, performed various database searches, created numerous tables, and also prepared the materials and documents for curation. Shannon Gray, current Laboratory Director, performed data verification and database management tasks, and drafted and finalized many drawings and tables used in the final report. She also performed editing and proofreading tasks for the draft final report. Steve Miller (formally with TRC) arranged and oversaw the delivery of soil samples to e-Lab Analytical, Inc., in Houston. Meghan Bruckse Bury sorted multiple heavy fractions from the flotation process, used her artistic skills in drawing the Big Sandy and Bell points scar by scar, skillfully accentuated and make the stone boiling sketch come to life, plus conducted many editing tasks to various sections and chapters during the preparations of the draft chapters.
Dr. Robert Ricklis and Paul Matchen contributed significantly to multiple drafts and final research design, multiple meetings with TxDOT staff, and letter reports concerning the direction of the research to guide analyses. Paul Matchen contributed to several chapters of the report, and edited various drafts for content. Benjamin Bury employed his lithic expertize and conducted the detailed lithic debitage analysis and wrote the two component lithic analysis sections, wrote the comparisons of the two component lithic assemblages. Barrett Clark, former biologist for TRC, conducted the mussel shell identifications and is thanked for his knowledge and expertise. He also provided plant identifications and educated me concerning plants. Ashleigh Knapp formatted the draft and final reports, conducted multiple edits on various chapters, and oversaw final document production. She also exchanged ideas and commented of the direction of the draft report and provided helpful comments to the lead author.

Many professional individuals and technical institutions also supported this project by conducting highly specialized technical analyses on diverse samples. Without their individual skills and knowledge, many specific aspects of the prehistoric peoples represented at these components would not have been discovered.

I thank artist/school teacher Shayne Tolman of Cardston, Alberta, Canada for allowing me to use his exquisite watercolor paintings of bone grease extraction in this report.

Geoarcheologist Dr. Charles Frederick is thanked for his thorough understanding and documentation of the lower Onion Creek valley deposits and his helpful comments and directions following the fieldwork. Dr. Frederick's contributions to the geoarcheological chapter are greatly appreciated.

Projectile point identification specialist and renowned Texas archeologist Elton Prewitt of Austin was kind enough to verify the types of 
projectile points recovered. Thank you Elton for your time, insights and comments.

Dr. Phil Dering, of Shumla Archeobotanical Services in Comstock, Texas, conducted the flotation of the initial sediment samples and performed individual macrobotanical identification of the charcoal and plant remains, wrote an appendix for the 2007 interim report, and also wrote Appendix $\mathrm{H}$ in this final report.

Dr. Steven Bozarth, of Lawrence, Kansas, conducted the initial presence/absence of phytolith samples in the sediments, made recommendation for further analysis and provided his results and recommendations in Appendix $\mathrm{K}$. $\mathrm{He}$ also conducted the processing of samples to assess the presence/absence of pollen in samples with his findings presented in Appendix L.

Two soil laboratories provided their technical expertise for conducting chemically analyses of feature sediments. Thank you Hector Coronado of e-Lab Analytical, Inc. of Houston, and Mary Schabel of Milwaukee Soil Laboratory, LLC for your skills and dedication to your work, and supplying the data results presented in Appendix M.

Dr. Linda Perry, Executive Director of The Foundation for Archaeobotanical Research in Microfossils, in Fairfax, Virginia conducted the starch grain analysis and wrote Appendix D. Her continued interest and support towards a greater understanding of the microfossil record in archeological sites is commended. I also thank Linda for her willingness to answer my multitude of questions and continue to educate me on the various complexities of starch grains.

Dr. Byron Sudbury, at J. S. Enterprises in Oklahoma, conducted the detailed phytolith analysis and wrote Appendix F. His dedication to the science is a tribute to his interest in the past. Thank you Byron for taking the time to clarify many aspects of the phytolith assemblage for me.
Dr. Bruce Hardy, professor in the Department of Anthropology at Kenyon College in Ohio, is thanked for his expertise in conducting the highpowered microscopic use-wear analyses and his tremendous experience in identifying microfossils on selected chipped stone tools. He reported his findings in Appendix D.

Dr. M. Steven Shackley, Director Geoarchaeological XRF Laboratory Albuquerque, New Mexico is thanked for his X-ray analysis of two rocks covered in resides and his results are presented Appendix I.

I thank the scientists at Wildlife Analytical Labs, LLC in Burnet, Texas for their willingness and attempt at determining the age of two very old deer incisors. Their aging process involves the cementum annuli method, but the teeth failed to retain any signs of growth rings. Their methods are presented in Appendix $\mathbf{J}$.

I would also like to thank Dr. Dean A. Hendrickson, Curator of Ichthyology, University of Texas at Austin, Texas Natural Science Center, Texas Natural History Collections, in Austin and Collections Manager J. Chris Sagebiel for their information, comments, directions, and possible use of those facilities for identification of prehistoric fish vertebrae. Mr. Sagebiel commented that fish vertebrae are not usually diagnostic below the ordinal level and saved TRC considerable time and effort in attempts of identifications.

Dr. Chris Lintz of the Parks and Wildlife Department is thanked for facilitating and identifying fish experts, and passing along fish vertebrae to individuals for possible identification. A few fish vertebrae were eventually passed to Bob Betsill, Research Program Director, Inland Fisheries Division for Texas Parks and Wildlife and he reviewed and commented on the small specimens. He commented that no one on their staff feels confident in identifying these vertebrae 
to species. Thank you all for your willingness to review the samples and provide comments.

Dr. Leland C. Bement of Oklahoma Archeological Survey is thanked for his identification of deer incisors from photographs presented to him. Following his direct comparison with their collections he commented "my best fit is with a 5+ year old whitetail". Most mule deer incisors are broader at the occlusal surface. Thank you Lee for you time and effort to help out in the identifications.

Dr. Carl R. Falk, a renowned zooarchaeologist for Paleocultural Research Group, in Pennsylvania was kind enough to examine a couple photographs of bird bones and provide his expert opinion on what species they represented. Thank you Carl for your willingness to advise me on these rare and fragmentary elements.
The technical staff at Beta Analytic Inc. in Florida, especially the helpful comments and professional direction received from Ron Hatfield, is thanked for their expertise in handling the multiple and diverse samples submitted for radiocarbon dating and providing technical advice along the way. I also want to thank the scientific staff for their dedication to providing solid results in a timely manner, with the final results presented in Appendix A.

I thank these many skilled individuals and institutions for their professionalism and willingness to partner with TRC and conduct specific technical analyses for this project. Their collective knowledge and dedication within their areas of expertise made significant contributions to furthering our understanding of prehistoric populations in central Texas during the middle Holocene, and they are thanked for their efforts.

\section{Mike Quigg}

Project Manager 


\subsection{INTRODUCTION}

\author{
J. Michael Quigg
}

\section{$1.1 \quad$ INTRODUCTION}

This technical report describes the methods, findings, results, and interpretations from data recovery excavations at the Big Hole site (41TV2161) in eastern Travis County, Texas (CSJ: 0440-06-006). During April and May 2006, TRC Environmental Corporation (TRC) archeologists conducted hand-excavations at this deeply buried, multi-component prehistoric cultural resource site. This project was sponsored by the Texas Department of Transportation (TxDOT). Investigations were necessary to mitigate negative impacts from the construction of State Highway 130 (SH 130), which were part of TxDOT responsibilities under the National Historic Preservation Act (NHPA) of 1966, as amended through 1992 (PL-89-665; 80 Stat. 915; 16 U.S.C. $\S 470$ et seq.), the Department of Transportation Act of 1966 (PL 89-670), and the Antiquities Code of Texas (Texas Natural Resource Code, Title 9, Chapter 191 as amended). This project was conducted on an accelerated schedule driven by the design-build plan for SH 130, a fact that shaped many of the decisions made during the course of the excavation and analysis.

TxDOT issued a Work Authorization (No. 57511SA008) to the Planning, Permitting and Licensing (PPL) practice of TRC's Austin office under TxDOT Scientific Services Contract No. 575XXSA008 to conduct data recovery excavations prior to direct impact from this road construction project. TRC's investigations were also conducted under a Texas Antiquities Committee (TAC) Permit No. 4064, issued by the Texas Historical Commission (THC) to Principal Investigator J. Michael Quigg.

\subsection{SITE AND PROJECT LOCATION}

This prehistoric site lies in southeastern Travis County, just east of Austin-Bergstrom International Airport (Figure 1-1). It sits in a low, broad, deep alluvial terrace on the right, descending bank of the lower Onion Creek valley. The creek flows northeastward from the Edwards Plateau on to the Blackland Prairie and into the Colorado River about six kilometers $(\mathrm{km})$ downstream. This expansive alluvial terrace is flat with a few shallow swales at elevations between 137 and 140 meters (m) above mean sea level (amsl). The eastern valley margin is marked by a sloping hill that extends east, northeastward. Pleistocene gravel deposits cap the ridge and higher terraces. Approximately 1,220 m northwest of the site, the channel of Onion Creek is entrenched into the alluvial surface, and lies at an elevation some $15 \mathrm{~m}$ below the elevation of the terrace surface of site 41TV2161. This valley exhibits bedrock exposures of soft mudstones and marl as part of the Sprinkle Formation of the Upper Cretaceous Taylor Group (Barnes 1974, 1981). Site 41TV2161 lies in a shallow swale near what once was a meander channel or loop of Onion Creek that is currently filled. Soils across the site are classified as Lewisville silty clays (Werchan et al. 1974).

Prior to road construction activities, the historical land use was dominated by intensive cultivation and pasture land. Those activities followed land clearing that undoubtedly removed much of the woodlands along and throughout the Onion Creek valley. Riparian woodlands currently exist only along the very margins of Onion Creek and along number of property and pasture boundaries. The Big Hole site was discovered in a cattle pasture. Nearby land use includes gravel mining, specifically a gravel mine just south of the site in Onion Creek valley. 


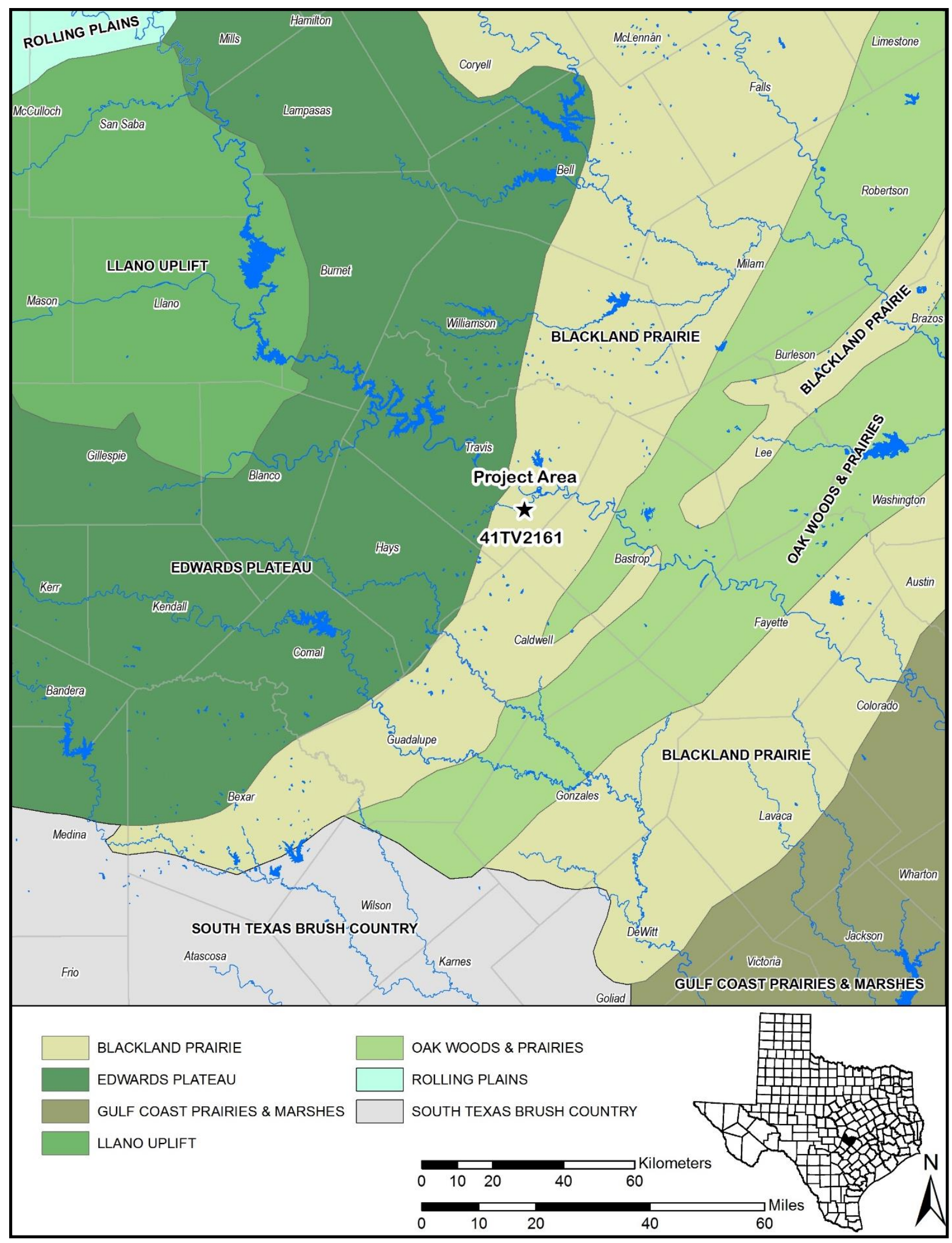

Figure 1-1. General site location in Travis County, Texas. 


\subsection{PROJECT BACKGROUND}

From November 1, 2005 through January 26, 2006, an archeological crew from Hicks \& Company conducted and intensive cultural resource survey of 2.08 ha (5.16 ac.) in parcels 365 and 365E south of Pearce Lane. This survey along proposed SH 130 right-of-way in eastern Travis County was part of the required investigation prior to development of SH 130 (Campbell et al. 2007). Following the initial discovery of prehistoric site 41TV2161, investigations were expanded westward across the SH 130 right-of-way. In total, Hicks \& Company personnel excavated 10 backhoe trenches (BTs), 13 shovel tests (STs), and 11 test units (TUs) into site 41TV2161. Investigations yielded positive results, with at least seven buried cultural features and 1,034 artifacts discovered, mostly in good context throughout the $3.5 \mathrm{~m}$ deep deposits investigated (Table 1-1). In a short draft archeological survey report to TxDOT, the results and site recommendations were presented (Campbell et al. 2006). The Environmental (ENV) Affairs Division of TxDOT and the THC reviewed the recommendations and determined site 41TV2161 was eligible for listing in the National Register of Historic Places (NRHP) and as State Antiquities Landmark (SAL).

In March 2006, ENV Affairs Division of TxDOT contracted with TRC to conduct mitigation excavations at a small portion (maximum $40 \mathrm{~m}^{3}$ ) of site 41TV2161 prior to any disturbance from proposed road construction activities. TxDOT personnel informed TRC archeologists the upper deposits contained vertically dispersed cultural materials in generally poor context and data recovery should focus on one or more deeply buried cultural zones with better context. Consequently, our objective was to try an isolate one narrow vertical zone and extract information concerning human behaviors represented during that time.
TRC archeologists reviewed and assessed Hicks \& Company interim report (Campbell et al. 2006) to identify one zone with good context and potential to yield important information. A concentration of cultural materials and intact burned rock features were apparent in a relatively narrow 30 to $50 \mathrm{~cm}$ thick zone between 250 and $300 \mathrm{cmbs}$, particularly in BTs 21 and 25 (Figure 1-2). This roughly $50 \mathrm{~cm}$ thick zone became the target for the data recovery plan submitted by TRC and approved by TxDOT.

The construction of a large concrete box culvert crossing the northern part of 41TV2161 was scheduled at the same time as the archeological excavations (see Figure 1-2). Therefore, the proposed target block for hand-excavations was positioned along the western side of the site (south of BTs 22 and 24, north of BTs 21 and 25) at a safe distance from the culvert construction. TRC proposed to mechanically strip a large block area down to the top of the targeted cultural zone (ca. 240 to $250 \mathrm{cmbs}$ ), then hand-excavate horizontal blocks in order to recover cultural remains from that zone. This excavation plan was designed to uncover broad activity areas to facilitate interpretation of human behaviors.

Following the completion of the 2006 fieldwork, numerous Work Authorizations were issued by TXDOT to TRC for multiple stages of investigation. The initial stages of investigation included the development of an interim report concerning the findings from the data recovery, combined results from six feasibility studies and soil chemistry (Quigg et al. 2007), an annotated bibliography focused on the time represented by the cultural materials recovered during data recovery, and a detailed research design to guide analyses. Subsequent Work Authorizations were issued for additional technical analyses, final artifact analyses, and preparation of the draft and final reports, and curation of materials. 
Table 1-1. Summary of Trench and Unit Data from Site Discovery by Hicks \& Company (from Campbell et al. 2007).

\begin{tabular}{|c|c|c|c|c|}
\hline Unit, Size in $\mathbf{c m}$ & $\begin{array}{l}\text { Depth } \\
\text { (cmbs) }\end{array}$ & $\begin{array}{l}\text { Cultural } \\
\text { Material } \\
\text { Present }\end{array}$ & $\begin{array}{l}\text { Artifact Depths } \\
\text { (cmbs) }\end{array}$ & $\begin{array}{c}\text { Total } \\
\text { Artifacts } \\
\text { from Unit }\end{array}$ \\
\hline BT 3 & $0-675$ & Positive & $0-90$ & 184 \\
\hline TU-F $50 \times 50$ & $0-120$ & Positive & $30-70$ & 3 \\
\hline TU-G $100 \times 100$ & $35-80$ & Positive & $35-70$ & Feat 1,168 \\
\hline BT 4 & $0-169$ & Positive & $40-150$ & 705 \\
\hline TU-I $50 \times 50$ & $0-80$ & Positive & $60-80$ & 79 \\
\hline TU-J $50 \times 50$ & $64-160$ & Positive & $40-150$ & 57 \\
\hline BT 5 & & Positive & $40-80$ & 2 \\
\hline BT 20 & $0-145$ & Positive & & 84 \\
\hline TU-1 $50 \times 50$ & $0-145$ & Positive & $40-80$ & \\
\hline BT 21 & $0-273$ & Positive & $60-290$ & 33 \\
\hline TU-2 $50 \times 50$ & $126-128$ & Positive & $126-228,220-275$ & Feat 2,4 \\
\hline TU-6 $100 \times 100$ & $267-296$ & Positive & $267-296$ & Feat 2 \\
\hline TU $4 \quad 100 \times 50$ & $251-254$ & Positive & $247-276$ & Feat 4 \\
\hline BT 22 & $0-250$ & Positive & $190-230$ & Feat 3 \\
\hline TU-3 100x100 & $180-235$ & Positive & $200-230$ & 262 , Feat 3 \\
\hline BT 23 & & Negative & & 0 \\
\hline BT 24 & $0-260$ & Positive & $175,235,260$ & 3 \\
\hline BT 25 & $0-353$ & Positive & $173,210-353$ & $\begin{array}{c}45 \text {, Feat } 5,6 \text {, } \\
7\end{array}$ \\
\hline TU-5 $100 \times 100$ & $112-194$ & Positive & 170- 175 & Bones \\
\hline TU-7 100x100 & 244-260 & Positive & $244-260,353$ & Feat5, 6, 7, \\
\hline BT 26 & & Negative & & \\
\hline
\end{tabular}

$\mathrm{BT}=$ backhoe trench; $\mathrm{TU}=$ test unit; cmbs = centimeters below surface, Feat $=$ Feature

\subsection{CONTENTS OF REPORT}

Following this introductory chapter, Chapter 2.0 presents a brief overview of the modern environmental setting and a broad regional paleoenvironmental section for the projected time represented by the investigated cultural components. Chapter 3.0 provides an in-depth, regional review of the Bell/Andice and Martindale intervals for cultural background of the recovered cultural materials. Chapter 4.0 presents the research design that guided and directed the subsequent analyses and reporting. Chapter 5.0 describes the field methods implemented in 2006, plus the subsequent laboratory procedures, terminology employed, and analytical techniques conducted to extract data from the findings. Chapter 6.0 addresses the geoarcheology identified at the Big Hole site and surrounding area. The geophysical investigation conducted prior to handexcavations is presented in Chapter 7.0. Chapter 8.0 provides the cultural stratigraphy, context, and 


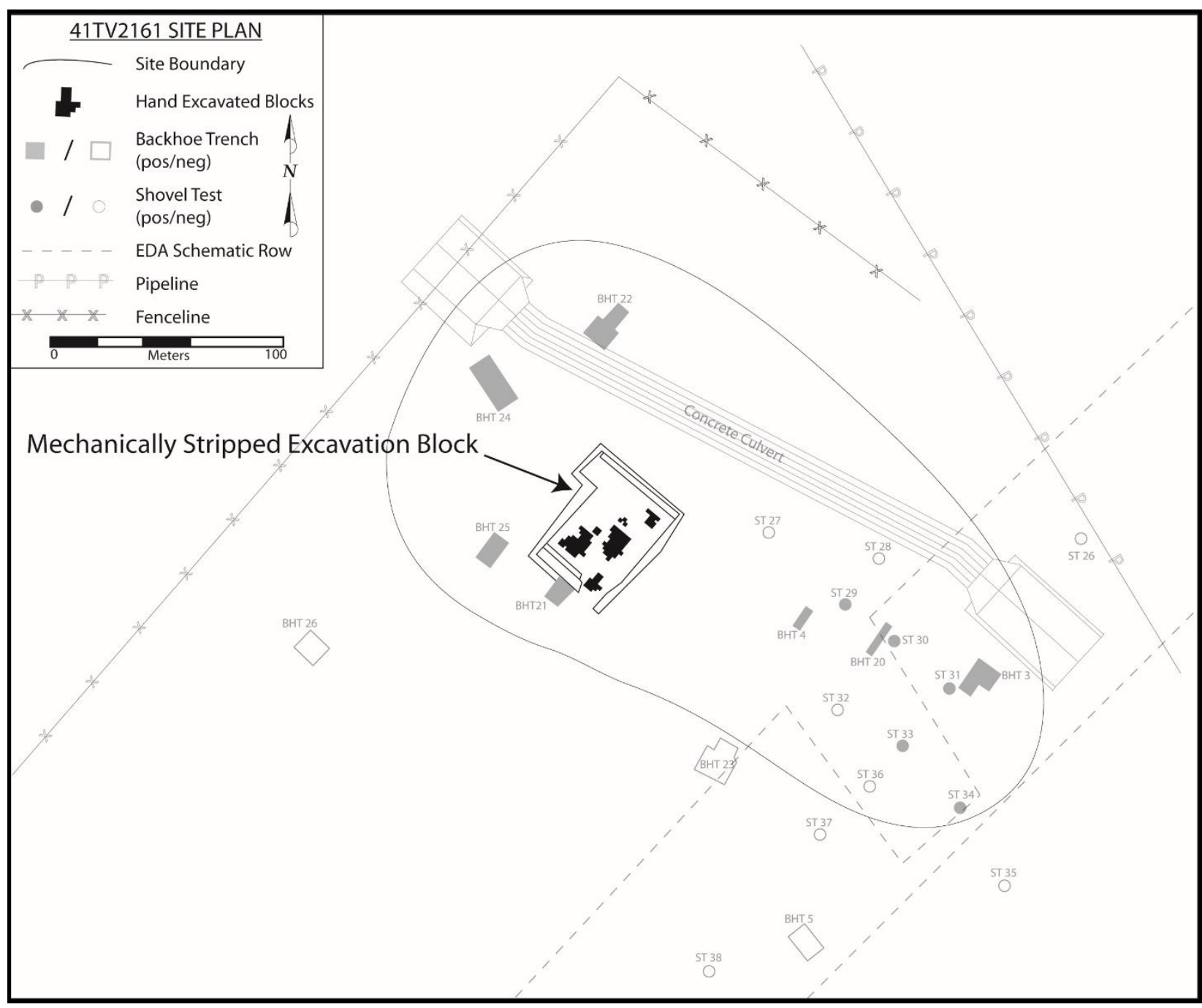

Figure 1-2. General location of hand-excavations within the mechanically stripped block in relations to Hicks \& Company previous investigations.

discusses the assigned analytical units used in the analysis and reporting. Data and interpretations concerning the Bell/Andice component are presented in Chapter 9.0. This is followed by data presentations and interpretations concerning the Martindale/Early Corner-notched component in Chapter 10.0. Chapter 11.0 compares the results from and presents discussions concerning the two identified components. Unassigned cultural materials are presented in Chapter 12.0. A summary, discussion, and conclusions are presented in Chapter 13.0. Chapter 14.0 provides recommendations. These chapters are followed by a list of references cited throughout the document.
Finally, a glossary of technical terms used in this report, which may not be familiar to all potential readers is presented.

Following the glossary, 15 appendices (designated A through O) are presented. These provide detailed data by technical experts who served as subconsultants and address specific types of analyses. Appendix A presents the radiocarbon laboratory results, the procedures used in the dating process, and individual results from Beta Analytic Inc. Macrobotanical data and procedures employed by Dr. Phil Dering are presented in Appendix B. Dr. Barbara Winsborough provides the diatom 
procedures and results in Appendix C. Appendix D contains the processing procedures and individual results of high-powered microscopic lithic usewear analysis performed on stone tools by Dr. Bruce Hardy. Appendix E presents the procedures and findings from the phytolith analysis on selected samples by Dr. Byron Sudbury. Appendix F presents the detailed procedures, handling, and individual results for artifacts subjected to starch grain analysis by Dr. Linda Perry. The lipid residue analyses procedures and results are presented in Appendix G. The procedures and results from instrumental neutron activation analysis conducted by the University of Missouri are provided in Appendix H. Dr. Steven Shackley preformed X-ray fluorescence (XRF) analysis on two rocks and the results are provided in Appendix I. Appendix $\mathbf{J}$ provides the results of an attempt to determine the season of death from two deer incisor. Dr. Steven Bozarth provides presence/absence results for the initial phytolith assessment in Appendix K. Dr. Bozarth methods and presence/absence findings concerning pollen are presented in Appendix L. Appendix $M$ provides the findings from two difference laboratories concerning analyses of the dark stained soil samples submitted. Appendix N is the "TxDOT Lithic Protocol Version 2.1, Chipped Stone Analytical Protocol" followed during lithic analysis. Metric and nonmetric data concerning the Martindale edge-modified flakes is presented in Appendix O. 


\subsection{ENVIRONMENTAL SETTING}

\author{
J. Michael Quigg
}

\subsection{PHYSIOGRAPHY AND HYDROLOGY}

Travis County is located along the eastern edge of the Edwards Plateau, an upland region with hilly to rolling topography demarcated by the Balcones Escarpment on the eastern side. The Big Hole site (41TV2161), which is located in eastern Travis County, lies near the escarpment and ecotonal boundary in the Blackland Prairie region. The Blackland Prairie ecological region, a relatively flat and narrow zone, parallels the Balcones Escarpment and extends northward to the Red River (see Figure 1-1). The Blackland Prairie is characterized by tall grasses that are the southern extension of the True Prairie of the Midwest, and it is a western inclusion of the Gulf Coast section of the Coastal Plain Physiographic Province (Fenneman 1931).

This funnel-shaped region expands from south to north with to the widest segment at the Red River in northern Texas. The Blackland Prairie is bordered by the Balcones Escarpment to the west and the Oak Woodlands to the east. Elevations across the region range from 91 to $244 \mathrm{~m}$ (300 to $800 \mathrm{ft}$.) above sea level. Blair (1950) considers this region within the Texan Biotic Province, an ecozone with interdigitating woodland and prairie, wherein the Blackland is a major prairie component. The Blackland Prairie is dissected by numerous creeks and rivers flowing eastwards from the Edwards Plateau.

Onion Creek flows eastward from the Edwards Plateau, cuts through the Balcones Escarpment fault zone and onto the Blackland Prairie (see Figure 1-1). The Big Hole site lies in the lower Onion Creek drainage basin nearly $6 \mathrm{~km}$ upstream from the confluence with the Colorado River. The site is located on a low, broad, flat alluvial terrace with shallow swales and elevations between 137 and $140 \mathrm{~m}$ (449.5 and $460 \mathrm{ft}$.) above mean sea level (Figure 2-1). The site occupies a filled swale near what was previously a meander channel or loop of Onion Creek. The entrenched Onion Creek is 1.2 $\mathrm{km}$ northwest and roughly $15 \mathrm{~m}$ lower in elevation than the site surface. The Big Hole site now lies under SH 130 east of the Austin Bergstrom International Airport, constructed after archeological mitigation clearance for this project.

\subsection{GEOLOGY AND QUATERNARY STRATIGRAPHY}

The Big Hole site is in Quaternary (Qt) deposits that fill the lower Onion Creek valley (Barnes 1974, 1981). The eastern valley wall consists of the Navarro Group and Marlbrook Marl (Knb) (Figure 2-2). Recent archeological and geoarcheological investigations in this region (e.g., Blum 1992; Campbell et al. 2005, 2007; Frederick 2006, 2011) have unequivocally demonstrated this age interpretation is incorrect in many areas, and the Bureau of Economic Geology has miss-mapped the majority of the Holocene-age deposits as Pleistocene. West of the valley is higher fluviatile terrace with gravel deposits (Qhg), and to the southwest, a small prominent knoll of Cretaceous igneous rocks (Ki), locally known as Pilot Knob. The high gravel deposits commonly expose surface gravels (Barnes 1974, 1981). The high upland gravel deposit immediately northwest of the Big Hole site was sampled for cherts to compare to the prehistoric cherts recovered from the site utilizing Instrumental Neutron Activation Analyses (see Appendix H).

Frederick (2011) investigated and recorded the stratigraphy of 12 localities just north of the Big Hole site. His investigations included the sampling of various exposures for particle size analysis, calcium carbonate ratio analysis, magnetic susceptibility analysis, and organic carbon and total 


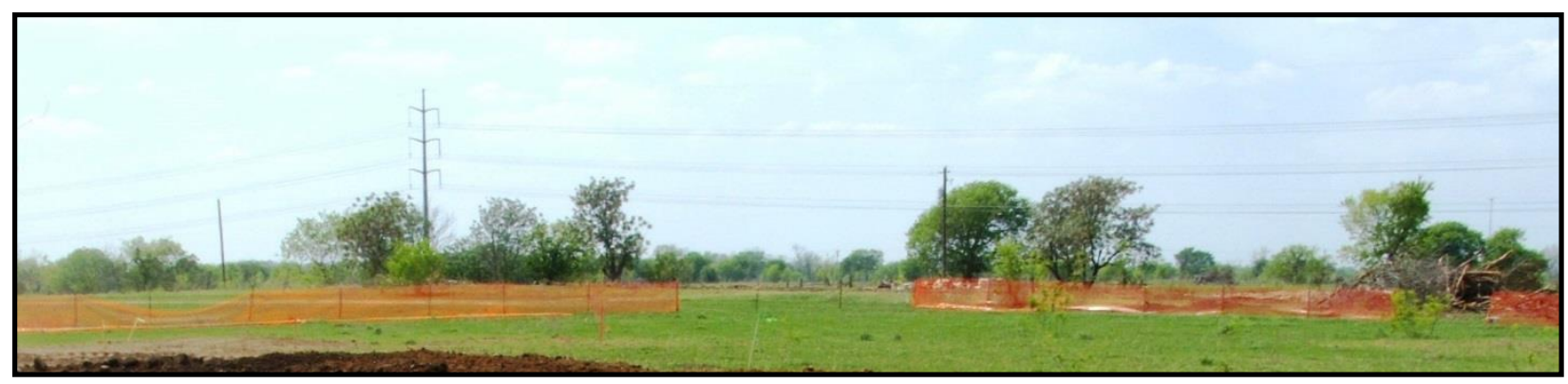

Figure 2-1. General site environment across expansive, flat alluvial terrace of lower Onion Creek valley. Landscape has been altered in historic times.

Figure 2-2. Geology of the area surrounding the Big Hole site east of the Austin Bergstrom International Airport (ABIA) in eastern Travis County (after Barnes 1974, 1981).

Figure 2-3. Soils map (after Werchan et al. 1974). 
carbon analyses. Frederick also submitted numerous bulk sediment samples, Rabdotus snail shells, and wood charcoal for radiocarbon analysis. With these results, Frederick devised a new stratigraphic framework for the late Quaternary deposits of lower Onion Creek valley, applicable to the Big Hole site (see Chapter 6.0).

\subsection{SOILS}

The majority of soils in the immediate vicinity of the Big Hole site are classified as Lewisville silty clay (LcB), with 0 to 2 percent slopes that are frequently flooded by Onion Creek (Figure 2-3) (Werchan et al. 1974). The Lewisville series consists of deep, nearly level to gently sloping soils on bottom lands along major streams. These are well-drained and developed in alluvium under tall grasses and sparse trees. A typical profile has a dark grayish-brown (10YR 4/2) silty clay A horizon to a depth of approximately $33 \mathrm{~cm}$ (13 in.). The B horizon is brown (10YR 5/3) silty clay to a depth of approximately $73.7 \mathrm{~cm}$ (29 in.), above a very pale brown (10YR 8/3) silty loam $\mathrm{C}$ horizon. These soils are calcareous and moderately alkaline throughout.

\subsection{CLIMATE}

Travis County has a humid, subtropical climate with short mild winters and long hot summers (Werchan et al. 1974). The monthly averages temperature varies from $11.2^{\circ} \mathrm{C}\left(52^{\circ} \mathrm{F}\right)$ in January, to $31.6^{\circ} \mathrm{C}\left(88.8^{\circ} \mathrm{F}\right)$ in August (Figure 2-4) (Werchan et al. 1974). The growing season averages 180 days annually (April through September). Winter precipitation is mostly in the form of fog, drizzle, and light showers. Spring rains of short duration, with high intensity are frequent (Figure 2-5). Rainfall is usually sufficient to leach calcium carbonate from the uppermost horizons, although not enough to leach it out entirely. Rainfall is distributed throughout the year with the annual average approaching $82.8 \mathrm{~cm}$ (32.6 in.).
Repeated wetting and drying of the clay soils creates cracks that deeply penetrate the profile (Werchan et al. 1974). Consequently, most soils exhibit specks, threads, or nodules of calcium carbonate throughout the profile.

\subsection{FLORA AND FAUNA}

The region is a relatively mesic zone referred to as the Texan biotic province (Blair 1950) (Figure 2-6). The Texan province lies to the east of the Kansan province, a massive mesquite (Prosopis) grassland; the Balconian province, a transition zone centered on the Edwards Plateau; and the Tamaulipan province, a semiarid province with extensive bushlands. The Big Hole site (41TV2161) is located in proximity to the boundaries of the Texan, Balconian, and Tamaulipan provinces; thus, the local area may see a mixture of biota from these adjacent regions.

\subsubsection{Vegetation}

Tharp (1939) designates this area as an extension of the Andropogon-Stipa-Aristida (bluestems, grasses, and three-awns) association of the Tall Grass Prairie. Presently, the bottomlands (floodplains) have had vegetation significantly altered from their original state and are comprised of ground-cover regrowth understory (Kenmotsu 1982). These areas are presently dominated by introduced species such as brome grasses, rescuegrass (Bromus unioloides) and Japanese brome (Bromus japonicas). Canadian wildrye (Elymus canadensis) and Johnson grass (Sorghum halepense) are found in shaded areas by midsummer, along with scattered populations of Texas Wintergrass (Stipa leucotricha). The overstory includes netleaf hackberry (Celtis reticulate), ceder elm (Ulmus crassifolia), pecan (Carya sp.), red ash (Fraxinus pensylvanica), red mulberry (Morus rubra), eastern cottonwood (Populus deltoids), prickly ash (Xanthoxylum clava-herculis), deciduous holly (Ilex decidua), black willow (Salix nigra), osage 


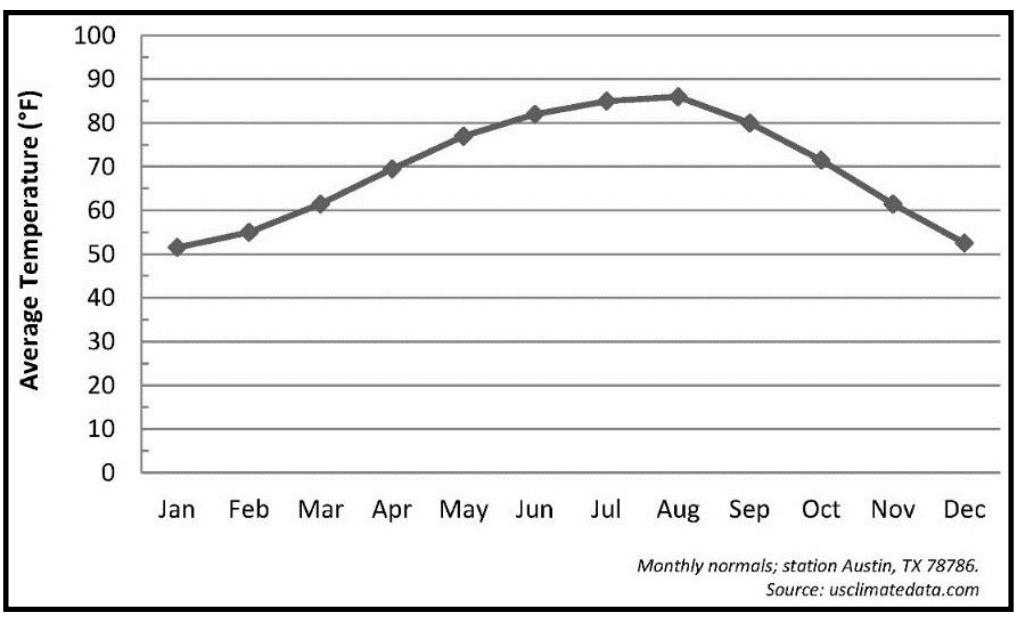

Figure 2-4. Area average temperature in degrees Fahrenheit (usclimatedata.com, after NOAA data).

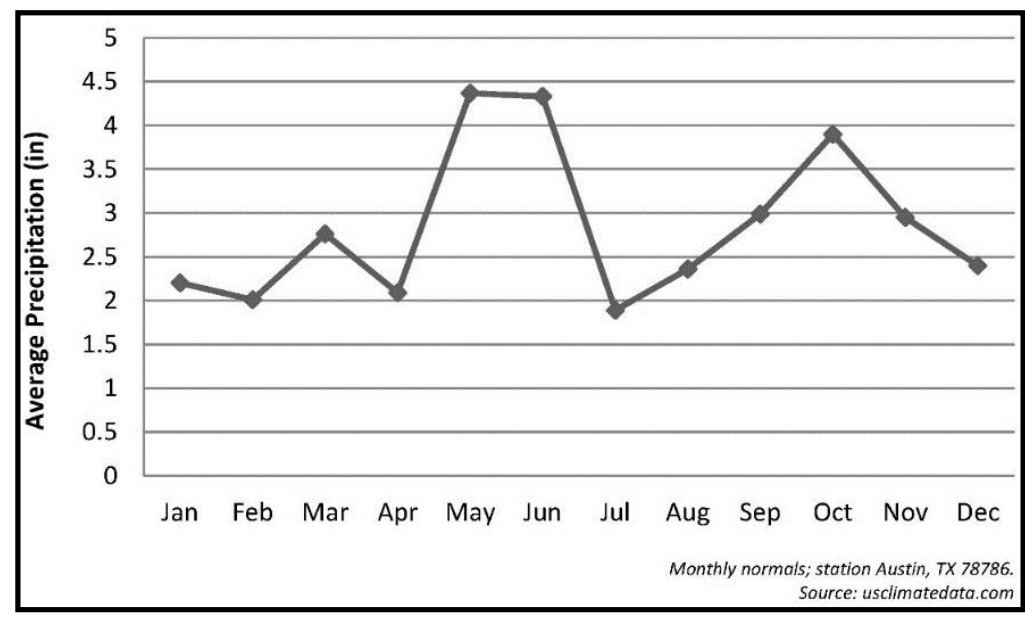

Figure 2-5. Average area precipitation in inches (usclimatedata.com, after NOAA data).

orange (Maclura pomifera), box elder (Acer negundo), soapberry (Sapindus saponaria), and chinaberry (Melia azearach) (Kenmotsu 1982:3$15)$.

The upland prairie assemblages also reflect disturbed conditions with Johnson grass (Sorghum halepense), Roosevelt weed (Baccharis neglecta), hedge parsley (Torilis arvensis), yellow sweet clover (Melilotus officinalis), and silver-leaf nightshade (Solanum elaeagnifolium). Other major species in the area include prairie three-awn (Aristida oligantha) and little bluestem (Schizachyrium scoparium) (Kenmotsu 1982).
Although most discussions of the Blackland Prairie do not provide potential plant food resources, archeobotanists such as Phil Dering (2000b:219) have suggested several geophyte resources in the Blackland Prairie region potentially utilized in prehistoric times (Figures 2-7 and 2-8). These include bulbs of eastern camas (Camassia scilloidies), garlic (Allium spp.), false garlic (Nothoscoardum bivalve), rain lily (Cooperia drummondii), dog's tooth violet (Erythronium albidum), yellow-eyed grass (Hypoxis hirsute), along with tubers such as prairie turnips (Psoralea spp.), groundnut (Apios americana), and spring beauty (Claytonia virginica). 
Gould (1975) lists numerous forbs found in the Blackland Prairie. These include, but are not limited to, bluebonnet (Lupinus texensis), Mexican hat (Ratibida columnaris), sunflower (Helicanthus annuus), Indian paintbrush (Catilleja indivisa), western ragweed (Ambrosia psilostachya), and milkweed (Asclepias sp.).

\subsubsection{Fauna}

As part of the Texan biotic province, Blair (1950:101) lists minimally 49 species of mammals as present in this province. Most species are not restricted to this one province. Common species of mammals include Whitetail deer (Odoccoleos virginianus), Virginia opossums (Didelphis virginiana), eastern mole (Scalopus aquaticus), fox squirrel (Sciurus niger), Louisiana pocket gopher (Geomys breicepus), western harvest mice (Reitbrodontomys fulvescens), White-footed mice (Peromyscus leucopus), hispid cotton rat (Sigmodon hispidus), eastern cottontail (Sylvilagus floridanus), blacktailed jackrabbit (Lepus californicus), and swamp rabbit (Sylvilagus aquaticus). Two species of turtles, ornate box turtle (Terrapene ornata) and Florida box turtles (Terrapene carolina), occur here. Sixteen species of lizards and some 39 species of snakes occur in this province.

One of the largest land mammals across much of North America and throughout the entire Holocene period was the bison (Bison sp.), which was probably present in Texas during much of the Holocene. Alford (1973) indicated bison numbers peaked between 9000 and 6000 B.P., decreased drastically in the mid-Holocene, and increased again 3000 B.P. Bison range and size fluctuations are due to environmental stresses (Alford 1973; Hill et al. 2008; Lewis et al. 2007; McDonald 1981). Geist (1996) discusses the morphological changes in bison due to varying climate and vegetation changes.

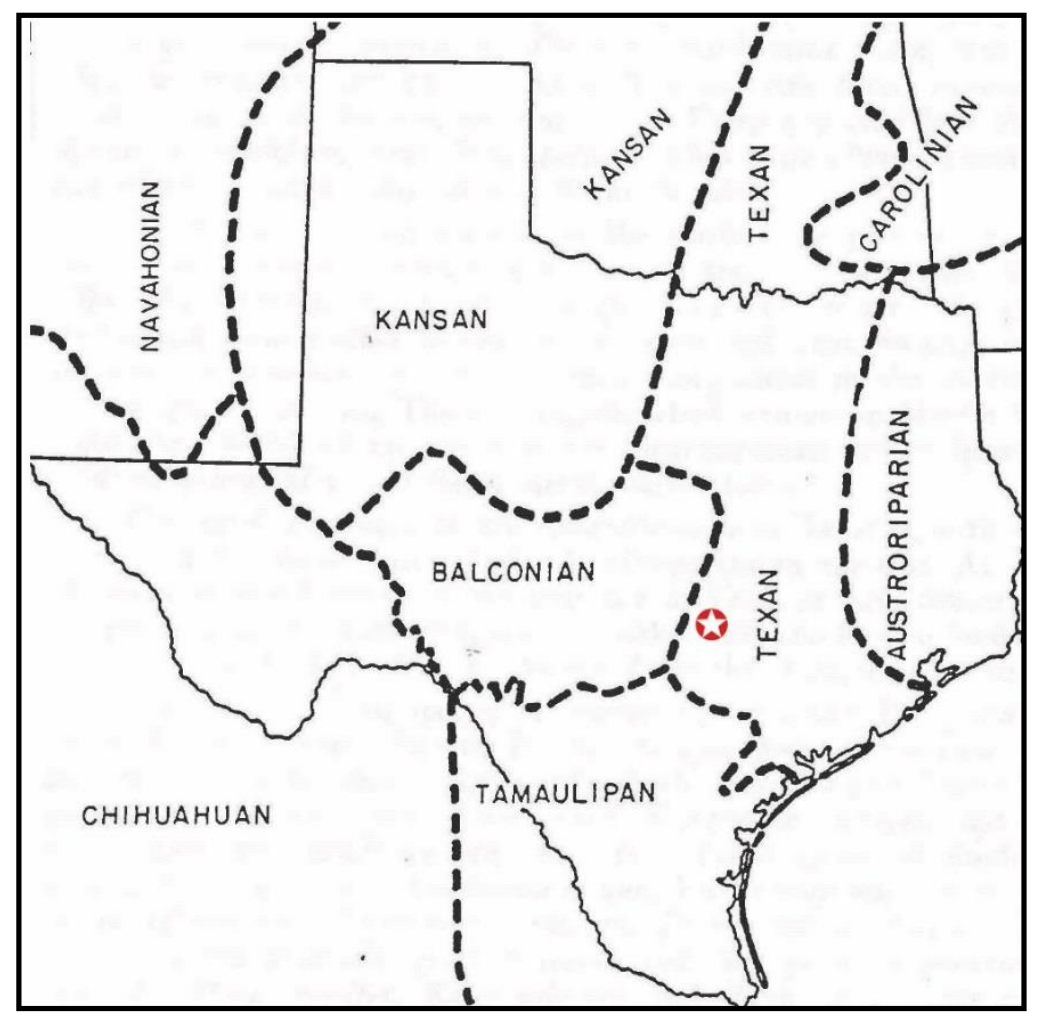

Figure 2-6. Biotic regions across Texas with site location (modified from Blair 1950). 


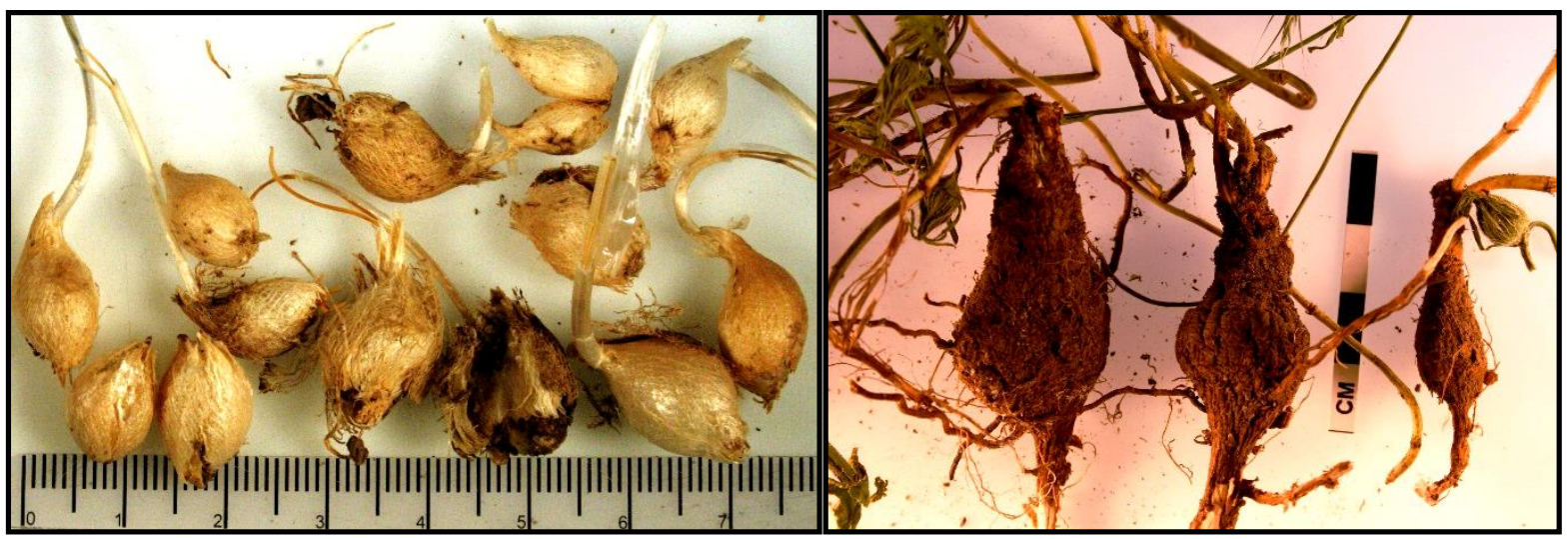

Figure 2-7. Geophytes: wild onion (left) and winecup (right). Scale in centimeters, photographs by M. Quigg.

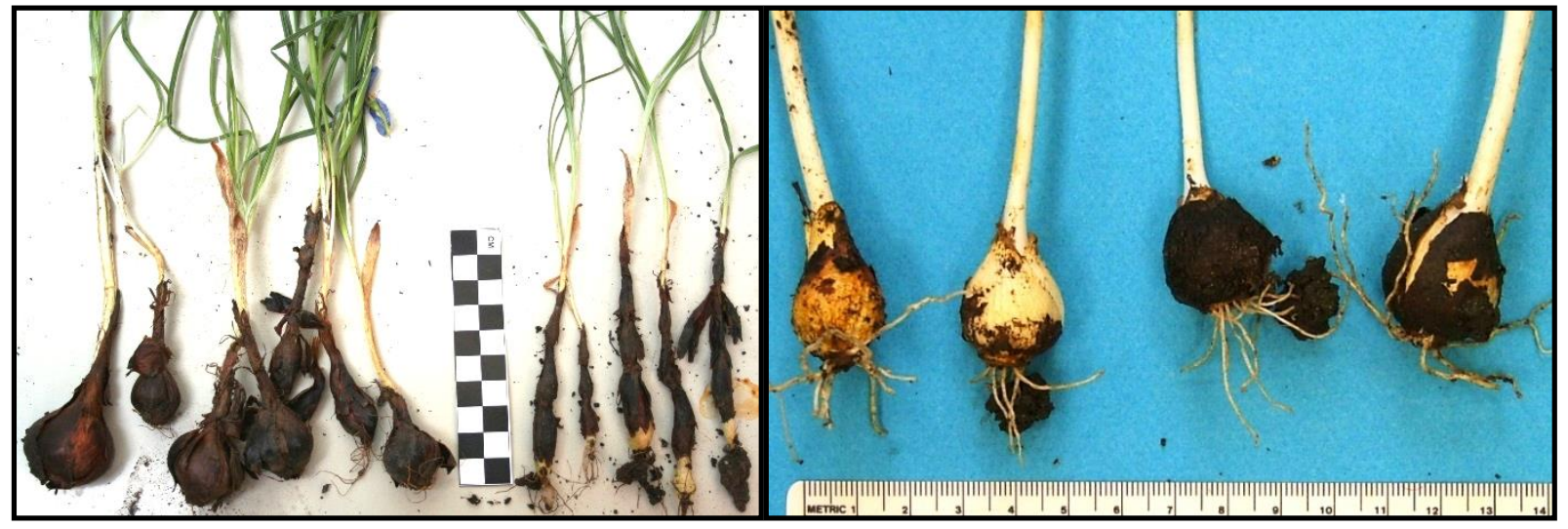

Figure 2-8. Geophytes: prairie pleatleaf (left photo, left side) and false onion (left photo, right side) and eastern camas (right photo). Scale in centimeters, photographs by M. Quigg (left), and A. Thoms (right).

\subsection{PALEOENVIRONMENT AND INDICATIONS OF PALEOCLIMATE DURING THE MIDDLE HOLOCENE}

J. Michael Quigg and Paul M. Matchen

\subsubsection{Introduction}

Paleoenvironmental studies across Texas and adjacent states are spotty and incongruent especially for the middle Holocene period (Figure 2-9). Paleoenvironmental reconstructions are a very complex endeavor with many interpretative pitfalls, potential problems, and interpretative biases to be overcome. Reconstructive interpretations of paleoenvironmental conditions vary due to a range of geographical factors that include the research setting within specific ecoregions and proximity to ecotonal boundaries, the localized topo-edaphic setting, and the mosaic of habitats represented in the immediate regions of the study area. The paleoenvironmental reconstructions are based on one or more environment indicators or proxies that rely on localized sediments (e.g., geomorphological setting, depositional rates, and pedogenic developments), macrobotany and microbotany (e.g., pollen, phytoliths), macrofaunal bone elements and microfaunal remains (e.g., diatoms, foraminifera), as well as carbon and nitrogen 


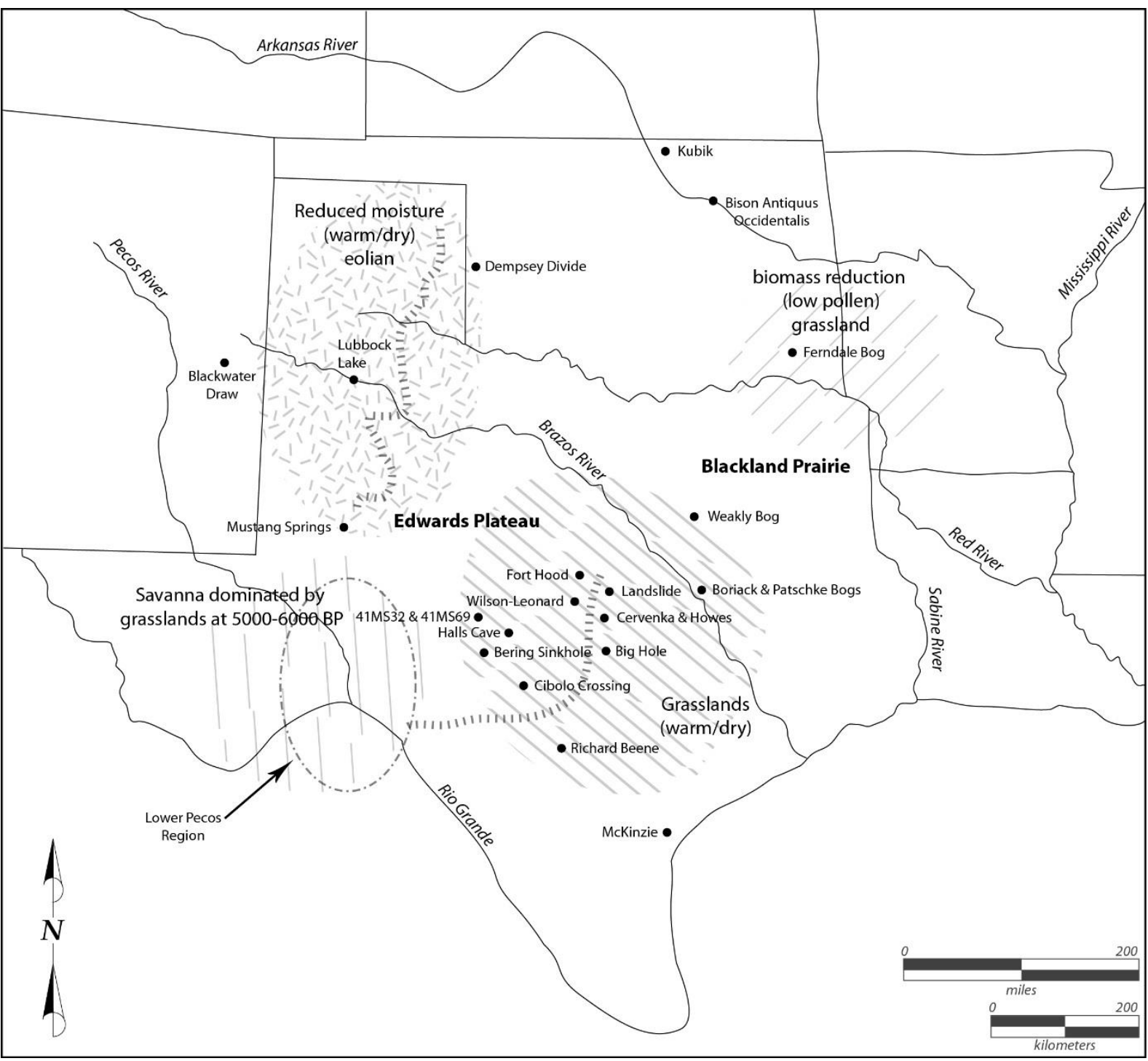

Figure 2-9. Map depicting generalized interpretations of middle Holocene paleoenvironments and data collection localities.

isotopic signatures in soils, plants and animals arising from plant decomposition or ingestion fractionation resulting in concentrations of carbon isotopes in the food chain.

Considerations must also account for the context and integrity of the recovered proxy samples (i.e., a single event episode, hearth feature, random charcoal, composite/dispersed charcoal, a broad geological zone, single or multiple samples, or vertical column. Context relates to the genesis of the deposit matrix and the proxy samples of interest. Some kinds of proxy indicators, such as wind-borne pollens or bones and soil humates in alluvial settings, can travel scores to hundreds of miles from their primary setting of origin, while other kinds of proxy indicators remain at or near the site of origin. Similarly, the study of faunal or macrobotanical remains inside sinkholes have to distinguish between the remains of resident occupants of the sinkhole, and those proxy remains that washed into the cave along with older sediments from the surrounding surfaces.

If the results of a particular proxy study are to be combined with other lines of paleoenvironmental 
proxy evidence, then context and ages of respective proxy samples should be acquired to ensure integration of results are on the basis of contemporaneous periods. Dating is especially critical even within single sites due to differential rates of sediment deposition. Unfortunately, too often sequences with high resolution paleoenvironmental results or trends are inadequately dated so reliable comparisons are not possible. Worse yet are instances where paleoenvironmental data lack adequate dating points so researchers engage in tautological or circular reasoning exercises and extrapolate comparable environmental trends with an unknown degree of reliability or validity. Further chronological considerations must address the kinds of dateable materials selected (i.e., chunk or dispersed charcoal, carbonates, pedogenic humate carbonates, annual vs perennial plant parts, carbonate genesis and fractionation of animal bones, and snails, etc.), the validity of the association of the date to the studied environmental proxy samples (including considerations of old dead wood, and cross-sectional effects), and the accuracy and precision of the chronometric methods.

These and other factors affect the usefulness, data reliability and interpretative potential facing efforts to reconstruct the paleoenvironmental record. This chapter summarizes the paleoenvironmental studies conducted in Texas and adjacent areas for the middle Holocene period to provide an interpretative context that probably influenced the adaptive responses of prehistoric populations to the region.

For the Great Plains and western United States, geologist E. Antevs (1955) proposed in very general terms the climatic period comparable to the middle Holocene (roughly 7000 to 4500 B.P.) was a time of drier and warmer conditions compared to the present. He designated this period the Altithermal. Antevs' model is based on evidence of erosional and depositional cycles observed in geologic strata across the western United States. In the eastern United States, the contemporaneous period from ca. 7000 to 4000 B.P. correlates to the warm Hypsithermal interval (Deevey and Flint 1957). Ever since Antevs' (1955) publication, greater interest has been directed towards understanding past environmental/climatic conditions in general and specifically for the middle Holocene period. Early research indicated the Great Plains were drier and/or warmer during this period than today (Webb and Bryson 1972). Subsequently, Reeves (1973) argued the short grass Plains expanded during the middle Holocene and offered potentially a larger region for grasses to support bison populations. The paleoenvironment plays a major role in the formation of human ecological models created to understand past human behaviors. These models are based on the assumption the environment influenced human behavior, social organization, resource procurement strategies, activities, and even technologies.

Paleoenvironmental reconstruction is often based on collaborative trends evident in diverse datasets (e.g., aspects of geomorphology; frequency and diversity of pollen, short cell phytolith ratios, spores, charcoal, diatoms, faunal remains, snail types, and changes in stable carbon and oxygen isotope values, etc.) with diverse specialists employing different analytical techniques to extract specific kinds of information to reconstruct paleoenvironmental facets. Many researchers proposed changes in the environment or speculated on climatic conditions from a narrowly selected dataset. In most instances these datasets represent second, third, or higher order extrapolations from the paleoenvironmental condition (Caran 1998). Quaternary deposits provide context from which various kinds of proxy datasets are extracted. A clear and accurate understanding of the context and age of the deposits are critical for interpretations of proxy data. The following discussions present diverse paleoenvironmental proxy datasets and interpretations by researchers from across the 
Southern Plains. Regional variation probably occurred across the Plains region and may provide different or even conflicting regional interpretations depending upon preservation conditions and the kinds of data employed relative to the paleo ecoregions boundaries.

\subsubsection{Paleoenvironmental Conductions in Central and Southeastern Texas}

In central Texas, specifically across the Edwards Plateau and along its eastern margins in the adjacent Post Oak Savanna region, paleoclimatic records are comparatively better known in comparison to available from adjacent regions (Figure 2-10). Various proxy data for reconstructing the paleoenvironment includes pollen cores from bogs in the Post Oak Savanna (Bousman 1998; Bryant 1977; Holloway et al. 1987; Larson et al. 1972), geomorphic data from river valleys (Abbott 1994; Blum 1987; Blum and Valastro 1989; Frederick 2011; Johnson 1995; Johnson and Goode 1994; Mear 1998; Nordt 1992, 1993; Nordt et al. 1994; Toomey et al. 1993), and microtine mammal remains from cave deposits (Toomey 1993; Toomey et al. 1993), and deep open sites (Balinsky 1998).

Bousman (1998) recounted previously reported pollen results from Boriack and Weakly bogs in the oak-woodlands region of eastern Texas to obtain a 16,000 year sequence of grass-arboreal pollen fluctuations, which indicate shifts between forest, woodland, and open plant communities. Bousman's interpretations are based on his recalculations and indicate open plant communities were present during the Late Glacial Maximum, between 13,000 B.P. and 12,000 B.P., 10,000 B.P. and 9000 B.P., and 8000 B.P. and 2500 B.P. (Figure 2-10). A key factor in understanding shifts in vegetation communities is mesic (moist) woody species invaded grassland regions during moist climate intervals, in contrast woody species died during extended xeric (drought) periods (Coupland
1958). Apparently, between 8000 B.P. and 7000 B.P., the Post Oak region of central Texas died back and grasslands became more prominent.

Bousman (1998) presented pollen data to indicate a possible two-phased, middle Holocene dry interval, with extremes recorded at ca. 6500 B.P. and 5000 B.P. Some evidence exists in the pollen data for a brief arboreal pollen event at 6000 B.P., which supports a hiatus in the long drying event. Bousman also found that alluvial pedogenesis occurred concurrently with pollen spikes in the Weakly Bog. Therefore, Bousman interprets dry, grassy intervals are associated with alluvial landscape stability and pedogenesis in eastern Texas. This is a key point when interpreting geomorphic deposits. During the period from roughly 6000 B.P. to 5000 B.P., the pollen record documents extreme changes with the arboreal cover changing from about 25 percent to 0 percent during that 1,000-year period.

Nordt (1992) presented geoarcheological data combined with stable carbon isotope values on stream sediments in the Fort Hood area, located in Bell and Coryell Counties of central Texas and on the Edwards Plateau. He identified a specific Fort Hood alluvium underlying the $\mathrm{T}_{1}$ surface, which radiocarbon dated between 8000 and 4800 B.P. This depositional unit is characterized by finegrained sedimentation from meandering and abrading streams. Paleosols (buried soils) are absent from this ca. $10 \mathrm{~m}$ thick alluvium, which indicates a moderately rapid deposition, which lacked long periods of stability. Nordt (1993) and Nordt et al. (1994) interpreted vegetation changes for this middle Holocene depositional unit using stable carbon isotope data from dated alluvial deposits. In these studies, the $\mathrm{C}_{4}$ warm season grasses increase from 65 to 70 percent prior to 6000 B.P., to between 85 and 95 percent of the total vegetation during a period from 6000 to 4000 B.P. This pattern signals a warmer and drier than today, and generally supports the pollen findings presented by Bousman (1998). 


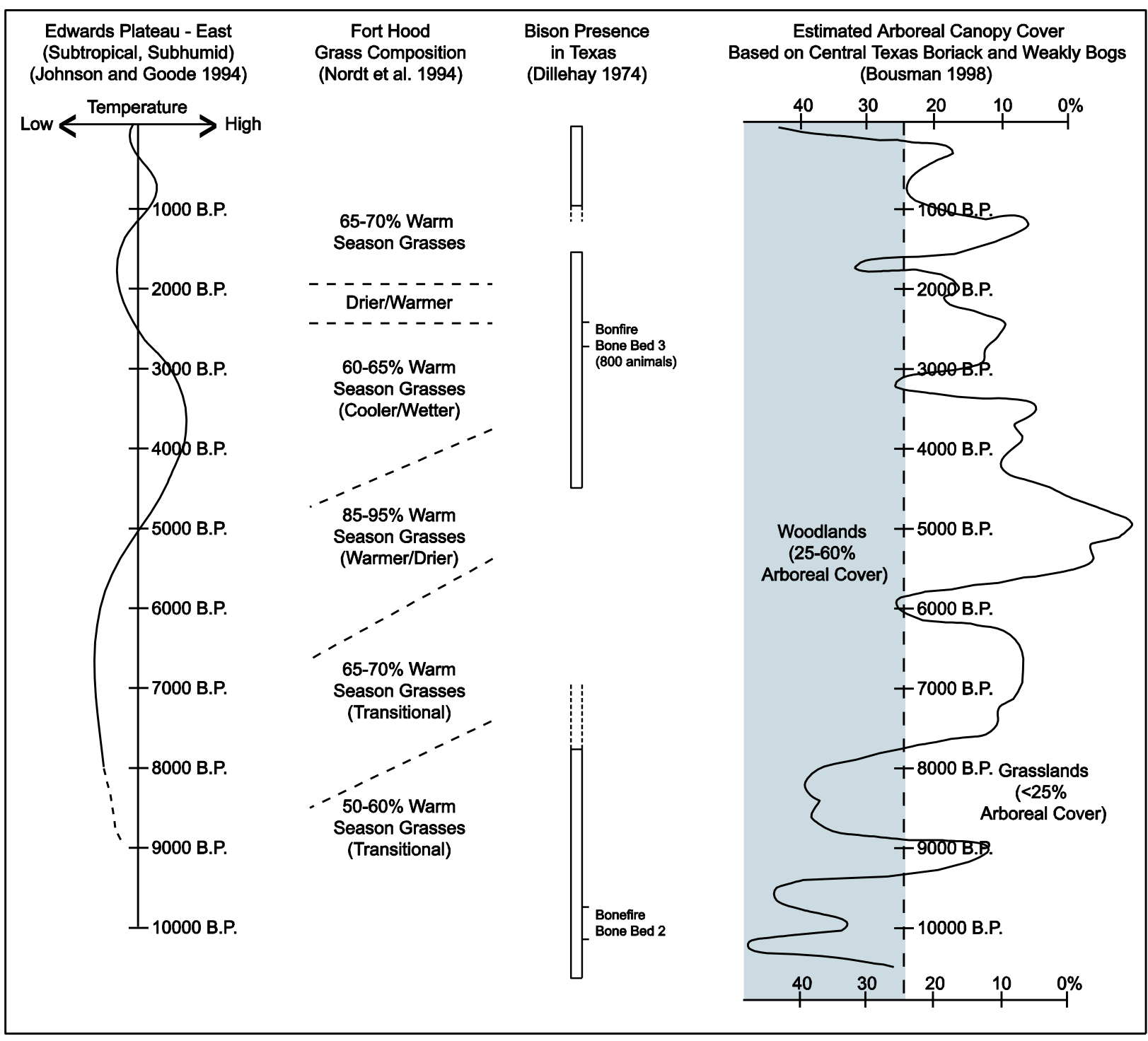

\section{Figure 2-10. Comparison of multiple interpretations of environmental variation in central Texas based on different kinds of proxy data.}

Other stable isotope data reflects slightly different conditions. For example, at O. H. Ivie Reservoir located at the confluence of the Concho and Colorado rivers in the northwestern edge of the Edwards Plateau in Coleman County, a radiocarbon date from humate sediments yielded a stable carbon isotopic signal interpreted as reflecting a 60 percent $\mathrm{C}_{4}$ plant contribution to the soil matter at 6000 B.P. (Lintz et al. 1993). This single isotope value supports isotope data from Fort Hood, even though the amount of $\mathrm{C}_{4}$ warm season grasses is some 20 to 30 percent less than those documented at Fort
Hood for this same time. Although the isotope value obtained from $\mathrm{O}$. H. Ivie is comparably less, it does support the dominance of short grass vegetation at that time. This value and the extrapolation of the $\mathrm{C}_{4}$ grasses reflect a general warm period with $\mathrm{C}_{4}$ grasses dominating local vegetation.

Stable carbon isotopic data from middle Holocene sediments (IIIb deposits, ca. 6000 to 4000 B.P.) at the Wilson-Leonard site (41WM235) in Williamson County of central Texas reflect little change in the amount of $\mathrm{C}_{4}$ matter into the 
sediments. However, the $\delta^{13} \mathrm{C}$ values of ca. $-23 \%$ are unusually negative and reflect nearly 90 percent $\mathrm{C}_{3}$ vegetation proportion during this 2000 year time span (Fredlund and Tieszen 1998). This is opposite the stable isotope data from Fort Hood and reflects a very low percentage of $\mathrm{C}_{4}$ warm season grasses at that time, as well as limited change from the proceeding period. Findings from Fort Hood do not support the data from the Wilson-Leonard site. It is the first author's opinion the stable carbon isotope values from the Wilson-Leonard site are suspect, since they also contradict the phytolith record from the Wilson-Leonard site for this same period. If the Fort Hood dataset accurately reflects the past environment, then that environment was dominated by $\mathrm{C}_{4}$ grasses, which increased during extended periods of warm and dry climates.

Recent research that employed stable carbon and nitrogen isotope data from 61 radiocarbon dated bison bones across central and south Texas has revealed relative temperature and moisture conditions during four time intervals defined by four bone date clusters (Lohse et al. 2014b). The interpretations were derived from central Texas bison bones with $\delta^{13} \mathrm{C}$ values that range from $-9.4 \%$ through $-11.9 \%$ with an outlier at $-19.0 \%$. The $\delta^{15} \mathrm{~N}$ values range from $5.7 \%$ to $9.59 \%$ with no apparent outliers (Lohse et al. 2014b). The earliest cluster of dates, a Calf Creek interval (ca. 5120 to 5205 B.P.) is identified by elevated $\delta^{15} \mathrm{~N}$ values to indicate the driest of the four periods, whereas the $\delta^{13} \mathrm{C}$ values indicate the coldest of the four periods. The authors used these data to reconstruct mean annual temperature for that period at $14.87 \pm$ $1.13^{\circ} \mathrm{C}\left(58.8^{\circ} \mathrm{F}\right)$ on the basis of stable carbon isotope data (Lohse et al. 2014b). Employing the $\delta^{15} \mathrm{~N}$ results based on a linear regression model they derive precipitation approximations at ca. 40 to 45 $\mathrm{cm}$ per year. Data relevant to the Calf Creek interval was therefore characterized by cool, but dry conditions. The authors also suggest the data indicates this period was more volatile, with greater variation in rainfall over short periods. They summarize by providing a broad climate reconstruction with other stable isotope data to reveal a broad cold period across North America just prior to the Calf Creek interval, which was the coldest and driest of the three subsequent periods (Lohse et al. 2014b).

The $\delta^{13} \mathrm{C}$ isotope values on a column of organic carbon from sediments through the Medina pedocomplex (Unit A5, about 7000 to 4400 B.P.) along the Medina River just south of San Antonio indicates a steady increase in the amount of $\mathrm{C}_{4}$ organic plant residues from about 40 percent at 7000 B.P. to about 62 percent at 5000 B.P. (Figure 2-11). Following this latter period, the $\mathrm{C}_{4}$ isotope values decrease to about 50 percent at 4500 B.P (Mandel et al. 2007). This isotope data again provides support for a warmer climate period peaking at around 5000 B.P. These values are 30 to 40 percent less than isotope values obtained from the Fort Hood alluvium for this same period, although they still reflect dominance of short $\mathrm{C}_{4}$ grasses. However, that same isotope data also reveals support for Johnson and Goode's (1994) Edwards Interval, with even greater peaks in $\mathrm{C}_{4}$ values slightly later in time, with a peak near 75 percent $\mathrm{C}_{4}$ grasses between ca. 3000 and 1500 B.P. The dates from the Medina pedocomplex are not in total agreement with Johnson and Goode (1994). The increase in $\mathrm{C}_{4}$ plants does support a drying interval following the Altithermal period.

In geomorphic studies directed towards the upper Colorado River drainage across the Edwards Plateau, Blum (1992) interpreted the broad depositional sequence dating from 11,000 to 5000 B.P. as slow accumulation, with slow valley widening through lateral migration. Deposition was dominated by sediments from relatively proximal sources within the drainage, and sediment supply exceeded transport capacity. At O. H. Ivie Reservoir, the middle Holocene deposits occur at the confluence of the Concho and Colorado River channels aggrading with gravel and sand deposits. 


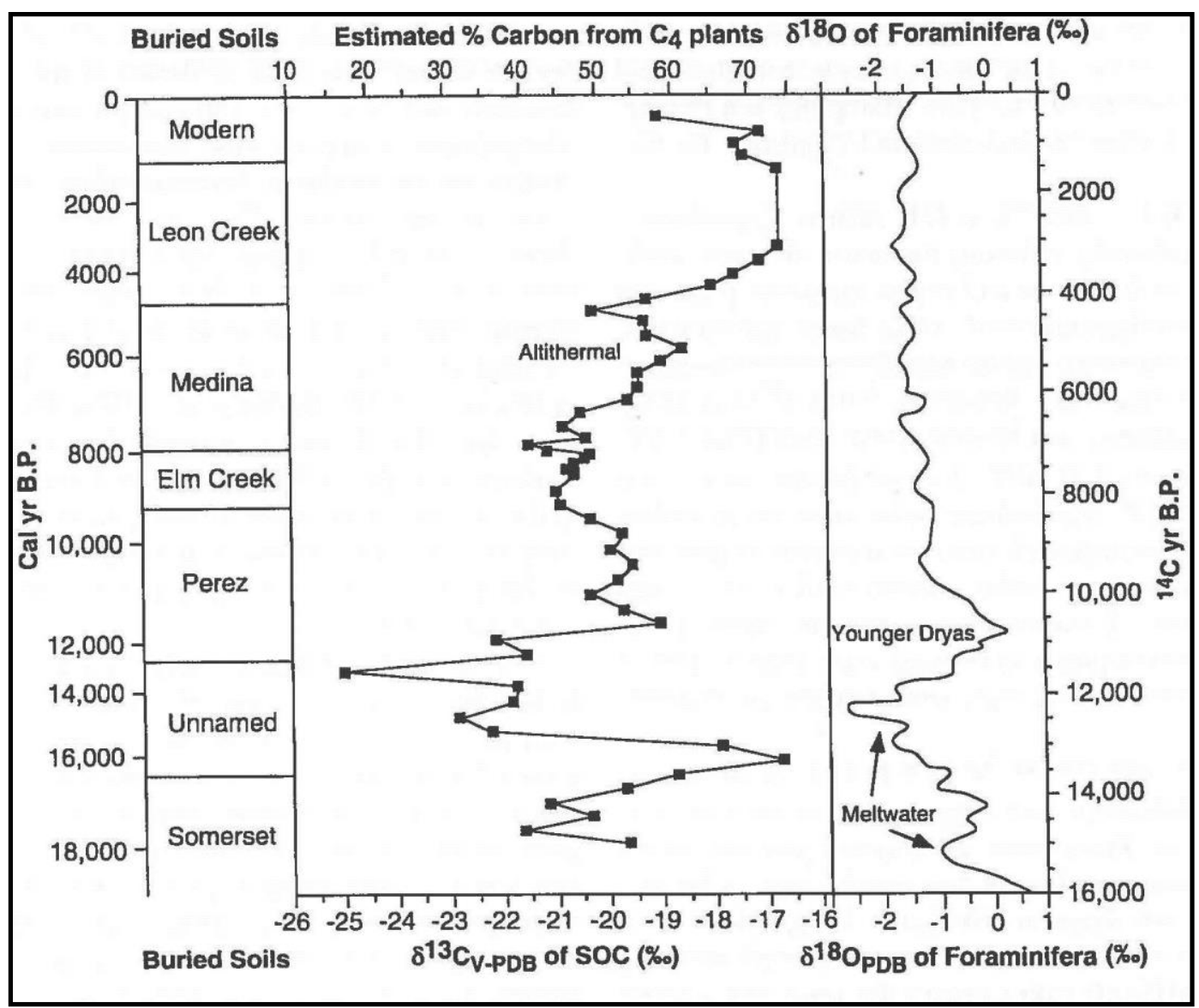

Figure 2-11. Carbon isotope values of soil organic carbon (SOC) in the sequence of buried soils at the Richard Beene site in Southern Texas (from Mandel et al. 2007, Figure 3.20).

Around 5000 B.P., the channel terraces were incised to bedrock and floodplain deposition temporarily ceased. This was interpreted to represent a severely arid period (Blum and Lintz 1993). Blum's work (1987) in the Pedernales River Valley, also in the Edwards Plateau, observed changes in channel sediments, which revealed a middle Holocene erosional unconformity. Blum and Valastro (1989) interpreted this to reflect a dry climate around 4500 B.P. Near the eastern margin of the Edwards Plateau and upstream from the Big Hole site, the Onion Creek valley deposits near Buda, revealed an erosionally truncated B soil horizon of a $20 \mathrm{~cm}$ thick paleosol radiocarbon dated by soil humates to $5310 \pm 90$ B.P. (Abbott 1994). This paleosol indicates a period of stability. Just south of the Edwards Plateau, along the Medina River south of San Antonio, Mandel et al. (2007) defined the Medina pedocomplex (Unit A5) in geomorphic studies. This is a cumulic soil under development from about 7000 B.P. to about 4400 B.P. This soil was buried soon after 4400 B.P. The Medina pedocomplex was $4.5 \mathrm{~m}$ thick and characterized by an Ak-ABk-Bk soil profile. The particle size does not change significantly throughout this period. Consistency in texture generally reflects a long stable environment over the 2,600-year period with periodically flooded on a regular basis. Obvious erosional episodes were not observed in the profiles.

At the Hall's Cave site (41KR474), near the center of the Edwards Plateau in Kerr County northwest of San Antonio, Unit 3 deposits ranged from 1 to 1.5 $\mathrm{m}$ in depth were consistent, horizontally stratified, with a sharp base and distinctive top (Toomey et al. 


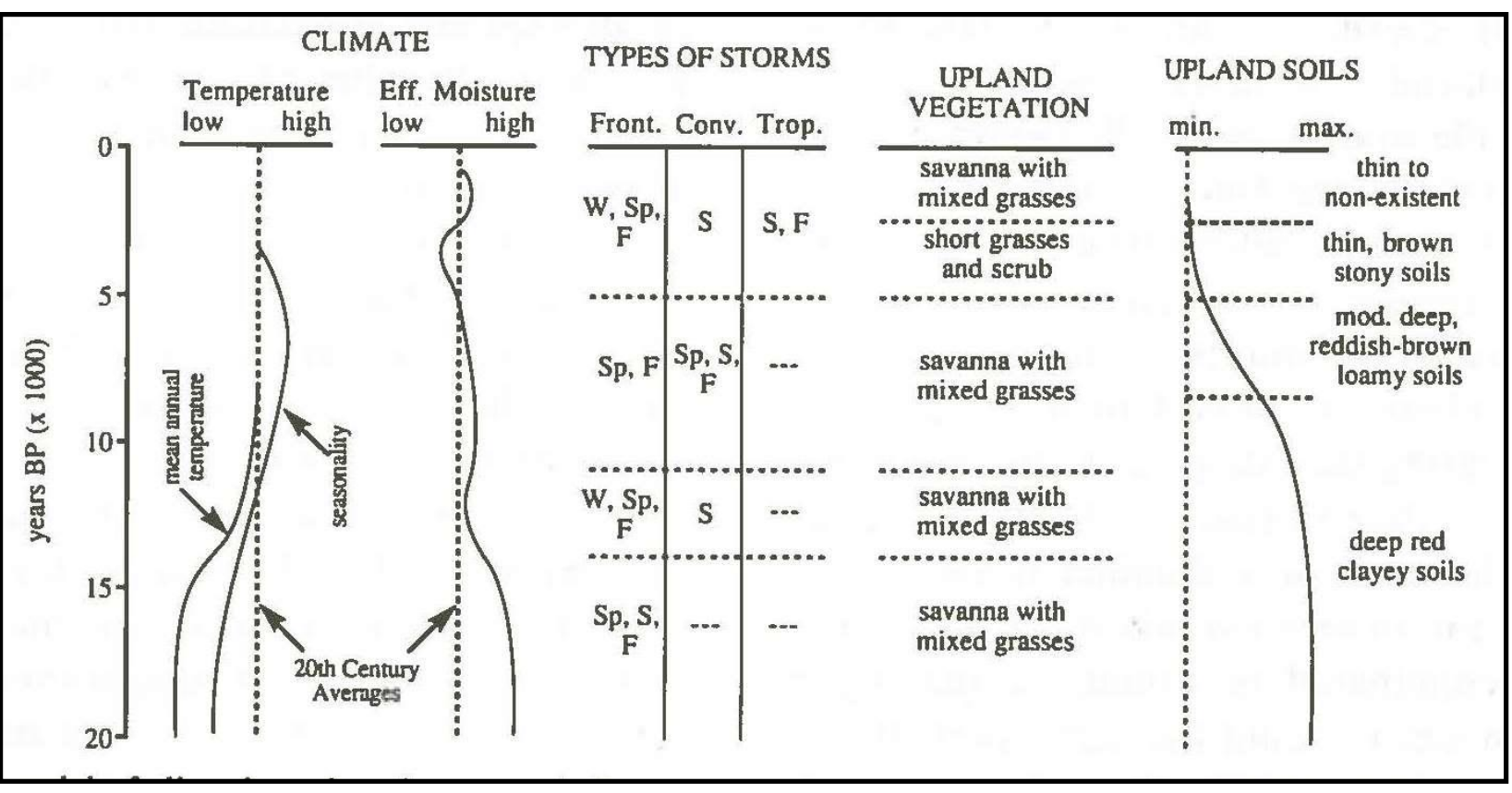

Figure 2-12. Summary model of climatic and environmental changes projected from the Hall's Cave data in the Edwards Plateau (from Toomey et al. 1993:315, Fig.9).

1993). This deposit was radiocarbon dated between 7320 and 4850 B.P. Unfortunately, Unit 3 lacks sufficient radiocarbon dates to document changes at specific times. The authors concluded during this early to middle Holocene period, the upland soil mantles were undergoing progressive dissection and/or downwasting and became darker, thinner, and stonier. This was based on rates of vertical accretion at the Hall's Cave site that were twice the previous period, as the amount of clay decreased, soil color changed, and the percentage of transported limestone clasts increased (Toomey et al. 1993). Toomey et al. (1993) reviewed accumulated data from central Texas and found a protracted decrease in effective moisture from the early to middle Holocene reflecting conditions somewhat drier than modern times (Figure 2-12). This xeric trend persisted until roughly 2500 B.P., a much later date than most previous researchers have proposed.

Geomorphic research has not clearly demonstrated whether flood plain aggradation is related to dry periods or moist conditions (Johnson and Goode 1994). However, Blum and Valastro (1989) and
Blum and Lintz (1993) support the notion fluvial systems reflect channel aggrading during more humid periods, and incision following a transition to drier conditions.

Insights gleaned from sparse middle Holocene faunal assemblages have been employed to characterize paleoenvironmental conditions. For example, at the Wilson-Leonard site Baker (1998) did not identify any bison remains from the Middle Archaic deposits. This trend is also apparent in the period ca. 7000 to 3000 B.P., Unit IIIb at the Wilson-Leonard site. The representative faunal inventory was dominated by rabbits and medium mammals along with fish, snakes, and turtles (Baker 1998). The Wilson-Leonard data support the assertion the period from 8000 to 4500 B.P. was a period of bison absence or scarcity based on data from 28 paleofaunal assemblages across Texas (Dillehay 1974). If it is assumed bison frequency is environmentally influenced, then frequency of bison in archeological deposits potentially serves as a gross indicator of paleoenvironmental conditions. Conflicting interpretations concerning bison ecology, however, have identified both wet and dry 
conditions as suitable scenarios for bison herd populations in a particular area (Collins 1995, 2004; Johnson and Goode 1994), leaving the distinction somewhat unclear.

In direct contrast to the Wilson-Leonard faunal data for the middle Holocene and Dillehay's (1974) earlier work, the Spring Lake site (41HY160) data from Hays County south of Austin, documents bison presence between 5060 and 5180 B.P. on the basis of $11 \delta^{13} \mathrm{C}$ corrected radiocarbon dates on bison bones from a $50 \mathrm{~cm}$ thick zone (Lohse et al. 2013, 2014a). This zone also yielded turtles, deer (Odocoileus), pronghorn (Antilocapra americana), Canidae, cottontail rabbits, bird (Aves), fish, and snake.

During a period from 10,500 to 5000 B.P., the faunal record from the Hall's Cave site reveals a progressive extirpation of microvertebrate taxa with higher moisture requirements, such as the eastern mole (Scalopus aquaticus), mole salamanders (Ambystoma sp.) and the short-tailed shrew (Blarina sp.), along with an increasing importance of species such as the desert shrew (Notiosorex crawfordi) that tolerate drier conditions (Figure 2-13) (Toomey 1993; Toomey et al. 1993). The faunal records from Hall's Cave are superb, although the deep deposits representing long depositional history lacks published radiocarbon dates. The lack of published dates reduces the importance and timing of changes in this faunal record.

Indicators of plant resources within dated deposits also provide insight into paleoenvironmental conditions. For example, indirect plant data via fossilized plant silicates (e.g., phytoliths) from the Wilson-Leonard site implies a significant increase in the rate of grassland expansion around 8700 B.P. (Figure 2-14). Grassland composition during the period from ca. 8700 to 6000 B.P. (Fredlund 1998) was similar to existing conditions. The phytolith record indicates a relatively stable period between about 6000 and 4000 B.P. dominated by grasslands,

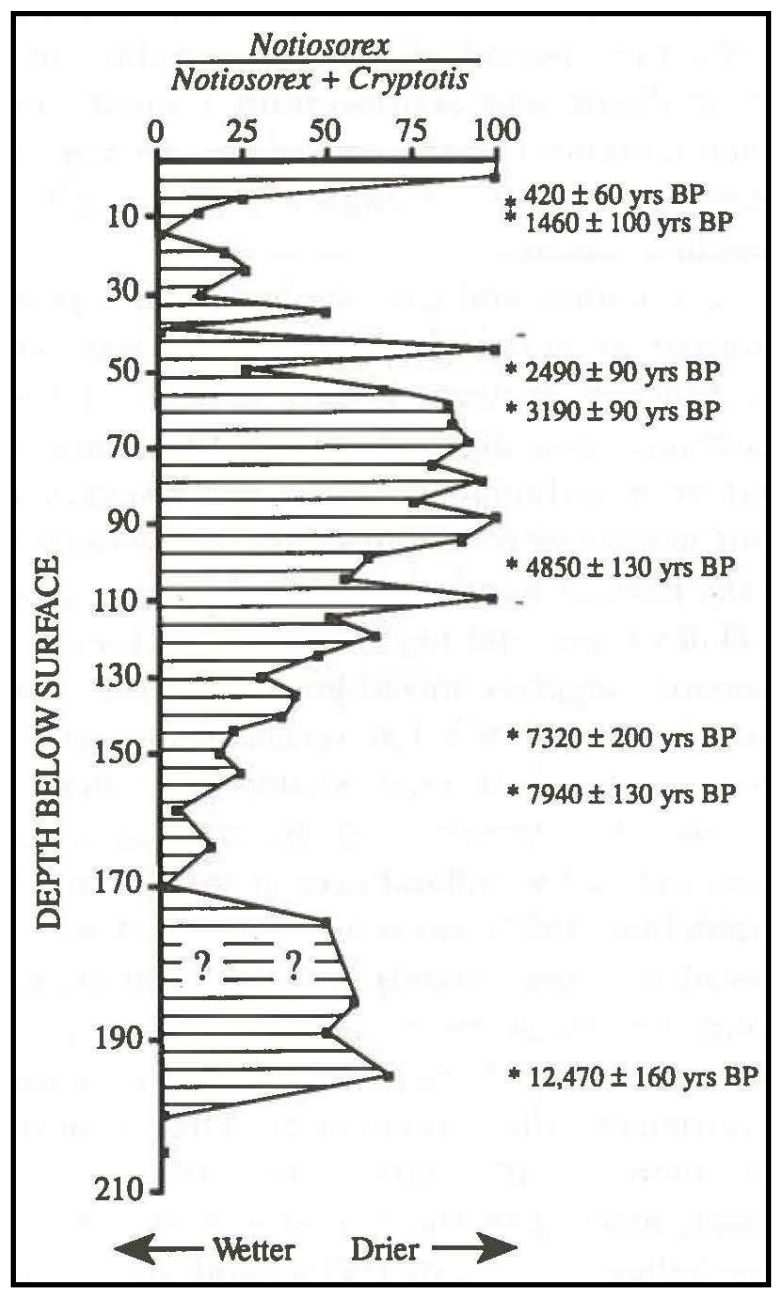

Figure 2-13. Changes in the ratio of Notiosorex crawfordii to Notiosorex crawfordii

+ Cryptotis parva at the Hall Cave site and available radiocarbon dates (from Toomey et al. 1993:308, Fig.6).

with most grasslands peaking just after about 4000 B.P. Fredlund (1998) also theorizes the overall vegetative composition of central Texas reached its modern balance of woodlands and grassland by about 4000 B.P.

Few macrobotanical records are available for the middle Holocene period from central Texas. One record from the Wilson-Leonard site, dating between ca. 7000 and 3000 B.P., revealed live oak (Quercus virginiana), juniper (Juniperus Mexicana), walnut (Juglans major), elm (Ulmus sp.), and mulberry trees (Morus spp.) (Dering 


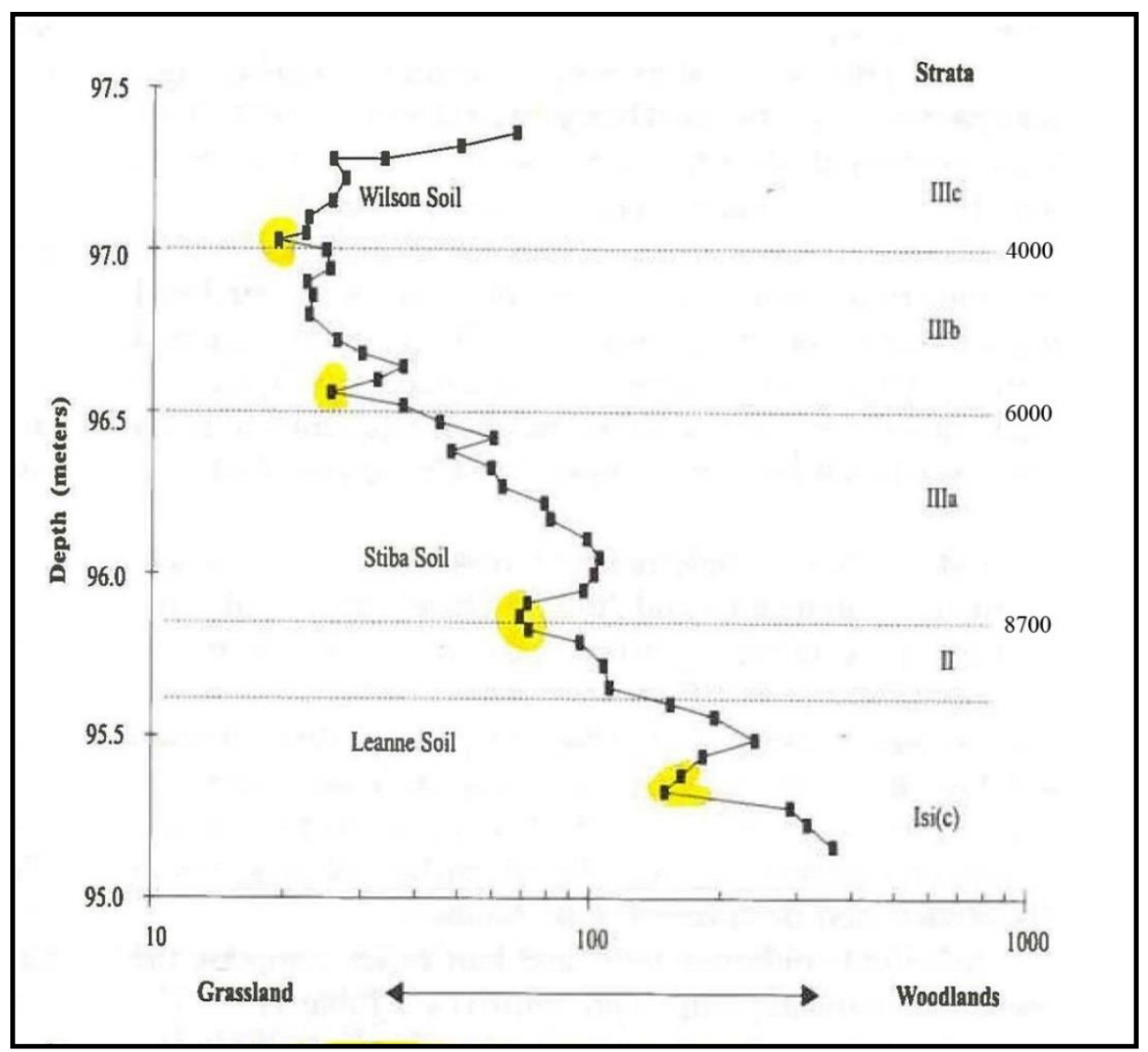

\section{Figure 2-14. Phytolith changes over time that demonstrate woodland to grassland ratio by depth and time (from Fredlund 1998:1643, Figure 41-2). Note log scale used for ratio.}

1998). Macrobotanical remains from other early and middle Holocene archeological sites are nearly nonexistent, evidenced by the lack of carbonized organic remains from the excavated components at the Landslide site (Sorrow et al. 1967), the Cibolo Crossing site (Kibler and Scott 2004), the Richard Beene site (Dering and Bryant 2007), the Holt site (Iruegas and Brownlow 2004), among other locations reflecting this period.

In summarizing the central Texas area, the collective body of diverse proxy data presented above indicates a drying trend during the middle Holocene all across the region and included central and southeastern Texas, which roughly corresponds to Antevs' (1955) Altithermal period. These diverse studies reveal expressions of the Altithermal, which reflect some potentially variability across the mosaic of microhabitats of
Edwards Plateau and through time (see Figure 2-9). Johnson and Goode (1994) see no long-lasting, dry Hypsithermal (their term for the Altithermal) climate in the eastern Edwards Plateau based largely on the faunal data derived from the Hall's Cave site and geomorphic data from San Geronimo and Cow House Creek valleys. They proposed, however, the existence of a dry Edwards Interval separate from the Altithermal period peaked around ca. 4000 B.P. and lasted from about 5000 to 3000 B.P. This time frame is based on some 50 calibrated wood-charcoal radiocarbon assays from archeological and geomorphic context in the Jonas Terrace site (41ME29) along the San Geronimo Creek and Fort Hood Military Reservation. Johnson and Goode (1994) interpret little change in the climate record between the Early and Middle Archaic cultural periods. They also propose bison were present in central and southeastern Texas 
during the Middle Archaic on the basis of very limited bison data from questionable contexts, contradicting data presented by Dillehay (1974). The proposed dry conditions are postulated to have promoted the spread of yuccas and sotol $\left(\mathrm{C}_{4}\right.$ and CAM plants), and it was these xeric-adapted plants collected by humans and cooked in large burned rock ovens during Middle Archaic times (Johnson and Goode 1994).

Data from the Texas Gulf Coast near Corpus Christi indicates between about 6700 and 6000 B.P., the sea level was rising rapidly (Ricklis and Blum 1997). Ricklis and Cox (1998) interpreted stratigraphic evidence to indicate widespread erosion of upland margins prior to 5000 B.P. implying sparse vegetation cover and possibly patterns of relatively low precipitation. This implies regional, if not a probable global warming trend that supports Antevs' (1955) Altithermal model, as well as the timing of this event. Ricklis (1993) presents archeologically derived radiocarbon dates and faunal evidence to indicate a middle Holocene period of relative aridity with an ocean stillstand in the Corpus Christi area. This was accompanied by shell fishing by the human population between roughly 6000 and 4000 B.P. Nordt et al. (2002) suggested little association between marine and adjacent continental ecosystems as a result of reduced glacial meltwater entering the Gulf of Mexico during the Holocene, and a shift in global circulation patterns. With the waning meltwater flow, $\mathrm{C}_{4}$ production generally increased throughout the Holocene, culminating in peak warm intervals at ca. 5000 B.P. and 2000 B.P.

Also towards the coast, along the Guadalupe River south of Victoria, Texas, the Buckeye Knoll site (41VT98) yielded a pollen sequence interpreted by Albert (2012) to indicate the middle Holocene was dry, similar to central Texas (e.g., Collins 2004; Johnson and Goode 1994), though it was interrupted by a wet period from ca. 5500 to 5000 B.P.

\subsubsection{Paleoenvironmental Conditions in Northern Texas, Oklahoma, and Adjacent Areas}

The areas north of the Edwards Plateau encompass several different biotic regions, which include the short grass plains (Texas and Oklahoma panhandles), the mixed grass prairies (north central Texas and central Oklahoma), and the Cross Timbers (eastern Texas and Oklahoma). A few archeological and geomorphic studies have encountered middle Holocene deposits across this expansive region. From published data, the more prominent investigations include work by Albert (1981); Albert and Wyckoff (1984); Ferring (2001); Hall (1988); Haynes 1995; Holliday (1985a, 1989, 1995a); Humphrey and Ferring (1994, 2001); Johnson and Holliday (1986); and Meltzer (1999). These studies are presented to illuminate the overall paleoenvironmental picture of the middle Holocene.

Hall (1988), in discussing environmental conditions across much of Oklahoma derived from geomorphic data, stated the middle Holocene (ca. 7000 to 5000 B.P.) climate was exceptionally dry and characterized by stream valley erosion. His assertion supported previous work in a comprehensive evaluation of river response to Holocene climates by Knox (1983) who noted "between 6000 and 4500 B.P. significant erosion of early Holocene alluvial fills was occurring in most regions." They both contend stream valley erosion removed middle Holocene sediments and associated archeological sites from the record. This extensive area-wide erosion accounts for their low density of middle Holocene sites across the region. In western Oklahoma the paleoenvironmental reconstruction was developed from four datasets from the riparian setting along the Bull Creek site (34BV176) near the eastern end of the panhandle. Diverse data reflects greater effective moisture in the pollen samples (46 and 47) with a mixed-grass phytolith assemblage dominated by $\mathrm{C}_{3}$ grasses (ca. 60 percent). The latter indicates a moist condition 
at about 6200 B.P., trending toward drying conditions (Bement et al. 2007). This locality also revealed a nearly $80 \mathrm{~cm}$ thick deposit of loess (Unit III) dated between $8670 \pm 90$ and $6200 \pm 90$ B.P. to demonstrate the area received eolian sediments during this early period (Bement et al. 2007).

Multiple pollen records from Ferndale Bog, along the western edge of the Ouachita Mountains in southeastern Oklahoma, provide a significant, wellstudied, and dated source for the Holocene from which to reconstruct the paleoenvironment (Albert 1981; Albert and Wyckoff 1984). At Ferndale Bog, the lowest dated zone is close to 12,000 B.P. (Bryant and Holloway 1985). The pollen record following the Pleistocene documents a loss of pine (Pinus) and oak (Quercus sp.), with very minor amounts of hickory (Carya sp.), combined with significant increases in grasses (Poaceae) and weeds (Ambrosineae). It is not clear if these changes document the short Younger Dryas, a cold period that saw the return of glaciers between 11,000 and 10,000 B.P. During the early
Holocene/Early Archaic, pine and hickory pollen remained very limited, whereas oak peaked at some point following a peak in the grasses. Both the later peaks in pine, oak and plus weeds continued to decline into the middle Holocene. Prior to about ca. 5200 B.P. Albert's (1981) core data reveals a significant dominance of nonarboreal pollen over arboreal pollen with oaks the dominate tree. Accordingly, in far southeastern Oklahoma a grassland region with scattered oaks was well established by Calf Creek horizon times. Subsequently, oaks gradually increased as did the overall arboreal composition. In light of the postulated grasslands in southeastern Oklahoma by minimally 5200 B.P., the vegetation further west was probably dominated by grasslands as well.

The Aubrey site in north-central Texas contributes geomorphic and isotopic data to the paleoenvironmental discussion (Ferring 2001; Humphrey and Ferring 1994, 2001). From that data, Humphrey and Ferring (1994, 2001) suggest the middle Holocene (8000 to 4000 B.P.) experienced

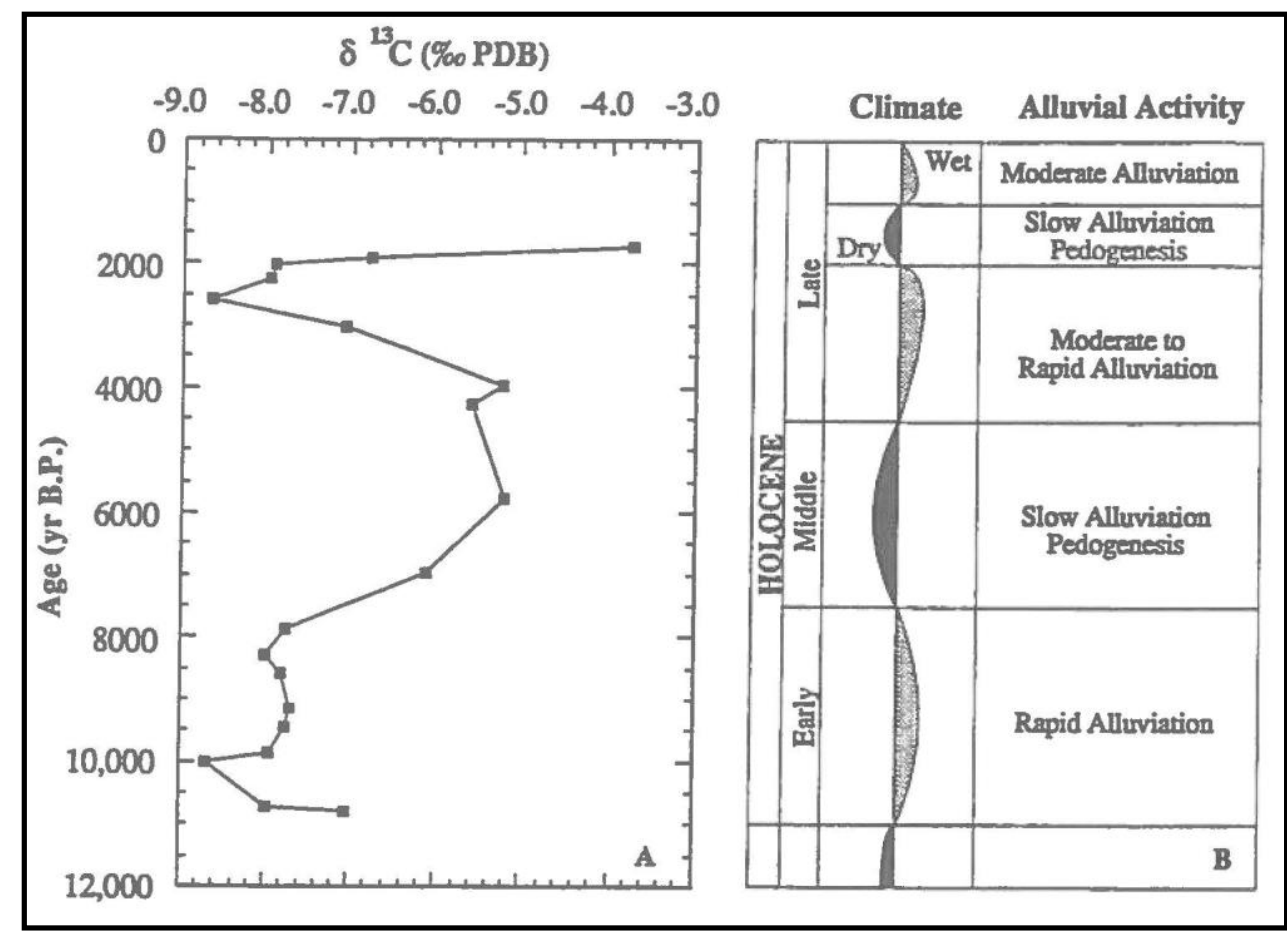

Figure 2-15. Pedogenic carbon isotope data and inferred climate history (from Humphrey and Ferring 2001:67, Figure 4-6). 
a decrease in $\mathrm{C}_{3}$ plant composition, as revealed in the floodplain stable carbon isotope record (Figure 2-15). Ferring and Yates (1997) consider the middle Holocene as a transitional period between the earlier period with high pollen representing prairie-step vegetation, and later low pollen amounts representing mixed forest vegetation, on the basis of data from Ferndale Bog in southeastern Oklahoma. Ferring and Yates (1997) indicate the very low pollen influx during the middle Holocene, especially between 6500 and 5500 B.P., reflects a significant biomass reduction. They presume this was caused by lower annual precipitation.

The intensively investigated site of Lubbock Lake (41LU1) is located in northwestern Texas at the edge of the High Plains is (e.g., Holliday 1985a, 1985b, 1989, 1995a, 1995b; Johnson and Holliday 1986). Multiple analyses directed at diverse datasets contribute significant data to the paleoenvironment there and the surrounding region. At the Lubbock Lake site the weakly developed Yellowhouse Soil (Substratum 31) formed at the top of Stratum 3 dated between ca. 6300 and 5000 B.P. (Holliday 1985b). The Yellowhouse Soil has a weak A-C profile which developed over an estimated 500 years in calcareous lacustrine and sandy eolian deposition sediments with and subsequent pedogenesis. Holliday (1995a) indicates the water table was high during this period of soil formation. Sediments similar to the Yellowhouse Soil are widespread in Southern High Plains and support an interpretation for a regional wide climate change toward conditions of increased eolian activity, reduced effective moisture, and possibly warmer temperatures. He also states the most intense deflation was between 6500 and 4500 B.P., which he attributes to a reduction in plant cover with little moisture on the valley floors, except in restricted marsh muds (stratum 4m) at Lubbock Lake (Holliday 1995a). Johnson and Holliday (1986) reported the Middle Archaic was mostly a dry period from roughly 6300 to 4500 B.P. with a slightly cooler and more moist period with a cessation of blowing dust lasting for 500 years, between 5500 and 5000 B.P. as represented by the Yellowhouse Soil (Johnson 1987b: 99). They interpret the 500-year moist period to coincide when bison were present.

Faunal remains can also be indicators of the environment and potentially reflect environment conditions. Johnson and Holliday (1986) indicate the archeological record from the Southern Plains is scarce for this estimated 2,000-year Middle Archaic period from ca. 6400 to 4500 B.P. The Middle Archaic cultural events at the Lubbock Lake site reflect small groups of people subsisting on sparse bison. With the lack of excavated sites and components dated to the Altithermal period, faunal records are also nearly nonexistent. Between 5500 and 5000 B.P. the limited faunal assemblage at the Lubbock Lake site indicates a period of landscape stability (Johnson 1987b:95, Table 8.2). Other faunal remains from the Middle Archaic period (ca. 6300 to 4500 B.P.) at Lubbock Lake includes the presence of frogs, mud turtles, box turtles, Texas horned lizards, bull snakes, jackrabbits, prairie dogs, pocket gophers, rats, coyotes, and pronghorn (Johnson 1987a). Most species except rats, gophers, coyotes, and the previously mentioned bison were recovered from noncultural contexts. Therefore, most of these species may be considered to be natural background fauna from the general site environment. No extinct species are apparent in the Lubbock Lake faunal assemblage. As expected, the faunal assemblage reflects the grassland dominated, water-edge habitats of the site setting. The presence of bison documented for this middle Holocene period at the Lubbock Lake site is indicated. Specific bone dates were not obtained to pinpoint precisely when bison were present at the Lubbock Lake site.

The gastropod assemblage from the Lubbock Lake site provides excellent clues to the paleoenvironmental conditions. Specifically 
Substratum 31, the buried A horizon referred to as the Yellowhouse Soil, from ca. 5500 to 5000 B.P., yielded a gastropods assemblage markedly different from the more mesic strata 1 and 2. The terrestrial species is essentially a modern population, with 86 percent derived from six very tolerant and drought-resistant species making their first appearance (Pierce 1987). Another indication of a severely altered environment was the very limited occurrence of aquatic gastropods as represented by only six species. Pierce (1987) interpreted the assemblage as reflecting an environment with the possibility of higher temperatures and less effective moisture. This pattern potentially resulted from decreased rainfall or increased evaporation. $\mathrm{He}$ proposed an environment similar to southeastern New Mexico with a probable average annual precipitation that did not exceed $40 \mathrm{~cm}$ per year.

Pollen studies conducted at the Lubbock Lake site reveal varying degrees of success due to differential preservation and low densities of recovered pollen (Bryant and Schoenwetter 1987). Researchers agreed the pollen record is so suspect the pollen should not be employed to reconstruct the local paleoenvironment (Bryant and Schoenwetter 1987). No other, lengthy well-dated pollen records exist from northwestern Texas due primarily to poor pollen preservation.

Hall (1995) has made various attempts to recover pollen from late Quaternary valley fills across the Southern High Plains. Overall, pollen preservation was poor, and primarily characterized by low pollen concentrations, low taxa diversity, and high proportions of corroded grains. This makes the interpretations of paleoenvironmental conditions on the basis of pollen proxy data impossible.

Holliday (1989) employed geomorphic data to indicate the Altithermal period was present in the western United States between 7500 and 5000 B.P. Stratigraphic data from many Southern Plains draws indicate eolian deposits to about 5000 B.P.
Other researches regard significant portions of dune fields in the Great Plains were created between 7000 and 4000 B.P. (Arbogast and Muhs 2000; Forman et al. 2001; Holliday 1989; Miao et al. 2005). In western Oklahoma, evidence exists for stabilized surfaces buried by dune deposits (Brady 1989; Thurman and Wyckoff 1994; Wyckoff 1990).

Winsborough (1997) conducted diatom analysis from ten localities within draws across northwestern Texas. She discovered similar assemblages in all draws and suggested a great similarity in the lacustrine habitats in the draws. She claims evidence for synchronous, region-wide deterioration of aquatic habitat associated with drying conditions occurred between about 8000 and 6500 B.P.

The Clovis type site, Blackwater Draw (LA3224), in northeastern New Mexico provides significant soil stratigraphy data to indicate paleoenvironmental changes on the Southern High Plains. During the middle Holocene a large blowout remained exposed in the top of Unit $\mathrm{F}$ from roughly 8500 to 6500 B.P. The blowout was filled by reddish-brown dune sand of Unit G (the Jointed Sands) bracketed by radiocarbon dates on bulk soil of $4855 \pm 90$ B.P. at the top of Unit $G$ and four dates from the Unit $\mathrm{F} / \mathrm{G}$ contact that range from $8730 \pm$ 90 to $6720 \pm 80$ B.P. (Seeback 2002). After approximately 5000 B.P. the depression filled with eolian sand of Unit $\mathrm{G}$, and the presence of a weak brown paleosol at the top of Unit G indicates dune stabilization by vegetation under conditions slightly more mesic than today (Haynes 1995). This paleosol is truncated in places by the deflational contact with overlying Unit G2 dune sand. Burned bison bone dated to $4950 \pm 150$ B.P. documents the presence of bison (Haynes 1995). During the dry period, which persisted from ca. 8000 to 5000 B.P., Archaic peoples apparently utilized the blowout as a place to trap and kill bison (Haynes 1995). The prehistoric water wells at the Clovis site described by Evans (1951) and Green (1962) were excavated 
from the surface of this blowout. The presence of water wells indicate a drop in the water table and present compelling evidence for the dry Altithermal period proposed by Antevs (1955), an interpretation also emphasized by Meltzer and Collins (1987) and Holliday (1989b).

The Mustang Springs site (41MT2) northeast of Midland in extreme Southern High Plains also makes significant contribution to the early to middle Holocene environment via prehistoric water well data (Meltzer 1999). Excavations there yielded a relatively fine-grained record of middle Holocene environments and climate (Meltzer 1991, 1995, 1999; Meltzer and Collins 1987). The site contains over 60 Altithermal-age (7000 to 4500 B.P.) water wells hand-excavated by humans into the bottom of a dry Mustang Draw stream bed to obtain underground fresh water. Less than 50 nonculturally diagnostic artifacts were recovered from the Altithermal surface and no other recognizable cultural features were associated with the wells. The wells at the Mustang Springs site plus other documented wells across the Southern Plains (Evans 1951; Green 1962; Quigg et al. 1994; Smith et al. 1966) reflect definite use of a clear adaptive human strategy to the region during dry periods when the water table is thought to have dropped by some $3 \mathrm{~m}$. The shallowest wells are the earliest, indicating as the water table dropped the wells got deeper. Most water wells at the Mustang Springs site were dug over a 200 year period from 6600 to 6800 B.P., during what Meltzer considers the maximum dryness. Meltzer speculated the well digging reflects a generalized strategy by highly mobile people as an adaptive response to the drying environment. Meltzer (1999) postulated a reduction in effective moisture, surface water, and resource abundance, and an increase in resource patchiness, sediment weathering, erosion, and eolian activity.

Stable carbon isotope data from the middle Holocene period across northern Texas and Oklahoma is very limited and such kinds of information from well-dated, stratified contexts is absent. Isotope analyses are available from several draws across northwestern Texas, although isotope data directly related to this targeted period is limited to only a few samples from the Lubbock Lake site (Holliday 1995b).

Macrobotanical remains from archeological features dating to the Altithermal are nearly nonexistent for this region as excavated sites/components dated to this period are extremely rare. In most instances, the few excavations conducted did not conduct flotation of feature sediments to acquire this kind of information. The plant assemblage from the Middle Archaic Stratum 4 at the Lubbock Lake site was considered to be too small to be regarded as significant (Thompson 1967).

Well-dated, extensive phytolith analyses for the middle Holocene period across northern Texas and the adjacent Oklahoma regions have not been conducted. A feasibility study directed towards extracting fossil biosilicates was conducted in the northwestern part of Texas (Bozarth 1995). Five of eight investigated localities yielded good phytolith preservation dating to at least 8300 B.P. Diatom preservation was also good in all eight samples. However, no samples dated between 6500 and 4500 B.P. (Bozarth 1995).

Morgan playa in the Rolling Plains of northwest Texas yielded a $220 \mathrm{~cm}$ deep sediment column containing a well-represented phytolith record (Figure 2-16). Unfortunately only a single radiocarbon date was obtained to chronologically place the phytolith assemblages (Fredlund et al. 1998). Warm season shortgrass phytoliths dominate the profile from 15 to $145 \mathrm{cmbs}$. Despite the near absence of dates to determine the rate of sedimentation, the authors divided the column into four parts and interpreted the different zones. Zone B, a period estimated to date between 5600 and 7900 B.P., significantly reflects more mesic conditions than today. On the other hand, the 


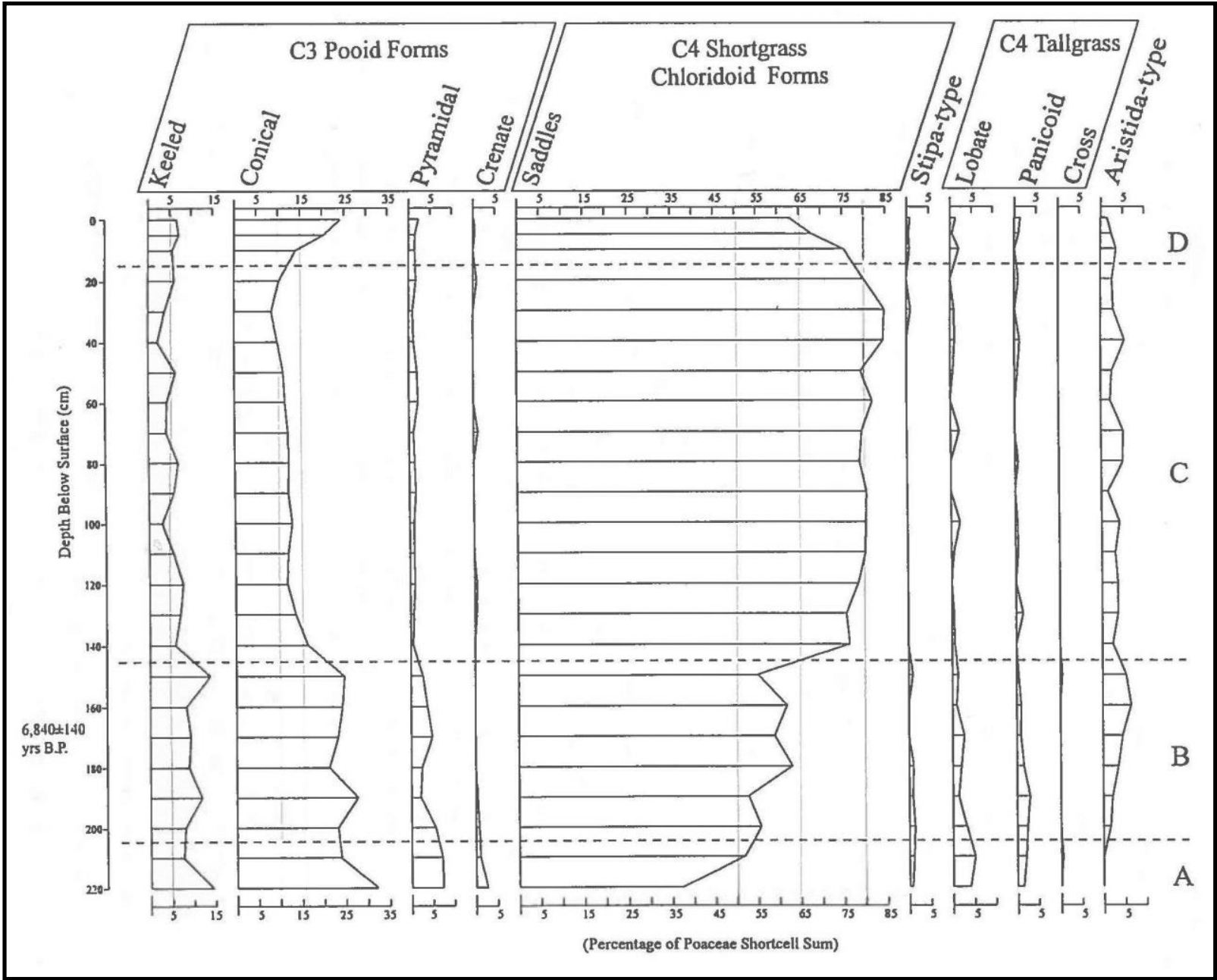

Figure 2-16. Short cell grass phytolith assemblages from Morgan Playa (from Fredlund et al. 1998:195, Figure 3).

relatively high percentage of threeawn (Aristida)type phytoliths indicates increased surface disturbance and aridity (Fredlund et al. 1998). Lacking solid chronological control the interpretations are not time-specific. The analysis demonstrates the merit of phytolith analysis for the Southern Plains.

Stafford (1981) studied the correlation between the geomorphology conditions and archeological potential of valleys crossing the Texas High Plains to demonstrate certain periods had significantly different archeological potential. He indicated sediments dating from 6000 to 2000 B.P. have a distinctly lower archeological potential.
As in other areas, the environmental record for the Altithermal period across Kansas is sparse. Stites (2006) summarizes the environmental data and draws heavily on Grueger's (1973) pollen sequences spanning the last 25,000 years from two marshes in Atchison County, northeastern Kansas. "Prairie vegetation, with perhaps a few trees along the valleys, covered the region until about 5,000 years ago, when a re-expansion of deciduous trees began in the lowlands" (Grueger 1973:239). Subsequently, quantities of ragweed (Ambrosia) and sagebrush (Artemisia) combined with sparse deciduous tree pollen signaled the end of the prairie interval. 
Mandel (1992, 1995, 2006) interprets the Kansas Altithermal geomorphic data as marked by upland erosion and sediment removal from low-order streams valleys and sediment increases in highorder stream valleys. This generally supports early interpretations by Haynes (1967) where he observed arroyo cutting after 7500 B.P. and channel filling between 6000 and 4000 B.P.

In Missouri, the remains of prairie faunal taxa are common in western Missouri archeological sites (Wolverton 2002). Interestingly, no reported prairie taxa such as bison are from sites in the northern Ozark Highlands of Missouri. Wolverton (2002) indicates new data reveals prairie taxa inhabited central Missouri during the warm and dry middle Holocene (8500 to 5000 B.P.).

Studies concerning the middle Holocene environment on Northern Plains and its margins are many and wider spread than those in the Southern Plains. It is not the intention of this summary to cover the broad Northern Plains region. However, one excellent example from the region reveals the common theme most studies revealed. In a high resolution study of diatom assemblage sediments from Moon Lake in southwestern North Dakota served as a gauge for water salinity changes to infer past climatic conditions (Laird et al 1996). The authors interpreted their data to reflect high salinity during the middle Holocene from 7300 to 4700 B.P. as evidence of a period of low effective moisture.

In summary, the diverse proxy datasets overwhelmingly reflect a mostly warm, dry Altithermal period between ca. 7000 to 4500 B.P. in the northerly regions of Texas, in Oklahoma, and across the plains. However, the lack of tight chronological control for many reported records hinders correlation of precise timing for the onset and duration of this warm, dry period or the potential for reversion to short moist period(s) within this long, arid period. Lack of well-dated assemblages also prevents researchers from refining the probable short-term fluctuations within the Altithermal period. The best evidence for the peak in the dry period may be from the dated handdug water wells at the Mustang Springs site in northwestern Texas, which places the principal well digging period between ca. 6800 and 6500 B.P.

\subsubsection{Regional Context and Discussion}

The following discussion considers environmental reconstructions and interpretations of paleoenvironments by multiple researchers employing diverse datasets gathered from across Texas and adjacent regions. It is unclear as to which specific dataset or analytical technique best represents the middle Holocene paleoenvironment. It is important to note that these datasets represent second, third, or higher order extrapolations from diverse content (Caran 1998). Also, just because warmer or drier conditions are detected in one part of the Southern Plains or in northwestern Texas does not necessarily mean that those conditions were applicable to central Texas. One should expect local and/or regional differences.

The use of different kinds of proxy data and the ambiguities in chronological controls probably account for slightly different interpretations and the slightly different ages. Johnson's and Goode's (1994) temperature shift projections contradict Bousman's (1998) canopy cover estimates and other studies in central Texas. This contradiction is difficult to resolve since Johnson and Goode (1994) employed calibrated radiocarbon dates in contrast to the dates presented by all other researchers. The radiocarbon assays from humates or bulk soils may be a major factor underlying the different ages of interpreted events as humate dates are composite organic matter in soils, which do not reliably date specific events (e.g., Brock et al. 2010; Frederick 2011). More precise dating through use of wood charcoal or even carbonized annual seeds from specific context provide greater precision in timing events in most instances. 
If the period of paleoenvironmental interest, roughly ca. 6000 to 4800 B.P., was arid, then dry conditions probably created great variation in types and density of vegetation as documented by empirical ground-cover observation during droughts in Kansas when large areas became devoid of plants (Weaver and Albertson 1956). The above ground net production of both $\mathrm{C}_{3}$ and $\mathrm{C}_{4}$ grasslands is strongly influenced by the amount and distribution of annual precipitation (Sala et al. 1988). Although the number, height and diversity of many grasses decline during drought phases, erosion and forbs increase and fuel limitations reduce fire importance (Clark et al. 2002). When precipitation is less than $37 \mathrm{~cm}$ per year, sandy soils with low water-holding capacity yield more productivity than loamy soils with higher waterholding capacity (Sala et al. 1988). Short grasses $\left(\mathrm{C}_{4}\right.$ photosynthetic pathways species) growing on stable lands would be the first to manifest drought effects, with many mesic plants $\left(\mathrm{C}_{3}\right.$ photosynthetic pathway species) disappearing completely. Bluestem grasses would decrease rapidly in two years of drought and would give way to $\mathrm{C}_{4}$ grama grasses, which increase during drought (Weaver and Albertson 1956). Various weeds would also start growth promptly and develop vigorously. The reduced vegetation cover would cause excessive runoff when infrequent rains occurred. The reduced carrying capacity would adversely affect Plains bison and pronghorn ranges which would undoubtedly disperse into smaller herds and reduce in number. Reduction in herbivore numbers has been postulated by Dillehay's (1974) documentation of bison absence or scarcity during the dry periods of the Altithermal (ca. 7000 to 4500 B.P.). Collins (1995:384, 2004:120) indicated the "climate during the cultural interval reflected by the Bell/Andice/Calf Creek projectile points was somewhat mesic" and a time when bison were hunted. He equates bison with mesic periods. Recently, Lohse et al. (2014a) radiocarbon dated 13 bison bones from Bell/Andice/Calf Creek components across Texas and demonstrated their presence during a very narrow 200 year period between 5060 through 5205 B.P. (cal 5815 through 5955 B.P.).

McDonald (1981) also proposed a general reduction in frequency of bison across the Plains during the middle Holocene, which is generally supported by their scarcity or absence at many localities. Bamforth (1987) states bison migrate from place to place: to search for food and water, to search for other members of their species, and to escape dangerous circumstances. The distribution of relatively high quality forage in a region at any given time forms a mosaic of patches of varying forage quality, with the distribution of high quality patches largely controlled by spatial distribution of available moisture. Thus, the distribution of high quality food patches control where bison would move. If moisture was limited, then bison were undoubtedly dispersed across the landscape and directed towards isolated patches of good forage. Better range conditions probably occurred along major river valleys and around large water bodies, and therefore, provided suitable range for bison present in a given region.

The above data strongly indicates dry and warm conditions existed across the Southern Plains, which includes central Texas during a period from ca. 7000 to 4500 B.P., with the region dominated by short $\mathrm{C}_{4}$ grasses that flourish in warm dry environments. Although increased warming and drying during the middle Holocene is documented, the intensity probably varied across space and over time. This sets the broad climate and paleoenvironmental conditions affecting human populations. Human populations living during this general period, and using a Bell/Andice/Calf Creek point technology were operating within and adapting to a relatively open grassland region during a warm and dry period. Bison were present during this period, although apparently in relatively low numbers. Most published data and interpretations derived from this data have demonstrated this period was generally warm. 
What is not well-documented at present, however, is the type and amount of changes to the animal and plant communities. Data concerning those changes will probably be derived from future archeological investigating at prehistoric sites of this age.

The limited prehistoric sites radiocarbon dated to a period between 6700 and 6500 B.P. across the Northern Plains (Walker 1992), combined with the radiocarbon dated 200-year period from ca. 6700 to 6500 B.P. for the hand-dug water wells at the Mustang Springs site in west Texas (Meltzer 1991, 1999), may actually mark the period of greatest aridity during the Altithermal.

Multiple local researchers (Albert 2012; Bousman 1998; Collins 2004; Holliday 1985a; Hudler 2000; Johnson and Holliday 1986; Nordt et al. 1994 and Nordt et al. 2007) have suggested a two-peak Altithermal, with a possible moist period in between. The Yellowhouse Soil at the Lubbock Lake site reflects a moist or stable period between two dryer events. Similar floodplain stability as reflected by soil development around 5100 and 5000 B.P. for the east-central Plains states of Kansas, Nebraska, Missouri, and Oklahoma supports a brief moist period in the late Altithermal (Johnson and Martin 2010). Albert (2012) also identified a wet period from ca. 5500 to 5000 B.P. in pollen data from Vitoria County, Texas.

Two rapid sea level rises discussed by Ricklis and Blum (1997), one between 7000 and 6000 B.P., and a later one between 4000 and 3000 B.P., support the notion of a two-drought interval. In the Estancia Basin of central New Mexico, the eolian landforms studied reveal two episodes of extreme drought and low groundwater levels during the middle Holocene (ca. 7000 to 5400 B.P.), followed by a rise in the water table through the late Holocene (Menking and Anderson 2003). Still, the pollen records from Boriack and Weakley bogs reveal a single peak in grass pollen at ca. 5000 B.P. (see Figure 2-10) (Bousman 1998). This peak in grass pollen occurs at the time Lohse et al. (2014a) documents the presence of bison in central Texas. Consequently, although some datasets correlate, many mixed signals exist in the proxy data. Much additional research is required to answer specific questions concerning the middle Holocene warm period, clearly document when it occurred, and the timing of probable wet period(s) within the Altithermal.

Paleoclimatologists have a much better understanding of the root cause to mid-Holocene aridity. Changes in the Earth's orbit have altered the amount of solar radiation reaching each latitudinal bond on earth (National Oceanic and Atmospheric Administration 2007). By calculating these changes, the northern hemisphere was probably warmer in the summer during the middle Holocene than today, and colder in the winter. However, paleoenvironmental evidence is present to demonstrate the magnitude of the average annual middle Holocene temperature was warmer than today's temperature. Nordt et al. (2007) presented the first comprehensive late Quaternary temperature record for the Great Plains by assessing trends of 64 published stable carbon isotopic values from buried soils. The estimated temperature was $+1.0^{\circ} \mathrm{C}\left(1.8^{\circ} \mathrm{F}\right)$ above modern July temperatures for the middle Holocene between 6000 and 4500 B.P.

In contrast, Poore et al. (2005) analyzed variations in the planktic foraminifera (unicellular organisms) from Gulf of Mexico sediment cores to indicate these species are an environmental proxy for the southwest monsoon on millennial and submillennial time scales. The marine record indicates monsoon circulation, or summer rainfall in the Gulf of Mexico, was enhanced in the middle Holocene (ca. 6500 to 4500 B.P.).

Most data support a drier and warmer period across much of the Southern Plains for the middle Holocene, or Early and Middle Archaic periods. A few studies indicate greater moisture at specific periods as indicted by Bement et al. (2007) for western Oklahoma with more volatility and greater variation in rainfall over short periods. In contrast, 
Lohse et al. (2014b) indicates the region was cooler in central Texas near the end of the Altithermal (ca. 5100 to 5400 B.P.). To what degree is yet to be clearly established. With the mostly dry, and probably warmer conditions, the climate had a profound effect on the foraging patterns and overall lifeways of middle Holocene populations. Studies have indicated a correlation between effective moisture and forage quality and bison herd size as periods of high effective moisture were times of large bison herds as reflected by extensive bison kills in Wyoming (Reher 1977). It also stands to reason the opposite occurred, when periods of low moisture dominated such as was apparent during the Altithermal, bison herds were smaller and more dispersed. It is clear from diverse datasets and variable interpretations concerning paleoenvironmental conditions that additional datasets, better context, and more precise dating are needed from middle Holocene deposits. Further knowledge must also be gained about environmental episodes before and after the middle Holocene to further refine interpretations about past climatic conditions and human responses to the changes. 
This page intentionally left blank. 


\subsection{CULTURAL BACKGROUND}

\section{$3.1 \quad$ INTRODUCTION}

This data recovery excavation occurred in the Central Texas Archeological Region as defined by Collins (1995, 2004), Prewitt (1981, 1985), and Weir (1976). The Big Hole site contains multiple components in a 3.5+ $\mathrm{m}$ deep stratified context as demonstrated during site discovery (Campbell et al. 2007). Subsequent hand-excavations, however, targeted a relatively narrow $50 \mathrm{~cm}$ thick vertical zone in the deep deposits thought to reflect middle Holocene events between roughly 5000 and 6000 B.P. Excavations yielded Martindale/Early Cornernotched and Bell points in separate, well-defined components. Therefore, the following cultural background section focuses on these two specific cultural intervals related to those two point types. The Bell/Andice/Calf Creek interval is presented first with an in-depth review of this poorly known cultural manifestation followed by a review of the more localized and localized Martindale interval.

\subsection{BELL/ANDICE/CALF CREEK HORIZON BACKGROUND}

J. Michael Quigg and Paul M. Matchen

\subsubsection{Introduction}

This section summarizes information concerning the Bell/Andice (Calf Creek in Oklahoma) horizon of the middle Holocene period. Initially a background review and brief history of investigations concerning this cultural horizon is presented for the Southern Great Plains, Texas, and Oklahoma. Next are discussions, which focus on their geographic distribution, chronometric age, cultural assemblage composition, lithic procurement strategies, material use, technical observations on knapping production, cooking technologies and processes. Then the subsistence base, landscape setting selection and mobility, possible trade and exchange interactions, and mortuary treatment practices are summarized. These discussions provide the foundation for advancing interpretations about human behaviors.

\subsubsection{Background and History}

This section provides an overview of what is known concerning this cultural horizon across Texas, Oklahoma, and surrounding states with selected inclusions of other pertinent materials. The following observations provide a foundation for framing future research. Such a framework encompassing various research topics provides the basis for applying various analytical techniques and methods to collect human behavioral information from contemporaneous but diverse occupations across the region.

Most reported artifacts from this middle Holocene manifestation are from surface sites, especially in Oklahoma. Few extensive archeological excavations in Texas and Oklahoma have encountered intact deposits that contained artifacts, which manifest this interval. In fact, less than two dozen excavated archeological components have been directly attributed to this horizon. Many diagnostic projectile points of this horizon have come from poorly defined excavated context. In the few investigated sites, the context of recovered material has often been unclear or ambiguous as artifacts are rarely derived from well-defined, stratigraphically-segregated occupation layers. Problems arise from interpreting surface-collected materials due to their lack of primary context and frequent mixture of artifacts from multiple time periods.

Limited excavations also severely constrain interpretations due to the discontinuous sampling from widely-distributed test units. Most published literature concerning this cultural horizon focuses on the diagnostic projectile points of this culture (Figure 3-1). 


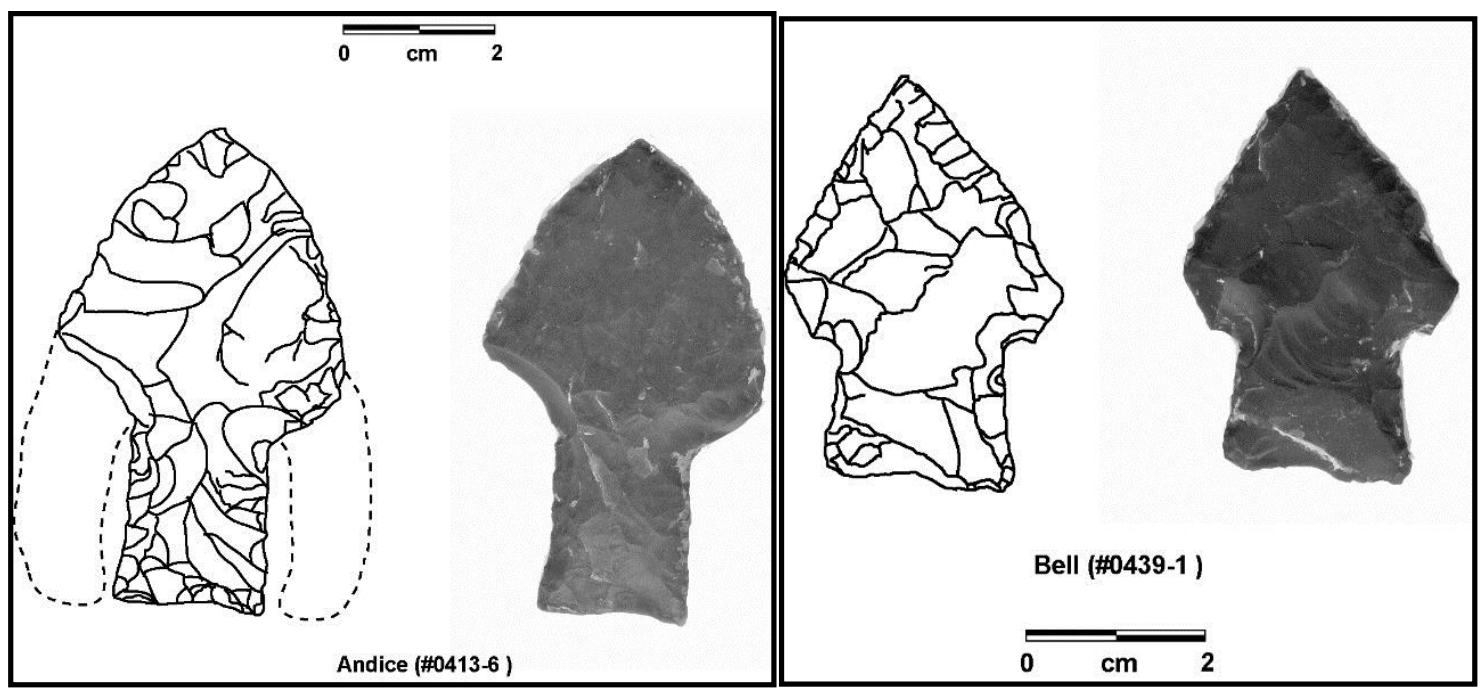

Figure 3-1. Diagnostic Andice and Bell point examples from the Edwards Plateau in central Texas (from Quigg and Lintz et al. 2011b).

\subsubsection{Investigations across the Southern Great Plains}

Although first noted by Adams (1958) in southwestern Missouri and again by Roberts in 1965 at Tick Creek Cave in Missouri, the Calf Creek point was subsequently named and described by Dickson (1968) as a provisional type based on projectiles from the Calf Creek Cave in Searcy County, northwestern Arkansas. Although the stratum from which these new and distinctive, deep basally-notched points were recovered was not directly radiocarbon dated, the points occurred below a zone that yielded Rice (wide cornernotched), Big Sandy (early side-notched) and Searcy (stemmed lanceolate) dart points, all part of the middle Holocene. The names and forms were subsequently discussed and illustrated by Bell (1960) and Perino (1968:14) and the Calf Creek name has been employed for these points since that time. Perino describes Calf Creek points as follows:

“... deep but narrow basal notches and barbs which are usually aligned with the base of the parallel-sided to slightly expanding stems. Bases vary from straight to slightly convex in outline. A slight angle is usually formed where the parallel sides of the barbs and the triangular shaped blade unite, but some point edges are convex or recurved due to resharpening of damaged specimens. Calf Creek points were roughly formed by percussion and excellently finished by a pressure flaking technique that often produced delicate serrations along the edges of the blade and barbs. In cross section, the points range from very thin to medium lenticular, the thicker specimens being made from poor grades of flint. The base and sometimes the edges of the stems were usually smoothed by grinding or dulling. Most specimens are resharpened; ranging from 43 to $63 \mathrm{~mm}$ in length" (Perino 1968:14).

During the mid-1980 and 1990s, the U.S. Army Corps of Engineers financed a multi-volume syntheses for both the southern and northern Great Plains region. The region-wide synthesis of hunter- 
gatherer culture history for the southern Plains includes a summary of the Archaic (Hofman 1989). The general Archaic patterns for the region involved the acquisition of a diverse range of modern species using a diffuse foraging pattern. Most Archaic lithic assemblages relied on local resource usage, although some tool forms occurred across extensive regions. The widespread pattern in tools may indicate a sharing of ideas and technological advances (Hofman 1989). An important technological development during the Archaic involved the intentional thermal alteration of cherts to improve flaking quality. Hofman lists five assumptions believed to be common characteristics of Archaic groups across the Southern Great Plains region, which include the following: 1) seasonally varied economies, 2) flexible group structure with periods of aggregation and dispersal, 3) seasonally variable needs such as fuel, shelter, and clothing, 4) a variety of site types which result from diverse economic, social, and maintenance activities, and 5) a variety of alternative strategies for coping with seasonal, or yearly, economic shortfalls or windfalls (Hofman 1989). He briefly describes selected complexes defined across the region. Only those complexes, pertinent to the time period from ca. 6000 to 4500 B.P., are presented below.

The Black Vermillion phase was defined by Schmits (1978 as cited in Hofman 1989) for components at the Coffey site, in northeastern Kansas and provide a range of radiocarbon dates between 5700 to 4900 B.P. This phase is distinguished by triangular basal- and cornernotched dart points or knives (Figure 3-2). The Coffey site yielded a wide range of plant and animal resources that included weedy plants, fish, reptiles, birds, small animals, deer, and bison (Hofman 1989). Blackmar and Hofman (2006) indicate a similar age for this phase, from 5650 to 4850 B.P. The basally-notched points from Horizons III-5 and III-7 at the Coffey site were stratigraphically associated with several kinds of corner-notched forms (Schmits 1976, 1978).

Hofman (1989) also discusses the Munkers Creek phase in northeastern and east-central Kansas and also dates to 5500 to 5000 B.P. (Blackmar and Hofman 2006; Witty 1969, 1982). The economy of this group was apparently based on hunting and foraging, with deer and small animals serving as the primary meat sources. Distinctive artifacts include the Munkers Creek gouges, Munkers Creek bifaces, chipped double bitted axes, and long and narrow lanceolate dart points with small, broad stems and primarily straight bases (Figure 3-3) (Witty 1982). Neither the Black Vermillion nor Munkers Creek phases have yielded deeply basal-notched points similar to the Calf Creek style. These phases overlap in time with Calf Creek materials, which increases the potential for group interactions to have occurred.

Stites (2006) summarized the Calf Creek horizon in Kansas and acknowledged formal research on this complex in Kansas is lacking. His review draws primarily on data from Coffey, William Young, and Site 98. A distribution map of known Calf Creek points is restricted to the southeastern corner of Kansas. What little is known about the cultural technology in Kansas is consistent with data recorded further south in Oklahoma.

\subsubsection{Bell/Andice in Texas}

In Texas, the Bell projectile point was first described as a provisional type by Sorrow et al. in 1967. This first description was based on the recovery of 10 similarly-crafted, deep basallynotched projectile points from Stratum III-b at the Landslide site (41BL85) in Bell County, central Texas (Figure 3-4). Stratum III-b was roughly 30 to $40 \mathrm{~cm}$ thick and yielded two Bulverde and two triangular projectile points. Four burned rock concentrations (Features 1, 6, 8, and 9) were present in Stratum III-b. Just below in Stratum IV, 


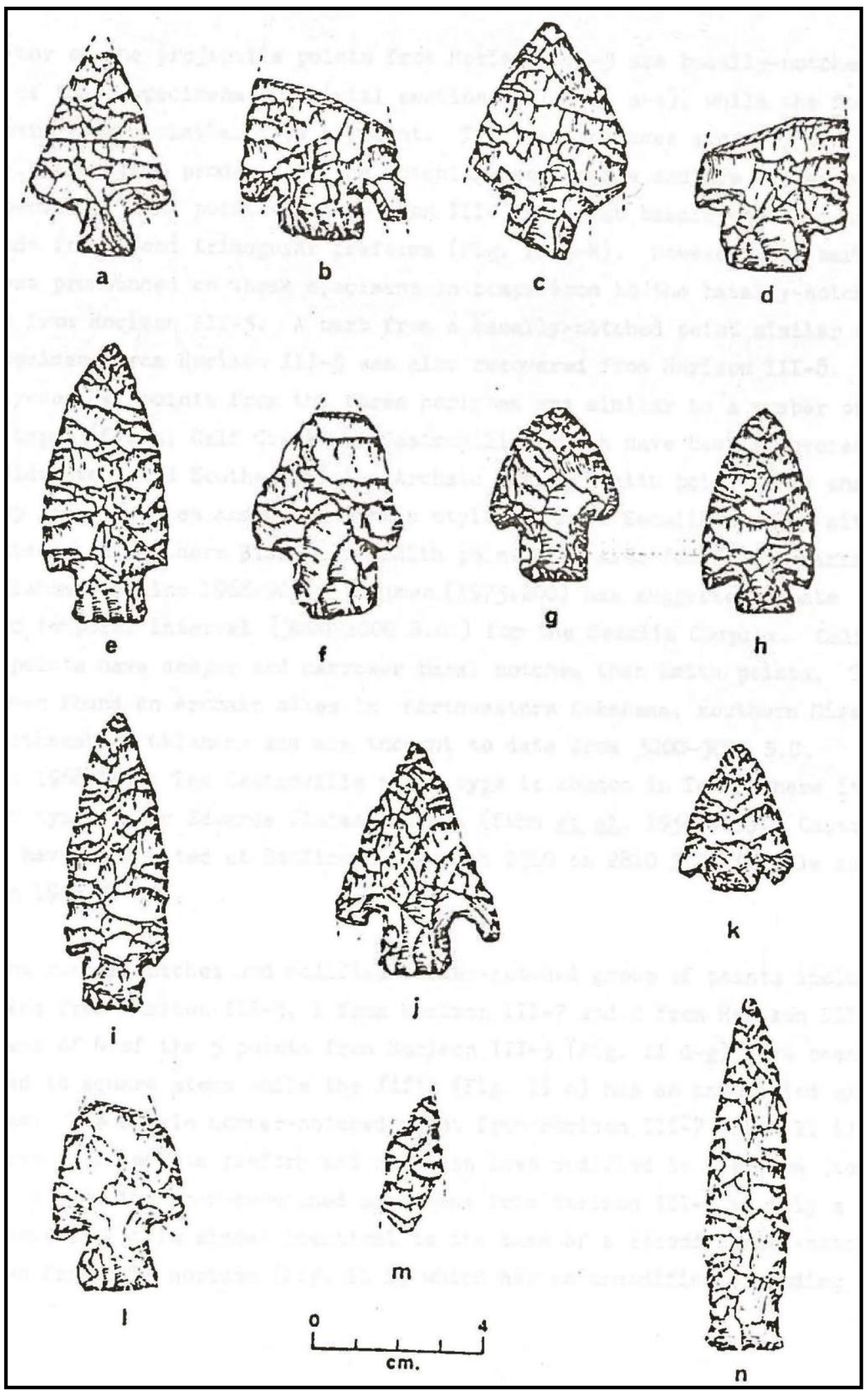

Figure 3-2. Projectile points from the Coffey site in Kansas (from Schmits 1976, 1978). Top two rows (a-h) from Horizon III-5, next row (i-k) from Horizon III-7, and bottom row (I-n) from Horizon III-8. 


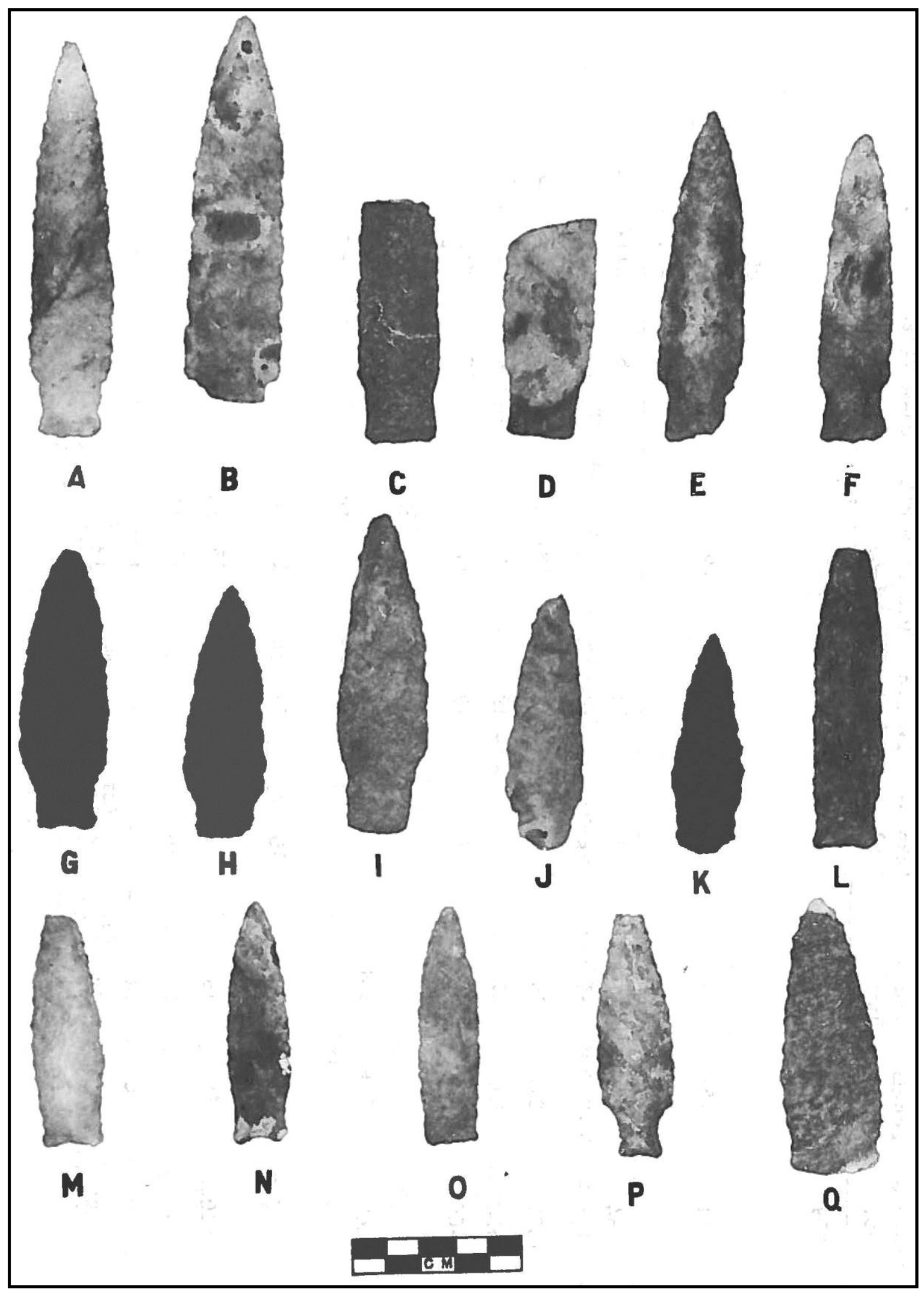

Figure 3-3. Munkers Creek points from Witty (1982:171). 


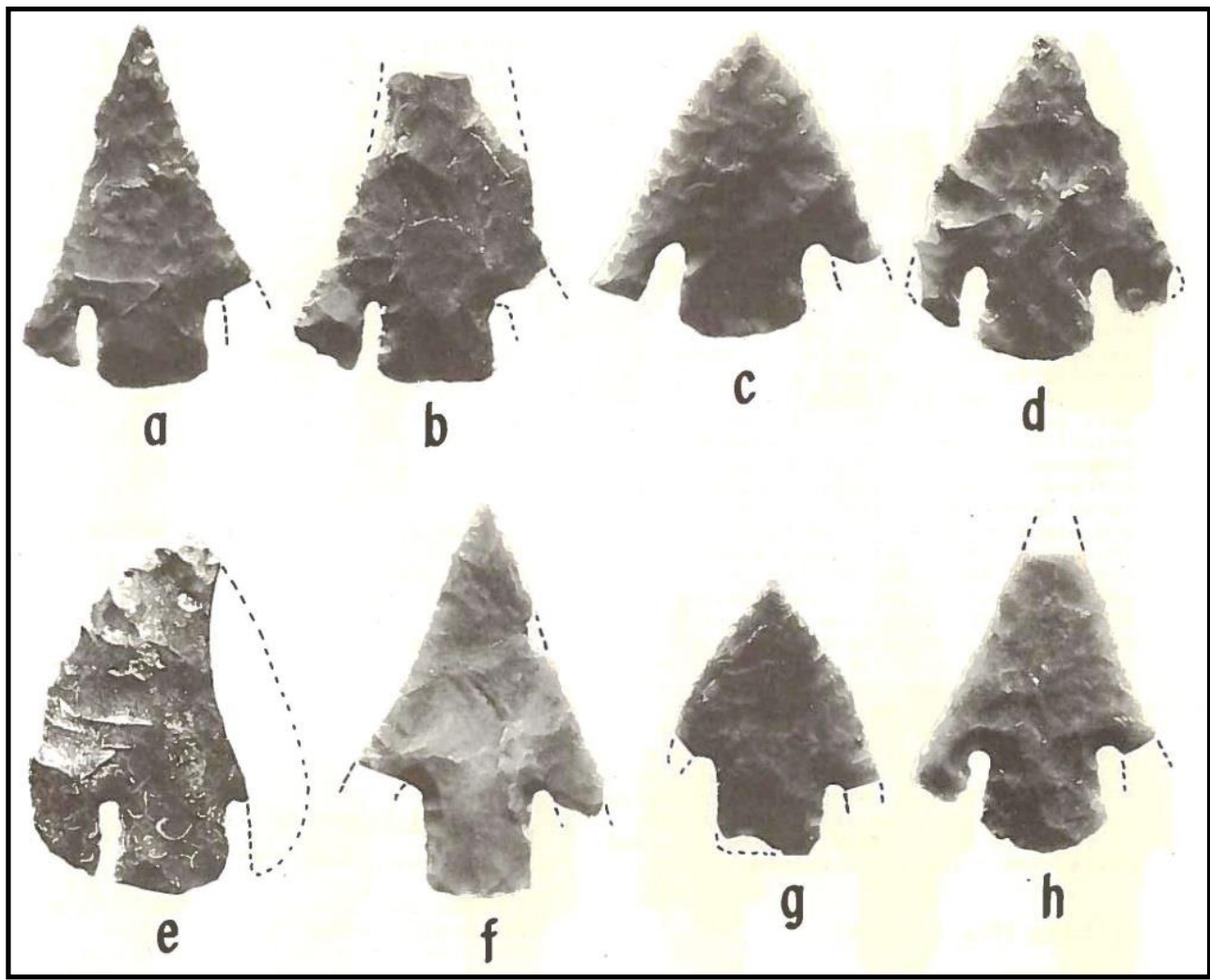

Figure 3-4. Bell points from the Landslide site (from Sorrow et al. 1967: Fig.10).

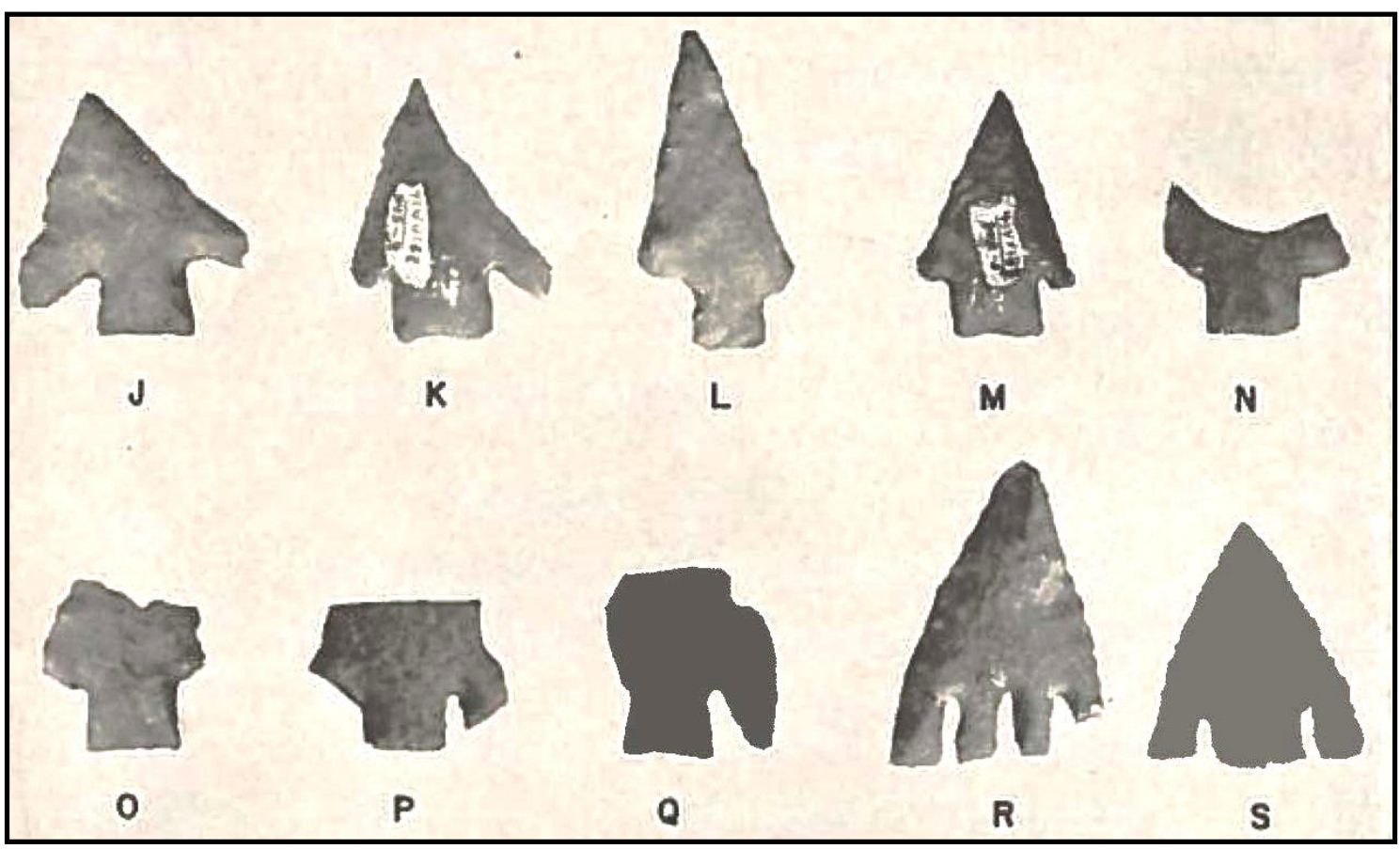

Figure 3-5. Johnson's "Early Barbed" points from the Devil's Mouth site (Johnson 1964, Figure 11; E. Prewitt 1981a considers Bell/Andice varieties). 
a $150 \mathrm{~cm}$ diameter, circular mass of burned rock (Feature 2) was excavated with multiple bison bones on top and a Bell point possibly associated. This lower stratum yielded a mixture of projectile point types that included two Bell, one Martindale, and one Merrell point. Bison bones resting on Feature 2 were radiocarbon dated to $>3520$ B.P. (Tx-289) without a $\delta^{13} \mathrm{C}$ correction value; and the material dated was the organic fraction (Valastro et al. 1967).

Although the Landslide site was the first to yield the Bell point type in Texas, excavations at the Devil's Mouth site (41VV188) in the Lower Pecos of southwestern Texas in 1959 and 1961-62 yielded 10 basal-notched points similar to Bell points (Johnson 1994). These points were called "Early Barbed" points and exhibit long barbs formed by deep basal notches; many have asymmetrical blades and rectangular stems (Figure 3-5) (Johnson 1964, Figure 11). These "Early Barbed" points came from the deepest part of the Early Archaic deposits (Levels 17 through 21 in Area A), just above Paleoindian points, and just below Pandale points at the Devil's Mouth site (Johnson 1964). Johnson reported these "Early Barbed" styles had not been previously reported in Texas or adjacent regions. Other artifacts associated with these "Early Barbed" points (presently considered Bell/Andice varieties by Prewitt 1981a) were bifaces, side scrapers, drills, burin and burin spalls, core tools, manos and metates, scratched pebbles, and edgemodified flakes.

Frank Weir (1976) reviewed and revised the central Texas Archaic into five named phases (San Geronimo, Clear Fork, Round Rock, San Marcos, and Twin Sisters) based on specific changes in projectile point forms (Figure 3-6). Weir listed only two sites with Bell points, which also contained at least four other point types: Gower, Martindale, Uvalde, and Tortugas (Wier's "Tortugas" is probably equivalent to the Baird and Taylor point types in existing usage). He attributed these four recognized types to the San Geronimo phase of the Early Archaic.

In a more ambitious effort to synthesize the cultural chronology of central Texas, Prewitt (1981) attributed the Bell and Andice points to his newly named Jarrell phase of his Early Archaic period (see Figure 3-6). Although Prewitt employed the term Andice, this point type was not formally defined until 1983 (Prewitt 1983; see discussion below). Prewitt listed large flat hearths under feature types for the Jarrell phase, but did not list burned rock middens as one of the key index markers. Other characteristic artifacts in addition to the point types include Clear Fork gouges, bifaces, scrapers, hammer stones, and grinding stones. The sites with Jarrell components included the Landslide site (41BL85), the Gault site (41BL323), site 41TV17, the Tombstone Bluff site (41WM165), the Merrell site (41WM2), the La Jita site (41UV21) and the Jetta Court site (41TV151) (Prewitt 1985). No radiocarbon dates were available in 1985. Prewitt estimated the phase's age at ca. 6000 to 5000 B.P. Chronologically, he attributed Gower, Hoxie, and Wells point types to the preceding San Geronimo phase. He attributed the Baird and Taylor triangular point forms to the following Oakalla phase. Prewitt (1981) also noted Bell and Andice types are possibly related to the same tradition represented by the Calf Creek points in northeastern Oklahoma, northwestern Arkansas, and southwestern Missouri. Despite the absence of absolute dates, many Texas archeologists employed Prewitt's chronology, terminology, and projectile point-related sequence.

In 1981, McKinney (1981) published a distribution map of selected sites with Bell or Bell-like points. Seven sites were listed as having yielded Bell points and another nine sites yielded Bell-like points. Their geographical range reveals a concentration along the Balcones Escarpment in central and west Texas and southeast to the Texas coast. One exception was five Bell/Bell-like points in Crosby 


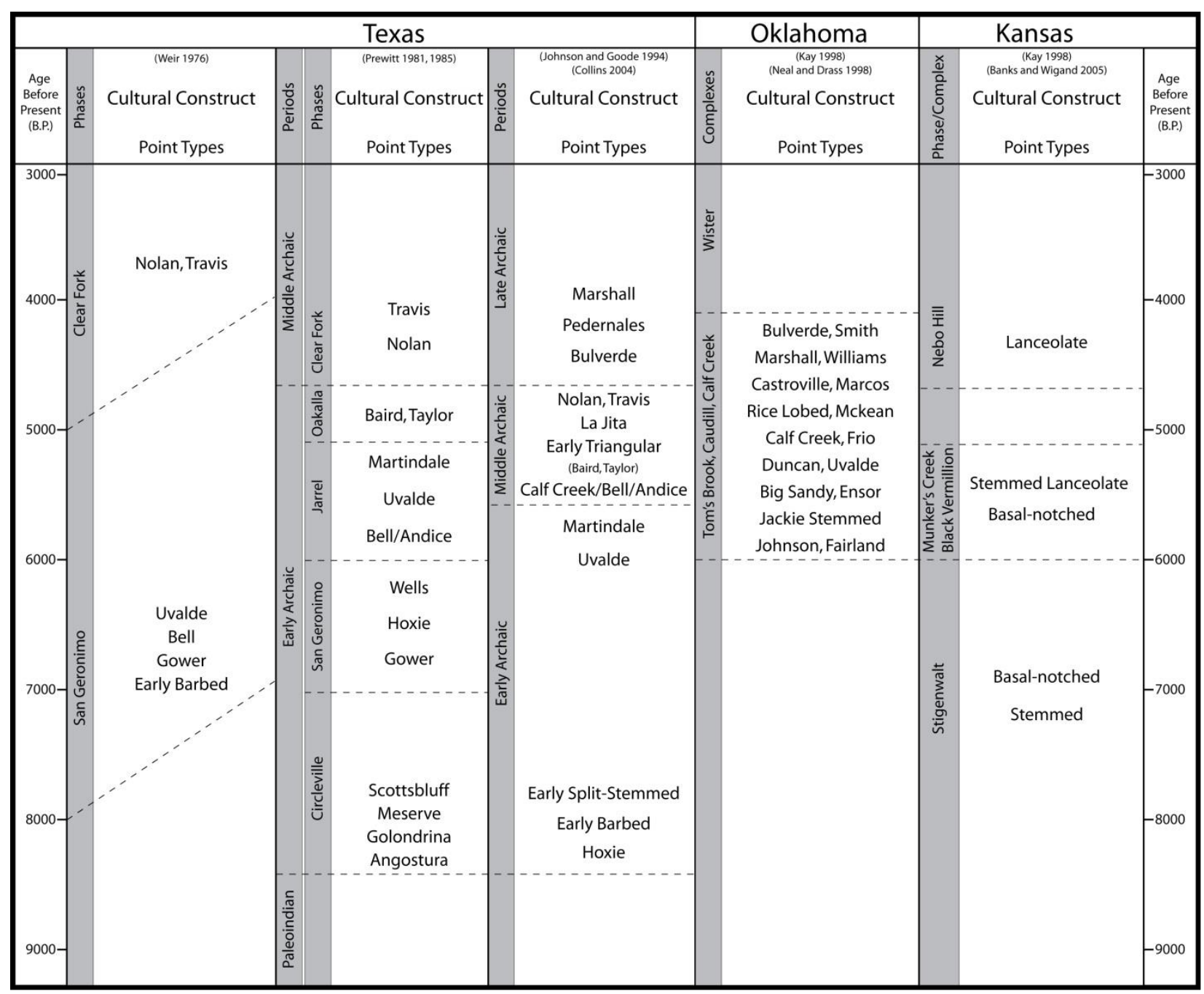

Figure 3-6. Middle Holocene cultural constructs for the Southern Plains region.

County on the eastern edge of the Llano Estacado and near the head waters of the Brazos River (Parker and Mitchell 1979). McKinney comments Bell points appear to have a wider distribution than the earlier Gower points.

Prewitt (1983) described and formally named the Andice point as an Early Archaic type in Texas. His type Andice specimens were excavated by J. E. Pearce in 1929 from the Gault site (41BL323), the type site for this form in Texas. Prewitt recognized the following attributes as distinctly separating Andice from Bell: greater size, greater stem and barb lengths. The primary difference between the two types is a shorter stem length for Bell points as compared to the Andice type. Prewitt acknowledged both Andice and Bell types may be related to the Calf Creek point type of eastern Oklahoma and western Arkansas as measurements of those points cluster mid-range between the Texas Bell and Andice types. Prewitt (1983) cautions Andice points are frequently misidentified as Bulverde type.

In a qualitative analysis of Bell and Andice projectile points, Weber and Patterson (1985) determined these two point types are statistically distinct based on stem length and blade thickness. Even though these points can be classified into two groups, the general similarity in form, distribution, and presumed ages, Weber and Patterson (1985) concluded Andice and Bell types may represent a 
"single technological continuum" with a close technological relationship with Calf Creek point type in Oklahoma and other states. However, in a subsequent study using discriminant function analysis, Andice and Bell points were determined not to support the postulated typological separation (Weber 1986). Although these two point types have been attributed separate names in Texas, statistically they have been shown to represent the same point with slight alterations during their uselife, which can be metrically-documented (Weber 1986).

Prewitt (1985) compiled and published 147 radiocarbon dates in an attempt to support his 1981 chronology for central Texas. He provides just two assays for his Jarrell phase, and both came from Williamson County. One date of 5285 B.P. was from $41 \mathrm{WM} 73$ and the other date was 4970 B.P. derived from Feature 19 at the Cervenka site (41WM267). Based on these two dates, he attributed the Jarrell phase to a period dating from about 6100 to 5100 B.P. The date from the Cervenka site was based on charcoal from Feature 19 (ca. $93.5 \mathrm{~m}$ in elevation) in Area D, about $5 \mathrm{~cm}$ above an undated Feature 26, which yielded bison bones. In Area A at the Cervenka site, one Andice point (97.0 $\mathrm{m}$ in elevation) was from a nonfeature context in Level 22 (Peter et al. 1982).

Since the Cervenka site was reported, a few tested or excavated sites add significant information towards understanding populations that employed Bell and Andice points (i.e., Collins 1994a; Kibler and Scott 2000; Lohse et al. 2013; Quigg and Frederick 2005; Quigg et al. 2007). Collins (1994) presents data recovery results from a $104 \mathrm{~m}^{2}$ excavation block, which represented a mixed or palimpsest Early Archaic component in Area B at the Barton site (41HY202-B) in Hays County. A series of Early Archaic events accumulated on a stable or slowly aggrading clay loam zone less than $50 \mathrm{~cm}$ thick, overlying archeologically sterile gravels. The mixed materials consisted of nine Early Archaic features along with 15,468 pieces of lithic debitage, and 98 chipped stone tools made of uniformly patinated chert. The 23 diagnostic artifacts are dominated by the 11 Bell/Andice points along with 4 Uvalde 3 Early Triangular, 1 Wilson, 1 Gower, 1 Angostura, and 2 unknown dart point types. Feature 19 contained 2,055 chert debitage pieces concentrated in a 100-by-185 cm area. Although no diagnostic tools were from this lithic concentration, four distinctive Bell/Andice/Calf Creek "notching flakes" derived from knapping narrow, deep basal-notches characteristic of this point type were found (Collins 1994a). Based on these distinctive flakes and their stratigraphic position, this feature was attributed to the Bell/Andice interval. Collins (1994) also suggested Prewitt's $(1981,1985)$ Jarrell phase be split into an earlier interval containing Uvalde and Martindale style dart points and a later interval for which Bell/Andice dart points are diagnostic.

In their synthesis of central Texas Archaic, Johnson and Goode (1994) assign the Calf Creek/Bell types into the early part of the Middle Archaic period with a projected starting date of ca. 5600 B.P. (see Figure 3-6). Interestingly, they fail to include or even mention the Andice point type in these discussions. They suggest this distinctive assemblage represents large game hunting groups from the Eastern Woodland margins, which followed expansion of bison to this region during a brief moderately moist, but drying climatic episode. They also state Early Triangular forms, Baird and Taylor point types, are approximately contemporaneous with or slightly post-date the Calf Creek cultural interval (Johnson and Goode 1994).

Collins (1995, 2004) more recent syntheses of central Texas prehistory groups Bell/Andice/Calf Creek types together as Johnson and Goode (1994) did, and places these types into one style interval attributed to the early part of the Middle Archaic, which he estimated to date ca. 6000 to 5000 B.P. (see Figure 3-6). Collins claims the Bell/Andice component at the Landslide site has high integrity, although he does not list any other sites with high- 
to-moderate degrees of integrity. $\mathrm{He}$ also recognizes a subsequent Middle Archaic cultural interval characterized by triangular Taylor point type. He feels these two Middle Archaic cultural intervals reflect a lithic technological shift from relatively specialized dart points employed during the terminal Early Archaic to these initial Middle Archaic styles. The Taylor points could have served as knives or as tips of lances, spears, or darts. Collins (1995, 2004) states the Bell/Andice/Calf Creek period occurred when climatic conditions were somewhat mesic, and bison were the dominant species hunted. He also agrees with Johnson and Goode (1994) these thin bifaces were part of a specialized bison-hunting toolkit, probably brought by people moving southwesterly from the prairie, prairie margins, and woodlands areas west of the Ozarks.

Additional Bell/Andice sites and components have been investigated since these last syntheses, some with excellent context and associations (e.g., Kibler and Scott 2000; Quigg and Frederick 2005; Quigg et al. 2007) and other investigated sites or components with questionable stratigraphy and poor contexts (e.g., Collins 1994a, 1998; Decker et al. 2000; Houk et al. 2008; Johnson 1991; and others). Sites with better context are selected and discussed below.

Excavations within a $59 \mathrm{~m}^{2}$ area at the Cibolo Crossing site (41BX377) in Bexar County near San Antonio encountered a deeply stratified Bell/Andice component in creek terrace deposits (Kibler and Scott 2000). Seven cultural components were delineated despite the lack of obvious component separation by sterile zones between components. A series of 4 sediment-based and 16 charcoal radiocarbon dates provide a chronological sequence for the deposits and associated materials. The Bell/Andice component $\left(59 \mathrm{~m}^{2}\right.$ or $\left.16.8 \mathrm{~m}^{3}\right)$ was defined just above a Martindale component and just below a Late Archaic component with Castroville, Ensor, and Frio points. The Bell/Andice component provides significant data derived from an assemblage with 55 chipped stone tools 897 pieces of lithic debitage, 12 cultural features, 41 animal bones, $715 \mathrm{~kg}$ of burned rocks, and stratigraphic information supported by radiocarbon dates. Eight radiocarbon dates from the Bell/Andice component are probably intrusive charcoal; the sediment dates are at least 1,000 years too young for the projected age of the component. Three charcoal $\delta^{13} \mathrm{C}$-corrected dates of $4420 \pm 50$ B.P. (Beta126362), $4400 \pm 60$ B.P. (Beta-126364), and $4370 \pm$ 80 B.P. (Beta-126367) are statistically contemporaneous. All three were from burned rock discard pile Feature 19, directly associated with two Bell points and an Andice point barb, and are accepted by the authors as reasonable dates for the Andice/Bell component (Kibler and Scott 2000:74). Seven calendrical dates were also derived from mean Dalloisoleucine/L-isoleucine (A/I) values using the racemization dating method on Rabdotus snail shells and range from 4528 to 5503 B.P. Four A/I racemization dates on Rabdotus snails from Feature 19 have projected ages of 5128 B.P., 5128 B.P., 5165 B.P., and 4528 B.P. (Kibler and Scott 2000:74). Diagnostic projectiles include nine Bell point fragments, one Andice barb, two triangular Baird points, and one untyped point midsection. Some may argue the identification of some Bell points. Cooking is inferred from multiple burned rock features as reflected by two rock-filled basin-shaped pits. Direct evidence of subsistence resources is limited to a few unidentifiable bone scraps and one freshwater mussel shell valve. The authors provide metric data on the stone tools (i.e., points, bifaces, and unifaces), descriptions and discussion of features, brief discussions of the unmodified debitage, raw material types, and some discussions on site function and interpretations for this hunting group using the Cibolo Creek site data (Kibler and Scott 2000).

In 2004, limited assessment investigations at the Slippery Slope site (41MS69) in Mason County of the Edwards Plateau yielded an Andice point fragment at the same elevation and $50 \mathrm{~cm}$ from dense burned rock Feature 1 eroding on a steep 
slope (Quigg and Frederick 2005). Eight radiocarbon dates on charcoal from Feature 1 average 4993 B.P. (Table 3-1). Burned rock Feature 2, stratigraphically $15 \mathrm{~cm}$ below Feature 1 was radiocarbon dated by seven charcoal samples to an average age of 5305 B.P. (Table 3-1) (Quigg et al. 2015). Despite differences in depths, Features 1 and 2 were thought to represent the same culture, although restricted excavations did not yield any diagnostic projectile points associated with Feature 2.

Limited excavations at the Ticket Kiosk at the Spring Lake site (41HY160) in Hays County, just south of Austin, yielded two Bell/Andice, three Merrell, one Martindale and two Uvalde projectile points from a $50 \mathrm{~cm}$ thick cultural zone (Lohse et al. 2013). The investigations document a more or less continuous occupation sequence spanning the Early Archaic to the latest Late Prehistoric period, and are supported by 14 radiocarbon dates derived from mammal bones combined with 59 projectile points and point fragments (Table 3-1). The Bell/Andice/Calf Creek component was defined between roughly 135 and $185 \mathrm{cmbd}$ with the multiple point types ( 3 Merrell, 1 Martindale, and 2 Uvalde). The faunal assemblage from this $50 \mathrm{~cm}$ thick zone includes bison, turtles (Testudines), deer (Odocoileus), pronghorn (Antilocapra americana), Canidae, cottontail rabbits (Sylvilagus), bird (Aves), fish (Osteicthyes), and snake (Serpentes). Ten $\delta^{13} \mathrm{C}$ corrected radiocarbon dates on bison bones range between 5060 B.P. and 5180 B.P. at the two sigma range (see Table 3-1) (Lohse et al. 2014a).

The Gatlin site (41KR621) along the upper Guadalupe River in Kerr County, Texas is example of a site with questionable stratigraphy and context (Houk et al. 2008:7-7 through 7-10). Eighteen of 46 wood charcoal and two bulk soil radiocarbon dates from this site fall between 5570 and 4930 B.P. Most dates were derived from cultural features associated with a variety of point types such as Bandy, Gower, and Martindale types, but not directly with Bell/Andice points. Four dates were from Feature 2 directly associated with three Martindale and one Gower point. Although not directly associated with features, 18 Bell and Andice points (including 2 "Western Bell or Early Barbed" points [Prewitt 1981]) were among the 342 identified points from the roughly $145 \mathrm{~m}^{3}$ excavation area. The site was interpreted as having a mixture of temporal indicators as represented by four subjectively defined occupation zones or analytical units with compressed stratigraphy. The four zones were not always distinguishable and lacked stratigraphic separation by sterile fill (Houk et al. 2008). The suite of radiocarbon ages per zone overlap the ages of more than one zone. Sixteen Bell/Andice points were attributed to the top three occupation zones and the two Early Barbed or Western Bell point varieties were from the lowest occupation zone.

We currently know very little about the precise age of the Bell/Andice interval. As future excavations encounter Bell/Andice components with good context across Texas and more technical analyses are conducted on those assemblages, a significant increase in knowledge and understanding of this cultural interval is anticipated. How the Texas Bell/Andice manifestations relate to similar cultures in the surrounding region remains to be thoroughly studied.

\subsubsection{Bell/Andice in Oklahoma}

In Oklahoma the term Calf Creek is employed to describe essentially the same projectile points referred to as the Bell and Andice types in Texas (Perino 1968). The last state-wide synthesis of prehistory in Oklahoma was published 30 years ago (Bell 1984) and discusses only three extensively excavated sites in western Oklahoma for general Archaic periods ca. 9000 to 1400 B.P. (Hughes 1984). Little was known about the Archaic period as a whole, only four Archaic period radiocarbon dates were available for interpretation. Despite 
Table 3-1. Radiocarbon Dates for Sites Associated with Bell/Andice/Calf Creek Points.

\begin{tabular}{|c|c|c|c|c|c|}
\hline Site Name/No. & $\begin{array}{l}\text { Uncalibrated } \\
\text { (B.P.) }\end{array}$ & $\begin{array}{l}\text { Laboratory } \\
\text { Number }\end{array}$ & $\begin{array}{l}\text { Associated Point } \\
\text { Types }\end{array}$ & Reference & Context and Comments \\
\hline \multicolumn{6}{|c|}{ OKLAHOMA } \\
\hline $\begin{array}{c}\text { Arrowhead } \\
\text { Ditch, 34MS174 }\end{array}$ & $5730 \pm 160$ * & Beta-28192 & $\begin{array}{l}\text { Indirectly } 1 \text { Calf } \\
\text { Creek above }\end{array}$ & $\begin{array}{l}\text { Wyckoff et al. } \\
1994\end{array}$ & $\begin{array}{l}\text { Calf Creek point was } 50 \mathrm{~cm} \text { above charcoal date, } \\
\text { date from salvaged Feature } 2 \text { (burned rock oven), } \\
290-320 \mathrm{cmbs} \text {, }\end{array}$ \\
\hline Near Tulsa, OK & $5120 \pm 25$ & $\begin{array}{l}\text { UCI AMS- } \\
11696\end{array}$ & 1 Calf Creek & $\begin{array}{l}\text { Bement et al. } \\
2005\end{array}$ & $\begin{array}{l}\text { Petrous bone from Bison antiquus occidentalis skull } \\
\text { with Calf Creek point imbedded in skull }\end{array}$ \\
\hline $\begin{array}{l}\text { Kubik site, } \\
\text { 34KA354 }\end{array}$ & $5050 \pm 60$ & Beta-98146 & No points & $\begin{array}{l}\text { Neal } 1999 \\
2002\end{array}$ & $\begin{array}{c}\text { Level } 16 \text {, Unit 0,0, charred nut husk, same sample as } \\
\text { below, split }\end{array}$ \\
\hline $\begin{array}{l}\text { Kubik site, } \\
34 \mathrm{KA} 354\end{array}$ & $5020 \pm 120$ & NZA-6602 & No points & $\begin{array}{l}\text { Neal } 1999 \\
2002\end{array}$ & $\begin{array}{l}\text { Level 16, Unit 0,0, charred nut husk, same sample as } \\
\text { above, split }\end{array}$ \\
\hline $\begin{array}{l}\text { Kubik site, } \\
\text { 34KA354 }\end{array}$ & $4990 \pm 100$ & NZA-6601 & No points & $\begin{array}{l}\text { Neal } 1999 \\
2002\end{array}$ & Level 15 , Unit 0,0 , scattered charred wood \\
\hline $\begin{array}{l}\text { Bellcow, } \\
\text { 34LN29 }\end{array}$ & $4190 \pm 80$ * & Beta-31404 & $\begin{array}{l}6 \text { Calf Creek point } \\
\text { fragments }\end{array}$ & $\begin{array}{c}\text { Girard and Carr } \\
1994\end{array}$ & $\begin{array}{l}\text { All bone from } 140 \text { to } 160 \mathrm{cmbs} \text { in northern trench, } \\
\text { collagen was of poor quality for dating, }\end{array}$ \\
\hline \multicolumn{6}{|c|}{ TEXAS } \\
\hline $\begin{array}{l}\text { Devils } \\
\text { Rockshelter, } \\
\text { 41VV264 }\end{array}$ & $7430 \pm 240 *$ & TX-314 & Bell & Prewitt 1966 & $\begin{array}{l}\text { Charcoal from Zone V I Test Pit 2, which contained } \\
\text { "Early Barbed" points that Prewitt now calls "western" } \\
\text { Bell. }\end{array}$ \\
\hline $\begin{array}{c}\text { Arenosa } \\
\text { Shelter, 41VV99 }\end{array}$ & $5360 \pm 170$ & TX-313 & $\begin{array}{l}4 \text { Early barb, Early } \\
\text { Triangular }\end{array}$ & $\begin{array}{l}\text { Dibble 1967, } \\
1997\end{array}$ & $\begin{array}{l}\text { Looks like Martindale, but not directly associated, } \\
\text { scattered charcoal near base of Stratum } 32\end{array}$ \\
\hline 41WM73 & $5285 \pm 726$ & UGa-2482 & $\begin{array}{l}1 \text { Bulverde-like, } 1 \\
\text { Nolan, } 1 \text { Group 2, }\end{array}$ & $\begin{array}{c}\text { Peter \& Hays } \\
\text { 1982, Johnson } \\
\text { 1987, Sorrow } \\
\text { et al. } 1987\end{array}$ & $\begin{array}{c}\text { In stratum 6, a compact yellowish brown clayey } \\
\text { matrix, charcoal from stratum, Level 19, Area B, } \\
\text { initial occupation of site. }\end{array}$ \\
\hline $\begin{array}{l}\text { Slippery Slope, } \\
41 \mathrm{MS} 69\end{array}$ & $5000 \pm 40$ & $\begin{array}{c}\text { Beta- } \\
233355\end{array}$ & 1 Andice & $\begin{array}{l}\text { Quigg et al. } \\
2015\end{array}$ & Charcoal from Feature 1 in Unit 5, at $152 \mathrm{cmbs}$ \\
\hline $\begin{array}{l}\text { Slippery Slope, } \\
41 \mathrm{MS} 69\end{array}$ & $5070 \pm 40$ & $\begin{array}{l}\text { Beta- } \\
233352\end{array}$ & 1 Andice & $\begin{array}{l}\text { Quigg et al. } \\
2015\end{array}$ & $\begin{array}{l}\text { Charcoal from Feature } 1 \text { in Unit 5, level } 14 \text { at } 134 \\
\text { cmbs }\end{array}$ \\
\hline
\end{tabular}


Table 3-1. Radiocarbon Dates for Sites Associated with Bell/Andice/Calf Creek Points (continued).

\begin{tabular}{|c|c|c|c|c|c|}
\hline Site Name/No. & $\begin{array}{l}\text { Uncalibrated } \\
\text { (B.P.) }\end{array}$ & $\begin{array}{l}\text { Laboratory } \\
\text { Number }\end{array}$ & $\begin{array}{l}\text { Associated Point } \\
\text { Types }\end{array}$ & Reference & Context and Comments \\
\hline $\begin{array}{l}\text { Slippery Slope, } \\
41 \mathrm{MS} 69\end{array}$ & $3910 \pm 40$ * & $\begin{array}{c}\text { Beta- } \\
370497\end{array}$ & 1 Andice & $\begin{array}{l}\text { Quigg et al. } \\
\quad 2015\end{array}$ & Ashy sediments from around Feature 1 burned rocks \\
\hline $\begin{array}{l}\text { Slippery Slope, } \\
41 \mathrm{MS} 69\end{array}$ & $4890 \pm 40$ & UGA-14116 & 1 Andice & $\begin{array}{l}\text { Quigg et al. } \\
2015\end{array}$ & Charcoal from Feature 1 in Unit 5 Ext.at $152 \mathrm{cmbs}$ \\
\hline $\begin{array}{l}\text { Slippery Slope, } \\
41 \mathrm{MS} 69\end{array}$ & $4920 \pm 40$ & $\begin{array}{c}\text { Beta- } \\
370495\end{array}$ & 1 Andice & $\begin{array}{l}\text { Quigg et al. } \\
2015\end{array}$ & Charcoal from Feature 1 Unit 6 at $130-140 \mathrm{cmbs}$ \\
\hline $\begin{array}{l}\text { Slippery Slope, } \\
41 \mathrm{MS} 69\end{array}$ & $4960 \pm 40$ & $\begin{array}{l}\text { Beta- } \\
370496\end{array}$ & 1 Andice & $\begin{array}{l}\text { Quigg et al. } \\
2015\end{array}$ & Charcoal from Unit 5 Ext. at 151-154 \\
\hline $\begin{array}{l}\text { Slippery Slope, } \\
41 \mathrm{MS} 69\end{array}$ & $5120 \pm 40$ & UGA-14110 & 1 Andice & $\begin{array}{l}\text { Quigg et al. } \\
2015\end{array}$ & Charcoal from Feature 1 Unit 5 at $134 \mathrm{cmbs}$ \\
\hline $\begin{array}{l}\text { Slippery Slope, } \\
41 \mathrm{MS} 69\end{array}$ & $4890 \pm 40$ & UGA-14116 & 1 Andice & $\begin{array}{l}\text { Quigg et al. } \\
2015\end{array}$ & Charcoal from Feature 1, Unit 5 Ext at $152 \mathrm{cmbs}$ \\
\hline $\begin{array}{l}\text { Slippery Slope, } \\
41 \mathrm{MS} 69\end{array}$ & $4740 \pm 40$ & UGA-14107 & No points & $\begin{array}{l}\text { Quigg et al. } \\
2015\end{array}$ & $\begin{array}{l}\text { Black sediment from Feature } 2 \text { Unit } 5 \text { Ext at } 180 \\
\text { cmbs }\end{array}$ \\
\hline $\begin{array}{l}\text { Slippery Slope, } \\
41 \mathrm{MS} 69\end{array}$ & $4550 \pm 40$ * & $\begin{array}{c}\text { Beta- } \\
370492\end{array}$ & No points & $\begin{array}{l}\text { Quigg et al. } \\
2015\end{array}$ & $\begin{array}{l}\text { Ashy sediment form Feature } 2 \text { Unit } 5 \text { at 170-174 } \\
\text { cmbs }\end{array}$ \\
\hline $\begin{array}{l}\text { Slippery Slope, } \\
41 \mathrm{MS} 69\end{array}$ & $5420 \pm 40$ & $\begin{array}{c}\text { Beta- } \\
370493\end{array}$ & No points & $\begin{array}{l}\text { Quigg et al. } \\
2015\end{array}$ & Charcoal from Feature 2 Unit5 at $177 \mathrm{cmbs}$ \\
\hline $\begin{array}{l}\text { Slippery Slope, } \\
41 \mathrm{MS} 69\end{array}$ & $5100 \pm 40$ & UGA-14111 & No points & $\begin{array}{l}\text { Quigg et al. } \\
2015\end{array}$ & $\begin{array}{l}\text { Charcoal from below Feature } 2 \text { Unit } 6 \text { at } 190-200 \\
\text { cmbs }\end{array}$ \\
\hline $\begin{array}{l}\text { Slippery Slope, } \\
41 \mathrm{MS} 69\end{array}$ & $5360 \pm 40$ & $\begin{array}{c}\text { Beta- } \\
233356\end{array}$ & No points & $\begin{array}{l}\text { Quigg et al. } \\
\quad 2015\end{array}$ & $\begin{array}{l}\text { Charcoal from Feature } 2 \text { in Unit 6, level } 17 \text { at } 166 \\
\text { cmbs }\end{array}$ \\
\hline $\begin{array}{l}\text { Slippery Slope, } \\
41 \mathrm{MS} 69\end{array}$ & $5390 \pm 40$ & $\begin{array}{c}\text { Beta- } \\
233353\end{array}$ & No points & $\begin{array}{l}\text { Quigg et al. } \\
2015\end{array}$ & $\begin{array}{l}\text { Charcoal from Feature } 2 \text { in Unit } 5 \text {, level } 19 \text { at } 180 \\
\text { cmbs }\end{array}$ \\
\hline $\begin{array}{l}\text { Slippery Slope, } \\
41 \mathrm{MS} 69\end{array}$ & $5320 \pm 50$ & $\begin{array}{c}\text { Beta- } \\
233354\end{array}$ & No points & $\begin{array}{l}\text { Quigg et al. } \\
\quad 2015\end{array}$ & $\begin{array}{l}\text { Charcoal from Feature } 2 \text { in Unit 5, level } 19 \text { at } 189 \\
\text { cmbs }\end{array}$ \\
\hline $\begin{array}{l}\text { Spring Lake, } \\
41 \mathrm{HY} 160\end{array}$ & $5050 \pm 20$ & $\begin{array}{l}\text { UCIAMS- } \\
111178 \\
\end{array}$ & 1 Nolan & $\begin{array}{l}\text { Lohse et al. } \\
\text { 2013:51 }\end{array}$ & Mammal bone from Unit 3, $125-135 \mathrm{cmbd}$ \\
\hline $\begin{array}{l}\text { Spring Lake, } \\
41 \mathrm{HY} 160\end{array}$ & $5140 \pm 20$ & $\begin{array}{l}\text { UCIAMS- } \\
106473\end{array}$ & $\begin{array}{c}1 \text { Bell, } 1 \\
\text { Martindale }\end{array}$ & $\begin{array}{l}\text { Lohse et al. } \\
\text { 2013:51 }\end{array}$ & Bison bone from Unit 4, $145-155 \mathrm{cmbd}$ \\
\hline
\end{tabular}


Table 3-1. Radiocarbon Dates for Sites Associated with Bell/Andice/Calf Creek Points (continued).

\begin{tabular}{|c|c|c|c|c|c|}
\hline Site Name/No. & $\begin{array}{l}\text { Uncalibrated } \\
\text { (B.P.) }\end{array}$ & $\begin{array}{l}\text { Laboratory } \\
\text { Number }\end{array}$ & $\begin{array}{l}\text { Associated Point } \\
\text { Types }\end{array}$ & Reference & Context and Comments \\
\hline $\begin{array}{l}\text { Spring Lake, } \\
41 \mathrm{HY} 160\end{array}$ & $5140 \pm 20$ & $\begin{array}{l}\text { UCIAMS- } \\
106468\end{array}$ & 1 Merrell & $\begin{array}{l}\text { Lohse et al. } \\
\text { 2013:51 }\end{array}$ & Bison bone from Unit 3, $155-165 \mathrm{cmbd}$ \\
\hline $\begin{array}{l}\text { Spring Lake, } \\
41 \mathrm{HY} 160\end{array}$ & $5145 \pm 20$ & $\begin{array}{l}\text { UCIAMS- } \\
106469\end{array}$ & 1 Merrell & $\begin{array}{l}\text { Lohse et al. } \\
\text { 2013:51 }\end{array}$ & Bison bone from Unit 3, $155-165 \mathrm{cmbd}$ \\
\hline $\begin{array}{l}\text { Spring Lake, } \\
41 \mathrm{HY} 160\end{array}$ & $2415 \pm 20^{*}$ & $\begin{array}{l}\text { UCIAMS- } \\
106470\end{array}$ & 1 Merrell & $\begin{array}{l}\text { Lohse et al. } \\
\text { 2013:51 }\end{array}$ & Bison bone from Unit 3, $155-165 \mathrm{cmbd}$ \\
\hline $\begin{array}{l}\text { Spring Lake, } \\
41 \mathrm{HY} 160\end{array}$ & $5290 \pm 20$ & $\begin{array}{l}\text { UCIAMS- } \\
111178\end{array}$ & $\begin{array}{l}1 \text { Bell, } 1 \text { Merrell, } 2 \\
\text { Uvalde }\end{array}$ & $\begin{array}{l}\text { Lohse et al. } \\
\text { 2013:51 }\end{array}$ & $\begin{array}{c}\text { Mammal bone from Unit } 4,175-185 \mathrm{cmbd} \text {, specimen } \\
\text { below Calf Creek Zone }\end{array}$ \\
\hline $\begin{array}{l}\text { Spring Lake, } \\
41 \mathrm{HY} 160\end{array}$ & $5060 \pm 40$ & $\begin{array}{l}\text { UCIAMS- } \\
80999\end{array}$ & & $\begin{array}{l}\text { Lohse et al. } \\
\text { 2014, Lohse et } \\
\text { al. } 2014\end{array}$ & Bison bone from Unit 9, level 14 \\
\hline $\begin{array}{l}\text { Spring Lake, } \\
41 \mathrm{HY} 160\end{array}$ & $5110 \pm 15$ & $\begin{array}{l}\text { UCIAMS- } \\
95717\end{array}$ & $\begin{array}{c}1 \text { Bell, } 1 \\
\text { Martindale }\end{array}$ & " & Bison bone from Unit 14, Level 13 \\
\hline $\begin{array}{l}\text { Spring Lake, } \\
41 \mathrm{HY} 160\end{array}$ & $5115 \pm 20$ & $\begin{array}{l}\text { UCIAMS- } \\
80139\end{array}$ & & $"$ & Bison bone from Unit 7, Level 14 \\
\hline $\begin{array}{l}\text { Spring Lake, } \\
41 \mathrm{HY} 160\end{array}$ & $5120 \pm 20$ & $\begin{array}{l}\text { UCIAMS- } \\
80136\end{array}$ & & " & Bison bone from Unit 7, Level 15 \\
\hline $\begin{array}{l}\text { Spring Lake, } \\
41 \mathrm{HY} 160\end{array}$ & $5120 \pm 20$ & $\begin{array}{l}\text { UCIAMS- } \\
80998\end{array}$ & & $"$ & Bison bone from Unit 7, Level 14 \\
\hline $\begin{array}{l}\text { Spring Lake, } \\
41 \mathrm{HY} 160\end{array}$ & $5155 \pm 15$ & $\begin{array}{l}\text { UCIAMS- } \\
81000\end{array}$ & & $"$ & Bison bone from Unit 16, Level 13. \\
\hline $\begin{array}{l}\text { Spring Lake, } \\
41 \mathrm{HY} 160\end{array}$ & $5165 \pm 15$ & $\begin{array}{l}\text { UCIAMS- } \\
81001\end{array}$ & & $"$ & Bison bone from Unit 16, Level 13. \\
\hline $\begin{array}{l}\text { Spring Lake, } \\
41 \mathrm{HY} 160\end{array}$ & $5180 \pm 15$ & $\begin{array}{l}\text { UCIAMS- } \\
80997\end{array}$ & & $"$ & Bison bone from Unit 7 , Level 9. \\
\hline $\begin{array}{l}\text { Stinnet Pool, } \\
41 \mathrm{HC} 220\end{array}$ & $4950 \pm 40$ & $\begin{array}{c}\text { Beta- } \\
169973\end{array}$ & 1 Bell & Unpublished & $\begin{array}{l}\text { Bison bone date thought to be associated with Bell } \\
\text { point }\end{array}$ \\
\hline $\begin{array}{l}\text { Devils Mouth, } \\
\text { 41VV188 }\end{array}$ & $4900 \pm 100$ & TX-525 & 10 Bell & Sorrow 1968 & $\begin{array}{l}\text { Flecks of charcoal from in and around Feature } 3 \text { in } \\
\text { Stratum N, in Area D, just below Group 3- Early- } \\
\text { barbed (Prewitt =Bell) points }\end{array}$ \\
\hline
\end{tabular}


Table 3-1. Radiocarbon Dates for Sites Associated with Bell/Andice/Calf Creek Points (continued).

\begin{tabular}{|c|c|c|c|c|c|}
\hline Site Name/No. & $\begin{array}{l}\text { Uncalibrated } \\
\text { (B.P.) }\end{array}$ & $\begin{array}{l}\text { Laboratory } \\
\text { Number }\end{array}$ & $\begin{array}{l}\text { Associated Point } \\
\text { Types }\end{array}$ & Reference & Context and Comments \\
\hline $\begin{array}{l}\text { Cervenka, } \\
41 \mathrm{WM} 267\end{array}$ & $4970 \pm 90$ & TX-3684 & $\begin{array}{l}1 \text { Andice, not } \\
\text { directly associated }\end{array}$ & $\begin{array}{l}\text { Peter et al. } \\
1982\end{array}$ & $\begin{array}{c}\text { Stratum 5, charcoal from hearth Feature } 19 \text { near } \\
\text { bison bones, Level 117/118, Area D, Andice point } \\
\text { from Area A }\end{array}$ \\
\hline $\begin{array}{l}\text { Cervenka, } \\
41 \text { WM267 }\end{array}$ & $5135 \pm 20$ & $\begin{array}{l}\text { UCIAMS- } \\
129248\end{array}$ & No points & $\begin{array}{l}\text { Lohse et al. } \\
\text { 2014, Lohse et } \\
\text { al. 2014; }\end{array}$ & $\begin{array}{c}\text { Bison bone from Area D, Unit E-6, } 120-121 \mathrm{cmbd} \text {, at } \\
\text { hearth Feature } 26\end{array}$ \\
\hline $\begin{array}{l}\text { Granberg II, } \\
41 \mathrm{BX} 271\end{array}$ & $4770 \pm 110$ & TX-3606 & 1 Bell & $\begin{array}{c}\text { Black \& } \\
\text { McGraw } 1985 \\
\end{array}$ & charcoal, Stratum V \\
\hline $\begin{array}{l}\text { Panther Springs } \\
\text { Creek, 41BX228 }\end{array}$ & $4720 \pm 170$ & TX-3912 & Numerous types & $\begin{array}{c}\text { Black \& } \\
\text { McGraw } 1985 \\
\end{array}$ & Area A, $84 \mathrm{cmbs}$ \\
\hline $\begin{array}{l}\text { Cibolo Crossing, } \\
\quad 41 \mathrm{BX} 377\end{array}$ & $\begin{array}{l}4420 \pm 50 \\
4400 \pm 60 \\
4370 \pm 80\end{array}$ & $\begin{array}{l}\text { Beta-126362, } \\
\text { Beta-126364, } \\
\text { Betta-126367 }\end{array}$ & $\begin{array}{l}9 \text { Bell, } 2 \text { Baird, } 1 \\
\text { Andice }\end{array}$ & $\begin{array}{l}\text { Kibler \& Scott } \\
2000\end{array}$ & $\begin{array}{c}\text { Bell component, Rabdotus snail A/I ratio projected } \\
\text { dates }\end{array}$ \\
\hline $\begin{array}{l}\text { McKinzie, } \\
41 \text { NU221 }\end{array}$ & $4450 \pm 90^{*}$ & Tx-5263 & $\begin{array}{l}1 \text { Bell, } 1 \text { Catan, } 1 \\
\text { Tortugas }\end{array}$ & Ricklis 1988 & $\begin{array}{c}\text { Raw date from Rangia flexuosa shells from Zone III, } \\
\text { roughly between } 37-55 \mathrm{cmbs} \text { in shell midden on top } \\
\text { of tan B horizon, Pleistocene clay, possible reservoir } \\
\text { error }\end{array}$ \\
\hline $\begin{array}{l}\text { McKinzie, } \\
41 \text { NU221 }\end{array}$ & $4630 \pm 90^{*}$ & Tx-5264 & $\begin{array}{l}1 \text { Bell, } 1 \text { Catan, } 1 \\
\text { Tortugas }\end{array}$ & Ricklis 1988 & $\begin{array}{l}\text { Raw date from Rangia flexuosa shells from Zone III, } \\
\text { roughly between } 37-55 \mathrm{cmbs} \text { in shell midden on top } \\
\text { of tan B horizon, Pleistocene clay, possible reservoir }\end{array}$ \\
\hline $\begin{array}{l}\text { McKinzie, } \\
41 \text { NU221 }\end{array}$ & $4410 \pm 90^{*}$ & Tx-5265 & $\begin{array}{l}1 \text { Bell, } 1 \text { Catan, } 1 \\
\text { Tortugas }\end{array}$ & Ricklis 1988 & $\begin{array}{l}\text { Raw date from Rangia flexuosa shells from Zone III, } \\
\text { roughly between } 37-55 \text { cmbs in shell midden on top } \\
\text { of tan B horizon, Pleistocene clay, possible reservoir }\end{array}$ \\
\hline 41ME147 & $5205 \pm 20$ & $\begin{array}{l}\text { UCIAMS- } \\
111182\end{array}$ & ?? & $\begin{array}{l}\text { Lohse et al. } \\
\text { 2014, Lohse et } \\
\text { al. 2014; }\end{array}$ & Bison bone from N802 E631 Level 4 \\
\hline $\begin{array}{l}\text { Landslide, } \\
\text { 41BL85 }\end{array}$ & $>3520$ * & TX-289 & $\begin{array}{l}2 \text { Bell/Andice, } 1 \\
\text { Martindale, } 1 \\
\text { Merrell }\end{array}$ & $\begin{array}{l}\text { Sorrow et al. } \\
\quad 1967\end{array}$ & $\begin{array}{c}\text { Bison bones from Feature 2, Horizon IV, Stratum IIIa } \\
\text { with a mixture of point types, organic fraction of } \\
\text { bone, not comparable }\end{array}$ \\
\hline
\end{tabular}

${ }^{*}$ = Date is rejected as not associated. $\quad{ }^{* *}=$ date not associated with Bell/Andice/Calf Creek points; $\mathrm{cmbs}=$ centimeters below surface; $\mathrm{cmbd}=$ centimeters below datum 
recognition of the Calf Creek point type, no specific western Oklahoma cultures were mentioned in the discussion on the Archaic period.

In the eastern half of the state, where more investigations occurred in conjunction with reservoir developments, hunter-gatherers employed notched points to kill modern game animals (Wyckoff 1984). Thirteen Archaic period sites in eastern Oklahoma contained 75 radiocarbon dates, although no dates were reported for the period between ca. 9400 and 4700 B.P. The Tom's Brook complex was the only manifestation believed to include remains from 5000 to 6000 B.P even though it lacked radiocarbon dates (Wyckoff 1984). This complex, however, contains many kinds of projectile point types to indicate probable mixed assemblages had accumulated over a very long period as reflected by an occasional Calf Creek, Bulverde, and Big Sandy point types. The poorly defined Tom's Brook complex extends into Arkansas where at least two sites revealed similar materials.

In the mid-1990s, the Oklahoma Anthropological Society published two significant volumes dedicated to the Calf Creek horizon (Wyckoff and Shockey 1994, 1995). These volumes present 26 papers by numerous authors, which reveal Calf Creek materials occur across the state. They also present information on several important Calf Creek sites as well as updated information about Calf Creek artifacts, and inferred behavioral models. Unfortunately, nearly all information presented was derived from surface collections, with only five sites where hand-excavations encountered Calf Creek materials. Limited salvage excavations conducted at the Arrowhead Ditch site (34MS174) targeted an eroding burned rock concentration (Feature 2) about $3 \mathrm{~m}$ below the surface (Wyckoff et al. 1994b). Hand-excavations of 24 test units were conducted at the Hester/Adams site (34ML83) at a disturbed upland setting (Cestaro and Carrell 1994). In 1941, the Works Progress Administration (WPA) conducted excavations at the Lamar site (34BR8), currently inundated by Lake Texhoma, where a lithic tool cache possibly associated to a single, badly deteriorated human skeleton were recovered (Neal 1994a).

Salvage excavations included multiple backhoe trenches, eight test pits and two blocks of units for a totaled $37 \mathrm{~m}^{2}$ in the Bellcow Creek reservoir in central Oklahoma investigated a $3 \mathrm{~m}$ deep stratified site at 34LN29. Colluvial deposits yielded a Calf Creek point and other artifacts between 130 and 185 cmbs. The collagen extracted from multiple bison bones from 140 to $180 \mathrm{cmbs}$ were radiocarbon dated to $4190 \pm 80$ RCYBP (Beta-31404), and the recent age was attributed to dating poorly degraded collagen (Girard and Carr 1991, 1994:199).

Since the mid-1990s, several articles on Calf Creek finds have appeared in various journals (Andrews 1999; Bement et al. 2004, 2005; Duncan 1996; Neal 1999, 2002; Wyckoff 1993, 2005). Neal and Drass (1998) provided a summary of the Middle Archaic period in northeastern Oklahoma and included a discussion on the Calf Creek materials. Thurmond and Wyckoff (1999) presented a specific overview of Calf Creek manifestations.

Andrews (1999) analyzed data sheets from 808 Calf Creek points and point fragments from across Oklahoma to extract patterns. In studying metric measurements, point outlines, basal outlines, stem forms, and resharpening frequencies he determined no morphological differences exists in Calf Creek point forms across Oklahoma. Also Andrews discovered as Wyckoff (1995) before him, raw chert material exploitation occurred in five distinct regions across the state. One particular raw material type dominated each of the five regions with limited percentages of other material types also present. 
Excavated and reported sites with good contextual Calf Creek horizon are still limited in Oklahoma. However, sporadic excavations conducted at the Kubik site (34KA354) in Kay County, Oklahoma has contributed 11 radiocarbon dates, a final report has not been prepared (Duncan 1996; Neal 1999, 2002; Neal and Drass 1998; Neal and Duncan 1998). Neal (2002) reports five notching flakes, one late stage preform, bifaces, flakes, and bison bones from the Calf Creek levels at the Kubik site. One possible feature (Feature 02-14) was discovered $2.3 \mathrm{~m}$ below datum. Neal (2002) presents 11 radiocarbon dates from the Kubik site along with the kinds of material dated and unit and level provenience locations and feature associations; three dates of ca. 5020 B.P. are associated with the Calf Creek artifacts (Neal 1999:1).

In summary, the broad regions of Texas, Oklahoma, and neighboring states have focused significant attention on the form and technology of the diagnostic projectile points (e.g., Anderson 1999; Weber 1986, 1991, 2000, 2002; Weber and Patterson 1985; Wyckoff et al. 1994a; Wyckoff et al. 1995; and others). However, most studies provide very limited data about the overall lifeways, behaviors, settlement patterns, interactions of the people possessing the Bell/Andice/Calf Creek points. This lack of information about the horizon arises from the paucity of excavation results. Until additional sites with isolatable components in good context are intensively excavated, analyzed, and reported, this cultural phenomenon will only be discussed in general terms.

Based on the preceding regional background and history concerning Bell/Andice/Calf Creek projectile points (here discussed as one group) and associated sites, the following sections provide an overview of specific aspects of what is known for this horizon.

\subsubsection{Geographical Distribution}

Bell/Andice/Calf Creek projectile points have been recovered from across most of Texas, which include the eastern Trans-Pecos (Gray 2013), Big Bend (Walter 2013), Lower Pecos region, specifically at Zones IV, V, VI in the Devils Rockshelter (Prewitt 1966) and the lowest levels at the Devils Mouth site (Johnson 1964), the Edwards Plateau, with limited materials from south Texas (Chandler 1983; Chandler and Kumpe 1993; Cox 1996; Ricklis 1988), far west Texas in Ward County (Wyckoff 2005), and the Texas panhandle region (Calame et al. 2002; Hester and McReynolds 2003; McReynolds 2002; Prewitt 1995) (Figure 37), plus all across Oklahoma, northwestern Arkansas, southern Missouri, southeastern Kansas, and possibly into southeastern Colorado, and northeastern New Mexico (Figure 3-8). A synthesis of Colorado archeology by Cassells (1997) does not mention the Calf Creek projectile points. However,

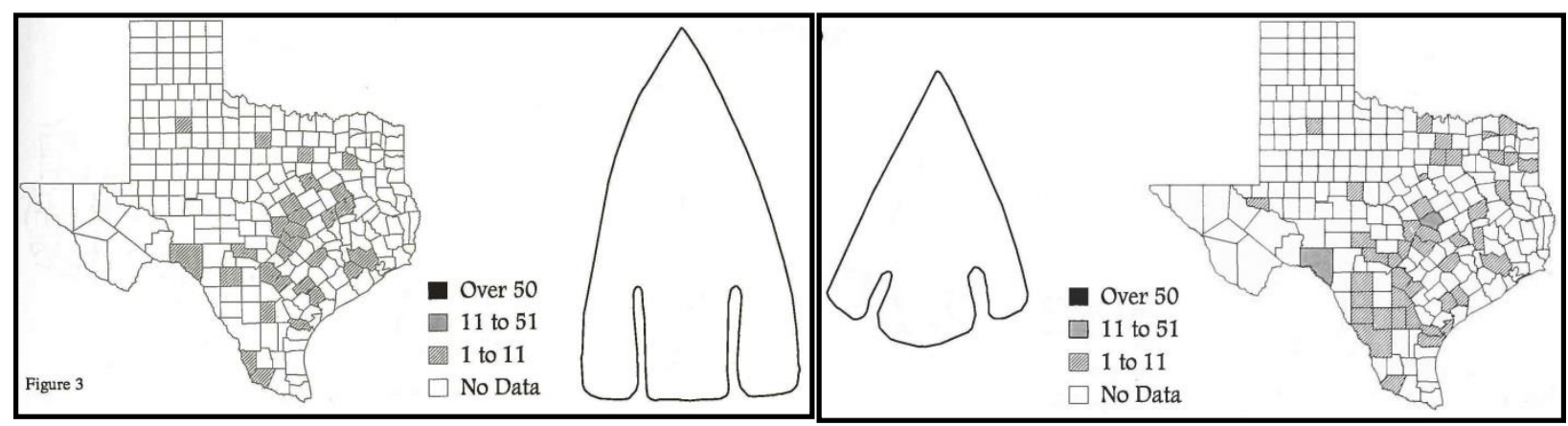

Figure 3-7. The 1995 distribution of Andice (left) and Bell (right) points across Texas (after Prewitt 1995). 


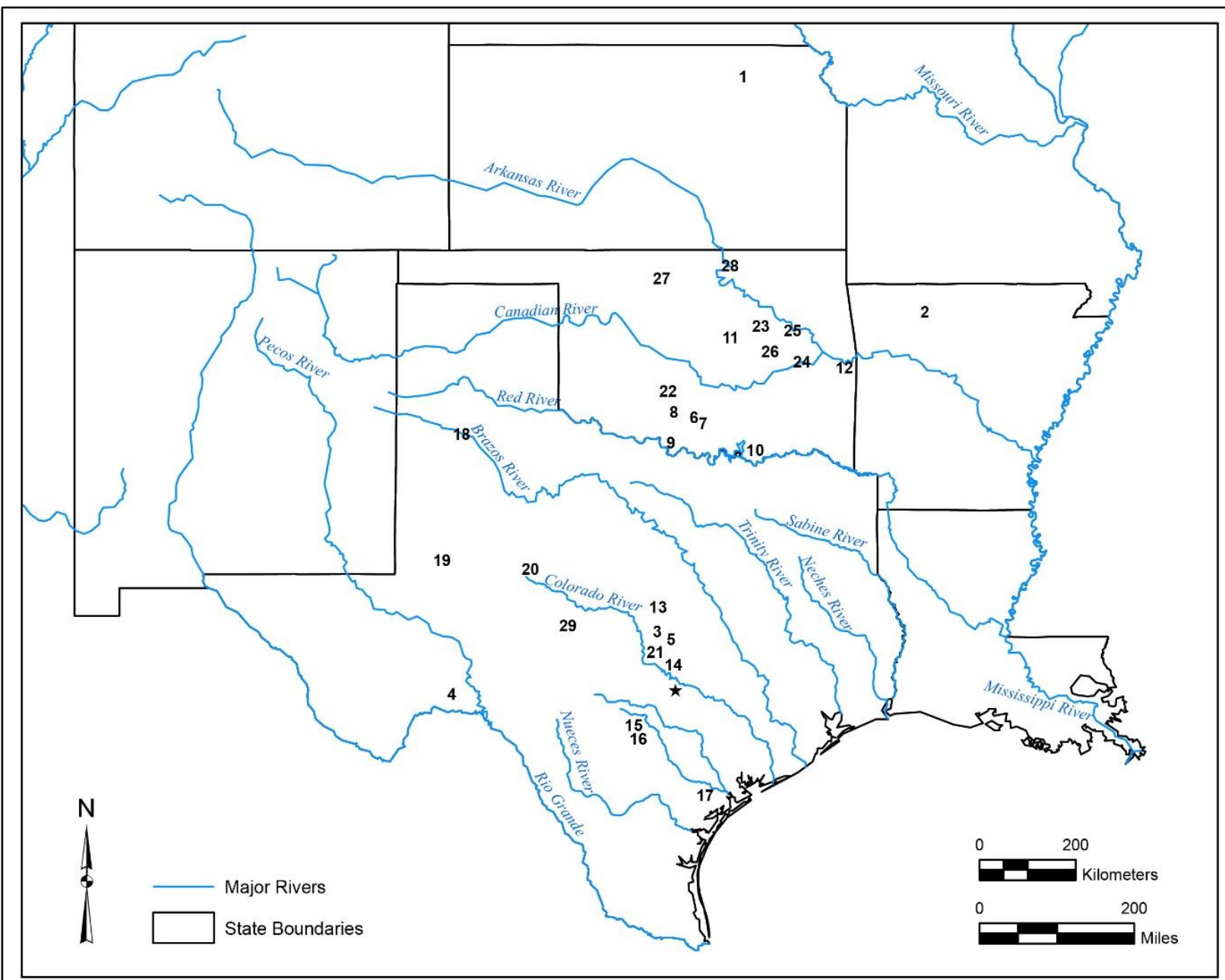

$\star$ Big Hole (41TV2161)

1. Coffey (14PO1)

2. Calf Creek Cave

3. Landslide (41BL85)

4. Devils River sites (41VV188 \& 41VV264)

5. Cervenka (41WM267)

6. Primrose (34MR65)

7. Stilman (34MR71)

8. Williams Orchard (34GV37)

9. Frazier (34JF43)
10. Lamar (34BR8)

11. Bellcow (34LN29)

12. Red Clay (34HS29)

13. Fort Hood (various sites)

14. Wilson-Leonard (41WM235)

15. Cibolo Crossing (41BX377)

16. Richard Beene (41BX831)

17. McKinzie (41NU221)

18. Lubbock Lake (41LU1)

19. Mustang Springs (41MT2)
20. O.H. Ivie Reservoir (various sites)

21. Gault (41WM9)

22. Hester/Adams (34ML83)

23. Brandon (34TU82)

24. Mckellips (34MI136)

25. Arrowhead Ditch (34MS174)

26. Woodard-Benefield (34MS258)

27. Hunter (34GT6)

28. Kubik (34KA354)

29. Slippery Slope (41MS69)

Figure 3-8. Location of key Bell/Andice/Calf Creek sites in the Southern Plains region. 
Rhoton (1995) indicates he knows of three surface collected specimens from Baca County, southeastern Colorado. A Calf Creek point form has even been reported from Utah, even though this specimen appears far removed from comparable specimens (Wyckoff 2010). These points appear concentrated over widely distributed areas across Oklahoma and Texas with significant decreases stretching northward and eastward from Kansas.

They are apparently quite sparse in northern New Mexico and most of Colorado. In sheer number, these points appear less frequent in northwestern Texas and the Oklahoma panhandle regions (Thurmond and Wyckoff 1999), although fewer archeological projects have occurred in these regions. The existing distribution is undoubtedly biased and reflects surface visibility, since so few sites or components with these point types has been recovered. Consequently, areas where these point styles are absent, may merely reflect poor surface visibility combined with few excavation projects in that region. Calf Creek sites are frequent and widespread enough across Oklahoma, for Wyckoff (1995) to identify site clusters from four major geographical regions across Oklahoma. Using distribution data from two volumes of reported site data published in the Oklahoma Anthropological Society Bulletins XL and XLII, Wyckoff (1995) notes regional differences in the use of different raw materials for the manufacture of their stone tools. Preferential use of lithic materials in different areas across Oklahoma provides insights into different group's movements, exchanges and territories. The four territories in Oklahoma consist of 1) the Arbuckle Mountains in south-central Oklahoma, 2) the Arkoma Basin in east-central Oklahoma, 3) the Osage Plains and Osage Hills in north-central Oklahoma, and 4) the western Osage Plains and southern High Plains in southwestern Oklahoma. Distribution studies have not been conducted in Texas.

\subsubsection{Chronometric Age}

The age of the Bell/Andice/Calf Creek projectile points are chronometrically documented and/or bracketed by some 52 radiocarbon assays (see Table 3-1). This compilation of published radiocarbon dates indicates not only an expansive time range exists for this cultural manifestation, but over 21 percent are potentially not associated with these materials. Multiple dates are of questionable reliability because of poor or uncertain contexts, poor association, mixed assemblages, and/or poor choices of materials dated. For example, the radiocarbon dates on bones of an unidentified species from the Bellcow Shelter site (34LN29) in Oklahoma yielded a relatively recent date of 4190 B.P. (Girard and Carr 1994:199). This date was apparently based on degraded collagen; thus, poor preservation of bone collagen potentially caused problems with the date (Girard and Carr 1994). At the Arrowhead Ditch site (34MS174) the recovered Bell point was $50 \mathrm{~cm}$ above the date of 5730 B.P. which is potentially not associated with the point (Wyckoff et al. 1994b). Therefore, the obtained date does not directly reflect the age of the Bell point.

Another potentially unreliable date of $>3520$ B.P. was obtained from bison bones associated with Feature 2, Stratum IV at the Landslide site (Sorrow et al. 1967:9). The date is not only imprecise, the published materials dated was reportedly organic (collagen) bone fraction (Valastro et al. 1967). Subsequent discussions with Valastro indicated he only processed apatite or the mineral components of bones in his laboratory to obtain dates (Quigg, personal communication 1989). Another example is the 5770 B.P. date derived from six Rabdotus shells from the Big Hole site in central Texas. Chronometric studies on single Rabdotus snail shells have documented the ingestion of ground carbonates into the shell formation and consequently the ages may be in error at a 
magnitude of several hundred years (Ellis et al. 1996). Rabdotus shell dates and probably sediment dates are considered suspect and do not precisely document the true age range of this population. To strengthen the reliability of the assignment of the age range for Bell/Andice/Calf Creek occupation in Texas, the context and associations should be reassessed.

Probably the best dated contexts for the Calf Creek interval is on collagen from the petrous/inner ear bone of a bison skull with a broken Calf Creek point embedded in the skull of a young Bison antiquus occidentalis (Bement et al. 2004, 2005). The obtained date of 5100 B.P. fits with most other dates for the culture (see Table 3-1). However, the identification of this bison species is quite interesting as it postdates the commonly accepted age range for $B$. occidentalis on the southern Plains. The date indicates a co-existence of modern bison with Bison occidentalis. In general terms, Bell/Andice/Calf Creek projectile points in Oklahoma reliably range from ca. 5800 to 5000 B.P. based on just a few dates.

In Texas the age range is not as easily determined as additional dates on a wider array of organic materials and debates over the accuracy and reliability of the different materials combined with context issues are a major concern. The Bell/Andice component at the Cibolo Crossing site is one of the better defined components, but provides ambiguous results as multiple charcoal dates from Feature 19 range from ca. 4370 to 4420 B.P. which are considered too young for this horizon. These young charcoal dates contrast with seven calendrical dates derived from mean amino acid epimers D-alloisoleucine/L-isoleucine (A/I) values derived from Rabdotus snail shells. The seven calendrical dates range from 4528 to 5390 B.P. (Kibler and Scott 2000). Three bulk organic sediment dates from Features 9, 10, and 11, also attributed to the Bell/Andice component, are at least 1,000 years younger than the three charcoal dates from Feature 19. These bulk sediment-derived dates do not appear as accurate as charcoal dates from the same contexts, if all the dated features were properly attributed to this component and the charcoal was not intrusive.

The most reliable context between a projectile point directly associated with a radiocarbon date may be the seven charcoal dates from Feature 1 directly associated with an Andice point $50 \mathrm{~cm}$ away at the Slippery Slope site (41MS69) in central Texas (Quigg and Frederick 2005; Quigg et al. 2015). The multiple dates provide an excellent, narrow time frame of 230 years or an average of 4993 B.P. for that particular component (see Table 3-1). The Bell/Andice/Calf Creek component at the Ticket Kiosk at Spring Lake, defined as a $50 \mathrm{~cm}$ thick zone between roughly 135 and $185 \mathrm{cmbd}$ with the multiple point types ( 3 Merrell, 1 Martindale, and 2 Uvalde) yielded a diverse faunal assemblage that included many bison bones. Ten $\delta^{13} \mathrm{C}$ corrected radiocarbon dates on bison bones from this thick component range between 5060 B.P. and 5180 B.P. at the two sigma range (see Table 3-1; Lohse et al. 2014a).

More precise direct radiocarbon dating of known or newly discovered components with good context will enable a more refined timeframe for these point styles. As additional dates become available from sites and components across this broad use region, researchers can pursue the possibility of contemporaneity of cultural artifacts and the rate of spread of these peoples across the landscape. Precise dating will also allow researchers to explore regional settlement patterns of population differences during this period.

The multiple projectile point styles from the Coffey site in northeastern Kansas are not part of the Bell/Andice Calf Creek horizon. However, the radiocarbon dates, centered on ca. 5240 B.P. with a smaller concentration of dates at ca. 5640 B.P., overlap dates obtained for the Calf Creek horizon (Banks and Wigand 2005). This indicates 
contemporaneous groups in the region could have potentially interacted with populations from the Calf Creek horizon.

\subsubsection{The Artifact Assemblage}

Much of what is known about this phase/complex/horizon is based on diagnostic projectile points with their characteristic deep basal-notches creating a rectangular stem and long, broad barbs. The original description for the 14 Bell points from the Landslide site in central Texas is provided by Sorrow et al. (1967:12):

"The distinctive attributes of these specimens are their large barbs and overall thinness. They have been fashioned by chipping two deep, narrow notches into the base of an otherwise trianguloid blade. The resulting barbs (when present) extend into line with the basal corners. Stems generally expand faintly and the often broad blades have straight or less often convex lateral edges. The blade on one specimen is asymmetrical (Fig. 10. i). On the specimens with straight blade edges, the barbs are wider at the tip (Fig. 10; a, b, c, h). By contrast, the barbs are pointed on the examples with convex lateral edges and are wider at the point in line with the inception of the base (Fig. 10, e). The blade edges of one specimen are serrated (Fig. 10, c). Bases on all are very carefully thinned and are either convex, or less frequently straight. Workmanship is excellent."

Although Bell and Andice projectile points have received separate names in most Texas reports, Weber (1986) employed a discriminant function analysis to statistically document metric data does not support the separation into different point types. No comparable studies have been conducted to demonstrate the Bell and Andice forms are typologically identical to Calf Creek points, although they appear similar. Prewitt (1983) suggested the Calf Creek type is intermediate between the Andice and Bell morphology. Wyckoff (1994a) and other authors agree the Bell, Andice, and Calf Creek forms generally overlap and are part of a single biface production form. Differences detected generally relate to the amount of resharpening and reworking during the use-life of the artifact. It is important to note morphological variations in projectile points remain the primary method for determining archeological groupings or complexes and comparing assemblages.

At a minimum three excavated Bell components in Texas, at the Cibolo Crossing site (Kibler and Scott 2000), the Landslide site (Sorrow et al. 1967), and potentially the Barton site Area B (Collins 1994a), yielded triangular dart points in association with the Bell points. In these and other instances, those assemblages possibly represent mixed events, or reflect trade and interaction with contemporaneous groups, which employed different point types.

Since most information about the Andice/Bell/Calf Creek complex, especially from the Oklahoma sites, is derived from surface collections, the delineation of assemblage composition other than the deep basal-notched bifaces may be unreliable. Broader tool assemblages are most often from excavated contexts, where artifact association problems and assemblage mixing are more limited, but are also real possibilities. Sites containing tools such as ground stone and bone tools are rare (e.g., Bellcow Shelter). Other recognized tools include unnotched ovate and rectangular bifaces, end scrapers, drills, cores, "deep notched practice pieces," edge-modified flakes, gouges, choppers, burin spalls, hammer stones, occasionally adzes and rarely pitted stones (Wyckoff 1995). Stratum III-b at the Landslide site yielded a broad range of stone tools purportedly associated with basal-notched 
projectiles. That assemblage consists of 72 utilized flakes, 3 hammer stones, 2 gouges, many burins and burin spalls, 2 choppers, 1 quartzite mano, and 1 sandstone grinding slab (Sorrow et al. 1967). Unfortunately, the cultural attribution is questionable, as these materials came from a $40 \mathrm{~cm}$ thick zone and probably mixed as indicated by multiple point types.

In much of the Oklahoma literature concerning Calf Creek points, these are often referred to as bifacial projectile points and/or knives (Thurmond and Wyckoff 1999). These characteristic artifacts often exhibit multiple resharpening and breakage episodes, which shorten and narrow the original blade and often shorten or remove the broad barbs. The young Bison occidentalis skull embedded with the broken Calf Creek point (Bement et al. 2004, 2005) is irrefutable evidence this broad-bladed, deep basal-notched biface was employed as a projectile. How it was propelled and whether it was attached to a dart shaft or a spear is not clear. Functional interpretations of this tool as a knife are primarily based on their asymmetrical outlines with shorter and/or narrower blades due to extensive blade resharpening. No detailed high-powered usewear studies have been conducted to verify if knife resharpening occurrence is real.

Unnotched bifaces constitute a considerable percentage of many assemblages. If the Stilman cache (34MR71) is a representative sample of late stage bifaces for this culture, then overall shapes are ovate-to-roughly triangular. These pieces have thicknesses between 11 and $15 \mathrm{~mm}$. Often, broken bifaces exhibit resharpened areas and/or bending stress breaks (Wyckoff et al. 1994a). Broken specimens in caches indicate those items have not completed their life cycles and were of sufficient size and form suitable for further use.

Scrapers are a common tool type in many Calf Creek sites in Oklahoma, as revealed at the Arrowhead Ditch site (Wyckoff et al. 1994b) and the Hunter site (34GT6, Brooks 1995). At least one scraper is circular and manufactured from heattreated Keokuk chert (Wyckoff et al. 1994b). Others are of various shapes, generally with one steeply worked distal end, although a side scraper is reported at the Cibolo Crossing site (Kibler and Scott 2000). Brooks (1995) examined 53 scrapers from surface contexts at the Hunter site in northcentral Oklahoma, which he attributes to a Calf Creek occupation. He observed extensive edge crushing, smoothing, polishing, and step fracturing on the worked edges. He interpreted edge damages to use-wear from dry-hide processing, probably of bison hides (Brooks 1995). All scrapers are oval to rectangular in outline, generally with thick cross sections; they are unifacially worked, with steeply retouched and modified edges. A few have spokeshave and graver functions (Brooks 1995). Scrapers at the Hunter site were manufactured from regionally available tabular nodules of Florence A chert. Flakes selected to make ended scrapers were removed from near the exterior of cobbles as 42 percent exhibit some cobble cortex. Macroscopic wear patterns on nine specimens (17 percent) indicate they were hafted, whereas three retained traces of an adhesive substance (Brooks 1995).

A few chipped stone drills came from the Primrose and Arrowhead Ditch sites in Oklahoma (Wyckoff et al. 1994a, 1994b). The contexts and affiliation of most drills to the Andice/Bell/Calf Creek horizon are questionable. These drills generally have long, tapered, and pointed, slightly and lack clear evidence of hafting modifications. Gouges are relatively rare in most assemblages; however, excavations at Stratum II-b at the Landslide site yielded at least two gouge specimens (Sorrow et al. 1967). Hammer stones have been infrequently identified in assemblages at a few sites (Sorrow et al. 1967). Expediently made edge-modified flakes are frequently reported, although they have been minimally studied to determine their range of functions. Expedient tools are modified along one or more edges and come in a wide range shapes and sizes. Cobble tools of various shapes and sizes have 
been identified at the few excavated sites (i.e., Kibler and Scott 2000; Sorrow et al. 1967). Cores are relatively common in excavated assemblages and surface collections. Most are multidirectional cores with few platforms, and exhibit no culturally distinctive systematic preparation.

One characteristic and unique artifact group has multiple, deep, well-executed notches randomly knapped around the perimeter of broken bifaces, point fragments, or flakes. The notches on these "eccentrics" resemble the depth and width of notches on Bell/Andice/Calf Creek projectile points and have been interpreted as training or practices pieces (e.g., Collins 1994a; Hester 1990; Weber and Collins 1994). Collins (1994), Hester (1990), and Walter (2013) illustrate selected "practice pieces" from across Texas. Practice pieces are also found at the Primrose and other sites in Oklahoma (Duncan 1994; Wyckoff et al. 1994a).

Ground stone is rarely reported from sites in Oklahoma or Texas. Girard and Carr (1994:203) recovered 10 pieces from the Calf Creek zone at Bellcow Shelter in central Oklahoma. A single quartzitic sandstone grinding slab fragment and a single mano came from questionable context in Stratum III-b at the Landslide site in central Texas (Sorrow et al. 1967). No ground stone implements were from the Bell/Andice component at the Cibolo Crossing site (Kibler and Scott 2000).

Worked bones are rarely recovered due to poor preservation conditions. An eroded flat awl and a grooved and snapped long bone fragment were from the Calf Creek zone at the Bellcow site (Girard and Carr 1994:203). Shell tools are represented by a perforated oyster valve and a rectangularly-shaped piece of oyster from Zone III Rangia shell midden at the McKinzie site near Corpus Christi in conjunction with a broken Bell, a Catan dart, and two unnamed, unnotched points similar to Catan points (Ricklis 1988:18). Freshwater mussel shell tools are absent from other excavated Bell/Andice/Calf Creek components.
Several stone tool caches have been recovered in southern Oklahoma. The Lamar site (34BR8) in south-central Oklahoma yielded 38 stone implements (Calf Creek points, bifaces, scrapers, and modified flakes) from a $50 \mathrm{~cm}$ diameter by 15 $\mathrm{cm}$ deep cache (Neal 1994a). The Stilman Pit site (34MR71) also in south-central Oklahoma contained a cache of 21 large bifaces and 8 large flakes (Bartlett 1994). Three Frisco chert caches are reported from the Primrose site (Bartlett 1994). In Texas one cache is known: the Benton-Whiteside cache along the Red River from Montague County (illustrated in Walter 2013:65).

It is paramount that components with good context and large tool assemblages are excavated and needed to expand our understanding of the tool diversity and range of task employed by these populations.

\subsubsection{Lithic Procurement and Knapping Observations}

Despite the abundance of raw lithic material source areas are across Texas and Oklahoma (e.g., Banks 1984, 1990; Duncan 1995; Holliday and Welty 1981; Shaeffer 1958; Shideler 1970), lithic acquisition techniques are unknown. No quarries or pits are attributed to populations that employed the Bell/Andice/Calf Creek points. Quantities of preferred cherts from multiple source areas were selected and transported some distance for manufacturing stone tools. At the Primrose site more than $40 \mathrm{~kg}$ of large flakes, early stage bifaces made on flakes from residual tabular cobbles were transported $35 \mathrm{~km}$ eastward from the main Frisco chert bedrock exposures, and gravel-bearing source areas (Wyckoff et al. 1994a). The largest pieces from the Primrose site are secondary and tertiary decortication flakes that weigh greater than $100 \mathrm{~g}$. Bifacial preforms, unworked spalls, and cobbles were also cached for future use at the Primrose site. The 21 bifaces from the Stilman Pit cache represent late-stage bifaces manufactured from Frisco chert (Bartlett 1994). At the Gault site (41WM9) in 
central Texas, many interior biface thinning flakes and a few primary reduction flakes were from the Bell/Andice/Calf Creek component, which prompted Weber and Collins (1994) to infer raw materials were brought to the site in the form of large flakes and/or core bifaces.

The excavated Bell component at the Cibolo Crossing site yielded all reduction stages, from multidirectional cores, to complete and fragmentary flakes, chips, chunks, and bifaces in various stages of reduction, as well as formally and informally shaped tools (Kibler and Scott 2000). Biface reduction was a major activity accomplished by reduction of fully corticated cobbles. People at the Bell component at the Cibolo Crossing site employed local Edwards chert for the production of stone tools. No mention was made of heat treatment of Edwards chert or use of nonlocal materials (Kibler and Scott 2000). The 16 Bell/Andice points from the Gatlin site were manufactured exclusively from local Edwards chert (Houk et al. 2008).

Bell/Andice/Calf Creek projectile point production reflects a very precise sequence of steps particularly late in the primary trimming and throughout the secondary trimming stages (Weber and Collins 1994). Both qualitative and quantitative attributes are important in understanding what steps were taken in the manufacturing of these implements. Weber and Collins 1994 present a series of manufacturing steps of point production based on observations on nearly 400 Bell/Andice points, combined with experimental point replication. Weber systematically isolated flake scar attributes into a group derived from point manufacture and a second group from use and damage. Their detail study of attributes provides insights into the manufacturing of Bell/Andice/Calf Creek projectile points and the tool kit employed to produce this particular point style. Webber and Collins (1994) conclude the flint knappers were skilled craftsmen to produce large, pressure-flaked bifaces with very narrow notches using indirect percussion for notching. Notching and stem attributes have also been studied by Ayala (2014a, 2014b) who delineates possible differences in the creation of Bell and Andice points.

Debitage analysis was conducted on Feature 19, a lithic concentration at the Barton site in Hays County, Texas. This feature was attributed to the Bell/Andice horizon based on the recognition of four tiny notching flakes in the lithic debris (Collins 1994a). The 2,055 pieces $(9.2 \mathrm{~kg})$, range in size from less than $1 \mathrm{~cm}$ to greater than $10 \mathrm{~cm}$, were sorted into subjective morphological categories and the results indicate multiple large weathered nodules of local Edwards chert were knapped into multiple bifaces, and included at least one Andice point. Based on Collins's (1994) observations full biface reduction sequence was represented. Direct percussion was employed during the decortication stages, followed by direct billet percussion during biface thinning, and finishing with precise pressure flaking. During the biface thinning stage, flake platform edges were carefully prepared by grinding. Based on the color and four of 96 bifacial thinning flakes, Collins estimates at least 4 to 8 preforms may have been thinned at this work station. No mention was made of chert heat treating to enhance knapability in this assemblage.

After dulling from use, points were often resharpened along blade edges. Sometimes resharpening was so extensive the original form was nearly obliterated (Collins 1994a; Weber 2000). The overall debitage and stone tool assemblage reflects use of soft-hammer, bifacial thinning, which employed extensive grinding in preparation of multifaceted platforms (Collins 1994a; Weber and Collins 1994).

Weber (2000) attributed 13 qualitative traits to the Andice/Bell resharpening techniques and suggests resharpening happened in early, moderate, and late resharpening stages. He concludes points were employed in multiple ways and many were 
employed as knives. The extensive tool resharpening activities indicate a material conservation strategy.

Weber (2002) also studied the fracture patterns of $371 \mathrm{Bell} /$ Andice points to obtain clues to their use. Discriminant function value groups show very similar breakage patterns for Andice and Bell points. Broken specimens were often reworked for further use as projectiles, knives, or infrequently as drills. "Damage to stems varies by stem length and stem shape. Long stems are more likely snapped at the blade. Severe stem damage is relatively rare; most damage is to blades and barbs" (Weber 2002:44). Most specimens were employed as projectile points based on observed breakage patterns.

Production of Calf Creek end scrapers from the Hunter site in Oklahoma was from tabular nodules with flakes removed from near the exterior surface (Brooks 1995). Forty-two percent reveal cortex with an average of 13 percent cortex on dorsal surfaces. Scrapers were manufactured on thick flakes (mean thickness of nearly $13 \mathrm{~mm}$ ) and 40 percent were greater than $10 \mathrm{~cm}$. Most lengths range between 40 and $60 \mathrm{~mm}$. Eight-five percent striking platforms exhibit a single faceted implying a single blow removed the flake from the core nodule. A strong (0.85) correlation was demonstrated between striking platforms with bulbar characteristics of soft-hammer percussion. Brooks (1995) also noted the intentional removal of the striking platform and the bulb of percussion. Thirty-two percent show evidence of heat treatment to improve knapping.

Heat treating chert was a common practice in the Oklahoma Calf Creek assemblages. Heat treated tools manufactured from Frisco chert dominate the implements from the Primrose, Stilman Pit, Williams' Orchard, and Lamar sites in southcentral Oklahoma Calf Creek assemblages. For example, all 6 Calf Creek points, all 21 bifaces, and
3 of 8 flakes from the Stilman Pit cache were heat treated Frisco chert (Bartlett 1994). The different stages of cobble reduction, biface, and point manufacture reflect heat treatment, which indicates multiple heating episodes employed in production of a single artifact (Wyckoff et al. 1994a). Heat treatment was often conducted during initial stages and between manufacturing stages during tool production. Using a series of heat treatment experiments, Wyckoff et al. (1994a) observed changes in luster and color on artifacts, which probable occurred after two or three separate heating episodes. Heat treatment was not restricted to Frisco chert. Heat treatment has also been documented for Calf Creek bifaces, points and implements manufactured from Johns Valley chert, Boone chert, Keokuk, and Novaculite, various Ozarks chert, Florence-A/Kay County chert, Alibates agatized dolomite, Edwards chert, and Ogallala chert (e.g., Andrews 1999; Duncan and Wyckoff 1994; George 1995; Neal et al. 1994; Powell 1995; Sullivan 1995; Thurmond and Wyckoff 1999). From a sample of 808 Calf Creek points in Oklahoma, Andrews documents Edwards and John's Valley cherts were heat treated much less often (about 20 percent) than other cherts. He also states Edwards chert, John's Valley cherts and Alibates "lose the evidence of heat treatment relatively quickly" which may account for the low percentages of observed heat treatment (Andrews 1999:122).

Collins (1994) notes great skill and effort in the production of very thin bifaces with long deep, narrow notches on projectile points. He believes skill levels for manufacturing these artifacts were not possessed by everyone in the community and argues skilled flint knappers achieved a high degree of social status and perhaps intrinsic value was attributed to objects produced by such technological masters. The enhanced value may affect how the objects were treated, which might explain why broken projectile points were so 
extensively refurbished to the point of complete depletion prior to discard, even though raw material was plentiful.

\subsubsection{Cooking Processes}

Cooking activities are represented by various concentrations and clusters of burned rocks designated as features (Table 3-2). However, limited detailed macro-botanical analyses of feature contents, the sediments, or the burned rocks have been conducted to recover food resources processed by these thermal features.

Several different types of thermal features have been recognized at the few excavated sites. In eastern Oklahoma, a nearly $2 \mathrm{~m}$ diameter rock-lined feature (Feature 2) was identified at 260 to 330 cmbs at the Arrowhead Ditch site (Wyckoff et al. 1994b). This salvaged rock feature consisted of two layers of 86 sandstone clasts that completely lined a 10 to $13 \mathrm{~cm}$ deep shallow basin and was interpreted as a roasting pit. Numerous bits and chunks of charcoal were noted between and below the rocks, which yielded a radiocarbon date of 5730 \pm 160 B.P.

Mixed Stratum III-b at Landslide contained four recognizable features to reflect possible heating, cooking, and/or dumping activities (Sorrow et al. 1967). Three were dominated by burned and fractured rock and one (Feature 10) was an irregular carbon stain associated with burned rocks. The latter potentially represented a type of in situ warming facility. Recovery of two triangular dart points with Feature 10, probably indicates this feature was not part of the Bell/Andice interval. These burned rock and charcoal stained features were not attributed a functional interpretation.

At the Cibolo Crossing site near San Antonio, 12 burned rock features were identified in the Bell/Andice component (Kibler and Scott 2000). These features varied in shape, size, outline, contents, and were interpreted to reflect different types of cooking facilities, which ranged from rock hearths and rock grills to hearth dumping residue piles (Kibler and Scott 2005). Features with recognizable basins filled with broken rocks, were interpreted as ovens for plant-food cooking over a long period. Features that lacked recognizable basins or pits and comprised of large flat rock slabs were interpreted as grills and broilers. Small burned rocks clusters that lacked intact core or internal structure, observable charcoal, or oxidized sediment were interpreted as dumps or discard piles (Kibler and Scott 2005).

The small cooking facilities and dumps were probably employed to cook different food resources (see Wandsnider 1997 for discussion of cooking requirements on different foods to facilitate human digestion). As yet, no direct evidence from technical research (i.e., lipid residues and starch grain analyses) is currently available to support the interpretations of various kinds of cooking features.

The dominant thermal feature indicators are fractured burned rocks, which stored and transferred heat from the rock to the food. This cooking process might involve stone boiling, roasting, and oven baking with or without steam. Burned rocks are not normally curated by prehistoric groups and their accumulations on sites are suitable for multiple kinds of analyses to explore cooking technologies. After rocks expended their heat retention properties, they were often discarded in irregular clusters of randomly positioned burned rocks. These irregular clusters represent various cleaning and maintenance of cooking areas on site occupation surfaces.

\subsubsection{Subsistence Base}

Direct evidence for the subsistence base is limited to a few excavated sites (i.e., Bellcow, Big Hole, Cibolo Crossing, Landslide) reported in detail. Poor preservation however has often prevented recovery of organic remains. For example, only 41 unidentifiable bone fragments were from the $59 \mathrm{~m}^{2}$ Bell/Andice component excavated at the 
Table 3-2. Excavated Bell/Andice/Calf Creek Feature Data.

\begin{tabular}{|c|c|c|c|c|c|c|c|c|}
\hline $\begin{array}{l}\text { Site No./ } \\
\text { Name }\end{array}$ & $\begin{array}{c}\text { Feature } \\
\text { No. }\end{array}$ & $\begin{array}{l}\text { Feature } \\
\text { Type }\end{array}$ & $\begin{array}{l}\text { Feature } \\
\text { Size }(\mathrm{cm})\end{array}$ & Profile & Stained Matrix & $\begin{array}{l}\text { Depth }(\mathbf{c m}) \\
\text { below } \\
\text { surface }\end{array}$ & $\begin{array}{l}\text { Burned Rock } \\
\text { Counts/Wts. } \\
\text { (g) }\end{array}$ & $\begin{array}{c}\text { Material \& } \delta 13 C \\
\text { Corrected C14 Date } \\
\text { B.P. }\end{array}$ \\
\hline \multirow{12}{*}{$\begin{array}{c}\text { 41BX377 } \\
\text { Cibolo } \\
\text { Crossing }\end{array}$} & 9 & $\begin{array}{l}\text { Burned rock } \\
\text { concentration }\end{array}$ & $260 \times 160+$ & Sloping & & $\begin{array}{l}100.07- \\
99.83 \mathrm{~m}\end{array}$ & $205 / 46,000$ & $\begin{array}{c}\text { Sediment }= \\
3550 \pm 70 \text { B.P. }\end{array}$ \\
\hline & 10 & $\begin{array}{l}\text { Burned rock } \\
\text { concentration }\end{array}$ & $210 \times 100+$ & $\begin{array}{l}\text { Flat, } \\
\text { sloping }\end{array}$ & & $\begin{array}{c}99.83-9948 \\
\mathrm{~m}\end{array}$ & $111 / 3,225$ & $\begin{array}{c}\text { Sediment }= \\
3510 \pm 80 \text { B.P. }\end{array}$ \\
\hline & 11 & Burned rock & $43 \times 47$ & $\begin{array}{c}\text { Shallow } \\
\text { basin }\end{array}$ & & $\begin{array}{c}99.86-9978 \\
\mathrm{~m}\end{array}$ & $27 / 7,250$ & $\begin{array}{c}\text { Sediment }= \\
3060 \pm 70 \text { B.P. }\end{array}$ \\
\hline & 18 & $\begin{array}{l}\text { Burned rock } \\
\text { scatter }\end{array}$ & $380 \times 240$ & $\begin{array}{c}\text { Shallow } \\
\text { basin in } \\
\text { middle }\end{array}$ & & $\begin{array}{l}100.12- \\
99.85 \mathrm{~m}\end{array}$ & $403 / 124,500$ & \\
\hline & 19 & $\begin{array}{l}\text { Burned rock } \\
\text { concentration }\end{array}$ & $400 \times 275$ & flat, sloping & & $\begin{array}{c}99.95-99.21 \\
m\end{array}$ & $1,574 / 280,000$ & $\begin{array}{c}3 \text { Charcoal }= \\
4420 \pm 50,4400 \pm \\
60,4370 \pm 80 \text { B.P. }\end{array}$ \\
\hline & 24 & $\begin{array}{l}\text { Burned rock } \\
\text { cluster }\end{array}$ & $110 \times 65$ & Unreported & & $\begin{array}{c}99.95-99.78 \\
m\end{array}$ & $/ 28500$ & \\
\hline & $25 a$ & $\begin{array}{l}\text { Burned rock } \\
\text { cluster }\end{array}$ & $80 \times 35$ & Unreported & & \multirow{2}{*}{$99.95-99.83$} & $23 / 4500$ & \\
\hline & $25 b$ & $\begin{array}{l}\text { Burned rock } \\
\text { cluster }\end{array}$ & $40 \times 30$ & Unreported & & & $15 / 4000$ & \\
\hline & 27 & $\begin{array}{l}\text { Burned rock } \\
\text { cluster }\end{array}$ & $71 \times 65$ & & & $\begin{array}{c}99.70-99.50 \\
\mathrm{~m}\end{array}$ & $21 / 8,000$ & \\
\hline & 28 & $\begin{array}{l}\text { Burned rock } \\
\text { cluster }\end{array}$ & $100 \times 100$ & Unreported & & $\begin{array}{c}99.85-99.67 \\
m\end{array}$ & $16 / 3,000$ & \\
\hline & 29 & $\begin{array}{l}\text { Burned rock } \\
\text { cluster }\end{array}$ & $140 \times 100+$ & Flat & & $\begin{array}{c}99.71-99.52 \\
\mathrm{~m}\end{array}$ & $/ 4,750$ & \\
\hline & 30 & $\begin{array}{l}\text { Burned rock } \\
\text { cluster }\end{array}$ & $80 \times 20+$ & Unreported & & $\begin{array}{c}99.64-99.5 \\
m\end{array}$ & $4 /$ & \\
\hline
\end{tabular}


Table 3-2. Excavated Bell/Andice/Calf Creek Feature Data (continued).

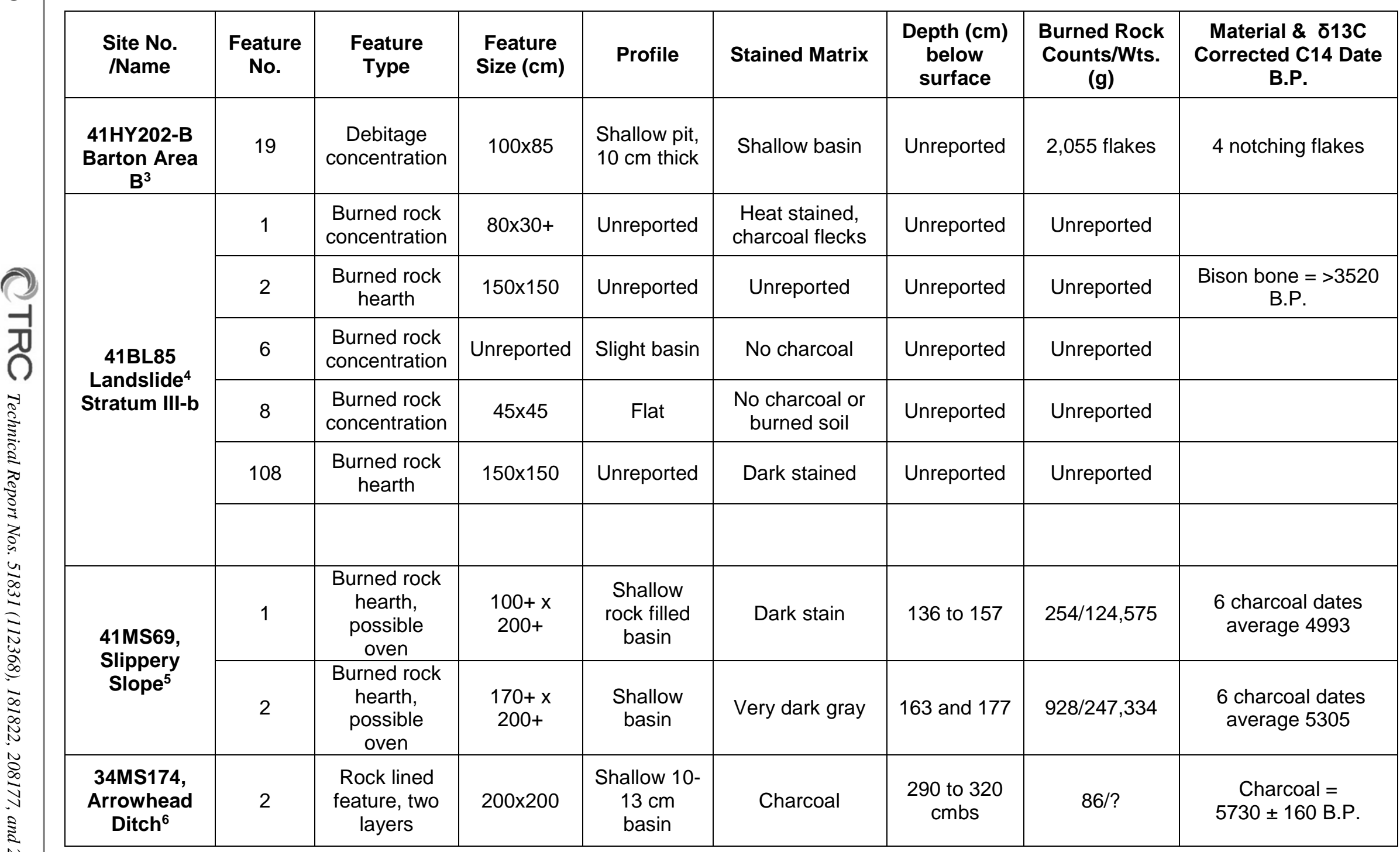

1 = Quigg et al. 2007; 2 = Kibler and Scott 2005; 3 = Collins 1994; 4 = Sorrow et al. 1967; 5 = Quigg and Frederick 2005 \& Quigg et al. $2015 ; 6=$ Wyckoff et al. 1994 
Cibolo Crossing site (Kibler and Scott 2000). Bison remains have been recovered from the Bellcow site (Girard and Carr 1994), the Frazier site (Spivey et al. 1994), the Snyder site, the Spring Lake site (Lohse et al. 2013), the Stinnett site, and the Landslide site (Sorrow et al. 1967) sites, and a Bison Antiquus occidentali skull with an embedded Calf Creek point came from the Arkansas River in northeastern Oklahoma (Bement et al. 2004, 2005). Bison remains from the Frazier site represent a single adult butchered animal (species unknown) as indicated by the association of a Calf Creek point with a bison scapula. Bison leg bones were from an 18 to $25 \mathrm{~cm}$ thick Stratum IV/Occupational Phase 3 zone at the Landslide site associated with Bell, Martindale, and Merrill points (Sorrow et al. 1967). Due to the thick cultural deposits and diversity of point types, the age of the bison bones and association with the points remain unknown. At the Bellcow site in central Oklahoma, the reported faunal remains from Middle Archaic Phase I levels include a broad assortment of fish, birds, mussel shells, turtles, snakes, small mammals, deer, and bison (Girard and Carr 1994:205). If all faunal remains reported from the Phase I levels were from the Calf Creek component, then a very diverse procurement and subsistence pattern is represented. The frequency of bison in Kansas and Oklahoma during the Middle Archaic period is unknown. Recently, bison bones have been generally associated with Bell and other point types at the Spring Lake site (41HY160) in San Marcos in central Texas (Lohse et al. 2013).

At the Cervenka site in central Texas, in a zone attributed to the middle Holocene period, a diverse faunal assemblage was identified by the presence of aquatic, grassland, and woodland species. The presence of Prairie chicken, turkey, quail, bison, pronghorn, deer, and rabbits indicate a diffuse, broad spectrum foraging subsistence strategy. The faunal assemblage consists of mammals (71\%), birds $(1.7 \%)$, reptiles $(25 \%)$, amphibians $(0.6 \%)$, and fish $(1.8 \%)$ with about 12 percent of the assemblage burned (Peter et al. 1982). Based on bison bone in Feature 26 and the recently obtained bone date of 5135 B.P. (see Table 3-1) bison are presently documented at multiple sites in central Texas almost five millennia ago.

Poor preservation conditions cannot account for the lack of bison from all Calf Creek/Andice/Bell assemblages. The thickness of bison long bone cortical walls is sufficiently dense to enable preservation longer than small mammal bones with thinner cortical walls. Multiple middle Holocene period (ca. 7000 to 4000 B.P.) occupations at Wilson-Leonard in central Texas yielded limited faunal assemblages, but reflect a broad range of exploitation of small mammals, turtles, rodents, and fish (Baker 1998). If bison populations were significantly reduced during this period, then hunters would have adjusted and broadened their diet to include such lower ranked faunal resources as deer, small mammals, turtles, birds, rodents, and fish.

The distribution of bison during this period is uncertain. Newman (1983) concluded bison probably had not expanded significantly east of Oklahoma and Texas and into the southeastern United States, since the warm-dry period of the middle Holocene. McDonald (1981) sees an overall reduction in the number of bison during the middle Holocene across the Plains. Excavations at the Lubbock Lake site in the lower Texas panhandle yielded a few bison from middle Holocene deposits (Johnson and Holliday 1986). Only localized individual bison kills and processing areas were represented in Stratum 4A and 4B of the Middle Archaic at the Lubbock Lake site. Unfortunately few diagnostic artifacts were present in the Middle Archaic assemblages and the archeological record between 8500 and 4500 B.P. is scarce (Johnson and Holliday 1986).

Mussel shells preserve better than bones, although mussel shells are rarely reported from surface collections across Oklahoma. A single shell was 
from the Bell component at the Cibolo Crossing site. In contrast, many mussel and snail shells were present in Stratum III-b at the Landslide site and are assumed discard debris from food resource procurement (Sorrow et al. 1967). The 10 to $15 \mathrm{~cm}$ thick shell midden, Zone III at the McKinzie site near Corpus Christi, yielded 12,722 valves of marine clams (Rangia flexuosa) from a possible mixed Bell component. Since two other point types were present with the Bell point in Zone III the association of abundant shell with the Bell component is uncertain. Zone III at the McKinzie site also yielded 32 marine fish otoliths from at least six different fish species. These saltwater remains are unique for Bell/Andice/Calf Creek assemblages. If the otoliths and shells were associated with the Bell assemblages, these resources increase the subsistence base diversity. Based on the growth patterns within the otoliths, Zone III was occupied at least during the late fall-winter season (Ricklis 1988).

Limited direct evidence of plant foods utilized by these people has been recovered. Lack of excavated sites is the principle reason for the absence of plant remains from Oklahoma sites. The few excavated sites in Texas have produced sparse plant remains from most Bell/Andice/Calf Creek assemblages and poor preservation is probably explains the absence (Kibler and Scott 2000; Lohse et al. 2013). Thus, macrobotanical food evidence is lacking. Despite the occurrence of thermal features at the Landslide site, no macrobotanical remains are reported (Sorrow et al. 1967).

Poor preservation in most excavated sites combined with often ambiguous contexts or associations hinders our understanding of the subsistence base of these people. Lack of preserved faunal and floral remains limit component and regional comparisons into subsistence practices or seasonal site usage. In light of poor bone and macrobotanical preservation, researchers should turn attention to such other technical analytical approaches such as starch grain and lipid residue analyses in order to address subsistence and seasonality issues.

\subsubsection{Site Selection and Mobility}

Most hunter-gatherer groups were mobile foragers and collectors who exploited mosaic of habitats across the landscape. Site location distributions across the southern plains regions provide insights into population movements. Frequently groups followed waterways through different regions. Many sites have been identified along terraces of large rivers and small creeks, such as at the Arrowhead Ditch site (Wyckoff et al. 1994b), the Cibolo Crossing site (Kibler and Scott 2000), the Landslide site (Sorrow et al. 1967), the Slippery Slope site (Quigg and Frederick 2005), and the Big Hole site (Quigg et al. 2007). Other site settings include occupations in sand dunes at the Stilman Pit site (34MR71) (Bartlett 1994), the Primrose site (34MR65) (Wyckoff et al. 1994a), and site 34GT6, (Wyckoff 1995); high ridges along the Dempsey Divide at sites 34RM334-B, 34RM804, 34RM785, and the Westfahl locality (Thurmond and Wyckoff 1999), and the Hester/Adams site (34ML83) (Cestaro and Carrell 1994); on open short grass plains at the Goff Creek locality in Texas County, Oklahoma (Rhoton 1995; Thurmond and Wyckoff 1999; White 1995); in the Osage Savannah at the Woodard-Benefield site (34MS258) and the Arrowhead Ditch site (34MS174) of northeastern Oklahoma (Shockey et al. 1994; Wyckoff 1994b; Wyckoff and Neal 1994; Wyckoff et al. 1994b); in the Tallgrass Prairie on the Arkansas River (Splawn 1995; Splawn and Wyckoff 1995); extreme eastern Oklahoma at the Red Clay site (34HS29) (Neal et al. 1994); near springs at the Hester/Adams site (Cestaro and Carrell 1994); and rockshelters at the Bellcow site (Girard and Carr 1994). Other settings include sites like Landslide (Sorrow et al. 1967), Slippery Slope (Quigg and Frederick 2005; Quigg et al. 2015), Gatlin (41KR621) (Houk et al. 2008), Spring Lake (Lohse et al. 2013), Cibolo Crossing (Kibler and Scott 2000), Gault (41BL323) and many others in the Edwards Plateau of central Texas; the Blackland Prairie at the Big Hole site (41TV2161) (Quigg et al. 2007) in central Texas; the McKinzie site in a hilltop remnant of a dissected 
Pleistocene river terrace along the Gulf coast (Ricklis 1988); and the Devil's Mouth site in a deep river terrace deposits in the desert canyon lands of the Lower Pecos (Johnson 1994). Central Texas sites are concentrated along the Balcones escarpment where abundant springs and biotic ecotones contain easy access to diverse biotic and chert resources. Site selections in diverse topographic situations indicate broad landscape use rather than a rigid use of specific landforms, an indication of large band territories. Diverse site settings may reflect individual choices, or seasonally determined localities for hunting, plant gathering, camping, or raw material acquisition across a mosaic of biotic resources.

A survey of Calf Creek artifacts in northwestern Oklahoma between the Caprock Escarpment and the Oklahoma and Texas line located only seven widely scattered isolated find localities and no sizeable campsites (Thurmond and Wyckoff 1999). These few isolated localities tended to occur high on the landscape, along major east-west interfluvial divides. They postulated Calf Creek bands employed interfluves as travel routes between the Southern High Plains to the west and the Redbed Plains to the east. These high settings are also apt to expose older surfaces rather than covered with more recent sediments (Thurmond and Wyckoff 1999).

Wyckoff (1995) delineated four geographical territorial regions for Oklahoma Calf Creek sites based on differences of knappable stone resources employed for manufacturing stone tools. The smaller sizes of implements are interpreted as evidence of resource curation behavior in the use of local tool stone in a territory and the presence of some lithic resources from adjacent areas were employed as evidence of territorial boundary limits (Wyckoff 1995). In Oklahoma, mobile groups apparently centered movements on specific regional raw material source areas and confined movement to a specific territory.
The occurrence of eight lithic caches from the Primrose, the Stilman Pit, the Benton-Whiteside, the Lamar, 34AT698 and 34MA4 sites in southcentral Oklahoma and north-central Texas (Bartlett 1994; Duncan 1994; Neal 1994a; Walter 2013; Wyckoff et al. 1994a; Brooks, personal communication) indicate a practice of deliberate stashing tool stone in order to "load a landscape" in relatively chert-poor areas for later use. The range of artifacts from these eight caches consists of large bifaces in various stages of reduction, scrapers, and large flakes suitable for shaping into tools. Most cached items represent Frisco chert known within the Arbuckle Mountain territory in south-central Oklahoma (Wyckoff 1995). Caching behavior indicates those peoples clearly understood the regional resource availability and employed predetermined travel routes during their seasonal movements.

Although some researchers refer to direct chert acquisition as a logistical procurement associated with a collector strategy, Bartlett (1994) believes the majority of evidence indicates a foraging strategy. Some sites such as Area B at the Barton site (Collins 1994a) in central Texas, with a relatively narrow range of tool types and the Hunter site in north-central Oklahoma with a high percentage of end scrapers (Brooks 1995) may represent remains of task-specific activities of short-term durations. Other sites such as the Primrose site in south-central Oklahoma appear intensively and repetitively occupied campsites (Wyckoff et al. 1994a).

The recovery of a single Bell point from the Zone III Rangia shell midden at the McKinzie site perhaps represents a fall seasonal use by a Bell/Andice/Calf Creek group along the coast. Unfortunately, poor preservation of floral and faunal remains from most sites has provided very limited insights into seasonal movement patterns of groups employing Calf Creek/Bell/Andice points. 
Duncan (1995) studied surface lithic assemblages from three Calf Creek sites in Oklahoma and concluded a foraging mobility strategy was in operation. She felt the entire group developed cognitive resource "maps" of exploitable resources and needed lithic resources were obtained during normal movement patterns as an embedded procurement strategy. The resharpening and recycling of broken tools reflect a highly curated assemblage, since various tool stone source areas were locally restricted yet widely scattered across the landscape (Duncan 1995).

\subsubsection{Trade and Interactions}

Indications of possible trade or interactions of Bell/Andice/Calf Creek populations internally or with other groups are quite limited and poorly documented. The diversity of lithic artifact raw materials, especially from distant sources and/or other exotic materials form the basis for interpreting cultural interactions. At the heart of the Frisco chert resource area in south-central Oklahoma, the Primrose site yielded six flakes $(<1 \%)$ of Johns Valley Flint from the western edge of the Ouachita Mountains and 66 artifacts (6\%) of Edwards chert from central Texas (Wyckoff et al. 1994a). Edwards and Tecovas cherts have also come from surface sites in central Oklahoma (e.g., Andrews 1999; Cestaro and Carrell 1994; Duncan 1995; Neal 1994a; Powell 1995; Wyckoff et al. 1994a). Alibates agatized dolomite from the Canadian River valley of the Texas Panhandle was employed to manufacture tools from sites in the Oklahoma Panhandle (White 1995; Thurmond and Wyckoff 1999), and near the Oklahoma-Kansas line in Kay County (Sullivan 1995). Alibates outwash cobbles occur along terraces in the Canadian River drainage as far east as the presentday Lake Eufaula (Kraft 1997; Wyckoff 1993) and potentially was the source of Alibates from sites in central Oklahoma (Powell 1995). Projectile points manufactured from Florence (Kay County) chert from the southern Flint Hills of northern Oklahoma were $250 \mathrm{~km}$ from their source areas in northwestern Oklahoma (Thurmond and Wyckoff 1999). Diverse nonlocal material types are present in Oklahoma sites even though quantities of suitable local cherts occur in the region.

Duncan (1995) has identified minimally seven chert resources from Oklahoma and two lithic resource areas in Texas among the 246 Calf Creek points from the Anthony site in Caddo County in westcentral Oklahoma. Within the context of regional territories delineated for Oklahoma, the occurrence of diverse chert resources in small numbers and distant source areas suggests intra-group interaction or exchange.

Whereas the dominance of different chert sources has been employed to delineate four potential territories employed by Calf Creek groups in Oklahoma (Wyckoff 1995) while Andrews (1999) delineated five exploitation regions, small percentages of other raw materials from distant regions also occur in site assemblages. Wyckoff postulates the low frequency of non-local cherts from sites in different territories provides evidence for exchange among contemporaneous groups. Where high quality chert was not immediately available in a region, local Ogallala quartzite was employed (Andrews 1999; Thurmond and Wyckoff 1999). Use of local raw materials is also evident at excavated Bell/Andice/Calf Creek components in central Texas, which are dominated by central Texas Edwards chert (Collins 1994a; Kibler and Scott 2000). However, the extensive availability of Edwards chert within a 260-by-100 km region across Texas may mask contact and exchange by groups in Texas, as exchange of Edwards chert may involve reasons more subtle than mere chert acquisition. The high value given to Edwards chert may be seen as it represents 1 to 11 percent in each of Andrews (1999) projected five exploitation regions across Oklahoma. 
Thus far, no obsidian has been attributed to an unmixed Bell/Andice/Calf Creek component. A single obsidian flake was found at the Rangia shell midden in Zone III at the McKinzie site (Ricklis 1988) near Corpus Christi; however, the occurrence of minimally two other identified point types from this zone may reflect component mixture of groups or assemblages. Lack of obsidian tools and debitage associated with the Calf Creek interval across Oklahoma probably reflects a lack of contact and/or travel to obsidian source areas in the northern New Mexico or the northwestern Plains.

\subsubsection{Treatment of the Dead}

No human remains are unambiguously attributed to Bell/Andice/Calf Creek components in Texas or Oklahoma. A partial human skeleton was found during Works Progress Administration (WPA) investigations at the Lamar site in Bryan County, Oklahoma, in 1941. Burial \#14 consisted of scattered fragments of cranium, long bones, and possible vertebrae from a single individual at a level slightly above a chipped stone tool cache (Neal 1994a). These remains were not retained and no formal physical anthropological study was conducted. Although artifacts, including the cache, represent the Calf Creek horizon, the association of Burial 14 with the component is not clear.

Six Bell/Andice point fragments were found in nonmortuary contexts at the substantial Buckeye Knoll cemetery (41VT98) along the Guadalupe River in Victoria County on the Texas coastal plain (Ricklis 2012a:189). Investigations documented some 69 human interments of 116 individuals. Radiocarbon dated bones and teeth from 13 human interments date between 5470 and 6075 B.P. (Table 3-2, Ricklis et al. 2012). The occurrence of other associated point types and artifacts with the interments might reflect cultural affiliations to groups contemporaneous with and in contact with the Bell/Andice people (Ricklis 2012b).
The absence of human remains from Bell/Andice/Calf Creek components precludes insights into the diversity of mortuary practices, social status, or health conditions within the multiple hunting-gathering populations in the southern plains.

\subsubsection{Summary}

As this summary has shown, Calf Creek, Bell and Andice point forms have been defined for about 40 years, and except for their recovery in a few excavations in Texas sites, little progress occurred in fleshing out the culture complex until 1991 and 1993, when Wyckoff and Shockey edited a series of 29 papers on Calf Creek sites in Oklahoma (Wyckoff and Shockey 1991, 1993). Most papers examined site distributions, landform utilization, raw material use and lithic technology, and diverse aspects of the diagnostic artifacts. Although the information was compiled from surface collections from 50 sites, most lack clear stratigraphic context; which undermines claims for isolating the associate assemblage parts. This ambiguity not only creates difficulty in identifying cultural assemblages but also limits researcher's ability to address specific aspects of human behavior beyond information from stone tools. Only a few limited excavations in Oklahoma have contributed insights into other aspects of this Middle Archaic cultural lifeways.

Considerably more excavations in Texas have encountered Bell/Andice/Calf Creek components and added diverse information on tool kits, subsistence, food processing and cooking processes. Excavations often encountered materials from stable or slowly accreting landforms, which resulted in mixed assemblages and questionable contexts. Most early excavated components with these artifacts were undated or poorly dated with their general chronological assignment defined primarily through multiple stratigraphic positions and other dated components. 
More recent excavated components have helped solidify their Middle Archaic assignment with stratigraphic control and increased radiocarbon dates, therefore the temporal framework and material assemblages are slowly being refined.

Radiocarbon assays indicate the Bell/Andice/Calf Creek culture generally dates between 5200 and 4990 B.P. in Oklahoma and between 5770 and 4720 B.P. in Texas (see Table 3-1). The few radiocarbon dates from Kansas are very similar to those in Texas. Several dates provide results, which are older or younger than the most clustered results. The outlier dates may be eventually verified by additional dates or reflect the expansion of populations at various times into different regions within the vast expansive territory. Existing data is not sufficient to document population movements across the broad region.

The Bell/Andice/Calf Creek culture flourished during part of the Altithermal climatic period, which is characterized by a warm and dry period across the southern Plains region. That environmental condition must have significantly influenced the habitat mosaics and decisions these people made in procuring animal and plant resources necessary to maintain their lifestyles.

After reviewing the archeological and environmental record from the middle Holocene, Meltzer (1999) lists several key points that emerged from the xeric period: 1) fewer archeological sites exist from this period throughout the Plains, 2) existing sites are widely scattered in space and time, resulting in few localities that can provide finegrained evidence of adaptive change through time, 3) adaptive strategies vary across different parts of the Plains, and 4) the apparent effects of the Altithermal are most extreme and evident on the Southern Plains.
As a result of these key points, Meltzer (1999) identifies several trends in human subsistence, technology, settlement, and demographics, which have been perceived and attributed to forger responses to Altithermal climates. The responses included the following: 1) an expansion of the diet breath to incorporate a variety of animals and plants, 2) the advent of new technologies to cope with diminished food and water resources, 3) large scale settlement shifts, from the Plains to the western foothills and mountains or to local refugia (springs and watercourses on the Plains), 4) reduced settlement mobility marked by increasing reliance on local stone sources and construction of residential pithouses, and 5) an overall decline in human population density. The relevance of these trends to the Calf Creek/Andice/Bell culture can serve as a basis for developing research designs to test the Middle Archaic responses in the southern Plains.

Further insights into the hunter-gatherers who employed Bell/Andice/Calf Creek points will require a commitment to conduct expansive excavations in sites with clear stratigraphy, isolation of component, good preservation of organic remains, and dateable remains. Additional detailed and technical analyses on multiple artifact classes are critical to understand human adaptive activities across areas of Oklahoma, Texas and beyond. Much remains to be understood concerning this period and how these people operated and survived during periods of environmentally stressful conditions. The following chapters contribute to a greater understanding of these people. Adaptive lessons learned may hold relevance to the climatic changes facing us during the existing cycle of global warming. 


\subsection{MARTINDALE BACKGROUND}

\section{J. Michael Quigg}

\subsubsection{Introduction, History, and Background}

The Martindale point was first named "Martindale Fishtail" by J. Charles Kelly for specimens recovered at Belton Reservoir in central Texas (Miller and Jelks 1952). Subsequently, the name was changed to Martindale and described by Suhm et al. (1954). Early excavations had no or few radiocarbon dates, even so, the Martindale point type was attributed to the Early Archaic period based primarily on stratigraphic positions in relationship with other point types (Suhm et al. 1954:446; Shum and Jelks 1962:213; Turner and Hester 1985:120-121). Chronological assignment remained with the Early Archaic as additional data with better stratigraphic context became available (Collins 1995:376, 2004:113; Johnson and Goode 1994:24; Turner et al. 2011:132), despite lack of radiocarbon dates directly associated with this point type. Suhm and Jelks (1962) provide the following description for Martindale points. In outline the point has:

"a triangular blade with edges sometimes straight, usually convex. Shoulders pronounced to well-barbed, but barbs seldom reach the base. Stem varies from nearly parallel-edged (d, G) to strongly expanding. The most distinguishing feature of these points is that the base is formed by two distinct convex curves meeting in a depression in the center, a "fish-tail" best seen in specimen C, D, E, G, I (Figure 3-9). On other specimens the base appears to be a simple recurve, but close examination reveals the doubleconvex 'fish- tail'. It is assumed that this form of base was the result of deliberate effort setting it apart from other bases (1962:213)."
In the late 1970s Weir (1976b) presented a reconsidered chronology for central Texas and divided the Archaic stage into five easily recognizable phases, partially supported by 44 radiocarbon dates, 31 of which were from sites in the Lower Pecos region. This early chronology was primarily based on radiocarbon dates from excavations at rock shelters in the Lower Pecos region. Weir's Archaic phases (1976b), from the earliest to the latest, included five phases: San Geronimo phase (dominated by Early Barbed', Bell, Gower, and Uvalde points), Clear Fork phase (dominate by Pandale, Bulverde, Travis and Nolan points), Round Rock phase (dominate by Pedernales, Langtry, and Val Verde points), San Marcos phase (dominate by Montell, Marcos, Marshall, Castroville, Frio, and Ensor points), and the Twin Sisters phase (dominated by Ensor, Darl, and Godley points). Of note is the absence of the term Martindale. Weir (1976a) included the Martindale type (Figure 3-10) into the San Geronimo phase and listed only six sites with Martindale points. The sites included Landslide (41BL85), Footbridge (41CM2), Williams (41TV75), Crumley (41TV86), McCann (41LM3), and Crystal Rivers (41BX195), with a relatively low frequency of 21 points, or 0.62 percent, of his total sample. With this low frequency it is obvious not many Martindale points had been recovered before 1976.

Subsequently, Prewitt (1981) created a new subdivision in the Early Archaic period, the Jarrell phase, which he estimated between ca. 6000 and 5000 B.P. He attributed the Martindale point type to this new_phase along with the Uvalde, Bell, and Andice types. Prewitt (1981) listed seven sites that represented the Jarrell phase: Landslide (41BL85) (Sorrow et al. 1967), Gault (41BL:232) (TARL), site 41VT17 (Fox and Hester 1976), Tombstone Bluff (41WM165) (Prewitt 1982), Merrell (41WM2) (Campbell 1948), La Jita (41UV31) (Hester 1971), Jetta Court (41TV151) (Wesolowsky et al. 1976). For this phase he listed 


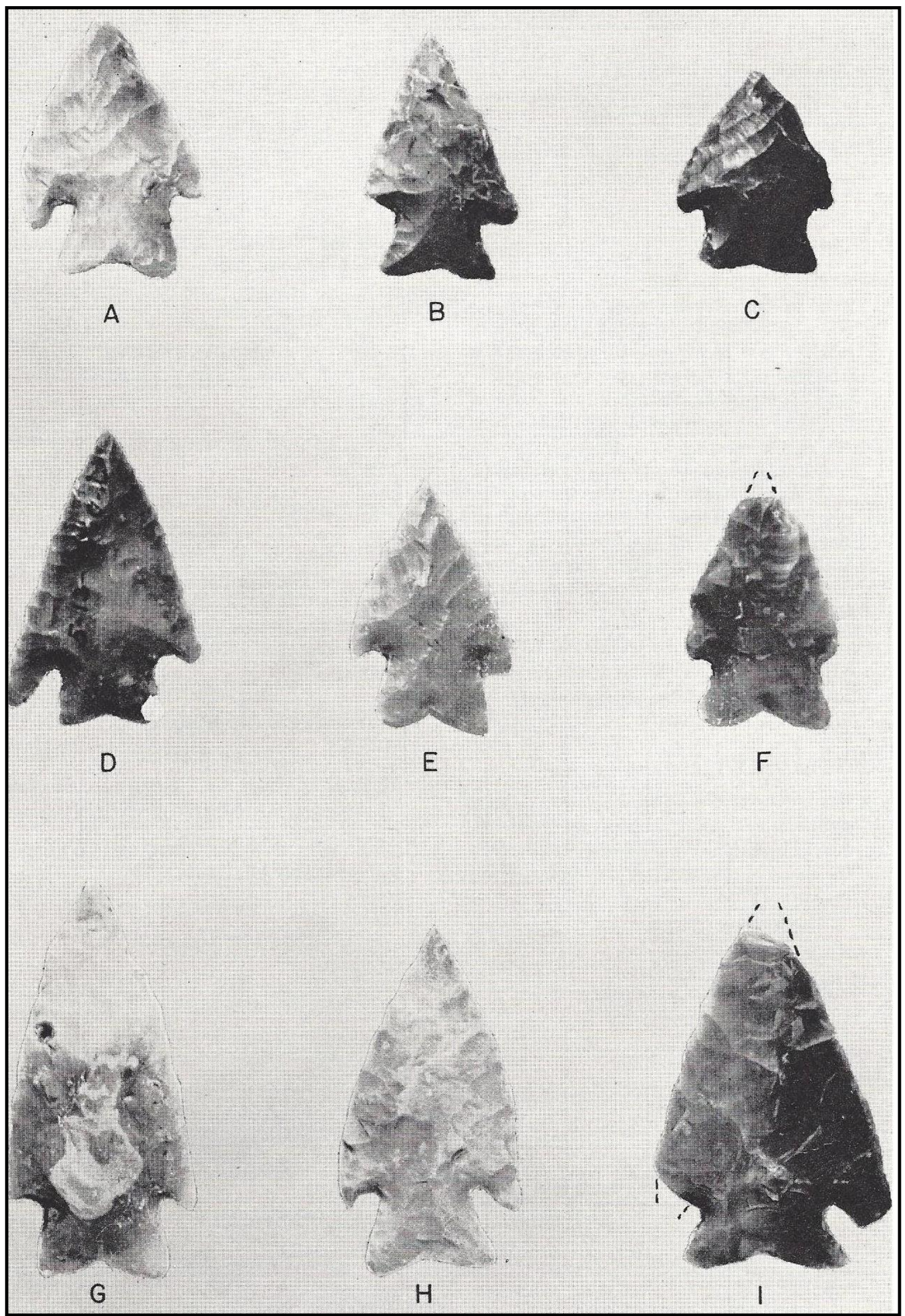

Figure 3-9. Martindale point forms from Suhm and Jelks 1962, Plate 107. 


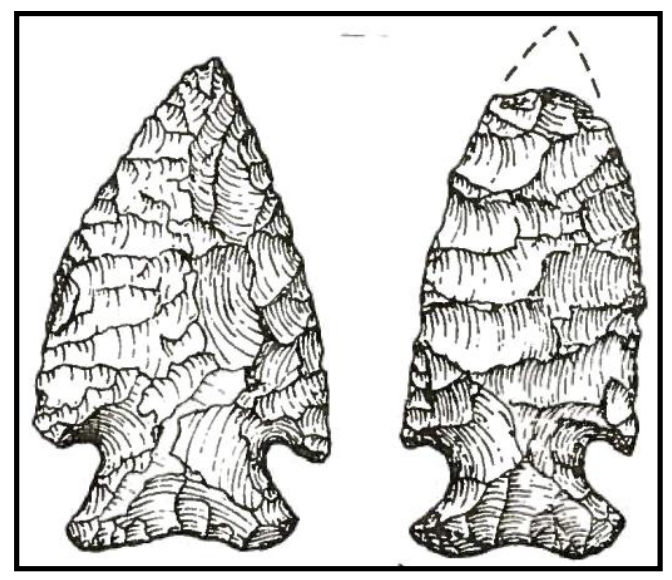

Figure 3-10. Martindale points forms from Weir (1976a, Figure 9).

the above projectile points, Clear Fork gouges, bifaces, scrapers, hammer stones, and grinding stones. The only feature type he included was a large flat hearth. He speculated subsistence was focused on hunting and gathering, with an emphasis on gathering, and included freshwater mussels as one resource (Prewitt 1981a:78). In 1985 Prewitt provided only two radiocarbon dates for his Jarrell phase, both from Williamson County. A date of $4970 \pm 90$ B.P. on charcoal from the Cervenka site (41WM267), and the other of $5285 \pm 725$ B.P. from 41WM73, both from fieldwork in the San Gabriel Reservoir Districts of central Texas.

Collins (1995, 2004) places Martindale, Uvalde, Angostura, and Early Split Stem point styles into the Early Archaic, which he postulates dates from ca. 8800 to 6000 B.P., with the Martindale and Uvalde the youngest. Collins et al. (1990) suggested the term 'interval' as a substitute for the term 'phase', arguing it was a more neutral term. Collins has continued to employ the term since (Collins 1995, 2004), although not all researchers have adopted his terminology.

Collins does not provide specific dates for the individual point styles/intervals nor specific discussions concerning lifeways or material culture assemblages for individual intervals or point groups. He lists three sites, Camp Pearl Wheat
(41KR243), Hall's Cave (41KR474), and Landslide (41BL85) in the Martindale-Uvalde style interval, with high integrity only at the Camp Pearl Wheat site, the other sites with moderate integrity. Although Camp Pearl Wheat and Hall's Cave sites exhibited good stratigraphy, neither site yielded large artifact assemblages associated with the few Martindale points recovered. Collins and Ricklis (1994) observed a technological difference as a reason for the break in styles between the earlier Martindale-Uvalde points and the later Bell/Andice/Calf Creek forms. This is also the reason they attributed the latter to the Middle Archaic.

Collins $(1995,2004)$ briefly summarized the Early Archaic with general statements concerning the 2800-year long period. He states the period included numerous dart point types, Clear Fork tools, and Guadalupe bifaces. $\mathrm{He}$ indicates subsistence data is sparse, although deer, various small animals including fish, and bulbs cooked in earth ovens were exploited, and specialization of cooking appliances occurred during that time. Collins (2004) states bison and antelope were scarce or absent. He sees a general correlation between bison occurrences and comparatively mesic conditions (Collins 2004:115). The existing paleoenvironmental evidence portrays an oscillation from mesic through extremely xeric and back to mildly xeric conditions existed (see Chapter 2.0).

Confusion in the Early Archaic point identification system stems from a variety of contributing factors. These include low frequencies of identified projectiles from any one site, poor or limited understanding of their context, no or few radiocarbon dates associated with individual types, inability of researchers to correctly identify morphological types because of reworking of specimens, poor or too restrictive type definitions in the literature, and considerable individual subjectivity inherent in classification of projectile 


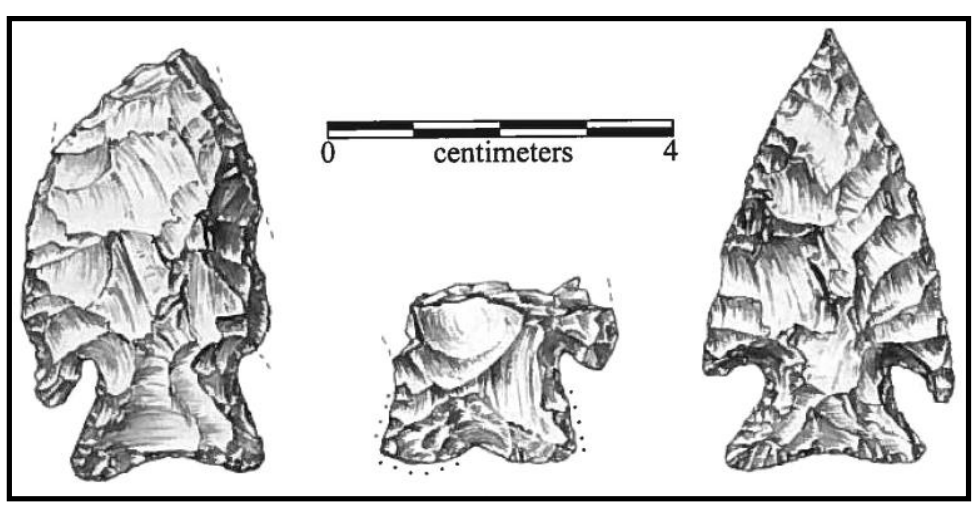

Figure 3-11. Group A Martindale points from the Wilson-Leonard site (from Dial et al. 1998:Figure 13-46).

points. To address identification problems at the Wilson-Leonard site (41WM245) in central Texas, Kerr and Dial (1998) employed rigorous statistical analyses to investigate complexity and help eliminate potential biases and confusion over these many Early Archaic point types. A total of 102 projectiles was subjected to cluster and discriminate function analyses, which resulted in the identification of 11 major morphological clusters or groups. These statistical analyses of Early Bifurcated Stem projectiles at the WilsonLeonard site exhibited several distinct clusters with varying degrees of similarity. However, many identified clusters contained several different point types, and some clusters yielded tight groupings. Martindale points (Figure 3-11) formed a comparatively distinct group (Cluster 2) that exhibited relatively thin, expanding stems with a low arc- to V-shaped base (Kerr and Dial 1998).

\subsubsection{The Sites}

Most excavated Early Archaic sites and components throughout central Texas and the adjacent Lower Pecos region have yielded multiple Early Archaic point types from mixed context (Figure 3-12). The context may reflect very slowly aggraded deposits, a thick zone that lacked stratigraphy or erosion/deflation that created a stable surface where multiple point types and events accumulated (Table 3-3). Some representative sites include the following: Jetta
Court (41TV151) (Wesolowsky et al. 1976), Footbridge (41CM2) (Suhm 1962), Eckols (41TV528) (Karbula 2000), Woodrow Herd (41UV88) (Decker et al. 2000), Baker Cave (41VV213) (Word and Douglas 1970), Higgins (41BX184) (Black et al. 1998), Sleeper (41BC65) (Johnson 1991), Landslide (41BL85) (Sorrow et al. 1967), Wilson-Leonard (41WM235) (Collins 1998a), Youngsport (41BL78) (Shafer 1963), Varga (41ED28) (Quigg et al. 2008), Pavo Real (41BX52) (Collins et al. 2003), Gatlin (41KR621) (Houk et al. 2008), Richard Beene (41BX831) (Thoms and Mandel 2007), and Barton (41HY202) (Collins 1994a).

As an example of compressed and mixed stratigraphy, the Gatlin site (421KR621) yielded some 61 Martindale points (subdivided into multiple subtypes) (Oksanen et al. 2008) (Figure 313). These were vertically dispersed in all four proposed occupation zones (OZs 1-4) spanning the Early Archaic through Late Archaic (Houk et al. 2008). The reported zones were not clearly distinguishable, as occupations were not vertically separated by obvious sterile levels. Occupation zones were defined as broad cultural or temporal zones correlated to the established prehistoric record (Smith and Oksanen 2008:7-21). Other point types in the Early Archaic Occupation Zone 1 included 1 Gower, 2 Early Barbed Devil's River variants, 1 untyped, and a 1 Pandale point. Most 


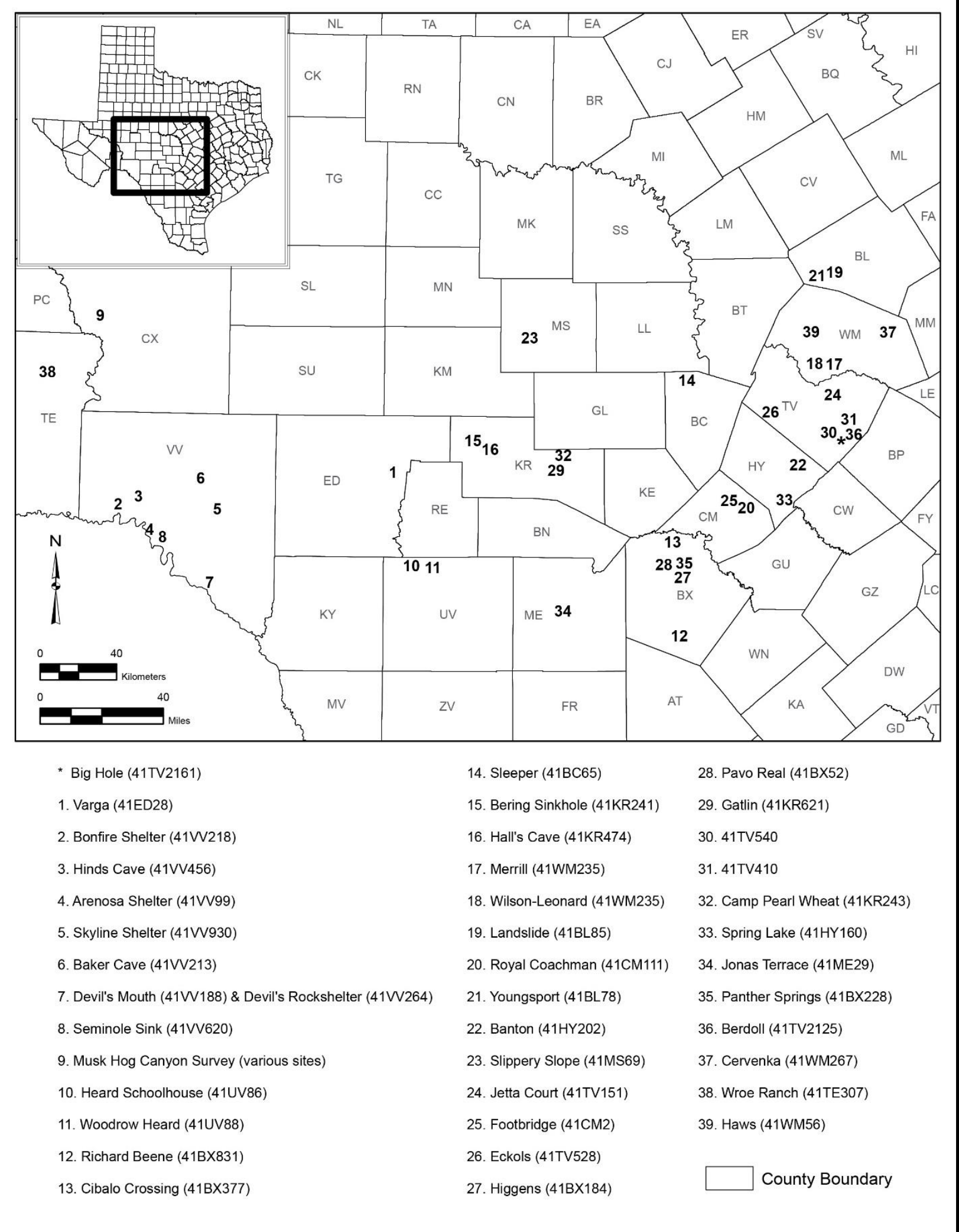

Figure 3-12. Selected Early Archaic sites in and near the Edwards Plateau and central Texas. 
Table 3-3. Martindale Site and Component Integrity Data.

\begin{tabular}{|c|c|c|c|c|c|c|}
\hline $\begin{array}{l}\text { Site Name and } \\
\text { Number }\end{array}$ & Location & $\begin{array}{l}\text { Component } \\
\text { Integrity }\end{array}$ & Point Types Present & $\begin{array}{l}\text { Radiocarbon } \\
\text { Dates (B.P.) }\end{array}$ & $\begin{array}{l}\text { Comments on } \\
\text { Date }\end{array}$ & References \\
\hline $\begin{array}{l}\text { Arenosa Shelter, } \\
\text { 41VV99 }\end{array}$ & Lower Pecos & Fair & 1 Early barb/Martindale & $5360 \pm 170$ & $\begin{array}{l}\text { Date from multiple } \\
\text { charcoal } \\
\text { concentrations }\end{array}$ & $\begin{array}{l}\text { Dibble 1997; } \\
\text { Valastro et al. } \\
1967\end{array}$ \\
\hline $\begin{array}{l}\text { Bering Sinkhole, } \\
\quad 41 \mathrm{KR} 241\end{array}$ & $\begin{array}{l}\text { Eastern } \\
\text { Edwards } \\
\text { Plateau }\end{array}$ & Good & 2 Martindale, 1 Uvalde & $\begin{array}{c}6860 \pm 170,6660 \\
\pm 110,5840 \pm \\
190\end{array}$ & $\begin{array}{l}\text { Dates on human } \\
\text { petrous bones }\end{array}$ & Bement 1994 \\
\hline $\begin{array}{c}\text { Camp Pearl } \\
\text { Wheat, } 41 \text { KR243 }\end{array}$ & $\begin{array}{l}\text { Central } \\
\text { Edwards } \\
\text { Plateau }\end{array}$ & Fair & $\begin{array}{l}3 \text { Martindale, } 1 \text { Uvalde, } 1 \\
\text { Uvalde-like, } 1 \text { Bell }\end{array}$ & None & NA & $\begin{array}{l}\text { Collins et al. } \\
\quad 1990\end{array}$ \\
\hline $\begin{array}{c}\text { Gatlin, } 41 \mathrm{KR} 621, \\
\text { Occupation Zone } \\
2,\end{array}$ & $\begin{array}{l}\text { Central } \\
\text { Edwards } \\
\text { Plateau }\end{array}$ & Poor & $\begin{array}{c}88 \text { points; } 64 \text { Early Archaic, } 34 \\
\text { Martindale, } 21 \text { Gower, } 10 \text { Early } \\
\text { Triangular, } 5 \text { Bandy, } 2 \text { Bell, } 1 \\
\text { Andice, } 1 \text { Baker, } 1 \text { Marcos }\end{array}$ & $\begin{array}{l}13 \text { dates }=6100 \\
\text { to } 4600\end{array}$ & $\begin{array}{l}13 \text { Dates on wood } \\
\text { charcoal }\end{array}$ & $\begin{array}{l}\text { Houk et al. } \\
2008\end{array}$ \\
\hline $\begin{array}{l}\text { Cibolo Crossing, } \\
\quad 41 \mathrm{BX} 377\end{array}$ & $\begin{array}{l}\text { Southeastern } \\
\text { Edwards } \\
\text { Plateau }\end{array}$ & Good & 2 Martindale and 1 Bandy & $\begin{array}{c}790 \pm 40^{*}, 820 \pm \\
50^{\star} 2110 \pm 40^{*} \\
\text { Mean A/I values } \\
=5953 \& 6290\end{array}$ & $\begin{array}{l}\text { Three charcoal } \\
\text { dates intrusive. } \\
\text { A/l values on } \\
\text { Rabdotus shells }\end{array}$ & $\begin{array}{l}\text { Kibler and } \\
\text { Scott } 2000\end{array}$ \\
\hline $\begin{array}{l}\text { Clear Creek Golf } \\
\text { Course, } \\
\text { 41CV413, Lower } \\
\text { Component }\end{array}$ & $\begin{array}{l}\text { Eastern } \\
\text { Edwards } \\
\text { Plateau }\end{array}$ & Poor & $\begin{array}{l}42 \text { points: } 22 \text { Early Archaic: } 8 \\
\text { Yarbrough, } 5 \text { Martindale, } 4 \\
\text { Wells, } 4 \text { Gower, } 1 \text { Merrell }\end{array}$ & $\begin{array}{c}4320 \pm 40,4580 \\
\pm 40,4280 \pm 40\end{array}$ & $\begin{array}{l}\text { Three dates on } \\
\text { charcoal }\end{array}$ & $\begin{array}{l}\text { Quigg et al. } \\
\qquad 2011\end{array}$ \\
\hline $\begin{array}{l}\text { Eckols, 41TV528, } \\
\text { Analytic Unit } 2\end{array}$ & $\begin{array}{l}\text { Edwards } \\
\text { Plateau }\end{array}$ & Mixed & $\begin{array}{c}1 \text { Pedernales, } 1 \text { Early Triangular, } \\
3 \text { Martindale. } 2 \text { Martindale-like, } 2 \\
\text { Bell-like }\end{array}$ & $5199 \pm 124$ & $\begin{array}{l}\text { Charcoal date } \\
\text { from zone }\end{array}$ & Karbula 2000 \\
\hline $\begin{array}{l}\text { Halls Cave, } \\
41 \mathrm{KR} 474\end{array}$ & $\begin{array}{l}\text { Central } \\
\text { Edwards } \\
\text { Plateau }\end{array}$ & Good & $\begin{array}{l}1 \text { Uvalde, } 1 \text { probable Uvalde, } 1 \\
\text { Martindale at } 102 \mathrm{cmbs}\end{array}$ & $4850 \pm 130$ & $\begin{array}{c}\text { Charcoal date } \\
\text { from hearth at } 100 \\
\text { cmbs }\end{array}$ & Toomey 1993 \\
\hline
\end{tabular}


Table 3-3. Martindale Site and Component Integrity Data (continued).

\begin{tabular}{|c|c|c|c|c|c|c|}
\hline $\begin{array}{l}\text { Site Name and } \\
\text { Number }\end{array}$ & Location & $\begin{array}{l}\text { Component } \\
\text { Integrity }\end{array}$ & Point Types Present & $\begin{array}{l}\text { Radiocarbon } \\
\text { Dates (B.P.) }\end{array}$ & $\begin{array}{l}\text { Comments on } \\
\text { Date }\end{array}$ & References \\
\hline $\begin{array}{l}\text { Haws, 41WM56, } \\
\text { Area C }\end{array}$ & $\begin{array}{l}\text { Eastern } \\
\text { Edwards } \\
\text { Plateau }\end{array}$ & Poor & 1 Martindale above 1 Nolan & None & NA & $\begin{array}{l}\text { Peter et al. } \\
1982\end{array}$ \\
\hline $\begin{array}{l}\text { Higgins, } \\
\text { 41BX184, Hearth- } \\
\text { Field, Slice } 1\end{array}$ & $\begin{array}{l}\text { Southeastern } \\
\text { Edwards } \\
\text { Plateau }\end{array}$ & Poor & $\begin{array}{c}3 \text { Martindale, } 2 \text { Early Triangular, } 1 \\
\text { Bell, } 1 \text { Early Stemmed, } 3 \\
\text { unnamed types }\end{array}$ & None & NA & $\begin{array}{l}\text { Black et al. } \\
\quad 1998\end{array}$ \\
\hline $\begin{array}{c}\text { Landslide, } \\
\text { 41BL85, Stratum } \\
\text { V }\end{array}$ & $\begin{array}{l}\text { Eastern } \\
\text { Edwards } \\
\text { Plateau }\end{array}$ & Fair & $\begin{array}{c}1 \text { Bell, } 4 \text { Gower, } 4 \text { Gower-like, } 6 \\
\text { Martindale }\end{array}$ & None & NA & $\begin{array}{l}\text { Sorrow et al. } \\
\quad 1967\end{array}$ \\
\hline $\begin{array}{l}\text { Panther Springs, } \\
\text { Area M, 41BX228 }\end{array}$ & $\begin{array}{l}\text { Southeastern } \\
\text { Edwards } \\
\text { Plateau }\end{array}$ & Poor & $\begin{array}{c}5 \text { Martindale, } 7 \text { Early corner- } \\
\text { notch, } 7 \text { Expanding stem, } 2 \text { Bell, } \\
\text { 3 Nolan, } 1 \text { Travis }\end{array}$ & $\begin{array}{l}4720 \pm 170 \text { from } \\
\text { Area I }\end{array}$ & $\begin{array}{c}\text { Charcoal from } \\
\text { Area I at } 98.60,84 \\
\text { cmbs, "transition" } \\
\text { zone, Local Period } \\
5\end{array}$ & $\begin{array}{l}\text { Black and } \\
\text { McGraw } \\
1985\end{array}$ \\
\hline $\begin{array}{l}\text { Richard Beene, } \\
\text { 41BX831, Lower } \\
\text { Medina } \\
\text { Component, } \\
\text { Block G }\end{array}$ & $\begin{array}{c}\text { Northern } \\
\text { Coastal Plain }\end{array}$ & Poor & $\begin{array}{c}\text { Stemmed corner-notched, straight } \\
\text { based, Martindale/Bandy-like, } \\
\text { stemmed corner-notched, Baker, } \\
\text { Pandale-like }\end{array}$ & $\begin{array}{c}6700 \pm 110 \\
6985 \pm 65,6900 \\
\pm 70,7000 \pm 70 \\
6930 \pm 65\end{array}$ & $\begin{array}{l}\text { Dates on charcoal } \\
\text { from Features } 30 \\
43, \& 44,1 \text { on } \\
\text { burned tree }\end{array}$ & $\begin{array}{l}\text { Thoms and } \\
\text { Mandel } 2007\end{array}$ \\
\hline $\begin{array}{l}\text { Seminole Sink } \\
41 \mathrm{VV} 620\end{array}$ & $\begin{array}{l}\text { Southwestern } \\
\text { Edwards } \\
\text { Plateau }\end{array}$ & Good & $\begin{array}{l}1 \text { Early corner-notched (not } \\
\text { Martindale) }\end{array}$ & $\begin{array}{c}3 \text { dates, } 4671 \text { to } \\
5750\end{array}$ & $\begin{array}{l}\text { Dates on human } \\
\text { bones }\end{array}$ & Turpin 1988 \\
\hline $\begin{array}{l}\text { Skyline Shelter, } \\
\text { 41VV930 }\end{array}$ & Lower Pecos & Poor & $\begin{array}{c}14 \text { devils Triangular, } 7 \text { triangular } \\
\text { preforms, } 6 \text { Martindale/Bandy, } 4 \\
\text { Baker/Uvalde, } 3 \text { Gower, } 1 \text { Early- } \\
\text { barbed, } 2 \text { Golondrina }\end{array}$ & $5920 \pm 70$ & $\begin{array}{l}\text { Wood charcoal } \\
\text { from central } \\
\text { pit/hearth }\end{array}$ & $\begin{array}{l}\text { Turpin and } \\
\text { Bement } 1992\end{array}$ \\
\hline Sleeper, 41BC65 & $\begin{array}{l}\text { Central } \\
\text { Edwards } \\
\text { Plateau }\end{array}$ & Poor & $\begin{array}{l}6 \text { Uvalde, } 4 \text { Gower, } 1 \text { Bell, } 1 \\
\text { Martindale, } 4 \text { Unclassified }\end{array}$ & None & No charcoal & $\begin{array}{l}\text { Johnson } \\
1991\end{array}$ \\
\hline
\end{tabular}


Table 3-3. Martindale Site and Component Integrity Data (continued).

\begin{tabular}{|c|c|c|c|c|c|c|}
\hline $\begin{array}{l}\text { Site Name and } \\
\text { Number }\end{array}$ & Location & $\begin{array}{l}\text { Component } \\
\text { Integrity }\end{array}$ & Point Types Present & $\begin{array}{l}\text { Radiocarbon } \\
\text { Dates (B.P.) }\end{array}$ & $\begin{array}{l}\text { Comments on } \\
\text { Date }\end{array}$ & References \\
\hline $\begin{array}{l}\text { Varga, 41ED28, } \\
\text { Early Archaic } \\
\text { Component }\end{array}$ & $\begin{array}{l}\text { Southwestern } \\
\text { Edwards } \\
\text { Plateau }\end{array}$ & Poor & $\begin{array}{c}179 \text { points: } 32 \text { Gower, } 22 \\
\text { Martindale, } 21 \text { Bandy, } 15 \text { Group } \\
\text { 1, } 12 \text { Baker, } 12 \text { Merrell, } 7 \text { Group } \\
\text { 2, } 3 \text { Carrizo, } 1 \text { Pandale }\end{array}$ & 6280 to 5200 & $\begin{array}{c}17 \text { Dates: } 2 \\
\text { sediment, } 3 \\
\text { bones, } 4 \text { charcoal, } \\
5 \text { walnut shells, } 1 \\
\text { Rabdotus, } 2 \\
\text { charred seeds }\end{array}$ & $\begin{array}{l}\text { Quigg et al. } \\
2008\end{array}$ \\
\hline $\begin{array}{l}\text { Wilson-Leonard, } \\
\text { 41WM235, Early } \\
\text { Archaic Period }\end{array}$ & $\begin{array}{l}\text { Eastern } \\
\text { Edwards } \\
\text { Plateau }\end{array}$ & Poor & $\begin{array}{c}266 \text { points, } 20 \text { Angostura, } 30 \\
\text { bifurcate stem, } 17 \text { Hoxie, } 16 \\
\text { thrall, } 11 \text { Gower, } 30 \text { lanceolate, } \\
10 \text { Martindale, \& others }\end{array}$ & 8700 to 6000 & $\begin{array}{l}\text { Charcoal and } \\
\text { charred geophytes }\end{array}$ & $\begin{array}{l}\text { Collins et al. } \\
\qquad 1998\end{array}$ \\
\hline $\begin{array}{l}\text { Woodrow Heard, } \\
\text { 41UV88, } \\
\text { Geological Unit } \\
\text { lla }\end{array}$ & $\begin{array}{l}\text { Southwestern } \\
\text { Edwards } \\
\text { Plateau }\end{array}$ & Poor & $\begin{array}{l}\text { Baker, Bell, Early Triangular, } \\
\text { Martindale, Uvalde }\end{array}$ & $\begin{array}{l}15 \text { dates, } 6500 \\
\text { to } 4400\end{array}$ & $\begin{array}{l}\text { Charcoal dates, } \\
\text { not directly } \\
\text { associated }\end{array}$ & $\begin{array}{l}\text { Decker et al. } \\
\quad 2000\end{array}$ \\
\hline
\end{tabular}

= date rejected by Author, $\mathrm{NA}=$ not applicable 


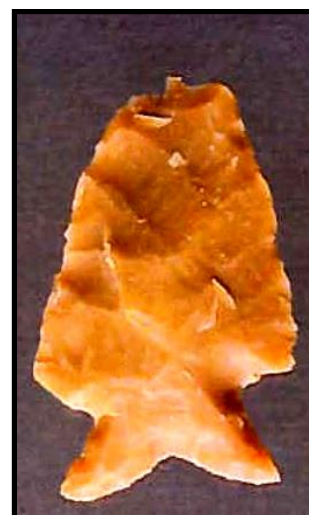

a)

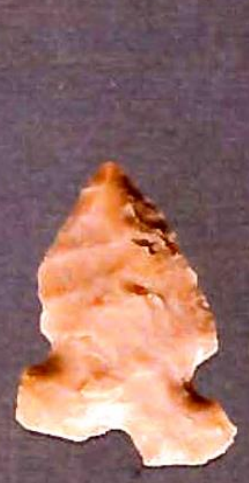

d)

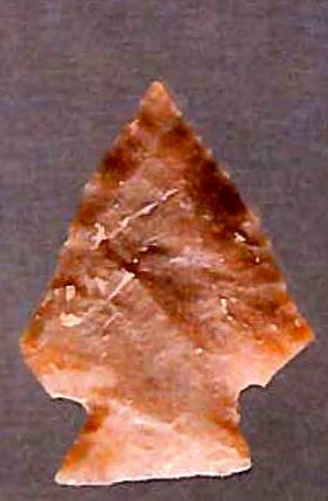

g)

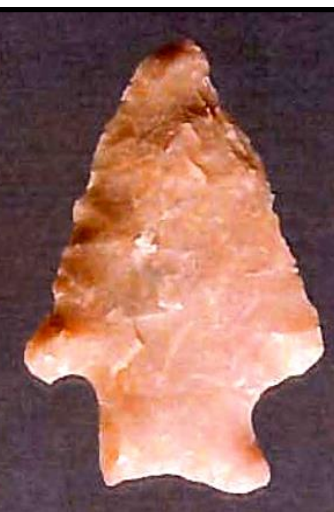

b)

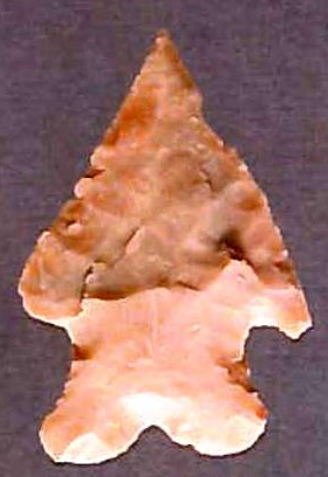

c)

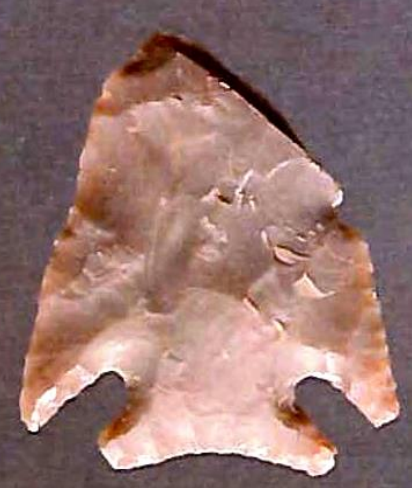

h)

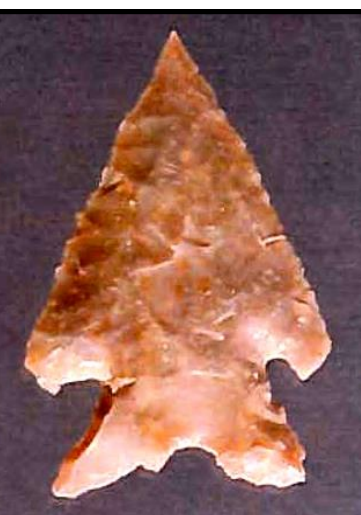

c)

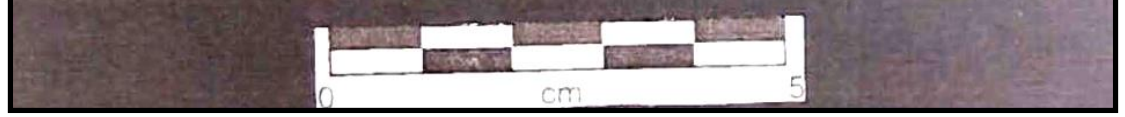

Figure 3-13. Selected Martindale points from the Gatlin site (from Oksanen et al. 2008, Figure 3). 


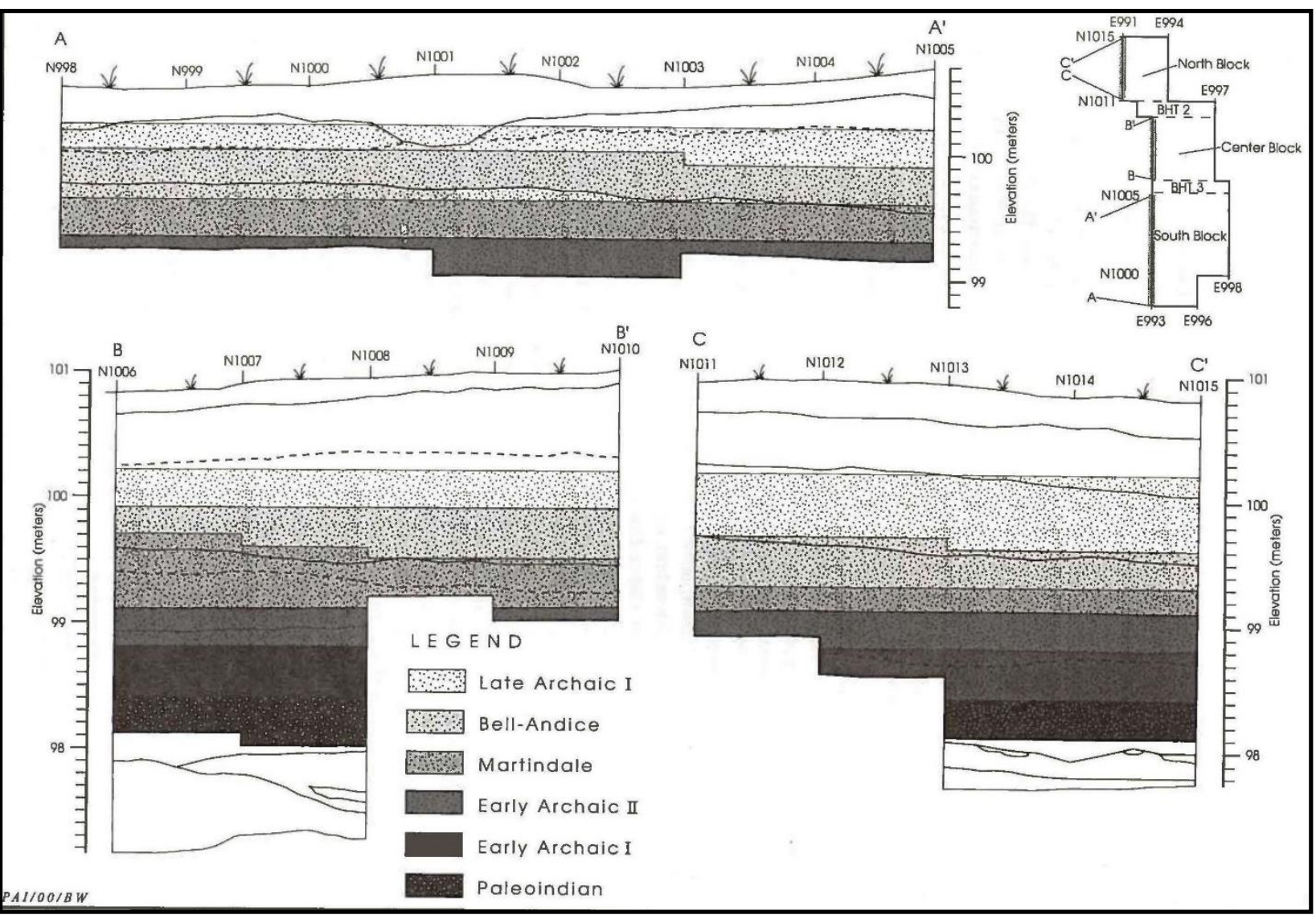

Figure 3-14. Stratified cultural components at the Cibolo Crossing site (from Kibler and Scott 2000, Figure 13).

Martindale points $(N=37)$, however, were from Occupation Zone 2, along with some 21 Gower, 5 Bandy, 10 Early Triangular, 1 Andice, 1 Baker, 2 Bell, and 1 Marcos, and 10 untyped points.

Very few excavated sites reveal good stratigraphic context with Martindale points in apparent isolated and/or discrete components. Even fewer sites or components provide radiocarbon dates in good association with Martindale points. Martindale components in six selected sites with relatively good context are presented below to provide assemblage characteristics and a broad general understanding of this interval.

The Cibolo Crossing site (41BX377) in Bexar County on the northern side of San Antonio has provided good stratigraphic context for Martindale points in a deep alluvial setting (Figure 3-14)
(Kibler and Scott 2000). The multiple components included materials from Paleoindian (ca. 10,000 B.P.) through Late Archaic (1400 B.P.) times in stratified zones. This includes a Martindale component below a Bell/Andice component and above two older Early Archaic components. The Martindale component was present in the upper deposits of geologic Unit 2, the $2 \mathrm{Bb}$ horizon in the north and south blocks, and the $2 \mathrm{Bb}-2 \mathrm{Bkb}$ in the central block in Levels 11 through 18. Unit 2 was light yellowish brown, silty clay, which apparently aggraded rapidly, as it lacked pedogenic development. Surface stability occurred during the Early to middle Holocene as evidenced by a welldeveloped soil formed on Unit 2, which was truncated at ca. 5000 to 6000 B.P. (Kibler and Scott 2000). The Martindale component encompassed a total area of $59 \mathrm{~m}^{2}$ or $21.2 \mathrm{~m}^{3}$. The associated Martindale artifact assemblages includes 2 
Martindale points (Figure 3-15), 1 Bandy point, 1 biface, 4 unifaces, 1 cobble tool, 7 edge-modified flakes, 1 ground/batter stone, 385 pieces of chert debitage, 1 tiny bone fragment, 1 freshwater mussel shell, $182 \mathrm{~kg}$ of burned rocks, and 5 burned rock features.

This assemblage was dated by three different mechanisms, with three charcoal radiocarbon dates yielding assays much too young and rejected by the authors. Mean A/I ratios from eight Rabdotus snail shells were employed to establish calendrical dates from 5953 to 6290 B.P. The diagnostic projectiles were also employed to estimate the use period (Kibler and Scott 2000). The authors interpret this event to represent a short-term occupation with diverse activities for hunter-gatherers with biface production as a major activity. Bifaces were reduced from fully corticated Edwards chert cobbles using a hard-hammer percussion tool, with limited soft-hammer or billet work. The five burned rock features represent general cooking activities, with one slab-lined, basin shaped oven or hearth (Feature 26) thought to represent plant cooking. The presence of Rabdotus snails is thought to indicate snails were a food resource. The authors speculate the season of use was late spring (Kibler and Scott 2000).

The Bering Sinkhole site (41KR241) in Kerr County yielded two Martindale points directly below one Uvalde point, to indicate Martindale points predate Uvalde points (Bement 1994). Both Martindale points were from Level 36 and another from Level 39, with both levels within the $1.2 \mathrm{~m}$ thick deposition Unit III (Levels 32-41). These two points were stratigraphically below all other points recovered. However, the two tentatively identified Martindale points have attributes that closely resemble Early Corner-notched forms, which had not yet been named. Level 37 was radiocarbon dated to $5840 \pm 190$ B.P. (Tx-6282), and Level 40 was radiocarbon dated to $6660 \pm 110$ B.P. (Tx$6831)$ and $6860 \pm 170$ B.P. (Tx-6526). These three

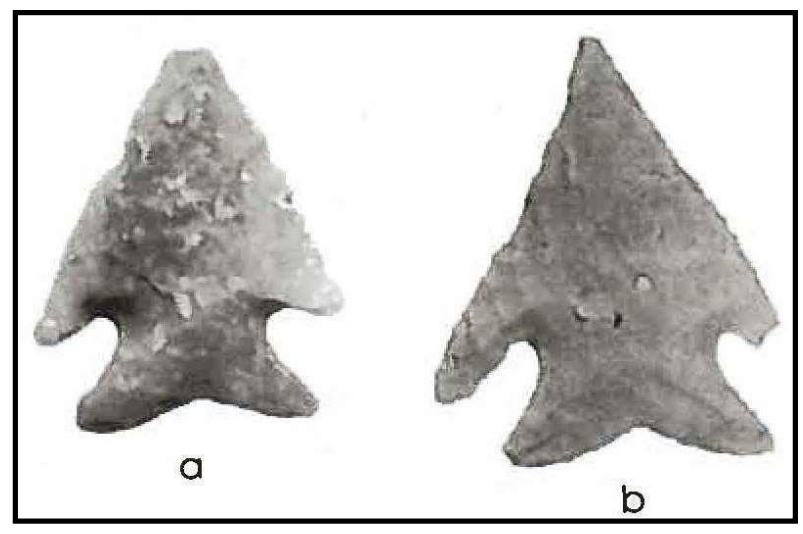

Figure 3-15. Martindale points from the Martindale component at the Cibolo Crossing site (Kibler and Scott 2000, Figure 22).

absolute dates provide a general time period for the Martindale point type and/or Early Corner-notched type attributed to the San Geronimo phase (8000 to 5000 B.P.). Associated tool assemblages were not in these burial contexts (Bement 1994).

The Landslide site (41BL85) in Bell County, specifically the Early Archaic Stratum V (Figure 316), is considered to have moderate integrity by Collins (1995, 2004). This stratum was described as 35 to $60 \mathrm{~cm}$ (1.1 to $2.0 \mathrm{ft}$.) thick, with abundant cultural debris and included; burned rocks, stone tools, mussel shells, and minimally two intact features (Features 3 and 9). Feature 3 was a concentration of flat lying burned rocks in no obvious pattern, a few mussel shells, and charcoalstained soil. The excavated portion was 90 -by-45 $\mathrm{cm}$. The rocks were between 5 and $15 \mathrm{~cm}$ in diameter. Feature 9 was a small concentration of burned limestone rocks in a semicircle. This concentration measured about 50-by-30 cm and contained rocks between 4 and $10 \mathrm{~cm}$ in diameter (Sorrow et al. 1967:9-11). Stratum V yielded at least four projectile point styles that included six Martindale (Figure 3-17), four Gower, four Gowerlike, and one Bell point, plus a couple of untyped expanding stem points (Sorrow et al. 1967). Also present were 19 bifaces, 18 scrapers, 1 uniface, 22 utilized flakes, 9 burins, and 13 burin spalls. 


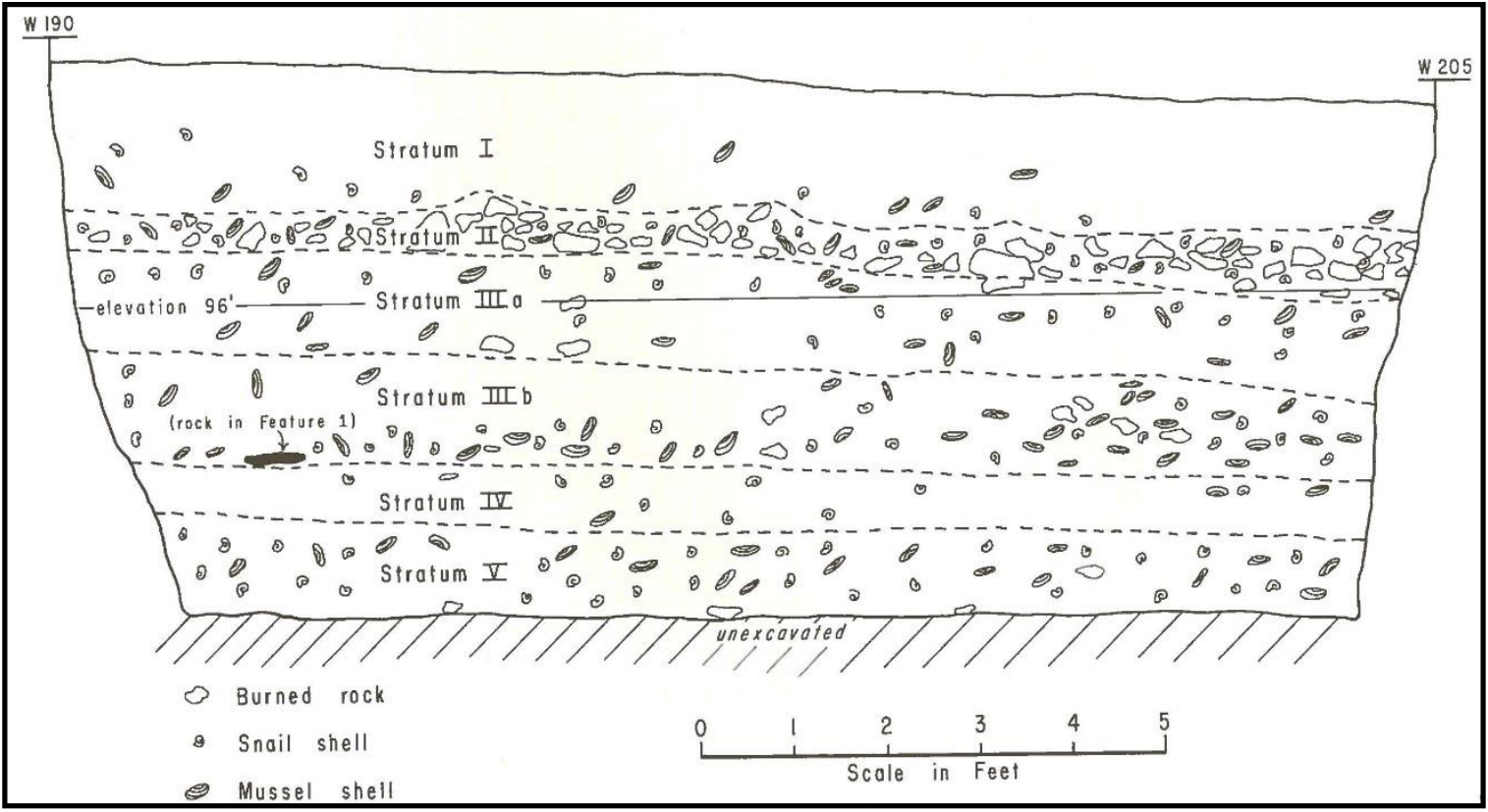

Figure 3-16. East-west profile along N190 at the Landslide site (from Sorrow et al. 1967:

Figure 4).

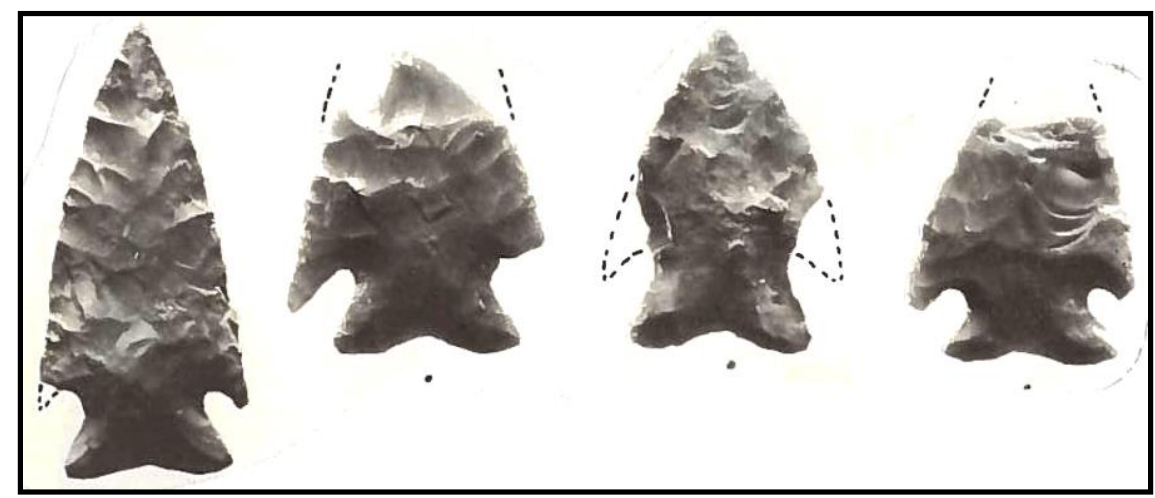

Figure 3-17. Martindale points from Stratum V at the Landslide site (41BL85) (from Sorrow et al. 1967:Figure 12).

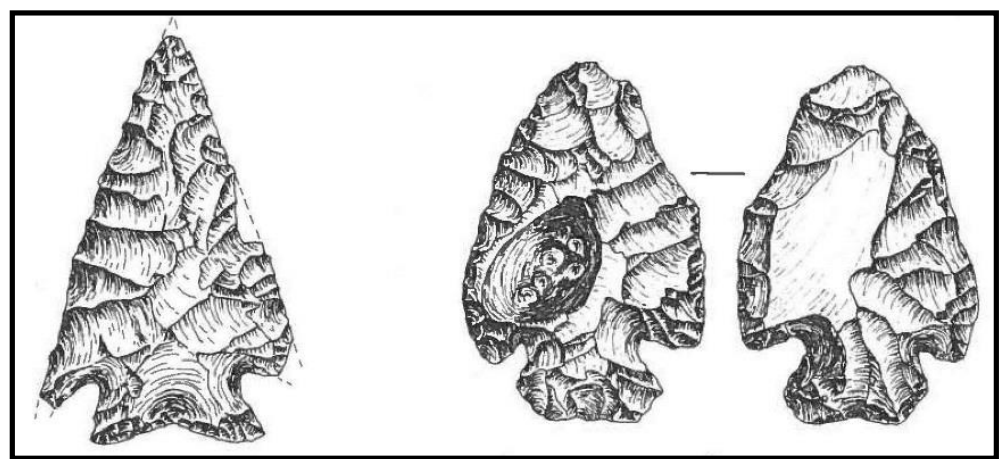

Figure 3-18. Martindale points from the Camp Pearl Wheat site (from Collins et al. 1990: Figure 19). 


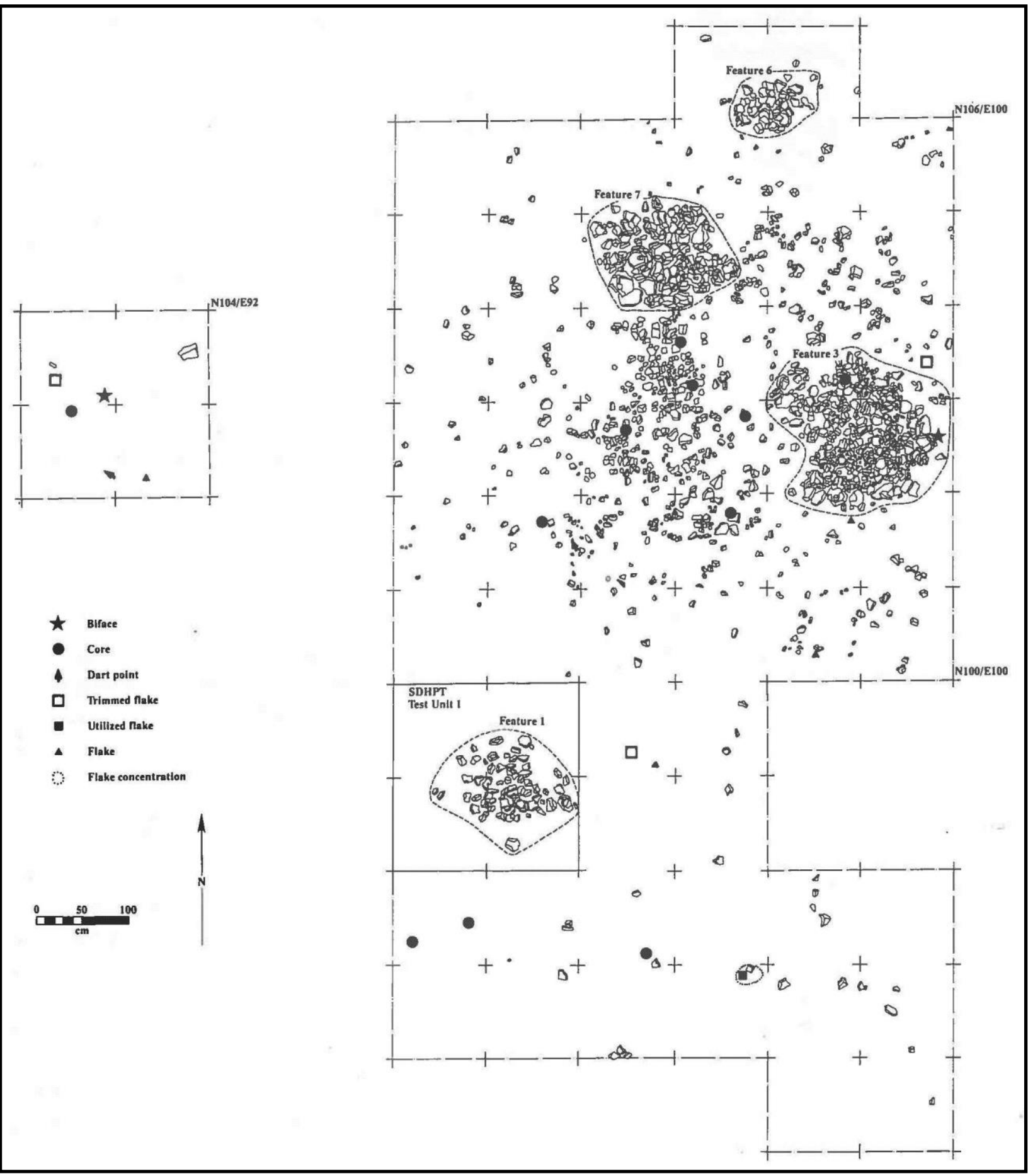

Figure 3-19. Plan map of south block depicting horizontal distribution of cultural features at the Camp Pearl Wheat site (from Collins et al. 1990:Figure 19). 
The Camp Pearl Wheat site (41KR243), also in Kerr County in the Edwards Plateau, yielded three Martindale points and two Uvalde points from 81 $\mathrm{m} 2$ in what Collins $(1995,2004)$ lists as a high integrity site for the Martindale-Uvalde interval (Figure 3-18). No radiocarbon dates were directly associated with this component, as vertebrate faunal, mussel shells and other organic remains were absent.

Six burned rock features were present, and appeared as circular or slightly ovate, and ranged from 70 to $160 \mathrm{~cm}$ in diameter (Figure 3-19). Most appeared to have shallow pits on the basis of rock elevation differences, with no obvious pit outline of organic stains (Collins et al. 1990). If the recovered artifacts are all directly associated with the Martindale component, then 16 bifaces and 12 edge-modified flakes constitute the entire stone tool assemblage, with some 2,877 pieces of debitage and 14 cores and core fragments.

Organic residue analysis yielded low frequencies of residues on burned rocks and stone tools. Residues on burned rocks indicated more meat processing than vegetable matter (Collins et al. 1990:84). The edge-modified flakes, however, yielded mostly residues from plants, in contrast to faunal residues on a biface and points. The authors interpret the remains to represent "a single array of human activities" (Collins et al. 1990:90). They also state the apparent 'meagerness' of the recovered nonperishable assemblage reflects a short-term utilization. The tasks represented included knapping activities directed towards production of bifaces, with unifaces representing specialized tasks (Collins et al. 1990:90). This is the first instance where the term 'interval' was proposed over the term 'phase' as a more appropriate term as it is culturally neutral.

The Hawes site (41WM56) in adjacent Williamson County is a stratified multicomponent site with data presented by cultural time and stratigraphic units (Peter et al. 1982). Only three charcoal dates were obtained, with the oldest being $3750 \pm 90$ (UGa2473) B.P. from Feature 14b in Level 8 (6 inch levels) in Area C. Level 8 contained Bulverde, Travis, and Nolan point styles. Area $\mathrm{C}$ yielded a Martindale point stratigraphically below these other points, to indicate an earlier age. Also in Area C were three small, well-defined burned rock features (Features 16, 17, and 20) in Levels 11 and 12, within an area of $15 \mathrm{~m} 2$ attributed to the late Early Archaic San Geronimo phase. Charcoal flecking was present in at least Features 16 and 17. None revealed prepared basins or burned earth. Although a Nolan point was in Feature 16, a Martindale point was from a level above these three hearths to create uncertainty the three features belong to the Nolan component. The authors believe the low density of associated cultural materials next to the three hearths indicates a short-term occupation and cooking/heating with small burned rock features. The Early Archaic San Geronimo phase bone assemblage, approximately 30 percent of which was burned, included deer (17.6\%), pronghorn $(0.6 \%)$, cottontail (7.0\%), jackrabbit (1.5\%), gopher $(7.0 \%)$, woodrat $(2.7 \%)$, various rodents (23\%), snake $(12.0 \%)$, turkey $(0.6 \%)$, reptiles (28.2\%), and fish (10.6\%), documenting environmental information and a diverse subsistence base (Yates 1982:15-77). This component indicates small, circular hearths without formal basins were potentially associated with the Martindale point.

In the Lower Pecos region, excavations at the Wroe Ranch site (41TE307) rock shelter in Terrell County of western Texas yielded 28 cultural features with 9 hearths or ash pits, 18 grass-lined basins or pads, and 1 rock alignment, plus 3 individual burials (Turpin 1998). Feature 21, a small $45 \mathrm{~cm}$ diameter prickly pear and grass-lined, basin-shaped pit on bedrock, yielded $\delta^{13} \mathrm{C}$ corrected prickly pear radiocarbon date of $6400 \pm 180$ B.P. (TX-9104) and a sotol stalk $\delta^{13} \mathrm{C}$ corrected date of $5730 \pm 70$ B.P. (TX-9180). A Martindale dart point (Figure 3-20) was on bedrock adjacent to Feature 


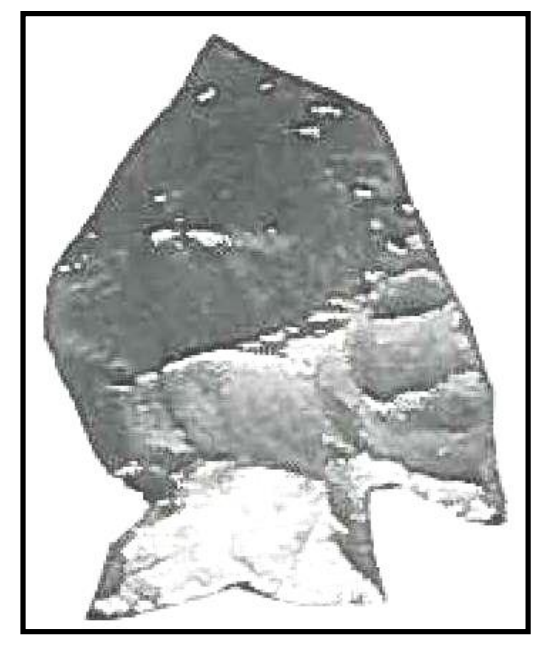

Figure 3-20. 'Martindale' point on bedrock next to fiber Feature 21 at Wroe Ranch shelter (from Turpin 1998:Figure 12).

21, although not demonstrably associated with Feature 21 (Turpin 1998). Although the association is not conclusive, the preserved organic feature provides evidence these feature types and multiple desert plants were utilized in the region beginning roughly 6400 years ago. Dry rock shelters such as this provide organic materials not otherwise preserved at open air sites. It also reveals occupation of arid environmental regions by these groups.

\subsubsection{Chronological Age Assessment}

Radiocarbon dates directly associated with Martindale points are rare as are Martindale points. In the few instances where charcoal was recovered and dated, as in the Martindale component at the Cibolo Crossing site, the three derived charcoal dates are all less than 2500 B.P. These results are much too young for the component assemblage, thus intrusive and rejected (Kibler and Scott 2000:61). Less than 15 dates from six different excavated sites are available. In most instances the context and/or association is not tight (see Table 33 ), and these dates provide a broad time frame for Martindale points across central and southwestern Texas, without specifically documenting when they were actually in use. As indicated in Table 3-4, the existing radiocarbon range encompasses nearly 2,200 years, from ca. 6860 to 4671 B.P., excluding the soil humate date from the Camp Pearl Wheat site. However, outliers within this group probably exist.

Too few dates from good enough context are available to identify outliers and potential age differences between the different regions. Curiously, the authors, in discussing a rather large Martindale point assemblage $(N=36)$ from subsurface context at Woodrow Heard, state "there is no evidence the site's Martindale points are older than 5000 B.P." (Decker et al. 2000:265). Although they may not have had any direct evidence, they present no convincing evidence to indicate Martindale points are younger than 5000 B.P.

At the Gatlin site (41KR621), a Martindale and two untyped points were from the $10 \mathrm{~cm}$ thick level in which Feature 22 was present (Houk et al. 2008). This burned rock feature in Occupation Zone 2 was radiocarbon dated on charcoal to $5260 \pm 50$ B.P. (Beta-206119). Because the materials were mixed in broad zones, it is unclear if Feature 22 truly is associated with the Martindale point since the point was not in the feature.

The Devil's Mouth site (41VV188) in Val Verde County had extremely deep deposits plus excellent stratigraphy, but no projectile points identified as Martindale/Early Corner-notched points (Johnson 1964). Paleoindian points (Golondrina) were at the bottom and 'Early Barbed', which Prewitt calls Western Bell points, were generally from levels with Bulverde points. The latter were below the Pandale points The Skyline Shelter site (41VV930), also in the Lower Pecos, exhibited deep, stratified deposits with only six Bandy and/or Martindale points, all from Levels 25 through 28. They occurred in the same levels as Gower, Early Barbed, Devil's Triangular, and possibly others, so context and point identifications may be questionable (Turpin and Bement 1992). Sites with 
Table 3-4. Radiocarbon Dates Generally Associated with Martindale Points.

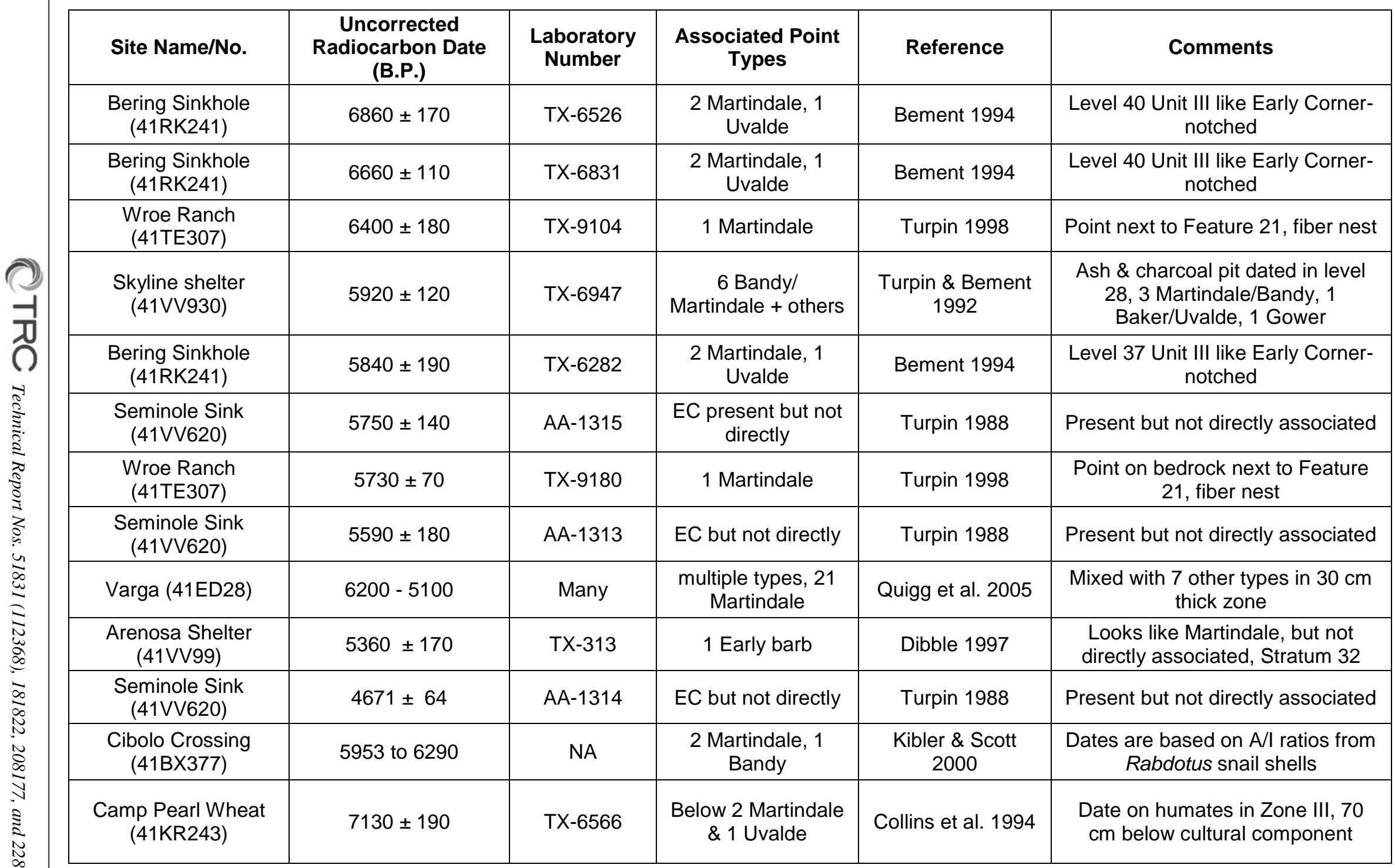


Table 3-4. Radiocarbon Dates Generally Associated with Martindale Points (continued).

\begin{tabular}{|c|c|c|c|c|c|}
\hline Site Name/No. & $\begin{array}{c}\text { Uncorrected } \\
\text { Radiocarbon Date } \\
\text { (B.P.) }\end{array}$ & $\begin{array}{l}\text { Laboratory } \\
\text { Number }\end{array}$ & $\begin{array}{l}\text { Associated Point } \\
\text { Types }\end{array}$ & Reference & Comments \\
\hline $\begin{array}{c}\text { Cervenka } \\
(41 \text { WM267) }\end{array}$ & $4970 \pm 90$ & Tx-3684 & $\begin{array}{l}\text { No diagnostics } \\
\text { associated }\end{array}$ & Peter et al. 1982 & $\begin{array}{l}\text { Charcoal from hearth Feature 19, } \\
\text { part of San Geronimo component }\end{array}$ \\
\hline 41WM73 & $5285 \pm 725$ & Uga-2482 & $\begin{array}{l}\text { Bulverde, Nolan, } \\
\text { Groups } 2\end{array}$ & Peter et al. 1982 & $\begin{array}{l}\text { Burned rock midden, charcoal from } \\
\text { the Clear Fork phase stratum } 6\end{array}$ \\
\hline $\begin{array}{l}\text { Halls Cave } \\
(41 \mathrm{KR} 474)\end{array}$ & $4850 \pm 130$ & $T x-6413$ & $\begin{array}{c}1 \text { Martindale, } 2 \\
\text { Uvalde }\end{array}$ & Toomey 1993 & $\begin{array}{l}\text { Date on charcoal from hearth at } \\
\text { same level as Martindale point }\end{array}$ \\
\hline \multicolumn{6}{|c|}{$\mathrm{EC}=$ Early Corner-notched } \\
\hline
\end{tabular}


deep, stratified deposits containing Martindale points are nearly absent, with extremely low frequency. Consequently, specific age of Martindale points remains unknown, as is good context from which their specific age can be derived.

\subsubsection{Subsistence Resources}

Direct subsistence data is nearly nonexistent in most Early Archaic components because of poorly preserved organic materials (Table 3-5). In 1981 Prewitt proposed hunting and gathering, with an emphasis on gathering, was the subsistence strategy for the period between about 6000 and 5000 B.P. However, lack of single component contexts with only Martindale points inhibits clear understanding of subsistence patterns for those populations even if preservation was good.

Data from the broader Early Archaic period provides a skewed picture of past subsistence patterns. Organic materials such as bones are rarely preserved or recovered, with only a few isolated exceptions. When vertebrate remains were recovered from an early occupation zone, researchers often fail to address the potential for incorporation of natural faunal remains into archeological deposits. However, deer, various small animals, turtles, birds, and fish are all indicated as probable resources by Collins (1995, 2004). An example of fish and turtle comes from the San Geronimo phase (6000 to 7000 B.P., Prewitt 1985) at 41WM57, on the eastern margin of the Edwards Plateau, where a burned catfish pectoral spine and burned turtle shell were from Area G (Yates 1982:15-108). Bison, deer, cottontail, jackrabbit, dog (sp.), rodent, snakes, turtle, birds and three different species of fish (gar, catfish, bass/perch) were represented in the San Geronimo phase at the Cervenka site (41WM267) in the Blackland Prairie (Yates 1982:15-186 through 15-198). Burned bones of deer, rabbit, turtle, cotton rat, fish, and snake were identified, which supports their cultural use at the Cervenka site (Yates 1982:15-194). At the Wilson-Leonard site, burned taxa, supporting cultural use, include turtle, rabbit, carnivore, and deer/antelope (Collins et al. 1998). A minimum of three bison were processed during at least one Early Archaic event radiocarbon dated between 8400 and 6500 B.P. at the Lubbock Lake site in northwest Texas (E. Johnson 1987). That discovery confirms bison were present in Texas at that time, and potentially in central Texas as well.

Deer and turtle bones were present in Martindale components, in stratigraphic association with the Martindale and Uvalde points, at the Bering Sinkhole site (41RK241, Bement 1994:82) and deer elements dominated in the lowest levels associated with 11 Uvalde/Martindale points at the Panther Springs site (41BX228, Black and McGraw 1985).

Mussel and snail shells are common in many Early Archaic components such as the Landslide site (Sorrow et al. 1967), the Cibolo Crossing site (Kibler and Scott 2000), the Berdoll site (Karbula et al. 2011), and the Sleeper site (Johnson 1991). Only a single mussel shell, however, was from the $59 \mathrm{~m}^{2}$ excavated at the Cibolo Crossing site (Kibler and Scott 2000), and no mussel shells were from the $81 \mathrm{~m}^{2}$ excavated at the Camp Pearl Wheat site (Collins et al. 1990). Therefore, mussels were apparently not a substantial food resource at all sites. Kibler and Scott (2000) suggest the Rabdotus snails were a human food resource, although they provided no direct supporting evidence.

Analyses of organic residues in burned rocks from the Camp Pearl Wheat site indicate plant and animal processing during this period (Collins et al. 1990). Direct evidence of specific plants utilized comes from 10 charred wild hyacinth bulbs from a burned rock oven (Feature 181) in the Early Archaic strata at the Wilson-Leonard site, with bulbs directly dated to 7870 B.P. and 8420 B.P. (Collins et al. 1998). The Angostura component at the Richard Beene site yielded charred sotol or yucca leaf bases dated to 8000 B.P. (Thoms et al. 
Table 3-5. Early Archaic and Martindale Subsistence Data.

\begin{tabular}{|c|c|c|c|c|c|c|c|c|c|c|c|}
\hline & 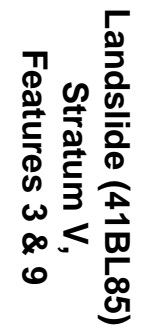 & 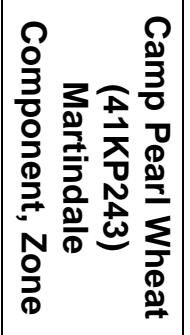 & 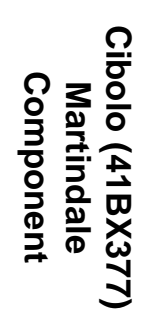 & 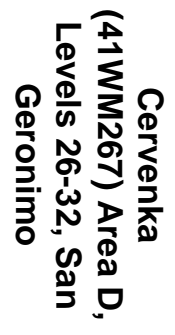 & 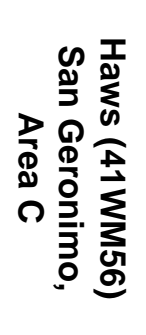 & 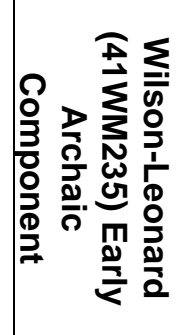 & 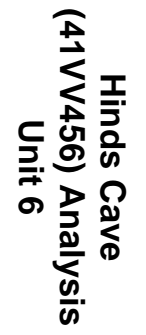 & 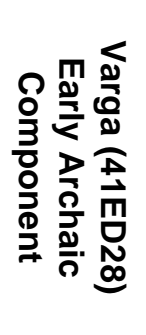 & 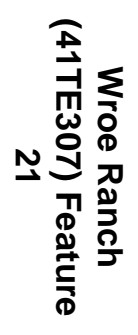 & 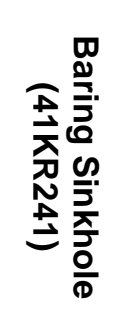 & $\frac{\overrightarrow{-1}}{\frac{0}{\omega}}$ \\
\hline Context & $\begin{array}{l}\text { Fair- } \\
\text { Poor }\end{array}$ & Fair & Good & Good & Good & $\begin{array}{l}\text { Fair- } \\
\text { Poor }\end{array}$ & $\begin{array}{l}\text { Fair- } \\
\text { Good }\end{array}$ & Poor & Poor & Fair & \\
\hline $\begin{array}{c}\text { Radiocarbon } \\
\text { Age (B.P.) }\end{array}$ & None & $\begin{array}{c}<7300 \pm \\
190\end{array}$ & $\begin{array}{l}5900- \\
6300\end{array}$ & 4970 & & $\begin{array}{l}8800- \\
6000\end{array}$ & $\begin{array}{l}6500- \\
6100\end{array}$ & $\begin{array}{c}5200- \\
6300\end{array}$ & $\begin{array}{c}6400 \pm \\
180\end{array}$ & $\begin{array}{c}6860 \pm \\
170, \\
6660 \pm \\
110 . \\
5840 \pm \\
190\end{array}$ & \\
\hline $\begin{array}{c}\text { Faunal } \\
\text { Assemblage }\end{array}$ & None & None & None & 3,240 & 135 & 796 & Yes & 229 & None & & \\
\hline Bison & & & & 15 & & 11 & & & & & 26 \\
\hline Deer & & & & 70 & 14 & & & 2 & & 1 & 87 \\
\hline Deer/Pronghorn & & & & 24 & & 15 & & & & & 39 \\
\hline Medium mammal & & & & 5 & 7 & 133 & & & & & 145 \\
\hline Small mammal & & & & 13 & 6 & 2 & & 2 & & & 23 \\
\hline Carnivore & & & & 40 & 1 & 2 & & & & & 43 \\
\hline Rabbit & & & & 87 & 9 & 5 & & & & & 101 \\
\hline Turtle & & & & 68 & 17 & 19 & & & & 1 & 105 \\
\hline Rodent & & & & 195 & 24 & & & & & & 219 \\
\hline Fish & & & & 9 & 27 & 5 & & & & & 41 \\
\hline Mussel & Yes & & Yes & Yes & & & & 7 & & Yes & 7 \\
\hline Bird & & & & 16 & & Yes & & & & 2 & 18 \\
\hline
\end{tabular}


Table 3-5. Early Archaic and Martindale Subsistence Data (continued).

\begin{tabular}{|c|c|c|c|c|c|c|c|c|c|c|c|}
\hline & 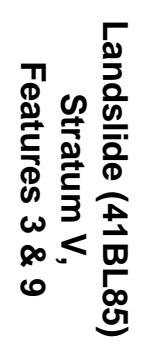 & 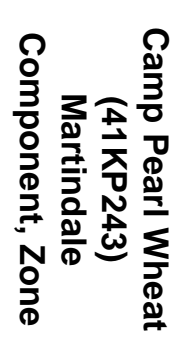 & 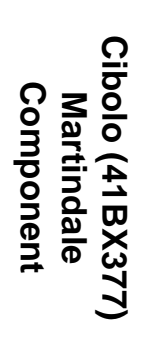 & 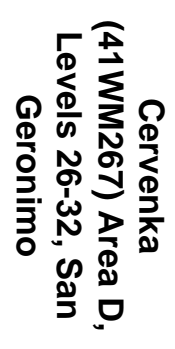 & 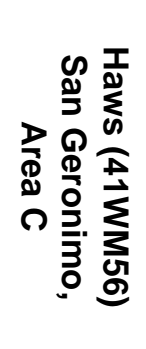 & 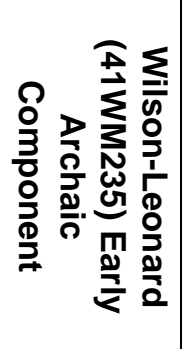 & 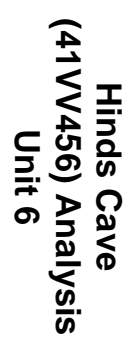 & 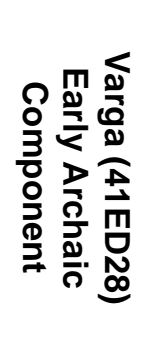 & 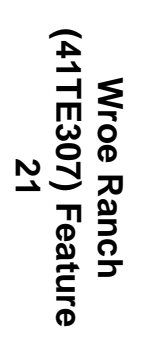 & 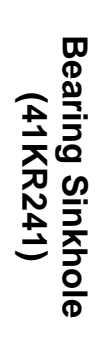 & $\frac{\vec{D}}{\frac{0}{\omega}}$ \\
\hline Plant Remains & None & None & None & Sparse & Sparse & Sparse & Good & Sparse & Good & None & \\
\hline Prickly pear & & & & & & & Yes & Yes & Yes & & \\
\hline Grass & & & & & & & & & Yes & & \\
\hline Sotol & & & & & & & Yes & & Yes & & \\
\hline Walnut & & & & & & Yes & Yes & Yes & & & \\
\hline Agave/lechuguilla & & & & & & & Yes & & & & \\
\hline Onion/geophytes & & & & & & Yes & Yes & & & & \\
\hline Oak nuts & & & & & Yes & & Yes & & & & \\
\hline Mesquite seeds & & & & & & & Yes & & & & \\
\hline Hackberry seed & & & & Yes & & & & & & & \\
\hline
\end{tabular}


1996). The Armstrong site in the Blackland Prairie near San Marcos yielded lily bulb fragments from a feature in Occupation Zone 3 dated to 6780 B.P. (Schroeder 2002:23). The Berdoll site, directly next to site 41TV2161, also yielded multiple charred lily bulbs and charred acorns from a large earth oven (Feature 11) dated to approximately 7854 to 8103 B.P. (Karbula et al. 2011). Site 41TV540, also next to 41TV2161, yielded charred onion bulbs from three features (Dering 2011a). Apparently geophytes, specifically in the lily family, and onions were intensively exploited in the lower Onion Creek valley, which, given its name, should not be a surprise.

Analyses of organic residues in burned rocks from the Camp Pearl Wheat site indicate plant and animal processing during this period (Collins et al. 1990). Direct evidence of specific plants utilized comes from 10 charred wild hyacinth bulbs from a burned rock oven (Feature 181) in the Early Archaic strata at the Wilson-Leonard site, with bulbs directly dated to 7870 B.P. and 8420 B.P. (Collins et al. 1998). The Angostura component at the Richard Beene site yielded charred sotol or yucca leaf bases dated to 8000 B.P. (Thoms et al. 1996). The Armstrong site in the Blackland Prairie near San Marcos yielded lily bulb fragments from a feature in Occupation Zone 3 dated to 6780 B.P. (Schroeder 2002:23). The Berdoll site, directly next to site 41TV2161, also yielded multiple charred lily bulbs and charred acorns from a large earth oven (Feature 11) dated to approximately 7854 to 8103 B.P. (Karbula et al. 2011). Site 41TV540, also next to 41TV2161, yielded charred onion bulbs from three features (Dering 2011a). Apparently geophytes, specifically in the lily family, and onions were intensively exploited in the lower Onion Creek valley, which, given its name, should not be a surprise.

The limited data available from a few scattered Early Archaic components indicates the subsistence base was very broad, with different populations exploiting diverse riverine and land resources.
Should Martindale components be discovered with preserved organic remains, it should be anticipated a broad subsistence pattern would emerge.

\subsubsection{Cooking Processes}

Many Early Archaic sites have yielded diverse cooking features, burned rock hearths or ovens (e.g., Sleeper, Richard Beene, Wilson-Leonard, Woodrow Heard, Berdoll, Armstrong, and Cibolo Crossing) to indicate quantities of plant foods were cooked (Table 3-6). Collins (1998b) sees the Early Archaic period as the advent of large rock-lined earth ovens (Figure 3-21). Small basin hearths such as those from the Sleeper site (Johnson 1991), the Camp Pearl Wheat site (Collins et al. 1990), and site 41TG307 (Quigg et al. 1996), may reflect different cooking processes for different types of foods. The "baking heaps" at the Sleeper site are said to have been mostly one stone thick, generally elongated, lack organic stains, and have flat bottoms (Johnson 1991:49) rather than particularly suitable for use as ovens. Three cooking hearths at the Sleeper site are smaller than "baking heaps", lined with flat slabs, and slightly basin shaped (Johnson 1991:51).

Large burned rock mounds or middens have not been directly associated with Martindale points in central Texas, with a few possible exceptions of Martindale points from Cedar Park and San Gabriel 'mounds' in Williamson County (Schuetz 1957), and Middens B and $\mathrm{C}$ at the Greenhaw site (41HY29) with points labeled as "Provisional Type I"/Early Barbed (Weir 1979). Martindale points, however, were below large burned rock middens in a couple of instances, at 41CV413 (Quigg, Lintz, et al. 2011b), and Honey Creek (41MS32, Black 1997), minimally in a midden at 41CM211 (Wilder et al. 2003), and a midden in Area B at Culebra Creek (Nickels et al. 2001).

The five burned rocks features (Features 22, 23, 26, 33, and 35) discovered in the Martindale component at the Cibolo Crossing site and believed 
Table 3-6. Early Archaic and Martindale-Related Feature Frequency and Types.

\begin{tabular}{|c|c|c|c|c|c|c|c|c|c|c|c|c|}
\hline & & 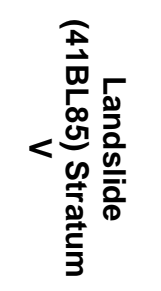 & 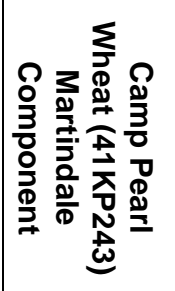 & 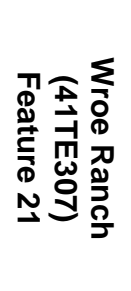 & 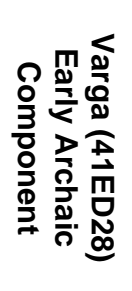 & 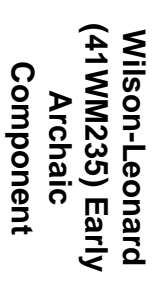 & 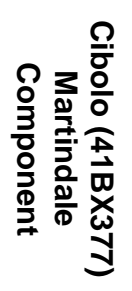 & 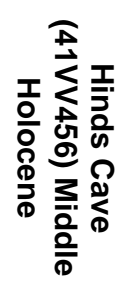 & 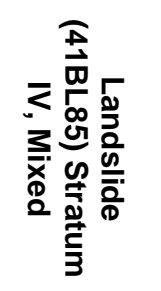 & 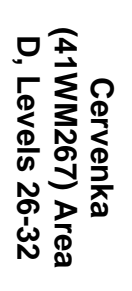 & 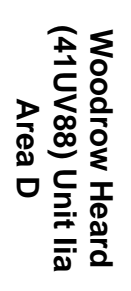 & $\begin{array}{l}\stackrel{-1}{\stackrel{0}{\Phi}} \\
\frac{\vec{\omega}}{\omega}\end{array}$ \\
\hline Cont & & Fair-Poor & Fair & Fair & Poor & Fair-Poor & Fair & Good & Fair-Poor & Good & Fair & \\
\hline $\begin{array}{l}\text { Radi } \\
\text { (B.P. }\end{array}$ & carbon Age & None & $\begin{array}{c}<7300 \pm \\
190\end{array}$ & $\begin{array}{c}6400 \pm \\
180\end{array}$ & $\begin{array}{l}5200- \\
6300\end{array}$ & $\begin{array}{l}8800- \\
6000\end{array}$ & $\begin{array}{l}5900- \\
6300\end{array}$ & $\begin{array}{l}6500- \\
6100\end{array}$ & $>3520$ & 4970 & $\begin{array}{l}6000- \\
6500\end{array}$ & \\
\hline Feat & re Frequency & 2 & 6 & 1 & 9 & 100 & 5 & 1 & 3 & 4 & 5 & 136 \\
\hline & $\begin{array}{l}\text { Burned rock } \\
\text { midden }\end{array}$ & & & & & & & 1 & & & & 1 \\
\hline & $\begin{array}{l}\text { Burned rock } \\
\text { sheet midden }\end{array}$ & & & & & & & & & & & 0 \\
\hline & $\begin{array}{l}\text { Burned rock } \\
\text { hearth }\end{array}$ & & 3 & & 2 & 14 & & & 3 & 1 & 4 & 27 \\
\hline & $\begin{array}{l}\text { Burned rock } \\
\text { dump }\end{array}$ & & 2 & & 2 & & 2 & & & & & 6 \\
\hline & $\begin{array}{l}\text { Burned rock } \\
\text { accumulation }\end{array}$ & & & & 5 & 71 & & & & & & 76 \\
\hline$\underset{0}{\stackrel{2}{1}}$ & $\begin{array}{l}\text { Burned rock } \\
\text { scatters }\end{array}$ & 2 & & & & 11 & 1 & & & & & 14 \\
\hline $\begin{array}{l}\sum_{\mathbb{N}}^{\bar{N}} \\
\text { L }\end{array}$ & $\begin{array}{l}\text { Stained/dark } \\
\text { sediment }\end{array}$ & & & & & 2 & & & & 1 & & 3 \\
\hline & $\begin{array}{l}\text { Burned rock } \\
\text { ring }\end{array}$ & & & & & 2 & & & & & & 2 \\
\hline & Fiber nest & & 1 & 1 & & & & & & & & 2 \\
\hline & $\begin{array}{l}\text { Burned rock } \\
\text { platform }\end{array}$ & & & & & & 1 & & & & & 1 \\
\hline & $\begin{array}{l}\text { Burned rock } \\
\text { oven }\end{array}$ & & & & & & 1 & & & & 1 & 2 \\
\hline & $\begin{array}{l}\text { Burned } \\
\text { clay/bone dump }\end{array}$ & & & & & & & & & 2 & & 2 \\
\hline
\end{tabular}




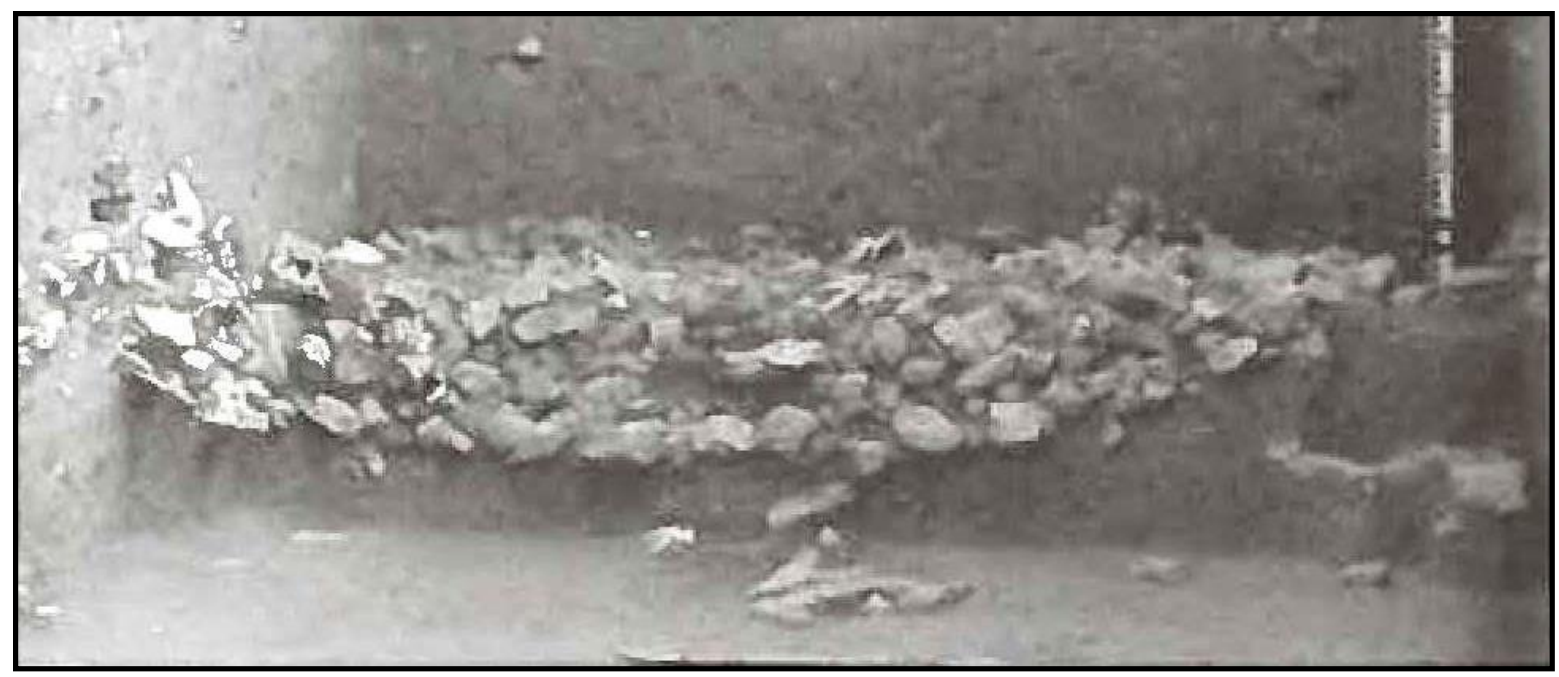

Figure 3-21. Photograph of earth oven profile of Feature 181 at the Wilson-Leonard site (from Collins et al. 1998b:237).

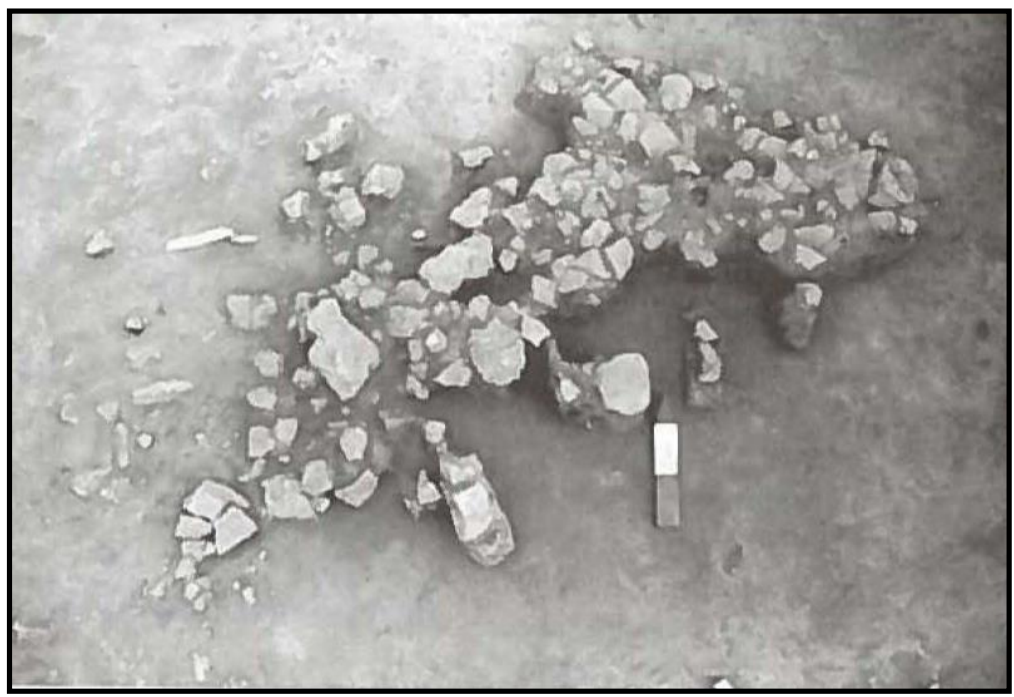

Figure 3-22. Photograph of Feature 22 from Martindale component at the Cibolo Crossing site (from Kibler and Scott 2000:Figure 24).

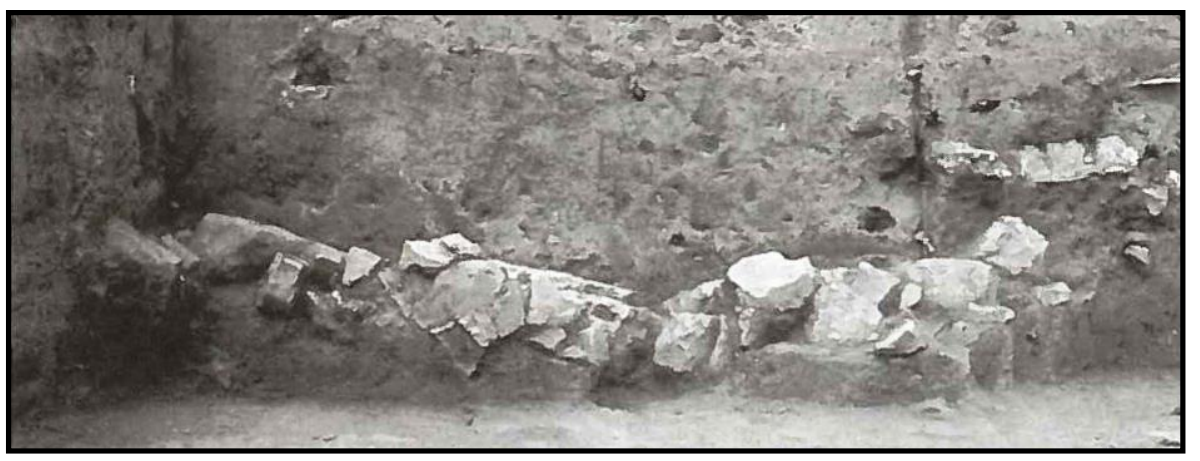

Figure 3-23. Profile photograph of Feature 26 from Martindale component at the Cibolo Crossing site (from Kibler and Scott 2000:Figure 26). 
to represent diverse cooking facilities, are discussed here as informative of Martindale feature characteristics. Feature 22 was a large, 200-by-380 $\mathrm{cm}$, flat concentration of 315 burned rocks $(40 \mathrm{~kg}$ ) and yielded 24 pieces of lithic debitage, an estimated 700 Rabdotus shells, tiny scattered charcoal flecks, but lacked charcoal staining (Figure 3-22). Fifteen burned rocks from the concentration were subjected to paleomagnetic analysis. Results indicate the rocks were heated to low temperatures $\left(150\right.$ to $\left.300^{\circ} \mathrm{C}\right)$, with all samples displaying minimally two temperature components, with 40 percent exhibiting three temperature components. This feature was interpreted for use with Rabdotus snails (Kibler and Scott 2000).

Feature 23, was a single layer of flat lying, scattered burned rock over an area 100-by-140 cm. No artifacts or charcoal were recovered. The 48 rocks $(11.25 \mathrm{~kg})$ were highly fragmented. The function was not known.

Feature 26 was a large, circular concentration with three layers of burned rocks over an area at least 65by-119 cm (Figure 3-23). The 132 rocks (32.5 kg) were very angular, fractured and broken in situ, with two large slabs in the southeastern part. No stained or oxidized sediment or charcoal was apparent. The profile revealed a shallow basin, potentially slab-lined, potentially an oven. Feature 33 was another flat lying burned rock concentration in a dispersed circular area that measured 55-by-56 $\mathrm{cm}$. No stained or oxidized sediment or charcoal was observed. This was probably a discard pile.

Feature 36 was a small cluster of eight burned rocks $(2.5 \mathrm{~kg})$ in a semicircular arrangement. No stained or oxidized sediment or charcoal was observed. Paleomagnetic analysis on two rocks reveled low, ca. 200 to $250^{\circ} \mathrm{C}$ heating temperatures, with one to three temperature components. This feature probably represented a discard pile (Kibler and Scott 2000).
Six burned rock features were attributed to the Early Archaic period at the Camp Pearl Wheat site. These appeared as circular to slightly ovate in shape and ranged from 70 to $160 \mathrm{~cm}$ in diameter (see Figure 3-19). Some exhibited evidence of dispersal by natural and cultural causes. Most revealed elevational differences to indicate they had shallow pits. Selected feature rocks were subjected to archeomagnetic analysis, which indicates rocks were heated to various temperatures, some in the low range of 350 to $375^{\circ} \mathrm{C}$, others to $550^{\circ} \mathrm{C}$, and a few in excess of $600^{\circ} \mathrm{C}$. Most sampled feature rocks apparently cooled in place to indicate their in situ nature (Collins et al. 1990). Organic chemical residue studies were also conducted on selected feature rocks. When residues were detected, most yielded both plant and animal products.

As more single Martindale components are encountered, it is anticipated the diversity of associated feature types will increase, and other cultural aspects will become clearer. Collins (1995, 2004) sees this as a period of specialized cooking appliances, which he projects as antecedents to the larger burned rock middens. Those specialize appliances may include the earth ovens, which have not yet been identified in Martindale components.

It is apparent small burned rock features are associated with Martindale points and they are generally interpreted to reflect in situ cooking of multiple plant and animal products, plus discarded cooking rocks. No organic stained pits or nonrock hearth features have been identified. Near absence of organic matter (e.g., bones, seeds, charcoal, etc.), especially as it relates to subsistence activities, combined with limited microfossil studies have not permitted food types to be clearly identified in these features. Poor preservation accounts for most absence, although greater effort is required to focus on the recovery and analyses of microfossils. More focused research is needed to identify potential differences in feature types/function. 


\subsubsection{Artifact Assemblage}

Most excavated components and zones that yielded Martindale points also contained multiple other point types to reflect mixed or palimpsests events. Consequently a clear understanding of what constitutes a pure assemblage directly associated with Martindale points is absent. Generally, excavated Early Archaic assemblages often contain chipped stone tools such as bifaces, scrapers, and utilized flakes. Only the undated and probable palimpsest occupations at the Sleeper site yielded ground stone tools (Johnson 1991). Given the poor context of those ground stone tools, it is unclear what populations employed them. Decker et al. (2000:290) stated ground stone tools do not appear characteristic of the Early Archaic (6500 to 4400 B.P.) at the Woodrow Heard site in the southwestern Balcones Canyonlands. At the Wilson-Leonard site, minimally 16 artifacts are listed as ground stone, with five others as metates pieces (Collins et al. 1998:217). Guadalupe tools, Clear Fork unifaces/gouges, edge-modified flakes, burins, and burin spalls, are all thought to be part of the Early Archaic tool assemblages. No apparent unique or specialized stone tools have been identified for this period, with the possible exception of pieces esquillees at the WilsonLeonard site (Collins et al. 1998:217). Three Waco sinkers and grooved stones are attributed to the Early Archaic at the Wilson-Leonard site, which Collins (1998b:282) suggest may represent net sinkers. Until single components with good context are encountered and reported in detail, the specific Martindale assemblage cannot be clearly defined. Although the context of many components with Martindale points appears mixed, no indication of extensive trading of nonlocal items or use of nonlocal lithic materials is evident.

Root et al. (2008) conducted functional analysis on 21 Martindale points from the Gatlin site. In summary, all except one were interpreted to have functioned as projectile points, with the exception likely having functioned as a biface in a cutting action. The use-wear on this point indicates it was used to cut wood. Impact fractures occur on minimally 52 percent, with 29 percent having evidence of being resharpened and reused, thus multifunctional tools. Nearly 10 percent have beveled edges, with two displaying serrated blade edges still in apparently pristine condition. Sixtyseven percent reveal notch and/or base dulling to facilitate hafting. Forty-three percent exhibit wear related to cutting soft material, such as animal hide and tissue, with another nearly 20 percent employed on hard or stiff materials such as wood or antler. Nineteen percent also exhibit burin scars (Root et al. 2008). These observations provide a good indication of the utilization patterns of Martindale points.

The 37 Martindale points from the Woodrow Heard site include a potentially distinctive subtype/substyle as 16 percent exhibit alternate basal edge-beveling of the fishtail-shaped ears, whereas another 46 percent have unifacially beveled bases (Figure 3-24) (Decker et al. 2000). This may be a local characteristic, however, with so few Martindale points studied in detail from Texas it is unclear.

The stone tool technology is not well-known, as most lithic debitage and cores have not been intensively scrutinized, although lithic debris is frequent at many Early Archaic sites. General observations indicate cobble reduction and biface manufacture were conducted. However, exploitation strategies and material use have not been addressed.

\subsubsection{Settlement Patterns and Distribution}

Many researchers have postulated broad regional distribution of Early Archaic sites and materials. Many artifact forms from this period appear widely distributed across much of Texas, with the exception of the Big Sandy points. For example, Gower and Gower-like points and Guadalupe tools have come from central and southwestern parts of 

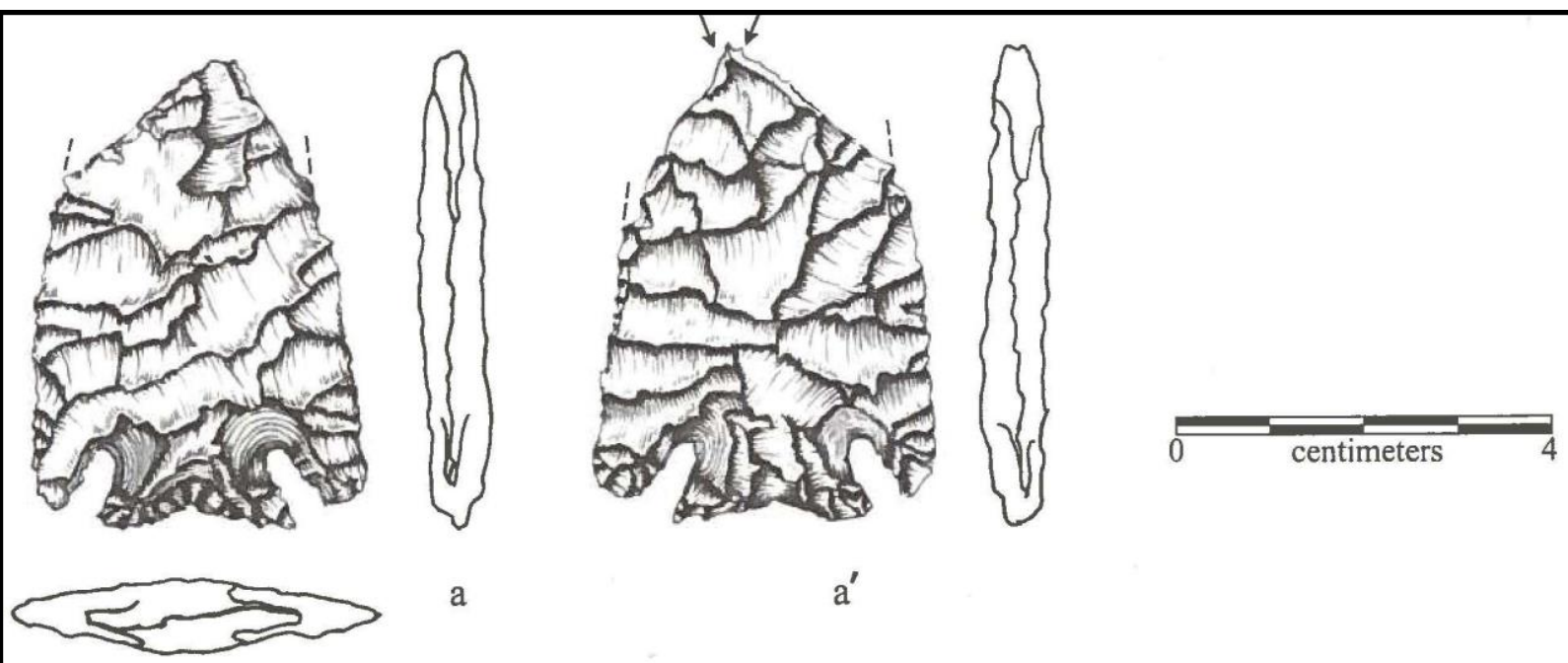

a
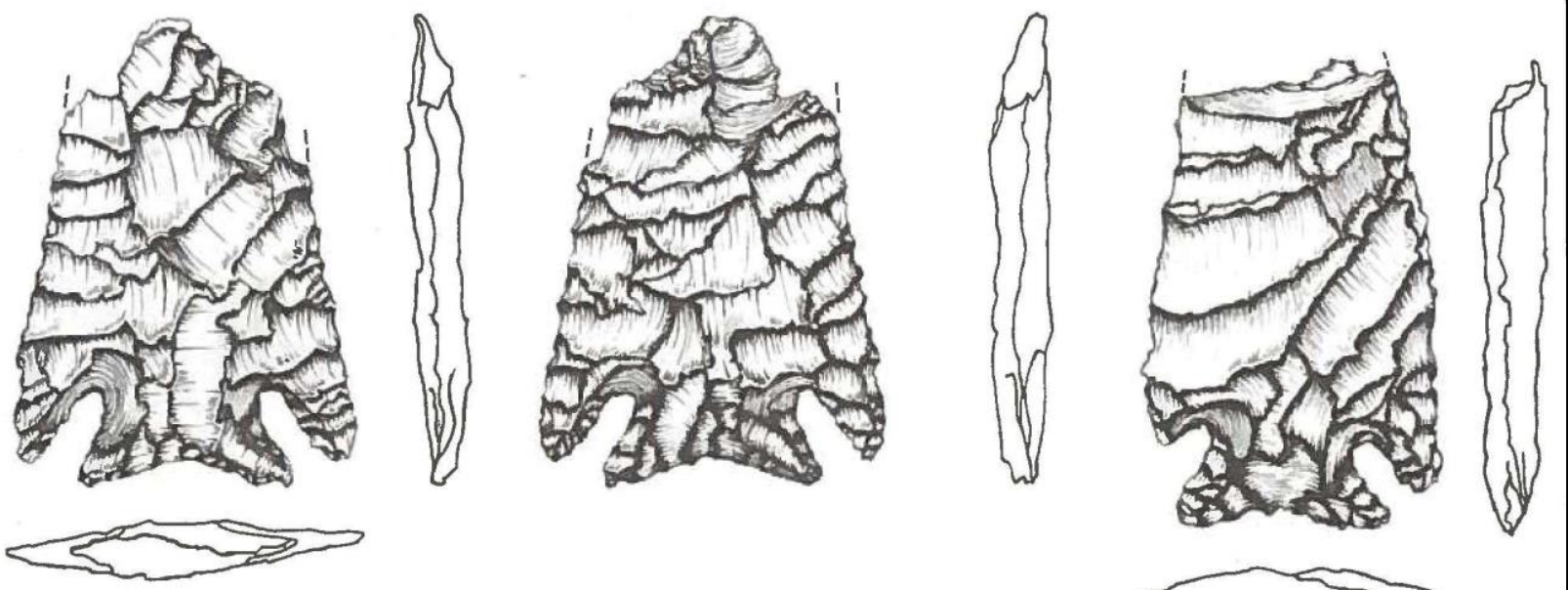

b

$b^{\prime}$
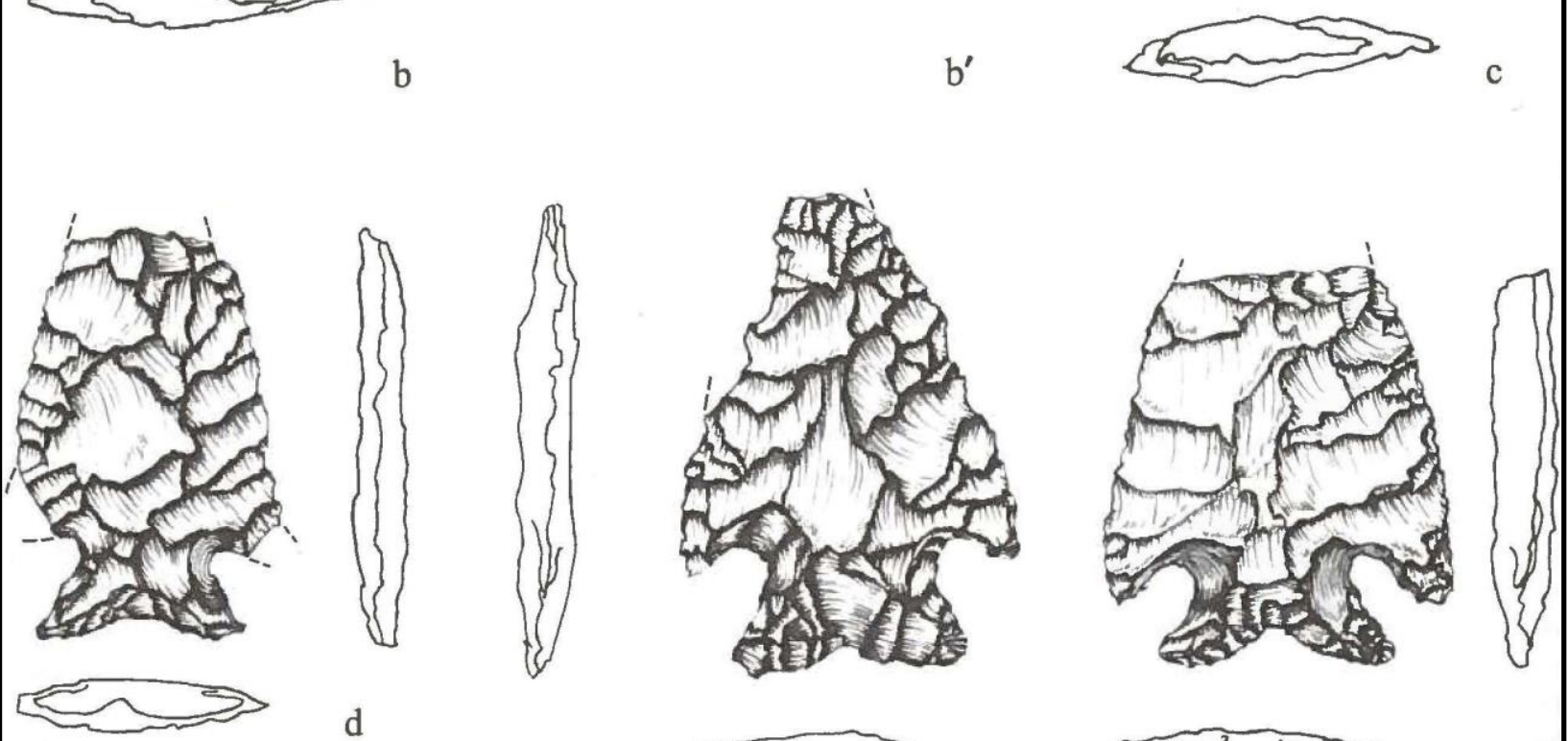

e
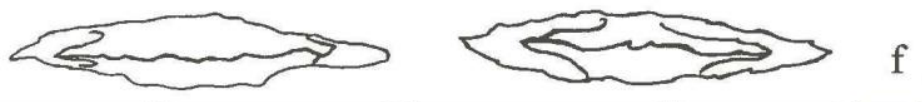

Figure 3-24. Martindale points from the Woodrow Heard site depicting impact fractures and beveled bases (Decker et al. 2000:Figure 184). 
Texas (McKinney 1981). Weir (1976a) postulated population density was low in central Texas and reflects small nomadic bands. In comparison to the previous Late Paleoindian period groups, mobility was apparently reduced. Using optimal foraging theory, Kibler and Scott (2000) speculate Early Archaic site distribution at Camp Bullis revolved around subsistence activities. In their site distribution analysis for Camp Bullis, Martindale site locations reflect concentrations in and along the Balcones Canyonlands fringe areas as groups employed a generalized foraging pattern. This is similar to the site pattern observed by McKinney (1981) for the earlier Gower points.

McKinney's (1981) findings revealed many known Early Archaic sites appear concentrated along the southern Edwards Plateau. This concentration might be linked to greater abundance of resources, including water, in this region at a time when the climate was somewhat dryer. In 1991, Johnson reviewed and discussed 11 important excavated Early Archaic sites distributed from the eastern edge of the Edwards Plateau around the Austin area, and westward across the Edwards Plateau to include the Lower Pecos region (Johnson 1991). The sites Johnson discussed form a 'crescent' shaped distribution pattern across an east-west section through central Texas. This distribution may or may not have real meaning, and may reflect a very biased sample of excavated sites. The two extreme ends of this crescent are in two different archeological regions, the Central Texas and Lower Pecos. The Big Hole site falls towards the northeast margin of the crescent, and provides a means of discussing and interpreting the findings from this site.

Prewitt (1995) documented Martindale points across 41 counties (Figure 3-25). Most were in central Texas and the Lower Pecos region, with occurrences in scattered counties north, south and northwest Texas. This dart point style appears under-represented from major excavated sites. For example, only four percent of the 263 identified dart points from Early Archaic context at the Wilson-Leonard site are listed as Martindale (Collins et al. 1998b:220). Of the 145 dart points attributed to the long Archaic period, at the Pavo Real site only two or three percent are listed as Early Corner-notched (Black 2003:210). At the Gatlin site, nearly 16 percent of the 342 typed points are classified as Martindale (Houk et al. 2008), although some researchers may question the

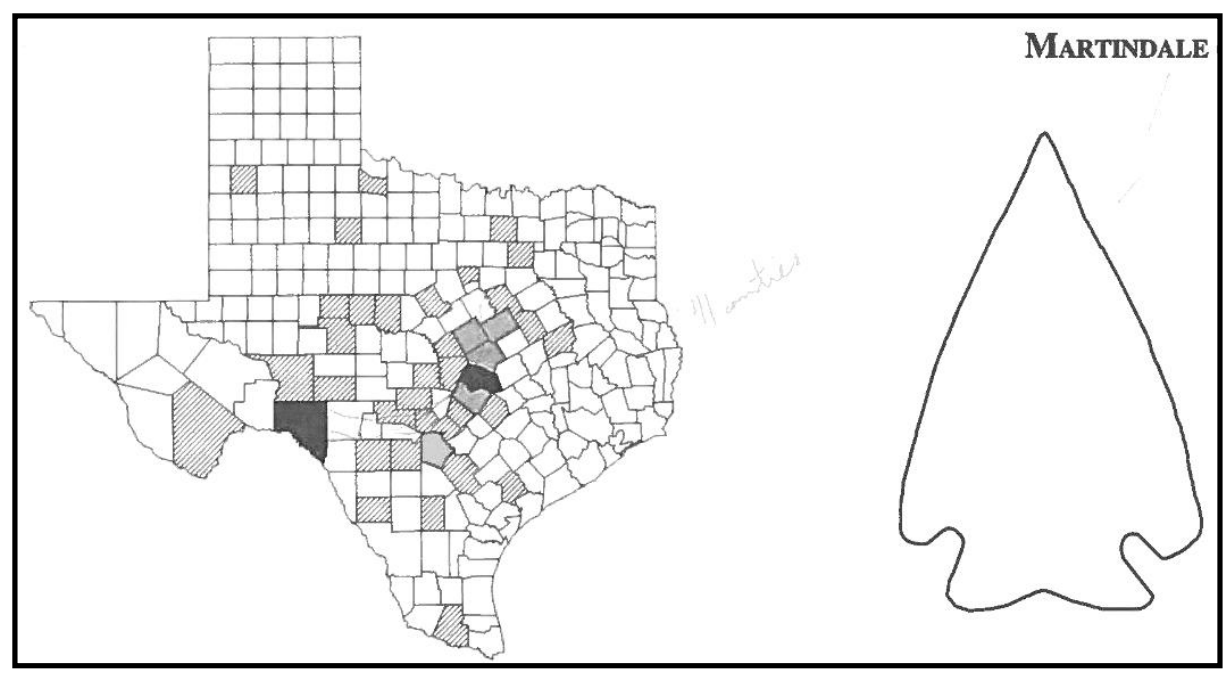

Figure 3-25. Martindale point distribution by county to 1995 (from Prewitt 1995:117, Black represents over 50 points, gray reveals 11 to 51 , hatched is 1 to 11 and white is no data). 
identifications. Similarly, at the Woodrow Heard site the 37 Martindale points account for six percent of the identified projectile points (Decker et al. 2000). Lack of identified nonlocal lithic materials in sites and components indicate limited trading of nonlocal materials or at least not in the form of tool stone.

\subsubsection{Burial Customs and Skeletal Data}

Human remains are scarce with the exception of those from sinkholes such as the Bering sinkhole site in Kerr County (Bement 1994). This sinkhole contains sufficient context and data to address mortuary practices and provides the bestdocumented evidence of human interment patterns for the Early Archaic period. Bement (1994) presents and discusses mortuary practices during the central Texas Archaic period, or roughly a 5500 year period, and includes data from other sites in the region. The evidence indicates human burials and offerings were simply dropped from the surface onto the sinkhole floor some 5 to $7 \mathrm{~m}$ below and accumulated on the talus cone below. Apparently 20 bodies, 1 bundle, and 2 cremations were attributed to depositional Unit III, which represent a period from 5100 to 7100 B.P. Dental analysis indicates the carries rate $(0.69 \mathrm{c} / \mathrm{p})$ is low for this San Geronimo phase, as opposed to a similar time period in the Lower Pecos region, and indicates substantially different diets in operation in the two adjacent regions (Bement 1994:93). The low ratio is indicative of a diet relatively low in carbohydrates. Stable carbon isotope analyses on human bones indicate the Martindale human population had a roughly 54 percent reliance on $\mathrm{C}_{3}$ plants (e.g., sotol, acorns, etc.) and animals consumed $\mathrm{C}_{3}$ plants (e.g., deer) and a 46 percent $\mathrm{CAM} / \mathrm{C}_{4}$ foodstuffs (e.g., prickly pear cactus, agave, and sotol). Stable nitrogen isotope analysis indicates plant foods consistently dominated animal foods in their diet. The human teeth also reveal grooves and abraded surfaces, which indicate plant or sinew processing was common.
Excavations at the Seminole Sink site, a single vertical shaft tomb (sinkhole) in the Lower Pecos region, yielded broken and dispersed fragments of minimally 21 human skeletons compacted into one stratum (Zone 2) beneath a talus cone below the surface opening (Turpin 1988). The burial episode dates primarily to the Early Archaic (Viejo subphase) by the presence of only one Early Corner-notched projectile from the very base of the cultural deposits directly associated with human burials (Figure 3-26). The three most acceptable radiocarbon dates for this population were obtained on human petrous (ear) bones from the lowermost stratum: $4671 \pm 64$ B.P. (AA-1314), $5590 \pm 180$ B.P. (AA-1313), and $5750 \pm 140$ B.P. (AA-1315). Age interpretations are derived from nine radiocarbon dates.

Bioarcheological data indicates this Early Archaic population was well-adapted to their desert environment and represented a normal huntergatherer population. The caries rate is a high 1.8 per person for the 10 Early Archaic bodies. No evidence of differential mortuary status was apparent due to the absence of grave goods from the differently aged and sexed bodies. This supports an egalitarian society in the Lower Pecos. The dental evidence indicates consumption of coarsely prepared high fiber diet with large quantities of

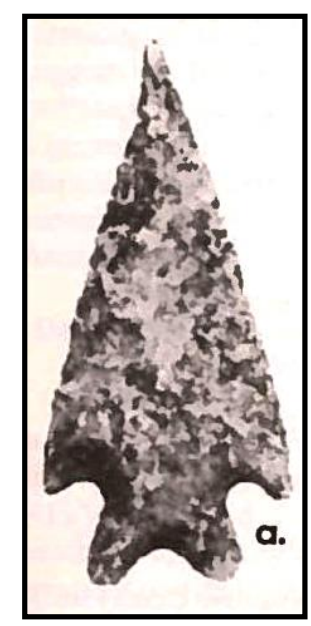

Figure 3-26. Early Corner-notched point from Seminole Sink (from Turpin 1988: Fig.12). 
carbohydrates. The stable carbon isotope data also supports a heavy reliance on $\mathrm{C}_{4} / \mathrm{CAM}$ vegetation such as prickly pear cactus, agave, and sotol. The authors' interpretations indicate the living group spent the majority of the year within a limited distance from the sinkhole (Turpin 1988). The social and status characteristics of Early Archaic populations at the Seminole Sink site appear similar in age and types of those encountered at Big Hole.

\subsubsection{Summary}

The Martindale point style has been defined for $60+$ years, although excavations across Texas have provided limited information concerning lifeways of populations which employed this point type and even the precise timing of its employment. Excavations that encountered this point type have often revealed contextual problems, limiting the information obtained. Most early excavated components with this point type were undated or poorly dated. The general chronological assignment of this style is defined primarily through multiple stratigraphic positions in relation to other dated components.
Radiocarbon assays generally associated with Martindale/Early Corner-notched/Early Barbed points encompass a time range over roughly 2,200 years, from ca. 6860 to 4671 B.P. Further dating will eventually determine which dates, if any, are outliers. Existing data is not sufficient to document possible population movements across Texas.

The groups utilizing Martindale points probably flourished during the Altithermal climatic period, which is characterized by a warm and dry period across the Southern Plains region. That environmental condition most certainly influenced the population's lifeway and the animal and plant resource utilization.

Greater understanding of these hunter-gatherers will require a commitment to conduct expansive horizontal excavations in sites and components, with clear stratigraphy, isolation of a Martindale component, good preservation of organic remains and dateable remains. Additional detailed and technical analyses on multiple artifact classes are critical to understanding human adaptive activities. Much remains to be understood concerning this culture and how these people adapted and survived during periods of environmentally stressful conditions. Chapter 10.0 contributes to a greater understanding of these populations. 
This page intentionally left blank. 


\subsection{RESEARCH DESIGN FOR ANALYSIS AND REPORTING}

Robert A. Ricklis, J. Michael Quigg, and Paul M. Matchen

\section{$4.1 \quad$ INTRODUCTION}

The data recovery excavations targeted rather deeply buried (ca. 200 to $300 \mathrm{cmbs}$ ), stratified prehistoric archeological components that had been identified during previous testing (Campbell et al. 2006). These components were recognized as containing a number of small burned rock features, lithic debitage, plus limited quantities of timediagnostic artifacts and faunal remains, mostly resting at a depth range of 200 to $300 \mathrm{cmbs}$ within Holocene alluvial fill adjoining the present channel of Onion Creek just upstream of its confluence with the Colorado River.

This research design for analysis of, and reporting on, was written and approved by TxDOT in 2011. It involves two hypotheses that are susceptible to testing with the data and materials recovered. Both hypotheses address what we believe is a key question for the pertinent period of central Texas prehistory, ca. 8000 to $6000 \mathrm{cal}$ B.P. (ca. 6000 to 4000 B.C.), namely, how did hunter-gatherer populations respond to the prevailingly dry climatic conditions during this part of the Holocene? The first hypothesis relates to the particular nature of site occupations during this temporal interval, while the second is directed toward elucidating how the kind of occupations fit into the overall patterns of regional human adaptation during this period.

In the subsequent pages, we provide the following:

- Statements of the two research hypotheses.

- A brief overview of the site's stratigraphy, cultural components, and the chronometric dating of those components.
- A postulation of the nature of human occupations of the site between ca. 7000 and $6000 \mathrm{cal}$ B.P. (ca. 5000 to 6000 B.P.), along with supporting lines of evidence.

- An overview of current models of regional Holocene climate history.

- A succinct summary of the data requirements for addressing each of the two hypotheses.

\subsection{THE HYPOTHESES}

\subsubsection{Hypothesis 1}

The middle Holocene occupations at 41TV2161 were non-intensive, short-term, and economically unspecialized encampments from which a broad range of subsistence resources were procured and processed; these occupations can be characterized as short-term residential encampments by small groups of nonspecialized foragers.

\subsubsection{Hypothesis 2}

The kind of occupation(s) represented at 41TV2161, namely, short-term residential encampments with nonspecialized and nonintensive reliance on a diverse range of resources, is essentially characteristic of this period of the Holocene in central Texas, ca. 7000 to $6000 \mathrm{cal}$ B.P. (ca 5000 to 6000 B.P.), and this represents an adaptive response on the part of regional human populations to the relatively dry climate of the period that involved a diversified reliance on a wide range of plant and animal food resources.

\subsection{PREHISTORIC CULTURAL COMPONENTS AT 41TV2161 AND THEIR CHRONOLOGICAL PLACEMENT}

Two distinct cultural components have been identified at the site (Quigg et al. 2007) (Figure 41). Defined in the field as stratigraphically separated by a thin lens of mottled light greenish- 
gray clay (Gley 27/1 5bg) that likely represents fine-grained sedimentation associated with a pond that once existed on the site, these components were targeted during the data recovery excavations. The earlier of the two components, underlying the light greenish-gray clay lens, produced a single timediagnostic lithic artifact, a corner-notched dart point of the Martindale type, sometimes referred to as Early Corner-notched type (Johnson 1964, 1967) (Figure 4-2, d). This form is generally ascribed to the later part of the Early Archaic period in central Texas (e.g., Collins 1995, 2004; Prewitt 1985). The later component, overlying the light greenish-gray clay lens, produced three diagnostic dart points, two of the basally-notched and heavily barbed Bell/Andice/Calf Creek series (Figure 4-2, a, b), and a single example of the side-notched Big Sandy type (Figure 4-2, c). Bell and Andice points, both widespread in Texas (see Prewitt 1995; Turner et al. 2011), are typologically closely related to the morphologically very similar Calf Creek point type widely distributed in Oklahoma and Missouri. These points were assigned by Prewitt (1985) to his central Texas Jarrell phase, which he estimated to date to between 6000 and 5000 B.P. (ca. 4100 and 3100 B.C.). At one time considered a chronological segment of the Texas Early Archaic (e.g., Hester 1980; Prewitt 1985; Turner and Hester 1999), the period represented by these point types has more recently been designated as the early part of the Middle Archaic (e.g., Collins 1995, 2004; Johnson and Goode 1994; Thoms and Mandel.

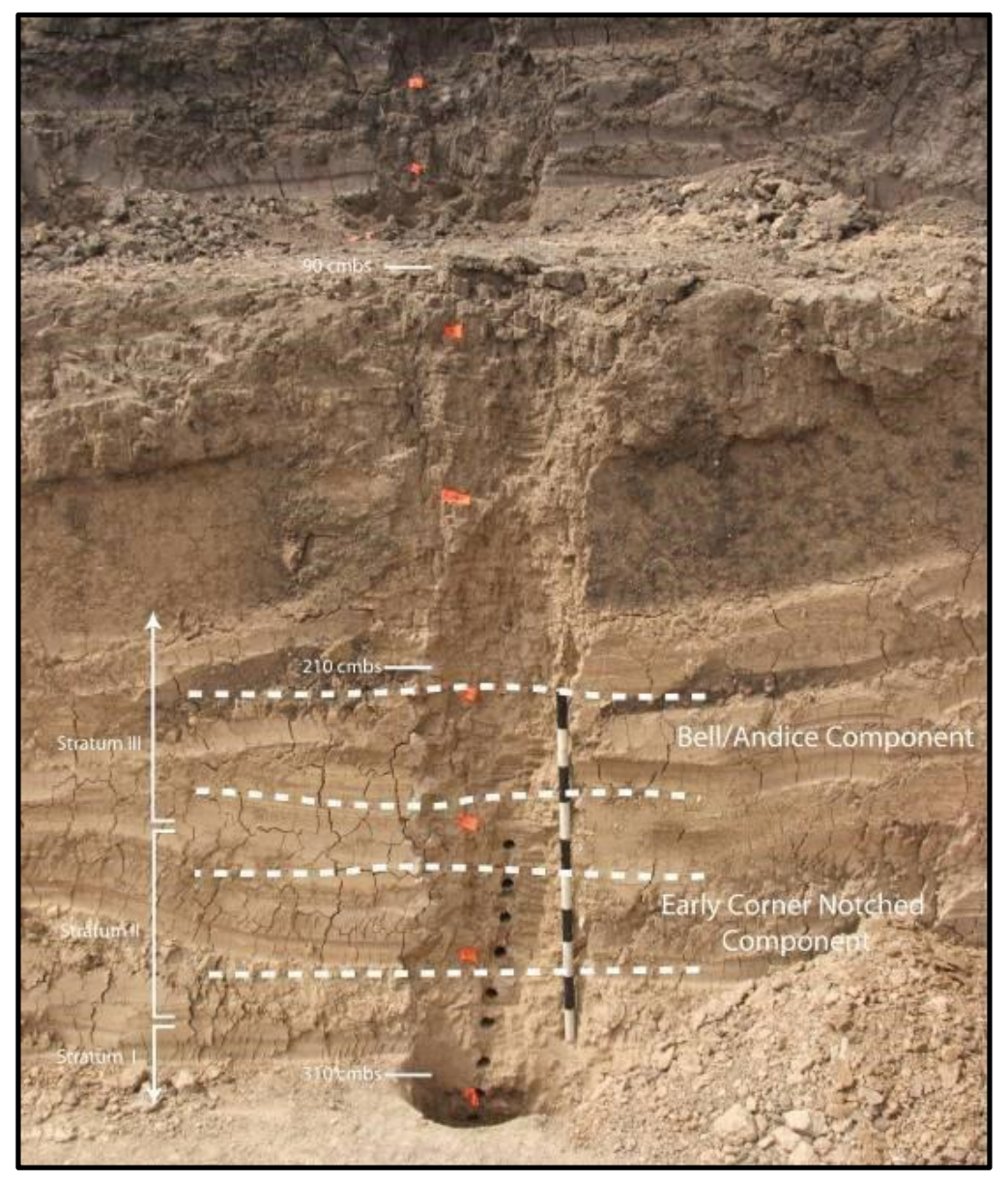

Figure 4-1. Profile of fine-grained alluvial sediments at the Big Hole site, 41TV2161, with the Bell/Andice and Martindale/Early Corner-notched components delineated by dashed lines (from Quigg et al. 2007). 


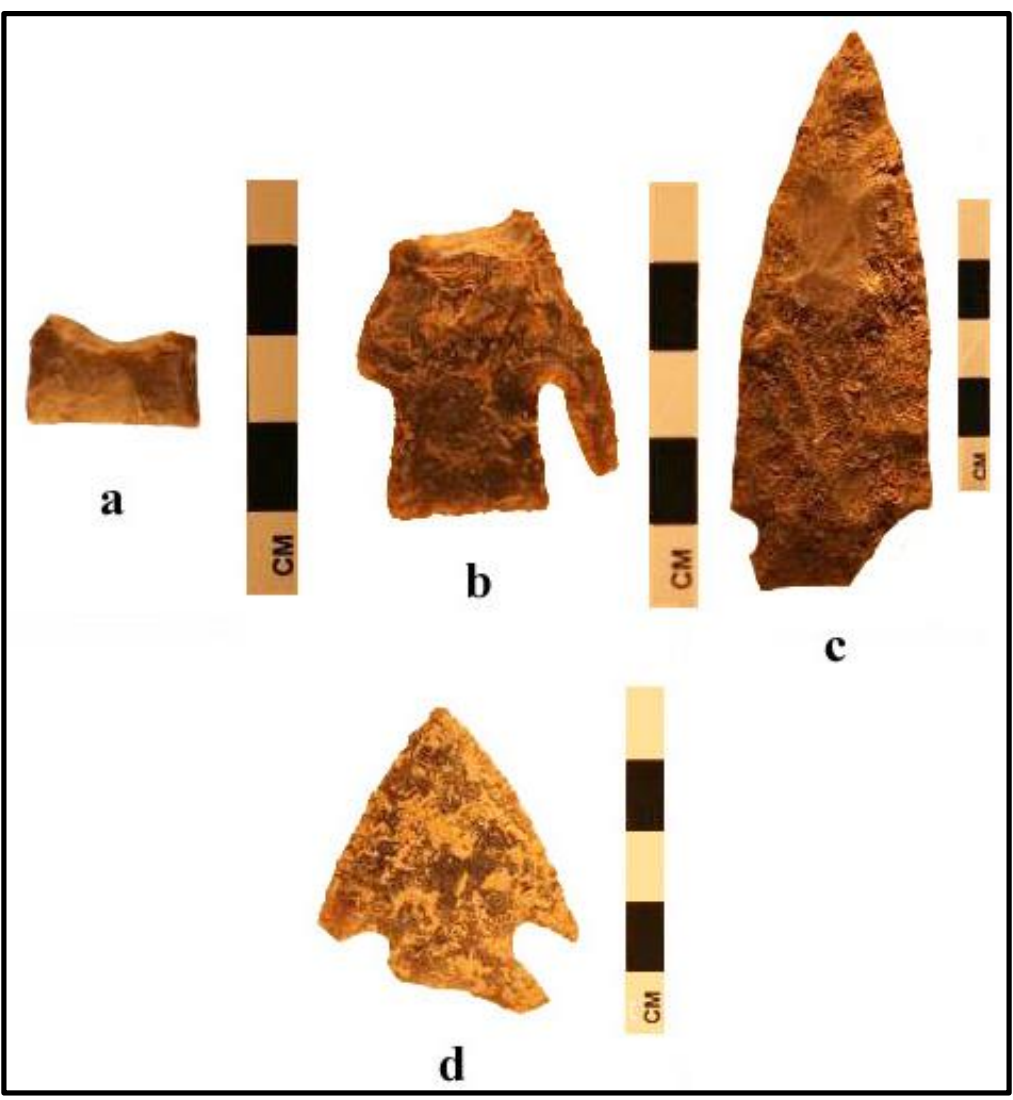

Figure 4-2. Dart points from 41TV2161. $a=$ basal stem fragment of a Bell point; $b=$ Bell point; $c=$ Big Sandy point; $d=$ Martindale point.

2007; see also Perttula 2004, Table 1-1). While the chronological and cultural contexts of Big Sandy points are not well-defined in Texas, and the type is generally only poorly represented in the State, they have been assigned to the same general temporal interval as have Bell and Andice points, ca. 4000 B.C. (Story et al. 1990, Figure 32). Big Sandy points are also identified in the bottom of Unit II at the Wilson-Leonard site to indicate a Paleoindian age (ca. 10,000 to 10,500, Collins 1998a). Although sometimes attributed to the Paleoindian period or discovered in mixed context, Bousman (2004) does not mention them in his in-depth discussion of the Paleoindian period in Texas

Initially in 2007, eight radiocarbon dates were obtained on materials that appeared associated with the two identified cultural components (Quigg et al. 2007). These dates were derived from organic materials from discrete features, or from depths within the site sediments that more or less closely correspond to the depths at which the timediagnostic artifacts and associated materials were recovered. The dated organic materials variously include Rabdotus land snail shells (which may or may not represent human predation upon land-snail populations), black-colored charred material thought to be degraded charcoal, and deer skeletal elements (in one case a radius, in the other, an incisor tooth). The combined, 2-sigma calibrated age range of all eight samples is 5270 to 3990 B.C., a 1,280 year interval that spans the culturaltaxonomic intervals thought to be represented by both Bell/Andice points as well as the slightly earlier Martindale points (cf. Collins 1995, 2004; Prewitt 1985). Three dates are associated with discrete features, mostly small clusters of burned rocks that are linked with one or another of the two identified components. A sample of charred material from Feature 3, tentatively assigned to the 
Bell/Andice component (Quigg et al. 2007), produced a conventional radiocarbon age of $5290 \pm$ 40 B.P. (Beta-214482), which yields a 2-sigma calibrated date range of 4240 to 3990 B.C. Two other dates, both obtained on samples of multiple Rabdotus land snails, are ascribed to the "Cornernotched" or Martindale component; one sample came from Feature 20 and the other is from Feature 26. Respectively, the ages in uncalibrated radiocarbon years are $5900 \pm 50$ B.P. and $5850 \pm 50$ B.P., which yield respective 2-sigma calibrated date ranges of 4850 to 4640 B.C. and 4800 to 4580 B.C.

While it is presently unclear whether or not the dates obtained on Rabdotus shell samples are affected by ingestion of old carbon by the snails, a factor which could account for their somewhat older ages than the date obtained on the charred material from Feature 3, the results do, in fact, conform to expectations for the ages of Bell/Andice and Martindale points. In any case, the occupations represented by the recovered cultural materials can be assigned, on the bases of both typology and chronometric dating, to the period of ca. 5000 to 6000 B.P. (ca. 3000 to 4000 B.C.).

\subsection{THE NATURE OF THE PERTINENT SITE OCCUPATIONS AS INFERABLE FROM FEATURES AND ARTIFACT ASSEMBLAGES}

Based upon the acquired data, it is inferred that the recovered occupation(s) at 41TV2161 were of relatively short durations, entailed correspondingly limited ranges of on-site activities, and involved relatively small resident groups of people. These suggested characteristics, evident in both identified components, are supported by the data from the site, as follows.

Short-term occupations are strongly suggested by the generally small size of features and their discrete morphologies resulting from a general lack of "smearing" or overprinting by other features, as would result from the redundant use of space in activities at relatively long-term encampments. Moreover, longer-term occupations can be expected to leave evidence for various types of features (e.g., storage facilities, and/or at least minimal evidence of domiciliary structures), none of which were in evidence at 41TV2161. Also indicative of short-term occupations is the low density of artifacts and ecofacts (only a very few projectile points or other formal tools, plus limited quantities of lithic debitage $(N=1,897)$, faunal bones $(N=1,259)$ and freshwater mussel shell fragments $(N=137)$; longer-term occupations would be expected to have resulted in much greater quantities/densities of these materials.

A corollary of occupations of limited duration is a limited range of on-site activities. Such a situation is indicated for the two occupations by the limited range of formal lithic tools recovered and the limited quantities of faunal remains, plus the narrow range of feature types.

As regards the inference of small resident groups, this derives from general theoretical perspectives on hunter-gatherer lifeways in which mobile small groups typically set up short-term residences in proximity to exploitable resource patches (e.g., Butzer 1982; Jochim 1981; Lee and DeVore 1968), as well as the assumption that a larger group would likely conduct a wider range of activities than appears to be evidenced at 41TV2161, even if occupation were only of relatively short duration.

In this context, it is worth noting that the sum of the geoarcheological evidence at 41TV2161 suggests that the site has sufficient integrity to permit inferences concerning occupational duration and intensity based on the excavated materials and data. As pointed out by Frederick et al. (2007), the cultural materials/features appeared to rest in secure, primary contexts, as they were sealed within fine-grained alluvial sediments that represent lowenergy overbank flood deposition, a circumstance 
which minimized postoccupational dislocation of cultural materials and left the archeological record at the site in a relatively undisturbed, and thus readily interpretable, condition (as a representative example, see the plan and profile drawings of Feature 18 shown in Figure 4-3).

\subsection{REGIONAL PALEOCLIMATE AND ENVIRONMENT, CA. 5000 TO 6000 B.P.}

As stated above, the present research design is directed toward exploring how prehistoric human populations responded to a regional climate marked by relative aridity and a corresponding reduction in animal biomass. It is posited that under such conditions, the food resources exploited by hunting and gathering populations would have in general been available in relatively patchy distributions, a situation that would have necessitated a relatively high degree of residential mobility, as human groups would have needed to move frequently in order to exploit spatially limited patches of food resources when and where they were available. It is further suggested that during extended periods of dryness, overall environmental productivity would have resulted in a reduced animal biomass, so that prehistoric peoples would have tended to base their subsistence strategies more on gathering of edible plant resources. Additionally, it is also posited that such groups would have been relatively small in size, given that the density and areal extent of exploitable resource patches would have been too limited to support large resident populations.

Given the significance, in this context, of the postulation of a dry climate during the period of site occupation, ca. 5000 to 6000 B.P., it is worthwhile to briefly consider the currently available information on climatic trends for this period in central Texas. We briefly review several reconstructions of broad patterns of Holocene climate change proposed in recent years and based variously on palynological studies (Albert 2007; Bousman 1998 as presented in Collins 2004), microfaunal analysis (Toomey et al. 1993), and shifts through time in the proportions of $\mathrm{C}_{4}$ plants during the Holocene (Thoms 2007b). Additionally, we refer to a postulated Holocene climate history presented by Johnson and Goode (1995), a synthetic interpretation that draws upon a number of different kinds of data, including palynology, microfauna (after Toomey et al. 1993), and geostratigraphic evidence from various locations in and around central Texas.

The studies based upon fossil pollen evidence are particularly instructive, as they document longterm shifts in species compositions of plant communities that were directly affected by climatically controlled temperature and moisture conditions. The climatic trends shown in Figure 44 are based on pollen sequences from radiocarbondated sediment cores from the floodplain of the Guadalupe River near the Buckeye Knoll site (41VT98) in Victoria County (Albert 2007) as well as dated cores from two east-central Texas bog sites (Bousman 1998; Collins 2004). Also compelling is the evidence from Hall's Cave on the Edwards Plateau, where documented changes in the relative abundances of the desert shrew (Notiosorex crawfordi) and the more xeric least shrew (Cryptotis parva) in the cave's sediment deposits (Toomey et al. 1993) serve as proxy indicators of long-term climate change and resultant environmental moisture levels.

Figure 4-4 shows that, while none of these five climate history reconstructions are identical, they all exhibit certain key similarities. In all but one case, extended periods of relatively dry environmental conditions are indicated for the temporal interval between ca. 7000 and $8000 \mathrm{cal}$ B.P. and ca. $5500 \mathrm{cal}$ B.P., followed by a relatively short interval of moister climate between ca. 5500 and $5000 \mathrm{cal}$ B.P. Between 5000 and $3000 \mathrm{cal} \mathrm{B.P.,}$ there is another extended dry interval (termed the "Edwards Interval" by Johnson and Goode 1995), except in the case of the climate history indicated by long-term shifts in abundances of $\mathrm{C}_{4}$ plants at the 


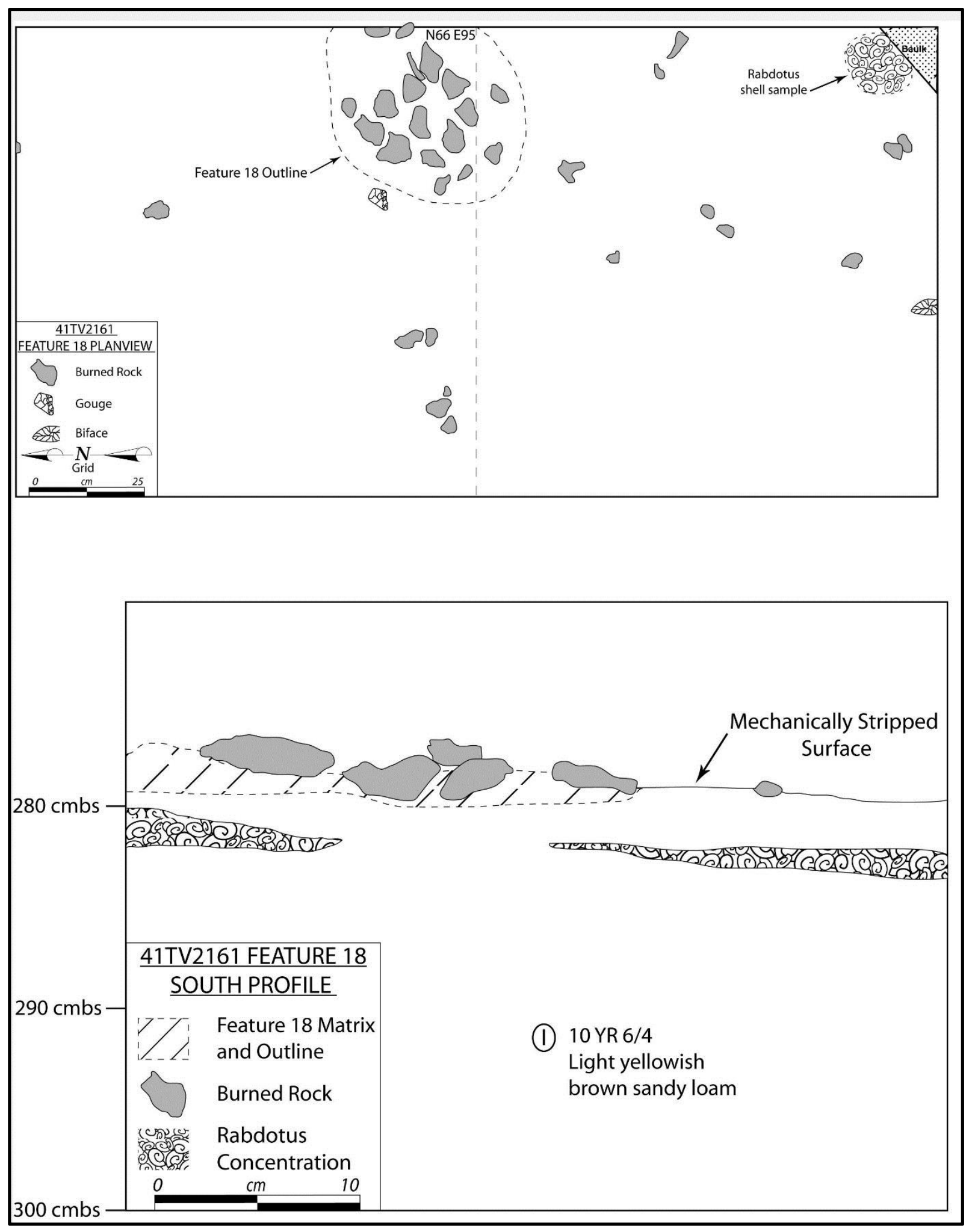

Figure 4-3. Plan (top) and profile (bottom) views of Feature 18, a burned rock cluster, showing the discrete and relatively intact condition typical of features at this site. 


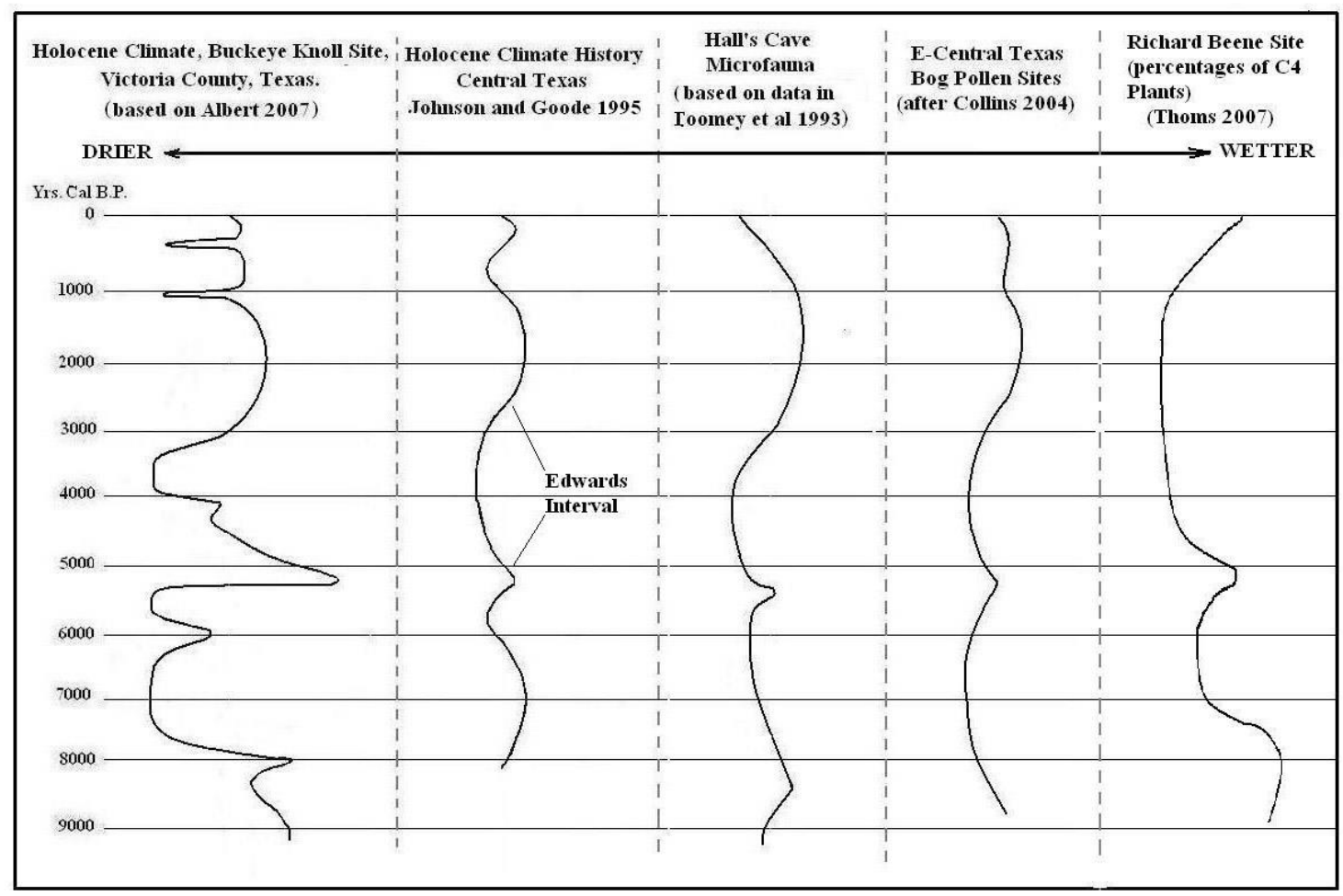

Figure 4-4. Non-scalar presentations of five basic patterns of Holocene climate fluctuations (relatively dry to relatively moist) based on pollen studies (Albert 2007; Bousman 1998, as summarized in Collins 2004) shifts in microfaunal species in cave deposits (Toomey et al. 1993), and fluctuations in percentages of $\mathrm{C}_{4}$ plants, based on carbon isotope values (Thoms 2007b). The Holocene climate history presented by Johnson and Goode (1995) draws upon various datasets, including sediment stratigraphy that reflect shifting hydrological conditions related to climate change.

Richard Beene site (Thoms 2007b), where the pertinent data suggest that this dry interval lasted until as recently as ca. $1500 \mathrm{cal}$ B.P.

The key point, for our present purposes, is that all five reconstructions of Holocene climate history reveal an extended period of relatively dry conditions between ca. 8000 and $7500 \mathrm{cal} \mathrm{B.P.} \mathrm{and}$ 5500 cal B.P. The only exception is the reconstruction offered by Johnson and Goode (1994), wherein the drying trend begins only at, or shortly after, ca. 7000 cal B.P., approximately a millennium later than in the other models. Since, among the various data represented in Figure 4-4, only the Hall's Cave data were available to Johnson and Goode (while the east-central Texas bog pollen data were available, they had not yet been adjusted by Bousman [1998]), we are inclined to believe that the longer period of dryness indicated by other models is probably a more accurate representation, and it is reasonable, on the preponderance of the evidence, to regard the two-millennia period between 7500 cal B.P. and 5500 cal. B.P. as an interval of sustained, relatively dry climate and correspondingly xeric environmental conditions.

\subsection{ANALYSIS FOR TESTING THE TWO HYPOTHESES}

Each of the two previously stated hypotheses will be addressed with specific kinds of analyses to be undertaken on the materials recovered from targeted zone at 41TV2161. These are listed and briefly discussed below for our two research hypotheses, each of which is restated here for the sake of clarity of exposition. 


\subsubsection{Hypothesis 1}

The middle Holocene occupations at site 41TV2161 were non-intensive, short-term, and economically unspecialized encampments from which a broad range of subsistence resources were procured and at which they were processed; these occupations can be characterized as short-term residential encampments by small groups of nonspecialized foragers.

\subsubsection{Proposed Analytical Approaches}

- Lithic-tool analysis (to follow TxDOT's 2010 Protocol for Lithic Analysis) will involve the identification of the range of tool forms and their functional purposes, plus use-wear studies and starch grain analysis to test the functional interpretations. In view of Hypothesis 1, the use-wear analyses should indicate a range of tasks rather than a focus on any particular kind of tool use, which is to say that the short-term use of the two components by nonspecialized forager groups should be reflected in use-wear data that indicates a variety of on-site tasks without particular emphasis on any one kind of activity.

- Lithic-debitage analysis (to follow TxDOT's 2010 Protocol for Lithic Analysis). This will entail the quantification of debitage and flake types in order to assess the kinds and stages of lithic tool production and/or refurbishing that took place on site. Given that Hypothesis 1 posits short-term occupations, debitage analysis is expected to reveal a greater emphasis upon tool refurbishing more than the primary production of tools, since relatively short-term occupations should not be expected to require significant on-site replacement of exhausted tools.

- Faunal analysis will involve the identification and quantification of the terrestrial and aquatic (fish, shellfish) taxa represented as a means to define the range of animal resources that was exploited. A more or less wide range of species should be represented, as opposed to a preponderance of any one species, such as would imply a specialized focus.

- Macrobotanical studies: Identifications and quantifications of the range of floral species represented by carbonized plant materials recovered in the light fractions of flotation of sediment samples, especially those associated with thermal features. In keeping with the Hypothesis 1, there should be evidence for the processing of a variety of plant resources, without intensive use of any one taxon.

- Otolith seasonality analysis. Otoliths have been repeatedly shown to be highly useful in as indicators of the age and season of death of fishes (Casteel 1976), and this has enabled archeologists to make reliable estimates of seasonality of fishing activities at prehistoric sites in Texas (e.g., Prewitt 1987; Ricklis 1996, 2011; Smith 1983; Wilson 2002). Microscopic examination of cross-sectioned fish otoliths will be undertaken to determine if fish procurement at 41TV2161 was seasonally dispersed or seasonally focused. Otolith seasonality will not, in itself, be diagnostic of short-term occupation(s) at the site, since a single short-term occupation for a given component should show a unimodal seasonal distribution, but two or more such occupations could easily result in a bimodal or multi-modal distribution. It is possible, however, that in conjunction with other possible seasonality indicators (e.g., use of certain seasonally available plant resources), reasonable inferences concerning restricted seasonality in a short-term encampment(s) can be made.

- Study of features in terms of their constituents (e.g., rock types and sizes), size, density, morphology, and associated materials such as artifacts, ecofacts, and charcoal, in order to obtain a preliminary assessment of their 
functions (e.g., cooking platforms, vs. boiling facilities, vs. discard piles). In accord with Hypothesis 1, features should be relatively small and contain relatively limited quantities of materials (e.g., fire-cracked rocks) so as to reflect short-term, non-intensive activities. In conjunction with the technical analyses discussed under the next bulleted section, it is expected that the specific functions of features will be definable. In keeping with Hypothesis 1 , this should reveal a limited range of functions (e.g., for roasting, boiling or as discard piles) in conjunction with the processing of an array of plant and animal resources.

- Technical analyses of burned rock samples from features: To include identification of microscopic residues such as phytoliths (to determine plant taxa that were processed and/or cooked), diatoms (to ascertain whether or not water was associated with feature use, as in stone-boiling), starch grain analysis (to document the use of various plant resources cooked and/or processed), and lipid residue analysis (to determine whether plants or animals, or both plants and animals, were cooked in conjunction with a given feature). These analyses have obvious relevance to the question of the diversity of resources that were procured and utilized.

- Instrumental neutron activation analysis is to be directed at a suite of lithic tools and debitage from the two components to assess the use of local versus nonlocal chert resources, and to examine how lithic technological organization operated within the relevant adaptive system.

The results of these various analyses will contribute to addressing the stated hypothesis. For example, the hypothesized short-term occupation(s) of the site will be supported by a) small feature sizes, limited quantities of stone tools and debitage, a restricted functional range of tools, low frequency of faunal remains, quantitatively and qualitatively limited plant species representations, and so on. The proposed corollary of a diverse but nonspecialized and non-intensive extraction of food resources will be supported by evidence for a diverse range of plant resources, and evidence for a diverse range of faunal species but a restricted quantity of any one species.

\subsubsection{Hypothesis 2}

The kind of occupation(s) represented at the two recovered components, namely short-term residential encampments with nonspecialized and non-intensive reliance on a diverse range of resources, is essentially characteristic of this period of the Holocene in central Texas, ca. 7000 to 6000 cal B.P., and this represents an adaptive response on the part of regional human populations to the relatively dry climate of the period that involved a diversified reliance on a wide range of plant and animal food resources.

\subsubsection{Proposed Analytical Approaches}

- Acquisition of an increased number of radiocarbon dates from the two recovered components, with which to more firmly define the chronological range of the two represented components (i.e., the so-called Martindale/Early Corner-notched component represented by a single Martindale point, and the Bell/Andice component, represented by Bell or Calf Creek-like points and a Big Sandy point, along with the associated materials assignable on the basis of stratigraphic positions to each of these two components as analytical units). The proposed radiocarbon dates will be obtained on additional samples of charcoal, bone, organic residues in burned rock specimens, Rabdotus, and the black residue associated with some of the features. The goal of obtaining these additional radiocarbon 
dates is to narrow the temporal interval represented by the two identifiable components which, on the basis of the presently available dates, are believed to both pertain to the roughly one millennium interval between ca. 7000 and $6000 \mathrm{cal}$ B.P. In particular, it will be desirable to ascertain whether or not the presumably slightly earlier of the two components, the so-called Martindale/Early Corner-notched component, in fact does predate the Bell/Andice component. As noted above, such a temporal succession appears to be indicated by their stratigraphic positions and the currently available radiocarbon dates (as well as previous age estimates for the pertinent point types), but the dates on the Martindale/Early Corner-notched component are exclusively derived from samples of Rabdotus snail shells, and additional dates derived from other organic materials are desirable, considering the possibility that Rabdotus shells may yield dates that are too old because of possible ingestion of older carbons by the living snails.

- Compilation and comparative discussion of archeological data from other central Texas sites of this time period, as well as immediately preceding and succeeding periods, to show that the currently available evidence suggests a pattern of relatively non-intensive occupation of sites and the absence of information that would imply any significant degree of intensification or specialization in resource use during this period. The purpose of this exercise will be to gather supporting evidence for a prevalence of forager-type adaptation that involved a relatively high degree of residential mobility during this period, as well as to discuss, to the degree possible, the place of this pattern in long-term development of human adaptive strategies. Attention will also be directed to ascertaining if there are discernible, contrasting differences in the nature of occupations on the Blackland Prairie as opposed to the nearby Balcones Escarpment such as would suggest spatial and/or seasonal variability within regional adaptive strategies. Site data for comparative purposes will be drawn from various published reports including, but not necessarily limited to, reports on pertinent components at the Barton site (Ricklis and Collins 1994), the Richard Beene site (Thoms and Mandel 2007), the Landslide site (Sorrow et al. 1967), the WilsonLeonard site (Collins 1998), and other recently reported sites in the lower Onions Creek valley (e.g., 41TV410 and 41TV540 [Figueroa et al. 2011], 41TV2125 [Campbell et al. 2010; Karbula et al. 2011]). It is expected that a thoughtful review of extant data from these sites, including sites and components of immediately preceding and succeeding periods, in conjunction with the paleoenvironmental data mentioned above, will provide synchronic and diachronic environmental and cultural contexts for the human-ecological patterns represented at the two recovered components at 41TV2161 and, possibly, allow for modeling of long-term trends in such patterns. 


\subsection{FIELD AND LABORATORY METHODS}

J. Michael Quigg, Paul M. Matchen, and Benjamin G. Bury

\subsection{FIELD METHODS}

The following field methods were implemented with TxDOT approval of the data recovery strategy and target block locations.

\subsubsection{Mechanical Stripping}

To prepare for hand-excavations designed to target a deeply buried zone of cultural materials below roughly $250 \mathrm{cmbs}$, TRC initiated investigations with the mechanical-removal of 230 to 260 centimeters $(\mathrm{cm})$ of alluvial sediment from a large block area some 40-by-30 meters (m). The massive mechanically-excavated area was prepared through the use of a track hoe with a 2.5 to 3 m wide bucket supplied by Lone Star Infrastructure and operated by their personnel (Figure 5-1).

Prior to the TRC excavation, Hicks \& Company recorded minimally seven cultural features (designated 1 through 7) during site discovery and assessment investigations (Campbell et al. 2007). The vertical positions of Features 3 through 6 were between 200 and 280 cmbs. This depth and apparent concentration of cultural features combined with the radiocarbon age obtained from Feature 3 was the stimulus for TRC's decision to target this deeply buried cultural zone. A few cultural burned rock features were partially exposed during mechanical stripping with those left in place on pedestals (see Figure 5-1).

TRC archeologists mapped the target area and guided the mechanical stripping with the use of a

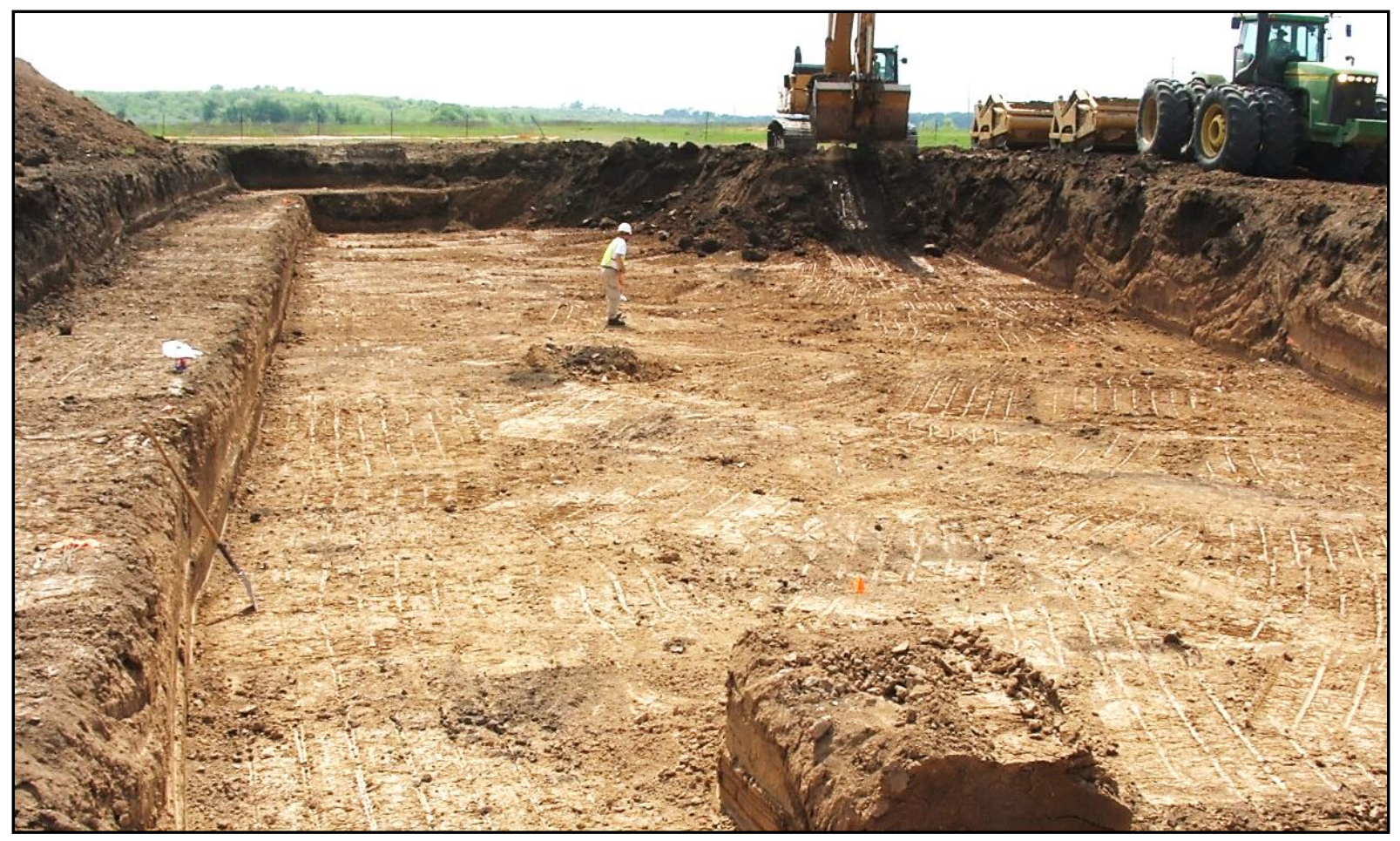

Figure 5-1. Southern view across mechanically stripped excavation block with track hoe and other earth removal equipment in background. Note safety bench on left side and pedestalled burned rock feature in foreground. 

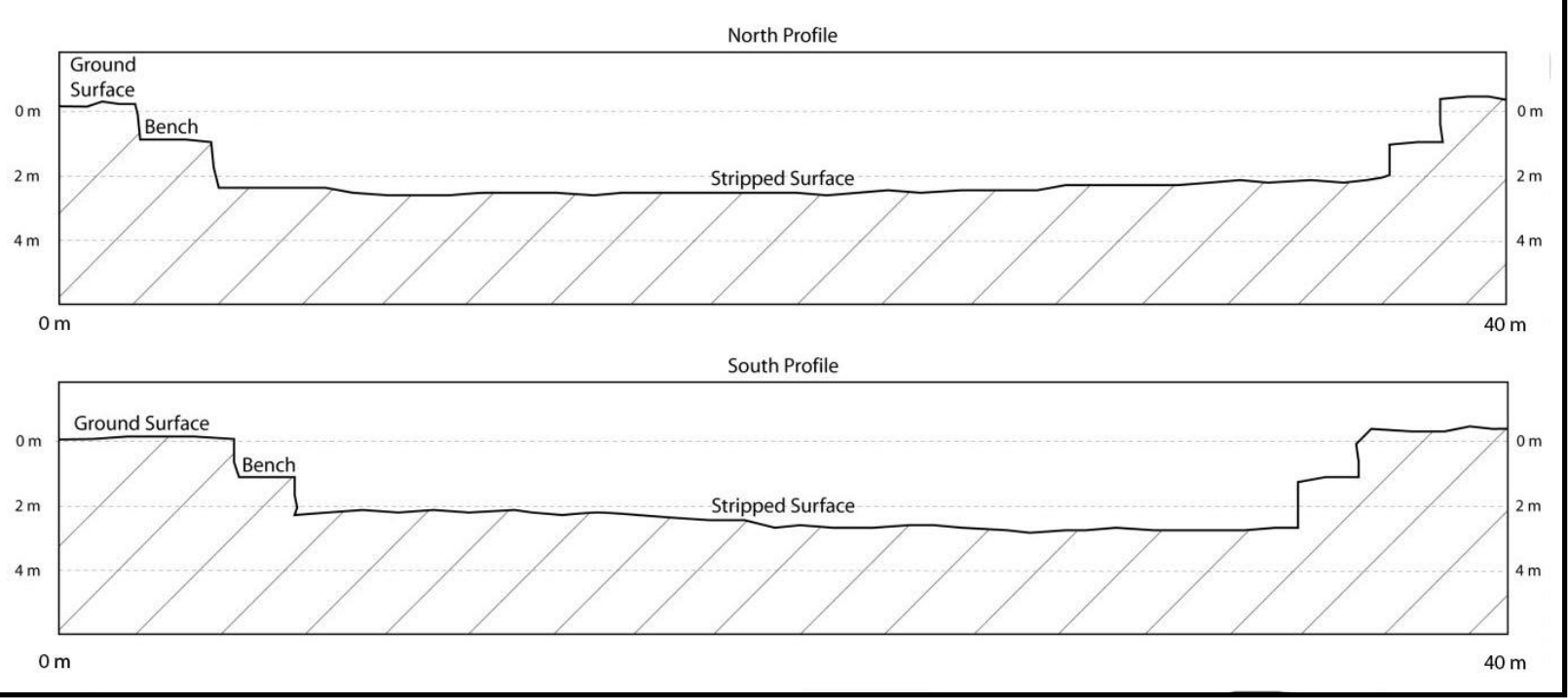

Figure 5-2. Profile of the Big Hole site target block showing depth of mechanically stripped surface to roughly 2.5 meters deep.

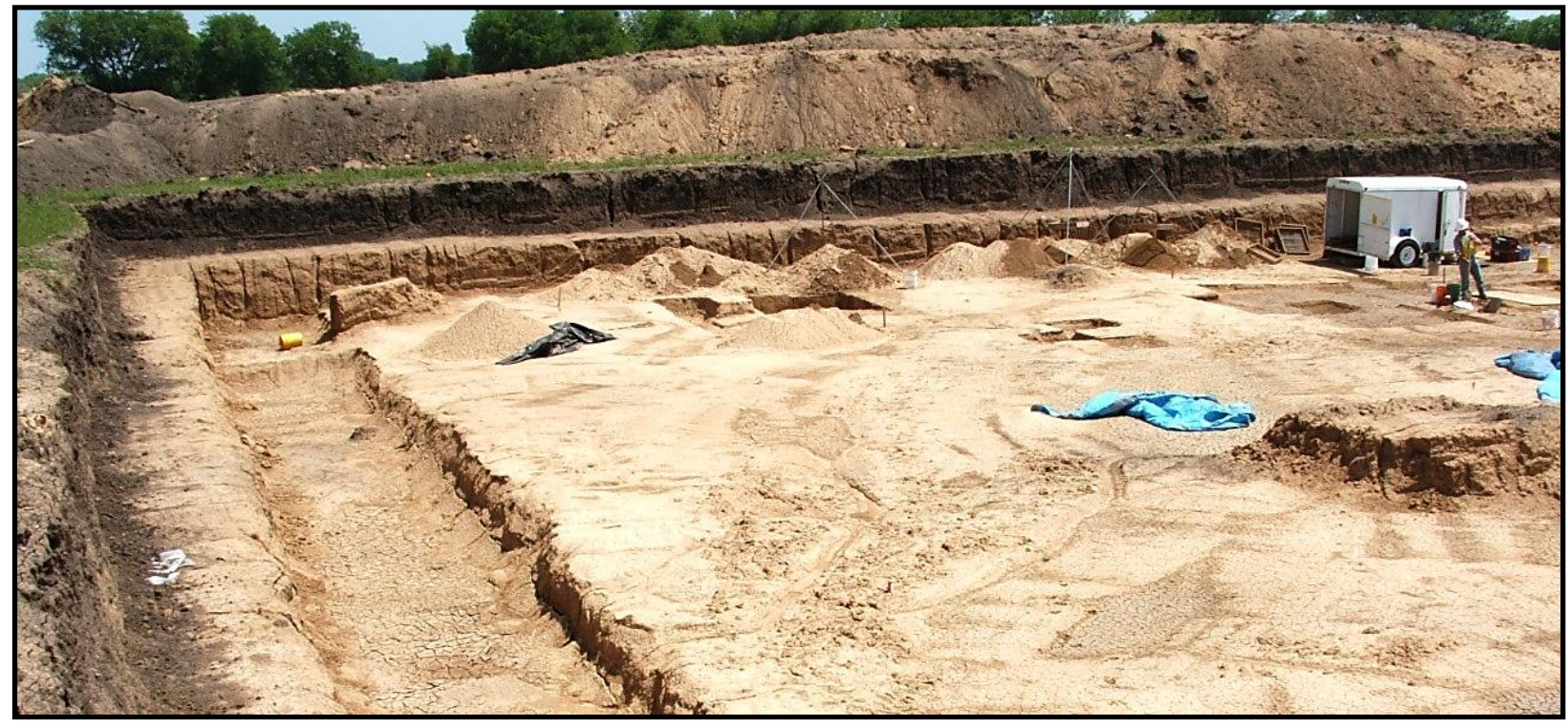

Figure 5-3. Northern end of excavation block showing safety bench and water collection trench. Note Block $A$ is on far side and Block $D$ on right.

total station theodolite (TST) and prism survey rod. At regular intervals, the TST was employed to check the relative elevation of the ground surface to the mechanically stripped surface, and ensure vertical proximity to the target zone of approximately $250 \mathrm{~cm}$ below surface $(\mathrm{cmbs})$. This method of mechanically stripping was only precise enough to get within $20 \mathrm{~cm}$ of the target depth in most places, and some areas dipped below the target depth. During the stripping process, a few burned rock clusters were recognized above the target zone and were flagged and pedestalled in anticipation of potential investigation. Areas surrounding those features were mechanically stripped down to the target depth (Figures 5-1 and 5-2). 
Following mechanical stripping the area measured approximately 30 -by- $40 \mathrm{~m}$ with a $2.5 \mathrm{~m}$ wide safety bench established around the margins at roughly 1.5 mbs. Inside the stripped area at the northern and southern ends, mechanically dug trenches, approximately $60 \mathrm{~cm}$ deep by $200 \mathrm{~cm}$ wide were created as potential water collectors in case of rain and also served to observe stratigraphy (Figure 53). Backdirt from the mechanical excavations was piled around the outer edges leaving a small, sloping access ramp at the southeastern corner. The irregular stripped surface ranged in depth between approximately 230 and $260 \mathrm{cmbs}$ with the occasional high spot of $220 \mathrm{cmbs}$, particularly in the southwestern quadrant. Generally the prepared surface was higher in elevation on the western side than the eastern side, but most initial starting depths for hand-excavations averaged roughly $250 \mathrm{cmbs}$.

\subsubsection{Geophysical Investigations}

Following mechanical stripping, large dirt clods and excess mounds of dirt were removed by hand and the stripped surface prepared and smoothed to facilitate the geophysical evaluation. Tiffany Osburn of Geo-Marine (Plano, Texas) conducted a geophysical survey across the stripped area using high resolution ground penetrating radar (GPR) (see Chapter 7.0 for more details). The radar was mounted on a wheeled cart sensitive to the uneven surface (Figure 5-4). The rougher the surface, the greater noise in the radar reflection data as the radar could not maintain constant contact with the bumpy ground.

Two GPR antennas (a 400 and 900 megahertz [MHz]) were employed to perform this task. Radar reflection data focused on a package of sediments that measured no greater than $60 \mathrm{~cm}$ deep by limiting the time window in which the system is "listening" for returning reflected waves. All GPR reflection data was first analyzed in twodimensions to determine the nature of subsurface reflections, their wavelength, depth of penetration, amount and nature of background interference, and

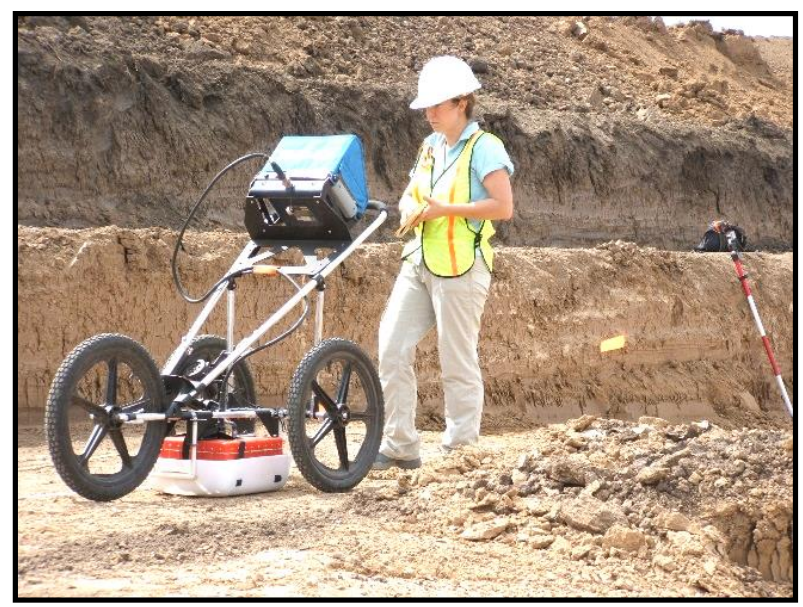

Figure 5-4. Tiffany Osburn employing the mounted ground penetrating radar.

the velocity of radar energy in the ground. The most visually interpretable method for GPR data processing was amplitude slice-mapping. This method produces a series of horizontal slices at different depths that illustrate all significant reflections within given layers in the ground. Four amplitude slice maps were produced for each antenna for the surveyed block and provided the best means of guiding hand-excavations based on subsurface geophysical data (Figures 5-5 and 5-6).

Numerous geophysical anomalies were detected through the GPR (see Chapter 7.0 for details). Following detailed processing and data analyses by Osburn, those anomalies were ranked with a numerical number (1 through 8 ). The highest ranked anomalies were thought to have the highest potential to represent cultural features. The anomalies were marked on each amplitude slice and detailed maps were provided to TRC archeologists prior to field excavations and became targets for hand-excavations (Figures 5-5 and 5-6).

\subsubsection{Establishment of Hand- Excavated Blocks}

Initially, selected GPR anomalies (1 through 6) were targeted by hand-excavations through the use of several 1-by-1 $\mathrm{m}$ units over the projected anomaly. Targeted anomalies were not clearly 


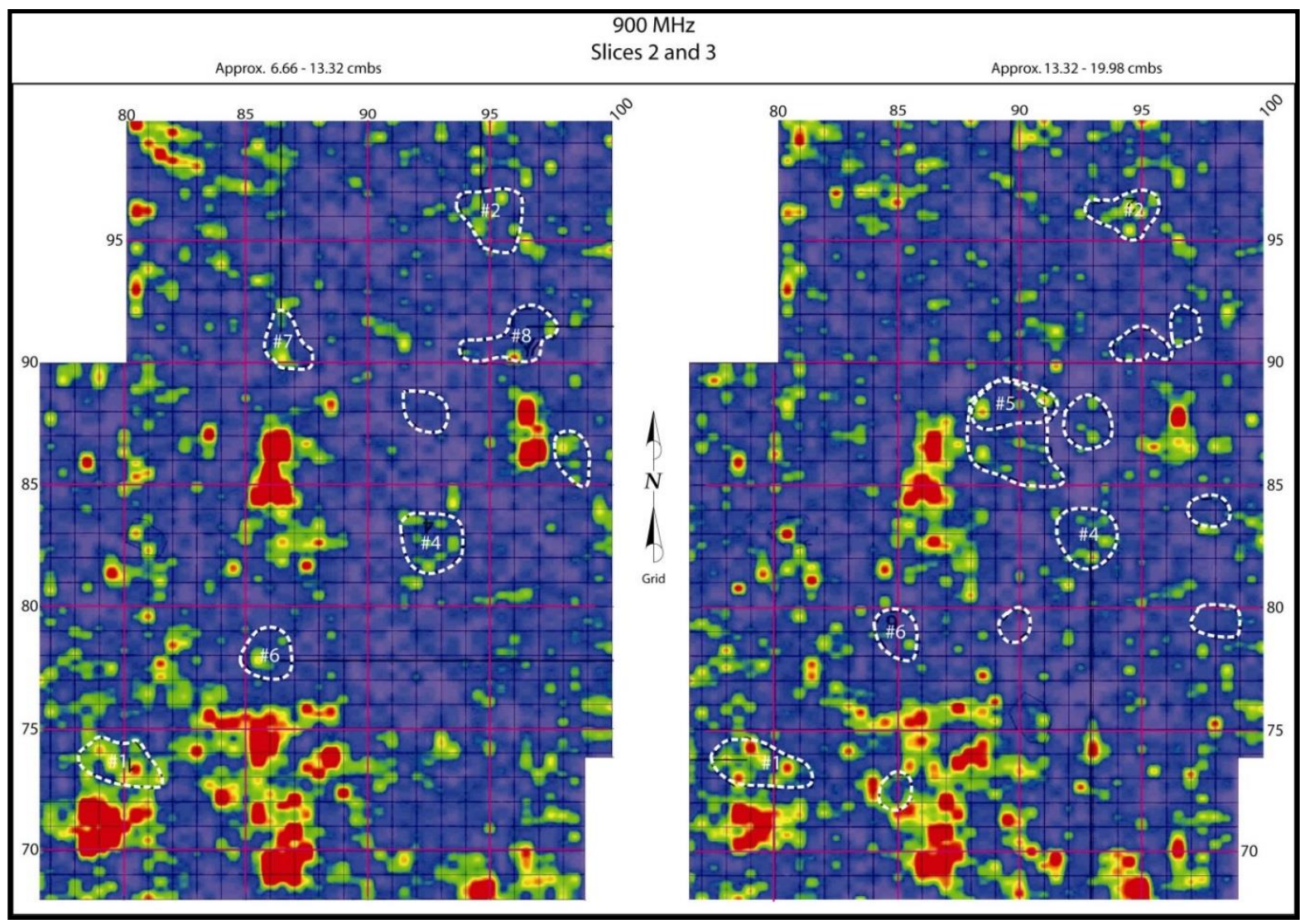

Figure 5-5. Ground penetrating radar scans from the $900 \mathrm{MHZ}$ antenna showing anomalies from 6.7 to $13.3 \mathrm{~cm}$ and 13.3 to $20.0 \mathrm{~cm}$ below stripped surface of investigation block.

discerned in individual 1-by-1 m units; therefore, hand-excavations often expanded horizontally to locate or expose possible features, and blocks of units were formed (assigned A through F) (Figure 5-7). In many instances the GPR anomalies did not depict a prominent or obvious recognizable feature (i.e., Blocks $\mathrm{E}$ and F). In those instances, our focus shifted to more promising areas with greater concentrations of cultural debris. The five blocks are presented and discussed below.

Block $\mathrm{A}$ in the northeastern corner targeted the second highest ranked anomaly, Anomaly 2. The mechanically stripped surface varied from 240 to $260 \mathrm{cmbs}$ with excavation units along the southern edge starting closer to $260 \mathrm{cmbs}$. Hand-excavations were designed to identify the anomaly, which first appeared as an amorphous, poorly defined, irregular shaped dark organic stain with mottling of orange and black colored sediment. Thirteen 1-by-
$1 \mathrm{~m}$ units were hand-excavated $\left(4.8 \mathrm{~m}^{3}\right)$ with four units excavated to $290 \mathrm{cmbs}$ to determine the vertical extent of the stained sediments (see Figure 5-7). The southernmost units were only excavated to $280 \mathrm{cmbs}$ while trying to define the boundary of the burned sediment and clarify its origin.

Block B in the southwestern corner targeted the highest ranked GPR anomaly, Anomaly 1 (see Figures 5-6 and 5-7). Mechanical stripping encountered several small burned rock clusters at roughly 215 to $220 \mathrm{cmbs}$ and stripping was stopped at this slightly higher elevation as opposed to most other stripped locations (see Figure 5-6). Stripping left an uneven surface with the southwestern corner at roughly $215 \mathrm{cmbs}$, whereas the eastern side of Block B was close to $250 \mathrm{cmbs}$ and deeper in spots. Hand-excavations began between 215 and 225 cmbs. 


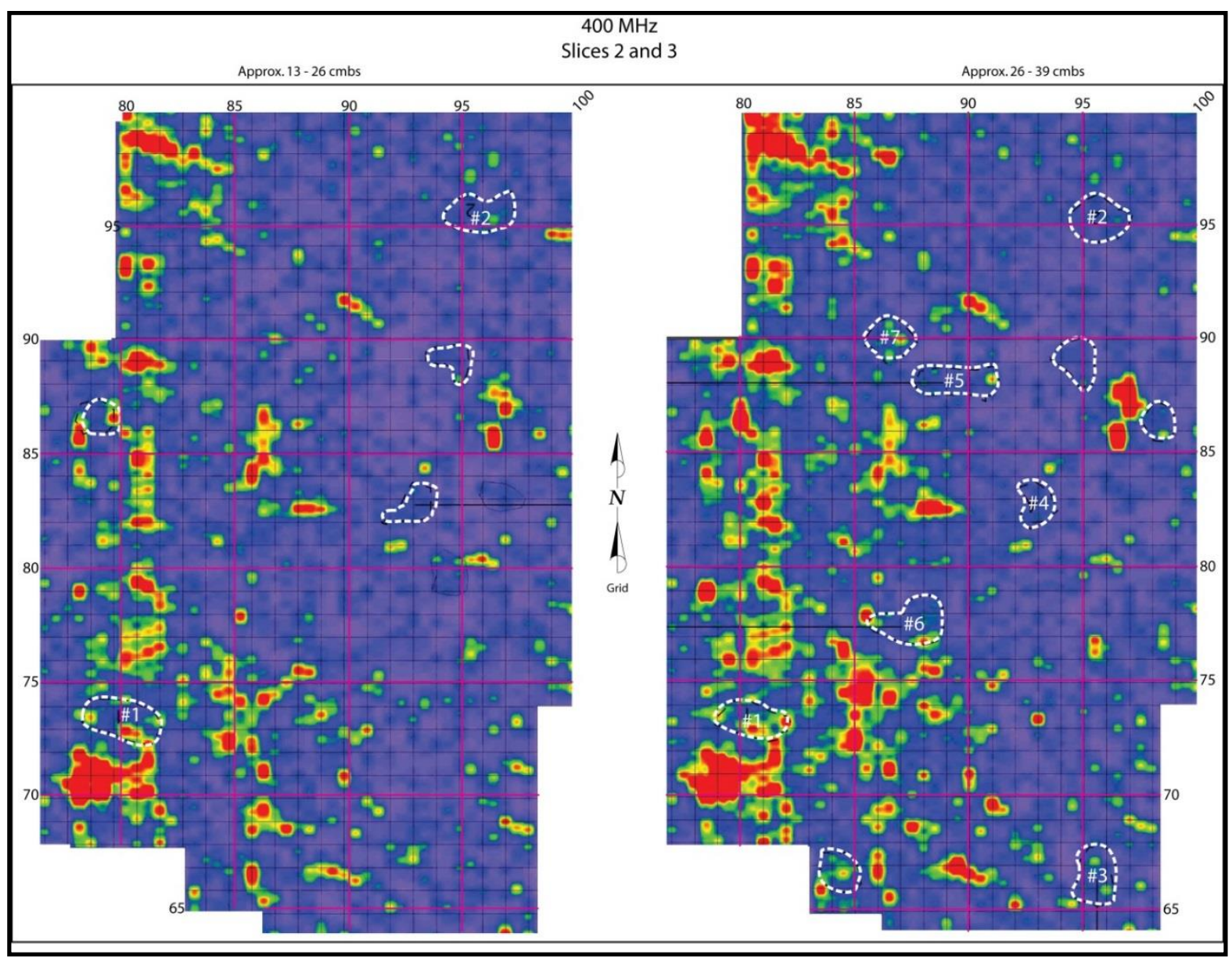

\section{Figure 5-6. Examples of ground penetrating radar scans from the $400 \mathrm{MHZ}$ antenna showing anomalies from 13 to $26 \mathrm{~cm}$ and 26 to $39 \mathrm{~cm}$ below stripped surface of investigation block.}

As hand-excavations proceeded, natural and cultural stratigraphy and the vertical distribution of cultural materials was revealed, and two significant cultural components were identified. Materials between 210 and $250 \mathrm{cmbs}$ became the principal target zone in Block B, which was expanded horizontally to capture this upper component in one large continuous block of $51 \mathrm{~m}^{2}$. One burned rock cluster encountered during mechanical stripping was initially left in place at roughly $210 \mathrm{cmbs}$ as it appeared slightly higher in elevation than most other encountered features nearby. Eventually, this burned rock cluster was assigned Feature 31 and hand-excavated.

In the southern part of Block B, 12 units were handexcavated to $290 \mathrm{cmbs}$ to sample a cultural component. The southernmost units encountered an in situ organic stained hearth (Feature 26) below 260 cmbs, and units north of Feature 26 were excavated to $290 \mathrm{cmbs}$ to capture part of that occupation in the time allotted. This occupation zone was stratigraphically below the upper occupation separated by a thin, mottled light greenish-gray clay lens.

Block $\mathrm{C}$ was initiated in the southeastern corner of the stripped area and targeted the third highest ranked anomaly (Anomaly 3) (see Figures 5-6 and 5-7). The mechanically stripped surface and the starting depth for hand-excavations varied from roughly 240 to 250 cmbs. This depth was stratigraphically below the upper, principally targeted cultural zone in Block B, but above the lower occupation sampled in Block B. 


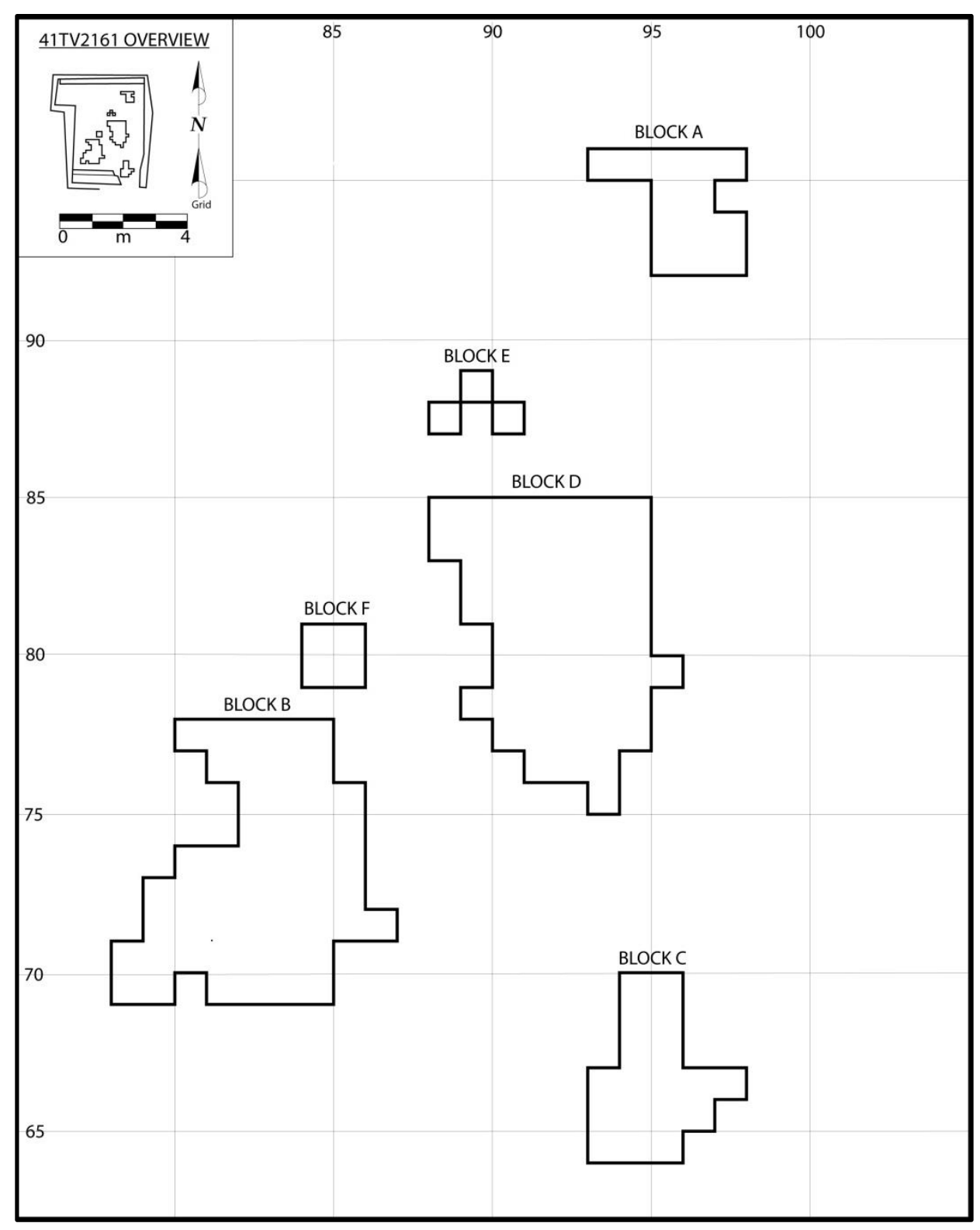

Figure 5-7. Plan map and distribution of completed hand-excavated blocks within mechanically stripped area.

A total of $18 \mathrm{~m}^{2}\left(7.2 \mathrm{~m}^{3}\right)$ was hand-excavated with most units dug with five to six $10 \mathrm{~cm}$ levels to a depth of $300 \mathrm{cmbs}$. Exceptions were a few units with only 1 or 2 hand-excavated levels in northern part to expand, follow and assess the burned area (Feature 21) encountered.

Block D, in the central area, targeted the fourth highest ranked anomaly (Anomaly 4) (see Figures 5-6 and 5-7). The mechanically stripped surface varied between 240 and 255 cmbs. Again, this depth was below the upper occupation targeted in Block B. Hand-excavations encountered a relatively well-defined occupation zone; therefore, Block D was expanded horizontally to $62 \mathrm{~m}^{2}$ to capture those cultural materials. The horizontal expansion was conducted in one to three levels across most of Block D. This component appeared relatively well-defined, intact with some slight vertical displacement of artifacts, and exhibited horizontal patterning of cultural materials 
associated with two small, dark stained cultural hearth features (Features 25 and 33). This same component was encountered in the lower levels in Block B associated with Feature 26. This cultural zone was apparently distributed across a wide area, although at slightly different depths in most handexcavated units. The cultural zone detected in Block D appeared just below a thin, mottled light greenish-gray clay lens as was the case in Block B.

Block E was located $2 \mathrm{~m}$ north of Block D (see Figure 5-7). This area targeted the fifth highest ranked anomaly (Anomaly 5) with only $3 \mathrm{~m}^{2}$ noncontiguous units excavated $\left(1.2 \mathrm{~m}^{3}\right)$. Lack of a recognizable feature and few artifacts curtailed expansion of this excavation block.

Block $F$ was on the northern edge of Block B targeted another GPR anomaly (Anomaly 6) (see Figure 5-7). The block lacked any clear sign of a cultural feature and the excavation was terminated with only $4 \mathrm{~m}^{2}\left(0.6 \mathrm{~m}^{3}\right)$ excavated.

Cultural materials were vertically distributed, starting from the mechanically stripped surface at roughly 220 and 260, to minimally $300 \mathrm{cmbs}$ in most localities where hand-excavations extended to that depth. No excavations penetrated below 300 cmbs, although cultural materials appeared to continue below that depth in multiple blocks. As previously stated, initial hand-excavation units directed at multiple electronic anomalies dispersed across the stripped area were subsequently expanded horizontally as the cultural and natural stratigraphy became clearer to capture horizontal zones of cultural material.

As indicated above in the different excavation blocks, well-defined cultural zones or components were recognized and followed within the excavation parameters. Diagnostic projectile points were recovered from each major zone. The upper component contained two Bell/Andice (B/A) and a Big Sandy point, whereas the lower component was associated with an Early Cornernotched $(\mathrm{CN})$ point later verified as a Martindale point. Consequently, in the field and many original references to the lower component employed the term corner-notched $(\mathrm{CN})$. Therefore, the Martindale/Early Corner-notch $(\mathrm{M} / \mathrm{CN})$ reference refers to the exact same component.

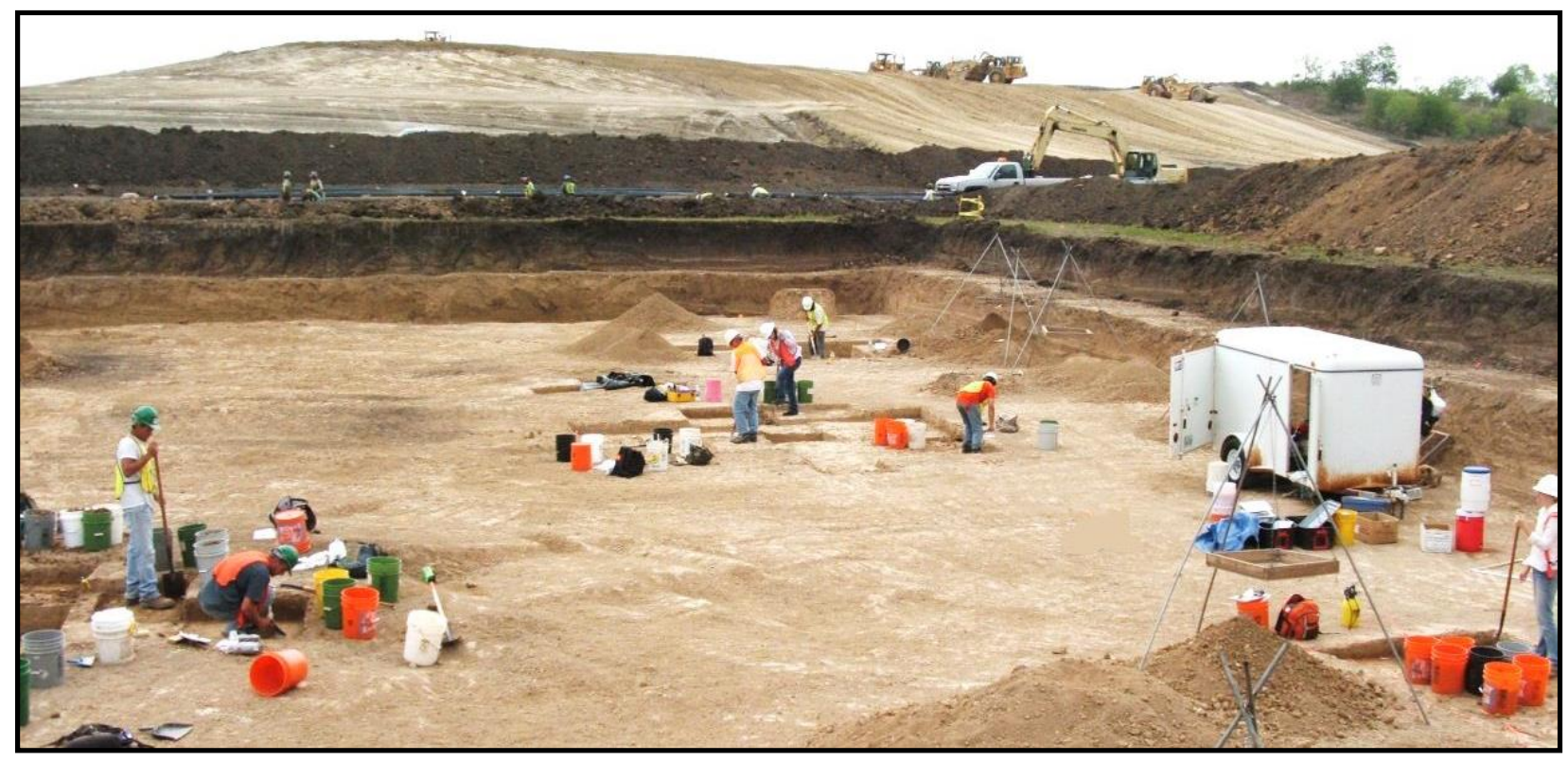

Figure 5-8. Northern view of ongoing hand-excavations at Blocks $B$ (left) and C (right) in the foreground, Block $D$ (in center), and $A$ in far corner. 


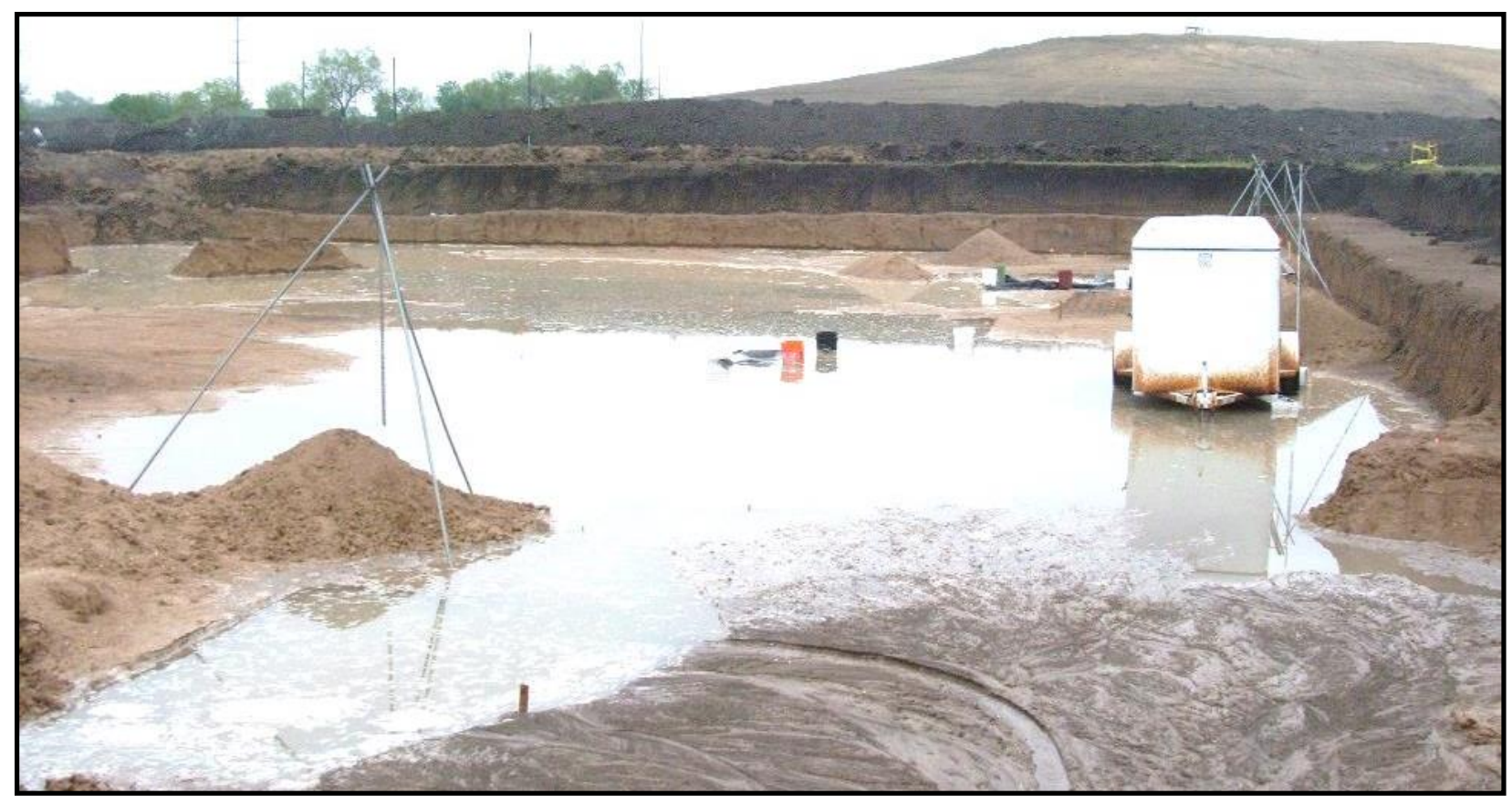

Figure 5-9. Northern view of flooded excavation area.

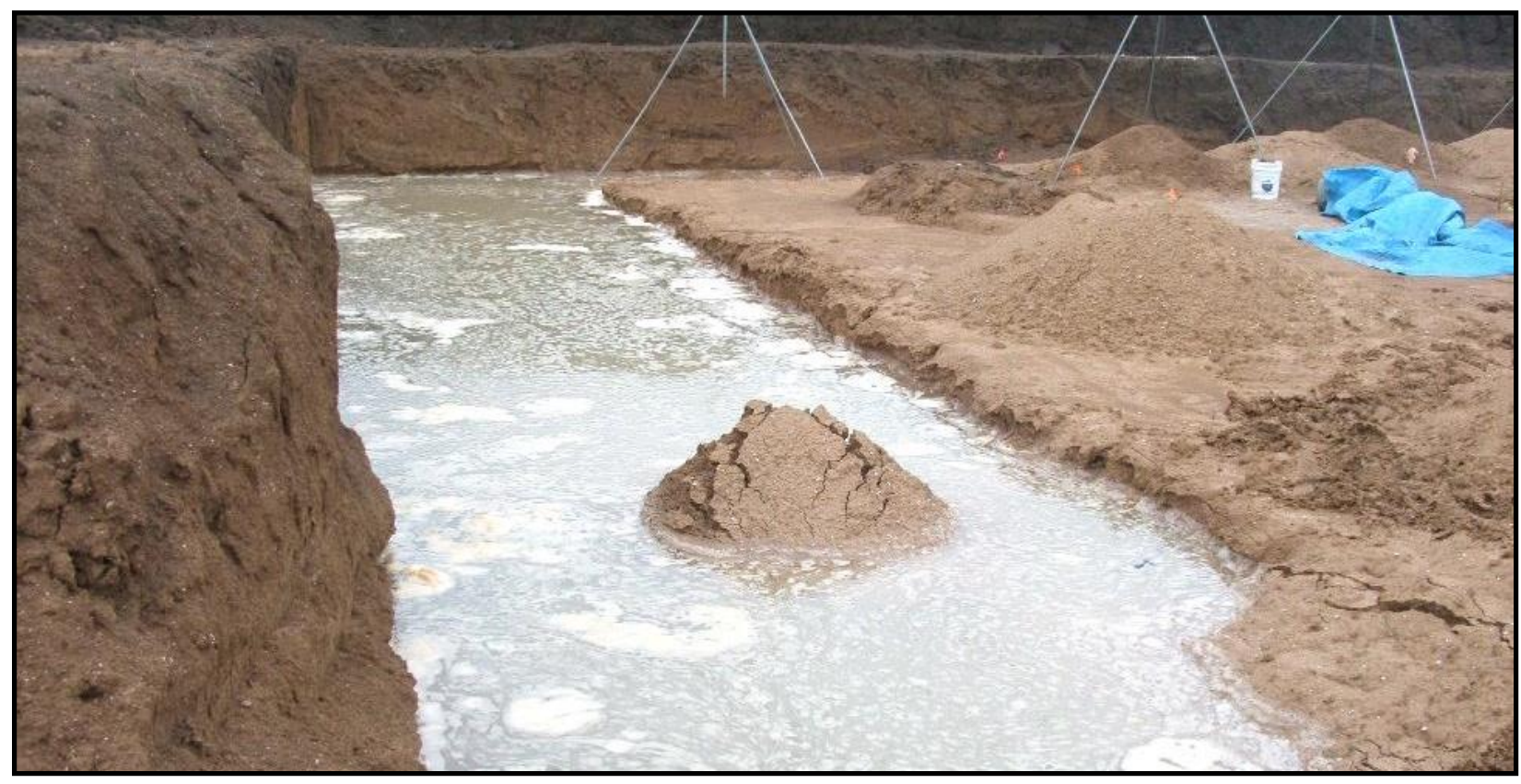

Figure 5-10. Western view of water filled trench along southern margin of excavation block. 
As required by TxDOT staff, the information retrieved from the first $10 \mathrm{~m}^{3}$ of hand-excavation was to be reviewed by TxDOT personnel in the field to determine if further excavations were warranted. That review entailed an onsite inspection by two or more TxDOT staff archeologists and/or supervisors, led by Jon Budd, to witness first-hand what had been recovered and to evaluate the context of materials. Following the first review, TRC was directed to excavate another $10 \mathrm{~m}^{3}$. This review process occurred three times with a maximum effort allocated by TxDOT staff not to exceed $40 \mathrm{~m}^{3}$. In total, $38.5 \mathrm{~m}^{3}$ or $150 \mathrm{~m}^{2}$ were hand-excavated in six separate blocks dispersed across the deep, mechanically stripped pit (Figure 5-8). During April and May 2006, fieldwork was interrupted because water from of intense rainstorms flooded the stripped area and filled the emergency water trenches (Figures 5-9 and 5-10). This not only stopped work for multiple days, but upon return, opened units had to have water bailed out to speed drying. The lingering damp conditions also hindered detection of potential cultural stains in and below opened water saturated units. The fieldwork was completed on May 4, 2006 and artifacts and equipment were returned to TRC facilities.

\subsubsection{Recovery Methods}

Horizontal control was maintained through handexcavation of 1-by-1 m units from the stripped surface in mainly $10 \mathrm{~cm}$ arbitrary levels with excavated sediment screened through $6.4 \mathrm{~mm}(0.25$ in.) hardware cloth. Some levels were handexcavated in 5 and $15 \mathrm{~cm}$ levels in specific areas and under certain circumstances. Depths and levels were measured from the original ground surface and measured in cmbs. When sizable pieces of cultural materials or features were encountered in situ, larger items were generally piece potted on TRC excavation level records and bottom elevations of objects were measured and recorded. A TRC excavation level form was completed for each hand-excavated and screened level. Level forms documented provenience information, starting and stopping depths below surface, excavation techniques employed, observations concerning sediment types, and types and quantities of cultural materials and samples from that level.

Recovered cultural materials (except for burned rocks) were counted and recorded on the level records, and bagged by unit and level in labeled, resealable plastic bags. As levels were completed, bagged and tagged materials were deposited in field boxes. Once full, boxes were transported to TRC's Cultural Resources laboratory in Austin for temporary storage. Burned rocks were generally sorted into pre-established size categories (i.e., 0 to $4 \mathrm{~cm}, 4.1$ to $9 \mathrm{~cm}, 9.1$ to $15 \mathrm{~cm}$, and greater than 15 $\mathrm{cm})$, counted, and weighed in the field, the data recorded on level record forms, and subsequently were discarded. Samples of burned rocks from feature and non-feature contexts were collected and transported to the laboratory for possible analyses and/or curation.

A running count of material classes was compiled from individual level records and generally checked against bag contents. Materials were initially divided into two broad recognizable components (Bell/Andice and Martindale/Early Corner-notch) with a vertical dividing line roughly estimated at $250 \mathrm{cmbs}$. This information was used to inform TxDOT personnel of the ongoing field results and guide block expansion.

Clusters of cultural materials recognized in excavation units were assigned feature numbers and recorded on appropriate level records plus a separate TRC feature form. TRC started numbering features at 15 , to ensure no overlap in numbering with previous work. Features were assigned numbers as they were encountered rather than sequential in the investigated areas or assigned by area. TRC's hand-excavations documented Features 15 through 33 . The feature recognized in a unit was drawn, digitally photographed, and artifact and sediment samples from the feature collected. 
Feature boundaries were subjectively determined and materials from inside feature boundaries were separated from materials beyond the feature. In most instances the feature was cross-sectioned (Figure 5-11) and sediment from the feature was collected for further processing in the laboratory rather than screened in the field.

Those features pedestalled (Figure 5-12) above the targeted components were assigned numbers from 50 and up to distinguish them from target zone features. Limited field time was dedicated to the features above the targeted zone.
Sediment samples were collected from within cultural deposits (i.e., feature context and occupation zones) with the intent to recover macrobotanical remains. Collection of charcoal and other macrobotanical samples from any and all proveniences was emphasized in anticipation that these were scarce and extremely important. Individual charcoal and macrobotanical samples were piece plotted, and collected as encountered with the goal to identify and gain understanding of woods used for fires, contribute to understanding the local environment, and potentially radiocarbon date.

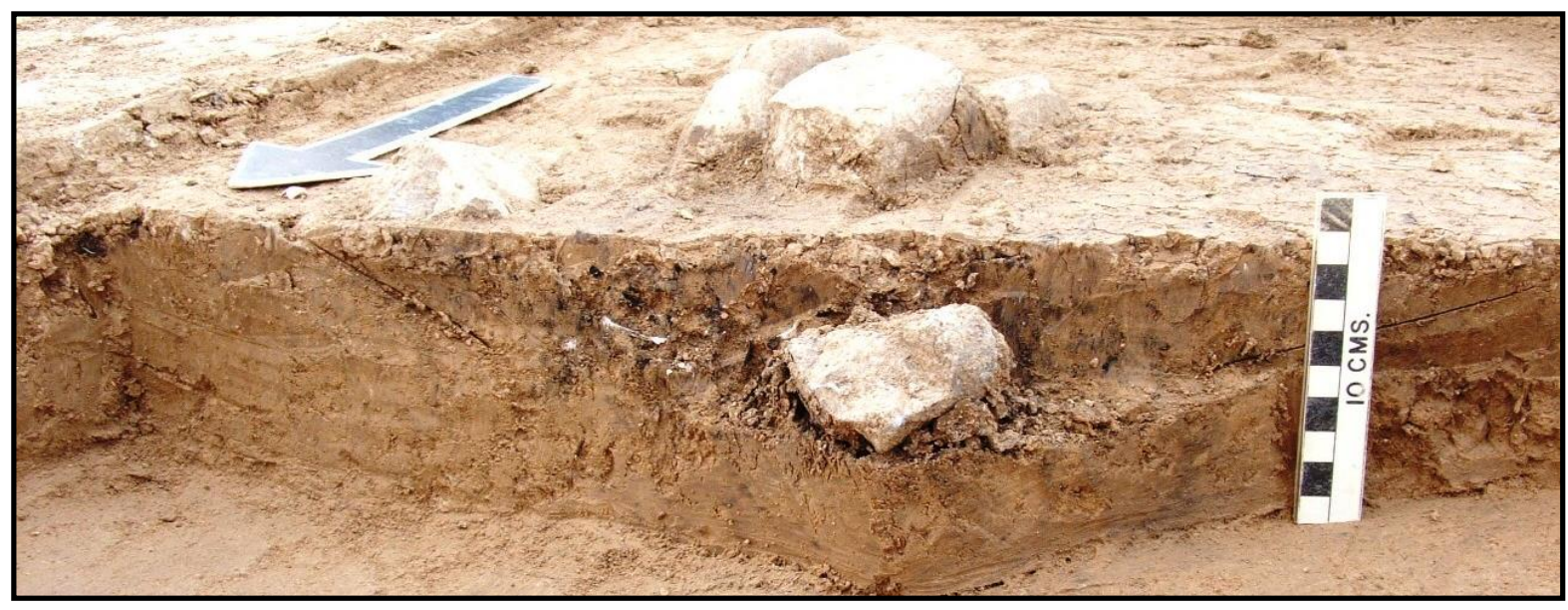

Figure 5-11. Example of profiled burned rock feature.

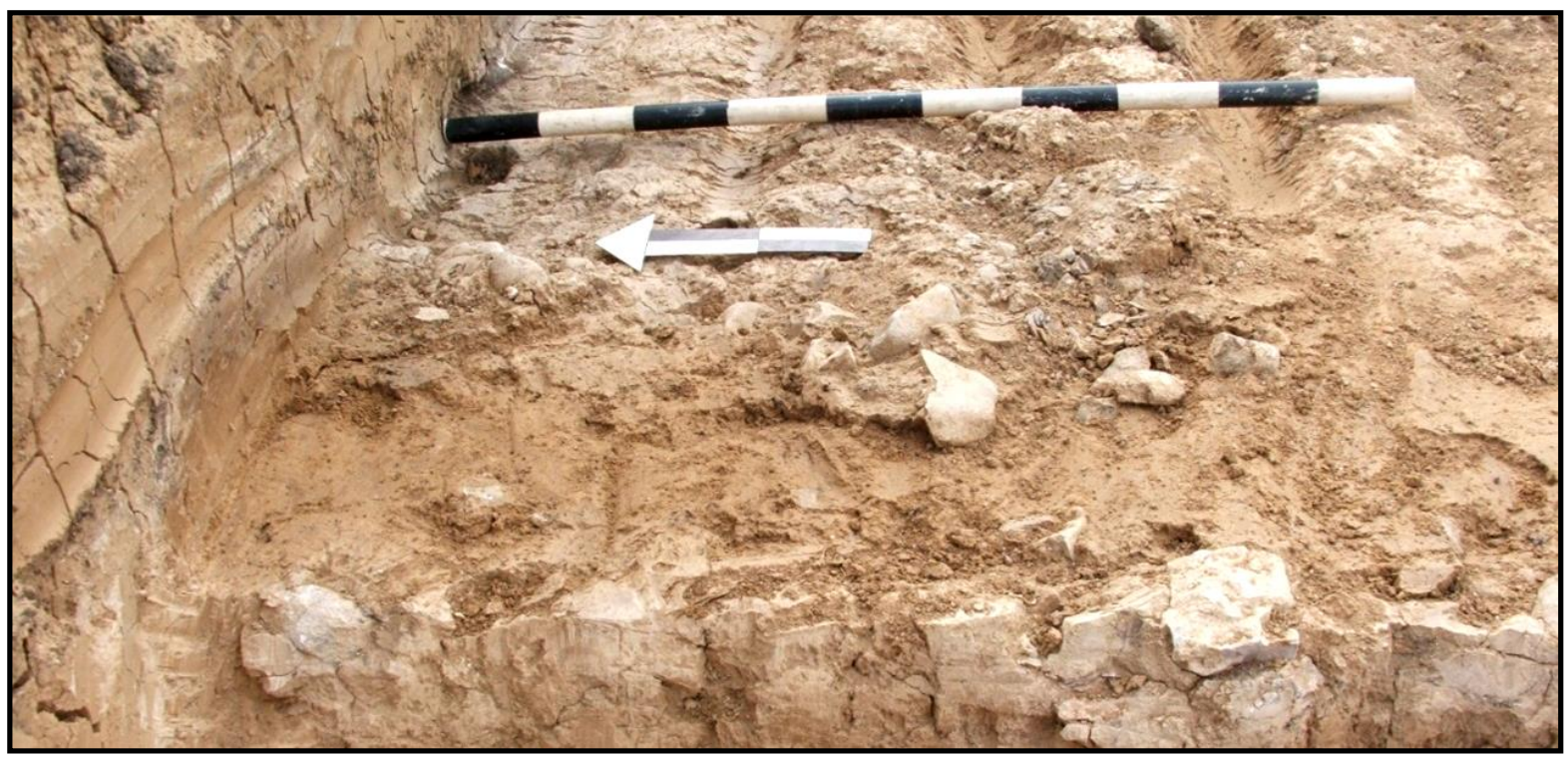

Figure 5-12. Example of burned rock feature encountered and pedestalled during stripping. 


\subsubsection{Stratigraphic and Sediment Sampling Column}

Along the south wall of the stripped area and towards the western side, a vertical column through the targeted cultural zone was cleaned and sampled (Figure 5-13). Geoarcheologist Eric Schroeder used this location to identify and describe the deposits as discussed in the geoarcheological Chapter 6.0. Sediments collected from this vertical profile were also analyzed for diatoms, starch, phytoliths, particle size, and soil chemistry. Samples were collected at roughly $10 \mathrm{~cm}$ increments numbered from 0 at $250 \mathrm{cmbs}(\# 2400)$ through sample 11 at $360 \mathrm{cmbs}$ (\#2411).

\subsection{LABORATORY PROCEDURES AND TECHNICAL ANALYSES}

Laboratory artifact processing included the initial check-in and sorting of bags as necessary. Before washing, all bags of lithic debitage were examined for both formal and informal tools, including flakes with modified edges. All identified stone tools were handled with nitrile gloves, bagged separately, and only cleaned where necessary to apply archivally stable provenience labels. These unwashed artifacts were projected for future usewear and/or residue analyses. Most other materials were washed, labeled, bagged, sorted into material classes, accessioned with catalog numbers assigned to each level and subcategory, and represented in an electronic database.

All provenience data available and any pertinent data from level records or collection bags were entered into a Microsoft Access format database at initial artifact check-in. TRC's cataloging system assigns strings of numbers to artifacts that encode, as a unique identifier, provenience information, artifact class, as well as denoting any subsamples extracted from the artifact(s) for technical analyses. All cultural materials were assigned Provenience Numbers (PNUMs) by site and entered into an electronic database. These unique PNUMs (e.g.,
\#250) were assigned to individual excavation levels, as well as any additional provenience, such as feature designations. PNUMs are sequential integers that designate the overall provenience unit (e.g., excavation unit, backhoe trench, modern ground surface), level and/or depth within that provenience unit, and can be cross referenced to a master list of PNUMs.

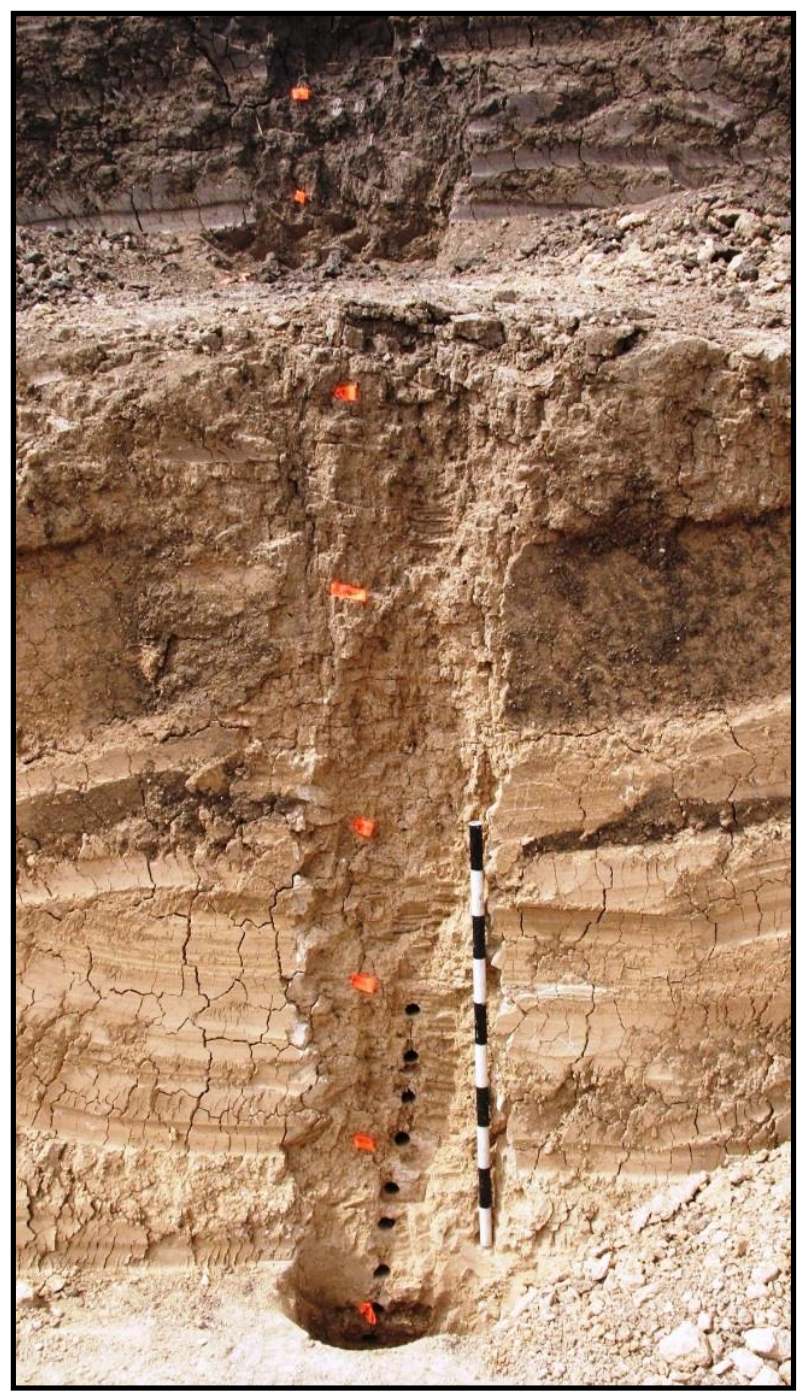

Figure 5-13. View south of cleaned and sampled vertical stratigraphic column through the targeted deposits with the top collection hole at $250 \mathrm{cmbs}$. Note the visible safety bench at the boundary of the dark soil (above), whereas the smooth flat area (foreground) is the base of the emergency water collection trench (scale is 1 meter in $10 \mathrm{~cm}$ increments). 
Within each PNUM, various artifact classes were assigned a secondary designation referred to as the artifact category number: lithic debitage (001), faunal bone (002), burned rock (003), soil samples (004), organic matter (005), shell (006), macrobotanical remains (007), ceramic sherds (008), and historic material (009). Unique items, such as tools, were assigned individual artifact identifiers by excavation unit and level, starting with the number 10 and increasing sequentially as needed for that particular provenience.

Therefore, individual tools were assigned a PNUM and an individual unique number appended to the PNUM (e.g., \#250-10, \#250-11, and \#250-12).

In selected instances, individual burned rocks were removed from the collection for technical analyses that included lipid residue and starch grain analyses. Burned rocks selected for analyses were broken into multiple pieces for multiple types of analyses. For example, a burned rock collected from within a specific provenience unit (\#250) is coded by class of artifact (003) in sequential order. The first burned rock from the provenience was assigned \#250-003-1 as the catalog number. If burned rock \#250-003-1 was subdivided into two pieces for two different types of analyses, such as lipid residue and starch grain analyses, then lower case letter designations (e.g., a and b) would be added after the last number in the sequence (i.e., \#250-003-1a and \#250-003-1b) to signify that two parts were removed from burned rock \#250-003-1. The complete two- or three-part number sequence assigned to each object or class of objects constitutes the accession number. This process allows individual pieces of large collections of various materials to be individually handled and tracked without risk of loss of provenience information.

About one in ten items (10 percent) in bulk material classes (e.g., chert debitage, mussel shells, faunal bones) within specific provenience units (e.g., a unit/level) were individually labeled as per Council of Texas (CTA) curation guidelines. Specimen size was also a major consideration for labeling purposes, as many lithic pieces are less than $1.0 \mathrm{~cm}$ in diameter and were not labeled. Artifact labeling consisted of inscribing the State of Texas Archeological Site Trinomial (41TV2161) and the PNUM number on designated artifacts using black indelible ink. After the ink was dry, artifact labels were coated with clear Acryloid B-72 with reagentgrade acetone solvent to preserve inscriptions.

Permanent paper tags were included with each individually bagged artifact or class of artifacts collected from a single provenience. Tags contain the site trinomial, provenience information (unit and depth), the class or type of artifact(s), the date of excavation, the excavator's initials, and the quantity of items in the bag. Tags were printed on acid-free, $30.4 \mathrm{~kg}(67 \mathrm{lb}$.) card stock and filled out with pencil.

\subsubsection{Technical Analyses}

In order to assess the potential for specific types of technical analyses to yield interpretable results and contribute towards addressing questions developed in the initial research design, TRC staff proposed the following six exploratory analyses. TxDOT staff agreed to allocate limited funds for soil chemistry, starch grain, fatty acid, phytolith, diatom, and pollen analyses. Additional samples were submitted and analyzed if the technique proved productive and TxDOT agreed to move forward. These analyses were conducted by highly trained professional individuals and technical institutions that provided their expertise and recommendations, and their results are included in attached appendices.

\subsubsection{Soil Chemistry Analysis}

TRC collected quantities of dark stained sediment from cultural feature contexts associated with heating and/or cooking features (i.e., hearths, burned rock concentrations, and burned locales). TRC's initial field interpretation was the dark 
sediment was degraded charcoal. TxDOT staff, however, had concerns over this identification as multiple dark stained sediment samples from Feature 3 originally sent to Beta Analytic (Beta) in Miami, Florida for radiocarbon analysis by Hicks \& Company in 2006 did not yield datable material. Given the lack of radiocarbon results from black stained sediment previously, TxDOT staff questioned whether or not the quantities of dark stained sediment collected by TRC was actually carbon. A small stained sediment sample from our excavations was sent to and examined by TxDOT archeologists, and the black matter did not float and had granular properties, which is not consistent with charcoal suitable for radiocarbon analysis.

To address the question as to what the black matter was, TxDOT required sediment samples to be sent to professional laboratories to determine the organic content. Two samples of dark stained sediment from two apparent intact cultural hearths were selected for analysis by the Walkley-Black method to e-Lab in Houston. The Walkley-Black acid digestion method has been employed since the 1930s and employs chromic acid to measure oxidizable organic carbon in a soil. This method has greater accuracy on soils with less than 2.0 percent organic matter. In situations of high soil organic matter, this method may result in low test results due to uneven oxidation of organic carbon in the sample (Agvise Labs 2007). TRC submitted two sediment samples (\#2223-4 from Feature 29 and \#2112-4 from Feature 26) to e-Lab and ten sediment samples to Milwaukee Soil Laboratory, LLC for chemical analysis (Table 5-1). In the event that the dark stained sediment samples proved positive for organic content, TRC proposed that Beta Analytic (Beta) in Miami, attempt to derive radiocarbon dates from similar dark sediment samples (see Appendix $\mathrm{M}$ for laboratory assessments).

\subsubsection{Starch Grain Analysis}

Starch grain analysis has become more widely employed in Texas archeology to identify plant resources used by early inhabitants (see Perry and Quigg 2011; Quigg 2011; Quigg et al. 2010, 2011a, 2013a, 2013b, 2014a, 2014b). A brief introduction is presented here as background to this discipline for those unfamiliar with this technique.

Starch grains are microscopic granules that serve as the principal food storage mechanism of plants (Figure 5-14). They primarily occur in roots, tubers (e.g., crow poison, rain lilies, false garlic, wine cup, and spring beauty), seeds of legumes, and grasses,

Table 5-1. Sediment Samples Sent to Milwaukee Soil Laboratory, LLC for Analyses.

\begin{tabular}{|c|c|c|c|}
\hline $\begin{array}{c}\text { Sample/ } \\
\text { PNUM No. }\end{array}$ & Unit N/E & $\begin{array}{c}\text { Depth } \\
\text { (cmbs) }\end{array}$ & $\begin{array}{c}\text { Feature } \\
\text { No. }\end{array}$ \\
\hline $2092-4$ & $71 / 80$ & $230-240$ & 30 \\
\hline $2112-4$ & $71 / 83$ & $270-280$ & 26 \\
\hline $2143-4 b$ & $72 / 83$ & $270-280$ & NA \\
\hline $2179-4$ & $73 / 86$ & $215-220$ & 24 \\
\hline $2223-4$ & $76 / 86$ & $220-230$ & 29 \\
\hline $2239-4$ & $78 / 83$ & $255-268$ & 25 \\
\hline $2367-4$ & $95 / 96$ & $270-280$ & 19 \\
\hline $2400-4 b$ & South Profile & 248 & NA \\
\hline $2402-4 b$ & South Profile & 270 & NA \\
\hline $2414-4$ & $79 / 93$ & $254-266$ & 25 \\
\hline
\end{tabular}




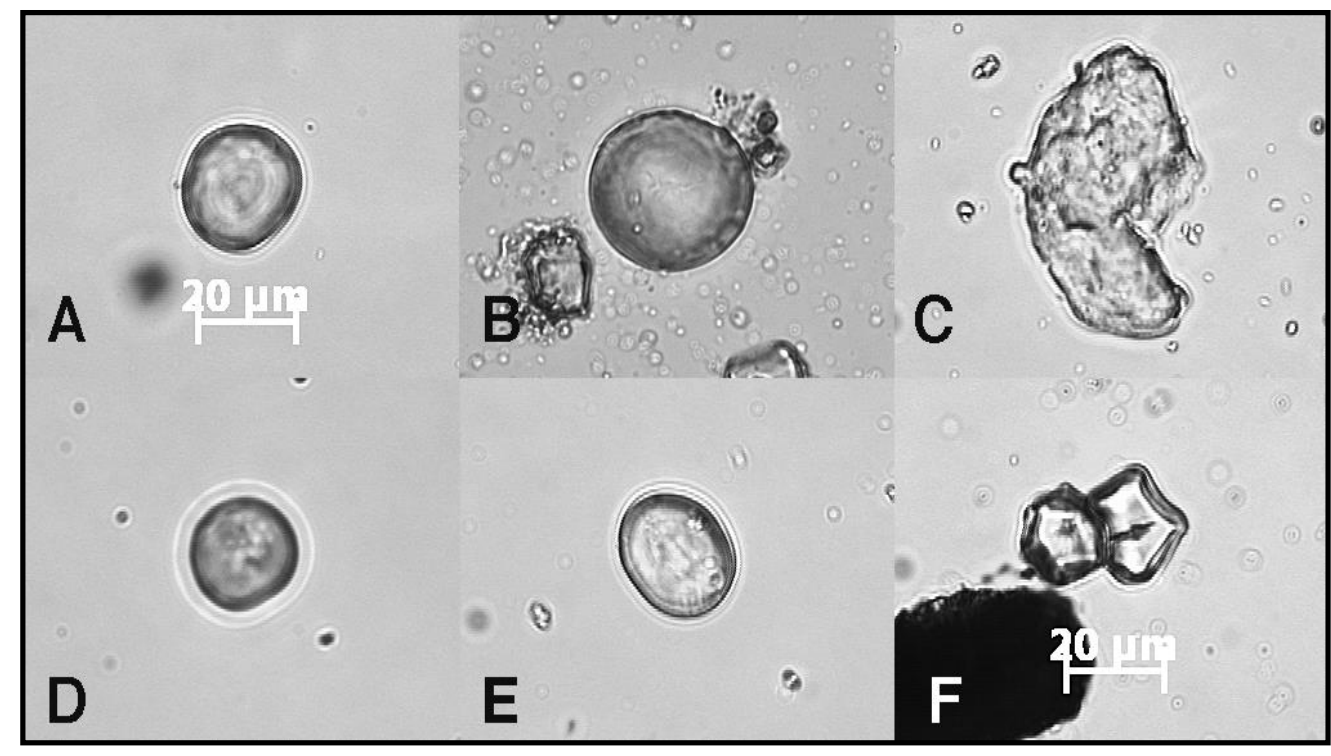

Figure 5-14. Examples of starch grains of wildrye grass recovered from burned rocks at an archeological site in Texas (photograph by Linda Perry).

where they are often produced in abundant numbers. Starch grains from different plants possess distinctive forms recognized for some time. Distinctive features of storage starch grains are genetically controlled and when carefully observed, are employed to identify plant taxa (e.g., Denniston 1907; MacMasters 1964; Reichert 1913). Minimally 300 species and varieties of important economic plants from around the world are described using this technique. In recent years there has been widespread acceptance that these materials do survive (Piperno and Holst 1998; Piperno et al. 2000). Since then researchers from around the world (particularly in the neotropics and in Australia) use these techniques with excellent results (Perry personal communication 2007). Specifically, starch grain remains have significantly increased the knowledge of plant domestication and crop plant dispersal in various regions (Perry et al. 2006:76-77). Researchers have employed starch grain analyses to study diet, plant processing, plant domestication and cultivation, tool use, and in ceramic residue analysis. Starch grains extracted from soil samples, ceramics, and chipped and ground stone tools are employed to address questions of resource procurement and food preparation. Intact starch grains have been extracted from formal and informal chipped tools, both washed and unwashed (Perry personal communication 2007). Starch grains have survived on tools from the Central American tropics for minimally 7,000 to 8,000 years (Piperno and Holst

1998). Starch grain presence in soil depends on preservation. Heat alone does not destroy starches as they are present in vessels, therefore burned rocks also have potential to yield starch grains.

Initially, presence or absence of starch grains was conducted by Dr. Linda Perry affiliated with the Smithsonian Institution on ten samples that included three ground stone tools, three chipped stone tools, three burned rocks, and one sediment sample. Based on the initial positive results, subsequently 42 additional samples were submitted for analysis. This latter suite included another 24 burned rocks and 10 stone tools divided between the Bell/Andice component (7 stone tools and 29 burned rocks) and the Martindale component with 3 stone tools and 13 burned rocks. Perry's procedures and individual findings are incorporated into Appendix F. 


\subsubsection{Fatty Acid Analysis}

Fatty acids are major constituents of fats and oils (lipids) and occur in nature as triglycerides, which consist of three fatty acids attached to a glycerol molecule by ester connections. Fatty acids are insoluble in water and relatively abundant as opposed to other classes of lipids, such as sterols and waxes, making fatty acids suitable for residue analysis. This proxy line of investigation is critical when environmental conditions lack conducive preservation of primary organic data, such as macrobotanical remains and vertebrate materials.

This chemical approach has been employed in Texas since about 2000 (Malainey 2000; Quigg and Cordova 2000) and in other areas. It has successfully demonstrated that organic residues are present and can be extracted and generally interpreted from burned rocks employed by prehistoric peoples to process foodstuffs (cf. Malainey 2003; Malainey and Malisza 2002, 2008; Quigg et al. 2000). Interpretations present only a general indication of what is chemically represented (i.e., plant, animal, or plant and animal) rather than precise species or taxa. The fatty acid analysis provides chemical results to help identify general food groups or types of resources cooked by burned rocks. This allows feature function to be addressed, what the rocks cooked, and what foods were processed.

Chunks of burned rocks, weighing from 9 to $80 \mathrm{~g}$, were broken from parent rocks for submission. The parent rock was retained and curated for future reference. Analyzed burned rocks were primarily from burned rock features in identified components.

Initially, back in 2006, 15 samples that included 12 burned rock fragments from Features 18, 20, 24, 26, 29, 30, 31, and 33 plus 3 ground stone fragments, were submitted to Dr. Mary Malainey of Brandon University for analysis. That initial report on the results from burned rocks from eight features and three ground stone tools was first reported in Quigg et al. (2007).
Since 2010, additional sophisticated techniques have been incorporated into lipid residue analysis by Dr. Malainey to improve and add accuracy to interpretations. The incorporation of high-temperature gas chromatography (HT-GC) and gas chromatography with mass spectrometry (GC-MS), to identify specific biomarkers have significantly enhanced residue identifications and interpretations. Based on positive results from 10 of 15 initial samples analyzed in 2007, the 10 positive plus 23 additional burned rocks, 15 from the Bell/Andice component and 8 from the Martindale component, from Features 16, $20,24,26,27,28,29,31,32$, and 33 were submitted for lipid analysis. The newer techniques were employed on all samples. Malainey's laboratory procedures, background or residues, and individual sample results are in Appendix G.

\subsubsection{Phytolith Analysis}

Phytolith studies are important in reconstructing grassland profiles in local environments through time. The Opal silicate bodies that compose phytolith entities form within plant cells. Distinctiveness of various types of bodies varies within cellulose structure. In grasses, however, phytoliths exhibit diversity and distinctiveness per grass species. The presence of certain phytolith groups (e.g., panicoids, festucoids, and chloridoids) in the paleoenvironmental record can enlighten researchers to general vegetative conditions such as forested habitats versus those of open grassland prairie.

In order to provide direction for conducting further phytolith analysis, preliminary assessments of phytolith presence were conducted. Nine matrix samples, three from the mottled greenish-gray clay lens at different locations, three from cultural Features 18,22 , and 26 , and three from the sediment column at the southern edge of the stripped area were submitted to and analyzed by Dr. Steven Bozarth. His presence absence findings and recommendations are presented in Appendix K. Based on Bozarth's positive findings and recommendations additional phytolith analysis was undertaken. A suite of 33 sediment samples were 
submitted to and analyzed by Dr. Byron Sudbury. The samples included material collected from Martindale cultural Features 26 and 33, Bell/Andice cultural Features 22, 24, 27, 28, 29, 30, and 32, three unassigned Features 18, 19 and 25, plus 12 samples from a vertical column through the two primary occupations. His detailed procedures and findings are presented in Appendix E.

\subsubsection{Diatom Analysis}

Diatoms are single celled algae with a siliceous cell wall and provide data concerning water and/or wet conditions. They grow in a wide range of aerophilous habits that include damp soils, wet plants and rocks, marshes, wetlands and mudlands, as well as in all types of aquatic habitats. Their silica cells are often preserved in sedimentary deposits. Because individual taxa have specific requirements and preferences with respect to water chemistry, hydrologic conditions and substrate characteristics, the presence of diatoms in archeological context can provide paleoecological insights concerning the local environments. Diatoms, when present, provide a proxy measure of water quality and degree of pollution and ultimately paleoenvironmental conditions. Ten samples were selected and submitted to Dr. Barbara Winsborough in Austin for assessment. These ten samples included three from the mottled greenish-gray clay lens, four cultural features, and three from a vertical natural column. Dr. Winsborough's initial results, interpretations, and recommendations were presented in Quigg et al. (2007). Insights obtained from the initial results led to subsequent diatom analysis of a total of 33 samples. Seven were from the Bell/Andice component and 13 were from the Martindale/Early Corner-notched component, whereas others were from multiple proveniences. Dr. Winsborough's background concerning diatoms, methods, and results are incorporated into Appendix C.

\subsubsection{Pollen Analysis}

Palynomorph spectrums can be derived from cultural and noncultural settings when reconstructing plant communities. Changes in pollen profiles can often be interpreted reliably as corresponding to changes in general character of an area's vegetation. Pollen may represent both cultural and natural environments and lead to discussions concerning the overall environment during the occupation. A suite of six samples, three ground stone tools and three sediment samples from the mottled greenish-gray clay lens were submitted to Dr. Steven Bozarth to determine presence or absence of pollen. His results and recommendations are presented in Appendix L. Basis on Bozarth's initial findings and recommendations no further pollen analysis was attempted.

\subsubsection{Additional Technical Analyses}

In conjunction with the initial six technical analyses employed to address feasibility and guidance for further analyses, other analytical techniques were subsequently implemented to extract detailed information from collected data. These include the following diverse techniques, which are briefly addressed below.

\section{Radiocarbon Dating}

Charcoal, the preferred material for radiocarbon dating, was sparse and often limited to tiny degraded flecks (Figure 5-15). Consequently, other organic substances with potential datable residues were employed to obtain absolute dates. Organic materials trapped in sediments, bones, snail shells, and sandstone burned rocks, may yield dates, although the obtained date may not provide as precise date as what one might obtain from charred seeds or wood. Direct dating of bones and Rabdotus snail shells has been previously accomplished with mixed results (see Frederick 2011 for an example of variable dates from the 


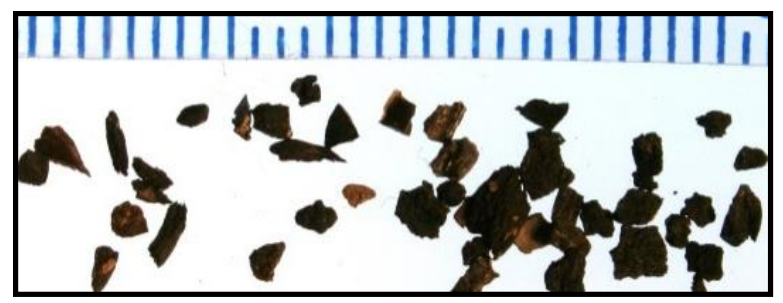

Figure 5-15. Example of tiny charcoal pieces submitted for radiocarbon dating (scale in millimeters).

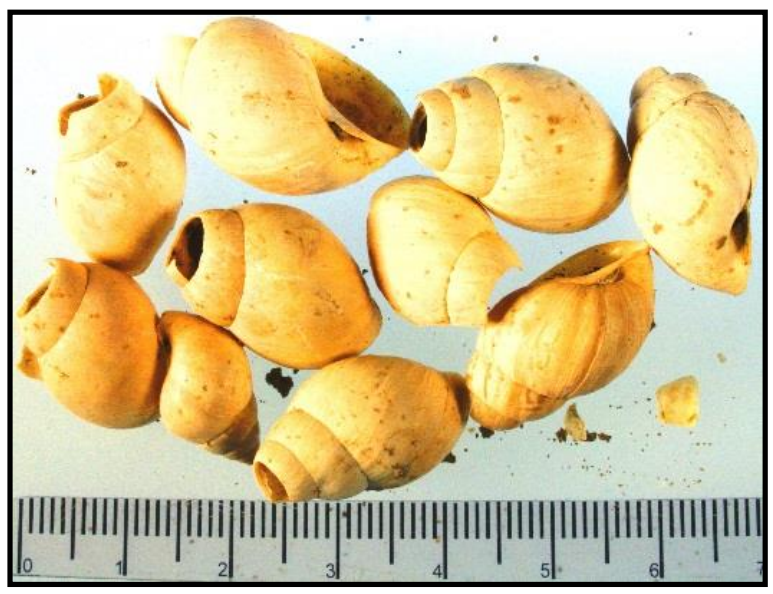

Figure 5-16. Example of Rabdotus snail shells with apex removed and submitted for radiocarbon dating (scale in centimeters).

area). Consequently, one must view dates derived from noncharcoal as approximate dates, rather than a narrowly definable point in time.

Following the 2006 fieldwork, TRC archeologists selected 11 samples (5 Rabdotus samples, 4 animal bones, and 2 burned hackberry seeds) for direct radiocarbon (C-14) dating. A request letter with justification of 11 samples was sent to Dr. James Abbott of TxDOT for approval. Once TxDOT approved the samples, they were prepared and laboratory forms completed by TRC staff and submitted to TxDOT. TxDOT submitted the 11 samples to Beta Analytic (Beta) in Miami, Florida for processing. The apex of each snail shell was removed before submission (Figure 5-16). Results from the 11 submitted samples varied (Quigg and et al. 2007) and guided selection of subsequent samples.
Two hackberry seed samples (\#M-6 and \#M-7 from Blocks A and C) totally dissolved during sample preparation as did two animal bone samples (\#M10 a deer ulna and \#M-11 a deer size long bone fragment, both from Block $\mathrm{B}$, one each per component) and yielded no dates. Two other bone samples (\#M-8 [\#2064-2a] a deer radius and \#M-9 [\#2025-2] a deer incisor, both from Block C) yielded acceptable dates. Five samples of multiple adult Rabdotus snail shells all yielded results that appeared in the range of what was expected.

Following the soil laboratories determination that the dark stained sediments in and around features was carbon based, a second batch of 15 samples, 14 organic rich sediments and one tooth, was requested for radiocarbon dating to TxDOT in August 2007. Once approved, the samples were prepared, and Beta forms completed and submitted to TxDOT. The tooth was a pronghorn $\mathrm{M}_{1}$ from Feature 25 in the Martindale/Early Corner-notch component. The organic sediments were from Bell/Andice Features 16, 24, 29, 30, Martindale/Early Cornernotch Features 25, 26, 33, plus unassigned Features 19, 21, 23. Beta indicated that these sediment samples provided plenty of carbon for accurate measurements.

In November 2014, during the initial stages of data analyses for the final reporting phase, another 20 samples ( 7 animal bones, 12 charcoal, and 1 sample of burned seeds) were requested to TxDOT for dating to support and hopefully narrow previous radiocarbon results. Eleven were selected from the Bell/Andice and nine from the Martindale component with a bias towards the use of animal bone and charcoal. Again, the request and justification for dating each was sent to Dr. Abbott of TxDOT and the samples were approved. Once again, TRC archeologist prepared the samples, completed the Beta Analytic laboratory forms and submitted all to TxDOT for shipping to Beta.

Beta dates are reported as radiocarbon years before present (RCYBP), with present being A.D. 1950, 


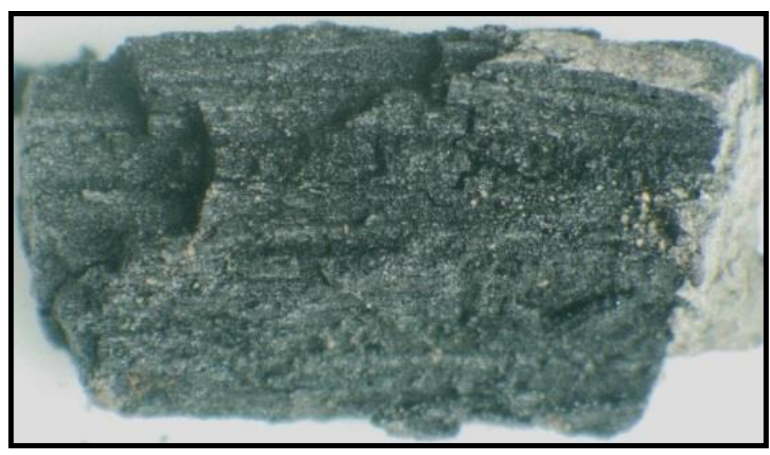

Figure 5-17. Example of piece plotted charcoal sent for identification.

and calculated using the Libby 14C half-life of 5,568 years. Quoted errors represent one relative standard deviation statistics (68\% probability) with counting errors on the basis of the combined measurements. Each sample was measured for C13 verses $\mathrm{C}-12$ ratios $(13 \mathrm{C} / 12 \mathrm{C})$ expressed as the delta 13 carbon $\left(\delta^{13} \mathrm{C}\right)$ and calculated relative to the internationally standard Cretaceous belemnite formation at Peedee, South Carolina (PDB or VPDB). The reporting forms present measured radiocarbon age (years B.P.), $\delta^{13} \mathrm{C}$ values obtained to derive the conventional radiocarbon ages, plus calibrated ages in terms of both cal B.C. and cal B.P. using Reimer et al. (2004; 2013).

Charcoal samples were pretreated with acid, alkali and acid washes, the snail shells were treated with mild acid, and collagen was extracted from bone samples prior to processing for accelerated mass spectrometry (AMS) dating. Beta's individual laboratory reports, with specific results concerning each sample, are presented in Appendix A. Individual sample results are also presented and discussed throughout the body of this report.

\section{Macrobotanical Analysis}

Seven sediment samples totaling 26.8 liters from Features 19, 24, 25, 26, 29, 30, and 31 were submitted to Dr. Phil Dering of Shumla Archeobotanical Services in Comstock in 2005. The samples were processed using Dering's standard bucket flotation method, and he collected and identified the macrobotanical remains. In addition, three samples previously floated by TRC staff using the Dousman flotation system and 36 point- or screen collected macrobotanical samples were submitted for analysis. Those results were presented previously (Quigg et al. 2007). Subsequently, another 16 point- or screen-collected macrobotanical samples were submitted to Dr. Dering for identification (Figure 5-17). All data stemming from these samples, plus his procedures, individual results, identifications, and discussions are presented in Appendix C. Individual sample results and identifications are also incorporated in the text body.

\section{High-Powered Microscopic and Residue Analysis}

Formal chipped stone tools are generally categorized by overall form, with an assumed function, such as pointed projectiles for tipping arrows or dart shafts, bifaces for cutting meat, and scrapers for scraping hides, etc. This generalized classification strategy was employed throughout this report. However, to gain insights into true tool function of certain tool classes, a suite of 33 mostly unwashed stone artifacts were judgmentally selected from the two identified components. This included 13 artifacts from the Bell/Andice and 20 from the Martindale/Early Corner-notched, and submitted to Dr. Bruce Hardy at Kenyon College in Gamber, Ohio for analyses. Most tools selected were minimally handled in the field and not washed in the laboratory. In order to track individual specimens, a small spot on one face of each artifact was cleaned and an ink provenience number applied and coated. Edge-modified flakes were intensively sampled as they presumably functioned in a variety of tasks on diverse materials. Therefore, the greatest functional diversity would be represented in this tool class. Hardy's analytical methods, individual observations, and results are presented in Appendix D. Use-wear results are also incorporated into appropriate sections in Chapters 9.0 and 10.0 . 


\section{Instrumental Neutron Activation Analysis}

Instrumental neutron activation analysis (INAA) provides a chemical fingerprint for a variety of different materials, such as ceramic sherds, tool stones, and clays (Figure 5-18). The chemical fingerprint aids in tracing material to a general region or sometimes a specific formation or locale. This technical analysis allows inquiry into origins of lithic acquisition areas and movements of lithic artifacts and populations. It is possible that quarry sources reflected in the manufactured lithic artifacts (tool stone employed in tool manufacturing) can be identified. If so, this contributes to our understanding of resource procurement strategies and movement of products through trade and/or population movements. INAA have been conducted and published concerning prominent local and regional Texas tool stone resources and includes, but not limited to multiple types of Edwards chert from Fort Hood (Frederick et al. 1994), the broader regional Edwards chert (Quigg et al. 2008), cherts from the Callahan Divide (Glascock 1995; Quigg et al. 2006), Alibates and Tecovas (Quigg et al. 2010; Quigg et al. 2011b) and various noncultural gravel outcrops scattered across central Texas. These multiple Texas datasets are on file at the Missouri University Research Reactor (MURR) laboratory and employed for comparisons to unknown cultural samples.

With the goal of identifying specific origins of chipped stone artifacts from the two identified components, 35 cultural pieces (MURR nos. TRC676-710) from the Martindale/Early Corner-notched and Bell/Andice components, plus 20 natural chert samples (TRC656675) collected from exposed gravel atop a hill immediately adjacent to 41TV2161 were submitted to Dr. Michael Glascock at MURR for analysis (Table 52). When formal tools were sampled a small roughly $1 \mathrm{~g}$ flake was knapped from the margin and the resulting new/fresh flake scar labeled for INNA. In instances of broken tools, a tiny section was removed from the broken area. The laboratory procedures and individual results are presented in Appendix $\mathrm{H}$.

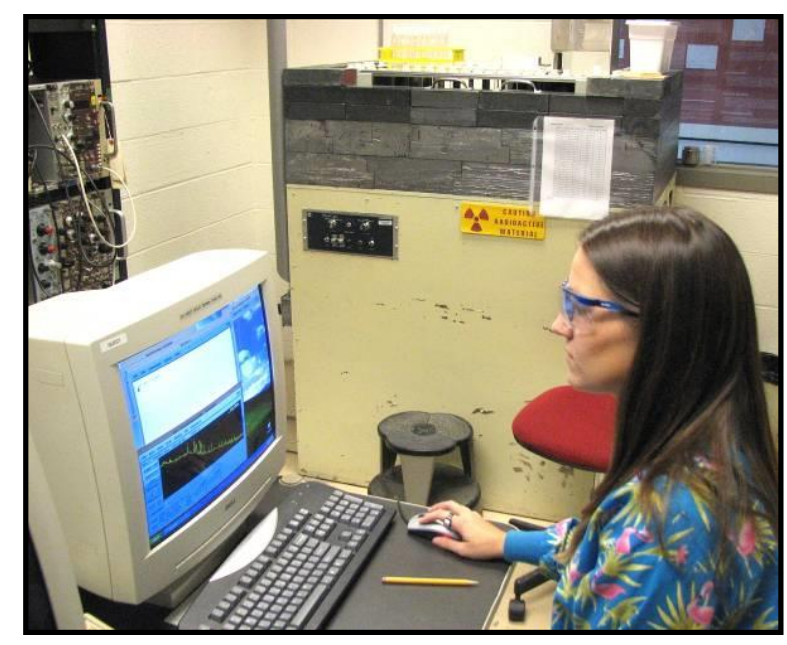

Figure 5-18. Missouri University Research Reactor (MURR) laboratory assistant compiling data (photograph furnished by Dr. Glascock).

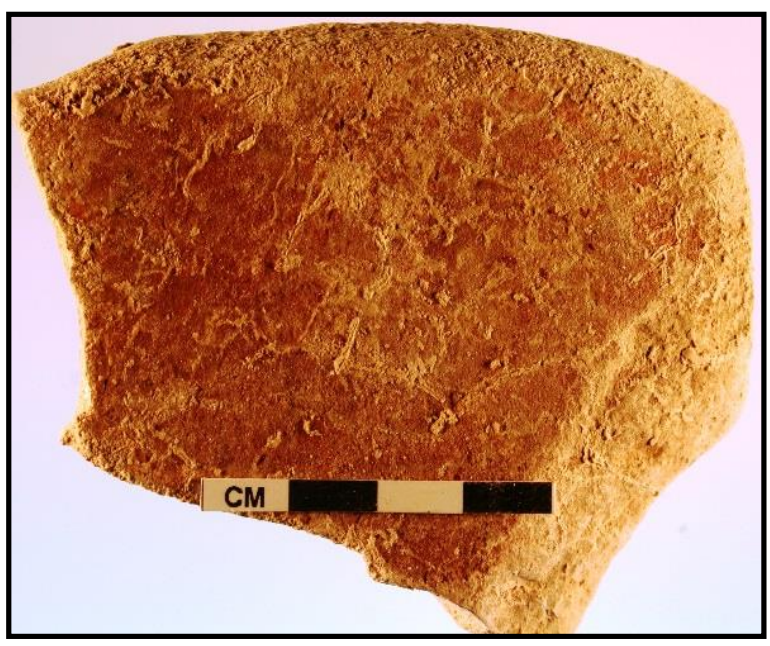

Figure 5-19. Rock with red pigment on surface (\#2396-10) subjected to XRF analysis.

\section{X-Ray Fluorescence Analysis}

Composition of pigments on two sandstone rock samples was investigated through the use of elemental analysis employing X-ray fluorescence (XRF) (Figure 5-19). Two possible metate fragments or burned rocks (samples \#2396-10 and \#2396-11) from between 248 and $260 \mathrm{cmbs}$ of N79/E85 in Block F with red and black pigments on their surfaces were sent to Dr. Steven M. Shackley, Director of Geoarchaeological XRF Laboratory in Albuquerque, New Mexico for analysis. His procedures and analytical results are presented in Appendix I. 
Table 5-2. INAA Sample Numbers, Component Associations, and Descriptions.

\begin{tabular}{|c|c|c|c|c|}
\hline PNUM & Component & INAA No. & Description & $\begin{array}{l}\text { Artifact } \\
\text { Class }\end{array}$ \\
\hline $2097-1-2 a$ & $\mathrm{~B} / \mathrm{A}$ & TRC690 & No color match, darker than Neutral 8/N & Flake \\
\hline 2097-1-3a & $\mathrm{B} / \mathrm{A}$ & TRC691 & $\begin{array}{l}2.5 \text { Y } 6 / 2 \text { light brownish gray mottled with } \\
7 / 4 \text { very pale brown with a crystal inclusion }\end{array}$ & Flake \\
\hline 2098-1-1a & $\mathrm{B} / \mathrm{A}$ & TRC681 & $\begin{array}{l}10 \text { YR } 6 / 2 \text { light brownish gray with darker } \\
\text { bands and pink inclusions }\end{array}$ & Flake \\
\hline $2103-1-3$ & $\mathrm{M} / \mathrm{CN}$ & TRC700 & $\begin{array}{c}10 \text { YR } 3 / 1 \text { very dark gray mottled with } 10 \\
\text { YR } 2 / 1 \text { black }\end{array}$ & Flake \\
\hline $2105-10 a$ & $\mathrm{~B} / \mathrm{A}$ & TRC677 & 10 YR 6/2 light brownish gray & Biface \\
\hline $2106-1-2 a$ & $\mathrm{~B} / \mathrm{A}$ & TRC692 & $\begin{array}{c}10 \text { YR 6/2 light brownish gray mottled with } \\
10 \text { YR } 8 / 2 \text { very pale brown }\end{array}$ & Flake \\
\hline 2109-11a & $\mathrm{M} / \mathrm{CN}$ & TRC697 & 10 YR 2/1 black & $\begin{array}{l}\text { Edge- } \\
\text { modified } \\
\text { Flake }\end{array}$ \\
\hline $2120-1-1 a$ & $\mathrm{~B} / \mathrm{A}$ & TRC686 & 10 YR 6/3 pale brown with darker inclusions & Flake \\
\hline $2126-1-a$ & $\mathrm{~B} / \mathrm{A}$ & TRC684 & 10 YR 7/1 light gray & Flake \\
\hline $2139-1-2 a$ & $\mathrm{~B} / \mathrm{A}$ & TRC687 & GLEY $15 / \mathrm{N}$ to $4 / \mathrm{N}$ dark gray to gray & Flake \\
\hline $2146-10 a$ & $\mathrm{~B} / \mathrm{A}$ & TRC679 & 10 YR 3/1 very dark gray & Bell biface \\
\hline 2146-1-1a & $\mathrm{B} / \mathrm{A}$ & TRC682 & $\begin{array}{c}2.5 \mathrm{Y} 7 / 3 \text { to } 6 / 3 \text { pale brown to light yellowish } \\
\text { brown }\end{array}$ & Flake \\
\hline $2149-1-3$ & $\mathrm{~B} / \mathrm{A}$ & TRC685 & 10 YR 4/1 dark gray & Flake \\
\hline 2150-1-a & $\mathrm{B} / \mathrm{A}$ & TRC688 & $\begin{array}{l}10 \text { YR } 7 / 3 \text { very pale brown mottled with } 7 / 1 \\
\text { light gray }\end{array}$ & Flake \\
\hline $2152-1-2 a$ & $\mathrm{~B} / \mathrm{A}$ & TRC689 & $\begin{array}{l}2.5 \text { Y } 7 / 1 \text { light gray with reddish brown } \\
\text { specks }\end{array}$ & Flake \\
\hline $2161-10 a$ & $\mathrm{M} / \mathrm{CN}$ & TRC696 & $2.5 Y 7 / 1$ to $6 / 1$ light gray to gray & $\begin{array}{l}\text { Medial dart } \\
\text { point }\end{array}$ \\
\hline $2172-1-2 a$ & $\mathrm{M} / \mathrm{CN}$ & TRC698 & $\begin{array}{c}10 \text { YR } 7 / 2 \text { light gray mottled with } 7.5 \text { YR } 6 / 3 \\
\text { light brown }\end{array}$ & Flake \\
\hline $2175-12 a$ & $\mathrm{~B} / \mathrm{A}$ & TRC678 & 10 YR 6/3 pale brown with darker banding & Biface \\
\hline $2182-1 a$ & $\mathrm{M} / \mathrm{CN}$ & TRC680 & $\begin{array}{c}10 \text { YR } 6 / 3 \text { pale brown with silica and white } \\
\text { inclusions }\end{array}$ & Core \\
\hline $2187-1-1 a$ & $\mathrm{~B} / \mathrm{A}$ & TRC683 & $\begin{array}{c}10 \text { YR 6/2 light brownish gray with pink } \\
\text { inclusions }\end{array}$ & Flake \\
\hline $2247-1-2$ & $\mathrm{M} / \mathrm{CN}$ & TRC706 & $\begin{array}{c}10 \text { YR } 4 / 4 \text { dark yellowish gray with } 10 \mathrm{YR} \\
3 / 1 \text { very dark gray inclusion }\end{array}$ & Flake \\
\hline $2247-1-3$ & $\mathrm{M} / \mathrm{CN}$ & TRC709 & $\begin{array}{c}10 \text { YR 3/1 very dark gray mottled with } 10 \\
\text { YR 5/4 yellowish brown }\end{array}$ & Flake \\
\hline 2254-1-1 & $\mathrm{M} / \mathrm{CN}$ & TRC704 & 2.5 Y 5/1 gray & Flake \\
\hline
\end{tabular}


Table 5-2. INAA Sample Numbers, Component Associations, and Descriptions (continued).

\begin{tabular}{|c|c|c|c|c|}
\hline PNUM & Component & INAA No. & Description & $\begin{array}{l}\text { Artifact } \\
\text { Class }\end{array}$ \\
\hline 2257-10a & $\mathrm{M} / \mathrm{CN}$ & TRC695 & $\begin{array}{l}2.5 \text { Y } 6 / 2 \text { light brownish gray with bands of } \\
2.5 \text { Y } 5 / 1 \text { gray and } 7.5 \text { YR } 6 / 3 \text { light brown }\end{array}$ & Biface \\
\hline $2261-1-2$ & $\mathrm{M} / \mathrm{CN}$ & TRC707 & $2.5 \mathrm{Y} 7 / 3$ pale brown & Flake \\
\hline $\begin{array}{l}2261-4- \\
1 \mathrm{~g} 1 \mathrm{a}\end{array}$ & $\mathrm{M} / \mathrm{CN}$ & TRC710 & $\begin{array}{c}10 \text { YR } 7 / 1 \text { light gray banded with } 10 \text { YR } 8 / 1 \\
\text { white }\end{array}$ & Flake \\
\hline $2292-1-2$ & $\mathrm{M} / \mathrm{CN}$ & TRC708 & 10 YR 4/1 dark gray & Flake \\
\hline $2300-1-2 a$ & $\mathrm{M} / \mathrm{CN}$ & TRC702 & 10 YR 3/1 very dark gray & Flake \\
\hline $2300-1-3$ & $\mathrm{M} / \mathrm{CN}$ & TRC705 & 2.5 Y 4/1 dark gray & Flake \\
\hline $2300-1-4 a$ & $\mathrm{M} / \mathrm{CN}$ & TRC703 & 10 YR 8/1 white & Flake \\
\hline $2310-1-2$ & $\mathrm{M} / \mathrm{CN}$ & TRC701 & 10 YR 5/2 grayish brown & Flake \\
\hline $2310-1-3$ & $\mathrm{M} / \mathrm{CN}$ & TRC699 & $\begin{array}{l}7.5 \mathrm{R} 5 / 4 \text { weak red mottled with } 10 \mathrm{YR} 6 / 3 \\
\text { pale brown and } 10 \mathrm{YR} 7 / 3 \text { very pale brown }\end{array}$ & Flake \\
\hline 2317-13a & $\mathrm{M} / \mathrm{CN}$ & TRC694 & $\begin{array}{c}2.5 \text { Y } 5 / 1 \text { gray mottled with } 2.5 \text { Y } 7 / 3 \text { pale } \\
\text { brown }\end{array}$ & $\begin{array}{l}\text { Edge- } \\
\text { modified } \\
\text { Flake }\end{array}$ \\
\hline $2317-14 a$ & $\mathrm{M} / \mathrm{CN}$ & TRC693 & 10 YR 5/1 grayish brown & Biface \\
\hline $2416-10 a$ & $?$ & TRC676 & $\begin{array}{l}\text { No color match, dark gray bluish tint with } \\
\text { white crystals }\end{array}$ & Biface \\
\hline
\end{tabular}

$\mathrm{B} / \mathrm{A}=\mathrm{Bell} /$ Andice, $\mathrm{M} / \mathrm{CN}=$ Martindale/Early Corner-notch component

\section{Tooth Cementation Analysis}

Aging of ungulates is possible through analysis of dental cementation in teeth. Two deer incisors (samples \#2246-2-10 and \#2246-2-11) from between 254 and $266 \mathrm{cmbs}$ of N79/E93 in Block D that represent the Martindale/Early Corner-notched component were submitted to Wildlife Analytical Labs, LLC in Burnet, Texas to determine the animals age from which the teeth represented (Figure 5-20). If cementation lines or rings were present, the animal's season of death and age could be projected. The laboratory procedures and results are presented in Appendix $\mathbf{J}$.

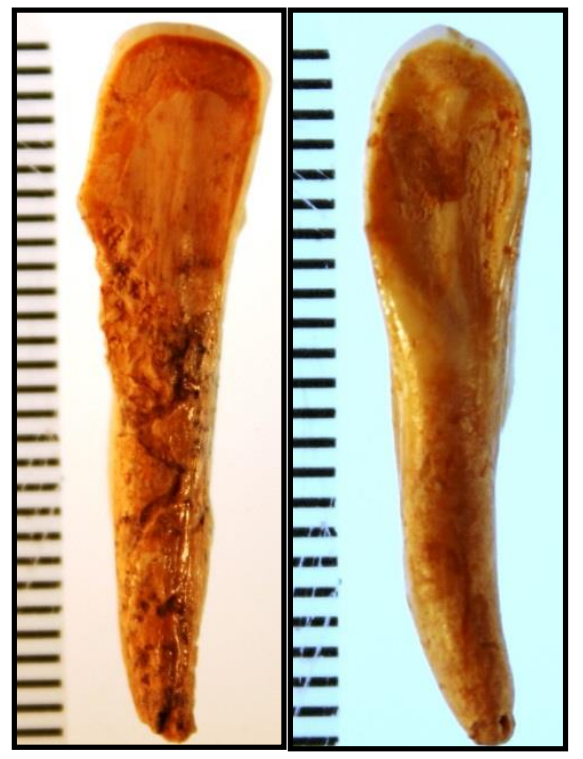

Figure 5-20. Deer incisors (\#2246-2-10 and \#2246-2-11) subjected to cementation analysis. 


\subsection{METHODS OF ARTIFACT ANALYSES}

Artifacts were subjected to different metric, nonmetric, typological, and technical analyses, such as starch grain and high-powered microscopic use-wear analysis. A set of predefined attributes for each material class were first encoded on paper, and subsequently entered into TRC's electronic database management system that utilized Microsoft Access 2010 software, which constitutes the master database for this site. The specific data recorded for each class of artifact are presented below. Analytical methods pertinent to each data class are discussed in detail in the appropriate parts of this report.

\subsubsection{Chipped Stone Artifact Analysis}

A protocol for analysis of lithic debitage and chipped stone tools has been developed by TxDOT archeological staff (TxDOT 2010) in an effort to standardize data collection and presentation in analytical and interpretive chapters of archeological reports sponsored by TxDOT. When possible, terminological and taxonomic uses follow those terms for this assemblage (Figure 5-21).

\subsubsection{Bifaces}

Bifacial tools, whether finely or roughly produced, appear to have completed the manufacturing process. This is evidenced by secondary retouch, edge straightening, hafting preparation, notching, and similar characteristics. Bifaces are defined predominantly on the basis of morphological characteristics, but they may also have functional associations (e.g., cutting, piercing, chopping, and drilling). Bifacial tools exhibit purposeful, usually patterned, flake removals on both faces (ventral and dorsal). Most or all of both faces may be covered with flake scars, and sometimes one face may be completely modified, whereas the opposite face may exhibit only partial modification. Bifaces may be fashioned either from large bifacial cores or from flakes. Included within this overall morphological category are diverse functional groups such as projectile points (see below).

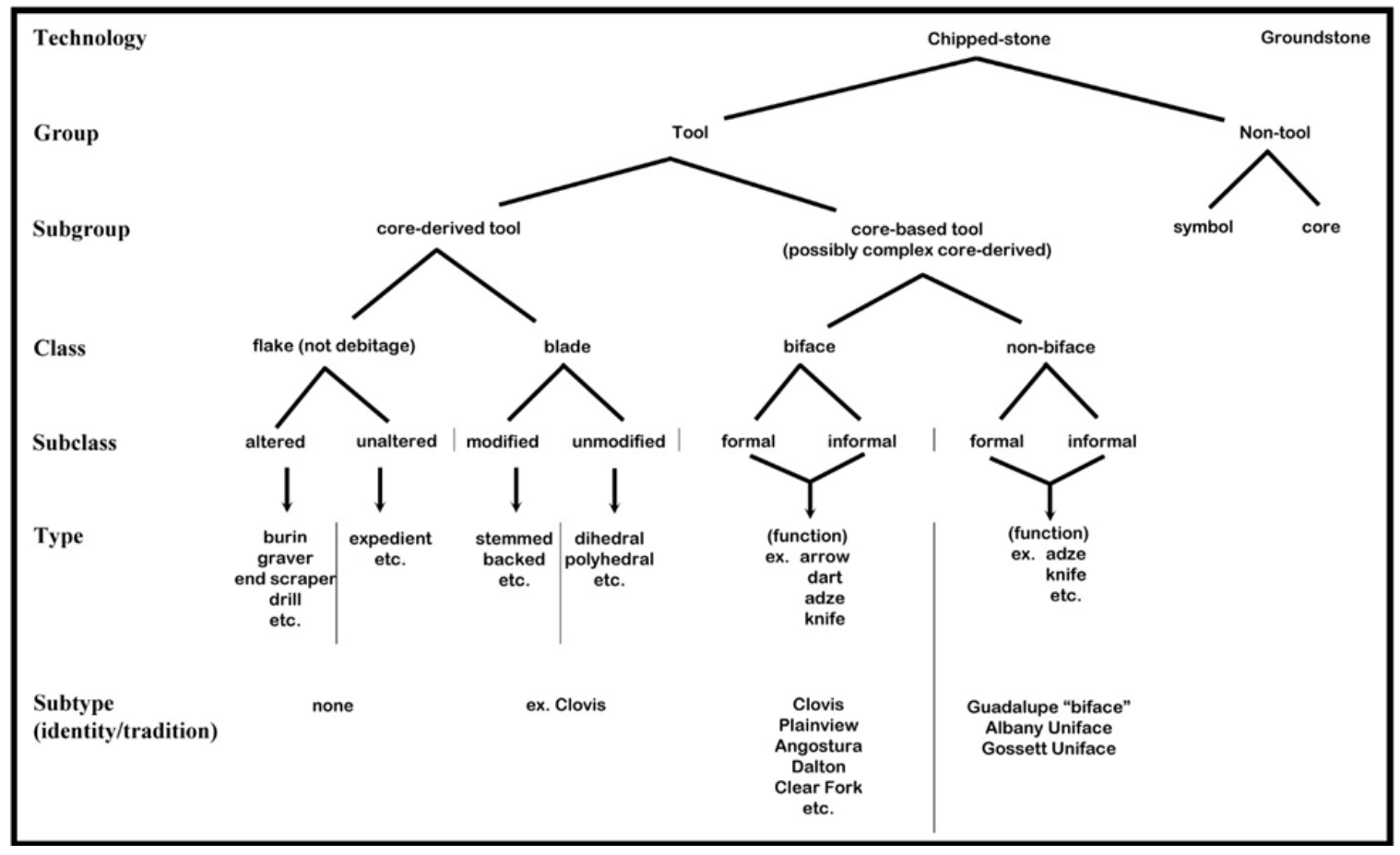

Figure 5-21. Chipped stone artifact analysis flowchart (from TxDOT 2010). 


\subsubsection{Projectile Points}

Projectile points are a functional subset of the biface class specifically designed to be hafted to the distal end of a shaft employed in stabbing, throwing, or shooting for hunting or warfare. Projectile points are bifacial tools given their final form by means of fine secondary retouch, usually with basal modification in the form of notching, stemming, or thinning the proximal end for purposes of hafting. Dart points, arrow points, and indeterminate dart/arrow points are all classes of projectile points. Dart points are those employed to tip hand-held darts or spears, arrow points are to tip arrows, and indeterminate points are, as the name implies, of uncertain usage. Whereas dart points are usually manufactured from bifacial preforms, arrow points are often manufactured on thin flakes.

Projectile points were assigned to published types whenever possible. In traditional archeological literature, projectile points are normally referred to by their typological designation, which are usually based on a set of morphological characteristics, shared in common by groups of similar points, which generally focus on haft modification. Point classifications were conducted by TRC's personnel in reference to established point typologies in use in Texas archeology (Suhm and Jelks 1962; Turner et al. 2011) and subsequently verified by Elton Prewitt, a well-known and longtime point typologist in Texas.

\subsubsection{Scrapers}

Scrapers are a specific type of unifacial tool that have minimally one intentionally modified working edge. In some instances, bifacial modification may be present, but in such instances the intentional retouch tends to be located on the dorsal flake surface whereas the ventral surface tends to exhibit primarily use-related flake scars. Based upon the primary working edge location, scrapers are subdivided into end, side, or combination types. End scrapers are pieces with retouch, primarily restricted to either the distal or proximal end of the flake blank, generally producing a convex working edge. The opposing end may bear some minimal retouch, presumably to facilitate hafting. Side scrapers are pieces with retouch present on one or both lateral edges. Working edges may be convex, straight, or concave. On combination scrapers, marginal retouch may appear along the end as well as along one or more lateral edges. As implied by the name of this tool, the primary function of scrapers is presumed to relate to scraping relatively soft materials such as animal hides or vegetable matter, or slightly harder materials, such as wood or possibly antler or bone.

\subsubsection{Unifaces}

Unifaces are those tools that exhibit flake scars on one face only. Like bifaces, unifaces are defined by predominantly on morphological characteristics, but they also tend to have functional associations (e.g., scraping, planing, cutting, engraving). Unifacial tools exhibit purposeful flaking across most or all of one face, whereas the opposite face most often remains flat and unmodified. Unifaces may be fashioned from cobbles or flakes and include such functionally diverse groups as scrapers, gouges, edge-modified flakes, gravers, and spokeshaves. One or more edges of a unifacial tool may exhibit manufacture and/or use-related flake removals that may be patterned or random. To some degree, unifacial tools form a continuum that range from formal tools with intentional, patterned, and manufacture-related edge flaking, to informal, expedient tools that reveal only userelated edge scarring. The former tend to fall in the scraper and gouge categories, whereas the latter are generally classified as edge-modified flakes.

\subsubsection{Edge-Modified Flakes}

Edge-modified flakes are minimally modified flakes, flake fragments, or pieces of angular debris that are characterized by one or more areas of flake scarring along margins. The edge flaking may be patterned or unpatterned, continuous or discontinuous, and may result from use-related 
activities or from intentional pressure retouch to prepare an edge for use. Many edge-modified flake tools exhibit combinations of these characteristics, and many have multiple worked edges. The modifications, however, usually are restricted to the edges and do not significantly alter the original flake form. Such edge modifications may be either unifacial or bifacial. Edge-modified flakes are usually considered 'expedient' tools, pieces of raw or minimally modified material that are utilized for a short time, and subsequently discarded soon after use.

\subsubsection{Lithic Debitage Analyses}

Following the typology outlined in the TxDOT Chipped Stone Protocol (TxDOT 2010), chipped stone or lithic debitage is defined here as the unmodified debris resulting from stone tool manufacture and maintenance. Various classification systems have been proposed for the analysis of lithic debitage. In addition to the typological methods outlined in the TxDOT Protocol, an attribute-level analysis was conducted on all complete flakes and proximal and lateral flake fragments.

During the analysis process, the laboratory technician uses macroscopic analysis for flake or use scars that indicate modification on each flake to separate any possible tools. All debitage from hand-excavated units was counted, whereas detailed analysis targeted materials from the two identified components to understand the general character of debris assemblage. Detailed analyses were not conducted on debitage from unassigned components.

Selected tools and lithic debitage were also examined under ultraviolet (UV) light to isolate raw materials inconsistent with the bright yellow UV fluorescence of Edwards Formation chert (see Collins and Headrick 1990; Hofman et al. 1991). A hand-held multiband UV-254/366NM (Model UVGL-58) light source with short and long wave capabilities was employed for materials analyzed.

\subsubsection{TxDOT Analytical Methods}

Following TxDOT protocol, the lithic debitage was first size graded as specified by screen manufacturer Gilson Company, Inc. into 6.3, 12.5, 19.0, and 26.5 mm size groups (TxDOT 2010). Each specimen was sorted into platform bearing and nonplatform-bearing groups. Nonplatform bearing specimens were treated as shatter and weighed in bulk by provenience (specific to level and test unit). Platform-bearing specimens were examined individually and sorted into one of four classes: flat, multi-faceted, crushed, and cortical. Other attributes documented included the presence/absence of heat alteration, cortex percentage (i.e., none, 1 to 25 percent, 26 to 50 percent, 51 to 75 percent, and 75 to 100 percent) and raw material type. Specimens were weighed and findings entered into a database spreadsheet. Those that lacked a platform were grouped together on a single line, counted and weighed.

When possible, debitage were categorized as core reduction flakes, biface thinning flakes, and tertiary thinning flakes to identify stages of tool production on-site. Items of debitage were designated as angular debris and indeterminate flakes if no diagnostic traits were visible.

\subsubsection{Core Reduction Flakes}

This category includes flakes, flake fragments, and pieces of angular debris associated with initial core preparation activities, such as test flakes that were removed to determine the quality of raw material within a cobble as well as to decorticate a cobble for further reduction. Items in this category tend to have cortex on greater than 50 percent of their dorsal surfaces. By definition, most core reduction flakes tend to be relatively large (smaller flakes with dorsal cortex often fall within other categories, such as early and late stage biface flakes or indeterminate flakes, depending on their diagnostic characteristics). Core preparation flakes may or may not exhibit pronounced platforms, bulbs of percussion, or ventral concussion rings, though most only have one of these characteristics. 


\subsubsection{Biface Thinning Flakes, Flakes with Complex Platforms}

Biface manufacture flakes were classified primarily based on the presence of complex striking platforms, multidirectional dorsal flake scars, parallel to slightly expanding flake margins, and slight to moderate longitudinal curvatures. This category was subdivided into early and late stage biface manufacture flakes. Early stage biface flakes tend to be somewhat larger than late stage biface flakes, have fewer and larger dorsal flake scars, and may retain a considerable amount of cortex on their dorsal surfaces. As employed in this analysis, early stage biface flakes correlate roughly with Callahan's (cf. 1979) revised Stage 1, 2, and 3 bifaces ("blank," "rough out," and "primary preform" stages) while late stage biface flakes correlate with Callahan's revised Stage 4 and 5 bifaces ("secondary preform" and "final preform" stages). In practice, Stage 1 ("blank") flakes normally fall within the core preparation flake category due to the lack of clear diagnostic characteristics on many such specimens. Final percussion thinning, pressure thinning, and retouch flakes that do not clearly exhibit biface manufacture characteristics due to their small size would probably be included in the tertiary thinning and retouch flakes category. The early and late stage biface flake categories may contain complete flakes, proximal and distal flake fragments, and/or small pieces of angular debris that exhibit clear biface manufacturing characteristics (in practice, the latter type of debitage - angular debris bearing bifacial traits - is rare in the biface manufacture flake categories).

\subsubsection{Tertiary Thinning, Retouch Flakes}

This category includes flakes and proximal and dorsal flake fragments resulting from the final stages of tool manufacture, including final percussion thinning and any subsequent pressure retouch. By definition, flakes in this category tend to be quite small, and it is difficult to distinguish whether they result from biface manufacture, uniface manufacture, or resharpening.

\subsubsection{Angular Debris}

Angular debris, or "shatter," includes angular pieces of lithic raw material that break away from the core as flakes are struck. In contrast to flakes, angular debris does not generally retain any diagnostic characteristics of the flint knapping process (i.e., platforms, bulbs of percussion, concussion rings, and definable dorsal or ventral surfaces). In this analysis, those few pieces of angular debris that exhibit characteristics diagnostic of biface manufacture were included in appropriate biface manufacturing category (i.e., early versus late stage biface flakes).

\subsubsection{Indeterminate Flakes}

This category includes flakes and flake fragments that lack diagnostic traits that would permit their placement into one of the other categories. Generally, these flakes are small fragments of flakes and/or thin pieces of angular debris that do not display clear evidence of a platform, concussion rings, or flake scar patterning on their dorsal surfaces. This category also includes a small number of potlid flakes and fractured heat spalls resulting from thermal alteration of raw materials.

\subsubsection{Attribute-Level Analysis}

In addition to the typological methods outlined in the TxDOT Protocol (TxDOT 2010), an attributelevel analysis was conducted with all complete flakes, and proximal and lateral flake fragments. Complete flakes are defined here as flakes that retain a platform remnant, dorsal and ventral surfaces, and an identifiable termination and lateral margins. The flake may not necessarily be 100 percent complete, as long as all attributes defined in the methodology can be accurately measured. Further, bulbs of force and eraillure scars are not always pronounced on flakes, especially within assemblages composed of poor quality raw materials, and should not be considered a necessary attribute for defining a flake. Proximal flake fragments have all the attributes of complete flakes 
except for an identifiable termination. In this analysis, all flakes with step terminations were classified as proximal fragments. Lateral flake fragments are all complete flakes or proximal flake fragments that split laterally.

The benefit of an attribute-level analysis is that a database of attribute measurements can be filtered by different technological or typological criteria at any time and by any researcher. Additionally, attributes measured on ratio scales can always be grouped into coarser classification systems to aid in comparison with other analyses. For example, the maximum dimension of objects measured on a ratio scale could be subdivided on an interval scale into size classes, or further grouped on an ordinal scale as small, medium, and large. Ideally, all attributes should be measured on a ratio scale if possible.

Attributes measured on a nominal scale included the absence or presence of an eraillure flake scar, termination type, and the type of platform preparation. Attributes measured on an ordinal scale included the relative size of the bulb of percussion, the relative degree of platform lipping and longitudinal curvature, and the number of dorsal flake scars. Attributes measured on a ratio scale included the platform length and width, the maximum dimension of the flake, and the oriented length, width and thickness.

Dorsal surface attributes recorded within the assemblage included scar counts and percentage of cortex. Only scars greater than one quarter the length of the flake were counted to avoid recording scars not related to primary flake removals prior to flake detachment (Andrefsky 2006:106; Crabtree 1972:14). In general, as the number of dorsal flake scars increase, and the percentage of dorsal cortex decreases, the reduction sequence progresses (Mauldin and Amick 1989:73; Tomka 1989:143). Cortex was placed into one of four categories as defined in the TxDOT Protocol and is described in the previous section.
Ventral surface attributes that were monitored included presence/absence of an eraillure flake, bulbar definition, and the presence/absence of platform-remnant lipping. The presence/absence or prominence of these attributes on the ventral surface of a flake may provide information on the amount of applied force or application load, striking angle, and physical characteristics of the percussor employed during flake initiation (Andrefsky 2006). Light or moderate force may leave a minimal eraillure flake scar or no scar at all. A large scar may indicate a high degree of applied force. These observations can be relevant to the interpretation of reduction techniques, with hard-hammer percussion generally equated with intense force when applied to flake dynamics. Energy from a softer hammer, such as an antler billet swung with equal force as a hard-hammer, may be absorbed and then dispersed, leaving a small eraillure flake, or perhaps none at all (Crabtree 1972:65; Hayden and Hutchings 1989:241).

Bulbar definition was monitored to evaluate the application load concept. Hard-hammer percussion tends to result in pronounced bulbs of force. Due to the displacement of force, soft-hammer percussion often produces a diffuse bulb of force. Three ordinal categories were created to evaluate this attribute: absent/weak, moderate, and pronounced. Platform lipping, which occurs at the juncture between the platform remnant and the ventral surface, is generally associated with bifacial reduction or pressure flaking, although some studies have determined no such relationship (Patterson and Sollberger 1978). Platform lipping was difficult to quantify, and was recorded as absent/weak, moderate or pronounced. Almost all flakes composed of good quality material will have a slight lip that can be felt while running a finger across the juncture between the platform remnant and ventral surface of the flake.

Metric attributes measured on platform remnants included maximum thickness and width, which relate to core size and reduction techniques 
(Andrefsky 2006:92; Kooyman 2002:79). The platform remnant is the segment of the flake or flake fragment that retains a portion of the surface or original platform of the objective piece. This attribute allows examining platform preparation on the objective piece, or core. Additionally, platform remnant analysis provided a method for investigating raw material selection, reduction strategies, and potentially the energy expended in tool manufacture.

Nonmetric platform remnant categories selected for this analysis included: 1) cortical, 2) collapsed, 3) crushed/abraded, 4) unifaceted, and 5) multifaceted (Andrefsky 2006:94). Cortical platform remnants retain a portion of the outer cortex of the parent material. Unifaceted platforms exhibit a smooth, noncortical flat surface. A multifaceted platform remnant contains portions of multiple truncated flake scars across its surface. A crushed/abraded platform remnant is similar to a multifaceted platform remnant, but characterized by a rough, irregular surface with poorly defined facets. Also recorded was the presence or absence of platform preparation along the juncture between the remnant surface and dorsal flake surface. These were categorized as the result of trimming or abrading. Trimming was defined as small, patterned flake removals intended to further shape the platform. Abrading allows for platform preparation by removing any overhangs, and tends to result in small, stepped-flake scars and abrasions. Removal of the overhang strengthens a platform, preventing collapse during flake removal. A collapsed platform remnant commonly results from insufficient preparation, resulting in the collapse or shearing of the platform.

Metric attributes including maximum dimension, length, width, maximum thickness, and weight were recorded on all complete flakes and proximal flake fragments. Lateral fragments were measured for all attributes except width.
The length of complete flakes was measured along the proximal-distal axis on the ventral surface of the flake. A measurement was taken from the point of force application to the extreme opposite end. Width was measured along the widest point perpendicular to the length axis. Maximum thickness was measured at the thickest point. Platform remnant width was measured from right margin to left margin, perpendicular to the axis measured for flake length. Platform remnant thickness measured the maximum dimensions perpendicular to the platform remnant width, from dorsal surface to ventral surface.

\subsubsection{Cores}

A core is a cobble, pebble, or other mass of lithic raw material that exhibits one or multiple platforms and flake scars resulting from the systematic removal of flakes by flint knappers (Parry and Kelly 1987). Technically, any chipped stone tool may properly be classified as a core as it is the object created through the removal of flakes from the exterior surface of the original mass of lithic material. In common terms, however, cores are generally considered to be those masses of material from which one or more flakes were removed. In other words, cores do not exhibit any intentional or use-related flake scarring along any edge, though scars resulting from platform preparation may be evident, and a core might be expediently used as a tool (e.g., extensive crushing damage along one or more thick edges of a core would probably result in classification as a chopper).

Cores are typed according to the degree of knapping and the flake removal strategy. Four basic types of cores are unifacial, bifacial, multidirectional, and blade core. The last named type often has a distinctive conical polyhedral shape, which resulted from repeated, parallel removal of long, narrow flakes known as prismatic blades.

A unifacial core is one that exhibits flake scars removed from only one face. The flake removals may be in various directions and exhibit no pattern 
or structure to the removals. There are usually only one or two platforms. A bifacial core exhibits flake removals from both faces and again these may be in multiple directions. The parent or objective rock is generally a cobble that exhibits two detectable faces. The flakes were driven from the lateral edges; thus, the platforms are along the edges.

The multidirectional core is generally a chunk of raw material that does not necessarily exhibit two obvious faces. Generally, several platforms are present from which flakes were removed. Most often, the flakes are removed in different directions.

Blade cores are chunks of raw material intentionally prepared to facilitate the removal of a specific kind of desired flake. These generally exhibit two or more parallel scars driven from the same platform in the same direction with the same overall shape.

\subsubsection{Ground Stone Tool Analyses}

This broad artifact class includes pieces of natural rock modified by grinding, pecking, or battering, either to intentionally shape an implement or as a by-product of use. Ground stone tools are discernable by the presence of intentional abrasions, grooves, and striations and/or smoothing. Significant rounding, flattening, and/or pitting of utilized surfaces can also be identified. Categories of ground stone tools can include manos, metates (milling stones or grinding slabs), abraders/shaft smoothers, and edge-ground cobbles.

Edges and surfaces of each rock were macroscopically examined for signs of use as a tool. If battered, smoothed, unnaturally flattened, pitted, ground, striated, incised, or pecked areas were identified, then the artifact was attributed to a morphological and/or functional category based on general form and inferred function. Multiple observations were recorded for the tool classes. The following subsection provides a definition of major tool class identified.

\subsubsection{Manos and Metates}

Manos and metates are generally used together to grind friable materials (nuts, seeds, other vegetal matter, and sometimes pigments) into powder. A mano is a hand-held grinding stone, generally characterized by a round to ovate shape, usually of hard, dense siliceous rock such as quartzite, dolomite, or sandstone. One or more surfaces exhibit a smoothed or polished, and/or possibly flattened area caused by grinding action against another hard surface (the metate). In some instances, the edges exhibit crushed or pitted areas that indicate possible use as hammerstones as well. Sometimes one or both faces may be pitted, which results from the user trying to rough up the smooth surface to facilitate grinding. Generally, water worn cobbles that exhibit no other alterations are employed for this tool.

A metate is often a large slab of a dense rock such as sandstone, cherty limestone, or dolomite, which has functioned as the base on which the mano was employed to grind materials. The grinding action most often wears down the natural surface and creates a shallow concave face that is smoothed and/or polished. Extensive and continued use creates a deeper concave basin and in some instances, both faces functioned as a base for grinding. Occasionally, the edges are artificially shaped, usually by direct percussion that removed flakes along the margins. Metric and nonmetric observations were recorded for manos and metates.

Three metate fragments (\#2175-13b [7MQ1], \#2289-10b [7MQ2], and \#1414-10b [7MQ3]) were sent to Dr. Malainey for lipid residue analysis in 2007. The detailed methods and results are presented in Appendix G. Three manos (\#2414-10c, \#2175-13c, \#2164-11) were sent to Dr. Bozarth for pollen presence/absence analysis. His procedures and assessments are presented in Appendix L. Three manos (\#2106-10, \#2143-10, and \#2164-10), three metate fragments (\#2175-13a, \#2223-10, and \#2414-10) were send to Dr. Perry for starch grain 
analysis. Her detailed methods, identifications and discussions are in Appendix F. Data and analyses results on these ground stone are presented in the appropriate component chapters.

\subsubsection{Mussel Shell Analysis}

Collected mussel shell was compared to TRC's extensive modern and prehistoric comparative collection identified and labeled by Dr. R. G. Howells. To confirm the initial identification, specimens were compared to modern pictures and finalized by our in-house specialist Barrett Clark. Habitat data were obtained from literature sources (e.g., Howells et al. 1996).

Freshwater mussel shells and shell fragments were relatively sparse from this alluvial setting. In the field, the more complete shells and larger shell fragments were collected and bagged, and transported to the laboratory for analysis. Pieces from the $6.4 \mathrm{~mm}$ screens consist mostly of small shell fragments that varied considerably in completeness from thin, tiny flakes of shell, to nearly complete specimens (Figure 5-22). Many larger shells were piece plotted on the level records and depths recorded to reflect their horizontal and vertical distribution patterns. Shells are generally well preserved.

In the laboratory, shells were separated from other artifact classes examined for signs of cultural modification (i.e., cut marks, ground edges, and heat alterations). Shells collected from individual proveniences were first weighed, with more complete shells and those with potential identifiable characteristics set aside for comparisons and identifications. The more complete shells were identified to the species level where possible, and counts were recorded by species. Data and identifications concerning these shells are presented in the appropriate component chapters.

Although larger fragments and more complete shells were brought back to the laboratory for analysis, not all were curated. THC was consulted and they approved the discard of most mussel shells. A small sample of shells from cultural features was selected for curation, along with shells that exhibit obvious burning or some other unusual characteristics. The shells curated were washed and 10 percent were labeled according to curation standards.

\subsubsection{Vertebrate Faunal Analyses}

The encountered vertebrate faunal assemblage was unexpected and highly significant, although small and extensively fragmented (Figure 5-23). Each piece was examined to identify specific taxa, anatomical elements, element symmetry, part, size, the represented animals gross weight, skeletal maturity, presence or absence of burning, and type of human modification (cuts, impacts, and/or use as a tool). If a bone tool was identified, that specimen was set aside for detailed observation and recorded as an artifact.

Faunal remains were divided into major taxon groups on the basis of size and type of animal represented. Groups identified include dog/coyote (Canis), deer (Odocoileus sp.), pronghorn (Antilocapra americana), cottontail rabbit (Sylvilagus cf.), jackrabbit (Lepus californicus), turtles (Testudines), snakes (Serpentes), small rodents, birds (Avian), and fish (Osteicthyes). A bone fragment was attributed to a specific taxon based primarily on overall size and cortical wall thickness, bone shape and structure, and other observed attributes. If observed attributes were not sufficient to confidently assign a bone to a specific taxon or general category, the fragment was assigned to an "unknown" category. Bones were identified as to element and symmetry where possible, but most are too small, often midsections of long bone fragments (LBF) that could not be identified to a specific taxon. The counts and weights of each group or taxon were recorded and assigned by taxon.

Bones were also recorded according to predetermined size categories and range from 0 to $3 \mathrm{~cm}, 3.1$ to 6.0 $\mathrm{cm}, 6.1$ to $9.0 \mathrm{~cm}, 9.1$ to $12.0 \mathrm{~cm}$, and greater than $12.1 \mathrm{~cm}$. Fragment size reveals an indication of how 


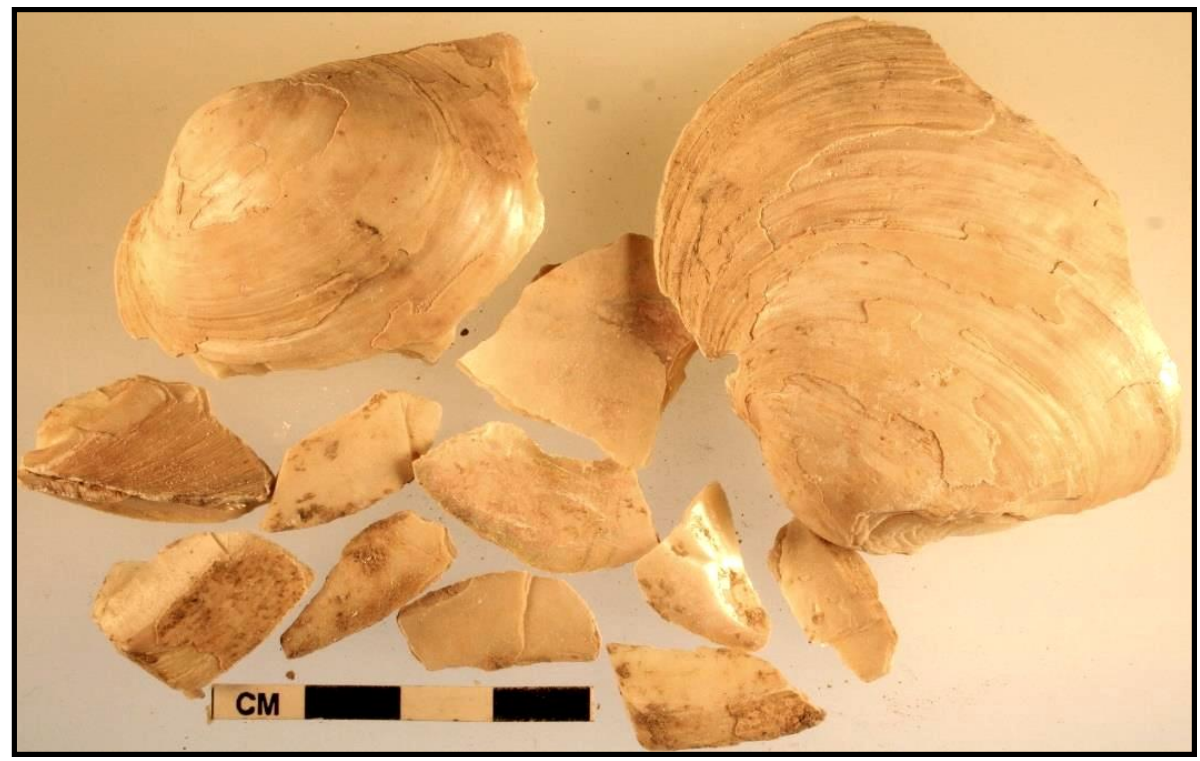

Figure 5-22. Example of unburned mussel shell fragments.

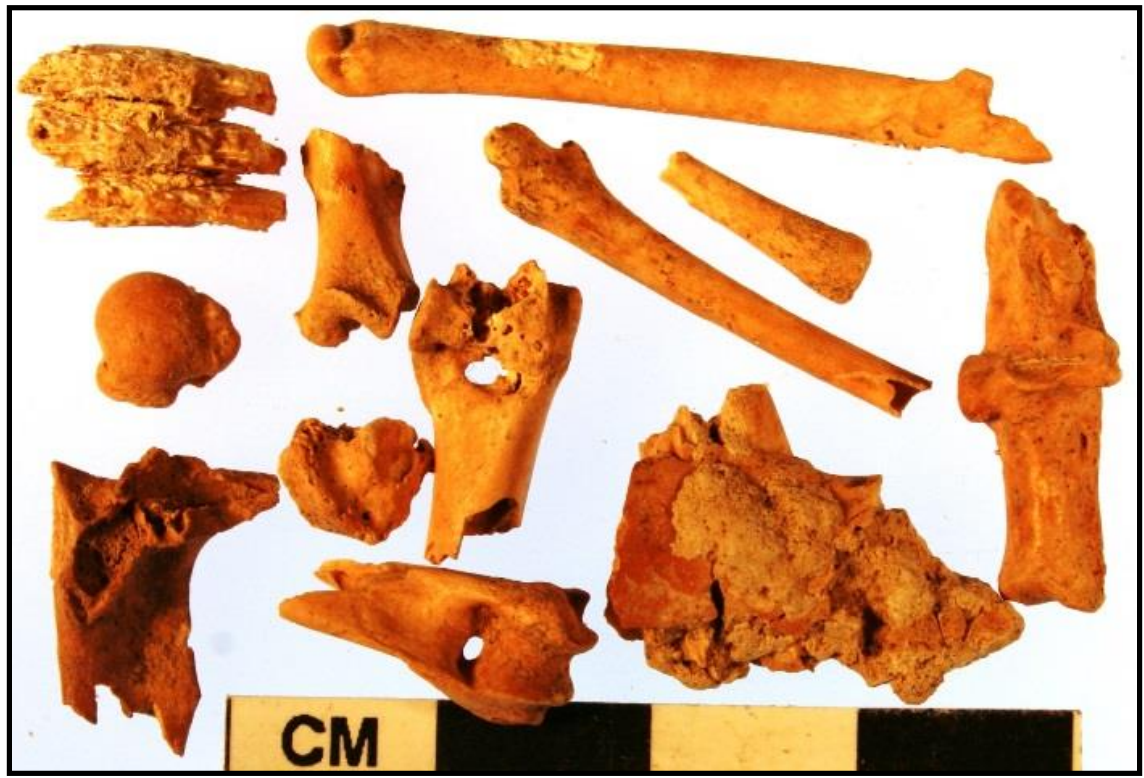

Figure 5-23. Example of small fragmented vertebrate bones.

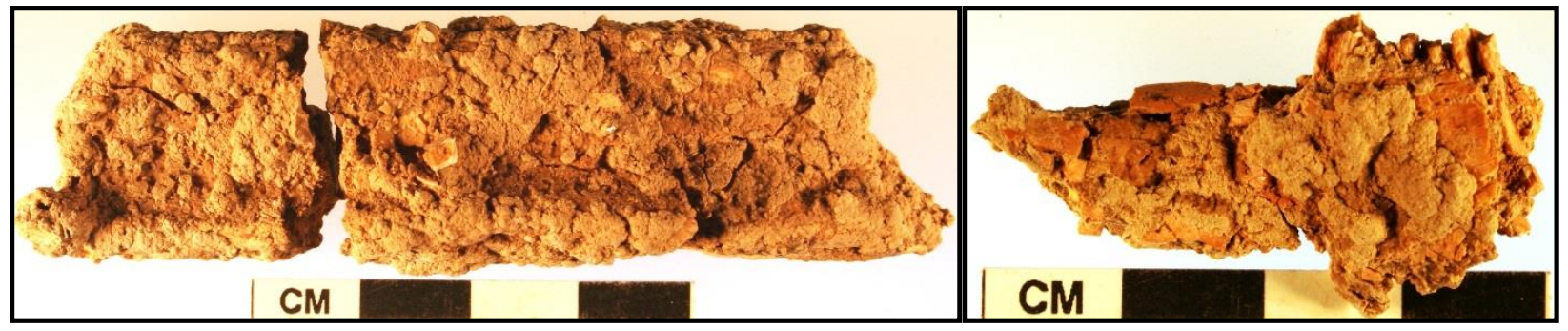

Figure 5-24. Examples of calcium carbonate adhering to a deer size long bone fragment (left \#2296) and rabbit mandible (right \#2193). 
intensively bones were processed. Highly fragmented bones may reflect bone grease rendering or the gross size of the processed animal.

Many fragments were partially or completely covered with calcium carbonate (Figure 5-24). Often this hindered species and even element identification, and detection of cultural alterations. The calcium obviously added weight to specimens so the total weight presented for groups or component is slightly exaggerated by the extra calcium carbonate weight. The calcium was not removed from individual bones.

Each bone was inspected for various cultural alterations that included burning, scrape marks, chop marks, blunt impacts, cut marks, and other possible cultural modifications (Fisher 1995). Cut marks include different types such as thin and thick cut lines from stone tools made during skinning, defleshing, and disarticulation (Figure 5-25). Cut mark morphology reflects the shape of the tool's edge, the angle at which the tools was held, and the force behind the tool. Broad chop marks or percussion pits are often linear depressions that generally exhibit a $\mathrm{V}$-shaped cross section caused by larger and heavier stone tools, often during disarticulation. Impact locations are characterized by conchoidal flake scars and bone flakes, created by heavy hammer stones that indicate point of impact where the element was struck to break the bone, as in marrow extraction (Figure 5-26).

Burning may result in a variety of visible colors that are generally related to the temperature (degrees Celsius $\left[{ }^{\circ} \mathrm{C}\right]$ ) that the bone was exposed to (Figure 5-27). This includes bones burned to a solid black, a solid brown, a mixture of brown and black,

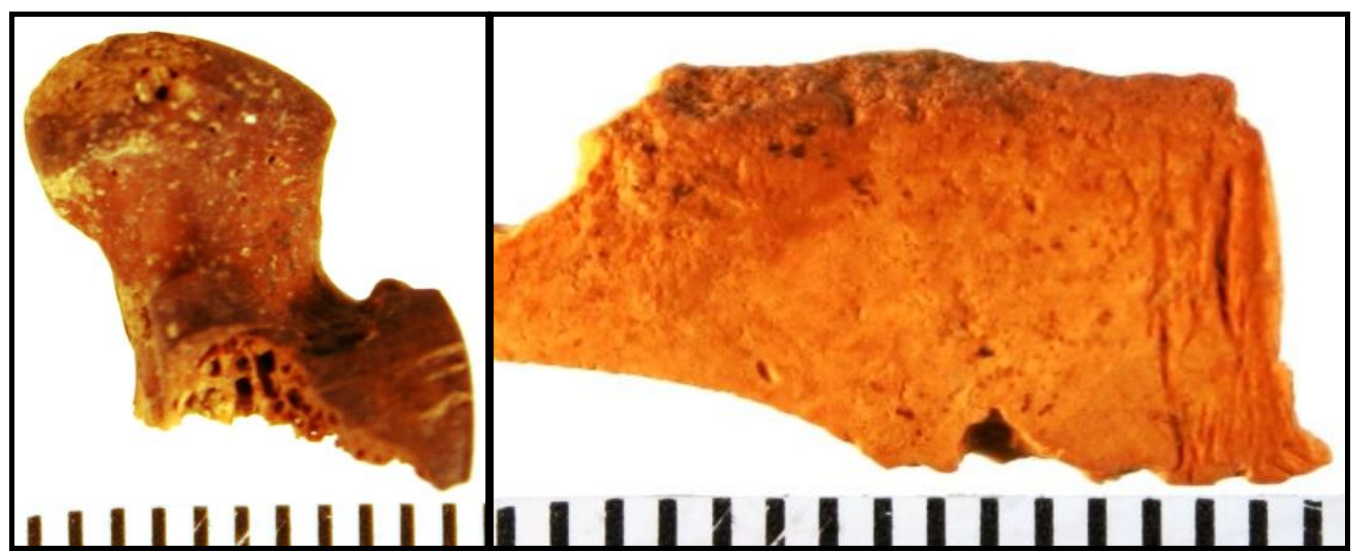

Figure 5-25. Examples of thin cut marks on bones (\#2292- left, \#2244-right; scale in millimeters).

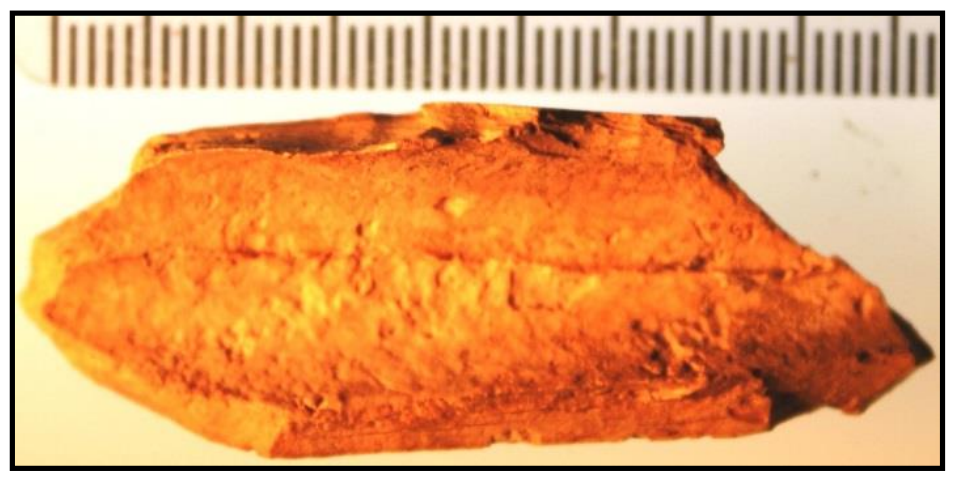

Figure 5-26. Examples of impact scar (middle top) on interior of deer size long bone (\#2250, scale in millimeters). 


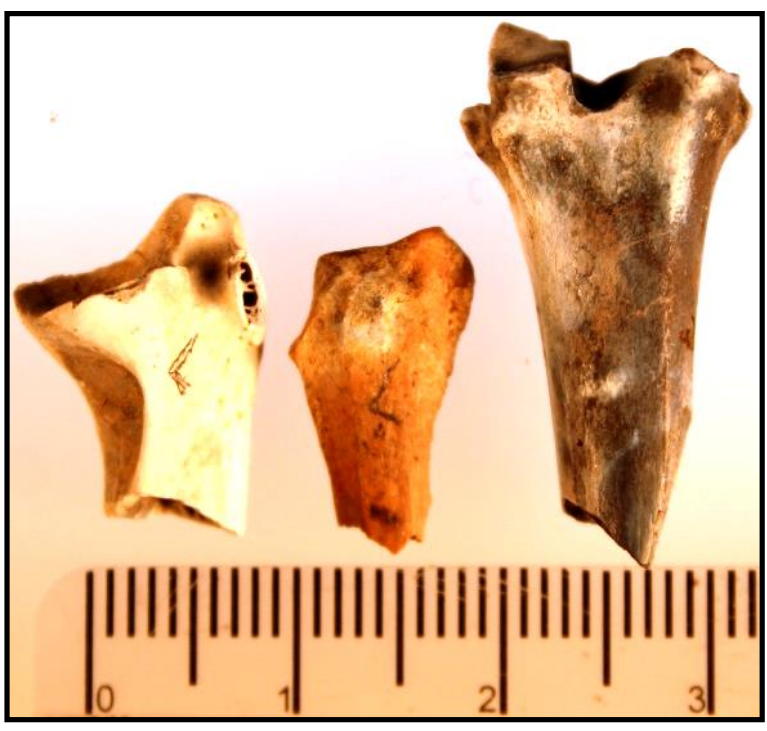

Figure 5-27. Examples of burned jackrabbit bones (\#2247, scale in centimeters).

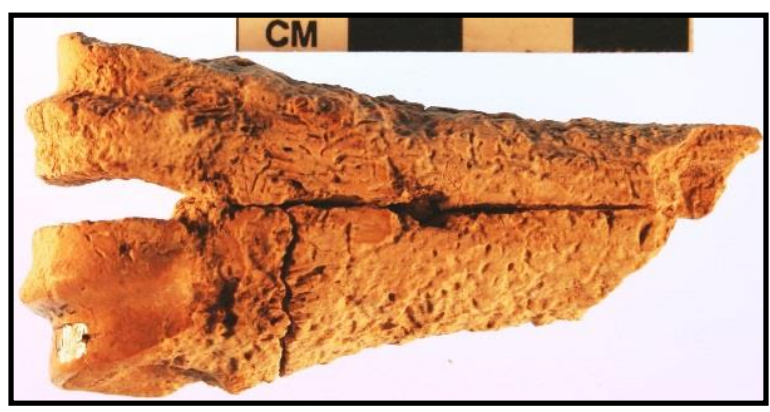

Figure 5-28. Example of root etched bone.

a calcined white, and a mixture of black and white. Generally speaking, bones of an ungulate turn a brown color in the temperature range around $200^{\circ} \mathrm{C}$, black in the $300^{\circ} \mathrm{C}$ range, gray in the 300 to $400^{\circ} \mathrm{C}$ range, and white above about $700^{\circ} \mathrm{C}$ range (Nicholson 1993).

Root etching, chemical degradation, is a separate noncultural process that causes narrow, shallow squiggly lines and pits etched into outer bone surfaces by acids associated with plant roots (Fisher 1995). These marks are sinuous or wavy, have Ushaped cross sections, and are easily identified (Figure 5-28). These are postdepositional processes.
Element maturity estimates (i.e., element not fully developed) are based on degree of fusion of articular/epiphyseal ends to the main bone shaft. Different bone elements are known to fuse at different times in an animal's life and vary between animals. However, very little is known about the exact timing of bone fusion rates in deer. Fetal bones are elements characterized by a gross structural morphology which mimics the same form as the mature animal, but are miniature in size. These pieces reveal a porous layered tissue or flakey structure unlike a solid cortical wall or the interior cancellous tissues from mature elements. Age of ungulates is often determined by tooth eruptions and/or tooth cementation analysis of individual teeth. Most mandible sections were very incomplete and often teeth were damaged so eruption and wear patterns to determine age were not possible. In a few instances a general age was possible for a couple of ungulate mandibles/teeth, but these are insufficient in number to have complete confidence in the final analysis.

Minimum number of individuals (MNI) by species was derived from the maximum number of discernable elements by side, combined with size, and age estimates also considered. Faunal identifications were conducted by Mr. Quigg using his personal comparative collection. An electronic scale was employed for weights. A paper recording sheet was employed during the analysis in which the above observations were recorded along with specific provenience information. Once analysis was completed the hand-written data sheets were entered into a database, and subsequently incorporated into the site's master database. Data analysis was performed for all faunal remains with results and discussions presented in appropriate chapters (Chapters 9.0, 10.0, and 11.0), with unassigned specimens presented and discussed in Chapter 11.0. 


\subsubsection{Burned Rock Analysis}

Burned rocks often account for the highest percentage of artifacts from open-air huntergatherer camps. These rocks were heated and often rapidly cooled as the result of use in transferring heat from a fire to the rock to food stuffs in cooking or other heating activities. While it is occasionally difficult to distinguish burned from unburned rocks in the field, especially if the rock has not broken during the heating and cooling process, many may exhibit cracks, discoloration, crazing, reddening, and/or if broken angular fragmented edges.

During excavation, burned rocks were treated as cultural artifacts. The larger pieces were most often mapped in situ. Burned rock pieces greater than 1 $\mathrm{cm}$ from each hand-excavated level were collected and recorded. Collected burned rocks were sorted into four previously established size categories (i.e., 0 to $4 \mathrm{~cm}, 4.1$ to $9 \mathrm{~cm}, 9.1$ to $15 \mathrm{~cm}$, and greater than $15 \mathrm{~cm}$ ) based on maximum dimensions, and counted and weighed by size class. Most burned rocks were in feature contexts, whereas scattered burned rocks from nonfeature contexts were also collected and recorded with samples bagged, and returned to the laboratory for processing, cataloging, and possible analysis. Following their documentation most burned rocks were discarded in the field. While the entire volume of burned rock encountered during hand-excavations is known, only a small sample, mostly from features, was retained for possible further analysis. Even a smaller sample was curated. Subsamples of rocks subjected to specific analyses such as lipid residue or starch grain analyses were curated. Larger pieces form various features were also selected and curated.

In 2006, 15 samples that included 12 burned rocks plus 3 ground stone tools were more or less randomly selected for lipid residue analysis. Selected rocks were often larger pieces that could be broken and subsampled to allow extraction of multiple subsamples from the same rock to be sent for different analyses. The fundamental assumption being multiple analyses on the exact same artifact strengthens the final interpretation of function and/or the foods cooked by the burned rocks. The 12 burned rocks represented 6 cultural features from both identified components as did the ground stone tools. Subsamples were sent to Dr. Mary Malainey in Winnipeg, Manitoba for analyses. Her original findings were presented in the interim report (Quigg et al. 2007). On the basis of positive findings from the initial assessments, another 28 burned rocks from mostly the same features plus 10 previous samples were again sent to Dr. Malainey. New and improved lipid analysis procedures were added to the previous procedures since the 2006 analysis and applied to all 38 samples. Dr. Malainey's detailed sample preparation and extraction methods, individual sample analyses, and interpretations are presented in Appendix G.

In most instances, subsamples subjected to lipid residue analysis were also subjected to starch grain analysis. Therefore, 28 subsamples of burned rocks were submitted to Dr. Linda Perry for starch grain analysis. Appendix F presents background on starch analyses, the methods used, and individual results of starch analysis.

Subsamples of nine burned rocks employed in the above starch grain analyses were also sent for diatom analysis to Dr. Winsborough in Austin. Results from the diatom analysis are presented in Appendix C.

\subsubsection{Sediment Analyses}

Multiple sediment samples from a wide variety of proveniences and context were collected during field investigations in anticipation of multiple types of laboratory analyses. Sample sizes varied tremendously depending on the projected type of analyses. Data and results concerning these sediments are incorporated into appropriate component chapters and discussions. 
Three small samples (\#2143-4c, \#2156-4b, and $\# 2262-4 b$ ) from the mottled greenish-gray clay zone were sent for pollen presence/absence to Dr. Bozarth. His procedures and assessments are presented in Appendix L.

Nine small samples, three from feature context (\#2024-4d -Feature 18, \#2112-4b-Featrue 26, and $\# 2146-4 b-F e a t u r e ~ 22)$, three from the mottled greenish-gray clay zone (\#2143-4d, \#2156-4c, and $\# 2262-4 c$ ), and three from the geoarcheological vertical column (\#2411-4c, \#2400-4c and \#2146$4 c)$ were sent for phytolith presence/absence to Dr. Bozarth. His procedures and assessments are presented in Appendix K.

One small sediment sample (\#2143-4b) was sent for starch analysis to Dr. Perry as a control and comparisons with results from cultural artifacts. Her detailed methods, identifications, results and discussions are in Appendix F.

Thirty-three sediment samples were submitted to Dr. Winsborough for diatom analysis. This included sediments from multiple features, three from the vertical geoarcheological column, six calcium carbonate flakes extracted from faunal remains, and one from a burned zone. She presents her diatom background, extraction methods, and individual results in Appendix C.

Thirty-six samples were submitted for detailed phytolith analysis to Dr. Sudbury. Those samples included 18 from features, 11 from the geoarcheological vertical column, 3 from artifact concentrations, 2 from the mottled greenish-gray clay zone and 2 were surface control samples. His detailed extraction methods, individual results and discussions are in Appendix E.

Ten small samples were submitted to Mary Schabel of the Milwaukee Soil Laboratory, LLC for multiple types of analysis that included particle size, organic carbon (Walkley-black method), percent organic matter, percent carbonates, percent magnesium, total phosphate, and $\mathrm{pH}$. Three of the samples were collected from the geoarcheological vertical column (\#2400-4, \#2402-4, and \#2414-4), one from the mottled greenish-gray clay (\#21434b), two from Martindale features (\#2239-4 Feature 25, and \#2112-4 - Feature 26), two from Bell/Andice features (\#2179-4 - Feature 24, and \#2223-4 - Feature 29), one from a unassigned feature (\#2367 - Feature 19), and one from the Bell/Andice cultural component (\#2094-4) Two sediment samples (\#2223-4 from Feature 29 and \#2112-4 from Feature 26) were submitted to e-Lab Analytical Inc., in Houston for total organic carbon through the Walkley-Black method plus metal tests. These were submitted to determine the nature of the dark organic stains around burned rocks features. The laboratory results are presented in Appendix M.

\subsubsection{Flotation Methods}

Bulk sediment samples that totaled 290.7 liters from 24 separate proveniences and features were mechanically floated with the Dousman flotation system at the TRC Austin facilities in south Austin (Figure 5-29). Dry sediment was first measured for volume, then slowly poured into churning water. To insure lightweight materials were not adhering

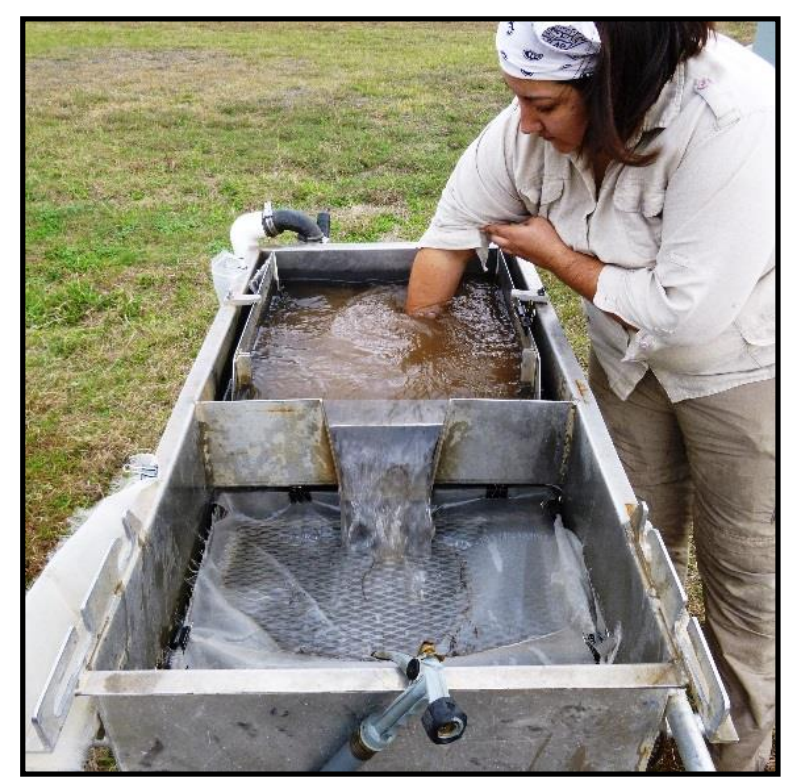

Figure 5-29. Dousman flotation system in operation to float sediment samples. 
to clay particles, hand agitation was often conducted. Each sample was in the float system for 15 to 30 minutes to insure complete separation of heavy and light fractions from the clayey sediments. The system allows for light and heavy fractions to be collected separately, which were bagged, tagged and dried, and returned to the laboratory for sorting. Floated sediment samples were from eight Bell/Andice features (Features 18, $22,24,27,28,29,30$, and 32) with a total of 83.9 liters, three Martindale features (Features 25, 26 and 33) with a total of 20.5 liters, four natural or unassigned features (Features 15, 19, 21, and 23) with 139.8 liters, and nine samples from nonfeature context with 46.5 liters.

In the laboratory, each heavy fraction was spread across clean white paper and sorted with the aid of lighted magnification into general artifact classes such as flakes, shells, burned rock fragments, charcoal, bone, etc. The frequencies and weights of each class were recorded on forms, bagged and tagged separately, and results incorporated into appropriate feature discussions. Light fractions were visually scanned under magnification searching for charred seeds, charcoal, and other possible cultural materials. The obvious tiny hairlike rootlets were most often removed and discarded from each sample. Those with apparent charred macrobotanical remains were sent to Dr.
Dering for identification. Most light fractions yielded no charcoal or charred materials and were not further analyzed.

\subsection{CURATION}

Artifacts collected during this 2006 data recovery project were temporarily stored at the offices of TRC in Austin. Most collected artifacts and samples, all field records, and digital photographs from this project are permanently curated at the Center for Archaeological Studies (CAS) at Texas State University in San Marcos, Texas. Curated items include material classes such as sediments, charcoal, burned rocks, stone tools, and snail and mussel shells. The discarded materials include mostly burned rocks, snail shells, mussel shell fragments, sediment samples, burned clay and most unassigned materials. Individual artifacts or artifact lots, (including stone tools, lithic debitage, mussel shells, animal bones, sediment samples, and burned rocks) are bagged in clear, zip-locking four millimeter thick archival polyethylene bags according to provenience. Each polyethylene bag contains an archival-quality, acid-free curation tag that lists the site number, provenience data, date of excavation, excavator(s) name, artifact type, and quantity in pencil. Digital photographs and the photograph catalog were submitted on compact disks (CD) along with a printed contact sheet. All original field records are on acid-free paper and were placed in acid-free file folders for curation. 
This page intentionally left blank. 


\subsection{GEOARCHEOLOGICAL EVALUATIONS}

Charles D. Frederick, Eric Schroeder, and J. Michael Quigg

\section{$6.1 \quad$ INTRODUCTION}

The site is situated in the lower reaches of the Onion Creek drainage basin, approximately $7 \mathrm{~km}$ upstream of its confluence with the Colorado River. From the point where it crosses U.S. Highway 183 to its mouth, Onion Creek appears to flow in an ancient Colorado River meander. The Bureau of Economic Geology (Barnes 1974, 1981) has mapped almost all deposits in this ancient meander, except the low floodplain surface of Onion Creek, as Pleistocene (map Unit Qt). Recent archeological and geoarcheological investigations in this region (e.g., Blum 1992; Campbell et al. 2005, 2007; Frederick 2006, 2011) have unequivocally demonstrated this age interpretation is incorrect in many areas, and the Bureau of Economic Geology has miss-mapped the majority of the Holocene-age deposits as Pleistocene.

\subsection{REGIONAL BACKGROUND}

Currently, few detailed studies of the alluvial stratigraphy of Onion Creek are available, and a significant portion of the existing literature directly correlates the Onion Creek deposits with the stratigraphy of the Colorado River. The late Quaternary stratigraphy of the Colorado River is one of the most studied in Texas, with investigations dating back to 1897, when Hill and Vaughan (1897) first identified five terraces of the Colorado River near Austin. Between then and the most recent work (Blum 1992; Blum and Valastro 1994; Blum et al. 1994), more than seven aerially extensive investigations have been published (Baker and Penteado-Orellana 1977, 1979; Looney 1977; Looney and Baker 1977; Urbanec 1963; Weber 1968; Weeks 1945) and if several site specific studies within the Colorado catchment, with relevant stratigraphic observations are included (e.g., Caran 1998b, 1999, 2003, 2009; Caran et al. 1992; Frederick 1987, 1996, 2006, 2011; Nordt 2002; Quigg and Peck 1995; Robinson and Caran 1995) the number of studies is larger still.

At this point in time the stratigraphic nomenclature for the Colorado River is a mixture of old and new studies. The most recent study is Blum's work, but this piece of research focused on the latest Pleistocene and Holocene and did not examine the older deposits, so the nomenclature for the older deposits must be sought from another, older work. Although the work of Victor Baker and students are next in line, Blum's work on the Colorado at disparate ends of the basin (upstream near O.H. Ivie Reservoir and downstream on the coastal plain southeast of Austin) has demonstrated that although much of Baker's work is, elegantly designed, it is inherently flawed, and for this reason it is excluded. Prior to Baker's work, the most recent and presumably accurate account of Colorado River terraces is Weber's (1968) Master's thesis. This work is only subtly different from the sequence described by Weeks (1945) and recognizes seven alluvial deposits in the Colorado valley near Austin. From oldest to most recent, these are Manor Lag Gravel, Asylum Terrace, Hornsby Terrace, Capitol Terrace, Montopolis Terrace, Sixth Street Terrace, and Floodplain. Weber lumped the last three deposits of Week's sequence (First Street Terrace, Riverview, and Sand Beach) into a single unit on the basis that all three had been inundated by modern flooding. At this point in time, it is assumed that Weber's nomenclature is the most accurate for the older terrace deposits.

The latest Pleistocene and Holocene deposits were revised by Blum (1992) and he recognized four allostratigraphic units. From oldest to most recent, the allostratigraphic units include the Eagle Lake Alloformation, and the Columbus Bend Alloformation members 1, 2, and 3. Blum (1992:198) correlated his most recent deposit (the Columbus Bend 
Alloformation Member 3) which represents deposition in the last millennium, with the youngest two terraces in Week's sequence (the Sand Beach and Riverview). Blum correlated the Columbus Bend Alloformation members 1 and 2 which represent deposition in the Holocene, with the First Street Terrace, and the Eagle Lake Alloformation with the Sixth Street Terrace. Although Blum's work has been repeatedly confirmed by archeological studies, it has not been universally accepted, with Caran (2003) presenting the most detailed rebuttal, specifically taking issue with the age of the First Street Terrace, which Caran argues spans a much longer period than Blum suggests.

There is a critical issue at the core of this dispute. The most detailed chronology of the First Street Terrace is from the Avenue Site (cf., Frederick 1987; Lundelius 1992) where it was demonstrated that the core of the First Street Terrace is fully Pleistocene where it lies adjacent to a buried bedrock scarp along the northern valley wall and abuts a much older Sixth Street Terrace deposit. The top of the First Street Terrace at the Avenue site is clearly cumulic during the Holocene. Blum (1992:163-165) acknowledged a potential problem between his correlations with older studies and his work, and suggested that the core of the First Street Terrace in this location may be the Eagle Lake Alloformation, but that in most places this surface is underlain by Holocene sediments. The chronology of the deposits from the Avenue site is consistent with such an interpretation. But if this is the case, then the Eagle Lake Alloformation is not correlative with the Sixth Street Terrace as Blum posits, but rather is a terrace fill that has not been previously recognized by the traditional alluvial terrace sequence defined for Austin.

\subsection{ALLUVIAL STRATIGRAPHY OF ONION CREEK}

The latest Pleistocene and Holocene alluvial deposits of Onion Creek have been examined in detail in three studies: by Abbott (1994) near Buda along Onion Creek, by Caran (1998b) at Austin-Bergstrom
International Airport, and by Frederick (2011) at sites 41TV410 and 41TV540 just east of the airport with limited work at the Berdoll site (41TV2125) of SH 130 (Campbell et al. 2005). The first three projects provide detailed discussions of the stratigraphy.

Abbott (1994) examined the deposits of Onion Creek and a tributary (Mustang Branch) at the Barton and Mustang Branch archeological sites in Hays County, Texas, approximately $30 \mathrm{~km}$ (linear) upstream from the confluence of Onion Creek and the Colorado River. Although the majority of the alluvial deposits examined by Abbott were Pleistocene-age Onion Creek deposits, both streams experienced deposition in the Holocene, albeit in a locally asynchronous manner. A total of five distinct geomorphic surfaces were present in the area Abbott examined, but only the lowest two terraces were of Holocene age. The second terrace of Onion Creek lies 9 to $10 \mathrm{~m}$ above the stream channel and the deposits beneath this surface were first deposited in the late Pleistocene and continued into the early Holocene. Abbott obtained a radiocarbon date of $5310 \pm 90$ B.P. (Tx-7170) from a buried soil B horizon at the top of the point bar deposits of $1.7 \mathrm{~m}$ below surface, and also noted that a Bell projectile point had been recovered from the same gravelly deposits elsewhere on the site. Abbott correlated this phase of deposition with Unit E on the Pedernales River (Blum 1987) and the Columbus Bend Alloformation Member 1 (early-middle Holocene fill) (Blum 1992).

During the middle to late Holocene Onion Creek was locally incising and the only significant deposits associated with this period were found in the lower parts of the abandoned channel associated with the second terrace. Bulk sediment samples derived from these deposits yielded radiocarbon dates of $3220 \pm 100$ B.P. (Tx-6650) and $2780 \pm 60$ B.P. (Tx-6649; Abbott 1994). After 2700 B.P., deposition within this slough shifted from overbank flooding by Onion Creek to local slope sources, and Abbott inferred this to mean that channel incision by the trunk channel of Onion Creek had proceeded to a point where flooding of this surface was no longer possible. 
Although the main channel of Onion Creek was not depositing sediment in this area in the late Holocene, the modern floodplain of Mustang Branch did form in this period. Radiocarbon dates on charcoal collected from this deposit indicate that this deposit began forming around 2000 B.P. and continued through at least 600 B.P. Significantly, Abbott (1994) demonstrated a significant difference in radiocarbon age between bulk sediment and charcoal ages within this alluvial fill.

Some of the more recent work on the alluvial stratigraphy of Onion Creek has been performed by Chris Caran (1998b; Campbell et al., 2006). Caran (1998b) recognized four geomorphic terrains in the vicinity of Austin-Bergstrom International Airport:

1) the stream banks and channel floor;

2) the active floodplain ( $T_{1} ; 4$ to $5 \mathrm{~m}$ above the channel floor);

3) the Onion Creek Terrace $\left(T_{2} ; 6\right.$ to $11 \mathrm{~m}$ above the channel floor); and

4) a late Holocene flood terrace developed on late Pleistocene to middle (?) Holocene channel deposits.

Caran (1998b) describes the majority of the Holocene deposits in the Onion Creek valley as part of the Onion Creek Terrace (his $\mathrm{T}_{2}$ surface) which he views as correlative with the First Street Terrace in downtown Austin because the Onion Creek terrace and the First Street Terrace merge near the mouth of Onion Creek. Caran specifically describes all of the deposits beneath the Onion Creek Terrace as part of a flood terrace that is a surface that has been abandoned by the stream channel, but continues to be inundated by floods. Such surfaces are separated from the floodplain by a steep bank, but continue to vertically aggrade through inundation by overbank flooding. Unfortunately, Caran makes no attempt to subdivide the deposits of the Onion Creek Terrace, and few of the deposits examined in this volume were radiocarbon dated.
More recently, Campbell et al., (2005) examined the deposits of Onion Creek along the margins of the proposed $\mathrm{SH}$ 130, immediately upstream from the mouth of Onion Creek. In this work Caran reiterates his impression of the Onion Creek Terrace, but for this project radiocarbon ages were obtained from two sites: 41TV2125 and 41TV540. Both sites are situated up against the bedrock valley wall, adjacent to a prominent paleochannel. At 41TV2125, Campbell et al., (2005) reports a series of radiocarbon ages on charcoal recovered at 4 to 5 $\mathrm{m}$ below the present ground surface of $9190 \pm 40$ B.P. (Beta-204632), $9420 \pm 60$ B.P. (Beta-204633) and $9540 \pm 40$ B.P. (Beta-204634). At 41TV540, about $1 \mathrm{~km}$ south of 41TV2125, four radiocarbon ages were obtained from charcoal recovered at depths between 3.8 and $4.8 \mathrm{~m}$, which ranged from $5310 \pm 40$ B.P. (Beta-209897; $3.81 \mathrm{~m}), 5400 \pm 40$ B.P. (Beta-209896; $4.02 \mathrm{~m}$ ), $5310 \pm 40$ B.P. (Beta209899; $4.15 \mathrm{~m}$ ), and 5350 \pm 40 B.P. (Beta-209898; $4.79 \mathrm{~m})$. The latter dates were not reported in the draft report, but provided by Dr. Jim Abbott (personal communication, TxDOT). Although seemingly from similar landscape positions, these two sites were clearly deposited at significantly different times.

The only date available from the alluvial deposits beneath the $\mathrm{T}_{1}$ surface was recently obtained by Hicks \& Company from work they performed at 41TV410, which is on the south (right) bank of Onion Creek where it is crossed by the path of $\mathrm{SH}$ 130. At this location Robert Rogers (2007, personal communication) obtained a charcoal date of $1290 \pm$ 40 B.P. (Beta-213174; $3.1 \mathrm{~m}$ depth) from cultural deposits approximately $3 \mathrm{~m}$ below the floodplain surface.

\subsection{LATE QUATERNARY ONION CREEK STRATIGRAPHY: A NEW MODEL}

Although Caran (1998b) suggests that the Onion Creek Terrace is a single flood terrace that is time transgressive from the late Pleistocene through to 


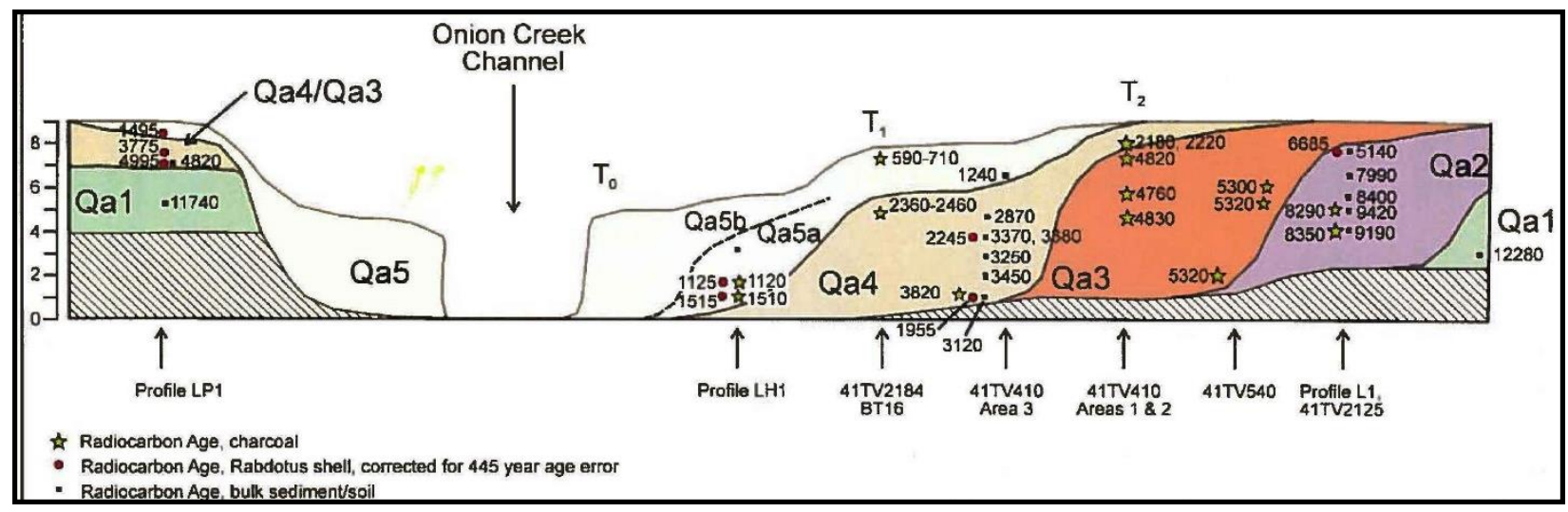

Figure 6-1. Schematic illustration showing the stratigraphic relationships observed in the lower Onion creek valley and selected radiocarbon ages. Horizontal is not to scale, and vertical scale is approximate (from Frederick 2011; Figure 8-10).

today, it seems more likely that the core of this terrace deposit was formed episodically at different times in different places. Whereas the upper portions of the terrace is comprised of vertically accreted sediment is time transgressive with the length of cumulic deposition being dependent upon the time the core at depth was abandoned and the elevation of the surface. In other words, the deposits of the Onion Creek Terrace at any single location are bipartite. Radiocarbon dating conducted at specific archeological sites in association with the construction of $\mathrm{SH} 130$ by various cultural resource management contractors, specifically University of Texas at San Antonio at 41TV410 and 41TV540 (Figueroa et al. 2011), TRC at 41TV2161 (this report), Hicks \& Company at 41TV410 (Campbell et al. 2005, 2006, 2007), and Hicks \& Company at 41TV2125 (Campbell et al. 2010; Karbula et al. 2011) appear to support this interpretation (Figure 6-1).

Figures 6-2 and 6-3 exhibit at least three discrete depositional episodes present at depth beneath the $\mathrm{T}_{2}$ or Onion Creek Terrace during the Holocene, and if the modern floodplain is considered, then there appear to be four Holocene aggradational events. With the evidence in hand, these events date to the following intervals:
1) 10,000 to 8,000 years B.P.,
2) 6000 to 4500 years B.P.,
3) 3800 to 300 years B.P., and
4) post 1300 years B.P.

At this time it is not clear if this is an accurate representation of all of the major depositional events as a prominent gap exists between 8000 and 6000 B.P. (see Figure 6-1). It is also interesting that this does not directly match the Colorado River alluvial chronology (Blum 1992).

Although many central Texas streams exhibit Holocene alluvial fills with cumulic treads, this trend appears considerably greater in this area. This may be attributable to hydraulic damming of Onion Creek in the vicinity of the confluence with the Colorado River. Hydraulic damming or back flooding is a common occurrence where a tributary joins a larger stream, and this situation often results in the deposition of slack water or suspended sediment, and the accumulation of flotsam (e.g., such as driftwood and snail shells) in the tributary valley (cf., Denlinger et al. 2002:130-131; House et al. 2002:277). Throughout the lower reaches of Onion Creek the vertically accreted alluvial deposits exhibit thin, but extensive burn zones that are probably associated with the burning of flotsam 


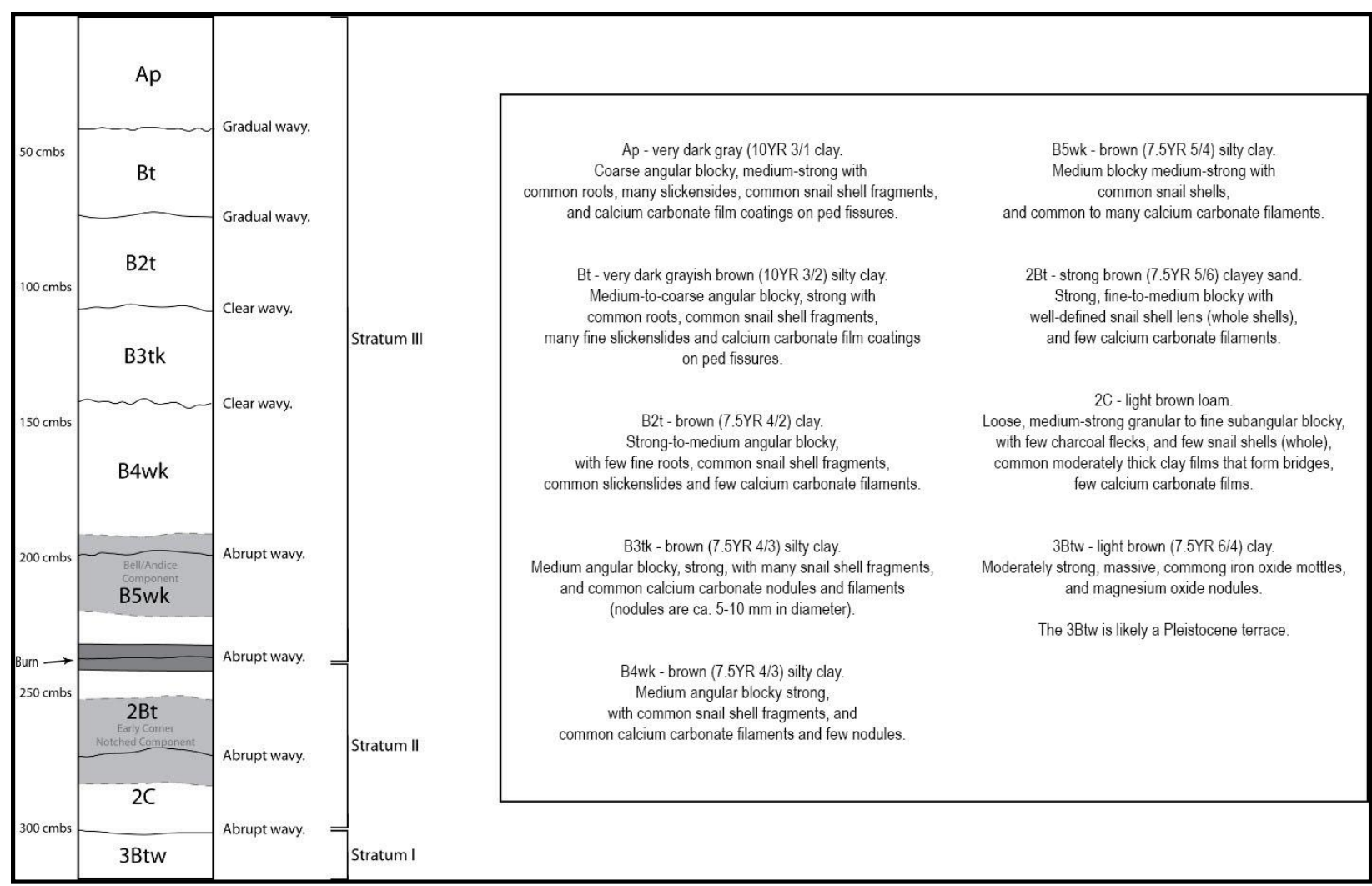

Figure 6-2. Soil column descriptions on south profile of mechanically stripped block at Big Hole.

deposited on the floodplain during large magnitude flood events (i.e., Mauldin et al. 2011b, Figure 621). There are two reasons this is the favored mechanism to explain these features. First, it seems unlikely that grass fires would have sufficient fuel to result in such intense and extensive burn zones; and second, these burns, although often stratigraphically close to prehistoric occupations, did not appear to be related to them. Given that the Colorado River is a much larger stream, it is probable that flooding on the Colorado would back up into the Onion Creek valley, resulting in higher flood levels than would be common in the main part of the Onion Creek valley at a distance from the confluence. It is uncertain how far up the valley this effect might reach, but it clearly affects the lower reaches of Onion Creek.

\subsection{GEOARCHEOLOGYOF SITE 41TV2161}

With the aforementioned issues in mind, the deposits exposed at 41TV2161 can be placed into a larger context. The targeted cultural deposits at 41TV2161 were near the bottom of a large mechanically dug pit that was ca. 2.0 to $2.5 \mathrm{~m}$ deep, and covered an area ca. 30-by-40 $\mathrm{m}$ in size. The archeological materials at the site were found throughout the depth of the pit, but were clearly concentrated between 2.0 and $3.0 \mathrm{~m}$ below surface. The geoarcheologist, Mr. Eric Schroeder, spent time inspecting the walls of the deep pit and open hand-excavated units, and then examined and documented one vertical column from the south wall of the deep pit. This profile revealed at least three alluvial strata referred to as Stratum I, II, and III (see Figures 6-2, 6-3 and 6-4). The following 


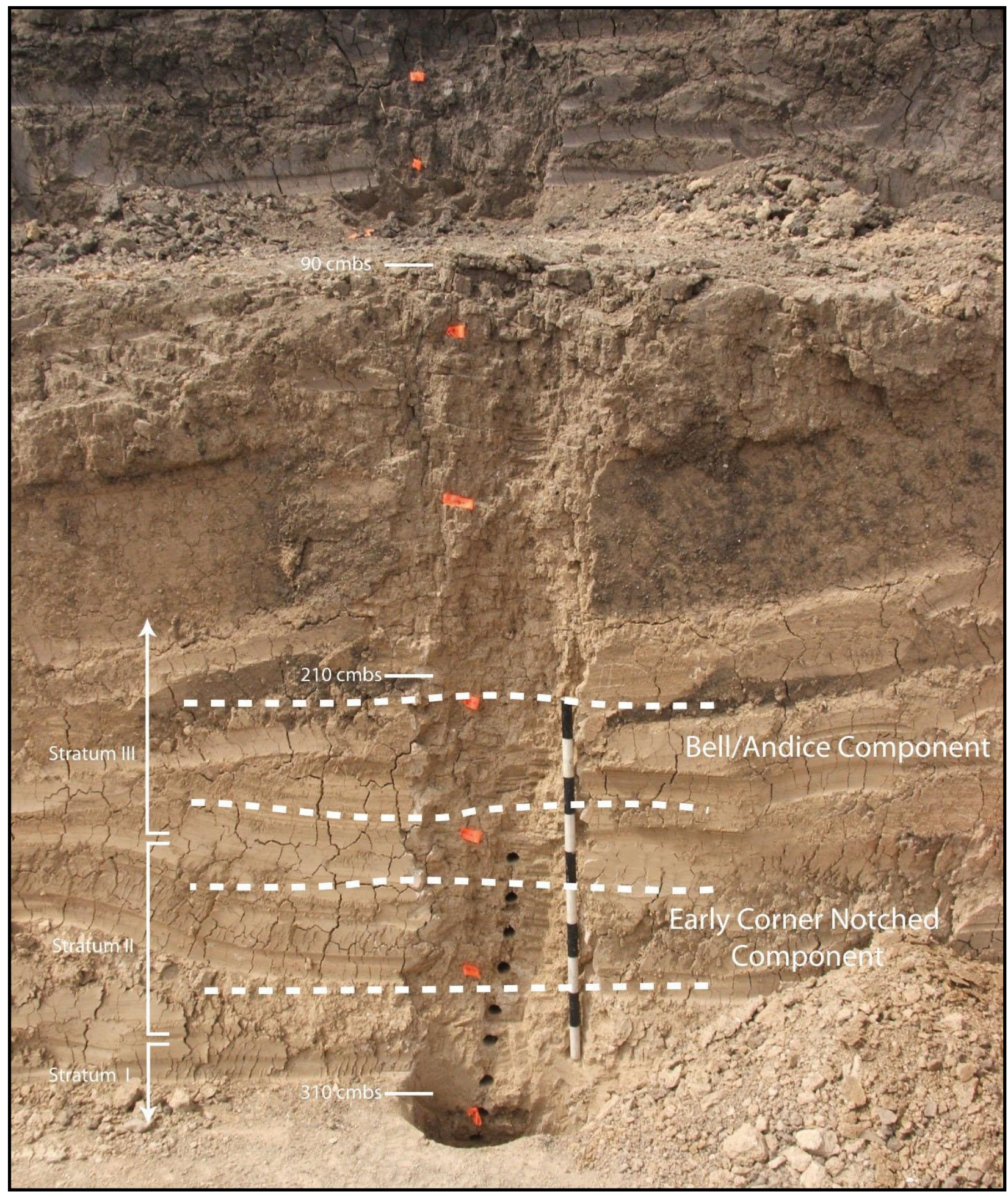

Figure 6-3. View of sediment column described by geoarcheologist on south wall of deep pit showing sample locations (top hole is at boundary of Stratum II and III, flat base is bottom of drainage trench, base of upper dark zone is top of safety bench). 


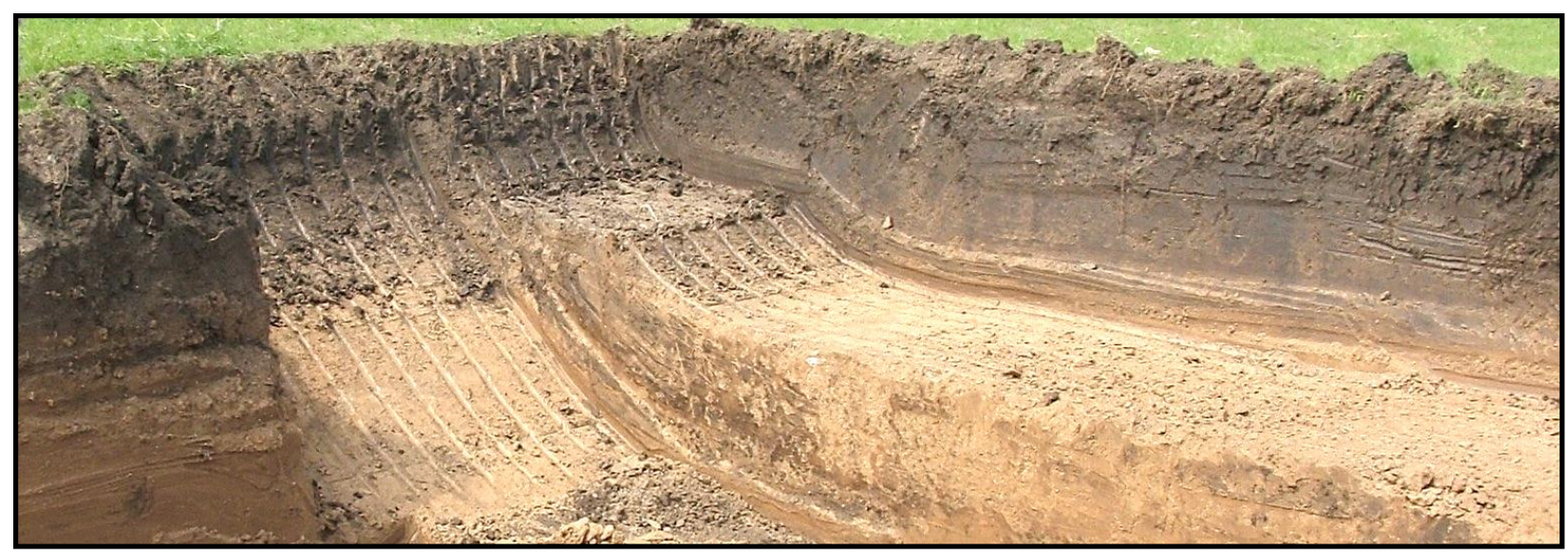

Figure 6-4. General view of upper Stratum II and III deposits above the targeted cultural deposits at bottom of photograph.

discussions and the representative vertical profile of the sediments were extracted from near the middle of the southern wall and near original BT 21. The identified strata are presented and discussed from the bottom to the top.

Stratum I consists of light brown (7.5YR 6/4) clay that was encountered at a depth of $3.0 \mathrm{~m}$ below the surface. The high degree of weathering in the profile, indicated by its strong brown color and mineral staining, all suggests that it is an alluvial terrace deposit of excessive age. The elevation of this stratum above the modern Colorado River channel indicates that it likely corresponds to an allostratigraphic unit of Pleistocene age. Stratum II overlies Stratum I across an unconformity and consists of a $0.6 \mathrm{~m}$ thick unit of brown (7.5YR 5/6) sandy loam. Stratum II is likely middle Holocene in age and represents overbank flood deposits that, based on diagnostic archeological materials recovered, likely dates to around 6000 B.P. Soil development within this stratum is relatively weak and consists of two horizons - the upper a strong brown (7.5YR 5/6) sandy Bt Horizon that overlies a light brown (7.5YR 6/4) sandy C Horizon (see Figures 6-2, 6-3, and 6-4). Other soil characteristics include few fine calcium carbonate $\left(\mathrm{CaCO}_{3}\right)$ filaments and moderately thick clay films in the Bt Horizon.
Stratum III overlies Stratum II and is ca. $2.4 \mathrm{~m}$ thick (see Figures 6-2, 6-3, and 6-4). Stratum III consists predominantly of a silty clay facies that likely represents relatively slow aggradation over several thousand years. The one notable difference is that the sedimentary facies is considerably finer grained than the lower strata, suggesting an abrupt change in the local depositional regime. The soil sequence in the stratum is characterized by at least six soil horizons that comprise Ap-Bt-B2t-B3tk-B4wkB5wk profile sequence (see Figure 6-3). In the lower three B Horizons, the calcium carbonate morphology primarily consists of common filaments with a few small (5 to $10 \mathrm{~mm}$ in diameter) soft nodules.

During the hand-excavations in Block B a very thin, mottled greenish-gray (Gley 2 7/1, 5 BG) clay lens was observed early on and it was eventually recognized in different areas (Figure 6-5). Archeologists used this thin often indistinguishable lens as a horizontal marker when observed. However, wet spring conditions that flooded the excavations on two occasions hampered visual observation of this mottled lens. This lens was not identified by Mr. Schroeder in the matrix column documented on the southern end of the block. This lens was initially interpreted as a pond deposit that varied from 1 to $3 \mathrm{~cm}$ in thickness, but was not always traceable across each excavation block. In 


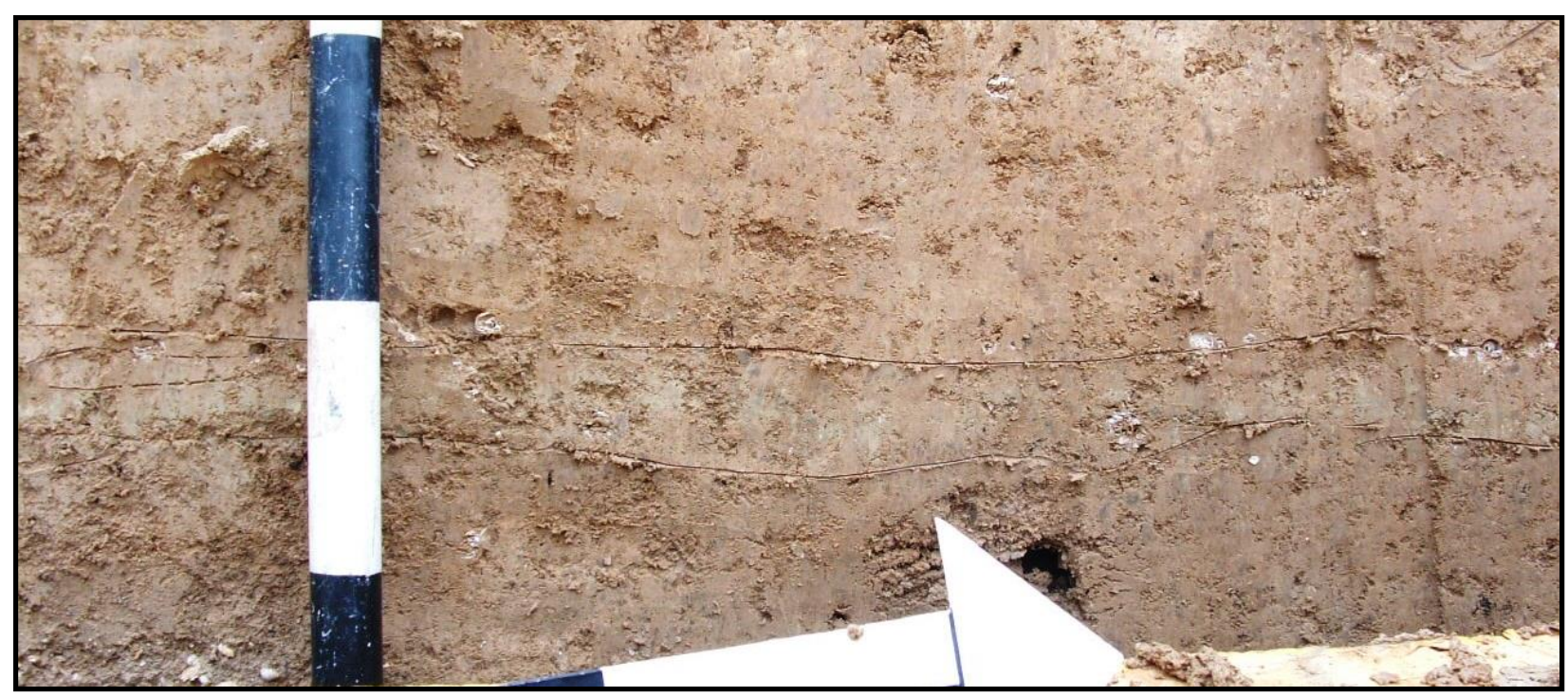

Figure 6-5. Very thin dispersed mottled greenish-gray clay lens that separated the Bell/Andice component from the lower Martindale component (bar scale in $10 \mathrm{~cm}$ increments).

certain areas the thin 1 to $2 \mathrm{~cm}$ deposits blended into the sediment above or below making it indistinguishable. When observable in multiple units it varied vertically across each block, between areas, and in thickness. Along the western side of Block B, the southern units (i.e., N71/E82) exhibited this lens at roughly 264 to $266 \mathrm{cmbs}$, which dipped northward to 283 to $284 \mathrm{cmbs} 2.0 \mathrm{~m}$ away in N73/E81. A nearly $20 \mathrm{~cm}$ change in vertical elevation over $2.0 \mathrm{~m}$. In Block C, this lens varied from $268 \mathrm{cmbs}$ along the eastern side to 280 cmbs along the western side. In Block D this same lens was partially removed during the initial mechanical stripping, but was detected from 270 to $290 \mathrm{cmbs}$ and undulated across Block D. The unevenness and variable depth provides a general understanding of the very unevenness of the surface the prehistoric groups likely occupied at this locality. Wet conditions during hand-excavations hampered excavators from always seeing or identifying this lens. In some instances, Rabdotus snail shells appeared concentrated immediately above and below this lens. It is not clear if these shell deposits resulted from water transport or some other unidentified action. However, this mottled lens was an important horizontal marker when observed and used to separate the two major occupations or components.

\subsection{INTERPRETATIONS}

The stratigraphic and pedogenic evidence encountered at site 41TV2161 suggests that Stratum I is likely an alluvial terrace of Pleistocene age. Given its presumed age, Stratum I can be considered archeological bedrock. Stratum II appears to represent fine-grained overbank flood deposits of Onion Creek. The weak soil development observed in Stratum II suggests that its accumulation occurred over a relatively short period of time. This implies that the archeological deposits within Stratum II also are constrained within a relatively brief period of time. The finegrained nature of Stratum II indicates that it is unlikely that the archeological deposits have been significantly affected by hydraulic forces, and that their context is largely the result of human activity.

Two interesting things are noteworthy about the stratigraphy of 41TV2161. One, an abrupt change in sediment texture is expressed between Stratum II 
and the overlying Stratum III, where the deposits of Stratum III are decidedly finer-grained than that observed within Stratum II. Furthermore, the density of archeological deposits is comparatively lower in Stratum III than that of Stratum II. This phenomenon may have something to do with site formation and paleoenvironmental conditions. What this would appear to indicate is that while Stratum II was being deposited, the site occurred relatively close to the active channel of Onion Creek, and thus, was likely a more favorable environment for human habitation. In fact, a remnant channel scar can be seen in the modern aerial photograph near the site (Figure 6-6). However, by the time Stratum III was deposited, the channel had shifted further to the northwest, thus explaining the finer-grained deposits and possibly the lack of human habitation. In essence this is the break between the formative deposition in the core of the terrace, and the cumulic tread which was discussed previously.

Currently, the age of Stratum I is unknown. If it is an ancient terrace remnant at only $3.0 \mathrm{~m}$ depth, this is significantly different than other sites that were examined in the lower reaches of Onion Creek. Nearly all prehistoric sites in or near the path of $\mathrm{SH}$ 130 (the floodplain of 41TV410 excavated by Hicks \& Company) had Holocene age deposits at depth resting upon bedrock. Examination of Stratum II at 41TV2161 appears to correlate with the alluvial fill event that is present at 41TV540 and 41TV410, although the widespread of radiocarbon ages in such a narrow depth interval poses some interpretive challenges (Frederick 2011).

Figure 6-6. Paleochannels of Onion Creek in vicinity of 41TV2161. 
Since 41TV2161 contains deposits representing the middle to late Holocene periods, it presents an opportunity to provide proxy datasets for paleoclimatic interpretation. Proxy datasets present at the site include fossil pollen and phytoliths (see Appendices E, K, and L) and soil for stable isotope analysis. In addition, these datasets can be compared to similar data recovered from other nearby sites (e.g., Armstrong [Schroeder 2002, 2011; Schroeder and Oksanen 2002], Little Barton Creek, 41WM1, etc.) to secure a more complete regional picture of paleoclimatic conditions. One feature of the 41TV2161 deposits, which hold considerable potential are the small ephemeral ponds which may be particularly amenable to preservation of paleoenvironmental information that corresponds to the middle Holocene occupations.

A series of recommendations were originally presented to TxDOT for more in-depth studies concerned with the paleoenvironment. These included a comprehensive dating of different materials from the site deposits, detailed examination of the lithology from the entire vertical deposit at 41TV2161 combined with a suite of technical analyses to address paleoenviromental conditions, and explore origins of the burned zone. These were not pursued by TxDOT at this time. 


\subsection{GEOPHYSICAL INVESTIGATION}

Tiffany Osburn

\section{$7.1 \quad$ INTRODUCTION}

A ground-penetrating radar (GPR) investigation was employed at 41TV2161 to guide the placement of hand-excavation blocks for data recovery and, ideally, provide preliminary feature data. The mechanically stripped pit was surveyed with the goal of identifying buried anomalies and possible archeological features that could be targeted for hand-excavation, thereby maximizing limited resources and field time. GPR is usually particularly well-suited for these goals given its ability to delineate buried features in threedimensions. Local ground conditions at the site, however, prevented quality GPR data acquisition. The geophysical survey was conducted in the field on April 5 and 6, 2006 (Figure 7-1).

\subsection{GROUND-PENETRATING RADAR METHODS}

GPR data are acquired by transmitting pulses of radar energy into the ground from a surface antenna. Transmitted pulses are reflected off buried objects, features or bedding planes and then detected back at the ground surface with a receiving antenna. The greater the contrast in electrical, magnetic, and physical properties between two materials at a buried interface, the stronger the reflected signal will be, and therefore the greater the amplitude of reflected waves. When collecting radar reflection data, the surveyor moves surface radar antennas along the ground following transects within a survey grid. The radar data are measured in temporal units called nanoseconds (ns). The resulting data are a series of reflections from each transect that are plotted to create a two-dimensional profile. Each profile is a graphic representation of a vertical face along a particular transect (Figure 72).
The success of GPR surveys in archeology is largely dependent on soil grain size and sediment mineralogy, ground moisture, depth of burial and surface topography and vegetation. Electrically conductive or highly magnetic materials in the ground will quickly dissipate radar energy and prevent its transmission to depth.

The best conditions for energy propagation are usually therefore dry sediments and soils. The greater the contrast in electrical (and to some extent magnetic) properties between two materials at an interface, the stronger the reflected signal, and therefore the greater the amplitude of reflected waves (Conyers and Goodman 1997:33-34).

When reflections from buried objects are viewed in two dimensions they often appear as hyperbolic reflections (see Figure 7-2). These reflections are produced from buried "point sources" such as pipes or large rocks as opposed to planar surfaces such as buried pathways or floors. The wide angle or "footprint" of the radar beam causes the antenna to "see" the object as it moves toward it and continues to "see" it as it moves over and away from the buried object, resulting in a hyperbolic reflection along the transect.

The GPR system used for the 41TV2161 survey was a Geophysical Survey Systems Inc. (GSSI) Subsurface Interface Radar (SIR) 3000 model mounted on a wheeled cart (see Figure 7-1). Prior archeological trenching at 41TV2161 indicated the potential for multiple buried components. Therefore, two radar antenna frequencies were used for this GPR survey in an attempt to locate shallowly buried features below the surface of the mechanically stripped block, as well as those more deeply buried. The two GPR antennas

used for the survey were a dual 400 megahertz $(\mathrm{MHz})$ antenna that produces a radar pulse of about $25 \mathrm{~cm}$ in wavelength and a dual $900 \mathrm{MHz}$ antenna that produces a radar pulse of about $10 \mathrm{~cm}$ in wavelength. The higher frequency antenna (900 


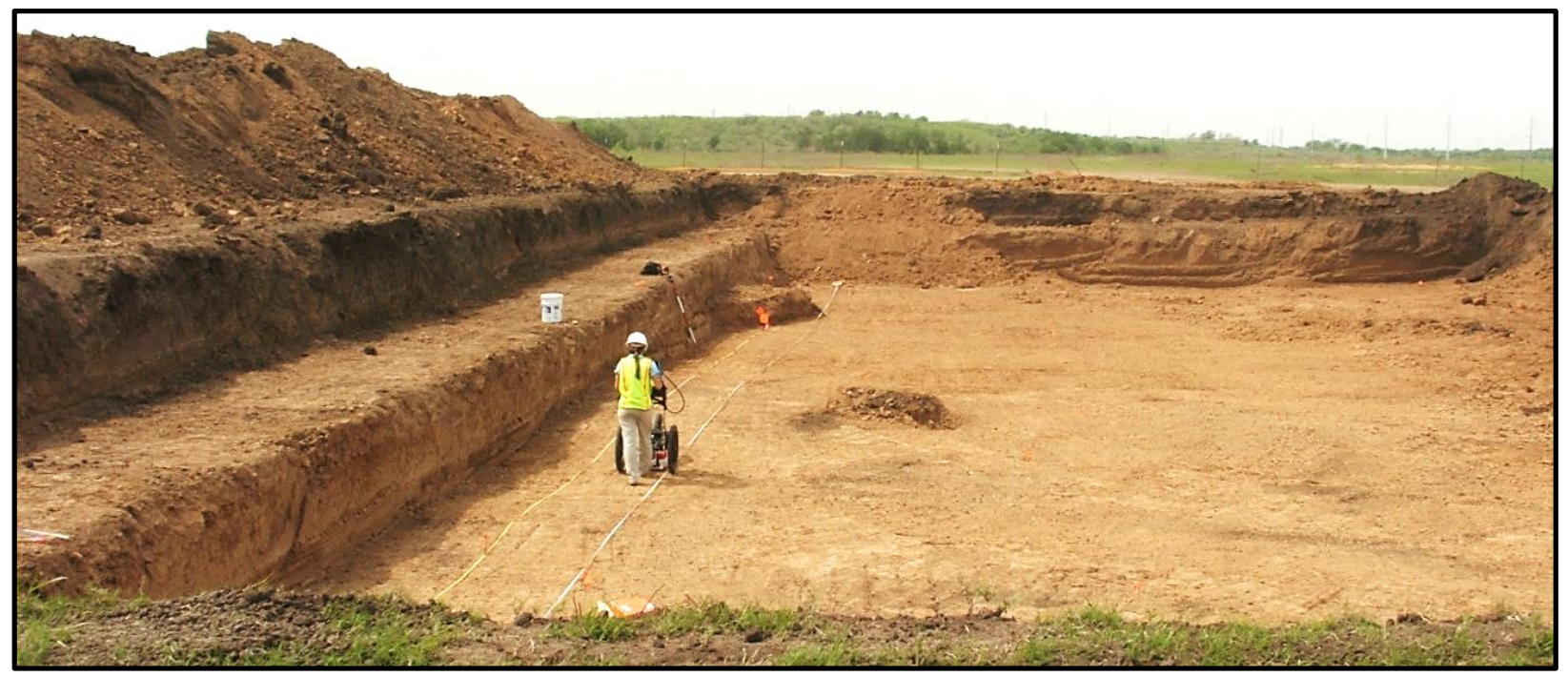

Figure 7-1. Excavation grid placed within the mechanically-stripped excavation block. Surveyed surface is $\mathbf{2 3 0 - 2 5 0} \mathrm{cm}$ below the modern ground surface.

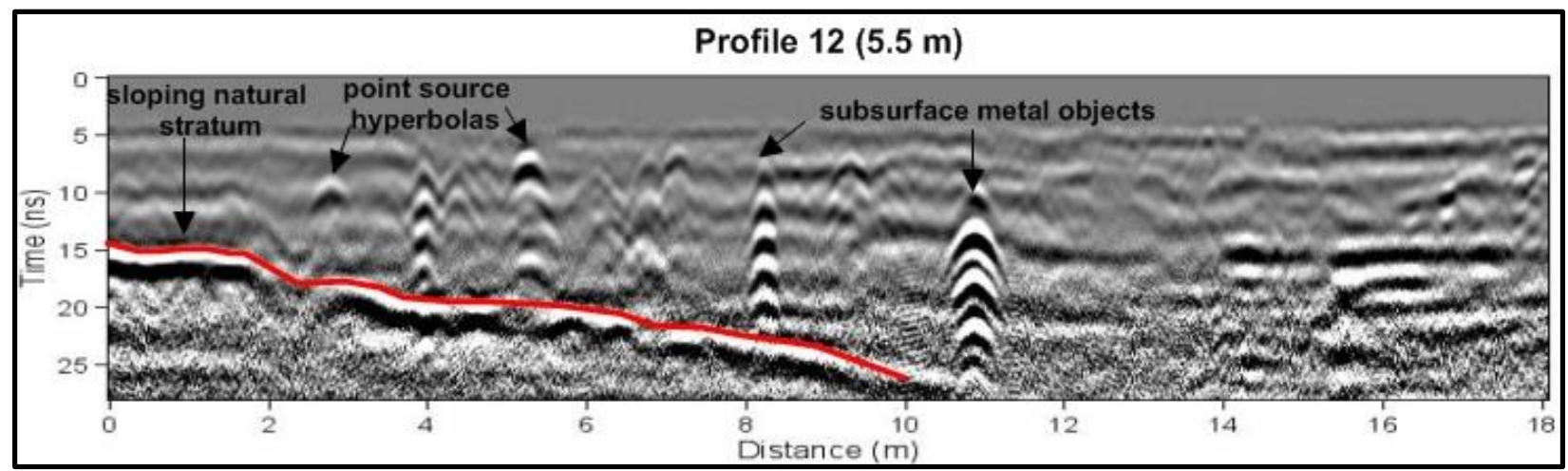

Figure 7-2. Example of a GPR profile showing hyperbolic reflections resulting from discrete point sources, reflection signature of metal objects, and a sloping planar surface.

$\mathrm{MHz}$ ) does not penetrate as deeply into the subsurface, but can resolve very small features, while the lower frequency (400 MHz) antenna allows deeper penetration. This survey grid was laid out within the mechanically stripped pit as a 36-by-24 m area; parallel transects were collected at $50-\mathrm{cm}$ spacing along the north-south axis.

All GPR reflection data were collected as 16-bit data, with 512 digital samples defining each reflection trace. The data were frequency-filtered to remove extraneous noise from the reflection traces. Range gains were applied in the field to enhance reflections from deeper within the profiles. All reflection data were first processed into two- dimensional profiles and analyzed as if looking at stratigraphy within a trench or cutbank. Amplitudeslice maps were also generated to aid in interpretation. These maps are analogous to arbitrary excavation levels in standard archeological terminology.

\subsection{DATA COLLECTION AT 41TV2161}

In addition to identifying the horizontal location of buried anomalies to target during data recovery, an attempt was made to identify the depths of anomalies for planning purposes. GPR has the capability to accurately map the subsurface in three 
dimensions. Reflections in GPR data are measured in two-way travel time and travel time can be converted to depth if the velocity of the material through which the radar energy is traveling can be calculated. The measurement of how well radar energy will be transmitted through any material is relative dialectric permattivity or RDP. In general, the greater the RDP of a material the slower the radar energy will move through it. Dry sand has an RDP of 3 to 5 while wet soils and clay-rich sediments or soils often have RDPs approaching 40 or 50 (Conyers and Goodman 1997:33). In most settings, soil and sediment layers that are deeper in the ground are more compact and have higher water saturation levels - both lead to higher RDP values. These factors were present and negatively affected data collection in the deep pit at 41TV2161.

A second major factor that affects the depth of penetration and amplitude of reflected radar waves is dispersion and energy attenuation through conductive and magnetic mediums. This commonly occurs when sediment is electrically conductive, which dissipates and absorbs propagating waves. In a highly conductive soil, the electrical portion of the electromagnetic wave is conducted away and the wave itself dissipates. As energy moves through the ground and is conducted away, less and less of it is available to be reflected back to the surface (Conyers 2004:50-51).

Very conductive ground will include soils containing electrically conductive clays, particularly if the clay is wet, soils containing soluble salts or electrolytes (e.g., agricultural runoff can increase the electrical conductivity due to dissolved nitrogen and potassium), and moist calcium carbonate impregnates soils in arid regions. Common mineralogical clay soils characterized as smectitic or bentonitic, for example, have a high ionic displacement; these are shrinking and swelling clays. The molecular structure of smectitic clay, when it contains water, allows the easy movement of ions, making it a good electrical conductor and hindering data collection and resolution (Conyers and Goodman 1997:32). Other nonswelling clays, by contrast, are relatively resistive and their presence in the soil will not impede radar energy transmission.

Werchan et al. (1974) depict site 41TV2161 within the Lewisville silty clay soil series. These soils develop under a cover of mid to tall grasses. This association consists of nearly level to gently sloping soils and gently sloping to rolling soil on terraces along creeks and rivers. These soils are characterized as well-drained silty clays, moderately permeable, and subject to shrink-swell. The alluvial strata encountered at $230 \mathrm{cmbs}$ during the geoarcheological investigation at 41TV2161 were described as blocky silty clay with common calcium carbonate (CaCO3) filaments representative of slowly aggrading fine overbank deposits. Considering the location within the Lewisville soil association, it is probable that the major constituent of this silty clay is the smectitic clay, and therefore, highly conductive due to its mineralogical clay component. In addition, the sediment within the pit assuredly retained a good deal of moisture, considering the mechanical stripping of the upper $230 \mathrm{~cm}$ of sediment was ongoing during the week of survey. These soils and moisture conditions proved difficult for obtaining clear, interpretable radar reflection data during the survey.

\subsubsection{Velocity Analysis}

While the Gradall@ completed excavation within the block on the first day of GPR survey, a bar test was conducted in an attempt to determine the velocity or speed at which the electromagnetic wave travels through the subsurface. This test was conducted to allow conversion of two-way radar wave travel time to depth at the site. The most accurate way to measure velocity is to identify reflections in a GPR profile that are produced from objects which occur at known depths. Because metal is a near perfect reflector, reflections generated in a profile crossing a metal object are 


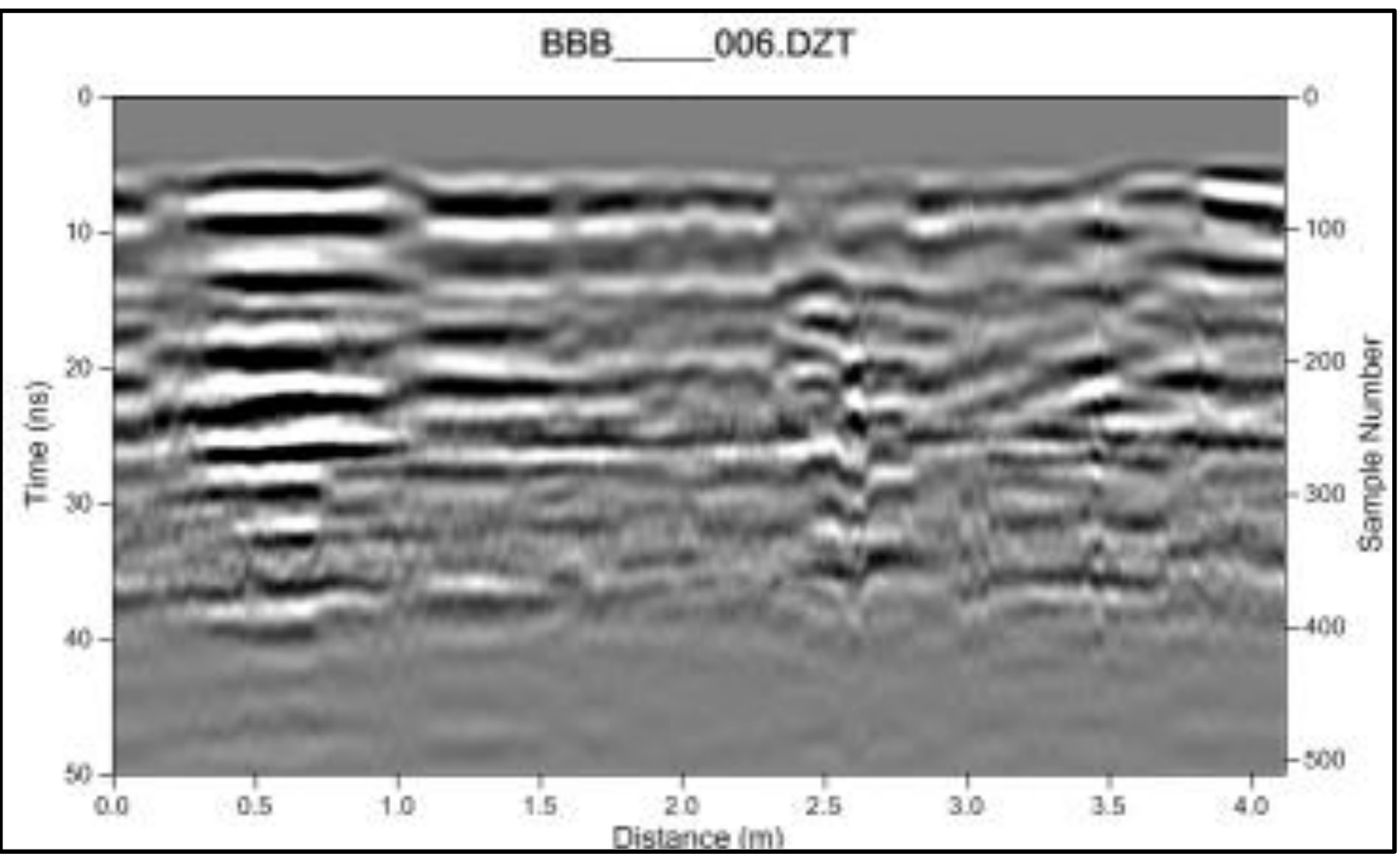

Figure 7-3. An attempted bar test to determine velocity. The metal bar should be visible at 2 meters along the profile.

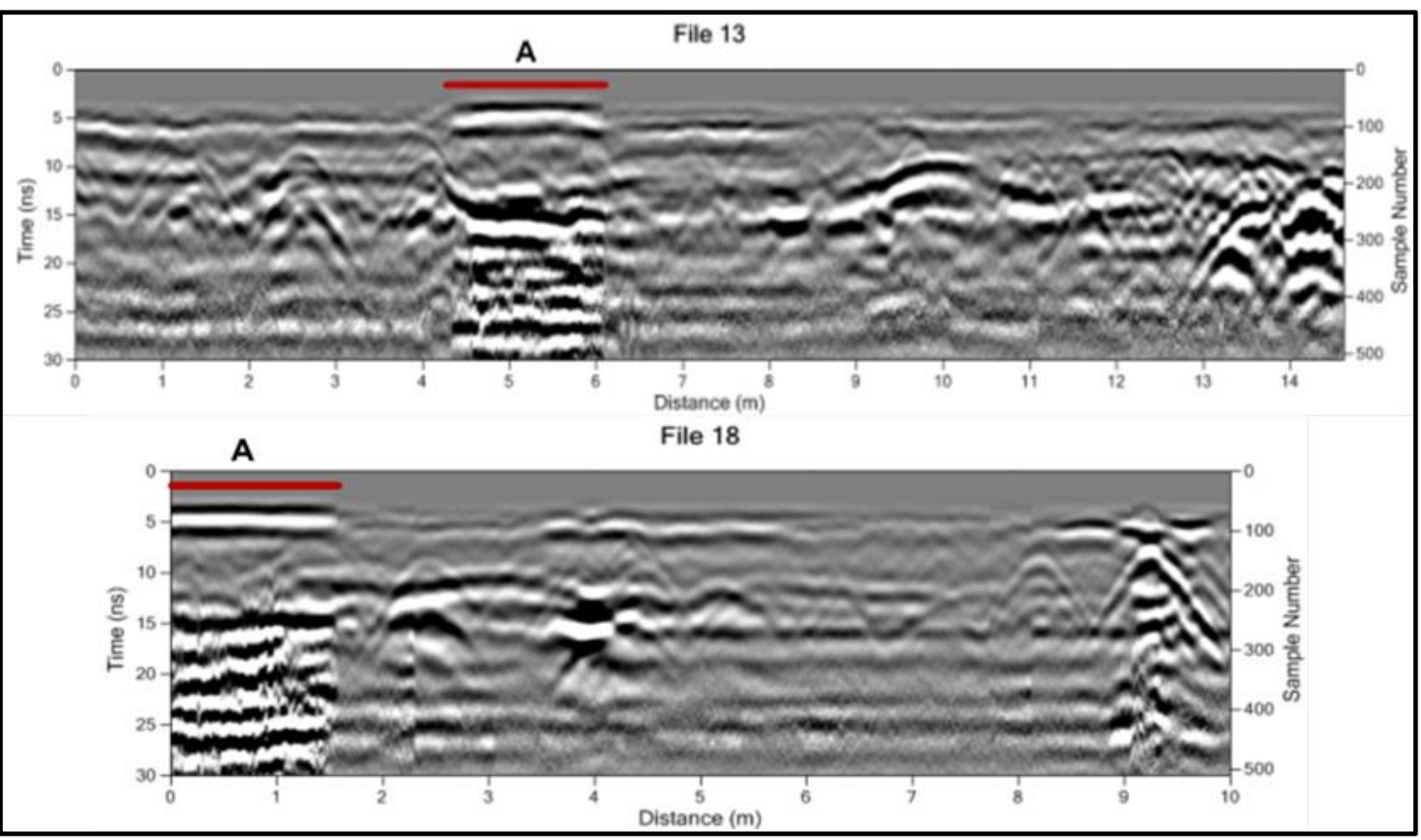

Figure 7-4. Two reflection profiles showing areas (A) where coupling changes occurred, adversely affecting the resulting reflection amplitudes and obscuring archeological features. 
easily identifiable on most GPR profiles as a distinct point source hyperbola. In the bar test, a metal bar or pipe is hammered horizontally into the vertical face of an open trench wall. Antennas are then pulled over the bar as subsurface reflections are recorded in a profile. The metal bar should be apparent as a distinct reflection hyperbola as shown in Figure 7-3. When time and distance are known, the average velocity of radar wave propagation in the ground can be calculated.

Both the $400 \mathrm{MHz}$ and the $900 \mathrm{MHz}$ antennas were used for the bar test and the bar was placed at $40 \mathrm{~cm}$ below the stripped surface in a trench. The bar was located at meter 2 along a 4-m transect. Although the bar was placed at only $40 \mathrm{cmbs}$, no reflection of the metal bar was visible in any of the 15 radar profiles pulled across it (see Figure 7-3). It is possible that the bar was not hammered in far enough or was not large enough to be detected, but it is more likely that this negative finding is a product of the ill-suited matrix and conditions for this GPR survey.

\subsubsection{Coupling}

The type of surface material within which the radar energy is coupled will also greatly affect the amplitude of reflected waves below it (Conyers 2004:68-69). When transmitting antennas are placed on the surface, variations in radar wave geometry occur as the electromagnetic waves move from transmission in the air to transmission within the ground. When constant ground contact is not maintained these variations can become an impediment to successful GPR survey. Although some attempt was made to smooth the surface using hand tools, the freshly scraped blocky clay still resulted in a surface that consisted of ridges and clods that caused the antenna to frequently lose contact or coupling with the ground.

Figure 7-4 illustrates the way surface coupling can drastically affect energy penetration depth and the resulting reflection amplitudes. Where the ground surface topography and matrix is consistent, clear reflections of discrete features are readily resolved to depth. Where coupling changes occur due to the antenna being pulled over unsuitable or uneven surfaces, the data are noisy and useful reflections are obscured. The ability to record reflected radar wave data at 41TV2161 was greatly impaired by coupling changes at the surface.

\subsection{RESULTS}

One large GPR grid was laid out within the mechanically excavated pit. On the first day of GPR survey the mechanical stripping was ongoing and the mechanical excavation was working in the west and southwest sides of the pit. The bar tests and a smaller grid on the eastern side of the pit were surveyed. On the second day, the entire freshly mechanically excavated pit was surveyed with both GPR antennas.

During the GPR survey, it was immediately apparent from the two-dimensional profiles that were visible on the digital screen of the data collector that conditions were less than optimal. The GPR data appeared "noisy" with coupling variations and background noise comprising most of what was visible in the profiles. Various filters, such as frequency filters and trace stacking, were applied during field work to mitigate these effects, but none were successful. Additional filters and smoothing were applied during post-processing of the data as well. Unfortunately, none of these improved the inherently poor quality of the data collected. Regardless, the goal of the survey was to select possible archeological anomalies to target during data recovery. In hindsight, perhaps this should have been avoided, but eight possible anomalies were selected nonetheless through review of the slice maps, profiles, and data collection notes. Each of the selected "anomalies", however, were very low probability of representing an archeological feature and could have easily been the result of coupling variations in the data due to the antenna passing over ridges on the surveyed 


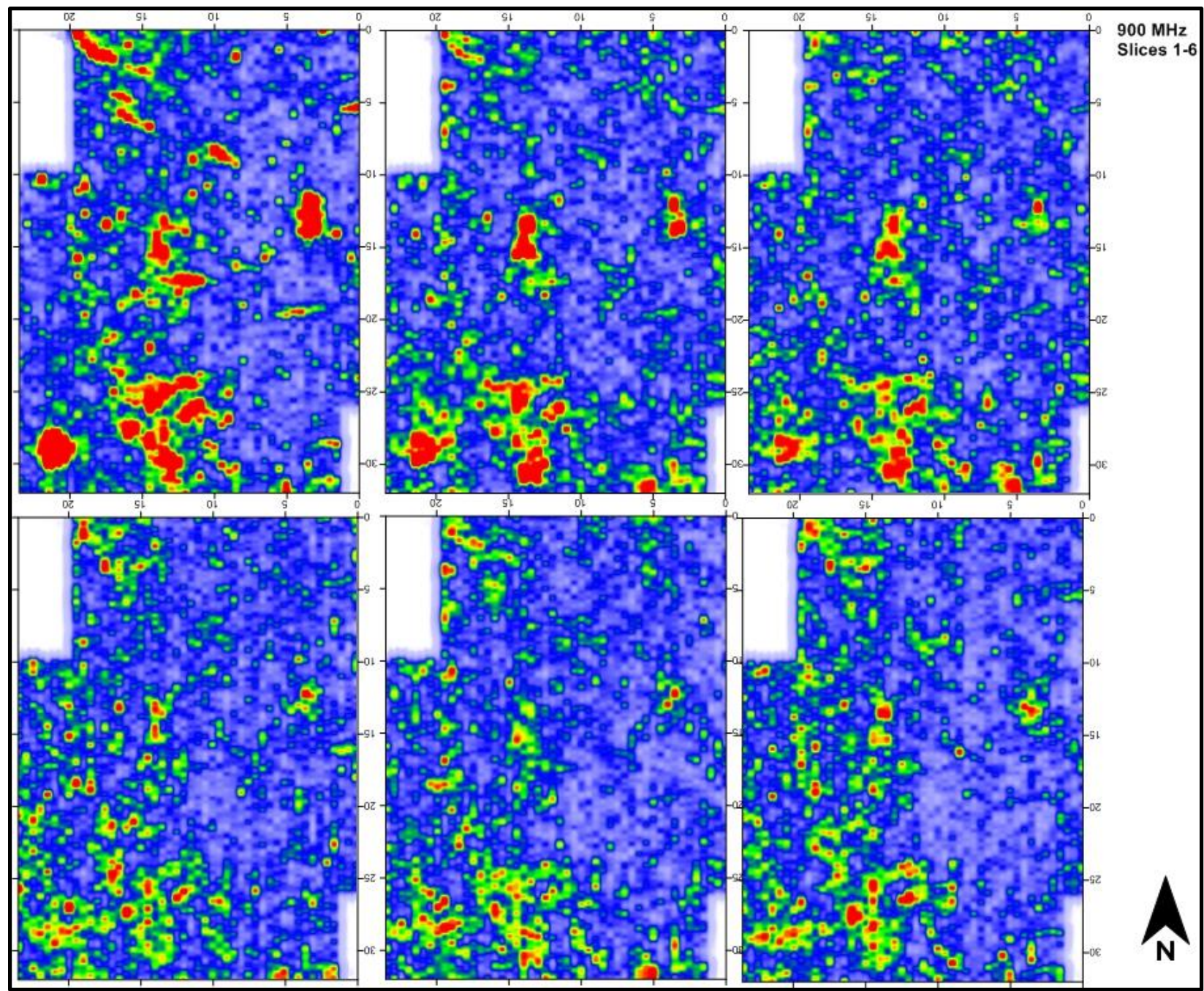

Figure 7-5. GPR amplitude slice maps resulting from the $900 \mathrm{MHz}$ antenna. High amplitude reflections are seen as "warmer" colors, greens, yellows, and reds.

surface, etc. Figures 7-5 and 7-6 depict all of the amplitude slice maps from the GPR data collected using the $900 \mathrm{MHz}$ and $400 \mathrm{MHz}$ antennas, respectively. Figures 7-7 and 7-8 show the locations of the recommended anomalies within the grid.

Figure 7-9 illustrates a sample of GPR profiles over transects where anomalies were recommended for targeting. The top profile (File 11), shows the transect over the area where Anomaly 2 was identified at 3 to 5 meters along the line. A slight, shallow disturbance is visible at 4.5 to 5 meters along the transect in this profile. Excavation in Block A tested the outlined footprint of Anomaly 2 (see Figures 7-7 and 7-8) and identified Features 15 and 19. Feature 15 was described as a natural cluster of Rabdotus snail shells and Feature 19 was an area of organic staining or burning that was suspected to be noncultural. Although these features were identified in the location of Anomaly 2 , they did not produce reflections that correlate with the GPR data; therefore, their discovery was simply fortuitous. Anomaly 3 was identified along transect 12 in Figure 7-9 at approximately 32 to 34.5 meters. During data recovery, Block C was 




Figure 7-6. Amplitude slice maps from the $400 \mathrm{MHz}$ antenna. Each slice is $4 \mathrm{~ns}$ thick.

placed to target this anomaly. Two cultural features were identified within the footprint of Anomaly 3. Feature 20 was a small cluster of four burned rocks and Feature 18 consisted of a circular concentration of 18 burned rocks. Once again, the discovery of these features does not appear to correlate in any meaningful way with reflections produced in the GPR data. A review of the profile (12) in Figure 79 does not show any subsurface reflection, only coupling variation and background noise. Transmitted radar energy was not reflected from subsurface material, only conducted away. This is also the case with Anomaly 5 in the bottom profile (File 21) of Figure 7-9. Block E was placed to test this proposed anomaly, but no cultural features were identified during excavation.

\subsection{CONCLUSION}

In summary, the moisture and mineral makeup of the soils combined with the uneven nature of the surface within the mechanically stripped pit at 41TV2161 were poorly suited to GPR data 


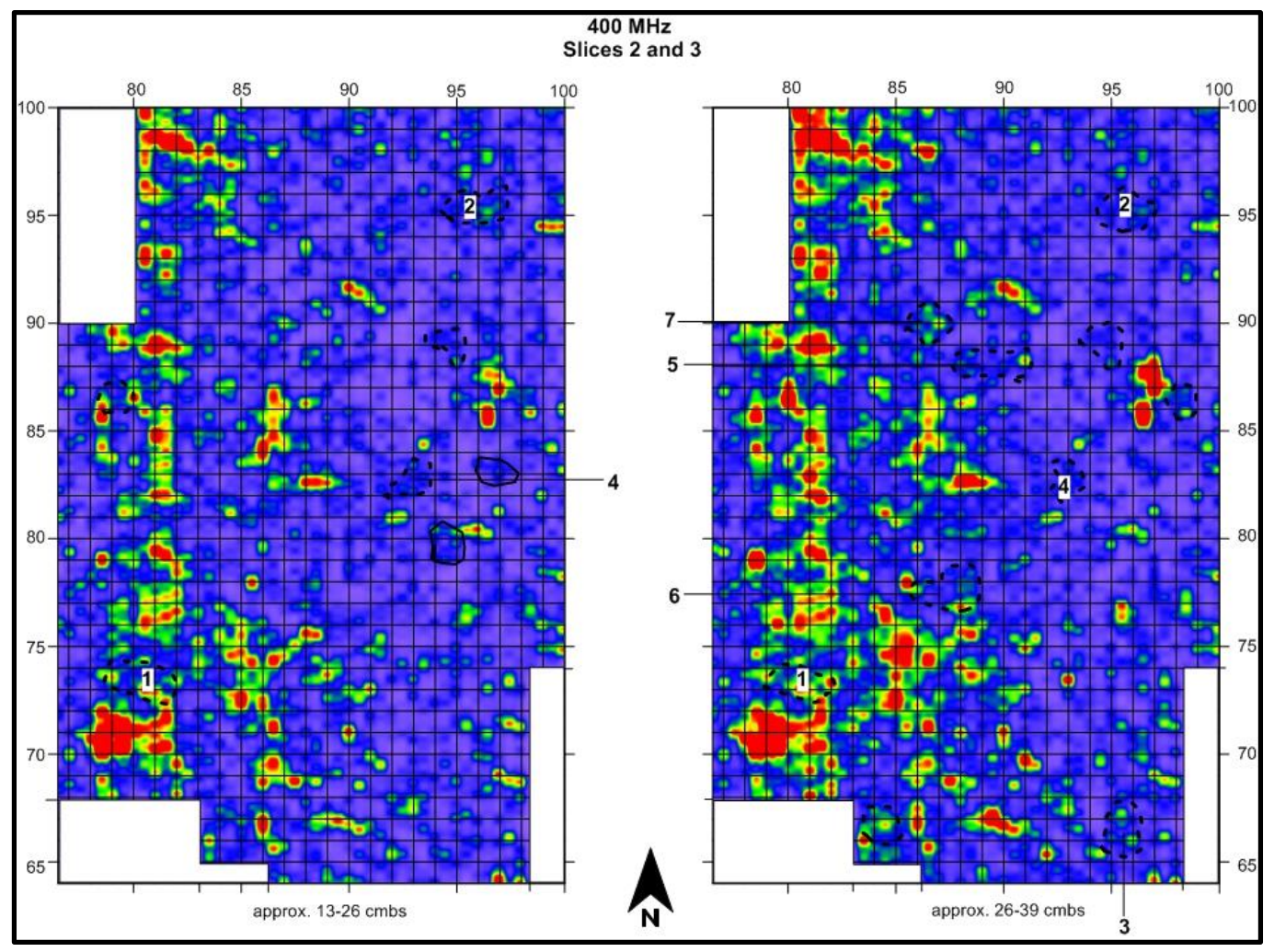

Figure 7-7. Indicates the location of 7 "anomalies" in the GPR data shown on slices 2 and 3 of the $400 \mathrm{MHz}$ data.

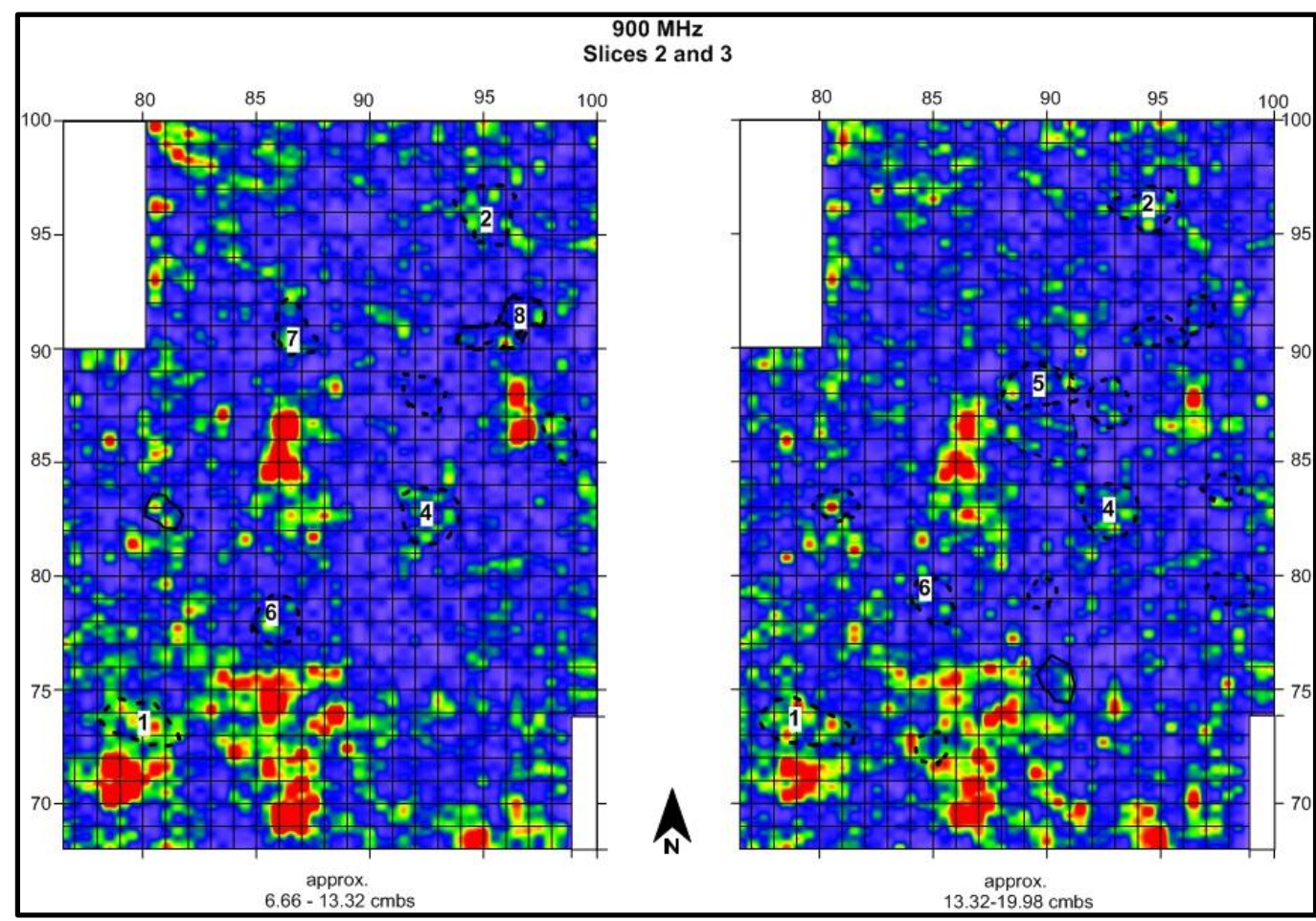

Figure 7-8. Indicates the location of 8 "anomalies" in the GPR data shown on slices 2 and 3 of the $900 \mathrm{MHz}$ data. 
collection. Continuous changes in coupling as the antenna moved over the surface, paired with a highly conductive medium resulted in an extremely rare incident where no real reflections from the subsurface were recorded. Filters and smoothing were used in the field in an attempt to increase data quality. In addition, similar filters were used in post-acquisition processing to improve data. Both failed. Since the goal of the GPR survey was to guide the placement of hand excavation units for data recovery, several areas to target were recommended. The recommended anomalies, while admittedly low probability, provided a good starting point to target, but did not correlate to features identified during excavation.

While GPR and other geophysical survey methods can be powerful tools for successfully identifying and mapping subsurface features in Texas, local conditions at site 41TV2161 during the GPR survey impeded the collection of subsurface reflection data. Additional GPR survey data should be collected in similar settings to determine whether these results are isolated to this site area or represent a rule of thumb within the alluvial clays derived from the Lewisville soil association.

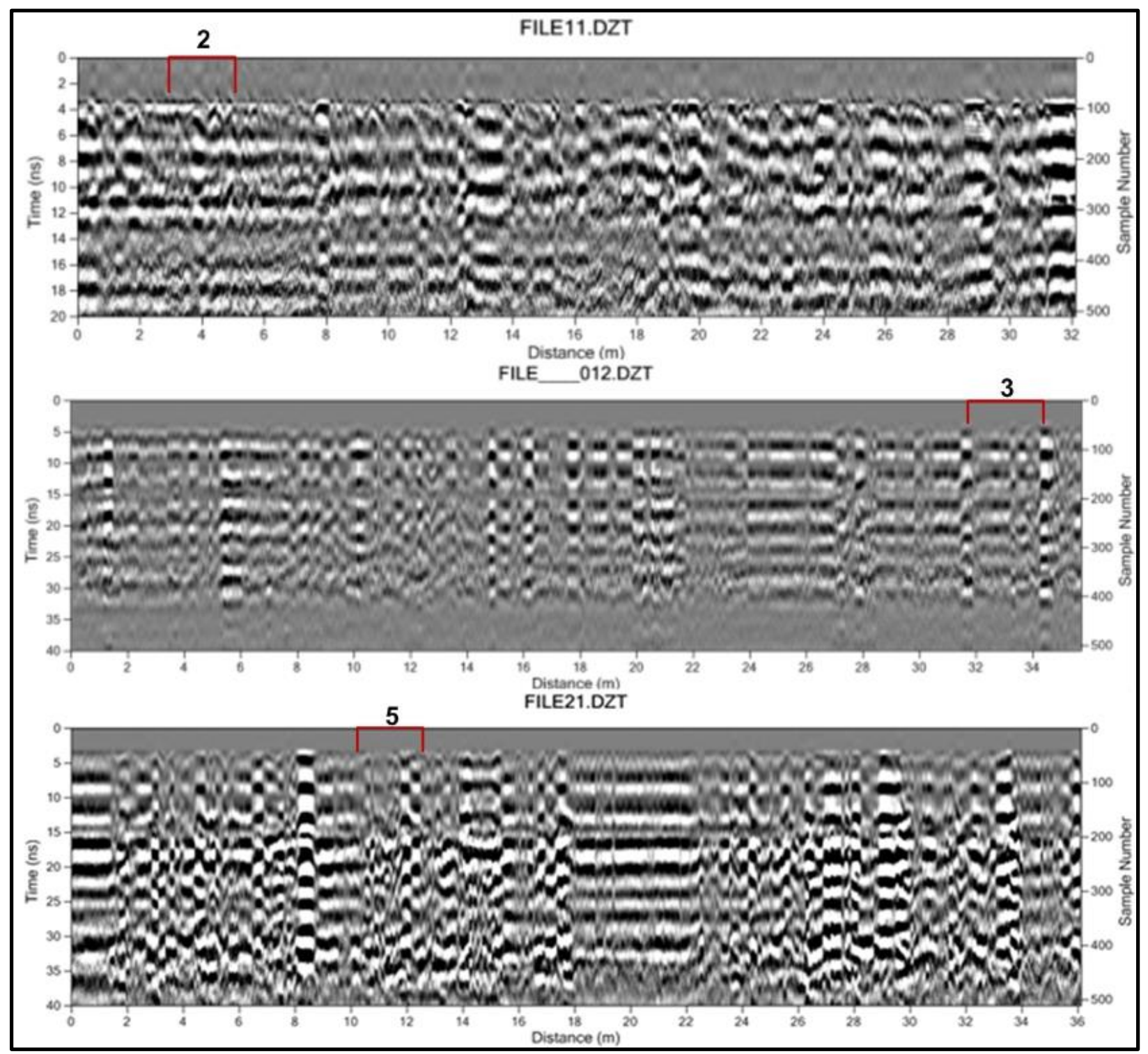

Figure 7-9. Sample of GPR transect profiles over recommended Anomalies 2, 3, and 5. 
This page intentionally left blank. 


\subsection{CULTURAL STRATIGRAPHY, INTEGRITY, AND COMPONENT ASSIGMENTS}

\section{J. Michael Quigg}

\subsection{INTRODUCTION: INTEGRITY AND PRESERVATION ISSUES}

Data recovery investigations targeted one deeply buried cultural zone, roughly $70 \mathrm{~cm}$ thick, between 220 and $290 \mathrm{cmbs}$ in this deeply stratified site. Initially, the zone was thought to represent one cultural component (Figure 8-1). Based on preliminary data collected during Hicks \& Company's initial site discovery phase (Campbell et al. 2006), the target zone selected centered on the deeply buried, well-preserved hearth Feature 3. This was a tight aggregated burned rock cluster in a shallow basin with a thin charcoal stained lens that lined the basin at 225 to $230 \mathrm{cmbs}$ (Figure 8-2). Charcoal from that hearth yielded a radiocarbon date of 5320 B.P. (Beta-214482, Campbell et al. 2006). The intact state of Feature 3 and the surrounding deposits indicated low-energy overbank deposits had encased and preserved this feature within a cultural zone of high integrity.

TRC excavations initially targeted six GPR anomalies at multiple, dispersed blocks. Two primary cultural components were encountered, both with horizontally scattered artifacts and features. Additionally, multiple unassigned artifacts and features were present in the fine-grained alluvial deposits (see Chapter 6.0 for geoarcheological description). The low-energy overbank deposits provided protective burial layers sheltering the intact cultural materials without significant displacement of artifacts on the prehistoric ground surface. Collins (1995:374; 2004:111) states these types of deposits afford optimum conditions for isolation of discrete cultural assemblages.
Hand-excavations during data recovery encountered eight well-defined small burned rock clusters, some with charcoal stained sediments, three organically stained hearth features, one cluster or cache of ground stone tools, one natural Rabdotus concentration, and three natural burned areas (Figure 8-3). Burned rock features exhibited tightly aggregated burned rocks with fractured in-place portions of larger burned rocks still adjacent to one another, and dark organic stains around the rocks. No significant displacement from rodent or other bioturbation was detected. In a few instances, the base of stained basins exhibited minor downward movement of stained sediment from worm or other insect activity. No obvious rodent disturbances or krotovina were encountered in unit profiles or during hand-excavation of units. Overall, post-depositional disturbances appeared minimal, and encountered features and associated cultural objects were well-preserved, which indicate high integrity deposits largely representative of cultural activities at abandonment.

Deposits that surrounded the intact cultural features contained various quantities and classes of cultural artifacts, which include lithic debitage, chipped and ground stone tools, mussel shell, burned rocks, and vertebrate faunal remains. Presence of vertebrate faunal is rather unique for this age deposit in central Texas. Sediment conditions across Texas for most late Early Archaic and Middle Archaic deposits are unfavorable for bone preservation, even at most younger open-air sites. Bone in this deposit is mostly well-preserved to allow pieces with specific characteristics to be identified to element, and potentially species identification. A portion of the faunal assemblage exhibits root etching, a few with cut marks, and some with spiral fractures. Bone condition did vary as some fragments are encased in calcium carbonate, whereas others exhibit clean surfaces. Calcium carbonate creates difficulty in identification, hides possible cultural cut marks, and may affect original bone chemistry in the dating process. 


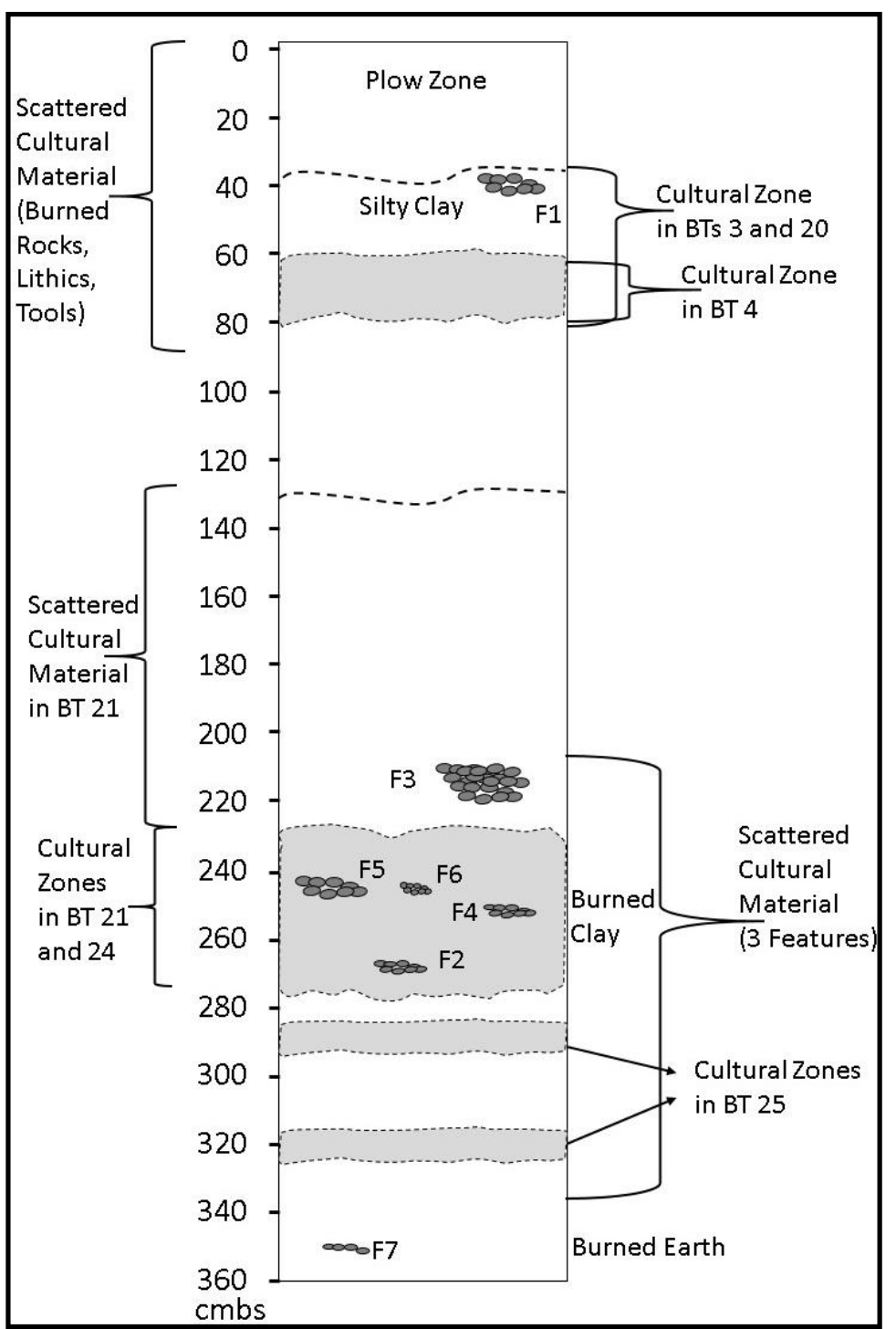

Figure 8-1. Composite schematic site profile (data derived from Campbell et al. 2007).

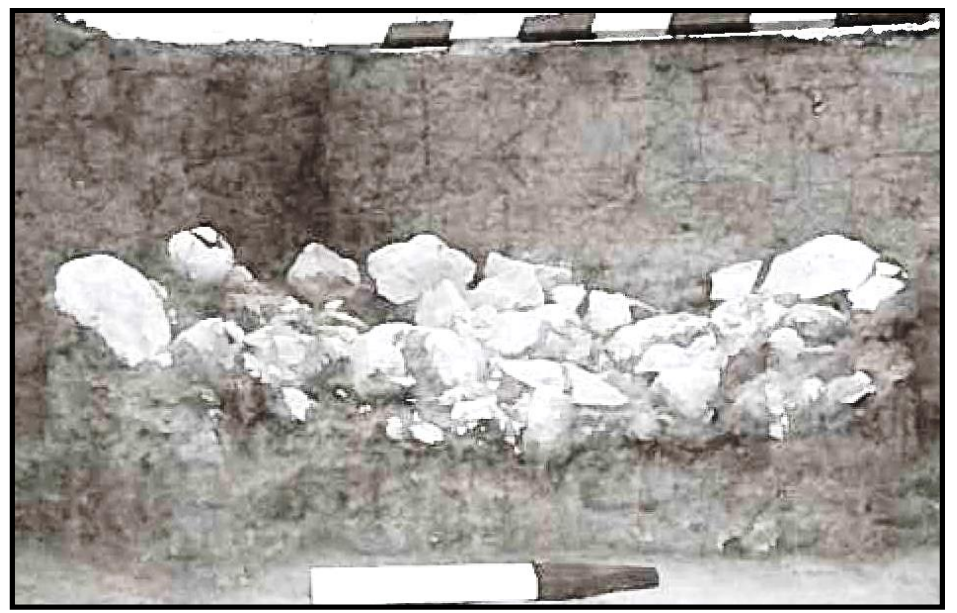

Figure 8-2. Profile of well-preserved, intact hearth Feature 3 (from Campbell et al. 2007:115). 
No cultural feature horizontally overlapped with any other cultural feature. Cultural debris did cooccur with non-cultural burned tree features in blocks A and C. Although hand-excavated units were not horizontally expansive, excavations at Blocks B and D were sufficiently broad to allow detection of horizontal patterning in artifacts and features. Patterns are indicative of specific human behaviors across space and probably represent single-event utilization, specialized feature function during limited use, and/or very short-term occupation(s). Reuse of the same surface was not indicated in either identified component.

The intact nature of individual features, absence of overlapping cultural features, low artifact density, combined with detectable horizontal patterns in feature and artifact distributions documents intact occupations, which lacked occupation overprinting often discovered in many sites. This documents high integrity components. It is projected the two identified components represent distinct living surfaces, although repeated short-term occupations of limited use of these two surfaces cannot be completely ruled out. However, data recovered supports the interpretation, both components are indicative of single, short-term events. Chapters 9 and 10 provide details on the two components with specific documentation of their ages.

Not only were features, bones, and mussel shell well-preserved in fine grained, low-energy overbank deposits, but periodic flood events were frequent enough and characterized by low-energy to allow sedimentation between individual cultural events. This facilitated in the detection of vertically separated occupations and components. Lowenergy alluvial deposits characterized by consistently similar hue and texture appeared throughout the excavated zone (Figure 8-4). This prevented visual differentiation of specific depositional events and/or traceable stable surfaces (i.e., buried A horizons). An horizontally

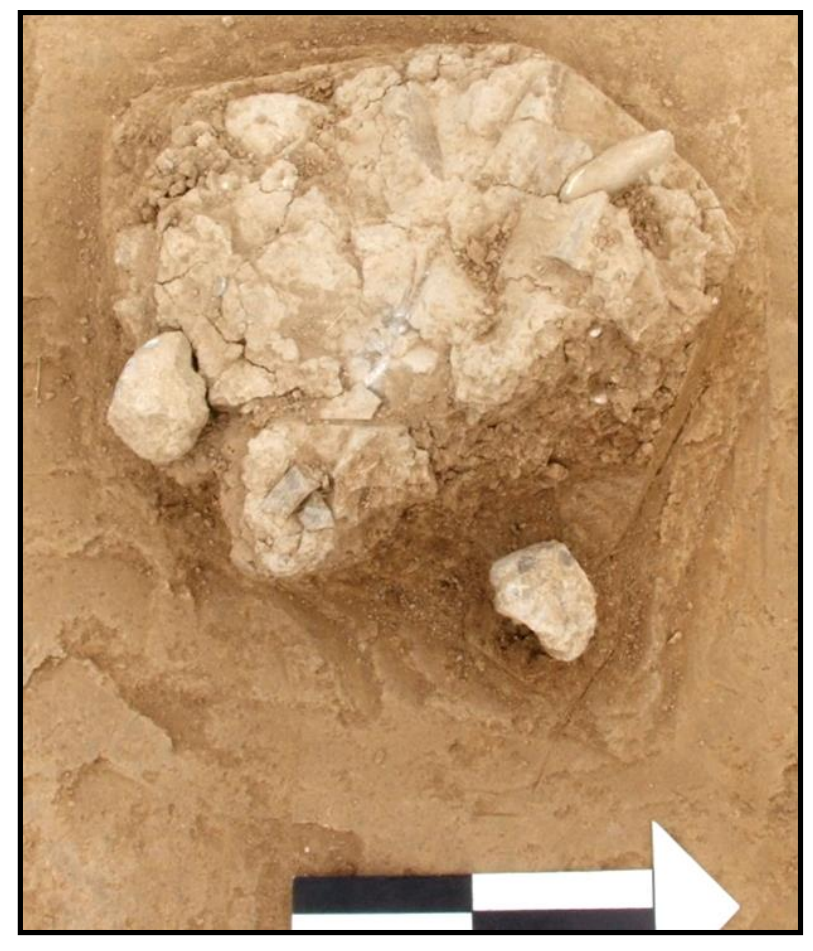

Figure 8-3. Plan view of intact rock feature.

intermittent green-gray clay lens was detected in some units and contributed towards our understanding where our excavations were vertically in the deposits and some knowledge as to the position of the different components.

\subsection{VERTICAL DISTRIBUTION OF COMPONENTS}

Cultural items discovered in situ during handexcavations were plotted on level records and their depth measurements recorded. These data allowed for the detection and measurement of the vertical separation of cultural materials in the field. Accumulated recordings allowed stratigraphic delineation of the two primary cultural zones and enabled more targeted excavations in Blocks B and D. Within the two distinct zones, horizontal distributions of features and classes of cultural material were observed. In the laboratory, data points for materials piece-plotted in the field allowed back plot distribution diagrams to depict overall vertical frequency of identified features and associated materials. Results indicate all features in 


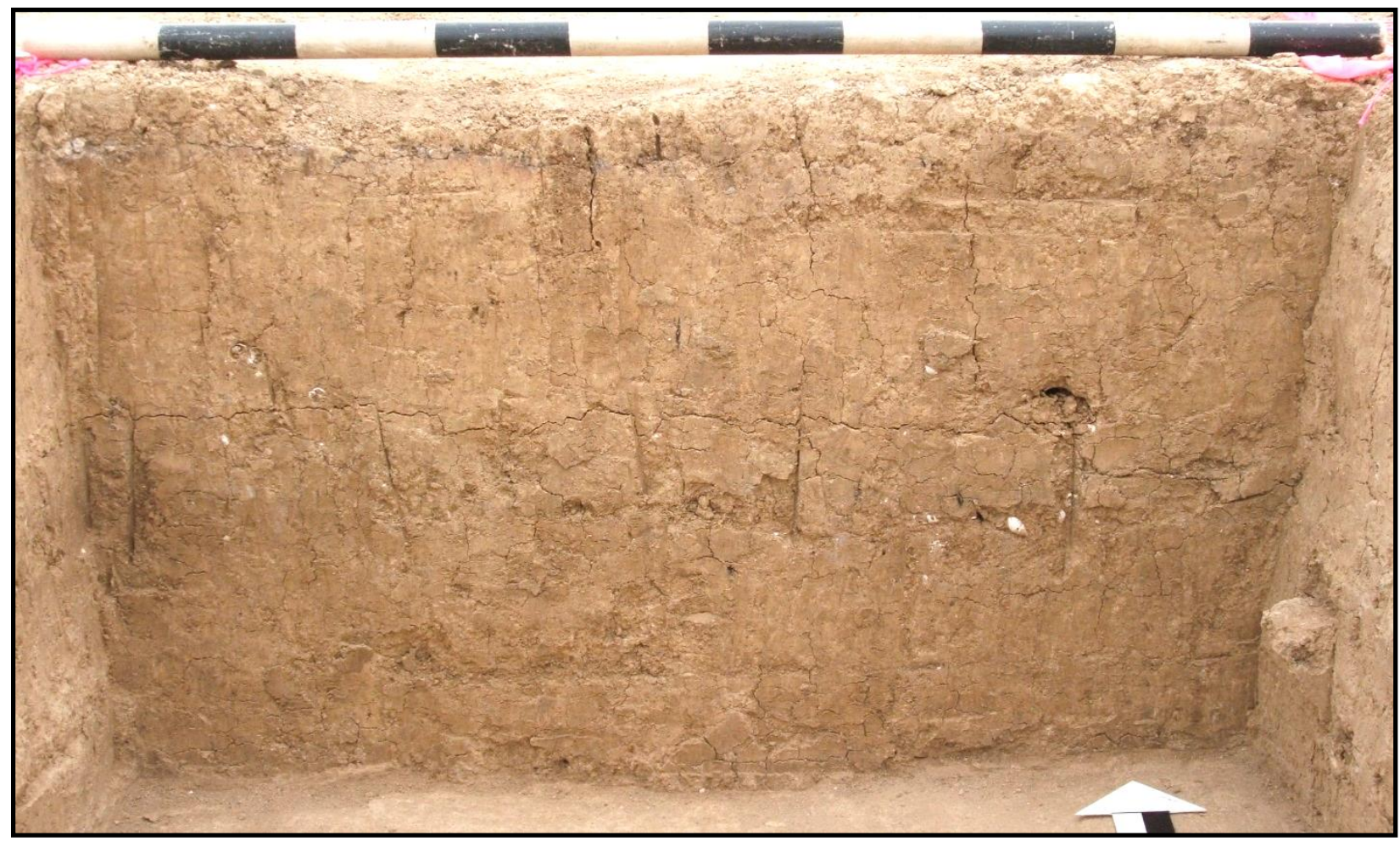

Figure 8-4. Profile of $50 \mathrm{~cm}$ thick silty clay deposits in north wall of N68/E96 in Block C. No obvious or traceable soil color changes are present.

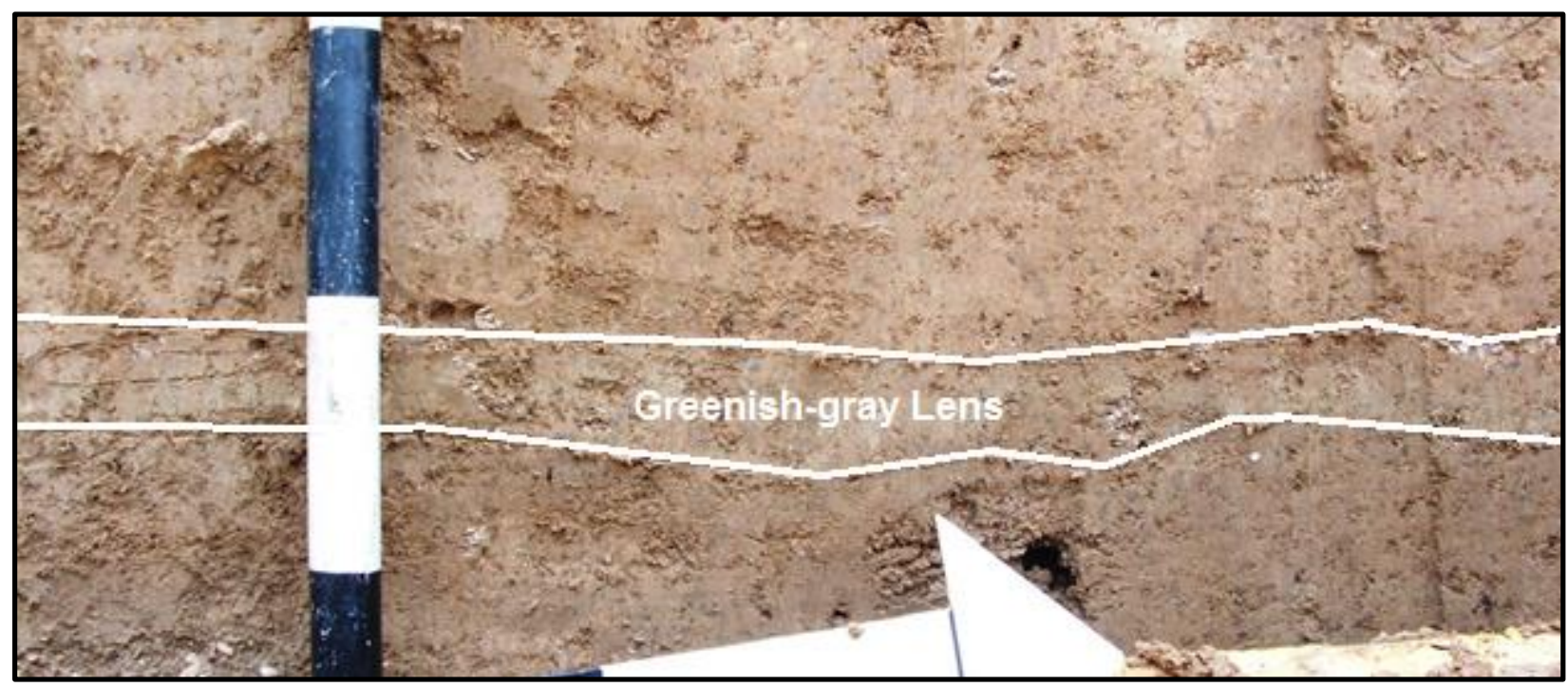

Figure 8-5. Profile of thin mottled greenish-gray lens that separates the two identified components. 
Blocks B and D, with the exception of Feature 31 in Block B (see below for further discussion), were restricted to the two identified components.

A thin, 1 to $3 \mathrm{~cm}$ thick, mottled light greenish-gray (Gley 2 7/1, 5 BG) clay lens was observed in many hand-excavated units across the blocks (Figure 85). This lens was a convenient stratigraphic marker that aided excavators with easily observable correlation of stratigraphic level and approximate age of materials targeted. Once this lens was identified below the recognized upper cultural component in a specific unit context, the comparative stratigraphic distribution and relative age of the cultural materials present above and below the lens was generally known. This stratigraphic marker guided excavators and in block expansion, especially in Block D. To identify the source of this light greenish-gray clay, three sediment samples (\#2143-4e, \#2156-43, and \#2262-4e) were submitted for diatom analysis. All three samples lacked sufficient diatoms to allow interpretation (Appendix C). Sample \#2143, collected from N72/E83 between 270 and 273 cmbs, was also analyzed for phytoliths. It also failed to yield sufficient phytoliths to assist in sediment interpretation (Appendix E). A subsample (\#2143-4b) yielded no starch grains (Appendix F). Two of the three samples from this lens contained a few aerial diatoms found in damp settings, splash zones, and newly wetted surfaces and are well-aerated. No evidence to support permanent water exists (Appendix C). The near absence of microfossils prevented our understanding of what this lens represented. It is suspected this indistinct and mottled lens combined with its distinctive color and mottling formed below standing water.

Recovery of diagnostic projectile points from each component, combined with multiple radiocarbon dates from Blocks $\mathrm{B}$ and $\mathrm{D}$, supports the identification of a well-defined Bell/Andice component in Block $\mathrm{B}$, and a stratigraphically distinct, likewise well-defined Martindale/Early
Corner-notched component in Block D and the lower part of Block B. Although the two components were vertically separated, inconsistency in mechanical stripping of approximately $200+\mathrm{cm}$ left high and low areas across the bottom of the stripped area. Consequently, mechanical stripping in the area of Block D unfortunately removed deposits that probably contained Bell/Andice component materials. The slightly stratigraphically higher area that remained in the vicinity of Block B yielded the Bell/Andice component identified in that block. The deposits yielded a Bell/Andice component situated directly above the light greenish-gray clay lens, and the stratigraphically lower Martindale component.

The four smaller blocks (A, C, E, and F) that initially targeted geophysical anomalies yielded few well-defined cultural features, limited cultural materials, and lacked diagnostic tools. Consequently, expansion of Blocks A, C, E, and F were generally determine unwarranted as further excavation efforts were thought to yield limited cultural data. Limited cultural materials from these four blocks preclude confident assignment to either component, therefore those materials were left unassigned. Stratigraphy of each excavation block is individually addressed below, starting with Block A and ending with Block F.

\subsubsection{Block A}

The northeastern corner of Block A was mechanically stripped to between 240 and $260 \mathrm{cmbs}$ (Figure 8-6). Block A was initiated to target the second highest ranked GPR anomaly (\#2). Upon excavation, the anomaly represented an amorphous dark, mottled organic stain with reddish, orange and black colored sediment and sparse cultural artifacts (Figure 8-7).

These mottled darken stained areas, subsequently designated as Features 19 and 23, are interpreted as non-cultural burned areas, probably from tree burns. Sparse and horizontally scattered cultural materials 
(less than 100 pieces of lithic debitage, burned rocks, and bone fragments) were across $13 \mathrm{~m}^{2}$ area. This material was scattered in and slightly above the mottled stained sediment. A horizontally distinct cultural component was not identified (Figure 8-8). A small Rabdotus concentration, Feature 15, was in the farthest northwestern unit (N96/E98), between 250 and $260 \mathrm{cmbs}$. This cluster was definitively higher stratigraphically in the profile than the broader, mottled burned area. The relatively complete snail shells were not associated with cultural materials, nor were cultural materials immediately adjacent in areas above or below the concentration. Consequently, the low-density, dispersed cultural materials from Block A are unassigned to a component, although vertical positioning indicates minimally a Martindale age or older.

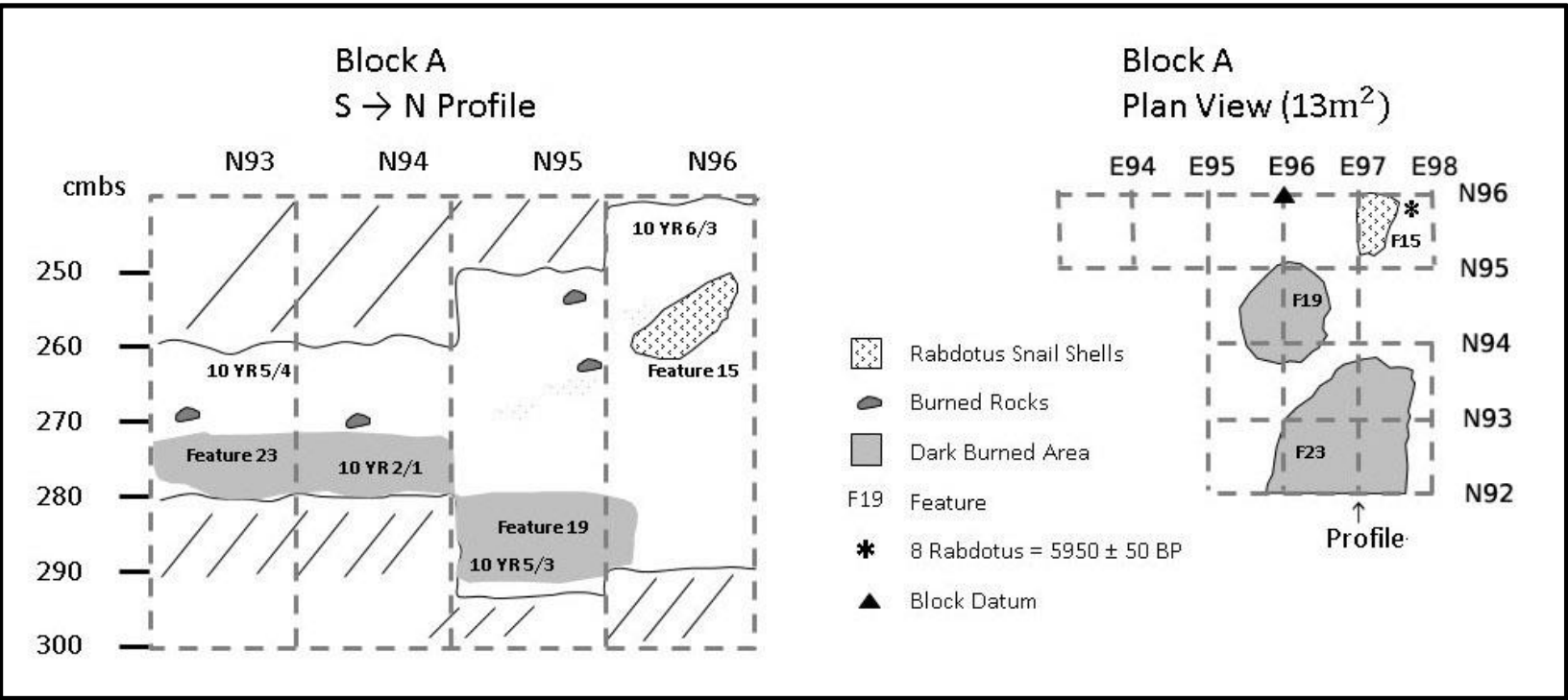

Figure 8-6. Profile (left) and plan (right) distribution of encountered features (Features 15, 19, and 23) in Block $A$.

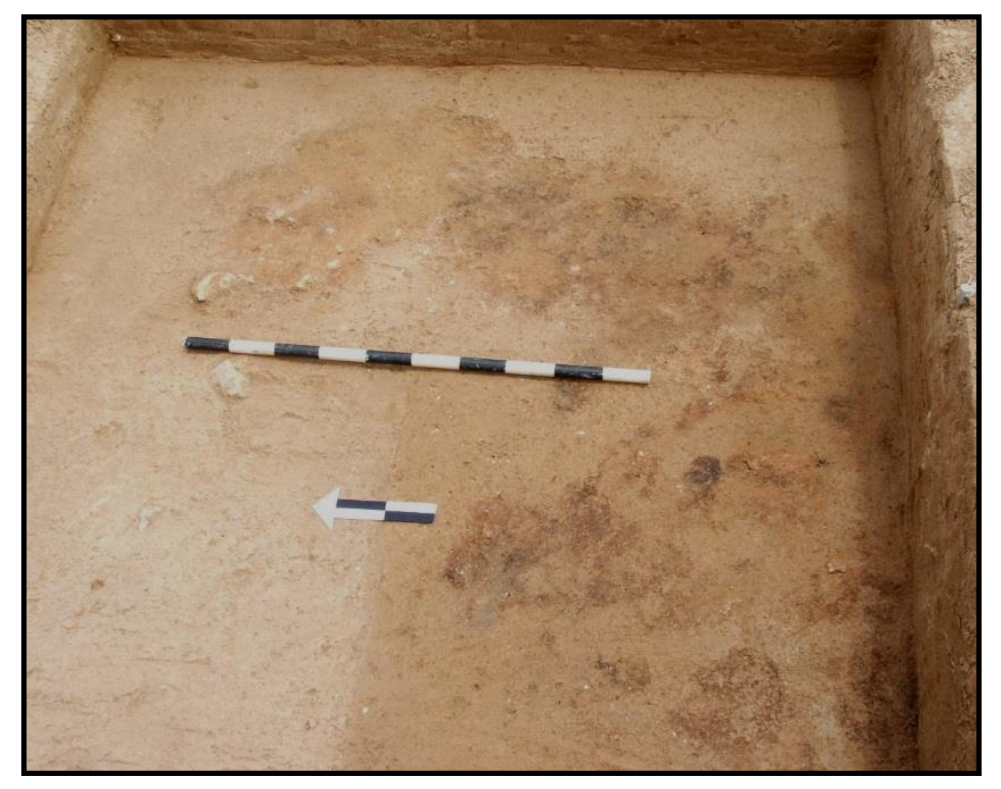

Figure 8-7. Overview of reddish and dark stained mottling of Feature 23 in Block A. 


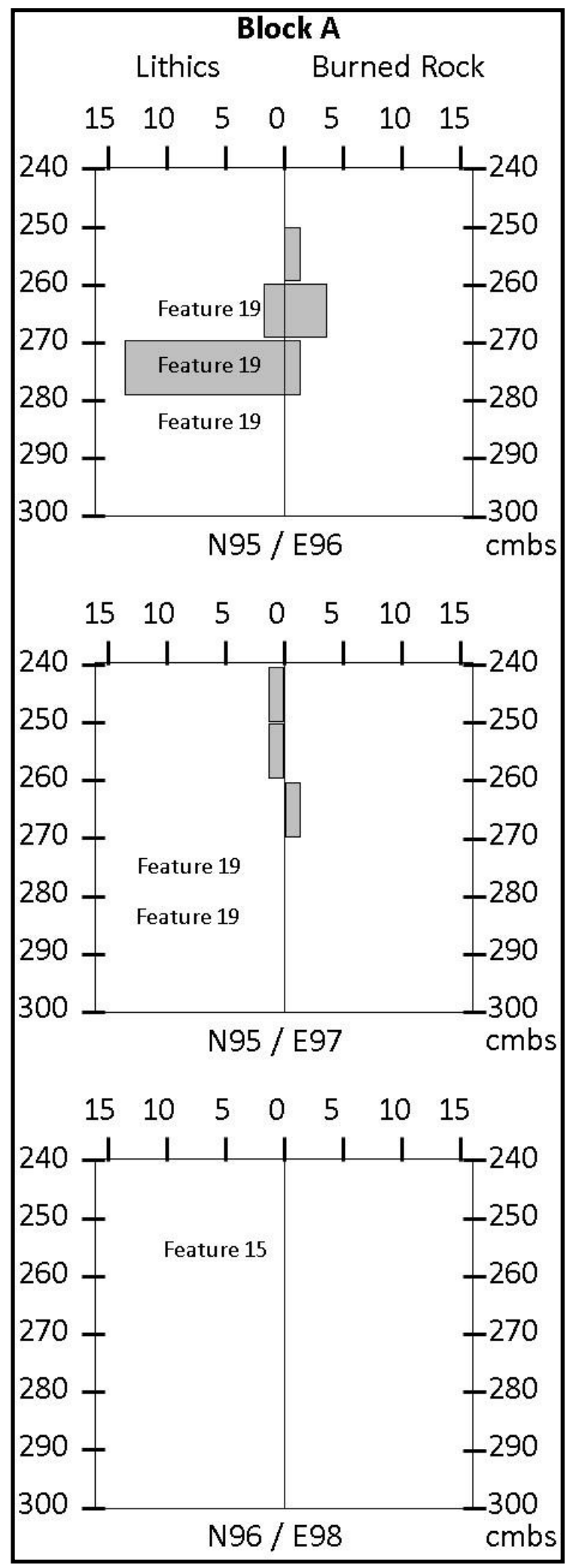

Figure 8-8. Vertical distribution of cultural materials from three selected units in Block $A$.

\subsubsection{Block B}

In Block $B$ hand-excavations were initiated between 215 and $225 \mathrm{cmbs}$, and targeted a cultural zone down to $250 \mathrm{cmbs}$. A total of $51 \mathrm{~m}^{2}$ were excavated in this block. Thirty-nine were excavated to roughly $250 \mathrm{cmbs}$. This zone yielded multiple burned rock features (Features 16, 22, 24, 27, 28, 29, 30, and 32) and associated artifacts, primarily between 220 and $240 \mathrm{cmbs}$ (Figure 8-9). A high spot in the extreme southwestern corner yielded Feature 31, a small burned rock concentration at $208 \mathrm{cmbs}$, some 12 to $15 \mathrm{~cm}$ higher in the profile than the majority of identified features.

Smaller artifacts, although potentially subject to greater vertical displacement and/or movement from various turbation actions (e.g., worms, insects, rodents, and roots), appeared to retain association with the majority of cultural features. Slight displacement probably occurred, although not to any significant extent. Features 16, 22, 24, 27, 28, 29,30 , and 32, in conjunction with a diverse artifact assemblage that consists of two broken Bell and one complete Big Sandy projectile points, lithic debitage, burned rocks, vertebrate faunal remains, and mussel shells surrounded the features in a horizontal plane. This association represents a single well-defined component with good integrity. The component was stratigraphically above the thin, mottled greenish-gray lens.

Twelve of the 51 units were excavated in a block to $290 \mathrm{cmbs}$ along the southern margin of Block B (see Figure 8-9). These units encountered an in situ organically stained hearth (Feature 26) associated with scattered burned rocks between 265 and 280 cmbs. Feature 26 was stratigraphically and immediately below the indistinct, 1 to $3 \mathrm{~cm}$ thick, mottled greenish-gray lens present at approximately 263 to $265 \mathrm{cmbs}$ (Figure 8-10). Feature 26 was roughly 20 to $25 \mathrm{~cm}$ below the Bell/Andice component. Subsequently, Feature 26 and associated materials were attributed to the 


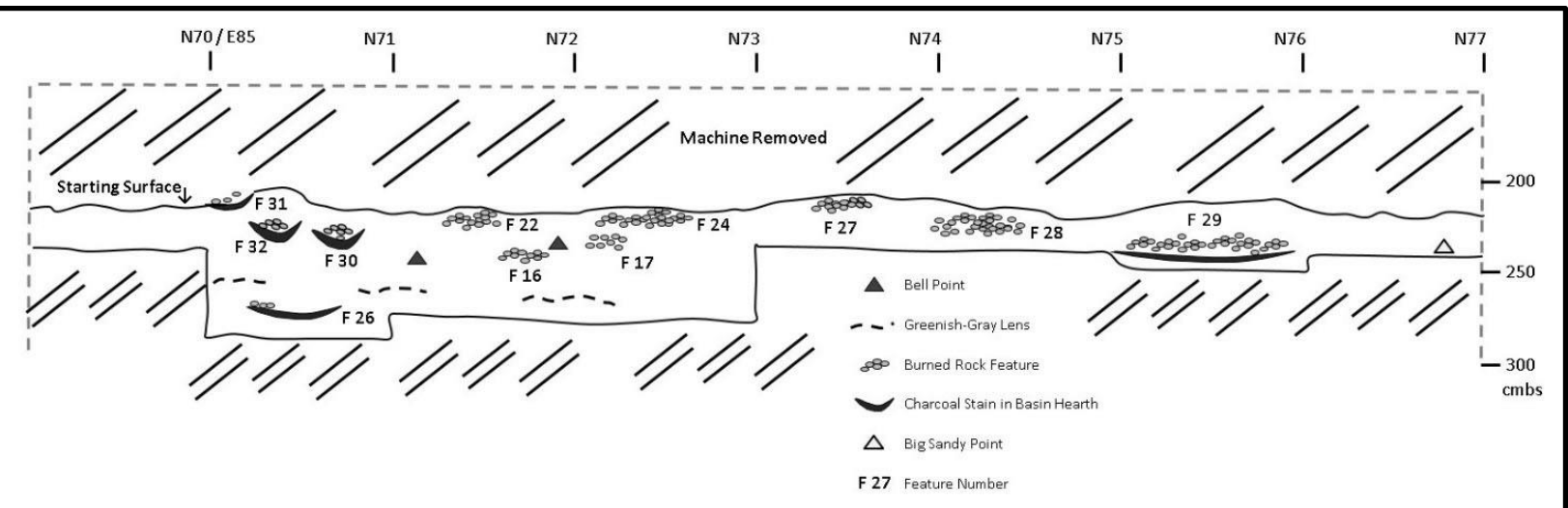

Figure 8-9. Vertical distribution of encountered features (Features 16, 22, 24, 27, 28, 29, and 30 through 32) across Block B. Note Feature 26 was stratigraphically below other features.

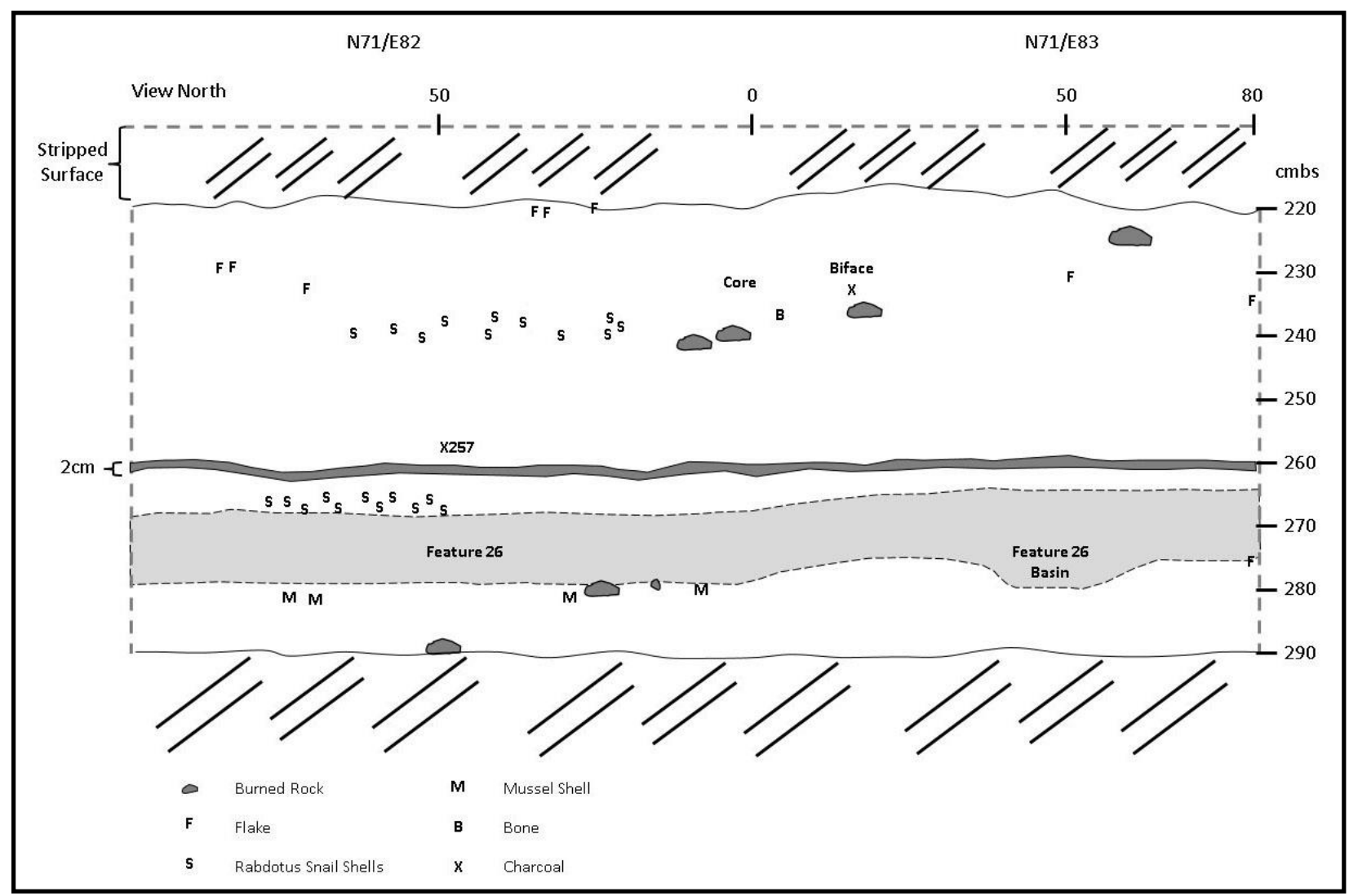

Figure 8-10. Piece-plotted materials from two units in Block $B$ (71N/82E and 71N/83E) that illustrate the vertical distribution of the Bell/Andice component stratigraphically above the light greenish-gray clay lens and the lower Feature 26 of the Martindale component. 
Martindale component based on vertical positioning and multiple radiocarbon dates. Block B results document a Bell/Andice component between 220 and $240 \mathrm{cmbs}$, stratigraphically separated by the thin mottled greenish-gray lens from the lower Martindale component between 265 and $280 \mathrm{cmbs}$ (see Chapter 9.0 for a detailed discussion of this component).

\subsubsection{Block C}

Block $\mathrm{C}$ in the southeastern corner of the stripped area yielded an ill-defined burned area thought to represent targeted GPR Anomaly \#3. Block C was subsequently expanded both vertically and horizontally to $18 \mathrm{~m}^{2}\left(7.2 \mathrm{~m}^{3}\right)$ to expose this poorly defined feature, and several vertically separated, sparse events (Figure 8-11). The broad amorphous burned area, designated Feature 21, resembled a tree burn (Figure 8-12). The mottled greenish-gray lens was observed in many units and was encountered at depths roughly 275 to $280 \mathrm{cmbs}$, dipping to nearly $290 \mathrm{cmbs}$ in N65/E96. Feature 21 was definitively above the greenish-gray lens. Cultural materials were thinly scattered both vertically and horizontally across this block. These materials were generally stratified both above and below the green-gray lens (Figure 8-11). Small concentrations of Rabdotus snail shells were

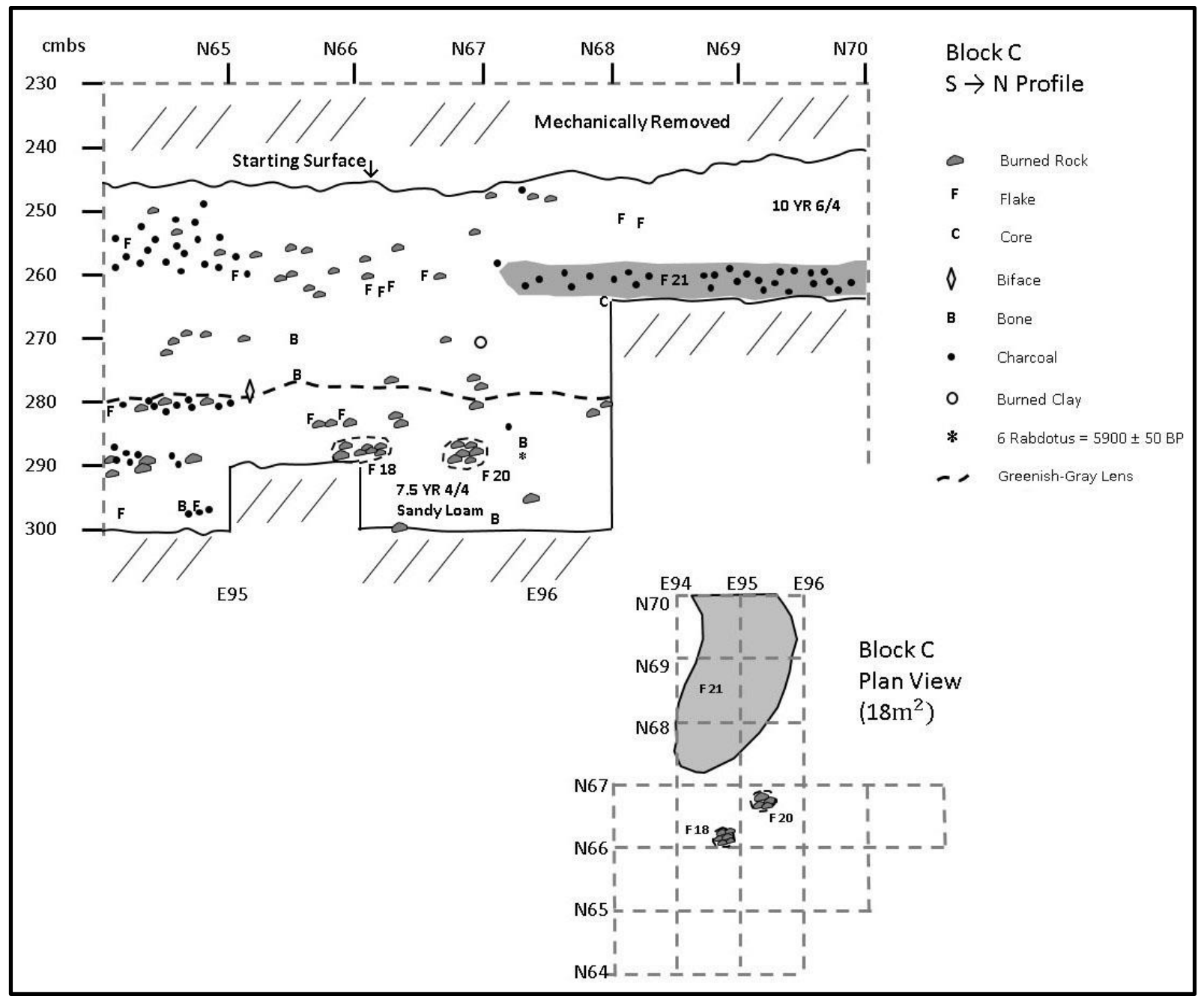

Figure 8-11. Plan and profile distribution of piece-plotted materials and features (Features 18, 20 and 21) in Block C. 


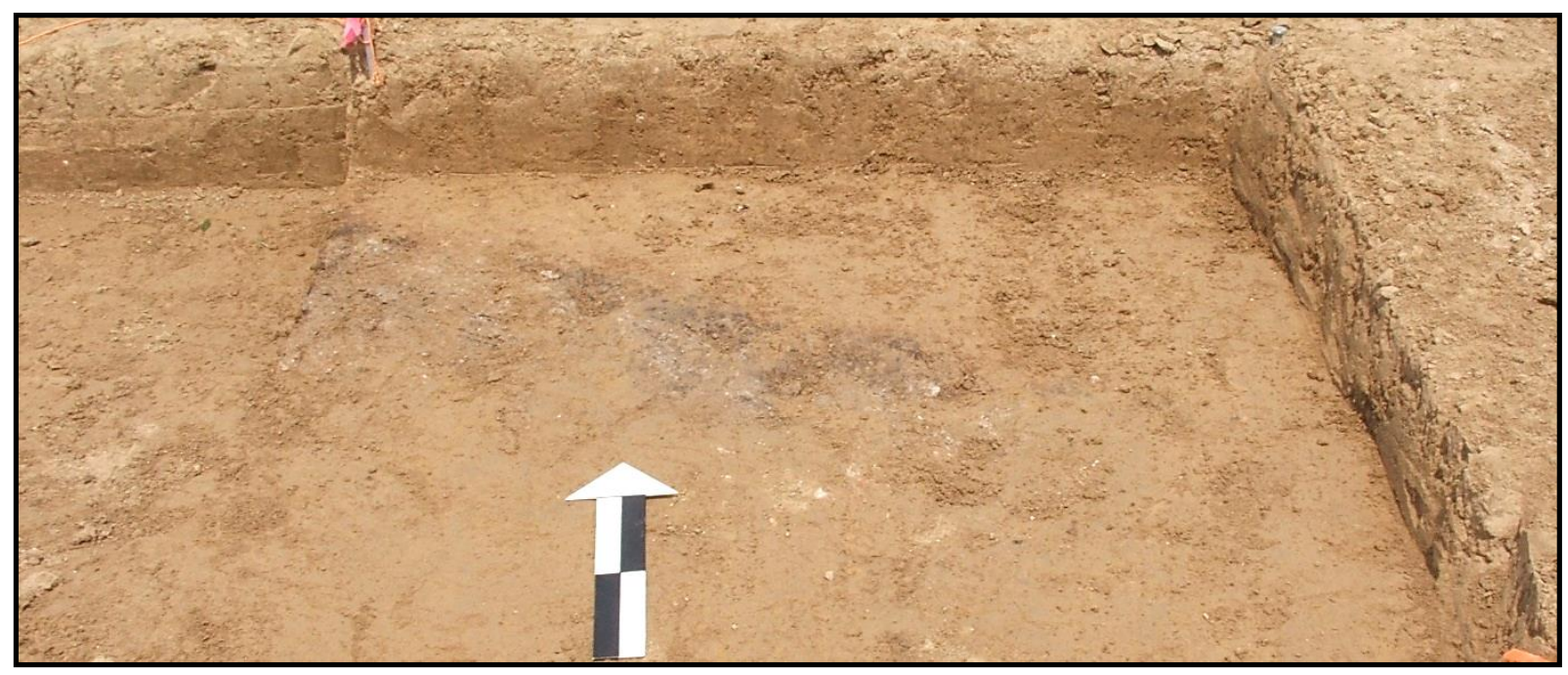

Figure 8-12. Oblique photograph of a portion of the mottled dark stained area of Feature 21.

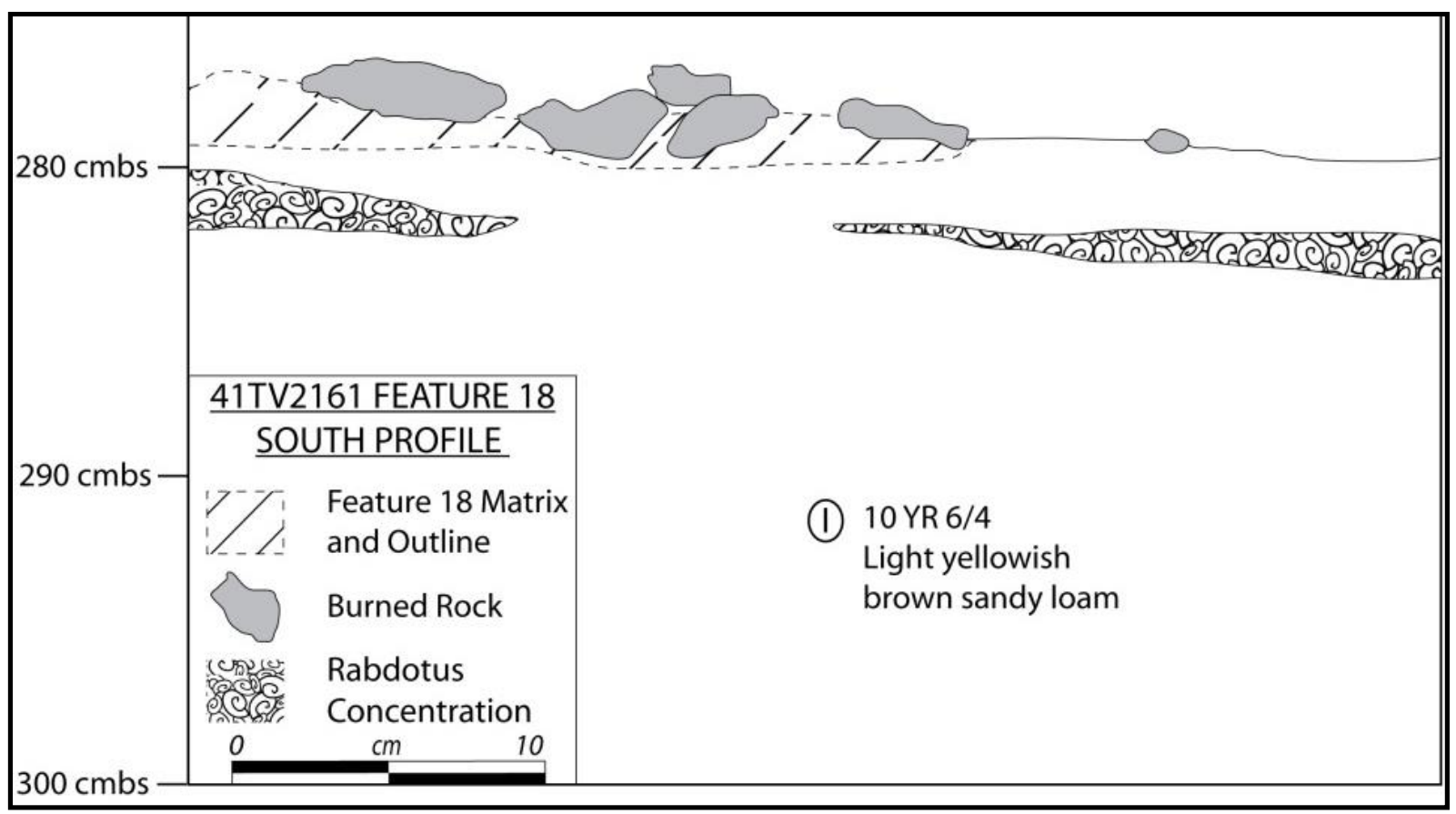

Figure 8-13. Profile of Feature 18 in N66/E95 of Block C, documenting noncultural Rabdotus concentrations not directly associated with cultural feature. 
encountered just above or just below the greenish-gray lens, Feature 21 and scattered cultural materials and features. The shell concentrations did not appear directly associated with the more frequent cultural materials (Figure 8-13). The sandy clay loam above the greenish-gray lens was a light yellowish-brown (10YR 6/4), whereas below it, the clay loam was a similar yellowish-brown (10YR 4/6).

Back plots developed from in situ plotted materials reveal minimally four thin cultural events between 250 and $300 \mathrm{cmbs}$ (Figure 8-11). Feature 21, encountered at approximately $260 \mathrm{cmbs}$, with an amorphous outline, was not clearly associated with the upper cultural event situated between 250 and $260 \mathrm{cmbs}$. It is unclear if this upper cultural event was part of the Bell/Andice component recognized in Block B. A second and more widely dispersed cultural event appeared at approximately $270 \mathrm{cmbs}$. A third and more intensively represented cultural event was associated with the thin, mottled greenish-gray lens thought to be the same lens detected in Block B. If this is the same greenish-gray lens, then the cultural material below the lens might represent the Martindale component identified in Blocks B and D. Stratigraphically below this cultural lens, two small burned rock clusters, Features 18 and 20, were present between 280 and $290 \mathrm{cmbs}$, along with other scattered burned rocks (see Figure 8-11). Below Features 18 and 20 were small concentrations of Rabdotus shells not associated with either cultural feature (see Figure 8-13). A sparse distribution of cultural artifacts were also present beneath Features 18 and 20. If the greenish-gray lens denotes the division between the higher Bell/Andice component and the lower Martindale component, then a general association can be postulated. The presence of multiple stratified events, which lack charcoal radiocarbon dates and diagnostic projectile points, creates uncertainty as to when those cultural events and natural tree burn occurred. This uncertainty prevented confident assignment of Block $\mathrm{C}$ cultural materials to components identified in Blocks B and D. Therefore, Block $\mathrm{C}$ materials were unassigned.

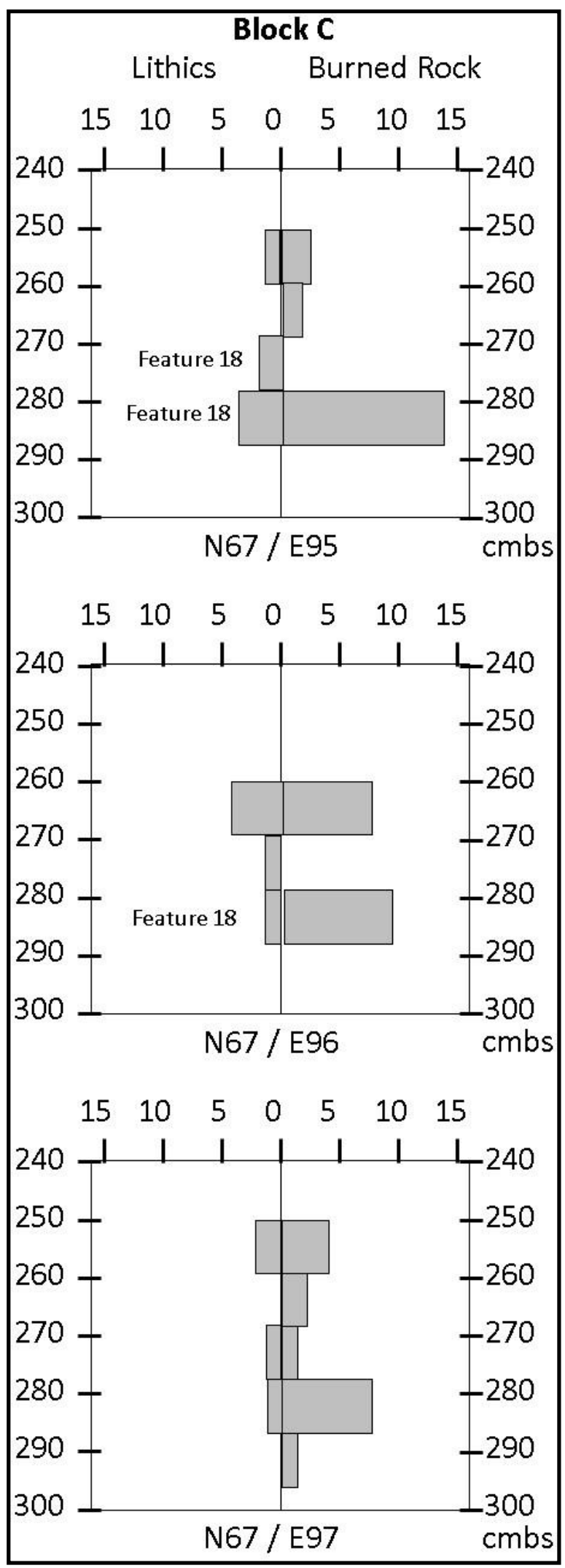

Figure 8-14. Vertical distribution of cultural materials from three selected units in Block $C$. 


\subsubsection{Block D}

A poorly defined, 1 to $3 \mathrm{~cm}$ thick, mottled greenishgray (Gley 2 7/1, 5 BG) clay lens was sporadically detected in upper deposits in this area. When observed, this lens was employed as a stratigraphic marker that allowed excavators to roughly determine their vertical position and to seek and follow the cultural materials below the lens. This thin natural lens was not always observable, or horizontally consistent, as it rose and dipped across Block D. At the southern end, N81/E92, the lens varied from 263 to $270 \mathrm{cmbs}$ (Figures 8-15 and 8-

16). Across the northern edge of Block $D$, it varied from 235 to $270 \mathrm{cmbs}$. This variability caused some confusion during excavations. When not obvious, this fostered uncertainty in vertical association of the artifacts encountered.

Cultural materials appeared concentrated just below the greenish-gray lens, and confined to a zone roughly between 250 and $280 \mathrm{cmbs}$. The identified artifacts in this $30 \mathrm{~cm}$ thick zone were vertically more concentrated in individual units. The cultural occupation had slightly uneven elevation differences across Block D (Figure 8-17). This cultural zone, a few centimeters below the greenish-gray lens, was associated with one Martindale/Early Corner-notched dart point and attributed to the Martindale component. In a few

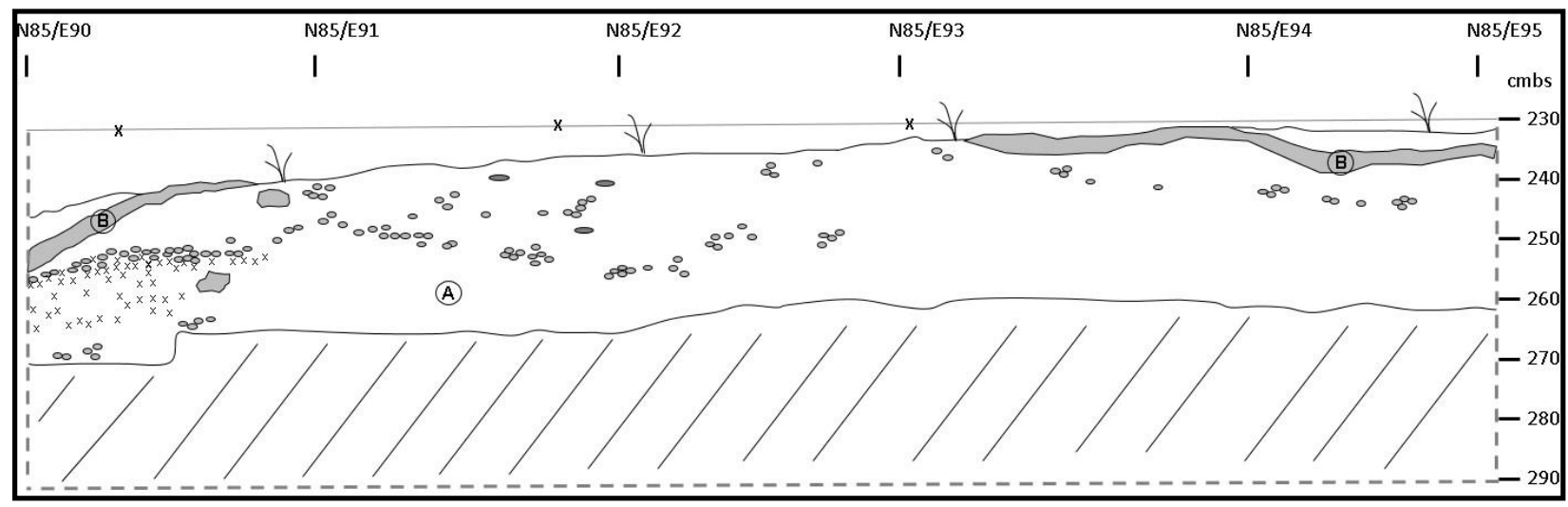

Figure 8-15. Profile of north wall across Block D demonstrating vertical variability of the greenish-gray clay lens.

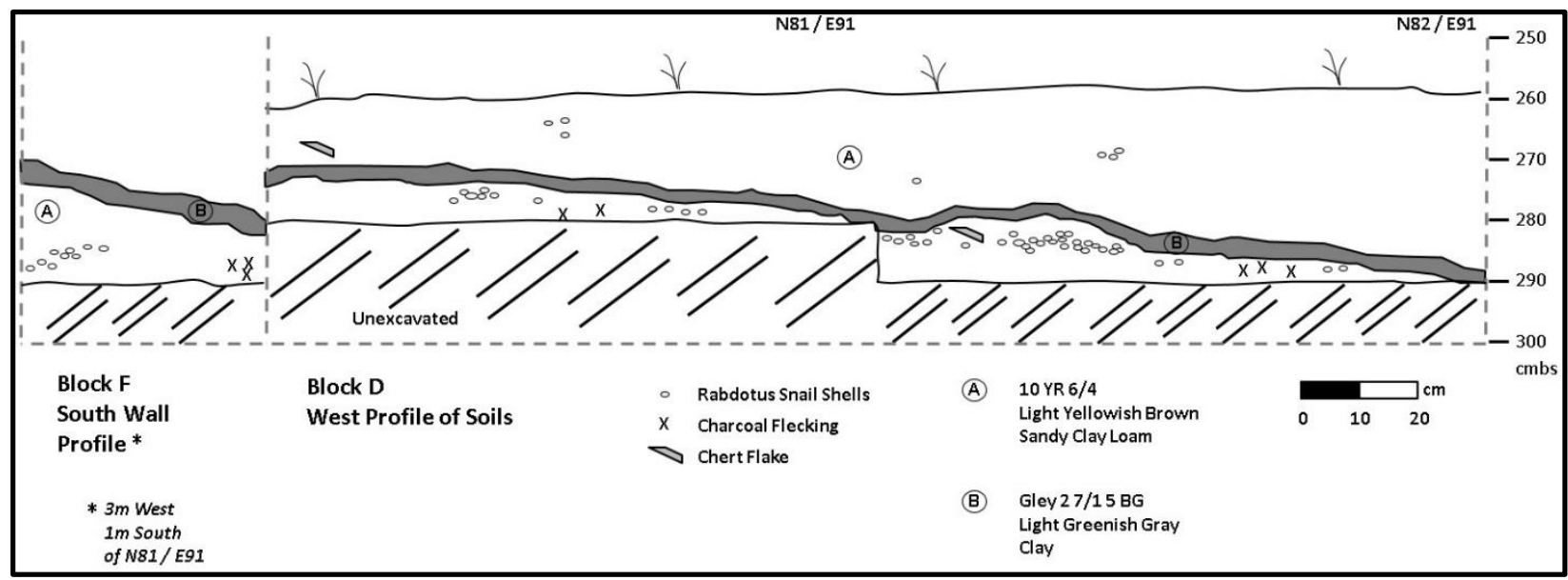

Figure 8-16. Profile of north wall of two units in Block D demonstrating vertical variability of the greenish-gray clay lens. 


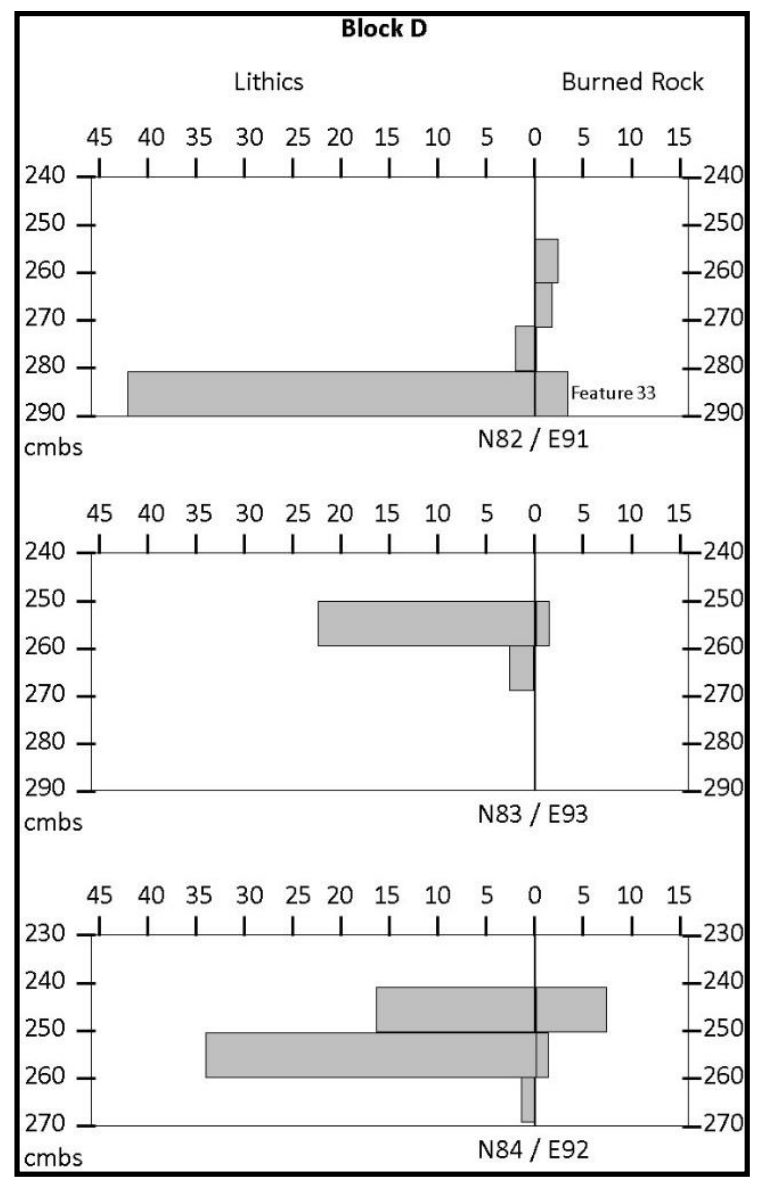

Figure 8-17. Vertical distribution of cultural materials in three selected units from Block $D$.

instances, where hand-excavations extended to 290 cmbs, a few stratigraphically lower artifacts were encountered. The limited lower materials were not considered part of this Martindale component and probably represent an older, unassigned occupation. The Martindale component in Block D yielded two organically stained features (Features 25 and 33) vertically equivalent with the Martindale point. Smaller cultural items (e.g., lithic debitage, bones, and scattered burned rocks) were encountered at roughly similar depths as Features 25 and 33.

Cultural materials surrounded those features in horizontal distribution patterns to indicate artifacts probably originated from the same uneven surface. The diverse cultural assemblage includes lithic debitage, butchered animal bones, scattered burned rocks, and infrequent stone tools. The material diversity reflects multiple different tasks by the occupants. Horizontal distribution of material probably reflects different activity areas across Block D. Absence of overlapping material concentration or features indicate the presence of a single Martindale component (Figure 8-18). Although a few rodent holes and burrows were observed, they did not appear to significantly disrupt the broader horizontal or vertical distribution of most materials in this component. Features 25 and 33, and associated surrounding cultural materials across Block D represent a welldefined and isolated Martindale/Early Cornernotched component of short-term duration. Feature 26 in the southern part of Block B, stratigraphically below the thin greenish-gray lens, was also attributed to this Martindale component based on its vertical position and radiocarbon dates (see Chapter 10.0 for a detailed discussion of this component).

\subsubsection{Block E}

This small $3 \mathrm{~m}^{2}\left(1.2 \mathrm{~m}^{3}\right)$ block, just $2 \mathrm{~m}$ north of Block D, targeted GPR Anomaly \#5. Stratigraphically, it was anticipated that Anomaly \#5 would have been associated with the Martindale component in Block D. The starting depth was roughly $250 \mathrm{cmbs}$, with two units dug to $280 \mathrm{cmbs}$ and one to $290 \mathrm{cmbs}$. Only Unit N89/E69 yielded point provenienced material and those few unburned rocks were between 279 and $287 \mathrm{cmbs}$. No features or diagnostic artifacts were encountered. This together with a low frequency of cultural materials present and the lack of radiocarbon dates precluded assignment of Block $E$ materials to any identified component.

\subsubsection{Block F}

Block F also targeted a GPR anomaly potentially associated with the Bell/Andice component in Block B. The anomaly was just beyond the northern edge of Block $B$, and electronically registered at the appropriate stratigraphic depth. 


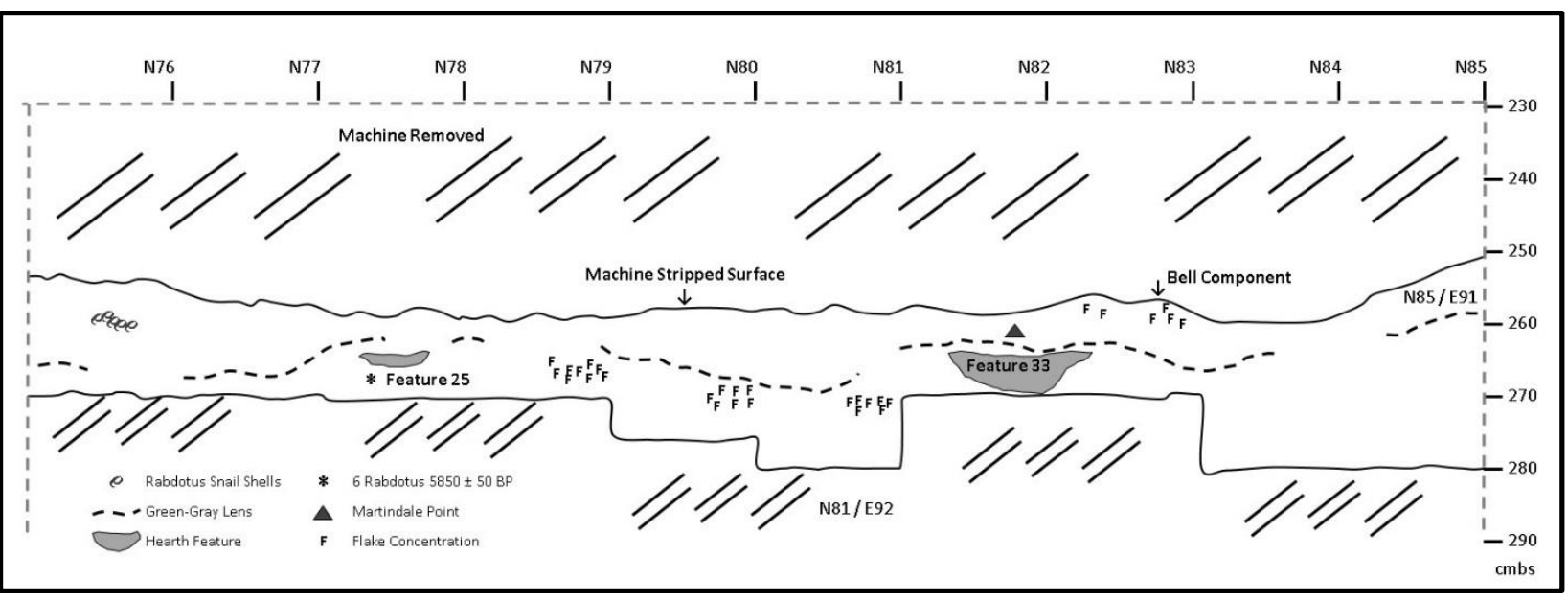

Figure 8-18. Vertical distribution Features 25 and 33 along with identified flake concentrations across Block D.

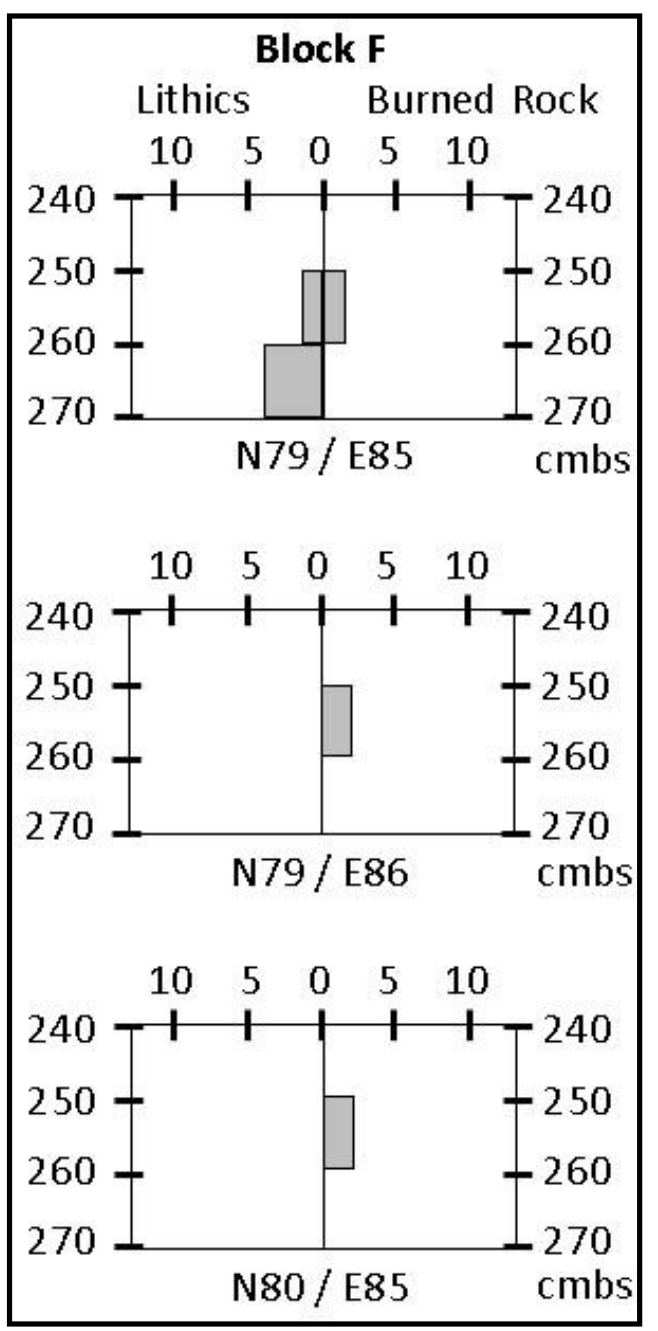

Figure 8-19. Vertical distribution of cultural materials within three selected units from Block F.
However, only three burned rocks and three flakes were recovered between 245 and $270 \mathrm{cmbs}$ within this limited at $4 \mathrm{~m}^{2}\left(0.6 \mathrm{~m}^{3}\right)$ block (Figure 8-19). No attempt was made to assign the very limited cultural material to a specific component.

\subsection{SITE STRATIGRAPHY SUMMARY AND COMPONENT ASSIGNMENT}

The above information allows the identification and recognition of two principal components, a Bell/Andice over a Martindale component, deeply buried between 220 and $280 \mathrm{cmbs}$ in low-energy overbank alluvium. The two components were recognized in Blocks $\mathrm{B}$ and $\mathrm{D}$. In Block $\mathrm{B}$, a Bell/Andice component was identified and consisted of eight burned rock features (Features 16, 22, 24, 27, $28,29,30$, and 32) and associated artifacts, primarily between 220 and $240 \mathrm{cmbs}$. Twelve deeper units towards the southern end of Block B yielded Feature 26 associated with scattered cultural materials stratigraphically below the

Bell/Andice component. This lower material was attributed to the Martindale component. The Martindale component was also encountered all across Block D between 250 and $280 \mathrm{cmbs}$. Unfortunately in the Block D area, deposits that may have contained 
Bell/Andice component materials were mechanically removed during the initial stripping.

Blocks $\mathrm{E}$ and $\mathrm{F}$ lacked features and yielded low frequencies of cultural material upon excavation. As result, these areas could not be classified into a designated component.

Excavations in Block $\mathrm{C}$ yielded multiple events or components in stratified context. Unfortunately, it also lacked diagnostic data to confidently assign the multiple cultural zones to either identified component. Block A contained scattered cultural materials unassignable to a component and a noncultural tree burn.

In summary, two stratigraphically well-defined cultural components are identified, a Bell/Andice component above a Martindale component in a targeted cultural zone between 210 to $290 \mathrm{cmbs}$ within a deep stratified site. The two components were separated by a thin, mottled light greenishgray clay lens that lacked cultural materials. The separation between two well-defined cultural components is quite significant. The excellent context and high integrity of the materials permits more detailed and in-depth analyses to focus on discrete assemblages that represent short-term occupations. It also allows comparisons between two specific discrete components with established typed projectile points, and affords more in-depth interpretations of cultural behaviors.

The following two chapters provides detailed information on the Bell/Andice and Martindale components at the Big Hole site (see Chapters 9.0 and 10.0, respectively). Cultural materials not attributed to either component are discussed in Chapter 12. 
This page intentionally left blank. 


\subsection{BELL/ANDICE COMPONENT}

\author{
J. Michael Quigg and Paul M. Matchen
}

\subsection{INTRODUCTION}

The following sections present cultural materials from a deeply buried (ca. 220 to $240 \mathrm{cmbs}$ ) very well-defined Bell/Andice component that yielded nine small features, a limited quantity of chipped and ground stone tool assemblage, quantities of scattered burned rocks, and a vertebrate faunal assemblage from $51 \mathrm{~m}^{2}$ excavated in Block B. The component age is presented first, followed by a description and discussion of each feature. Multiple technical analyses were conducted on an array of materials from features and those results are presented with each feature. Artifact group analysis is presented followed by a summary and interpretations section with concluding statements that address multiple research issues and the hypotheses stated in the research design.

\subsection{DATING THE COMPONENT}

Stratigraphically, the component is well-defined and isolated from any other identifiable component. Although this component is well-defined, preservation of organic debris and microfossils was poor, as one might expect of materials greater than 5,000 years old. Charcoal staining was observed in and around several burned rock features, however, the carbon staining was just that, with few charcoal chunks present, not even in floated feature sediment. Because charcoal was nearly nonexistent as only three of the eight burned rock features yielded charcoal, multiple materials were sent for dating and included three animal bone fragments, three organic rich sediments, and one Rabdotus snail shell sample, besides limited charcoal from three features. Twelve radiocarbon dates provide the age determination for this component (Table 9-1, Figure 9-1).
Charcoal, the more reliable index for age determination in most instances, yielded six dates from three cultural features with an average of 5322 B.P. and a very narrow 90 year radiocarbon age range (see Table 9-1). Dating charcoal directly associated with cultural features provides excellent context for the interpretation of results, and increases confidence in these dates. Given the age derived from the charcoal and limited range documented, the average is quite appropriate for what is currently known for the Bell/Andice interval (see Section 3.2.7).

The slight variation within the six charcoal dates may be explained by use of old dead wood. The two radiocarbon dates derived from charcoal from Feature 24 are slightly different with Sample \#2179-4-g being slightly older than the other four charcoal dates. A second slightly older date came from Sample \#24244-g out of Feature 29 and closely matches the older date from Feature 24. Given that these slight differences probably reflect old wood used in the fires, the charcoal dates do not refute the short-term nature of this occupation.

One obtained date on multiple Rabdotus snail shells from $2 \mathrm{~m}$ west of Feature 22 and between 235 and 241 cmbs is the oldest date by nearly 450 years as compared to the six charcoal dates. The sample consisted of six nearly complete shells from the same depth as most dated charcoal. If the shells accumulated at roughly the same time as the Bell/Andice cultural materials, then the shells provided a date older than the stratigraphically associated charcoal. The older age is potentially caused by incorporation of older carbon into their shells as a result of their feeding habits (e.g., Ellis et al. 1996). It is also possible that older shells were redeposited during the depositional processes (e.g., flood events) and are truly much older than the in situ cultural materials.

Two dates obtained on animal bones were derived from small fragments of what appeared as deer size long bone fragments. The dates of 4710 B.P. 
Table 9-1. Radiocarbon Dates from the Bell/Andice Component.

\begin{tabular}{|c|c|c|c|c|c|c|c|c|c|c|c|}
\hline $\begin{array}{c}\text { Catalogue } \\
\text { No. }\end{array}$ & Block & $\begin{array}{c}\text { Unit No. } \\
\text { N/E }\end{array}$ & $\begin{array}{l}\text { Depth } \\
\text { (cmbs) }\end{array}$ & $\begin{array}{c}\text { Feature } \\
\text { No. }\end{array}$ & $\begin{array}{l}\text { Material } \\
\text { Dated }\end{array}$ & $\begin{array}{l}\text { Wt of } \\
\text { Material } \\
\text { (g) }\end{array}$ & $\begin{array}{l}\text { Beta } \\
\text { Lab. } \\
\text { No. }\end{array}$ & $\begin{array}{l}\text { Measured } \\
\text { Age }\end{array}$ & $\begin{array}{c}\text { 13C/12C } \\
\text { Ratio } \\
(\%)\end{array}$ & $\begin{array}{l}\text { Conventional } \\
\text { Age (B.P.) }\end{array}$ & $\begin{array}{c}2 \text { Sigma } \\
\text { Calibration } \\
\text { Range }\end{array}$ \\
\hline TV2161/M-3 & $B$ & N71/E82 & $\begin{array}{l}235- \\
241\end{array}$ & & $\begin{array}{c}6 \text { Rabdotus } \\
\text { shells }\end{array}$ & 3.3 & 216370 & $5520 \pm 50$ & -9.5 & $5770 \pm 50^{1}$ & $\begin{array}{c}\text { BC } 4720 \text { to } \\
4490\end{array}$ \\
\hline $2176-4-b$ & $B$ & N73/E85 & $\begin{array}{l}218- \\
230\end{array}$ & 24 & $\begin{array}{c}\text { Partial } \\
\text { burned bone }\end{array}$ & 0.2 & 398641 & NA & NA & $5580 \pm 30^{1}$ & $\begin{array}{c}\text { BC } 4460 \text { to } \\
4350\end{array}$ \\
\hline $2176-4-g$ & $B$ & N75/E85 & $\begin{array}{l}218- \\
230 \\
\end{array}$ & 24 & Charcoal & 0.1 & 398642 & $5300 \pm 30$ & -26.3 & $5280 \pm 30$ & $\begin{array}{c}\text { BC } 4230 \text { to } \\
4190\end{array}$ \\
\hline 2179-4-g & B & N73/E86 & $\begin{array}{l}220- \\
230\end{array}$ & 24 & Charcoal & 0.1 & 398643 & $5340 \pm 30$ & -23.3 & $5370 \pm 30$ & $\begin{array}{c}\text { BC } 4325 \text { to } \\
4280\end{array}$ \\
\hline $2179-4-1 a$ & B & N73/E86 & $\begin{array}{l}223- \\
230 \\
\end{array}$ & 24 & $\begin{array}{c}\text { Organic } \\
\text { sediment }\end{array}$ & 200 & 234636 & $5530 \pm 40$ & -24.5 & $5540 \pm 40^{1}$ & $\begin{array}{c}\text { BC } 4460 \text { to } \\
4330\end{array}$ \\
\hline $2200-4-g$ & $\mathrm{~B}$ & N74/E86 & $\begin{array}{l}212- \\
230\end{array}$ & 28 & Charcoal & 0.1 & 398646 & $5240 \pm 30$ & -22.3 & $5280 \pm 30$ & $\begin{array}{c}\text { BC } 4230 \text { to } \\
3995\end{array}$ \\
\hline $2202-2-A$ & $B$ & N75/E83 & $\begin{array}{l}230- \\
240\end{array}$ & & $\begin{array}{c}\text { Bone } \\
\text { fragment }\end{array}$ & 13.4 & 398647 & $4580 \pm 30$ & -16.8 & $4710 \pm 30^{1}$ & $\begin{array}{c}\text { BC } 3630 \text { to } \\
3375\end{array}$ \\
\hline 2229-7-2 & B & N78/E81 & 241 & & Charcoal & 0.3 & 398648 & $5270 \pm 30$ & -23.9 & $5290 \pm 30$ & $\begin{array}{c}\text { BC } 4235 \text { to } \\
4000\end{array}$ \\
\hline $2424-4-1 \mathrm{~g}$ & B & N76/E86 & $\begin{array}{l}230- \\
235\end{array}$ & 29 & Charcoal & 0.1 & 398656 & $5260 \pm 30$ & -26.3 & $5340 \pm 30$ & $\begin{array}{c}\text { BC } 4315 \text { to } \\
4050\end{array}$ \\
\hline $2424-4-2 g$ & $B$ & N76/E86 & $\begin{array}{l}230- \\
235\end{array}$ & 29 & Charcoal & 0.1 & 398657 & $5290 \pm 30$ & -26.4 & $5370 \pm 30$ & $\begin{array}{l}\text { BC } 4325 \text { to } \\
4075\end{array}$ \\
\hline 4/1/2223 & B & N76/E86 & 235 & 29 & $\begin{array}{c}\text { Organic } \\
\text { sediment }\end{array}$ & 143 & 234637 & $5360 \pm 40$ & -25.2 & $5360 \pm 40$ & $\begin{array}{c}\text { BC } 4330 \text { to } \\
4050\end{array}$ \\
\hline $7 / 1 / 2092$ & $B$ & N71/E80 & $\begin{array}{l}234- \\
238\end{array}$ & 30 & $\begin{array}{c}\text { Organic } \\
\text { sediment }\end{array}$ & 73 & 234638 & $5650 \pm 50$ & -23.2 & $5680 \pm 50^{1}$ & $\begin{array}{c}\text { BC } 4670 \text { to } \\
4390\end{array}$ \\
\hline $\begin{array}{c}\text { TV2161/M- } \\
10\end{array}$ & $B$ & N72/E79 & 237 & & Deer ulna & 16.4 & & NA & & & \\
\hline
\end{tabular}




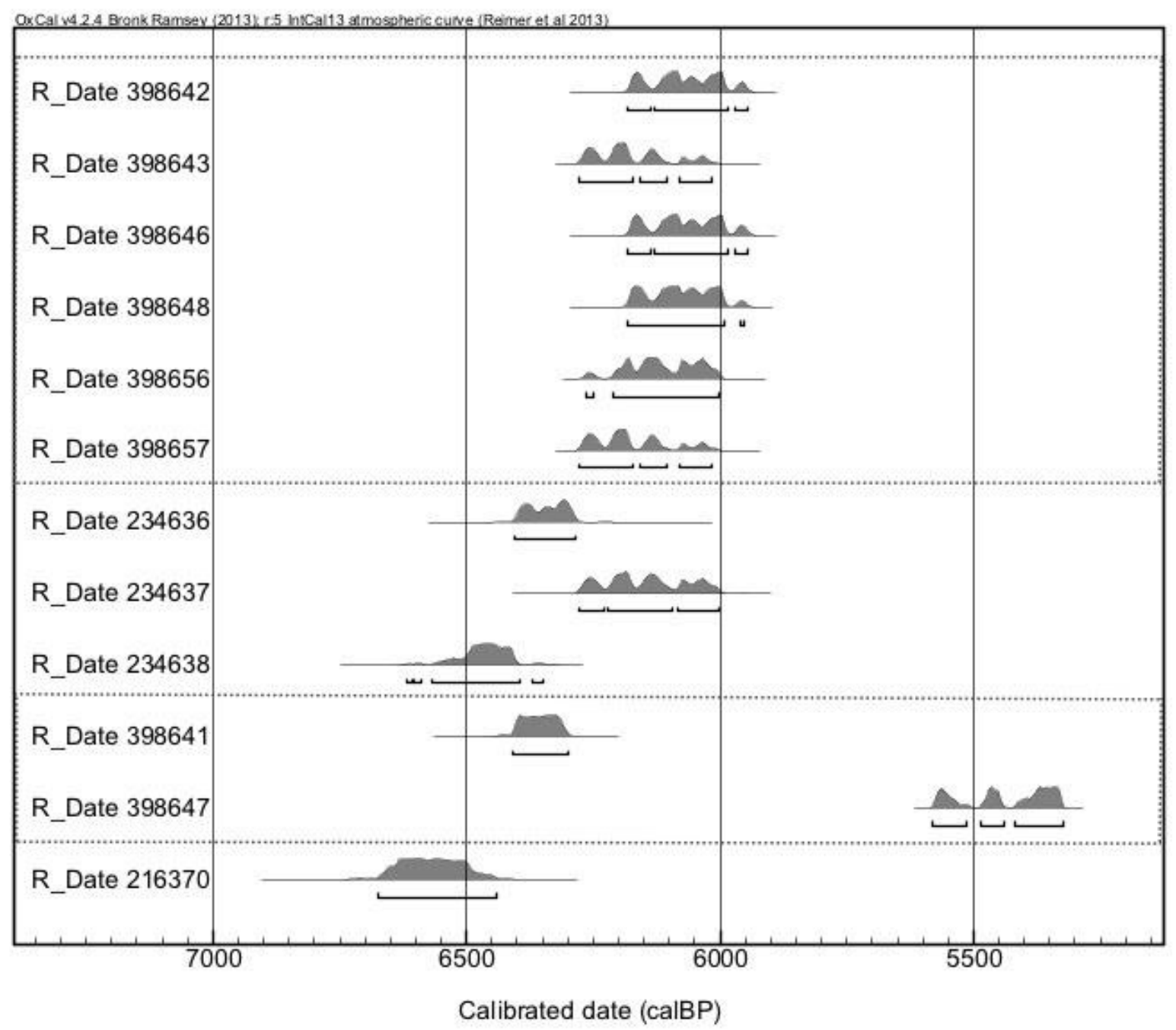

Figure 9-1. Plot of radiocarbon assays from the Bell/Andice component. Samples are presented from top to bottom in the following order: charcoal (6), organic sediment (3), bone (2), and Rabdotus shells (1).

and 5580 B.P. are younger and older respectively, than the averaged six charcoal dates. A reasonable explanation for their variability and deviation from the charcoal results is that the organic component in the bone tissue has been differentially altered from the original state through preservation issues. These bone dates provide a general indication or an approximation of when this cultural event occurred, but are not accepted as the actual age of the occupation.

Three results from organic sediments also yielded inconsistent dates, with two older and one similar to the charcoal dates. The date of 5540 B.P. from Feature 24 is ca. 215 years older in comparison to the averaged two charcoal dates from that same feature. It reveals an acceptable ballpark age, but is not as precise as the two charcoal dates. The organic sediment date of 5360 B.P from Feature 29 reflects the same age as the charcoal date from that feature, and is quite appropriate. The date of 5680 B.P. from Feature 30 is older than the average six charcoal dates for this component and probably reflects a ballpark age rather than the precise use age. 
Frederick (2011:80-82) provided analyses of radiocarbon dates from two prehistoric sites immediately adjacent to the Big Hole site and compared bulk sediment dates to charcoal dates and found that the bulk sediments were all in error by 940 to 1,490 years, and overestimate the age of sediments. Charcoal was also compared to Rabdotus snail dates with two results the same as charcoal and one 875 years older. However, he indicates snail dates may be just as problematic as bulk sediment ages. At Horn Shelter, four paired samples that compared charcoal to Rabdotus shells. The Rabdotus shells were always older by 180 to 530 years (Watt 1978). Discrepancies in materials dated were also documented at the adjacent Berdoll site (41TV2125). From the latter site, bulk sediment samples yielded ages of 9190 and 9540 B.P., whereas the charcoal samples from similar context ranged from 7606 to 7920 B.P., which indicate differences from 1,584 to 1,934 years on bulk sediments compared to charcoal dates (Karbula et al. 2011:159). This age discrepancy is not a new discovery as others have also shown that the sediment dates are often older than associated charcoal dates (e.g., Abbott 1994; Collins 1994b).

\subsection{CULTURAL FEATURES}

Ten features, including nine burned rock features (Features 3, 16, 22, 24, 27, 28, 29, 30, and 32) and one cluster of ground stone tools (Feature 17), associated with the Bell/Andice component are presented below. Feature 3 was investigated during the previous phase with the other nine from this data recovery. Each feature is described in numerical order with results presented from diverse technical analyses on individual feature samples. All features were encountered in Block B, with the exception of Feature 3. Sparse but diverse cultural materials surrounded the nine features in Block B and are presented following this section. A short feature summary follows the discussion of individual features.

\subsubsection{Feature 3}

Feature 3 was excavated in 2004 by Hicks \& Company and was discussed in their report concerning Parcels 365 and 365E (CSJ: 0440-06006) (Campbell et al. 2007). Feature 3, with the previously obtained charcoal radiocarbon date and overall intact nature of this well-defined feature, led TRC archeologists to target the zone that contained this feature within this multicomponent, deeply stratified site.

The following information concerning the description of Feature 3 was extracted from Campbell et al. (2007). Feature 3 was encountered in the side wall of BT 22 (ca. 90 m north of Block B) and subsequently, the remaining portion, roughly two-thirds of the feature, was handexcavated in TU 3, a $1 \mathrm{~m}^{2}$ unit. Feature 3 was recorded from 208 to $229 \mathrm{cmbs}$ and consisted of a dense concentration of burned rock, burned fossil oyster shells, lithic debitage, a few bone scraps, and a concentration of charcoal (Figure 9-2). The circular concentration was approximately $90 \mathrm{~cm}$ in diameter and exhibited a shallow saucer-shaped basin lined with 201 burned rocks above a 2 to $3 \mathrm{~cm}$ thick, dark organic stain (Figure 9-3). The burned rocks ranged from 6 to $15 \mathrm{~cm}$ in diameter and included pieces of chert, petrified wood, and 53 fossil oyster shells (Campbell et al. 2007).

Fossil oyster shells and other diverse rock types were common on the exposed surface in the nearby uplands (Campbell et al. 2007). Inside the western margin was a dense concentration of shattered chert flakes, probably caused from intense heat. Most feature fill was collected in seven flotation samples, although no results were presented.

At least five charcoal samples were piece plotted inside Feature 3 (Campbell et al. 2007), with eight collected throughout the feature and two humate samples collected from directly beneath the rocks. Of these, eight charcoal samples were submitted to Beta Analytic, Inc. for dating and seven failed to yield 


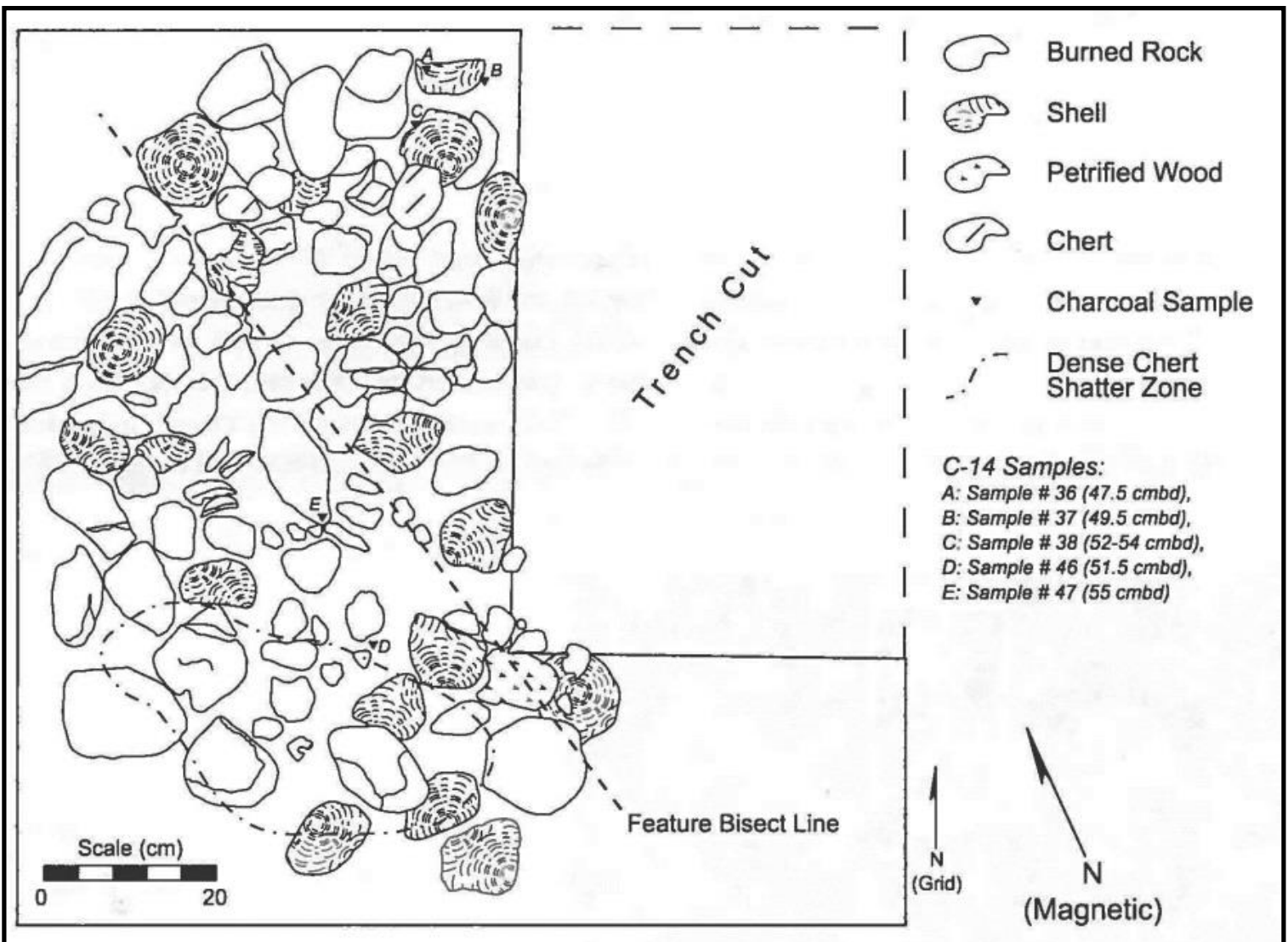

Figure 9-2. Plan view of Feature 3 (from Campbell et al. 2007, Figure 34).

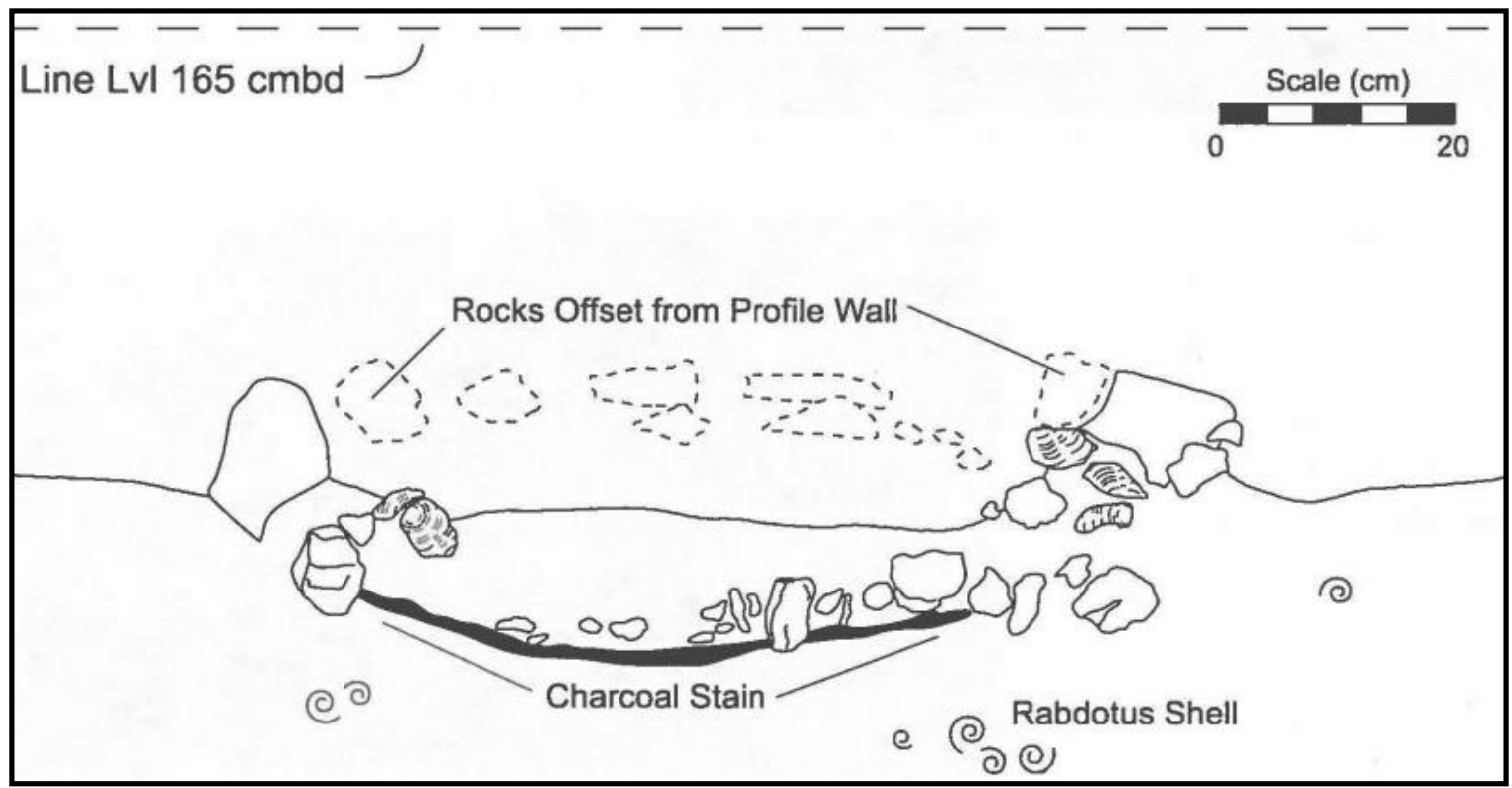

Figure 9-3. Profile of Feature 3 (from Campbell et al. 2007, Figure 35). 
radiocarbon assays. One dark organic stained sample (SH130-C14-2) contained a piece of charcoal large enough to yield a $\delta^{13} \mathrm{C}(-26.4 \%$ ) corrected AMS date of $5290 \pm 40$ B.P. (Beta-214482).

Due to the fact that only one of eight samples returned a radiocarbon date, TxDOT was concerned that the dark stained sediment was not degraded charcoal and requested TRC to submit similar stained sediment samples to outside laboratories to determine if the dark material was carbon based (see Appendix M). Beta Analytic, Inc. later explained that carbon stained sediment samples do contain datable material but are processed differently than charcoal samples (personal communication from D. Hood to M. Quigg, 7 August 2007) as stated here:.

"The samples were submitted as 'charcoal.' As such, the sediment matrix was considered contaminated and superfluous and was eliminated in the attempt to retrieve charcoal. When no discrete charcoal was available, the samples were deemed unsuitable. Had the samples been submitted as 'soot,' 'carbon dust,' 'carbon stained sediment,' or 'bulk sediment,' the matrix would have been considered the primary component for which you wished analyzed. A date would have been produced on the fine black component, or perhaps on the sediment itself, with your understanding of limited pretreatments or bulk-sediment subjectivity. Of course I do not remember the specific samples, but this is generally the case when this type of thing happens. You are always welcome to instruct us to attempt charcoal extraction, but if none is available, date the bulk sediment. In this case, you would have obtained dates either way."
Technical analyses on collected materials from Feature 3 were not conducted. Based on form alone, Feature 3 probably functioned as a cooking feature. This, combined with the radiocarbon date, indicates a probable association with the Bell/Andice interval. The obtained date fits very well with subsequent results obtained from charcoal dated features in Block B (see Table 9.1).

\subsubsection{Feature 16}

Feature 16 was measured between 247 and 254 cmbs at N72/E80, in the southwestern corner of Block B (Figures 9-4 and 9-5). Feature 16 consisted of a tight cluster of 10 burned rocks with dark stained sediment immediately surrounding and under the rocks. The stain and rocks were restricted to a roughly $25 \mathrm{~cm}$ diameter area in the northeastern quadrant. The base of the rocks varied from 252 to $254 \mathrm{cmbs}$, with one rock beneath the others that created a shallow basin. A small, dark charcoal stained sediment sample was collected below the clustered rocks for further processing. At least three pieces of heat altered chert and a few small unburned bone fragments were recovered adjacent to this feature in this same level.

Ten burned rocks (\#2122-3) weighed 1,766 g, for an average weight of $177 \mathrm{~g}$, with nine in the 4.1 to $9 \mathrm{~cm}$ size class (Table 9-2). All pieces were broken with no apparent refits. Three rocks had dark, organic stained areas on their surfaces. Three pieces also exhibited hackled edges, similar to what is often caused by the quick cooling of a hot rock. Burned rock subsamples were subjected to multiple technical analyses. Four different subsamples were sent for starch grain analysis. Three lacked starch grains, whereas one sample (\#2122-3-2) yielded a gelatinized grain, indicative of exposure to heat and water (Appendix F). Parts of those same burned rocks were sent for lipid residue analysis.

Subsample \#2122-3-5 yielded borderline moderatehigh and high fat content reflecting a possible plant and animal combination. Subsample \#2122-3-5 


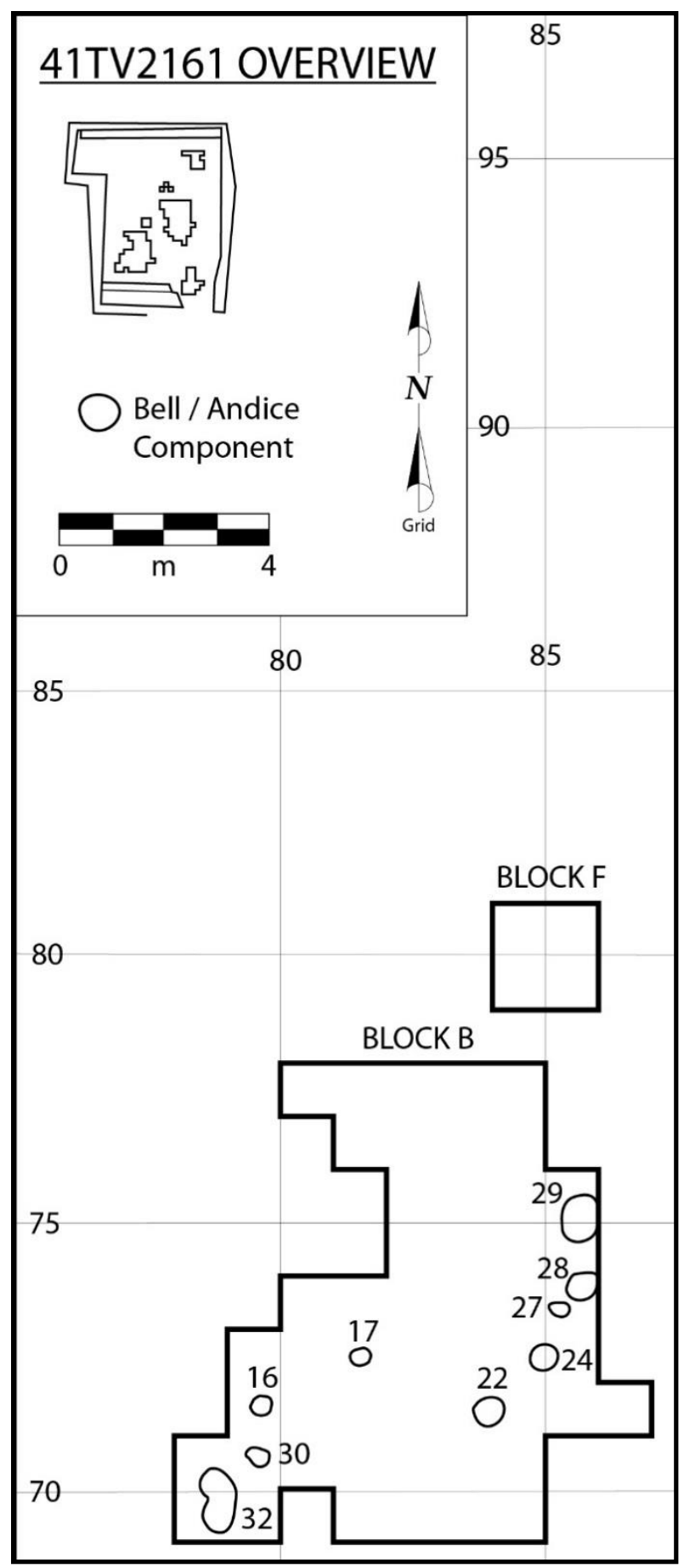

Figure 9-4. Plan view drawing of Bell/Andice feature locations across Block B.

yielded low fat plant and borderline medium and moderate-high fat content also indicative of possible plant and animal combinations (Appendix $\mathrm{G).}$

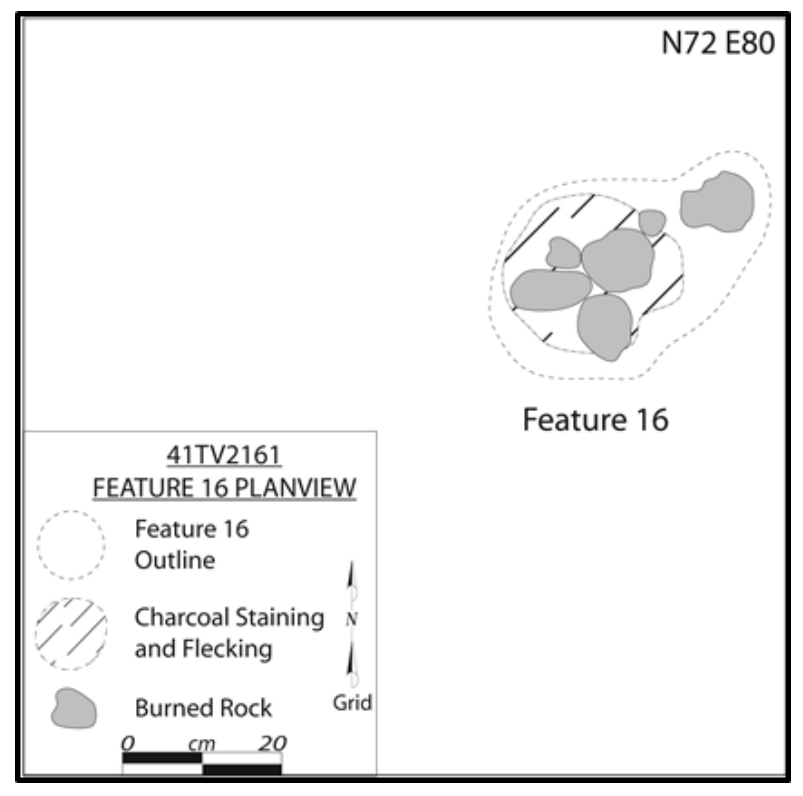

Figure 9-5. Plan view drawing of burned rock Feature 16.

The presence of plant and animal residue on the burned rocks, the broken nature and hackled edges, combined with the gelatinized starch grain, indicated the rocks were probably employed in a boiling process to cook plant and animal products. Feature 16 was interpreted as the remains of small in situ fire employed to reheat rocks previously employed in a cooking facility, probably a stone boiling process.

\subsubsection{Feature 17}

Feature 17 was recorded between 233 and $239 \mathrm{cmbs}$ at N73/E82 in Block B (see Figure 9-4). It consisted of a tight concentration of four rocks, including one flat piece that represents a metate fragment (\#2164-12) and three rounded pieces, with at least two probable manos (\#2164-10 and \#2164-11), within a roughly 20 to $24 \mathrm{~cm}$ diameter area (Figure 9-6). These clustered ground stones were in a brown $(7.5 \mathrm{YR} 4 / 4)$ silty clay loam with no visible sign of dark colored or stained sediments. The surrounding level yielded a single mussel shell fragment $40 \mathrm{~cm}$ east at $226 \mathrm{cmbs}$, with at least two chert flakes and ten faunal bone fragments that 
Table 9-2. Burned Rock Quantities and Weights from Bell/Andice Features.

\begin{tabular}{|c|c|c|c|c|c|c|c|c|c|c|c|c|c|}
\hline \multirow[t]{2}{*}{$\begin{array}{l}\text { Feature } \\
\text { No. }\end{array}$} & \multirow[t]{2}{*}{ Unit } & \multirow[t]{2}{*}{$\begin{array}{c}\text { Unit } \\
\text { Depth } \\
\text { (cmbs) }\end{array}$} & \multirow[t]{2}{*}{ PNUM } & \multicolumn{2}{|c|}{$\begin{array}{c}\text { Burned } \\
\text { Rocks 0-4 } \\
\text { cm }\end{array}$} & \multicolumn{2}{|c|}{$\begin{array}{c}\text { Burned } \\
\text { Rocks 4.1-9 } \\
\text { cm }\end{array}$} & \multicolumn{2}{|c|}{$\begin{array}{c}\text { Burned } \\
\text { Rocks } 9.1-15 \\
\text { cm }\end{array}$} & \multicolumn{2}{|c|}{$\begin{array}{c}\text { Burned } \\
\text { Rocks }>15 \\
\text { cm }\end{array}$} & \multicolumn{2}{|c|}{$\begin{array}{l}\text { Total Burned } \\
\text { Rocks }\end{array}$} \\
\hline & & & & Qty. & $\begin{array}{l}\text { Wt. } \\
\text { (g) }\end{array}$ & Qty. & $\begin{array}{l}\text { Wt. } \\
\text { (g) }\end{array}$ & Qty. & $\begin{array}{l}\text { Wt. } \\
\text { (g) }\end{array}$ & Qty. & $\begin{array}{l}\text { Wt. } \\
\text { (g) }\end{array}$ & Qty. & Wt. (g) \\
\hline 16 & $\begin{array}{l}\text { N72 } \\
\text { E80 }\end{array}$ & $\begin{array}{l}240- \\
250\end{array}$ & 2122 & 1 & 8 & 9 & 1758 & & & & & 10 & 1766 \\
\hline 22 & $\begin{array}{l}\text { N72 } \\
\text { E84 }\end{array}$ & $\begin{array}{l}216- \\
230\end{array}$ & 2146 & 5 & 75 & 6 & 1050 & & & & & 11 & 1125 \\
\hline 22 & $\begin{array}{l}\text { N72 } \\
\text { E85 }\end{array}$ & $\begin{array}{l}211- \\
230\end{array}$ & 2149 & 1 & 2 & 1 & 145 & & & & & 2 & 147 \\
\hline 24 & $\begin{array}{l}\text { N73 } \\
\text { E85 }\end{array}$ & $\begin{array}{l}218- \\
230\end{array}$ & 2176 & 4 & 300 & 13 & 3000 & 5 & 3000 & 2 & 2750 & 24 & 9050 \\
\hline 24 & $\begin{array}{l}\text { N73 } \\
\text { E86 }\end{array}$ & $\begin{array}{l}223- \\
230\end{array}$ & 2179 & 7 & 1000 & 7 & 1300 & 3 & 1750 & & & 17 & 4050 \\
\hline 27 & $\begin{array}{l}\text { N74 } \\
\text { E86 }\end{array}$ & $\begin{array}{l}212- \\
216\end{array}$ & 2197 & & & & & 4 & 24100 & & & 4 & 24100 \\
\hline 28 & $\begin{array}{l}\text { N74 } \\
\text { E86 }\end{array}$ & $\begin{array}{l}218- \\
231\end{array}$ & 2200 & 3 & 20 & 8 & 656 & & & & & 11 & 676 \\
\hline 29 & $\begin{array}{l}\text { N76 } \\
\text { E86 }\end{array}$ & $\begin{array}{l}220- \\
230\end{array}$ & 2223 & 22 & 486 & 61 & 10797 & 9 & 3920 & 0 & 0 & 92 & 15203 \\
\hline 30 & $\begin{array}{l}\text { N71 } \\
\text { E80 }\end{array}$ & $\begin{array}{l}230- \\
240\end{array}$ & 2092 & & & 6 & 1303 & & & & & 6 & 1303 \\
\hline 32 & $\begin{array}{l}\text { N70 } \\
\text { E79 }\end{array}$ & $\begin{array}{l}220- \\
230\end{array}$ & 2074 & & & 3 & 448 & 2 & 611 & & & 5 & 1059 \\
\hline 32 & $\begin{array}{l}\text { N70 } \\
\text { E80 }\end{array}$ & $\begin{array}{l}229- \\
236\end{array}$ & 2076 & 1 & 0 & 3 & 611 & & & & & 4 & 611 \\
\hline 32 & & $\begin{array}{l}230- \\
240\end{array}$ & 2078 & & & 5 & 996 & & & & & 5 & 996 \\
\hline 32 & $\begin{array}{l}\text { N71 } \\
\text { E79 }\end{array}$ & $\begin{array}{l}215- \\
220\end{array}$ & 2087 & & & 14 & 3505 & 3 & 1350 & & & 17 & 4854 \\
\hline 32 & $\begin{array}{l}\text { N71 } \\
\text { E80 }\end{array}$ & $\begin{array}{l}230- \\
240\end{array}$ & 2093 & 4 & 60 & 2 & 350 & & & & & 6 & 410 \\
\hline
\end{tabular}

Note: Feature 3 excavated by Hicks \& Company not included.

weighed $43 \mathrm{~g}$. The latter include fragments of a deer-size rib and pelvis and a rabbit-size tooth. This cluster was distinct and set apart from other features in Block B dominated by burned rocks.

The metate (\#2164-12) associated with Feature 17 is a thin (ca $25 \mathrm{~mm}$ ) flat sandstone slab that had many cracks and, subsequently, broke into multiple pieces when bagged. One edge is thicker than the middle section, creating the appearance of a worn central area of a grinding slab (Figure 9-7). This sandstone piece was covered in calcium carbonate, making it difficult to see wear and determine if it once functioned as a metate. The extensive cracking was reminiscence of burned rock and may have functioned as both, a heating rock and recycled metate. A $12 \mathrm{~cm}$ long sample was sent for starch grain analysis, however no starches were recovered (Appendix F).

An 89 mm long complete mano (\#2164-10) consists of hard sandstone partially covered in calcium carbonate (Figure 9-8). One surface is relatively flat, whereas the other unused surface is strongly 


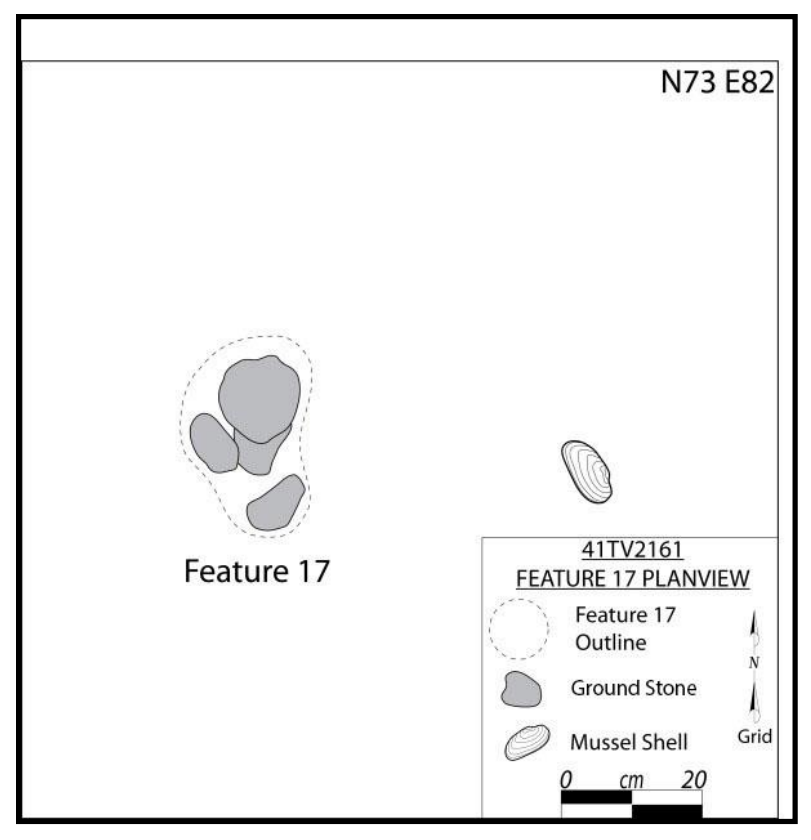

\section{Figure 9-6. Drawing of tightly clustered ground stone cache in Feature 17.}

convex. Heat alteration is not visible; therefore, this mano was probably employed for grinding only. The entire piece was sent for starch grain analysis and a single, damaged unknown grain was recovered (Appendix F).

A larger, $123 \mathrm{~cm}$ long by $64 \mathrm{~cm}$ thick complete mano (\#2164-11) exhibited one well-defined worked face opposite of an unworked convex face (Figure 9-9). The worked face is slightly convex and smoothed, with very thin and shallow striations across the surface. This face was originally covered in calcium carbonate. The complete mano was sent for pollen wash and the calcium carbonate was removed from the worked face. Borzarth determined this mano had very poor pollen preservation (Appendix L).

Although nearly absent of microfossils, one damaged starch grain indicates Mano \#2164-10 functioned as a grinding instrument. These clustered ground stones represent cached items, probably within or near a food processing area, situated away from the actual cooking and heating features.

\subsubsection{Feature 22}

Feature 22 was a tight cluster of thirteen small and medium-sized burned rocks, a burned mussel shell fragment, an edge-modified tool, and two chert flakes, all between 220 and $230 \mathrm{cmbs}$. These items were along the north-south line between N72/E84 and N72/E85 in Block B (Figure 9-10). Thirteen burned rocks weighed $1,272 \mathrm{~g}$, with six in the 0 to $4 \mathrm{~cm}$ size class and seven in the 4.1 to $9 \mathrm{~cm}$ size class (see Table 9-2). The diverse artifacts were in an oval area, roughly 35 to $40 \mathrm{~cm}$ in diameter. No visible dark stained sediment was under the rocks, as the soils were the same brown (7.5YR 4/4) silt loam as the rest of the level.

No burned rocks were submitted for technical analyses. Two sediment samples from below the rocks, one from N72/E84 (\#2146-4) and one from N72/E85 (\#2149-4) were sent for phytolith analysis. Sample \#2146-4 contained 14 tiny bone fragments, eight of which were burned, in addition to, a few tree phytoliths. No short cell phytoliths were present within the sample. Sample \#2149-4 yielded poor phytolith preservation, although five complete sponge spicules (Figure 9-10) were present (Appendix E).

Two other sediment samples (\#2146-4-g and \#2149-4-g) collected below the rocks in each unit were mechanically floated, which yielded minimal results, with only 4 flakes and 97 bone fragments less than $6.4 \mathrm{~mm}$ (Table 9-3). Included in the latter are two burned rabbit size phalanges, one calcined caudal vertebra, and five unburned rodent teeth less than $6.4 \mathrm{~mm}$.

Unit N72/E84 contained numerous cultural items that include a large, thin heat spalled biface (\#214610 ), at least six chert flakes, and scattered burned rock fragments. A flake (\#2145-10) from the unit was sent for starch grain analysis, but no starches were recovered (Appendix F). Much of N72/E85 was disturbed at this level from mechanical stripping, but the western and northern edges 
remained intact. The large, thin, heat exploited biface fragment was $60 \mathrm{~cm}$ southwest of this artifact cluster adjacent to Feature 22, with a Bell projectile point roughly $1 \mathrm{~m}$ west in N72/E83. Sediment samples from the clusters in both units were collected.

The cluster of relatively small burned rocks, along with bone fragments less than $6.4 \mathrm{~mm}$, probably represent a dump of cooking rocks, and from their appearance, they potentially were from a boiling activity or clean out of a small hearth area. The scattered cultural materials that surrounded this small feature also appear as discarded items.

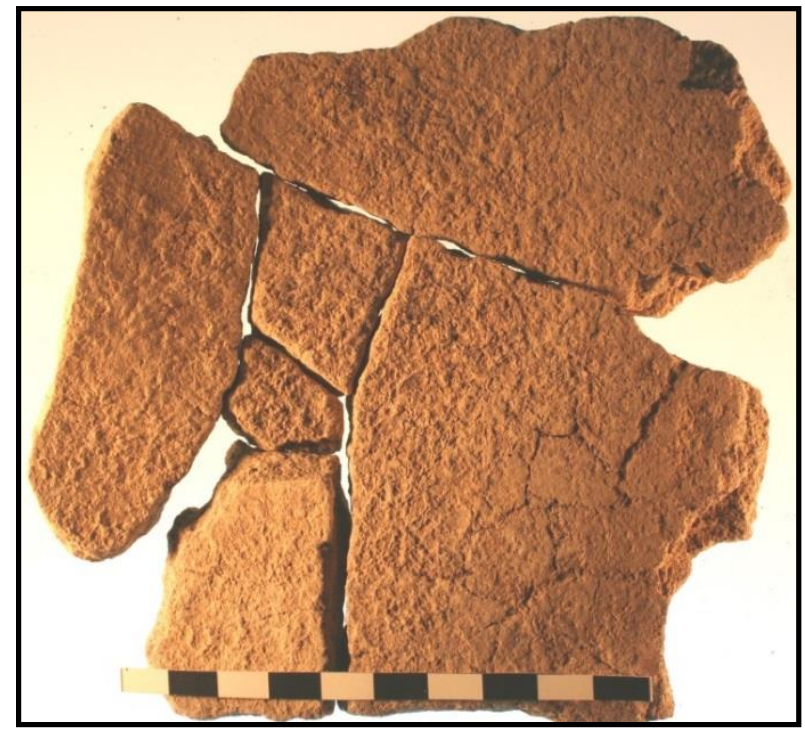

Figure 9-7. Photograph of tapered sandstone pieces covered in calcium carbonate, possible metate fragment (\#2164-12). Scale in centimeters.

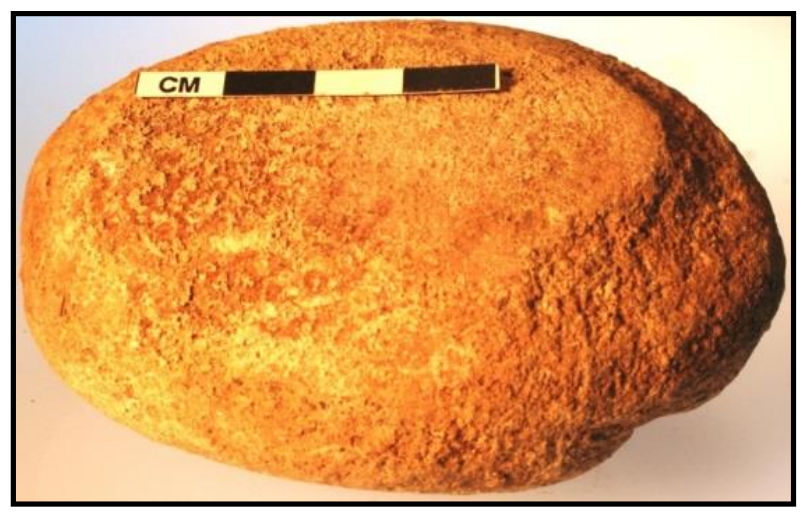

Figure 9-8. Photograph of complete mano (\#2164-10) from Feature 17.

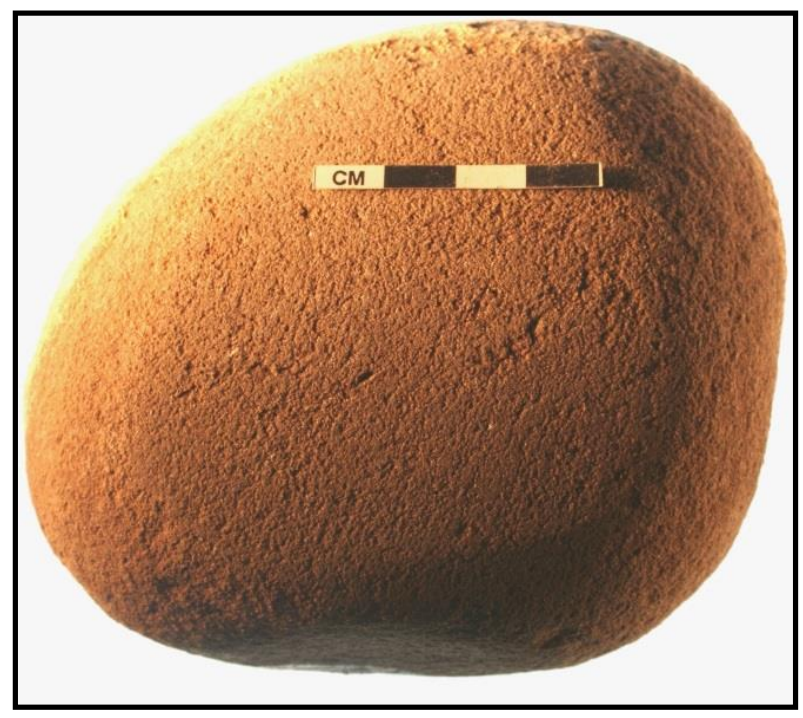

Figure 9-9. Photograph of complete mano (\#2164-11) from Feature 17.

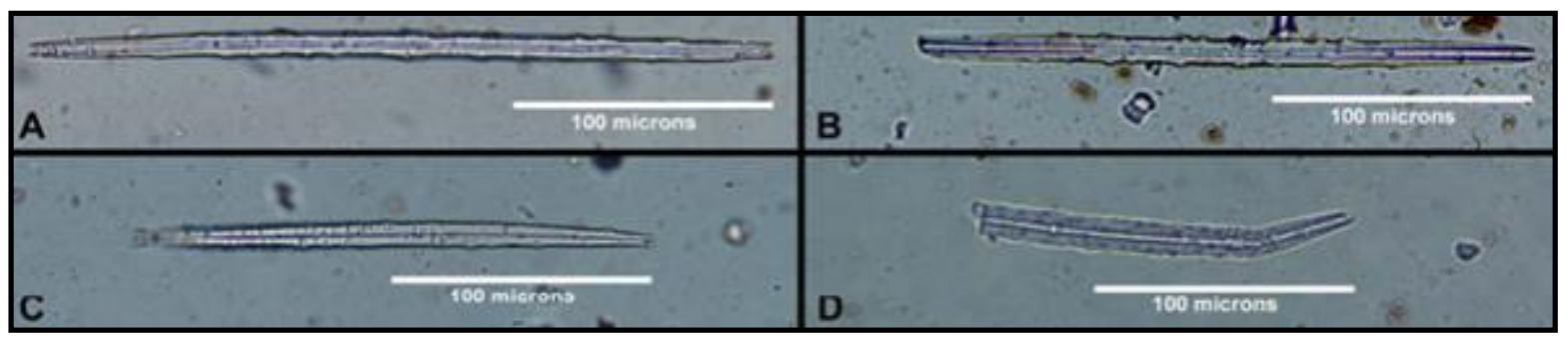

Figure 9-10. Examples of spined freshwater sponge spicules (Appendix D). 


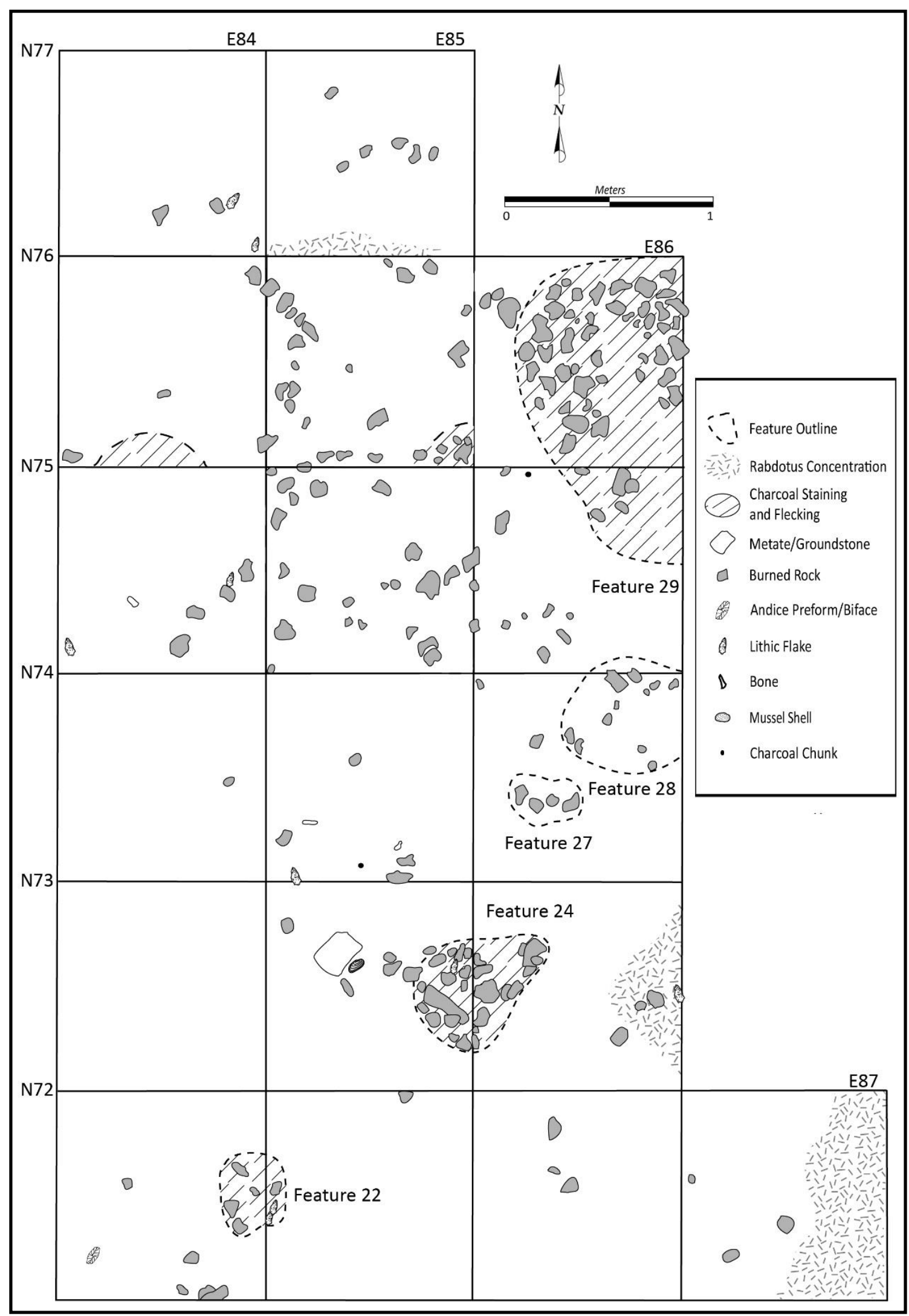

Figure 9-11. Plan view drawing of Features 22, 24, 28, and 29, Block B. 
Table 9-3. Flotation Results with Counts and Weights by Material Class for Bell/Andice Component.

\begin{tabular}{|c|c|c|c|c|c|c|c|c|c|c|c|c|c|c|c|c|c|c|c|c|c|c|}
\hline \multirow{2}{*}{ 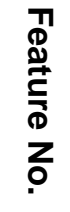 } & \multirow{2}{*}{$\begin{array}{l}\stackrel{8}{\oiint} \\
z \\
0\end{array}$} & \multirow{2}{*}{$\stackrel{c}{S}$} & \multirow{2}{*}{ 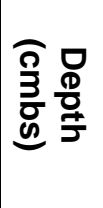 } & \multirow{2}{*}{$\begin{array}{l}\frac{\boldsymbol{w}}{\mathbf{0}} \\
\frac{\AA}{x}\end{array}$} & \multirow{2}{*}{ 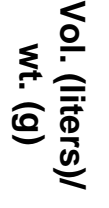 } & & \multicolumn{2}{|c|}{$\begin{array}{c}\text { Mussel } \\
\text { Shell }\end{array}$} & \multicolumn{2}{|c|}{$\begin{array}{c}\text { Burned } \\
\text { Rock }\end{array}$} & \multicolumn{2}{|c|}{ Debitage } & \multicolumn{2}{|c|}{ Charcoal } & \multicolumn{2}{|c|}{ Snail } & \multicolumn{2}{|c|}{$\begin{array}{c}\text { Macro- } \\
\text { botanical }\end{array}$} & \multicolumn{2}{|c|}{$\begin{array}{c}\text { Burned } \\
\text { Clay }\end{array}$} & \multicolumn{2}{|c|}{ Bone } \\
\hline & & & & & & & $1 / 4$ & $<1 / 4$ & $1 / 4$ & $<1 / 4$ & $1 / 4$ & $<1 / 4$ & $1 / 4$ & $<1 / 4$ & $1 / 4$ & $<1 / 4$ & $1 / 4$ & $<1 / 4$ & $1 / 4$ & $<1 / 4$ & $1 / 4$ & $<1 / 4$ \\
\hline \multirow{4}{*}{22} & $2146-4-g$ & $\begin{array}{l}\text { N72 } \\
\text { E84 }\end{array}$ & $\begin{array}{l}216- \\
230 \\
\end{array}$ & B & 1.8 & $\mathbf{N}$ & 0 & 0 & 0 & 0 & 0 & 0 & 0 & 1 & 0 & 2 & 0 & 0 & 0 & 0 & 0 & 56 \\
\hline & & & & & 5.5 & Wt. & 0.0 & 0.0 & 0.0 & 0.0 & 0.0 & 0.0 & 0.0 & 0.1 & 0.0 & 1.1 & 0.0 & 0.0 & 0.0 & 0.0 & 0.0 & 0.3 \\
\hline & $2149-4-g$ & $\begin{array}{l}\text { N72 } \\
\text { E85 }\end{array}$ & $\begin{array}{l}218- \\
230\end{array}$ & B & 2.0 & $\mathbf{N}$ & 0 & 0 & 0 & 0 & 0 & 2 & 0 & 3 & 0 & 3 & 0 & 5 & 0 & 0 & 0 & 41 \\
\hline & & & & & 1.3 & Wt. & 0.0 & 0.0 & 0.0 & 0.0 & 0.0 & 0.1 & 0.0 & 0.1 & 0.0 & 0.6 & 0.0 & 0.1 & 0.0 & 0.0 & 0.0 & 0.3 \\
\hline \multirow{4}{*}{24} & $2176-4-g$ & $\begin{array}{l}\text { N73 } \\
\text { E85 }\end{array}$ & $\begin{array}{l}218- \\
230\end{array}$ & B & 12.1 & $\mathbf{N}$ & 0 & 0 & 5 & 0 & 0 & 3 & 0 & 6 & 0 & 38 & 0 & 0 & 3 & 16 & 0 & 152 \\
\hline & & & & & 140.9 & Wt. & 0.0 & 0.0 & 98.3 & 0.0 & 0.0 & 0.1 & 0.0 & 0.1 & 0.0 & 5.9 & 0.0 & 0.0 & 8.2 & 1.5 & 0.0 & 1.7 \\
\hline & $2179-4-g$ & $\begin{array}{l}\text { N73 } \\
\text { E86 } \\
\end{array}$ & $\begin{array}{l}223- \\
230 \\
\end{array}$ & B & 7.4 & $\mathbf{N}$ & 0 & 0 & 3 & 12 & 0 & 4 & 0 & 14 & 0 & 23 & 0 & 0 & 3 & 0 & 0 & 78 \\
\hline & & & & & 39.6 & Wt. & 0.0 & 0.0 & 1.0 & 0.9 & 0.0 & 0.1 & 0.0 & 0.1 & 0.0 & 0.6 & 0.0 & 0.0 & 1.0 & 0.0 & 0.0 & 0.9 \\
\hline \multirow{2}{*}{27} & $2197-4-g$ & $\begin{array}{l}\text { N74 } \\
\text { E86 }\end{array}$ & $\begin{array}{l}215- \\
217\end{array}$ & B & 0.7 & $\mathbf{N}$ & 0 & 0 & 0 & 0 & 1 & 0 & 0 & 0 & 0 & 1 & 0 & 0 & 1 & 0 & 0 & 4 \\
\hline & & & & & 8.2 & Wt. & 0.0 & 0.0 & 0.0 & 0.0 & 0.4 & 0.0 & 0.0 & 0.0 & 0.0 & 0.1 & 0.0 & 0.0 & 0.5 & 0.0 & 0.0 & 0.1 \\
\hline \multirow{2}{*}{28} & $2200-4-g$ & $\begin{array}{c}\text { N74/75 } \\
\text { E86 }\end{array}$ & $\begin{array}{l}218- \\
231\end{array}$ & B & 5.0 & $\mathbf{N}$ & 0 & 3 & 1 & 2 & 1 & 19 & 0 & 2 & 0 & 36 & 0 & 2 & 0 & 0 & 0 & 148 \\
\hline & & & & & 17.1 & Wt. & 0.0 & 0.1 & 2.1 & 0.3 & 0.3 & 0.2 & 0.0 & 0.1 & 0.0 & 2.2 & 0.0 & 0.1 & 0.0 & 0.0 & 0.0 & 1.0 \\
\hline
\end{tabular}


Table 9-3. Flotation Results with Counts and Weights by Material Class for Bell/Andice Component (continued).

\begin{tabular}{|c|c|c|c|c|c|c|c|c|c|c|c|c|c|c|c|c|c|c|c|c|c|c|}
\hline \multirow{2}{*}{ 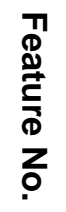 } & \multirow{2}{*}{$\begin{array}{l}\stackrel{8}{\dddot{z}} \\
\text { o }\end{array}$} & \multirow{2}{*}{ 돌 } & \multirow{2}{*}{ 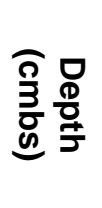 } & \multirow{2}{*}{$\begin{array}{l}\frac{m}{\circ} \\
\frac{\operatorname{c}}{x}\end{array}$} & \multirow{2}{*}{ 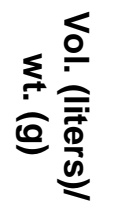 } & & \multicolumn{2}{|c|}{$\begin{array}{l}\text { Mussel } \\
\text { Shell }\end{array}$} & \multicolumn{2}{|c|}{$\begin{array}{l}\text { Burned } \\
\text { Rock }\end{array}$} & \multicolumn{2}{|c|}{ Debitage } & \multicolumn{2}{|c|}{ Charcoal } & \multicolumn{2}{|c|}{ Snail } & \multicolumn{2}{|c|}{$\begin{array}{c}\text { Macro- } \\
\text { botanical }\end{array}$} & \multicolumn{2}{|c|}{$\begin{array}{l}\text { Burned } \\
\text { Clay }\end{array}$} & \multicolumn{2}{|c|}{ Bone } \\
\hline & & & & & & & $1 / 4$ & $<1 / 4$ & $1 / 4$ & $<1 / 4$ & $1 / 4$ & $<1 / 4$ & $1 / 4$ & $<1 / 4$ & $1 / 4$ & $<1 / 4$ & $1 / 4$ & $<1 / 4$ & $1 / 4$ & $<1 / 4$ & $1 / 4$ & $<1 / 4$ \\
\hline \multirow[t]{4}{*}{29} & $2424-4-2 g$ & $\begin{array}{l}\text { N76 } \\
\text { E86 }\end{array}$ & $\begin{array}{l}230- \\
240\end{array}$ & B & 14.0 & $\mathbf{N}$ & 0 & 0 & 6 & 32 & 3 & 198 & 0 & 45 & 0 & 45 & 0 & 4 & 3 & 8 & 0 & 204 \\
\hline & & & & & 72.7 & Wt. & 0.0 & 0.0 & 10.5 & 1.7 & 1.2 & 3.0 & 0.0 & 0.1 & 0.0 & 8.4 & 0.0 & 0.1 & 1.5 & 0.4 & 0.0 & 1.3 \\
\hline & $2424-4-1 \mathrm{~g}$ & $\begin{array}{l}\text { N76 } \\
\text { E86 }\end{array}$ & $\begin{array}{l}230- \\
235\end{array}$ & B & 11.0 & $\mathbf{N}$ & 0 & 1 & 18 & 6 & 2 & 240 & 0 & 58 & 0 & 87 & 0 & 4 & 27 & 21 & 0 & 520 \\
\hline & & & & & 244.1 & Wt. & 0.0 & 0.1 & 80.1 & 0.6 & 1.6 & 4.7 & 0.0 & 0.1 & 0.0 & 13.7 & 0.0 & 0.1 & 63.4 & 2.2 & 0.0 & 4.1 \\
\hline \multirow[t]{2}{*}{30} & $2092-4-g$ & $\begin{array}{l}\text { N71 } \\
\text { E80 }\end{array}$ & $\begin{array}{l}235- \\
240\end{array}$ & B & 1.3 & $\mathbf{N}$ & 0 & 1 & 1 & 0 & 0 & 2 & 0 & 0 & 0 & 10 & 0 & 8 & 0 & 0 & 2 & 85 \\
\hline & & & & & 27.7 & Wt. & 0.0 & 0.7 & 23.3 & 0.0 & 0.0 & 0.1 & 0.0 & 0.0 & 0.0 & 0.5 & 0.0 & 0.1 & 0.0 & 0.0 & 0.4 & 0.3 \\
\hline \multirow[t]{4}{*}{32} & 2078-4-g & $\begin{array}{l}\text { N70 } \\
\text { E80 }\end{array}$ & $\begin{array}{l}230- \\
240\end{array}$ & B & 10.0 & $\mathbf{N}$ & 0 & 0 & 2 & 0 & 0 & 6 & 0 & 1 & 1 & 41 & 0 & 4 & 0 & 0 & 0 & 156 \\
\hline & & & & & 20.9 & Wt. & 0.0 & 0.0 & 5.6 & 0.0 & 0.0 & 0.1 & 0.0 & 0.1 & 0.1 & 3.8 & 0.0 & 0.1 & 0.0 & 0.0 & 0.0 & 1.1 \\
\hline & Tot & I Num & & & 67.1 & & 0 & 5 & 36 & 52 & 7 & 474 & 0 & 131 & 1 & 288 & 0 & 27 & 37 & 45 & 0 & $\begin{array}{c}144 \\
4\end{array}$ \\
\hline & Tota & Weigh & (g) & & 583.5 & & 0 & 0.9 & 221 & 3.5 & 3.5 & 8.2 & 0 & 0.9 & 0.1 & 38 & 0 & 0.6 & 75 & 4.1 & 0 & 11.1 \\
\hline
\end{tabular}




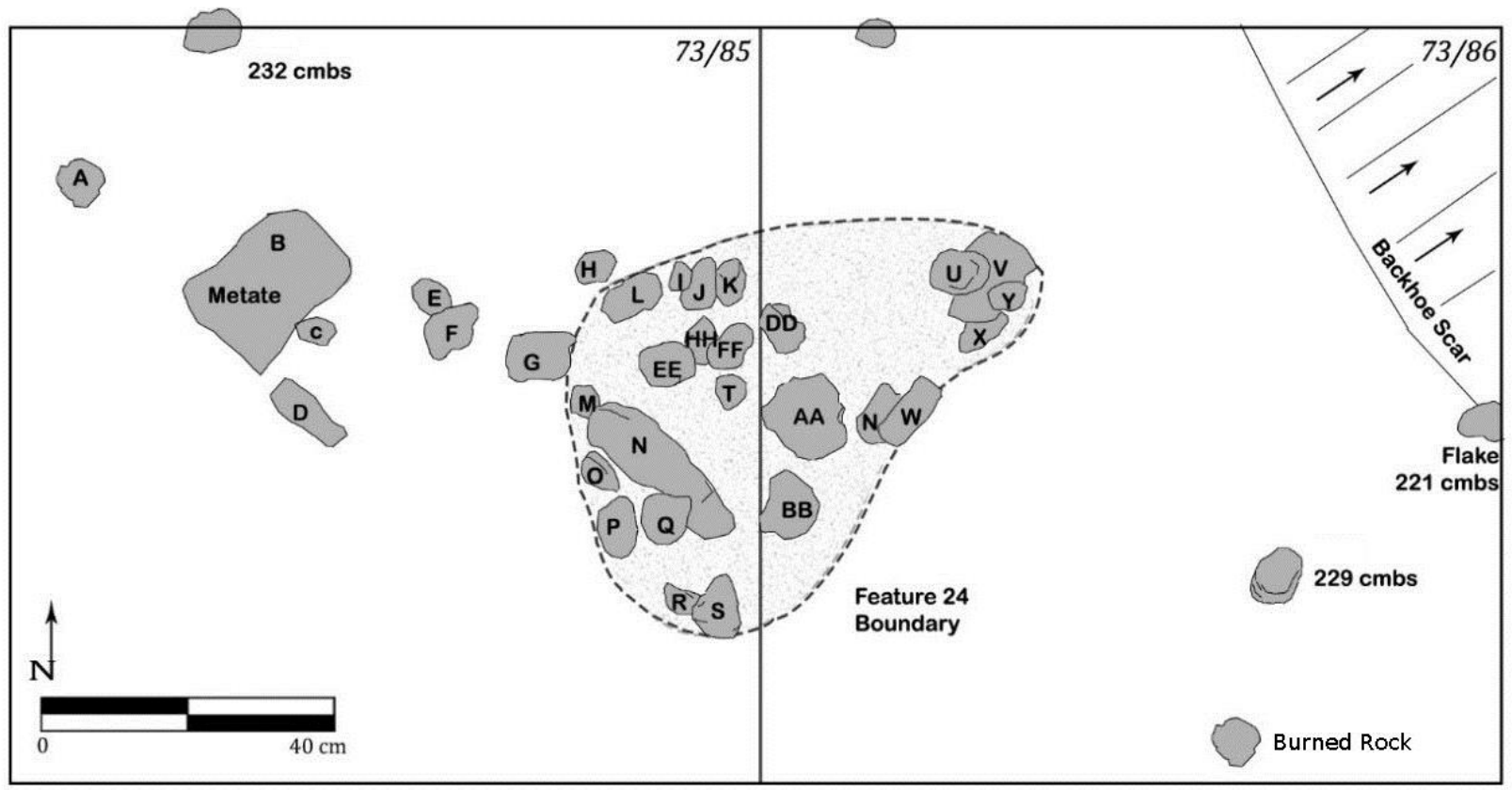

Figure 9-12. Plan view drawing of Feature 24 with broken metate just west.

\subsubsection{Feature 24}

Feature 24 was an oval shaped, burned rock cluster between 216 to $233 \mathrm{cmbs}$ within a 50-by-60 cm area. The feature was bisected by the north-south line between N73/E85 and N73/E86 in Block B (Figures 9-11 and 9-12). Feature 24 exhibited a tight cluster of 41 burned rocks over and surrounded by dark stained sediment (10YR 5/4 to 7.5YR 4/2). Some burned rocks were piled on top of one another, and some were complete. Sediment samples were collected below the burned rocks in each unit, and all rocks were also collected.

Four samples were radiocarbon dated, including two charcoal, one partially burned bone fragment, and a dark organic stained sediment sample from under the burned rocks (see Table 9-1). The bone and sediment results are older than the two charcoal results by at least 170 years. Given charcoal dates of $5280 \pm 30$ B.P. and $5370 \pm 30$ B.P. are generally considered more precise, the average of those two dates at $5325 \pm 30$ is considered acceptable as the true age of this feature.
The 41 burned rocks weighed 13,100 g and were multiple sizes (see Table 9-2). At least eight were complete, water rounded cobbles, six pieces refit into three rocks, four exhibited hackled edges, and multiple pieces had smears or dark organic stains on parts of their surface. Lipid analysis was attempted on five pieces of burned rocks. Three rocks (\#2176-3-1b, \#2179-3-1b, and \#2176-3-4) had no interpretable residues and two rocks (\#21763-3b, and \#2176-3-2) exhibited similar low fat plant and moderate-high fat content, indicating possible plant and animal residue combinations, with one dominated by plant (Appendix G). Two had biomarker dehydroabietic acid present, indicative of conifer products, like juniper (Juniperus) or bald cypress (Taxodium). Five rock fragments were subjected to starch grain analysis with only one not yielding any starches. The four rocks yielded two gelatinized grains, two unknown grains, one true lily (Lillium sp.) grain (Figure 9-13), and one eroded grain (Appendix F).

Sediment sample \#2179-4 was submitted to Dering for floatation, which resulted in no identifiable 


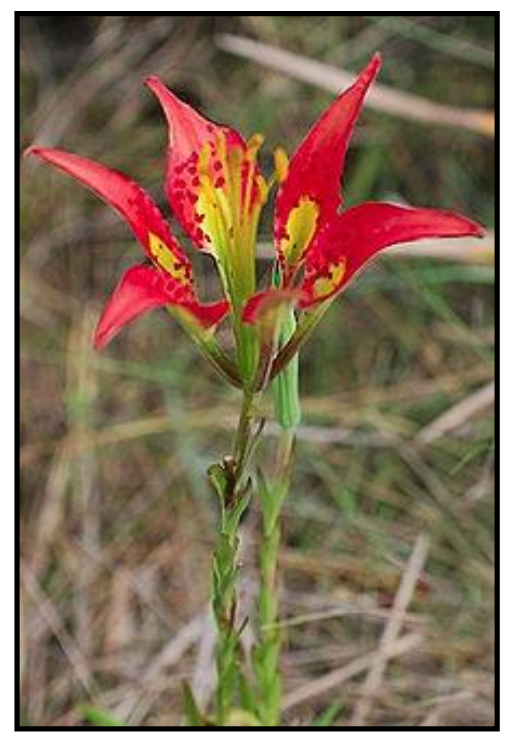

\section{Figure 9-13. Example of true lily (Catesby's lily or pine lily, Lilium catesbaei) flower.}

charred remains (Appendix B). Two other samples were mechanically floated and yielded 230 bone fragments, 20 flecks of carbonized matter, and 7 flakes less than $6.4 \mathrm{~mm}$ (see Table 9-3; Figure 9$14)$.

Identifiable bones from sediment sample \#2176 minimally include a burned third phalanx, burned rabbit-size feet and teeth, $3 \mathrm{~mm}$ burned fish vertebrae, and burned fossil oyster shell fragments.

Other cultural materials, such as burned rocks, flakes, a metate fragment, animal bone fragments, and Rabdotus snail shells were in the same vertical zone in brown (7.5YR 4/4), silty clay loam outside Feature 24. Mechanical stripping removed the northeastern corner of N73/E86, and only a couple of scattered burned rocks, 4 bone fragments, and 12 flakes were in the same level. The bone fragments represent deer and rabbit-size animals, with two deer size pieces burned to a mottled black and brown color. Unit N73/E85 also yielded a few scattered burned rocks, a mussel shell next to a metate fragment (\#2175-13), three heat spalled, crescent shaped fragments from the same early stage biface (\#2175-10, \#2175-11, and \#2175-12), and five flakes.
This dense cluster of burned rocks combined with dark stained sediments under the rocks indicates an in situ heating and/or cooking feature. The fact that many of the burned rocks were still complete indicates this group had not been subjected to many heating and cooling episodes or they would be broken (e.g., Jackson 1998; Leach et al. 1998, 2001; Stark 1997). This supports a short-term use episode for the rocks and feature. Lipid residues indicate plant and animal products were cooked. This is supported by the presence of lily starch grain and bone fragments. Gelatinized starch grains support cooking occurred in association with heat and water. Also, processing plant matter and seeds is supported by a damaged starch grain.

\subsubsection{Feature 27}

Feature 27 consisted of a narrow 15-by-40 cm linear row of four burned rocks at $212 \mathrm{cmbs}$ near the center of N74/E86 in Block B (see Figure 9-4). The rocks were roughly $10+\mathrm{cm}$ in diameter and weighed 24,100 $\mathrm{g}$ (see Table 9-2). These burned rocks were surrounded by a light yellowish brown (10YR 6/4) silty clay loam that exhibited no obvious sign of charcoal staining. When crosssectioned, no basin or dark stained sediment was discerned below the rocks (Figure 9-15). An edgemodified flake was next to the four burned rocks.

Two burned rocks were submitted for lipid residue analysis. Rock \#2197-3-1 yielded low fat plant and high fat content that reflects possible plant and animal residue combination. Rock \#2197-3-2 was only slightly different, with residue combinations indicative of low fat plant and moderate-high fat content, also indicative of plant and animal residues (Appendix G).

A small sediment sample was collected below the rocks and was subsequently sent for phytolith analysis. The sand fraction of phytolith processing yielded three microscopic bone fragments, two of which were burned, and only a few short cell phytoliths and no sponge spicules (Appendix E). A 
second 0.7 liter sediment sample (\#2197-4-g) was mechanically floated, which yielded one flake, a small burned clay lump and four bone fragments less than $6.4 \mathrm{~mm}$ (see Table 9-3).

This same unit yielded four flakes, a chert core and two animal bone fragments. The southwestern edge of Feature 28 was in the northeastern corner. The bones include one black and gray burned, deer-size long bone fragment and a jackrabbit calcaneus.
One flake (\#2197-01) was sent for starch grain analysis but no starches were detected (Appendix F).

This narrow row of four medium sized burned rocks that lacked dark stained sediment is thought to represent a dump from adjacent Features 28 and/or 29 , and were potentially rocks targeted to be reused in one of those two features, as they were all relatively large and could function for heat retention purposes.

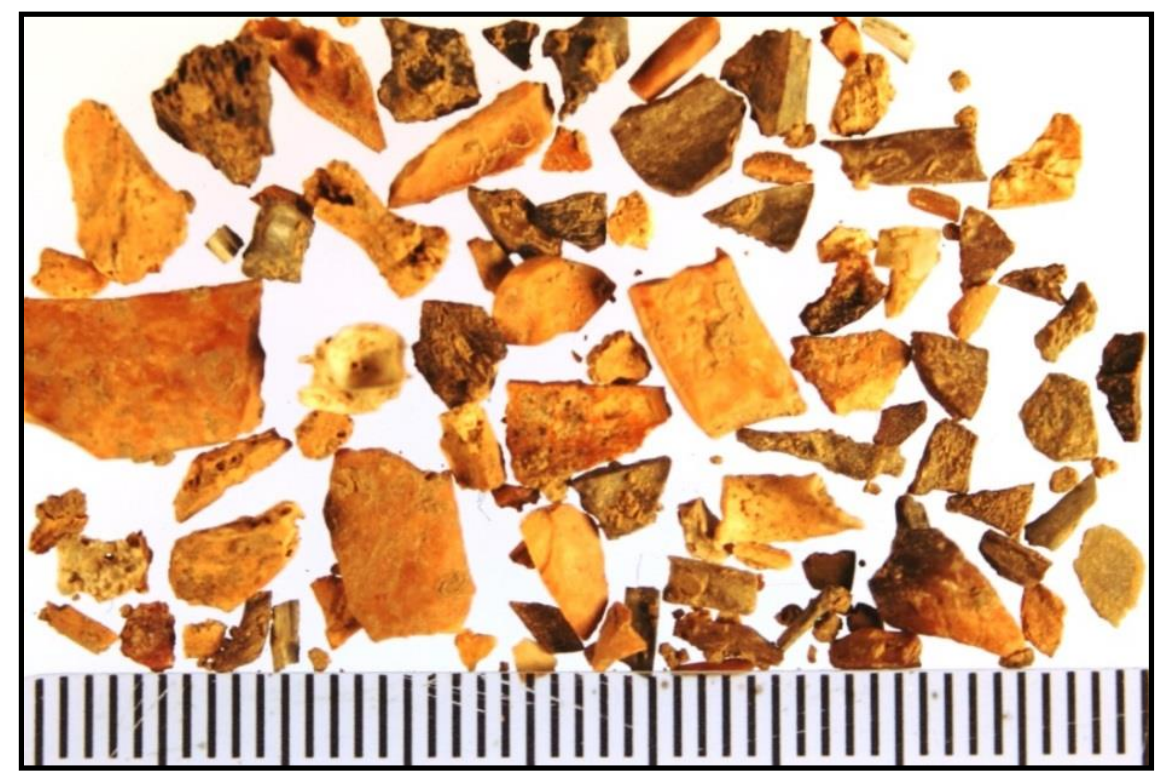

Figure 9-14. Burned and unburned faunal remains (\#2179-4-g) in heavy fraction from Feature 24 (scale in millimeters).

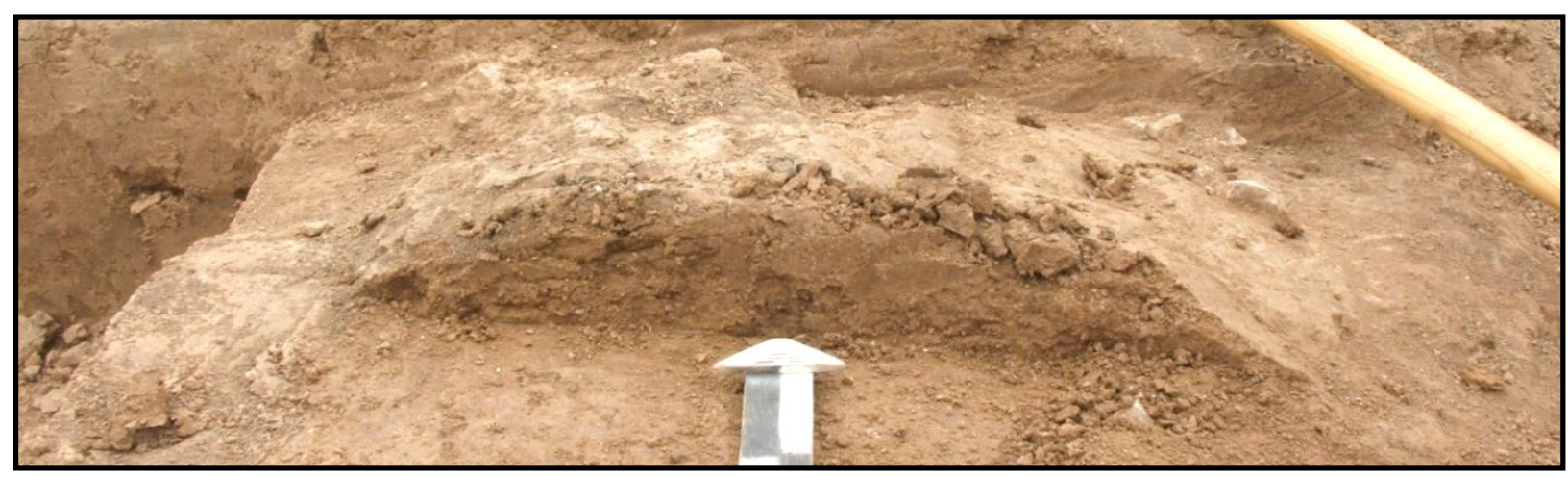

Figure 9-15. Photograph of profile under Feature 27 burned rocks (north arrow is 10 centimeters). 


\subsubsection{Feature 28}

Feature 28 consisted of 11 concentrated burned rocks in a small area, between 218 and $231 \mathrm{cmbs}$, in the northeastern corner of N74/E86, that extended $10 \mathrm{~cm}$ into N75/E86 (see Figures 9-4). The burned rocks were approximately $15 \mathrm{~cm}$ northeast of Feature 27 and $50 \mathrm{~cm}$ south of Feature 29. The amorphous concentration measured 40 to $45 \mathrm{~cm}$ in diameter, with no pattering and no visible basin or dark stained sediment (Figure 9-16). The 11 burned rocks varied in size from 5 to $20 \mathrm{~cm}$ and revealed no obvious arrangement. The 11 burned rocks weighed $676 \mathrm{~g}$, for an average weight of $61 \mathrm{~g}$ per rock (see Table 9-2). A bulk sediment sample (\#2200-4) was collected below the rocks in N74/E86.

A tiny charcoal sample from around the rocks yielded a date of $5280 \pm 30$ B.P. (see Table 9-1). This is an acceptable date for this feature.
Parts of three burned rocks were sent for lipid residues analysis. Two rocks (\#2200-3-1b and \#2200-3-3) yielded low fat plant and medium fat content indicating a possible plant and animal combination, with one dominated by animal residues. The third sample did not yield sufficient residues for interpretation (Appendix G). Chemical biomarker dehydroabietic acid present on at least one rock indicates conifer products present. For this regional area, probably juniper or bald cypress was employed to heat the rocks. An ambiguous mano fragment (\#2200-10), which was also a broken burned rock, was sent for starch grain analysis. No starch grains were recovered (Appendix F).

Sediment sample \#2200-4 was sent for phytolith analysis. Five microscopic bone fragments, one of which was burned, were observed in the sand fraction. Very limited short cell, a few tree phytoliths, and no sponge spicules were recovered

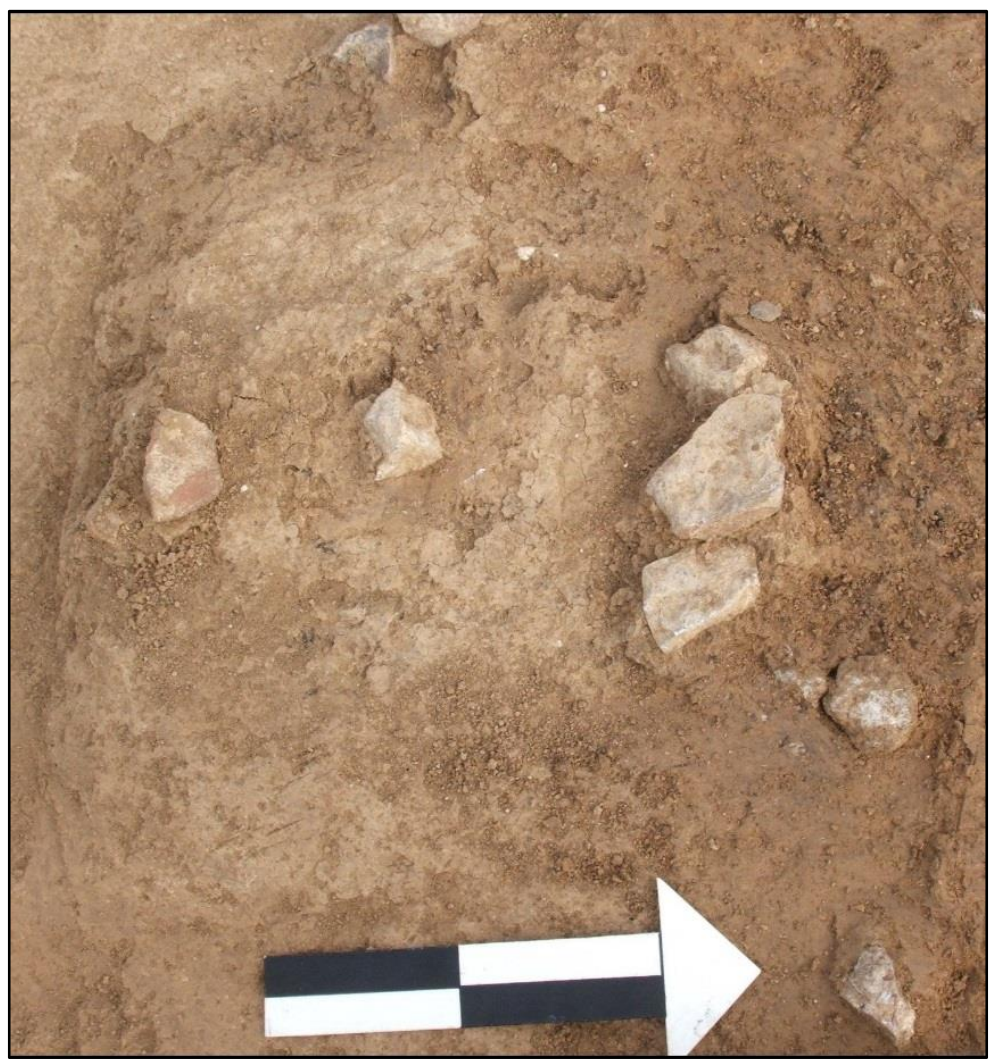

Figure 9-16. Overview photograph of burned rocks in Feature 28 in the northeastern corner of N74/E86 (north arrow is 20 centimeters). 


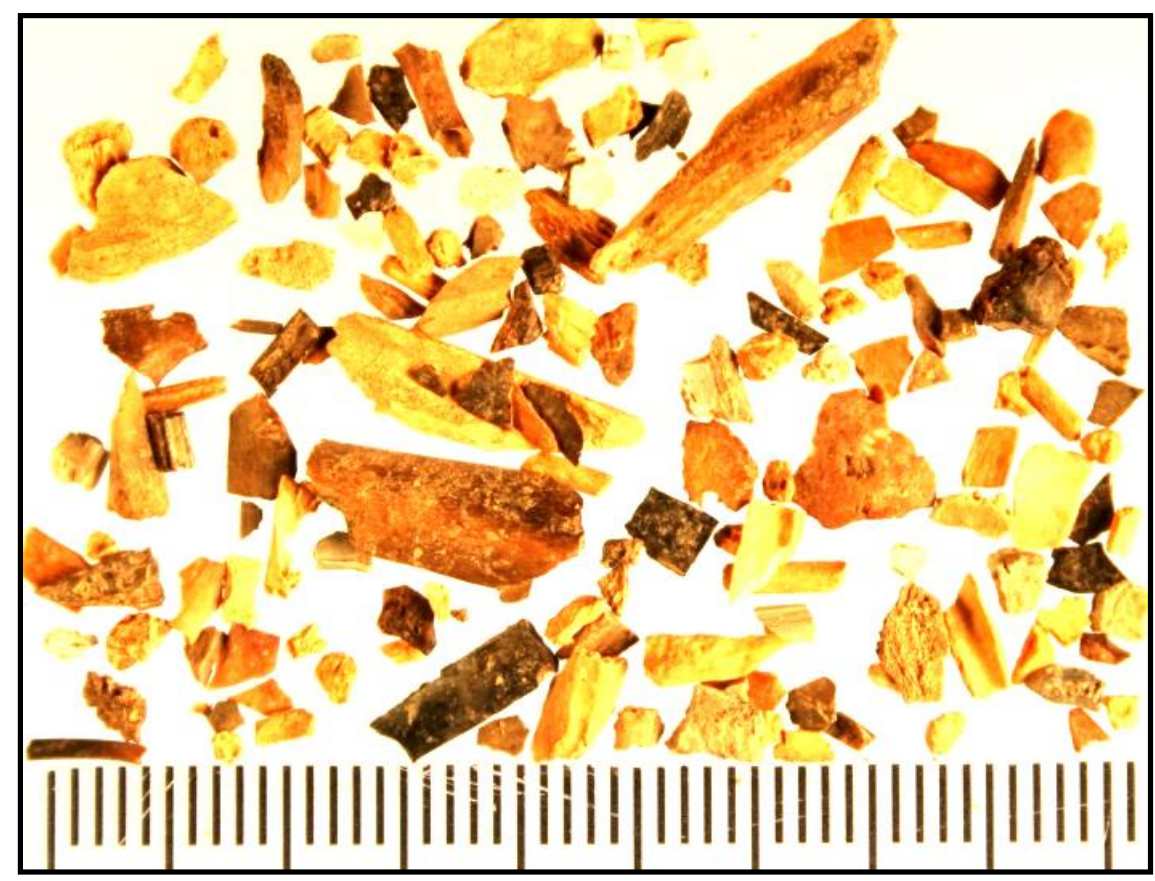

Figure 9-17. Example of faunal fragments (\#2200-4-g) in heavy fraction from Feature 28 (scale in millimeters).

(Appendix E). A second sediment sample (\#22004b) was also submitted for diatom analysis, but no diatoms were recovered (Appendix C).

A 5.0 liter sediment sample (\#2200-4) was mechanically floated and yielded 20 pieces of lithic debitage, a few mussel shell fragments, burned rock fragments, snail shell fragments, and 148 bone fragments all less than $6.4 \mathrm{~mm}$ (see Table 9-3; Figure 9-17). The latter include for the most part unburned small mammal and rodent bones and teeth, with 19 pieces burned and two with spiral fractures.

These clustered burned rocks served once for cooking plant and animal products, but their presence at this location is unclear. Their general small size and random pattern indicate they were discarded rocks, probably from adjacent Feature 29. The absence of dark stained sediment adjacent to or below the rocks support a dump of used rocks, rather than an in situ heating facility.

\subsubsection{Feature 29}

Feature 29 was a very well-defined rock filled, dark stained hearth area between 230 to $242 \mathrm{cmbs}$ along the western edge of the mechanically stripped surface in N76/E86 and N75/E86 in Block B (see Figures 9-4, 9-18, and 9-19). Mechanical stripping encountered the tops of the rocks and stopped, whereas the eastern margin of Features 28 and 29 were stripped below this level. Consequently, a limited part of Feature 29 was potentially removed during stripping, although Feature 29 exhibits a mostly intact feature. Feature 29 measured 100-by$70 \mathrm{~cm}$ and exhibited a strong brown (7.5YR 4/6) clay loam with very frequent mottles of black (10YR 2/1), decomposed charcoal stains surrounding and below the rocks. A shallow saucer-shaped 8 to $9 \mathrm{~cm}$ deep basin was surrounded by a natural yellowish brown (10YR 5/4) clay loam with frequent mottles of dark yellowish brown (10YR 3/4) clay (Figure 9-20). Visible chunks of charcoal were not observed in the fill, only dark 


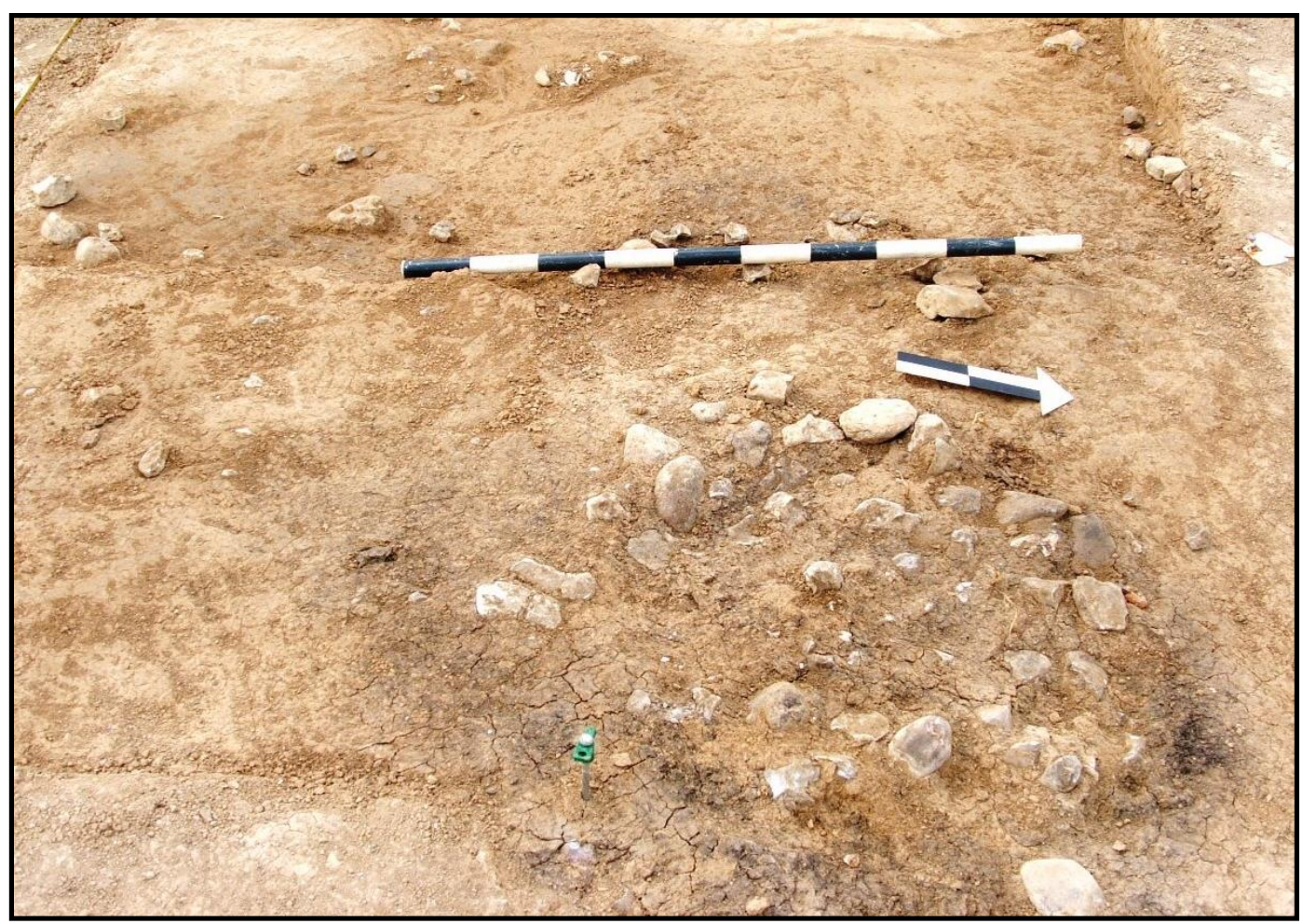

Figure 9-18. Oblique photograph of top of Feature 29 that shows mottled, dark stained sediment in and around a circular cluster of burned rocks surrounded by scattered burned rocks (scales in 10 centimeters increments).

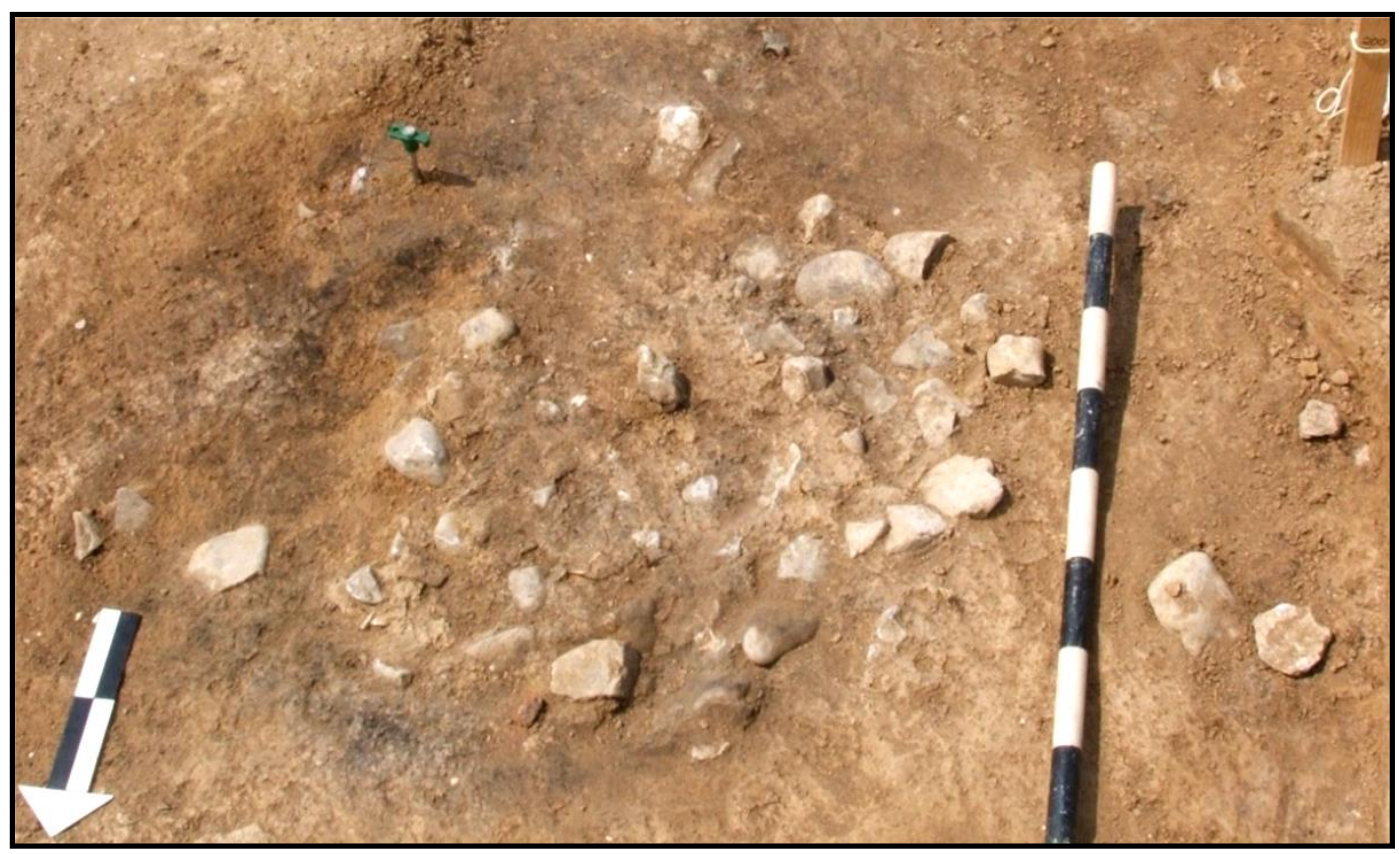

Figure 9-19. Oblique close-up of mottled, dark stained sediment in and around Feature 29 burned rocks (scales in 10 centimeters increments). 


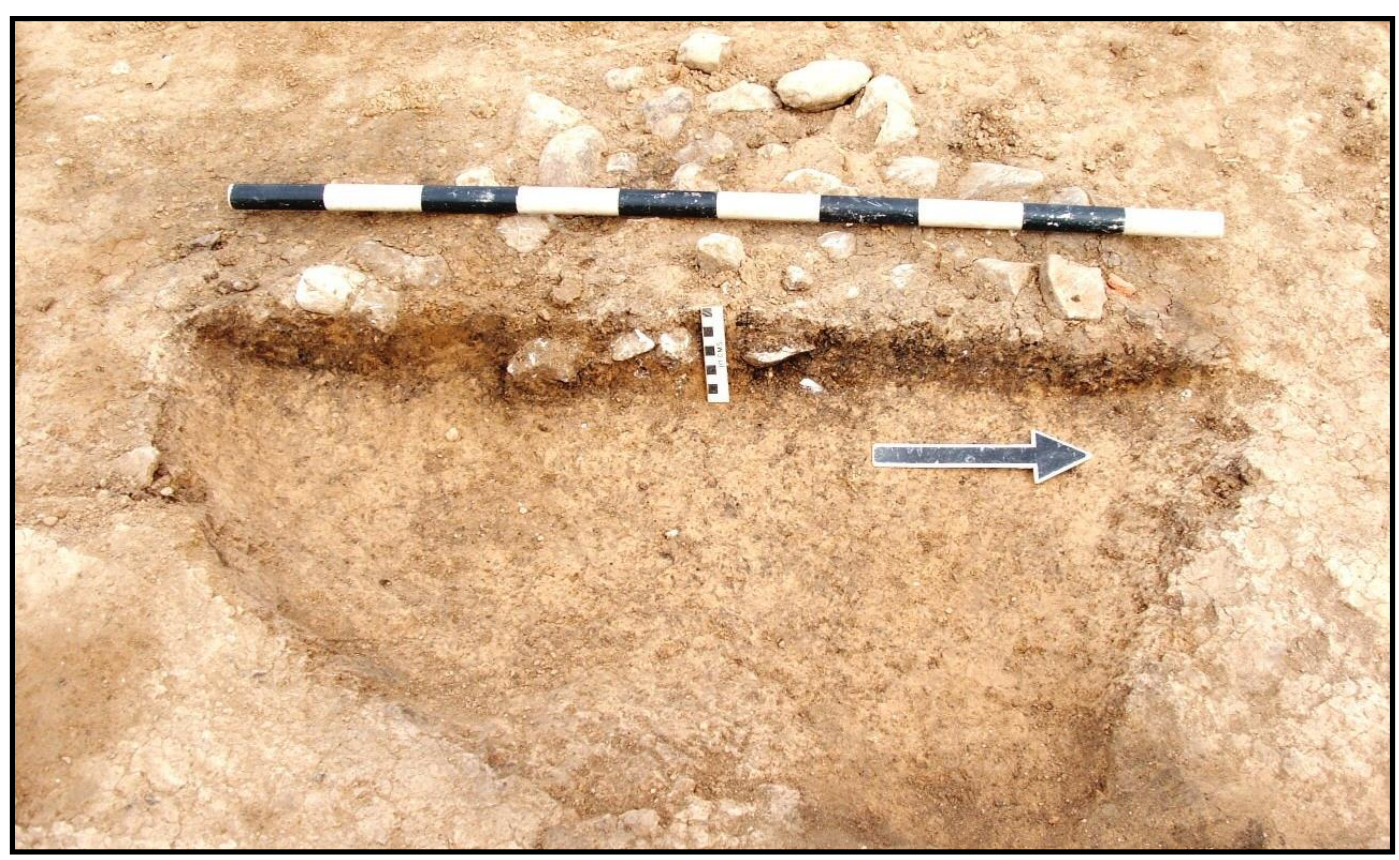

Figure 9-20. Oblique photograph of profile and excavated eastern half of shallow basin under Feature 29 (scale in 10 centimeter increments).

stained areas and patches. No burned or oxidized clay was observed at the base or around the edges. The burned rocks and the more intensive dark stained sediments were in a defined circular pattern. In places, some rocks were above one another in the dark fill sediment, with fewer and more dispersed rocks on the southern side. Dark stained sediments extended further south beyond most rocks (see Figure 9-19).

Two charcoal and one dark organic stained sediment sample from under the burned rocks were radiocarbon dated (see Table 9-1). The two charcoal dates of $5340 \pm 30$ B.P. and $5370 \pm 30$ B.P. are considered more precise, therefore their average of $5355 \pm 30$ is considered acceptable as the true age of this feature. The sediment date of $5360 \pm 30$ is statistically the same and is also acceptable.

The 92 burned rocks weighed 15,203 g and ranged from 2 to $15 \mathrm{~cm}$ in diameter, with an average weight of roughly $165 \mathrm{~g}$ (see Table 9-2). Nearly 66 percent $(N=61)$ were in the 4.1 to $9 \mathrm{~cm}$ size class. Most rocks exhibited dark stained organic spots adhering to their surfaces. The rocks were not laid flat, with a more jumbled, nonpatterned appearance across the stained area. Out of the 92 pieces, 9 were complete, rounded cobbles, and only two small refits were possible from the remaining 83 pieces. Multiple rock types were observed and included quartzites, sandstones, and limestones. Burned rocks and sediment were collected from the feature for diverse analyses.

Nine burned rocks were analyzed for starch grains and four yielded positive results. This included one damaged grain, one true lily grain, one unidentified grass grain, and three unidentifiable grains (Appendix F). An apparent recycled metate fragment (\#2223-10) (Figure 9-21) and an obvious burned rock was also examined for starch grains, with only one damaged grain identified (Appendix C). Four burned rocks were subjected to lipid residue analysis with insufficient residues to interpret on three. One rock (\#2223-23-1b) provided very high fat content that reflects possible plant and animal combination of residues (Appendix G). 


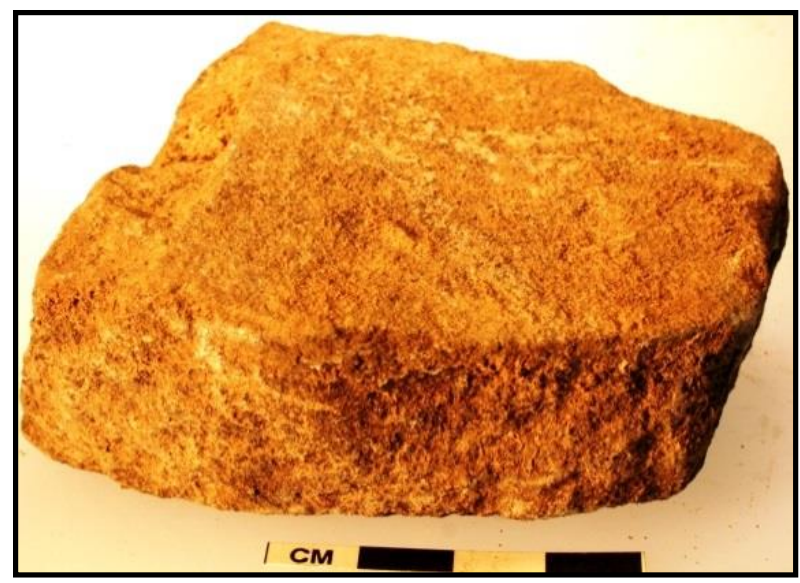

Figure 9-21. Oblique photograph of metate fragment (\#2223-10) recycled as a burned rock.

A small sediment sample (\#2424-4) was subjected to phytolith analysis. The sand fraction of the phytolith processing yielded nine microscopic bone fragments, three of which were burned. A few panicoids (100 percent burned), a few Chloridoids (none burned), and two sponge spicules were observed (Appendix E). A second small sediment sample (\#2424-4) was searched for diatoms, but none were recovered (Appendix C). Two sediment samples (\#2223-4 and \#2424-4) were sent to Dering for botanical analysis. No charred materials were observed, only dark soot (Appendix B).

Twenty-five liters from two bulk sediment samples (\#2424-4-1g and \#2424-4-2g) were mechanically floated. These yielded many burned rock fragments, 443 pieces of lithic debitage, 103 flecks of charcoal, 132 snail shell fragments, and 724 bone fragments all less than $6.4 \mathrm{~mm}$ (see Table 9-3). Bones included, minimally, one possible bird phalanx, many unidentified burned pieces, plus caudal and phalange fragments of rodents or small rabbit (Figure 9-22). Most flakes less than $6.4 \mathrm{~mm}$ are heat altered, angular shatter and spalls that exhibit a reddish color (Figure 9-23). Rabdotus shell fragments in the heavy fraction were not discolored from heat, which indicates they were not in the feature when hot and probably entered following the heating event.
Immediately outside Feature 29, N76/E86 revealed scattered burned rocks and dark stained patches, but very little other cultural debris. Three surrounding units had very limited lithic debitage and a few small bone fragments. The area immediately west and southwest also exhibited scattered burned rocks and charcoal flecks, with the occasional chert flake and mussel shell fragment that reflects the broader occupation area. Feature 28 is less than $50 \mathrm{~cm}$ south of Feature 29 and probably associated with Feature 29. Most scattered rocks were in the small, 0 to 4 $\mathrm{cm}$ size class and probably represent discarded pieces.

This well-defined heating element, with its shallow basin and medium-sized burned rocks, was probably too shallow with too short of a fire to have functioned as the base of an earth oven. The rocks also appeared randomly distributed, with surface too irregular to reflect a flat open griddle. The combination of a few complete medium size rocks and many medium size fragment that do not refit indicated these rocks were collected from other areas and activities, and brought here to heat. The presence of grass and lily starch plus damaged starch grains, combined with lipid residues from both plant and animal remains on the rocks support the rocks once were employed in the cooking process and left to possibly reheat and use again. It is possible the fire was employed to heat the rocks to use in a stone boiling process, and camp was abandoned prior to their use. The high counts of burned and unburned bone and lithic debitage from the floated fill sediments probably reflects discarding of unwanted debris into the fire as a means of cleaning other areas.

\subsubsection{Feature 30}

Feature 30 was a small dark stained basin with a few burned rocks between 229 and $238 \mathrm{cmbs}$ on the eastern side of N71/E80, along the western margin of Block B (see Figure 9-4). The stained surface measured 30-by-47 cm and exhibited dark charcoal 


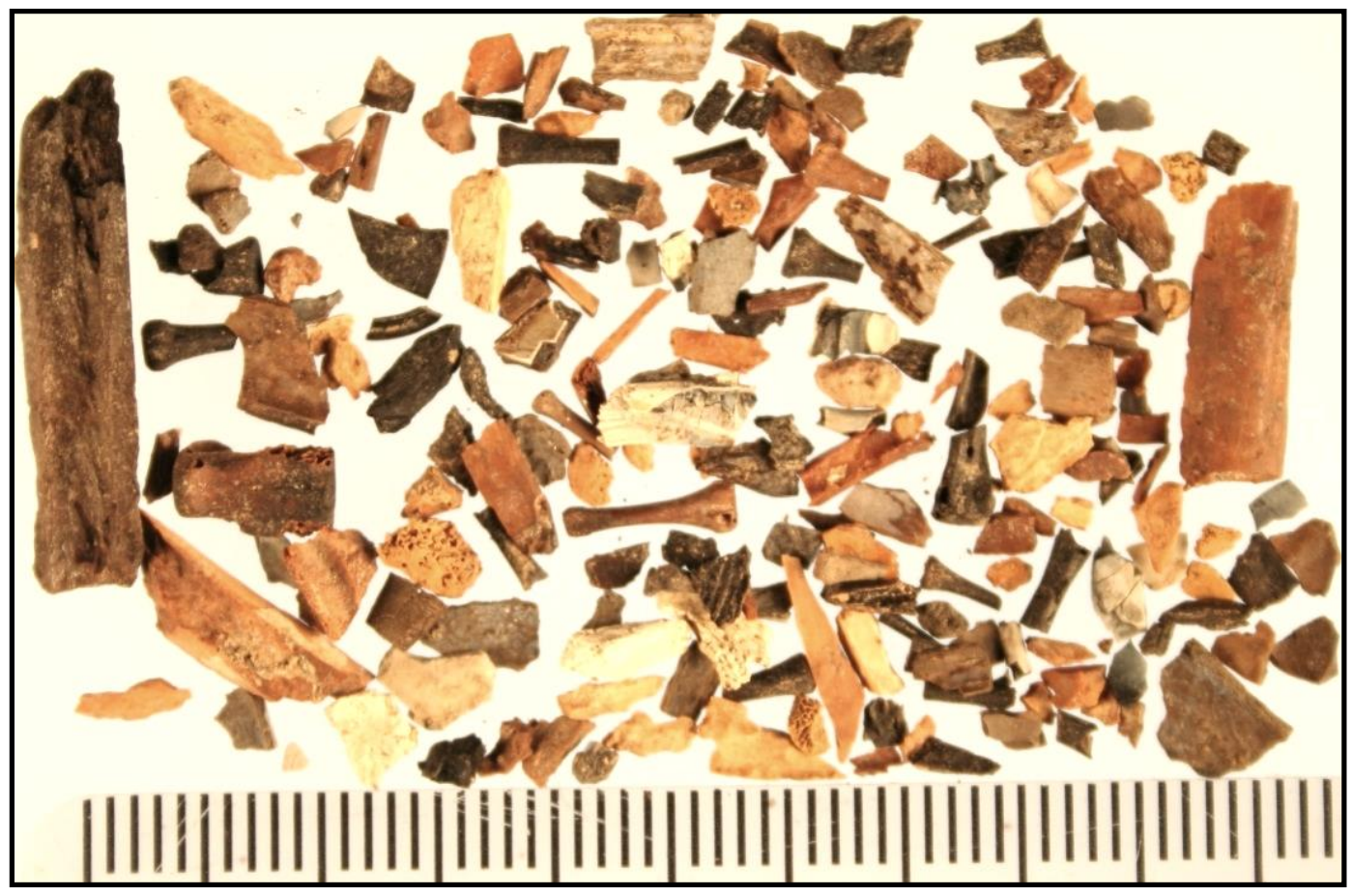

Figure 9-22. Close-up of burned and unburned bone fragments (\#2424-4-2g) from floated Feature 29 sediment (scale in millimeters).

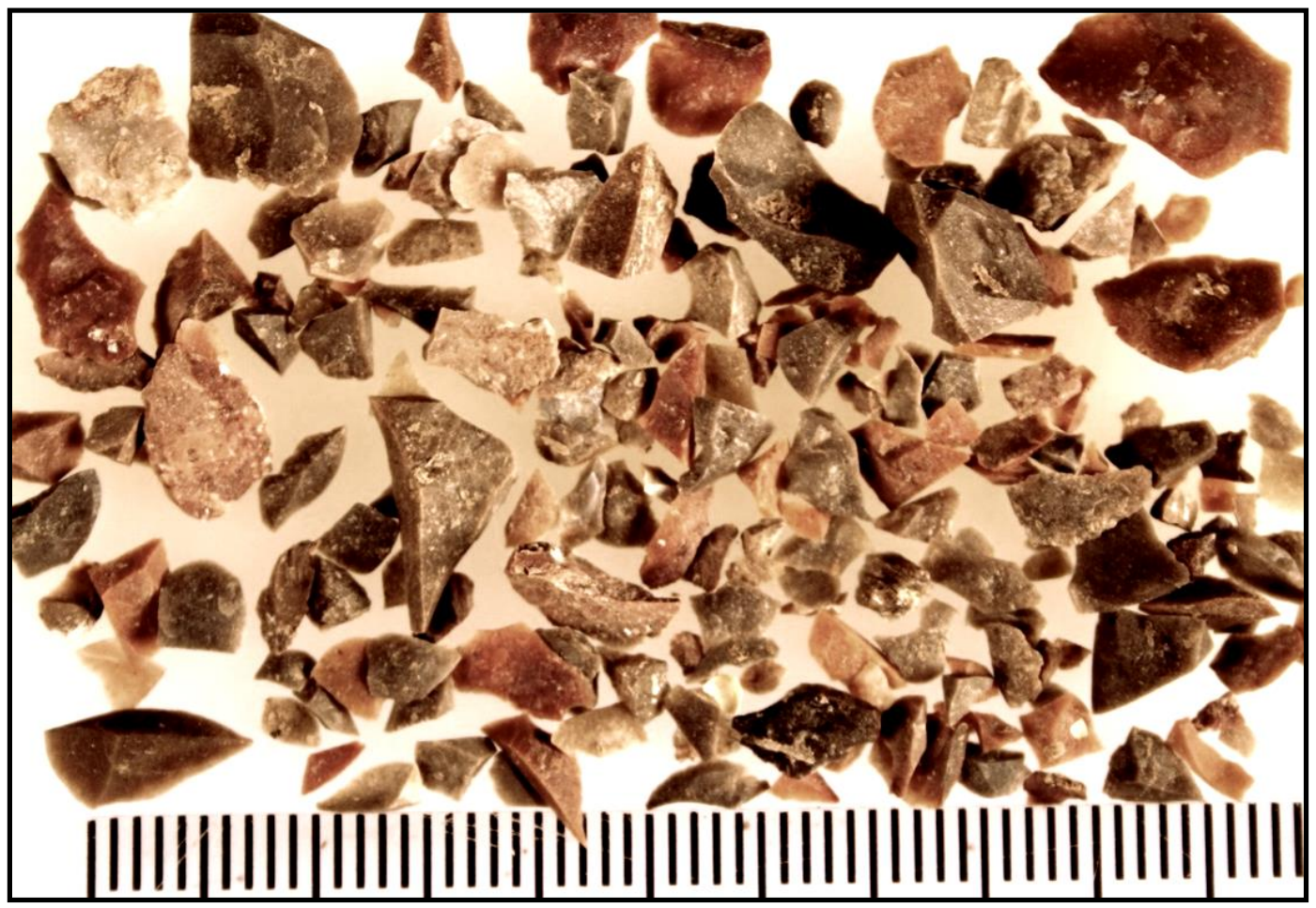

Figure 9-23. Close-up of tiny lithic debitage (\#2024-4-g) from floated Feature 29 sediment (scale in millimeters). 


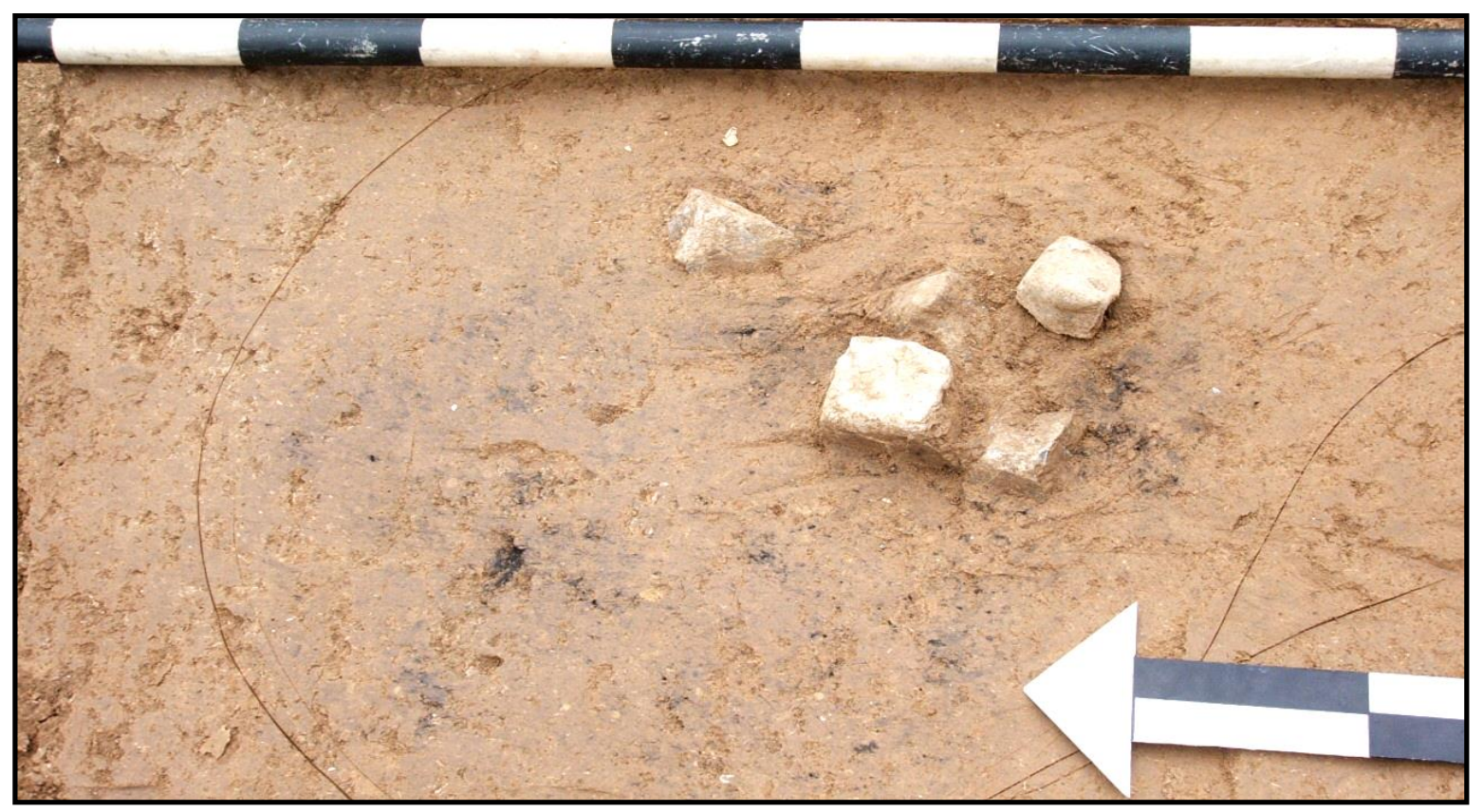

Figure 9-24. Overview photograph of Feature 30 that shows dark stained sediment around five clustered burned rocks (scales in 10 centimeter increments).

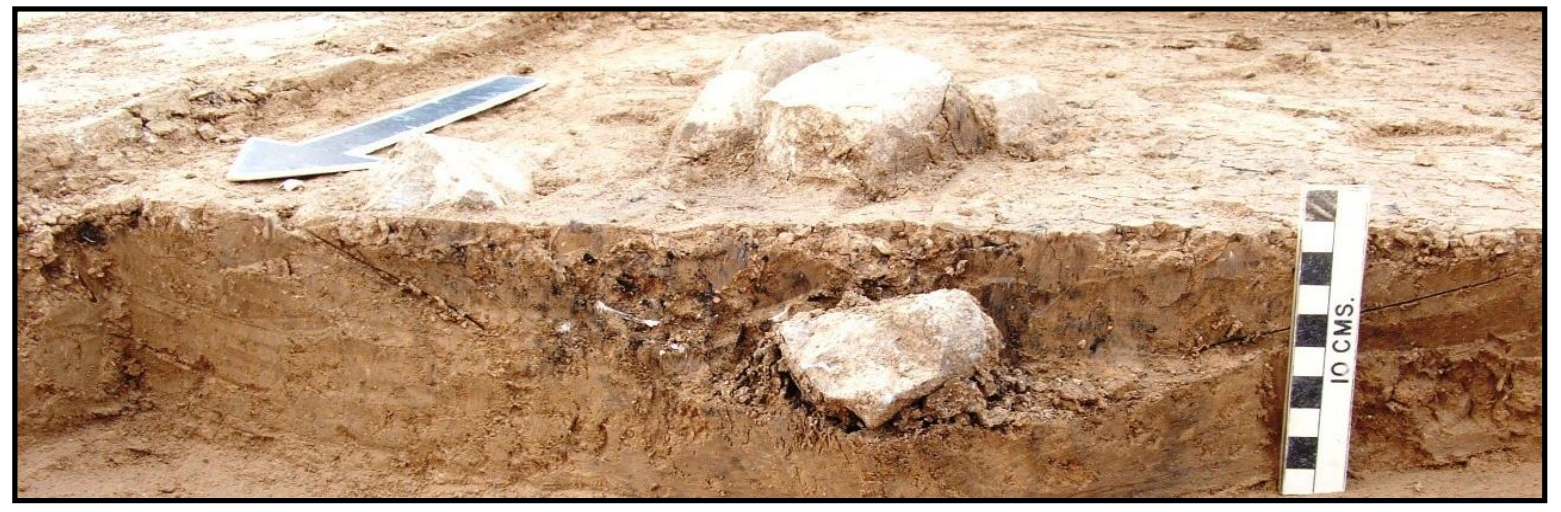

Figure 9-25. Profile photograph of Feature 30 that shows shallow basin with mottled dark stained sediment and no oxidation rim (scale in 1 centimeter increments).

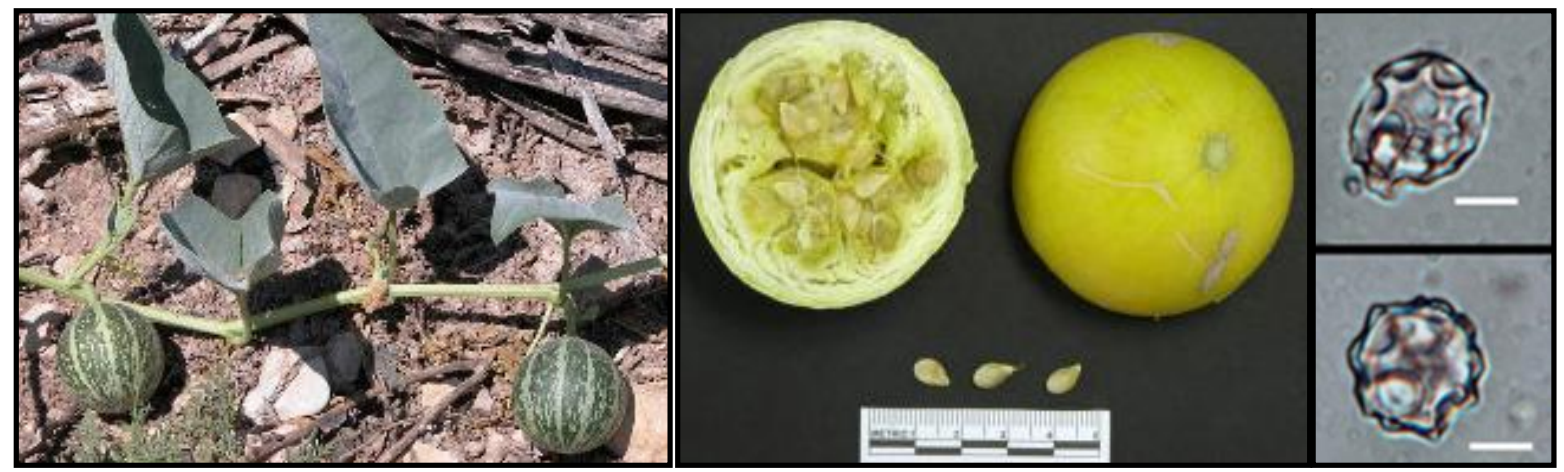

Figure 9-26. Buffalo gourd fruit and leaves on vine (left), ripe fruit and seeds (right, from Texas Beyond History 2015) and gourd phytoliths (Appendix D). 
stained sediment (7.5YR 5/4), with six burned rocks (Figure 9-24). The base of the shallow $7 \mathrm{~cm}$ deep, stained basin yielded no burned or oxidized soil (Figure 9-25).

No chunks of charcoal were observed in the basin. Consequently, a dark stained sediment samples from around the rocks was submitted for dating. This yielded a date of $5680 \pm 50$ B.P. (see Table 91). As sediment dates are generally older than the actual event represented, this date is considered to represent only an approximation of the age of this feature. This date is some 358 years older than the six charcoal dates derived from the entire component.

Six burned rocks measured 4.1 to $9 \mathrm{~cm}$ in diameter and weighed $1,303 \mathrm{~g}$, for an average weight of 217 g per rock (see Table 9-2). Four rocks exhibited hackled edges indicative of stone boiling and two pieces refit. Three rocks also had dark stained areas on their surfaces. The rocks, and sediment directly below the rocks, were collected.

Portions from two burned rocks were submitted for starch analysis. Rock \#2092-3-1c yielded a gelatinized grain, which reflects changes from heat and water. No starch was recovered from the other (Appendix F). Parts of the same two rocks sent for starch analysis were also subjected to lipid residue analysis. Both rocks (\#2092-3-1b and \#2092-3-2b) yielded very similar results, with very high fat content that indicates a combination of plant and animal, with one dominated by plant residues (Appendix G).

Four liters of sediment (\#2092-4) were sent to Dering for flotation, but failed to yield any burned organic matter (Appendix B). A subsample of \#2090-4 was sent for diatom analysis also did not yield any diatoms (Appendix C). Another subsample (\#2090-4) was examined for phytoliths with its sand fraction yielding 14 less than $6.4 \mathrm{~mm}$ bones, 10 of which were calcined, but no short cell phytoliths were present and only a few tree phytoliths, two gourd (Cucurbita foetidissima or buffalo gourd) (Figure 9-26) phytoliths, and one sponge spicule (see Figure 9-10) were identified (Appendix E).

A second 1.3 liter sediment sample (\#2092-4-g) was mechanically floated and yielded a few unburned mussel shell fragments, unburned snail shells, one flake, and 85 bone fragments, the majority of which were burned (see Table 9-3). One possible head of a rib fragment less than 6.4 $\mathrm{mm}$ represents a small mammal and exhibits thin, short, barely visible cut marks (see Figure 5-25).

Feature 30 represented an in situ fire where a few medium size limestone rocks were heated. The presence of a gelatinized starch grain indicates water was present and the rocks were probably employed in a boiling process. Tree phytoliths present support wood was used to heat the rocks. The presence of gourd phytoliths probably reflects a fall season when gourds were ripe.

\subsubsection{Feature 32}

Feature 32 was a cluster of burned rocks at the intersection of four units in the southwestern corner of Block B (Figures 9-4, 9-27 through 9-31). These units included the southwestern corner of N71/E80, southeastern corner of N71/E79, northwestern corner of N70/E80, and northeastern corner of N70/E79. This feature was only $40 \mathrm{~cm}$ southwest of Feature 30. The cluster of 37 burned rocks was concentrated between 226 and $234 \mathrm{cmbs}$ in no apparent arrangement, surrounded by dark stained sediment. No chunks of charcoal were observed in the basin and no oxidation rim was visible. All burned rocks and feature sediment were collected.

Thirty-seven burned rocks weighed 7,930 g, for an average rock weight of $214 \mathrm{~g}$. Nearly 73 percent were in the 4.1 to $9 \mathrm{~cm}$ size class (see Table 9-2). Seven rock samples, a few from each unit, were submitted for starch analysis. Only two rocks yielded starch grains, one gelatinized grain and on 


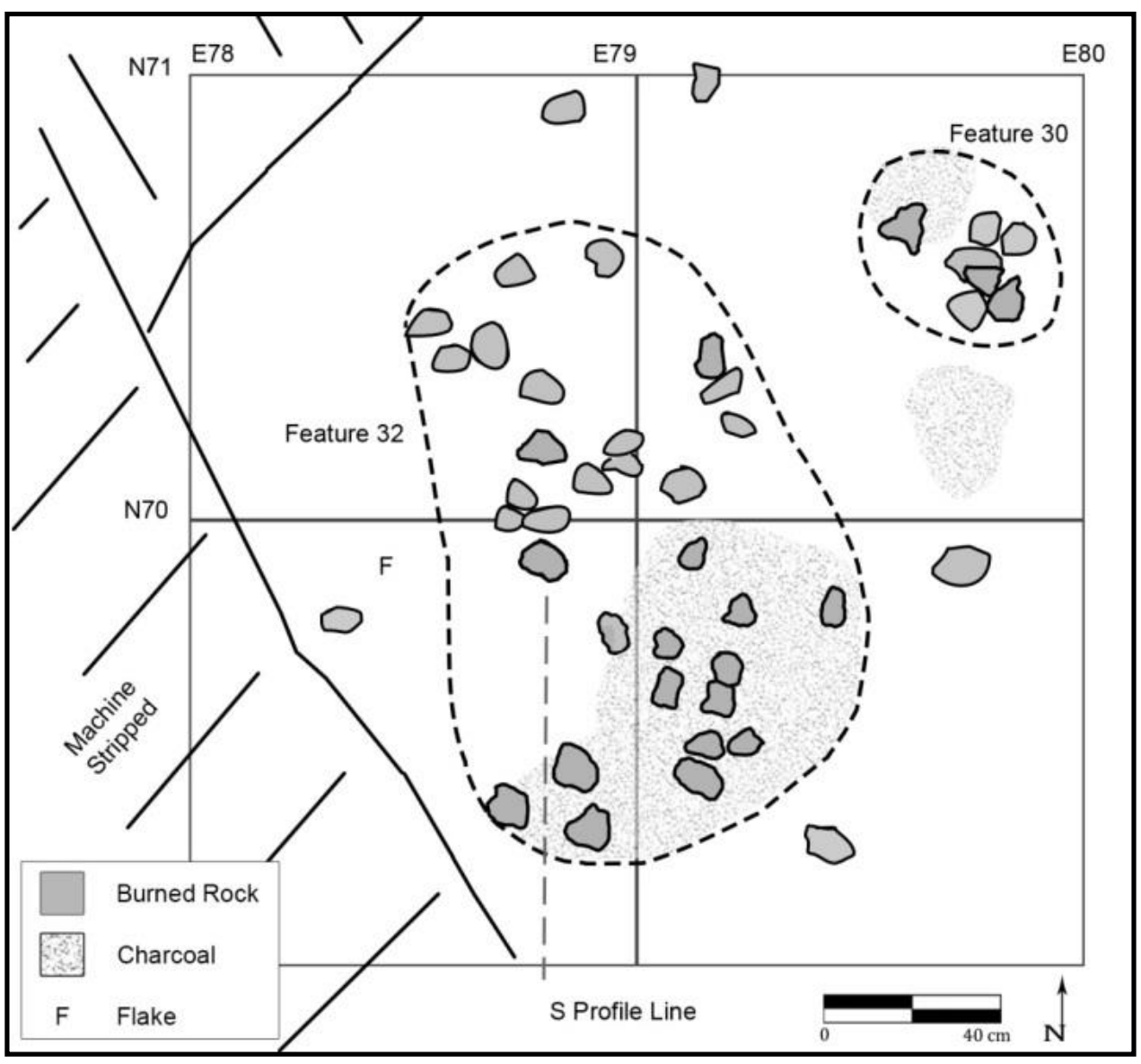

Figure 9-27. Plan view drawing of Feature 32 and its relation to Feature 30.

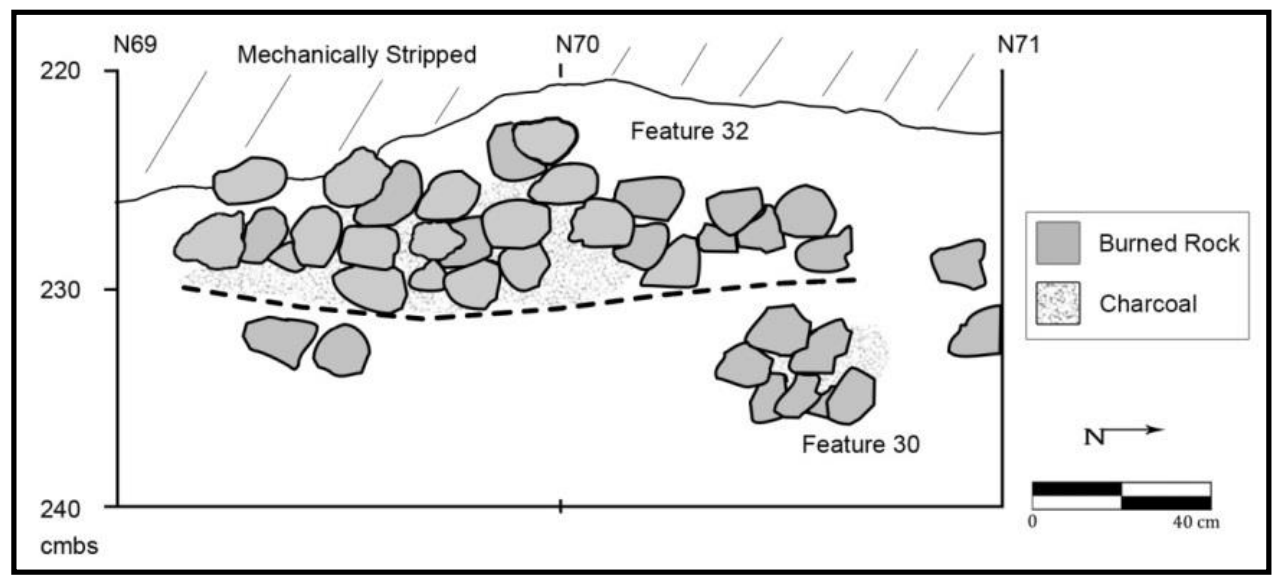

Figure 9-28. Profile drawing of Feature 30 and 32. 


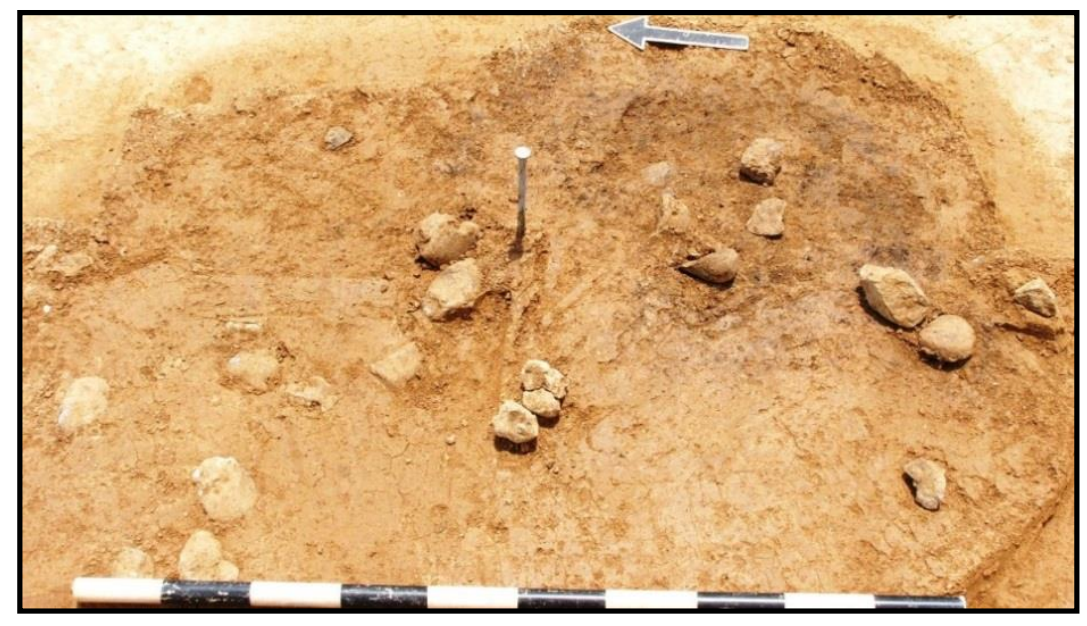

Figure 9-29. Photograph of burned rocks and dark stained sediment in and around Feature 32 (scale in 10 centimeter increments).

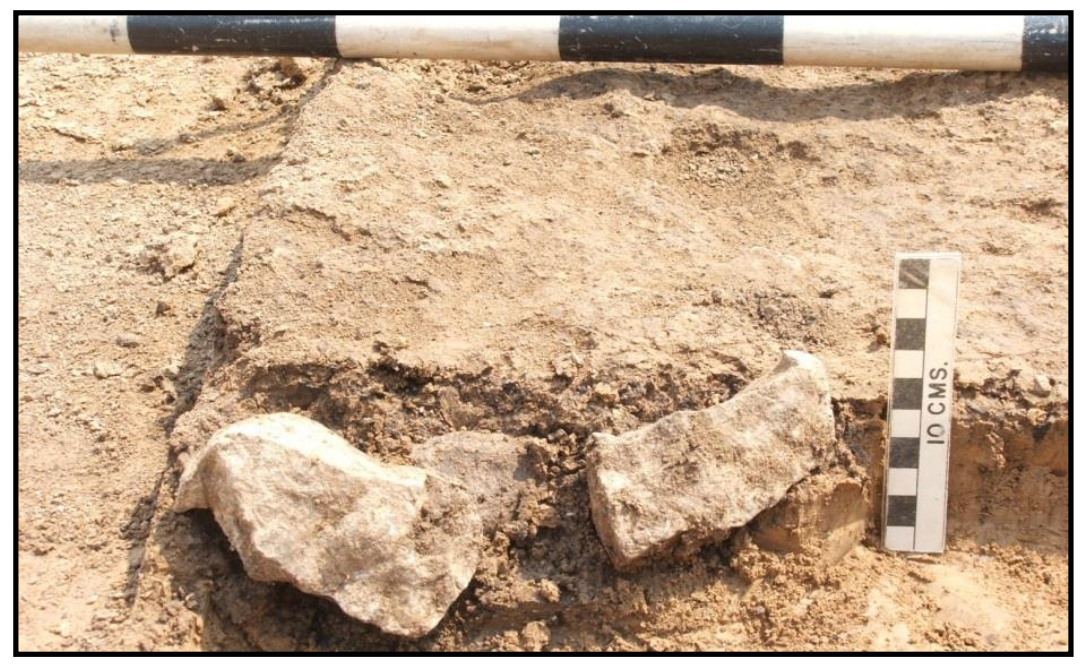

Figure 9-30. Close-up of profile of burned rocks in of Feature 32 (scale in 10 centimeter increments).

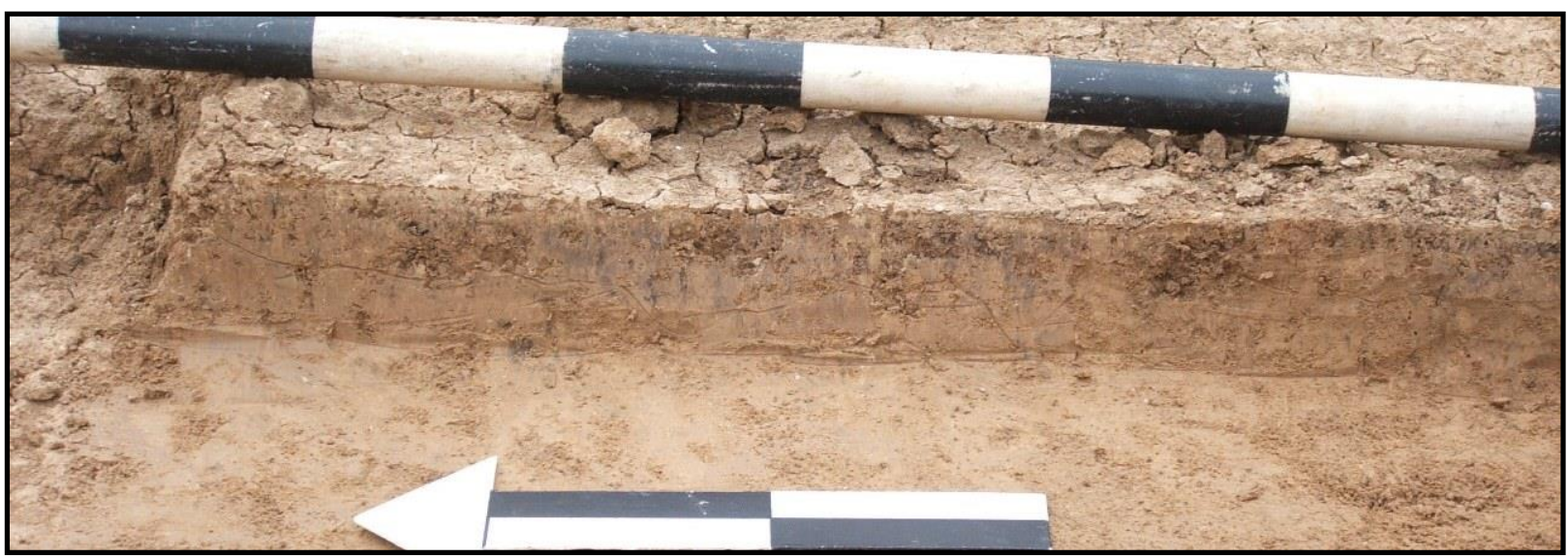

Figure 9-31. Profile photograph of dark mottled stained sediment under Feature 32 burned rocks (scale in 10 centimeter increments). 


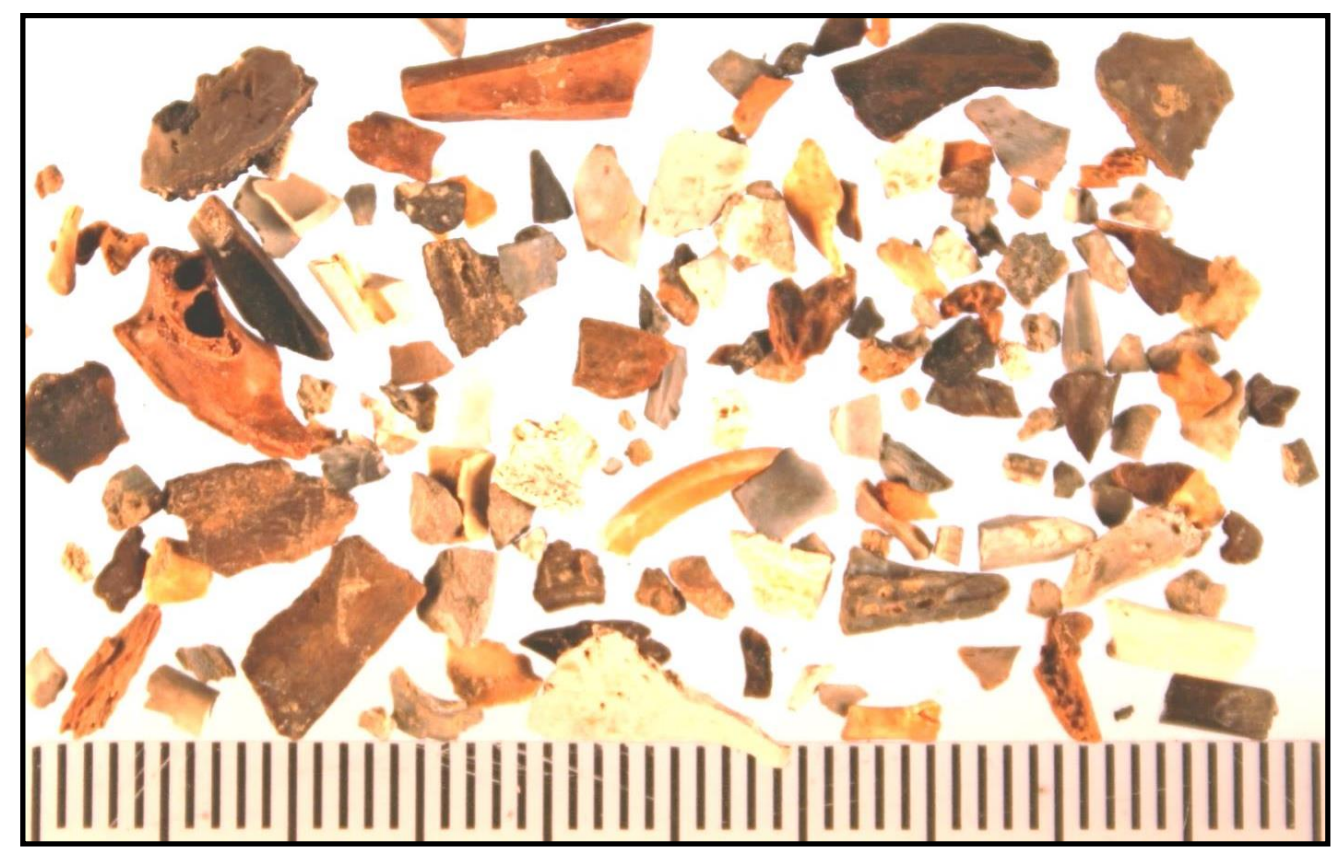

Figure 9-32. Close-up of tiny burned and unburned bone fragments (\#2074-4-g) from floated Feature 32 sediment (scale in millimeters).

eroded grain (Appendix F). Two rock subsamples were sent for lipid analysis and both revealed similar results. The obtained residues reflected low fat plant and moderate-high fat content that indicate a combination of plant and animal residues, both dominated by plant residues (Appendix G).

Samples of sediments were sent for diatom and phytolith analyses. No diatoms were detected (Appendix C). A few short cell grass and tree phytoliths were present, with some burned tree phytoliths (Appendix E). Ten liters of feature sediment (\#2078-4-g) were mechanically floated and the heavy fraction contained minor amounts of burned rocks, debitage, charcoal flecks, snail shell fragments, and macrobotanical remains, with 156 bone fragments less than $6.4 \mathrm{~mm}$, of which many were burned to different colors (Figure 9-32; see

Table 9-3). The light fraction had some carbonized material, which was sent to Dering for identification. He identified a hardwood, a carbonized hackberry nutlet, and a burned unidentifiable animal bone (Appendix B).
Feature 32 represented a heating area where cooking rocks were probably reheated as they yielded starch grains from previous cooking events. The presence of a gelatinized starch grain indicates water was present and the rocks were probably employed in a boiling process in which animal and plant products were cooked. The burned tree phytoliths support a wood fire was employed to heat the rocks. Gourd phytoliths and burned hackberry nutlet support a fall season when gourds were ripe.

\subsubsection{Feature Summary and Discussion}

Eight burned rock (not counting Feature 3 previously excavated) and one ground stone feature, between 212 and $254 \mathrm{cmbs}$ in Block B, represent the Bell/Andice component. The burned rock features were all less than $120 \mathrm{~cm}$ in diameter, and dominated by burned rocks less than $10 \mathrm{~cm}$ in diameter. Five exhibited dark stained sediment or degraded organic matter that surrounded the rocks (Table 9-4) and were considered in situ cooking 
Table 9-4. Bell/Andice Feature Summary.

\begin{tabular}{|c|c|c|c|c|c|c|c|}
\hline $\begin{array}{c}\text { Feature } \\
\text { No. }\end{array}$ & Feature Type & $\begin{array}{l}\text { Feature } \\
\text { Size }(\mathbf{c m})\end{array}$ & Profile & $\begin{array}{l}\text { Stained } \\
\text { Matrix }\end{array}$ & $\begin{array}{c}\text { Depth }(\mathrm{cm}) \\
\text { below surface }\end{array}$ & $\begin{array}{c}\text { Burned Rock } \\
\text { Counts/Wt. } \\
\text { (g) }\end{array}$ & $\begin{array}{l}\delta^{13} \mathrm{C} \text { Corrected C14 } \\
\text { Date B.P. (Material) }\end{array}$ \\
\hline 3 & $\begin{array}{l}\text { Burned rock cooking } \\
\text { feature }\end{array}$ & 90 & $\begin{array}{c}\text { Shallow } \\
\text { basin }\end{array}$ & Charcoal & $209-229$ & 201/unknown & $5290 \pm 40$ (charcoal) \\
\hline 16 & Burned rock hearth & $25 \times 25$ & $\begin{array}{c}\text { Shallow } \\
\text { basin }\end{array}$ & Charcoal & $247-254$ & $10 / 1,766$ & \\
\hline 17 & Ground stone Cache & $24 \times 20$ & Flat & None & 233-239 & & - \\
\hline 22 & Burned rock dump & $35 \times 40$ & Flat & None & $220-230$ & $13 / 1,272$ & - \\
\hline 24 & $\begin{array}{l}\text { Burned rock cooking } \\
\text { feature }\end{array}$ & $50 \times 60$ & Flat & Dark stain & $216-233$ & $41 / 13,100$ & $\begin{array}{c}5580 \pm 30 \text { (bone) } \\
5370 \pm 30 \text { (charcoal) } \\
5280 \pm 30 \text { (charcoal), } \\
5540 \pm 40 \text { (sediment) }\end{array}$ \\
\hline 27 & Burned rock dump & $15 \times 40$ & Flat & None & $212-217$ & $4 / 24,100$ & - \\
\hline 28 & Burned rock dump & $40 \times 45$ & Flat & None & $218-231$ & $11 / 676$ & $5280 \pm 30$ (charcoal) \\
\hline 29 & $\begin{array}{l}\text { Burned rock cooking } \\
\text { feature }\end{array}$ & $100 \times 70$ & $\begin{array}{l}\text { Shallow } \\
\text { saucer } \\
\text { basin }\end{array}$ & $\begin{array}{l}\text { Dark mottles, } \\
\text { charcoal }\end{array}$ & $230-242$ & $92 / 15,203$ & $\begin{array}{l}5340 \pm 30 \text { (charcoal) } \\
5370 \pm 30 \text { (charcoal) } \\
5360 \pm 40 \text { (sediment) }\end{array}$ \\
\hline 30 & Burned rock hearth & $30 \times 47$ & Basin & Dark stained & $229-238$ & $6 / 1,303$ & $5680 \pm 50$ (sediment) \\
\hline 32 & Burned rock hearth & $120 \times 83$ & Flat & Dark stain & $226-234$ & $37 / 7,930$ & - \\
\hline
\end{tabular}


Table 9-5. Summary of Analytical Results from Bell/Andice Features.

\begin{tabular}{|c|c|c|c|c|c|c|c|c|c|}
\hline $\begin{array}{c}\text { Feature } \\
\text { No. }\end{array}$ & Feature Type & $\begin{array}{c}\text { Depth }(\mathrm{cm}) \\
\text { below } \\
\text { surface }\end{array}$ & $\begin{array}{l}\text { Feature } \\
\text { Size }(\mathrm{cm})\end{array}$ & $\begin{array}{l}\text { Burned Rock } \\
\text { Counts/Wt. } \\
\text { (g) }\end{array}$ & $\begin{array}{c}\delta^{13} \mathrm{C} \text { Corrected } \\
\text { C14 Date B.P. } \\
\text { (Material) }\end{array}$ & $\begin{array}{l}\text { Starch } \\
\text { Analysis } \\
\text { Results }\end{array}$ & $\begin{array}{l}\text { Lipid } \\
\text { Analysis } \\
\text { Results }\end{array}$ & $\begin{array}{l}\text { Phytolith } \\
\text { Results }\end{array}$ & $\begin{array}{l}\text { Macro- } \\
\text { botanical } \\
\text { Results }\end{array}$ \\
\hline 3 & $\begin{array}{l}\text { Burned rock } \\
\text { cooking feature }\end{array}$ & $209-229$ & 90 & $\begin{array}{c}201 / \\
\text { unknown }\end{array}$ & $\begin{array}{l}5290 \pm 40 \\
\text { (Charcoal) }\end{array}$ & NA & NA & NA & NA \\
\hline 16 & $\begin{array}{l}\text { Burned rock } \\
\text { hearth }\end{array}$ & $247-254$ & $25 \times 25$ & $10 / 1,766$ & NA & $1 \mathrm{GL}$ & $\begin{array}{l}\text { Plant \& } \\
\text { animal }\end{array}$ & NA & NA \\
\hline 17 & $\begin{array}{l}\text { Ground stone } \\
\text { cache }\end{array}$ & $233-239$ & $24 \times 20$ & & NA & $1 \mathrm{D}$ & NA & NA & NA \\
\hline 22 & $\begin{array}{l}\text { Burned rock } \\
\text { dump }\end{array}$ & $222-230$ & $35 \times 40$ & $13 / 1,275$ & NA & NA & NA & $\begin{array}{l}\text { Few tree, } \\
\text { no short } \\
\text { cells, } \\
5 \text { spicules }\end{array}$ & NA \\
\hline 24 & $\begin{array}{l}\text { Burned rock } \\
\text { cooking feature }\end{array}$ & $216-233$ & $50 \times 60$ & $41 / 13,100$ & $\begin{array}{l}5280 \pm 30 \\
5370 \pm 30 \\
5580 \pm 30 \\
\text { (Charcoal) }\end{array}$ & $\begin{array}{c}2 \mathrm{GL}, 2 \\
\text { Un, } 1 \text { lily, } \\
1 \mathrm{E}\end{array}$ & $\begin{array}{l}\text { Plant \& } \\
\text { animal }\end{array}$ & $\begin{array}{l}\text { Many tree, } \\
\text { many } \\
\text { spicules, } \\
1 \text { gourd }\end{array}$ & NA \\
\hline 27 & $\begin{array}{l}\text { Burned rock } \\
\text { dump }\end{array}$ & $212-217$ & $15 \times 40$ & $4 / 24,100$ & NA & NA & $\begin{array}{l}\text { Plant \& } \\
\text { animal }\end{array}$ & $\begin{array}{l}\text { Few short } \\
\text { cells }\end{array}$ & NA \\
\hline 28 & $\begin{array}{l}\text { Burned rock } \\
\text { dump }\end{array}$ & $218-231$ & $40 \times 45$ & $11 / 676$ & $\begin{array}{l}5280 \pm 30 \\
\text { (Charcoal) }\end{array}$ & NA & $\begin{array}{l}\text { Plant \& } \\
\text { animal }\end{array}$ & $\begin{array}{l}\text { Limited } \\
\text { short cell, } \\
\text { few tree }\end{array}$ & NA \\
\hline 29 & $\begin{array}{l}\text { Basin burned } \\
\text { rock cooking } \\
\text { feature }\end{array}$ & $230-242$ & $100 \times 70$ & $92 / 15,203$ & $\begin{array}{c}5340 \pm 30,5370 \\
\pm 30 \text { (charcoal) } \\
5360 \pm 40 \\
\text { (sediment) }\end{array}$ & $\begin{array}{c}1 \text { D, } 1 \text { Lily, } \\
4 \text { Un }\end{array}$ & $\begin{array}{l}\text { Plant \& } \\
\text { animal }\end{array}$ & $\begin{array}{l}\text { Few } \\
\text { Panicoids \& } \\
\text { Chloridoids, } \\
2 \text { spicules }\end{array}$ & none \\
\hline 30 & $\begin{array}{l}\text { Basin burned } \\
\text { rock hearth }\end{array}$ & $229-238$ & $30 \times 47$ & $6 / 1,303$ & $\begin{array}{l}5680 \pm 50 \\
\text { (Sediment) }\end{array}$ & $1 \mathrm{GL}$ & $\begin{array}{l}\text { Plant \& } \\
\text { animal }\end{array}$ & $\begin{array}{l}\text { Few tree, } \\
2 \text { gourd, } \\
1 \text { spicule }\end{array}$ & Wood \\
\hline 32 & $\begin{array}{l}\text { Burned rock } \\
\text { hearth }\end{array}$ & $226-234$ & $120 \times 83$ & $37 / 7,930$ & NA & $1 \mathrm{GL}, 1 \mathrm{E}$ & $\begin{array}{l}\text { Plant \& } \\
\text { animal }\end{array}$ & $\begin{array}{l}\text { Few tree, } \\
\text { few short } \\
\text { cells }\end{array}$ & $\begin{array}{c}\text { Hardwood, } \\
\text { Hackberry } \\
\text { nutlet }\end{array}$ \\
\hline
\end{tabular}


features with defined shallow basins. The basins were no deeper than $20 \mathrm{~cm}$, with only two greater than $10 \mathrm{~cm}$. Three burned rock clusters are considered dumps or secondary disposals of used rocks from other facilities located elsewhere. Burned rock frequency and size was quite variable, with counts that ranged from 4 up to 92 rocks and sizes from 2 to $16 \mathrm{~cm}$. Rock material types varied as well, with limestones, quartzites, fossil oyster shell, and chert all employed.

All features generally lacked sizeable pieces (greater than $6.4 \mathrm{~mm}$ ) of other artifact classes such as flakes, bones, or mussel shells, although most floated sediments from around and below the rocks yielded many bone fragments and occasional chert debitage less than $6.4 \mathrm{~mm}$. Eight features lacked any quantity of lithic flakes under $6.4 \mathrm{~mm}$, with the exception of Feature 29, which yielded nearly 450 pieces of lithic debris measuring less than $6.4 \mathrm{mmin}$ size. Most flakes were burned and/or heat altered. These tiny artifacts are assumed to be discarded debris, deposited in features as a means of disposal and/or cleaning activities, as their presence in an apparent hearth likely does not reflect an in situ knapping activity. Rabdotus shells from the features did not reveal any signs of heat alteration and are probably not associated with the cultural deposit.

Burned rock sizes in the dumps or discard piles are generally smaller than the rocks from the in situ cooking features. Scattered burned rocks are dominated by the smallest size category ( 0 to 4.0 $\mathrm{cm})$, which accounts for at least 50 percent. Feature burned rocks reveal that approximately 51 percent were in the 4.1 to $9 \mathrm{~cm}$ size class, just the opposite of scattered pieces. Rocks in dumps or discard piles were most often lighter in average weight compared to those rocks in the hearths. One exception includes the four rocks in Feature 27, which were all in the 9.1 to $15 \mathrm{~cm}$ size class, with average weights of greater than $6,000 \mathrm{~g}$. Therefore, these rocks were probably set aside for reuse rather than discarded. Features that exhibited basins were defined by dark-stained sediment with no visible yellowish-orangish oxidation rim along basin margins.

Technical analyses provide evidence as to what burned rocks probably cooked, and in some instances, indicate how cooking occurred. True lily starch (Lilium, definitely not onion, garlic, camas, or rain lily) was present in Features 24 and 29 and indicate lily bulbs were one of the represented plant food resources (Table 9-5). An unidentified grass starch was recovered from a burned rock from Feature 29. Gourd phytoliths were identified in Features 24 and 30 to document their use. Apparently lily bulbs and grass seeds were two plants cooked, with the possibility gourds as well, or they potentially served as utensils.

Four gelatinized grains from four separate features support cooking occurred with heat and water, most probably stone boiling. A damaged grain was also identified in Feature 32 supporting plant processing activities. The use of grass seeds processed and cooked in features is supported by two metate fragments that yielded unidentified grass starches and one edge-modified flake with grass starch (see Table 9-5). Three damaged grains from processing were present on three ground stone tools to support processing activities. Lipid residue analysis on feature burned rocks documents plant and animal residues in nearly all cases (see Table 9-5). Stone boiling was probably the primary means of cooking, as this technique is a suitable means in which plant and animal products would be cooked together and have their residues retained on the rocks.

Those features that represent in situ heating and/or cooking elements were probably employed for short-periods, as they lacked obvious oxidation rinds that are expected with long-term heating over hours of intense use (Bond 1978:114-118). 
The one cluster of three ground stone tools (Feature 17 ) is interpreted as a discard locality or a potential cache, although the fragmented metate does not appear reusable. One mano yielded damage starch grains, in contrast to the metate that did not yield any starches (see Table 9-5). The presence of ground stone tools document plant processing through grinding.

\subsection{CHIPPED STONE TOOL ASSEMBLAGES}

Six formal chipped stone tools were recovered and represent two tool classes. Each class and tool is described below beginning with projectile points followed by bifaces.

\subsubsection{Projectile Points}

Three projectile points were recovered, two classify as Bell points (Sorrow et al. 1967) and one is a large Big Sandy point (Bell 1960:8). Specimen \#213911 represents three-quarters of a small, shortstemmed Bell point with deep basal notches, missing a distal end and one lateral barb (Figure 933, Table 9-6). It was discovered in situ at 238 cmbs in N72/E83 of Block B, collected, and left unwashed. It is possible that the broken distal end resulted from impact during use, but the fracture point on the distal end closely resembles a lipped, bending break, which commonly occurs during flaking and reworking (Whittaker 1994:163). The deep (14.8 $\mathrm{mm}$ from base), narrow (3.0 to $5.8 \mathrm{~mm}$ ) basal notches are well-executed; the lateral margins reveal thin pressure flake scars and the $17.8 \mathrm{~mm}$ long slightly expanding straight base was also thinned by pressure flakes and then ground. The neck width is $16.2 \mathrm{~mm}$ and the stem is $14.5 \mathrm{~mm}$ long and $5.8 \mathrm{~mm}$ thick at the neck. The remaining $11.3 \mathrm{~mm}$ long barb is relatively narrow and biconvex in cross section and tapers from 5.7 to 3.5 mm wide.

This Bell point (\#2139-11) was manufactured from a high quality dark gray $(2.5 \mathrm{Y} 4 / 0)$ chert that fluoresces an orange color, similar to some local Edwards chert. It has close visual comparison to some Georgetown chert. No heat treatment is apparent. The unwashed point was sent for highpowered use-wear analysis, which yielded haft polish, possible starch grains on the stem section, and high silica polish, and striations that document whittling hard materials (Appendix D).

Specimen \#2150-10 is the base of a Bell point nearly identical in size and shape to the base of Specimen \#2139-11 discussed above (see Figure 933, see Table 9-6). This partial base has the same flaking pattern and notching pattern as the

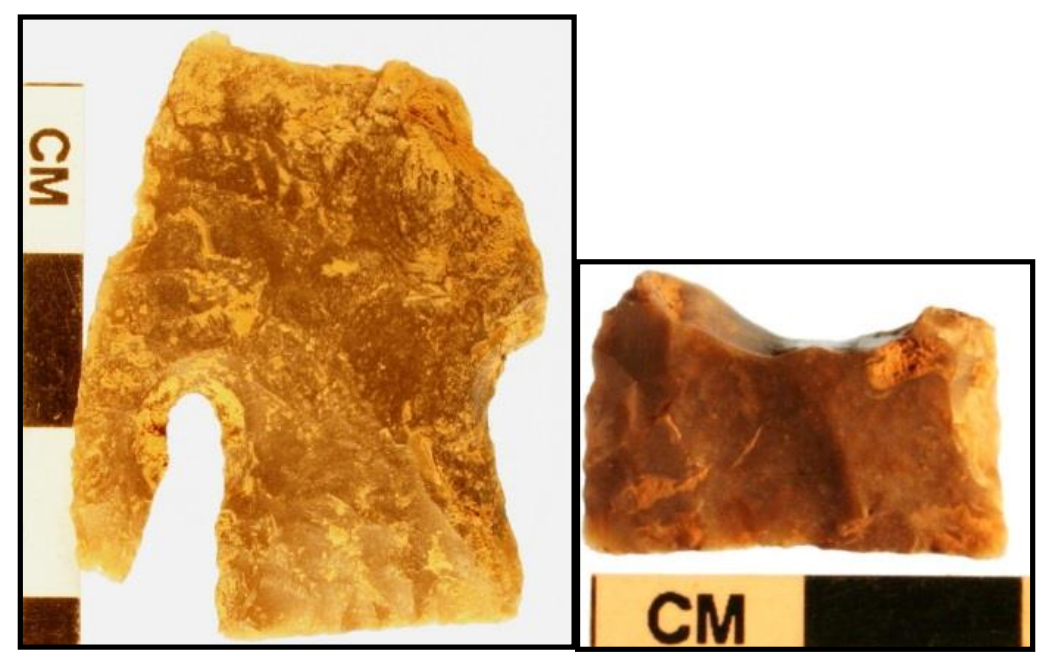

Figure 9-33. Photographs of Bell points (\#2139-11, left and \#2150-10, right). 
Table 9-6. Bell/Andice Formal Chipped Stone Tool Data.

\begin{tabular}{|c|c|c|c|c|c|c|c|c|c|c|c|c|c|}
\hline $\begin{array}{l}\text { D } \\
\text { C } \\
\text { ב }\end{array}$ & $\begin{array}{l}\text { 들 } \\
\text { 而 } \\
\text { 足 }\end{array}$ & 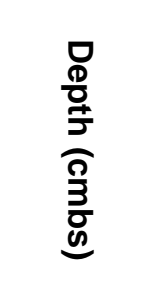 & 方 & 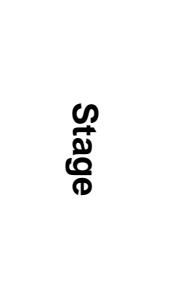 & $\begin{array}{l}\frac{0}{0} \\
\frac{0}{0}\end{array}$ & $\begin{array}{l}\frac{D}{\bar{\Phi}} \\
\frac{0}{0} \\
\frac{\overrightarrow{0}}{0}\end{array}$ & $\begin{array}{l}\text { 달 } \\
\frac{0}{8} \\
\stackrel{\varrho}{\varrho}\end{array}$ & 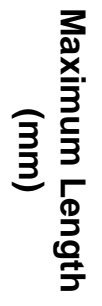 & 氕 & 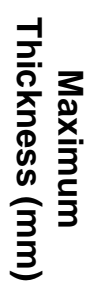 & 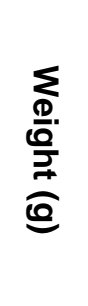 & 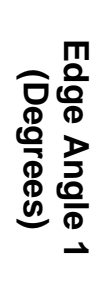 & 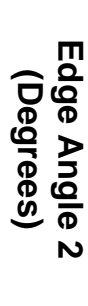 \\
\hline $2105-10$ & $71 / 83$ & $225-230$ & Biface & $\begin{array}{c}\text { Initial } \\
\text { Reduction }\end{array}$ & Distal & None & $\begin{array}{c}\text { Edwards } \\
\text { Chert }\end{array}$ & 32.0 & 36.7 & 10.8 & 12.0 & 70 & 66 \\
\hline 2139-11 & 72 / 83 & $237-238$ & $\begin{array}{l}\text { Bell } \\
\text { Point }\end{array}$ & Finished & $\begin{array}{l}\text { Proximal } \\
\text {-medial }\end{array}$ & None & $\begin{array}{c}\text { Edwards } \\
\text { Chert }\end{array}$ & 34.7 & 27.0 & 6.8 & 6.2 & 47 & 50 \\
\hline $2146-10$ & 72 / 84 & 226 & $\begin{array}{c}\text { Biface } \\
\text { Stage } 4\end{array}$ & Finished & $\begin{array}{l}\text { Distal- } \\
\text { medial }\end{array}$ & None & $\begin{array}{c}\text { Edwards } \\
\text { Chert }\end{array}$ & 89.9 & 68.8 & 7.7 & 38.5 & 56 & 57 \\
\hline $2150-10$ & $72 / 85$ & 248 & $\begin{array}{c}\text { Bell } \\
\text { Point }\end{array}$ & Finished & $\begin{array}{l}\text { Base/ } \\
\text { stem }\end{array}$ & $\begin{array}{c}\text { Possible } \\
\text { heated }\end{array}$ & $\begin{array}{c}\text { Edwards } \\
\text { Chert }\end{array}$ & 12.9 & 19.6 & 5.8 & 1.5 & 75 & 72 \\
\hline 2175-12 & $73 / 85$ & 224 & $\begin{array}{l}\text { Biface } \\
\text { Stage } 3\end{array}$ & $\begin{array}{l}\text { Partially } \\
\text { Finished }\end{array}$ & $\begin{array}{l}\text { Lateral } \\
\text { edge(s) } \\
\text { missing }\end{array}$ & Thermal & $\begin{array}{c}\text { Edwards } \\
\text { Chert }\end{array}$ & 67.4 & 21.8 & 20.7 & 10.4 & 61 & 61 \\
\hline $2224-10$ & 77 / 82 & 236 & $\begin{array}{l}\text { Big } \\
\text { Sandy } \\
\text { Point }\end{array}$ & Finished & $\begin{array}{l}\text { Base } \\
\text { corners } \\
\text { missing }\end{array}$ & $\begin{array}{l}\text { Carbonate } \\
\text { build-up }\end{array}$ & $\begin{array}{c}\text { Edwards } \\
\text { Chert }\end{array}$ & 91.9 & 33.4 & 8.2 & 24.6 & 61 & 64 \\
\hline
\end{tabular}




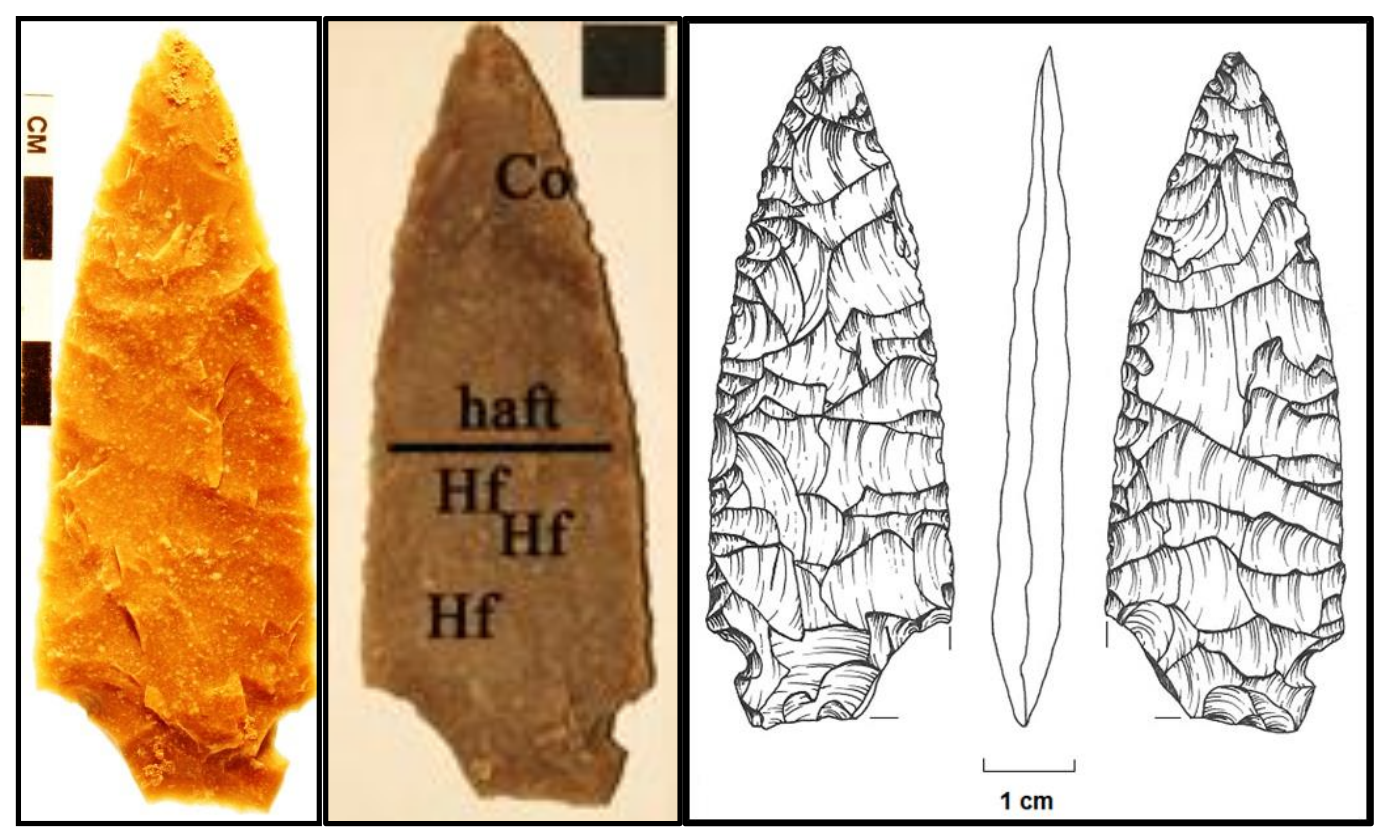

Figure 9-34. Photographs of washed Big Sandy point (\#2224-10, left), use-wear results (middle), and drawings (right).

more complete Bell point. This was again collected and left unwashed from $248 \mathrm{cmbs}$ in N72/E85, roughly 2 m north of the other Bell point. All three intact basal margins exhibit small pressure flake scars and ground edges. The break is laterally concave, created from one lateral edge potentially spalled from heat or similar to a burin blow. The neck width is minimally $18.3 \mathrm{~mm}$ as the break is below the maximum width. The stem length is unknown and stem thickness is minimally $5.64 \mathrm{~mm}$ at the break. The chert is a light gray to gray (10YR 6/1) with a light reddish hue apparent, thus potentially heat treated or at least in a fire at some point. The light gray chert fluoresces an orange color, similar to some local Edwards chert. This unwashed stem was sent for high-powered usewear analysis, which revealed no use-wear or residues (Appendix D).

The nearly complete, side-notched Big Sandy point (\#2224-10) (Bell 1960) was discovered in situ at $236 \mathrm{cmbs}$ in N77/E82 of Block B, collected, not handled, and left unwashed when in the laboratory. It was associated with a smear or scatter of dark organic stained sediment. The point is very symmetrical, not beveled, lacks patination, and does not appear reworked; therefore, it is not considered an older curated point (Figure 9-34, see Table 9-6). The specimen is very well-made from a light brownish-gray (10YR 6/2) chert with tiny light spots, and exhibits moderate size billet scars across both faces with small retouched scars along the edges. It lacks cortex and does not appear heat treated or heat altered. Both basal corners are broken. The base is slightly concave to straight, unground, and thinned with pressure flakes. The neck width is $21.1 \mathrm{~mm}$, stem length is $11.5 \mathrm{~mm}$, and the stem thickness is $7.8 \mathrm{~mm}$. The point was subjected to starch analysis, but no starches were recovered (Appendix F). After washing, the point was also subjected to residue and use-wear analysis, which revealed haft wear (Hf) polish that extends over about half the length of the point and collagen (Co) residues towards the distal end near the tip (see Figure 9-33, Appendix D). The presence of collagen residue documents this point was engaged in processing animals. Under UV light this point fluoresces a light yellow, similar to the local Edwards chert. 


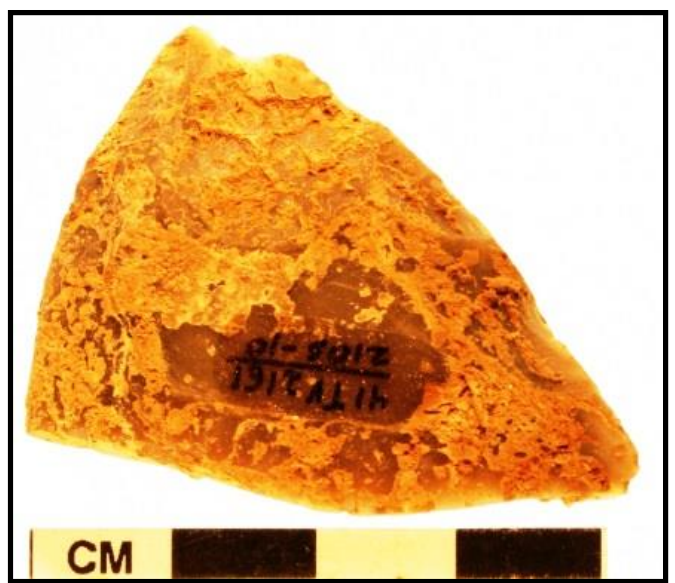

Figure 9-35. Photograph of unwashed, distal biface fragment (\#2105-10).

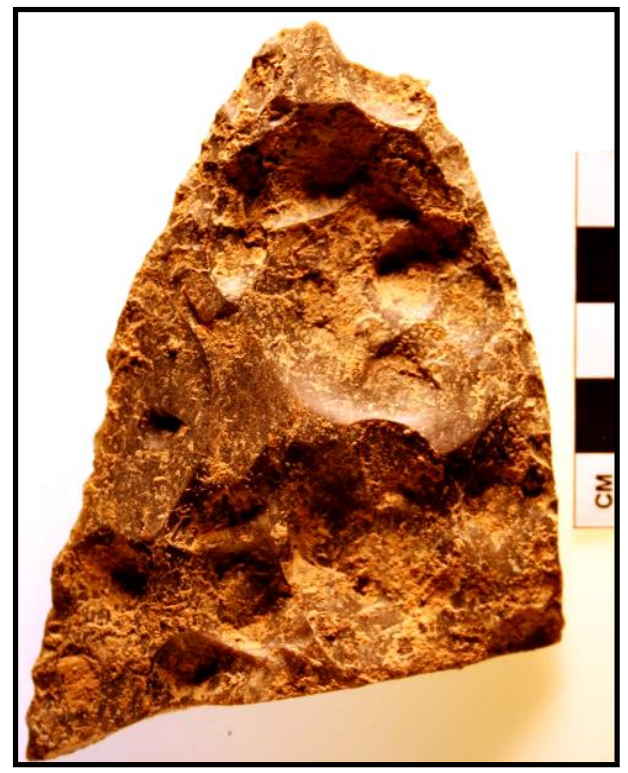

Figure 9-36. Photograph of unwashed, heat spalled, distal biface fragment (\#2146-10) adjacent to burned rock Feature 22.

\subsubsection{Bifaces}

Parts of three bifaces were recovered: one distal tip, a large intensively burned medial section, and three refit lateral edge fragments from a third specimen. Specimen \#2105-10 is the distal end of a biface from between 225 and 230 in N71/E83 in Block B (Figure 9-35, see Table 9-6). It was collected and left unwashed for possible residue analysis. The lateral edges are thinned and worn, and both faces exhibit broad billet scars. No cortex is present and the very distal tip is broken and reveals a major concave scar on one face. Manufactured from a high quality, dense, light brownish-gray (10YR $6 / 2$ ) chert that does not appear heat treated. A tiny $(0.6 \mathrm{~g})$ piece was removed from the medial break area and sent for INAA (TRC677), with chemical results that indicate it was manufactured from locally derived chert, specifically the adjacent gravel hilltop (Appendix H). This unwashed distal end was sent for high-powered use-wear analysis, but it did not reveal any use-wear or micro-residues (Appendix D).

Specimen \#2146-10 is a medial and distal end of a very large, thin biface (Figure 9-36, see Table 9-6). It was discovered in situ at $226 \mathrm{cmbs}$ in N72/E84 of Block B, collected, minimally handled, and left unwashed. Both faces are completely covered with potlids, many of which are overlapping. Exposure to heat also probably caused the breakage at the distal and proximal ends. What is visible is a wellmanufactured biface with relatively large billet flake scars across both faces and well-executed pressure flaked edges. The lateral edges appear lightly worn indicative of use. It was manufactured from a very dark gray (10YR 3/1), homogenous, high quality chert. Under UV light the biface fluoresces a dark or nonreflective color indicative of a non-Edwards chert. About $1 \mathrm{~g}$ was removed for INAA (TRC679), with chemical results that indicate it was manufactured from locally derived chert from the adjacent gravel hilltop (Appendix $\mathrm{H})$. This finished, but burned, biface fragment was sent for high-powered micro-wear and revealed hard, high silica polish along one lateral edge indicative of cutting hard, high silica materials(Appendix D). Use-wear data indicates this piece was employed for cutting plants prior to being directly subjected to extreme heat. No obvious manufacturing debris from this biface was observed in the lithic debitage assemblage. 


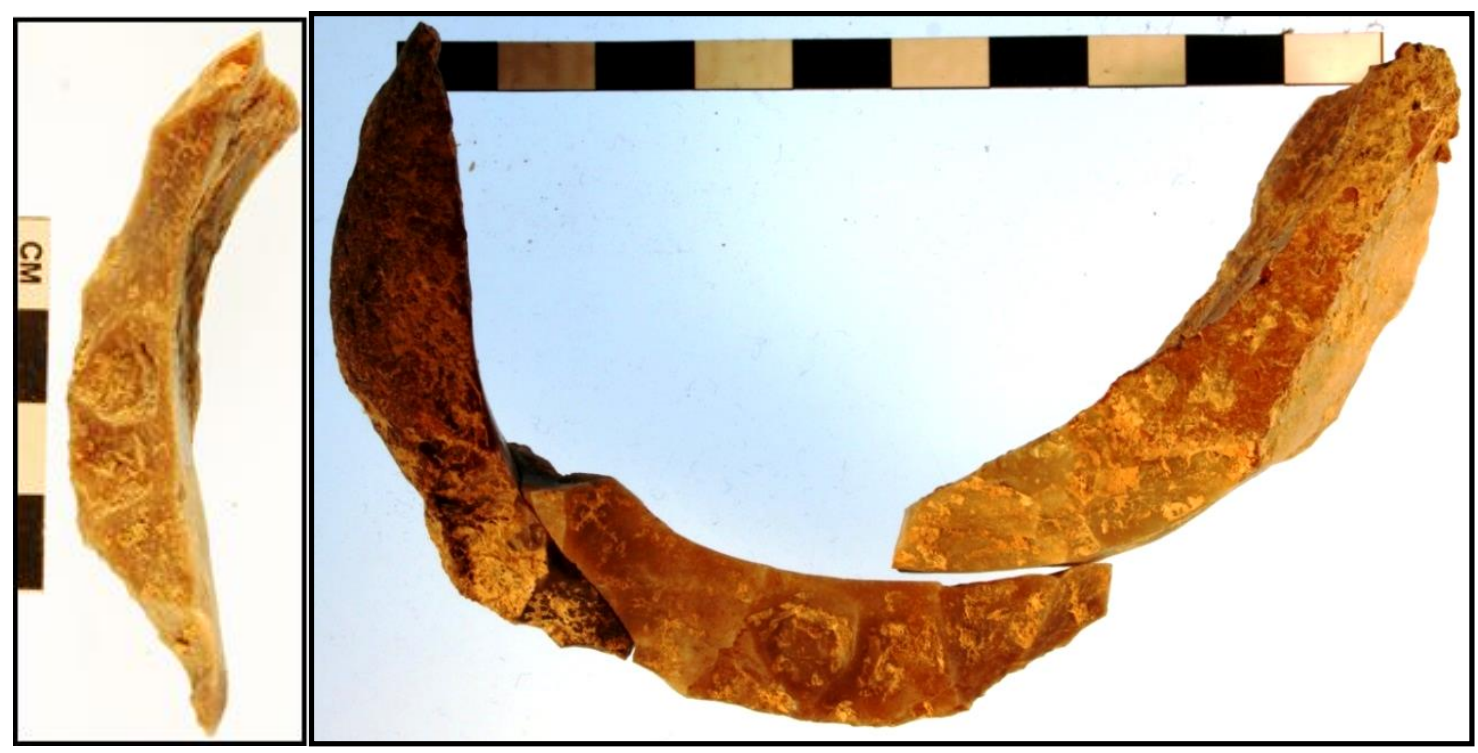

Figure 9-37. Photograph of unwashed, lateral biface fragment (\#2175-12, left); three refit pieces (\#2175-10, \#2175-11, and \#2175-12) on right reconstructing biface outline (scale in centimeters).

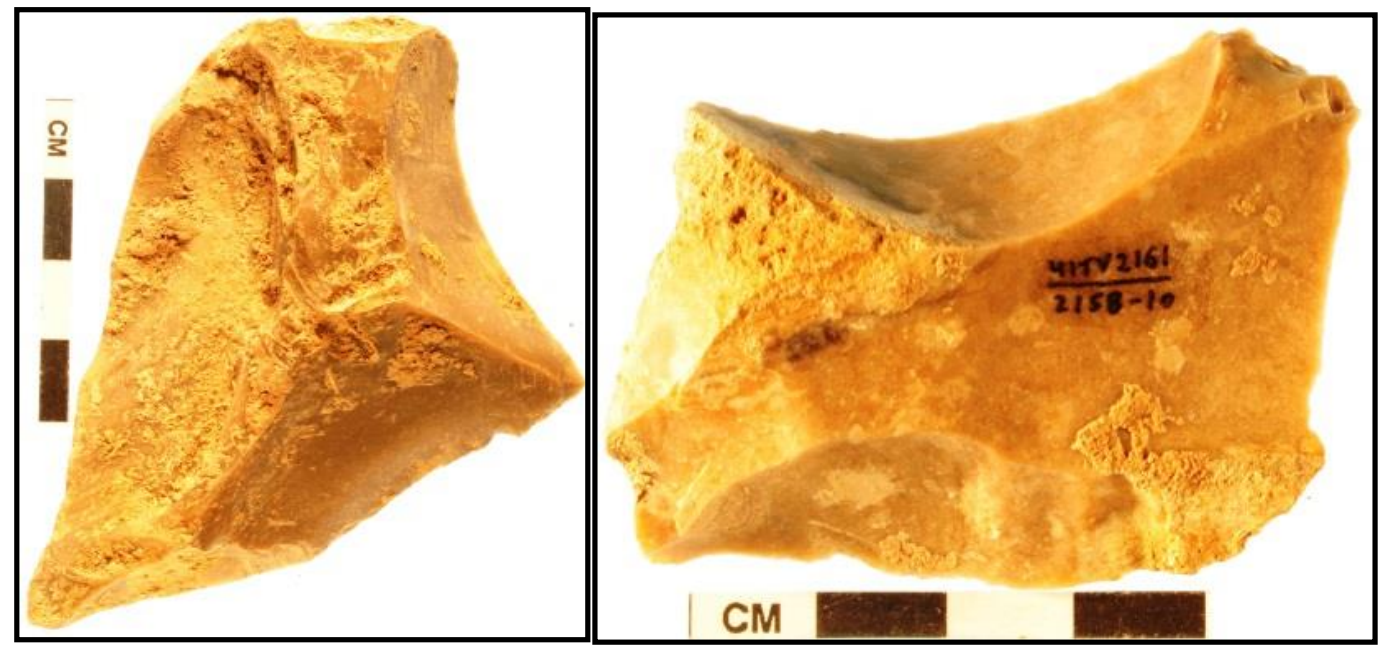

Figure 9-38. Photograph of two examples of edge-modified flakes (\# 2041-10, left and \#2158-10 right).

Specimen \#2175-12 is a crescent-shaped, lateral edge of a much larger, thick, presumably early to middle stage biface discovered between 216 and $230 \mathrm{cmbs}$ in N73/E85 of Block B along with two additional crescent lateral edge pieces (Specimens \#2175-10 and \#2175-11) that refit into one long curved edge (Figure 9-37, see Table 9-6). These three pieces all refit to create an unusual, $18 \mathrm{~cm}$ long curved outer edge of a biface roughly $12 \mathrm{~cm}$ across. On \#2175-12, multiple wide percussion scars are present on most of both faces that extend beyond the broken edge with tiny use scars at the very edge. A $9.5 \mathrm{~mm}$ wide pot lid is present on one face near the edge, with minimally two smaller pot lids on the opposite face and possibly along the distal edge. Specimen \#2175-10 is not bifacial, but exhibits a rounded water worn cortex over the outer edge. Specimen \#2175-11 is slightly towards the interior with broad flake scars and cortex on one face. 
Table 9-7. Bell/Andice Edge-Modified Flake Data.

\begin{tabular}{|c|c|c|c|c|c|c|c|c|c|c|c|c|c|}
\hline $\begin{array}{l}\text { D } \\
\text { c } \\
\underline{3}\end{array}$ & 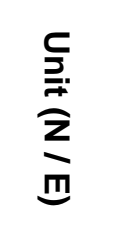 & 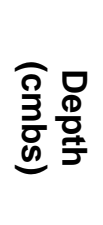 & 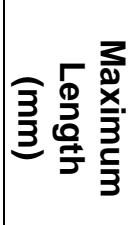 & 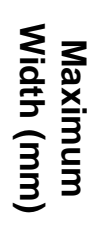 & 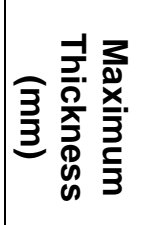 & 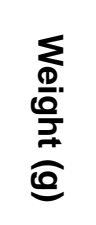 & 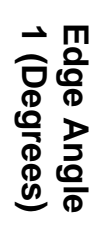 & 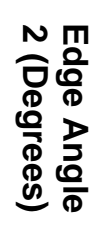 & 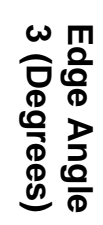 & $\begin{array}{l}\mathscr{P} \\
\mathbb{\Xi} \\
\stackrel{0}{0}\end{array}$ & $\frac{0}{\circ}$ & 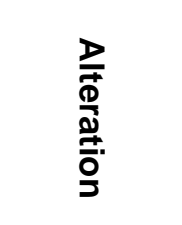 & $\begin{array}{l}\text { 달 } \\
\stackrel{0}{0} \\
\stackrel{0}{\gtrless}\end{array}$ \\
\hline 2074-10 & $70 / 79$ & $\begin{array}{l}220- \\
230\end{array}$ & 51.7 & 39.3 & 19.5 & 34.0 & 65 & 67 & & $\begin{array}{c}\text { Initial } \\
\text { Reduction }\end{array}$ & Complete & $\begin{array}{c}\text { None } \\
\text { observed }\end{array}$ & Chert \\
\hline 2114-10 & $71 / 84$ & $\begin{array}{l}220- \\
230\end{array}$ & 83.1 & 52.7 & 15.1 & 70.6 & 70 & 68 & & $\begin{array}{c}\text { Initial } \\
\text { Reduction }\end{array}$ & Complete & $\begin{array}{l}\text { Carbonate } \\
\text { build-up }\end{array}$ & Chert \\
\hline $2139-10$ & $72 / 83$ & $\begin{array}{l}230- \\
240\end{array}$ & 38.7 & 27.1 & 3.7 & 2.8 & 56 & & & $\begin{array}{c}\text { Initial } \\
\text { Reduction }\end{array}$ & $\begin{array}{c}\text { Proximal- } \\
\text { medial }\end{array}$ & $\begin{array}{c}\text { None } \\
\text { observed }\end{array}$ & Chert \\
\hline $2146-11$ & $72 / 84$ & $\begin{array}{l}230- \\
240\end{array}$ & 27.2 & 27.2 & 16.1 & 9.5 & 3.5 & 88 & & $\begin{array}{c}\text { Initial } \\
\text { Reduction }\end{array}$ & Medial & $\begin{array}{c}\text { None } \\
\text { observed }\end{array}$ & Chert \\
\hline $2149-10$ & $72 / 85$ & $\begin{array}{l}220- \\
230\end{array}$ & 55.3 & 34.2 & 8.6 & 12.3 & 50 & 54 & & $\begin{array}{c}\text { Initial } \\
\text { Reduction }\end{array}$ & $\begin{array}{l}\text { Distal- } \\
\text { medial }\end{array}$ & $\begin{array}{c}\text { Carbonate } \\
\text { build-up }\end{array}$ & Chert \\
\hline 2158-10 & $73 / 82$ & $\begin{array}{l}243- \\
244\end{array}$ & 59.0 & 41.9 & 14.5 & 32.7 & 59 & 52 & & $\begin{array}{c}\text { Initial } \\
\text { Reduction }\end{array}$ & Complete & $\begin{array}{c}\text { Carbonate } \\
\text { build-up }\end{array}$ & Chert \\
\hline 2193-10 & $74 / 85$ & $\begin{array}{l}220- \\
230\end{array}$ & 61.6 & 69.2 & 28.2 & 80.6 & 44 & 60 & 59 & $\begin{array}{c}\text { Initial } \\
\text { Reduction }\end{array}$ & Complete & $\begin{array}{l}\text { Carbonate } \\
\text { build-up }\end{array}$ & Chert \\
\hline 2197-10 & $74 / 86$ & $\begin{array}{l}212- \\
220\end{array}$ & 31.2 & 33.6 & 7.3 & 6.4 & 65 & 61 & & $\begin{array}{c}\text { Initial } \\
\text { Reduction }\end{array}$ & Complete & $\begin{array}{c}\text { None } \\
\text { observed }\end{array}$ & Chert \\
\hline 2204-10 & $75 / 84$ & $\begin{array}{c}130- \\
140\end{array}$ & 50.9 & 39.5 & 11.4 & 19.5 & 55 & 54 & & $\begin{array}{c}\text { Initial } \\
\text { Reduction }\end{array}$ & Complete & $\begin{array}{c}\text { None } \\
\text { observed }\end{array}$ & Chert \\
\hline $2209-10$ & $75 / 86$ & $\begin{array}{l}220- \\
230\end{array}$ & 32.5 & 39.1 & 14.2 & 15.1 & 57 & & & $\begin{array}{c}\text { Initial } \\
\text { Reduction }\end{array}$ & Complete & Thermal & Chert \\
\hline $2226-10$ & $77 / 84$ & $\begin{array}{l}222- \\
230\end{array}$ & 43.0 & 42.7 & 15.9 & 25.5 & 77 & & & $\begin{array}{c}\text { Initial } \\
\text { Reduction }\end{array}$ & Complete & $\begin{array}{c}\text { None } \\
\text { observed }\end{array}$ & Chert \\
\hline $2229-10$ & $78 / 82$ & 231 & 28.8 & 38.8 & 11.4 & 12.7 & 54 & & & $\begin{array}{c}\text { Initial } \\
\text { Reduction }\end{array}$ & Distal & $\begin{array}{c}\text { None } \\
\text { observed }\end{array}$ & Chert \\
\hline
\end{tabular}




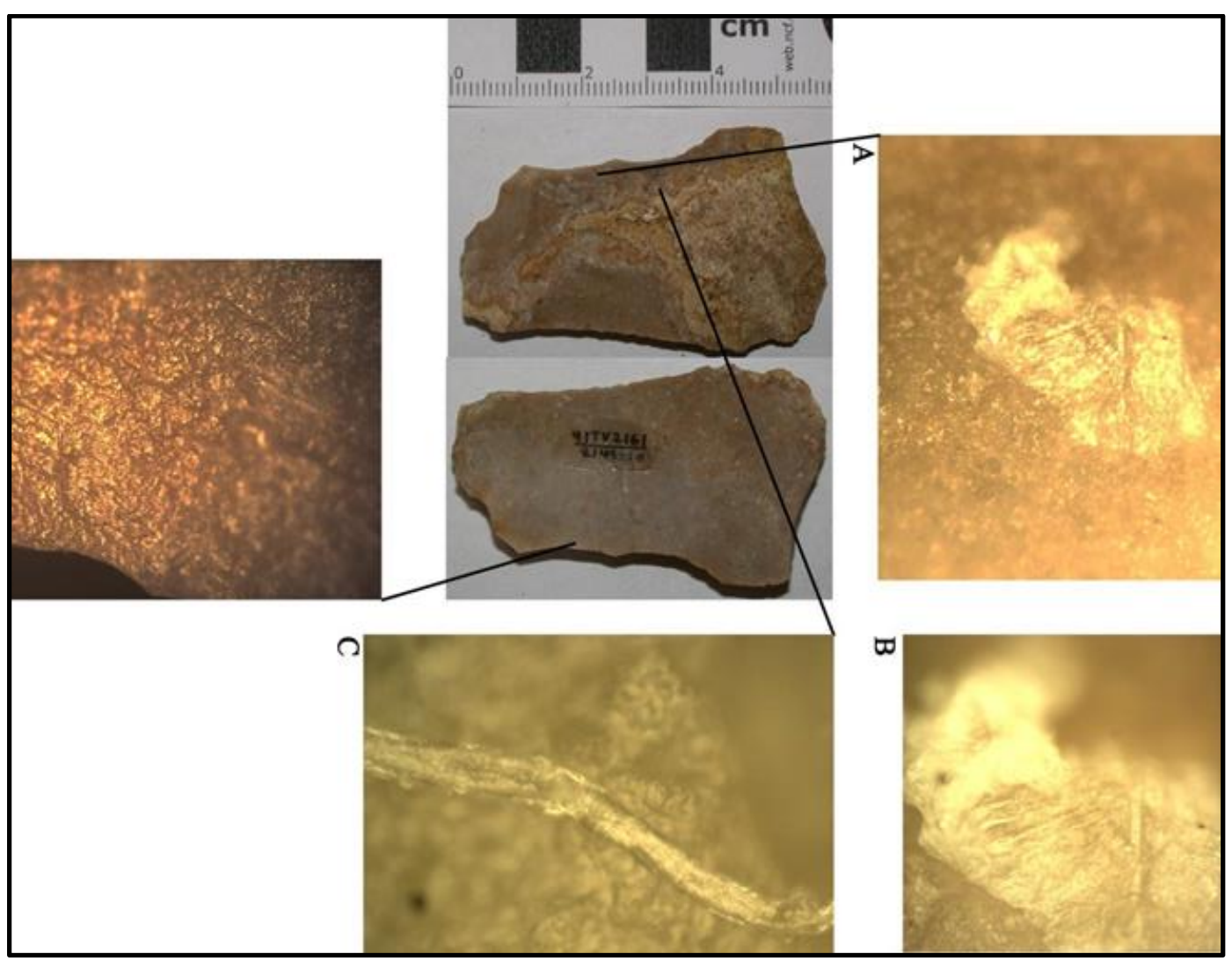

Figure 9-39. Multiple microphotographs of edge-modified flake \#2149-10 showing soft polish (far left), two views of ctenoid (toothed) fish scale (far right), and hair (bottom left), (see Appendix D).

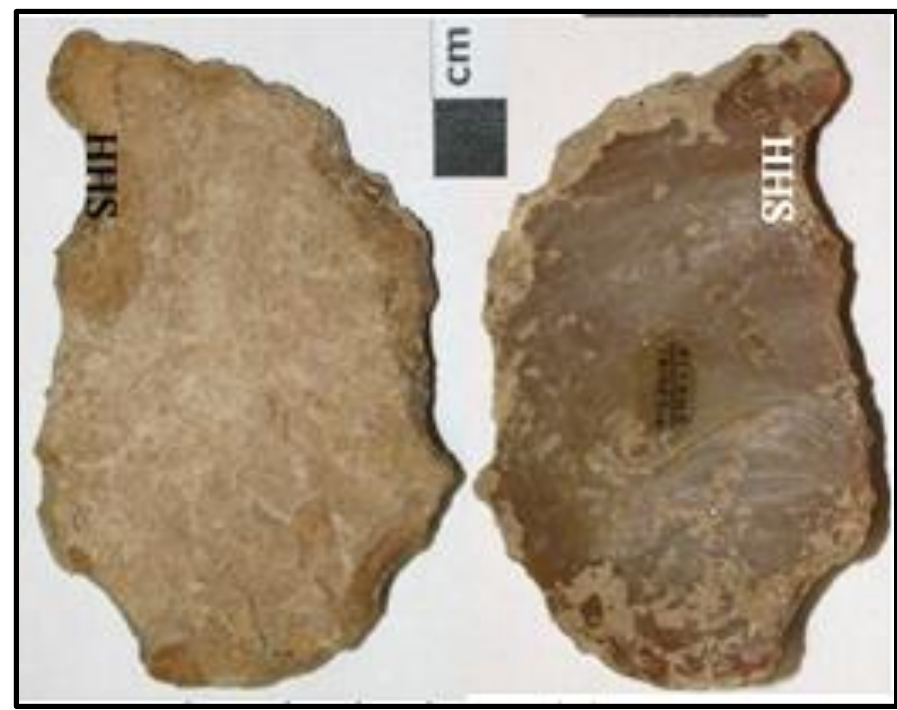

Figure 9-40. Dorsal and ventral views of an unwashed edge-modified flake (\#2114-10) depicting location of hard, high silica (HHS) polish in small concave notch area. 
Table 9-8. Summary of Bell/Andice High-Powered Use-Wear Analyses.

\begin{tabular}{|c|c|c|c|c|c|c|c|c|c|c|c|c|c|c|c|c|c|c|c|c|}
\hline \multirow[b]{2}{*}{$\begin{array}{c}\text { Artifact } \\
\text { Classes } \\
\text { Analyzed }\end{array}$} & \multicolumn{9}{|c|}{ Inferred Function } & \multicolumn{11}{|c|}{ Contact Material and Observed Residues } \\
\hline & $\begin{array}{l}\frac{c}{3} \\
\frac{1}{c} \\
\frac{c}{c} \\
\frac{c}{J}\end{array}$ & $\begin{array}{l}\frac{9}{0} \\
\frac{\overline{0}}{\pi} \\
\frac{\pi}{0} \\
c\end{array}$ & 年 & 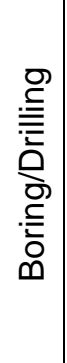 & 号 & $\begin{array}{l}\frac{0}{d} \\
\frac{\pi}{\pi} \\
\frac{\pi}{1}\end{array}$ & $\begin{array}{l}\frac{O}{\mathrm{C}} \\
\frac{\mathrm{U}}{\mathrm{C}}\end{array}$ & 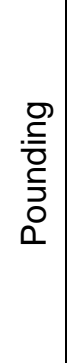 & $\begin{array}{l}\frac{0}{\bar{O}} \\
\frac{\overline{0}}{\circ} \\
\frac{0}{0} \\
\frac{1}{0}\end{array}$ & 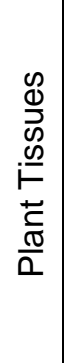 & $\frac{\frac{c}{0}}{\overline{0}}$ & $\begin{array}{l}0 \\
\frac{5}{0} \\
\varnothing\end{array}$ & 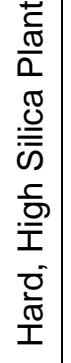 & $\begin{array}{l}\overline{0} \\
\vdots \\
3\end{array}$ & 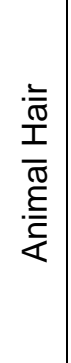 & 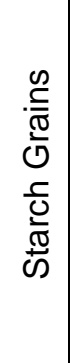 & 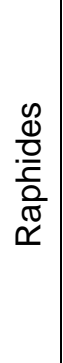 & $\stackrel{\frac{0}{0}}{\frac{0}{1}}$ & 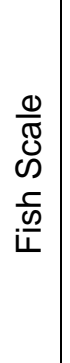 & $\begin{array}{l}\frac{5}{3} \\
0 \\
\frac{c}{c} \\
\frac{c}{5}\end{array}$ \\
\hline $\begin{array}{l}\text { Points } \\
(N=3)\end{array}$ & 1 & & & & & 2 & & & & & 2 & 1 & & & & & & & & 1 \\
\hline $\begin{array}{l}\text { Bifaces } \\
(N=3)\end{array}$ & 1 & & 2 & & & & & & & & 1 & & 2 & & & & 1 & & & \\
\hline $\begin{array}{l}\text { Edge- } \\
\text { Modified } \\
\text { Tools } \\
(N=7)\end{array}$ & & 2 & 2 & & 2 & & 1 & & & 1 & 1 & & 6 & 2 & 1 & & & & 1 & \\
\hline
\end{tabular}

The tool was manufactured from a pale brown (10YR 6/3) chert, and all three pieces have a slight reddish hue indicative of heat alteration. The extensive curvature is indicative of a heat spall from a larger partially, bifacially flaked tool as no obvious impact point was observed. Based on the thickness of the recovered pieces, the parent biface was minimally $2.5 \mathrm{~cm}$ thick and potentially much thicker. This three piece section is quite unusual and documents an early stage biface used, then subsequently discarded into a fire. These pieces were recovered immediately west of hearth Feature 24. High-powered use-wear analysis revealed soft polish and possible raphide residues along the very edge to indicate it was employed for cutting plants (Appendix D).

\subsubsection{Edge-Modified Flakes}

Edge-modified flakes are more numerous $(N=12)$ than the formal chipped tools. These informal tools reflect a variety of shapes and sizes, on relatively large chert flakes, generally with two modified edge. Figure 9-38 presents two examples of shape diversification supporting the lack of selection of a predetermined form for projected tasks. Table 9-7 presents basic observations and metric measurements with a few selected edge-modified flakes individually presented below as examples. Seven informal tools were sent for high-powered use-wear with individual details presented in Appendix D.

Edge-modified flake \#2149-10 was between 220 and $230 \mathrm{cmbs}$ in N72/E85 in Feature 22. This is a relatively large secondary decortification flake with nearly 50 percent cortex on the dorsal surface, parallel lateral edges, and a smooth slightly concave ventral face (Figure 9-39, see Table 9-7). The diffuse bulb of percussion indicates this flake was probably detached with a large billet on a thin, wide, ground, multifaceted platform. Both lateral edges exhibit tiny use-wear scars. High-powered use-wear analysis identified soft polish, a ctenoid (toothed/spiny) fish scale and hair fragment, which supports cutting of animals and fish (Appendix D). Ctenoid scales are usually found on fish with spiny fin rays, such as bass and crappie. 
Specimen \#2114-10 was between 220 and 230 cmbs in N71/E84. It is a large primary flake with calcium carbonate covered cortex, and short, broad flake scars along the lateral margins (Figure 9-40, see Table 9-7). The ventral surface is slightly concave with one major flake scar on the proximal end. Under UV light this flake fluoresces a dark orange unlike most Edwards chert.

\subsubsection{Summary of Chipped Stone Tools}

In summary, the small chipped stone tool assemblage consists of 3 projectile points, 3 bifaces, and 12 edge-modified flakes. The small and limited assemblage of chipped stone tools associated with the Bell/Andice component implies a short-term occupation and broad spectrum

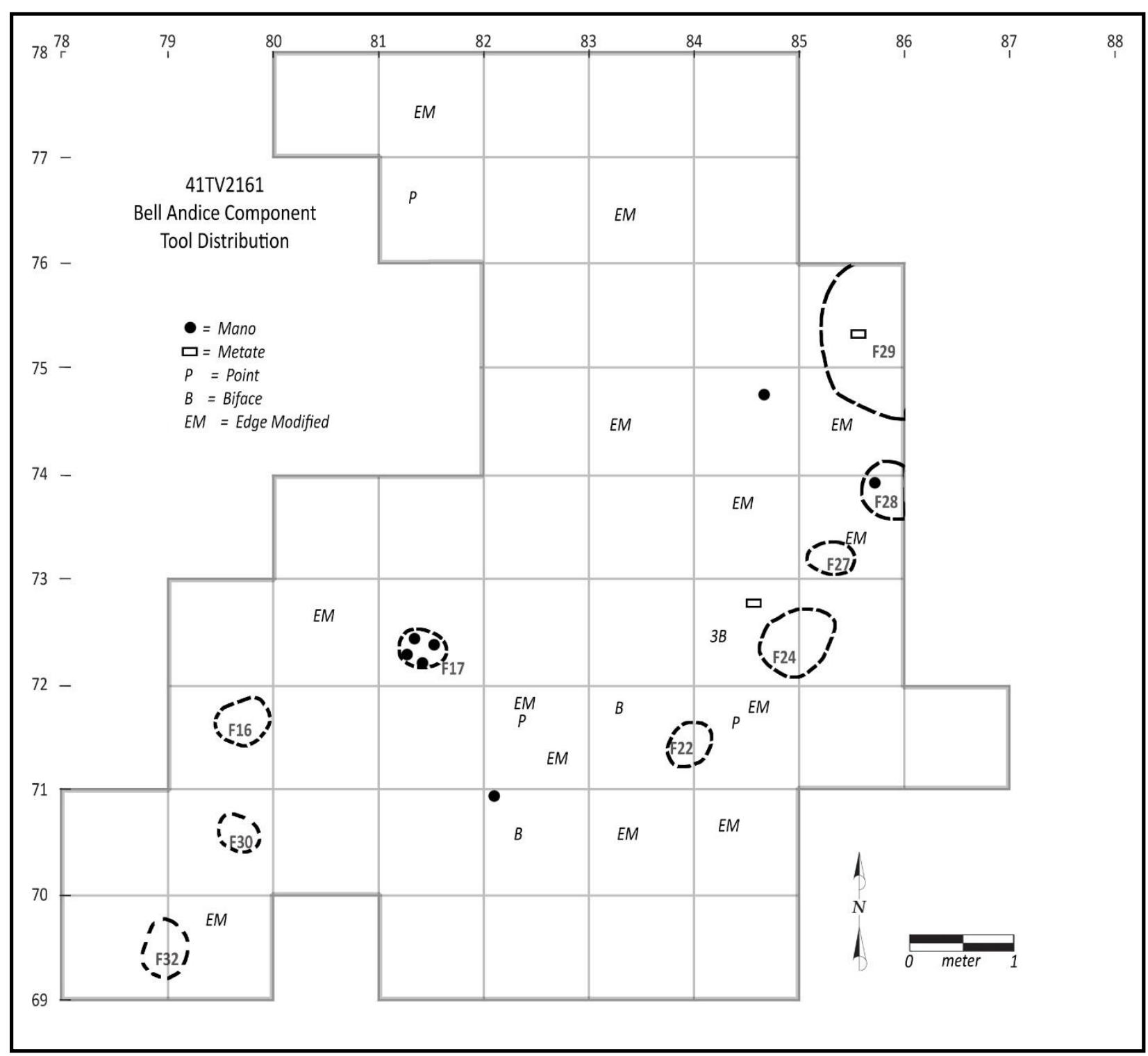

Figure 9-41. Horizontal distribution of Bell/Andice formal chipped stone tools, ground stone tools, and edge-modified flakes. 
foraging. High-powered use-wear analysis documented the more complete point was hafted as evident by polish in the haft area and also reveals bone collagen residues (Table 9-8). Bifaces were primarily employed for cutting hard, high silica materials (e.g.,bone, antler, wood, or plants with high silica content) rather than soft meat tissues, whereas the edge-modified flakes were employed in a variety of tasks such as, but not limited to, scraping, cutting, whittling on wood, cutting plants, cutting high silica materials, and cutting fish (Table 9-8). The INAA analysis reveals the adjacent hill top gravels are enriched in aluminum, as are the analyzed chert tools. Therefore, cherts in this assemblage are compositionally similar and probably from the hilltop or similar outcrops.

\subsubsection{Horizontal Distribution of Chipped Stone Tools}

The horizontal distribution of formal tools indicates a pattern of use and/or discards in an area near and between the multiple burned rock features (Figure 9-41). The Big Sandy point was north of the primary activity areas and appears isolated from the rest. The cluster of ground stone in Feature 17 was in the middle between two groups of burned rock features and may reflect a discarded cache of used tools that supported tasks in both areas. The limited excavated area of $51 \mathrm{~m}^{2}$ hinders our understanding of the adjacent work spaces and if other activities were present.

\subsection{GROUND STONE TOOL ASSEMBLAGE}

The ground stone assemblage includes eight pieces, five manos and three metate fragments. Each specimen is described below, beginning with the manos.

\subsubsection{Manos}

Specimen \#2106-10 is a complete, small onehanded sandstone mano from $237 \mathrm{cmbs}$ in N71/E83. Both faces exhibit light wear across slightly convex surfaces that are partially covered in calcium carbonate (Figures 9-42 and 9-43). Pecking to refurbish the surfaces is not apparent and no impacts or peck marks occur along the lateral edges. The entire piece was subjected to starch analysis and yielded evidence of grinding as well as a single, unidentified starch grain (Appendix F).

Specimen \#2164-10 is a small, complete onehanded mano from $237 \mathrm{cmbs}$ and part of Feature 17 in N73/E82 in Block B. One face is a worn slightly, convex, whereas the opposite face is very convex and lacks signs of use (Figures 9-43 and 9-44, Table 9-9). The surfaces are partially covered in calcium carbonate. No signs of peck marks or impacts are present. This mano was sent for starch analysis, but did not reveal starch grains (Appendix F).

Specimen \#2164-11 is a large, complete onehanded mano from $239 \mathrm{cmbs}$ and part of Feature 17 in N73/E82 in Block B. It exhibits one well-defined face that is slightly convex opposite a very convex face covered in calcium carbonate. This ovate mano has tiny linear striations across the worked face, but lacks any sign of impact or peck marks on the faces or lateral edges (Figures 9-43 and 9-45, see Table 9-9). The mano was subjected to pollen analysis, but pollen concentrations were extremely low (Appendix L).

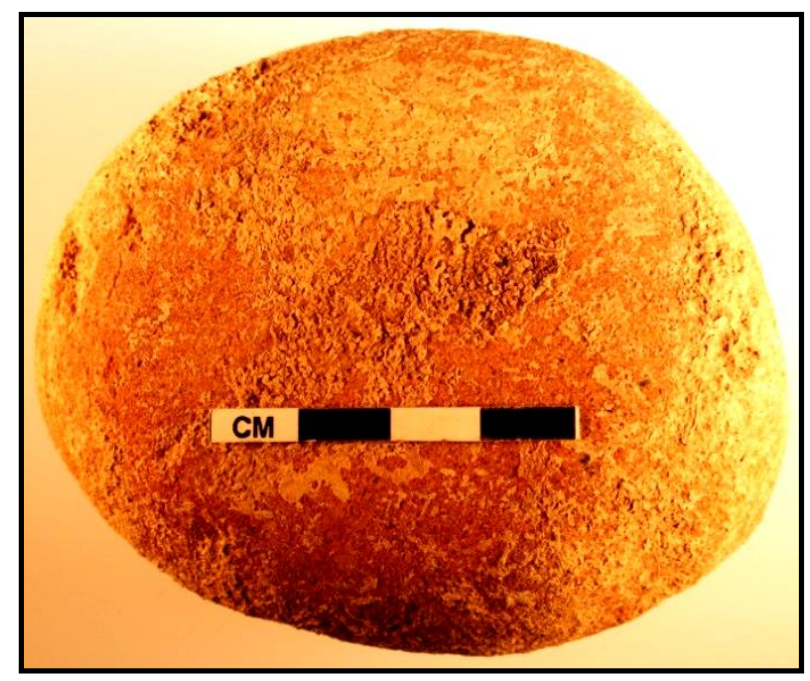

Figure 9-42. Complete mano (\#2106-10). 


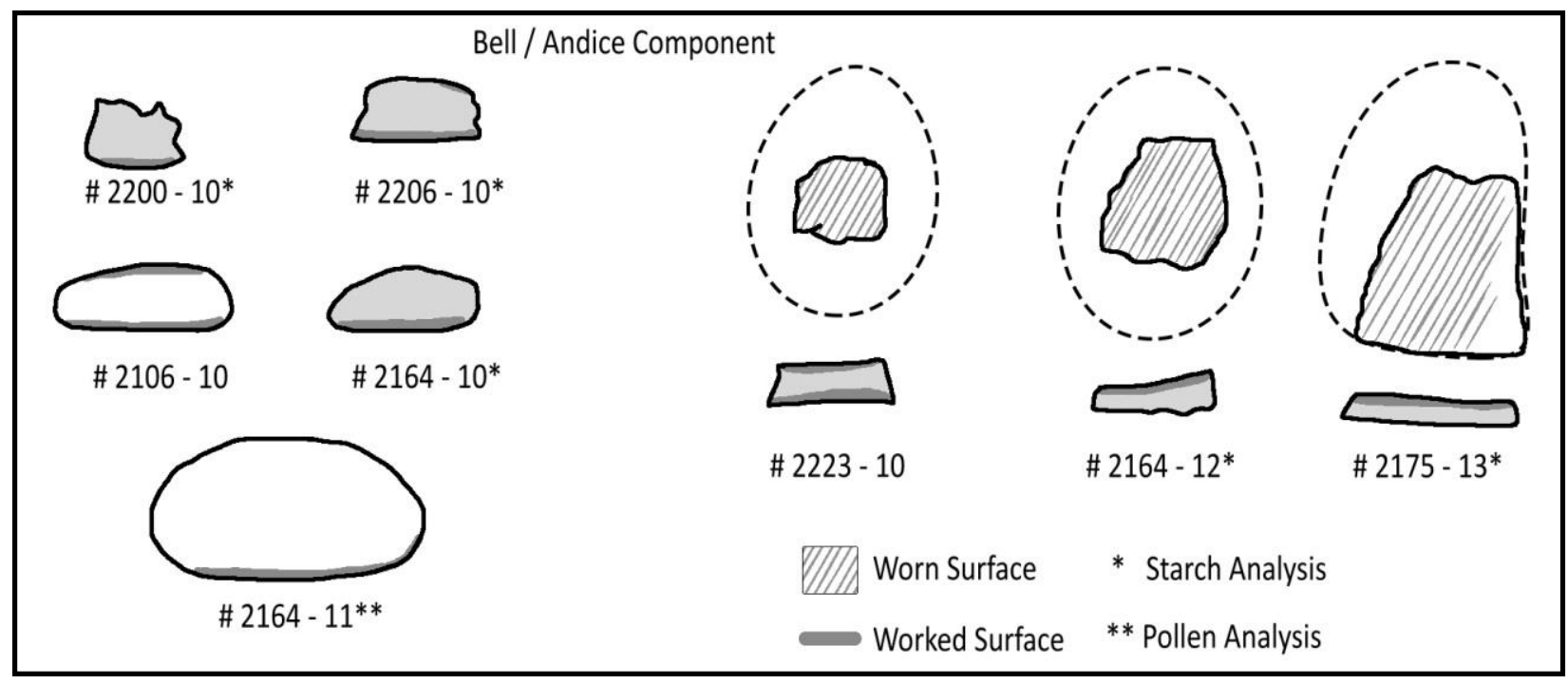

Figure 9-43. General profiles of manos and metates from Bell/Andice component.

Specimen \#2200-10 is a small mano fragment from between 218 and $231 \mathrm{cmbs}$ from Feature 28 in N74/E86 in Block B. This quartzite piece exhibits one relatively flat face with a light polish (see Figure 9-43), but also has two hackled edges to indicate it was in a fire, as well. This mano probably was recycled and incorporated into a heating element. Starch analysis failed to reveal starch grains (Appendix F).

Specimen \#2206-10 is a small mano fragment from between 230 and $235 \mathrm{cmbs}$ in N74/E86 in Block B. This course grained quartzite specimen has a weathered or potentially crushed distal end and lateral edges, with the opposite end broken (Figures 9-43 and 9-46, see Table 9-9). The two faces are only slightly convex with slight wear that lacks well-defined margins. No starch grains were detected (Appendix F).

\subsubsection{Metates}

Specimen \#2164-12 was from between 233 and 239 cmbs within Feature 17 in N73/E82 in Block B. This metate is fragmented and appears thermally fractured (Figures 9-43 and 9-47, see Table 9-9). The crumbly sandstone slab that is $25.8 \mathrm{~cm}$ thick, exhibits one slightly concave face opposite a nearly flat, rough bumpy face and both are covered in calcium carbonate. The main piece is wedgeshaped to indicate one worked face. The metate appeared discarded into a fire following it use as it appears heat fractured. Starch analysis failed to yield starch grains (Appendix F).

Specimen \#2175-13 is a metate fragment from 224 cmbs in N73/E825 and adjacent Feature 24 in Block B. This was an end section of a thin dense quartzite rock with one flat and smooth face opposite a flat and slightly irregular face (Figures 9-43 and 9-48, see Table 9-9). Three lateral edges appear natural and well-worn. The worked face is only slightly concave, but exhibits a well-defined ground margin towards the natural edge. No peck marks indicative of refurbishing are visible. Minor amounts of calcium carbonate are for the most part along the lateral edges and the unworked face.

This relatively large fragment was subdivided into multiple pieces for different technical analyses. One piece was subjected to starch analysis (\#217513a), one small piece for lipid residue (\#2175-13b), and another for a pollen wash (\#2175-13c) with one major section left unwashed. Starch analysis yielded two unidentified starch grains, a grass starch, and evidence of grinding as the original grain was altered (Appendix F). Lipid residue analysis of a $9.0 \mathrm{~g}$ piece yielded chemical residues 


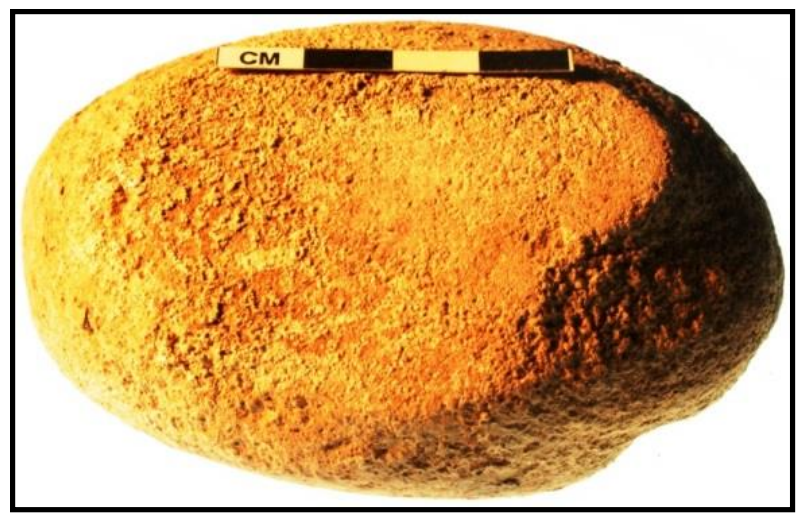

Figure 9-44. Complete mano (\#2164-10) from Feature 17.

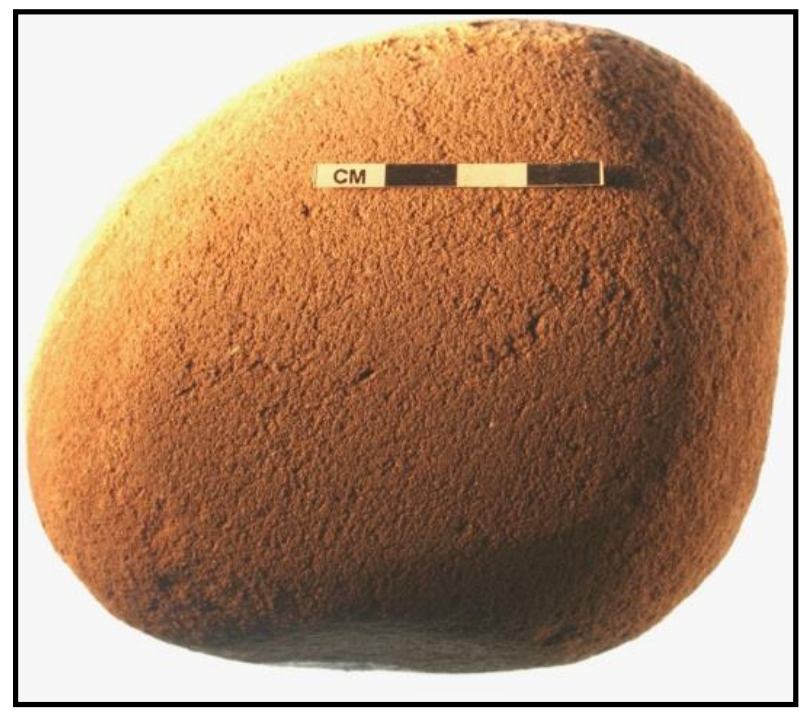

Figure 9-45. Complete mano (\#2164-11) from Feature 17 with use striations.

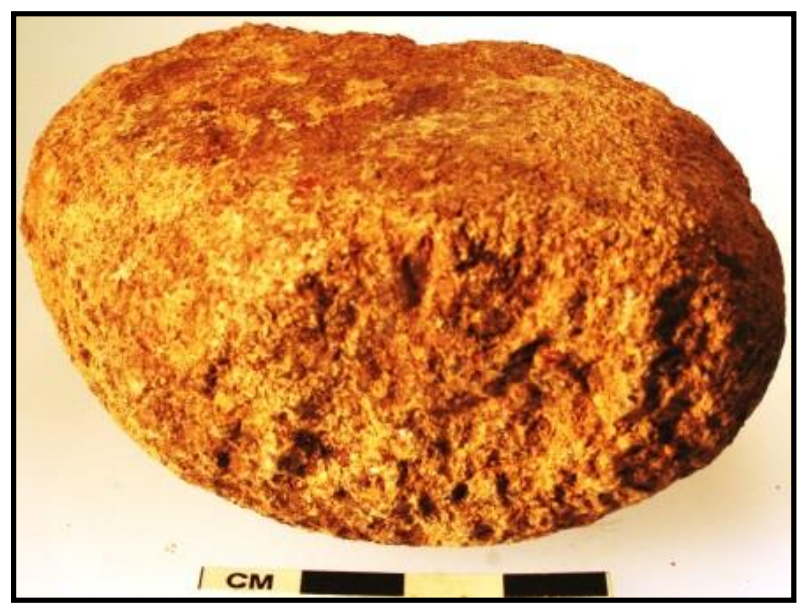

Figure 9-46. Mano fragmented (\#2206-10) with weathered end. from large herbivore meat mixed with animal fat and/or plants with biomarkers of cholesterol indicative of animal products and possibly dehydroabietic acid, which is from conifer products (Appendix G). The later residues probably reflect juniper or bald cypress trees in central Texas use as firewood to heat rocks. The pollen wash yielded no pollen concentration (Appendix L).

Metate fragment \#2223-10 was between 220 and 230 from Feature 29 in N76/E86 in Block B. This is a small, square-shaped sandstone fragment from the midsection of a metate with both faces worked as evidenced by slightly concave, smooth surfaces, but lacks visible striations or a distinctive polish (Figures 9-43 and 9-49, Table 9-9). All four edges are broken and the piece was probably recycled and discarded into a fire hearth. The specimen has a dark color reflecting organic residue from the fire.

\subsubsection{Summary of Ground Stone Tools}

In summary, this ground stone assemblage of five small one-handed manos and three metate fragments indicates food preparation through intentional grinding. Starch grain analysis on six of eight pieces supports this grinding function with observations of ground grains on two pieces, a damaged grain on another piece, three unidentifiable grains, and one grass grain on a metate. The presence of grass grains on a metate (\#2175-13) support grass was a food resource rather than just incidental or fuel for fires. The lipid residue analysis on metate fragment \#2175-13 indicate large herbivore meat mixed with animal fat and/or plants, plus biomarkers of cholesterol, documenting meat products. Possibly dehydroabietic acid, which is from conifer residues and probably reflects juniper or bald cypress trees in central Texas were employed as firewood to heat rocks. Apparently meat and plant products were placed on this metate. The horizontal distribution of these ground stone artifacts was generally associated with burned rock features with one apparent cache or discarded artifacts (Feature 17) 
Table 9-9. Bell/Andice Ground Stone Data.

\begin{tabular}{|c|c|c|c|c|c|c|c|c|c|c|c|c|c|}
\hline PNUM & Class & $\begin{array}{c}\text { Feature } \\
\text { No. }\end{array}$ & 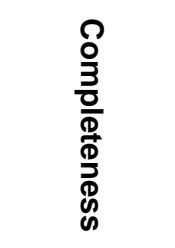 & 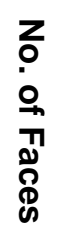 & 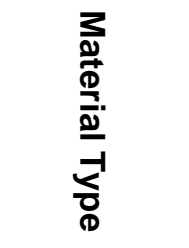 & 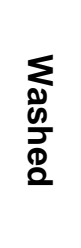 & 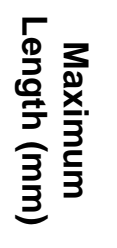 & 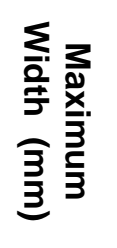 & 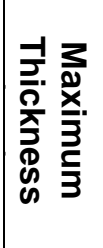 & $\frac{\substack{\Phi \\
\frac{0}{0}}}{\stackrel{0}{a}}$ & 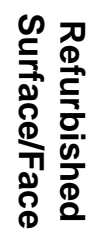 & 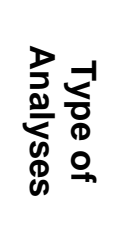 & Observations \\
\hline $2106-10$ & Mano & None & Complete & 2 & Sandstone & Yes & 88.0 & 76.1 & 41.3 & 386 & No & Starch & $\begin{array}{l}\text { Partially covered in } \\
\text { calcium carbonate, }\end{array}$ \\
\hline 2164-10 & Mano & 17 & Complete & 1 & Sandstone & No & 89.4 & 64.6 & 43.3 & 326 & No & Starch & $\begin{array}{l}\text { Partially covered in } \\
\text { calcium carbonate, }\end{array}$ \\
\hline 2164-11 & Mano & 17 & Complete & 1 & Sandstone & Yes & 122.9 & 101.8 & 64.3 & $\begin{array}{c}105 \\
5\end{array}$ & No & Pollen & $\begin{array}{l}1 / 2 \text { covered in } \\
\text { calcium carbonate }\end{array}$ \\
\hline 2164-12 & Metate & 17 & $\begin{array}{c}\text { Multiple } \\
\text { Fragments }\end{array}$ & 1 & Sandstone & No & 90.6 & 79.3 & 25.8 & 616 & None & Starch & $\begin{array}{l}\text { Likely recycled as } \\
\text { burned rock, } \\
\text { multiple fragments, } \\
\text { covered in calcium } \\
\text { carbonate, }\end{array}$ \\
\hline $2175-13$ & Metate & $\begin{array}{l}\text { Next to } \\
24\end{array}$ & $\begin{array}{l}\text { Lateral } \\
\text { Fragment }\end{array}$ & 1 & $\begin{array}{c}\text { Gray } \\
\text { Quartzite }\end{array}$ & Yes & 185.0 & 156.0 & 27.7 & $\begin{array}{c}140 \\
1\end{array}$ & No & $\begin{array}{l}\text { Starch, } \\
\text { Pollen, } \\
\text { Lipid }\end{array}$ & $\begin{array}{l}\text { Minor calcium } \\
\text { carbonate, broken } \\
\text { for different } \\
\text { analyses } \\
\end{array}$ \\
\hline $2200-10$ & $\begin{array}{c}\text { Mano/ } \\
\text { Burned } \\
\text { Rock }\end{array}$ & 28 & Fragment & 1 & Quartzite & Yes & 36.6 & 58.7 & 43.2 & 117 & No & Starch & $\begin{array}{l}\text { Partially covered in } \\
\text { calcium carbonate, } \\
\text { possible recycled } \\
\text { as burned rock }\end{array}$ \\
\hline $2206-10$ & Mano & None & Medial & 1 & Quartzite & No & 81.3 & 67.2 & 43.4 & 251 & No & Starch & $\begin{array}{c}\text { Partially covered in } \\
\text { calcium carbonate, } \\
\text { possible recycled } \\
\text { as burned rock }\end{array}$ \\
\hline $2223-10$ & $\begin{array}{c}\text { Metate/ } \\
\text { Burned } \\
\text { Rock }\end{array}$ & 29 & Fragment & 2 & Sandstone & No & 73.9 & 59.5 & 33.2 & 239 & None & Starch & $\begin{array}{c}\text { Likely heated, } \\
\text { recycled, partially } \\
\text { covered in calcium } \\
\text { carbonate }\end{array}$ \\
\hline
\end{tabular}




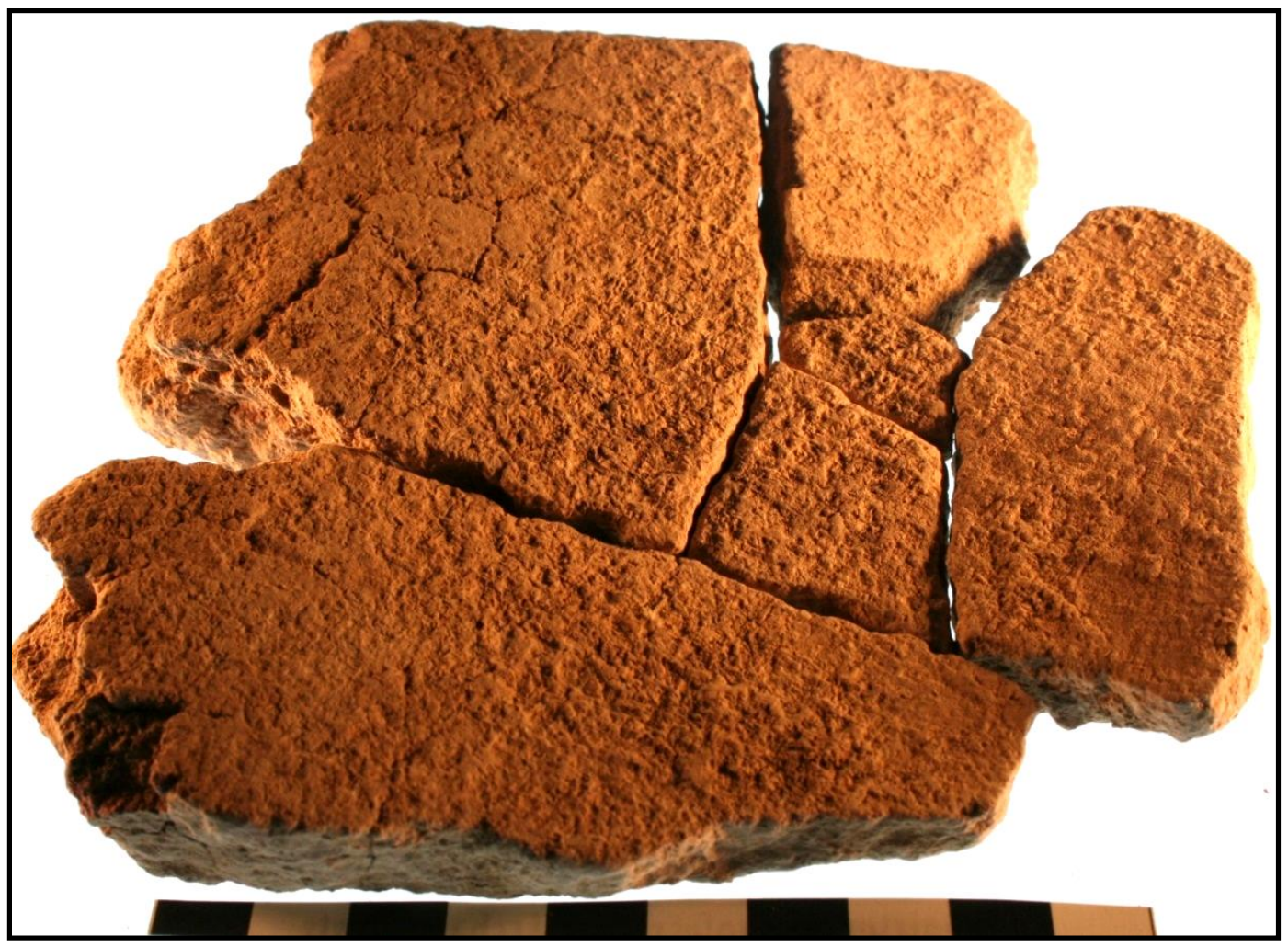

Figure 9-47. Oblique view of fragmented metate (\#2164-12) from Feature 17 (scale in centimeters).

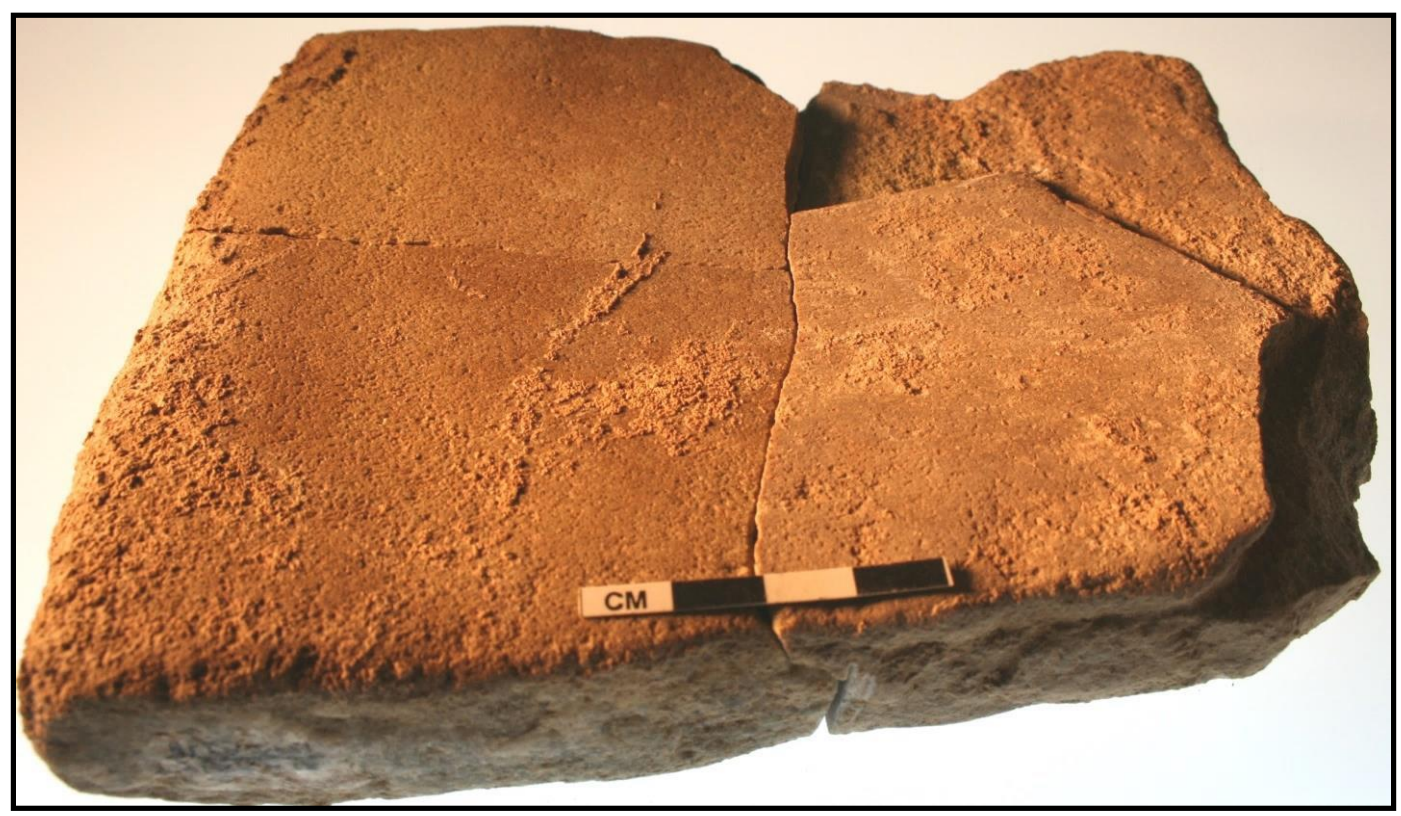

Figure 9-48. Oblique view of fragmented metate (\#2175-13) from next to Feature 24 (scale in centimeters). 


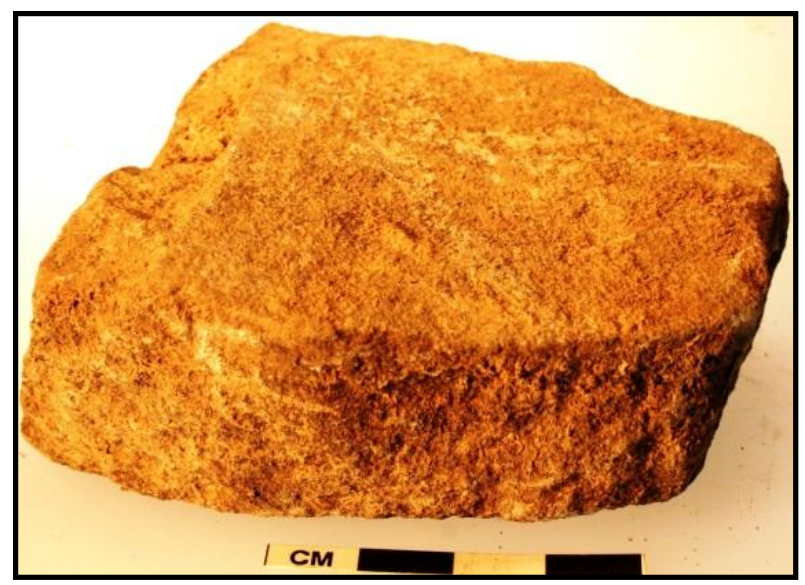

Figure 9-49. Oblique view of metate fragment (\#2223-10).

away from cooking features (see Figure 9-43). The fact that at least two, and possibly three, items were burned indicate either recycling of pieces following their use or the intentional discarding into fires as part of camp maintenance and cleaning.

\subsection{LITHIC DEBITAGE}

Benjamin G. Bury

\subsubsection{Introduction}

About 99 percent of the lithic debitage was determined to be local derived cherts from the adjacent gravelly hilltop as determined by INAA (Appendix H). No specifically identified nonlocal materials were identified in this assemblage. The analysis presented was structured around a free standing typology based on the morphological classification of debitage and the measurement of attributes (see Chapter 5.0). The benefit of an attribute-level approach is that different typologies can be applied within a single dataset and explored for patterning. The drawback of this approach is that it is time consuming. Therefore, platform bearing flakes (complete flakes and proximal flake fragments) were selected for in-depth analysis, and all attributes outlined in the TxDOT protocol were included. The specific observations and details concerning the flakes and their various attributes are presented followed by a brief discussion of other debitage classes and cores. A more detailed discussion of the Bell-Andice and Martindale components is included in Chapter 11.0. Lithic debitage recovered during excavation of the Bell/Andice component included 71 complete flakes, 95 incomplete flakes (distal flake fragments) and angular debris, and 14 proximal flake fragments. One flake fragment with edge modification was identified during the analysis and included in the following.

\subsubsection{Complete Flakes}

Table 9-10 lists the amount of cortex and number of flake scars recorded on the dorsal surface of complete flakes. The number of flake scars and the percentage of cortex is a good general indicator of the degree of reduction within an assemblage, with an increase in flake scars and corresponding decrease in cortex as raw materials are reduced (Odell 1989). From the perspective of a cortical typology, the assemblage of complete flakes is primarily composed of secondary flakes with less than 75 percent dorsal cortex, followed closely by tertiary flakes with no dorsal cortex, and primary flakes with 76 percent dorsal cortex or greater. The majority of the flakes have some dorsal cortex ( $N=$ $39 / 54.9$ percent). Correspondingly, the majority have between one and two dorsal flake scars, and only a small number were completely cortical with no scars. Based on the amount of dorsal cortex and the number of dorsal flake scars, the assemblage reflects a greater proportion of flakes from middle stages of reduction.

When an application load typology is applied to the database, a similar pattern is apparent. The majority of the flakes have pronounced bulbs of percussion (Table 9-11). A sizeable number have moderately developed bulbs, and only 19.7 percent $(N=14)$ have absent or slightly developed bulbs. Additionally, the presence or absence of eraillure flake scars was documented, with 29.5 percent $(N=$ 21) exhibiting a scar. In general, the size of the bulb and presence of an eraillure scar has been shown to 
Table 9-10. Dorsal Cortex and Scar Counts for Complete Flakes.

\begin{tabular}{|c|c|c|c|c|c|}
\hline \multicolumn{3}{|c|}{ Dorsal Cortex } & \multicolumn{3}{c|}{ Dorsal Scars } \\
\hline Class & Count & Percent & Class & Count & Percent \\
\hline 0 & 32 & 45.07 & 0 & 3 & 4.23 \\
\hline $1-25$ & 18 & 25.35 & $1-2$ & 43 & $60-56$ \\
\hline $26-50$ & 10 & 14.08 & $3-4$ & 22 & 30.99 \\
\hline $51-75$ & 6 & 8.45 & $5-6$ & 3 & 4.23 \\
\hline $76-100$ & 5 & 7.04 & $7+$ & 0 & \\
\hline Total & 71 & 100.00 & Total & 71 & 100.00 \\
\hline
\end{tabular}

Table 9-11. Bulb Size and Eraillure Scar Frequency for Complete Flakes.

\begin{tabular}{|c|c|c|c|c|c|}
\hline \multicolumn{3}{|c|}{ Bulb } & \multicolumn{3}{c|}{ Eraillure Scar } \\
\hline Class & Count & Percent & Class & Count & Percent \\
\hline Absent & 14 & 19.72 & Absent & 50 & 70.42 \\
\hline Moderate & 27 & 38.03 & Present & 21 & 29.58 \\
\hline Pronounced & 30 & 42.25 & & & \\
\hline Total & 71 & 100.00 & Total & 71 & 100.00 \\
\hline
\end{tabular}

Table 9-12. Lipping and Longitudinal Curvature for Complete Flakes.

\begin{tabular}{|c|c|c|c|c|c|}
\hline \multicolumn{3}{|c|}{ Lipping } & \multicolumn{3}{c|}{ Longitudinal Curvature } \\
\hline Class & Count & Percent & Class & Count & Percent \\
\hline Absent & 64 & 90.14 & Absent & 69 & 97.18 \\
\hline Moderate & 6 & 8.45 & Moderate & 2 & 2.82 \\
\hline Pronounced & 1 & 1.41 & Pronounced & 0 & 0.00 \\
\hline Total & 71 & 100.00 & Total & 71 & 100.00 \\
\hline
\end{tabular}

Table 9-13. Platform Class and Preparation for Complete Flakes.

\begin{tabular}{|c|c|c|c|c|c|}
\hline \multicolumn{3}{|c|}{ Platform Class } & \multicolumn{3}{c|}{ Platform Preparation } \\
\hline Class & Count & Percent & Class & Count & Percent \\
\hline Single Facet & 35 & 49.30 & Absent & 30 & 42.25 \\
\hline Multifaceted & 16 & 22.54 & Present & 37 & 52.11 \\
\hline Collapsed & 9 & 12.68 & N/A & 4 & 5.63 \\
\hline Cortical & 8 & 11.27 & & & \\
\hline Abraded & 3 & 4.23 & & & \\
\hline Total & $\mathbf{7 1}$ & $\mathbf{1 0 0 . 0 0}$ & Total & $\mathbf{7 1}$ & 100.00 \\
\hline
\end{tabular}


correspond to the amount of applied force and the nature of the percussor, with hard hammers tending to produce larger, more pronounced bulbs (Andrefsky 2005, 2006; Crabtree 1972). From the perspective of an application load typology, the majority of the assemblage is representative of frequent use of hard-hammer percussion during early and middle stages of reduction.

Technological typologies typically differentiate between bipolar flakes, core reduction flakes, bifacial thinning flakes, and more specific forms related to tool sharpening etc. Core reduction flakes are usually defined by single facet platforms, larger dimensions, greater amounts of dorsal cortex, and less pronounced platform lipping and longitudinal curvature. Biface thinning flakes are typically defined as having "curved longitudinal crosssections, extremely acute lateral and distal edge angles, feathered flake terminations, narrow faceted striking platforms, a lip, little or no cortex, and a small flattened or diffuse bulb of force" (Andrefsky 2006:118; Root 1992:83). Typically, these attributes are not measured separately, but assessed visually as a whole for individual flakes. During this analysis, a tentative technological category was assigned to each flake. In order of abundance, these included core reduction flakes $(\mathrm{N}=59 / 83.09$ percent), biface shaping or thinning flakes $(\mathrm{N}=$ $10 / 21.55$ percent), and one possible platform trimming flake ( $\mathrm{N}=1 / 1.40$ percent). Although a technological assessment of the assemblage is subjective, the general pattern is consistent with the other typologies.

Lipping of the ventral margin of the platform and longitudinal curvature were both documented independently (Table 9-12). Both attributes are not clearly understood and different studies have produced conflicting results (Andrefsky 2006). However, platform lipping is generally thought to occur more frequently with soft-hammer percussion (Andrefsky 2006; Hovers 2009). In some studies, longitudinal curvature has been shown to be associated with biface production (Hayden and Hutchings 1989). Lipping was placed into three ordinal classes. The majority of the platforms were categorized as slightly lipped or exhibited no lipping. Only 9 percent $(N=7)$ of the flakes exhibited a moderate to strongly developed lip. Longitudinal curvature was recorded on a nominal scale. The majority had absent or slightly discernable curvature. Only two (2.8 percent) had moderate to pronounced longitudinal curvature. The lack of lipping and longitudinal curvature in the assemblage suggests that most of the flakes may not have been produced with soft hammer percussion.

Platform characteristics can be applied within both an application load and a technological typology. Table 9-13 lists the platform classes and the presence or absence of preparation along the dorsal margin of the platform. With the exception of single facet platforms, platform types are often difficult to categorize, especially with smaller flakes. For example, it can be difficult to discern if facets on very small platforms were created from preparative abrasion, crushing of the surface during percussion, or the result of flake scars from previous flake removals (multifaceted). If abraded and multifaceted platforms are combined, then 26.76 percent $(N=19)$ of the platforms can be characterized as more complex than a single facet. Overall, the majority exhibit some form of preparation (trimming or abrasion) of the dorsal margin.

Table 9-14 lists counts of termination types and the distribution of flakes within five size classes. The majority have feathered terminations and fall into the $1 / 2$ inch size class. Hinged terminations are also present in significant quantities and generally occur more frequently with hard-hammer percussion (Andrefsky 2006; Patten 1999). Flakes with step terminations were classified as proximal flake fragments given the difficulty in distinguishing between step terminations and post-depositional breaks. 
Table 9-14. Size Class and Termination Types for Complete Flakes.

\begin{tabular}{|c|c|c|c|c|c|}
\hline \multicolumn{3}{|c|}{ Size Class } & \multicolumn{3}{c|}{ Termination } \\
\hline Class & Count & Percent & Class & Count & Percent \\
\hline$<1 / 4$ inch & 2 & 2.82 & Feather & 42 & 59.15 \\
\hline $1 / 4$ inch & 12 & 16.90 & Hinge & 24 & 33.80 \\
\hline $1 / 2$ inch & 22 & 30.99 & Axial & 3 & 4.23 \\
\hline $3 / 4$ inch & 16 & 22.54 & Overshot & 2 & 2.82 \\
\hline$>1$ inch & 19 & 26.76 & & & \\
\hline Total & 71 & 100.00 & Total & 71 & 100.00 \\
\hline
\end{tabular}

Table 9-15. Metric Dimensions for Complete Flakes.

\begin{tabular}{|c|c|c|c|c|c|c|c|}
\hline \multicolumn{3}{|c|}{ Platform Dimensions } & \multicolumn{4}{c|}{ Flake Dimensions } \\
\hline Attribute & Mean & StD & CV & Attribute & Mean & StD & CV \\
\hline Thickness & 9.25 & 14.83 & 1.60 & Maximum & 38.12 & 16.11 & 0.42 \\
\hline Width & 17.70 & 9.87 & 0.56 & Length & 31.16 & 14.91 & 0.48 \\
\hline \multicolumn{3}{|c}{} & Width & 31.19 & 13.58 & 0.43 \\
\cline { 4 - 7 } & & Thickness & 8.57 & 4.87 & 0.56 \\
\cline { 4 - 7 } & & Weight & 10.14 & 11.49 & 1.13 \\
\hline
\end{tabular}

Heat treatment within the assemblage was grouped within three ordinal categories. Flakes with obvious signs of thermal damage (e.g., potlidding, crazing, etc.) were categorized as thermally altered. Flakes with a waxy luster or traces of reddish colors were categorized as potentially heat treated. Flakes with no signs of heat treatment were categorized as not thermally altered. Within the sample of complete flakes, 73.23 percent $(N=52)$ are considered not thermally altered, 14.08 percent $(N=10)$ are potentially heat treated, and 12.67 percent $(N=9)$ are clearly thermally altered.

Table 9-15 lists the metric attributes measured within the assemblage, and their mean, standard deviation (StD) and coefficient of variation (CV). The maximum dimension of the flakes is the least variable of the attributes $(\mathrm{CV}=0.46)$, and platform thickness is the most variable $(\mathrm{CV}=1.6)$. Weight is also highly variable $(\mathrm{CV}=1.13)$. The degree of variability in the assemblage suggests that some flakes from all stages of reduction are likely represented in the sample.

\subsubsection{Proximal Flake Fragments}

Flakes with broken terminations are often indistinguishable from complete flakes with step terminations and were classified here as proximal flake fragments. Table 9-16 lists the nonmetric attributes measured within the assemblage $(N=14)$. The typical proximal flake fragment is nearly identical to the average complete flake, and has a single facet platform $(N=6 / 42.8$ percent $)$, an absence of dorsal cortex ( $N=7 / 50.0$ percent), a moderately developed bulb $(N=6 / 42.8$ percent $)$, absent or slight platform lipping $(N=11 / 78.5$ percent), platform preparation $(N=8 / 57.1$ percent $)$, between 1-2 dorsal flake scars $(N=10 / 71.4$ percent), absent to slight longitudinal curvature $(N$ $=13 / 92.8$ percent $)$, and no eraillure scar $(N=$ $11 / 78.5$ percent). 
Table 9-16. Nonmetric Attributes Recorded on Proximal Flake Fragments.

\begin{tabular}{|c|c|c|c|c|c|c|c|}
\hline Attribute & Category & Count & Percent & Attribute & Category & Count & Percent \\
\hline \multirow{6}{*}{ Cortex } & 0 & 7 & 50.00 & \multirow{6}{*}{ Scar Count } & 0 & 1 & 7.14 \\
\hline & $1-25$ & 3 & 21.43 & & $1-2$ & 10 & 71.43 \\
\hline & $26-50$ & 1 & 7.14 & & $2-3$ & 1 & 7.14 \\
\hline & $51-75$ & 2 & 14.29 & & $3-4$ & 2 & 14.29 \\
\hline & $76-100$ & 1 & 7.14 & & $5+$ & 0 & 0.00 \\
\hline & Total & 14 & 100.00 & & Total & 14 & 100.00 \\
\hline \multirow{4}{*}{ Bulb } & Absent & 4 & 28.57 & \multirow{4}{*}{ Eraillure } & Absent & 11 & 78.57 \\
\hline & Moderate & 6 & 42.86 & & Present & 3 & 21.433 \\
\hline & Pronounced & 4 & 28.57 & & & & \\
\hline & Total & 14 & 100.00 & & Total & 14 & 100.00 \\
\hline \multirow{4}{*}{ Lipping } & Absent & 11 & 78.57 & \multirow{4}{*}{ Long. Curv. } & Absent & 13 & 92.86 \\
\hline & Moderate & 3 & 21.43 & & Moderate & 1 & 7.14 \\
\hline & Pronounced & 0 & 0.00 & & Pronounced & 0 & 0.00 \\
\hline & Total & 14 & 100.00 & & Total & 14 & 100.00 \\
\hline \multirow{6}{*}{ Platform } & Single Facet & 6 & 42.86 & \multirow{6}{*}{ Size Class } & $<1 / 4$ inch & 1 & 7.14 \\
\hline & Multifacet & 3 & 21.43 & & $1 / 4$ inch & 3 & 21.43 \\
\hline & Collapsed & 1 & 7.14 & & $1 / 2$ inch & 2 & 14.29 \\
\hline & Abraded & 1 & 7.14 & & $3 / 4$ inch & 6 & 42.86 \\
\hline & Cortical & 3 & 21.43 & & $>1$ inch & 2 & 14.29 \\
\hline & Total & 14 & 100.00 & & Total & 14 & 100.00 \\
\hline \multirow{3}{*}{$\begin{array}{l}\text { Platform } \\
\text { Prep. }\end{array}$} & Absent & 6 & 42.86 & & & & \\
\hline & Present & 8 & 57.14 & & & & \\
\hline & Total & 14 & 100.00 & & & & \\
\hline
\end{tabular}

Table 9-17. Metric Dimensions for Proximal Flake Fragments.

\begin{tabular}{|c|c|c|c|c|c|c|c|}
\hline \multicolumn{3}{|c|}{ Platform Dimensions } & \multicolumn{4}{c|}{ Flake Dimensions } \\
\hline Attribute & Mean & StD & CV & Attribute & Mean & StD & CV \\
\hline Thickness & 11.65 & 6.01 & 0.52 & Maximum & 31.96 & 15.59 & 0.49 \\
\hline Width & 31.96 & 15.59 & 0.49 & Length & 26.49 & 14.22 & 0.54 \\
\hline \multicolumn{3}{|c}{} & Width & 25.60 & 11.00 & 0.43 \\
\cline { 4 - 7 } & & Thickness & 5.81 & 3.50 & 0.60 \\
\cline { 4 - 7 } & & Weight & 6.08 & 8.09 & 1.33 \\
\hline
\end{tabular}


Heat treatment within the sample shows a similar pattern to complete flakes, with 42.85 percent $(N=$ 6) not thermally altered, 35.71 percent $(N=5)$ potentially thermally altered, and 21.42 percent $(N$ $=3$ ) clearly thermally altered.

Table 9-17 lists the metric attributes measured within the assemblage, and their mean, standard deviation (StD) and coefficient of variation (CV). The width of the flakes is the least variable of the attributes $(\mathrm{CV}=0.43)$, and weight is the most variable $(\mathrm{CV}=1.33)$. As expected, the sample of proximal flake fragments is smaller in most dimensions than the complete flakes, but have larger platforms. This suggests that a portion of the proximal flake fragments are complete flakes with step terminations, and may have terminated prematurely due to excessive force or a mistake.

\subsubsection{Other Debitage}

In addition to complete flakes and proximal flake fragments, the Bell/Andice assemblage consists of 95 flake fragments and pieces of angular debitage averaging 3.0 grams in weight. Over 50 percent showed signs of thermal alteration. This is much higher than the amount of thermal alteration seen in the sample of complete flakes and proximal flake fragments, and suggests that smaller pieces of debitage may have been selectively disposed of in thermal features.

\subsubsection{Cores}

Seven cores were recovered during the excavation of the Bell/Andice component. Table 9-18 lists the mean, standard deviation (StD) and coefficient of variation $(\mathrm{CV})$ of the metric dimensions recorded within the assemblage. Overall, the cores are relatively consistent in their dimensions, with the most variation in weight $(\mathrm{CV}=0.74)$. Table 9-19 lists the nonmetric attributes documented within the assemblage. The majority had less than 50 percent total cortical cover, between 5 to 8 flake scars, and were reduced from multiple directions. Cortex on all of the cores was formed from a combination of fluvial processes and chemical weathering from surface exposure, and were produced from locally available cobbles. One core showed evidence of possible heat treatment in the form of reddening and a waxy luster (Figure 9-50). Overall, the ratio of cores to lithic debitage within the Bell/Andice component is $1: 25.71$.

\subsubsection{Summary and Discussion of Lithic Debitage}

The typical complete flake from the Bell/Andice assemblage has a single facet platform $(N=34 / 47.8$ percent), a feathered termination $(N=41 / 57.7$ percent), an absence of dorsal cortex $(N=32 / 45.0$ percent), a moderately developed bulb $(N=27 / 38.0$ percent), absent or slight platform lipping $(N=$ $62 / 87.3$ percent), platform preparation $(N=32 / 52.1$ percent), between 1-2 dorsal flake scars $(N=$ $43 / 60.5$ percent), absent to slight longitudinal curvature $(N=69 / 97.1$ percent), and no eraillure scar $(N=50 / 70.4$ percent $)$. Proximal flake fragments were found to be nearly identical. Overall, this assemblage is skewed towards early and middle stage reduction and the ratio of cores to debitage is correspondingly low. Rather than a complete reduction sequence where bifacial tools were produced from local cobbles, the assemblage appears to represent two separate reduction strategies, one that involved simple core-flake reduction of local cobbles, and a second that involved the maintenance of existing bifacial tools.

Even though lithic debitage was sparse and only averaged less than four pieces per square meter, the horizontal distribution reveals obvious patterning (Figure 9-51). The highest concentration $(N=21)$ of debitage was localized in one unit (2 percent of units) directly between the two spatially distinct feature concentrations just west of Feature 22, a concentration of discarded burned rocks. That unit yielded 11 flake fragments, one angular debris, and nine complete flakes. Of these, three were from core reduction, four were further down the line (potentially early to middle bifacial reduction), and 
Table 9-18. Metric Dimensions for Cores (measurements in $\mathrm{mm}$ ).

\begin{tabular}{|c|c|c|c|}
\hline Dimension & Mean & StD & CV \\
\hline Length & 63.43 & 14.24 & 0.22 \\
\hline Width & 49.25 & 10.17 & 0.20 \\
\hline Thickness & 32.60 & 10.56 & 0.32 \\
\hline Weight & 98.20 & 72.83 & 0.75 \\
\hline
\end{tabular}

Table 9-19. Non Metric Attributes Recorded on Cores.

\begin{tabular}{|c|c|c|c|c|c|}
\hline \multicolumn{3}{|c|}{ Cortex } & \multicolumn{3}{c|}{ Scar Count } \\
\hline Category & Count & Percent & Category & Count & Percent \\
\hline 0 & 0 & 0.00 & $1-2$ & 2 & 28.57 \\
\hline $1-25$ & 2 & 28.57 & $3-4$ & 1 & 14.29 \\
\hline $26-50$ & 2 & 28.57 & $5-6$ & 2 & 28.57 \\
\hline $51-75$ & 3 & 42.86 & $7-8$ & 2 & 25.57 \\
\hline Total & 7 & 100.00 & Total & 7 & 100.00 \\
\hline \multicolumn{7}{|c|}{ Type } & Percent & Category & Count & Percent \\
\hline Category & Count & 57.14 & Single & 1 & 14.29 \\
\hline Cobble & 4 & 28.57 & Multiple & 6 & 85.71 \\
\hline Macro Flake & 2 & 14.29 & & & 100.00 \\
\hline Fragment & 1 & 100.00 & Total & 7 & \\
\hline Total & 7 & \multicolumn{5}{l}{} \\
\hline
\end{tabular}

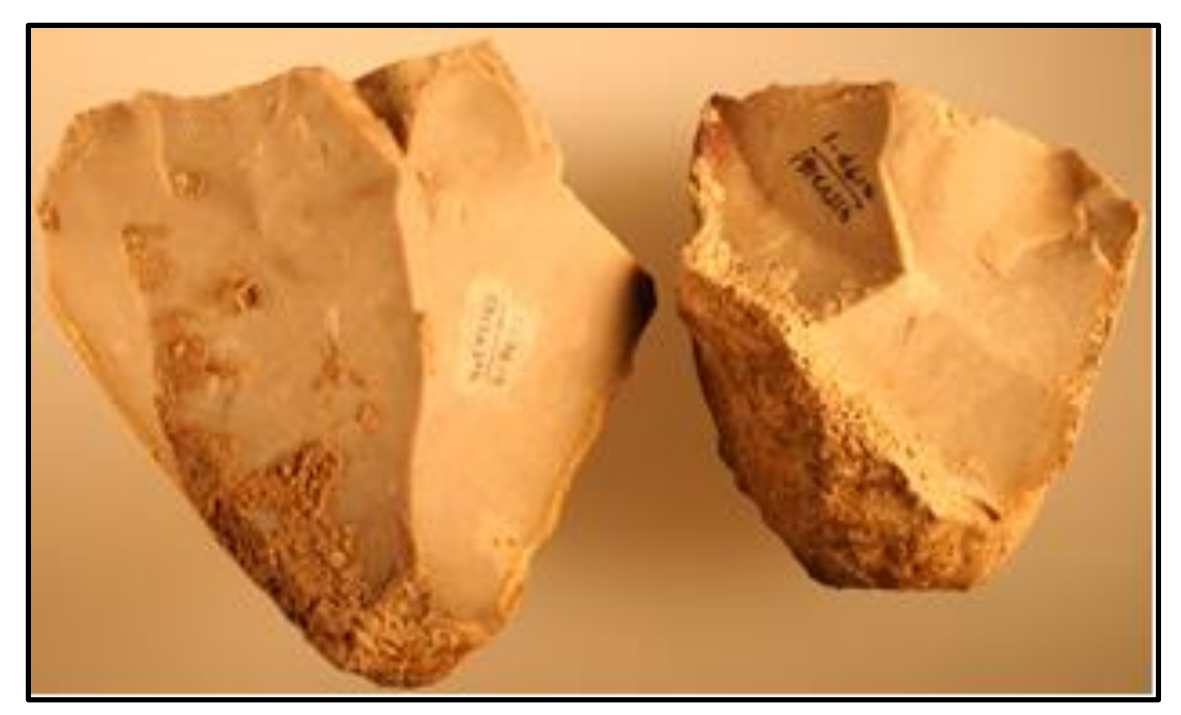

Figure 9-50. Two examples of Bell/Andice cores (\#2146-1 and \#2197-1). 


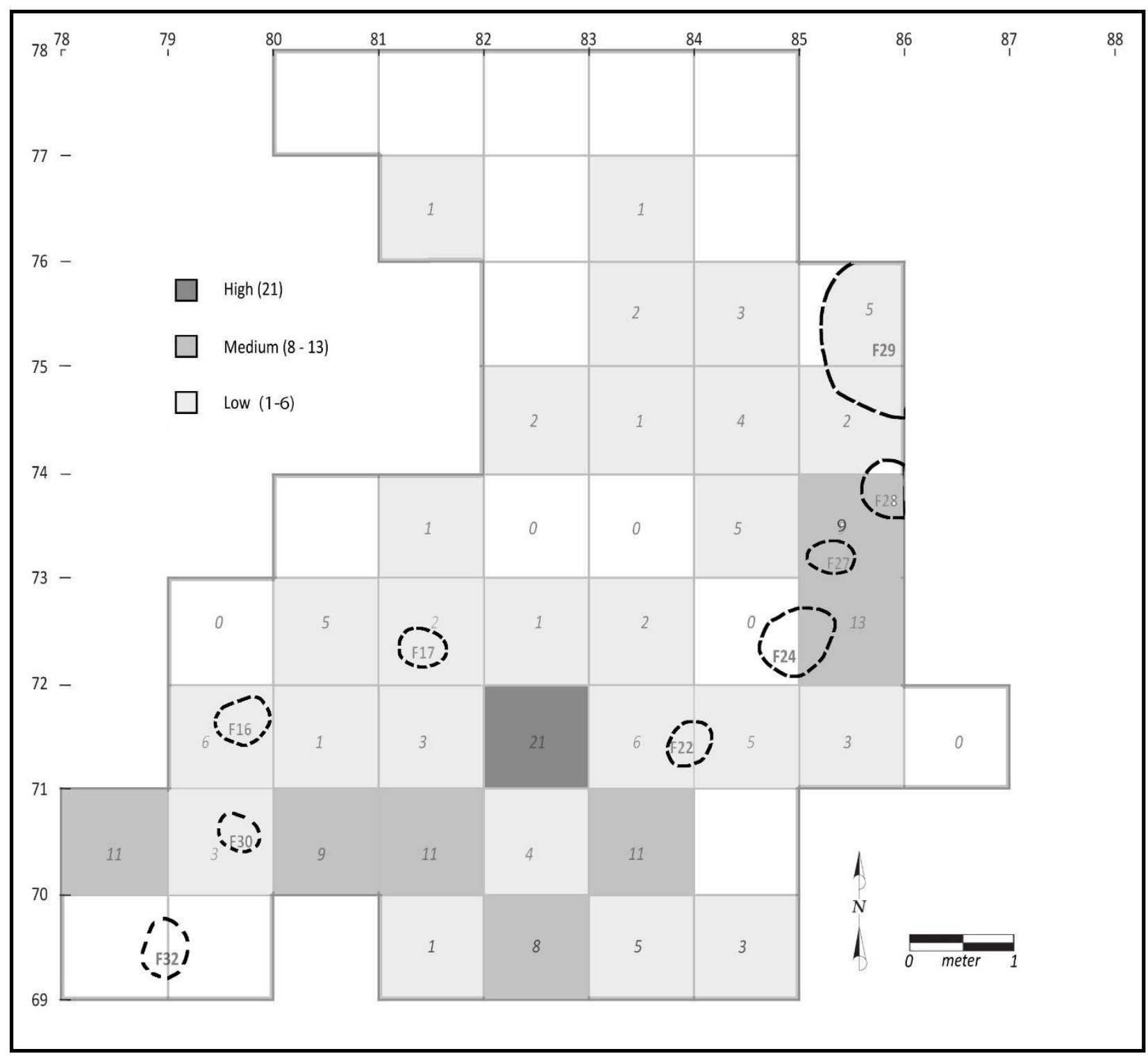

Figure 9-51. Horizontal distribution of the Bell/Andice lithic debitage across Block B.

one was smaller and either from platform preparation or edge modification. Two pieces are thermally damaged (burned) and five potentially thermally altered pieces. Eight units (16 percent) with moderate frequencies ( 8 to 13 pieces) yielded approximately 44 percent of the total debitage. Two units with modern frequencies were in and around three features (Features 24, 27, and 28) in the eastern concentration. One unit was west of Features 30 and 32 in the western concentration. Five units were at the southern end of Block B south of the unit with the highest frequency and again between the two feature concentrations (Figure 951). This area between the two feature concentrations apparently represented a discard area of unwanted debris. 
Table 9-20. Bell/Andice Bone Data.

\begin{tabular}{|c|c|c|c|c|c|c|c|c|}
\hline Taxon & Species & Count & $\begin{array}{l}\text { Weight } \\
\text { (g) }\end{array}$ & 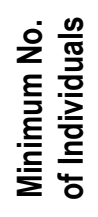 & 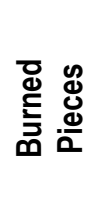 & 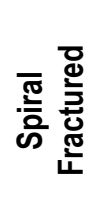 & 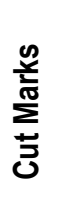 & 吾 \\
\hline Coyote & Indeterminate & 1 & 0.5 & 1 & & & & \\
\hline Deer & Indeterminate & 3 & 14.6 & 1 & & & & \\
\hline $\begin{array}{l}\text { Deer size } \\
\text { artiodactyl }\end{array}$ & Indeterminate & 95 & 128.9 & & 15 & 1 & & yes \\
\hline Rabbit family & Indeterminate & 316 & 68 & & 65 & 14 & 3 & \\
\hline Cottontail & & 5 & 3.5 & 1 & 2 & & & \\
\hline Jackrabbit & & 33 & 16.1 & 3 & 1 & 2 & & yes \\
\hline Rodent & - & 7 & 0.6 & 2 & & & & \\
\hline Fish & Gar & 1 & 0.1 & 1 & & & & \\
\hline Indeterminate & - & 130 & 46 & & 14 & & & yes \\
\hline \multicolumn{2}{|c|}{ Totals } & 591 & 278.3 & 9 & 83 & 17 & 3 & 40 \\
\hline
\end{tabular}

\subsection{VERTEBRATE FAUNAL ASSEMBLAGE}

A faunal assemblage of any sorts for this age component is rare and significant as only two or three assemblages with Bell/Andice points have been identified. This component yielded 591 bones and fragments that weighed $278.3 \mathrm{~g}$ for an average of $0.5 \mathrm{~g}$ per bone. Obviously the individual fragments are quite small and generally reflect small animals. This number and the following discussions do not include the 1,444 tiny fragments $(11.1 \mathrm{~g})$ from heavy fractions, some of which could be identifiable as microtines in seven floated feature sediments (see Table 9-3). No analyses were conducted on the floated materials beyond identification to bone, although some specific species were observed. Most all these bones present in the floated samples were less than 6.4 $\mathrm{mm}$ in size. These were not counted in the broader assemblage so as not to inflate the number, since float samples are not always analyzed from sites and features. Table 9-20 provides the taxa and species identified and recovered from the $6.4 \mathrm{~mm}$ mesh and other key data.
Nearly 22 percent were indeterminate as to possible taxon. The size of individual pieces are quite small with 99 percent less than $3 \mathrm{~cm}$ long (Figure 9-52). The largest specimen is a deer size artiodactyl proximal metatarsal that is $6.5 \mathrm{~cm}$ long. The overall small size combined with minimally 6.7 percent of the entire assemblage encrusted with calcium

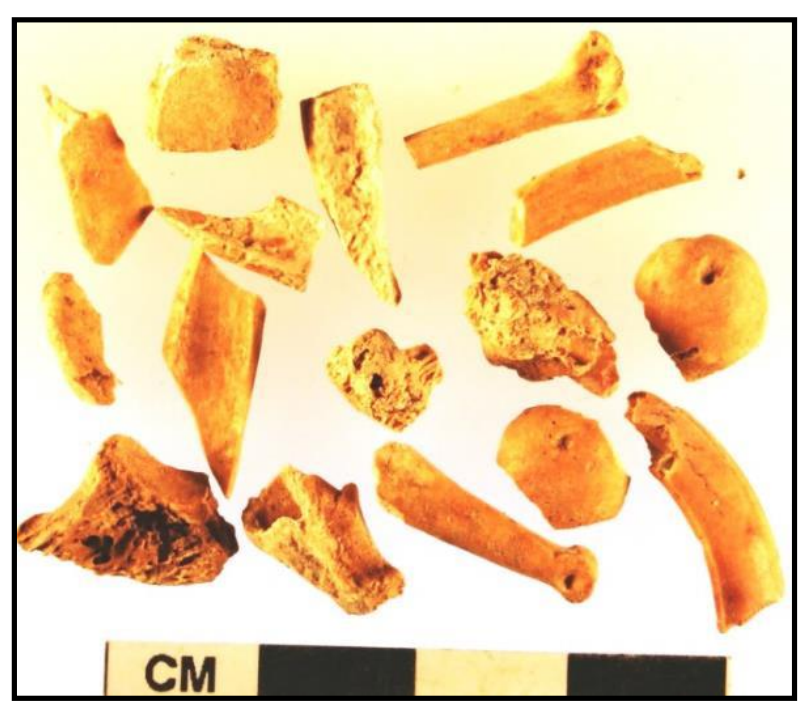

Figure 9-52. Example of small mammal remains (\#2202) between 230 and $240 \mathrm{cmbs}$ in N75/E83. 


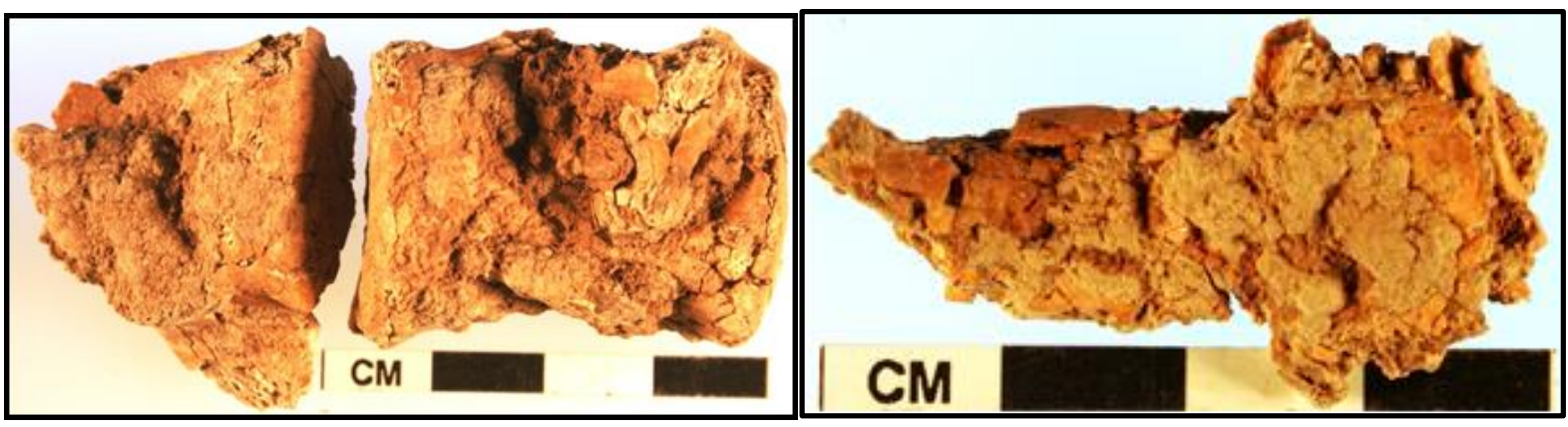

Figure 9-53. Two adjoining deer size vertebrae (left, \#2158) from $247 \mathrm{cmbs}$ in N73/E81 and rabbit mandible (right, \#2193) from 220 to $230 \mathrm{cmbs}$ in N74/E85 covered in calcium carbonate.

carbonate (Figure 9-53), plus some root etched pieces hindered bone identification and constrained observations of other key characteristics. No rodent gnawing or carnivore punctures were observed.

The identification of a single coyote tooth (Figure 9-54) and a single gar fish scale, calls into question the use of these two taxa as subsistence resources. Potentially these represent minor intrusion into the component. The tooth may represent something beside a food resource since no other parts of this animal were recognized.

Tiny rodent bones, commensal species that probably represent rats, voles, and similar animals may also be questioned, since these occur naturally in similar deposits. It is possible these were consumed for food as has been documented in coprolites in west Texas (e.g., Williams-Dean 1978), but rodents are burrowing animals and may be intrusive. If these are not considered cultural resources then the minimal number of individuals is one deer, one cottontail rabbit and three jackrabbits to constitute the meat resources utilized by this population. If all were food resources, then minimally five different species that includes the three just mentioned plus the fish and coyote reveal diverse land and aquatic resource base. Notably absent is bison or other large mammals, as indicated by the lack of thick-walled cortical bone specimens observed during analysis (Figure 9-55). Cottontail rabbits and jackrabbits combined with specimens representing rabbit bone fragments, account for nearly 60 percent the number of individual specimens (NISP). The minimal number of individual jackrabbits is three on the basis of three right astragalii, whereas one cottontail rabbit is represented. To support the cultural use of jackrabbits and cottontail rabbits and other small animals, nearly 83 percent of the rabbit family specimens are burned to a wide range of colors from a few calcined, to gray, dark gray, black, and primarily combinations of black and gray, and brown and black. All principal segments (e.g., head, vertebrae, legs and feet) of jackrabbits and cottontails are represented. Deer and deer size artiodactyl fragments that average only $1.5 \mathrm{~g}$ per bone were identified and in terms of counts,

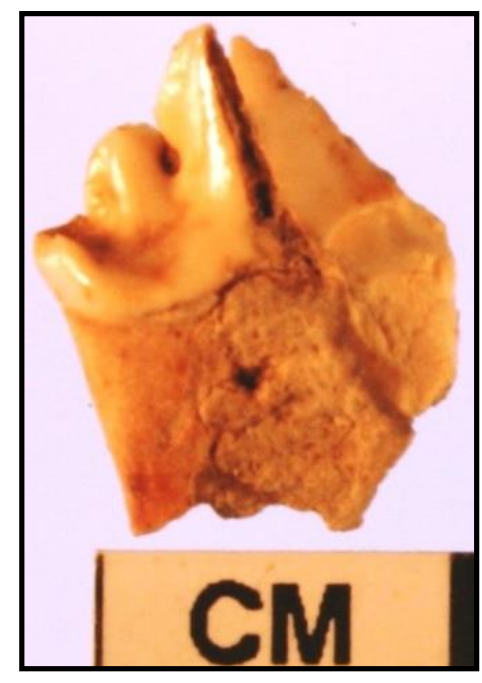

Figure 9-54. Close-up of loose coyote $P_{4}$ (\#2086) from 213 to $220 \mathrm{cmbs}$ in N71/E79 that shows no wear (scale in centimeters). 
represent nearly 17 percent of the total. Fifteen deer size artiodactyl fragments were burned with one spiral fractured specimen to support their cultural use. The identified deer remains and other and deer size elements include long bone fragments, rib fragments, vertebrae, sesamoids, distal femur, patella, and proximal metatarsal. No skull or antler specimens were identified. The entire deer carcass, with the exception of the skull, was apparently brought to camp as reflected by the bulky vertebrae column and ribs. The presence of the entire carcass indicates the kill was not far removed from the camp.

Calcium carbonate covers parts of the faunal material, restricted observations of stone tool cut marks on bones. A single piece however, exhibited tiny thin cut lines (Figure 9-56). The near absence of cuts may be partially attributed to the overall small size of most animals and the calcium carbonate and root etching observed.

On the basis of these data, it is apparent that small to medium animals were sought and processed with the exception of the fish and coyote. This probably included the internal organs such as the stomach, liver, heart, lungs and brain. Extensive butchering rather than choice meat selection is supported based on the extensive smashing of most individual elements into small bone fragments. Intensive smashing is indicative of extracting greater nutrients (i.e., bone marrow and bone grease) from each element (e.g., Binford 1978; Brackenridge 1904; Brink et al. 1985; Leechman 1951; Lowie 1954, 1963; Mandelbaum 1940; Mason and Holderby 1994; Newcomb 1993; Quigg and Peck 1995; Quigg 1997; Schilz 1983; Vehik 1977; Wissler 1910; Zierhut 1967). Boiling is the most efficient and reasonable means of extracting nutrient-rich bone grease, and this cooking process would account for the biomarker cholesterol detected in the lipid residues on nearly all burned rocks analyzed.

Extensive fragmentation increases the number of individual specimens (NISP), thus inflating counts.
Therefore, minimum number of individuals (MNI) is more suitable for understanding the meat resources in this excavation block. Not a lot of meat is projected if only one deer, three jackrabbits, and one cottontail rabbit were the only animals present.

The overall horizontal distribution of animal bones reflects clear indication of specific discard or work areas across the block (Figure 9-57). Four small, distinct areas $\left(4 \mathrm{~m}^{2}\right)$ exhibited high bone densities ( $N=57$ to 70 pieces) that contained 40 percent of all bones. Of the four concentrations, three were immediately adjacent to burned rock features, whereas the other was nearly $3 \mathrm{~m}$ from the nearest burned rock feature. Two limited areas $\left(2 \mathrm{~m}^{2}\right)$ of moderate densities ( $N=25$ to 43 pieces) account for about 12 percent of the total area. Therefore, low density units ( $N=4$ to 17 pieces) account for 48 percent of the area and were scattered across $30 \mathrm{~m}^{2}$ with almost no bone in the other $13 \mathrm{~m}^{2}$ (Figure 957). This obvious pattern reflects intentional discard of unwanted bone fragments around thermal cooking features, as well as the intact nature of the deposits and high integrity of the archeological materials.

Plotting identifiable taxa revealed no clear discard pattern with deer and rabbit fragments in the same units and scattered, inferring processing and foodsharing of whole carcasses rather than special portions or individual taxa preparation (Figure 958).

\subsection{MUSSEL SHELL ASSEMBLAGE}

Only 15 mussel shell fragments $(43.0 \mathrm{~g})$ were recovered from this component. None were identifiable to taxonomic group or showed characteristics of human alterations. The infrequent nature of this class probably indicates this was not a targeted resource and these fragments may be natural in origin or minor intrusions from above or below this component. 


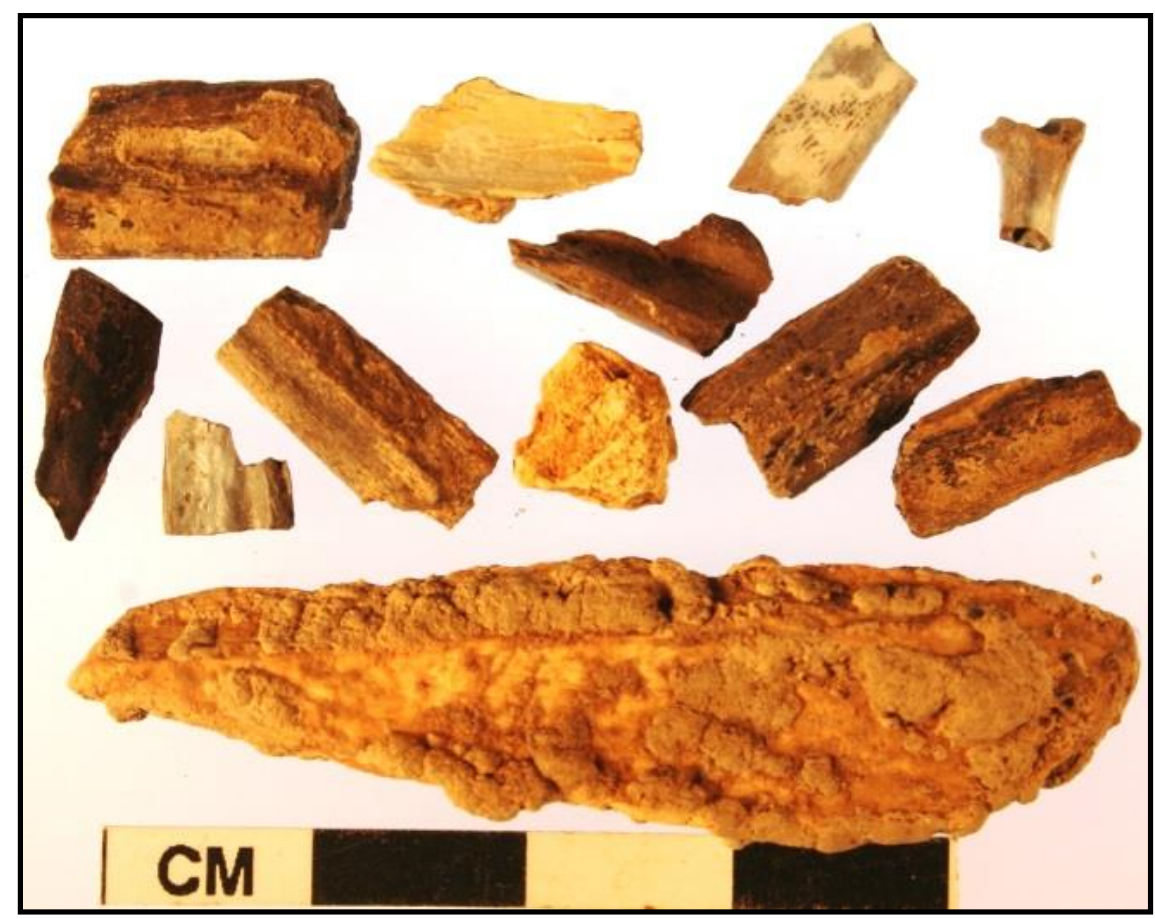

Figure 9-55. Example of bone fragments (\#2091) from 230 to $240 \mathrm{cmbs}$ in N71/E80.

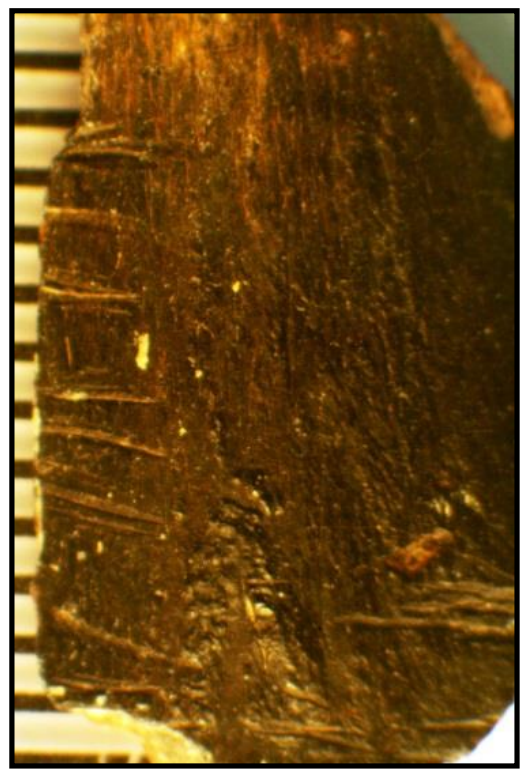

Figure 9-56. Close-up thin cut lines on tiny bone fragment (\#2202) from 230 to $240 \mathrm{cmbs}$ in N75/E83 (scale in millimeters). 


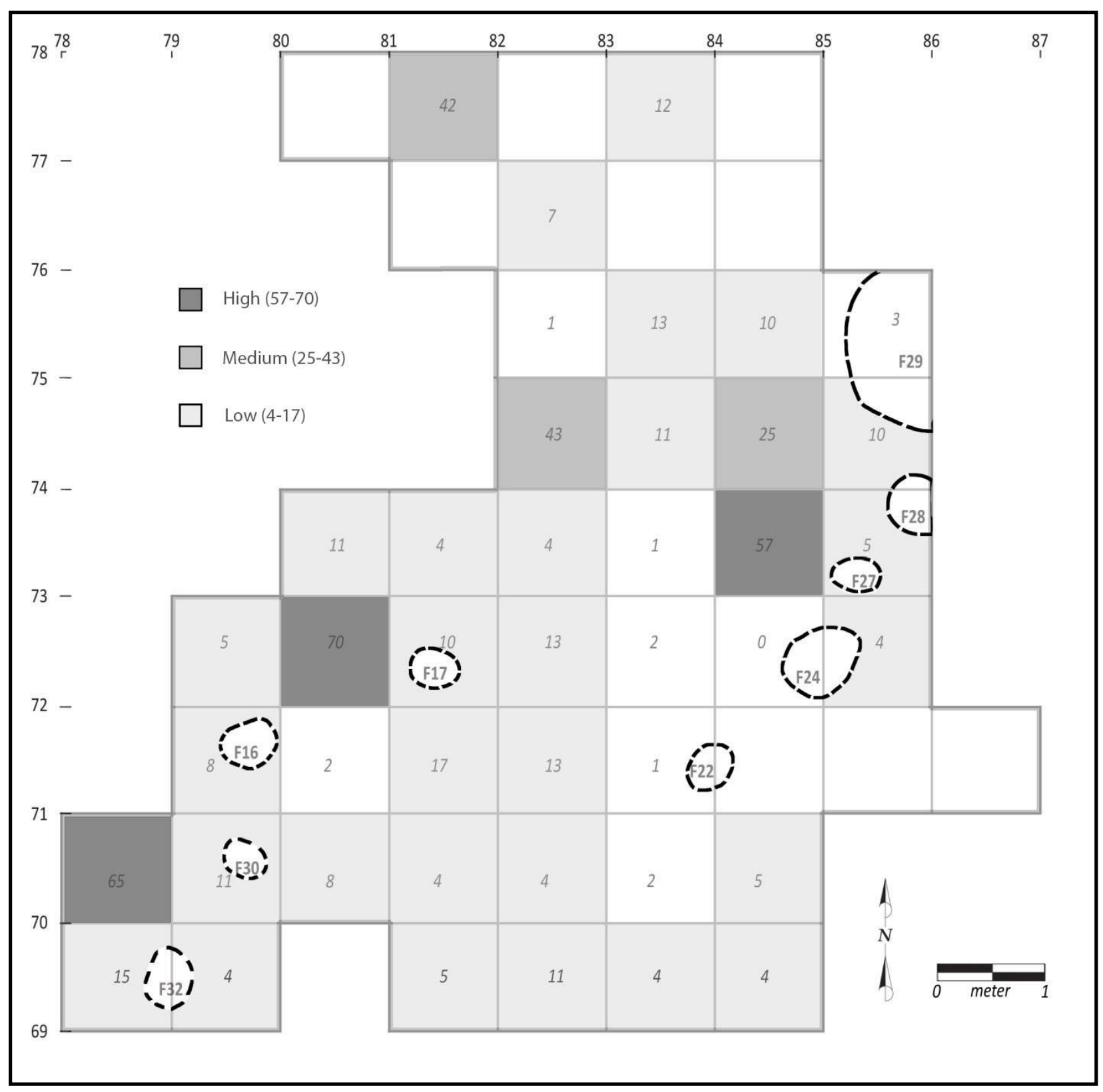

Figure 9-57. Vertebrate bone distribution across Block B for the Bell/Andice component. 


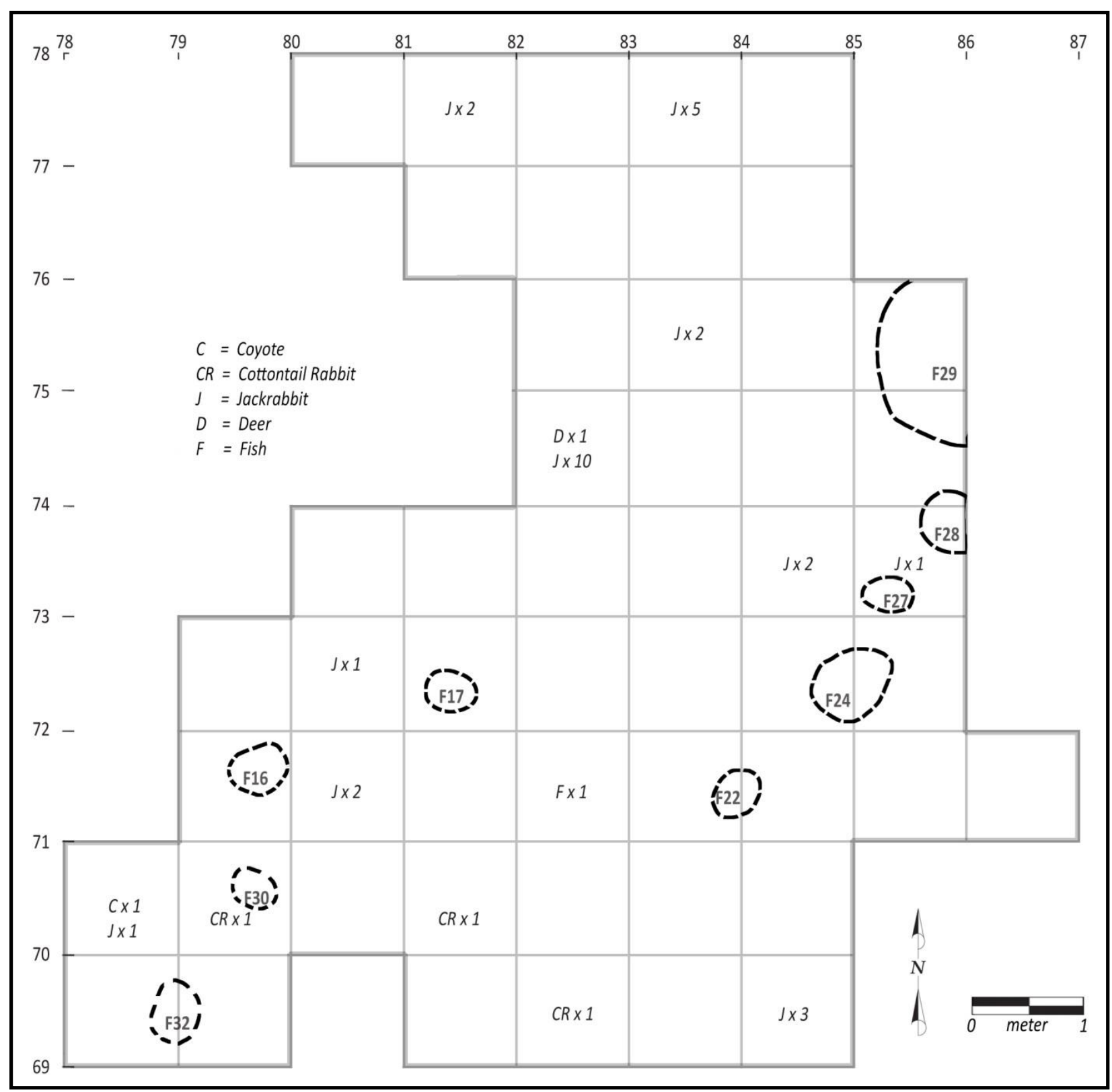

Figure 9-58. Identified taxa distribution across Block B for the Bell/Andice component. 


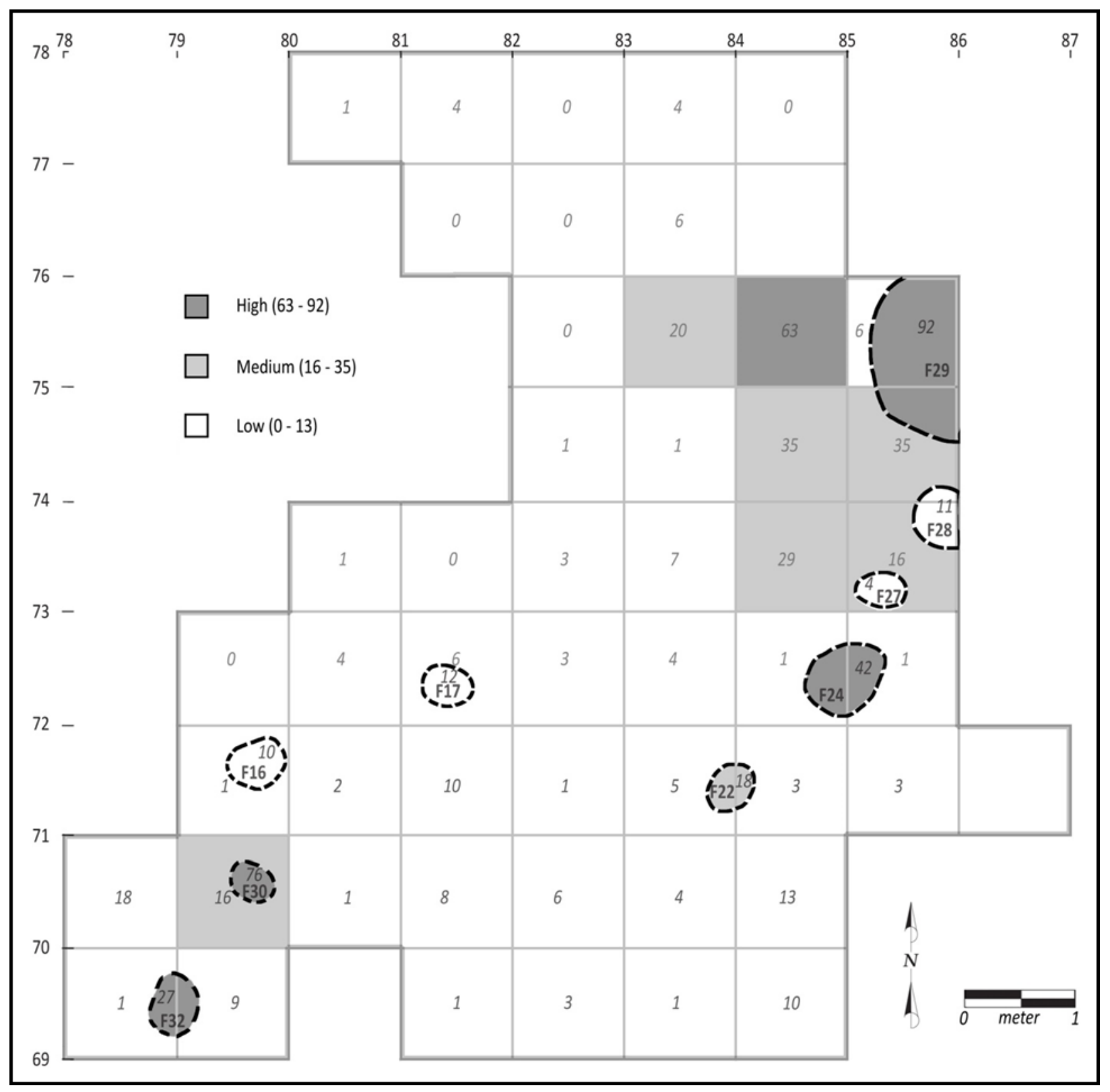

Figure 9-59. Bell/Andice burned rock distribution across Block B. 
Table 9-21. Bell/Andice Burned Rock Frequency, Size, and Weight Categories.

\begin{tabular}{|c|c|c|c|c|c|c|c|c|c|c|}
\hline Location & \multicolumn{2}{|c|}{$\begin{array}{c}\text { Burned Rocks } \\
\mathbf{0 - 4} \mathbf{~ c m}\end{array}$} & \multicolumn{2}{c|}{$\begin{array}{c}\text { Burned Rocks } \\
\mathbf{4 . 1 - 9} \mathbf{~ c m}\end{array}$} & \multicolumn{2}{c|}{$\begin{array}{c}\text { Burned Rocks } \\
\mathbf{9 - 1 - 1 5} \mathbf{~ c m}\end{array}$} & $\begin{array}{c}\text { Burned Rocks } \\
>\mathbf{1 5} \mathbf{~ c m}\end{array}$ & \multicolumn{3}{|c|}{ Total } \\
\hline & Qty. & Wt. (g) & Qty. & Wt. (g) & Qty. & Wt. (g) & Qty. & Wt. (g) & Qty. & Wt. (g) \\
\hline Feature & 48 & 1951.5 & 139 & $26,568.0$ & 29 & $36,081.0$ & 2 & 2750.0 & 218 & $67,350.5$ \\
\hline Scattered & 226 & 3380.8 & 94 & $17,884.0$ & 16 & 7200.0 & 0 & 0.0 & 336 & $28,464.0$ \\
\hline Total & $\mathbf{2 7 4}$ & $\mathbf{5 3 3 2 . 3}$ & $\mathbf{2 2 3}$ & $\mathbf{4 4 , 4 5 2 . 0}$ & $\mathbf{4 5}$ & $\mathbf{4 3 , 2 8 1 . 0}$ & $\mathbf{2}$ & $\mathbf{2 7 5 0 . 0}$ & $\mathbf{5 5 4}$ & $\mathbf{9 5 , 8 1 5 . 3}$ \\
\hline
\end{tabular}

\subsection{BURNED ROCK ANALYSIS}

Burned rocks were common across this component (Table 9-21). The majority were in the smallest size class $(0$ to $4 \mathrm{~cm})$ representing nearly 50 percent of the total, followed closely by 42 percent in the 4.1 to $9 \mathrm{~cm}$ class with only 8.5 percent greater than 9.1 $\mathrm{cm}$. This may indicate that larger cobbles were not readily available and, therefore, small rocks had to suffice for the tasks at hand. Alternatively, the burned rocks were highly fragmented from repeated heating and cooling episodes and represent the discard of exhausted rocks.

Burned rocks were in two major groups, those within the eight identified burned rock features and those scattered across the occupation floor (see Table 9-21). On the basis of size, scattered rocks composed 67 percent of the smaller pieces compared to only 22 percent in features. The opposite is true with the 4.1 to $9 \mathrm{~cm}$ size class with 64 percent in features, verses only 28 percent scattered rocks. Rocks greater than $9.1 \mathrm{~cm}$ were more frequent in the features, as well. With larger pieces most often in the features, and smaller rocks scattered over the surrounding area, a pattern of behavior becomes apparent. The smallest pieces were probably no longer suitable for their intended purpose of transferring heat and tossed and scattered beyond features.

Horizontal distribution of burned rocks indicate dense concentrations ( $N=63$ to 92$)$ in six of eight features and medium densities ( $N=16$ to 35$)$ in seven units (14 percent), with five units within $2 \mathrm{~m}$ west of Features 28 and 29 and just north of Feature
24, plus two units on the northern edge of Feature 32 (Figure 9-59). Beyond the recognized features, low densities were scattered across Block B in no recognizable pattern.

\subsection{BURNED CLAY AND DAUB}

Six tiny $(<1.0 \mathrm{~cm})$ burned clay lumps were encountered in four units with the largest a $1.8 \mathrm{~cm}$ ( $2.6 \mathrm{~g}$ ) irregular lump between 220 and $230 \mathrm{cmbs}$ in N75/E83. This latter piece exhibits a two-toned color, light beige and very dark brown, with no impressions to indicate it was attached to something. These small amorphous pieces were presumably formed due to exposure to intense heat. Burned clay was not observed in association with burned rock features, presumably reflecting low to moderate heat and/or short-term fires in the features.

\subsection{COMPONENT SUMMARY AND DISCUSSION}

A total of $51 \mathrm{~m}^{2}$ were hand-excavated across Block B and the cultural materials from ca. 212 to 254 cmbs in sloping deposits constitute those identified and attributed to the Bell/Andice assemblage. This well-defined component in excellent context yielded 2 Bell point fragments, 1 Big Sandy point, 5 bifaces, associated with at least 8 small burned rock features, 1 cluster of discarded ground stone tools, 180 pieces of lithic debitage, 15 fragments of mussel shells, 3 chipped stone biface fragments, 12 edge-modified flakes, 8 ground stone tools, quantities of burned rocks, and a rare vertebrate faunal assemblage of 591 pieces (Table 9-22). 
Bone preservation was generally good, but still reveals root etched and calcium carbonate covered pieces. Charcoal preservation was poor as it was reduced to dark organic stains and lacked structure. Microfossil preservation was also poor, but limited analytical results added greatly to understanding plant foods present and how they were processed, as well as some environmental information was obtained. Twelve radiocarbon dates were obtained, but six charcoal dates from three cultural features range from 5280 to 5370 B.P. for a variance of 90 radiocarbon years and provide an average age 5322 B.P. for this Bell/Andice event. Six other dates on diverse, noncharcoal materials do not provide precise event ages and are suspect to document the true age for this component.

Eight small burned rock features and moderate quantities of scattered burned rocks clearly document intensive hot rock cooking activities. Five were in situ hearths and three were dumps of discarded rocks. Lipid residues and chemical biomarkers extracted from feature burned rocks indicate both plant and animal products were cooked with feature rocks. The overall small size of the burned rock features, all less than $1.2 \mathrm{~m}$ in diameter, combined with smashed fragmented faunal remains in association with many, the presence of both plant and animals products on the rocks, coupled with gelatinized starch grains support cooking was probably accomplished through stone boiling.

The important faunal assemblage has diverse taxa that includes deer, cottontail rabbits, jackrabbits, coyote, and possibly fish. The latter is suspect as only one tiny element is represented near the bottom of the occupation and may represent a displaced item. The one coyote size tooth may also not represent a food resource. The entire assemblage is extremely fragmentary and following meat extraction the bones of both large and small animals were intensively smashed and boiled for bone marrow and bone grease. The absence of bison bones indicate the high ranked resource was probably not immediately available in the region or at least not at the season of this camp. Apparently higher ranked resources such as large game animals (e.g., bison, deer, and pronghorn) were not plentiful or preferably sought during the occupation of this camp as MNI is quite low for large game. Therefore, lower ranked resources such as small rabbits, grass seeds, and geophytes were targeted and incorporated into the diet at this event, thus documenting a broad diet breath.

Macrobotanical analysis documented nearly all charcoal was decomposed and/or soot, with the exception of four burned hackberry nutlets from hearth Feature 32. The latter probably originated

\section{Table 9-22. Summary of Bell/Andice} Component Materials.

\begin{tabular}{|c|c|}
\hline Cultural Material Classes & Counts \\
\hline Radiocarbon Dates & 12 \\
\hline Features & 9 \\
\hline $\begin{array}{l}\text { Burned Rock Dumps/Discard } \\
\text { Areas }\end{array}$ & $\begin{array}{c}\text { Features } \\
16,22,24, \\
27,28,29 \\
30,32^{*}\end{array}$ \\
\hline Ground Stone Cache & 1 \\
\hline Dart Points and Fragments & 3 \\
\hline Bell & 2 \\
\hline Big Sandy & 1 \\
\hline Bifaces & 3 \\
\hline Manos & 5 \\
\hline Metates & 3 \\
\hline Edge-Modified Flakes & 12 \\
\hline Lithic Debitage & 180 \\
\hline Cores & 7 \\
\hline Vertebrate Faunal Fragments & 591 \\
\hline Mussel Shells & 15 \\
\hline Burned Rocks & 554 \\
\hline Burned Clay & 6 \\
\hline Carbonized Plant Remains & 4 \\
\hline Total Materials & 1,398 \\
\hline Average Thickness (cm) & $10-15 \mathrm{~cm}$ \\
\hline Spatial Extent Excavated & $51 \mathrm{~m}^{2}$ \\
\hline Volume Excavated $\left(\mathrm{m}^{3}\right)$ & $10.2 \mathrm{~m}^{3}$ \\
\hline
\end{tabular}

This table does not include materials from float samples; ${ }^{*}$ = feature numbers, not counts. 
from wood used in the fires, but could potentially represent a food resource. No specific wood species were identified, but the burned nutlets document minimally hackberry trees were employed in fires and present in the immediate environment. The chemical dehydroabietic acid from conifer products was detected on feature burned rocks and probably represents bald cypress (Taxodium) and or juniper (Juniperus) in this setting and employed to heat the rocks. Additional support for the presence of wood fires was the identification of burned tree phytoliths from at least five burned rock features.

Microfossils were poorly preserved, but wild buffalo gourd phytoliths, tree phytoliths, sponge spicules, true lily (Lilium) starch, and multiple grass starches, were all detected. The different starches indicate two plant products that served as food resources with ground starch grains and unidentified grass starches on ground stones, and grass and true lily starches on the burned rocks to support their use as food rather than just fuel resources.

Local cherts from the gravel outcrop on the adjacent hill top dominate the sparse chipped stone assemblage as chemically demonstrated by INAA. A single piece of Georgetown chert that lacks cortex is represented, but no nonlocal materials were identified. The lithic debitage reflects limited cobble and core reduction with minimal early stage bifacial production. Roughly 30 percent of the debitage appears heat treated. The apparent discard of chipped stone tools (i.e., two bifaces) into hot fires as evidenced by two intensively burned and spalled biface fragments, which were then subsequently removed from the fires and discarded, is one interestingly observed behavioral strategy.

The Big Sandy point from excellent in situ context in this occupation was a unique discovery. Big Sandy point ages and distributions are not welldefined in Texas and are poorly represented across Texas (see Prewitt 1995). They have been attributed to the same general temporal interval as Bell/Andice points, ca. 6000 B.P. (ca. 4000 B.C.) for northeastern Texas (Story et al. 1990, Figure 32). Story et al. (1995) acknowledged that few absolute ages are available for chronological placement of this point type, but it is represented in the Archaic sequence. They go on to assign Early Side-notched points closer to ca. 8000 B.P. (ca. 6000 B.C.) in southeast Texas (Story 1990, Figure 33). Turner et al. (2011:66) place this type in the Late Paleoindian period. The Big Sandy from this component appears fresh, lightly used, is not reworked or patinated, and lacks any indication that it might represent an older curated specimen. Its vertical position and context in the component is secure as it was found in situ without any obvious disturbances. Therefore, the documented time for this component, at ca. 5322 B.P. also reflects the probable age of this Big Sandy point in central Texas.

Seasonality was explored through multiple lines of diverse evidence in different classes of materials. Charred hackberry nutlets present in Feature 32 are most prominent and abundant in fall. Other evidence such as tooth eruption and wear on deer teeth were examined with no specific season identified. Ground and damaged starch grains provide excellent evidence from at least three manos (\#2106-10, \#2175-13, and \#2414-10) that document seed processing. Three burned rocks from Feature 24 (\#2176-3-5 and \#2179-3-2) and Feature 29 (\#2223-3-8) yielded true lily (Lilium) and lenticular grass (probably Wildrye) (Figure 960) starch grains. Wildrye (Elymus sp.) grass seeds would be most abundant and ripe in the fall with true lily bulbs a possibility in the fall or early spring. Also, gourd (Cucurbita foetidissima or buffalo gourd) phytoliths were identified from Features 24 and 30 and again gourds are primarily available in the fall. This wide array of evidence is not overwhelming or in great quantities, but what is present supports a fall encampment. 


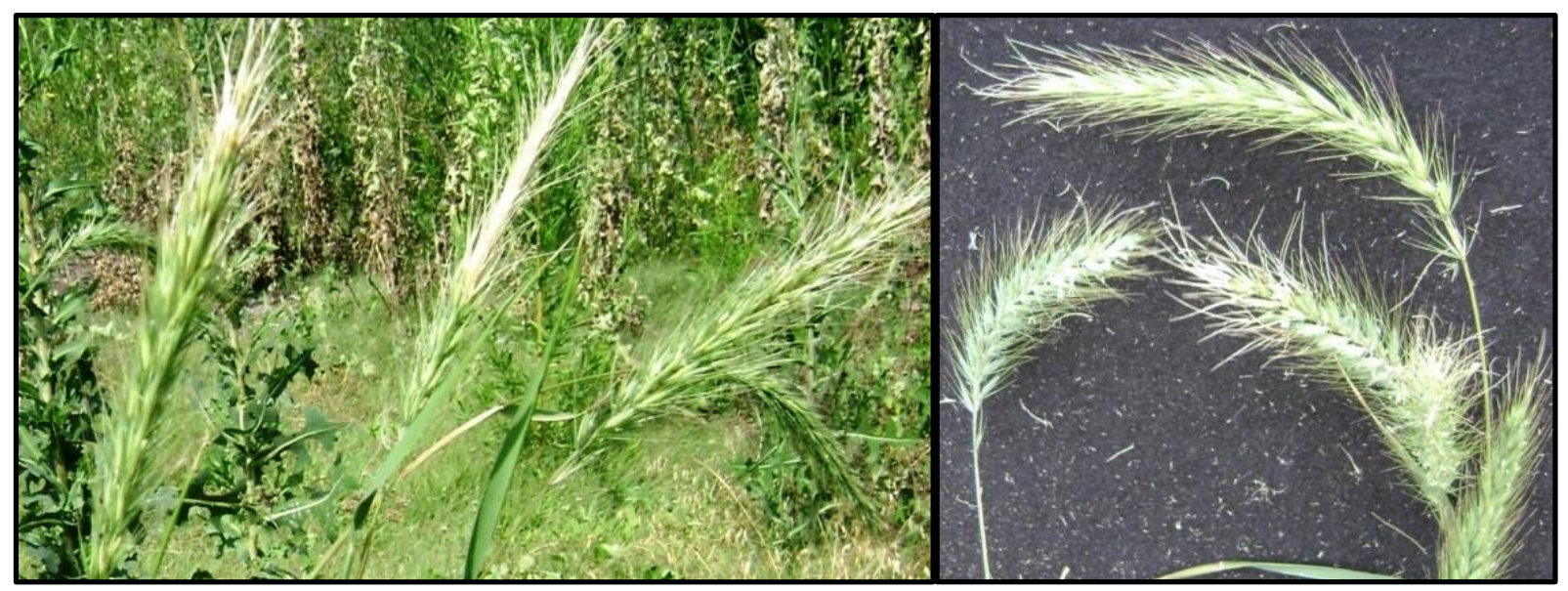

Figure 9-60. Example of wildrye (Elymus sp.) in natural setting (left) and close-up of ripe seed head (right, photographs by M. Quigg).

The high frequency of small burned rock cooking features, combined with eight ground stone tools, a high percentage of small game, the collecting and processing of multiple plant resources, few formal chipped stone tools and limited frequencies of lithic debitage, combined with minimal formal stone tool production probably reflect a small, female dominated task group focused on plant and small game procurement with subsequent cooking of those resources during a short-term occupation in the fall. This contrasts with a generalized camp where multiple, diverse tasks such as big game hunting and butchering, formal stone tool manufacturing and retooling, plant collecting, cooking, and hide working activities all occurred over a period of time (see Chapter 13 for detailed discussion).

\subsubsection{Chronology Issues}

Despite poor preservation of wood charcoal and microfossils, 12 radiocarbon dates were obtained on four different materials (i.e., charcoal, animal bones, Rabdotus snail shells, and organic sediment) to determine the age of this component. The 12 dates range over a 1,060 year period from 4710 to 5770 B.P., but the six dates derived from the more acceptable charcoal provide a much narrower range of 90 radiocarbon years from 5280 to 5370 B.P. and document the occupation occurred around ca. 5322
B.P. in comparison to the much broader time range represented by from other materials. In future work, if charcoal, seeds, and/or bulbs are recovered from good context, these materials would provide a much more acceptable age range over other organic materials. For more detailed discussion of the chronology see Chapter 11.0.

\subsubsection{Subsistence Issues}

The rare vertebrate faunal remains minimally include deer, cottontail rabbits, jackrabbit, and possibly coyote and fish. Combined with identified plant remains, such as wild buffalo gourds (Cucurbita foetidissima), grass seeds, true lily (Lilium) bulbs, and potentially hackberry nutlets, the subsistence data document broad spectrum foraging by this group. True lily starch grains were in hearth Features 24 and 25. Wild gourd phytoliths were also in hearth Features 24 and 30, rather than in nonfeature context, therefore considered cultural. The burned hackberry nutlets may be questioned as a food resource since these trees were probably employed as fuel for fires. Not only was animal meat targeted, but so was bone marrow and bone grease. The surprise was the absence of bison, which is significant since bison is considered a high valued resource and usually procured if available during this general period (see Chapter 3). Short cell grass phytoliths were nearly 
absent, but a high incidence of burned panicoid grass phytoliths in Feature 29 indicate use of either Panicoid biomass for fuel, or harvesting and processing Panicoid grasses for food. Drying Panicoid grasses for food would have occurred in the fall. This was a broad spectrum foraging group that demonstrated generalized hunting and gathering in a flexible mobility pattern with sufficient adaptive strategies to live and thrive in hot and dry conditions prevalent likely at that time. It is suspected that this population expanded their diet breath in response to limited availability of high value resources such as bison and regional climate conditions.

\subsubsection{Technology Issues}

Technologies surrounding preparation and cooking foods are most apparent. Grass seeds were collected and ground, whereas animal bones were processed (smashed) prior to cooking. Gelatinized starch grains, a reflection of a combination of heat and water, combined with multiple small burned rock hearth features and discard dumps, crushed animal bones, the presence of plant and animal lipid residues on the rocks, allude to stone boiling as the principle means of cooking food. Aerial diatoms recovered from burned rock discard Features 22 and 24 probably reflect temporary wet intervals, or derived from stream margin muds. This temporary wetting may be attributed to dumping water and/or wet rocks plus other contents from the stone boiling process. The relatively high sponge spicule frequency in burned rock Features 22 and 24, compared to frequencies recovered in the natural column samples also implies water was employed with these features. The shallow saucer shaped basins lacked visible oxidation rims, which reflect short-term heating and/or low temperature fires (e.g., Bond 1978) that potentially would be for heating rocks employed in stone boiling or broiling. These shallow basin hearths, combined with the absence of oxidation rims, and charcoal lenses, with limited and small rocks, are normally not associated with earth oven cooking.
Natural chert resources were procured from the local gravel covered hill adjacent to this site as INAA documented the chemical signatures of the cultural debitage and tools were similar to the hilltop cherts analyzed. The somewhat limited occurrences of heat altered debitage (ca. 30 percent) imply intentional heat treatment to improved knapping capabilities was not a frequent technology employed to this assemblage. This may reflect favorably on the high quality of the local chert resources employed or the near absence of formal chipped stone tool production, therefore reducing the need to improve the chert for knapping. The absence of late stage bifaces, notching flakes, and practice pieces also support the nonproduction of finished projectile points. Generic cobble and core reduction was evident, and reflects focus on production of informal flake tools for procurement of plants and processing small game rather than formal tool manufacturing that targeted thin bifaces.

The formal tool assemblage supports minor hunting activities (three points), animal processing (three biface fragments), and lacks other formal tool types such as scrapers, gouges, and awls to imply a variety of other activities. Meat procurement was conducted, but rabbits probably were procured with nets, traps, or clubs with that specific technology not preserved. Once collected, meat was probably stripped from the bones and then bones were smashed into small pieces to extract bone marrow and bone grease as reflected by the average weight of $0.47 \mathrm{~g}$ per bone. This smashing may have occurred on a slab metate as one metate yielded both animal and plant lipid residues.

Little attention has been paid to informal tools and their functions. Here, they account for the most frequent single tool class and high-powered usewear analyses on seven edge-modified flakes documents numerous tasks performed and the different materials involved. These flakes were employed for scraping plants, cutting hard high silica plants, whittling hard materials, and slicing. 
Worked materials included primarily plants, wood, some animals, and fish. Interestingly, informal tools were not documented during use-wear analysis as having scraped hides and combined with the absence of formal scrapers indicates this often conducted task was not performed at this camp.

Evidence of hafting was detected on two points with the Big Sandy point depicting haft wear that extended over half the point length, whereas the Bell/Andice point showed haft wear just pass the deep notches. Considerable neck width differences of $6.8 \mathrm{~mm}$ between these two points, $16.2 \mathrm{~mm}$ on the Bell and $21.1 \mathrm{~mm}$ on the Big Sandy, probably indicate shaft width differences. Potentially, this might reflect the Bell point was hafted to dart shafts, whereas the Big Sandy potentially was employed with a heavier spear shaft. Possible starch grains were observed on the Big Sandy stem, potentially indicating fibrous materials were also present. The impact fractures on the distal end of the Bell point and base specimens support the idea that these points were used, broken, discarded, and a retooling of dart shafts occurred.

Small plant processing technology was evident through the presence of multiple types of grass seeds, the cutting of true lily bulbs by informal flakes, five manos, and three metate fragments. The ground stone reveals only limited use-wear supporting short-term use. Plant grinding process is also documented by the ground and damaged starch grains on four ground stone tools.

Wood procurement apparently was limited to collecting small pieces of dead wood for fuel, as heavy wood chopping tools such as large chert choppers or adzes were not recovered and structures not detected. Wood was worked as evident through wood tissue on a couple of informal tools and in combination with hard, high silica polish to imply whittling and scraping wood products. Wood was employed as fuel for fires as reflected by the presence of burned tree phytoliths in five burned rock features and supported by the presence of chemical biomarker dehydroabietic acid that represents residues from conifer products (here probably bald cypress or juniper trees), on chemically analyzed burned rocks.

\subsubsection{Intra-Component Patterning}

Eight small burned rock features appeared in two principal clusters across the excavated block, five features towards eastern side and three features towards the southwestern corner (Figure 9-61). These two clusters indicate two separate work areas as both contained in situ heating and/or cooking features and burned rock discard piles. It is unclear if this division of space represents two family units or just spatially divided work space for multiple individuals. The roughly $5 \mathrm{~m}$ space between feature clusters contained the only the ground stone feature (Feature 17) and a few scattered artifacts. Feature 22 at the southern end of the eastern cluster was a dump of used burned rocks surrounded by a high concentration of broken tools that included two broken points, two broken bifaces, plus four edge-modified tools, one complete mano, and the highest concentration of lithic debitage.

The central area between the two projected cooking areas clearly represents a dump or discard area. As an example, two broken bifaces in that area had previously been discarded into hot fires, heat fractured, and subsequently retrieved, then discarded in the vicinity of dump Feature 22. Only two other edge-modified tools were scattered between the two major cooking areas. Four other broken ground stone tools were in or immediately adjacent the eastern burned rock features (see Figure 9-41). Just over 50 percent of the edgemodified tools were discarded around the eastern cluster of features.

Scattered burned rocks were primarily in seven units (14 percent) with high $(N=63$ pieces $)$ concentrations in one unit and moderate frequencies ( $N=17$ to 35 pieces) in six units. Six of those seven units were immediately west and 


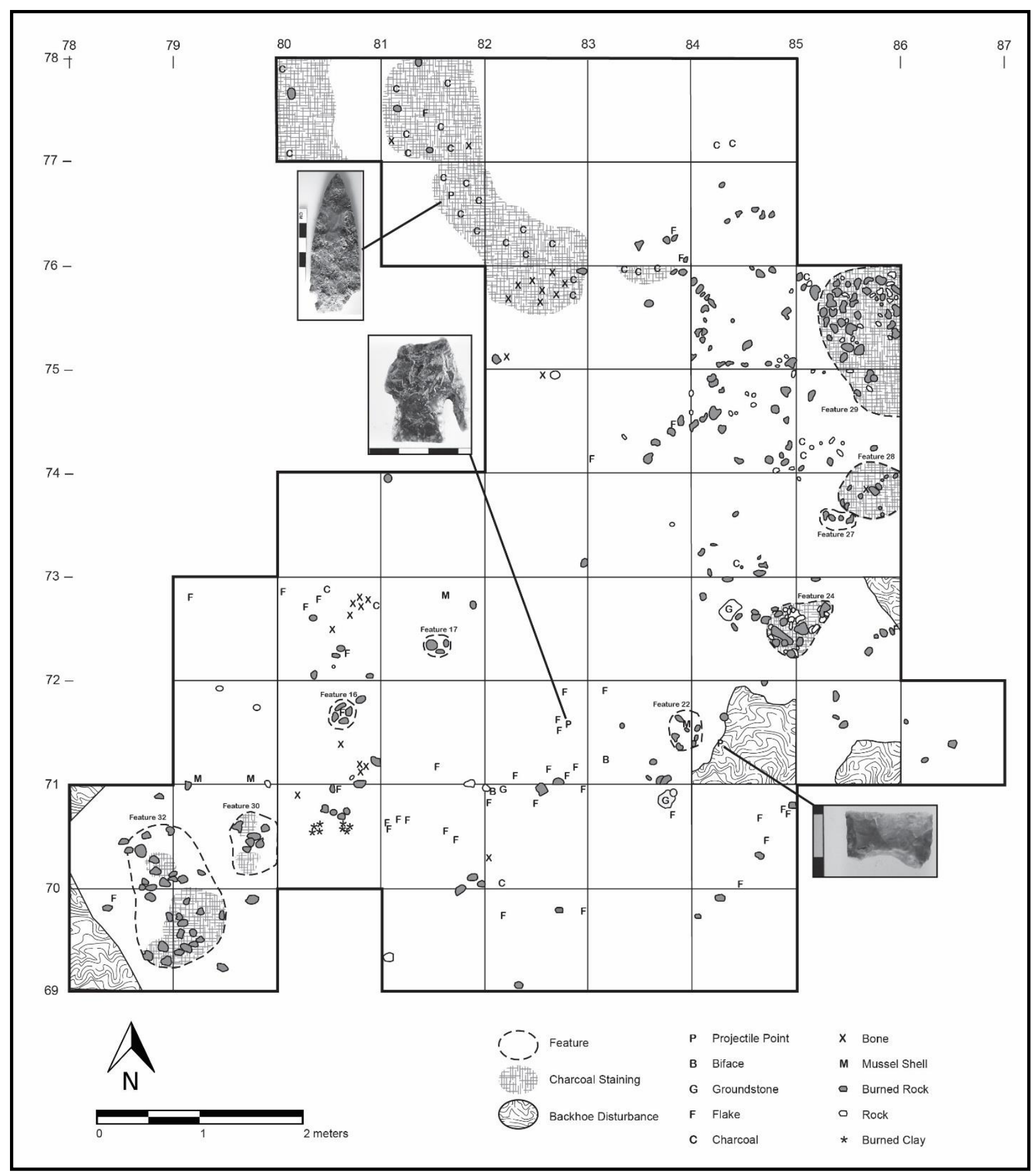

Figure 9-61. Distribution of Bell/Andice materials across Block B. 
south of in situ hearth Feature 29, between the two work areas (see Figure 9-59). These scattered rocks clearly document intensive heating and/or cooking activities associated with Feature 29. One moderately dense unit was north of in situ hearth Feature 32 and west of in situ hearth Feature 30.

The bone fragment distribution clearly reflects intentional well-defined discard patterns with over 32 percent of the fragments in 6 percent of the units $\left(3 \mathrm{~m}^{2}\right)$. These three high density areas $(N=57$ to 70) were immediately adjacent to identified burned rock features with two between the two major burned rock features. One was west of Features 24, 27 and 28, the second west of Feature 30 and 32, and the third northeast of in situ hearth Feature 16 (see Figure 9-57). This observed pattern reflects the fragmented bones and burned rocks were directly related to one another and support their heating and/or cooking with burned rocks, followed by their discard immediately adjacent hearth or cooking features.

Although lithic debitage was in low frequencies, the horizontal distribution of those pieces reveal a clear, recognizable pattern of discarding the debitage in a generalize location between the two feature clusters (see Figures 9-51 and 9-61). The clear horizontal patterning of different classes of cultural materials definitely documents the intact nature of this Bell/Andice occupation and reflects specific human behaviors related to cooking plant and animal food resources and discarding used no longer desired materials.

Overall artifact density of 27.4 items per meter square is somewhat deceiving as tiny bone fragments are generally not preserved in most sites, if compared to other sites that lack bone preservation, especially sites of this age or older, the data could be significantly skewed. Bone fragments account for 42 percent of the total materials, which makes a major difference in the total count and density. Bone is nearly three times more frequent than lithic debitage, which is often recovered at other sites and components. Formal tool frequency accounts for 1.0 percent of the total; therefore it may not be appropriate to compare this occupation to other hunter-gatherer components, which often report tool frequency in palimpsest conditions. If bone is removed from the total count, the remaining materials reveal a low density of only 16 items per meter square that includes the burned rocks. Again, the low density reflects the shortterm nature of the occupation and what should be expected in single use hunter-gatherer camp.

\subsubsection{Trade and Interaction}

The paucity of nonlocal raw materials in this assemblage implies limited contact, travel, or interactions with distant groups. This might reinforce that the occupants were regionally localized with a relatively restricted seasonal range. It may also indicate a relatively low regional population density with groups so dispersed they had minimal contact with each other. A third possibility was regional populations all utilized the same high quality Edwards chert, and followed similar lifeways. If so, potentially trade items were perishable goods rather than visible in the archeological records.

The Big Sandy point is the only artifact that might indicate interactions with other populations. The reasoned assumption discussed above being this artifact was not an old curated item, stimulates the question as to how it arrived at his location. Did it arrive through trade or was it carried in by an individual from another group? The fact it was manufactured from Edwards chert documents it was locally manufactured and the material not brought in from great distances. Not much is understood concerning this specific point type in Texas and the groups that employed this type, consequently this limits our understanding as to how it arrived in this context. For background concerning the age and distribution of early sidenotched projectiles see Walker 1980 and 1992 and Chapter 13.0. 


\subsubsection{Paleoenvironmental Conditions.}

Minimal direct evidence was recovered to document specific environmental conditions as microfossils and macrobotanical remains were poorly preserved. Identified wood was unspecified hardwood and hackberry, although biomarker dehydroabietic acid in the lipid residues extracted from burned rocks indicate conifer products (e.g., bald cypress [Taxodium] or juniper [Juniperus]) were probably employed to heat the rocks. Grass phytoliths and starch grains, probably wildrye grass, true lily starches, and wild buffalo gourds were identified and generally represent open, dry grassy regions. The probable wildrye (Elymus sp.) grass would have grown in moist, shady areas along the riparian zone adjacent Onion Creek. The true lily bulbs also support moist areas in the riparian setting. This sparse data reveals the local environment contained multiple tree and grass species that probably represent ecotones along the creek. The trees were probably restricted to the riparian settings along the creek. In support of open grasslands beyond the riparian zone is the presence of cottontail rabbits and jackrabbits in the cultural faunal assemblage, which both prefer open grasslands in combination with low brush and open spaces.

One noncultural sediment sample at $290 \mathrm{cmbs}$ from the stratigraphic column in the south wall of the large pit yielded a high count of diatoms in comparisons to other samples from this wall. Thirty-one percent of those types are found in standing water, eight percent in permanent water, and eight percent in flowing water. This one sample, although not from the greenish-gray clay lens observed in the excavation blocks below the Bell/Andice component, documents standing water was present prior to the occupation of the Martindale component. This may be similar to what was reflected by the greenish-gray clay lens, just a different expression at a different time.
A second sediment sample from $310 \mathrm{cmbs}$ in the same noncultural stratigraphic column and below the Martindale component yielded a moderate frequency $(N=10)$ of sponge spicules from major sandy deposits. These potentially were deposited during flood events, supported by the fact most spicules are abraded and chemically weathered. If the interpretations of the environment are correct, on the basis of the above presence of diatom and sponge spicules, flooding events occurred prior to the Martindale component, which would be pre-ca. 5500 B.P.

Diatoms recovered from calcium carbonate adhering to two separate deer bones indicate water at the time of the occupation was slightly alkaline, had low dissolved oxygen water with organic pollution, vegetated, highly degraded conditions, and clear enough to permit light to reach the bottom. The nutrient preferences of the identified diatoms indicate moderate to highly eutrophic conditions with phosphate and nitrate concentrations.

Diatoms recovered in Bell/Andice Features 22 and 24 are aerial diatoms, either from temporary wet intervals or from stream margin muds. All aquatic diatoms are primarily benthic species, typically found in vegetated, shallow streams and ponds. These are generally attached to or crawling on aquatic plants, larger algae, rocks, pebbles, sand, mud and debris. Closely related to the aquatic diatoms and well-represented in this assemblage are aerial species found on moss, soil and mud of marginal, occasionally or temporarily wet and damp habitats. These aerial taxa live along the banks of streams, the upper margins of ponds and lakes and in wetlands and depressions. Aerial and aquatic species are often in very shallow habitats with fluctuating water levels. The water had a residence time sufficiently short that did not allow common planktonic diatoms to grow. If the one gar fish represented was definitely part of the cultural component rather than displaced, it supports a flowing Onion Creek at that time of occupation. 


\subsection{RESEARCH DESIGN QUESTION}

How did hunter-gatherer populations respond to the prevailingly dry climatic conditions during this part of the middle Holocene (see Chapter 4.0)?

\subsubsection{Hypothesis 1}

The middle Holocene Bell/Andice occupation at 41TV2161 was nonintensive, short-term, and economically unspecialized encampment from which a broad range of subsistence resources were procured and processed. This occupation can be characterized as short-term residential encampment by small group of nonspecialized foragers.

The excavations and analyses partially support Hypothesis 1 . The $51^{2} \mathrm{~m}$ excavation yielded nine small cultural features, limited lithic debitage $(N=$ 203), less than a dozen formal chipped stone tools, 12 edge-modified flakes, three projectile points, 591 bone fragments, and eight ground stone tools that document a very limited, and generalized cultural assemblage that reflects short-term occupation. Lack of oxidation rims under the shallow, rock filled basin features reflects low heat and short-term use. The eight, small burned rock features revealed stages of cooking process, such as in situ heating of rocks, discarding used rocks and smashed bone fragments, and these probably reflect stone boiling. The limited lithic debitage assemblage reflects no or very limited formal stone tool production. These component characteristics more accurately depict a specialized encampment focused on plant gathering, hunting small and large mammals, (i.e., cottontail, jackrabbits, and deer), intensive processing bones, and cooking those diverse resources. The broad spectrum subsistence documented reflects this groups' response to the projected hot dry conditions as does their desired to camp near a water course such as Onion Creek that would have provided multiple plant and animal resources as well as necessary water, wood and rocks for their utilization in cooking the food resources obtained. The high frequency of burned rock features, numerous ground stone tools, plant resources, combined with very limited formal chipped stone tool production, reflects a female dominated specialized task group focused on gathering, processing, and cooking food resources.

This is not the only example of a probable specialized task group focused on gathering, processing, and cooking food resources in the immediate area as the adjacent Berdoll component radiocarbon dated to ca. 8000 B.P. also exhibited similar characteristics, such as a limited amount of manufacturing debris, multiple cooking features, no projectile points, and few formal tools to indicate a short-term, focused occupation(s) (Karbula et al. 2011).

\subsubsection{Hypothesis 2}

The kind of Bell/Andice occupation represented at 41TV2161, namely, short-term residential encampments with nonspecialized and nonintensive reliance on a diverse range of resources, is essentially characteristic of this period of the Holocene in central Texas, ca. 4000 to 6000 B.P., which represents an adaptive response on the part of regional human populations to the relatively dry climate of the period that involved a reliance on a wide range of plant and animal food resources.

The results and analyses partially support Hypothesis 2. This was a short-term occupation, but the remains document a very limited, specialized encampment focused on plant gathering, hunting small animals and not a residential encampment. The reliance on diverse food resources with no obvious specialized tools reveals continuity in subsistence practices documented in most other components and sites that are older or similar in age across central Texas (Table 9-23). Therefore, as the body of archeological evidence grows for this general period, it continues to strengthen the nonintensive adaptive nature of these mobile hunter-gatherers. 


\begin{tabular}{|c|c|c|c|c|c|c|c|c|c|c|c|}
\hline $\begin{array}{l}8 \\
\frac{0}{2} \\
\frac{\mathbb{D}}{3} \\
3 \\
\frac{0}{0} \\
\frac{0}{01} \\
\frac{0}{7} \\
\stackrel{0}{\gtrless}\end{array}$ & 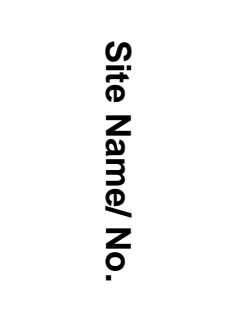 & 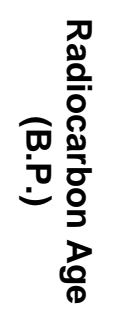 & 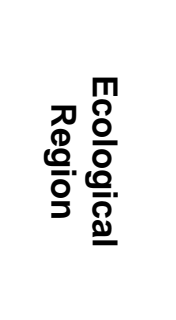 & 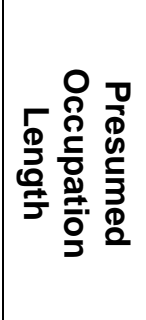 & 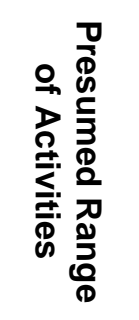 & 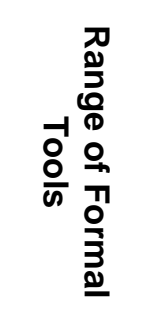 & 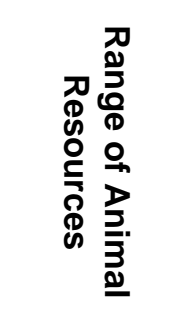 & 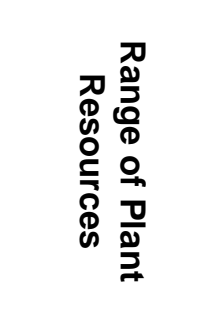 & 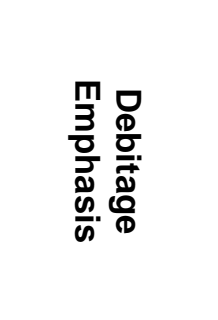 & 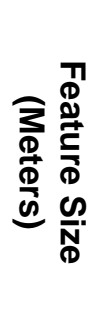 & 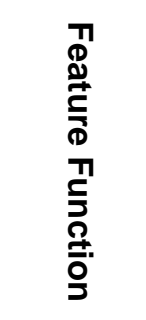 \\
\hline \multirow{3}{*}{$\begin{array}{l}\text { Prior } \\
\text { Period }\end{array}$} & $\begin{array}{l}\text { Berdoll/ } \\
\text { 41TV2125 }\end{array}$ & $\begin{array}{l}7854- \\
8291\end{array}$ & $\begin{array}{l}\text { Blackland } \\
\text { Prairie }\end{array}$ & $\begin{array}{l}\text { Short- } \\
\text { term }\end{array}$ & Narrow & $\begin{array}{c}\text { Narrow }= \\
4 \text { types }\end{array}$ & Broad & $\begin{array}{l}\text { Broad - bulbs, } \\
\text { nuts, acorns }\end{array}$ & NA & $<1.5$ & Cooking \\
\hline & $\begin{array}{c}\text { Holt/ } \\
\text { 41HY341/ } \\
\text { Zone II }\end{array}$ & None & $\begin{array}{l}\text { Blackland } \\
\text { Prairie }\end{array}$ & $\begin{array}{l}\text { Short- } \\
\text { term }\end{array}$ & Narrow & $\begin{array}{c}\text { Narrow }= \\
3 \text { types }\end{array}$ & $\begin{array}{c}\text { Narrow }= \\
1\end{array}$ & $\begin{array}{l}\text { Narrow }=1 \\
\text { poor } \\
\text { preservation }\end{array}$ & $\begin{array}{c}\text { Tool } \\
\text { refurbishing }\end{array}$ & $<1.5$ & $\begin{array}{l}\text { Heating/ } \\
\text { Cooking }\end{array}$ \\
\hline & $\begin{array}{l}\text { Richard } \\
\text { Beene/ } \\
\text { 41BX831/ } \\
\text { Lower } \\
\text { Medina }\end{array}$ & $\begin{array}{l}7000- \\
6400\end{array}$ & $\begin{array}{l}\text { Coastal } \\
\text { Plain }\end{array}$ & $\begin{array}{l}\text { Short- } \\
\text { term }\end{array}$ & Broad & $\begin{array}{l}\text { Broad }= \\
7 \text { types }\end{array}$ & $\begin{array}{c}\text { Broad }= \\
12\end{array}$ & $\begin{array}{c}\text { Not } \\
\text { Preserved }\end{array}$ & $\begin{array}{l}\text { Core \& biface } \\
\text { reduction, } \\
\text { manufacturing }\end{array}$ & $<1.5$ & $\begin{array}{l}\text { Heating/ } \\
\text { Cooking }\end{array}$ \\
\hline \multirow{4}{*}{$\begin{array}{l}\text { Same } \\
\text { Period }\end{array}$} & $\begin{array}{c}\text { Barton South/ } \\
41 \mathrm{HY} 202-\mathrm{B} / \\
\text { Area B }\end{array}$ & None & $\begin{array}{l}\text { Edwards } \\
\text { Plateau }\end{array}$ & $\begin{array}{l}\text { Short- } \\
\text { term }\end{array}$ & Narrow & Narrow & $\begin{array}{c}\text { Not } \\
\text { Preserved }\end{array}$ & $\begin{array}{c}\text { Not } \\
\text { Preserved }\end{array}$ & $\begin{array}{l}\text { Full bifacial } \\
\text { reduction }\end{array}$ & $<1.5$ & $\begin{array}{l}\text { Heating/ } \\
\text { Cooking }\end{array}$ \\
\hline & $\begin{array}{c}\text { Big Hole/ } \\
\text { 41TV2161/ } \\
\text { Martindale \& } \\
\text { Bell/Andice }\end{array}$ & $\begin{array}{l}4800- \\
6000\end{array}$ & $\begin{array}{l}\text { Blackland } \\
\text { Prairie }\end{array}$ & $\begin{array}{l}\text { Short- } \\
\text { term }\end{array}$ & Narrow & Narrow & Broad $=7$ & $\begin{array}{l}\text { Narrow }=2, \\
\text { bulbs, } \\
\text { grasses }\end{array}$ & $\begin{array}{l}\text { Tools } \\
\text { production, } \\
\text { refurbishing }\end{array}$ & $<1.5$ & $\begin{array}{l}\text { Heating/ } \\
\text { Cooking }\end{array}$ \\
\hline & $\begin{array}{l}\text { Landslide/ } \\
41 \mathrm{BL} 85 / \\
\text { Stratum IIIb }\end{array}$ & None & $\begin{array}{l}\text { Edwards } \\
\text { Plateau }\end{array}$ & $\begin{array}{c}? \\
\text { Short- } \\
\text { term }\end{array}$ & Broad & $\begin{array}{l}\text { Broad }= \\
8 \text { types }\end{array}$ & $\begin{array}{c}\text { Narrow }= \\
2\end{array}$ & $\begin{array}{c}\text { Not } \\
\text { Preserved }\end{array}$ & Not Analyzed & $<1.5$ & $\begin{array}{l}\text { Cooking } \\
\text { /Heating }\end{array}$ \\
\hline & $\begin{array}{c}\text { Cervenka/ } \\
\text { 41BL267/ } \\
\text { Area D, E-6, } \\
\text { level 120-121 }\end{array}$ & 5135 & $\begin{array}{l}\text { Blackland } \\
\text { Prairie }\end{array}$ & $\begin{array}{l}\text { Short- } \\
\text { term }\end{array}$ & Narrow & Narrow & Broad $=7$ & Unknown & Not Analyzed & $<1.5$ & $\begin{array}{l}\text { Cooking } \\
\text { /Heating }\end{array}$ \\
\hline
\end{tabular}


Table 9-23. Relevant Excavated Hunter-Gatherer Occupations in the Central Texas Region.

\begin{tabular}{|c|c|c|c|c|c|c|c|c|c|c|c|}
\hline 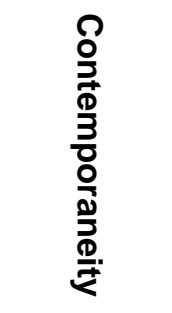 & 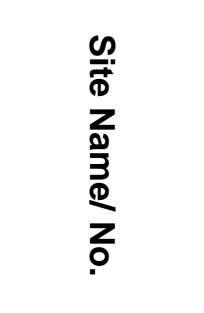 & 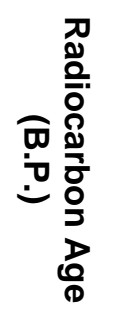 & 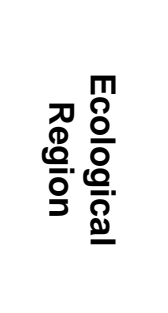 & 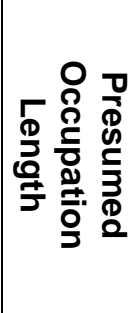 & 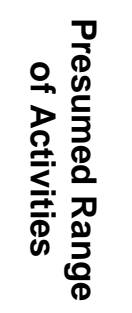 & 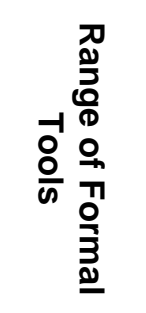 & 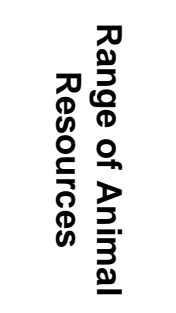 & 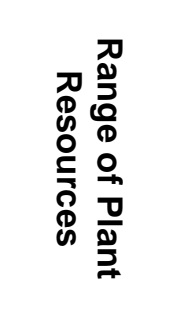 & 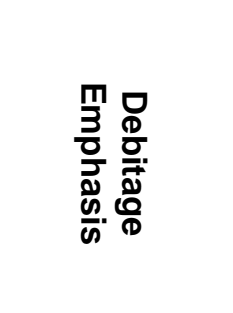 & 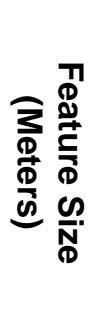 & 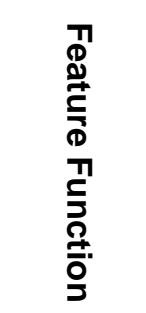 \\
\hline & $\begin{array}{l}\text { Landslide/ } \\
\text { 41BL85/ } \\
\text { Stratum V }\end{array}$ & None & $\begin{array}{l}\text { Edwards } \\
\text { Plateau }\end{array}$ & $\begin{array}{c}? \\
\text { Short- } \\
\text { term }\end{array}$ & Broad & $\begin{array}{l}\text { Broad }= \\
6 \text { types }\end{array}$ & $\begin{array}{c}\text { Not } \\
\text { Preserved }\end{array}$ & $\begin{array}{c}\text { Not } \\
\text { Preserved }\end{array}$ & Not Analyzed & $<1.5$ & $\begin{array}{l}\text { Cooking } \\
\text { /Heating }\end{array}$ \\
\hline & 41TV540 & $\begin{array}{l}5240- \\
5320\end{array}$ & $\begin{array}{c}\text { Blackland } \\
\text { Prairie }\end{array}$ & $\begin{array}{l}\text { Short- } \\
\text { term }\end{array}$ & Narrow & $\begin{array}{c}\text { Narrow }= \\
15 \text { total }\end{array}$ & Broad $=5$ & $\begin{array}{l}\text { Narrow }=1, \\
\text { bulbs }\end{array}$ & $\begin{array}{l}\text { Early \& middle } \\
\text { core reduction, } \\
\text { expedient tools }\end{array}$ & $<1.5$ & $\begin{array}{l}\text { Heating/ } \\
\text { Cooking }\end{array}$ \\
\hline \multirow{5}{*}{$\begin{array}{l}\text { Following } \\
\text { Period }\end{array}$} & $\begin{array}{c}\text { Holt/ } \\
\text { 41HY341/ } \\
\text { Zone III }\end{array}$ & 4740 & $\begin{array}{c}\text { Blackland } \\
\text { Prairie }\end{array}$ & $\begin{array}{l}\text { Short- } \\
\text { term }\end{array}$ & Narrow & $\begin{array}{c}\text { Narrow }= \\
2 \text { types }\end{array}$ & $\begin{array}{c}\text { Narrow }= \\
1\end{array}$ & Narrow $=1$ & $\begin{array}{l}\text { Core \& biface } \\
\text { reduction, } \\
\text { manufacturing }\end{array}$ & $>1.5$ & Oven \\
\hline & $\begin{array}{c}\text { Barrett/ } \\
41 \mathrm{MM} 382\end{array}$ & $\begin{array}{l}3310- \\
4040\end{array}$ & $\begin{array}{c}\text { Blackland } \\
\text { Prairie }\end{array}$ & $\begin{array}{l}\text { Short- } \\
\text { term }\end{array}$ & Narrow & Narrow & $\begin{array}{c}\text { Narrow }= \\
2\end{array}$ & $\begin{array}{c}\text { Not } \\
\text { Preserved }\end{array}$ & $\begin{array}{c}\text { Cobble } \\
\text { reduction, } \\
\text { biface \& flake } \\
\text { production }\end{array}$ & $<1.5$ & $\begin{array}{l}\text { Heating/ } \\
\text { Cooking }\end{array}$ \\
\hline & 41TV410 & $\begin{array}{c}4760- \\
4830\end{array}$ & $\begin{array}{c}\text { Blackland } \\
\text { Prairie }\end{array}$ & $\begin{array}{l}\text { Short- } \\
\text { term }\end{array}$ & Narrow & Narrow & $\begin{array}{c}\text { Narrow }= \\
2\end{array}$ & $\begin{array}{c}\text { Not } \\
\text { Preserved }\end{array}$ & No analysis & $<1.5$ & $\begin{array}{l}\text { Heating/ } \\
\text { Cooking }\end{array}$ \\
\hline & $\begin{array}{c}\text { Royal } \\
\text { Coachman/ } \\
41 \mathrm{CM} 111\end{array}$ & $\begin{array}{c}4770- \\
4990\end{array}$ & $\begin{array}{l}\text { Edwards } \\
\text { Plateau }\end{array}$ & $\begin{array}{l}\text { Short- } \\
\text { term }\end{array}$ & Narrow & Narrow & $\begin{array}{c}\text { Narrow }= \\
2\end{array}$ & $\begin{array}{c}\text { Not } \\
\text { Preserved }\end{array}$ & Lithic retooling & $<1.5$ & $\begin{array}{l}\text { Heating/ } \\
\text { Cooking }\end{array}$ \\
\hline & $\begin{array}{l}\text { Richard } \\
\text { Beene/ } \\
\text { 41BX831/ } \\
\text { Upper } \\
\text { Medina }\end{array}$ & $\begin{array}{l}4100- \\
4600\end{array}$ & $\begin{array}{c}\text { Coastal } \\
\text { Plain }\end{array}$ & $\begin{array}{l}\text { Short- } \\
\text { term }\end{array}$ & Broad & $\begin{array}{l}\text { Broad }= \\
6 \text { types }\end{array}$ & Narrow & $\begin{array}{c}\text { Not } \\
\text { Preserved }\end{array}$ & $\begin{array}{l}\text { Manufacturing } \\
\& \\
\text { reconditioning } \\
\text { of tools }\end{array}$ & $<1.5$ & $\begin{array}{l}\text { Heating/ } \\
\text { Cooking }\end{array}$ \\
\hline
\end{tabular}


One exception, is this Bell/Andice component probably does not represent a residential camp occupied by multiple families. The combined evidence supports limited tasks performed, which were most often performed by females who pursued a specific set of resources, plants and primarily small game, away from the primary camp (e.g., Dempsey 2001; Ewers 1982; Fowler and Flannery
2001; Grinnell 1972; Hughes 1991; Lowie 1963; Newcomb 1993; Parks 2001; Voget 2001). The high density of cooking and discard features combined with well-patterned distribution of specific cultural material classes reflect a close nit cultural group in a localized area, which focused on food preparation, cooking and related activities. 


\subsection{MARTINDALE COMPONENT}

\author{
J. Michael Quigg and Paul M. Matchen
}

\subsection{INTRODUCTION}

The following chapter presents materials recovered from a deeply buried (ca. 250 to $275 \mathrm{cmbs}$ ), very well-defined Martindale component. This component yielded three small features, with associated chipped and ground stone tool assemblages, moderate frequencies of lithic debitage, few mussel shells, scattered burned rocks, and a diverse and significant vertebrate faunal assemblage. The component was recognized across Block D and 12 units in the southwestern part of Block B with $74 \mathrm{~m}^{2}$ excavated. Component age is addressed first, followed by a description and discussion of the cultural features. Then, chipped stone tools and lithic debitage classes are presented, followed by ground stone tools, vertebrate faunal remains, mussel shell remains, and, finally, burned rocks. A summary and discussion follow at the end, with several research issues summarized along with addressing the two hypotheses presented in the research design (see Chapter 4.0).

\subsection{DATING THE COMPONENT}

The Martindale component was stratigraphically below the Bell/Andice component, hence it is older. The preservation of organic remains within this component was quite varied. The near absence of charcoal was frustrating; therefore, multiple materials (i.e., Rabdotus shells, organic sediment, and faunal specimens), were submitted for dating. Fifteen assays were obtained from multiple blocks, with five dates derived from vertebrate faunal material, five dates from organic sediment, two derived from Rabdotus shells, two from wood charcoal, and one from charred cheno-am and grass seeds (Table 10-1; Figure 10-1). The results provide a broad range of over 1,000 years from 5260 B.P. on a bone to 6310 B.P. on organic sediment. Consequently, only the more reliably dated materials charcoal and seeds were considered to establish the actual age of this component.

Two charcoal and one seed sample from two Martindale features (Features 26 and 33) horizontally separated by nearly $13 \mathrm{~m}$, provide an average age of 5393 B.P. with a narrow radiocarbon date range of 110 years. The range is excellent, but the average is younger than anticipated (see Section 3.3.3). Only 71 radiocarbon years separate this average from the Bell/Andice component average age of 5322 B.P., also derived from charcoal. As documented in the geomorphological section above (see Chapter 6.0) with roughly $20 \mathrm{~cm}$ of sediment between the two cultural components, this average charcoal age is acceptable for this Martindale component.

If the date derived from the charred seeds is considered most likely to represent the true age of the actual event, as the seeds likely represent annuals charred at the specific time, then old wood may be the principal cause for the two charcoal dates being slightly older than the seed date. Collecting and employing old dead and dried wood would certainly make for good fire wood and the resulting radiocarbon dates would definitely not reflect the time of the cultural event that used the old wood.

The five dates obtained from faunal material also from Block D yielded a narrow range of 250 years or from 5260 to 5510 B.P. with an average of 5382 B.P. (see Table 10-1). The statistical mean of these faunal results is comparable to the three charcoal dates. These dates are much more consistent than the bone dates from the Bell/Andice component. Hence, the five bone dates from Block D are also accepted as representing the age of this Martindale component. 
Table 10-1. Radiocarbon Dates from the Martindale Component.

\begin{tabular}{|c|c|c|c|c|c|c|c|c|c|c|c|}
\hline $\begin{array}{c}\text { Catalogue } \\
\text { No. }\end{array}$ & Block & Unit No. & $\begin{array}{l}\text { Depth } \\
\text { (cmbs) }\end{array}$ & $\begin{array}{l}\text { Feature } \\
\text { No. }\end{array}$ & $\begin{array}{l}\text { Material } \\
\text { Dated }\end{array}$ & $\begin{array}{l}\text { Wt. of } \\
\text { Material } \\
\text { (g) }\end{array}$ & $\begin{array}{l}\text { Beta } \\
\text { Lab. } \\
\text { No. }\end{array}$ & $\begin{array}{l}\text { Measured } \\
\text { Age }\end{array}$ & $\begin{array}{c}\text { 13C/12C } \\
\text { Ratio } \\
(\%)\end{array}$ & $\begin{array}{l}\text { Conventional } \\
\text { Age (B.P.) }\end{array}$ & $\begin{array}{c}2 \text { Sigma } \\
\text { Calibration } \\
\text { Range } \\
\end{array}$ \\
\hline TV2161/M-4 & $\mathrm{D}$ & N83/E93 & $260-270$ & & $\begin{array}{l}6 \text { Rabdotus } \\
\text { shells }\end{array}$ & 3.6 & 216371 & $5920 \pm 50$ & -10.1 & $6160 \pm 50^{1}$ & $\begin{array}{c}\text { BC } 5270 \text { to } \\
4940\end{array}$ \\
\hline $2239-4-1$ & $D$ & N78/E93 & $264-269$ & 25 & $\begin{array}{l}\text { Organic } \\
\text { sediment }\end{array}$ & 96.0 & 234630 & $6230 \pm 40$ & -20.4 & $6310 \pm 40^{1}$ & $\begin{array}{c}\text { BC } 5360 \text { to } \\
5330\end{array}$ \\
\hline $2241-4-1$ & D & N78/E94 & $270-275$ & 25 & $\begin{array}{l}\text { Organic } \\
\text { sediment }\end{array}$ & & 234631 & $5180 \pm 40$ & -19.9 & $5260 \pm 40^{1}$ & $\begin{array}{c}\text { BC } 4230 \text { to } \\
3980\end{array}$ \\
\hline TV2161/M-5 & B & N71/E82 & $270-280$ & 26 & $\begin{array}{l}6 \text { Rabdotus } \\
\text { shells }\end{array}$ & 3.4 & 216372 & $5600 \pm 50$ & -10 & $5850 \pm 50^{1}$ & $\begin{array}{c}\text { BC } 4800 \text { to } \\
4580\end{array}$ \\
\hline $2112-4-3$ & $B$ & N71/E83 & $270-280$ & 26 & $\begin{array}{l}7 \text { cheno-am } \\
\text { seeds }\end{array}$ & 0.1 & 398638 & $5350 \pm 30$ & -25.7 & $5340 \pm 30$ & $\begin{array}{c}\text { BC } 4315 \text { to } \\
4050\end{array}$ \\
\hline $2112-4-3 g$ & $B$ & N71/E83 & $270-280$ & 26 & Charcoal & 0.2 & 398639 & $5390 \pm 30$ & -25 & $5390 \pm 30$ & $\begin{array}{c}\text { BC } 4330 \text { to } \\
4175\end{array}$ \\
\hline 2112-4-1 & B & N71/E83 & $268-277$ & 26 & $\begin{array}{c}\text { Organic } \\
\text { sediment }\end{array}$ & 97 & 234632 & $5420 \pm 40$ & -24.7 & $5420 \pm 40^{1}$ & $\begin{array}{c}\text { BC } 4340 \text { to } \\
4230\end{array}$ \\
\hline 2136-7-1a & B & N72/E82 & $271-277$ & 26 & $\begin{array}{c}\text { Organic } \\
\text { sediment }\end{array}$ & 172 & 234634 & $5430 \pm 40$ & -24.3 & $5440 \pm 40^{1}$ & $\begin{array}{c}\text { BC4350 to } \\
4240\end{array}$ \\
\hline 2144-7-1 & $D$ & N72/E83 & $280-290$ & & Charcoal & 35.9 & 398640 & $5460 \pm 30$ & -25.4 & $5450 \pm 30$ & $\begin{array}{c}\text { BC } 4345 \text { to } \\
4260\end{array}$ \\
\hline $2280-4-1 a$ & $D$ & N82/E91 & $274-280$ & 33 & $\begin{array}{c}\text { Organic } \\
\text { sediment }\end{array}$ & 132 & 234633 & $6120 \pm 50$ & -21.9 & $6170 \pm 50^{1}$ & $\begin{array}{c}\text { BC } 5290 \text { to } \\
4990\end{array}$ \\
\hline $2250-2-c$ & $D$ & N79/E95 & $270-275$ & & $\begin{array}{c}\text { Bone } \\
\text { fragment }\end{array}$ & 4.3 & 398650 & $5440 \pm 30$ & -21 & $5510 \pm 30$ & $\begin{array}{c}\text { BC } 4440 \text { to } \\
4330\end{array}$ \\
\hline $2251-2-3$ & $D$ & N80/E91 & $257-270$ & & $\begin{array}{c}\text { Bone } \\
\text { fragment }\end{array}$ & 6.2 & 398651 & $5330 \pm 30$ & -19.9 & $5410 \pm 30$ & $\begin{array}{c}\text { BC } 4335 \text { to } \\
4235\end{array}$ \\
\hline $2280-2$ & $D$ & N82/E91 & $270-280$ & & $\begin{array}{c}\text { Bone } \\
\text { fragment }\end{array}$ & 4.2 & 398652 & $5160 \pm 30$ & -18.9 & $5260 \pm 30$ & $\begin{array}{c}\text { BC } 4225 \text { to } \\
3985\end{array}$ \\
\hline TV2161/M-8 & C & N68/E96 & 286 & & Deer radius & 13.9 & 216375 & $5370 \pm 40$ & -20.3 & $5440 \pm 40$ & $\begin{array}{c}\text { BC } 4350 \text { to } \\
4230\end{array}$ \\
\hline TV2161/M-9 & C & N66/E95 & $280-290$ & & Deer incisor & 0.9 & 216376 & NA & & & \\
\hline
\end{tabular}

${ }^{1}$ questionable results based on material dated in comparison to the charcoal dates. 


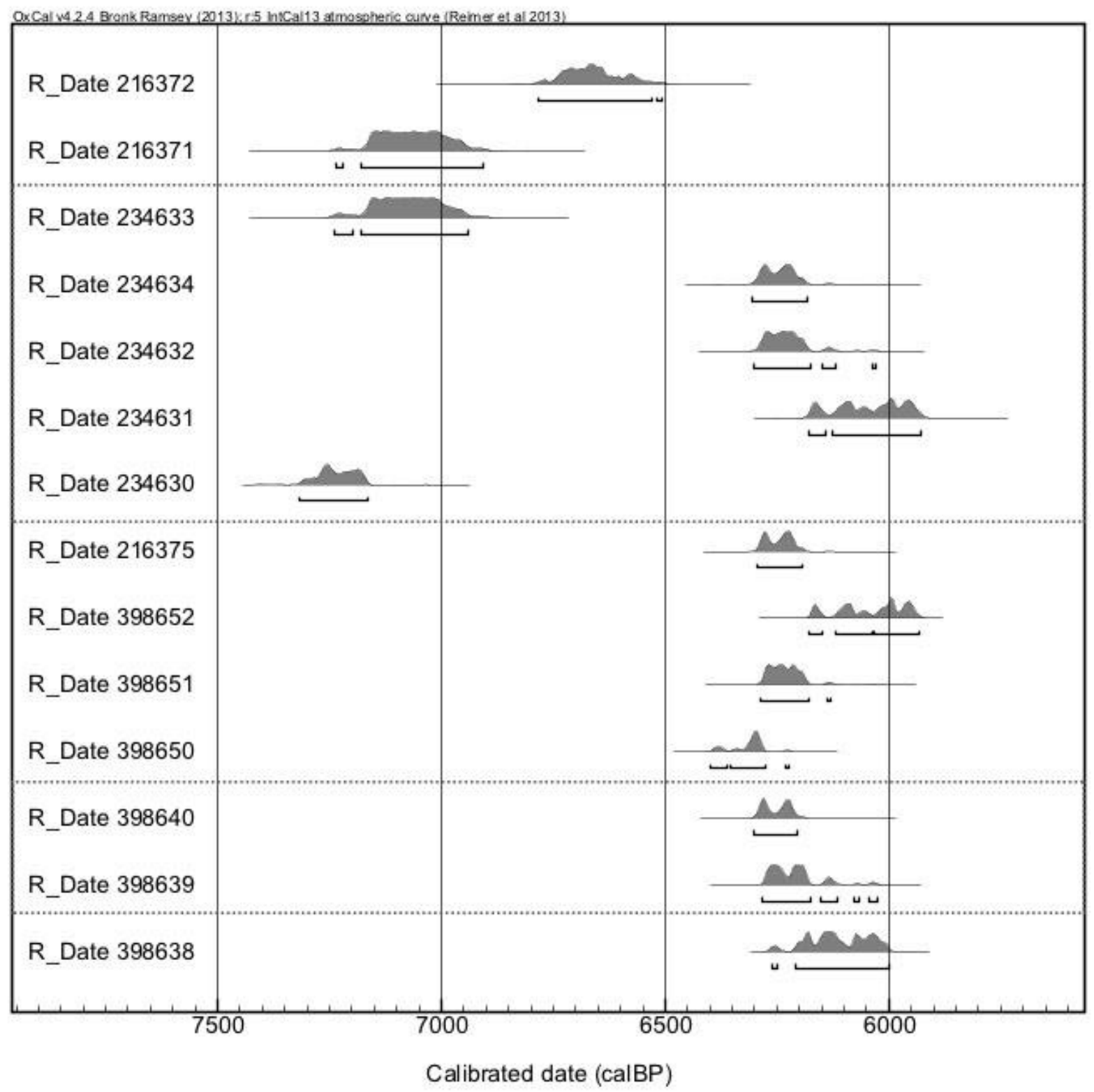

Figure 10-1. Plot of radiocarbon assays from the Martindale component. Samples are presented from top to bottom in the following order: Rabdotus shells (2), organic sediment (5), bone (4), charcoal (2), and seeds (1). 


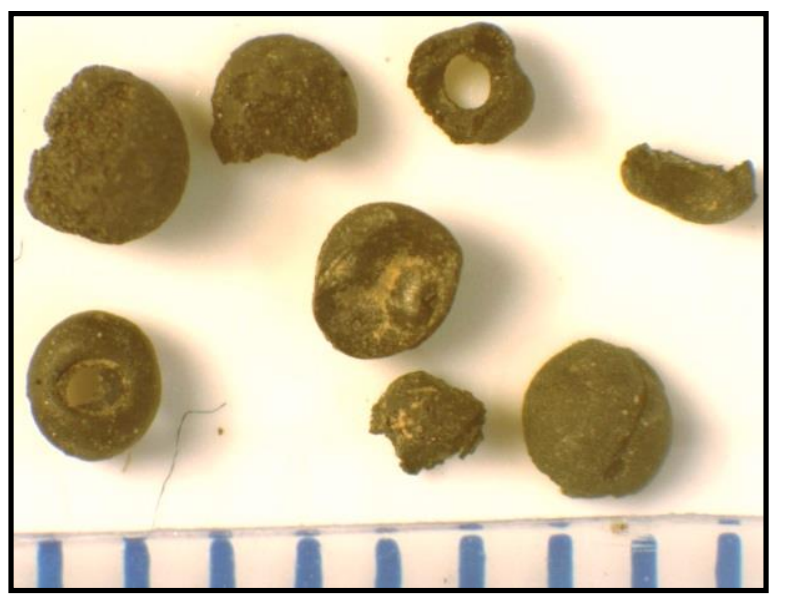

Figure 10-2. Charred cheno-am and grass seeds from Feature 26 radiocarbon dated to document the specific age of the Martindale component (scale in millimeters).

The date of 5340 B.P. derived from seven charred cheno-am (Figure 10-2) and grass seeds from Feature 26 is significant (). These annual seeds are probably the best means of determining the true cultural age or the specific time represented as the seeds were charred during the occupation. This may also be the oldest date on such seeds in central Texas, and reflects a very early use of cheno-am and grasses. In general terms charred seeds are not often preserved and less likely to have been radiocarbon dated.

The two bone dates of 5440 and 5290 B.P., from Block C may also reflect the age of the Martindale component, as they are within 100 years of the charcoal and seed dates from Feature 26. This potential association cannot be confirmed by the date alone as no other diagnostic materials were recovered in Block $\mathrm{C}$.

Rabdotus shells provided two of the four oldest dates obtained with dates ranging from 5850 to 6160 B.P. (see Table 10-1). Although their results are remarkably close to one another, the average at 5970 B.P. is 577 years older than the average derived from the three charcoal results from similar stratigraphic positions. The date of 5850 B.P. from shells in Feature 26 is from the same feature dated by charcoal to 5390 B.P. and charred cheno-am and grass seeds to 5340 B.P. This discrepancy indicates the shells, although from the same stratigraphic context as the charcoal and bones, provide ages not directly linked to the age of the cultural occupation. This probably results from snails incorporating older carbon into their shells as part of their development (see Goodfriend 1987 for age anomalies in land snails; Ellis and Goodfriend 1994 for assessment of dating land snails; Quigg and Ellis 1994; and Frederick 2011 for chronometric dating comparisons). As previous authors have suggested, radiocarbon ages derived from Rabdotus shells will probably provide approximations usually slightly older than the preferred wood charcoal assays.

Organic rich sediment dates provide the two oldest dates at 6170 B.P. and 6310 B.P. along with three dates that fit nicely with the charcoal dates. The oldest date of 6310 B.P. is from Feature 25 and the same context as a second organic sediment date of 5260 B.P. The latter is 133 years younger than the average charcoal dates for this component and as such are more reasonable than the date of 6310 B.P. Therefore, one of the two sediment dates from Feature 25 appears acceptable. The difference between the two highlights the problem with organic sediment dates as the two are 1,050 radiocarbon years apart from the same exact context.

Two organic sediment dates from Feature 26 can be compared to two charcoal and seed dates from the same feature. The organic sediment dates of 5420 B.P. and 5440 B.P. are consistent and only ca. 100 years older than the two charcoal and seed dates of 5340 B.P. and 5390 B.P. On the other hand, the organic sediment date of 6170 B.P. from Feature 33 is 720 years older than the charcoal date of 5440 B.P. from the same feature. The former date is not acceptable. Again, the organic sediment date is much older than the charcoal date and is less likely to represent to true age of this event. 
As results indicate, radiocarbon data from organic sediment samples provide an imprecise dating index in comparison to the data yielded from charcoal and seeds. As previous researchers have documented (e.g., Abbott 1994; Collins 1994b; Frederick 2011) organic sediment dates are generally older than the cultural occupations and should be only dated when other more suitable materials such as charred seeds and charcoal for dating are not available.

Comparing the different materials dated, the charcoal and seeds are consistent with one another and provide a solid index for the age of this Martindale component. Organic sediment dates are often inconsistent and provide reasonable, but generally older ages when compared to charcoal results. Rabdotus shells provide older and ballpark dates, which may be useful in certain circumstances. In this case the Rabdotus dates do not reflect the true age of the component when compared to the charcoal and seed results. Potential reasons for their older ages are that the shells are much older than the cultural occupation, or the shells have incorporated old carbon during the life spans.

\subsection{MARTINDALE CULTURAL FEATURES}

Three cultural features were identified for this component, two in Block D (Features 25 and 33) and one in Block B (Feature 26) (Figure 10-3). Feature 25 was observed between 264 and 269 cmbs in the southeastern half of N79/E93 and northeastern corner of N78/E93 in Block D. Feature 33 was observed at the juncture of four excavation units, the southeastern corner of N83/E90, the northeastern corner of N82/E90, the northwestern corner of N82/E91, and fades out in the southwestern corner of N83/E91. It was approximately 4 m north-northwest of Feature 25. Feature 33 was encountered between 273 and 280 cmbs, just below the 1 to $2 \mathrm{~cm}$ thick light greenish- gray clay lens. The light greenish-gray lens slopes up towards the north and Block B.

Feature 26, and associated cultural material, was uncovered in N71/E83 and N71/E82 of Block B between 264 and 267 cmbs. This feature was in clear stratigraphic context below the Bell/Andice component and the light greenish-gray clay lens (observed ca. 264 to $266 \mathrm{cmbs}$ in Block B). Each feature is described and discussed in numerical order below.

\subsubsection{Feature 25}

Feature 25 consisted of a small oval of dark stained sediment with a shallow $5 \mathrm{~cm}$ deep, saucer shaped basin that measured approximately 70 -by $-55 \mathrm{~cm}$ in diameter. The irregular ovate area exhibited scattered degraded charcoal with yellowish brown (10YR 5/8) silty clay mottled with reddish yellow clay spots, which roughly defined the feature. Areas of darker concentrated degraded charcoal were about 30-by-40 $\mathrm{cm}$ in size near the middle and within an oval area about 15-by-20 cm towards the northeastern margin. A natural light yellowish brown (10YR 6/4) sandy clay loam surrounded Feature 25 and contrasted with the mottled dark brown, reddish yellow clays in the feature (Figures 10-4 and 10-5). The oval, dark stained sediment with a shallow basin feature typically represents a hearth.

Additional materials included four burned rocks scattered to the western side, a small chert pebble in the lighter stained area on northern side, and a deer or pronghorn axis on the southern margin. The burned rocks were between 6 and $9 \mathrm{~cm}$ in diameter (Table 102). No burned rocks were sent for technical analysis.

Approximately 5.6 liters of feature fill (\#2239-7-2 and \#2239-7-3) from the southern half were collected and floated by Dr. Dering. No charred plant remains were recovered (Appendix B). At least two pieces of chert debitage and numerous fragments of small animal bones (40 to 50 tiny pieces) were recovered from feature fill. 


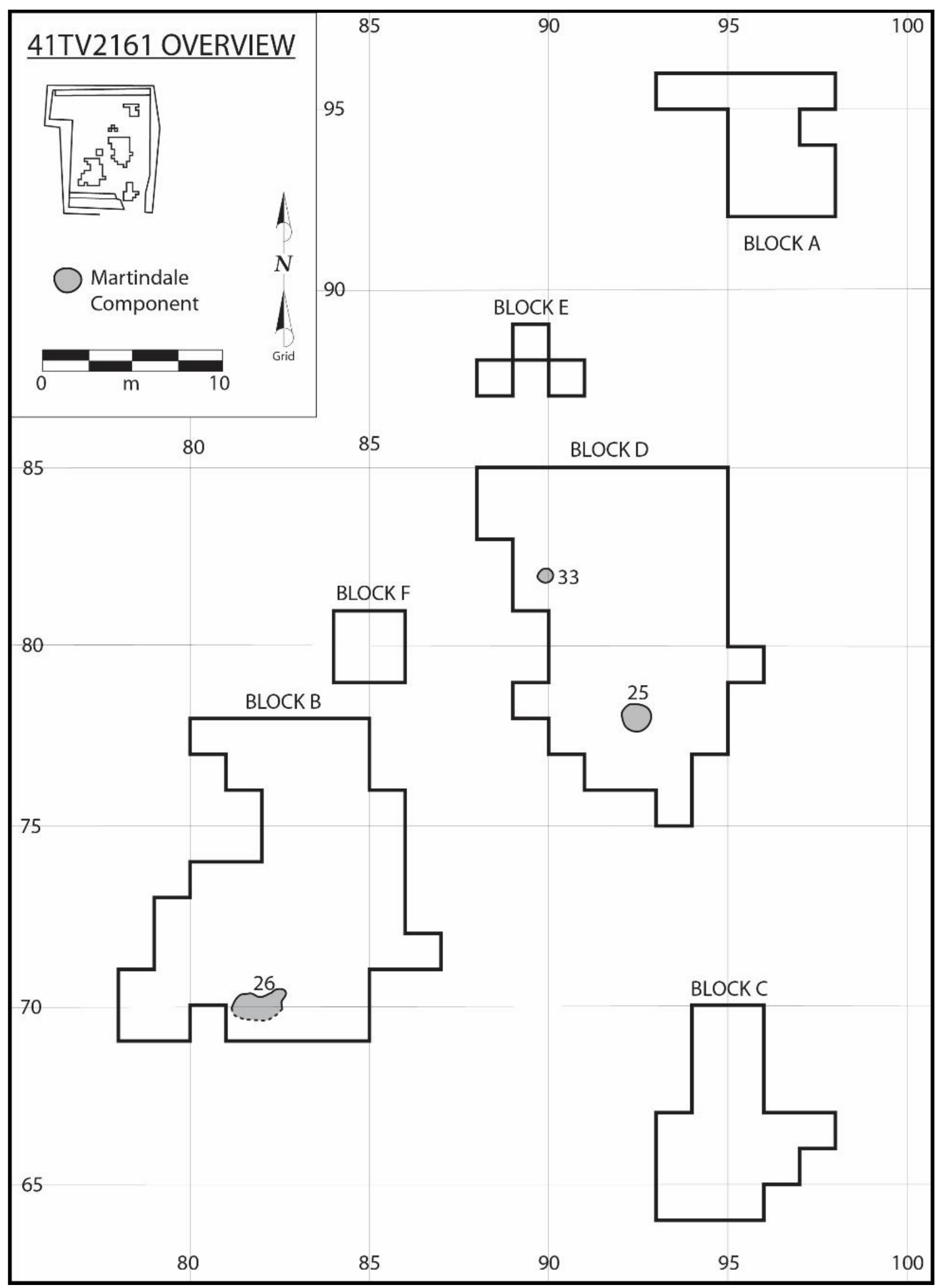

Figure 10-3. Location of Martindale features across Blocks B and D. 


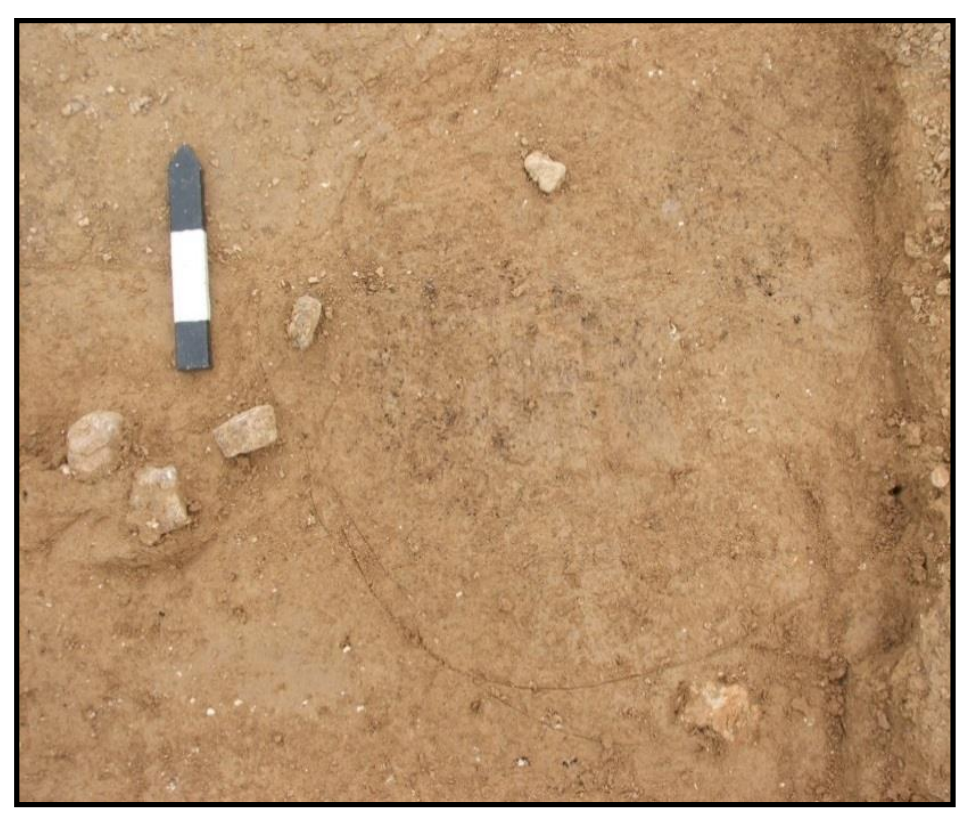

Figure 10-4. Overhead photograph of charcoal stained sediment of Feature 25 with burned rocks along the outer western margin.

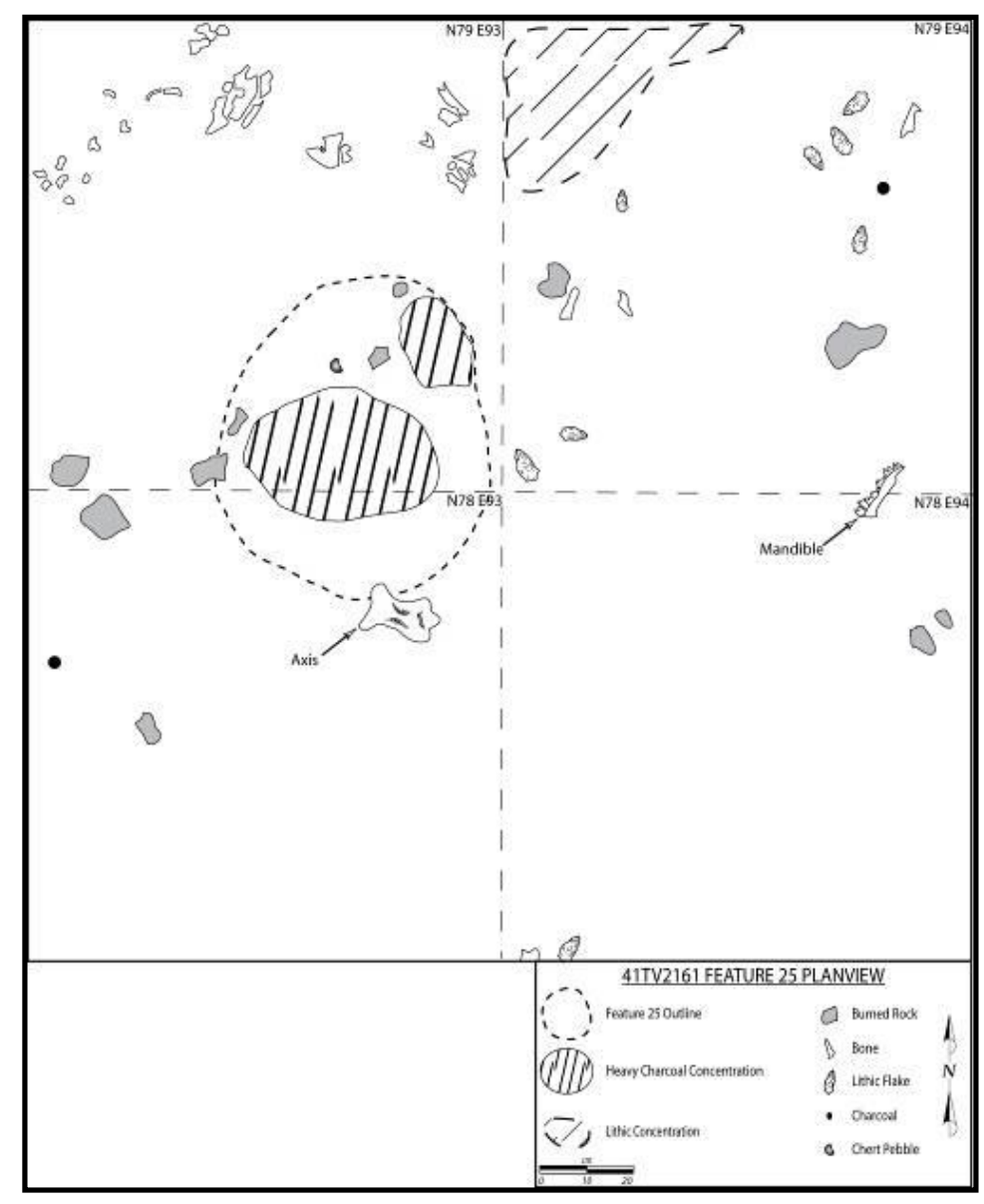

Figure 10-5. Plan view drawing of Feature 25 and cultural materials in four units that surrounded the small circular stained sediment. 
Table 10-2. Burned Rock Counts and Weights for Each Martindale Feature.

\begin{tabular}{|c|c|c|c|c|c|c|c|c|c|c|c|c|c|}
\hline $\begin{array}{c}\text { Feature } \\
\text { No. }\end{array}$ & Unit & $\begin{array}{l}\text { Depth } \\
\text { (cmbs) }\end{array}$ & PNUM & $\begin{array}{l}\text { BR 0-4 } \\
\text { cm } \\
\text { (Count) }\end{array}$ & $\begin{array}{l}\text { BR 0-4 } \\
\text { Wt. (g) }\end{array}$ & $\begin{array}{c}\text { BR 4.1-9 } \\
\text { cm } \\
\text { (Count) }\end{array}$ & $\begin{array}{c}\text { BR 4.1-9 } \\
\text { Wt. (g) }\end{array}$ & $\begin{array}{c}\text { BR 9.1-15 } \\
\text { cm } \\
\text { (Count) }\end{array}$ & $\begin{array}{c}\text { BR 9.1-15 } \\
\text { Wt. (g) }\end{array}$ & $\begin{array}{c}B R>15 \\
c m \\
\text { (Count) }\end{array}$ & $\begin{array}{l}\text { BR >15 } \\
\text { Wt. (g) }\end{array}$ & $\begin{array}{l}\text { Total } \\
\text { Count }\end{array}$ & $\begin{array}{c}\text { Total } \\
\text { Wt. (g) }\end{array}$ \\
\hline 25 & N79 E93 & $254-266$ & 2413 & & & 4 & 700 & & & & & 4 & 700 \\
\hline \multirow{3}{*}{26} & N71 E82 & $260-270$ & 2102 & 12 & 180 & 3 & 525 & & & & & 15 & 705 \\
\hline & N71 E83 & $260-269$ & 2110 & 18 & 50 & 2 & 194 & & & & & 20 & 244 \\
\hline & & $267-274$ & 2112 & 4 & 62 & 18 & 3162 & & & & & 22 & 3224 \\
\hline \multirow{2}{*}{33} & N83 E90 & $270-280$ & 2289 & 2 & 50 & & & 1 & 450 & & & 3 & 500 \\
\hline & N83 E91 & $270-272$ & 2292 & 4 & 60 & & & & & & & 4 & 60 \\
\hline
\end{tabular}


A small sediment sample (\#2239) from inside the southwestern quadrant was subjected to phytolith analysis. This yielded seven bone fragments, three of which were burned, abundant tree phytoliths, and three gourd (Cucurbita foetidissima) or buffalo gourd phytoliths (see Figure 9-26) (Appendix E). Also present was a single abraded sponge spicule (see Figure 9-10).

Two samples (both \#2239), one from sediment and one from calcium carbonate of a deer bone, were subjected to diatom analysis and yielded very similar results. The analysis of both samples yielded abundant benthic diatoms, which are found in shallow water and tolerate substantial organic pollution. There is not enough data to determine how the diatoms arrived in the feature, either with water for part of a cooking process or by some other means.

A concentration of approximately 150 pieces of chert debitage (\#2248) was found in the adjacent unit (N79/E84), just 30 to $40 \mathrm{~cm}$ to the northeast (see Figure 10-5). More than 300 animal bone fragments less than $6.4 \mathrm{~mm}$ were also in the four adjoining units outside the feature. Species identified include deer, pronghorn, jackrabbit, cottontail, rodent, and gar, with at least 13 rabbit size pieces burned. The highest frequency of bones was northeast in N79/E94 and the fewest northwest in N79/E93.

It is not clear if this hearth feature functioned for warming and/or cooking. The high concentration of butchered bone and lithic debitage immediately outside the feature boundary indicates multiple butchering and knapping tasks were conducted next to the hearth. Tiny cultural materials observed within Feature 25 were likely dumped into the hearth as a means of disposal.

\subsubsection{Feature 26}

Feature 26 consisted of two concentrations, a small burned rock cluster next to a dark stained basin hearth. The southern portion, maybe half, was not excavated as it extended into the southern wall of the excavation block. Therefore, the overall size and shape of the feature was not clear. Feature 26 measured at least 160 $\mathrm{cm}$ east-west and at least $76 \mathrm{~cm}$ north-south.

This complex feature exhibited a small cluster of burned rocks and dark stained and mottled sediments that surrounded a shallow $7 \mathrm{~cm}$ deep basin filled with dark organic matrix, a few small burned rocks, and many bone fragments and lithic debitage less than 6.4 mm (Figures 10-6 and 10-7). The basin hearth sediment was a brown (10YR 5/4) to black (10YR 2/1) silty clay, which contained charcoal flecks and chunks, small burned clay nodules, small burned rock fragments, and some chert debitage over a thin lens of darken matrix (degraded charcoal) at the bottom of the basin. The basin was about $7 \mathrm{~cm}$ deep with the top few centimeters blending into the surrounding dark stained matrix (Figures 10-8, 10-9, and 10-10).

The area immediately surrounding the hearth yielded a few broken mussel shell valves, small burned clay pieces, lithic debitage, small animal bone fragments, many Rabdotus snail shells, and scattered burned rocks (see Figure 10-6). Rabdotus shells were concentrated across the western margin, although their association with the cultural materials is not clear as the shells did not appear heat altered.

Large matrix samples, multiple burned rocks, and samples of Rabdotus snail shells from the western concentration were collected for future analyses. Six, more or less complete Rabdotus shells from the concentration were submitted for radiocarbon dating. The apex of each shell was removed before submission. The six shells weighed $3.4 \mathrm{~g}$ and yielded a $\delta^{13} \mathrm{C}$ corrected age of $5850 \pm 50$ B.P. (Beta-316372). Seven charred cheno-am and grass seeds (\#2112-4-3) derived from floated feature matrix yielded a $\delta^{13} \mathrm{C}$ corrected radiocarbon date of $5340 \pm 30$ B.P. A charcoal sample from the same provenience yielded a $\delta^{13} \mathrm{C}$ corrected date of $5390 \pm 30$ B.P. (see Table 101). The seed and charcoal dates are accepted as the age of this feature, whereas the Rabdotus date is not acceptable as to when this feature was in use. 


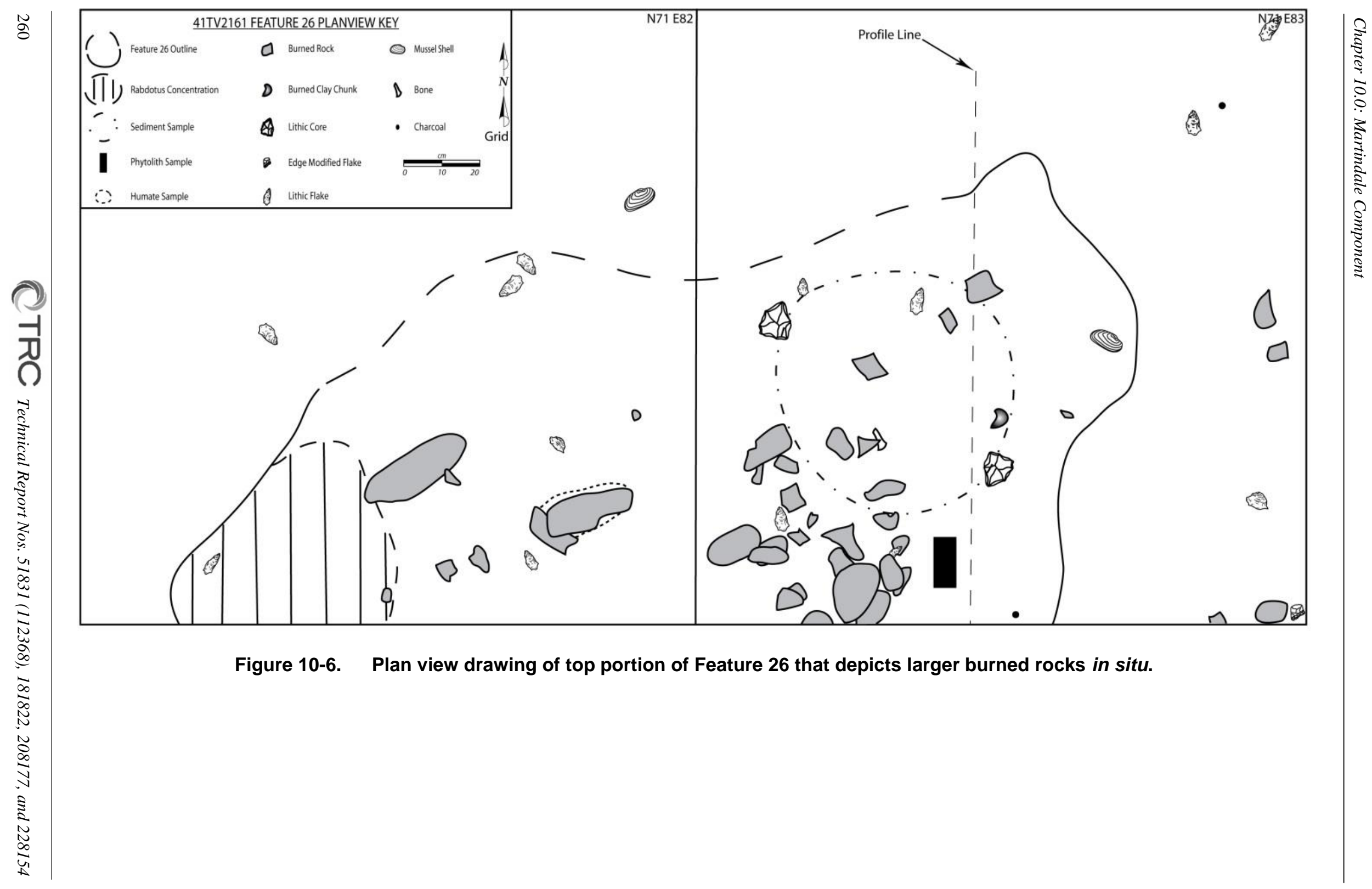




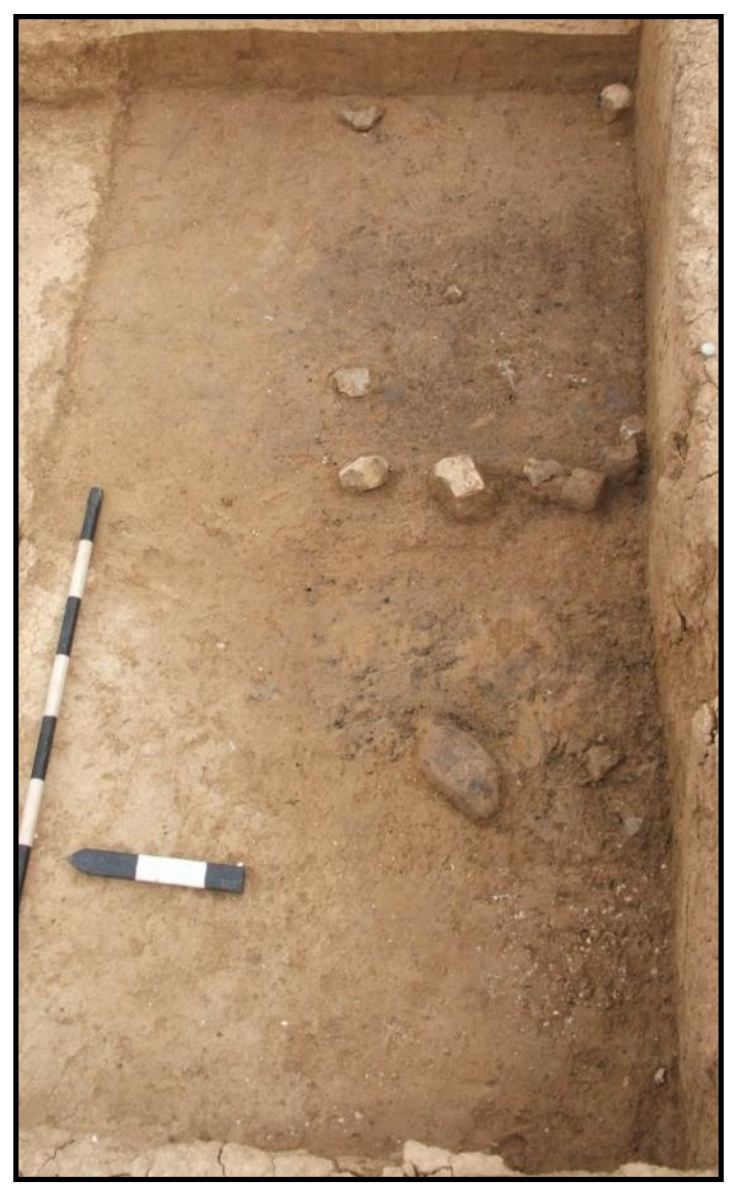

Figure 10-7. Oblique photograph of exposed top of Feature 26 depicting scattered burned rocks and dark stained sediments. Note complete oblong cobble is possible mano (\#2101-11). Scales in $10 \mathrm{~cm}$ intervals.

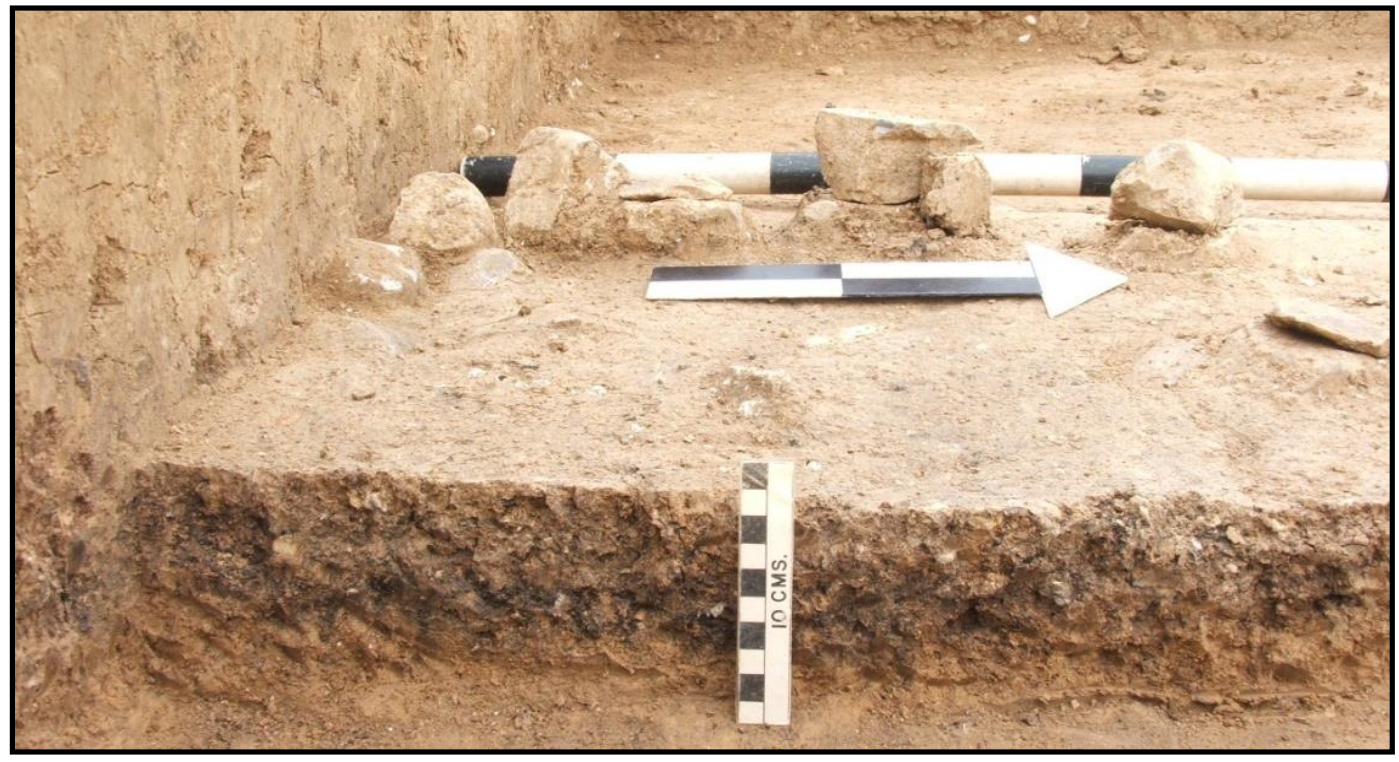

Figure 10-8. Oblique profile photograph of Feature 26 that depicts dark stained feature contents in shallow basin with burned rocks only along western margin. 


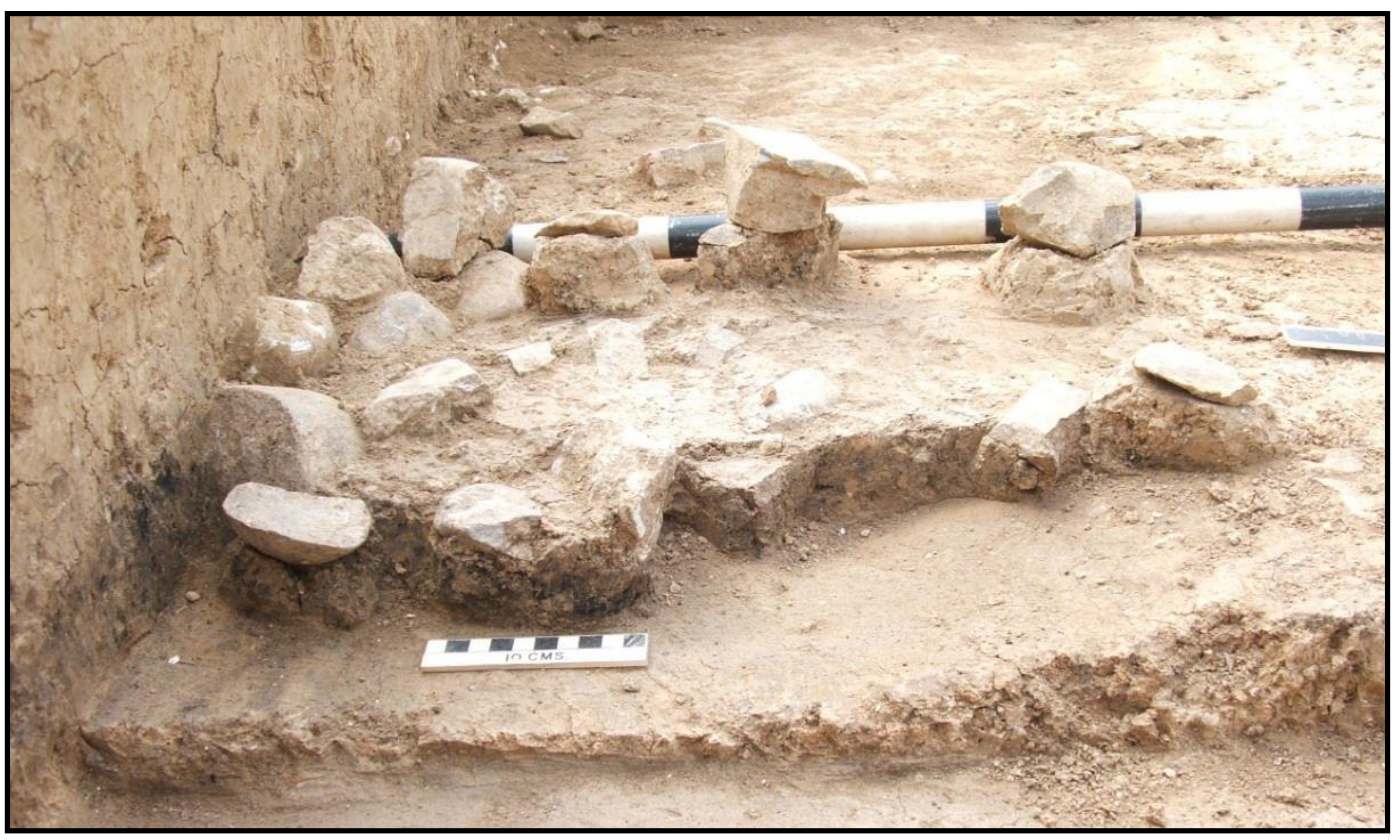

Figure 10-9. Oblique profile photograph of Feature 26 that depicts shallow baked base with burned rocks concentrated on western margin of basin.

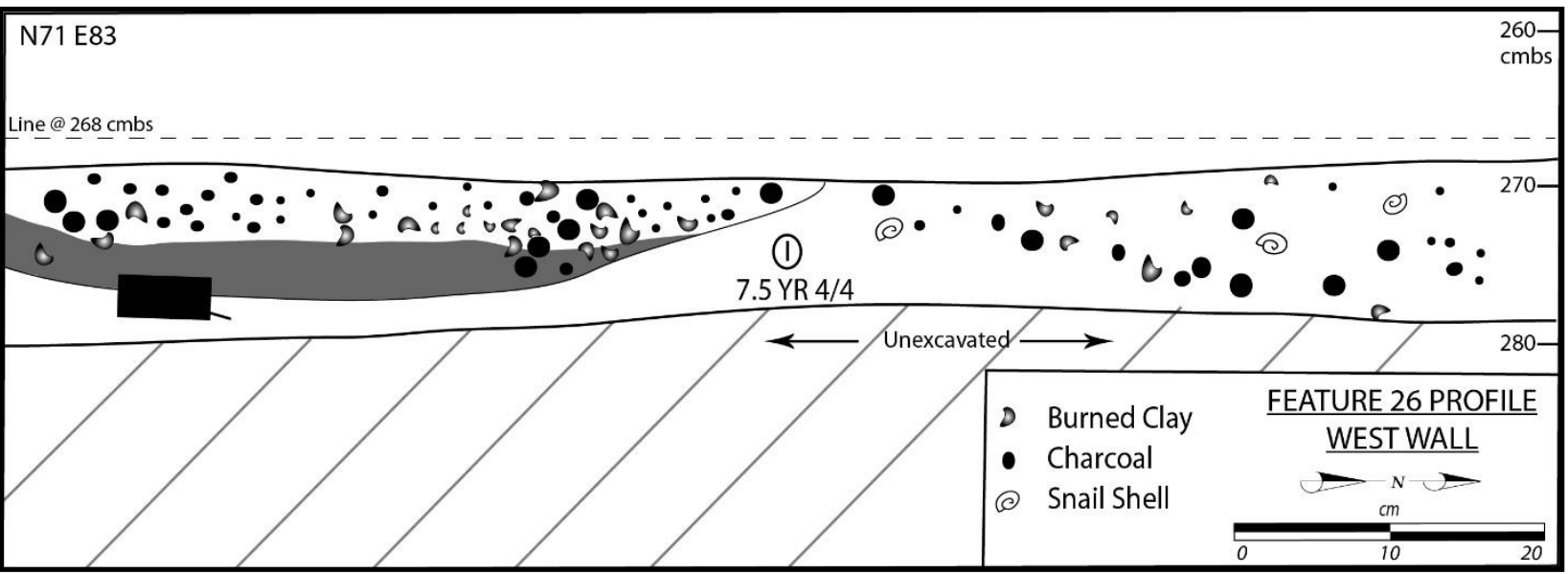

Figure 10-10. Drawing of north-south profile through dark stained basin of Feature 26.

A total of 57 burned rocks that weighed $4,172 \mathrm{~g}$ were from the stained sediments over parts of two units. These were less than $10 \mathrm{~cm}$ in diameter with 34 between 0 and $4 \mathrm{~cm}$ and weighed $291 \mathrm{~g}$, whereas 23 were 4.1 to $9 \mathrm{~cm}$ in diameter and weighed 3,881 $\mathrm{g}$ (see Table 10-2). One thin, tabular burned rock and possible metate fragment (\#2101-3-5) and a second rounded burned rock (\#2102-3-4) were stained with red hematite (Figure 10-11).
Multiple sediment samples were submitted for different technical analyses. Sediment sample \#2110-4 was subjected to diatom analysis, but yielded no diatoms (Appendix C). Phytolith analyses were conducted on two sediment samples (\#2110-4 and \#2112-4) and yielded different results from slightly different proveniences in Feature 26. 
Sample \#2110-4, collected from 266 to $268 \mathrm{cmbs}$ at the eastern side next to some burned rocks, yielded relatively good grass phytoliths with rare Pooids (from cool moist environments), a few Panicoids (intermediate environments) with 42 percent burned, and mainly Chloridoids (from hot dry environment), with 20 percent of the latter burned. This sample also yielded the highest frequency of sponge spicules $(N=65)$ (Appendix E), likely implying water was used in the feature. Sample \#2112-4, collected from 275 to $275 \mathrm{cmbs}$ in $\mathrm{N} 71 / \mathrm{E} 83$ at the base of the dark stained sediment, yielded mostly tree phytoliths, with 30 percent burned.

Starch grain analysis on seven burned rocks (\#2112-3) yielded two unknown grains, two lenticular grains likely from wildrye grass, and three gelatinized grains from exposure to heat and water (Appendix F). One edge-modified flake (\#2112-10) from Feature 26 did not yield any starch grains. One complete $15 \mathrm{~cm}$ long by $4.5 \mathrm{~cm}$ thick ovate rock and possible mano (\#2102-11) (Figure 10-12) also lacked starch grains.

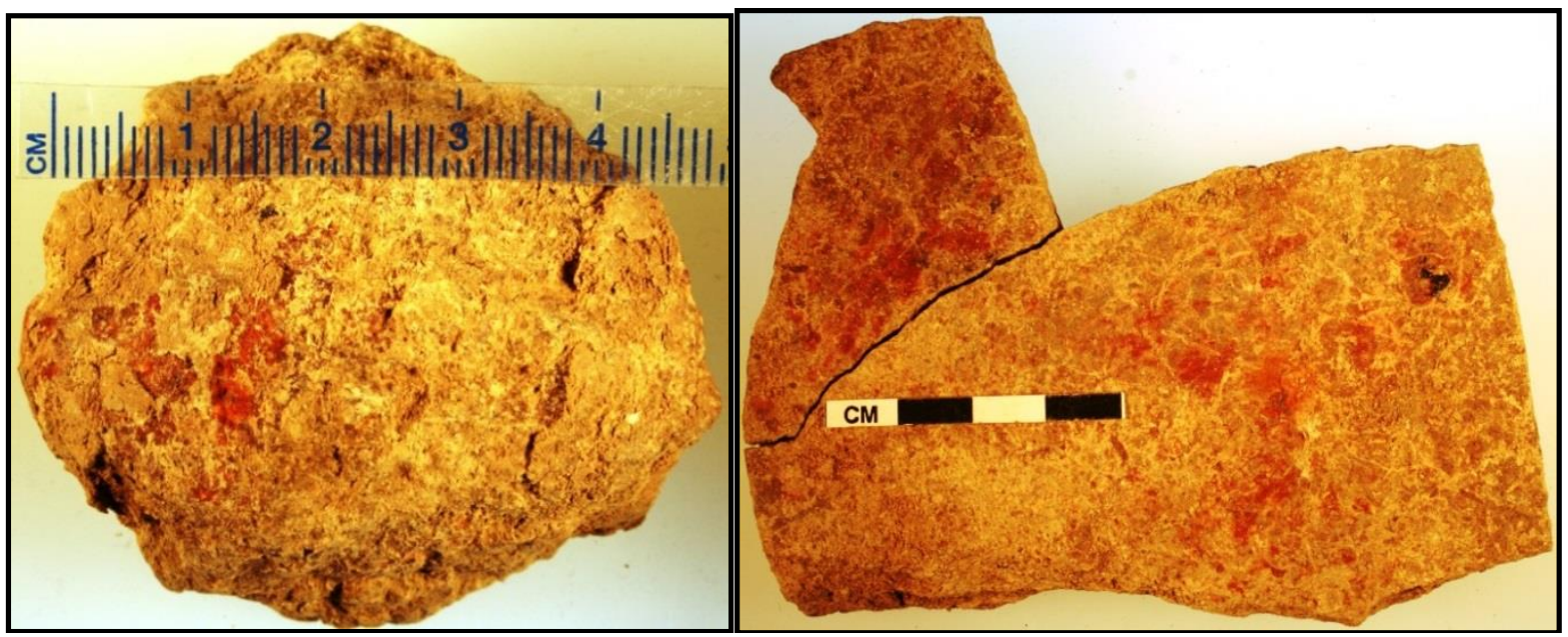

Figure 10-11. Burned rocks from inside Feature 26 with red hematite stained surfaces (\#2102-3-4 on left and \#2102-3-5 on right).

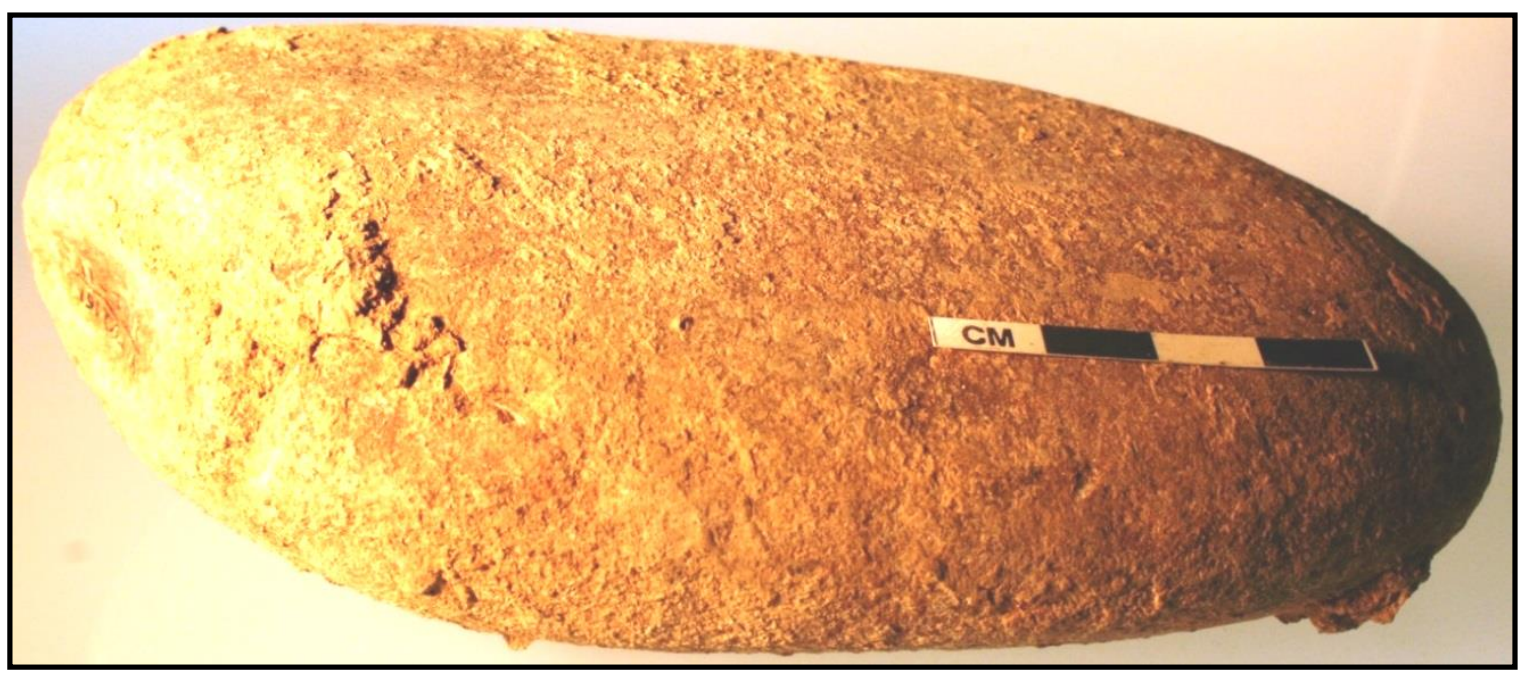

Figure 10-12. Possible mano (\#2101-11) from Feature 26. 
Table 10-3. Flotation Results with Counts and Weights by Material Class for Martindale Component.

\begin{tabular}{|c|c|c|c|c|c|c|c|c|c|c|c|c|c|c|c|c|c|c|c|c|c|c|}
\hline \multirow{2}{*}{ 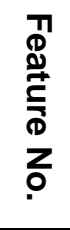 } & \multirow{2}{*}{$\begin{array}{l}\stackrel{8}{\not} \\
z \\
\stackrel{0}{z}\end{array}$} & \multirow{2}{*}{$\stackrel{ }{ }$} & \multirow{2}{*}{ 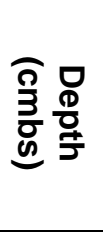 } & \multirow{2}{*}{$\begin{array}{l}\frac{m}{0} \\
\frac{\Gamma}{\lambda}\end{array}$} & \multirow{2}{*}{ 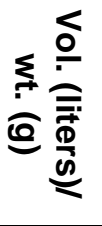 } & & \multicolumn{2}{|c|}{$\begin{array}{l}\text { Mussel } \\
\text { Shell }\end{array}$} & \multicolumn{2}{|c|}{$\begin{array}{l}\text { Burned } \\
\text { Rock }\end{array}$} & \multicolumn{2}{|c|}{ Debitage } & \multicolumn{2}{|c|}{ Charcoal } & \multicolumn{2}{|c|}{ Snail } & \multicolumn{2}{|c|}{$\begin{array}{l}\text { Macro- } \\
\text { botanical }\end{array}$} & \multicolumn{2}{|c|}{$\begin{array}{c}\text { Burned } \\
\text { Clay }\end{array}$} & \multicolumn{2}{|c|}{ Bone } \\
\hline & & & & & & & $1 / 4$ & $<1 / 4$ & $1 / 4$ & $<1 / 4$ & $1 / 4$ & $<1 / 4$ & $1 / 4$ & $<1 / 4$ & $1 / 4$ & $<1 / 4$ & $1 / 4$ & $<1 / 4$ & $1 / 4$ & $\begin{array}{c}<1 / \\
4\end{array}$ & $1 / 4$ & $<1 / 4$ \\
\hline \multirow{4}{*}{26} & $2110-4-g$ & $\begin{array}{l}\text { N71 } \\
\text { E83 }\end{array}$ & $\begin{array}{l}266- \\
268\end{array}$ & B & 0.9 & $\mathbf{N}$ & 0 & 0 & 0 & 0 & 0 & 44 & 0 & 10 & 0 & 11 & 0 & 0 & 1 & 8 & 0 & 69 \\
\hline & & & & & 8.3 & Wt. & 0.0 & 0.0 & 0.0 & 0.0 & 0.0 & 0.4 & 0.0 & 0.1 & 0.0 & 1.9 & 0.0 & 0.0 & 0.5 & 0.4 & 0.0 & 0.4 \\
\hline & $2112-4-3 g$ & $\begin{array}{l}\text { N71 } \\
\text { E83 }\end{array}$ & $\begin{array}{l}268- \\
277\end{array}$ & B & 9.0 & $\mathbf{N}$ & 1 & 1 & 3 & 3 & 3 & 199 & 0 & 1 & 0 & 83 & 0 & 11 & 13 & 10 & 0 & 385 \\
\hline & & & & & 93.8 & Wt. & 0.2 & 0.1 & 2.3 & 0.3 & 1.6 & 4.4 & 0.0 & 0.2 & 0.0 & 14.0 & 0.0 & 0.1 & 10.8 & 0.8 & 0.0 & 1.8 \\
\hline \multirow{4}{*}{33} & $2275-4-g$ & $\begin{array}{l}\text { N82 } \\
\text { E90 }\end{array}$ & $\begin{array}{l}270- \\
280\end{array}$ & D & 0.6 & $\mathbf{N}$ & 0 & 0 & 0 & 0 & 0 & 0 & 0 & 0 & 0 & 1 & 0 & 0 & 11 & 0 & 0 & 2 \\
\hline & & & & & 176.5 & Wt. & 0.0 & 0.0 & 0.0 & 0.0 & 0.0 & 0.0 & 0.0 & 0.0 & 0.0 & 0.1 & 0.0 & 0.0 & 174.1 & 0.0 & 0.0 & 0.1 \\
\hline & $2280-4-g$ & $\begin{array}{l}\text { N82 } \\
\text { E91 }\end{array}$ & $\begin{array}{l}274- \\
280\end{array}$ & D & 4.5 & $\mathbf{N}$ & 0 & 0 & 2 & 2 & 22 & 103 & 0 & 20 & 101 & 0 & 0 & 4 & 2 & 10 & 26 & $\begin{array}{c}163 \\
7\end{array}$ \\
\hline & & & & & 272.1 & Wt. & 0.0 & 0.0 & 15.6 & 0.5 & 53.9 & 2.3 & 0.0 & 0.1 & 73.4 & 0.0 & 0.0 & 0.1 & 2.7 & 1.8 & 17.2 & 10.5 \\
\hline & \multicolumn{4}{|c|}{ Total Number } & 55.75 & & 1 & 2 & 5 & 5 & 25 & 346 & 0 & 21 & 101 & 95 & 0 & 15 & 27 & 28 & 26 & 2093 \\
\hline & \multicolumn{4}{|c|}{ Total Weight (g) } & 1058 & & 0.2 & 0.1 & $\begin{array}{c}17 . \\
9\end{array}$ & 0.8 & $\begin{array}{c}55 . \\
5\end{array}$ & 7.1 & 0 & 0.4 & $\begin{array}{c}73 . \\
4\end{array}$ & 16 & 0 & 0.2 & 188.1 & 3 & 17.2 & 12.8 \\
\hline
\end{tabular}




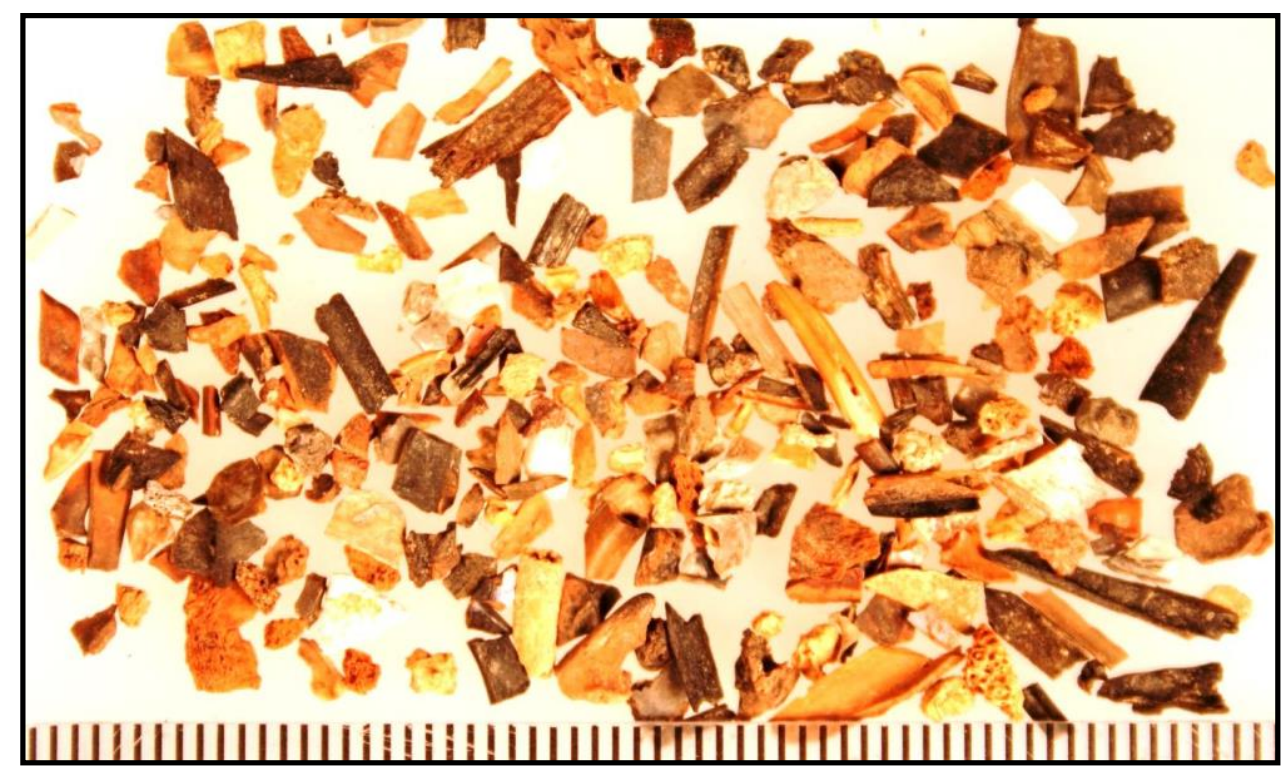

Figure 10-13. Burned, calcined, and unburned animal bone fragments from floated Feature 26 sediment. Note small size pieces and small animal elements represented (scale in millimeters).

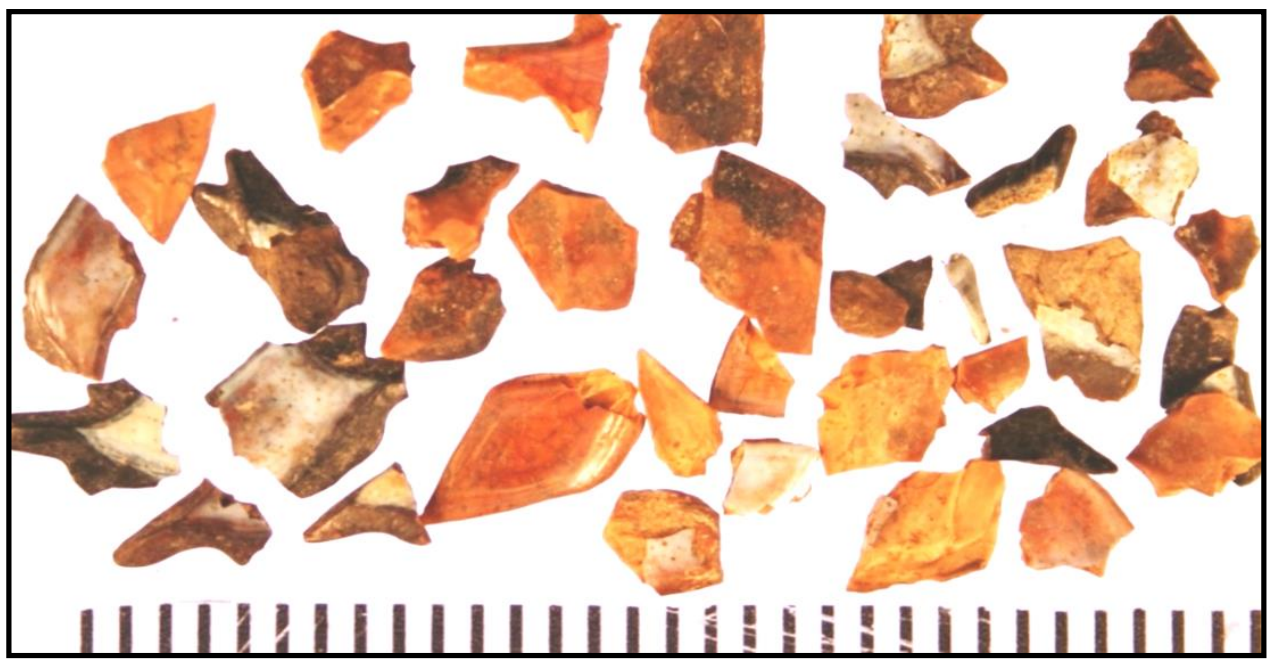

Figure 10-14. Close-up of burned and unburned gar fish scales from floated Feature 26 sediment (scale in millimeters).

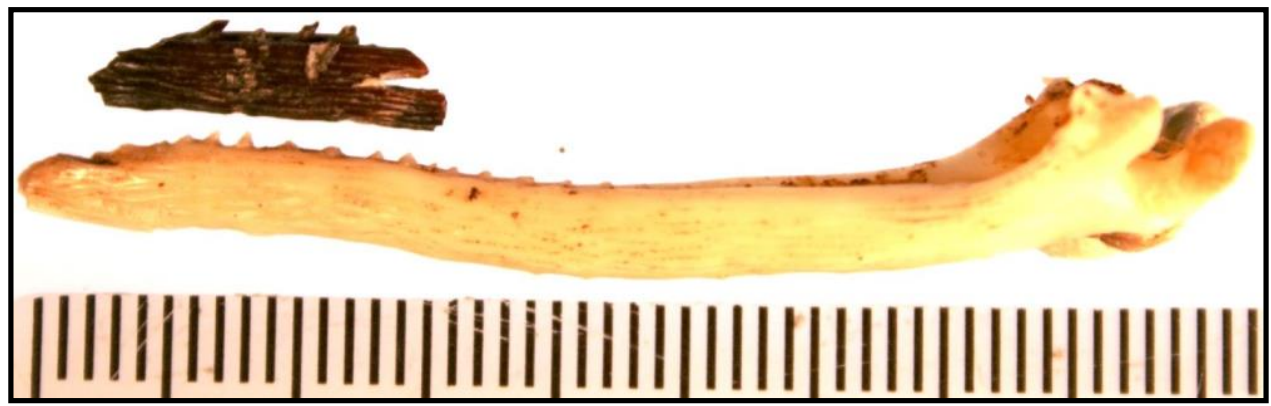

Figure 10-15. Close-up of burned catfish spine (top) compared to modern unburned catfish spine (bottom). Burned spine fragment from Feature 26 (scale in millimeters). 


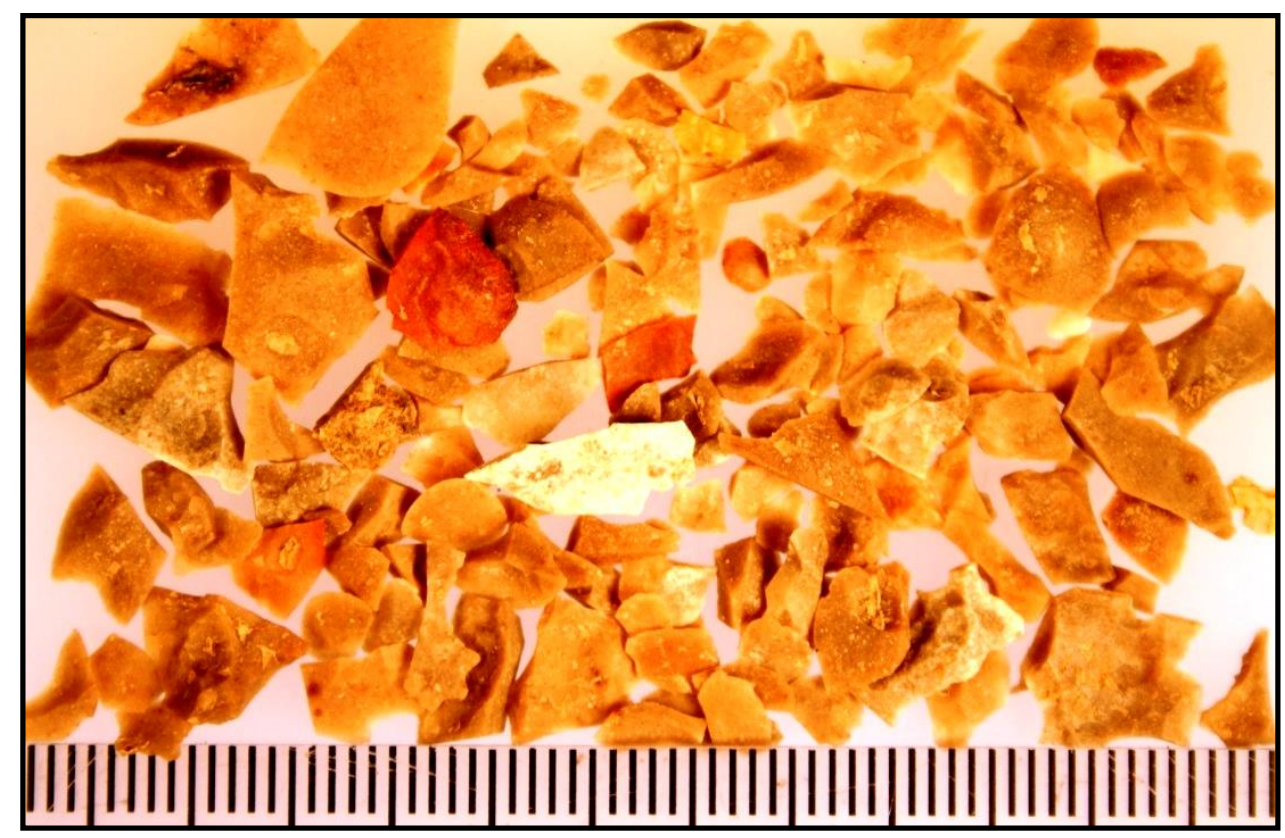

Figure 10-16. Micro debitage from floated Feature 26 sediment. Note consistency of material type (scale in millimeters).

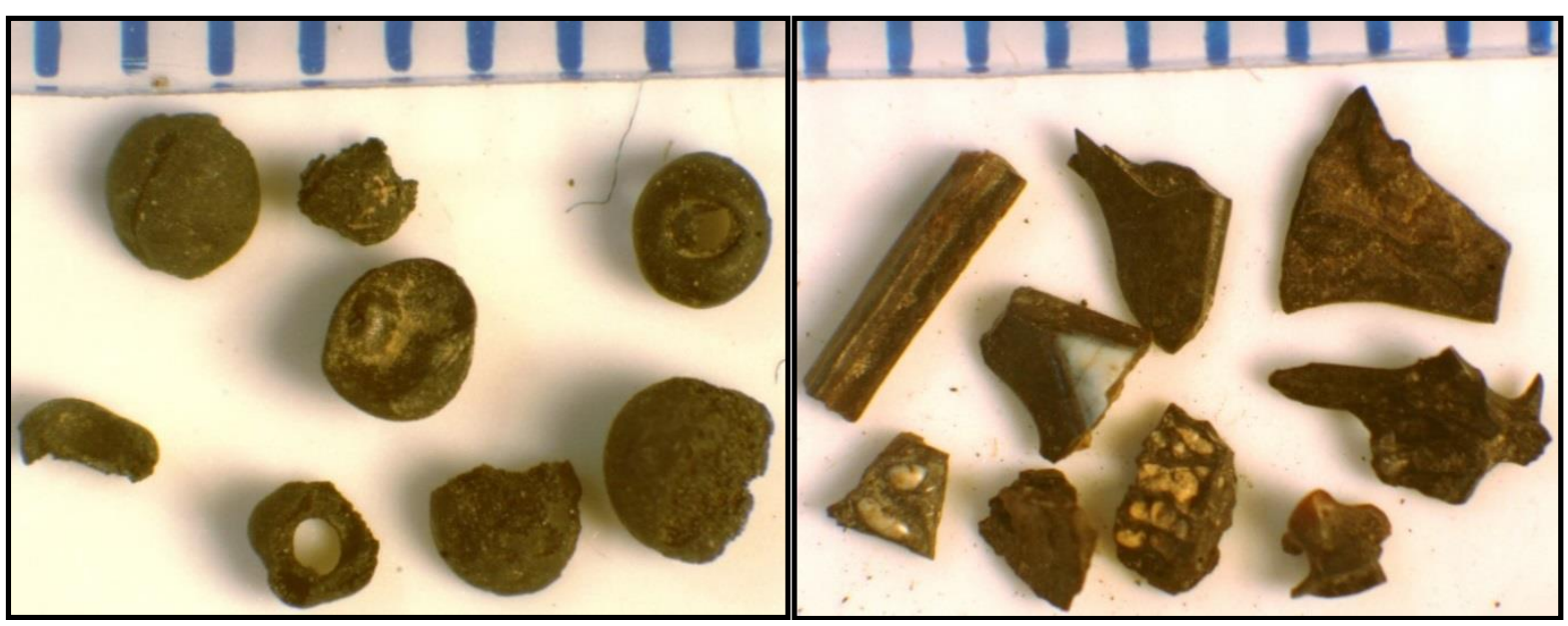

Figure 10-17. Burned organic material (cheno-am and grass seeds, left) and burned fish bones and gar scales (right) from floated Feature 26 sediment (scale in millimeters). 
Four burned rocks from Feature 26 were subjected to lipid residue analysis. Sample \#2112-2b (7MQ10) yielded very high fat content, implying possible plant and animal combination. Sample \#2112-3-10 (13MQ19) yielded low fat plant and borderline medium and moderate-high fat content, which indicates possible plant and animal combination. The other two samples (\#2112-3-9 [13NQ18] and \#2112-3-11 [13MQ20]) did not yielded sufficient residues for interpretations (Appendix G).

Two sediment samples (\#2110-4 and \#2112-4) that totaled nearly 10 liters were mechanically floated. These yielded over 200 gray (10YR 5/1) less than $6.4 \mathrm{~mm}$ in size chert pieces, over 450 bone fragments less than $6.4 \mathrm{~mm}$, a significant amount of snail shell fragments, 11 macrobotanical remains, and small burned clay pieces (Table 10-3). The bones minimally represent rabbit size mammals, gar and catfish, and a bird (Figures 10-13, 10-14, and 10-15). The tiny debitage indicates a very similar material, if not the same original core, as alldebitage is visually identical and has a dark orangish fluoresce (Figure 10-16). The macrobotanical remains were identified as two charred cheno-am and eight grass seeds, but no identifiable wood charcoal (Figure 10-17; Appendix B).

This elongated stained area with a shallow basin and scattered burned rocks represent a Martindale hearth based on its vertical position just under the light greenish-gray clay lens and two charcoal and seed radiocarbon dates. Tiny cultural materials were dumped in Feature 26 as a means of disposal from another area(s). The presence of sponge spicules and multiple gelatinized starch grains support water usage with this feature, likely in conjunction with boiling water, such as stone boiling. Microfossils off the burned rocks indicate both plant and animal products were present in this feature supported by the presence of burned and fragmented faunal remains and the charred chenoam and grass seeds. Cheno-am and grass seeds (likely wildrye) would be ripened in the late summer or fall, thus supporting a fall seasonal use event.

\subsubsection{Feature 33}

Feature 33 was a circular to oval, dark, orangish/black mottled stain with the hint of a shallow $7 \mathrm{~cm}$ deep saucer shaped basin at the juncture of four units in Block D (Figure 10-18). This rich, charcoal stained area measured roughly 100-by-100 $\mathrm{cm}$ in diameter with minimally ten scattered burned rocks, and a burned clay lump measuring nearly $10 \mathrm{~cm}$ in diameter along the southwestern edge. Numerous chert flakes and animal bone fragments were recovered in the feature fill. Multiple sediments samples were collected for processing.

A small bone fragment (\#2280-2) from N82/E91, just outside and southeast of Feature 33, yielded a $\delta^{13} \mathrm{C}$ corrected date of $5260 \pm 30$ B.P. (see Table 101). An organic rich sediment sample from the southeastern feature quadrant (\#2280-4-1a) yielded a $\delta^{13} \mathrm{C}$ corrected date of $6170 \pm 50$ B.P. This latter date is unacceptable compared to the former bone date and other dates derived from charcoal.

Ten scattered burned rocks from Feature 33 weighed $817 \mathrm{~g}$, for an average of $82 \mathrm{~g}$ per rock. The smallest burned rock size class represents 70 percent of the pieces (see Table 10-2).

At least 55 pieces of lithic debitage and approximately 200 animal bone fragments were in the four units that contained this feature. Animal bones from these same four units include parts of a butchered deer mandible with cut marks (Figure 1019), butchered deer size phalanges, jackrabbit long bones, two of which were burned, cottontail elements, turtle shell fragments, canid first, second, and third phalanges, fish skull and vertebrae fragments, three grouse size bird elements, and small rodent bones. Most bone fragments were in the southeastern unit, whereas the majority of debitage was in the southern two units. 


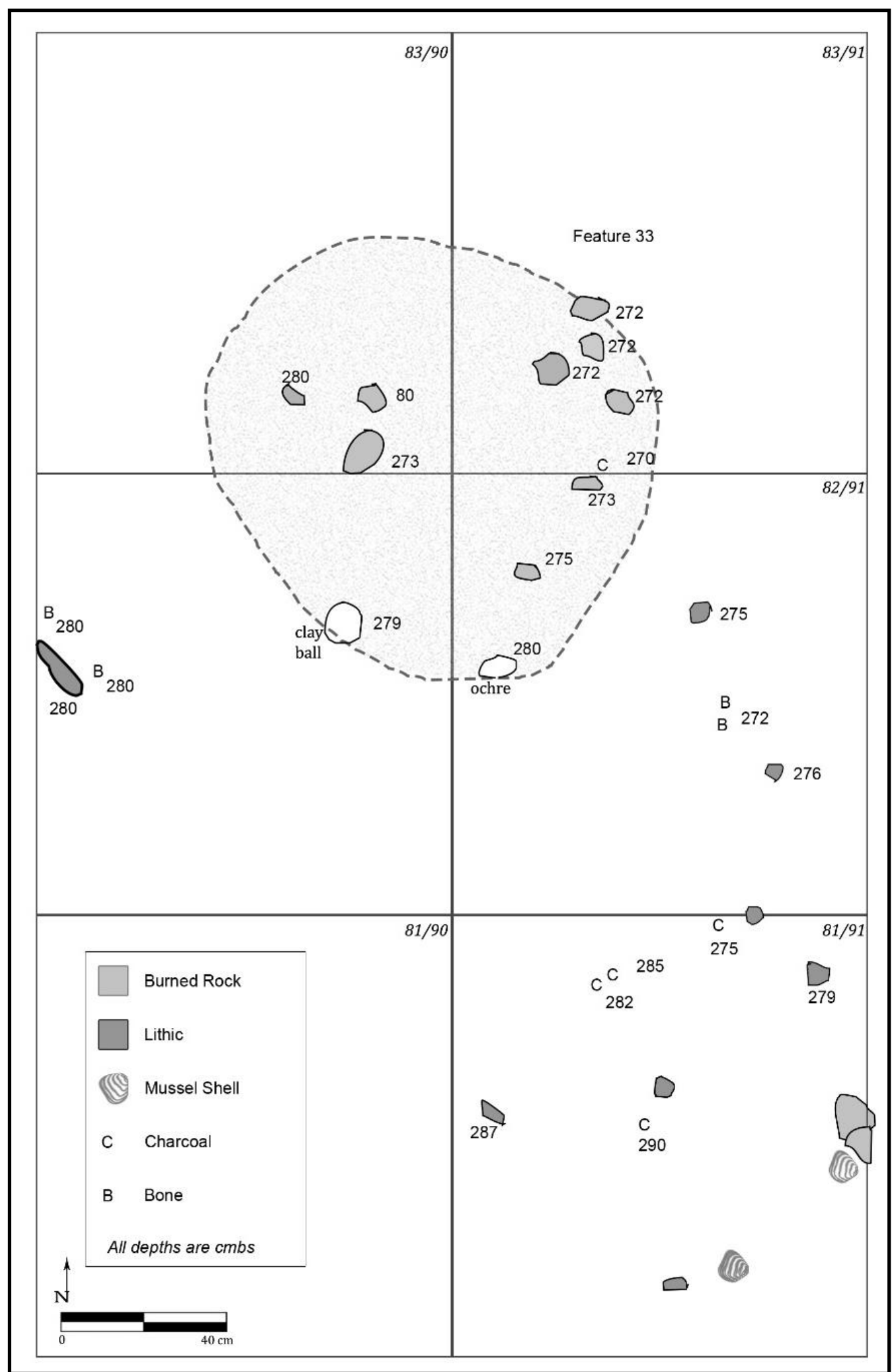

Figure 10-18. Composite drawing of piece plotted cultural materials in and around hearth Feature 33. 


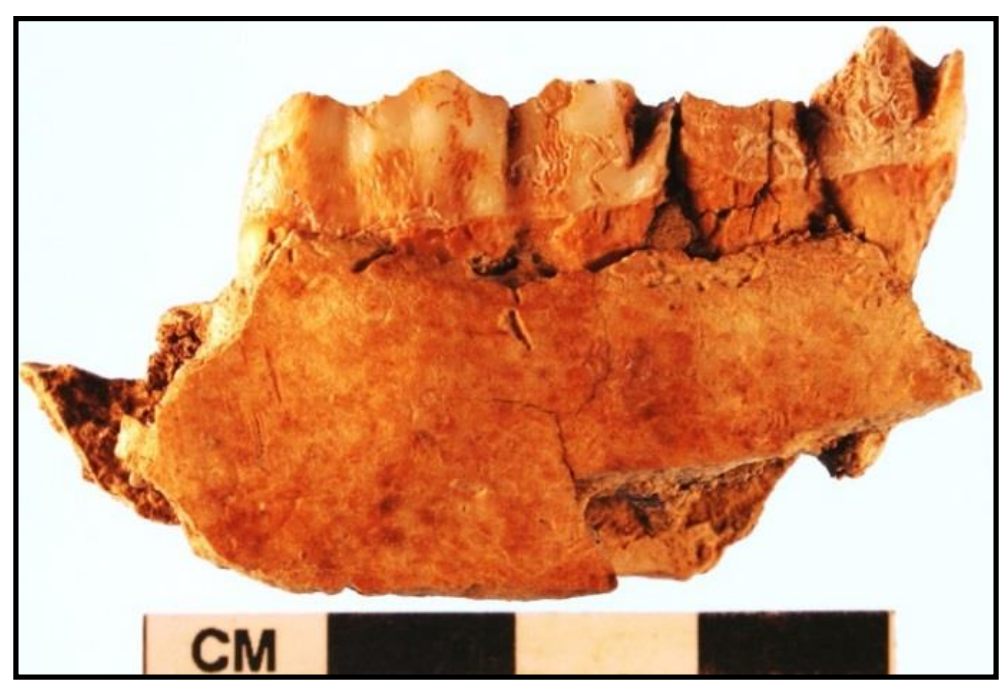

Figure 10-19. Deer mandible (\#2275) fragment with two socketed molars and thin cut marks below M3 on outside edge.

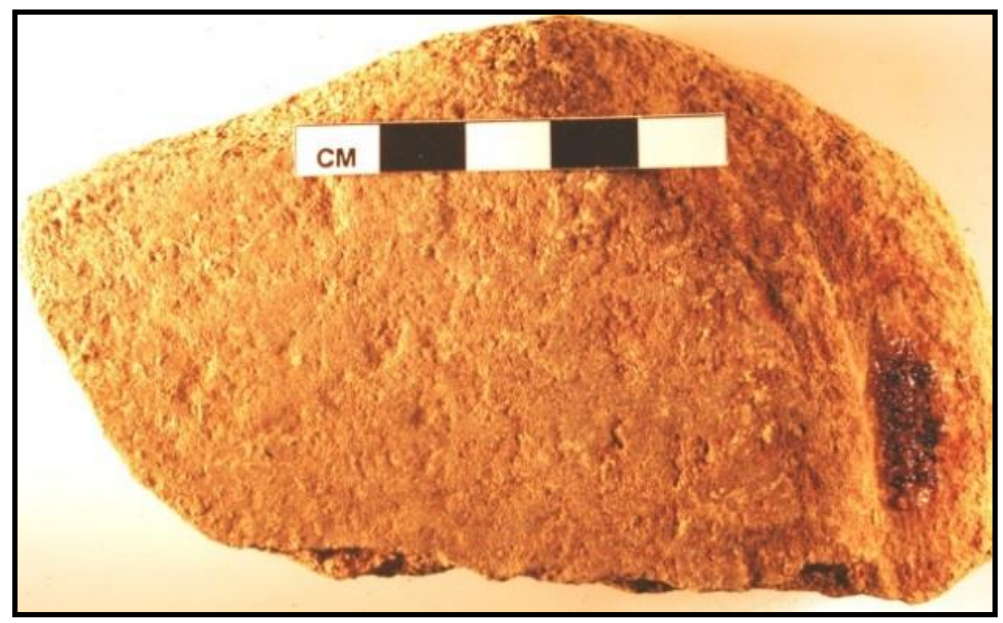

Figure 10-20. Metate fragment (\#2289-10) from inside Feature 33.

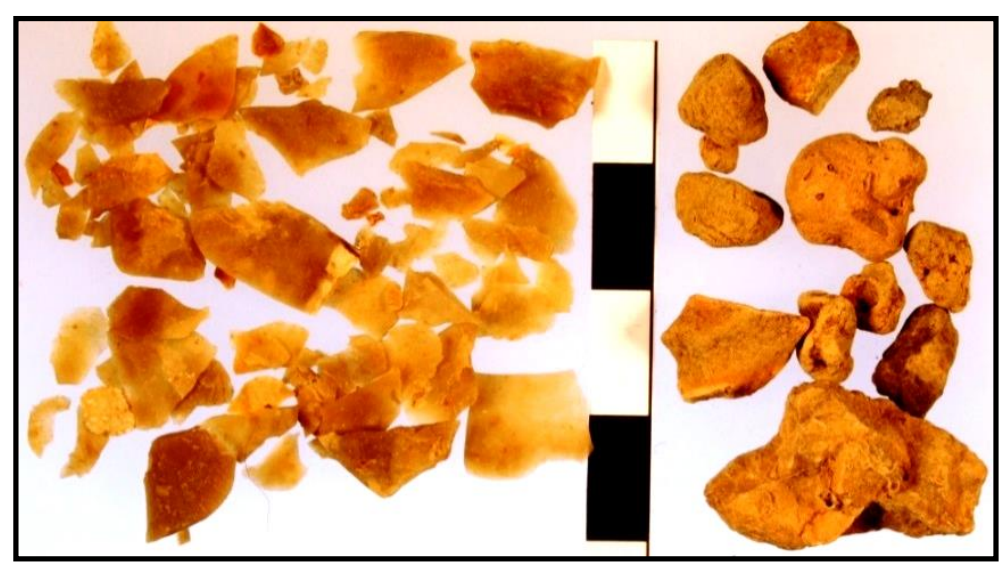

Figure 10-21. Lithic debitage (left) and burned clay chunks (right) from floated Feature 33 sediments (Scale in centimeters). 


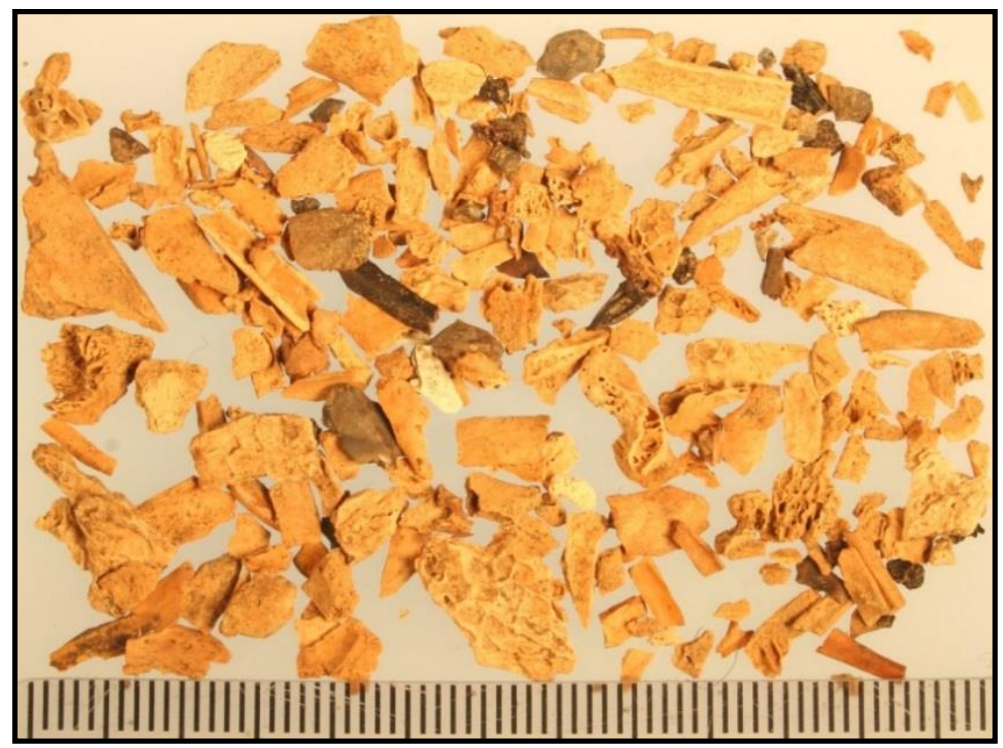

Figure 10-22. Bone fragments from heavy fraction from Feature 33 sediments (Scale in millimeters).

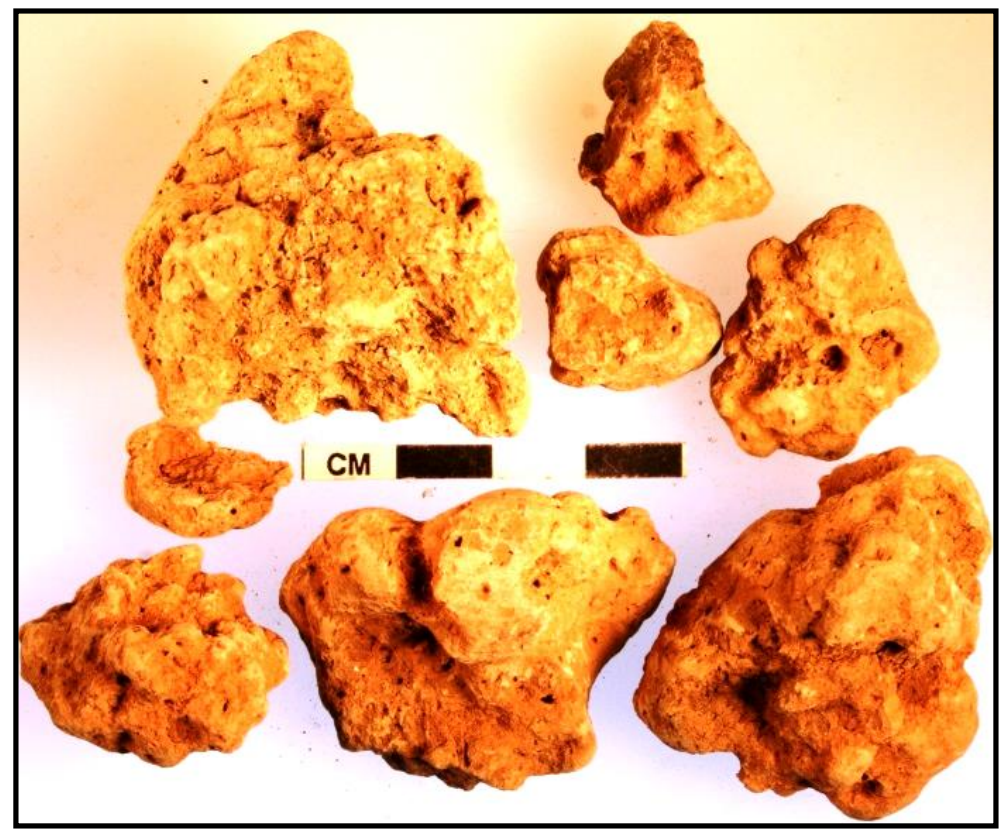

Figure 10-23. Photograph of burned clay nodules from Feature 33.

Metate fragment (\#2289-10a) (Figure 10-20), possibly a recycled burned rock from inside Feature 33 was sent for starch analysis and yielded a gelatinized starch grain (Appendix F). Part of that same artifact (\#2280-10b) was subjected to lipid residues analysis and yielded very high fat content indicative of plant and animal residues (Appendix $\mathrm{G})$.
Burned rock (\#2280-3-3) was also subjected to starch analysis, but lacked starch grains (Appendix F). A section of burned rock (\#2080-3-1) and second burned rock (\#2275-3) were also subjected to lipid residue analysis. Rock \#2275-3 yielded low fat plant and high fat content indicative of possible plant and animal combination, but dominated by 
animal residues (Appendix G). Burned rock \#20803-1 yielded insufficient fatty acids, but did reveal a biomarker indicative of conifer products.

A sediment sample (\#2280-4) was sent for phytolith analysis and yielded 15 sponge spicules, abundant tree phytoliths, four gourd phytoliths, and eight bone fragments. A second sediment sample (\#2275-4) yielded eight sponge spicules, abundant tree phytoliths, of which some are burned, and a gourd phytolith (Appendix E).

Two sediment samples (\#2075-4-g and \#2080-4-g) that totaled 5.1 liters from Feature 33 were mechanically floated. Heavy fractions yielded 22 chert flakes and 26 faunal bone fragments over 6.4 $\mathrm{mm}$, and 123 very pale brown (10YR 7/3) flakes and 1,637 faunal bone fragments less than $6.4 \mathrm{~mm}$ (Figures 10-21 and 10-22; see Table 10-3). Tiny pieces of unburned snail shells, flecks of charcoal, and small burned clay pieces were all recovered. Thirteen pieces (177 g) of irregular and amorphous shaped were also recovered and appear to reflect burned clay nodules (Figure 10-23). Bones include at least 30 burned pieces, at least 10 gar scale fragments, 3 turtle carapace, 2 unburned fish vertebrae, 2 burned gar skull fragments, rabbit size bones, deer incisors, and deer size pieces. The light fractions revealed no chunks of charcoal, just 6 to 10 flecks of likely charcoal, and a number of complete, unburned Rabdotus shells.

This ovate stain with shallow basin is thought to represent a hearth assigned to the Martindale component based on its depth and radiocarbon dates. Tiny cultural debris were dumped in Feature 33 as a means of disposal from another area. The presence of sponge spicules and a gelatinized starch grain support water usage with this feature, either in dousing the fire or in conjunction with boiling water. Multiple microfossils indicate plant and animal products in this feature, all likely residues from cooking. The plants cooked included chenoam and grass seeds, and likely gourds, which all support a fall use event.

\subsubsection{Feature Summary and Discussion}

Three dark, organic stained, shallow basin hearths were between 263 and $280 \mathrm{cmbs}$ with Features 25 and 33 in Block D and Feature 26 in Block B. These were primarily defined by dark stained sediments and degraded organic matter concentrated in a restricted area with relatively few burned rocks in the features (Table 10-4). Shallow basins were also defined by dark stained sediment with no visible yellowish or orangish oxidation rim detected along the basin margins. Features 25 and 33 were small, less than $100 \mathrm{~cm}$ in diameter, with shallow, less than $8 \mathrm{~cm}$ deep basins. Feature 26 was less than $2 \mathrm{~m}$ in diameter with a much smaller, shallow basin and scattered burned rocks. All three features are considered in situ heating facilities. Burned rock frequency varied from no rocks in Feature 25, to 57 in Feature 26, with no patterning detected in their distribution. Fifty-eight percent of feature rocks fell within the smallest size class, whereas 97 percent were less than $10 \mathrm{~cm}$ in diameter, likely indicating they had exhausted their use life and were no longer suitable for transferring heat.

Floated feature sediments from all three features yielded many burned and unburned bone fragments less than $6.4 \mathrm{~mm}$ from multiple species and high frequencies of chert debitage less than $6.4 \mathrm{~mm}$, many of which are burned and/or actual heat spalls and shatter. These tiny artifacts are thought to reflect unwanted cultural debris deposited into features as a means of disposal and/or cleaning other activity areas as they are too small to represent actual in situ activities. No sizeable chunks of charcoal were recovered from these basins. Features 26 and 33 yielded pea, marble and golf ball size burned clay nodules. Fragments of Rabdotus snail shells were in all the samples, but none appeared burned or heat altered. This probably implies intrusion following human abandonment. 


\begin{tabular}{|c|c|c|c|c|c|c|c|c|c|c|c|c|c|c|c|c|c|}
\hline \multicolumn{9}{|c|}{ Table 10-4. } & \multicolumn{9}{|c|}{ Martindale Feature Summary. } \\
\hline 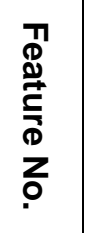 & $\begin{array}{l}\frac{m}{8} \\
\frac{\operatorname{c}}{x}\end{array}$ & $\stackrel{c}{S}$ & $\begin{array}{l}\widehat{9} \\
3 \\
30 \\
0 \\
0\end{array}$ & \multicolumn{2}{|c|}{ 귱 } & 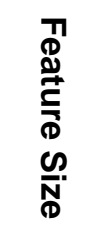 & $\begin{array}{l}\frac{0}{0} \\
\frac{\mathbb{D}}{0} \\
\frac{1}{5} \\
0 \\
\frac{0}{0} \\
\frac{0}{3} \\
3\end{array}$ & \multicolumn{2}{|c|}{ 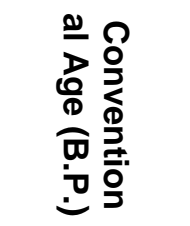 } & 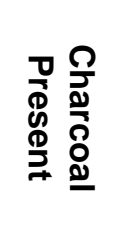 & 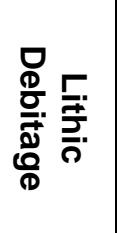 & $\begin{array}{l}0 \\
8\end{array}$ & 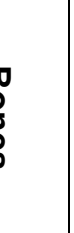 & 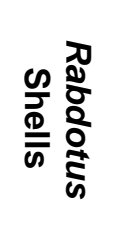 & 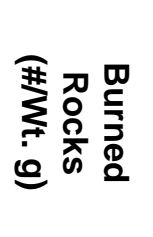 & 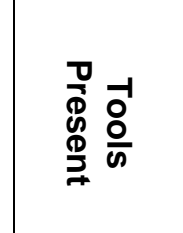 & 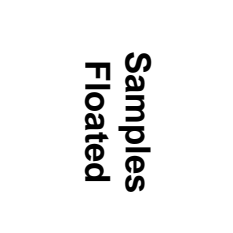 \\
\hline 25 & $\mathrm{D}$ & $\begin{array}{l}\text { N79/E93 } \\
\text { N78/E93 }\end{array}$ & $\begin{array}{l}264- \\
266\end{array}$ & \multicolumn{2}{|c|}{$\begin{array}{l}\text { Burned } \\
\text { basin } \\
\text { hearth }\end{array}$} & $\begin{array}{c}70 x \\
55\end{array}$ & 5 & \multicolumn{2}{|c|}{ NA } & Flecks & 2 & \multicolumn{2}{|c|}{$\begin{array}{l}\text { Next } \\
\text { to }\end{array}$} & No & $4 / 700$ & no & yes, 2239 \\
\hline 26 & $B$ & $\begin{array}{l}\text { N71/E82 } \\
\text { N71/E83 }\end{array}$ & $\begin{array}{l}263- \\
267\end{array}$ & \multicolumn{2}{|c|}{$\begin{array}{c}\text { Burned } \\
\text { basin } \\
\text { hearth }\end{array}$} & $\begin{array}{l}140 x \\
60+\end{array}$ & 7 & \multicolumn{2}{|c|}{$\begin{array}{l}5240 \pm 30 \\
5390 \pm 30\end{array}$} & Flecks & Few & & Yes & $57 / 4173$ & $\begin{array}{l}\text { Possible } \\
\text { Mano, } \\
\text { metate }\end{array}$ & $\begin{array}{l}\text { 2, lots of tiny } \\
\text { flakes \& } \\
\text { bones }\end{array}$ \\
\hline 33 & $\mathrm{D}$ & $\begin{array}{l}\text { N83/E90 } \\
\text { N82/E91 }\end{array}$ & $\begin{array}{l}273- \\
280\end{array}$ & \multicolumn{2}{|c|}{$\begin{array}{l}\text { Burned } \\
\text { basin } \\
\text { hearth }\end{array}$} & $\begin{array}{c}100 x \\
100\end{array}$ & 7 & \multicolumn{2}{|c|}{$\begin{array}{l}5450 \pm 30 \\
5320 \pm 50 \\
5340 \pm 30\end{array}$} & Flecks & Few & \multicolumn{2}{|c|}{ Many } & No & $10 / 817$ & no & $\begin{array}{l}2, \text { lots of tiny } \\
\text { flakes \& } \\
\text { bones }\end{array}$ \\
\hline & & & & \multicolumn{3}{|c|}{ Table 10-5. } & \multicolumn{10}{|c|}{ Summary of Analytical Results from Martindale Features. } & \\
\hline \multicolumn{2}{|c|}{$\begin{array}{l}\text { Feature } \\
\text { No. }\end{array}$} & $\begin{array}{l}\text { Feature } \\
\text { Type }\end{array}$ & \multicolumn{2}{|c|}{$\begin{array}{l}\text { Feature } \\
\text { Size } \\
(\mathrm{cm})\end{array}$} & \multicolumn{2}{|c|}{$\begin{array}{l}\text { Depth } \\
\text { (cm) } \\
\text { Below } \\
\text { Surface }\end{array}$} & \multicolumn{2}{|c|}{$\begin{array}{l}\text { Burned Rock } \\
\text { Counts/Wts. } \\
\text { (g) }\end{array}$} & \multicolumn{2}{|c|}{$\begin{array}{l}\text { Starch } \\
\text { Analysis } \\
\text { Results }\end{array}$} & \multicolumn{2}{|c|}{$\begin{array}{l}\text { Lipid } \\
\text { Analysis } \\
\text { Results }\end{array}$} & \multicolumn{3}{|c|}{$\begin{array}{l}\text { Phytolith Analysis } \\
\text { Results }\end{array}$} & $\begin{array}{l}\text { Macro- } \\
\text { Botanical } \\
\text { Results }\end{array}$ & $\begin{array}{c}\delta^{13} \mathrm{C} \text { Corrected } \\
\text { C14 Date B.P. } \\
\text { (Material) }\end{array}$ \\
\hline \multicolumn{2}{|c|}{25} & $\begin{array}{l}\text { Shallow } \\
\text { basin hearth }\end{array}$ & \multicolumn{2}{|c|}{$70 \times 55$} & \multicolumn{2}{|c|}{$264-266$} & \multicolumn{2}{|c|}{$4 / 700$} & \multicolumn{2}{|c|}{ NA } & \multicolumn{2}{|l|}{ NA } & \multicolumn{3}{|c|}{$\begin{array}{l}\text { tree phytoliths, } \\
\text { cucurbits }\end{array}$} & NA & NA \\
\hline \multicolumn{2}{|c|}{26} & $\begin{array}{l}\text { Shallow } \\
\text { basin hearth }\end{array}$ & \multicolumn{2}{|c|}{$\begin{array}{c}160 x \\
76+\end{array}$} & \multicolumn{2}{|c|}{$263-278$} & \multicolumn{2}{|c|}{$56 / 4,172$} & \multicolumn{2}{|c|}{$\begin{array}{c}2 \text { lenticular } \\
\text { grass, } 1 \\
\text { unidentified, } 2 \\
\text { gelatinized }\end{array}$} & \multicolumn{2}{|c|}{$\begin{array}{l}\text { Plant }+ \\
\text { Animal }\end{array}$} & $\begin{array}{r}\operatorname{tr} \\
\mathrm{b} \\
\text { Chlo }\end{array}$ & $\begin{array}{l}\text { ee phytc } \\
\text { one, bu } \\
\text { ridoids, } \\
\text { Panicoi }\end{array}$ & $\begin{array}{l}\text { liths, } \\
\text { rned } \\
\text { burned } \\
\text { ds, }\end{array}$ & $\begin{array}{l}8 \text { grass } \\
\text { seeds, } 2 \\
\text { cheno-am } \\
\text { seeds, } \\
\text { wood }\end{array}$ & $\begin{array}{c}5340 \pm 30 \\
5390 \pm 30 \\
\text { (Charcoal) } \\
5850 \pm 50 \\
\text { (Rabdotus) }\end{array}$ \\
\hline 33 & & Basin hearth & $\begin{array}{r}100 \\
100\end{array}$ & & $273-$ & 280 & $40 / 7,0 \mathrm{C}$ & & & A & $\begin{array}{l}\text { Plant } \\
\text { Anime }\end{array}$ & & $\begin{array}{l}\mathrm{S} \\
\text { phy }\end{array}$ & $\begin{array}{l}\text { picules, } \\
\text { toliths, } \\
\text { cucurb }\end{array}$ & $\begin{array}{l}\text { tree } \\
\text { one, \& } \\
\text { its }\end{array}$ & NA & $\begin{array}{l}5450 \pm 30 \\
\text { (Charcoal) }\end{array}$ \\
\hline
\end{tabular}

NA = Not applicable as no samples analyzed, NR = nothing recovered 
Results from multiple technical analyses provide clues to what was in and possibly how these features functioned. Lipid residue analysis targeted burned rocks and results document combinations of plant and animal residues (Table 10-5). Tree phytoliths document wood was used in the fires associated with these features. Gourd phytoliths were identified in Features 25 and 33 and document their presence, potentially as utensils.

Starch off Feature 26 rocks documents lenticular grass (likely wildrye), which is supported by charred grass and cheno-am seeds in the macrobotanical results from Feature 26. Gelatinized starch grains, created from heat and water, indicate rocks were likely used in stone boiling activities.

No detectable sediment color changes or oxidation along basin margins were observed, which indicate these were short-term fires, likely less than 1 hour (see Bond 1978:114-118 for experimental fire results). Wood used to fuel fires is documented by presence of tree phytoliths, and chemical biomarkers, specifically dehydroabietic acid in the lipid residues, indicate the woods were probably conifer, likely bald cypress (Taxodium) and/or juniper (Juniperus sp.) trees.

These three features were surrounded by various quantities of diverse cultural debris, which included, chert debitage, both scattered and in concentrations, fragmented animal bones from multiple species, small scattered burned rocks, and the occasional chipped and ground stone tools. The diversity of associated and surrounding artifacts support many activities centered on the cooking of plant and animal foods.

\subsection{MARTINDALE CHIPPED STONE TOOL ASSEMBLAGE}

The chipped stone tool assemblage is represented by two projectile point fragments, four bifaces, and 83 informal edge-modified flakes. Each formal tool is discussed below starting with the projectile points, with selected characteristics and measurements provided in Table 10-6.

\subsubsection{Projectile Points}

Specimen \#2282-10 is a nearly complete Martindale point from between 250 and $260 \mathrm{cmbs}$ in N82/E93 of Block D. Following recovery, this point was minimally handled and left unwashed to preserve potential residues for the purposes of future analysis. This short, triangular dart point is well-made with a sharp tip, $9.4 \mathrm{~mm}$ deep and 8.3 $\mathrm{mm}$ wide U-shaped corner-notches, one intact pointed medium long barb, a pointed basal edge, and a strongly concave base (Figure 10-24, see Table 10-6). One basal corner and one barb are missing. The corner-notches are relatively broad, slightly expanding U-shape, and distinct. The base exhibits two sizable flake scars on one face that potentially occurred when the base was fractured. A portion of the base appears lightly ground. The neck width is $16.6 \mathrm{~mm}$ indicative of a broad shaft. Broad flake scars extend across both faces with small pressure retouch scars along the sharp and unground lateral edges. This was manufactured from a very dark gray (10YR 3/1) homogenous chert. Under UV light this point fluoresces a dark orangish color similar to some varieties of Edwards cherts. This point was subjected to high-powered use-wear analysis and revealed hafting polish, striations, and soft polish near the middle of one face above the notches, which supports the projectile was hafted (Appendix D).

Specimen \#2161-10 is a medial section of an unidentified dart point (Figure 10-24, see Table 106). It was recovered from $274 \mathrm{cmbs}$ in N73/E81 of Block B, $150 \mathrm{~cm}$ northwest of Feature 26, and minimally unhandled and unwashed. The distal tip and stem are missing. The concave remnants of notches are present on either side of the stem break leaving slight shoulders visible. The neck width of $16.3 \mathrm{~mm}$ is quite similar to the Martindale point. 
Table 10-6. Martindale Chipped Stone Tool Data.

\begin{tabular}{|c|c|c|c|c|c|c|c|c|c|c|c|c|}
\hline PNUM & $\begin{array}{l}\text { Unit } \\
\text { N/E }\end{array}$ & $\begin{array}{l}\text { Depth } \\
\text { (cmbs) }\end{array}$ & Type & Stage & Portion & 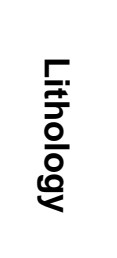 & 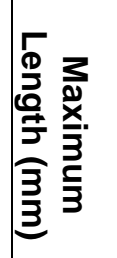 & 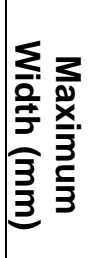 & 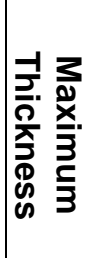 & 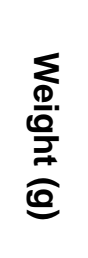 & 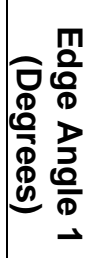 & 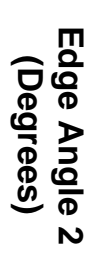 \\
\hline $2282-10$ & 82 / 93 & $250-260$ & Martindale & $\begin{array}{l}\text { Finished } \\
\text { Product }\end{array}$ & $\begin{array}{c}\text { Proximal- } \\
\text { medial }\end{array}$ & Chert & 41.3 & 36.9 & 6.7 & 7.9 & 53 & 55 \\
\hline $2161-10$ & 73 / 81 & $265-275$ & Untyped Point & $\begin{array}{l}\text { Late } \\
\text { Stage }\end{array}$ & Medial & Chert & 24.1 & 29.5 & 8.3 & 8.4 & 76 & 68 \\
\hline $2244-10$ & 79 / 91 & $258-270$ & Biface & Stage 4 & Medial & Chert & 14.7 & 31.0 & 7.9 & 2.9 & 51 & 0 \\
\hline $2257-10$ & $80 / 95$ & 273 & Biface & Stage 4 & Complete & Chert & 58.7 & 69.1 & 16.6 & 56.1 & 68 & 78 \\
\hline $2326-10$ & $85 / 95$ & $246-250$ & Biface & Stage 1 & Complete & Chert & 67.6 & 52.8 & 13.9 & 53.6 & 57 & 0 \\
\hline $2317-14$ & $85 / 90$ & $260-270$ & Biface & $\begin{array}{l}\text { Late } \\
\text { Stage }\end{array}$ & $\begin{array}{l}\text { Distal- } \\
\text { medial }\end{array}$ & Chert & 40.5 & 39.0 & 8.4 & 12.9 & 61 & 63 \\
\hline
\end{tabular}




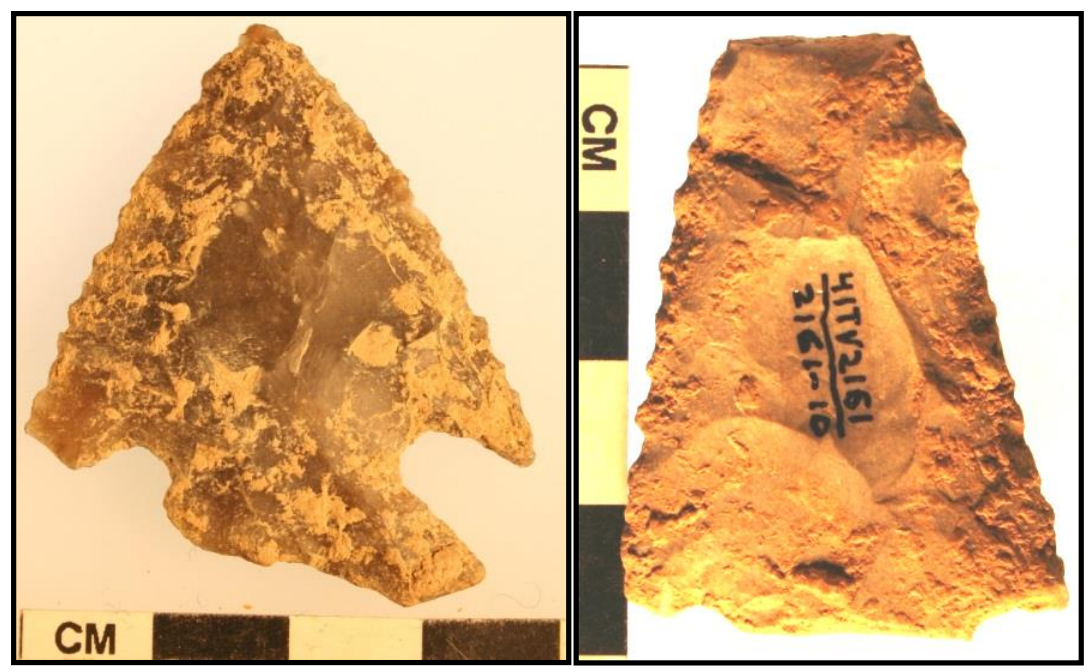

Figure 10-24. Unwashed Martindale point (\#2282-10, left) and possible corner-notched point
medial fragment (\#2161-10, right).

The lateral edges are slightly serrated, whereas the faces exhibit broad billet scars with no cortex present. The overall shape is slightly asymmetrical with one more pronounced concave face as opposed to the opposite convex face indicative of a point manufactured from a flake. This point was manufactured from a dull, light gray to gray $(2.5 \mathrm{Y}$ $7 / 1$ to $2.5 \mathrm{Y} 6 / 1$ ) homogenous chert. Under UV light this fluoresces an orangish color similar to some varieties of Edwards cherts. After photographing, a $1 \mathrm{~g}$ piece broken from the distal end was sent for INAA (TRC696). Chemical results indicate it was similar to cherts sampled from the adjacent gravel hilltop (Appendix $\mathrm{H}$ ).

\subsubsection{Bifaces}

Specimen \#2244-10 is a small, wedge-shaped lateral section of a well-made, $7.3 \mathrm{~mm}$ thick biface from between 258 and $170 \mathrm{cmbs}$ in N79/E91 in Block D. This $2.9 \mathrm{~cm}$ long wide section exhibits no cortex, with one well-thinned bifacial edge still sharp (see Table 10-6). It was made from a homogenous, medium brown chert. Under UV light this biface wedge fluoresces a yellowish orangish indicative of Edwards chert. This fragment has undetermined breaks and was in the same unit as a small lithic concentration.
Specimen \#2257-10 is an irregularly-shaped flake with limited bifacial working along one edge from $273 \mathrm{cmbs}$ in N80/E95 in Block D. Light yellowish cortex covers about 30 to 35 percent of one face, whereas the opposite face exhibits a massive, concave flake scar across about 80 percent. This piece may be the broken edge of an early stage biface or a rejuvenation flake from a large bifacial core (Figure 10-25, see Table 10-6). It is a mottled, partially stripped light brownish-gray (2.5Y 6/2) with gray (2.5Y 5/1) and light brown (7.5YR 6/3) bands. A $0.9 \mathrm{~g}$ chunk from one edge was sent for INAA (TRC695), which revealed a chemical signature similar to locally derived chert from the adjacent gravel hilltop (Appendix H). Under UV light this piece fluoresces a yellowish orangish indicative of Edwards chert.

Specimen \#2317-14 is the distal end of a biface from between 260 and $270 \mathrm{cmbs}$ in N85/E90 in Block D. This well-formed relatively thin fragment exhibits broad percussion scars across both faces, nearly straight lateral edges, with short broad billet scars along the edges (Figure 10-26, see Table 10$6)$. This unwashed fragment was subjected to highpowered use-wear analysis and revealed soft polish near the very distal end on the lateral edge indicating use on soft materials (Appendix D). 


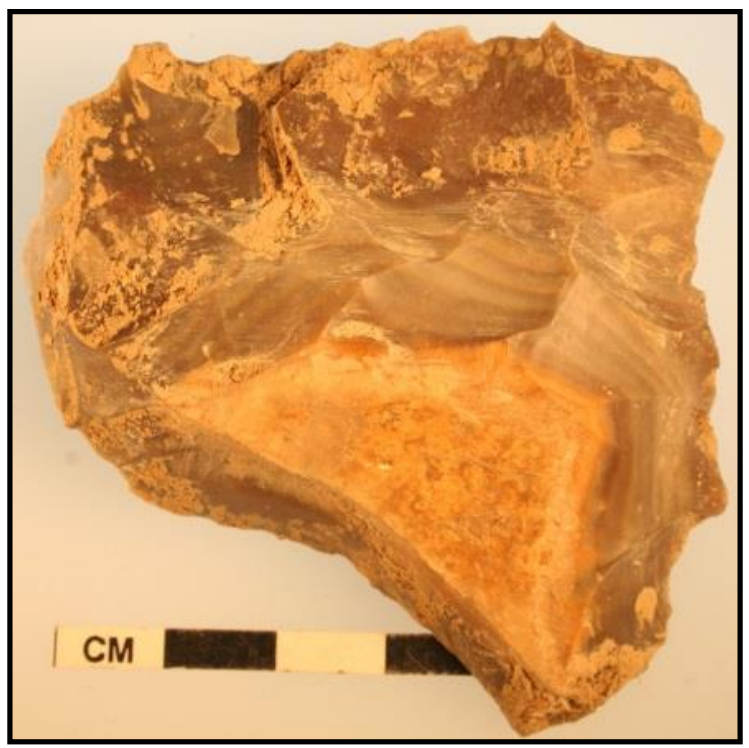

Figure 10-25. Unwashed Martindale biface fragment (\#2257-10).

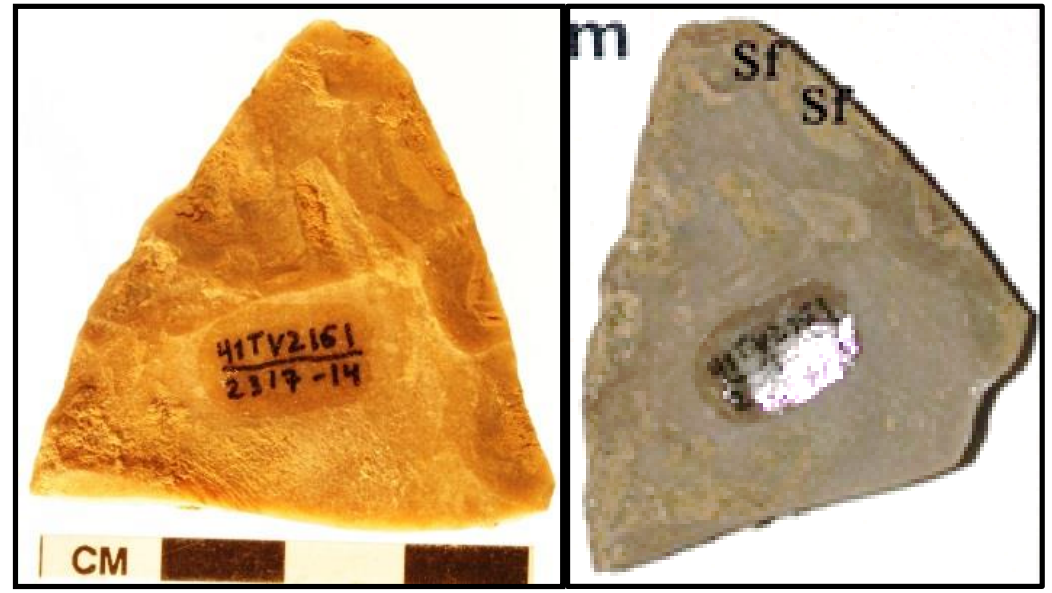

Figure 10-26. Unwashed Martindale biface fragment (\#2317-14).

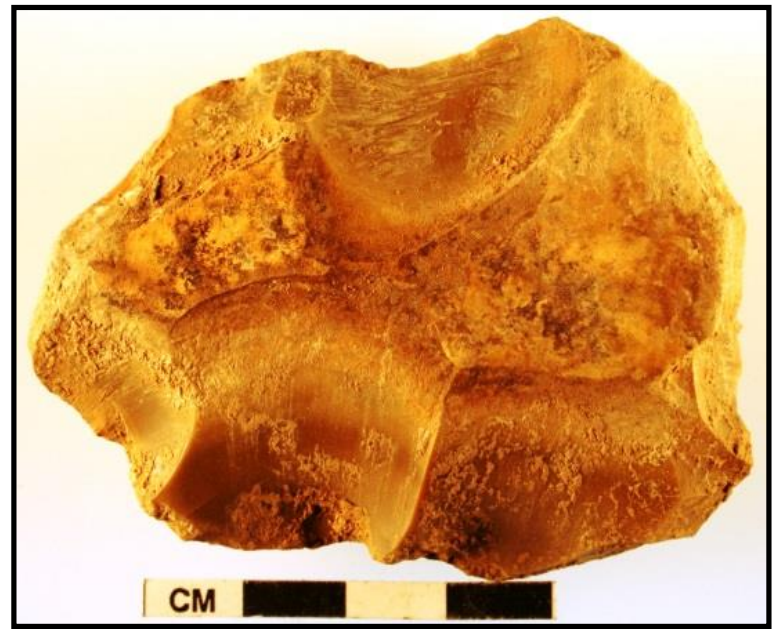

Figure 10-27. Unwashed Martindale biface (\#2326-10). 
Specimen \#2326-10 is a small early stage biface based on thickness and presence of cortex from between 246 and $250 \mathrm{cmbs}$ in N85/E95 in Block D. It was manufactured from a small, thin chert pebble with cortex still remaining on parts of both faces (Figure 10-27, see Table 10-6). This thin, roughly shaped, ovate biface exhibits broad percussion scars across both faces, and lacks small pressure flake scars along the lateral edges.

\subsubsection{Edge-Modified Flakes}

Edge-modified flakes are the most numerous tool class $(N=83)$ recovered. These informal tools occur in a wide variety of shapes and sizes generally with just the very edges revealing limited modification by a few and mostly tiny flake scars. Appendix $\mathrm{O}$ presents the basic observations and selected metric measurements with only a few selected edge-modified flakes individually presented below as examples. Andrefsky (1994:23) suggested the access to high quality abundant chert appears to have promoted quantities of informal tools, which may account for the high frequency. Figures 10-27 and 10-28 present examples of the shape diversity in this class, which suggests form was not a limiting factor in tool selection. A moderate percentage of the total is small, very thin flakes or flake fragments with tiny patterned retouch along one edge (Figure 10-29). Thirteen informal tools were sent for high-powered use-wear with individual details presented in Appendix D. Those analyzed reveal a dominance of cutting hide and/or butchering, followed by wood working and cutting plants. Two edge-modified flakes (\#210911 [TRC697] and \#2317-13 [TRC694]) were subjected to INAA and results are presented in Appendix H. Specimen \#2109-11 was sourced closest to the Segovia Formation materials. Edgemodified flake \#2317-13 was sourced to the hill top gravels adjacent to this site.

One edge-modified flake (\#2219-11) is an uncommon shinny jet black (10YR 2/1) piece from between 260 and $270 \mathrm{cmbs}$ in N71/E83. One edge on the ventral surface exhibits two flake scars that exhibit use. Under UV light this flake did not fluoresce or had a very dark purple or mauve response unlike Edwards chert. A 0.9 g piece was sent for INAA (TRC697) and the chemical results indicate this material was similar to those of the adjacent hilltop (Appendix $\mathrm{H}$ ).

Edge-modified flake \#2325-10 is a complete, 3.8 $\mathrm{cm}$ long, thin, blade shaped thinning flake from 259 cmbs in N85/E94. It has a thin, wide, ground platform with no adjacent bulb at the proximal end. The dorsal surface has a single ridge the entire length of the flake, whereas the dorsal surface is flat with ripple ridges. The distal end terminates in a very thin hinge fracture. The lateral edges taper to feather edges with the right lateral edge exhibiting very tiny scars and a dulled edge. This unwashed flake was subjected to high-power use-wear and revealed microscopic downy feather barbule attached to the distal end on the ventral surface, possible starch grains on the dorsal surface, plus striated polish to indicate multiple uses (Figure 1030, Appendix D). Under UV light this flake fluoresces a yellowish color that is similar to most Edwards chert.

\subsection{GROUND STONE ASSEMBLAGE}

Three ground stone artifacts, two manos and one metate, were directly part this component and each is described below with general profiles presented in Figure 10-31. Two small hammerstones were also recovered from the Martindale component.

\subsubsection{Manos}

One mano (\#2143-10) from $270 \mathrm{cmbs}$ in N72/E83 in Block B is about one-half of a relatively large, thick, and rounded quartzite cobble originally covered in a thin coating of calcium carbonate (Table 10-7, Figure 10-32). One convex face exhibits a ground surface, slight polish with tiny striations across the face. The remaining portion of the worked face measures about $93 \mathrm{~mm}$ by $70 \mathrm{~mm}$ 


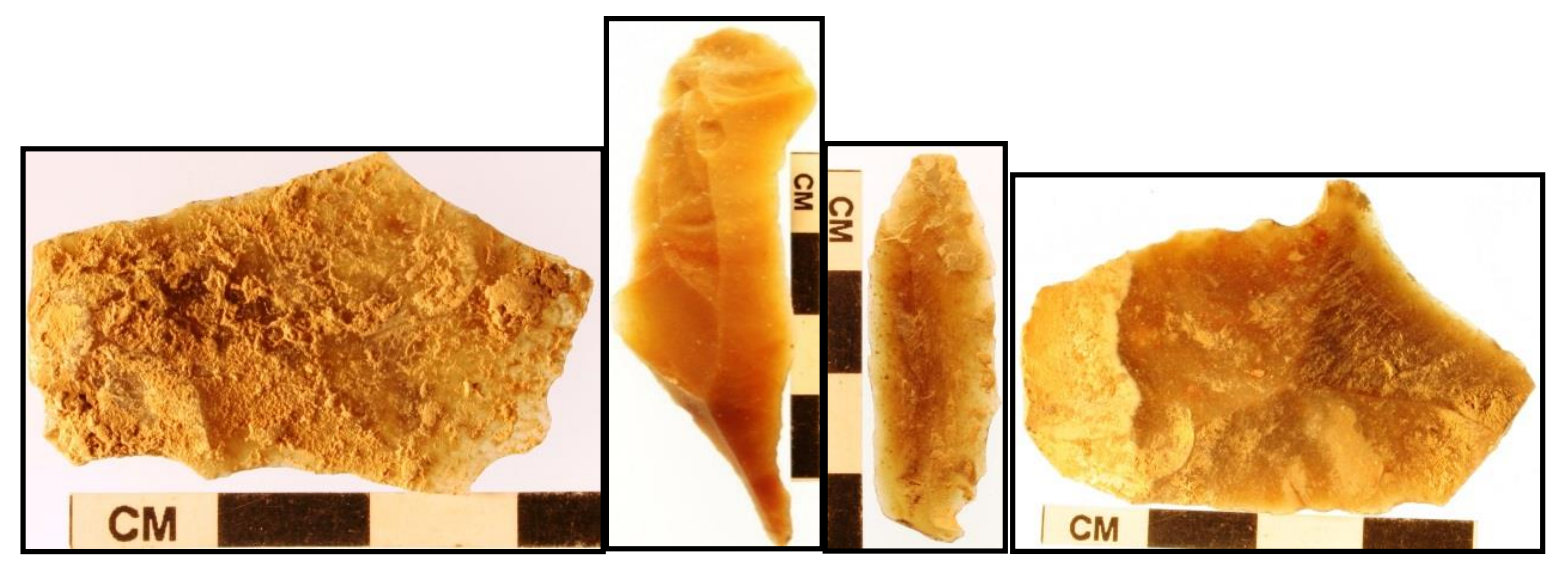

Figure 10-28. Four examples of Martindale edge-modified flakes (left to right \#2338-10, \#2304-10, \#2325-10, and \#2318-10).

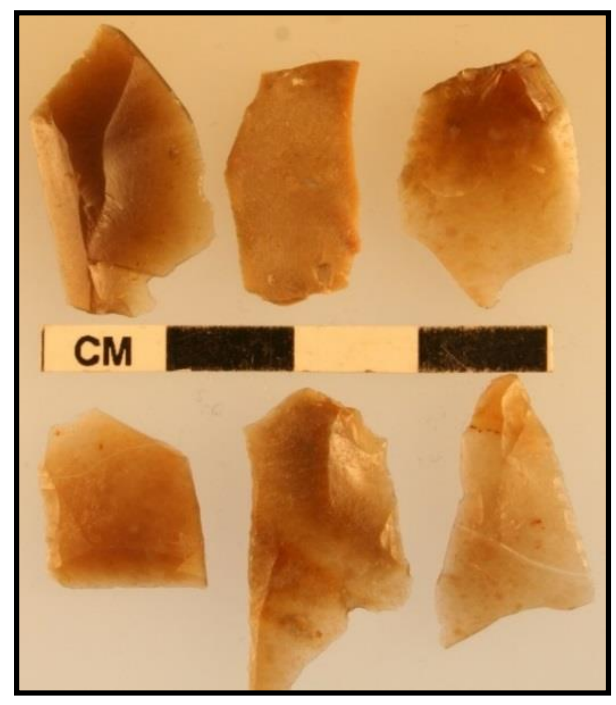

Figure 10-29. Six examples of small thin edge-modified flakes with pattern retouch (Top left to right \#2260-14, \#2260-13, \#2247-14, bottom left to right \#2246-13, 2254-10, and 2247-15).

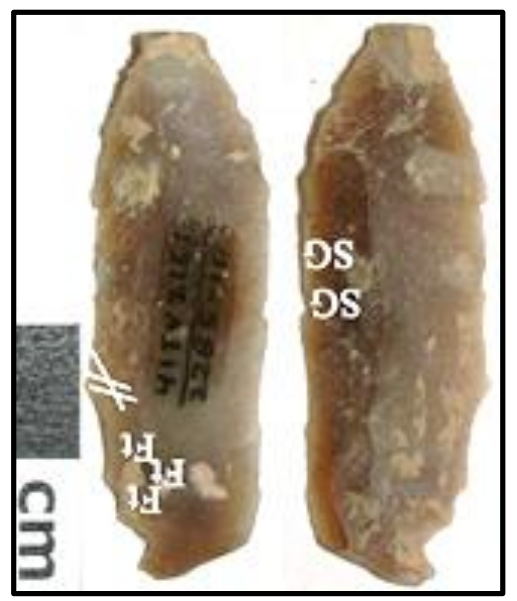

Figure 10-30. Photograph of edge-modified flake (\#2325-10) with tiny downy feather barbule attached to distal end and possible starch grains documenting multiple uses (Appendix D). 


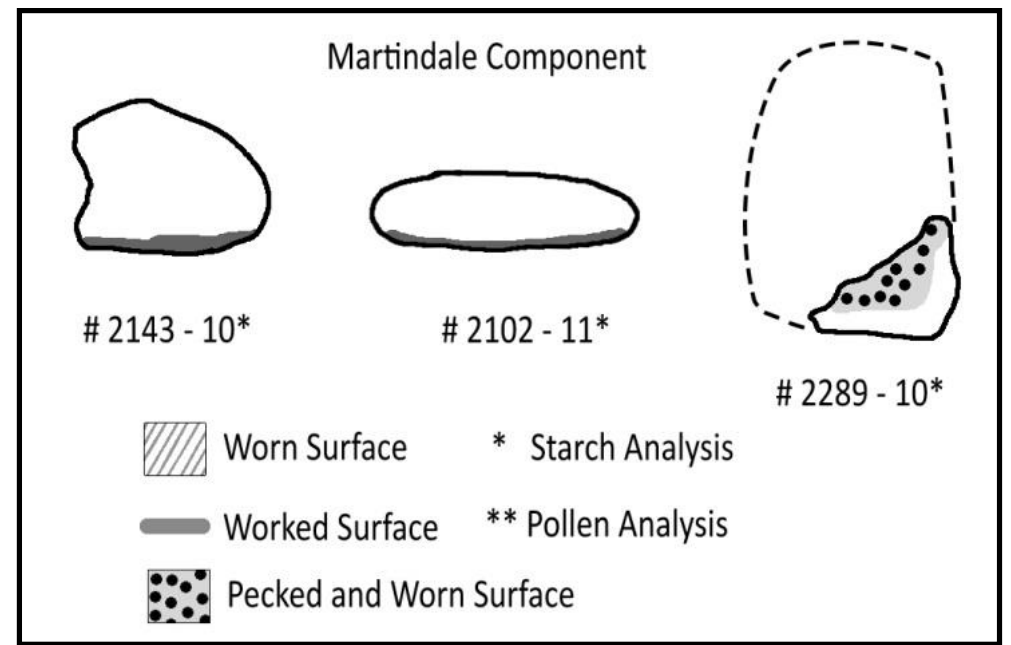

Figure 10-31. General profiles of manos and metates in Martindale component.

Table 10-7. Martindale Ground Stone Tool Data.

\begin{tabular}{|c|c|c|c|c|c|c|c|c|c|c|c|c|c|}
\hline PNUM & Class & $\begin{array}{l}\text { Feature } \\
\text { No. }\end{array}$ & 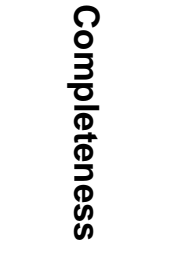 & 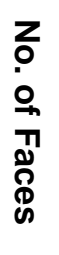 & 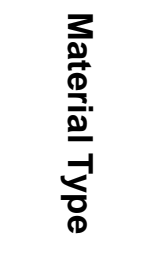 & 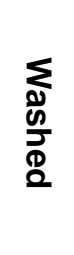 & 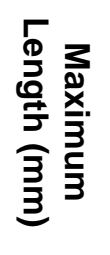 & 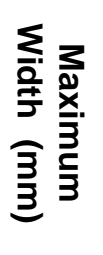 & 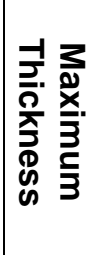 & 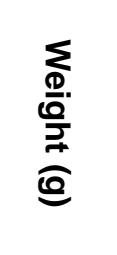 & 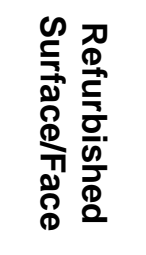 & 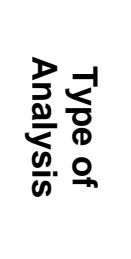 & Observations \\
\hline 2143-10 & Mano & None & Half & 1 & Quartzite & No & 86.2 & 106.7 & 64.2 & 801.0 & No & Starch & $\begin{array}{l}\text { Mostly covered in } \\
\text { calcium carbonate }\end{array}$ \\
\hline 2102-11 & Mano & 26 & Complete & 1 & Quartzite & No & 154.1 & 88.3 & 47.8 & 924.9 & Possible & Starch & $\begin{array}{l}\text { Poorly defined grinding } \\
\text { surface, light calcium } \\
\text { carbonate }\end{array}$ \\
\hline $2289-10$ & Metate & 33 & Fragment & 1 & Quartzite & Yes & 117.3 & 68.8 & 25.3 & 317 & No & $\begin{array}{l}\text { Starch, } \\
\text { Lipid }\end{array}$ & $\begin{array}{c}\text { Slightly smoothed } \\
\text { surface }\end{array}$ \\
\hline
\end{tabular}




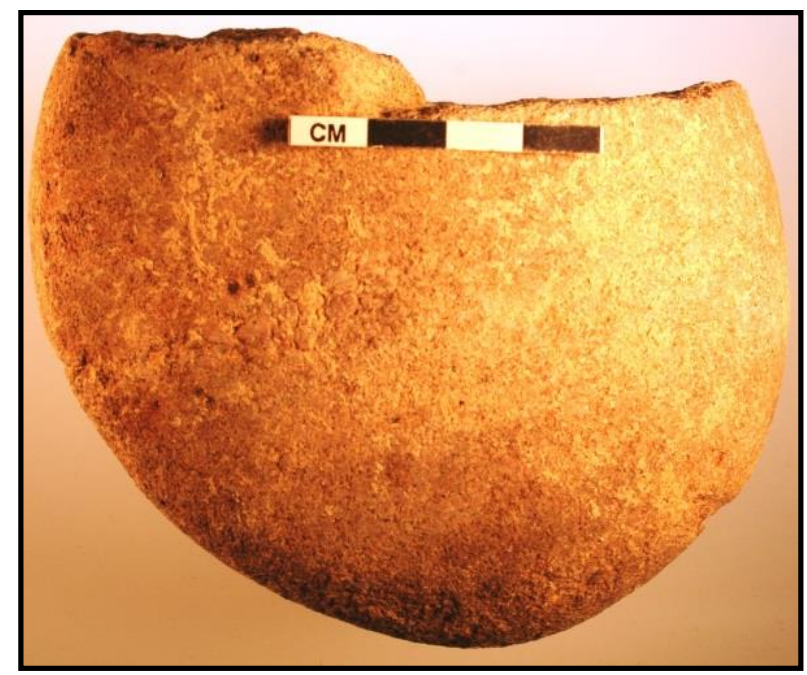

Figure 10-32. Photograph of half a mano (\#2143-10).

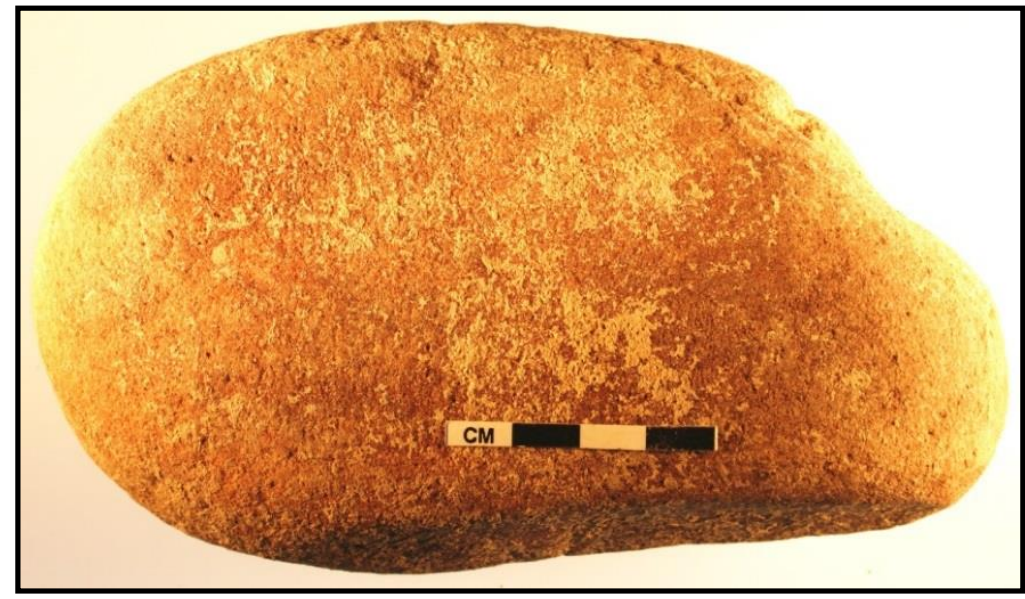

Figure 10-33. Photograph of complete mano (\#2102-11) from Feature 26.

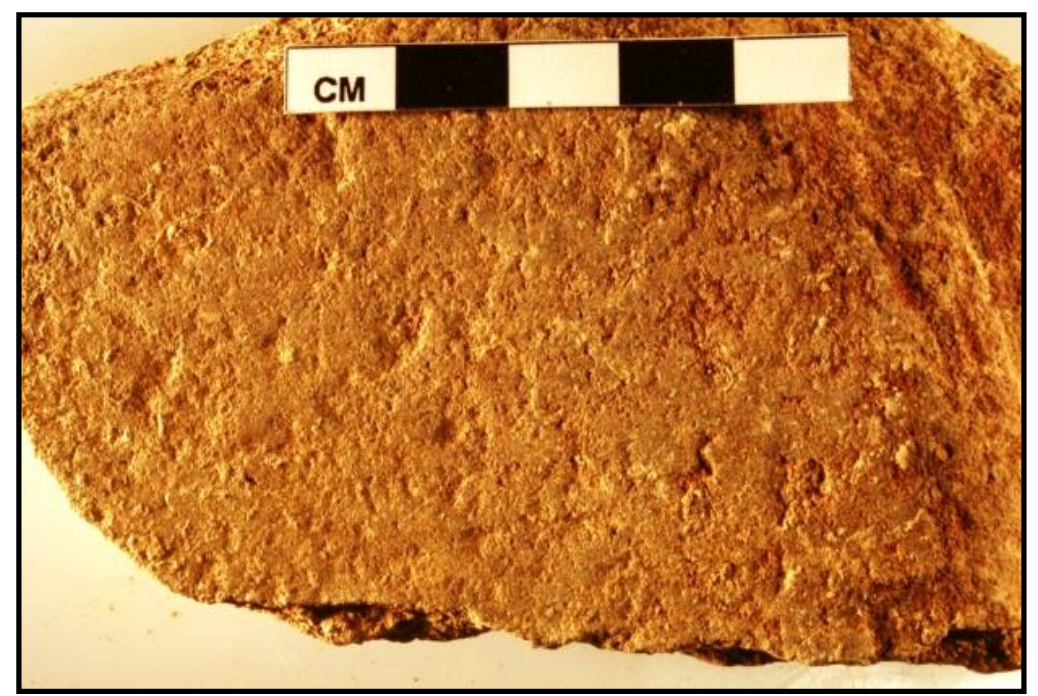

Figure 10-34. Close-up photograph of pecked surface of metate fragment (\#2289-10). 
across. This piece was sent for starch analysis and an unidentified ground starch grain was recovered to support its use in grinding tasks (Appendix F).

Specimen (\#2102-11) is a mano from $172 \mathrm{cmbs}$ in N71/E82, within Feature 26. This is a relatively large complete, elongated, oval specimen with one worked surface and spotty dark staining on both faces, plus a light calcium carbonate covering. The flatter face appears slightly smoothed with no obvious striations or refurbishing indentations (Figure 10-33, Table 10-7). The opposite face is more convex with seven small dimples or rock imperfections along the long axes. No obvious peck or impact marks were observed along the lateral edges. This mano was subjected to starch analysis, but no starch grains were recovered (Appendix F).

\subsubsection{Metates}

This small, possible metate (\#2289-10) fragment was between 270 and $280 \mathrm{cmbs}$ in N83/E90 and in Feature 33. This potentially served as a ground stone then recycled as a component of a thermal feature, but it is difficult to state with certainty. The one flat, slightly smooth face with multiple small pits lacks a convex surface, but no observed striations (see Figures 10-29 and 10-33). The opposite face is a rough more or less flat surface covered in calcium carbonate. One lateral edge exhibits a natural worn edge, whereas the other is a curved broken edge and possibly thermally fractured. About one half (\#2289-10a) was sent for starch analysis and yielded a gelatinized grain indicative of change from heat and water (Appendix F). The other half (\#2289-10b) was sent for lipid residues analysis and yielded very high fat content to indicate possible plant and animal combination (Appendix G). Since the gelatinization results from heat and water and residues from plant and animal remains are present, these results document this fragment once served as for cooking.
The small ground stone assemblage documents plant processing and potentially meat processing activities. The absence of starches on the complete mano (\#2102-11) from Feature 26 hinders its interpretive use. The presence of striations combined with the ground starch grain on \#214310 supports the use of grinding implements at this component. It is probable specimen \#2289-10 was used in a thermal cooking feature evidenced by the residue of plant and animal products. The peaking and ground starch grains however, supports its use as a metate.

\subsection{HAMMER STONES}

Two small, complete semirounded clasts served as hammers. Each is described below with metric and other data presented in Table 10-8.

Specimen \#2265-10 is a small, $6.8 \mathrm{~cm}$ water smoothed clast with 95 percent of the surface covered in cortex from between 250 and $260 \mathrm{cmbs}$ in N81/E93 of Block D. It exhibits a number of rounded ridges and projections with four projections that exhibit small crushed areas and wide impact scars, less than $1 \mathrm{~cm}$ long (Figure 1035). This dense fine-grained material has a pale yellowish (2.6YR 8/4) cortex and a slightly darker brownish yellow (10YR 6/6) interior.

Specimen \#2226-11, similar to the previous specimen, is a small $5.7 \mathrm{~cm}$ long, pear-shaped, cortex-covered cobble from between 246 and 250 cmbs in N85/E95 of Block D. It has three rounded protuberances with each projection exhibiting impact scars and restricted crushed area (see Figure 10-35). Two protuberances have flake scars, one on the more pointed end is large, whereas the one corner has only a crushed area. The material is identical to the first hammer stone and is a dense fine-grained, cherty limestone with a brownish yellow (10YR 6/8) cortex and a yellow (10YR 7/6) interior. 


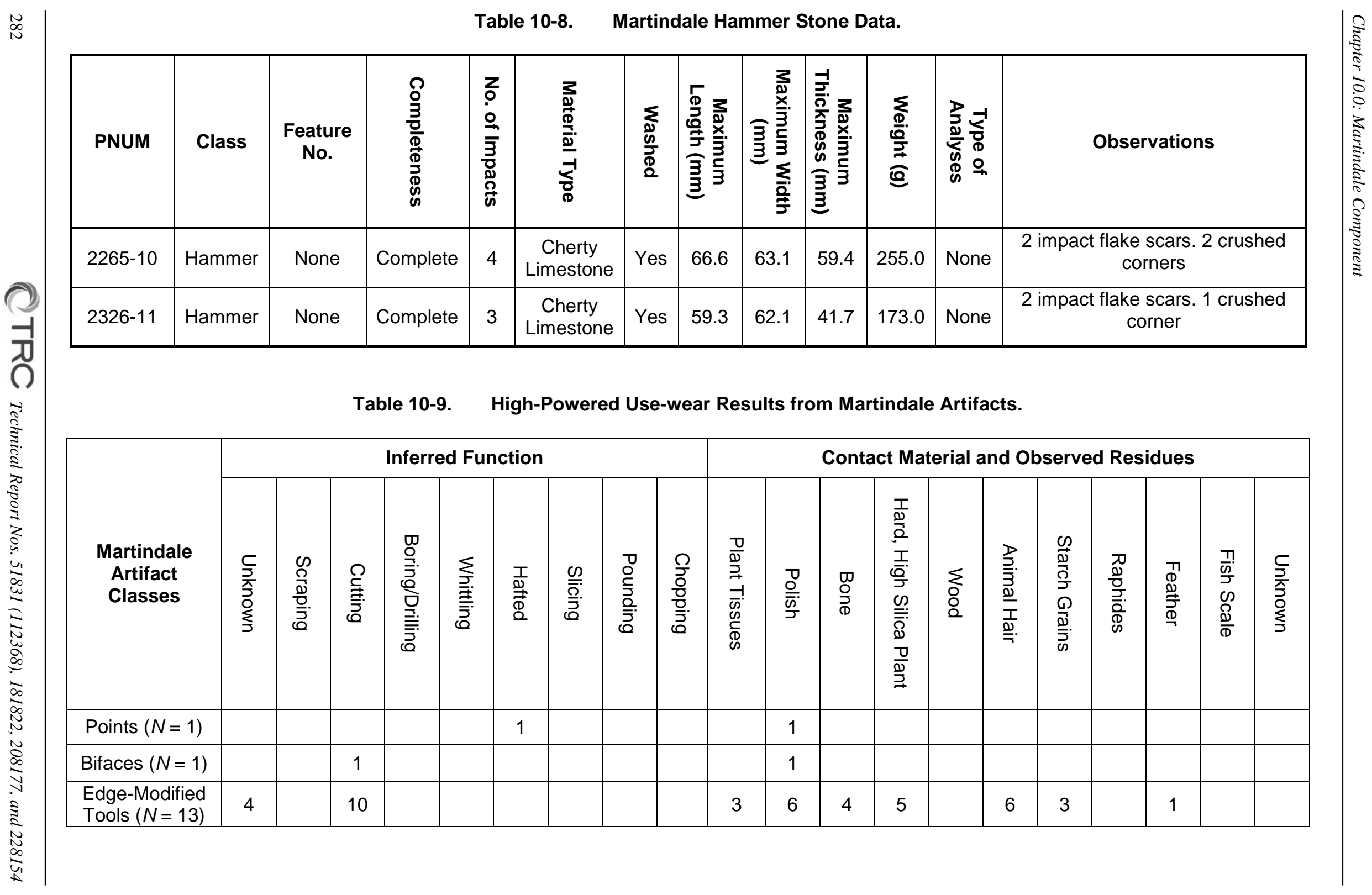




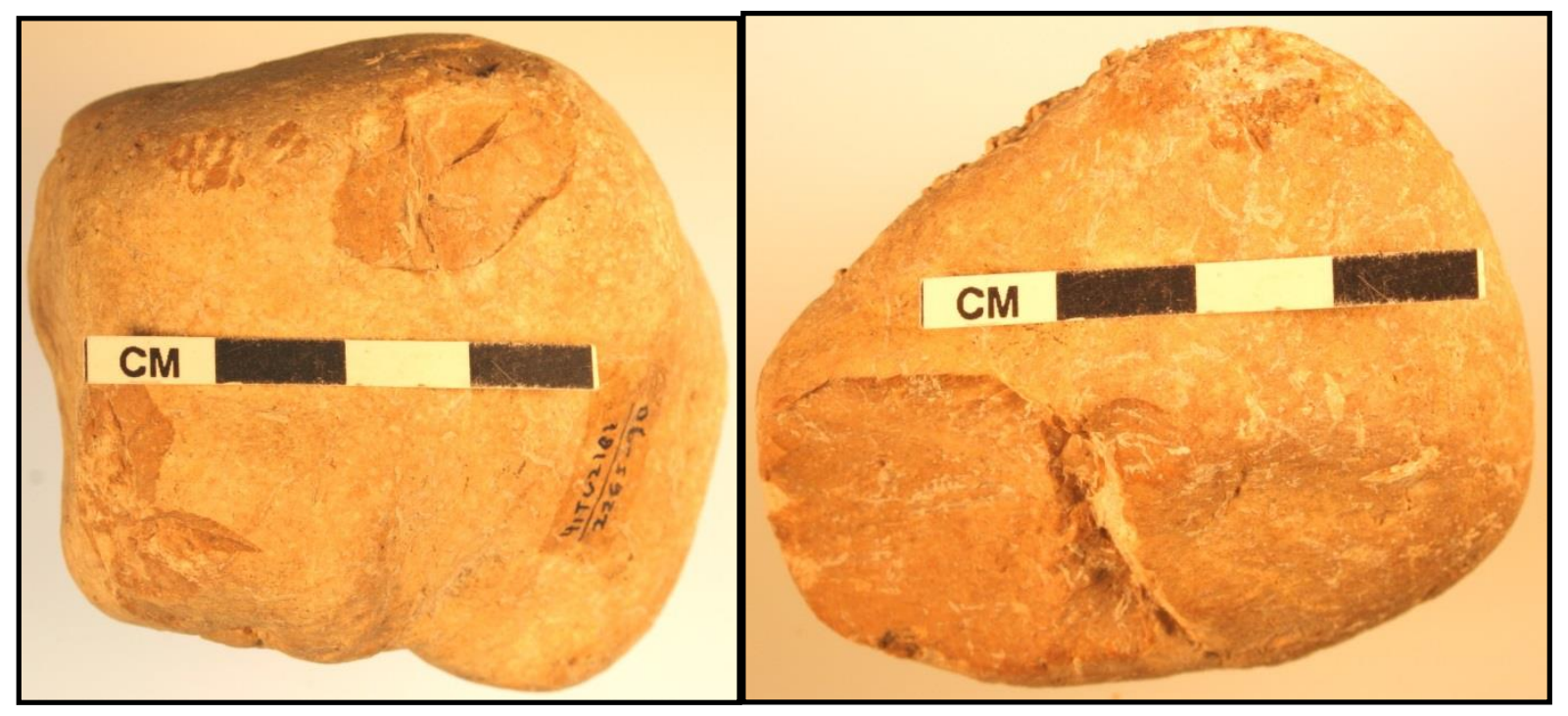

Figure 10-35. Photograph of hammer stones (\#2265-10 left and \#2226-10 right).

Both hammer stones are relatively small (173 $\mathrm{g}$ and $255 \mathrm{~g}$, see Table 10-8), and probably served well as hammers on hard substances as evident from multiple impact points and flake scars on both. The impact scars support the hammers probably served in knapping chert cobbles and tools. Both are of identical material and under UV light fluoresce a dull brown unlike Edwards chert. This nonEdwards material is likely from the exposed gravels from the adjacent hill top.

\subsection{SUMMARY}

In summary, this well-defined Martindale component yielded only six formal chipped tools, plus three ground stone tools and two hammer stones. The formal tools consists of projectile points and bifaces used in hunting and butchering. Scraping and special functional tools (e.g., scrapers, drills, Guadalupe bifaces, Clear Fork tools, and spokeshaves) are absent in both formal and informal classes. Absence of hide preparation and hide working tools (i.e., scrapers, bone fleshers and awls) to create other items from hides indicate these tasks were limited or not performed. The three ground stones, combined with analytical results from those tools, document grinding and processing of plants - grass and cheno-am seeds as indicated by the macrobotanical remains. The two hammer stones with the relatively high frequency of lithic debitage, plus three lithic debitage concentrations, reflect core and biface reduction was a primary task at this camp.

The 15 tools subjected to high-powered use-wear and residue analysis reveal cutting was a principal task, with edge-modified flakes having been used on diverse materials (Table 10-9). The identified residues include plant tissues, bone, animal hair, possible starchy plants, and the most unusual, feather. This documents cutting tasks that targeted multiple resources.

\subsection{HORIZONTAL DISTRIBUTION OF STONE TOOLS}

The horizontal distribution of formal chipped and ground stone tools reveals a pattern of use and/or discard adjacent, but not immediately next to hearth features (Figure 10-36). Interestingly, the two hammer stones were not in immediate proximity to the three lithic concentrations, although broken bifaces were. Either the hammer stones were tossed following their use, or the debitage concentrations reflect disposal or dump areas as an intentional cleaning process. The overall paucity of formal tools demonstrates the component is discrete and probably represents a short-term occupation. 


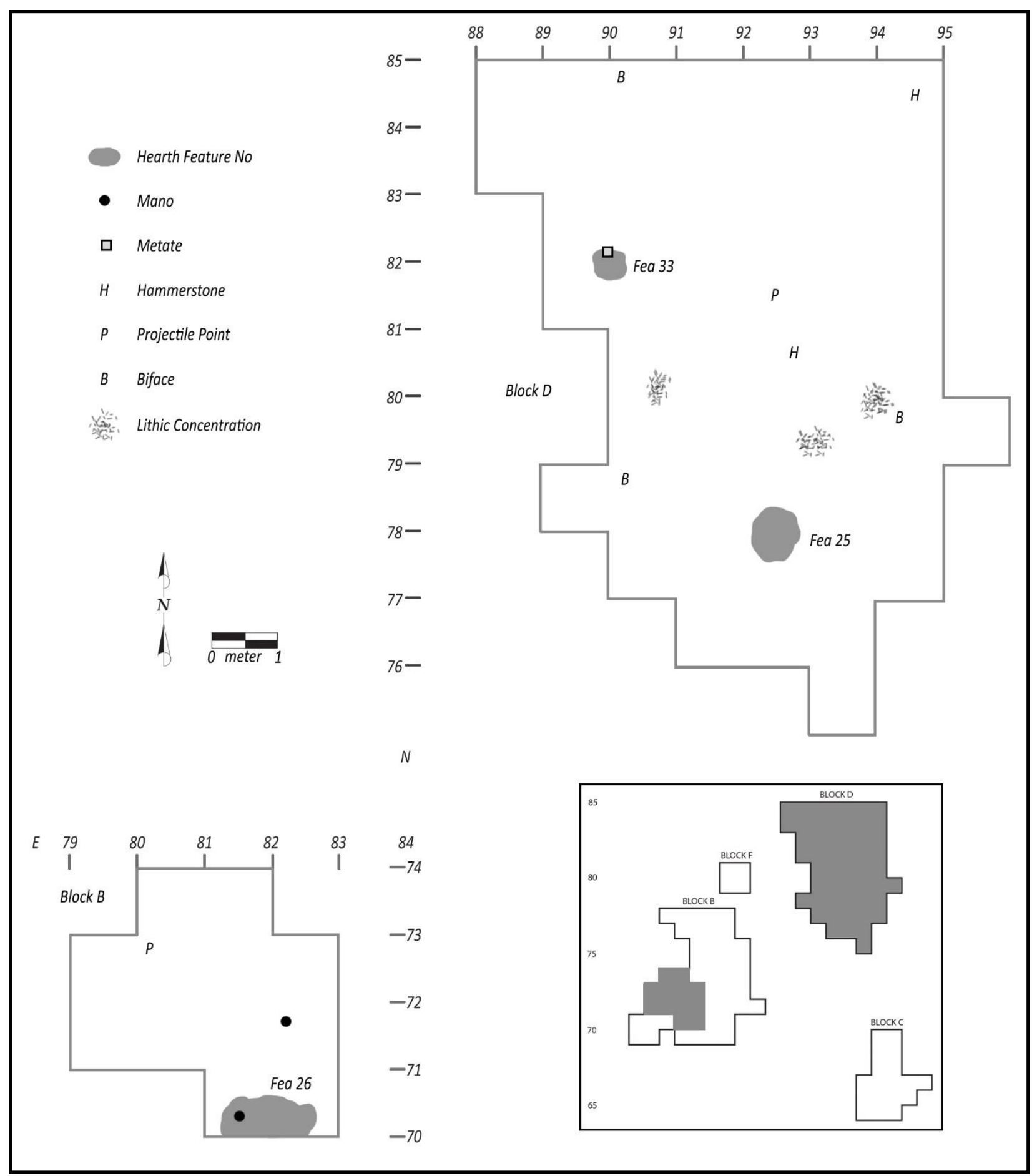

Figure 10-36. Horizontal distribution of Martindale features and formal stone tools. 


\subsection{LITHIC DEBITAGE}

Benjamin G. Bury

\subsubsection{Introduction}

The analysis presented here was structured around a free standing typology based on the morphological classification of debitage and the measurement of attributes (see Chapter 5.0). The analysis of lithic debitage associated with the Martindale component follows the similar methods as the analysis for the Bell/Andice component (see Section 9.6). Platform bearing flakes (complete flakes and proximal flake fragments) were selected for in-depth analysis, and all attributes outlined in the TxDOT protocol were included. The specific observations and details concerning the flakes and their various attributes are presented followed by a brief discussion of other debitage classes and cores. A more detailed discussion of the Bell/Andice and Martindale components is included in Chapter 11.0. Lithic debitage recovered during excavation of the Martindale component include 682 complete flakes, 607 incomplete flake fragments or unidentifiable pieces of manufacturing debris, and 140 proximal flake fragments. An additional 14 complete flakes and 7 proximal flake fragments with edge modification were identified during the analysis and include in the following statistics.

\subsubsection{Complete Flakes}

Table 10-10 lists the amount of cortex and number of flake scars recorded on the dorsal surfaces of complete flakes. The number of flake scars and the percentage of cortex is a good general indicator of the degree of reduction within an assemblage, with an increase in flake scars and corresponding decrease in cortex as raw materials are reduced (Odell 1989). From the perspective of a cortical typology, the assemblage of complete flakes is primarily composed of tertiary flakes with no cortex, followed by secondary flakes with less than 75 percent dorsal cortex, and primary flakes with greater than 75 percent dorsal cortex.
Correspondingly, only 2.8 percent had no dorsal flake scars (100 percent cortex), 49.7 percent had between 1 and 2 scars, and almost as many had three or more scars $(N=330,47.2$ percent). Based on the amount of dorsal cortex and the number of dorsal flake scars, the assemblage reflects a greater proportion of flakes from middle and later stages of reduction.

When an application load typology is applied to the assemblage, a similar pattern is apparent. The majority of the flakes have absent or slightly developed bulbs of percussion (Table 10-11). A sizeable number have moderately developed bulbs, and a smaller number have noticeably pronounced bulbs. Additionally, the presence or absence of eraillure flake scars was also recorded, with 19.5 percent $(N=136)$ exhibiting a scar. In general, the size of the bulb and presence of an eraillure scar has been shown to correspond to the amount of applied force and the nature of the percussor, with hard hammers tending to produce larger, more pronounced bulbs (Andrefsky 2006, 2005; Crabtree 1972). From the perspective of an application load typology, the majority of the assemblage is representative of middle to late stages of reduction.

Technological typologies typically differentiate between bipolar flakes, core reduction flakes, bifacial thinning flakes, and more specific forms related to tool sharpening and tool manufacture. Core reduction flakes are usually defined by single facet platforms, larger dimensions, greater amounts of dorsal cortex, and less pronounced platform lipping and longitudinal curvature. Biface thinning flakes are typically defined as having "curved longitudinal cross-sections, extremely acute lateral and distal edge angles, feathered flake terminations, narrow faceted striking platforms, a lip, little or no cortex, and a small flattened or diffuse bulb of force" (Andrefsky 2006:118; Root 1992:83). Typically, these attributes are not measured separately, but assessed visually as a whole for individual flakes. During this analysis, a tentative technological category was assigned to each flake. 
Table 10-10. Dorsal Cortex and Scar Counts for Complete Flakes.

\begin{tabular}{|c|c|c|c|c|c|}
\hline \multicolumn{3}{|c|}{ Dorsal Cortex } & \multicolumn{3}{c|}{ Dorsal Scars } \\
\hline Class & Count & Percent & Class & Count & Percent \\
\hline 0 & 507 & 72.84 & 0 & 20 & 2.87 \\
\hline $1-25$ & 91 & 13.07 & $1-2$ & 347 & 49.86 \\
\hline $26-50$ & 32 & 4.60 & $3-4$ & 297 & 42.67 \\
\hline $51-75$ & 22 & 3.16 & $5-6$ & 32 & 4.60 \\
\hline $76-100$ & 44 & 6.32 & $7+$ & 0 & 0.00 \\
\hline Total & 696 & 100.00 & Total & 696 & 100.00 \\
\hline
\end{tabular}

Table 10-11. Bulb Size and Eraillure Scar Frequency for Complete Flakes.

\begin{tabular}{|c|c|c|c|c|c|}
\hline \multicolumn{3}{|c|}{ Bulb } & \multicolumn{3}{c|}{ Eraillure } \\
\hline Class & Count & Percent & Class & Count & Percent \\
\hline Absent & 331 & 47.56 & Absent & 547 & 78.59 \\
\hline Moderate & 249 & 58.78 & Present & 136 & 19.54 \\
\hline Pronounced & 112 & 16.09 & N/A & 13 & 1.87 \\
\hline N/A & 4 & 0.57 & & & \\
\hline Total & 696 & 100.00 & Total & 696 & 100.00 \\
\hline
\end{tabular}

Table 10-12. Lipping and Longitudinal Curvature for Complete Flakes.

\begin{tabular}{|c|c|c|c|c|c|}
\hline \multicolumn{3}{|c|}{ Lipping } & \multicolumn{3}{c|}{ Longitudinal Curvature } \\
\hline Class & Count & Percent & Class & Count & Percent \\
\hline Absent & 595 & 85.49 & Absent & 478 & 68.68 \\
\hline Moderate & 55 & 7.90 & Moderate & 140 & 20.11 \\
\hline Pronounced & 35 & 5.03 & Pronounced & 78 & 11.21 \\
\hline N/A & 11 & 1.58 & & & \\
\hline Total & 696 & 100.00 & Total & 696 & 100.00 \\
\hline
\end{tabular}

Table 10-13. Platform Class and Preparation for Complete Flakes.

\begin{tabular}{|c|c|c|c|c|c|}
\hline \multicolumn{3}{|c|}{ Platform Class } & \multicolumn{3}{c|}{ Platform Preparation } \\
\hline Class & Count & Percent & Class & Count & Percent \\
\hline Single Facet & 289 & 41.52 & Absent & 410 & 58.91 \\
\hline Multifacet & 157 & 22.56 & Present & 271 & 38.94 \\
\hline Collapsed & 77 & 11.06 & N/A & 15 & 2.16 \\
\hline Abraded & 73 & 10.49 & & & \\
\hline Cortical & 51 & 7.33 & & & \\
\hline Crushed & 49 & 7.04 & & & 100.00 \\
\hline Total & 696 & 100.00 & Total & 696 & \\
\hline
\end{tabular}


In order of abundance, these include core reduction flakes $(N=317,45.54)$, biface shaping flakes $(N=$ $150,21.55$ percent $)$, biface thinning flakes $(N=$ $120,17.24$ percent), platform trimming flakes $(N=$ $65,9.33$ percent $)$, retouch flakes $(N=43,6.17$ percent), and notching flakes ( $N=1,0.14$ percent). Although a technological assessment of the assemblage is subjective, the general pattern is consistent with the other typologies.

Lipping of the ventral margin of the platform and longitudinal curvature were both recorded independently (Table 10-12). As stated in Section 9.6.2, platform lipping is generally thought to occur more frequently with soft-hammer percussion (Andrefsky 2005; Hovers 2009); and in some studies, longitudinal curvature has been shown to be associated with biface production (Hayden and Hutchings 1989). Lipping and longitudinal curvature were recorded in three ordinal classes absent, moderate, and pronounced. The majority of the platforms was categorized as slightly lipped or exhibited no lipping. Only 6.5 percent $(N=90)$ of the flakes exhibited a moderate to strongly developed lip. The majority have no visually discernable longitudinal curvature. However, a significant number have a moderate to pronounced curve $(N=218,31.1$ percent). The lack of lipping and longitudinal curvature in the assemblage suggests that a significant proportion of the flakes were not produced with soft hammer percussion.

Platform characteristics can be applied within both an application load and technological typologies. Table 10-13 lists the platform classes and the presence or absence of preparation along the dorsal margin of the platform. With the exception of single facet platforms, platform types are often difficult to categorize, especially with smaller flakes. If crushed, abraded, and multifaceted platforms are combined, then 40 percent $(N=279)$ of the platforms can be characterized as complex platforms. Platform preparation was recorded on the dorsal margin of the platform and consisted of either trimming, abrasion, or a combination of both. Overall, 39.0 percent $(N=272)$ exhibited some form of preparation of the dorsal margin and 58.8 percent $(N=410)$ had none.

Table 10-14 lists counts of termination types and the distribution of flakes within five size classes. The majority has feathered terminations and occur in the $6.4 \mathrm{~mm}(1 / 4$ inch $)$ size class. Hinged terminations are also present in significant quantities and generally occur more frequently with hard-hammer percussion (Andrefsky 2006; Patten 1999). Flakes with step terminations were classified as proximal flake fragments given the difficulty in distinguishing between step terminations and postdepositional breaks.

Heat treatment within the assemblage was grouped within three nominal categories. Flakes with obvious signs of thermal damage (e.g., potlidding, crazing, etc.) were categorized as thermally altered. Flakes with a waxy luster or traces of reddish colors were categorized as potentially heat treated. Flakes with no signs of heat treatment were categorized as not thermally altered. Within the sample of complete flakes, 70.11percent $(N=488)$ are potentially heat treated, 25.0 percent $(N=174)$ are not thermally altered, and 4.89 percent $(N=34)$ are clearly thermally altered.

Table 10-15 presents the descriptive statistics of metric attributes measured within the assemblage, including mean, standard deviation (StD) and coefficient of variation (CV). The maximum dimension of the flakes is the least variable of the attributes $(\mathrm{CV}=0.46)$, and the weight is the most variable $(\mathrm{CV}=2.00)$. Platform thickness was also highly variable $(\mathrm{CV}=0.95)$. The length and widths of the flakes are nearly identical, indicating that many are broadly shaped, a characteristic that is associated with bifacial reduction. The degree of variability in the assemblage suggests that flakes from all stages of reduction are represented in the sample. 
Table 10-14. Size Class and Termination Types for Complete Flakes.

\begin{tabular}{|c|c|c|c|c|c|}
\hline \multicolumn{3}{|c|}{ Size Class } & \multicolumn{3}{c|}{ Termination } \\
\hline Class & Count & Percent & Class & Count & Percent \\
\hline$<1 / 4$ inch & 29 & 4.17 & Feather & 529 & 76.01 \\
\hline $1 / 4$ inch & 276 & 39.66 & Hinge & 159 & 22.84 \\
\hline $1 / 2$ inch & 190 & 27.30 & Axial & 7 & 1.01 \\
\hline $3 / 4$ inch & 98 & 14.08 & Overshot & 1 & 0.14 \\
\hline$>1$ inch & 103 & 14.08 & & & \\
\hline Total & 696 & 100.00 & Total & 696 & 100.00 \\
\hline
\end{tabular}

Table 10-15. Metric Dimensions for Complete Flakes (measurements in $\mathrm{mm}$ ).

\begin{tabular}{|c|c|c|c|c|c|c|c|}
\hline \multicolumn{3}{|c|}{ Platform Dimensions } & \multicolumn{4}{c|}{ Flake Dimensions } \\
\hline Attribute & Mean & StD & CV & Attribute & Mean & StD & CV \\
\hline Thickness & 4.50 & 4.31 & 0.95 & Maximum & 27.28 & 12.76 & 0.46 \\
\hline Width & 12.91 & 8.90 & 0.65 & Length & 22.49 & 11.25 & 0.50 \\
\hline \multicolumn{3}{|c}{} & & Width & 21.77 & 10.74 & 0.49 \\
\cline { 4 - 7 } & & Thickness & 5.23 & 3.94 & 0.75 \\
\cline { 4 - 7 } & & Weight & 3.91 & 7.84 & 2.00 \\
\hline
\end{tabular}

\subsubsection{Proximal Flake Fragments}

Flakes with broken terminations are often indistinguishable from complete flakes with step terminations and were classified here as proximal flake fragments. Table 10-16 lists the nonmetric attributes measured on nominal or ordinal scales within the assemblage of proximal flake fragments $(N=147)$. The typical proximal flake fragment is nearly identical to the average complete flake, and had a single facet platform $(N=70 / 47.6$ percent $)$, an absence of dorsal cortex $(N=99,67.34$ percent $)$, an absent or weakly developed bulb $(N=67,45.5$ percent), absent or slight platform lipping ( $N=116$, 78.9 percent $)$, no platform preparation $(N=79,53.7$ percent), between 1 to 2 dorsal flake scars $(N=92$, 62.5 percent), absent to slight longitudinal curvature $(N=127,86.3$ percent), and no eraillure scar $(N=113,76.8$ percent $)$.
Table 10-17 presents the descriptive statistics of metric attributes measured within the assemblage, including mean, StD and $\mathrm{CV}$. The maximum dimension of the flakes is the least variable of the attributes $(\mathrm{CV}=0.40)$, and weight is the most variable $(\mathrm{CV}=2.2)$. As expected, the proximal flake fragments are smaller than the complete flakes in all dimensions.

\subsubsection{Other Debitage}

In addition to complete flakes and proximal flake fragments, the Martindale assemblage includes 607 flake fragments and pieces of angular debitage averaging 2.3 grams in weight. One notching flake were detected. Over 50 percent showed signs of thermal alteration. This is much higher than the amount of thermal alteration observed in the sample of complete flakes and proximal flake fragments, and suggests that smaller pieces of debitage may have been selectively disposed of in thermal features. 
Table 10-16. Nonmetric Attributes Recorded on Proximal Flake Fragments.

\begin{tabular}{|c|c|c|c|c|c|c|c|}
\hline Attribute & Category & Count & Percent & Attribute & Category & Count & Percent \\
\hline \multirow{6}{*}{ Cortex } & 0 & 99 & 67.35 & \multirow{6}{*}{ Scar Count } & 0 & 4 & 2.72 \\
\hline & $1-25$ & 21 & 14.29 & & $1-2$ & 92 & 62.59 \\
\hline & $26-50$ & 12 & 8.16 & & $2-3$ & 47 & 31.97 \\
\hline & $51-75$ & 8 & 5.44 & & $3-4$ & 4 & 2.72 \\
\hline & $76-100$ & 7 & 4.76 & & $5+$ & 0 & 0.00 \\
\hline & Total & 147 & 100.00 & & Total & 147 & 100.00 \\
\hline \multirow{4}{*}{ Bulb } & Absent & 68 & 46.26 & \multirow{4}{*}{ Eraillure } & Absent & 113 & 76.87 \\
\hline & Moderate & 60 & 40.82 & & Present & 31 & 21.09 \\
\hline & Pronounced & 19 & 12.93 & & $\mathrm{~N} / \mathrm{A}$ & 3 & 2.04 \\
\hline & Total & 147 & 100.00 & & Total & 147 & 100.00 \\
\hline \multirow{4}{*}{ Lipping } & Absent & 119 & 80.95 & \multirow{4}{*}{ Long. Curv. } & Absent & 127 & 86.39 \\
\hline & Moderate & 13 & 8.84 & & Moderate & 13 & 8.84 \\
\hline & Pronounced & 15 & 10.20 & & Pronounced & 7 & 4.76 \\
\hline & Total & 147 & 100.00 & & Total & 147 & 100.00 \\
\hline \multirow{7}{*}{ Platform } & Single Facet & 70 & 47.62 & \multirow{6}{*}{ Size Class } & $<1 / 4$ inch & 6 & 4.08 \\
\hline & Multifacet & 33 & 22.45 & & $1 / 4$ inch & 62 & 42.18 \\
\hline & Abraded & 18 & 12.42 & & $1 / 2$ inch & 40 & 27.12 \\
\hline & Crushed & 11 & 7.48 & & $3 / 4$ inch & 27 & 18.37 \\
\hline & Collapsed & 8 & 5.44 & & $>1$ inch & 12 & 8.16 \\
\hline & Cortical & 7 & 4.76 & & Total & 147 & 100.00 \\
\hline & Total & 147 & 100.00 & & & & \\
\hline \multirow{3}{*}{$\begin{array}{l}\text { Platform } \\
\text { Prep. }\end{array}$} & Absent & 80 & 54.42 & & & & \\
\hline & Present & 67 & 45.58 & & & & \\
\hline & Total & 147 & 100.00 & & & & \\
\hline
\end{tabular}

Table 10-17. Descriptive Statistics of Metric Dimensions for Proximal Flake Fragments (measurements in $\mathrm{mm}$ ).

\begin{tabular}{|c|c|c|c|c|c|c|c|}
\hline \multicolumn{3}{|c|}{ Platform Dimensions } & \multicolumn{4}{c|}{ Flake Dimensions } \\
\hline Attribute & Mean & StD & CV & Attribute & Mean & StD & CV \\
\hline Thickness & 3.53 & 3.00 & 0.85 & Maximum & 24.19 & 9.90 & 0.41 \\
\hline Width & 10.79 & 7.81 & 0.72 & Length & 19.01 & 7.97 & 0.42 \\
\hline \multicolumn{3}{|c}{} & Width & 20.47 & 9.33 & 0.46 \\
\cline { 4 - 7 } & & Thickness & 4.65 & 3.34 & 0.72 \\
\cline { 5 - 7 } & & Weight & 2.98 & 6.56 & 2.20 \\
\hline
\end{tabular}


Table 10-18. Metric Dimensions for Cores (measurements in $\mathrm{mm}$ ).

\begin{tabular}{|c|c|c|c|}
\hline \multicolumn{4}{|c|}{ Metric Dimensions } \\
\hline Dimension & Mean & StD & CV \\
\hline Length & 69.48 & 8.53 & 0.12 \\
\hline Width & 47.49 & 9.35 & 0.19 \\
\hline Thickness & 31.55 & 16.95 & 0.53 \\
\hline Weight & 101.50 & 81.13 & 0.79 \\
\hline
\end{tabular}

Table 10-19. Nonmetric Attributes Recorded on Cores.

\begin{tabular}{|c|c|c|c|c|c|}
\hline \multicolumn{3}{|c|}{ Cortex } & \multicolumn{3}{c|}{ Scar Count } \\
\hline Category & Count & Percent & Category & Count & Percent \\
\hline 0 & 1 & 14.29 & $1-2$ & 0 & 0.00 \\
\hline $1-25$ & 1 & 14.29 & $3-4$ & 2 & 28.57 \\
\hline $26-50$ & 5 & 71.43 & $5-6$ & 2 & 28.57 \\
\hline $51-75$ & 0 & 0.00 & $7-8$ & 3 & 42.86 \\
\hline Total & 7 & 100.00 & Total & 7 & 100.00 \\
\hline \multicolumn{7}{|c|}{} & \multicolumn{3}{|c|}{ Direction } \\
\hline Category & Type & Percent & Category & Count & Percent \\
\hline Cobble & 4 & 57.14 & Single & 1 & 14.29 \\
\hline Macro Flake & 2 & 28.57 & Multiple & 6 & 85.71 \\
\hline Fragment & 1 & 14.29 & & & 100.00 \\
\hline Total & 7 & 100.00 & Total & 7 & \\
\hline
\end{tabular}

\subsubsection{Cores}

Seven cores were recovered during the excavation of the Martindale component. Table 10-18 lists the mean StD and CV of the metric dimensions recorded within the assemblage. Overall, the cores are relatively consistent in their dimensions, with the most variation in weight $(\mathrm{CV}=0.79)$. Table 10 19 lists the nonmetric attributes recorded within the assemblage. All of the cores have less than 50 percent cortical cover. The majority have between 26-50 percent ( $N=5,71.4$ percent), more than 5 flake scars $(N=5,62.5$ percent $)$, and were reduced from multiple directions $(N=6,85.7$ percent $)$. Cortex on all of the cores was formed from a combination of fluvial processes and chemical weathering from surface exposure. Cores were produced from locally available cobbles. Overall, the ratio of cores to lithic debitage within the Martindale Component is 1:206.57 and reflects the substantial amount of lithic reduction that occurred at the site during the Martindale period.

\subsubsection{Summary of Martindale Lithic Debitage}

The typical complete flake from the Martindale component has a single facet platform $(N=289$, 41.4 percent $)$, a feathered termination $(N=526$, 75.4 percent), an absence of dorsal cortex ( $N=507$, 72.7 percent), an absent or weakly developed bulb ( $N=332,47.6$ percent), absent or slight platform lipping $(N=596,85.5$ percent $)$, no platform preparation $(N=410,58.8$ percent), between 1 to 2 
dorsal flake scars $(N=347,49.7$ percent $)$, absent to slight longitudinal curvature $(N=479,68.7$ percent), and no eraillure scar $(N=548,78.6$ percent). Proximal flake fragments were found to be nearly identical. Overall, this assemblage is skewed towards middle and later stages of reduction and the ratio of cores to debitage is correspondingly high. Combined, the various typologies indicate that biface production was a significant element of the lithic reduction that occurred at the site during the Martindale period.

\subsubsection{Samples from Lithic Concentration}

Three localized lithic debitage concentrations were recognized in the field (see Figure 10-36) and bulk sediment samples were randomly collected from those for laboratory processing. The remaining debitage were collected in the standard screening and recorded in that unit. Three samples from three different locations in Block D were mechanically floated and their results presented below.

A 4.25 liter sediment sample (\#2248-4-g) from between 270 and $272 \mathrm{cmbs}$ in N79/E94, some 30 to 40 $\mathrm{cm}$ northeast of Feature 25 yielded quantities of chert microflakes and bone fragments (Table 10-20, Figure 10-37). This sample was from 2 to $3 \mathrm{~cm}$ below a recognized lithic concentration between 265 and 270 in an area roughly 30 -by- $50 \mathrm{~cm}$ in the northwest corner of the unit. The lithic debitage from the entire $10 \mathrm{~cm}$ level in this unit (\#2247-1 and \#2248-1) includes 163 pieces. Minimally four edge-modified flakes and some 160 bone fragments were also in this level. The actual horizontal dispersal of this concentration in the adjacent units was not clearly recorded given slightly different excavation methods. The heavy fraction yielded 307 unburned bone fragments less than 6.4 $\mathrm{mm}$ in size that reflect minimally small and midsize mammals, with numerous gar scales and fish vertebrae (Figure 10-37. Over 100 chert flakes less than $6.4 \mathrm{~mm}$ in size were present and potentially represent two separate parent objects.
A second lithic debitage concentration (\#2257) was encountered roughly $1 \mathrm{~m}$ northeast of the first lithic concentration and nearly $2 \mathrm{~m}$ northeast of Feature 25 in N80/E95 at around $270 \mathrm{cmbs}$ (see Figure 10-36). A 5.5 liter bulk sediment sample from an area roughly 30-by-30 cm just on the eastern edge of the lithic concentration in the adjacent unit N80/E94 was collected and mechanically floated. The heavy fraction was dominated by 206 chert flakes and 214 unburned bone fragments less than $6.4 \mathrm{~mm}$ in size (see Table 10-20). The tiny lithic debitage was nearly all light colored tan chert and represents pressure flakes or shatter probably from a single tool. Bone fragments include four fish vertebrae, which represent two different size fish, and probably a couple of other fish bones. Snail shells, mostly Rabdotus fragments were also present with no observed signs of heat alteration. This same $10 \mathrm{~cm}$ level in Unit N80/E95 also yielded a total of 23 pieces of debitage and 17 bone fragments. It is possible these first two lithic concentrations document were an individual sat while knapping with the space between being where the individual sat. However, the presence of smashed bone fragments in and around this concentration more than likely supports this locality served as a dump area following bone grease extraction.

A third lithic debitage concentration (\#2261) was less well-defined and identified between 275 and $278 \mathrm{cmbs}$ in the southeastern quadrant of N81/E91, about $3 \mathrm{~m}$ northwest of hearth Feature 25 and nearly $2 \mathrm{~m}$ south of hearth Feature 33 (see Figure 10-36). A six liter sediment sample from the concentration was mechanically floated. The heavy fraction yielded 245 pieces of chert debitage less than $6.4 \mathrm{~mm}, 118$ bone fragments, and quantities of snail shell fragments (Table 10-20, Figure 10-38). Light and dark colored cherts represent minimally two distinct tools and reflect mostly resharpening flakes less than $10 \mathrm{~mm}$. Roughly 98 percent of the bone scraps are unburned. This same level and unit also yielded 71 chert flakes, 16 bone fragments, three small burned rocks, plus one edge-modified flake. 
Table 10-20. Float Results from Three Martindale Lithic Concentrations.

\begin{tabular}{|c|c|c|c|c|c|c|c|c|c|c|c|c|c|c|c|c|c|c|c|c|c|c|}
\hline \multirow{2}{*}{$\begin{array}{l}\stackrel{8}{\$} \\
z \\
\stackrel{0}{z}\end{array}$} & \multirow{2}{*}{$\stackrel{\text { c }}{\mathrm{C}}$} & \multirow{2}{*}{ 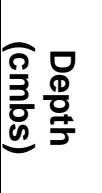 } & \multirow{2}{*}{$\begin{array}{l}\text { 罝 } \\
\frac{\Gamma}{x}\end{array}$} & \multirow{2}{*}{$\frac{8}{\stackrel{8}{3}}$} & \multirow{2}{*}{ 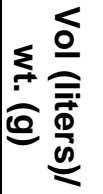 } & & \multicolumn{2}{|c|}{$\begin{array}{l}\text { Mussel } \\
\text { Shell }\end{array}$} & \multicolumn{2}{|c|}{$\begin{array}{l}\text { Burned } \\
\text { Rock }\end{array}$} & \multicolumn{2}{|c|}{ Debitage } & \multicolumn{2}{|c|}{ Charcoal } & \multicolumn{2}{|c|}{ Snail } & \multicolumn{2}{|c|}{$\begin{array}{c}\text { Macrobo } \\
\text { tanical }\end{array}$} & \multicolumn{2}{|c|}{$\begin{array}{l}\text { Burned } \\
\text { Clay }\end{array}$} & \multicolumn{2}{|c|}{ Bone } \\
\hline & & & & & & & $1 / 4$ & $<1 / 4$ & $1 / 4$ & $<1 / 4$ & $1 / 4$ & $<1 / 4$ & $1 / 4$ & $<1 / 4$ & $1 / 4$ & $<1 / 4$ & $1 / 4$ & $<1 / 4$ & $1 / 4$ & $<1 / 4$ & $1 / 4$ & $<1 / 4$ \\
\hline $2248-4-g$ & $\begin{array}{l}\text { N79 } \\
\text { E94 }\end{array}$ & $\begin{array}{l}270- \\
272 \\
\end{array}$ & D & $\mathrm{CN}$ & 4.25 & $\mathrm{~N}$ & 0 & 0 & 0 & 0 & 0 & 102 & 0 & 0 & 0 & 48 & 0 & 0 & 0 & 0 & 0 & 307 \\
\hline \multicolumn{5}{|c|}{ Light Fraction } & 19.5 & Wt. & 0.0 & 0.0 & 0.0 & 0.0 & 0.0 & 1.5 & 0.0 & 0.0 & 0.0 & 12.1 & 0.0 & 0.0 & 0.0 & 0.0 & 0.0 & 2.2 \\
\hline $2257-4-g$ & $\begin{array}{l}\text { N80 } \\
\text { E95 }\end{array}$ & $\begin{array}{l}270- \\
272.5\end{array}$ & D & $\mathrm{CN}$ & 5.5 & 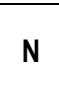 & 0 & 0 & 0 & 0 & 15 & 191 & 0 & 4 & 0 & 57 & 0 & 0 & 0 & 0 & 0 & 214 \\
\hline \multicolumn{5}{|c|}{ Light Fraction } & 48.1 & Wt. & 0.0 & 0.0 & 0.0 & 0.0 & 12.2 & 2.8 & 0.0 & 0.1 & 0.0 & 19.2 & 0.0 & 0.0 & 0.0 & 0.0 & 0.0 & 1.6 \\
\hline $2261.4-1 \mathrm{~g}$ & $\begin{array}{l}\text { N81 } \\
\text { E91 }\end{array}$ & $\begin{array}{l}275- \\
278\end{array}$ & D & $\mathrm{CN}$ & 6.0 & N & 0 & 0 & 0 & 0 & 11 & 234 & 0 & 0 & 0 & 194 & 0 & 0 & 0 & 0 & 0 & 118 \\
\hline \multicolumn{5}{|c|}{ Light Fraction } & 118.6 & Wt. & 0.0 & 0.0 & 0.0 & 0.0 & 8.3 & 4.2 & 0.0 & 0.0 & 0.0 & 86.6 & 0.0 & 0.0 & 0.0 & 0.0 & 0.0 & 1.6 \\
\hline
\end{tabular}




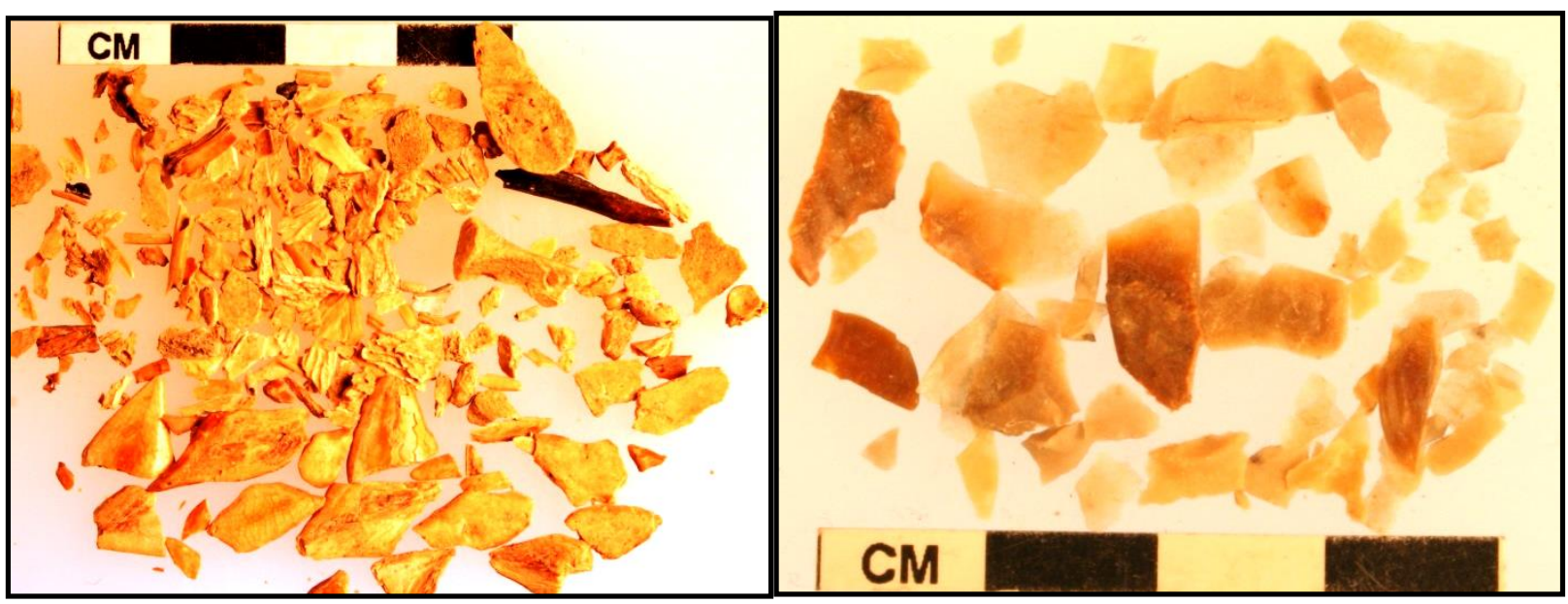

Figure 10-37. Photograph of unburned bone fragments (left) and lithic debitage (right) from heavy fraction (\#2248-4-g).

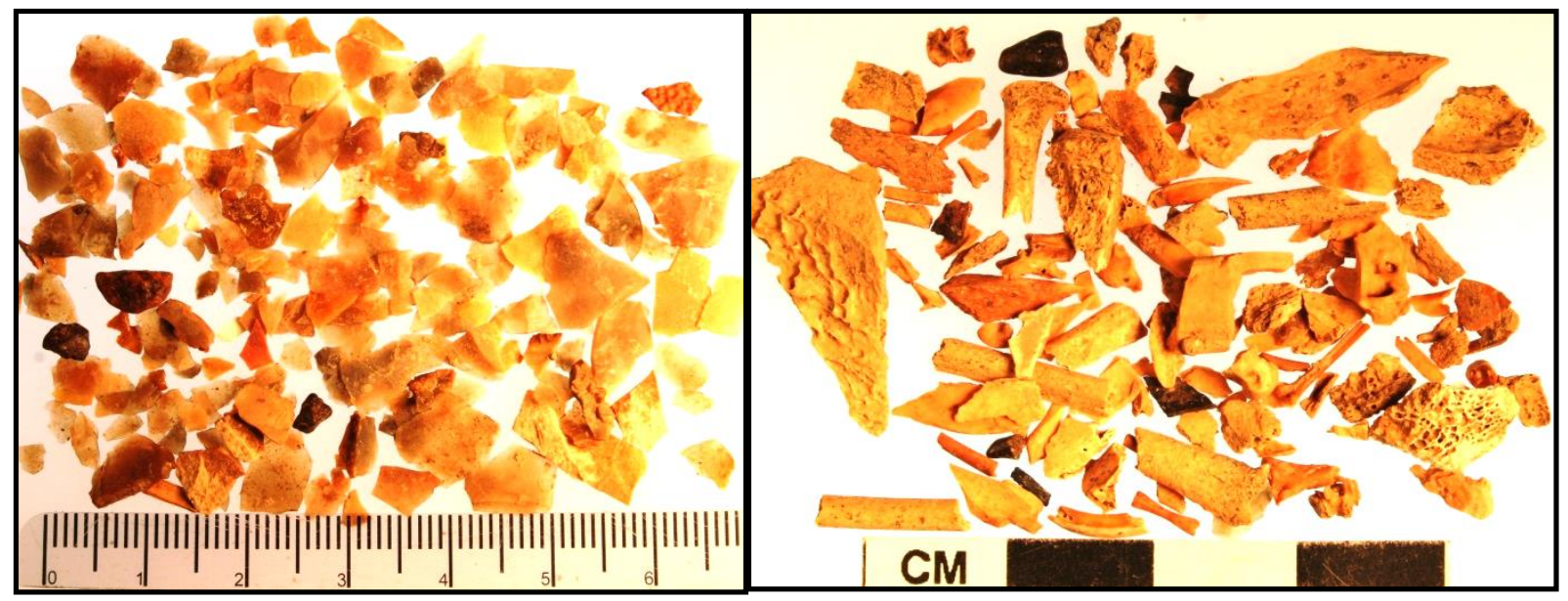

Figure 10-38. Photograph of lithic debitage (left) and bone fragments (right) from heavy fraction (\#2261-4-g).

\subsubsection{Horizontal Distribution of Lithic Debitage}

The horizontal distribution of the lithic debitage across Block D is depicted in Figure 10-39. High concentrations ( $N=114$ to 158 pieces/unit) were in four units (6.5 percent of the total) and yielded 37 percent of the total debitage. Three of those units actually contained field identified concentrations just addressed, but in more restricted areas of those units. The more eastern concentration, just northeast of Feature 25 apparently represents a couple of dumps of debitage or potentially an in situ knapping area with two closely spaced clusters of materials. The other concentration, nearly $2 \mathrm{~m}$ south of Feature 33, also contained a field identified concentration. In both instances, the observed concentrations were small restricted areas, which more than likely represented discarded knapping debris collected and dumped, rather than in situ knapping locals,. The latter would probably have had a broader dispersal. In these instances small bone fragments were also present in with the lithic debris to support these as dump areas rather than stickily knapping locals. As depicted in Figure 1039 moderate scatters of debitage $(N=41$ to 62 


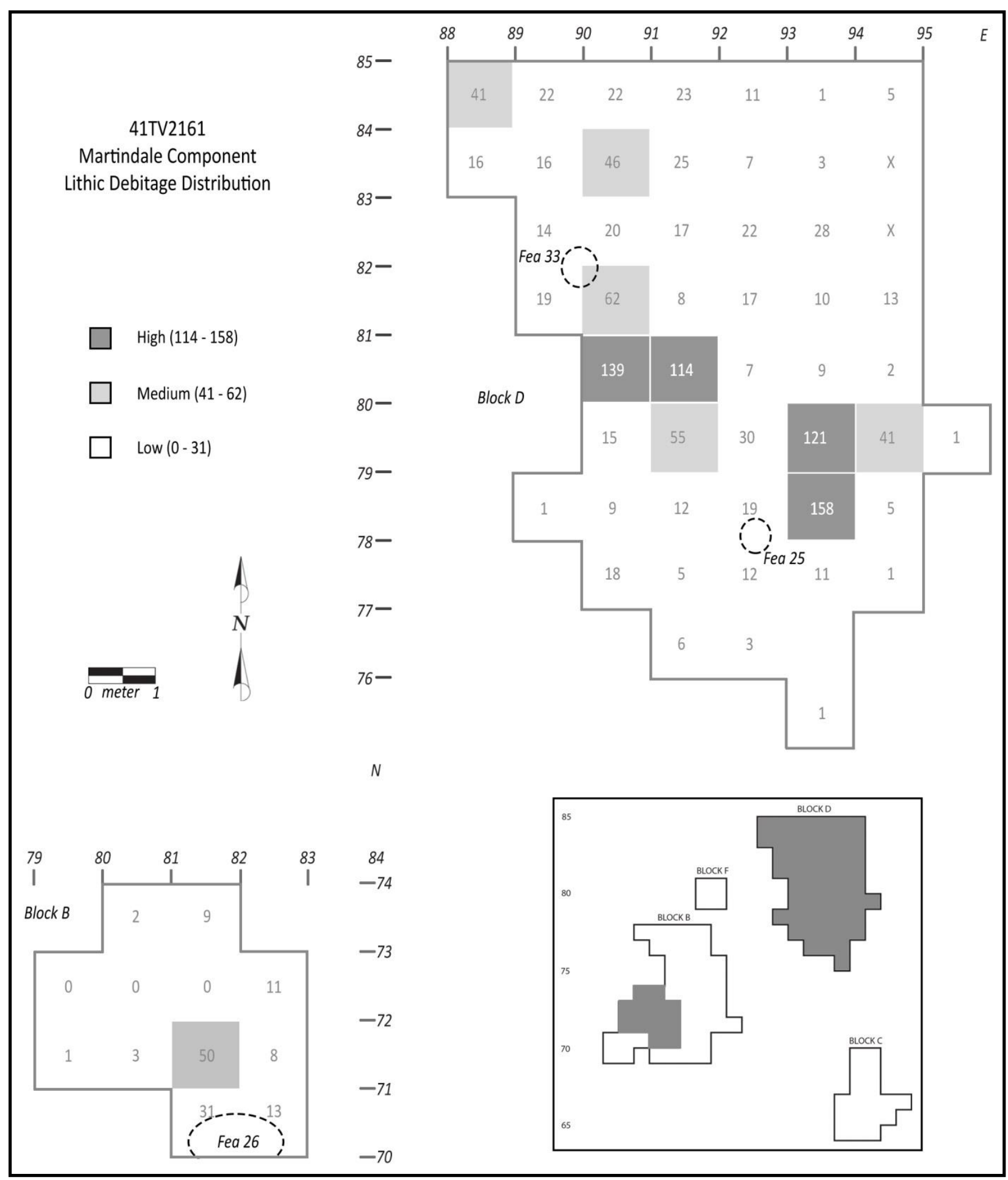

Figure 10-39. Horizontal distribution of Martindale lithic debitage across Blocks B and D. 
pieces/unit) were only in 5 units (roughly 8 percent of the total) and these were mostly around the two dense concentrations. Only one unit with moderate frequencies was isolated from the rest and was $1 \mathrm{~m}$ north of Feature 33. In Block B, only one unit yielded a moderate concentration $(N=50$ pieces $)$ just north of Feature 26. The identified high and moderate lithic concentrations were clearly distinct, which continues to support this distribution reflects purposeful human behaviors and the overall high integrity of the component.

\subsection{VERTEBRATE FAUNAL ASSEMBLAGE}

This rare faunal assemblage is one of the first for the Martindale interval in Texas, and is extremely significant as it is from a well-defined, dated component. This assemblage consists of 2,082 complete bones and fragments that weigh $832 \mathrm{~g}$, for an average of $0.4 \mathrm{~g}$ per bone. The pieces are quite small with 99 percent less than $3 \mathrm{~cm}$ long and only nine (less than 1 percent) greater than $6.1 \mathrm{~cm}$. These small pieces reflect mostly small animals with deer and pronghorn as the large animal exceptions. This number and the following discussions do not include another 5,260 tiny bone fragments $(35.8 \mathrm{~g})$ from nine floated sediment samples, two of which were from hearth features (see Table 10-3). Table 10-21 provides counts and weights, plus other key observations by taxa and species identified.

The overall minimum number of specimens (MNSP) is limited $(N=2,082)$ for the $74 \mathrm{~m}^{2}$ excavated, but the assemblage is very informative. Their extensive fragmented nature, with minimally 3.2 percent of the assemblage encrusted with various amounts of calcium carbonate, combined with root etched surfaces on many others, definitely hindered species identification and restricted observations of other key characteristics such as cut and impact marks, and fracture patterns. Obvious rodent gnawing or carnivore punctures were not detected.
Based on counts, nearly 50 percent are indeterminate to species, mainly due to their small, crushed nature and similarity in the sizes of a number of species represented. Jackrabbits, cottontail rabbits and other similar size specimens $(N=535)$ account for 25.7 percent of the total bone assemblage or 51 percent of those identified to species and a minimum of nine different individuals (Figure 10-40). Five jackrabbits are present based on five, left proximal radius, whereas four cottontails are reflected by four right calcanei. Mature and immature elements are represented. Sixteen pieces were burned and exhibit a range of colors from white, brown, black, and combinations of white with gray and black and brown. Plus 21 fragments exhibit spiral fractures to support their cultural use and intentional green bone breakage. The apparent absence of cut marks may reflect limited cutting in the dismemberment of these small mammals; however, their recognition may also have been hampered by the presence of root etching and calcium carbonate on faunal specimens.

Deer size artiodactyl elements include both unidentified deer and pronghorn elements as both were positively identified, primarily from teeth, antler fragments, and third phalanges. These artiodactyl fragments $(N=152)$ account for 7.3 percent of the total or 14.5 percent identified to taxa. Extensive processing, butchering, and cooking activities resulted in a very fragmented assemblage. These artiodactyls - deer and pronghorn - appear processed in similar fashion with very few complete elements. Extensive bone fragmentation and extraction of marrow was observed in nearly every element, even the tiny marrow cavity in deer phalanges was opened (Figure 10-41). However, most skeletal sections, front and hind legs, skull and axial fragments were identified, which indicate entire artiodactyl carcasses were brought to camp. Given all parts of these carcasses were present, indicates these animals were not transported very far from the actual kill site. 
Table 10-21. Martindale Bone Data and Observations.

\begin{tabular}{|c|c|c|c|c|c|c|c|c|}
\hline Taxon & Species & Count & $\begin{array}{l}\text { Weight } \\
\text { (g) }\end{array}$ & 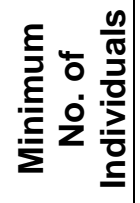 & 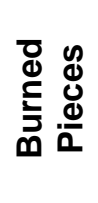 & 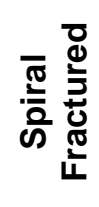 & 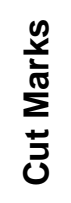 & 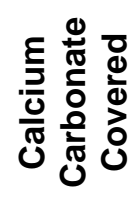 \\
\hline \multirow[t]{2}{*}{ Deer size artiodactyl } & Indeterminate & 139 & 434.9 & & 17 & 3 & 2 & 28 \\
\hline & Whitetail & 5 & 7.4 & 3 & 1 & & & 3 \\
\hline Pronghorn & & 8 & 70.7 & 2 & & & & 1 \\
\hline $\begin{array}{c}\text { Canid size } \\
\text { (dog/coyote) }\end{array}$ & - & 3 & 0.9 & 1 & & & & \\
\hline Rabbit family & Indeterminate & 423 & 86.3 & & 16 & 18 & & 19 \\
\hline Cottontail & & 50 & 12.2 & 4 & & 1 & & 1 \\
\hline Jackrabbit & & 62 & 44.4 & 5 & & 2 & & \\
\hline \multirow[t]{4}{*}{ Rodent } & Indeterminate & 137 & 11.1 & & 2 & & & 1 \\
\hline & Gopher? & 2 & 0.2 & 1 & & & & \\
\hline & Vole? & 2 & 0.1 & 1 & & & & \\
\hline & Rat? & 1 & 0.1 & 1 & & & & \\
\hline \multirow[t]{2}{*}{ Turtle } & Indeterminate & 37 & 11.6 & & 2 & & & 1 \\
\hline & Softshell & 16 & 4.2 & 1 & 1 & & & \\
\hline Snake, miscellaneous & - & 35 & 4.5 & 1 & & & & \\
\hline \multirow[t]{3}{*}{ Bird } & Indeterminate & 8 & 1.6 & & & & & \\
\hline & Grouse & 6 & 0.9 & 4 & & & & \\
\hline & Grouse size & 4 & 3.1 & & & & & \\
\hline Bird, miscellaneous & Indeterminate & 1 & 0.4 & & & & & \\
\hline \multirow[t]{4}{*}{ Fish } & Indeterminate & 42 & 5 & & 2 & & & 2 \\
\hline & Catfish & 2 & 0.4 & 1 & & & & \\
\hline & Crappie? & 2 & 0.3 & 1 & & & & \\
\hline & Gar & 64 & 3.9 & 2 & & & & \\
\hline \multirow[t]{2}{*}{ Indeterminate } & - & 1035 & 127.8 & & 24 & & 1 & 11 \\
\hline & Totals & 2084 & 832 & 28 & 65 & 24 & 3 & 67 \\
\hline
\end{tabular}


Deer and pronghorn mandibles and maxillaries were also reduced to small sections with only a few partially intact tooth rows and a number of loose teeth. These teeth and mandible sections provide an opportunity to investigate animal ages at the time of death and perhaps identify the season of this camp. As deer age, the teeth erupt in a sequential pattern and begin to wear, therefore if young individuals are represented, then potentially rough ages can be established based on the eruption sequence. Although this technique has been used extensively in archeology and wildlife biology, Gee et al. (2002) critically evaluated this technique and suggest their results do not support the efficacy of using tooth wear to accurately age deer beyond the general categories of fawn, yearling, and adult. Consequently, with a 5,000 year old assemblage, combined with environmental condition that differ from today, plus weathered and root etched teeth in various conditions, the accuracy of age determination based on eruption sequence and wear patterns on individuals older than yearlings can be questioned and potentially exhibit greater variation.

Along with loose deer incisors, three partially intact deer maxillary sections and two mandible section are presented below. A left deer mandible section with a socketed $M_{2}$ and $M_{3}$ represents an adult animal roughly 3.5 years or older. A more precise age could not be determined as the cusps are root etched (Figure 10-42). The boney exterior below $\mathrm{M}_{3}$ exhibits 7 to 11 tiny, shallow cut lines perpendicular to the long axis. This mandible was from $280 \mathrm{cmbs}$ in N82/E90 in Block D, less than 1 m southwest of hearth Feature 33.

The most intact tooth row is represented by a complete right deer maxilla (\#2268) with all six teeth and minimal bone attached, held together with calcium carbonate (Figure 10-43). This was recovered between 270 and $275 \mathrm{cmbs}$ in N81/E94 in Block D, $2.5 \mathrm{~m}$ northeast of hearth Feature 25. This maxilla represents another adult animal roughly 3.5 years or older as $\mathrm{M}_{3}$ is fully erupted and in wear.

A second maxillary section (\#2255) came from 272 cmbs in N80/E94, about $1 \mathrm{~m}$ north of the previous maxilla. This consists of five weathered, loose teeth from the left side (Figure10-43). The teeth include $\mathrm{P}^{2}, \mathrm{P}^{3}, \mathrm{P}^{4}, \mathrm{M}^{1}$, and $\mathrm{M}^{2}$ and based on wear represent an individual minimally 3.5 years old. Although opposite the previous maxilla, it potentially represents a different individual based

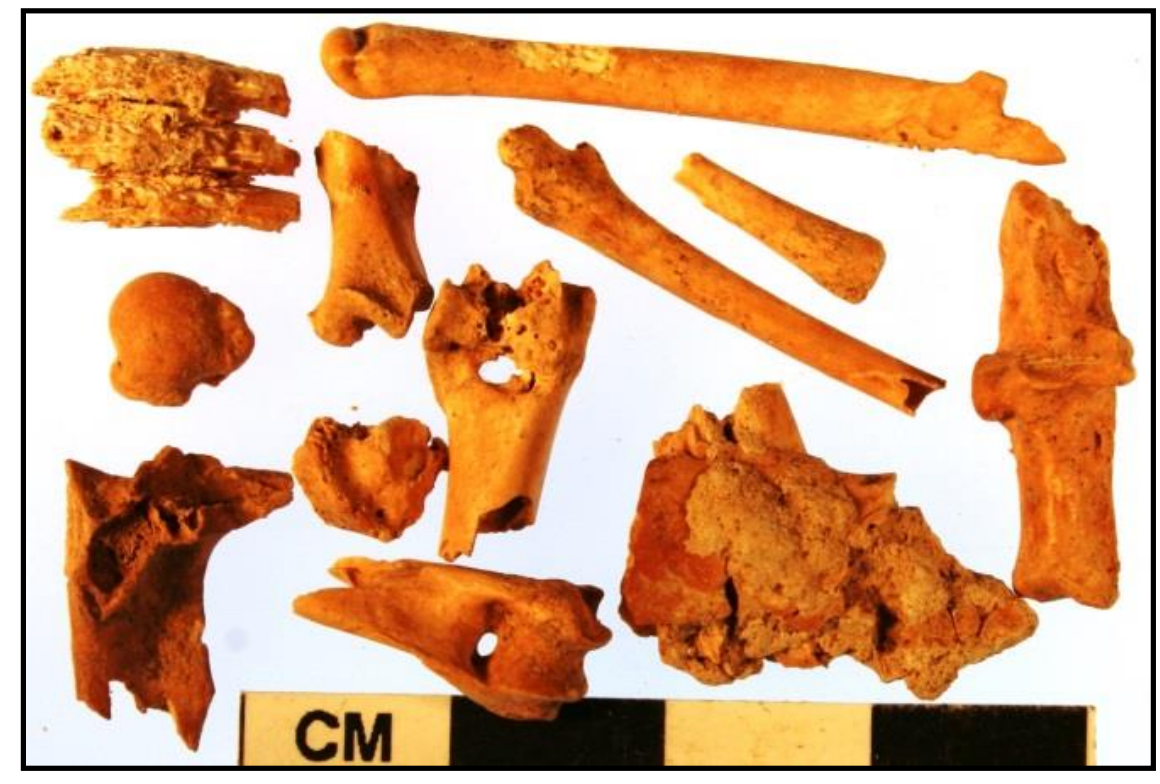

Figure 10-40. Example of rabbit element fragments (\#2237). 


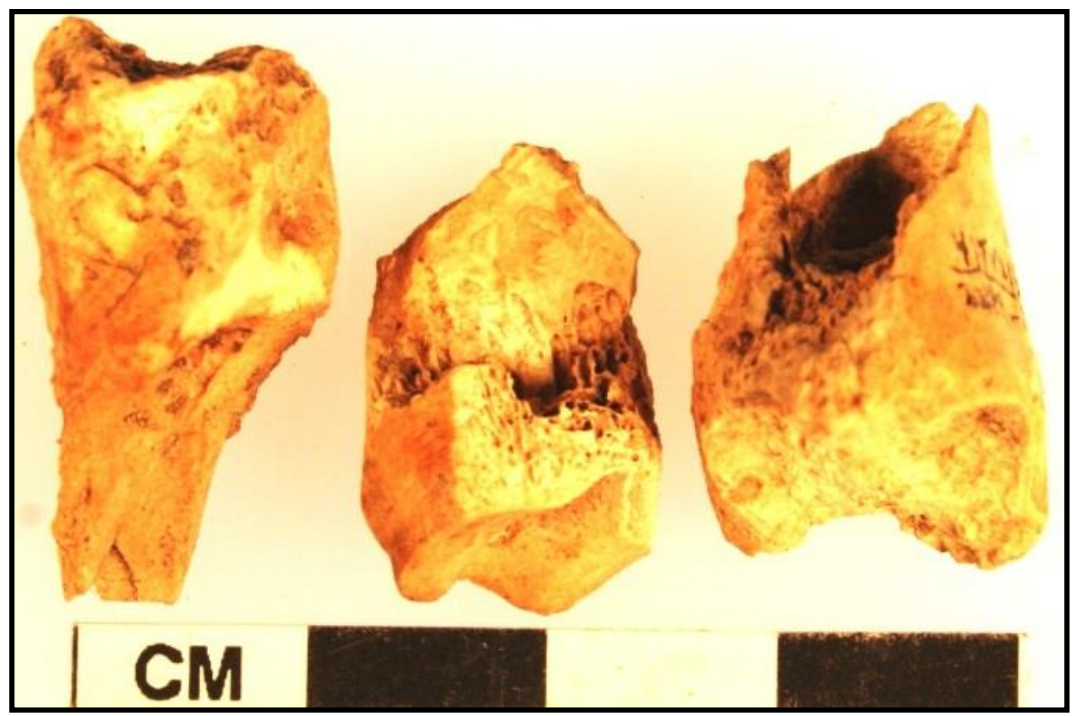

Figure 10-41. Extensively butchered deer phalanges (\#2279).

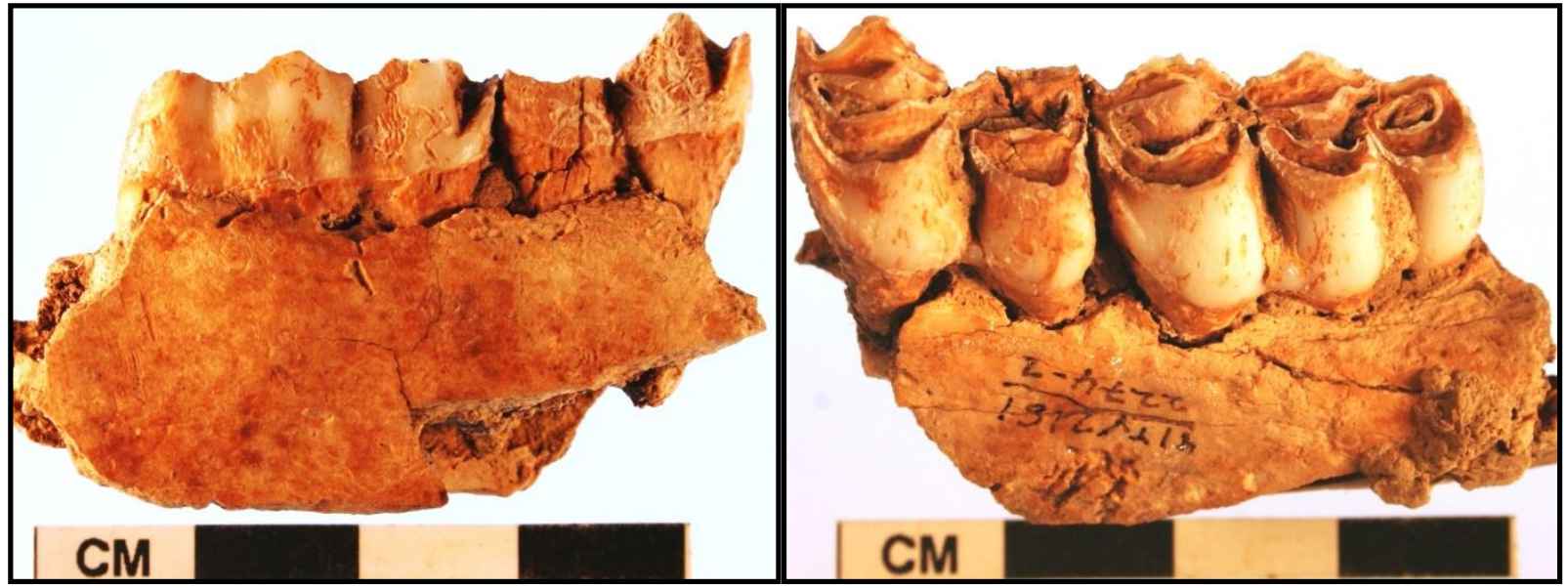

Figure 10-42. Two views, exterior (left) and interior (right) of $\mathbf{M}_{2}$ and $\mathbf{M}_{3}$ socketed in deer mandible fragment (\#2274) that exhibits multiple thin cut marks (front left).

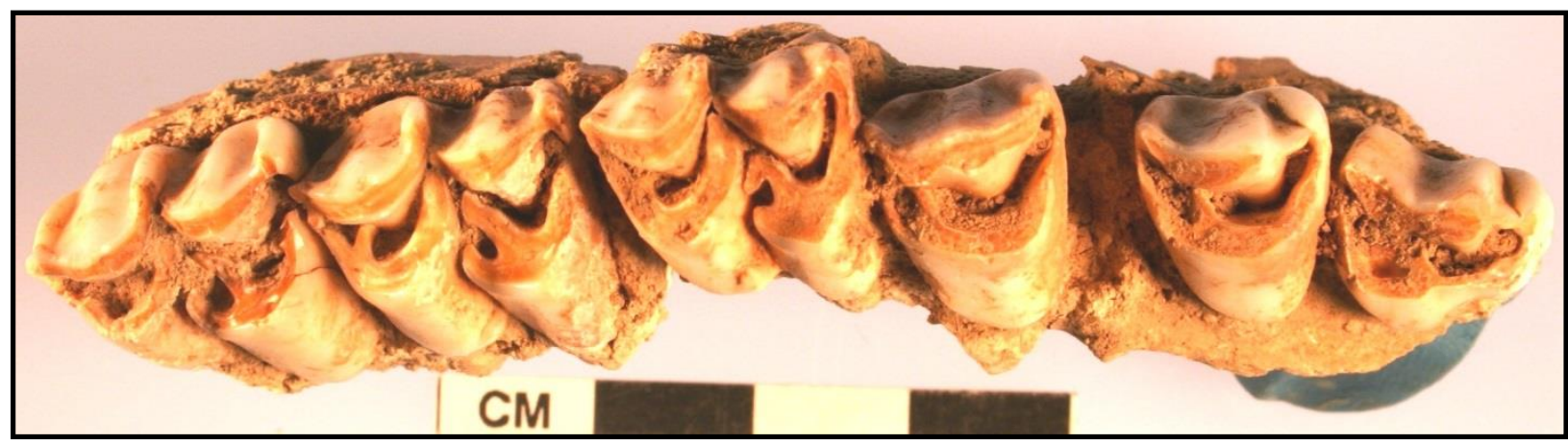

Figure 10-43. Well preserved right deer maxilla with socketed tooth row depicting tooth wear (\#2268). 


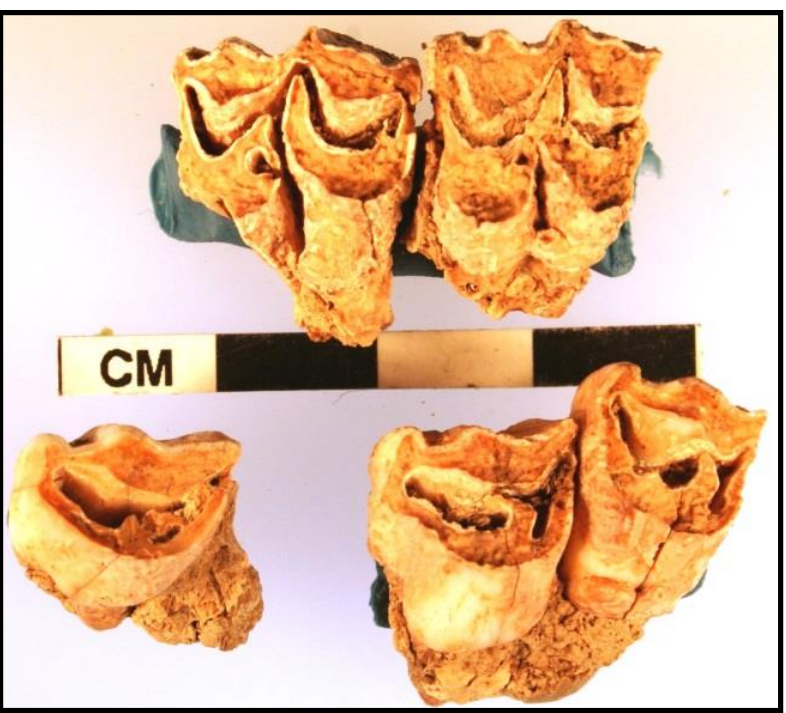

Figure 10-44. Poorly preserved, weathered and worn left deer maxilla with individual teeth from same maxilla (\#2255).

on slightly different wear patterns and slightly smaller teeth. The two maxilla sections possibly represent the same season of death as wear patterns are quite similar.

A single loose, right deer $\mathrm{M}_{1}$ was between 255 and 270 cmbs in N77/E93, about 1 m south of hearth Feature 25. It reflects a tooth in the eruption stage as it lacks a root or wear on the very tip of the enamel crest (Figure 10-45). This stage of eruption and wear represents a deer fawn roughly three to six months old (Harlow and DeFoor 1962), making the time of death in late summer early fall, September through December (Harlow, R. and M. DeFoor 1962). This is based on an average birth date around early June in the Edwards Plateau (Cook 1975).

Seven loose probable deer incisors also represent at least two individuals (Figure 10-46). Two probable deer incisors (samples \#2246-2-10 and \#2246-2-11, see Figure 5-20) between 254 and $266 \mathrm{cmbs}$ of N79/E93 in Block D were submitted for cementum annuli analysis to investigate their ages at death. Unfortunately, no growth lines were observed and no age was determined. Based on comparison with known aged whitetail deer, minimally five incisors represent individuals greater than 5.0 years old (L. Bement, personal communication).

Pronghorn mandibles and teeth are represented by individual loose teeth with only a couple of partially intact mandible section with two and three teeth. Pronghorn teeth, unlike deer teeth, continue to grow over time and are not as sensitive indicators of age, although some researchers have demonstrated this as an effective means of aging animals (Lubinski 2001). By the age of 3.5 years all permanent teeth are in place and from that age on, estimating age is based largely on rate of tooth wear (Jenson 1998). The more intact sections are discussed below followed by a couple of examples of individual teeth.

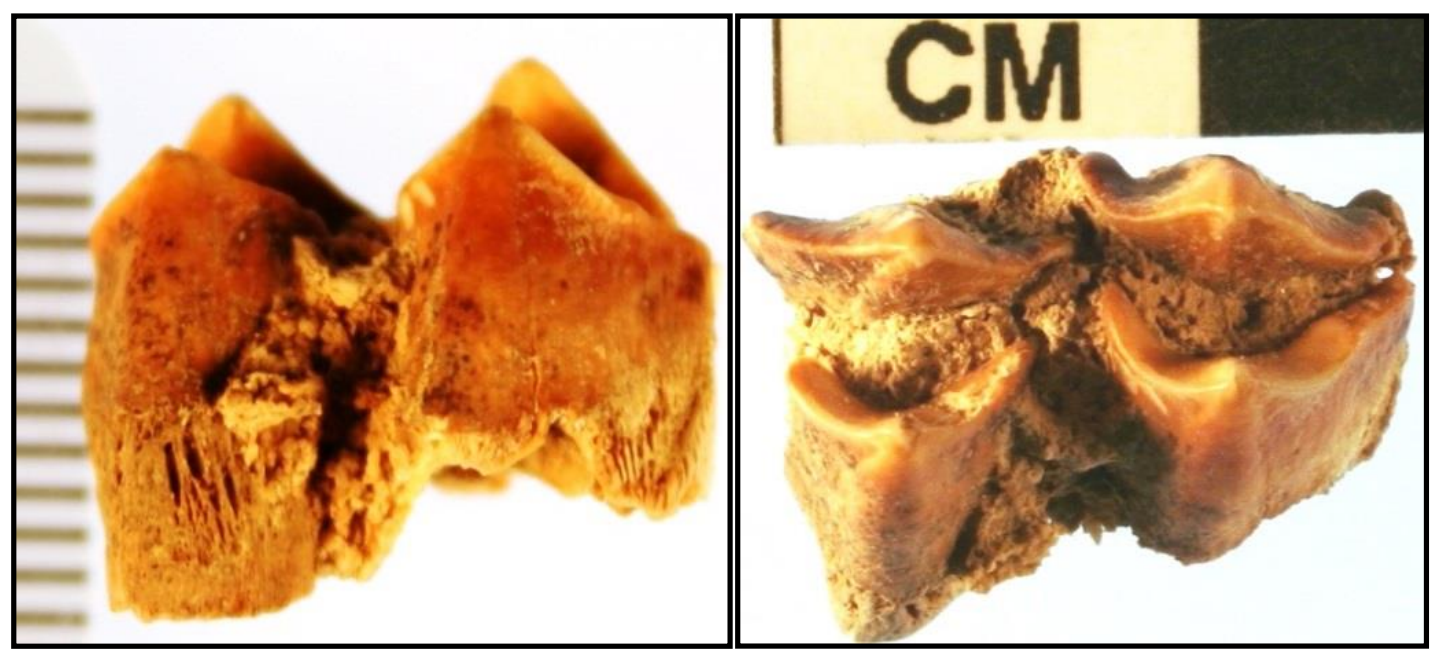

Figure 10-45. Two views of loose, right deer fawn $M_{1}$ (\#2234) in eruption stage with no wear. 


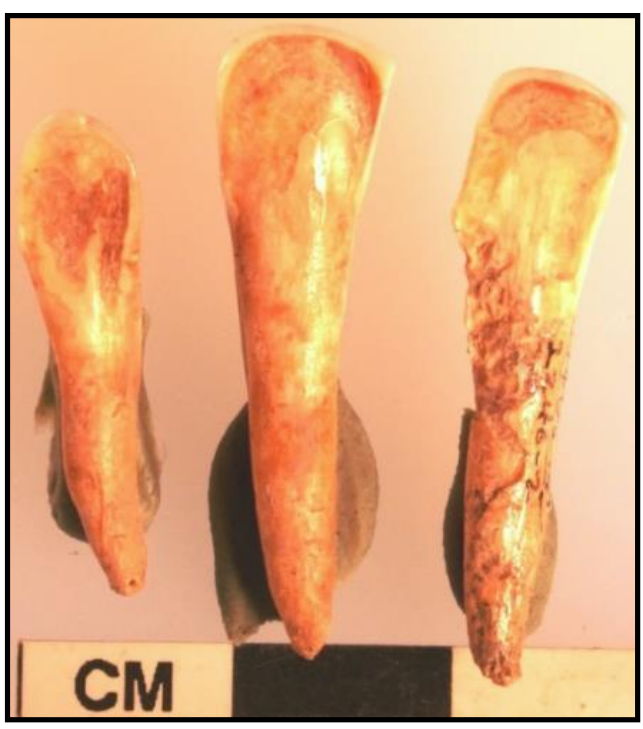

Figure 10-46. Examples of loose, individual deer incisors (\#2246).

A left pronghorn mandible section from between 270 and $275 \mathrm{cmbs}$ in N78/E94 in Block D was less than 1 $\mathrm{m}$ southeast of hearth Feature 25. This fragmented, root etched section consists of one loose $\mathrm{P}_{2}$ and socketed $\mathrm{P}_{3}$ and $\mathrm{P}_{4}$ (Figure 10-47). This represents an adult and is unclear as to a specific age or establishing seasonality.

A second pronghorn mandible section was from 265 cmbs in N79/E93 in Block D about $50 \mathrm{~cm}$ north of hearth Feature 25. This consists of a right, fragmented section with socketed $\mathrm{P}_{2}, \mathrm{P}_{3}$ and $\mathrm{P}_{4}$ in weathered bone, held together with calcium carbonate (Figure 10-48). The small size and moderate wear on the cups indicate an adult that could not be identified to a specific age. This right and the above left mandible sections appear very similar in age and likely represent the same individual.

A number of individual pronghorn teeth with bits of bone still attached were also recovered (Figure 10-49). The presence of three, loose right molars, one $\mathrm{M}^{3}$ from the maxilla and two $\mathrm{M}_{3}$ from mandibles all exhibit similar wear and may represent similar aged adult individuals. Based on dentition, the minimum number of individuals (MNI) is three deer, a fawn and two adults, and two adult pronghorns.

The canid elements (dog/coyote) include three complete, unburned first, second, and third phalanges. These were in two adjacent units, N82/E90 and $\mathrm{N} 82 / \mathrm{E} 91$, and likely represent the same toe. As these could represent three elements from one toe and no other canid bones were identified, this individual species may not represent a food resource.

Although 53 turtle shell fragments (2.7 percent of the total assemblage), mostly carapace with a few plastron pieces, were identified, the actual MNI is unclear. Minimally one and more likely two are represented. A soft shell turtle was identified. Three turtle pieces were burned, again testifying to their cultural use.

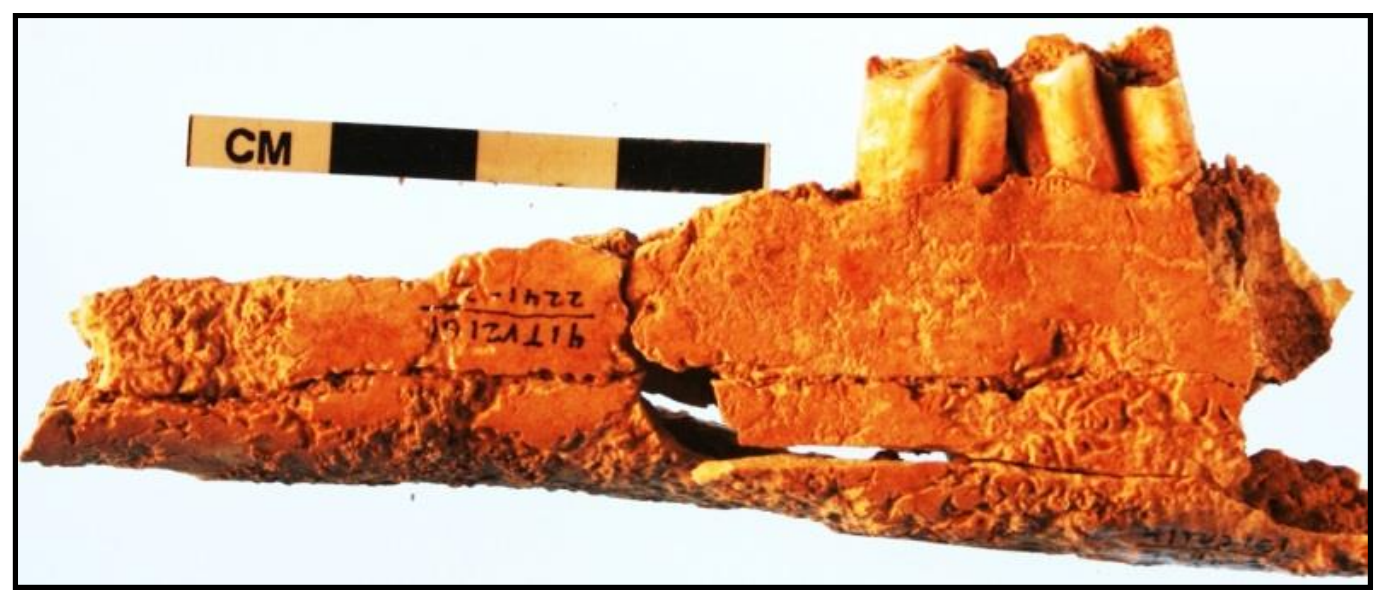

Figure 10-47. Left pronghorn left mandible section (\#2241) with socketed $P_{3}$ and $P_{4}$ that exhibits severe root etching. 


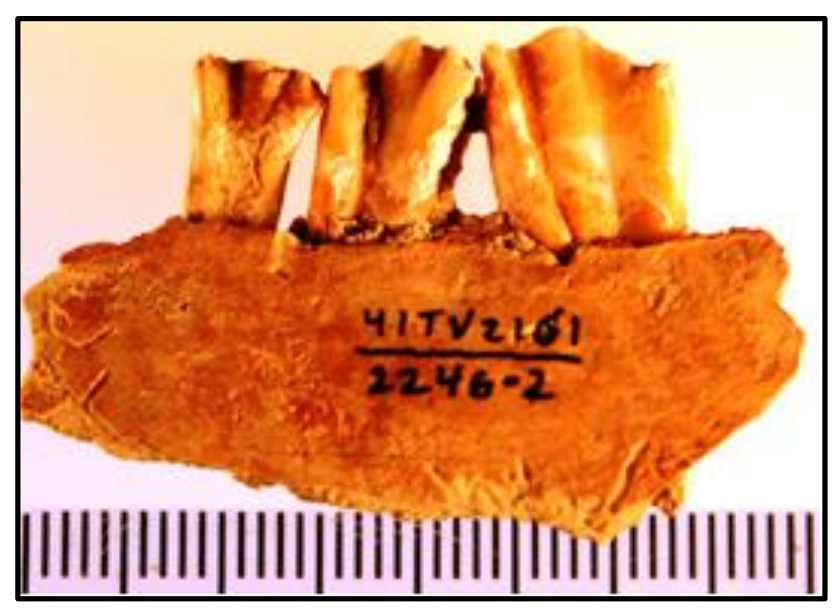

Figure 10-48. Right pronghorn mandible section with $P_{2}, P_{3}$ and $P_{4}$ (\#2246, scale in millimeters).

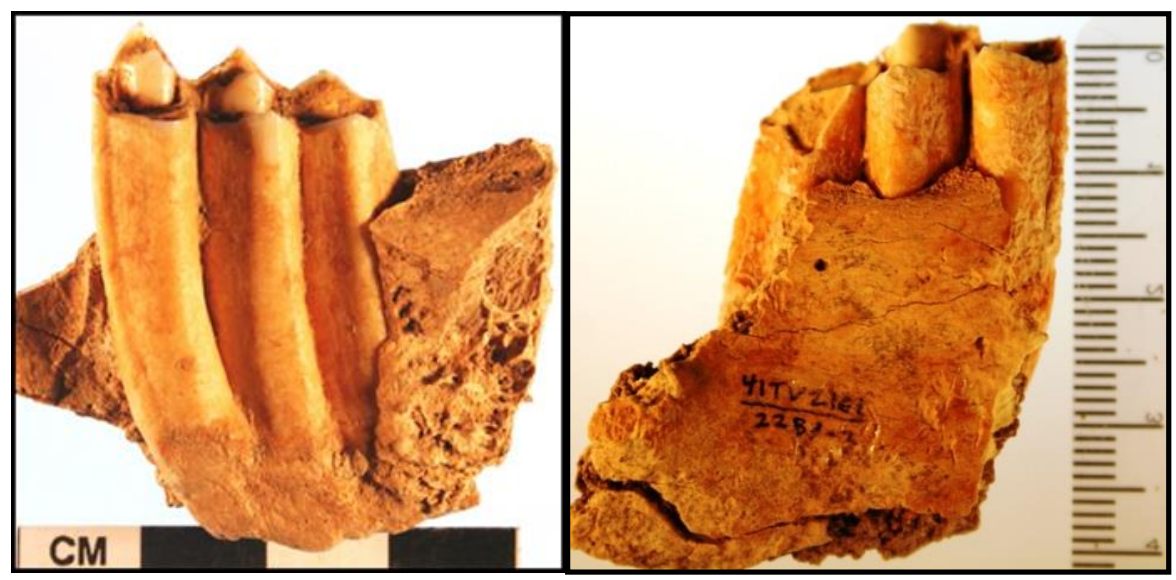

Figure 10-49. Socketed left pronghorn mandible $M_{3}$ (\#2246) and socketed right pronghorn $M_{3}$ (\#2281, right; scale in centimeters).

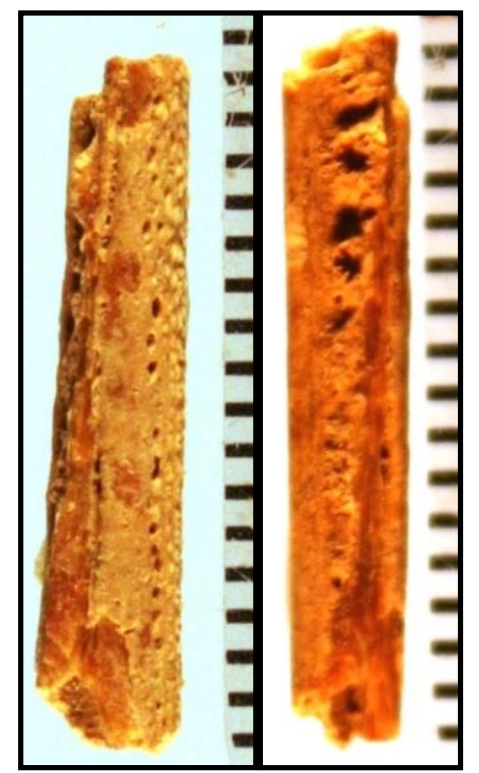

Figure 10-50. Two views of an unburned gar mandible (\#2233-2) fragment (scale in millimeters). 


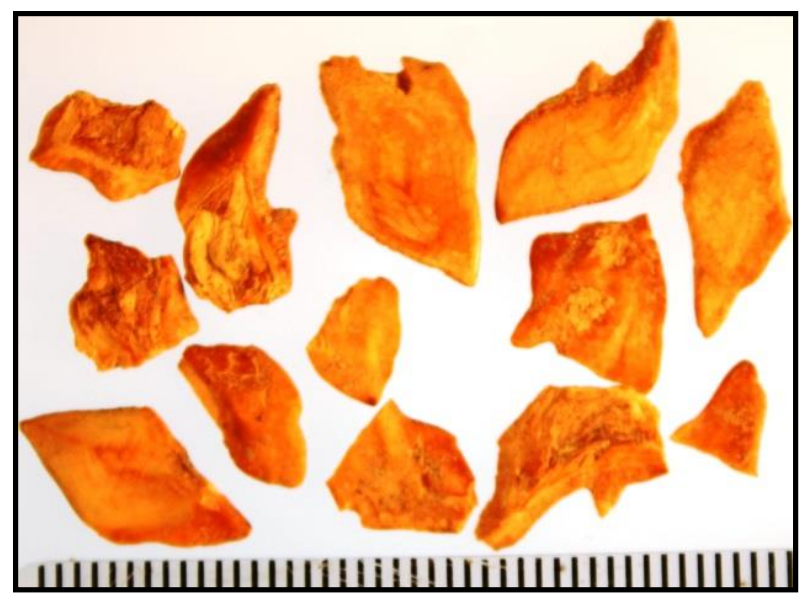

Figure 10-51. Examples of unburned gar scales (\#2238) (scale in millimeters).

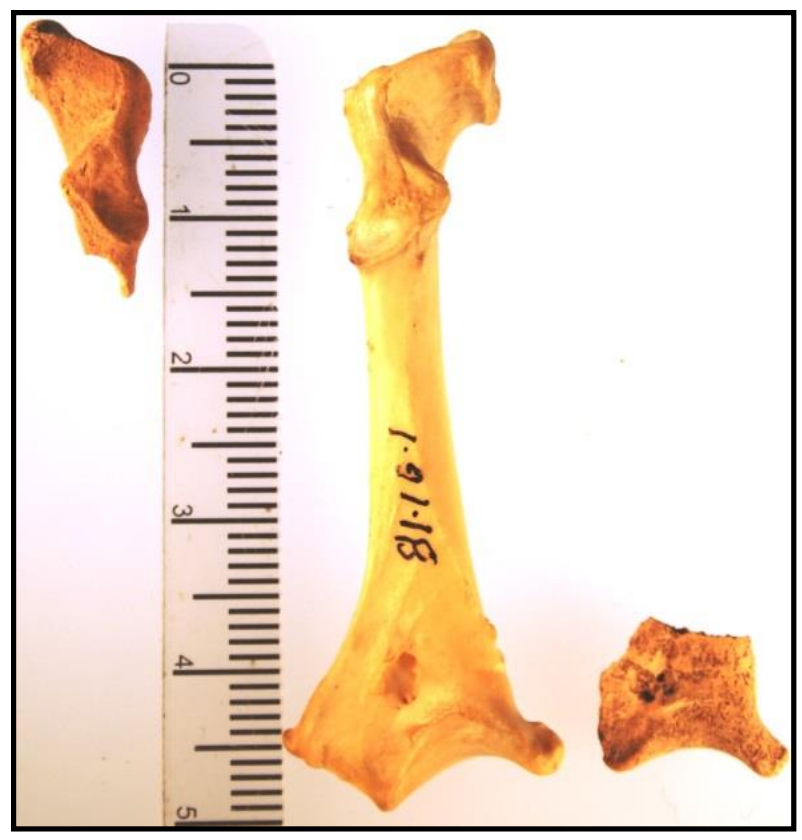

Figure 10-52. Prairie chicken (Tympanuchus sp.) proximal humeral facet (upper left, \#2305) and distal sternal facet (lower right, \#2260) of coracoid element with complete modern grouse comparative specimen (middle) (scale in centimeters).

The 110 fish bones (5.7 percent of the total bones or 10.5 percent of the bones identifiable to class) represent a MNI of one for each of three fish species. The identified fish bones include 1 catfish (Siluriformes) spine, 7 catfish skull fragments, 2 gar mandible fragments, 78 gar scales, multiple gar vertebrae, plus multiple unidentifiable vertebrae, some in the size range and shape of crappie (Figures 10-49 and 10-50). In addition to the fish bones recovered from the standard $6.4 \mathrm{~mm}$ screens, small fish bones less than $6.4 \mathrm{~mm}$ in length were also recovered from multiple proveniences, which had sediments floated. Catfish (mandible and burned spine), and gar (vertebrae, and burned and unburned scales) were in Feature 26. An additional gar vertebra and minimally 24 gar scales were in a unit adjacent Feature 26. Burned fish skull fragments, unburned vertebrae fragments, and burned gar scales were also in Feature 33. Unburned fish vertebrae were also recovered from a lithic concentration (\#2257-4) about 2 m northeast of Feature 25 in N80/E95 and gar scales were from a lithic concentration (\#2248-4) just $50 \mathrm{~cm}$ northeast of Feature 25. Catfish, gar, and probably one other species based on tiny unidentifiable vertebrae were definitely utilized as food resources. Multiple burned fish elements support their use as a food resource. Presence of small fish vertebra indicate these were collected through a technique that employed nets or weirs, as small fish would have been impossible to catch with hooks, shot, or by hand.

Seventeen bird bones represent less than one percent of the total bones. Six coracoid fragments are present with four right proximal fragments (Figure 10-52), one left proximal section, a left distal fragment, plus one medial section, all closely resemble prairie chicken (Tympanuchus sp.) (Figure 10-53). In addition to the coracoid elements, fragments of a sacrum, scapula, and a distal tibia represent a bird in the size range of a prairie chicken or grouse (Figures 10-53, 10-54, and 10-55). The MNI is four based on four right proximal coracoid fragments. No bird elements were burned or exhibit cut marks.

Tiny rodent bones $(N=142$ or 6.8 percent of the total or 13.6 percent of the identified taxon) potentially represent rats, voles, gophers, and similar small rodents likely were not culturally 
used, as these occur naturally in similar deposits. Only two indeterminate rodent fragments were burned and possibly represent something other than rodents. If rodent bones and snake vertebrae are not considered cultural resources, then minimally 24 different animals represent at least seven taxa constitute the meat resources utilized. Bison and bison size pieces are notably absent and preservation is not the issue as thick-walled bison elements would have preserved longer than the much thinner fish, bird, and rabbit bones.

Small fragmented bones were concentrated less than $1 \mathrm{~m}$ southeast of Feature 25 between 254 and $270 \mathrm{cmbs}$ in N78/E94. Four liters of bulk sediment was collected from that concentration and mechanically floated. The heavy fraction yielded 264 tiny bone fragments that weigh $2.6 \mathrm{~g}$ and nearly all are unburned with many small rabbit family and/or rodent fragments represented. One chert flake was also recovered. Beyond the float sample, regular dry screening of the rest of this same level and unit yielded 73 fragmented bones and seven chert flakes.

The overall bone distribution pattern across Blocks $\mathrm{B}$ and $\mathrm{D}$ reveals five units with localized high concentrations ( $N=125$ to 168 pieces), three units on the north-northeast edge of Feature 25, one just on the southern margin of Feature 33, and one $2 \mathrm{~m}$ north of Feature 33 (Figure 10-57). These high density areas account for 39 percent of the total bones and were generally surrounded by moderate high densities ( $N=95$ to 97 pieces) south of Feature 25 , to medium frequencies ( $N=29$ to 64 pieces) that account for 33 percent of the total, which were mostly around and between Features 25 and 33 with an area of medium density also north of Feature 33 (see Figure 10-57). Four units in Block B also reflect medium density units and those were on the north and eastern side of Feature 26 (see Figure 1057). This reflects a prominent association between hearth features and bone fragments. It is not clear if this pattern reflects a disposal process from cooking and/or heating bones in the features, or processing (smashing) bones around the hearths.

The three highest density units on the northeastern corner of Feature 25 were dominated (nearly 66 percent) by indeterminate pieces, although six different taxa are present in these three units. The high density on the southeastern corner of Feature 33 contained the fifth highest count $(N=125)$ of any unit and was dominated by 52 indeterminate pieces, 30 artiodactyl (deer/pronghorn) fragments, 22 rabbit family fragments, plus canid, bird, fish, turtle and rodent fragments. The diverse species represented in this one unit (N82/E91) supports this was likely a discard or processing (smashing) location as opposed to a place where animals were butchered. Unlike Block D, bone distribution on the north and northeastern side of Feature 26 in Block B reveal only medium ( $N=30$ to 51 pieces) to low ( $N=1$ to 21 pieces) frequencies (Figure 1057). The medium density units contained diverse species with jackrabbits, cottontails, and deer represented.

Deer, pronghorn, and deer size artiodactyl fragments were widely scattered across 26 units (35 percent of the total) and were in high and moderate dense areas (Figure 10-58). This reflects fragments of larger animals were discarded in the same areas as other taxa rather than separated from the smaller animals.

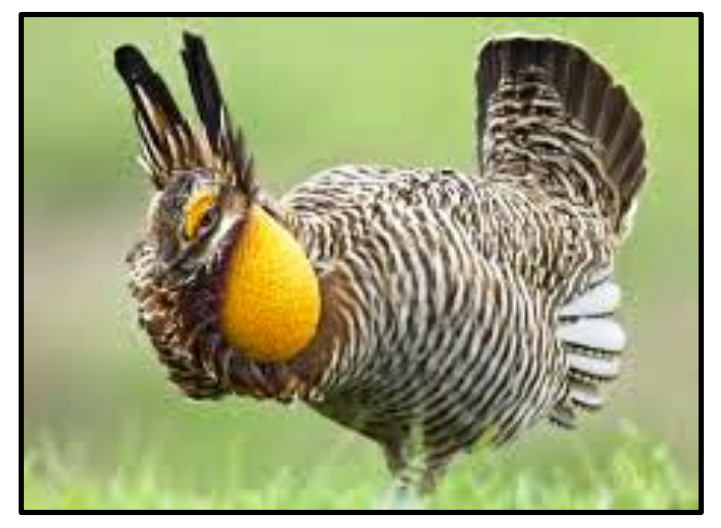

Figure 10-53. Male Attwater Prairie Chicken (Tympanuchus sp.). 


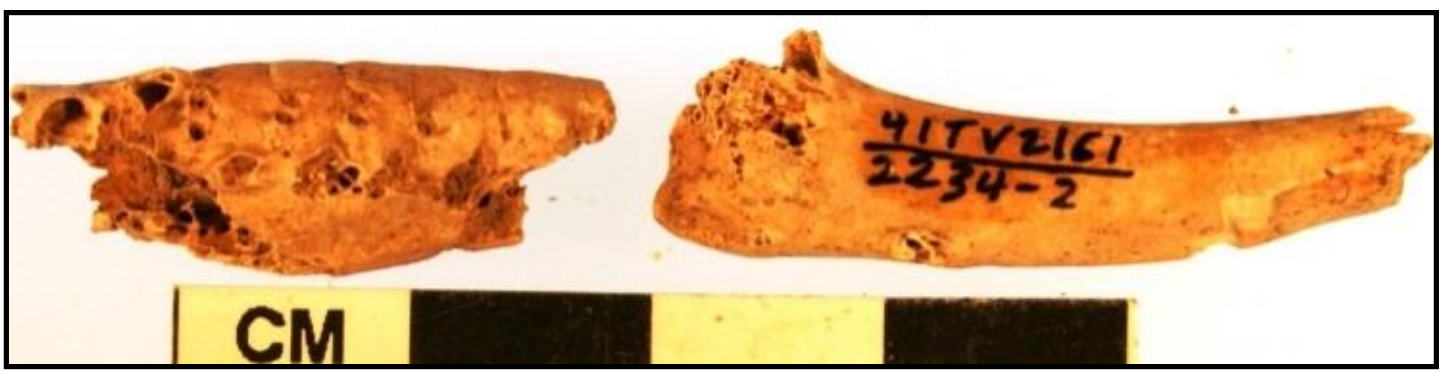

Figure 10-54. Bird elements (\#2234, medial sacrum fragment left and scapula fragment right).

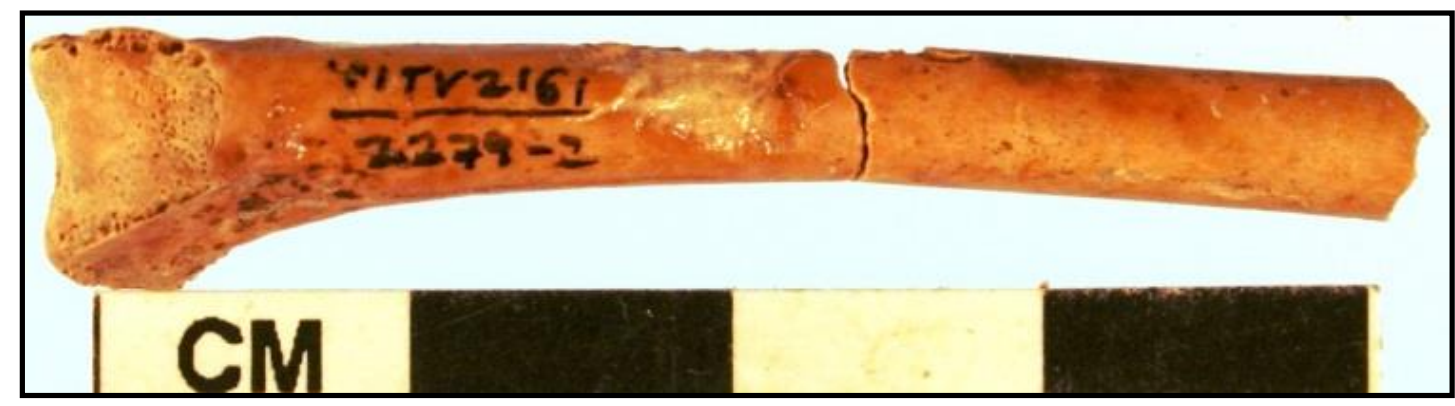

Figure 10-55. Bird distal tibia (\#2279).

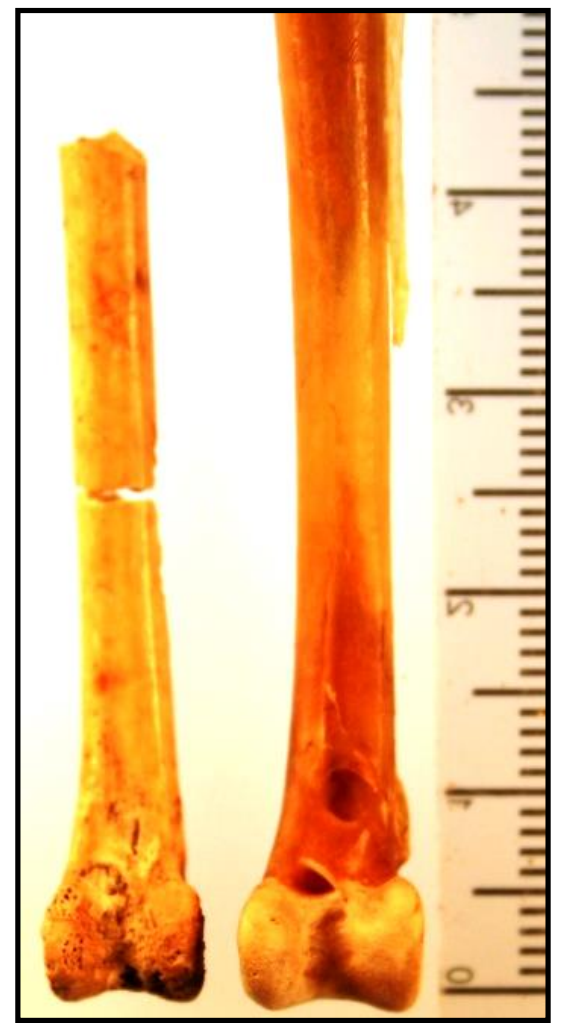

Figure 10-56. Large bird distal tibia (left, \#2279) compared to modern grouse distal tibia (right, scale in centimeters). 
Jackrabbits, cottontails and general rabbit family fragments $(N=535)$ were widely scattered across Block D (39 units) and parts of Block B (7 units). Only three units (4 percent), two in Block D and one in Block B, contained high density of elements ( $N=45$ to 51$)$ with only one unit (N81/E94) directly next to a feature. These three dense units likely reflect dumping locations where rabbit family fragments were discarded. Seventy units (23 percent of the total) had medium densities $(N=10$ to 28) and were widely scattered, mostly across Block D (Figure 10-59). Some scattered units also contained other discarded species, whereas most scattered rabbit family bones may be best explained as scattered from human traffic. Rabbit family fragments definitely occurred with other species in the highest density units, which reflect the overall distributions. Rabbit bones do not appear differently processed, although some fragments were scattered.

Fish bones were distributed across 22 of the 74 units (30 percent of the total) in Blocks B and D with 11 units within roughly $2 \mathrm{~m}$ of hearth Feature 25, two units between Features 25 and 33, three scattered units north of Feature 33, with one to the eastern side (Figure 10-60). In Block B, four units on the north and northeastern side of Feature 26 contained over 50 tiny fish bones with a catfish spine and many gar scales identified (Figure 10-60). Burned and unburned fish bones, specifically gar scales and mandible fragments, and unidentified skull fragments were also in Features 26 and 33.

The 17 bird elements were in only six of the 74 units (8.3 percent of the total) in Blocks B and D, three of which were within $2 \mathrm{~m}$ south of Feature 33, one unit within $1.5 \mathrm{~m}$ northwest of Feature 25, and one unit about $2 \mathrm{~m}$ north of hearth Feature 26 in Block B (Figure 10-61). The bird bone distribution south of Feature 33 corresponds with a high bone concentration area, whereas the broad nature of the horizontal scatter likely reflects multiple birds present and a single processing or discard area for birds was not employed.
The 53 turtles shell fragments were in 15 of the 74 units (nearly 20 percent) and all but one unit was in Block D. Two units in Block D contained 43 percent of the total with one unit $2 \mathrm{~m}$ north of Feature 25 and the other unit 2.5 to $3 \mathrm{~m}$ north of Feature 33 (Figure 10-62). The latter concentration yielded 13 fragments in one unit with nine fragments in three units to the east (Figure 1062). Another four units between Features 25 and 33 yielded 11 fragments. Other fragments were scattered about revealing no particular association with the three cooking features, processing, or discard location.

\subsubsection{Summary of Vertebrate Faunal Remains}

In summary, this vertebrate faunal assemblage is limited in quantity, not counting the faunal material from the floated feature sediments, with 2,082 fragments from $74 \mathrm{~m}^{2}$. The faunal assemblage while extremely fragmentary as the average weight is only $0.4 \mathrm{~g}$ still consists of very substantial data concerning resource exploitation, processing and cultural behaviors. Even though nearly 50 percent of the fragments are indeterminate to taxa, the MNI includes minimally 24 individuals of eight different taxa, if snakes and small rodents are not culturally derived. These eight identified taxa represent a very diverse meat resource with the unusual absence of highly ranked bison. The absence of bison probably reflects its scarcity in the immediate region or specifically during the fall season of this camp. Large game animals include deer and pronghorn, whereas jackrabbits and cottontails dominate the small game, supplemented by multiple fish and game birds. Apparently this human group sought and procured diverse species from minimally three different ecological settings. The presence of pronghorn, jackrabbits, and prairie chicken or grouse reflect an environment dominated by open grassland and/or desert scrub habitat (Martin 1974). Deer would occur in wooded areas along the creek or in the adjacent 


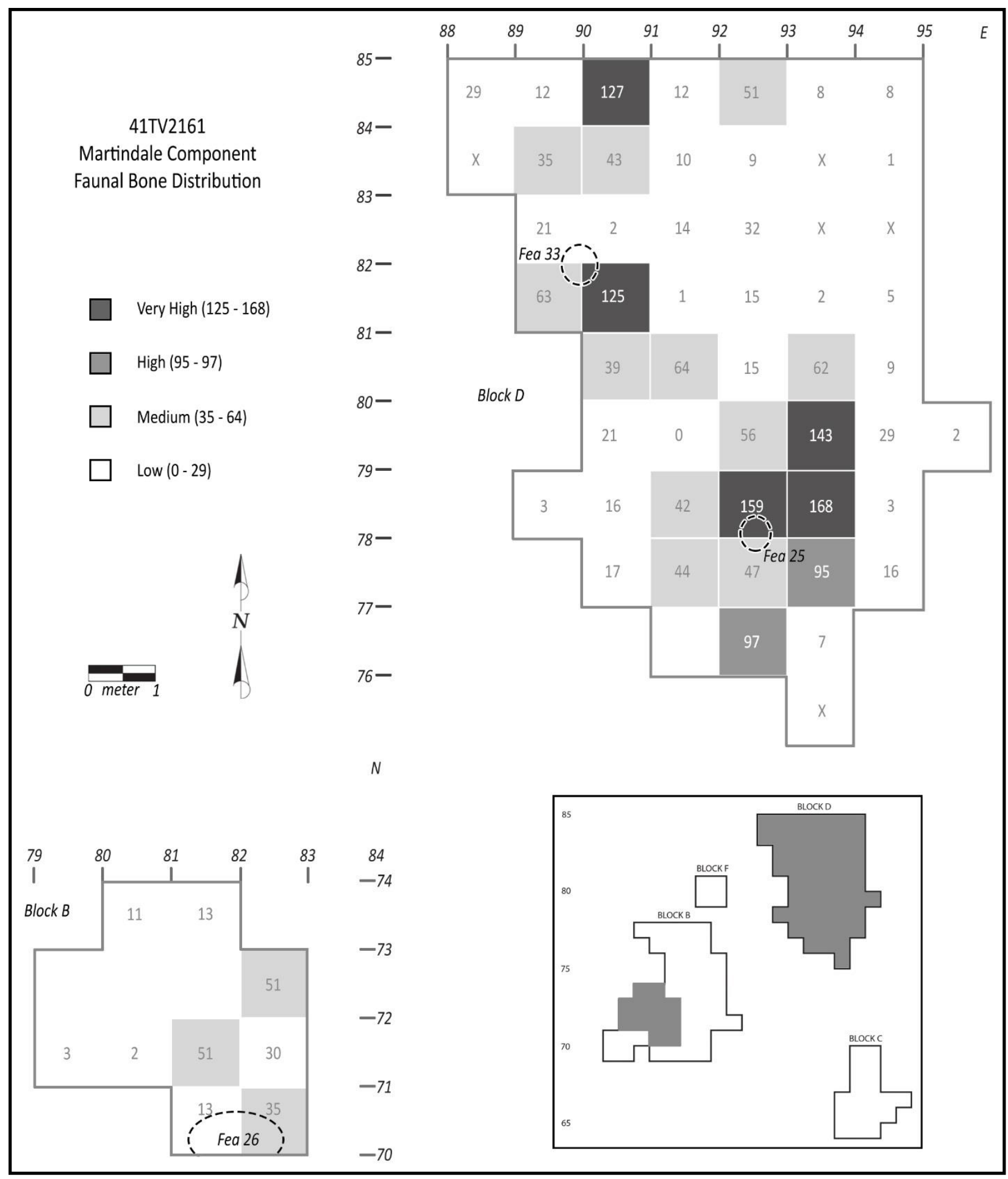

Figure 10-57. Martindale bone distribution across Blocks B and D. 


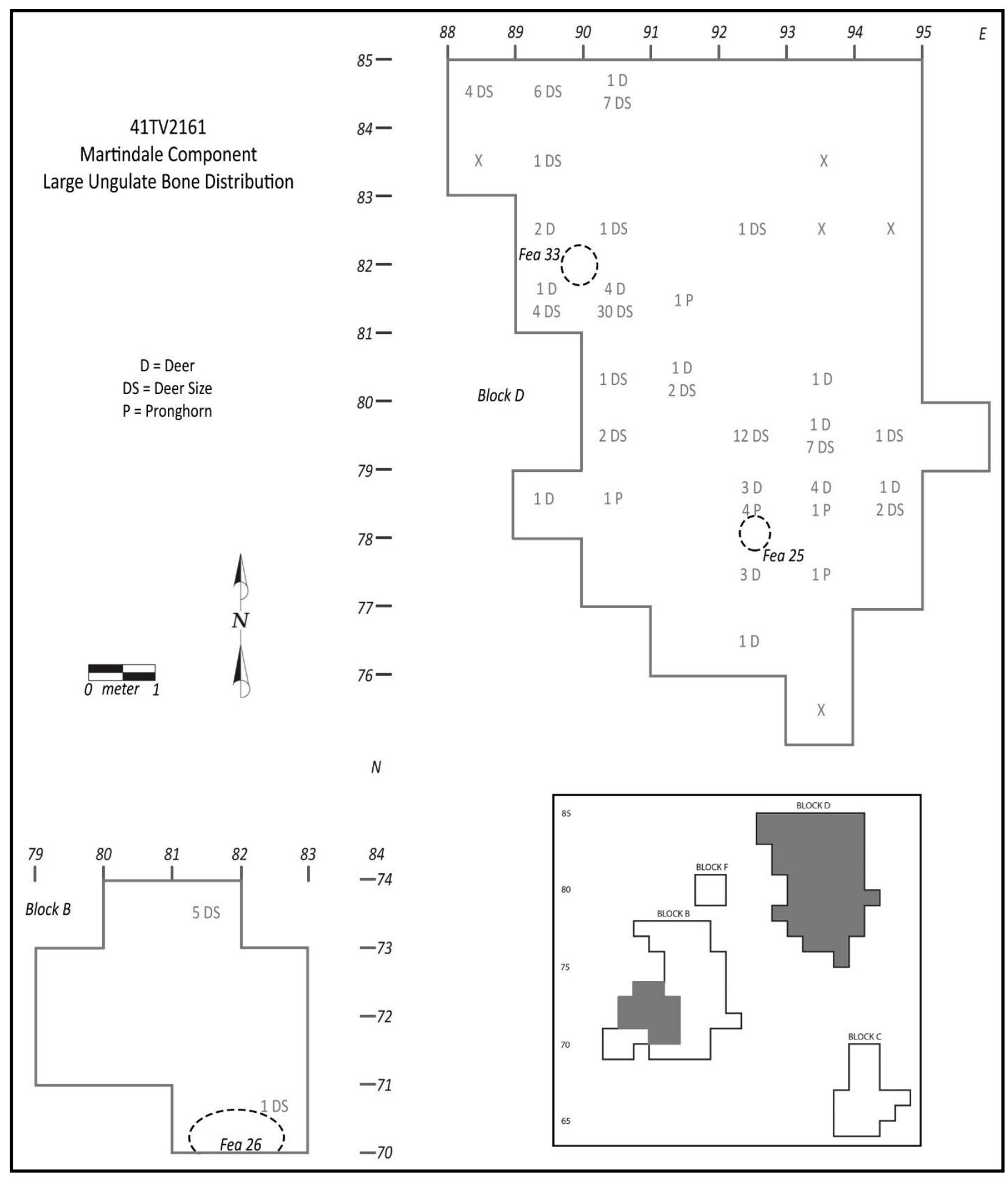

Figure 10-58. Martindale deer, pronghorn and deer size bone distribution across Blocks B and D. 


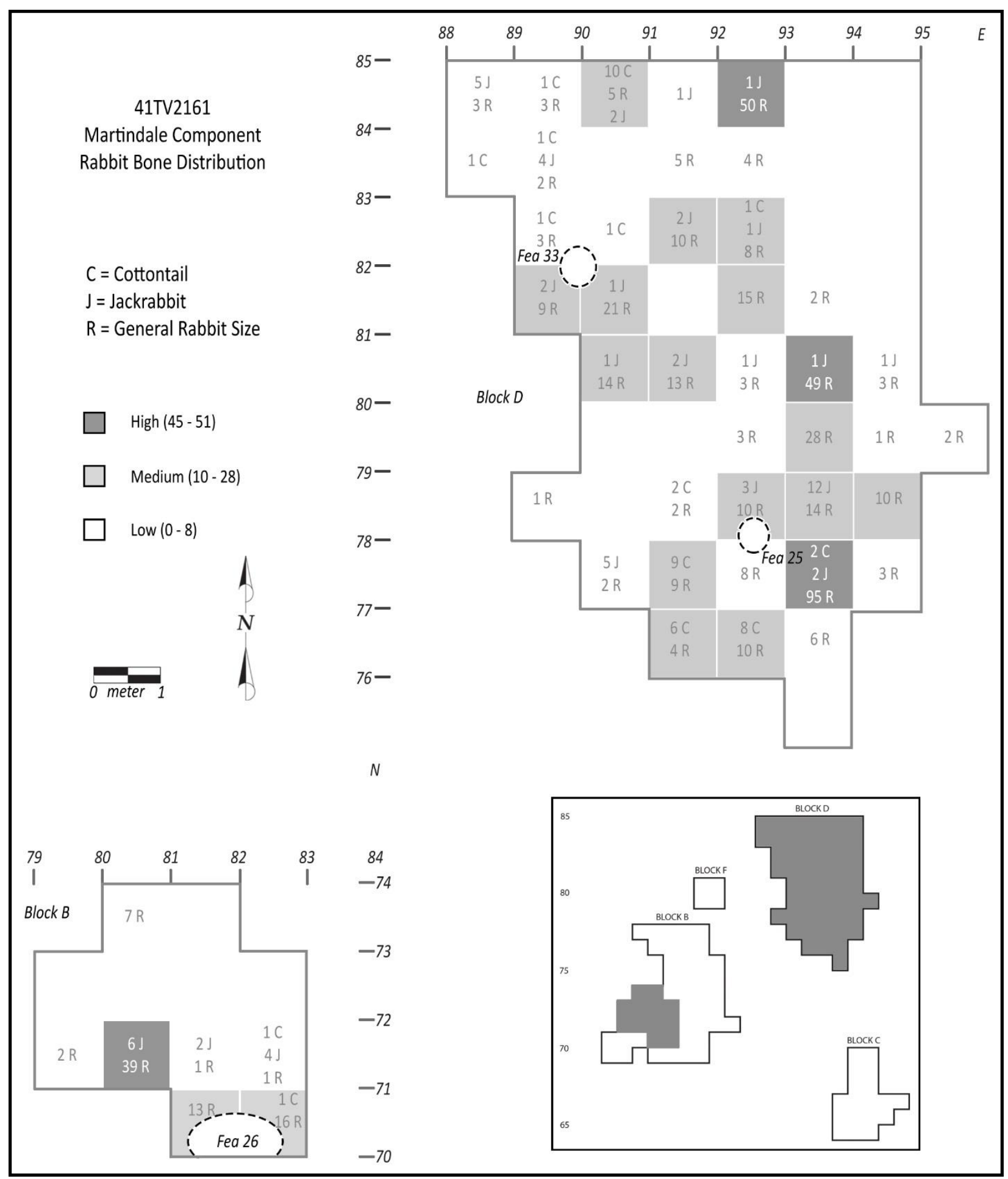

Figure 10-59. Martindale rabbit bone distribution across Blocks B and D. 


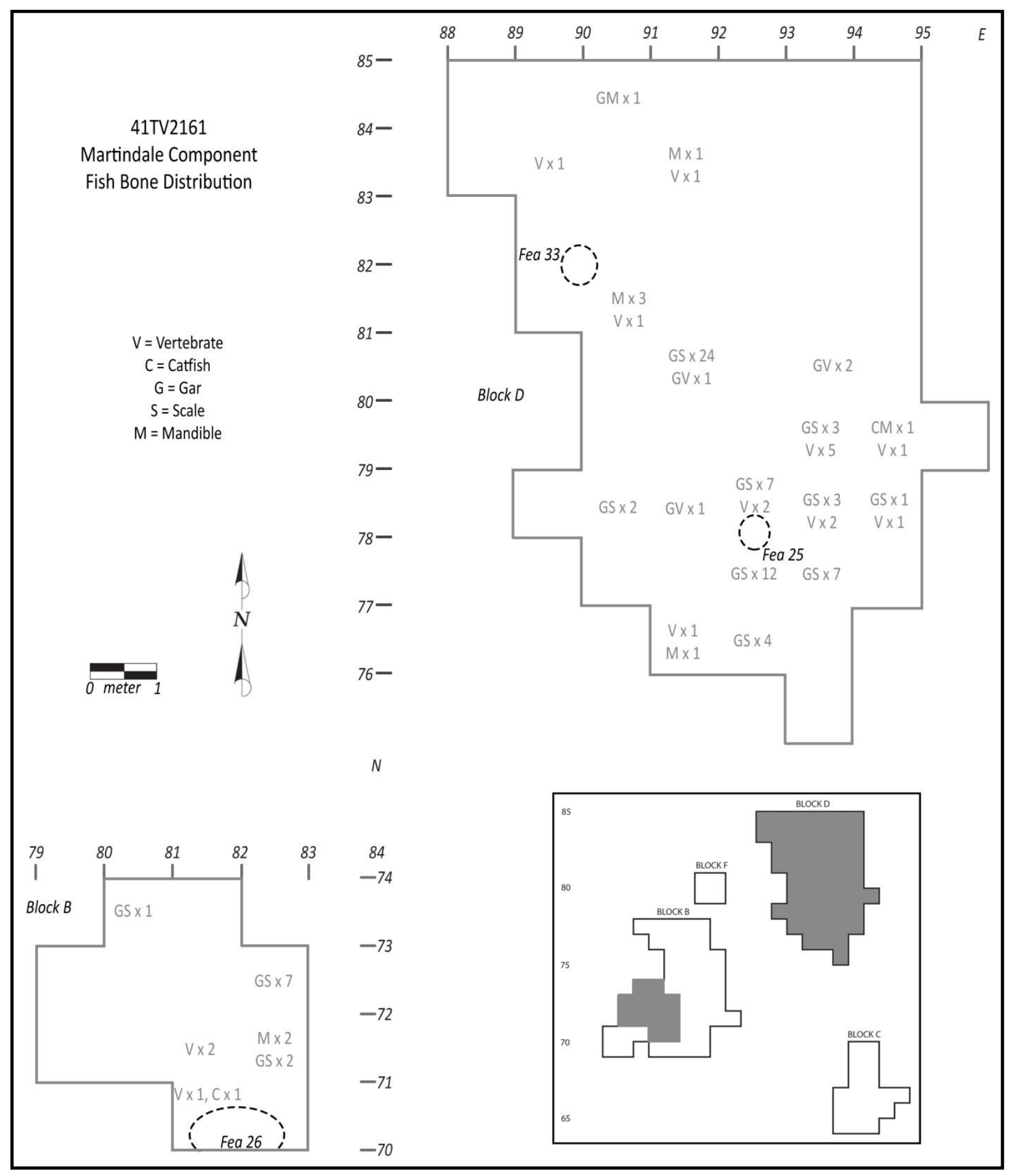

Figure 10-60. Martindale fish bone distribution across Blocks B and D. 


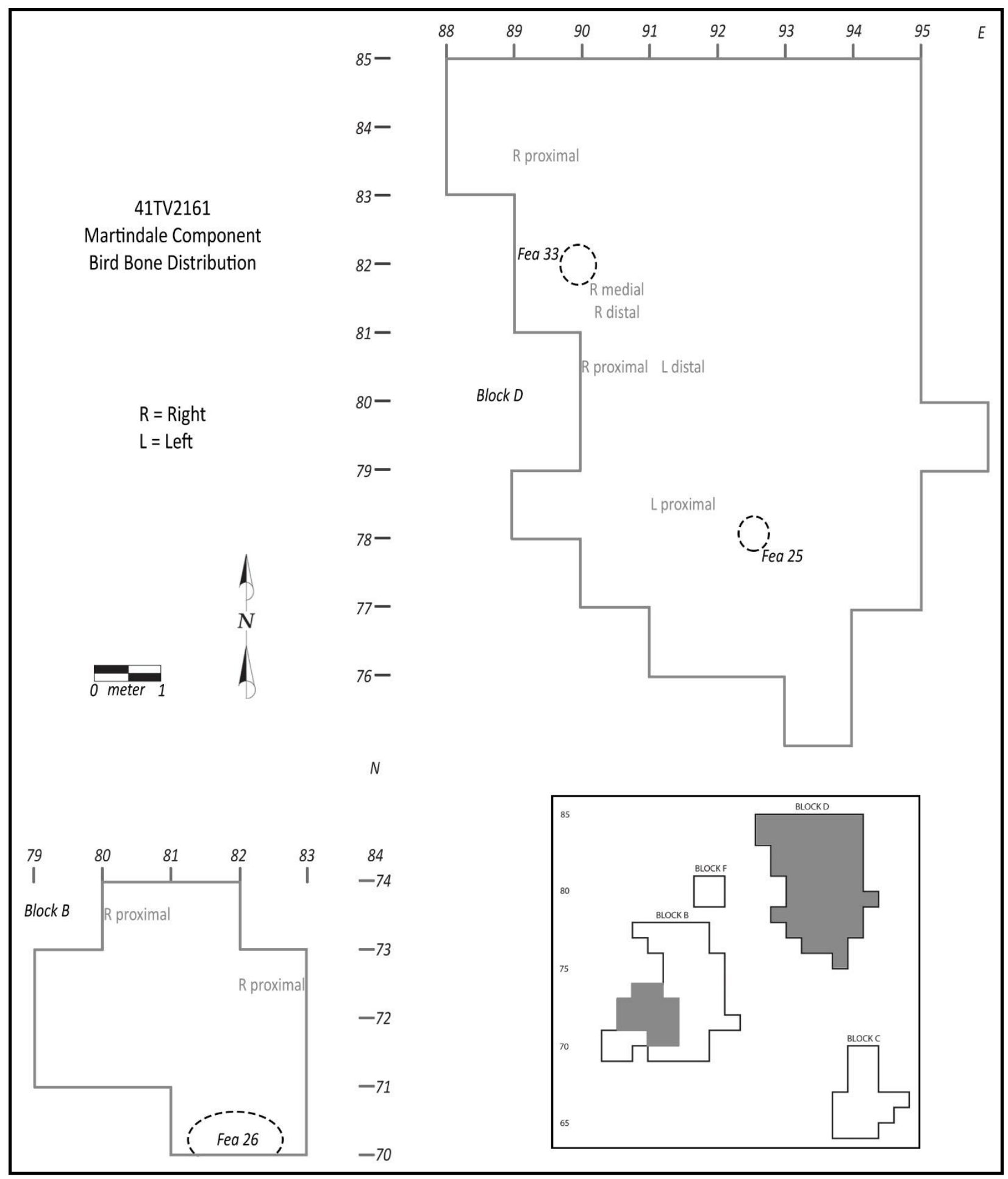

Figure 10-61. Martindale bird bone distribution across Blocks B and D. 


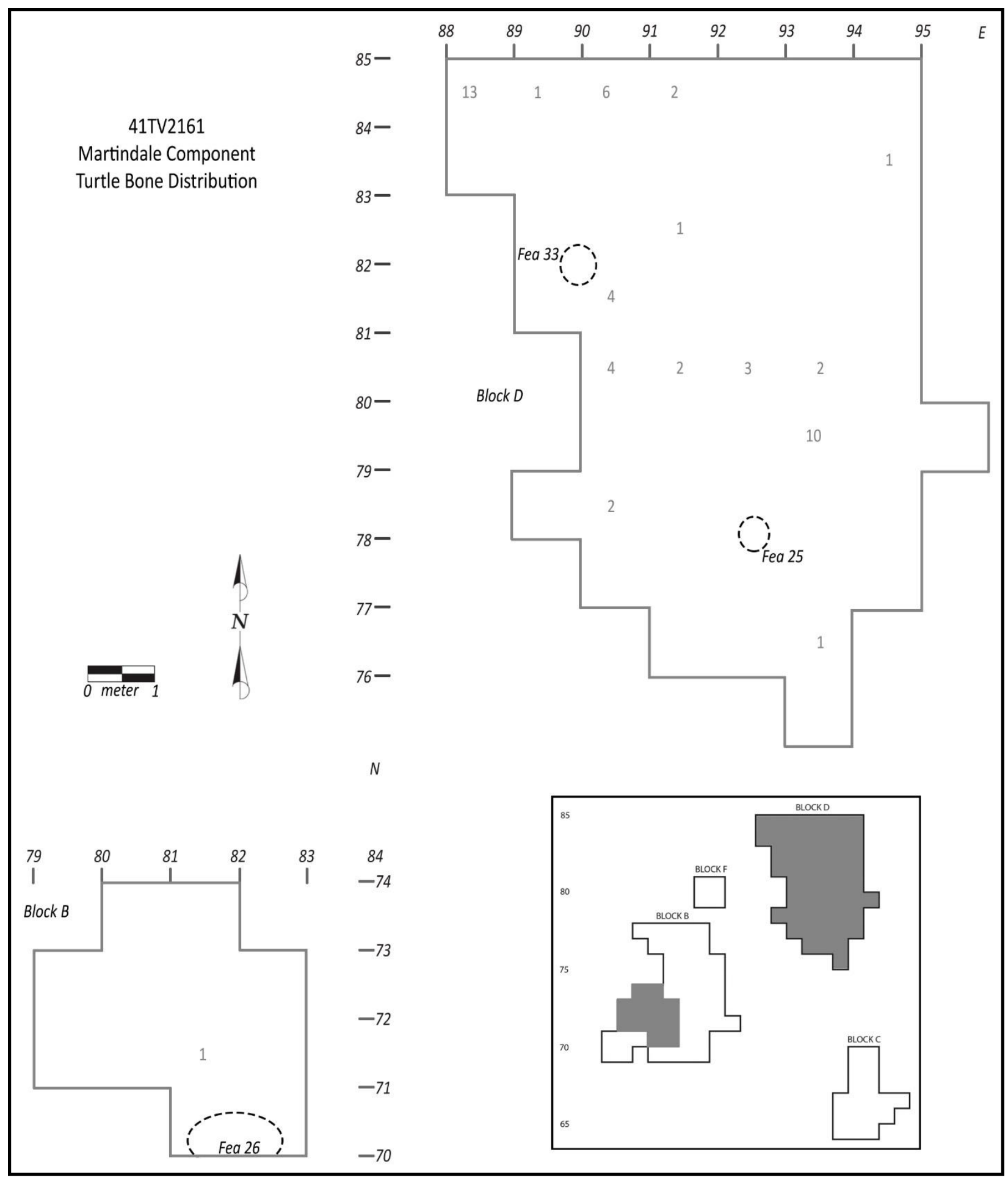

Figure 10-62. Martindale turtle bone distribution across Blocks B and D. 
Edwards Plateau. The multiple fish species document nearby Onion Creek had permanent water suitable for maintaining fish populations.

The extreme fragmentary nature of the vertebrate assemblage documents the human population not only sought meat resources, but also targeted bone marrow and bone grease. Few spiral fractures or impact marks were observed on the larger artiodactyl bones and fewer on the smaller bones. The apparent absence of cut marks may reflect the absence of complete defleshing of the bones and/or in the case of small rabbits and other rodents their dismemberment was conducted without sharp cutting tools or were cooked intact. However, root etching and calcium carbonate covered fragments makes it difficult to observe cut marks. The burned fragments from diverse species indicate many bones were often discarded directly into fires.

The molar $\left(\mathrm{M}_{1}\right)$ of a deer fawn in an erupting stage is perhaps the best indication of seasonality and supports a fall, September-October season of death (Harlow and DeFoor 1962) and the season of this camp. The deer and antelope teeth and mandible sections reveal adult individuals are not as sensitive to a specific season of death, however, they generally support a fall occupation.

\subsection{MUSSEL SHELL ASSEMBLAGE}

Mussel shells are minimally represented with only 54 fragments $(549.4 \mathrm{~g})$ total and few of sufficient size to attempt identification. Three species, Yellow Sandshell (Lampsilis teres), Tampico Pearlymussel (Cyrtonaias tampicoensis), and Texas Pimpleback (Quadrula petrina) were identified with eight, six, and five respectively. None appear culturally altered or heat damaged. If part of the food resource, they served a minimal role in the overall diet. These species are known to occur in the region with the Texas Pimpleback restricted to the Colorado and Guadalupe River systems. The Tampico Pearlymussel, however, is not associated with the Colorado River system (Howells et al. 1996).

\subsection{BURNED ROCK ANALYSIS}

Limited quantities of burned rocks were scattered across the excavation (Table 10-22). Surprisingly, no specific burned rock features similar to those in the Bell/Andice component were identified. Seventy-one burned rocks or 22 percent were from the three shallow basin hearth features. Those rocks beyond feature boundaries (78 percent) were dominated by small pieces ( 0 to $4 \mathrm{~cm}$ in size) and

Table 10-22. Martindale Burned Rock Frequency, Size, and Weight Categories.

\begin{tabular}{|c|c|c|c|c|c|c|c|c|c|c|}
\hline Location & 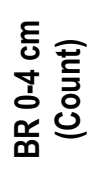 & 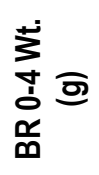 & 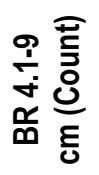 & 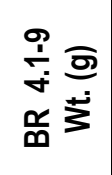 & 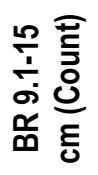 & $\begin{array}{l}\frac{n}{5} \\
\frac{5}{0} \\
\frac{\alpha}{0}\end{array}$ & 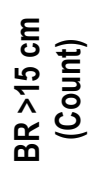 & $\underset{\substack{\hat{\alpha} \\
\frac{1}{0}}}{\stackrel{5}{0}}$ & 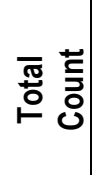 & 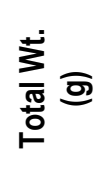 \\
\hline Feature Burned Rocks & 41 & 416 & 28 & 4661 & 2 & 612 & & & 71 & 5689 \\
\hline $\begin{array}{l}\text { Scattered Burned } \\
\text { Rocks }\end{array}$ & 178 & 2672 & 65 & 10439 & 7 & 3146 & & & 250 & 16257 \\
\hline Total Burned Rock & 219 & 3088 & 93 & 15100 & 9 & 3758 & & & 321 & 21946 \\
\hline
\end{tabular}




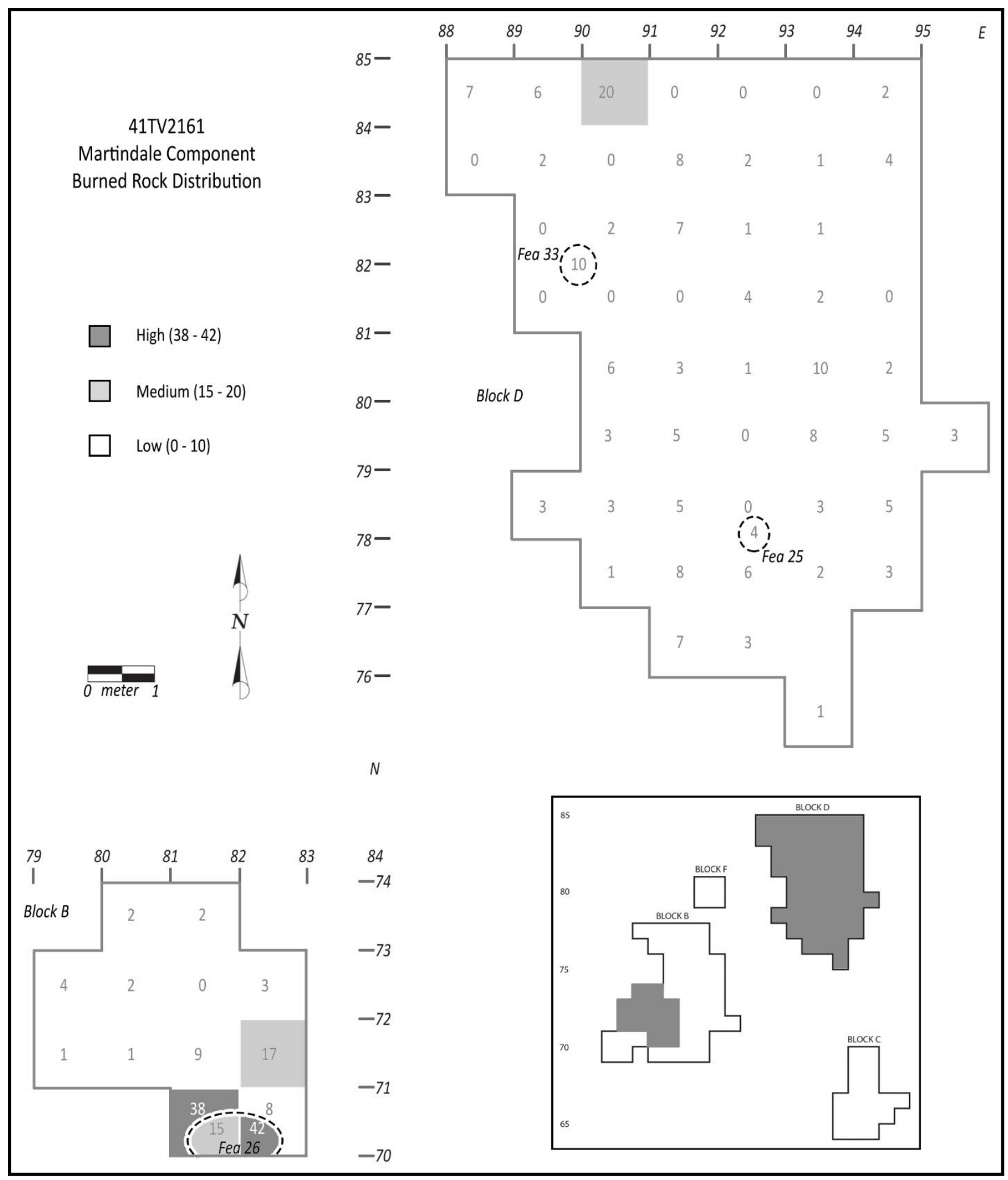

Figure 10-63. Martindale burned rock distribution across Blocks B and D. 
represent nearly 55 percent of the total. Only 32 percent were greater than $4.1 \mathrm{~cm}$, with no rocks greater than $15 \mathrm{~cm}$. The absence of largest size rocks may reflect their availability or their reduction through heating and cooling processes. The smallest pieces probably reflect rocks, which had exhausted their heating capacity and no longer suitable for that task, thus discarded.

Relatively few burned rocks $(N=321)$ were scattered across both blocks with two units (3 percent) that yielded high concentrations ( $N=38$ to 42 pieces) in and on the margin of Feature 26. Four units (5 percent) had medium quantities $(N=5$ to 20 pieces), with two of those units in and around Feature 26 and the higher concentrations, whereas the other two units were widely dispersed. One was more than $2 \mathrm{~m}$ north of Feature 25 and the other 2 $m$ north of Feature 33 at the northern edge of Block D (Figure 10-63). The latter was in the same unit as a high frequency of crushed bone and supports this was a discard location for multiple classes of debris.

\subsection{BURNED CLAY AND DAUB}

Irregular burned lumps of clay were in and around hearth Feature 26 in the southern edge of Block B. Thirty-six tiny pieces that weigh $31.9 \mathrm{~g}$ were in Feature 26 with 31 specimens less than $1 \mathrm{~cm}$ and five specimens between 1 and $4 \mathrm{~cm}$ in size. None exhibited any type of grass, rounded stick, or flat impressions to indicate they were attached to anything. These small irregular shaped pieces are assumed to have formed from intense heat generated by the hearth.

\subsection{MARTINDALE COMPONENT SUMMARY AND DISCUSSION}

The Martindale component was recognized in the lower, southwestern part of Block B $\left(12 \mathrm{~m}^{2}\right)$ and across all of Block D $\left(62 \mathrm{~m}^{2}\right)$ for a total of $74 \mathrm{~m}^{2}$ excavated. It was stratigraphically between 250 and $275 \mathrm{cmbs}$ in uneven, horizontally dispersed but intact deposits below a thin, mottled, greenish-gray clay lens. Component age was determined by 15 radiocarbon dates on multiple materials, although only two charcoal and one seed date provide a confident age of ca. 5393 B.P.

Three small, organically stained, basin hearth features were recognized along with three clusters of lithic debitage and one cluster of smashed bones. The formal stone tool assemblage is meager and consists of one corner-notched Martindale dart point, two point fragments, five biface fragments, two hammer stones, 83 edge-modified flakes, and three ground stone tools (Table 10-23). Minimally, 250 burned rocks were scattered across the occupation with 71 additional burned rocks (22 percent of total) in the three hearths. No burned rock features were identified. A moderate quantity of lithic debitage ( $N=1,429$ pieces) reflects cobble core reduction, early and middle biface production, and some tool maintenance.

The chipped stone tools were manufactured from local cherts procured from the adjacent gravelly hilltop and are of high quality chert, chemically similar to Edwards chert. A single, very dark-gray (10YR 3/1) chert flake (\#2300-1-2a) from between 250 and $260 \mathrm{cmbs}$ in N83/E94 subjected to INAA was a chemical anomaly compared to the remaining INAA samples. It yielded an exceptionally low probability of belonging to any source groups in MURRs analyses. This flake exhibited a yellow UV fluorescence similar to Edwards chert, although it is chemically unique from the other samples.

The component includes a sizeable vertebrate faunal assemblage $(N=2,082)$. The remains identified include fish, turtles, birds, and mammals - including a type of Canidae (e.g., dogs, coyotes, foxes, etc.). Faunal material identified to species include, pronghorn, jackrabbit, and cottontail (Table 10-23). Interestingly, the highly ranked bison were not among the individuals identified, which probably reflects its scarcity during this season or in the broader region. Rabdotus shells were common in restricted areas and scattered, mostly above or below, but infrequently associated 
Table10-23. Summary of Materials Remains from the Martindale Component.

\begin{tabular}{|c|c|}
\hline Cultural Material Classes & Counts \\
\hline Radiocarbon Dates & 16 \\
\hline Features & 3 \\
\hline Hearths, Organic Stained & 3 \\
\hline \multicolumn{2}{|l|}{ Dart Points and Fragments } \\
\hline Martindale & 1 \\
\hline Fragments & 2 \\
\hline Bifaces & 4 \\
\hline Manos & 2 \\
\hline Metates & 1 \\
\hline Hammer Stones & 2 \\
\hline Edge-Modified Flakes & 83 \\
\hline Lithic Debitage & 1,429 \\
\hline Cores & 7 \\
\hline $\begin{array}{l}\text { Vertebrate Faunal } \\
\text { Fragments }\end{array}$ & 2,082 \\
\hline Mussel Shells & 54 \\
\hline Burned Rocks & 321 \\
\hline Burned Clay & 36 \\
\hline Carbonized Plant Remains & $\begin{array}{c}8 \text { grass seeds, } 2 \\
\text { cheno-am } \\
\text { seeds, live oak } \\
\text { frags. }\end{array}$ \\
\hline Total Materials & 3,998 \\
\hline Average Thickness (cm) & $10-15$ \\
\hline Spatial Extent Excavated & $74 \mathrm{~m}^{2}$ \\
\hline Volume Excavated $\left(\mathrm{m}^{3}\right)$ & $8.6 \mathrm{~m}^{3}$ \\
\hline
\end{tabular}

This table does not include materials from float samples.

with the cultural component. Mussel shell fragments were present in limited frequencies. Plant procurement, processing, and use is evident by the presence of two manos and one metate fragment, charred grass and cheno-am seeds, starch grains from grasses and true lily bulbs (Lilium), and gourd phytoliths. This animal and plant diversity document a broad diet breath for this group who not only hunted very diverse meat resources (predominately conducted by males), but also gathered and processed a suite of plants, (predominately conducted by females) all probably necessary for subsistence. Procurement of diverse plant and animal food resources associated with cooking and/or heating in three small basin hearths (predominately conducted by females) combined with multiple knapping or lithic discard concentrations (predominately conducted by males), reflect a diverse male and female population at this generalized camp (see Chapter 13 for more detailed discussion).

Seasonality determination was attempted through exploring cementation of two deer incisors from the Martindale component. Unfortunately, the laboratory was unable to identify growth lines in the two samples (Appendix J). Other lines of evidence such as the tooth eruption, wear on deer and pronghorn teeth, and charred plant remains were pursued. The $\mathrm{M}_{1}$ of a deer mandible (\#2234-2-A) in an erupting stage indicate a three to six month old fawn and indicates occupation in the fall, roughly September through November (see Figure 10-45). Charred cheno-am and grass seeds, and even charred hackberry nutlets from Feature 26, are most abundant and mature in late summer and fall. The collection, processing, and cooking of true lily (Lilium) bulbs is indicated by six true lily starch grains on a flake stone tool (\#2103-10). Another flake tool (\#2021-10) and a burned rock (\#2112-33) from Feature 26 yielded lenticular grass starch (probably wildrye). Wildrye is mature and most abundant in fall season. Wild buffalo gourd phytoliths were also identified from Features 25 and 33 and are most readily available in the fall. Although the evidence is not overwhelming, the combined data strongly supports a fall encampment for this occupation.

The crushed and unburned nature of the vertebrate faunal assemblage combined with the meat resources present are most often cooked by boiling (e.g., Wandsnider 1997). Identification of gelatinized starch grains, plus meat and plant residues on burned rocks, all support the use of 
boiling as the primary cooking method. Although grasses potentially were incorporated into fire as a nonfood resource, the recovery of grass starch on the ground stones, an edge-modified flake, on multiple burned rocks, and identification of damaged grains clearly document grass as one plant resource intentionally processed and cooked.

\subsubsection{Chronology Issues}

Component age was determined through radiocarbon dating 15 samples of five diverse materials. Materials include five animal bones, two Rabdotus snail shell clusters, five organic rich sediments, two charcoal, and one charred seed sample. The results provide a broad range of over 1,000 years from 5260 B.P. on a bone to 6310 B.P. on organic sediment. Consequently, only the more reliably dated charcoal and seeds were considered to establish the age of this component. The latter three dates provide an average age of 5393 B.P. with a 110 radiocarbon year difference in the three samples. This later age is the best indication of when this Martindale component actually occurred. For more detailed discussion of the chronology see Chapter 11.0.

\subsubsection{Subsistence Issues}

The vertebrate faunal assemblage combined with macrobotanical and microfossil results provide evidence for a broad diet breath for this population. The vertebrate fauna includes deer, pronghorn, jackrabbits, cottontail rabbits, canid, turtles, fish, and birds. It is presumed the rats, mice, other small rodents, and snakes, were not part of the resources exploited by humans, but were natural inclusions in the deposits. Fish remains document minimally catfish (probably Bullhead [Ameriurus sp.]), gar, and some smaller fish(s). Birds are represented by 17 butchered elements, which represent minimally three prairie chickens (Tympanuchus sp.) or grouse. The low frequency of mussel shells, which lack any sign of cultural use, may or may not be part of the targeted food resources. It is possible these shells were noncultural and derived on site from other means (e.g., birds, raccoons, etc.).
Macrobotanical results include multiple charred grass and cheno-am seeds. The latter refers to the seeds of weedy annuals, specifically goosefoot (Chenopodium) and amaranth (Amaranthis). Microfossil results include multiple grass starches (probably wildrye or Elymus sp.), true lily bulb starches (potentially pine lily or Lilium catesbaei), and wild buffalo gourd phytoliths. Lily starches were on an edge-modified flake and are considered culturally procured and processed. Gourd phytoliths were in Features 25 and 33, but it is unclear if these were food resources or utensils. Short cell grass phytoliths were nearly absent from all contexts due to poor preservation. The high incidence of burned panicoid grass phytoliths in Features 26 and grass starch grains (probably wildrye) on burned rock from Feature 26 imply employment of either Panicoid grasses for fuel or harvesting and processing this grass for their seeds to use as food. The latter is supported with ground starch grain on one ground stone. Drying Panicoid grasses for food would have occurred in the fall, whereas dry Panicoid grass employed for fuel could occur anytime from fall to spring. Chenoams reflect disturbed and generally open areas. These types of plants yield thousands of seeds and are easily collected when ripe in late summer and fall. This combined data documents a broad diet breath of a mixture of low-ranked, high-cost plants and potentially similar animal resources. It is surprising this assemblage lacks the highly-ranked bison resource (see Chapter 3.0 for discussion). Apparently, bison were absent from this immediate area in the fall; therefore, the resident population resorted to hunting and gathering a broad range of other resources in the immediate area.

\subsubsection{Technology Issues}

The following discussions address multiple technological systems, resource procurement implements, and resource consumption strategies as evident from the recovered cultural assemblage and other relevant data. In terms of raw stone, the high quality chert employed to manufacture 
chipped stone tools and probably various limestones, sandstones, other rock, and fossil oyster shells used in the cooking process and grinding implements were procured from the gravel outcrop on the nearby hilltop, as the Onion Creek alluvial deposits in this section are naturally cobble-free. The hilltop source was verified as the chert source by INAA through analyzing a sample of 20 natural chert cobbles from the hilltop and a selection of 18 cultural chert pieces from this occupation. Nearly 98 percent of the cultural pieces tested had chemical signatures similar to those from the hilltop. The collected hilltop cherts were characterized by small, less than $15 \mathrm{~cm}$ diameter water-worn cobbles with smooth, dark cortices.

Natural cobbles were reduced by hard-hammers, various antler billets, and pressure flakers employed in the process of manufacturing chipped stone tools. Clues to the knapping technology employed include the identification of two small hammer stones used to reduce cobbles and manufacture chipped stone tools. Debitage analysis revealed different types of platforms from use of hard and soft-hammer stones were present, as were small resharpening flakes removed through pressure flaking, probably with an antler tine. Moderate quantities of chert debitage, both scattered and in minimally three concentrations, combined with two cherty limestone hammers and a fragmented antler, document multiple knapping episodes that targeted cobble reduction and biface production. Roughly 75 percent of the platformbearing debitage (roughly 37 percent of the total debris assemblage) have some degree of thermal alteration.

Limestone and other rock types dominated the stones employed to transfer heat in the cooking activities. Nearly all burned rocks were less than $15 \mathrm{~cm}$ in diameter and this small size may reflect the limited size of the local natural rock exposed on the adjacent uplands. Sixty-eight percent of the burned rocks were less than $4.1 \mathrm{~cm}$, an indication they had undergone multiple heating and cooling episodes and reduced in size, and were probably discarded as no longer suitable to retain and transfer heat as desired. This is supported by the lipid residue analysis, which documented burned rocks contained chemical residues from both plants and animals. Hearth Feature 26 yielded high sponge spicule content (which implies the presence of water), more than three times higher than any other sample, to support water use with the rocks. The concentration of fragmented bones $(N=125$ pieces $)$ on the southern edge of Feature 33 contained multiple taxa, and included canis, bird, turtle, fish, jackrabbit, and deer. On the basis of these multiple taxa in the same bone concentrations and the fact that lipid residue analysis identified animal products on the burned rocks, these species were probably cooked together and thereby supports a boiling process. Further support is from the presence of gelatinized starch grains on a rock from hearth Feature 33 and three rocks from hearth Feature 26. These facts, combined with the absence of large, deep-basin rock cooking ovens or flat, rock griddle features, coupled with the extremely fragmented bone, support a stone boiling technology for cooking multiple food resources. Plus, most hunter-gatherers cooked various game represented at the components by boiling (Wandsnider 1997).

Fuel employed in the fires was wood, although the macrobotanical analysis was hard pressed to identify specific woods from the weathered and degraded charcoal. Live oak was the only wood identified. Other wood phytoliths, many of which were burned, were in analyzed phytolith samples from all three hearth features. Also, the chemical biomarker dehydroabietic acid representing residues from conifer products, probably bald cypress (Taxodium), and/or juniper (Juniperus), was present on chemically analyzed burned rocks. As expected, wood resources were employed and probably available in the immediate area along Onion Creek. Lack of large chopping tools and adzes indicate wood procurement for fuel occurred 
in forms other than chopping or felling trees (e.g., deadwood gathering). The absence of heavy chopping tools may also indicate green wood for building structures was not necessary and no structures were identified.

Plant collecting and processing is well represented in the assemblage. Two edge-modified flakes yielded starch grains to testify that plants were procured and cut with informal tools. Highpowered use-wear also documented informal tools were employed to cut plants, possibly starchy plants and hard woody plants. It is often assumed, but not frequently demonstrated, basketry was the primary equipment necessary for collecting grass seed, although skin bags could have served this same task (e.g., Geib and Jolie 2008). Interestingly, most ethnographic literature and collecting observations do not mention cutting grass, only seed collection (e.g., Muir 1979). The two manos and one metate fragment with damaged starch grains document grinding of starchy plants such as grass and chenoam seeds.

Meat procurement and processing were readily evident in the vertebrate faunal assemblage combined with the presence of projectile points. Hunting large game such as deer and pronghorn would be most appropriate with darts tipped with stone projectile points. The Martindale point exhibits polish and use-wear on the stem, which extended just past the deep notches, to indicate it was hafted. The impact fracture on the distal end support its use as a projectile prior to discard. Two other point fragments testify to retooling and replacing broken points from their shafts. These three projectiles are the only artifacts directly related to game procurement.

Small game animals like cottontail rabbits and jackrabbit, however, were probably procured through other means, such as nets, traps, snares, or clubs. No direct evidence exists as to how small game was captured. This is also true for turtles and fish as these were potentially collected by hand or other means. Consequently, the hunting and collecting of meat resources undoubtedly was through multiple technologies. One edge-modified flake (\#2325-10) with a downy feather barbule fragment adhering to it documents butchering of birds with informal tools and their cultural use (see Figure 10-30). The dismemberment process is not readily discernable as few cut marks were detected. Following defleshing, bones were smashed into small pieces not only to extract the marrow, but to also extract bone grease. In most instances bone grease is extracted through boiling smashed bones (e.g., Brackenridge 1904; Leechman 1951; Quigg 1997; Vehik 1977; Zierhut 1967), with boiling also supported by other datasets. Obvious large chopping tools or mauls were not recovered, so it is not clear what tool performed the smashing task. It is possible manos were employed in this capacity, although the two hammer stones potentially served for this task. One metate fragment (\#2289-10) yielded both plant and animal lipid residues, which supports that artifact also served as a butcher block or anvil for processing (smashing) animal bones.

Although meat was procured, processed, and cooked, no direct evidence exists for scraping or processing animal hides. In fact, no scraping activities were even identified during the high-powered use-wear analysis on 13 edge-modified flakes (see Table 10-9). The latter exhibit minimal edge-modification on diverse shaped flakes or shatter, and are documented to have performed multiple tasks. High-powered use-wear documented woodworking, whittling plants and hard materials, cutting hide or butchering, cutting soft materials, and cutting plants, some probably starchy plants. These same informal tools also revealed microfossils, such as plant tissues, hair, feather, collagen, resin, and possible starch grains, which document use on a wide array materials. The one flake with the downy feather fragment is most unusual and highly informative as it directly links processing birds with informal flakes. These informal tools document site specific tasks that targeted diverse resources, which otherwise would have gone undetected. 


\subsubsection{Intra-Component Patterning}

The excavation of $74 \mathrm{~m}^{2}$ exposed only a small portion of a larger Martindale component, which likely extended over a much broader area. Therefore, observations presented represent a small part of a more expansive campsite. The three identified small, shallow basin hearths were dispersed over the $74 \mathrm{~m}^{2}$, with roughly $4 \mathrm{~m}$ separating them, to reflect broad use of space by multiple people. Their positions reflect work areas as other cultural debris was around these cooking features (see Figures 10-35). Three lithic concentrations were identified in the vicinity of Feature 25: one less than $1 \mathrm{~m}$ northeast, a second about $2 \mathrm{~m}$ northeast, and the third some $2 \mathrm{~m}$ northwest. Hammers, which potentially were employed in the creation of these concentrations or knapping areas, were beyond those concentrations. A single specimen was recovered a couple of meters north of Feature 25, and another was in the very northeastern corner of Block D, minimally 4+ $m$ north of the nearest lithic concentration. Two broken bifaces were in the immediate vicinity of two lithic concentrations with one other biface fragment at the northern end of Block D. The Martindale point was more or less between Features 25 and 33, and about $1 \mathrm{~m}$ north of the hammer. A point midsection was a couple of meters north northwest of Feature 26 in Block B with no other chipped tools in that $12 \mathrm{~m}^{2}$ area. Obviously, retooling of dart shafts was not conducted in the same place or the discarded broken points did not end up in the same place, which ended up meters away from the features.

Lithic debitage patterning reflects high $(N=114$ to $158)$ and moderate ( $N=41$ to 62$)$ densities adjacent to Features 25 and 33 (see Figure 10-39). This pattern reflects either direct tool manufacturing immediately next to these features or convenient places to dispose of unwanted debitage from other areas. Only a moderate lithic debitage concentration was just north of Feature 26. The detected pattern indicates the high integrity and intact nature of the cultural deposits. It also documents specific patterned human behavior rather than random dispersal of items.

The smashed faunal remains also revealed wellpatterned, horizontal distributions and interesting associations. The highest frequencies $(N=125$ to 168 pieces) were concentrated in five units $\left(5 \mathrm{~m}^{2}\right.$ or 7 percent of the area), with three units on the northeastern edge of hearth Feature 25, one unit (1 $\mathrm{m}^{2}$ ) on the southeastern edge of hearth Feature 33, and one unit on the northern edge of Block D (see Figure 10-57). The bone distribution document most smashed fragments were directly associated with hearth Features 25 and 33, with one concentration $2 \mathrm{~m}$ north of Feature 33. The high concentrations and moderate densities around the high concentration on the northern edge of Block D probably represent a specific discard area.

The lack of any bone concentration around Feature 26 is intriguing, and may reflect Feature 26 primarily served a function other than boiling bones for grease rendering (see Figure 10-57). Potentially, Feature 26 functioned more for cooking plants as gourd phytoliths, burned grass seed phytoliths, charred cheno-am, and grass seeds were recovered from this feature. In contrast to Features 25 and 35, interpreted as features for processing animal bone, Feature 26 likely served as a plant cooking feature as indicated by two mano fragments observed in and near this feature.

The roughly 150 large ungulate bone fragments, including deer, pronghorn, and unidentified ungulate, were scattered across 26 units (35 percent). These fragments were generally concentrated in the three highest and moderate dense areas (see Figure 10-58). Bird bone distribution revealed seventeen pieces in six units (8 percent) localized in Blocks B and D (see Figure 10-61). The highest concentrations were in the same units as bones from multiple other species to reveal direct association with other meat resources. 
Scrutinizing fish bone distribution showed them scattered across 22 units (30 percent) in both Blocks B and D with significant concentrations, mostly gar scales, in Features 26 and 33 (see Figure 10-60). Also fish bones were in five units that yielded high concentrations of other bones next to Features 25 and 33, documenting their association with other taxa. These distributions indicate these large animals were treated similarly to the smaller animals, birds, and fish, implying all were cooked together. Following cooking, these fragments were in two instances primarily discarded next to cooking Features 25 and 33, with one cluster $2 \mathrm{~m}$ north of Feature 33.

The 53 turtle shell bones were scattered across 15 units (20 percent) to reflect original whole shells did not weather in place (see Figure 10-62). Recognizable turtle shells were not in the three features. Four fragments were in the high bone concentration on the southern edge of Feature 33. Another 10+ fragments were clustered in one unit in the high concentration $1+\mathrm{m}$ northeast of Feature 25 (see Figures 10-57 and 10-62). Again, turtle bones were in direct association with other discarded species revealing no preferential treatment.

The two manos were in Block B, one within Feature 26 debris and the other about $1 \mathrm{~m}$ north (see Figure 10-36). The mano association with Feature 26 is interesting as this feature alone yielded charred grass and cheno-am seeds, and its immediate surroundings lacked high or even moderate frequencies of faunal remains. The manos and seed association may reflect activities around Feature 26 were directed towards plant processing and cooking, contrasting with Features 25 and 33 (see Figure 10-36). The probable metate fragment in Feature 33 was probably recycled as a burned rock.

The horizontal distribution of burned rocks reveals only two units with high concentrations, with one on the eastern side of hearth Feature 26 and one on the northwestern boundary of that same feature in Block B (see Figure 10-63). Only two units yielded medium concentrations, one $2 \mathrm{~m}$ beyond Feature 33 in Block D. Burned rocks were directly linked to all three features with the most intense use of rock associated with Feature 26. Interestingly, the rocks were not filling any of the three features.

The clear and distinct horizontal pattern revealed by different cultural material classes definitely reflects the intact nature and high integrity of these materials. It also documents multiple, specific human behaviors for processing and discarding bones, cooking bones from multiple species together at Features 25 and 33, cooking plants in Feature 26, either intact knapping or intentional discard areas for lithic debris, and discarding mixed debris to one side of hearth features. Such patterns document multiple human behaviors during a short-term camp where multiple activities occurred.

The overall artifact density across the excavated area was 54 items per square meter and is skewed to a higher density in comparison to many other early components because of the hundreds of bone fragments not often preserved in other sites. Bone fragments account for nearly 52 percent of the total materials, which makes a major difference in the total count and density. If bone is excluded, the remaining classes of materials reveal a much lower density of only 26 items per meter square. This more moderate density is thought to reflect the relatively short-term nature of this huntergatherer occupation in what is considered an intact single event. Formal tool frequency $\left(0.3 / \mathrm{m}^{2}\right)$ accounts for less than 1.0 percent of the total; therefore it may not be appropriate to compare this occupation to other hunter-gatherer components, which often report tool frequency in palimpsest conditions. 


\subsubsection{Paleoenvironmental Conditions}

Specific environmental conditions could not be precisely identified as most microfossils (specifically phytoliths and starches), macrobotanical remains, and wood charcoal were poorly preserved. This unfortunate fact significantly limited direct information pertaining to the grassland vegetation of the past environment. Consequently, bits and pieces of other data from various technical analyses provide spotty, but useful information to partially reveal the paleoenvironment. Identified wood was limited to live oak, although chemical biomarker dehydroabietic acid in the lipid residues from most burned rocks indicate conifer products (i.e., bald cypress and/or juniper) were probably employed to heat rocks and were, therefore, in the immediate vicinity.

In Feature 26, Chloridoid grass phytoliths that represent hot dry $\mathrm{C}_{4}$ grasses, such as grama grass (Bouteloua sp.) and buffalo grasses (Bouchloe sp.) thrive in warm, arid to semiarid regions, imply the region in the vicinity was a short grass prairie. Burned grass and cheno-am seeds document grasses and weedy plants in seed were present, and sufficiently abundant to purposely gather and process for food. Wild buffalo gourd phytoliths, which were in Features 25 and 33, require little water and grow well in semiarid and desert environments. These three plant groups generally represent open, dry regions and support the spotty phytolith evidence for a grassland environment. The support of open grasslands in this immediate vicinity is the presence of cottontail rabbits, jackrabbits, pronghorn, and prairie chicken remains in the faunal assemblage, all of which prefer open grasslands in combination with low brush and open spaces (Figure 10-64). These multiple species are good indicators the region beyond the moist riparian zone along Onion Creek was probably open grasslands with some brush.
Lenticular grass starch grains, probably wildrye (Elymus sp.), adhering to one informal tool and on burned rocks from Feature 26 prefer shady moist areas. Wildrye is a cool season $\mathrm{C}_{3}$ grass (ca. $27.6 \%$, $-27.1 \%$, Bender 1971) that produces short cell phytoliths, with large seed heads available during late summer and fall, and would have grown along the riparian zone. True lilies (Lilium sp.) also reflect moist areas in the riparian setting in Onion Creek valley. The chemical biomarker dehydroabietic acid represents residues from conifer products (locally probably bald cypress or juniper trees) support multiple tree species were present, which would have probably grown along the creek. The identified live oak trees potentially were interspersed across the grassland or restricted along the creek margins. This sparse, but diverse data reveals the local environment contained multiple tree and grass species, which probably represent multiple ecotones immediately along Onion Creek. The creek flowed from the Edwards Plateau just to the west, eastward through a broader grassland region.

The diatom assemblage indicates that the nearby Onion Creek was a permanent, relatively shallow, flowing stream. The most abundant diatoms were in hearth Feature 25 and represent forms in shallow water, through multiple species tolerate substantial organic pollution. The available water also contained fresh water sponges. This was a similar water condition as projected during the Bell/Andice

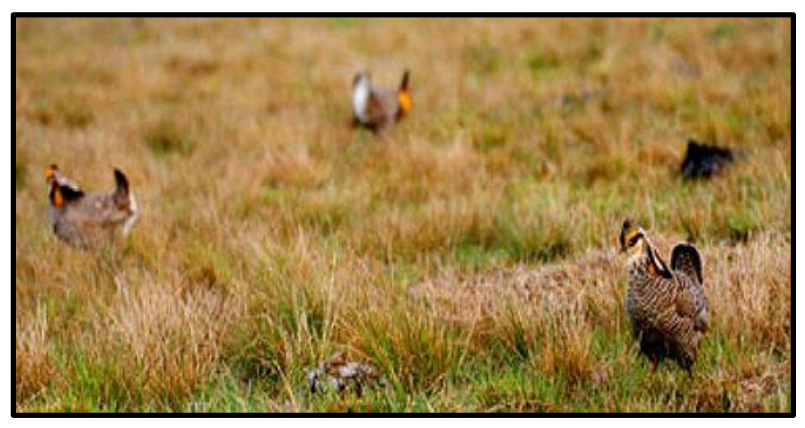

Figure 10-64. Attwater prairie chicken in their natural coastal prairie habitat (courtesy of the Nature Conservancy). 
period. Catfish (probably Bullhead) are bottom feeders in warm freshwater as they prefer muddy bottoms probably found in Onion Creek. Gar fish generally prefer sluggish areas of rivers, oxbow lakes, or pools (Thomas et al. 2007). The three species of mussels generally tolerate many water conditions, and generally avoid deep, shifting sands or deep silts. The Yellow Sandshells are very drought tolerant. The Tampico Pearly mussel is not known from the Colorado River system and maybe present in slow flowing, mud-bottom rivers (Howells et al. 1996:48).

One very intriguing discovery was the presence of $30+$ marine diatoms from $248 \mathrm{cmbs}$ in the column of natural sediment on the south side of the deep pit. These are projected to have occurred between the Bell/Andice and Martindale components rather than directly associated with cultural materials. These constitute unusual and rare occurrences a long distance from the coast, although not the first or only marine diatoms recovered inland across Texas (e.g., Frederick et al. 2008; Munoz et al. 2009; Oksanen et al. 2002; Quigg et al. 2011a; Schroeder 2011; Schroeder and Oksanen 2002; Winsborough 2002a, 2002b, 2008, 2011a, 2011b; Winsborough and Quigg 2012). Two marine species were identified along with eight small corroded fragments of planktonic diatoms. Dr. Winsborough (Appendix C) states the ecological affinities of these 38 marine diatoms are not compatible with the rest of the diatom assemblage. These exclusive marine species occur in the Gulf of Mexico, and one possible explanation for their presence is human transport from the Gulf in the form of raw salt or salted fish, or other meat resources traded inland.

One noncultural sediment sample at $290 \mathrm{cmbs}$ from the stratigraphic column in the south wall of the large pit yielded a high count of diatoms. Thirtyone percent of those types are found in standing water, eight percent in permanent water, and eight percent in flowing water. This one sample, although not from the greenish-gray clay lens observed in the excavation blocks below the Bell/Andice component, documents standing water was present prior to the occupation of the Martindale component. This may be similar to what was reflected by the greenish-gray clay lens, just a different expression at a different time.

Soil texture analysis as part of the phytolith study on samples from the natural sediment column south of Block B clearly document a significant increase in sand content at the expense of silt between 300 and $330 \mathrm{cmbs}$. The increased sand is thought to reflect a flood episode along Onion Creek. This depth is projected just below and prior to the Martindale component radiocarbon dated to ca. 5400 B.P. This supports interpretation of a wetter interval with greater sand deposition via flood episodes along Onion Creek, which is also supported by a high frequency of sponge spicules at $310 \mathrm{cmbs}$. At $340 \mathrm{cmbs}$ and prior to that wet period, the silt deposition rate was higher, which possibly reflect eolian deposition during a relatively dry interval. Following the wetter interval and the projected period of the Martindale component between 260 and $290 \mathrm{cmbs}$, the silt component in the soil again increased to offset the decrease in sand deposition implying a drying interval. If the interpretations of the environment are correct, based on the above presence of diatom and sponge spicules, then flooding events occurred prior to the Martindale component, which would be pre-ca. 5500 B.P. It is significant that an apparent wet period is documented by soil texture data to support dry periods just before and after, to lend support for a two part Altithermal as discussed in Chapter 2.0 and a wet interval towards the end of the Altithermal.

\subsection{RESEARCH DESIGN QUESTION}

How did hunter-gatherer populations respond to the prevailingly dry climatic conditions during the middle Holocene (see Chapter 4.0)? 


\subsubsection{Hypothesis 1}

The middle Holocene Martindale occupation at 41TV2161 was nonintensive, short-term, and economically unspecialized encampment from which a broad range of subsistence resources were procured and processed. The occupation can be characterized as short-term, residential encampment by small group of nonspecialized foragers.

This hypothesis is clearly supported by data obtained from the Martindale component that yielded the following: three small hearth features, a very limited but standard hunting and processing formal stone tool kit (points and, bifaces) with a restricted functional range, a chert debitage assemblage that documents core and biface reduction with some late stage biface manufacturing, plus a broad diet breath (i.e., deer, pronghorn, jackrabbits, cottontails, birds, fish, cheno-ams, grasses, and lily bulbs). These food resources support a broad spectrum, unspecialized range of subsistence resources. The support for a single season of use - fall, coupled with the low diversity of formal tool classes, few cooking and/or heating features, stone tool production, the exceptional high integrity of the horizontal distribution of artifact classes, testify to a shortterm residential camp occupied by multiple family units, which conducted an array of activities by nonspecialized hunter-gatherers. This pattern implies this group was primarily "mapped onto the resources" and were foragers (Binford 1980:5-10).

\subsubsection{Hypothesis 2}

This kind of occupation, namely, short-term residential encampment with nonspecialized and nonintensive reliance on a diverse range of resources, is essentially characteristic of this period of the Holocene in central Texas, ca. 5000 to 5500 B.P., and represent an adaptive response on the part of regional human populations to the relatively dry climate of the period that involved a diversified reliance on a wide range of plant and animal food resources.

Hypothesis 2 is also supported, as this Martindale component reflects a short-term fall event, nonspecialized occupation dated to the middle Holocene dry period (ca. 5000 to 5500 B.P.). The recovered artifact assemblage is very similar to artifact assemblages from other excavated early and middle Holocene sites from across the region (see Table 9-23). The close similarity in all Early Archaic assemblages presented above documents and supports the regional human population adapted to and maintained a similar lifeway during the projected long, relatively dry climate. 
This page intentionally left blank. 


\subsection{COMPONENT COMPARISONS}

J. Michael Quigg and Benjamin G. Bury

\subsection{INTRODUCTION}

The two identified components were both were deeply buried between 220 and $280 \mathrm{cmbs}$, vertically separated, well-defined, in good context with high depositional integrity in stratified alluvial fines. Both also had moderate organic preservation and yielded significant vertebrate faunal assemblages although very limited macrobotanical and microfossil remains. The Bell/Andice component was stratigraphically 20 to $25 \mathrm{~cm}$ above the Martindale component. The two components were in the exact same location and close in radiocarbon age, and these circumstances promote direct comparisons of two distinct human behaviors over a relatively short period of time.

The total area excavated in the Martindale component $\left(74 \mathrm{~m}^{2}\right)$ was greater than the area excavated in the Bell/Andice component $\left(51 \mathrm{~m}^{2}\right)$ by 50 percent. Several broad topics or classes of data are addressed below beginning with an age comparison and discussion, followed by comparisons of component stone tool and lithic debitage assemblages, the burned rock assemblages, features, vertebrate faunal assemblages, plant remains, patterns of human behaviors, characteristics of paleoenvironment, and ending with conclusions.

\subsection{CHRONOLOGY}

The Bell/Andice component age was determined through 12 radiocarbon assays on four different materials: six charcoal, two animal bones, three organic sediments, and one Rabdotus shell (Figure 11-1). Since radiocarbon dating at other sites has demonstrated charcoal is most reliable, the six dates derived from charcoal are accepted as the most likely to reflect the true age of this component.
Those six charcoal dates average 5322 B.P. with a very narrow 90 year radiocarbon age range. The age is appropriate for this specific cultural interval for what is known in Texas (see Section 3.2.7). Radiocarbon control for Big Sandy points in Texas is lacking; therefore, its presence does not contribute to our understanding of the age of this Bell/Andice component. Beyond Texas this point type reveals a broad time span that includes this time interval.

The Martindale component age was determined by 15 radiocarbon assays on similar diverse materials: two charcoal, one charred seeds, five animal bones, five organic sediments, and two Rabdotus shell samples (Figure 11-1). The two charcoal and single seed date yielded an average of 5393 B.P. with a radiocarbon date range of 110 years. This average age is considerably younger compared to most previous dates associated with this point type (see Table 3-3), although the context and average age associations between previous dates and Martindale points may be questioned in most cases (see Chapter 3.0). Surprisingly, the nine charcoal dates from the two components are less than 100 years apart and are statistically the same (Figure 11-2).

One may question if mixing of charcoal between the two components created the narrow time difference or overlapping age of occupations; however, the obtained dates appear accurate and represent two events very close in time. Charcoal samples were collected from good context and in association with cultural features. Burned rock features are resilient to site formation processes that could affect errors in dating. Further, cultural features in the two components were separated spatially as exemplified by Martindale Feature 26 and Bell/Andice Feature 24.

In the southern portion of Block B, the charcoal stained and dated Martindale Feature 26 was roughly $25 \mathrm{~cm}$ below the Bell/Andice component. The Bell/Andice component contained no charcoal or cultural features directly above Feature 26 (see Figure 8-10). The dated charcoal and seeds were in good context within Feature26 matrix. The average of the latter two 


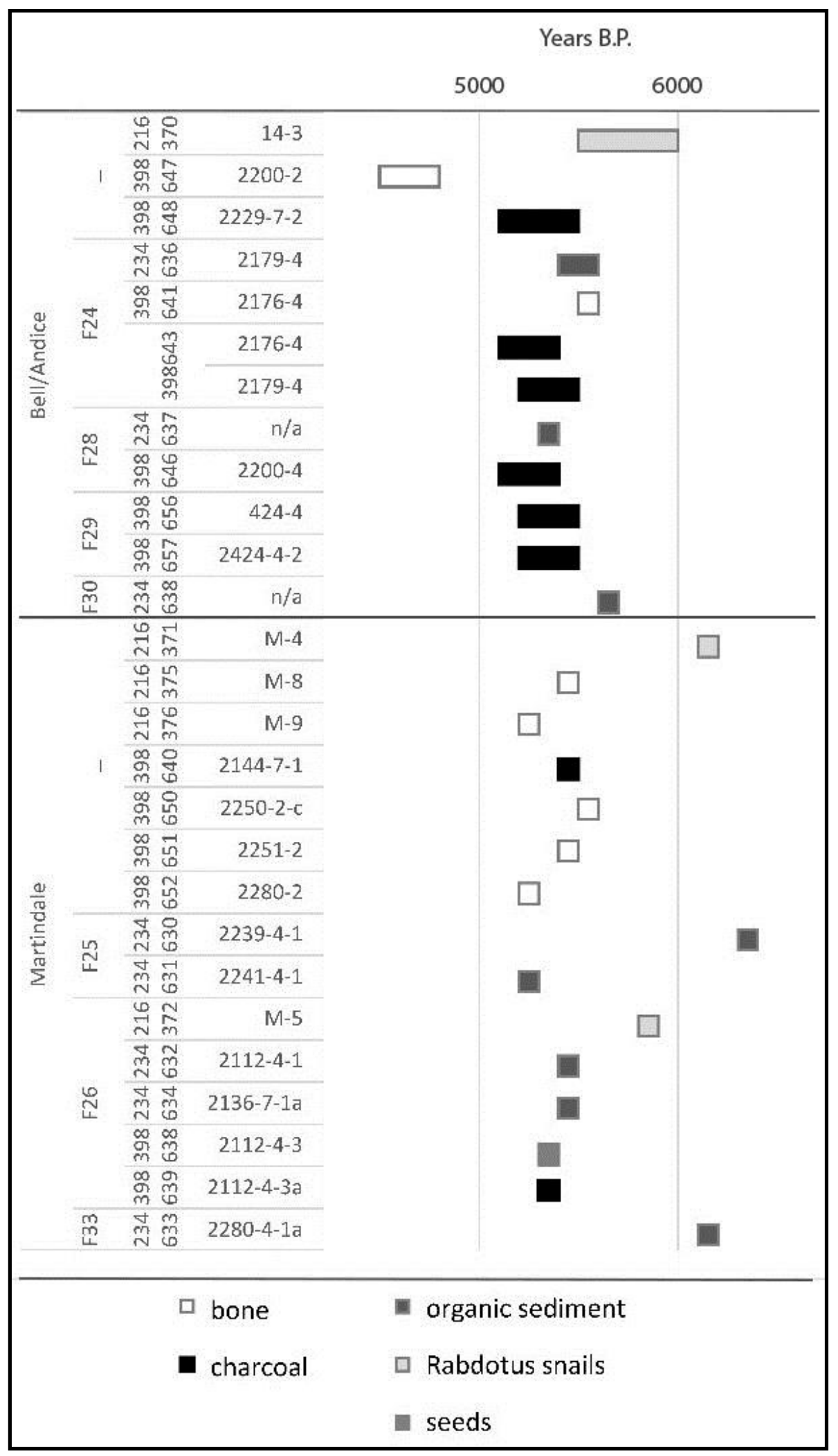

Figure 11-1. Plots of radiocarbon dates from Bell/Andice compared to Martindale dates. 


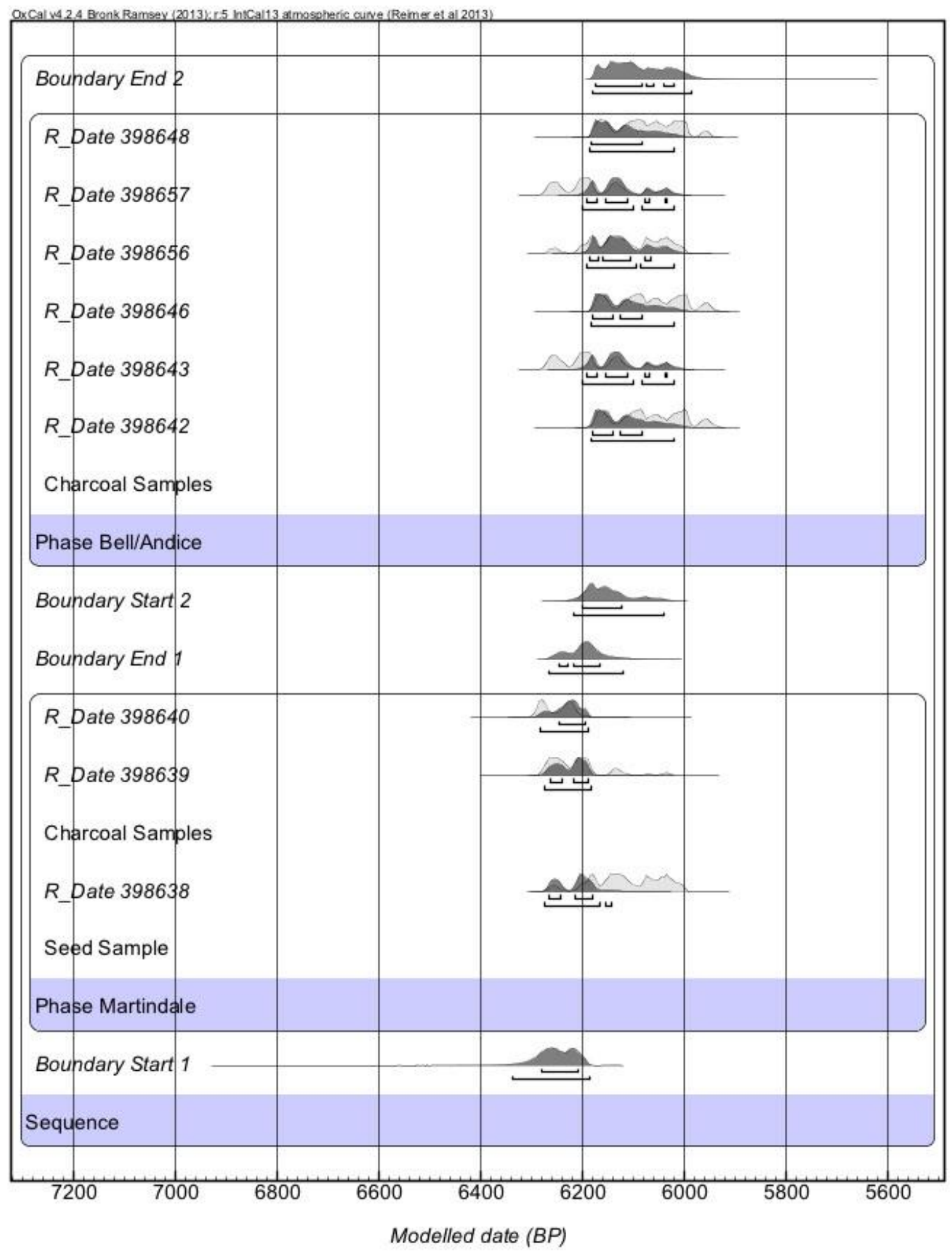

Figure 11-2. Calibrated radiocarbon assays from charcoal samples from the Bell/Andice and Martindale components. 
dates from Martindale Feature 26 is 5365 B.P. Two charcoal radiocarbon dates from Bell/Andice Feature 24 averaged 5325 B.P. Feature 24 was horizontally separated from Feature 26 by approximately $3 \mathrm{~m}$ as well as vertically separated by some 20 to $25 \mathrm{~cm}$. The tight context of the dated samples from the two features and their positions support the accurate dating of the components. The 40 radiocarbon year's difference between them signifies two well-dated, stratigraphically separate, and well-defined human occupations in a close time interval.

The obtained conventional radiocarbon dates for both components have been calibrated and corrected employing OxCal version 4.2.2 (Ramsey 2013). Examination of the modelled results from the two components show a narrow time interval for both components (see Figure 11-2). They do however, reveal that the deeper Martindale component is slightly older than the higher (later) Bell/Andice component. Statistically the end date for the Martindale component is older than the beginning date for the Bell/Andice beginning date. However, a slight overlap does occur revealing the two occupations were very close in time. According to the modelled radiocarbon data, the Bell/Andice occupation occurred between approximately 6000 to 6100 B.P., while the Martindale occupation occurred between roughly 6250 and 6200 B.P.

\subsection{ARTIFACT ASSEMBLAGES}

The two assemblages are similar in the kinds of material represented, including features, chipped and ground stone tools, lithic debitage, scattered mussel shells, and burned rocks (Table 11-1). Both components also contain remarkable vertebrate faunal assemblages. The differences between the assemblages are subtle and vary mostly in frequencies (Table 11-1). Formal chipped stone tools include projectile points and bifaces, which were rare in both components. Both lack other formal chipped stone tools, like scrapers and other unifacial tools. Both components also contained formal ground stone tools.
Table 11-1. Comparison of Material Remains from the Two Identified Components.

\begin{tabular}{|c|c|c|}
\hline \multirow{2}{*}{$\begin{array}{l}\text { Cultural Material } \\
\text { Classes }\end{array}$} & Bell/Andice & Martindale \\
\hline & Counts & Counts \\
\hline Radiocarbon Dates & 12 & 15 \\
\hline Features & 9 & 3 \\
\hline $\begin{array}{l}\text { Hearths, Organic } \\
\text { Stained }\end{array}$ & 0 & 3 \\
\hline $\begin{array}{c}\text { Burned Rock } \\
\text { Dumps/Discard Areas }\end{array}$ & $\begin{array}{l}16,22,24,27, \\
28,29,30,32\end{array}$ & 0 \\
\hline $\begin{array}{l}\text { Ground Stone } \\
\text { Cache }\end{array}$ & 1 & 0 \\
\hline $\begin{array}{l}\text { Dart Points and } \\
\text { Fragments }\end{array}$ & 3 & 3 \\
\hline Bell & 2 & 0 \\
\hline Big Sandy & 1 & 0 \\
\hline Martindale & 0 & 1 \\
\hline Fragments & 0 & 2 \\
\hline Bifaces & 3 & 4 \\
\hline Manos & 5 & 2 \\
\hline Metates & 3 & 1 \\
\hline Hammer Stones & 0 & 2 \\
\hline Edge-Modified Flakes & 12 & 83 \\
\hline Lithic Debitage & 180 & 1,429 \\
\hline Cores & 7 & 7 \\
\hline $\begin{array}{l}\text { Vertebrate Faunal } \\
\text { Fragments }\end{array}$ & 591 & 2,082 \\
\hline Mussel Shells & 15 & 54 \\
\hline Burned Rocks & 554 & 321 \\
\hline Burned Clay & 6 & 36 \\
\hline $\begin{array}{l}\text { Carbonized Plant } \\
\text { Remains }\end{array}$ & $\begin{array}{l}4 \text { hackberry } \\
\text { nutlets }\end{array}$ & $\begin{array}{c}8 \text { grass seeds, } \\
2 \text { cheno-am } \\
\text { seeds, live } \\
\text { oak frags }\end{array}$ \\
\hline Total Materials & 1,390 & 4,027 \\
\hline $\begin{array}{l}\text { Average Thickness } \\
(\mathrm{cm})\end{array}$ & $10-15$ & $10-15$ \\
\hline $\begin{array}{l}\text { Spatial Extent } \\
\text { Excavated }\end{array}$ & $51 \mathrm{~m}^{2}$ & $74 \mathrm{~m}^{2}$ \\
\hline $\begin{array}{c}\text { Volume Excavated } \\
\left(\mathrm{m}^{3}\right)\end{array}$ & $10.2 \mathrm{~m}^{3}$ & $8.6 \mathrm{~m}^{3}$ \\
\hline
\end{tabular}

This table does not include materials from float samples 
Table 11-2. Summary of Tool Function from Use-Wear Analysis.

\begin{tabular}{|c|c|c|c|c|c|c|c|c|c|c|c|c|c|c|c|c|c|c|c|c|}
\hline \multirow[b]{2}{*}{$\begin{array}{c}\text { Artifact Classes } \\
\text { Analyzed }\end{array}$} & \multicolumn{9}{|c|}{ Inferred Function } & \multicolumn{11}{|c|}{ Contact Material and Observed Residues } \\
\hline & 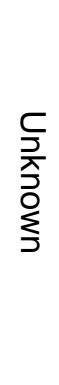 & 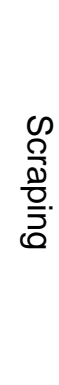 & 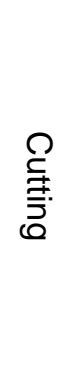 & 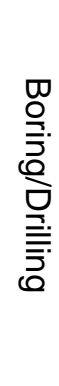 & & 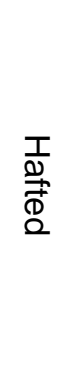 & $\frac{\mathscr{O}}{\overline{\bar{a}}}$ & 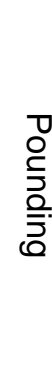 & $\begin{array}{l}\text { 음 } \\
\text { 음 } \\
\text { 음. }\end{array}$ & 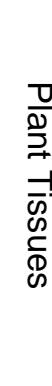 & $\frac{\overline{0}}{\frac{0}{\bar{\rho}}}$ & 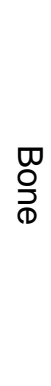 & 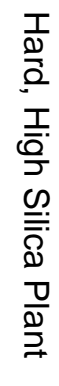 & 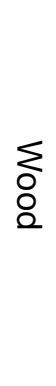 & 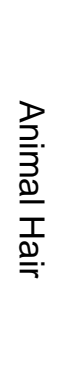 & 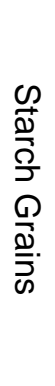 & $\begin{array}{l}\text { गD } \\
\frac{0}{0} \\
\frac{0}{\bar{D}} \\
\frac{\mathbb{D}}{\infty}\end{array}$ & 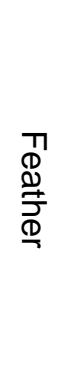 & 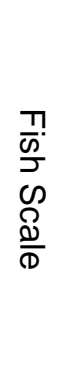 & 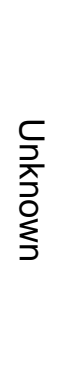 \\
\hline \multicolumn{21}{|c|}{ Bell/Andice Component } \\
\hline Points $(N=3)$ & 1 & & & & & 2 & & & & & 2 & 1 & & & & & 1 & & & 1 \\
\hline Bifaces $(N=3)$ & 1 & & 2 & & & & & & & & 1 & & 2 & & & & & & & \\
\hline $\begin{array}{l}\text { Edge-Modified } \\
\text { Tools }(N=7)\end{array}$ & & 2 & 2 & & 2 & & 1 & & & 1 & 1 & & 6 & 2 & 1 & & & & 1 & \\
\hline \multicolumn{21}{|c|}{ Martindale Component } \\
\hline Points $(N=1)$ & & & & & & 1 & & & & & 1 & & & & & & & & & \\
\hline Bifaces $(N=1)$ & & & 1 & & & & & & & & 1 & & & & & & & & & \\
\hline $\begin{array}{l}\text { Edge-Modified } \\
\text { Tools }(N=13)\end{array}$ & 4 & & 10 & & & & & & & 3 & 6 & 4 & 5 & & 6 & 3 & & 1 & & \\
\hline
\end{tabular}


In total, the Bell/Andice component yielded 14 formal tools, and the Martindale component yielded 12. The Bell/Andice component has 2.5 times the frequency of ground stone, while the Martindale component has twice the number of chipped stone tools. Minor differences in tool classes may not reflect anything more than sampling biases; and, the overall similarities in stone tool diversity reflect common lifeways or circumstances of single shortterm hunter-gatherer occupations.

High-powered use-wear on selected chipped stone tools (points, bifaces, and edge-modified flakes) from the two components indicates similar tool functions between components (Table 11-2). Edgemodified flakes from the Bell/Andice component exhibit a diversity of functions, with emphasis on cutting and scraping plants. Martindale edgemodified flakes reveal similar functions with slightly greater diversity in materials contacted. The use-wear on this tool class reveal multiple tasks on multiple materials that would not have otherwise been detected.

The types of ground stone tools are similar and exhibit only minimal differences, which probably reflect their functions and short life spans. No two handed manos or deep basin grinding slabs were present to indicate extensive grinding great quantities over extended periods. Metate surfaces exhibit limited wear as actual utilized surfaces are poorly defined. Since these tools were not curated, they were obviously expendable and replaced when needed. The seasons of occupations could have contributed to the limited use-life and decision to discard ground stone, as seeds and nuts that require grinding would not have been available during the following months.

In the chipped stone assemblages, both components reveal use of a single local chert resource as determined by INAA, which originated from the adjacent hill or minimally the same origin as the hilltop cherts. Aside from the raw material source, the lithic assemblages reflect different behaviors.
The Martindale component yielded nearly eight times the amount of debitage (see Table 11-1) and documents substantially more knapping activities compared to the Bell/Andice component. Martindale knapping focused on cobble and middle stage biface reduction and some late stage finishing, in contrast to the more general cobble/core reduction and flake production in the Bell/Andice component.

\subsection{LITHIC DEBITAGE ASSEMBLAGE}

\subsubsection{Introduction}

The well-defined context and integrity of the two components represents an excellent opportunity to advance our understanding of two different populations in the same setting that employed the same lithic resource. The specific observations and details concerning the complete flakes and proximal flake fragments and the various selected attributes are presented and contrasted between the two components. This is followed by a brief discussion of other debitage classes and cores. A general discussion is presented at the end of this section.

\subsubsection{Complete Flakes}

Table 11-3 lists the attributes and associated categories for the majority of flakes from the two assemblages. Overall, the two assemblages are relatively similar largely because the majority of both assemblages were produced from locally available chert cobbles. Notable differences between assemblages are outlined in the following discussion of individual attributes, namely the different stages of reduction represented. In general, the complete flakes from the Martindale component exhibit characteristics associated with a greater degree of reduction. This includes less dorsal cortex, more dorsal flake scars, less pronounced bulbs, fewer eraillure scars, increased longitudinal curvature, more feathered 
terminations, as well as a smaller average size. These attributes are discussed in greater detail below.

Table 11-4 lists the amount of cortex and the number of flake scars recorded on the dorsal surfaces of complete flakes from both assemblages. From the perspective of a cortical typology based on the amount of dorsal cortex and the number of dorsal flake scars, the Martindale assemblage reflects a greater proportion of flakes from middle and later stages of reduction, and the Bell/Andice assemblage reflects a greater proportion of flakes from early and middle stages of reduction. The number of flake scars and the percentage of cortex is a good general indication of the degree of reduction within an assemblage, with an increase in flake scars and corresponding decrease in cortex as raw materials are reduced (Odell 1989).

When an application load typology is applied to the database, a similar pattern is apparent (Table 11-5). In general, the size of the bulb and presence of an eraillure scar has been shown to correspond to the amount of applied force and the nature of the precursor, with hard-hammers tending to produce larger, more pronounced bulbs (Andrefsky 2005, 2006; Crabtree 1972).

From the perspective of an application load typology, the majority of the Martindale assemblage was likely produced with a softhammer and represent middle to late stages of reduction. The Bell/Andice assemblage is representative of more frequent use of a hardhammer during early and middle stages of reduction. Choosing a hard-hammer over a softhammer generally depends on what stage of reduction you were at in the tool making process.

Technological typologies typically differentiate between bipolar flakes, core reduction flakes, bifacial thinning flakes, and more specific forms related to tool sharpening etc. Core reduction flakes are usually defined by single facet platforms, larger dimensions, greater amounts of dorsal cortex, and less pronounced platform lipping and longitudinal curvature. Biface thinning flakes are typically defined as having "curved longitudinal crosssections, extremely acute lateral and distal edge angles, feathered flake terminations, narrow faceted striking platforms, a lip, little or no cortex, and a small flattened or diffuse bulb of force" (Andrefsky 2006:118; Root 1992:83). Typically, these attributes are not measured separately, but assessed visually as a whole for individual flakes. During this analysis, a tentative technological category was assigned to each flake (Table 11-6). Although a technological assessment of the assemblage is subjective, the general pattern is consistent with the other typologies, and indicates a greater frequency of bifacial reduction and a more complete reduction trajectory within the Martindale component.

Additionally, lipping and longitudinal curvature were recorded independently within the assemblages and placed within three categories (Table 11-7). Both attributes are not clearly understood and different studies have produced conflicting results (Andrefsky 2006). However, platform lipping is generally thought to occur more frequently with soft-hammer percussion (Andrefsky 2006; Hovers 2009). In some studies, longitudinal curvature has been shown to be associated with biface production (Hayden and Hutchings 1989). The frequency of lipping within the two assemblages in nearly identical, suggesting equal use of soft-hammer percussion. However, the amount of longitudinal curvature indicates more bifacial reduction is represented in the Martindale assemblage.

Platform characteristics can be applied within both an application load and a technological typology (Table 11-8). If crushed, abraded, and multifaceted platforms are combined, then a nearly equal number of the platforms with the Martindale assemblage ( $N$ $=279,40.0$ percent) can be characterized as more complex than a single facet $(N=289,41.4$ percent). In contrast, when these categories are combined 
within the Bell/Andice assemblage, only 26.76 percent $(N=19)$ can be characterized as complex. Additionally, cortical and single facet platforms are both more abundant within the Bell/Andice assemblage. These observations fit with the previously discussed attributes and indicate the Bell/Andice assemblage reflects a greater proportion of early stage reduction. However, for unknown reasons the Bell/Andice assemblage has a greater frequency of flakes with platform preparation along their dorsal margins.
Table 11-9 lists the frequency of size classes and termination types within the two assemblages. The majority of both assemblages have feathered terminations. However, the Bell/Andice component has a noticeably greater proportion of hinged terminations $(N=24,33.8$ percent $)$ and the Martindale component has a much larger proportion of feathered terminations $(N=526,75.4$ percent). Hinged terminations generally occur more frequently with hard-hammer percussion and support the interpretation that the Bell/Andice

Table 11-3. Dominant Attribute Classes in the Samples of Complete Flakes.

\begin{tabular}{|c|c|c|c|c|c|c|c|}
\hline \multicolumn{5}{|c|}{ Bell/Andice } & \multicolumn{4}{c|}{ Martindale } \\
\hline Attribute & Majority & Count & Percent & Attribute & Majority & Count & Percent \\
\hline Platform Type & Single Facet & 35 & 49.31 & Platform Type & Single Facet & 289 & 41.52 \\
\hline Platform Prep. & Present & 37 & 52.11 & Platform Prep. & Absent & 410 & 58.91 \\
\hline Dorsal Context & None & 32 & 45.07 & Dorsal Context & None & 507 & 72.84 \\
\hline Scar Count & $1-2$ & 43 & 60.56 & Scar Count & $1-2$ & 347 & 49.86 \\
\hline Bulb & Pronounced & 30 & 42.25 & Bulb & Absent & 331 & 47.56 \\
\hline Eralliure & Absent & 50 & 70.42 & Eralliure & Absent & 547 & 78.59 \\
\hline Lipping & Absent & 62 & 87.32 & Lipping & Absent & 595 & 85.49 \\
\hline Curvature & Absent & 69 & 97.18 & Curvature & Absent & 478 & 68.68 \\
\hline Size Class & $1 / 2$ inch & 22 & 30.99 & Size Class & $1 / 4$ inch & 276 & 39.66 \\
\hline Termination & Feather & 42 & 59.15 & Termination & Feather & 529 & 76.01 \\
\hline
\end{tabular}

Table 11-4. Dorsal Cortex and Scar Counts for Complete Flakes.

\begin{tabular}{|c|c|c|c|c|c|}
\hline \multirow{3}{*}{ Attribute } & \multirow{2}{*}{ Category } & \multicolumn{2}{|c|}{ Bell/Andice } & \multicolumn{2}{c|}{ Martindale } \\
\cline { 2 - 6 } & & Count & Percent & Count & Percent \\
\hline \multirow{4}{*}{ Cortex } & 0 & 32 & 45.07 & 507 & 72.84 \\
\cline { 2 - 6 } & $1-25$ & 18 & 25.35 & 91 & 13.07 \\
\cline { 2 - 6 } & $26-50$ & 10 & 14.08 & 32 & 4.60 \\
\cline { 2 - 6 } & $51-75$ & 6 & 8.45 & 22 & 3.16 \\
\cline { 2 - 6 } & $76-100$ & 5 & 7.04 & 44 & 6.32 \\
\cline { 2 - 6 } & Total & 71 & 100.00 & 696 & 100.00 \\
\hline \multirow{4}{*}{ Scar Count } & 0 & 3 & 4.23 & 20 & 2.87 \\
\cline { 2 - 6 } & $1-2$ & 43 & 60.56 & 347 & 49.86 \\
\cline { 2 - 6 } & $3-4$ & 22 & 30.99 & 297 & 42.67 \\
\cline { 2 - 6 } & $5-6$ & 3 & 4.23 & 32 & 4.60 \\
\hline
\end{tabular}


Table 11-5. Bulb Size and Eraillure Scar Frequency for Complete Flakes.

\begin{tabular}{|c|c|c|c|c|c|}
\hline \multirow{3}{*}{ Attribute } & \multirow{2}{*}{ Category } & \multicolumn{2}{|c|}{ Bell/Andice } & \multicolumn{2}{c|}{ Martindale } \\
\cline { 3 - 6 } & & Count & Frequency & Count & Frequency \\
\hline \multirow{4}{*}{ Bulb } & Absent & 14 & 0.20 & 331 & 0.48 \\
\cline { 2 - 6 } & Moderate & 27 & 0.38 & 249 & 0.36 \\
\cline { 2 - 6 } & Pronounced & 30 & 0.42 & 112 & 0.16 \\
\cline { 2 - 6 } & N/A & 0 & 0.00 & 4 & 0.01 \\
\cline { 2 - 6 } & Total & 71 & 1.00 & 696 & 1.00 \\
\hline \multirow{4}{*}{ Eraillure } & Absent & 50 & 0.70 & 547 & 0.79 \\
\cline { 2 - 6 } & Present & 21 & 0.30 & 136 & 0.20 \\
\cline { 2 - 6 } & N/A & 0 & 0.00 & 13 & 0.02 \\
\cline { 2 - 6 } & Total & 71 & 1.00 & 696 & 1.00 \\
\hline
\end{tabular}

Table 11-6. Complete Flakes by Technological Class.

\begin{tabular}{|c|c|c|c|c|}
\hline \multirow{2}{*}{ Type } & \multicolumn{2}{|c|}{ Bell/Andice } & \multicolumn{2}{c|}{ Martindale } \\
\cline { 2 - 5 } & Count & Percent & Count & Percent \\
\hline Core Reduction & 59 & 83.1 & 317 & 45.55 \\
\hline Bifacial Reduction & 10 & 14.08 & 270 & 38.79 \\
\hline Core Platform Trimming & 1 & 1.41 & 65 & 9.34 \\
\hline Bifacial Retouch & 1 & 1.41 & 43 & 6.18 \\
\hline Notching & 0 & 0.00 & 1 & 0.14 \\
\hline Total & 71 & 100.00 & 696 & 100.00 \\
\hline
\end{tabular}

Table 11-7. L Lipping and Longitudinal Curvature for Complete Flakes.

\begin{tabular}{|c|c|c|c|c|c|}
\hline \multirow{3}{*}{ Attribute } & \multirow{2}{*}{ Category } & \multicolumn{2}{|c|}{ Bell/Andice } & \multicolumn{2}{c|}{ Martindale } \\
\cline { 3 - 6 } & & Count & Percent & Count & Percent \\
\hline \multirow{4}{*}{ Lipping } & Absent & 62 & 87.32 & 595 & 85.49 \\
\cline { 2 - 6 } & Moderate & 6 & 8.45 & 55 & 7.90 \\
\cline { 2 - 6 } & Pronounced & 1 & 1.41 & 35 & 5.03 \\
\cline { 2 - 6 } & N/A & 2 & 2.82 & 11 & 1.58 \\
\cline { 2 - 6 } & Total & 71 & 100.00 & 696 & 100.00 \\
\hline \multirow{4}{*}{ Long. Curv. } & Absent & 69 & 97.18 & 478 & 68.68 \\
\cline { 2 - 6 } & Moderate & 2 & 2.82 & 140 & 20.11 \\
\cline { 2 - 6 } & Pronounced & 0 & 0.00 & 78 & 11.21 \\
\cline { 2 - 6 } & Total & 71 & 100.00 & 696 & 100.00 \\
\hline
\end{tabular}


Table 11-8. Platform Class and Preparation for Complete Flakes.

\begin{tabular}{|c|c|c|c|c|c|}
\hline \multirow{2}{*}{ Attribute } & \multirow{2}{*}{ Category } & \multicolumn{2}{|c|}{ Bell/Andice } & \multicolumn{2}{c|}{ Martindale } \\
\cline { 2 - 6 } & & Count & Percent & Count & Percent \\
\hline \multirow{4}{*}{ Platform Type } & Single Facet & 35 & 49.30 & 289 & 41.52 \\
\cline { 2 - 6 } & Multifaceted & 16 & 22.54 & 157 & 22.56 \\
\cline { 2 - 6 } & Collapsed & 9 & 12.68 & 77 & 11.06 \\
\cline { 2 - 6 } & Cortical & 8 & 11.27 & 51 & 7.33 \\
\cline { 2 - 6 } & Abraded & 3 & 4.23 & 122 & 17.53 \\
\cline { 2 - 6 } & Total & 71 & 100.00 & 696 & 100.00 \\
\hline \multirow{4}{*}{ Platform Prep. } & Absent & 30 & 42.25 & 410 & 58.91 \\
\cline { 2 - 6 } & Present & 37 & 52.11 & 272 & 39.08 \\
\cline { 2 - 6 } & N/A & 4 & 5.63 & 15 & 2.16 \\
\cline { 2 - 6 } & Total & 71 & 100.00 & 697 & 100.00 \\
\hline
\end{tabular}

Table 11-9. Size Class and Termination Types for Complete Flakes.

\begin{tabular}{|c|c|c|c|c|c|}
\hline \multirow{3}{*}{ Attribute } & \multirow{2}{*}{ Category } & \multicolumn{2}{|c|}{ Bell/Andice } & \multicolumn{2}{c|}{ Martindale } \\
\cline { 2 - 6 } & & Count & Percent & Count & Percent \\
\hline \multirow{4}{*}{ Size Class } & $<1 / 4$ inch & 2 & 2.82 & 29 & 4.17 \\
\cline { 2 - 6 } & $1 / 4$ inch & 12 & 16.90 & 276 & 39.66 \\
\cline { 2 - 6 } & $1 / 2$ inch & 22 & 30.99 & 190 & 27.30 \\
\cline { 2 - 6 } & $3 / 4$ inch & 16 & 22.54 & 98 & 14.08 \\
\cline { 2 - 6 } & $>1$ inch & 19 & 26.76 & 103 & 14.80 \\
\cline { 2 - 6 } & Total & 71 & 100.00 & 696 & 100.00 \\
\hline \multirow{5}{*}{ Termination } & Feather & 42 & 59.15 & 529 & 76.01 \\
\cline { 2 - 6 } & Hinge & 24 & 33.80 & 159 & 22.84 \\
\cline { 2 - 6 } & Axial & 3 & 4.23 & 7 & 1.01 \\
\cline { 2 - 6 } & Overshot & 2 & 2.82 & 1 & 0.14 \\
\cline { 2 - 6 } & Total & 71 & 100.00 & 696 & 100.00 \\
\hline
\end{tabular}

assemblage has a greater proportion of hardhammer flakes produced during earlier stages of reduction than in the Martindale assemblage (Andrefsky 2006; Patten 1999).

Table 11-10 lists the metric attributes measured on complete flakes from both components. Flakes from the Martindale component are smaller in all dimensions. The weight of flakes within the Martindale component is also the single most variable attribute within the two samples $(\mathrm{CV}=$ 2.0), followed closely by platform thickness within the Bell/Andice component $(\mathrm{CV}=1.6)$. To evaluate the perceived differences between the two samples, a two-sample t-test was conducted on maximum dimension and weight, with a null hypothesis that Martindale flakes are larger. The test returned a tvalue of -10.835 for maximum dimension $(90 \% \mathrm{CI}$ $=-14.118,-7.551)$ and -6.2181 for weight $(90 \% \mathrm{CI}$ $=-8.54,-3.89)$, and the null hypothesis can be 
Table 11-10. Metric Dimensions for Complete Flakes (in millimeters).

\begin{tabular}{|c|c|c|c|c|c|c|c|c|}
\hline \multirow{2}{*}{ Component } & \multicolumn{4}{|c|}{ Platform Dimensions } & \multicolumn{4}{|c|}{ Flake Dimensions } \\
\hline & Dimension & Mean & StD & CV & Dimension & Mean & StD & CV \\
\hline \multirow{5}{*}{ Bell/Andice } & Thickness & 9.25 & 14.83 & 1.60 & Maximum & 38.12 & 16.11 & 0.42 \\
\hline & Width & 17.70 & 9.87 & 0.56 & Length & 31.16 & 14.91 & 0.48 \\
\hline & & & & & Width & 31.19 & 13.58 & 0.43 \\
\hline & & & & & Thickness & 8.57 & 4.87 & 0.56 \\
\hline & & & & & Weight & 10.14 & 11.49 & 1.13 \\
\hline \multirow{5}{*}{ Martindale } & Thickness & 4.50 & 4.31 & 0.95 & Maximum & 27.28 & 12.76 & 0.46 \\
\hline & Width & 19.91 & 8.90 & 0.68 & Length & 22.49 & 11.25 & 0.50 \\
\hline & & & & & Width & 21.77 & 10.74 & 0.49 \\
\hline & & & & & Thickness & 5.23 & 3.94 & 0.75 \\
\hline & & & & & Weight & 3.91 & 7.84 & 2.00 \\
\hline
\end{tabular}

rejected at the .05 level of significance (Figures 113 and 11-4). Given that raw material availability was equal for both groups, these results provide additional lines of evidence that the Bell/Andice assemblage is representative of earlier stages of reduction than the Martindale assemblage.

Heat treatment within the assemblages was grouped within three ordinal categories. Flakes with obvious signs of thermal damage (e.g., potlidding, crazing, etc.) were categorized as thermally altered. Flakes with a waxy luster or traces of reddish colors were categorized as potentially heat treated. Flakes with no signs of heat treatment were categorized as not thermally altered. A similar pattern can be seen in the sample of proximal flake fragments. Previous studies of Bell/Andice assemblages in Oklahoma have shown the production of formal bifaces involved the use of heat treatment and often in multiple stages (e.g., Duncan 1995, 2014; Wyckoff 1994b, 1995). Within the Bell/Andice assemblage, 73.23 percent $(N=52)$ of the complete flakes show no evidence for thermal alteration, as opposed to 70.11 percent $(N=488)$ of the complete flakes within the Martindale assemblage that are thermally treated (Tables 11-11 and 11-12). Less heat treatment within the Bell/Andice component suggest that most lithic reduction was not oriented towards producing bifaces.

\subsubsection{Other Debitage}

In addition to complete flakes, the remaining Bell/Andice assemblage includes: 85 flake fragments and angular debitage, 14 proximal flake fragments, and 7 lateral flake fragments, all composed of chert. The remaining Martindale assemblage, also composed of chert, includes 569 incomplete flake fragments and angular debitage, 147 proximal flake fragments, and 34 lateral flake fragments. Excluding proximal flake fragments, the remaining indeterminate flake fragments and angular debitage averaged 3.0 grams within the Bell/Andice assemblage and 2.3 grams within the Martindale assemblage. The typical proximal flake fragment within both assemblages is nearly identical in nonmetric attributes to the average complete flake.

Table 11-13 lists the metric attributes measured within the proximal flake fragments from both assemblages. As with the samples of complete flakes and flake fragments, the mean dimensions of 


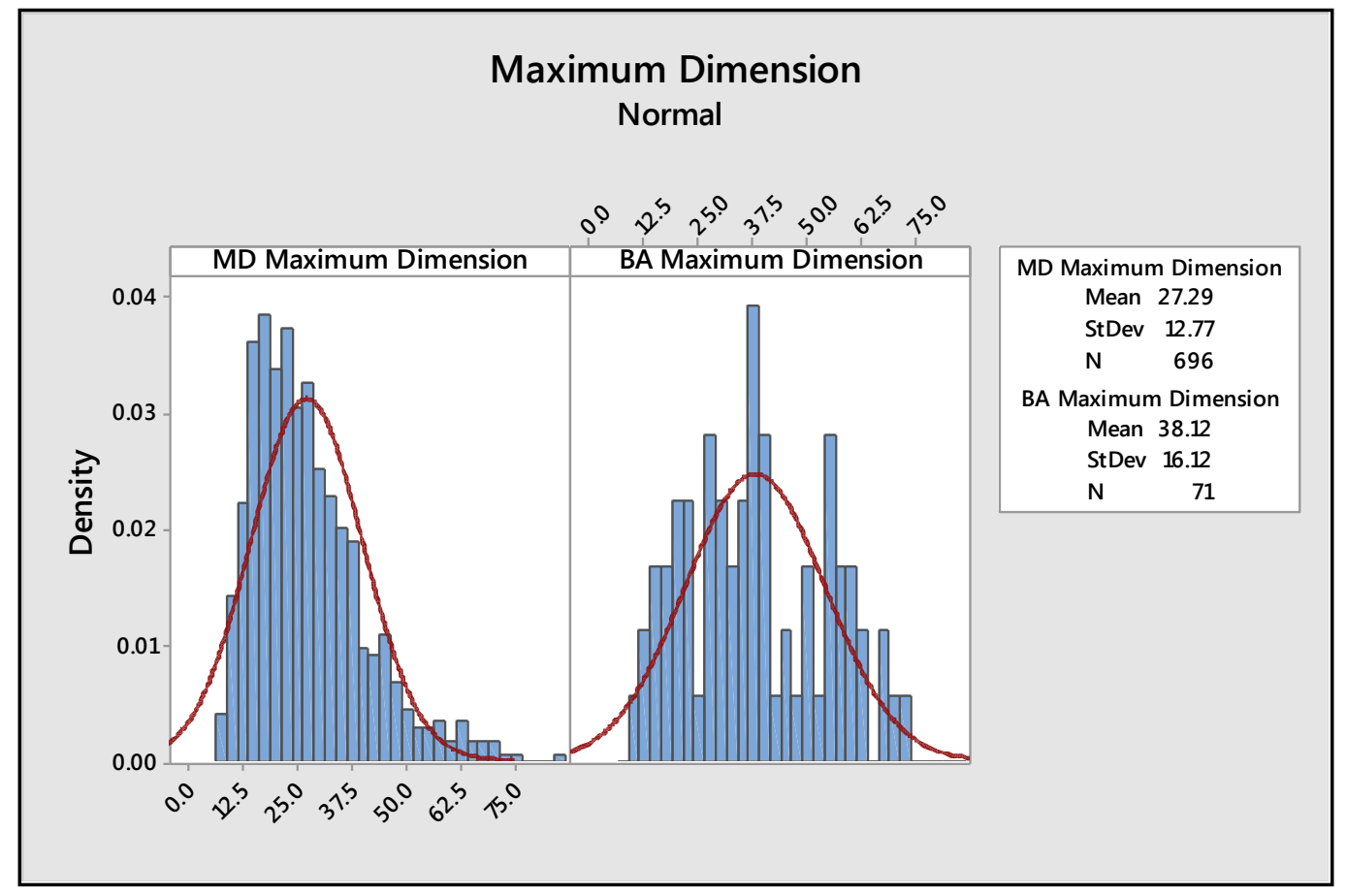

Figure 11-3. Distribution of flakes by maximum dimension within the Martindale (MD) and Bell/Andice (BA) components.

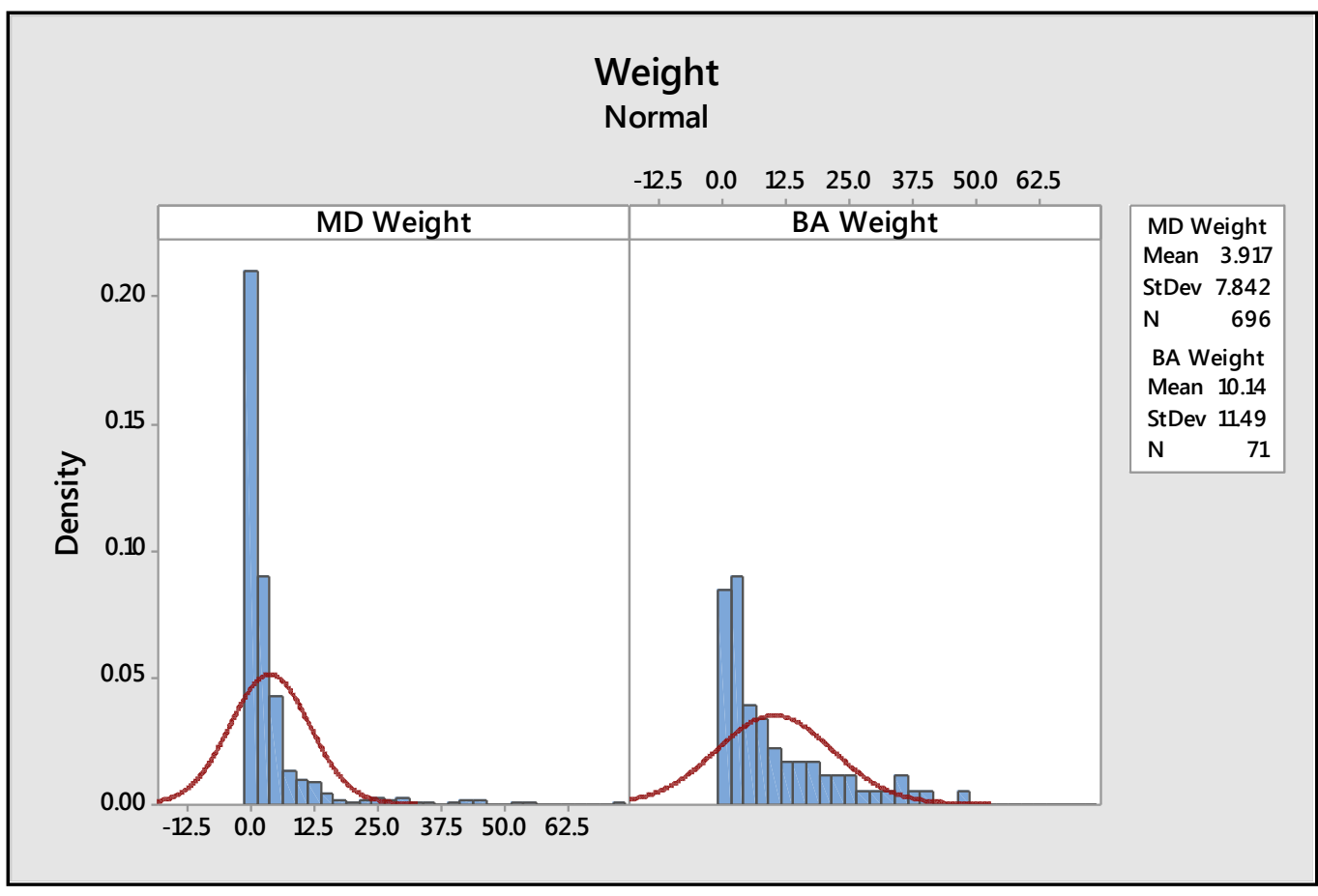

Figure 11-4. Distribution of flakes by maximum dimension within the Martindale (MD) and Bell/Andice (BA) components. 
Table 11-11. Heat Treatment within Complete Flakes.

\begin{tabular}{|c|c|c|c|c|}
\hline \multirow{2}{*}{ Category } & \multicolumn{2}{|c|}{ Bell/Andice } & \multicolumn{2}{c|}{ Martindale } \\
\cline { 2 - 5 } & Count & Percent & Count & Percent \\
\hline Thermally Altered & 9 & 12.68 & 34 & 4.89 \\
\hline Potentially Heat Treated & 10 & 14.08 & 488 & 70.11 \\
\hline Not Thermally Altered & 52 & 73.24 & 174 & 25.00 \\
\hline Total & 71 & 100.00 & 696 & 100.00 \\
\hline
\end{tabular}

Table 11-12. Heat Treatment within Proximal Flake Fragments.

\begin{tabular}{|c|c|c|c|c|}
\hline \multirow{2}{*}{ Category } & \multicolumn{2}{|c|}{ Bell/Andice } & \multicolumn{2}{c|}{ Martindale } \\
\cline { 2 - 5 } & Count & Percent & Count & Percent \\
\hline Thermally Altered & 3 & 21.43 & 12 & 9.16 \\
\hline Potentially Heat Treated & 5 & 35.71 & 80 & 61.07 \\
\hline Not Thermally Altered & 6 & 42.86 & 39 & 29.77 \\
\hline Total & 14 & 100.00 & 131 & 100.00 \\
\hline
\end{tabular}

Table 11-13. Metric Dimensions for Proximal Flake Fragments (in millimeters).

\begin{tabular}{|c|c|c|c|c|c|c|c|c|}
\hline \multirow{2}{*}{ Component } & \multicolumn{4}{|c|}{ Platform Dimensions } & \multicolumn{4}{|c|}{ Flake Dimensions } \\
\hline & Dimension & Mean & StD & CV & Dimension & Mean & StD & CV \\
\hline \multirow{5}{*}{ Bell/Andice } & Thickness & 3.53 & 3.00 & 0.85 & Maximum & 24.19 & 9.90 & 0.41 \\
\hline & Width & 10.79 & 7.81 & 0.72 & Length & 19.01 & 7.97 & 0.42 \\
\hline & & & & & Width & 20.47 & 9.33 & 0.46 \\
\hline & & & & & Thickness & 4.65 & 3.34 & 0.72 \\
\hline & & & & & Weight & 2.98 & 6.56 & 2.20 \\
\hline \multirow{5}{*}{ Martindale } & Thickness & 11.65 & 6.01 & 0.52 & Maximum & 31.96 & 15.59 & 0.49 \\
\hline & Width & 31.96 & 15.59 & 0.49 & Length & 26.47 & 14.22 & 0.54 \\
\hline & & & & & Width & 25.60 & 11.00 & 0.43 \\
\hline & & & & & Thickness & 5.81 & 3.50 & 0.60 \\
\hline & & & & & Weight & 6.08 & 8.09 & 1.33 \\
\hline
\end{tabular}

proximal flake fragments from the Bell/Andice component are larger in all dimensions, which are consistent with the analysis of complete flakes. Nonmetric attributes measured on proximal fragments are discussed individually in Chapters 9.0 and 10.0. The attributes were found to be nearly identical to the respective samples of complete flakes from both assemblages and serve as a control group for evaluating the consistency of the analysis.

Overall, the ratio of complete flakes to all other debitage is 1:1.07 within the Martindale assemblage, and 1:1.49 within the Bell/Andice assemblage. The Bell/Andice assemblage is much 
smaller, but the ratio is significantly higher. In general, a larger knapping event would be expected to produce a higher ratio of

incomplete flakes and angular debitage. Given both assemblages were produced from the same local raw materials, this suggests either natural or behavioral processes contributed to this difference. The most likely natural processes that could potentially produce a greater frequency of incomplete flakes in this context is trampling by animals. Potential behavioral processes include a difference in the skills of the knappers or the techniques employed. Assuming that both groups had equally skilled knappers, it is probable the more skilled knappers were present in the group that produced the Martindale assemblage. Alternatively, this difference could be attributed to site cleaning and maintenance. A greater frequency of broken flakes would be expected where knapping activities were not separated from living areas, or if the debitage was not routinely removed from living areas.

\subsubsection{Cores}

Seven cores were recovered from each component. Table 11-14 lists the mean, standard deviation $(\mathrm{StD})$ and coefficient of variation $(\mathrm{CV})$ of the metric dimensions recorded within the two assemblages. The cores are relatively consistent in their dimensions, with the most variation in the weight of cores from the Bell/Andice component $(\mathrm{CV}=0.79)$, and the least amount of variation within the length of cores from the Bell/Andice component $(\mathrm{CV}=0.12)$. Overall the cores from the two components are nearly identical in all dimensions. These similarities are expected given the cores were likely derived from locally available cobbles, and the initial reduction techniques were the same.

Table 11-15 lists the nonmetric attributes monitored within the assemblage. All cores within the Martindale component have less than 50 percent cortical cover and at least three flake scars. In contrast, three ( 43.0 percent) of the cores within the Bell/Andice component have more than 50 percent cortical cover, and two have only one to two flake scars, suggesting they were slightly less worked than in the Martindale component. Both assemblages have the same proportion of core types and were flaked from multiple directions, and indicate the same strategy was employed in each assemblage. Overall, the ratio of cores to lithic debitage within the Martindale component is $1: 206.57$ and the ratio of cores to debitage within the Bell/Andice component is 1:25.57, and reflects the considerable difference in the amount of lithic reduction between the two assemblages.

Table 11-14. Metric Dimensions for Cores (in millimeters).

\begin{tabular}{|c|c|c|c|c|}
\hline \multirow{3}{*}{ Component } & \multicolumn{4}{|c|}{ Metric Dimensions } \\
\cline { 2 - 5 } & Dimension & Mean & StD & CV \\
\hline \multirow{3}{*}{ Bell/Andice } & Length & 69.48 & 8.53 & 0.12 \\
\cline { 2 - 5 } & Width & 47.49 & 9.35 & 0.19 \\
\cline { 2 - 5 } & Thickness & 31.55 & 16.95 & 0.53 \\
\cline { 2 - 5 } & Weight & 101.50 & 81.13 & 0.79 \\
\hline \multirow{3}{*}{ Martindale } & Length & 63.43 & 14.24 & 0.22 \\
\cline { 2 - 5 } & Width & 49.25 & 10.17 & 0.20 \\
\cline { 2 - 5 } & Thickness & 32.60 & 10.56 & 0.32 \\
\cline { 2 - 5 } & Weight & 98.20 & 72.83 & 0.74 \\
\hline
\end{tabular}


Table 11-15. Nonmetric Attributes Recorded on Cores.

\begin{tabular}{|c|c|c|c|c|}
\hline \multirow{2}{*}{ Cortex } & \multicolumn{2}{|c|}{ Bell/Andice } & \multicolumn{2}{|c|}{ Martindale } \\
\hline & Count & Frequency & Count & Frequency \\
\hline 0 & 0 & 0.00 & 1 & 0.14 \\
\hline $1-25$ & 2 & 0.29 & 1 & 0.14 \\
\hline $26-50$ & 2 & 0.25 & 5 & 0.71 \\
\hline $21-75$ & 3 & 0.43 & 0 & 0.00 \\
\hline Total & 7 & 1.00 & 7 & 1.00 \\
\hline \multirow{2}{*}{ Type } & \multicolumn{2}{|c|}{ Bell/Andice } & \multicolumn{2}{|c|}{ Martindale } \\
\hline & Count & Frequency & Count & Frequency \\
\hline Cobble & 4 & 0.57 & 4 & 0.57 \\
\hline Macro Flake & 2 & 0.29 & 2 & 0.29 \\
\hline Fragment & 1 & 0.14 & 1 & 0.14 \\
\hline Total & 7 & 1.00 & 7 & 1.00 \\
\hline \multirow{2}{*}{ Scar Count } & \multicolumn{2}{|c|}{ Bell/Andice } & \multicolumn{2}{|c|}{ Martindale } \\
\hline & Count & Frequency & Count & Frequency \\
\hline $1-2$ & 2 & 0.29 & 0 & 0.00 \\
\hline 3-4 & 1 & 0.14 & 2 & 0.29 \\
\hline $5-6$ & 2 & 0.29 & 2 & 0.29 \\
\hline $7+$ & 2 & 0.29 & 3 & 0.43 \\
\hline Total & 7 & 1.00 & 7 & 1.00 \\
\hline \multirow{2}{*}{ Direction } & \multicolumn{2}{|c|}{ Bell/Andice } & \multicolumn{2}{|c|}{ Martindale } \\
\hline & Count & Frequency & Count & Frequency \\
\hline Single & 1 & 0.14 & 1 & 0.14 \\
\hline Multiple & 6 & 0.86 & 6 & 0.86 \\
\hline Total & 7 & 1.00 & 7 & 1.00 \\
\hline
\end{tabular}

Table 11-16. Metric Dimensions Recorded on Edge-Modified Flakes (in millimeters).

\begin{tabular}{|c|c|c|c|c|c|c|c|}
\hline \multicolumn{5}{|c|}{ Bell/Andice } & \multicolumn{4}{c|}{ Martindale } \\
\hline Dimension & Mean & StD & CV & Dimension & Mean & StD & CV \\
\hline Maximum & 49.14 & 15.90 & 0.32 & Maximum & 42.26 & 15.56 & 0.37 \\
\hline Length & 46.93 & 16.62 & 0.35 & Length & 39.32 & 16.19 & 0.41 \\
\hline Width & 39.50 & 12.94 & 0.33 & Width & 31.49 & 12.80 & 0.41 \\
\hline Thickness & 13.27 & 6.37 & 0.48 & Thickness & 9.26 & 5.85 & 0.63 \\
\hline Weight & 26.31 & 25.28 & 0.96 & Weight & 13.78 & 16.89 & 1.23 \\
\hline Edge Angle & 61.67 & 12.18 & 0.02 & Edge Angle & 55.32 & 11.48 & 0.21 \\
\hline
\end{tabular}




\subsubsection{Lithic Debitage Discussion}

The analyses of cores and debitage from the two components indicate both assemblages contain debitage from most stages of core reduction. However, flakes from the Bell/Andice assemblage are larger in all dimensions and skewed towards early and middle stages of biface reduction. The ratio of cores to debitage within the Bell/Andice assemblage (1:25.6) is also significantly lower than in the Martindale assemblage (1:206.6), and the ratio of bifaces to cores is slightly lower in the Bell/Andice assemblage (0.85:1) than in the Martindale assemblage (1.14:1).

Rather than a complete reduction sequence where bifacial tools were produced from local cobbles, the Bell/Andice assemblage most likely represent two separate reduction strategies; one that involved simple core-flake reduction of local cobbles to create informal tools, and a second involved a limited amount of maintenance of imported bifacial tools. In contrast, the majority of the Martindale assemblage reflects a complete bifacial reduction trajectory.

In exactly what sequence the process of tool manufacture and use unfolds has been linked to several different design constraints (Hayden et al. 1996). Some constraints include; the time and risk involved in the completion of a particular task (Torrence 1989), group mobility (Parry and Kelly 1987; Shott 1986), the characteristics of the available raw material (Andrefsky 1994; Shelley 1993), the skills of the individual knapper (Stout 2002), and the range of techniques and technology available (Andrefsky 2006; Odell 2006). Given the presence of large bifaces in both assemblages and nearly identical cores, it is assumed that reduction techniques and technological methods were relatively similar between the two groups. Additionally, chert cobbles of medium quality were locally available near the site and the primary source for the cores and debitage within both components, with few exceptions. The overall mobility of the two groups was also probably similar given the absence of any distantly sourced raw materials or exotic items.

Given design constraints were relatively equal for both components, the Martindale assemblage probably represents a variety of daily activities associated with multiple tasks that included manufacture and maintenance of stone tools for future, high risk activities, which included hunting. In contrast, stone tool production within the Bell/Andice component was likely related to immediate, low risk needs involved specifically in the processing of plants and small animals with stone boiling techniques. Edge-modified flakes from the Bell/Andice component are also both larger and less variable in all dimensions (Table 1116). This implies larger flakes were intentionally selected for use as informal tools within this component, whereas within the Martindale assemblage waste flakes from a wider range of the reduction sequence were selected for informal tools. With raw material availability, mobility, and technology having been relatively equal constraints, the differences detected between the two assemblages are likely related to the nature of the specific tasks performed at the site.

\subsection{BURNED ROCK ASSEMBLAGES}

Considerable differences were detected between the frequencies and sizes of burned rocks. First and foremost, eight burned rock features were identified in the Bell/Andice component, while no clear burned rock features were identified in the Martindale component. Burned rocks were present in the Martindale component, even some scattered in two of three basin-shaped, charcoal-stained hearths (Features 26 and 33), but no Martindale features contained a sufficient number of burned rocks to be classified as burned rock features. The Bell/Andice component had 1.7 times the quantity and 4.2 times the mass of burned rocks in a third less area (see Table 11-1; Figures 11-5 and 11-6). 


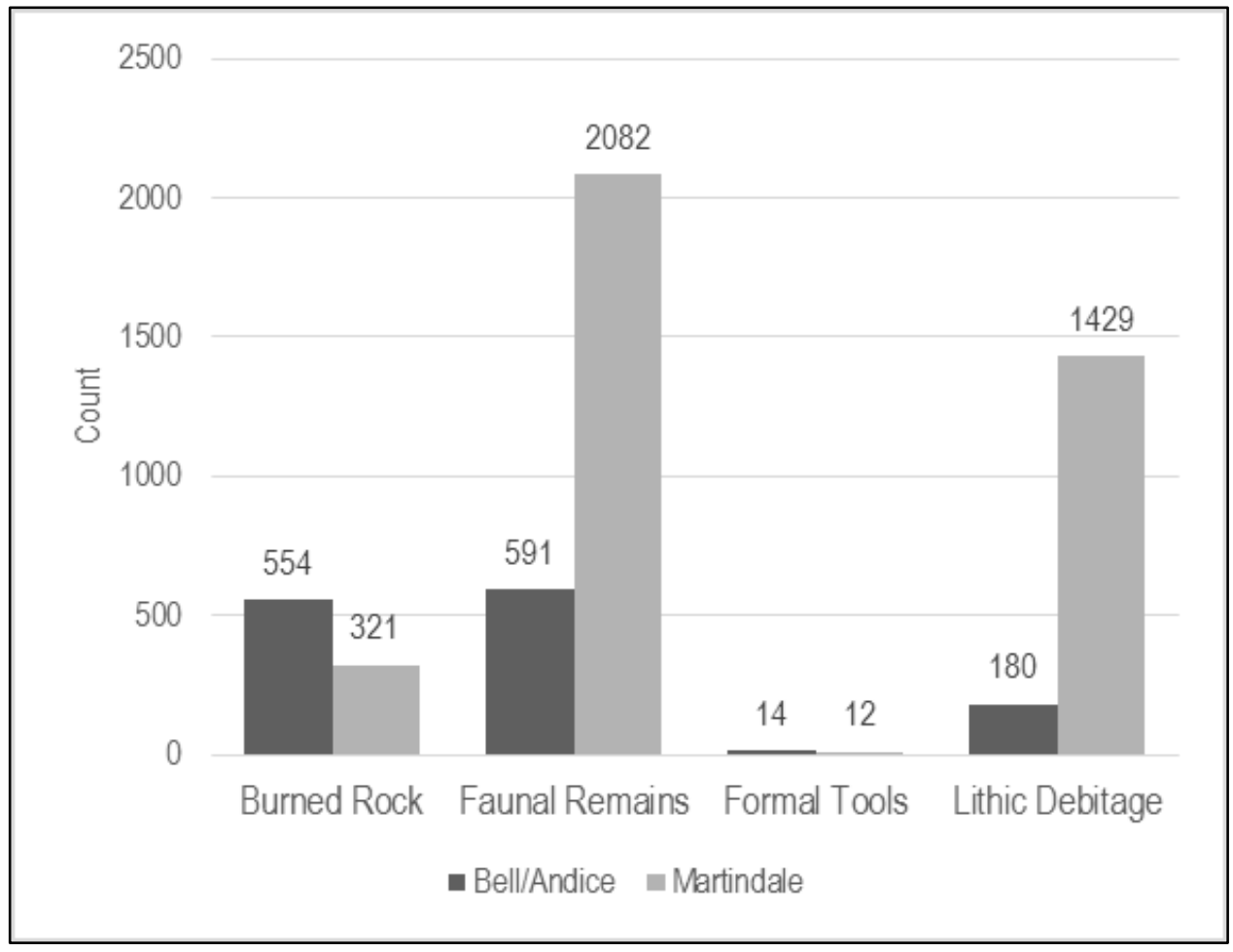

Figure 11-5. Comparison of materials classes of the two components.

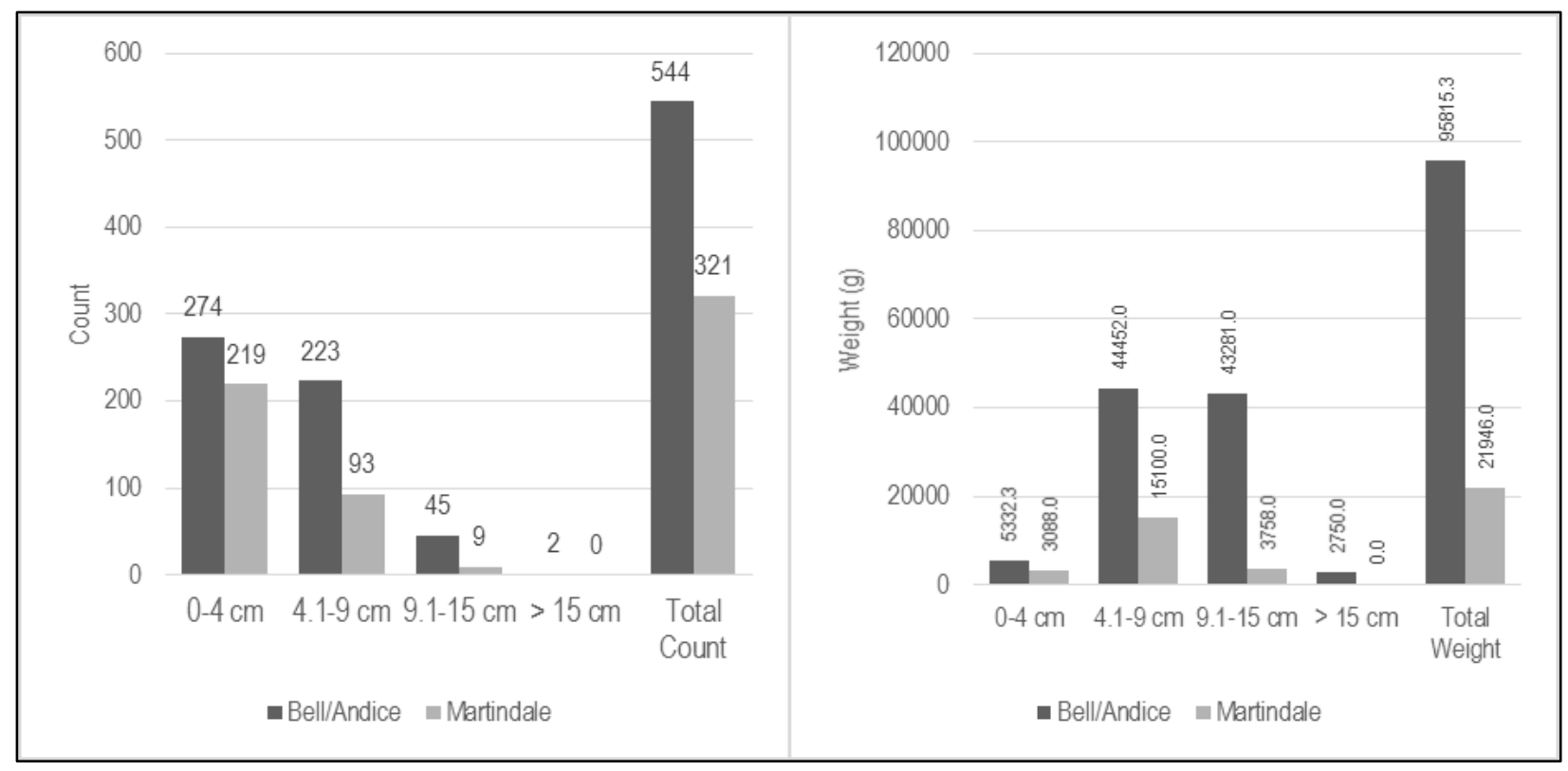

Figure 11-6. Comparison of burned rock by count and weight between components. 
Also the Bell/Andice component contained larger rocks in the burned rock features with smaller pieces scattered beyond the features. Overall the Martindale component exhibited relatively small, scattered burned rocks as though they were employed beyond the point they were no longer suitable to transfer heat and discarded. Rock types were similar as they probably originated from the same adjacent hilltop since the alluvial deposits in the immediate area appear nearly absent of rocks.

\subsection{FEATURE COMPARISONS}

Although both yielded features, the Bell/Andice component had 2.5 times the number in a third less area, with eight burned rock features. The Martindale component had three shallow basin hearths evidenced by circular stained sediments and no true burned rock features (Table 11-17). This indicates the Bell/Andice occupation focused more on heating and cooking with rocks, likely stone boiling as argued in previous chapters. The features in the Bell/Andice component probably represent both the heating rocks in in situ fires followed by dumping used rocks in small clusters. In contrast, the hearth features in the Martindale component exhibited no burned rock concentrations, and overall fewer rocks scattered in shallow basins. Although the Martindale component revealed rocks, it appears a slightly different cooking strategy was employed that involved less burned rocks. The small organic stained hearths probably functioned for general heating and cooking. The overall lower density of features in the Martindale component may reflect a more dispersed campsite or greater diversity in tasks.

\subsection{VERTEBRATE FAUNAL REMAINS}

Major and significant differences are reflected in the two faunal assemblages excavated at a single location separated by a short period of time. The Martindale component yielded 3.5 times the number of bone fragments and twice as many species to reflect a much greater diversity of faunal resources than the Bell/Andice component (see Tables 11-1 and 11-18, see Figure 11-6). Populations in the Martindale component procured fish, birds, turtles, rabbits, canid, deer, and pronghorn, which represent multiple environmental zones and most probably were employed for food (Table 11-18). The few rodent and snake remains can be questioned as to their cultural use, as they often occur naturally in similar deposits.

The younger Bell/Andice component yielded only three taxa (i.e., deer, cottontail, and jackrabbits) considered probable food remains, as the single canid and fish bones were potentially displaced and the few tiny rodent bones may be natural (see Table 11-18). These three taxa are also represented in the Martindale component, which exhibits at least four additional taxa. Apparently, the population that occupied the Bell/Andice component focused mostly on procurement of small game, or that was all available in the immediate environment. The major frequency and species differences between the two components may relate to the differences in overall group composition, and a shorter length of occupations during the Bell/Andice period. Potentially, the differences may also reflect the amount of area excavated, which was one-third less in the Bell/Andice than the Martindale component.

One notable similarity in the faunal assemblages was both lack bison remains, but this is particularly significant for the Bell/Andice component. As discussed previously, bison are documented in the central Texas region for this general Bell/Andice period (see Chapter 3.0). Populations employing Bell/Andice projectile points are noted for bison exploitation, so the absence of bison is truly unexpected and unusual. Here the Bell/Andice faunal assemblage represents the exploitation of a range of primarily same game resources as the Martindale component instead of specialized bison hunting. 
Table 11-17. Comparison of Feature Characteristics.

\begin{tabular}{|c|c|c|c|c|c|c|c|c|}
\hline Component & $\begin{array}{c}\text { Feature } \\
\text { No. }\end{array}$ & Feature Type & $\begin{array}{l}\text { Feature } \\
\text { Size } \\
(\mathrm{cm})\end{array}$ & Profile & $\begin{array}{l}\text { Stained } \\
\text { Matrix }\end{array}$ & $\begin{array}{l}\text { Depth } \\
\text { (cm) } \\
\text { below } \\
\text { surface }\end{array}$ & $\begin{array}{c}\text { Burned Rock } \\
\text { Counts/Wt. } \\
\text { (g) }\end{array}$ & $\begin{array}{c}\delta^{13} \mathrm{C} \text { Corrected C14 } \\
\text { Date B.P. } \\
\text { (Material) }\end{array}$ \\
\hline \multirow{10}{*}{ Bell/Andice } & 3 & $\begin{array}{l}\text { Burned rock cooking } \\
\text { feature }\end{array}$ & 90 & $\begin{array}{l}\text { Shallow } \\
\text { basin }\end{array}$ & Charcoal & $209-229$ & 201/unknown & $\begin{array}{l}5290 \pm 40 \\
\text { (Charcoal) }\end{array}$ \\
\hline & 16 & Burned rock hearth & $25 \times 25$ & $\begin{array}{l}\text { Shallow } \\
\text { basin }\end{array}$ & Charcoal & $247-254$ & $10 / 1,766$ & \\
\hline & 17 & $\begin{array}{c}\text { Ground stone } \\
\text { Cache }\end{array}$ & $24 \times 20$ & Flat & None & $233-239$ & $4 / 2,300$ & - \\
\hline & 22 & Burned rock dump & $35 \times 40$ & Flat & None & $220-230$ & $13 / 1,272$ & - \\
\hline & 24 & $\begin{array}{l}\text { Burned rock cooking } \\
\text { feature }\end{array}$ & $50 \times 60$ & Flat & Dark stain & $216-233$ & $41 / 13,100$ & $\begin{array}{c}5580 \pm 30 \text { (bone) } \\
5370 \pm 30 \\
\text { (charcoal), } 5280 \pm \\
30 \text { (charcoal), } 5540 \\
\pm 40 \text { (sediment) }\end{array}$ \\
\hline & 27 & Burned rock dump & $15 \times 40$ & Flat & None & $212-217$ & $4 / 24,100$ & - \\
\hline & 28 & Burned rock dump & $40 \times 45$ & Flat & None & $218-231$ & $11 / 676$ & $5280 \pm 30$ (charcoal) \\
\hline & 29 & $\begin{array}{l}\text { Burned rock cooking } \\
\text { feature }\end{array}$ & $100 \times 70$ & $\begin{array}{c}\text { Shallow } \\
\text { saucer basin }\end{array}$ & $\begin{array}{l}\text { Dark } \\
\text { mottles, } \\
\text { charcoal }\end{array}$ & $230-242$ & $92 / 15,203$ & $\begin{array}{c}5340 \pm 30 \\
\text { (charcoal), } 5370 \pm \\
30 \text { (charcoal), } 5360 \\
\pm 40 \text { (sediment) }\end{array}$ \\
\hline & 30 & Burned rock hearth & $30 \times 70$ & Basin & None & $229-238$ & $6 / 1303$ & $\begin{array}{l}5680 \pm 50 \\
\text { (sediment) }\end{array}$ \\
\hline & 32 & Burned rock hearth & $120 \times 83$ & Flat & Dark stain & $226-234$ & $37 / 7,930$ & - \\
\hline
\end{tabular}




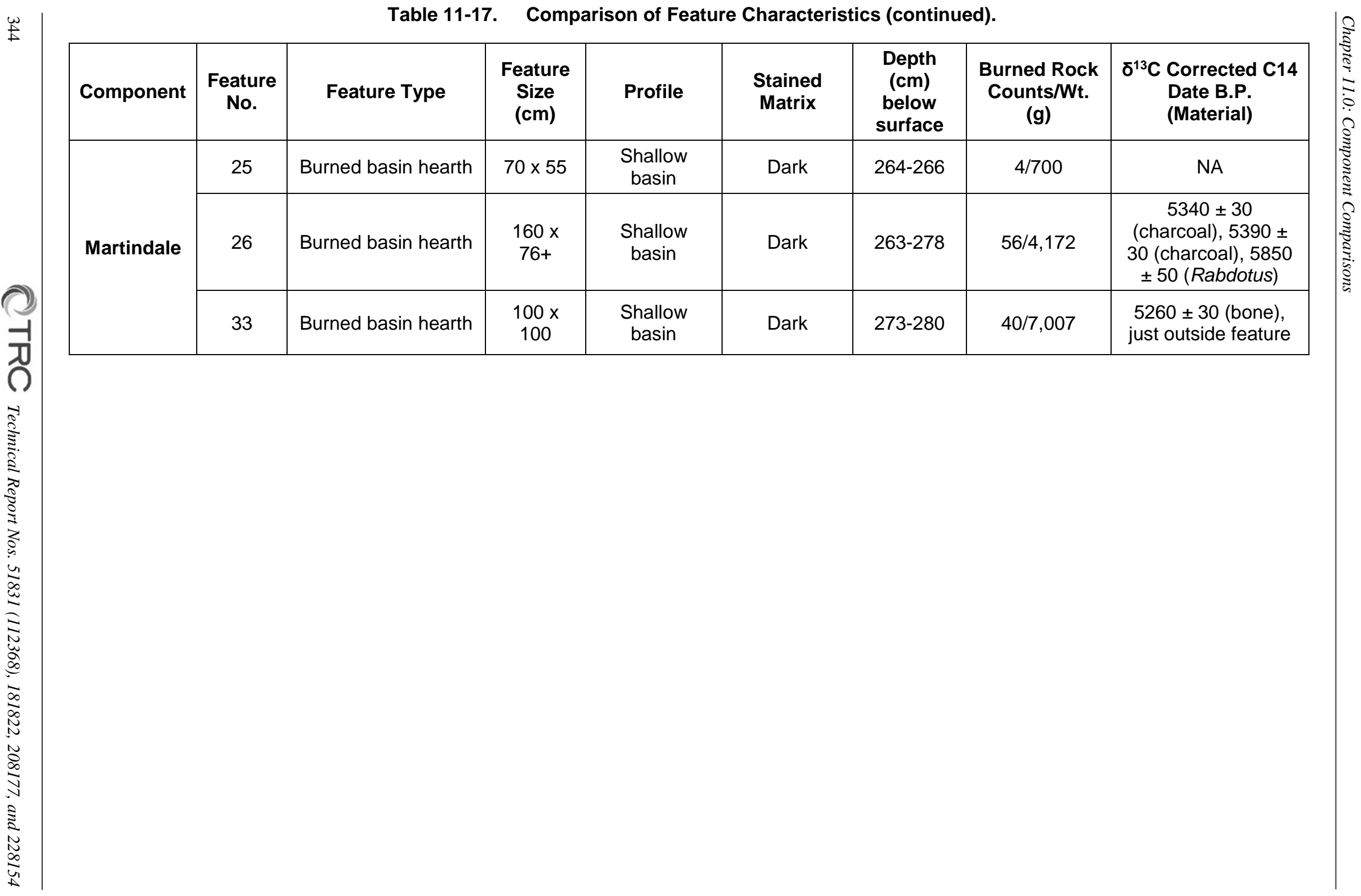


Table 11-18. Comparison of Vertebrate Faunal.

\begin{tabular}{|c|c|c|c|c|c|c|c|}
\hline \multirow[b]{2}{*}{ Taxon } & \multirow[b]{2}{*}{ Species } & \multicolumn{3}{|c|}{ Bell/Andice Component } & \multicolumn{3}{|c|}{ Martindale Component } \\
\hline & & Count & Weight (g) & $\begin{array}{l}\text { Minimum No. of } \\
\text { Individuals }\end{array}$ & Count & Weight (g) & $\begin{array}{l}\text { Minimum No. of } \\
\text { Individuals }\end{array}$ \\
\hline Dog/Coyote & Indeterminate & 1 & 0.5 & 1 & 3 & 0.9 & 1 \\
\hline Deer & Indeterminate & 3 & 14.6 & 1 & & & \\
\hline \multirow[t]{2}{*}{ Deer size artiodactyl } & Indeterminate & 95 & 128.9 & & 139 & 434.9 & \\
\hline & Whitetail & & & & 5 & 7.4 & 3 \\
\hline Pronghorn & Indeterminate & & & & 8 & 70.7 & 2 \\
\hline \multirow[t]{3}{*}{ Rabbit family } & Indeterminate & 316 & 68 & & 423 & 86.3 & \\
\hline & Cottontail & 5 & 3.5 & 1 & 50 & 12.2 & 4 \\
\hline & Jackrabbit & 33 & 16.1 & 3 & 62 & 44.4 & 5 \\
\hline \multirow[t]{4}{*}{ Rodent } & Indeterminate & 7 & 0.6 & 2 & 137 & 11.1 & \\
\hline & Gopher? & & & & 2 & 0.2 & 1 \\
\hline & Vole? & & & & 2 & 0.1 & 1 \\
\hline & Rat? & & & & 1 & 0.1 & 1 \\
\hline Snake & Indeterminate & & & & 35 & 4.5 & 1 \\
\hline \multirow[t]{2}{*}{ Turtle } & Indeterminate & & & & 37 & 11.6 & 1 \\
\hline & Softshell & & & & 16 & 4.2 & 1 \\
\hline \multirow[t]{4}{*}{ Fish } & Indeterminate & & & & 42 & 5 & \\
\hline & Catfish & & & & 2 & 0.4 & 1 \\
\hline & Crappie? & & & & 2 & 0.3 & 1 \\
\hline & Gar & 1 & 0.1 & 1 & 64 & 3.9 & 2 \\
\hline \multirow[t]{3}{*}{ Bird } & Indeterminate & & & & 8 & 1.6 & 1 \\
\hline & Grouse & & & & 4 & 0.9 & 3 \\
\hline & c.f. Grouse & & & & 4 & 3.1 & \\
\hline Bird & Indeterminate & & & & 1 & 0.4 & \\
\hline Indeterminate & - & 130 & 46 & & 1035 & 127.8 & \\
\hline Total & & 591 & 278.3 & 9 & 2082 & 832 & 29 \\
\hline
\end{tabular}


A second similarity in the two assemblages is the fact bones from the diverse species were intensively smashed, which resulted in very small (less than 3 $\mathrm{cm}$ ) fragments. This indicates similar processing of meat resources, and bone marrow and grease were also targeted by both populations. This probably reflects similarity in the cooking process, boiling to extract the grease, or similar nutritional needs. A third similarity is both assemblages represent a fall season of occupation. Although the data is limited, what is available indicates two fall events.

\subsection{PLANT ASSEMBLAGES}

Plant preservation was notably poor in the macrobotanical and microfossil analyses, which limit our understanding and the overall frequencies of prehistorically exploited plant resources. Microfossil analyses indicate plant use occurred in both components, and similar plants were employed, including wild gourds, multiple grasses, and geophytes (Table 11-19). The relative roles of plant resources in the Martindale and Bell/Andice economies are unclear due to poor preservation.

Starch grains on an edge-modified flake and on burned rocks revealed that true lily (Lilium sp.) bulbs were collected, sliced, and cooked. Bulb starches were not recovered from grinding/pounding artifacts, which makes sense from a processing standpoint as cooking lily bulbs do not require grinding for consumption. Damaged and ground starch grains also testify to processing techniques. Gelatinized starch grains indicate plants were cooked with heat and water. Lipid residue analysis targeted mostly burned rocks and consistently documented both plant and animal resources cooked with the rocks.

A few charred cheno-am seeds - weedy annuals (Chenopodiaceae and Amaranthaceae) and grass seeds were recovered from the Martindale component, in contrast to no cheno-am seeds in the Bell/Andice component. Although these plants are not technically the same, both types produce thousands of tiny seeds and are easily collected when mature in late summer and fall. Although charred grass and cheno-am seeds were not in the Bell/Andice component, starch grain analyses documents the use of grass seeds. The latter seeds document procurement of these low-ranked, high cost resources employed in conjunction with mostly low-ranked meat resources. Obviously, the need for low ranked resources out weighted procurement and processing costs.

Processing plants was similar as the high-powered use-wear results on multiple edge-modified flakes from both components depict plant residues on similar tools. In support of plant processing, each component yielded multiple manos and metates, some that reveal grinding of grass starches as evidenced by damaged starch grains from processing. Similar starch damage occurred in both components. The only difference in the two phytolith assemblages is an absence of wellpreserved warm, humid Panicoid-type grasses in the earlier Martindale component. The presence of wild gourds in both supports their cultural use and availability.

\subsection{PATTERNS OF HUMAN BEHAVIOR}

Clear vertical separation of each component combined with excellent horizontal context allowed detection of multiple behavioral patterns in both components, which strongly support each represented a single use episode. The Bell/Andice component revealed a broad pattern of intensive cooking with rocks and discarding used materials across $51 \mathrm{~m}^{2}$ in Block B (Figure 11-7). The eight burned rock features clustered in two areas, one with three features towards the western side and another with five towards the eastern side. The high density burned rock areas were nearly all recognized features with one exception of scattered burned rocks concentrated immediately west of cooking Feature 29. Three dense, smashed bone concentrations were immediately adjacent to 
Table 11-19. Comparison of Plant Indicators from Technical Analyses.

\begin{tabular}{|c|c|c|}
\hline Technical Analyses & Bell/Andice & Martindale \\
\hline \multicolumn{3}{|l|}{ Starch Grain } \\
\hline Lenticular Grass Starch & 1 & 7 \\
\hline Other Grass Starch & 2 & \\
\hline Unidentified Starch & 8 & 2 \\
\hline Liliaceae Starch & 2 & 6 \\
\hline Damaged & 3 & 1 \\
\hline \multicolumn{3}{|l|}{ Eroded } \\
\hline Ground & 2 & 1 \\
\hline Gelatinized & 5 & 4 \\
\hline \multicolumn{3}{|l|}{ Lipid Residue } \\
\hline$\beta$-Stosterol & yes & yes \\
\hline Cholesterol & yes & yes \\
\hline Dehydroabietic acid & yes & yes \\
\hline Interpretation & plant \& animal & plant \& animal \\
\hline \multicolumn{3}{|l|}{ High-Powered Use-Wear } \\
\hline Plant Residues & 5 = starch, tissue, raphides, wood & 7 = starch, tissue, wood \\
\hline Animal Residues & $2=$ bone, hair & $6=$ bone, hair \\
\hline Scraping functions & $2=$ plant, wood & \\
\hline Cutting Functions & $3=$ plant hard & $9=$ hide, plant, soft \\
\hline \multicolumn{3}{|l|}{ Slicing Functions } \\
\hline Whittling Functions & 2 = wood, hard & $3=$ wood, plant \\
\hline Unknown Functions & 2 & 5 \\
\hline Feather & & 1 \\
\hline Fish Scale & 1 & \\
\hline \multicolumn{3}{|l|}{ Phytolith } \\
\hline Wild gourd (Cucurbits) & yes & yes \\
\hline Grass & yes & yes \\
\hline Tree & burned, unburned & burned, unburned \\
\hline Chloridoid Grass & yes, some burned & yes, some burned \\
\hline Panicoid Grass & burned, unburned & yes, some burned \\
\hline Pooids Grass & & rare \\
\hline \multicolumn{3}{|l|}{ Sedges } \\
\hline Sponge Spicules & yes & yes \\
\hline \multicolumn{3}{|l|}{ Macrobotanical } \\
\hline Wood Identified & hardwood & Oak \\
\hline Grass Seeds & & 8 \\
\hline Cheno-am Seeds & & 2 \\
\hline Hackberry Nutlets & 4 & \\
\hline
\end{tabular}


burned rock features, usually on the northern side. This close association of clustered bone fragments next to heating and/or cooking features supports a direct connection between the two. This implies following the bone cooking process, the undesired waste fragments were discarded close to the cooking facility. The relatively small clusters of dense bone fragments indicate they were probably dumped from small containers, which occurred minimally three times.

In the Bell/Andice component, the area between the two feature clusters yielded the only relatively dense lithic debitage concentration and discarded formal and informal tools, plus a ground stone cache, Feature 17 (Figure 11-7). Two thermally fractured bifaces were some of the chipped stone tools discarded around the discarded burned rocks in Feature 22. The two previously used bifaces, as evident from use-wear, exhibited heat fractures which indicates they were likely once discarded into hot fires. Since both were recovered away from heating elements it is assumed they were removed from the fire and discarded. This discard pattern is unique and unlike that observed in the Martindale component. Also the single lithic concentration in N72/E83, scattered debitage that surrounded that concentration, the broken discarded stone tools, and the ground stone tool cache were discarded between the two feature clusters.

The Martindale component also revealed specific patterns of material use and discard across the 74 $\mathrm{m}^{2}$ in Blocks B and D (Figure 11-8). The close spatial association of dense concentrations of small bone fragments to cooking Features 25 and 33 implies that animal resources were processed at these features then discarded nearby. The one cluster of bone fragments and burned rock at the northern end of Block D may indicate another hearth just beyond the excavation. The cluster of burned rock and bone may also be interpreted as a discard zone. Areas of discard are noted elsewhere on site.
The overall pattern indicates that three cooking stations were in operation at the same time, most likely reflecting individual work areas and potentially nuclear families. Each work area was similar representing cooking stations for the boiling bone fragments to render grease, followed by the discard of bone fragments.

Two hammer stones not directly associated with the three lithic debris clusters or the smashed bone concentrations imply the lithic and bone clusters probably represent discarded materials, rather than in situ knapping and pounding stations. This is supported by their positions with other discarded materials.

Both components revealed quite similar bone processing, cooking, and discard patterns as dense concentrations of highly fragmented bones, which represent multiple diverse species, were generally deposited next to cooking features or discarded in small concentrations. This reflects a broad repeated pattern employed over time by different groups. Both populations also targeted bone grease in the fall season and extracted it through a similar stone boiling process, followed by discarding residues in similar manner.

Recycling of ground stone was also practiced by both populations, as minimally two pieces that apparently functioned as ground stone, were recovered from heating and cooking features and exhibited evidence they had been heated. If needed or handy, ground stone pieces were sometimes recycled as burned rocks following their original grinding use.

A major difference in the two components was the relationship between the classes and frequency of lithic debitage. The high frequency in the Martindale component revealed a well-defined horizontal distribution pattern. Three localized concentrations of lithic debitage were documented and reflect either individual in situ knapping areas, or where waste lithic debris from knapping task(s) 


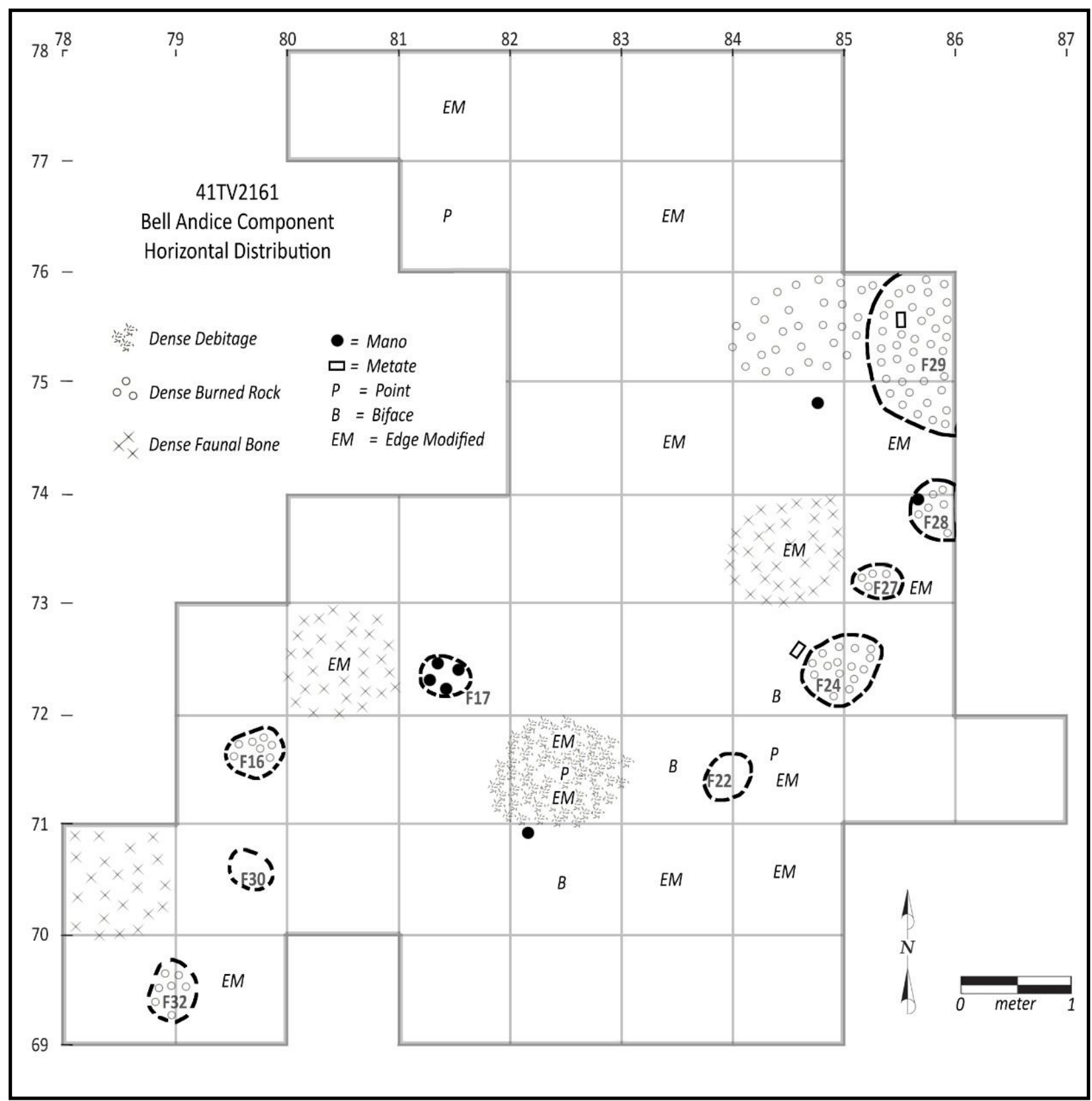

Figure 11-7. Composite of horizontal distribution of major materials classes in Bell/Andice component. 


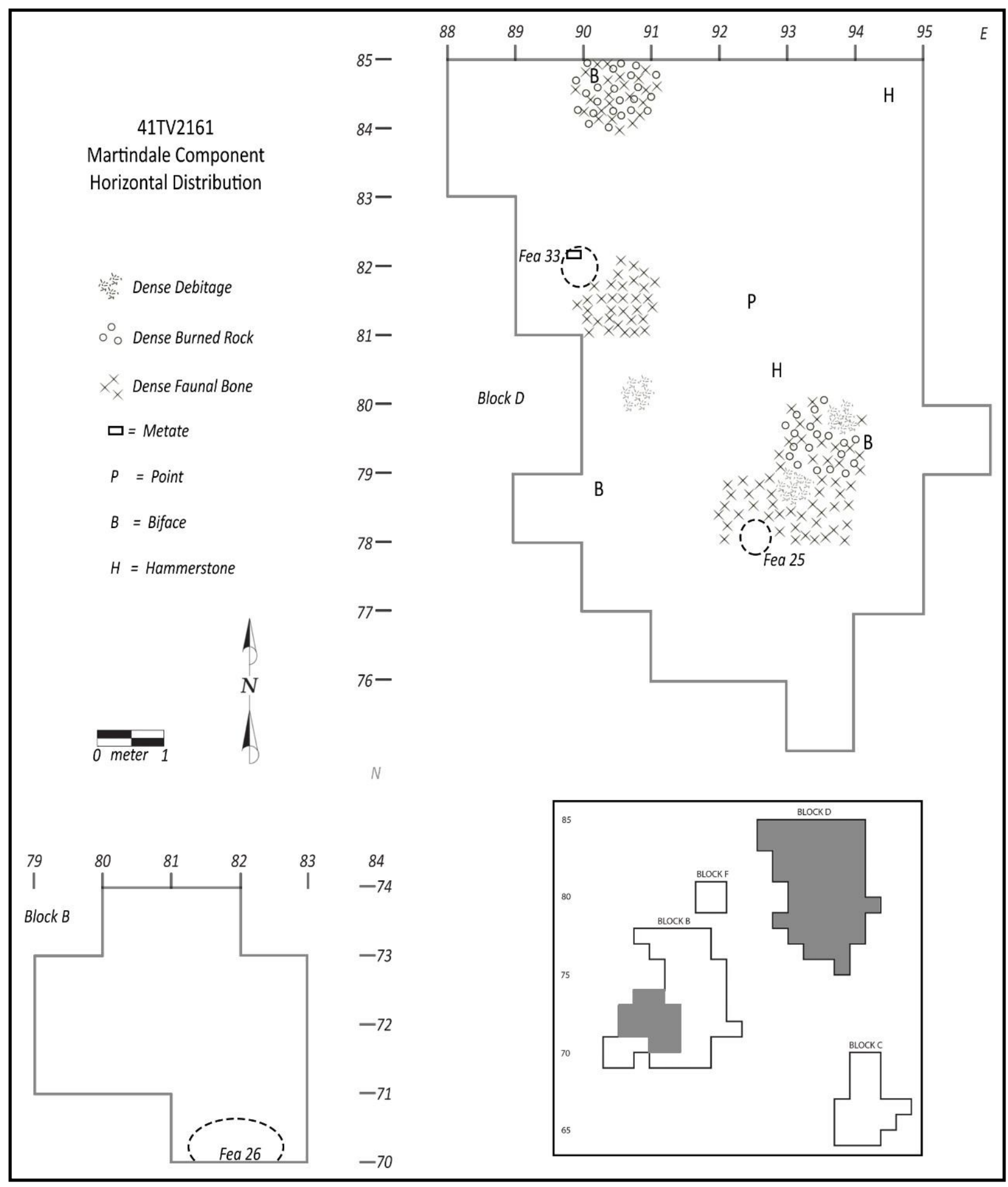

Figure 11-8. Composite of horizontal distribution of major materials classes in Martindale component. 
was dumped. The latter more likely as these lithic concentrations also yielded burned rocks and bone scraps. This discard pattern reflects intentional cleaning/maintenance, not unlike the Bell/Andice component where diverse debris was also selectively discarded between the two burned rock clusters. The Martindale debitage classes reflect a greater commitment towards formal tool production and refurbishing in contrast to the Bell/Andice lithic debris that focused mostly on core reduction and production of large flakes.

Fine screening sediments from nearly every hearth and burned rock feature in both components yielded smashed bone fragments and small lithic debitage, nearly all less than $6.4 \mathrm{~mm}$ (Table 11-20), though the frequency of these small items varied considerably between features. Interestingly, these materials were present in in situ hearths and burned rock dumps. Since deposits outside features were not fine screened, it is unclear if this represents a discard pattern or a reflection of the entire area.

Table 11-20. Comparison of Chert Debitage and Bone Fragments less than $6.4 \mathrm{~mm}$ in Size Recovered in Cultural Features.

\begin{tabular}{|c|c|c|}
\hline \multicolumn{3}{|c|}{ Bell Andice Component } \\
\hline Feature No. & Chert (Qty.) & Bone (Qty.) \\
\hline 16 & $?$ & $?$ \\
\hline 22 & 2 & 97 \\
\hline 24 & 7 & 230 \\
\hline 27 & 0 & 4 \\
\hline 28 & 19 & 148 \\
\hline 29 & 448 & 724 \\
\hline 30 & 2 & 85 \\
\hline 32 & 6 & 156 \\
\hline \multicolumn{3}{|c|}{ Martindale Component } \\
\hline Feature No. & Chert (Qty.) & Bone (Qty.) \\
\hline 25 & 2 & 50 \\
\hline 26 & 24 & 454 \\
\hline 31 & 125 & 1,663 \\
\hline
\end{tabular}

\subsection{CONCLUSIONS}

These two well-defined components are quite similar in age (less than 200 years difference) and season of occupation (fall). Both assemblages contain similar materials, including chipped stone tools (i.e., points, bifaces, and edge-modified flakes), and ground stone tools (i.e., manos and metates). Similarities are also present in plant resources (grass seeds, true lilies, and gourds) and the vertebrate faunal resources with extensively fragmented remains. Burned rocks and small cooking features were present in both. Local Edwards chert dominated both assemblages. The absence of recognized formal special purpose tools, the absence of trade goods, and no objects such as gaming pieces or ornaments, were also similar. The material classes support similar activities in each to reflect similar lifeways as both populations practiced hunting and gathering activities, procured and processed food resources, conducted knapping activities, and were highly mobile as the occupations represent short-term, fall season events. This location was repeatedly sought as it obviously provided the desired natural resources during the fall season.

Major differences occur in the frequency of similar artifact categories, overall subsistence strategies, feature types and frequencies, and intrasite debris pattern. The Bell/Andice component with the high frequency of small burned rock cooking and discard features, high frequency of ground stone tools, combined with limited chipped stone tools and formal tool production, and relatively restricted meat resources, probably reflects a small, specialized task group focused on plant collection and small game procurement with subsequent processing and cooking those resources during a very short-term fall occupation. In contrast, the Martindale component yielded higher frequencies of formal tool manufacturing debris, greater frequency and more diversified vertebrate faunal remains, few ground stone tools, fewer burned rocks and no formal burned rock features, 
horizontally distributed across a broader area to reflect nonspecialized activities, conducted for a somewhat longer period.

The sparse but diverse proxy data derived from the investigations implies the local environment that surrounded this site during the documented occupation period, ca. 5200 to 5400 B.P. was quite similar. The faunal and plant assemblages, which include multiple grasses and cheno-ams, jackrabbits and cottontail rabbits, Prairie chickens, and pronghorn, generally reflect an open grassland and low brush dominated vegetation community. The grassland environment bordered the riparian zone along the flowing Onion Creek that contained aquatic plants and fishes. The derived ages document less than a 200-year difference between the two components with little to no environmental change in this specific location over that short period. Consequently, given the environmental setting stayed constant, the detected differences and similarities between the two cultural assemblages and debris patterns directly relate to human behavioral patterns. 


\subsection{UNASSIGNED MATERIALS}

\section{J. Michael Quigg and Paul M .Matchen}

\subsection{INTRODUCTION}

The following presents materials that could not be confidently assigned to one of the two identified cultural components. These materials primarily came for small blocks of units (i.e., Blocks A, C, E, and F) with the exception of Feature 31 in Block B, from which no diagnostic materials and few radiocarbon dates were obtained. These smaller blocks were isolated from larger Blocks B and D and could not be stratigraphically linked with confidence to either defined cultural component, so it was unclear as to how these materials related to Bell/Andice and Martindale components. Additionally, some scattered materials from below the Martindale component in Blocks B and D cannot be attributed to either component with no diagnostic materials and few radiocarbon samples associated with these strata. It is possible some features and artifacts discussed below were part of one of the identified components. However, lacking direct and solid indicators such as diagnostic projectile points, well-defined stratigraphic markers, and radiocarbon dates from charcoal.

Below the radiocarbon dates obtained from areas other than the two identified components are presented followed by the feature descriptions and discussion. The different classes of unassigned cultural materials recovered are presented starting with chipped stone tools, ground stone tools, followed by vertebrate faunal remains, mussel shells, burned rocks, and ending with burned clay.

\subsection{DATES DERIVED FROM UNASSIGNED FEATURES AND MATERIALS}

Ten radiocarbon assays originated from five separate features (i.e., Features 15, 19, 20, 21, and 31) and from nonfeature context in four blocks. Of these, one date was from a deer radius, five dates on organic sediment, and three derived from multiple Rabdotus shells (Table 12-1). The dating of alternate organic materials, in this case due to the lack of recovered charcoal, created some uncertainty as to which of the two diagnostic components (Bell/Andice or Martindale) these deposits are attributed.

Seven of the ten assays yielded ages older than the established dates for the two diagnostic components. Dates obtained from organic sediment typically yield dates older than the accepted dates of the known components; therefore, the radiocarbon assays from Features 19 and 21 should be evaluated accordingly. Presently, these features cannot be linked to the Bell/Andice or Martindale components. Likewise, the radiocarbon date of organic sediment from Feature 31 places it in the time interval for the Bell/Andice component. Feature 31 was roughly $10 \mathrm{~cm}$ higher in the profile than the rest of the Bell/Andice features. Therefore, younger than the identified dated Bell/Andice component below it. Although slightly higher in profile, it is possible Feature 31 was constructed by peoples using the Bell/Andice point if the sediment date is accepted as reflecting the true age of the features.

The two deer bone dates derived from depths similar to those in the Martindale component identified in Block D, may reflect that component. However, the presence of multiple, closely spaced occupations in Block $\mathrm{C}$ make a direct and confident association with the Martindale component in Block D uncertain.

\subsection{UNASSIGNED FEATURES}

Seven features were unassigned with four (Features $15,19,21$, and 23) probably not cultural in origin, although six (Features 18, 19, 20, 21, 23, and 31) were associated or at least mixed with scattered cultural materials, with Features 18, 20, and 31 definitely cultural. Each feature is presented and discussed in numerical order. 
Table 12-1. Radiocarbon Dates from Multiple Blocks and Unassigned Features.

\begin{tabular}{|c|c|c|c|c|c|c|c|c|c|c|c|}
\hline $\begin{array}{c}\text { Catalogue } \\
\text { No. }\end{array}$ & Block & Unit No. & $\begin{array}{l}\text { Depth } \\
\text { (cmbs) }\end{array}$ & $\begin{array}{c}\text { Feature } \\
\text { No. }\end{array}$ & $\begin{array}{l}\text { Material } \\
\text { Dated }\end{array}$ & $\begin{array}{l}\text { Weight of } \\
\text { Material } \\
\text { (g) }\end{array}$ & $\begin{array}{l}\text { Beta } \\
\text { Lab. } \\
\text { No. }\end{array}$ & $\begin{array}{l}\text { Measured } \\
\text { Age }\end{array}$ & $\begin{array}{c}13 \mathrm{C} / 12 \mathrm{C} \\
\text { Ratio }(\%)\end{array}$ & $\begin{array}{l}\text { Conventional } \\
\text { Age (B.P.) }\end{array}$ & $\begin{array}{c}2 \text { Sigma } \\
\text { Calibration } \\
\text { Range }\end{array}$ \\
\hline$M-1$ & $A$ & N96/E98 & $\begin{array}{l}263- \\
267\end{array}$ & 15 & $\begin{array}{l}8 \text { Rabdotus } \\
\text { shells }\end{array}$ & 4.8 & 216368 & $\begin{array}{c}5710 \pm \\
50\end{array}$ & -10.3 & $5950 \pm 50^{1}$ & $\begin{array}{c}\text { BC } 4940 \text { to } \\
4710\end{array}$ \\
\hline $2366-4-1$ & $A$ & N95/E96 & $\begin{array}{l}270- \\
280\end{array}$ & 19 & $\begin{array}{c}\text { Organic } \\
\text { sediment }\end{array}$ & 112 & 234643 & $\begin{array}{c}6210 \pm \\
50\end{array}$ & -21.7 & $6260 \pm 50^{1}$ & $\begin{array}{c}\text { BC } 5220 \text { to } \\
5070\end{array}$ \\
\hline $2366-4-2 a$ & $A$ & N95/E97 & $\begin{array}{l}270- \\
280\end{array}$ & 19 & $\begin{array}{c}\text { Organic } \\
\text { sediment }\end{array}$ & 99 & 234644 & $\begin{array}{c}6400 \pm \\
50\end{array}$ & -23.3 & $6460 \pm 50^{1}$ & $\begin{array}{c}\text { BC } 5490 \text { to } \\
5230\end{array}$ \\
\hline $2414-7-1$ & B & N71/E79 & 213 & 31 & $\begin{array}{c}\text { Organic } \\
\text { sediment }\end{array}$ & 10.9 & 234640 & $\begin{array}{c}5390 \pm \\
50\end{array}$ & -25 & $5390 \pm 50$ & $\begin{array}{c}\text { BC } 4340 \text { to } \\
4680\end{array}$ \\
\hline TV2161/M-9 & C & N66/E95 & $\begin{array}{l}280- \\
290\end{array}$ & & $\begin{array}{l}\text { Deer } \\
\text { incisor }\end{array}$ & 0.9 & 216376 & NA & NA & $5290 \pm 40$ & $\begin{array}{c}\text { BC } 4240 \text { to } \\
3990\end{array}$ \\
\hline TV2161/M-8 & C & N68/E96 & 286 & & $\begin{array}{c}\text { Deer } \\
\text { radius }\end{array}$ & 13.9 & 216375 & $5370 \pm 40$ & -20.3 & $5440 \pm 40$ & $\begin{array}{c}\text { BC } 4350 \text { to } \\
4230\end{array}$ \\
\hline 2066-4-1a & C & N68/E95 & $\begin{array}{c}251- \\
153 \\
\end{array}$ & 21 & $\begin{array}{c}\text { Organic } \\
\text { sediment }\end{array}$ & 177 & 234641 & $5820 \pm 50$ & -22.1 & $5870 \pm 50$ & $\begin{array}{c}\text { BC } 4840 \text { to } \\
4610\end{array}$ \\
\hline $2072-4-1 a$ & C & N70/E95 & $\begin{array}{l}242- \\
260 \\
\end{array}$ & 21 & $\begin{array}{c}\text { Organic } \\
\text { sediment }\end{array}$ & 245 & 234642 & $\begin{array}{c}5840 \pm \\
50\end{array}$ & -21.9 & $5890 \pm 50$ & $\begin{array}{c}\text { BC } 4880 \text { to } \\
4680\end{array}$ \\
\hline TV2161/M-2 & C & N67/E96 & $\begin{array}{l}283- \\
290 \\
\end{array}$ & 20 & $\begin{array}{c}6 \text { Rabdotus } \\
\text { shells }\end{array}$ & 2.3 & 216369 & $5650 \pm 50$ & -9.9 & $5900 \pm 50^{1}$ & $\begin{array}{c}\text { BC } 4850 \text { to } \\
4680\end{array}$ \\
\hline TV2161/M-4 & $D$ & N83/E93 & $\begin{array}{l}260- \\
270\end{array}$ & & $\begin{array}{c}6 \text { Rabdotus } \\
\text { shells }\end{array}$ & 3.6 & 216371 & $5920 \pm 50$ & -10.1 & $6160 \pm 50^{1}$ & $\begin{array}{c}\text { BC } 5270 \text { to } \\
4940\end{array}$ \\
\hline
\end{tabular}




\subsubsection{Feature 15}

Feature 15 was roughly between 253 and $262 \mathrm{cmbs}$ in N96/E98 in Block A (Figure 12-1). Stratigraphically, Feature 15 was not directly associated with cultural materials and deposited above burned Features 19 and 23 in that same block (see Chapter 8.0). It was dominated by mostly complete Rabdotus snail shells clustered in a relatively small amorphous shaped area, which measured roughly 98 -by-58 cm in the northwestern corner of this unit (Figure 12-2). No burned rocks or chert flakes were detected in the shell concentration, although one burned rock was in the very southeastern corner of the same unit at approximately the same depth. The shells were within a brown yellowish (10YR 6/3), hard compacted clay loam with a few scattered dark charcoal flecks. Samples of sediment, charcoal and snail shells were collected. Upon sorting heavy fractions from these sediment samples, no lithic debitage or bones were recovered in the level between 250 and $260 \mathrm{cmbs}$ and only one small burned rock fragment was recorded from 260 to 270 cmbs, whereas a few small burned rock fragments were between 270 and $280 \mathrm{cmbs}$. Although the shell concentration is not considered cultural, scattered cultural materials were in the vicinity.

Eight, more or less complete Rabdotus shells were selected from the collected shells and radiocarbon dated. The apex of each shell was removed before submission. These eight Rabdotus shells weighed $4.7 \mathrm{~g}$ and yielded a $\delta^{13} \mathrm{C}$ corrected $(-10.3 \%$ ) age of $5950 \pm 50$ B.P. (Beta-216368, see Table 21-1). The shells however, probably date older than the projected age of this deposit, thus unlikely to represent the age of the deposit.

This Rabdotus shell concentration is considered noncultural as the shells were mostly complete with no obvious signs of being burned or otherwise culturally altered. It is perceived these shells may have become concentrated in this manner from flood waters evaporating as the shells are lightweight and float, easily transported by water. When overbank waters collect these lightweight objects they float around, and as the pools of water evaporate, the shells become concentrated.

\subsubsection{Feature 18}

Feature 18 was between 281 and $283 \mathrm{cmbs}$ in N66/E95 and N67/E95 of Block C and part of a broader occupation at that elevation (see Figure 121). A circular concentration of 24 burned rocks was in an area about $40 \mathrm{~cm}$ in diameter in a light yellowish brown (10YR 6/4) sandy clay loam (Figures 12-3 and 12-4). Rocks (\#2044-3) varied in size from 3 to $15 \mathrm{~cm}$ with a total weight of 5,804 $\mathrm{g}$ for an average of $241 \mathrm{~g}$ (Table 12-2). One burned rock exhibited a hackled/blocky edge. No charcoal staining was observed and no pit was visible under the rocks. Two small sediment samples (\#2026 and \#2044-4) were collected below the rocks. Other scattered burned rocks were in the two adjacent units, as were a few chert flakes, animal bone fragments, a biface fragment (\#2043-10 at 279 cmbs), and a scraper/gouge (\#2043-10). A cluster of Rabdotus snail shells were just below the feature rocks ( $275 \mathrm{cmbs}$ ) at 280 to $285 \mathrm{cmbs}$. Feature 18 was part of a more extensive and diverse occupation zone.

Multiple sediment samples from Feature 18 were submitted to various specialists for analyses. Subsample \#2044-4 yielded a gourd phytolith, numerous tree phytoliths, some of which were burned, 10 sponge spicules, but no short cell phytoliths (Appendix E). Another subsample (\#2044-4e) yielded 32 diatoms, the second highest frequency of the 10 samples analyzed for diatoms (Appendix C).

Fragments of four burned rocks were subjected to starch grain analysis. Only one rock (\#2026-3-4) yielded any results with one gelatinized and one eroded starch grain, but no identifiable starches (Appendix F). Fragments of two burned rocks (\#2044-3-1b and \#2044-3-2b) were subjected to 


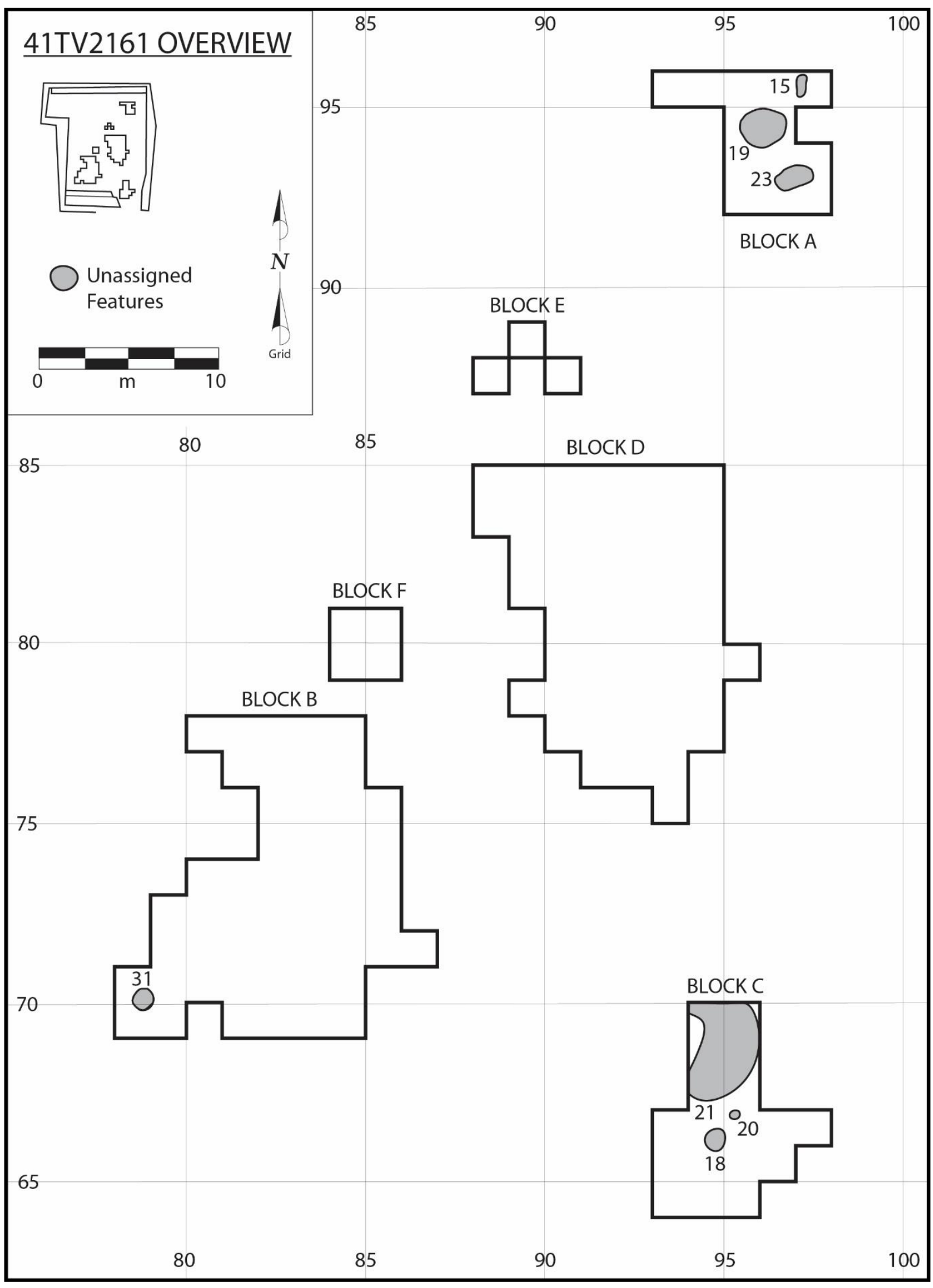

Figure 12-1. Drawing depicting the location of nonassociated features in various blocks. 


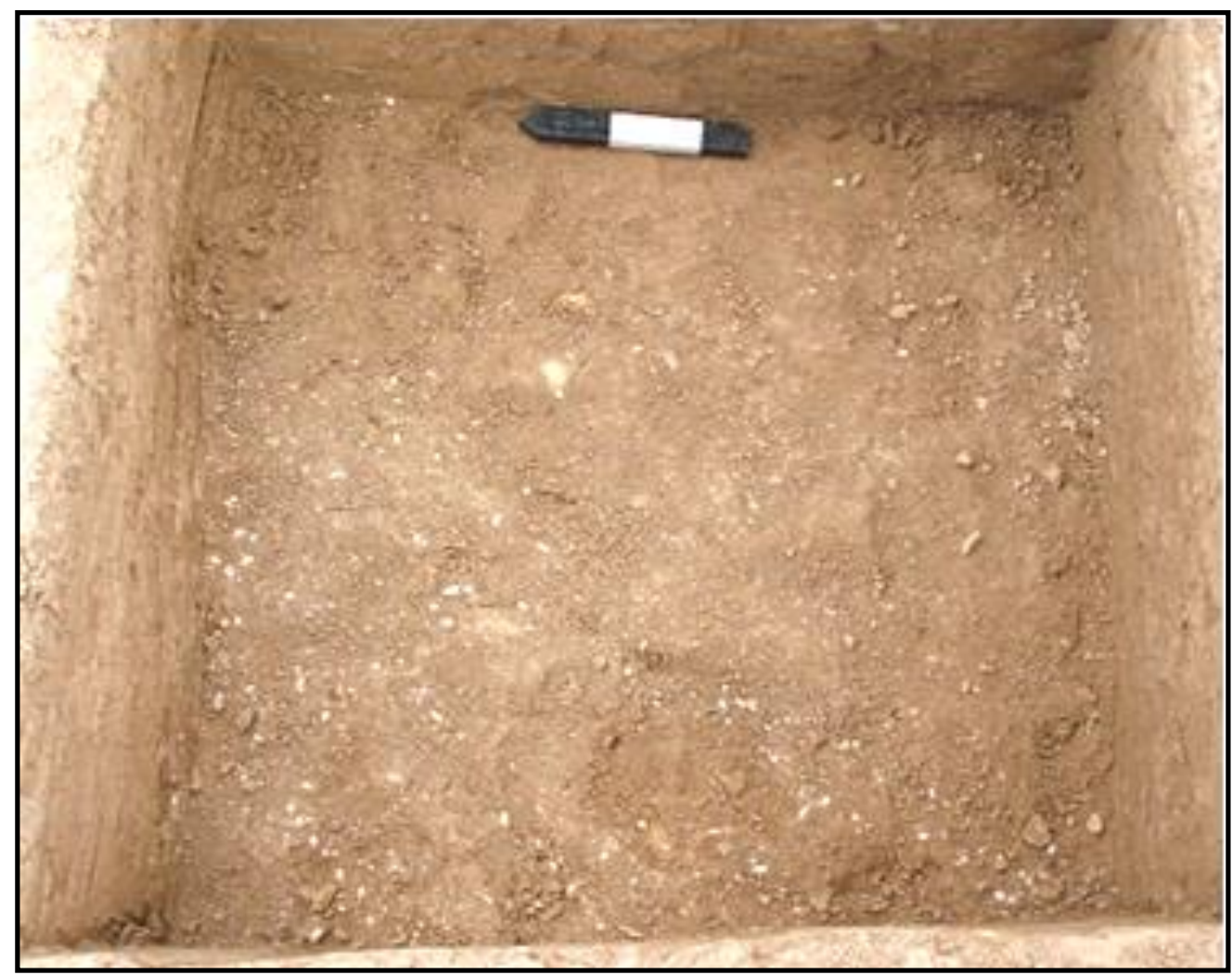

Figure 12-2. Overhead photograph of Rabdotus shell concentration, Feature 15 (scale in 10 centimeter increments).

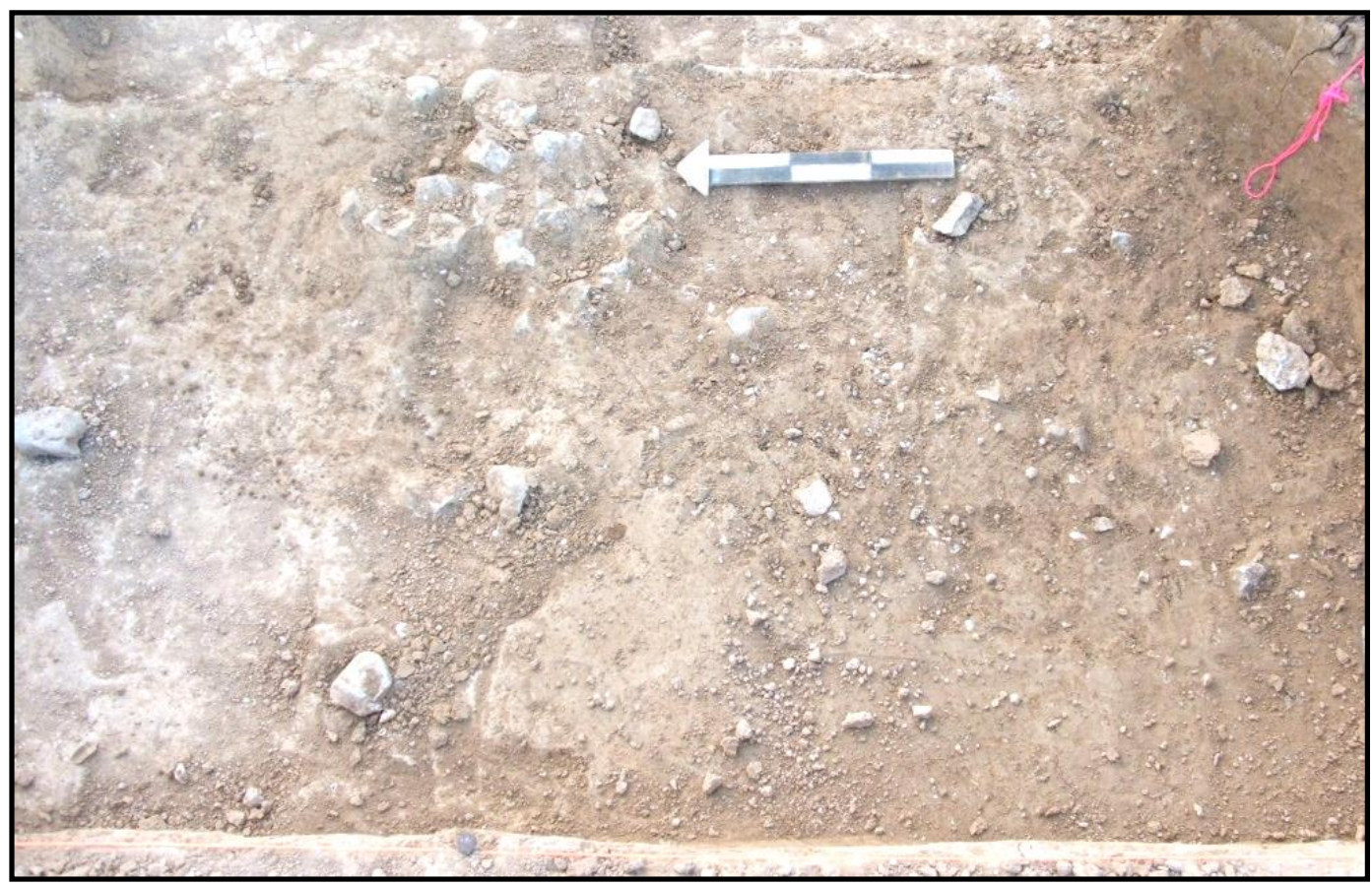

Figure 12-3. Overview photograph of exposed burned rock cluster of Feature 18 (left of arrow) and surround scattered cultural materials in Block $C$ (scale in 10 centimeter increments). 


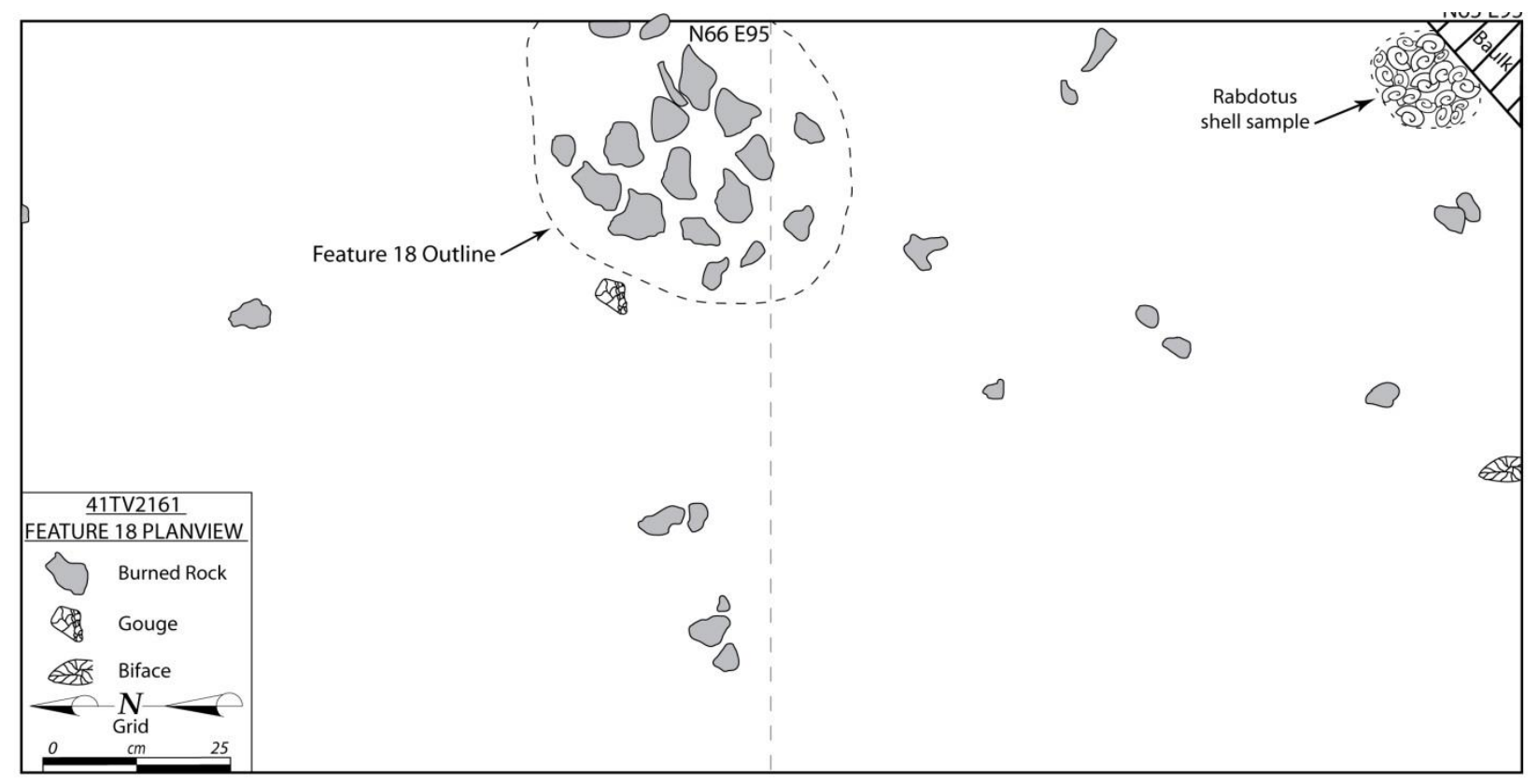

Figure 12-4. Plan view drawing of Feature 18 in Block C.

lipid residue analysis, and both revealed very high fat content, indicating possible plant and animal combination, but dominated by plant (Appendix $\mathrm{G})$.

The heavy fractions from both mechanically floated sediment samples (\#2026-4-g and \#2044-4-g) yielded mostly lithic debitage $(N=35)$, fragments of Rabdotus snail shells, plus 13 faunal bone fragments less than $6.4 \mathrm{~mm}$ (Table 12-3). The faunal material includes three unburned fish vertebrae less than $2 \mathrm{~mm}$ in size, and 10 other small unidentifiable fragments.

Feature 18 is interpreted as a discard pile of burned rocks previously employed in a boiling process as evidenced by the gelatinized starch grains, sponge spicules, and diatoms, combined with the plant and animal resides off the rocks. The stratigraphic position was very near the Martindale component and potentially associated with that component. The lack of direct supporting evidence, a radiocarbon date or diagnostic prevented assignment to the Martindale component.

\subsubsection{Feature 19}

Feature 19 was a relatively large amorphous shaped, orangish and black sediment with mottled dark brown (10YR 5/3) burned clay areas, with scattered charcoal and ash that measured roughly 130-by-160 cm in diameter dispersed across parts of four adjacent units in Block A (see Figures 12-1 and 12-5). The surrounding sediment was a yellowish brown (10YR 6/4) silty clay loam. An irregular, shallow, poorly defined, basin-shaped dark stained depression was in N95/E97 and may have represented a burned tree throw or root mass. This mottled area was about $12 \mathrm{~cm}$ thick in spots with an apparent shallow depression about $20 \mathrm{~cm}$ thick. At 270 to $296 \mathrm{cmbs}$ it was below Features 15 and 23 , rather than associated with significant quantities of cultural material, although a few scattered burned rocks and occasional chert flakes were in the vicinity. Rabdotus snail shells and a rodent burrow were observed in the sediments. Quantities of fill (about 111 liters) were collected for subsequent fine screening/floating. Numerous dark stained spots (presumably degraded charcoal), 
Table 12-2. Burned Rock Counts and Weights for Unassigned Features.

\begin{tabular}{|c|c|c|c|c|c|c|c|c|c|c|c|c|c|}
\hline Feature & Unit & $\begin{array}{c}\text { Depth } \\
\text { (cmbs) }\end{array}$ & PNUM & 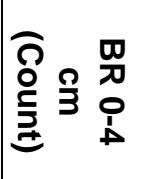 & 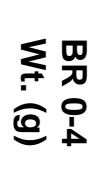 & 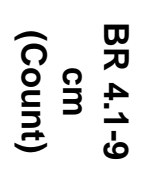 & 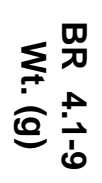 & 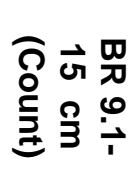 & 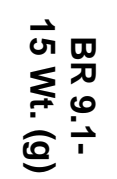 & 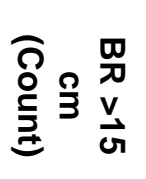 & 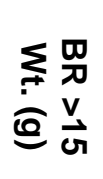 & $\begin{array}{l}\text { Total } \\
\text { Count }\end{array}$ & $\begin{array}{c}\text { Total } \\
\text { Wt. (g) }\end{array}$ \\
\hline 15 & $\begin{array}{l}\text { N96 } \\
\text { E98 }\end{array}$ & $\begin{array}{l}260- \\
270\end{array}$ & 2393 & 1 & 15 & & & & & & & 1 & 15 \\
\hline 18 & $\begin{array}{l}\text { N66 } \\
\text { E95 }\end{array}$ & $\begin{array}{l}280- \\
290\end{array}$ & 2026 & 5 & 70 & 9 & 734 & & & & & 14 & 804 \\
\hline 18 & $\begin{array}{l}\text { N67 } \\
\text { E95 }\end{array}$ & $\begin{array}{l}280- \\
290\end{array}$ & 2044 & & & 2 & 350 & 7 & 3150 & 1 & 1500 & 10 & 5000 \\
\hline 19 & $\begin{array}{l}\text { N95 } \\
\text { E96 }\end{array}$ & $\begin{array}{l}260- \\
270\end{array}$ & 2419 & & & 3 & 666 & 1 & 1042 & & & 4 & 1708 \\
\hline 20 & $\begin{array}{l}\text { N67 } \\
\text { E96 }\end{array}$ & $\begin{array}{l}283- \\
290\end{array}$ & 2050 & & & 4 & 617 & & & & & 4 & 617 \\
\hline 21 & $\begin{array}{l}\text { N68 } \\
\text { E95 }\end{array}$ & $\begin{array}{l}250- \\
260\end{array}$ & 2060 & 2 & 30 & 1 & 175 & & & & & 3 & 205 \\
\hline 21 & $\begin{array}{l}\text { N70 } \\
\text { E95 }\end{array}$ & $\begin{array}{l}240- \\
260\end{array}$ & 2070 & 1 & 15 & & & & & & & 1 & 15 \\
\hline 23 & $\begin{array}{l}\text { N94 } \\
\text { E97 }\end{array}$ & $\begin{array}{l}270- \\
280\end{array}$ & 2351 & 1 & 15 & 1 & 95 & 1 & 568 & & & 3 & 678 \\
\hline 31 & $\begin{array}{l}\text { N71 } \\
\text { E79 }\end{array}$ & $\begin{array}{l}208- \\
218\end{array}$ & 2414 & & & 1 & 142 & 22 & 9359 & & & 23 & 9500 \\
\hline \multicolumn{4}{|c|}{ Total from Features } & 10 & 145 & 21 & 2779 & 31 & 14119 & 1 & 1500 & 63 & 18543 \\
\hline
\end{tabular}


Table 12-3. Flotation Results from Unassigned Features.

\begin{tabular}{|c|c|c|c|c|c|c|c|c|c|c|c|c|c|c|c|c|c|c|c|c|c|c|}
\hline \multirow{2}{*}{$\begin{array}{l}\text { 足 } \\
\text { Ch }\end{array}$} & \multirow{2}{*}{$\stackrel{ᄃ}{\leftrightarrows}$} & \multirow{2}{*}{ 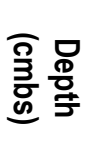 } & \multirow{2}{*}{$\begin{array}{l}\frac{m}{\circ} \\
\frac{\Gamma}{x}\end{array}$} & \multirow{2}{*}{ 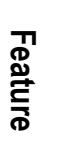 } & \multirow{2}{*}{$\underline{\underline{a}} \frac{\underline{o}}{\underline{e}}$} & & \multicolumn{2}{|c|}{$\begin{array}{l}\text { Mussel } \\
\text { Shell }\end{array}$} & \multicolumn{2}{|c|}{$\begin{array}{l}\text { Burned } \\
\text { Rock }\end{array}$} & \multicolumn{2}{|c|}{ Debitage } & \multicolumn{2}{|c|}{ Charcoal } & \multicolumn{2}{|c|}{ Snail } & \multicolumn{2}{|c|}{$\begin{array}{c}\text { Macro- } \\
\text { botanical }\end{array}$} & \multicolumn{2}{|c|}{ Burned Clay } & \multicolumn{2}{|c|}{ Bone } \\
\hline & & & & & & & $1 / 4$ & $<1 / 4$ & $1 / 4$ & $<1 / 4$ & $1 / 4$ & $<1 / 4$ & $1 / 4$ & $<1 / 4$ & $1 / 4$ & $<1 / 4$ & $1 / 4$ & $<1 / 4$ & $1 / 4$ & $<1 / 4$ & $1 / 4$ & $<1 / 4$ \\
\hline 2026-4-g & $\begin{array}{l}\text { N67 } \\
\text { E95 }\end{array}$ & $\begin{array}{l}281- \\
283\end{array}$ & C & 18 & 0.7 & $\mathbf{N}$ & 0 & 0 & 0 & 0 & 1 & 19 & 0 & 1 & 16 & 0 & 0 & 0 & 0 & 0 & 0 & 1 \\
\hline \multicolumn{5}{|c|}{ Light Fraction $0.3 \mathrm{~g}$} & 11.5 & Wt. & 0.0 & 0.0 & 0.0 & 0.0 & 0.3 & 0.1 & 0.0 & 0.1 & 9.7 & 0.0 & 0.0 & 0.0 & 0.0 & 0.0 & 0.0 & 0.1 \\
\hline 2044-4-g & $\begin{array}{l}\text { N67 } \\
\text { E95 }\end{array}$ & $\begin{array}{l}281- \\
283\end{array}$ & C & 18 & 0.8 & $\mathbf{N}$ & 0 & 0 & 0 & 0 & 0 & 16 & 0 & 1 & 19 & 1 & 0 & 0 & 0 & 0 & 0 & 12 \\
\hline \multicolumn{5}{|c|}{ Light Fraction $0.6 \mathrm{~g}$} & 10.0 & Wt. & 0.0 & 0.0 & 0.0 & 0.0 & 0.0 & 0.3 & 0.0 & 0.1 & 5.4 & 4.0 & 0.0 & 0.0 & 0.0 & 0.0 & 0.0 & 0.2 \\
\hline $\begin{array}{c}2367-4- \\
1 \mathrm{~g}\end{array}$ & $\begin{array}{l}\text { N95 } \\
\text { E96 }\end{array}$ & $\begin{array}{l}270- \\
280\end{array}$ & A & 19 & 109.5 & $\mathbf{N}$ & 0 & 4 & 0 & 0 & 13 & 170 & 0 & 0 & 0 & 126 & 0 & 536 & 82 & 53 & 0 & 326 \\
\hline \multicolumn{5}{|c|}{ Light Fraction $14.2 \mathrm{~g}$} & 811.4 & Wt. & 0.0 & 0.7 & 0.0 & 0.0 & 8.0 & 8.0 & 0.0 & 0.0 & 0.0 & 35.5 & 0.0 & 11.2 & 66.2 & 7.5 & 0.0 & 4.7 \\
\hline $\begin{array}{c}2367-4- \\
2 g\end{array}$ & $\begin{array}{l}\text { N95 } \\
\text { E96 }\end{array}$ & $\begin{array}{l}270- \\
280\end{array}$ & A & 19 & 1.45 & $\mathbf{N}$ & 0 & 0 & 0 & 0 & 0 & 0 & 0 & 0 & 0 & 1 & 0 & 89 & 0 & 0 & 0 & 11 \\
\hline \multicolumn{5}{|c|}{ Light Fraction $0.2 \mathrm{~g}$} & 6.4 & Wt. & 0.0 & 0.0 & 0.0 & 0.0 & 0.0 & 0.0 & 0.0 & 0.0 & 0.0 & 0.3 & 0.0 & 0.2 & 0.0 & 0.0 & 0.0 & 0.1 \\
\hline $\begin{array}{c}2060-4- \\
1 \mathrm{~g}\end{array}$ & $\begin{array}{l}\text { N68 } \\
\text { E95 }\end{array}$ & $\begin{array}{l}251- \\
253\end{array}$ & C & 21 & 1.8 & $\mathbf{N}$ & 0 & 0 & 0 & 0 & 116 & 2 & 0 & 12 & 0 & 2 & 0 & 8 & 3 & 2 & 0 & 14 \\
\hline \multicolumn{5}{|c|}{ Light Fraction $0.2 \mathrm{~g}$} & 11.8 & Wt. & 0.0 & 0.0 & 0.0 & 0.0 & 1.1 & 0.7 & 0.0 & 0.1 & 0.0 & 0.2 & 0.0 & 0.1 & 6.9 & 0.1 & 0.0 & 0.1 \\
\hline $\begin{array}{c}2068-4- \\
2 g\end{array}$ & \begin{tabular}{|l|} 
N69 \\
E96 \\
\end{tabular} & $\begin{array}{l}244- \\
253 \\
\end{array}$ & C & 21 & 1.6 & $\mathrm{~N}$ & 0 & 0 & 0 & 0 & 0 & 17 & 0 & 0 & 0 & 5 & 0 & 8 & 0 & 0 & 0 & 21 \\
\hline \multicolumn{5}{|c|}{ Light Fraction $0.3 \mathrm{~g}$} & 1.2 & Wt. & 0.0 & 0.0 & 0.0 & 0.0 & 0.0 & 0.1 & 0.0 & 0.0 & 0.0 & 0.5 & 0.0 & 0.1 & 0.0 & 0.0 & 0.0 & 0.1 \\
\hline 2066-4-g & $\begin{array}{l}\text { N69 } \\
\text { E95 }\end{array}$ & $\begin{array}{l}251- \\
253\end{array}$ & C & 21 & 1.6 & $\mathbf{N}$ & 0 & 0 & 0 & 0 & 0 & 2 & 0 & 0 & 0 & 1 & 0 & 0 & 4 & 0 & 0 & 22 \\
\hline \multicolumn{5}{|c|}{ Light Fraction $0.2 \mathrm{~g}$} & 58.5 & Wt. & 0.0 & 0.0 & 0.0 & 0.0 & 0.0 & 0.1 & 0.0 & 0.0 & 0.0 & 0.2 & 0.0 & 0.0 & 55.5 & 0.0 & 0.0 & 0.1 \\
\hline $\begin{array}{c}2070-4- \\
1 \mathrm{~g}\end{array}$ & $\begin{array}{l}\text { N70 } \\
\text { E95 } \\
\end{array}$ & $\begin{array}{l}242- \\
260 \\
\end{array}$ & C & 21 & 4.0 & $\mathbf{N}$ & 0 & 0 & 0 & 0 & 0 & 0 & 0 & 0 & 0 & 11 & 0 & 3 & 11 & 6 & 0 & 33 \\
\hline \multicolumn{5}{|c|}{ Light Fraction $0.1 \mathrm{~g}$} & 68.9 & Wt. & 0.0 & 0.0 & 0.0 & 0.0 & 0.0 & 0.0 & 0.0 & 0.0 & 0.0 & 0.5 & 0.0 & 0.1 & 61.9 & 0.5 & 0.0 & 0.1 \\
\hline
\end{tabular}


Table 12-3. Flotation Results from Unassigned Features (continued).

\begin{tabular}{|c|c|c|c|c|c|c|c|c|c|c|c|c|c|c|c|c|c|c|c|c|c|c|}
\hline \multirow{2}{*}{ 忍 } & \multirow{2}{*}{$\stackrel{ᄃ}{\stackrel{c}{F}}$} & \multirow{2}{*}{ 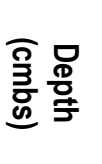 } & \multirow{2}{*}{$\begin{array}{l}\frac{w}{0} \\
\frac{\Gamma}{x}\end{array}$} & \multirow{2}{*}{ 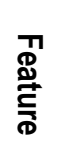 } & \multirow{2}{*}{ 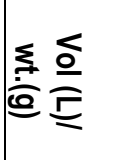 } & & \multicolumn{2}{|c|}{$\begin{array}{l}\text { Mussel } \\
\text { Shell }\end{array}$} & \multicolumn{2}{|c|}{$\begin{array}{l}\text { Burned } \\
\text { Rock }\end{array}$} & \multicolumn{2}{|c|}{ Debitage } & \multicolumn{2}{|c|}{ Charcoal } & \multicolumn{2}{|c|}{ Snail } & \multicolumn{2}{|c|}{$\begin{array}{l}\text { Macro- } \\
\text { botanical }\end{array}$} & \multicolumn{2}{|c|}{ Burned Clay } & \multicolumn{2}{|c|}{ Bone } \\
\hline & & & & & & & $1 / 4$ & $<1 / 4$ & $1 / 4$ & $<1 / 4$ & $1 / 4$ & $<1 / 4$ & $1 / 4$ & $<1 / 4$ & $1 / 4$ & $<1 / 4$ & $1 / 4$ & $<1 / 4$ & $1 / 4$ & $<1 / 4$ & $1 / 4$ & $<1 / 4$ \\
\hline 2072-4-g & $\begin{array}{l}\text { N70 } \\
\text { E96 }\end{array}$ & $\begin{array}{l}242- \\
260\end{array}$ & C & 21 & 2.1 & N & 0 & 0 & 0 & 0 & 0 & 0 & 0 & 0 & 0 & 11 & 0 & 3 & 60 & 0 & 0 & 0 \\
\hline \multicolumn{5}{|c|}{ Light Fraction $0.1 \mathrm{~g}$} & 336.9 & Wt. & 0.0 & 0.0 & 0.0 & 0.0 & 0.0 & 0.0 & 0.0 & 0.0 & 0.0 & 2.4 & 0.0 & 0.1 & 304.8 & 0.0 & 0.0 & 0.0 \\
\hline 2357-4-g & $\begin{array}{l}\text { N94 } \\
\text { E98 }\end{array}$ & $\begin{array}{l}270- \\
280\end{array}$ & A & 23 & 6.0 & $\mathbf{N}$ & 0 & 0 & 0 & 0 & 0 & 0 & 0 & 0 & 0 & 96 & 0 & 3 & 0 & 0 & 0 & 1 \\
\hline \multicolumn{5}{|c|}{ Light Fraction $1.9 \mathrm{~g}$} & 177.4 & Wt. & 0.0 & 0.0 & 0.0 & 0.0 & 0.0 & 0.0 & 0.0 & 0.0 & 0.0 & 43.9 & 0.0 & 0.1 & 0.0 & 0.0 & 0.0 & 0.1 \\
\hline 2351-4-g & $\begin{array}{l}\text { N94 } \\
\text { E97 }\end{array}$ & $\begin{array}{l}270- \\
280 \\
\end{array}$ & A & 23 & 6.0 & $\mathbf{N}$ & 0 & 0 & 0 & 0 & 0 & 0 & 0 & 0 & 0 & 119 & 0 & 2 & 0 & 0 & 0 & 0 \\
\hline \multicolumn{5}{|c|}{ Light Fraction $3.4 \mathrm{~g}$} & 167.1 & Wt. & 0.0 & 0.0 & 0.0 & 0.0 & 0.0 & 0.0 & 0.0 & 0.0 & 0.0 & 33.4 & 0.0 & 0.1 & 0.0 & 0.0 & 0.0 & 0.0 \\
\hline
\end{tabular}




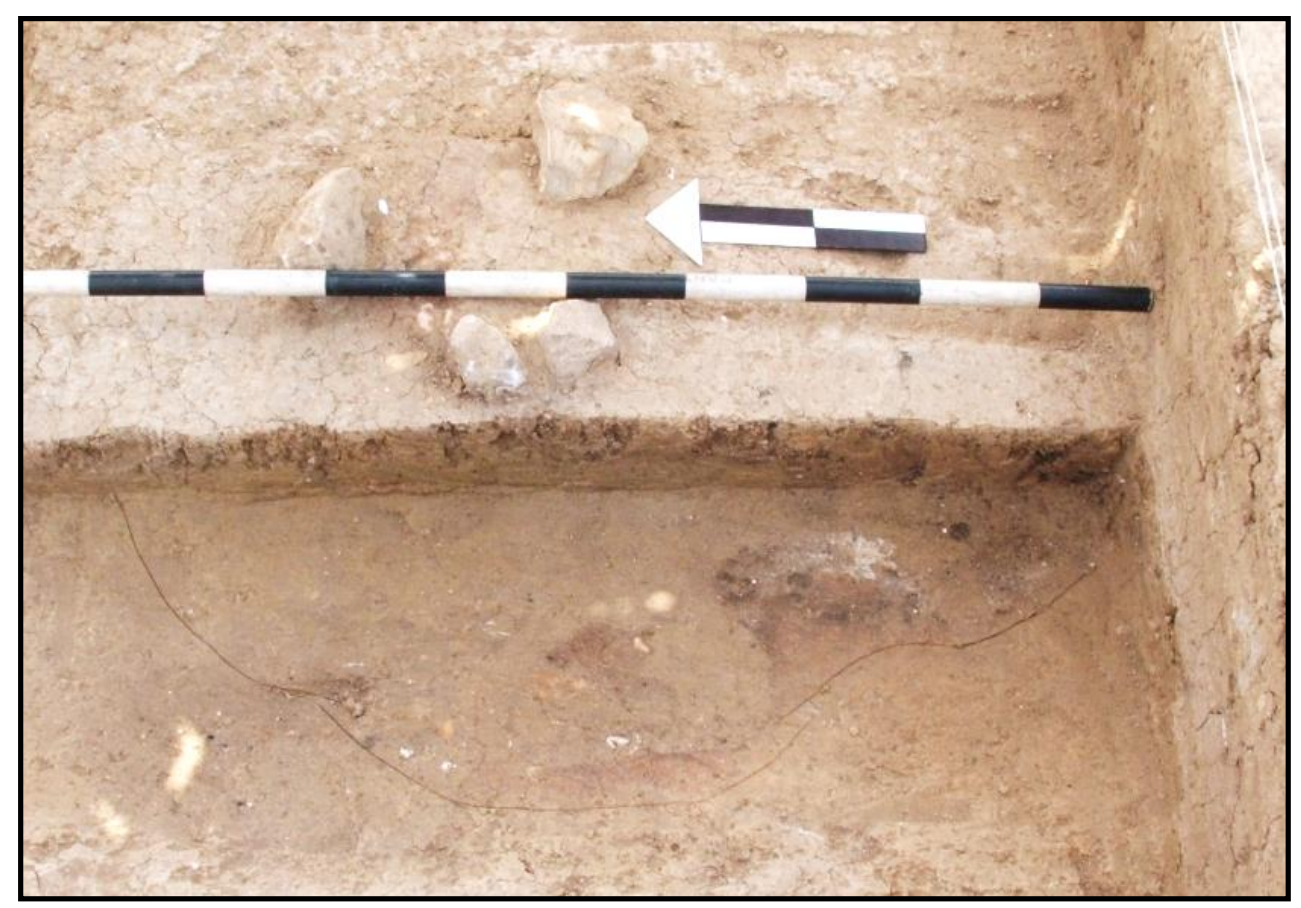

Figure 12-5. Oblique view of partially excavated Feature 19 in Block A (scale in 10 centimeter increments).

along with a few scattered burned rocks, a few tiny faunal bone fragments, a sample of Rabdotus shells, and some burned hackberry seeds were collected. Rocks (\#2419-3) varied in size from 4 to $10 \mathrm{~cm}$ with a total weight of $1,708 \mathrm{~g}$ for an average of $427 \mathrm{~g}$ (see Table 12-2). None of these materials were subjected to further analysis.

Two bulk organic rich sediment samples were dated. One sample (\#2366-4-1) yielded a $\delta^{13} \mathrm{C}$ corrected $(-21.7 \%$ ) age of $6260 \pm 50$ B.P. (Beta234643). The other (\#2366-4-2a) from the come context yielded a $\delta^{13} \mathrm{C}$ corrected $(-21.3 \%$ ) age of $6460 \pm 50$ B.P. (Beta-234644) (see Table 12-1). The two dates represent a general age for this amorphous burned area, and as discussed previously, organic sediments dates are considered older than the actual event dated, so the exact timing of this burn is unclear. These dates are definitely older than the charcoal dated Martindale component.

The floated sediment samples yielded hundreds of tiny lithic debitage, many unburned Rabdotus snail fragments, and hundreds of tiny faunal bone fragments (see Table 12-3). This burned area is thought to represent a natural burn, probably from a tree, just below the Martindale component.

\subsubsection{Feature 20}

Feature 20 was a tight cluster of four burned rocks between 283 and $286 \mathrm{cmbs}$ in the northwestern corner of N67/E96 in Block C (see Figures 12-1 and 12-6). Stratigraphically, it was at the same elevation as Feature 18 (see above and Chapter 8.0) and was generally associated with other cultural materials as part of an occupation zone. Rabdotus snail shells were also clustered adjacent to the burned rocks. No charcoal staining was visible in the light yellowish brown (10YR 4/6) sandy clay. A chert flake, charcoal chunks, and a deer bone fragment were in this same level in this unit. Sediment, snail shells, and charcoal were collected from around the burned rocks. The four burned rocks (\#2050-3) varied in size from 4.1 to $9 \mathrm{~cm}$, with a total weight of $617 \mathrm{~g}$ for an average weight of $154 \mathrm{~g}$ per rock (see Table 12-2). 
Six, more or less complete Rabdotus shells selected from the adjacent Rabdotus concentration were radiocarbon dated. The apex of each shell was removed prior to submission. These six Rabdotus shells weighed $2.3 \mathrm{~g}$ and yielded a $\delta^{13} \mathrm{C}$ corrected ($9.9 \%$ ) age of $5900 \pm 50$ B.P. (Beta-216369, see Table 12-1). The shells are not considered cultural, although they provide a general age for the adjacent Feature 20 and cultural materials.

Three burned rocks (\#2050-3) were subjected to lipid residue analysis. Sample \#2050-3-3 yielded low fat plant and moderate-high fat content to indicate possible residues from plant and animal combination. Sample \#2050-3-1b yielded similar results of very high fat content and again indicates a combination of plant and animal residues (Appendix G). A subsample of \#2050-3-1c sent for starch analysis yielded no starches (Appendix F).

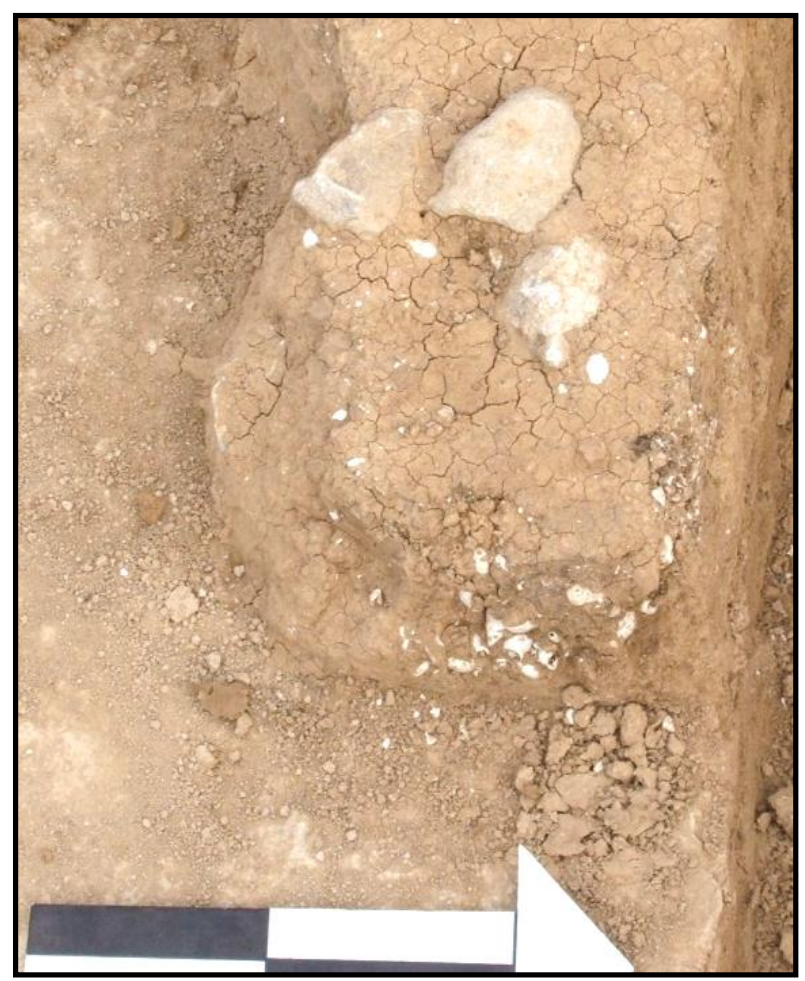

Figure 12-6. Photograph of overhead view of clustered burned rocks in Feature 20 (scale in 10 centimeter increments).
Feature 20 is interpreted as discarded rocks from a cooking event as indicated by the plant and animal residues detected. Features 18 and 20 appear part of the same broader occupation on the basis of general depth. These were unassigned since they could not be directly linked to the Martindale component in Block D.

\subsubsection{Feature 21}

Feature 21 was a large $(150+\mathrm{cm})$ amorphous shaped, charcoal stained and burned sediment area dispersed across parts of at least five units in the northern part of Block C (Figures 12-1, 12-7, 12-8, and 12-9). The diffuse and multiple colored areas were between 251 and $253 \mathrm{cmbs}$. Stratigraphically, Feature 21 was approximately at the Martindale component depth and above burned rock Features 18 and 20 (see Chapter 8.0, Figure 8-11). This burned area exhibited a diffuse linear shape in some areas, whereas other parts were irregular and difficult to distinguish. Quantities of chert debitage $(N=191)$ and at least three burned rocks were in the immediate vicinity. Sediment samples and charcoal spots along with the cultural materials from these units were collected.

Two organic rich sediment dates were obtained. One sample (\#2066-4-1a) yielded a $\delta^{13} \mathrm{C}$ corrected $(-22.1 \%)$ age of $5870 \pm 50$ B.P. (Beta-234641). The other (\#2072-4-1a) from nearby yielded a $\delta^{13} \mathrm{C}$ corrected $(-21.9 \%$ ) age of $5840 \pm 50$ B.P. (Beta234642) (see Table 12-1). The two dates are statistically the same and provide a general age for Feature 21. Again, bulk sediment dates probably do not provide the specific date of the actual event.

The four burned rocks (\#2060-3 and \#2070) were dominated by small pieces with a total weight of $220 \mathrm{~g}$, for an average of $55 \mathrm{~g}$ per rock (see Table 12-2).

Five sediment samples of 11.1 liters were floated and sorted from units that exhibited this burned area. Only one sample (\#2060-4) yielded a 


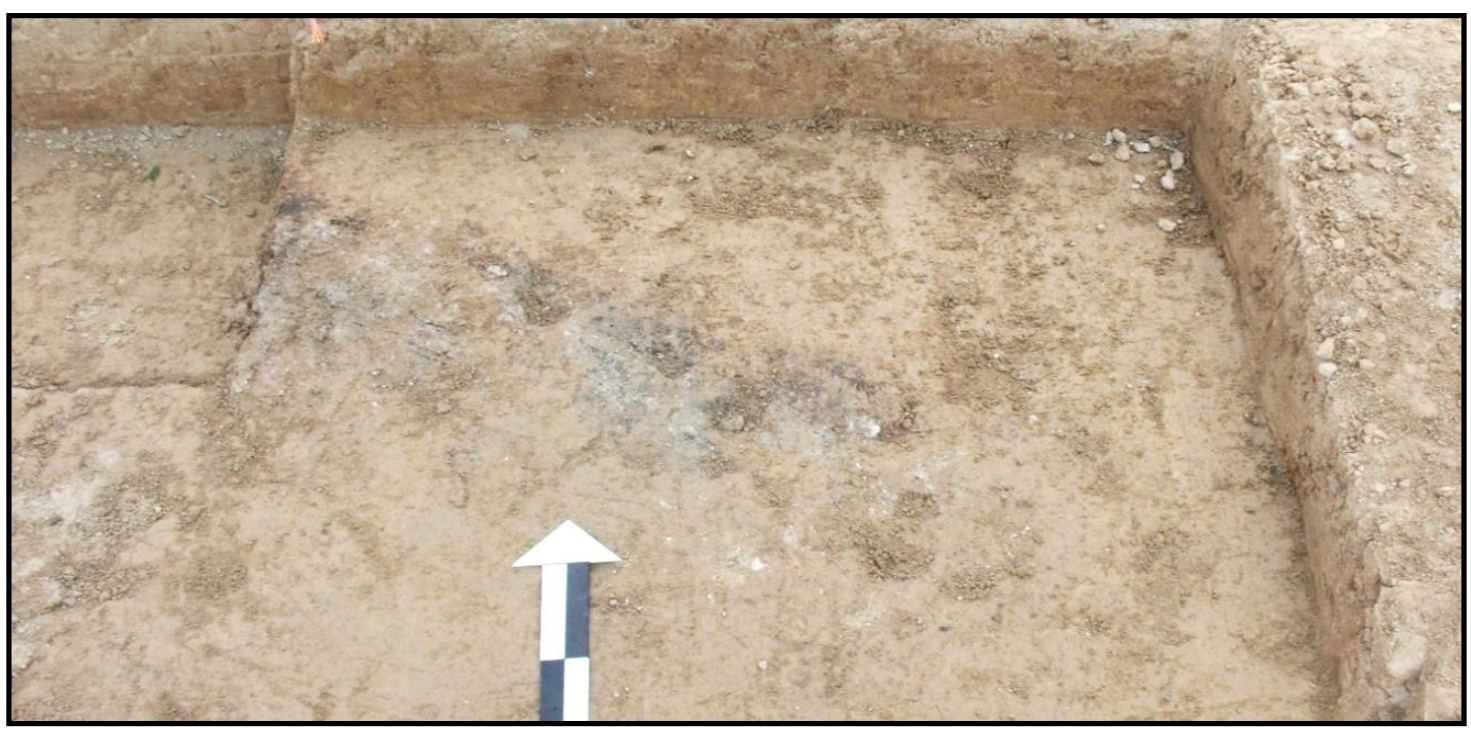

Figure 12-7. Top part of Feature 21 showing mottled dark, linear stained sediment in Block C (scale in 10 centimeter increments).

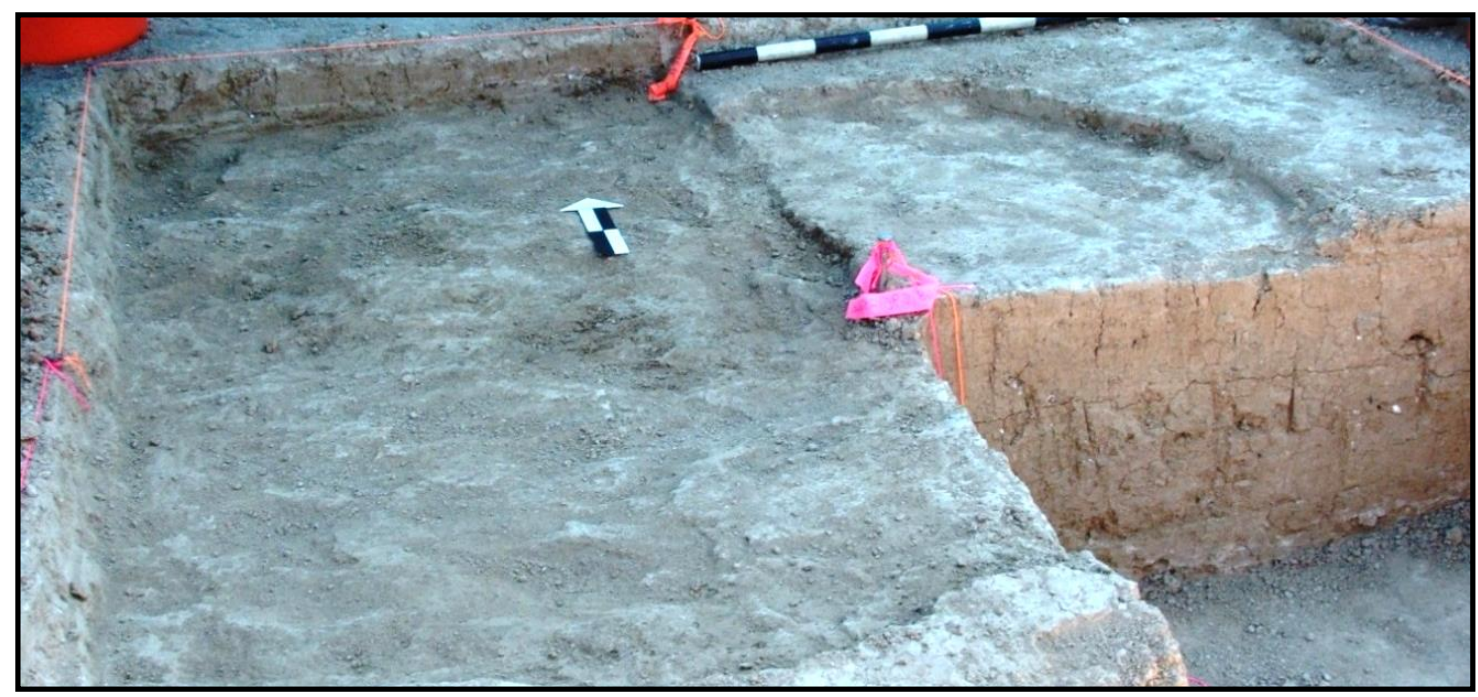

Figure 12-8. Overview of mottled dark stained sediment of Feature 21 across multiple units in Block C (scale in 10 centimeter increments).

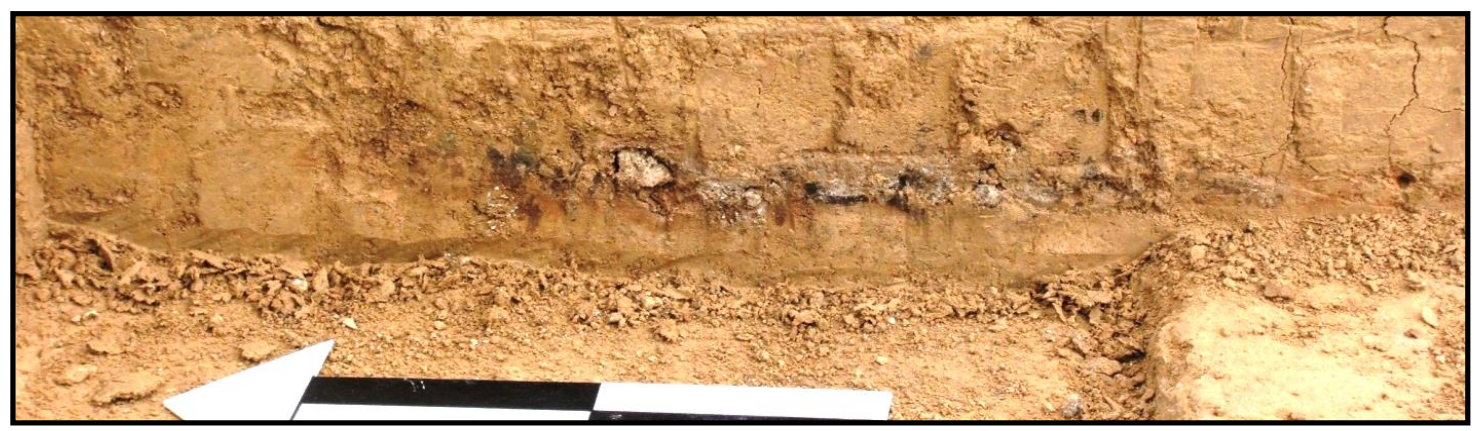

Figure 12-9. Close-up profile of part of Feature 21 in Block $C$ (scale in 10 centimeter increments). 
significant quantity of lithic debitage $(N=118)$, whereas three other samples yielded mostly tiny faunal bone fragments $(N=76)$, and two other samples mostly tiny burned clay pieces (see Table 12-3). The tiny bones include several identifiable burned fragments such as four fish vertebrae, a rodent bone, catfish mandible, plus five burned hackberry nutlets. Unburned bones of fish and rodents were also present.

Small sediment samples were sent for diatom and phytoliths analyses. No diatoms were recovered from sample \#2060-4 (Appendix C). In processing for phytoliths, 10 bone fragments, seven of which were burned, were observed the sand fraction, whereas the overall phytolith preservation was poor, although numerous sponge spicules $(N=26)$ and abundant tree phytoliths were present (Appendix C).

This poorly defined feature is not considered cultural as it lacked characteristics of intentional construction or evidence of a particular function, although sparse and scattered cultural remains were in the immediate vicinity. Feature 21 probably represents a natural tree burn, potentially in the general time frame as the Martindale component or slightly older.

\subsubsection{Feature 23}

Feature 23 appeared as a mottled and dispersed burned area across at least portions of four units between 274 and $280 \mathrm{cmbs}$ and less than $110 \mathrm{~cm}$ south southeast of burned area Feature 19 in Block A (see Figures 12-1 and 12-10). This irregular shaped burned area measured at least 170 -by- $180 \mathrm{~cm}$. Its surface was irregular, with some patchy areas of ashy sediment, orangish (10YR 6/3) burned clay and dark charcoal stained (10YR 2/1) sediments, surrounded by the natural yellow brown (10YR 5/4) sandy clay. Very few burned rocks, almost no chert debris and no sizable animal bone fragments, were detected. Minor rodent disturbances were also observed. At least two large sediment samples and a sample of various sizes of Rabdotus shells were collected.

Two sediment samples (\#2351-4 and \#2357-4) that totaled 115.5 liters were mechanically floated and sorted. Very limited cultural materials were

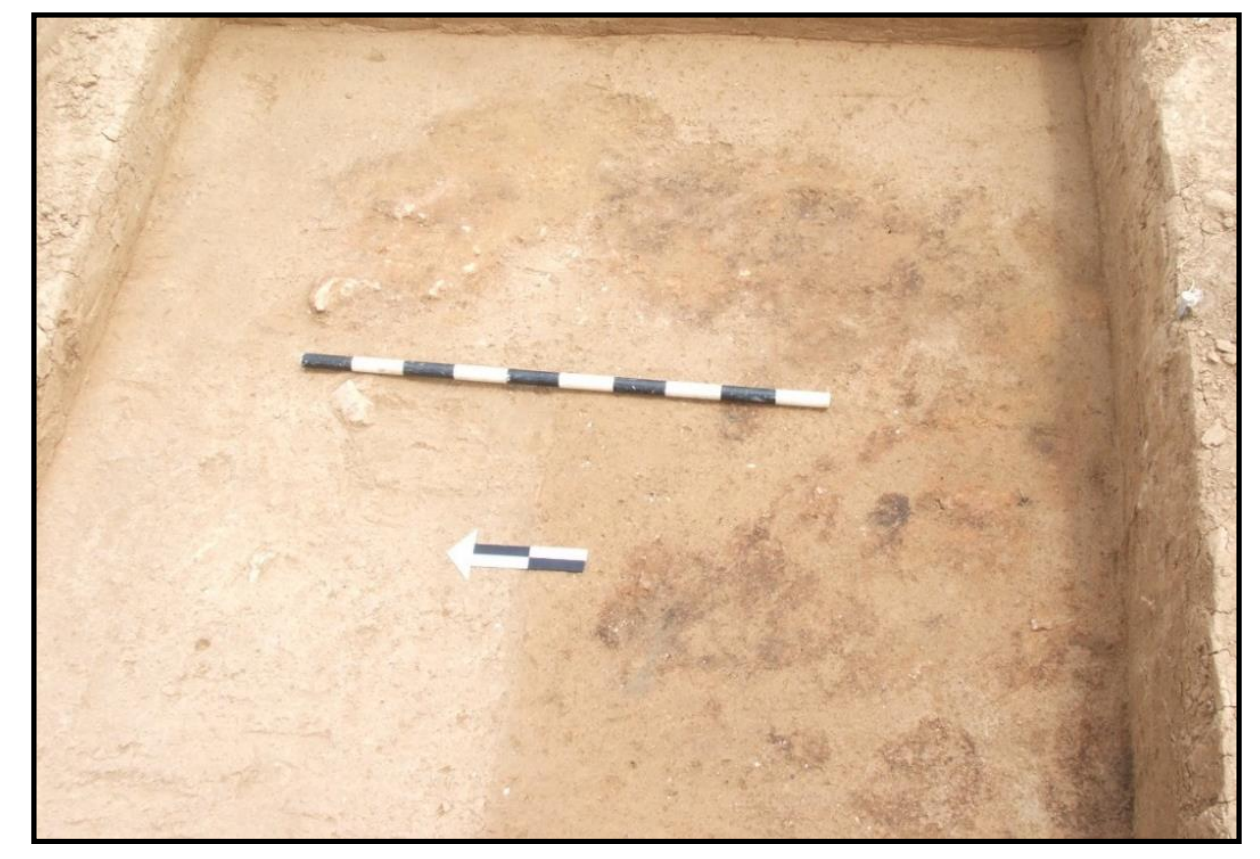

Figure 12-10. Oblique view of mottled reddened and dark stained sediment in Feature 23 in Block C (scales in 10 centimeter increments). 
present, although quantities of snail shell fragments and some burned clay pieces were recovered (see Table 12-3).

Stratigraphically, this burned area was slightly above burned area Feature 19 and below Rabdotus Feature 15 in Block A (see Chapter 8.0, Figure 86). During fieldwork, parts of Feature 23 resembled a dispersed clean out of a hearth and even a disturbed hearth area. As excavations preceded, this poorly defined area revealed limited burned rocks and few chert flakes and became confusing to the excavators. It was finally determined to reflect a noncultural event, the same as Features 19 and 23. The lack of diagnostic artifacts and overall sparse cultural materials directly associated and the adjacent two noncultural features contributes to the interpretation this was not a cultural feature. Without precise radiocarbon dates, it is unclear how old this apparent natural burn is.

\subsubsection{Feature 31}

Feature 31 was left on a high spot in the southeastern corner of Block B during the initial mechanical stripping (see Figure 12-1). It was subsequently excavated from 208 to $217 \mathrm{cmbs}$ in N71/E79. This was a well-defined, burned rock basin/hearth that measured about $45-$ by $-55 \mathrm{~cm}$ in diameter, which exhibited a shallow $10 \mathrm{~cm}$ deep by $55 \mathrm{~cm}$ wide basin (Figures 12-11, 12-12, and 1213). The dark stained matrix (10YR 2/1), dotted with charcoal flecks around and below the burned rocks, defined the shallow basin. The clustered burned rocks were in no apparent arrangement, but filled the western side of the basin. The 23 burned rocks (\#2414-3) were mostly in the 4.1 to $9.0 \mathrm{~cm}$ size class, weighed 9,500 $\mathrm{g}$ for an average of $413 \mathrm{~g}$ per rock (see Table 12-2). Most were angular chunks of limestone that exhibited internal cracks from heating. Rocks were not stacked, although minimally three were in a partial vertical position

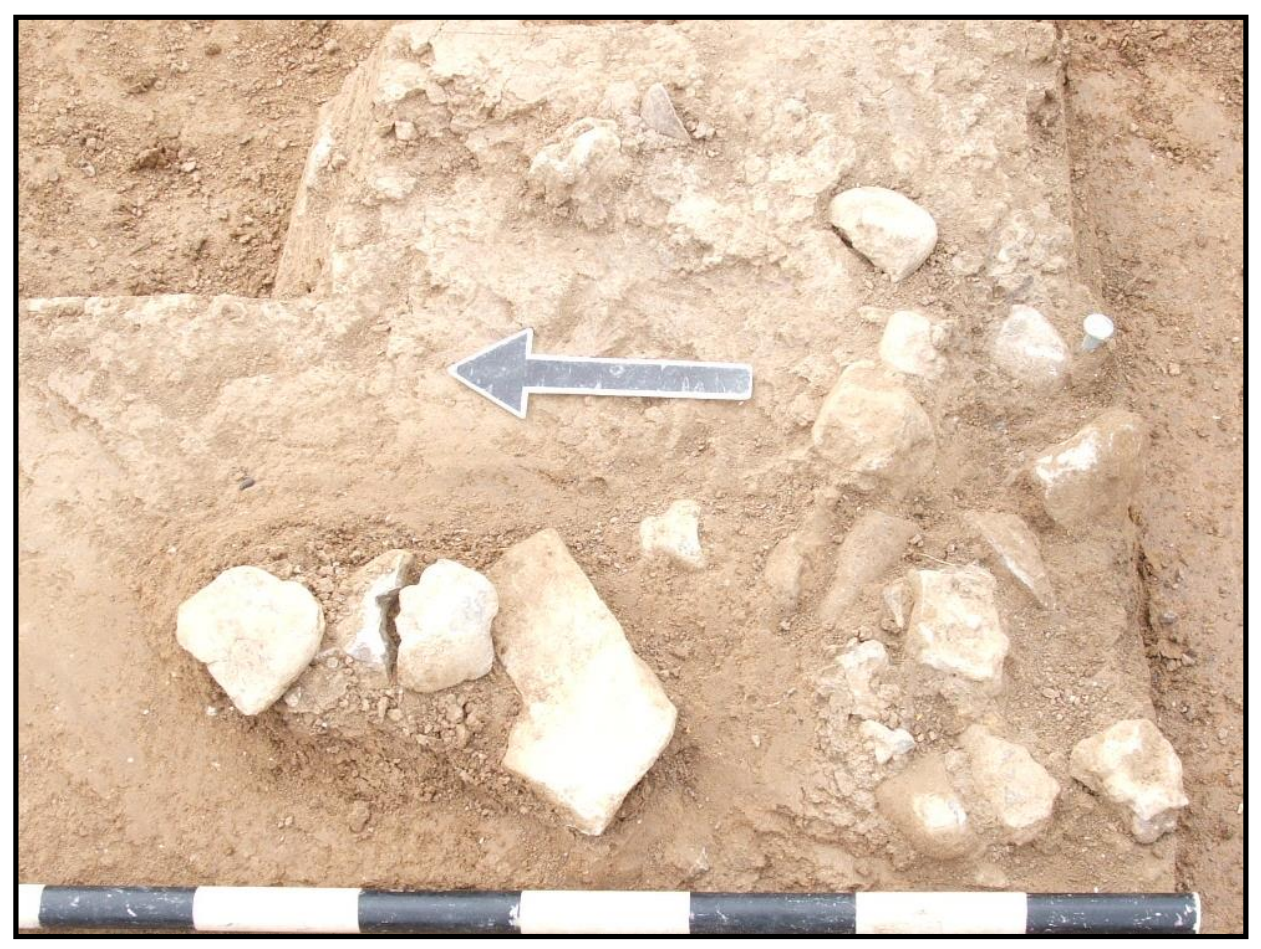

Figure 12-11. Plan view of exposed burned rocks in northern half of Feature 31 with metate fragment just north (scale in 10 centimeter increments). 


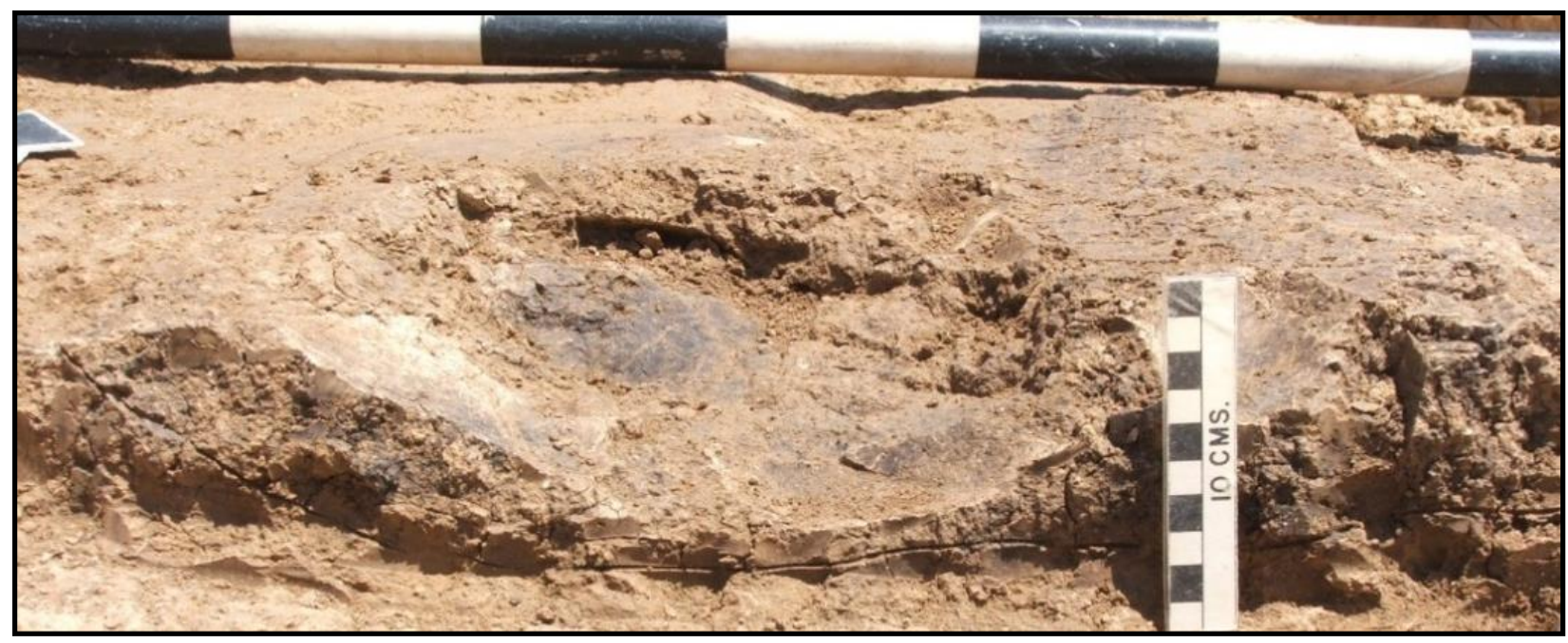

Figure 12-12. Profile of northern half of shallow dark stained basin of Feature 31.

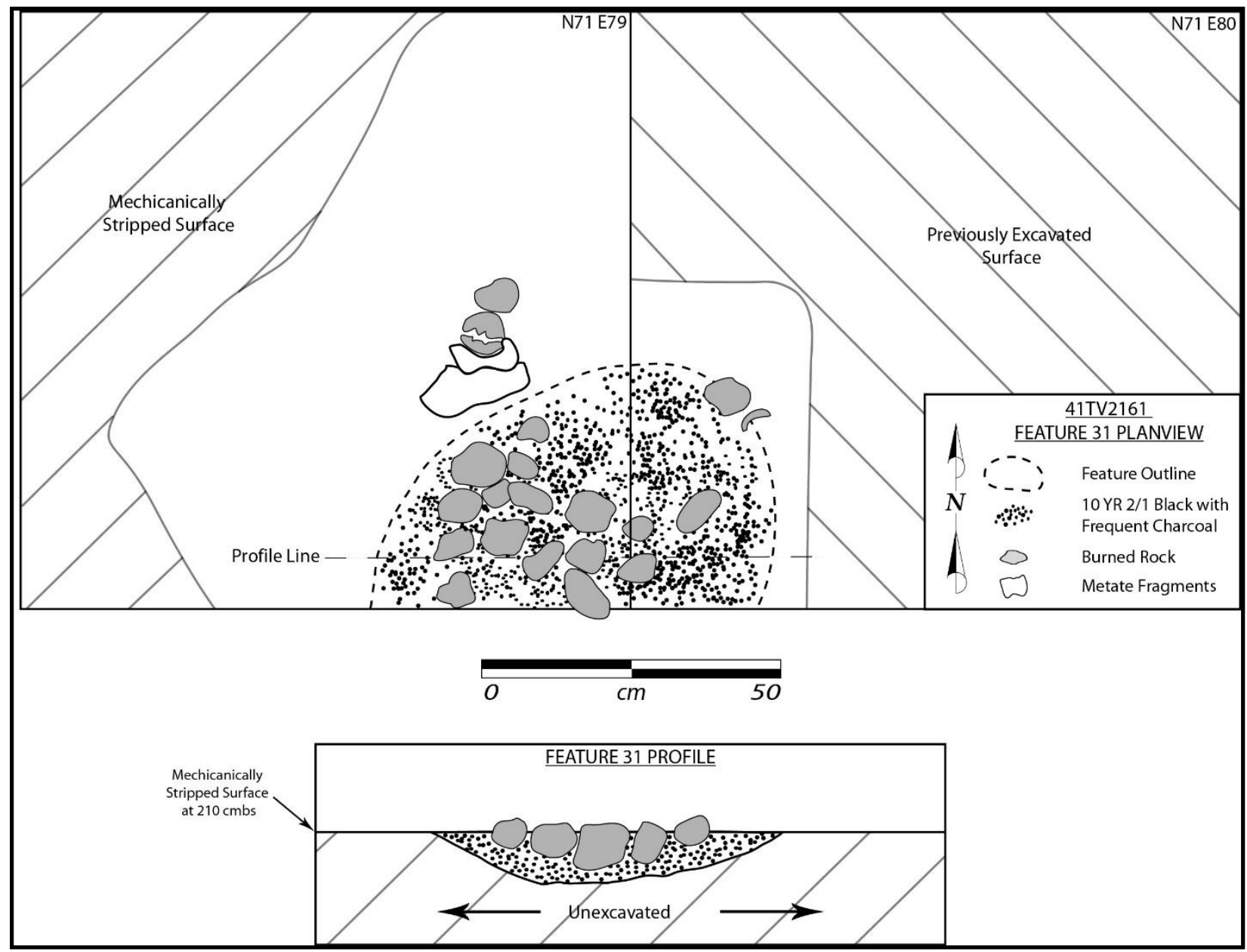

Figure 12-13. Plan view and profile drawing of northern half of Feature 31. 
inside the basin. These mostly intact rocks indicate they were subjected to very few, if more than one heating and cooling event. A few burned chert debitage pieces were also in the basin fill.

Two organic rich sediment samples from the basin were dated. One sample (\#2414-7-1) yielded a $\delta^{13} \mathrm{C}$ corrected $(-25.0 \%)$ age of $5390 \pm 50$ B.P. (Beta234640). The other (\#2414-7-2) yielded a $\delta^{13} \mathrm{C}$ corrected (-24.5\%) age of $5310 \pm 50$ B.P. (Beta234639) (see Table 12-1). The two dates are statistically the same and provide an acceptable age for this feature. These dates place Feature 31 within the Bell/Andice component age range. Feature 31 could be part of that component, although it was 10 to $15 \mathrm{~cm}$ higher in the profile and should be definitely younger given its stratigraphic position in comparison to adjacent Features 30 and 32 (see Chapter 8.0, Figure 8-9. The elevation difference indicates it was not directly part of the same Bell/Andice component represented across the larger part of Block B, but still may represent that time interval.

A 1.0 liter sediment sample (\#2424-4) was floated by Dr. Dering and yielded no charred organic remains (Appendix B). One piece plotted fragmented charcoal sample (\#2414-7) could only be identified as hard wood (Appendix B).

Multiple subsamples of burned rocks were subjected to multiple technical analyses. One of the two samples submitted for starch analysis yielded a gelatinized grain (Appendix F). Both rocks submitted for lipid residue analysis provided insufficient results for a clear interpretation, although chemical biomarkers present indicate the presence of plant and animal residues along with conifer products (Appendix G).

One $3.7 \mathrm{~cm}$ thick, flat slab (\#2414-10) at 214 to 216 cmbs $10 \mathrm{~cm}$ beyond the northern margin of Feature 31 (see Figures 12-11 and 12-13) and functioned as a grinding slab (Figure 12-14). This metate was subsampled in two pieces (\#2414-10 and \#241410a) for starch analysis and yielded ground starch, one lenticular grain, two unidentified grass starches, and two unknown starches (Appendix F). A third piece (\#2414-10b) revealed lipid residues of low fat plant and moderate-high fat content to indicate possible plant and animal combination (Appendix G). A piece submitted for pollen

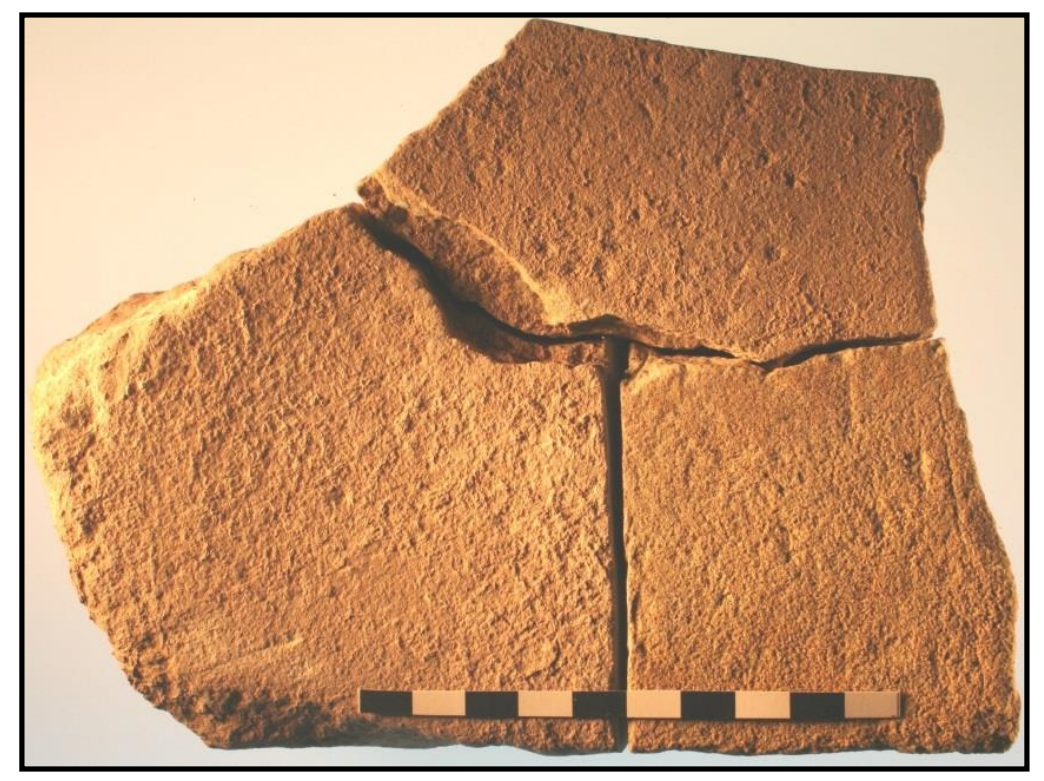

Figure 12-14. Metate fragment (\#2414-10) adjacent to burned rocks in Feature 31 (scale in centimeters). 
analysis yielded very poor pollen preservation (Appendix L). This metate fragment served for grinding minimally grass seeds and other products.

Feature 31 is interpreted as an in situ basin shaped heating facility to minimally heat rocks probably for stone boiling foods. The burned rocks were probably employed in a boiling process as evidenced by the gelatinized starch grains combined with the plant and animal resides off the rocks. The stratigraphic position was 10 to $15 \mathrm{~cm}$ above the Bell/Andice component. On that basis and the obtained radiocarbon date, may be part of that time interval.

\subsection{UNASSIGNED CHIPPED STONE ARTIFACTS}

Formal chipped stone tools not assigned to either identified component include four bifaces, two gouges, and one drill fragment. Each formal tool is discussed below with measurements provided in a Table 12-4. The informal tools and lithic debitage have no clear association and, therefore, not discussed.

\subsubsection{Bifaces}

Specimen \#2023-10 is a complete biface from 279 cmbs in N66/E95, within $1 \mathrm{~m}$ of burned rock Feature 18 in Block C. The piece was collected and left unwashed in anticipation of potential residues analyses. The biface was decortified with both faces completely worked exhibiting large, broad flake scars. A single broad flake scar along the left lateral edge has altered the overall appearance (Figure 12-15, Table 12-4). It is possible the sizable scar on the lateral edge occurred near the final finishing stages of this biface. Lateral edges are slightly sinuous with few small scars, although many of the projections are dulled. It was made of a light gray, high quality chert, probably Edwards chert.

Specimen \#2060-12 is a tiny pointed tip or possibly a barb from a projectile point. It was between 151 and $153 \mathrm{cmbs}$ in unit N68/E95 in Feature 21, a probable tree burn. It is worked on both faces and has tiny pressure flake scars on the lateral edges. It is a dark brown, high quality chert, which under UV light fluoresces a yellowish color indicative of Edwards chert.

Specimen \#2416-10 is the distal end of an early stage biface from the mechanically stripped surface at $233 \mathrm{cmbs}$. This is an elongated section that is also quite thick, with sinuous lateral edges (Figure 12-16). The workmanship exhibits large broad scars with almost no small scars along the lateral edges. The break type appears to be a bending fracture. This piece was manufactured from a fine grained, dark gray with a bluish tint and white crystals. Under UV light this biface does not fluoresce possibly due to the dark color of the material and is not considered Edwards chert. A 0.9 $\mathrm{g}$ chunk off the broken end was sent for INAA (TRC676) and the chemical data indicates it was similar to the local materials on the adjacent hilltop (Appendix H).

Specimen \#2418-10 is a large, complete, early stage biface from roughly $260 \mathrm{cmbs}$ from the mechanically stripped surface. One face exhibits nearly 60 percent cortex with short, broad flake restricted along the lateral edge. The reverse face exhibits broad flake scars that extend toward the middle of the piece, and one prominent knob exhibits hinge scars along the margins in an apparent and failed attempt to remove it.

The pointed distal end has numerous small flake and hinge scars on both faces and the edge is worn to indicate this end was engaged in a pounding motion. This is a homogenous gray chert with no apparent flaws. Under UV light this biface fluoresces a yellowish orange indicative of Edwards chert. A $1.6 \mathrm{~g}$ flake was removed from the interior surface on the reverse face and intended to be submitted for INAA, and the reset retained for later analysis. 
Table 12-4. Unassigned Chipped Stone Tool Data.

\begin{tabular}{|c|c|c|c|c|c|c|c|c|c|c|c|c|c|c|}
\hline PNUM & $\begin{array}{l}\text { Unit } \\
N / E\end{array}$ & $\begin{array}{l}\text { Depth } \\
\text { (cmbs) }\end{array}$ & Type & Stage & Portion & Alteration & Lithology & 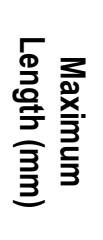 & 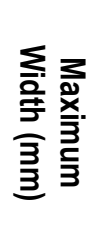 & 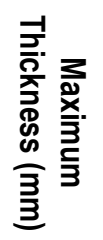 & 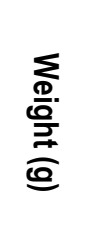 & 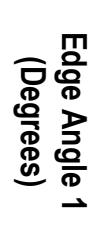 & 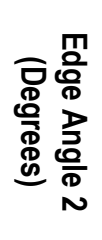 & 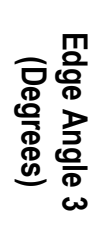 \\
\hline $2023-10$ & 6695 & 279 & $\begin{array}{l}\text { Stage } 3 \\
\text { Biface }\end{array}$ & $\begin{array}{c}\text { Late Stage } \\
\text { Preform }\end{array}$ & Complete & None & $\begin{array}{c}\text { Edwards } \\
\text { Chert }\end{array}$ & 93.3 & 54.2 & 17.1 & 96.3 & 69 & 62 & 71 \\
\hline $2060-12$ & 6895 & $\begin{array}{l}251- \\
253\end{array}$ & $\begin{array}{c}\text { Stage } 3 \\
\text { Biface }\end{array}$ & $\begin{array}{l}\text { Late Stage } \\
\text { Preform }\end{array}$ & Distal & None & $\begin{array}{c}\text { Edwards } \\
\text { Chert }\end{array}$ & 13.28 & 12.26 & 4.19 & 0.6 & 62 & 50 & 0 \\
\hline $2416-10$ & Surface & Surface & $\begin{array}{c}\text { Stage } 2 \\
\text { Biface }\end{array}$ & $\begin{array}{l}\text { Late Stage } \\
\text { Preform }\end{array}$ & $\begin{array}{l}\text { Distal- } \\
\text { medial }\end{array}$ & $\begin{array}{c}\text { Carbonate } \\
\text { buildup }\end{array}$ & $\begin{array}{l}\text { Unknown } \\
\text { Chert }\end{array}$ & 65.85 & 40.19 & 20.31 & 40.7 & 60 & 65 & 0 \\
\hline $2418-10$ & Surface & Surface & $\begin{array}{c}\text { Stage } 2 \\
\text { Biface }\end{array}$ & $\begin{array}{c}\text { Early Stage } \\
\text { Forming }\end{array}$ & Complete & None & $\begin{array}{c}\text { Edwards } \\
\text { Chert }\end{array}$ & 144.04 & 70.54 & 37.29 & 386.8 & 76 & 76 & 0 \\
\hline $2417-10$ & 7694 & 260 & Gouge & $\begin{array}{l}\text { Finished } \\
\text { Product }\end{array}$ & Complete & None & $\begin{array}{c}\text { Edwards } \\
\text { Chert }\end{array}$ & 75.34 & 44.32 & 15.17 & 48.8 & 78 & 73 & 0 \\
\hline $2043-10$ & 6795 & 287 & Gouge & $\begin{array}{l}\text { Finished } \\
\text { Product }\end{array}$ & Complete & None & $\begin{array}{l}\text { Edwards } \\
\text { Chert }\end{array}$ & 40.01 & 49.58 & 14.55 & 28.8 & 72 & 0 & 0 \\
\hline $2162-11$ & 7381 & 294 & Drill & $\begin{array}{l}\text { Finished } \\
\text { Product }\end{array}$ & $\begin{array}{c}\text { Proximal- } \\
\text { Medial }\end{array}$ & $\begin{array}{l}\text { Carbonate } \\
\text { buildup }\end{array}$ & $\begin{array}{c}\text { Unknown } \\
\text { Chert }\end{array}$ & 68 & 20.27 & 8.17 & 8.9 & 83 & 78 & 0 \\
\hline
\end{tabular}




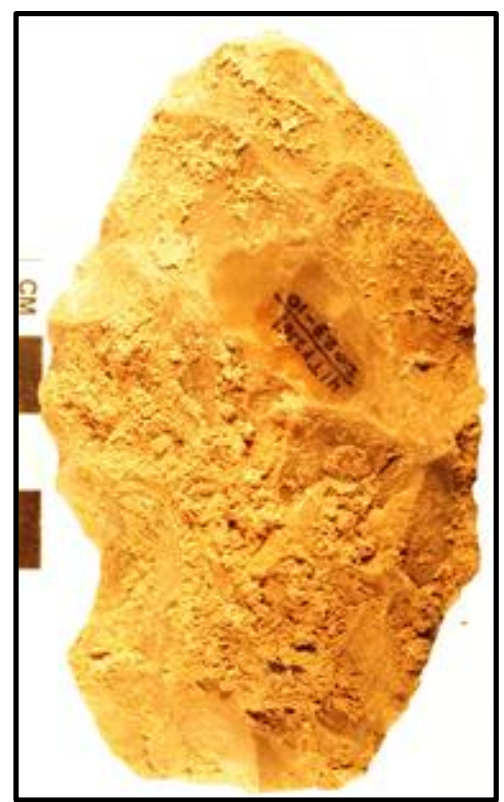

Figure 12-15. Complete unwashed biface (\#2023-10).

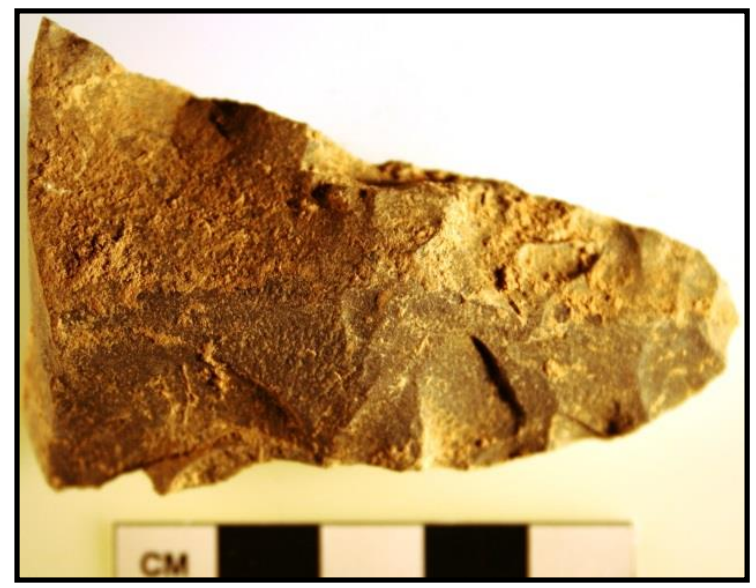

Figure 12-16. Distal end of unwashed, early stage biface fragment (\#2416-10).

\subsubsection{Gouges}

Specimen \#2417-10 is a complete unifacial gouge from $260 \mathrm{cmbs}$ on the mechanically stripped surface in Block D. The location is below the Bell/Andice component in Block B. Since it was lying on the surface is it not clear if the piece was in context and, thus, it was left unassigned. This triangular shaped gouge has a slightly concave ventral surface with short flake scars along the very margin, except at the distal end were there are no scars. The original bulb of percussion is partially present at the narrow proximal end, indicating this specimen was produced from a large flake blank. The dorsal surface is covered in broad flake scars with a high spot near the middle. The lateral edges exhibit short scars from percussion work, which create edge angles between 50 and 72 degrees (Figure 12-17). The distal end exhibits one large continuous, lateral flat scar without an obvious impact mark, which implies the end was rejuvenated. This created a 60 to 64 degree edge angle. The very distal edge has a few tiny use scars and is slightly rounded. The artifact was sent for high-powered use-wear analysis and revealed soft polish (Sf) with plant tissue $(\mathrm{Pt})$, and resin $(\mathrm{Re})$ residues to imply it was employed on plants and probably hafted (Appendix D). It was then subjected to starch analysis, although no starches were recovered (Appendix F). Under UV light this gouge fluoresces a yellow indicative of Edwards chert.

Specimen \#2043-10 is a short gouge from $287 \mathrm{cmbs}$ in N67/E95 of Block C. This is a triangular cortical flake with the wide distal end and exhibits a steeply worked edge angle (72 degrees) with multiple scars parallel to the long axis. Two areas on the worked face show short flakes scars with hinge terminations. The pointed proximal end has an impact scar and bulb of percussion on the ventral side. The dorsal surface contains about 50 percent, light colored cortex, whereas the remaining dorsal face has three broad flake scars. The edge of the distal end is relatively sharp. This is a multicolored light gray chert with some mottling and dark sports. This piece could have also functioned in a scraping tool on hard materials. Under UV light this gouge fluoresces a mottled orangish similar to some Edwards chert. This artifact was sent for starch analysis and revealed no starches (Appendix H). 


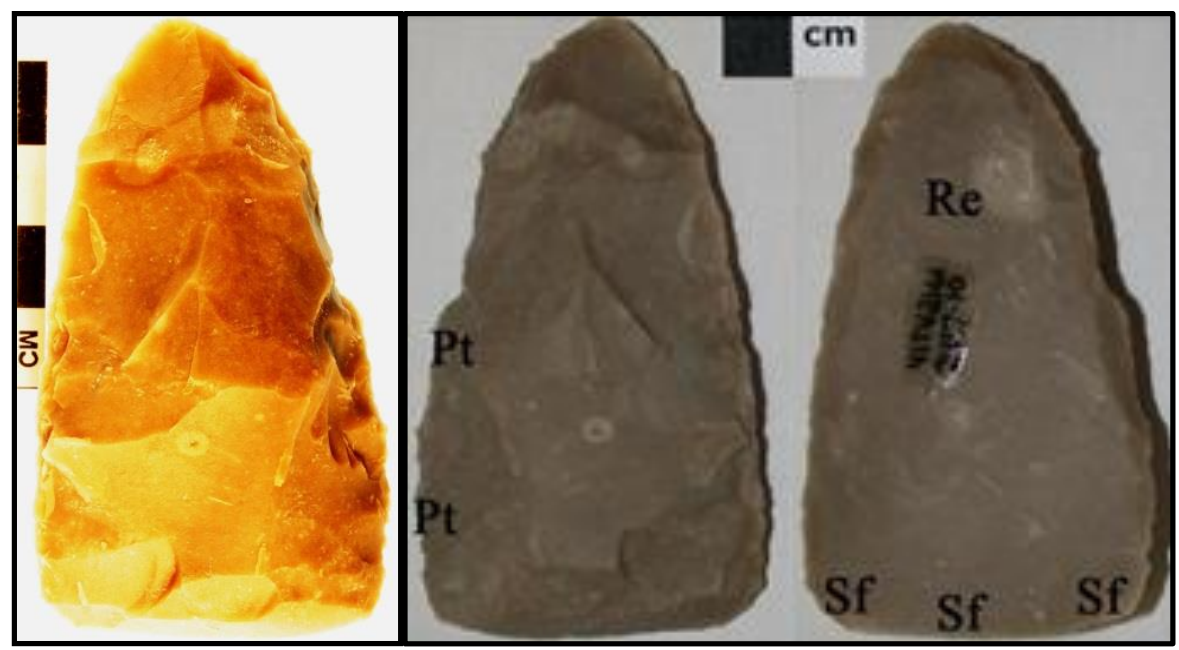

Figure 12-17. Photographs of complete washed gouge (\#2417-10, left) and use-wear results (right).

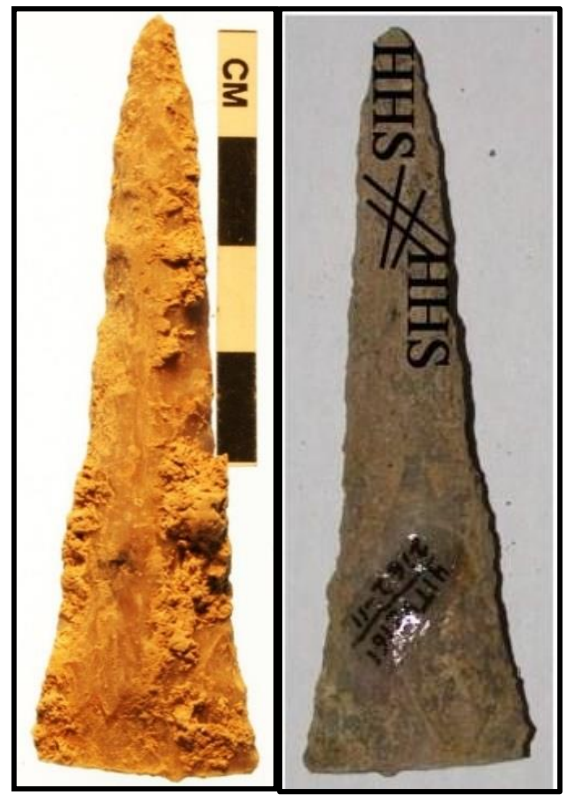

Figure 12-18. Complete unwashed drill stem (left, \#2162-11) depicting use-wear results (right) from just below the Martindale component.

\subsubsection{Drill}

Specimen \#2162-11 is the long distal/bit end of a drill from $294 \mathrm{cmbs}$ in N73/E81 of Block B (Figure 12-18). This unwashed bit was directly associated with other cultural items (i.e., mussel shells, lithic debitage, burned rocks, and bone fragments), just slightly below the Martindale component, and potentially part of that occupation. The proximal end exhibits a snap break, whereas the bit is diamond shaped in cross-section and thick enough to withstand considerable twisting and pressure on hard substances. Under UV light this drill section fluoresces an orangish color similar to some Edwards chert. This unwashed piece was subjected to high-powered use-wear analysis and revealed hard/high silica (HHS), striae (\#) on the very distal end indicative of boring hard/high silica material such as wood (Figure 12-18; Appendix D). 
Table 12-5. Unassigned Ground Stone Data.

\begin{tabular}{|c|c|c|c|c|c|c|c|c|c|c|c|c|c|}
\hline PNUM & Class & $\begin{array}{c}\text { Feature } \\
\text { No. }\end{array}$ & 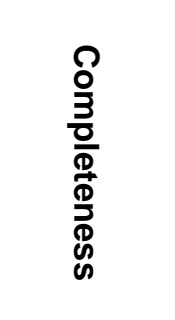 & 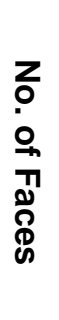 & 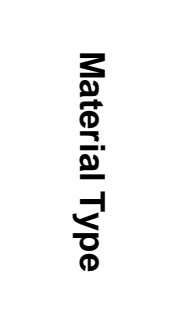 & 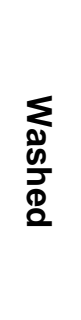 & 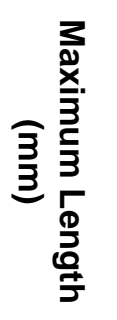 & 氕 & 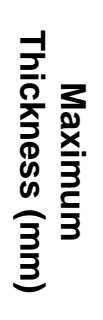 & $\frac{\substack{\Phi \\
\frac{\Phi}{2}}}{\stackrel{0}{a}}$ & 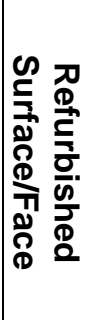 & 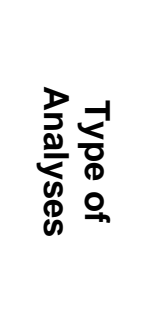 & Observations \\
\hline $2046-10$ & Mano & None & Complete & 1 & Quartzite & No & 111.3 & 94.9 & 48.4 & 693 & No & None & $\begin{array}{l}\text { Some calcium } \\
\text { carbonate }\end{array}$ \\
\hline 2104-11 & Mano & None & Complete & 1 & Sandstone & Yes & 62 & 60.5 & 43 & 199.1 & No & Starch & $\begin{array}{l}\text { Possibly recycled } \\
\text { as burned rock }\end{array}$ \\
\hline $2367-10$ & Metate & None & $\begin{array}{c}3 / 4 \\
\text { Fragment }\end{array}$ & 1 & Sandstone & No & 157 & 117 & 57.9 & 134.6 & No & None & $\begin{array}{l}\text { Partially covered } \\
\text { in calcium } \\
\text { carbonate }\end{array}$ \\
\hline $2396-10$ & $\begin{array}{c}\text { Mano/ Burned } \\
\text { Rock }\end{array}$ & None & Fragment & 1 & Sandstone & No & 90.7 & 75.1 & 24.3 & 243.7 & No & XRF & Red ocher stained \\
\hline $2396-11$ & $\begin{array}{c}\text { Metate/Burned } \\
\text { Rock }\end{array}$ & None & Fragment & 1 & Sandstone & No & 70.3 & 69.2 & 29.3 & 203.7 & Yes & XRF & $\begin{array}{l}\text { Red \& black ocher } \\
\text { stained }\end{array}$ \\
\hline $2414-10$ & Metate & 31 & Fragment & 2 & Sandstone & No & 118.6 & 111.0 & 36.0 & 710.7 & No & $\begin{array}{l}\text { Starch. } \\
\text { Lipids (b), } \\
\text { Pollen }\end{array}$ & $\begin{array}{l}\text { From Feature 31, } \\
\text { covered in } \\
\text { calcium } \\
\text { carbonate. Total } \\
\text { weigh of } 4 \text { pieces } \\
1584.0 \mathrm{~g} \text {. Bottom } \\
\text { dark stained \& flat }\end{array}$ \\
\hline
\end{tabular}




\subsection{UNASSIGNED GROUND STONE ARTIFACTS}

Ground stone artifacts not assigned to either component include three manos and three metate fragments. Each is presented and discussed below in order of its PNUM with metric measurements presented in Table 12-5 and general profiles depicted in Figure 12-19.

\subsubsection{Manos}

A complete mano (\#2046-10) was between 248 and $260 \mathrm{cmbs}$ in N67/E76 in Block C. This is an ovalshaped, river rounded, quartzite cobble. It lacks clear signs of intensive wear and use, although one limited area was possibly used. Both faces are convex and partially covered in calcium carbonate obscuring parts of the surface. No technical analyses were conducted on this unwashed artifact. Four burned rocks, a chert flake, a mussel shell fragment, Rabdotus shells, and charcoal were in this same level and unit. This artifact was slightly above burned rock Features 18 and 20 with the potential for an association with the Martindale component.
A small fragment of a possible mano (\#2104-11) was from $290 \mathrm{cmbs}$ in N71/E82 in Block B (Figure 12-20). It was nearly $20 \mathrm{~cm}$ below Feature 26 and the Martindale component and associated with 17 tiny burned rock fragments, a chert flake, an edgemodified flake, 34 bone fragments less than $3 \mathrm{~cm}$ in length, and 3 mussel shell fragments. This small sandstone fragment is burned and probably recycled as a burned rock. Both faces are restricted in area and are relatively flat and smooth. Actual wear and striations are difficult to observe. It was subjected to starch analysis, with no starches recovered (Appendix F).

Specimen (\#2396-10) is a possible mano fragment from $254 \mathrm{cmbs}$ in N79/E86 from Block $\mathrm{F}$ in a light yellow-brown (10YR 5/4) sandy clay loam. No other cultural materials were in this level or in the level immediately below, only 15 to 20 scattered Rabdotus shells. This relatively thin, rounded sandstone fragment is mostly covered in a thin layer of calcium carbonate with one relatively flat face with a red ochre stain visible (Figure 12-21). The opposite face is slightly convex and totally covered in calcium carbonate. This fine grained, dark colored sandstone appears heat altered and the

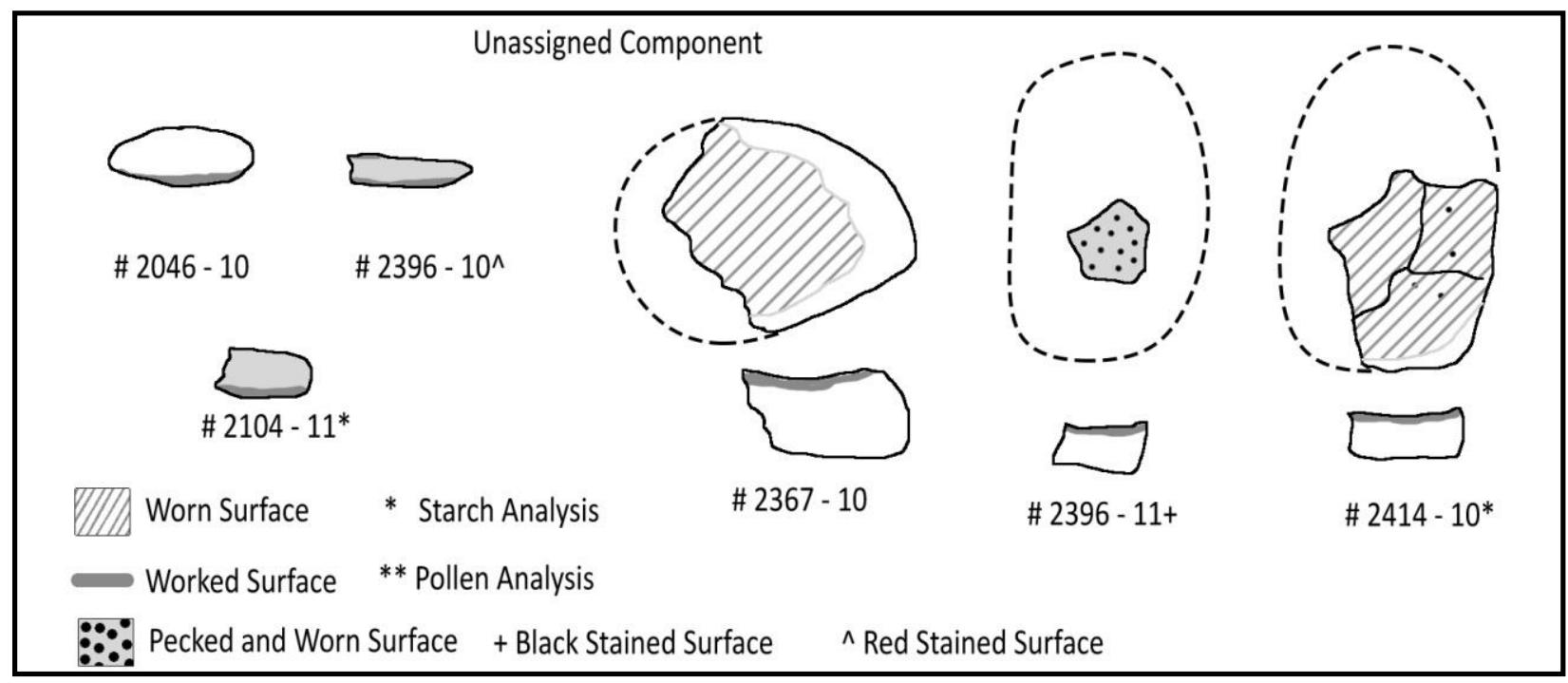

Figure 12-19. General profiles and plan views of manos and metates from unassigned context. 
broken edges are indicative of thermal fracturing. The piece was subjected to $\mathrm{x}$-ray fluorescence (XRF) analysis, which documented iron oxide was applied to one surface (Appendix I). Given the uncertainty of the context, it is also unclear what or how this specimen was employed.

\subsubsection{Metates}

A questionable metate fragment (\#2367-10) was from between 270 and $280 \mathrm{cmbs}$ in Feature 19 of Block A. No other cultural debris was in this unit level, only the dark stained sediments of Feature 19. This is a water-rounded sandstone cobble with one convex face and one concave face. The concave face is a possible grinding surface, although most surfaces are covered in a thin layer of calcium carbonate obscuring wear or polish. The usable surface area of the concave face on this thick rock is minimal, thus restricting the possible grinding surface. The rock is broken and burned exhibiting multiple tiny fissures and a discolored interior. This may be a recycled as a hot rock after use as a metate.

A possible metate fragment (\#2396-11) was 252 cmbs in N79/E86 in Block F. This piece was about $15 \mathrm{~cm}$ from the red stained mano fragment \#239610. This is the midportion of a relatively thin slab with breaks on all sides (Figure 12-22). One face is very flat with very small, shallow peck/pot marks, and is covered in a dark pigment. The opposite face is slightly irregular and the calcium carbonate covers a red stained surface (Figure 12-22). The piece was subjected to $\mathrm{x}$-ray fluorescence (XRF) analysis and documented iron oxide was applied to a single surface of this sandstone (Appendix I).

Metate fragment \#2414-10 associated with Feature 31 is also discussed in the Features section (see Figures 12-11, 12-13, and 12-14). This is a sandstone slab with two flat faces and natural appearing lateral edges. The worked face reveals a slight concave surface that tapers gradually towards the middle with no obvious striations or polish.

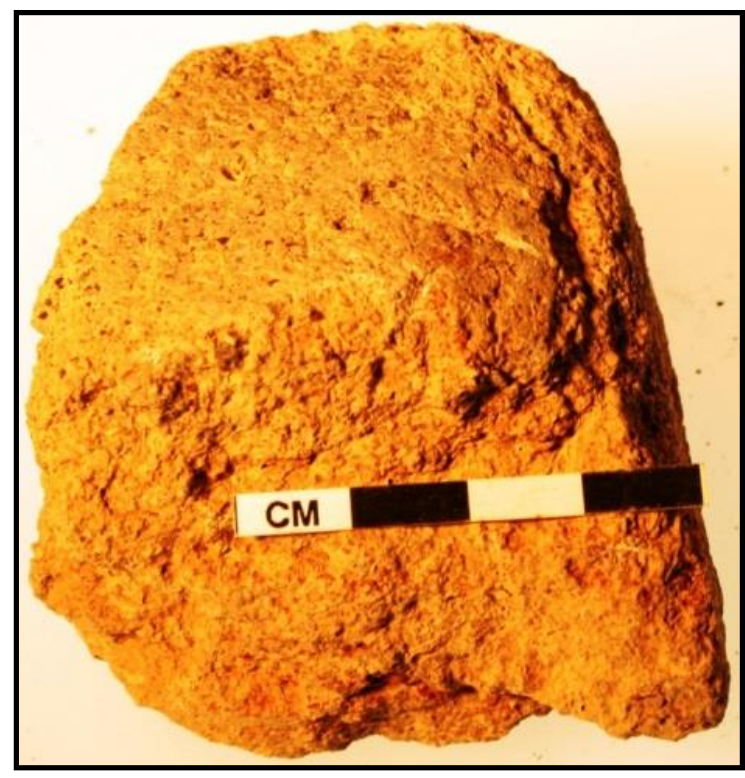

Figure 12-20. Oblique view of mano fragment (\#2104-11) from below Feature 26.

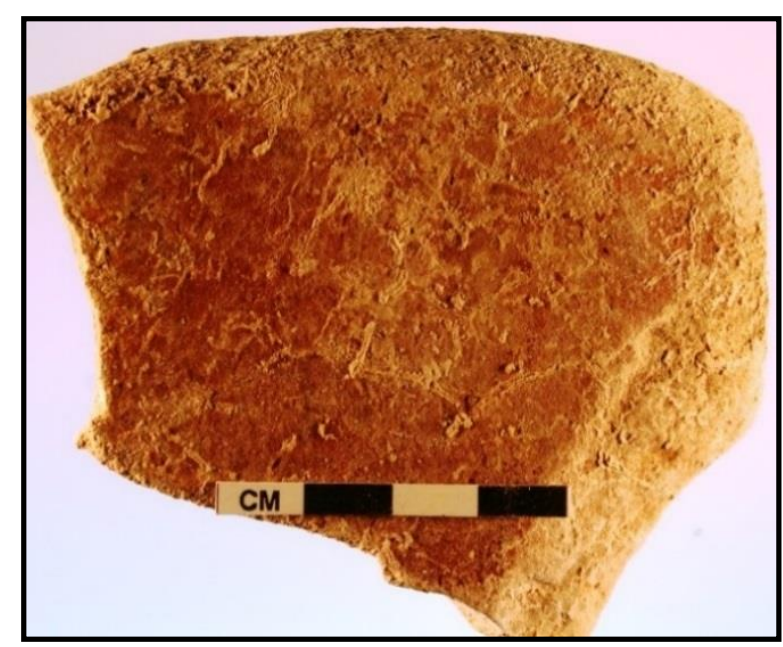

Figure 12-21. Plan view of possible mano fragment (\#2396-10) with red pigment staining from Block $F$ (scale in centimeters).

Tiny, shallow peck marks are towards the middle section. The worked face does not have a welldefined boundary. The opposite face is flat with a dark organic stained color. This metate fragment served for grinding minimally grass seeds and other products as documented by the starch grain analyses (Appendix F). 

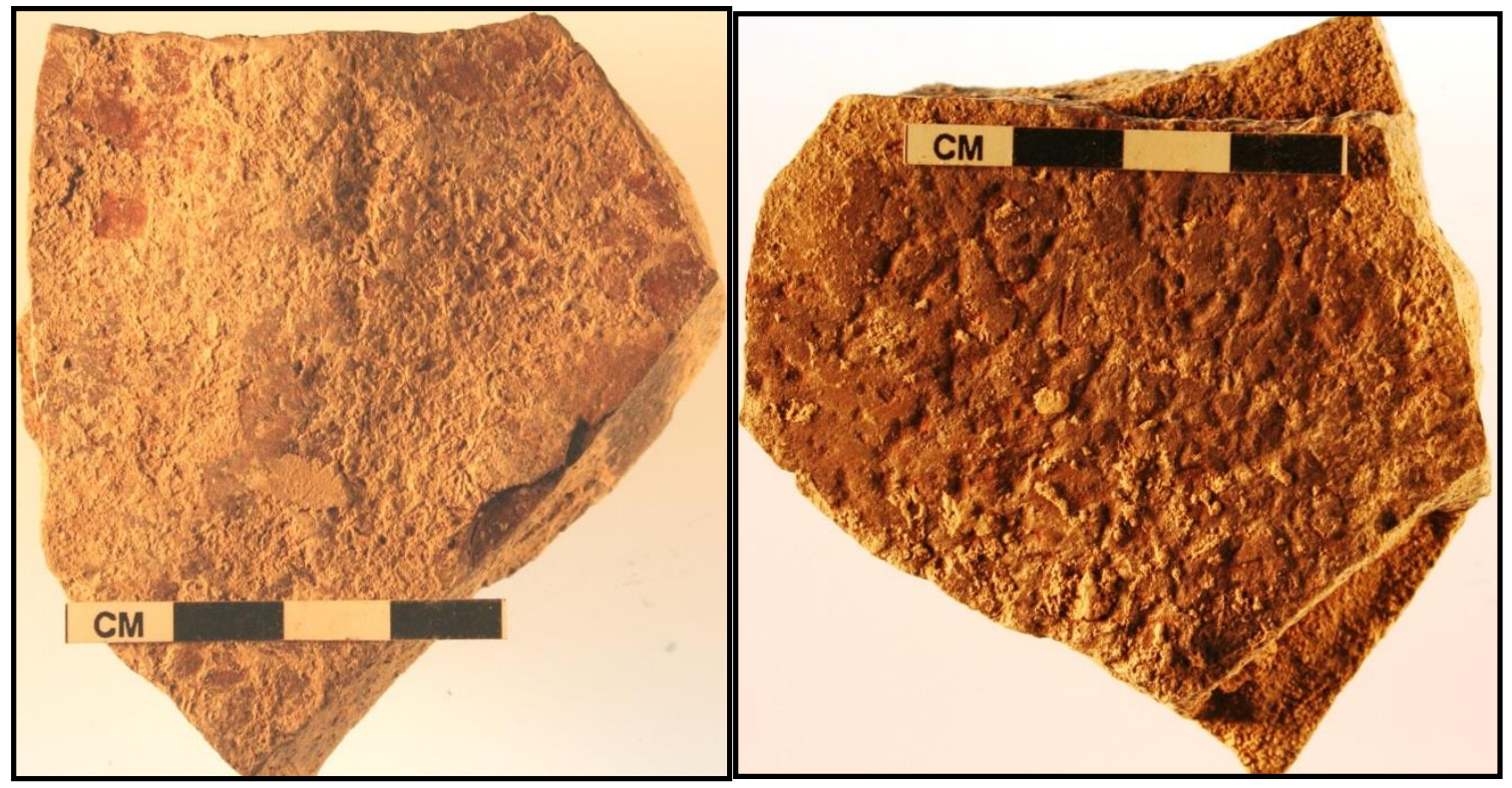

Figure 12-22. Two Plan views of fragmented metate (\#2396-11) with red (left) and black (right) pigment staining from Block $F$ (scale in centimeters).

Table 12-6. Unassigned Bone Data.

\begin{tabular}{|l|l|c|c|c|c|c|c|}
\hline \multicolumn{1}{|c|}{ Taxon } & Species & Count & $\begin{array}{c}\text { Weight } \\
\mathbf{( g )}\end{array}$ & $\begin{array}{c}\text { Minimum } \\
\text { No. of } \\
\text { Individuals }\end{array}$ & $\begin{array}{c}\text { Burned } \\
\text { Pieces }\end{array}$ & $\begin{array}{c}\text { Spiral } \\
\text { Fractured }\end{array}$ & $\begin{array}{c}\text { Cut } \\
\text { Marks }\end{array}$ \\
\hline Deer & - & 2 & 9.4 & 1 & & & \\
\hline $\begin{array}{l}\text { Deer/pronghorn } \\
\text { artiodactyl size }\end{array}$ & - & 15 & 23.3 & & 4 & & \\
\hline Pronghorn & & 3 & 12 & 1 & & & \\
\hline Cottontail & & 5 & 1.4 & 1 & & & \\
\hline Jackrabbit & & 19 & 14.3 & 1 & & & \\
\hline Rabbit family & Indeterminate & 29 & 4.5 & & & & \\
\hline Rodents & Indeterminate & 24 & 2.3 & 1 & & & \\
\hline Turtle & Indeterminate & 1 & 2.6 & 1 & & & \\
\hline Snake & Indeterminate & 2 & 0.4 & 1 & & & \\
\hline Bird & - & 3 & 1.1 & 1 & & & \\
\hline Fish & Indeterminate & 2 & 0.9 & & & & \\
\hline \multicolumn{2}{|l|}{ Black drum } & 1 & 0.8 & 1 & & & 1 \\
\hline Indeterminate & Catfish & 1 & 0.1 & 1 & & & $\mathbf{1}$ \\
\hline
\end{tabular}




\subsection{VERTEBRATE FAUNAL REMAINS}

The unassigned faunal assemblage includes 244 bone fragments with an average weight of $0.41 \mathrm{~g}$ and nearly 99 percent are under $3 \mathrm{~cm}$ long (Table 12-6). The small fragments make it difficult to identify cutmarks and decipher green bone breaks. The indeterminate pieces account for 56 percent of the total unassigned assemblage, whereas ten taxa are identified. This total and the following discussions do not include the 441 bone splinters from heavy fractions in 11 floated sediment samples (see Table 12-3).

The tiny bone fragments discussed in this section were recovered primarily from Block $\mathrm{C}$, and below the Martindale component in Blocks B and D. Those below the Martindale component in Blocks B and D could potentially be associated with the Martindale component, but were stratigraphically slightly below and could not be comfortably assigned to that component. The bones from Block $\mathrm{C}$ probably include a part of the Martindale component, however, since Block $\mathrm{C}$ lacked diagnostic points and secure radiocarbon dates, the materials from there could not be comfortably assigned (see section 8.2.3). Some bones in Block $\mathrm{C}$ were also stratigraphically below the Martindale component, and lack a known age.

Table 12-6 provides the taxon and species identified, plus other key aspects of the unassigned bones. The MNI is one in every instance. Many species are the same as identified in the Martindale component, and again the absence of bison is surprising.

Bird elements are again represented by a left (\#2057) and right (\#2162) proximal coracoid of a prairie chicken/grouse size bird. The former was from 290 cmbs in N67/E98 in Block C, whereas the later was from between 280 and $290 \mathrm{cmbs}$ in N73/E81 in Block B. These are nearly identical to those in the Martindale component and may have originated from that component.

Artiodactyl (deer/pronghorn) size pieces are mostly small long bone fragments with medial metatarsals, rib fragments, and a first phalange identified. Their small size and the elements represented are similar to those in the Martindale component.

Pronghorn is represented by multiple teeth and a third phalange. A complete left $\mathrm{M}_{3}$ in the eruption stage (Figure 12-23) (\#2019) was from $276 \mathrm{cmbs}$ in N66/E94 in Block C and represents an adult animal, whereas a root etched left $\mathrm{M}_{1}$ (\#2034) of an adult was from $283 \mathrm{cmbs}$ in N66/E97 of Block C. These elements may have also been part of the Martindale component, but this block lacked diagnostic points and secure radiocarbon dates to comfortably assign these to that component (see section 8.2.3).

Fish are also represented with one otolith (\#2066) of a black drum, unidentified skull fragments (\#2162 and \#2104), and a catfish spine (\#2060). This otolith was recovered from between 244 and $260 \mathrm{cmbs}$ in N69/E95 in Block $\mathrm{C}$ at a depth and potentially reflects the Martindale component.

Both cottontail rabbits and jackrabbits are again recognized; with vertebrae, teeth, phalanges, long bone fragments, metapodial fragments, calcanei, tibia, and radii all identified. These elements came from Blocks A, B, and C.

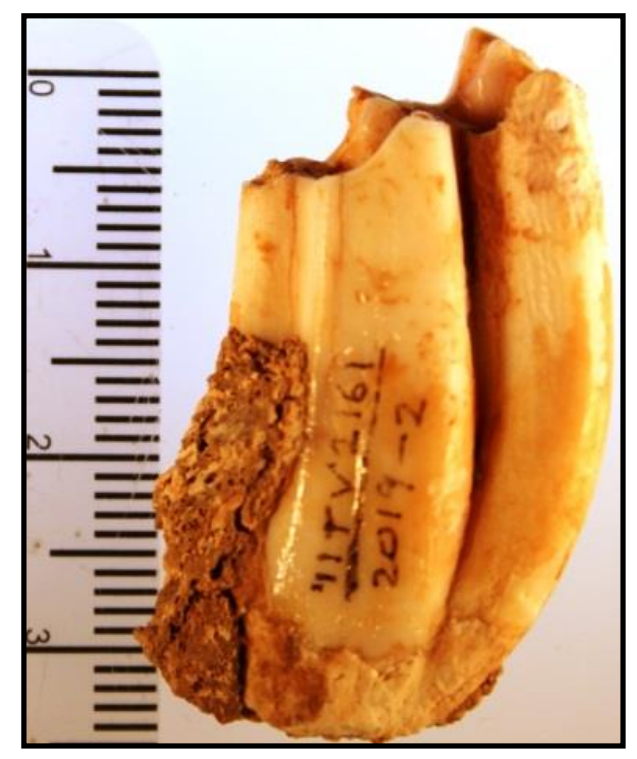

Figure 12-23. Loose pronghorn maxilla left $\mathrm{M}^{3}$ (\#2019, scale in centimeters). 
Table 12-7. Burned Rock Frequencies, Sizes and Weights from Unassigned Proveniences.

\begin{tabular}{|c|c|c|c|c|c|c|c|c|c|c|}
\hline \multirow[t]{2}{*}{ Location } & \multicolumn{2}{|c|}{$\begin{array}{c}\text { Burned Rocks } \\
0-4 \mathrm{~cm}\end{array}$} & \multicolumn{2}{|c|}{$\begin{array}{c}\text { Burned Rocks } \\
4.1-9 \mathrm{~cm}\end{array}$} & \multicolumn{2}{|c|}{$\begin{array}{c}\text { Burned Rocks } \\
9.1-15 \mathrm{~cm}\end{array}$} & \multicolumn{2}{|c|}{$\begin{array}{l}\text { Burned Rocks } \\
>15 \mathrm{~cm}\end{array}$} & \multicolumn{2}{|c|}{$\begin{array}{l}\text { Total Burned } \\
\text { Rocks }\end{array}$} \\
\hline & Count & Wt (g) & Count & Wt (g) & Count & Wt (g) & Count & $\mathrm{Wt}(\mathrm{g})$ & Count & Wt (g) \\
\hline Feature & 10 & 145 & 21 & 2,779 & 31 & 14,119 & 1 & 1,500 & 63 & 18,669 \\
\hline Scattered & 83 & 1,383 & 57 & 8,537 & 1 & 450 & 0 & 0 & 141 & 10,652 \\
\hline Total & 93 & 1,529 & 78 & 11,315 & 32 & 14,569 & 1 & 1,500 & 204 & 29,320 \\
\hline
\end{tabular}

Table 12-8. Summary of Unassigned Materials from Data Recovery.

\begin{tabular}{|c|c|}
\hline Cultural Material Classes & Counts \\
\hline Radiocarbon Dates & 10 \\
\hline Features & 7 \\
\hline Burned Rock Dumps/Discard & Features $18,20,31^{*}$ \\
\hline Tree Burns & Features $19,21,23^{*}$ \\
\hline Rabdotus Shell Concentrations & Feature 15 \\
\hline Bifaces & 4 \\
\hline Drills & 1 \\
\hline Gouges & 2 \\
\hline Manos & 3 \\
\hline Metates & 3 \\
\hline Edge-Modified Flakes & 19 \\
\hline Lithic Debitage & 339 \\
\hline Mussel Shells & 20 \\
\hline Burned Rocks & 204 \\
\hline Vertebrate Faunal Fragments & 244 \\
\hline Burned Clay & 38 \\
\hline Carbonized Plant Remains & 3 \\
\hline Total Materials & 887 \\
\hline Average Thickness (cm) & Variable \\
\hline Spatial Extent Excavated & 38 \\
\hline Volume Excavated $\left(\mathrm{m}^{3}\right)$ & 19.7 \\
\hline
\end{tabular}

This table does not include materials from float samples; ${ }^{*}=$ feature number, not count. 
The 24 rodent size and 2 snake fragments may not represent cultural use, as these species occur naturally in similar deposits and both live in the ground. It is acknowledged rodents were food resources in west Texas as demonstrated by their bones in coprolites (Williams-Dean 1978). Some rodent size bones in Block A (\#2388) exhibit fresh/recent surfaces calling into question their association with the cultural materials.

The single, unburned turtle carapace fragment (\#2131) was recovered between 280 and $290 \mathrm{cmbs}$ in N72/E81 in Block B, just below the Martindale component. It is possible this piece was displaced from the identified component immediately above.

\subsection{MUSSEL SHELLS}

Mussel shell fragments were recovered from Blocks B and C in very limited numbers. Block B yielded 11 pieces (266.9 g) of sufficient size to attempt identification. Six fragments reflect characteristics of Texas Pimpleback (Quadrula petrina). From Block $\mathrm{C}$ nine pieces ( $27.8 \mathrm{~g}$ ) were examined and only one was identifiable, and represents Yellow Sandshell (Lampsilis teres). Both species are known in the region (Howells et al. 1996).

\subsection{BURNED ROCKS}

Burned rocks not assigned to a cultural component include scattered burned rock in Blocks A, B, and $\mathrm{C}$, and two well-defined cultural features, Features 18 and 20. The burned rocks below the Martindale component in Block B could be associated with the Martindale component. Table 12-7 presents the frequencies and weights by the different size classes for features and scattered burned rocks.
Burned rocks within features tend be larger $(<9$ $\mathrm{cm})$, whereas scattered burned rocks are smaller and probably represent discarded items.

\subsection{BURNED CLAY}

Feature 19 (a tree burn) yielded at least 34 irregularly shaped lumps of burned clay, which collectively weigh $307 \mathrm{~g}$ for an average of $9 \mathrm{~g}$ each. All pieces are between 1 and $4.5 \mathrm{~cm}$ in diameter and lack surface impressions. These were undoubtedly created from the intense heat caused as a result of the tree burn. At least four other similar clay lumps were in the immediate vicinity of Feature 19.

\subsection{SUMMARY OF UNASSIGNED MATERIALS}

As stated above, these materials could not be confidently assigned to one of the two identified cultural components in Blocks $\mathrm{B}$ and $\mathrm{D}$, and primarily came from Blocks A, C, E and F, with the exception of Feature 31 in Block B. The smaller blocks (A, C, E, and F) were spatially isolated from larger Blocks (B and D) and could not be stratigraphically linked directly to either defined component. The dates obtained were from materials that provide only approximated ages rather than precise timing of cultural occupations. A few features and artifacts discussed above were potentially associated with the identified components. Without direct and solid indicators (i.e., diagnostic projectile points and dates on charcoal) these materials were left unassigned. The unassigned artifacts and features are as old or older than approximately 5300 B.P. The different classes and frequencies of cultural materials are summarized in (Table 12-8). 
This page intentionally left blank. 


\subsection{SUMMARY, DISCUSSION, AND CONCLUSIONS}

\author{
J. Michael Quigg
}

\subsection{PROJECT SUMMARY}

During April and May 2006, an archeological team from the Cultural Resources Section of the Planning, Permitting and Licensing Practice of TRC Austin office conducted geoarcheological documentation and data recovery excavations at 41TV2161 - the Big Hole site. Hand-excavations were restricted to a $70 \mathrm{~cm}$ thick target zone between ca. 220 and $290 \mathrm{cmbs}$ within a buried prehistoric deposit measuring $>3.5 \mathrm{~m}$ deep on the western side of the site. Site 41TV2161 is located in floodplain deposits of the lower Onion Creek valley in eastern Travis County, Texas. Targeted excavations were $10 \mathrm{~m}$ south of an east-west concrete box culvert under construction. The culvert crossed the site in the immediate vicinity of several positive backhoe trenches that yielded deeply buried cultural remains. Cultural resource investigations were necessary under requirements of Section 106 of the NHPA, the implementing regulations of 36CRF Part 800 and Antiquities Code of Texas (Texas Natural Resource Code, Title 9, Chapter 191 as amended) prior to impacts by planned construction of SH 130 by a private construction firm - Lone Star Infrastructure. The data recovery of a $38.5 \mathrm{~m}^{3}$ sample of prehistoric cultural materials was conducted for TxDOT Environmental Affairs Division under Scientific Services Contract No. 577XXSA003 (Work Authorization No. 57701SA003). Following the initial work authorization for fieldwork, multiple other work authorizations were implemented over the years towards specific aspects of analyses and reporting. Final analyses and report preparation were conducted under contract 57-3XXSA004 (Work Authorization 57-311SA004). All archeological work was under TAC Permit No. 4064 issued by the THC to Principal Investigator J. Michael Quigg.

Prior to data recovery, an archeological crew from Hicks \& Company of Austin encountered site 41TV2161 during an intensive cultural resource inventory south of Pearce Lane along the planned construction zone of SH 130 in the fall of 2005. Following initial discovery, archeologists expanded their investigations westward across SH 130 rightof-way, and completed excavation of 10 backhoe trenches, 13 shovel tests, and 11 test units. These investigations yielded 7 buried cultural features and 1,034 artifacts, some in relatively good context, which represent multiple occupations throughout $>3.5 \mathrm{~m}$ of alluvial deposits. The draft survey and testing report to TxDOT presented their findings and recommendations (Campbell et al. 2006). The Environmental Affairs Division of TxDOT and the THC reviewed the initial findings and recommendations, and determined site 41TV2161 was eligible for listing in the NRHP and as SAL. The proposed roadway development was to directly impact this eligible site and further mitigation excavations were required by THC. The final survey and testing report was published in 2007 (Campbell et al. 2007).

TRC archeologists employed the draft report produced by Hicks \& Company (Campbell et al. 2006) to develop a data recovery plan, subsequently presented to and approved by TxDOT in March 2006 (see Chapter 4.0). Data recovery investigations were initiated in April 2006 through the mechanical removal of between 230 and $250 \mathrm{~cm}$ of sediment from a 30-by-40 m area (roughly 3,000 $\mathrm{m}^{3}$ ) to facilitate hand-excavations targeting deeply buried cultural materials at roughly $250 \mathrm{cmbs}$. Mechanical stripping created a large, deep hole with an uneven bottom surface at depths between 220 and $260 \mathrm{cmbs}$. That depth became the starting point for hand-excavations. No hand-excavations exceeded $300 \mathrm{cmbs}$. 
To determine and isolate specific target areas for hand-excavations within the deep hole, a geophysical survey using GPR over the mechanically stripped surface was conducted by Tiffany Osburn, then with Geo-Marine in Plano, Texas. Numerous geophysical anomalies were detected. Following data processing and assessment, Osburn ranked the anomalies from high to low potential for representing cultural features. Consequently, anomalies 1 through 6 were targeted through the hand-excavation of 1-by$1 \mathrm{~m}$ units that formed continuous excavation blocks (A, B, C, D, E, and F). The type, nature, quantity, and context of cultural materials encountered in each block guided decisions whether or not to expand a block or redirect efforts towards other locations.

The entire $>3.5 \mathrm{~m}$ vertical and cultural material bearing deposit at 41TV2161 was not thoroughly or systematically investigated (see Figure 8-1). The targeted 50 to $70 \mathrm{~cm}$ thick zone between roughly 220 and $290 \mathrm{cmbs}$ yielded two well-defined, stratigraphically separate cultural components in excellent context with high integrity. These components provide significant and important contributions towards understanding two rare and poorly understood hunter-gatherer groups in central Texas and surrounding regions. Collected data also allows us to address environmental conditions during the last part of the Altithermal period of the middle Holocene. Data recovery excavations and subsequent artifact analyses, combined with diverse multiple technical analyses, yielded significant cultural information concerning human behaviors in two rarely encountered and or dated cultural components. The two components include a Bell/Andice component stratigraphically separated by 20 to $25 \mathrm{~cm}$ from a lower Martindale component, both in low-energy overbank deposits. Both components yielded multiple cultural features, well-preserved vertebrate assemblages, sparse organic remains, limited chipped and ground stone tool assemblages, and multiple radiocarbon dates. The assemblages consists of limited diagnostic projectile points, lithic debitage, and varying quantities of burned rocks and mussel shells.

Following 2006 field effort, an interim report was produced by TRC and submitted to TxDOT. The interim report briefly described the field methods, excavations, preliminary findings, initial results from seven technical analyses, and an initial proposed research design for data analyses (Quigg et al. 2007). Subsequently, multiple work authorizations were assigned to TRC for completion of various steps to progress towards completion of this final technical report.

\subsection{SUMMARY OF RESULTS}

The hand-excavation of $38.5 \mathrm{~m}^{3}\left(150 \mathrm{~m}^{2}\right)$ was completed in six excavation blocks (A through F) of different sizes, which resulted in the recovery of 19 features, and numerous artifacts, including 1,948 pieces of lithic debitage, 1,079 burned rocks, 2,917 vertebrate animal bone, 39 formal chipped stone tools, 114 edge-modified flakes, 10 manos, 7 metates, 2 hammer stones, and 89 mussel shell fragments (Table 13-1). Of the recovered features and artifacts, 86.4 percent were from two field identified and stratigraphically separated cultural components in Blocks B and D. The other 13.6 percent $(N=846)$ were from Blocks $\mathrm{A}, \mathrm{C}, \mathrm{E}$, and $\mathrm{F}$ with unidentified associations.

In the final cultural stratigraphic analyses of the roughly $70 \mathrm{~cm}$ thick restricted target zone, two vertically separate, well-defined cultural components in good context were roughly between 212 and $275 \mathrm{cmbs}$ in Blocks B and D. These components are represented by diagnostic projectile points and multiple radiocarbon dates, which constitute the most significant findings and are the primary focus of this final report.

Cultural materials from Blocks A, C, E, and F could not be securely and confidently attributed to one of the two recognized components as they lacked 
Table 13-1. Summary of Materials by Divisions.

\begin{tabular}{|c|c|c|c|}
\hline Cultural Material Classes & Bell/Andice & Martindale & Unassigned \\
\hline & Counts & Counts & Counts \\
\hline Radiocarbon Dates & 12 & 15 & 10 \\
\hline Features & 9 & 3 & 7 \\
\hline Hearths, Organic Stained & 0 & 3 & 0 \\
\hline Burned Rock Dumps/Discard Areas & $\begin{array}{c}16,22,24,27,28 \\
29,30,32\end{array}$ & 0 & $18,20,31$ \\
\hline Tree Burns & 0 & 0 & $19,21,23$ \\
\hline Rabdotus Shell Concentrations & 0 & 0 & 15 \\
\hline Ground Stone Cache & 1 & 0 & 0 \\
\hline Dart Points and Fragments & 3 & 3 & 0 \\
\hline Bell & 2 & 0 & 0 \\
\hline Big Sandy & 1 & 0 & 0 \\
\hline Martindale & 0 & 1 & 0 \\
\hline Fragments & 0 & 2 & 0 \\
\hline Bifaces & 3 & 4 & 4 \\
\hline Scrapers & 0 & 0 & 0 \\
\hline Drills & 0 & 0 & 1 \\
\hline Unifaces & 0 & 0 & 0 \\
\hline Gouges & 0 & 0 & 2 \\
\hline Manos & 5 & 2 & 3 \\
\hline Metates & 3 & 1 & 3 \\
\hline Hammer Stones & 0 & 2 & 0 \\
\hline Choppers & 0 & 0 & 0 \\
\hline Edge-Modified Flakes & 12 & 83 & 19 \\
\hline Lithic Debitage & 180 & 1,429 & 339 \\
\hline Cores & 7 & 7 & 0 \\
\hline Shell tools & 0 & 0 & 0 \\
\hline Bone Tools & 0 & 0 & 0 \\
\hline Vertebrate Faunal Fragments & 591 & 2,082 & 244 \\
\hline Mussel Shells & 15 & 54 & 20 \\
\hline Burned Rocks & 554 & 321 & 204 \\
\hline Burned Clay & 6 & 36 & 38 \\
\hline Socialtechnic Objects & 0 & 0 & 0 \\
\hline Carbonized Plant Remains & 4 hackberry nutlets & $\begin{array}{c}8 \text { grass seeds, } 2 \\
\text { cheno-am seeds, live } \\
\text { oak frags. }\end{array}$ & 3 \\
\hline Total Materials & 1,390 & 4,027 & 887 \\
\hline Average Thickness (cm) & $10-15$ & $10-15$ & Variable \\
\hline Spatial Extent Excavated & $51 \mathrm{~m}^{2}$ & $74 \mathrm{~m}^{2}$ & 38 \\
\hline Volume Excavated $\left(\mathbf{m}^{3}\right)$ & $10.2 \mathrm{~m}^{3}$ & $8.6 \mathrm{~m}^{3}$ & $19.7 \mathrm{~m}^{3}$ \\
\hline
\end{tabular}

This table does not include materials from float samples 
diagnostic projectiles, reliable radiocarbon dates, and unclear stratigraphy. These materials were left unassigned and addressed separately in Chapter 12.0. Unassigned materials include seven features: four were probably natural in origin (i.e., three tree burns, and one Rabdotus shell cluster) and three small cultural burned rock features (see Table 131). The unassigned artifact assemblage was quite limited in number and similar to materials from the identified components.

A Bell/Andice component was discovered in Block B, stratigraphically above a Martindale component in Blocks B and D. Following field efforts, 37 radiocarbon dates were obtained on 5 different material classes; 27 directly from the 2 identified components. Radiocarbon results derived from nine more acceptable and reliable charcoal samples define component ages to an infrequently identified period between ca. 5200 and 5400 B.P. Dating multiple substances such as Rabdotus shells, animal bones, and organic sediments along with charcoal allowed comparisons between the materials. The noncharcoal results provide a much broader time range, with most older than the accepted charcoal results.

Twelve diverse technical analyses were implemented on an array of materials with detailed methods, procedures, backgrounds, and results individually presented in separate appendices. Technical analyses included, radiocarbon dating, macrobotanical, diatom, high-powered use-wear with residue identification, phytolith, starch grain, lipid residue, instrumental neutron activation, x-ray diffraction, deer tooth cementation, pollen, and soil chemistry. Overall preservation of macrobotanical and microfossil remains was poor; however, important subsistence and environmental data were obtained through the diverse technical analyses employed.

The natural stratigraphy and field documentation, combined with results from technical analyses, contribute important and significant information to a greater understanding of Texas prehistory during a rarely encountered period. The two identified components are briefly summarized, beginning with the Bell/Andice and followed by the Martindale.

\subsubsection{Bell/Andice Component Summary}

The younger Bell/Andice component, as documented across $51 \mathrm{~m}^{2}$ of Block B yielded at least eight small burned rock features, and a cluster of ground stone tools. Recovered artifacts include, two Bell point fragments, one nearly complete Big Sandy point, three chipped stone biface fragments, eight ground stone tools, 12 edge-modified flakes, 180 pieces of lithic debitage, 15 fragments of mussel shells, quantities of burned rocks, and a rare vertebrate faunal assemblage (see Table 13-1). Bone preservation was generally good, although many bone fragments are root etched and or covered in calcium carbonate. Charcoal preservation was poor as charcoal was reduced to dark stains and lacked its original structure. Microfossil preservation was also poor; however, identified plant material added greatly to our understanding of utilized plant foods and how they were processed. Twelve radiocarbon dates were obtained on four different materials. Six charcoal dates from three separate burned rock features span 90 radiocarbon years with an average age of 5322 B.P. Six other noncharcoal dates reveal a broader time range and may not document the actual age of this cultural event. A small chipped stone assemblage is dominated by local cherts, 99 percent of which are chemically consistent with the gravel outcrop on the adjacent hill top. Only three animal species were identified and include, deer, cottontail, and jackrabbits. Diet breath is generally measured by animal taxa richness, and with only three species present would indicate a limited diet. However, the identification of multiple plant species (i.e., wild buffalo gourd phytoliths, true lily starch, and multiple grass starches), significantly broadens the diet breath. 
The excellent, well-defined horizontal patterning or activity areas documented in the Bell/Andice component (see Figure 9-61) clearly demonstrate the rare integrity and intact nature of the cultural component. The combined evidence solidifies this component represents a single event. The direct association of smashed bone fragments, burned rocks, and burned rock features in multiple locations strongly support multiple individuals cooking with stone boiling in a short period of time. The distinct activity areas reveal a pattern of expended materials (i.e., fractured bone and burned rocks) that were purposely and systematically discarded next to the cooking features.

If gender roles documented in the ethnographic literature hold true in prehistoric times, it is possible to project the composition of the group. The assumptions are that males primarily hunted and produced chipped stone tools, whereas females were responsible for processing meat, tanning hides, sewing garments, manufacturing and maintaining the tipi, collecting water and firewood, extracting bone marrow and grease, collecting plants, and preparing food (e.g., Dempsey 2001; Ewers 1982; Fowler and Flannery 2001; Grinnell 1972; Hughes 1991; Lowie 1963; Newcomb 1993; Parks 2001; Voget 2001). The high frequency of small burned rock cooking features in the Bell/Andice component combined with the presence of numerous ground stone tools, the dominance of small game, a restricted lithic debitage assemblage focused on producing flakes best reflect a female dominated task group focused on plant and small game procurement with subsequent processing and cooking those resources during a short fall occupation. In support of this interpretation as a female task focus group are multiple ethnographic accounts of similar group composition. As an example, Opler (1965:355) describes a specialty task group for the Apache as follows:
"They [women] go in groups of six or so and keep their finds separately. Should the destination be so distant that the group cannot hope to return for some days, men or youths go along to protect them and to assist them in the heavy work."

The data concerning this component best fits a collector strategy as Binford (1980) has modeled with a small logistical population, which moved to a specific resource local to target specific resources, here primarily plants. This collector strategy and settlement pattern potentially accounts for individual scattered or isolated burned rock cooking features in the immediate area and adjacent sites such as the Berdoll site (Karbula et al. 2011), sites 41TV410 and 41TV540 (Figueroa et al. 2011). Restricted and focused cooking features at these sites in the lower Onion Creek valley contrasts with larger, longer-term, artifact-rich campsites. The latter campsite types were locations where hunting and butchering large game, stone tool manufacturing and refurbishing, and diverse plant processing and cooking activities occurred by both sexes over a longer period. These more intensely occupied camps fit the forager role model (Binford 1980).

\subsubsection{Martindale Component Summary}

Roughly 20 to $25 \mathrm{~cm}$ below the well-defined Bell/Andice component in the southern part of Block B $\left(12 \mathrm{~m}^{2}\right)$, and horizontally distributed all across Block D $\left(62 \mathrm{~m}^{2}\right)$, was the Martindale component that extended across minimally $74 \mathrm{~m}^{2}$ area. This component yielded one corner-notched Martindale dart point and two dart point fragments associated with scattered burned rocks, three charcoal stained hearths, 2,082 vertebrate bone fragments, 54 mussel shell fragments, four biface fragments, 83 edge-modified flakes, two hammer stones, 1,429 pieces of lithic debitage, and three ground stone tools (see Table 13-1). Rabdotus snail 
shells were moderately common in localized areas, mostly above or below rather than directly associated with the cultural components. Fifteen radiocarbon dates were obtained on five different materials. Although only two charcoal and one charred seed date provide a confident average age of 5393 B.P., this is one of the best preserved and thoroughly dated Martindale components. It also yielded the first identified Martindale component to contain organic-stained, well-defined hearths and a diverse faunal assemblage. This occupation revealed evidence of a moderate lengthy stay during fall. Although Collins (1995, 2004) postulated that this period for development of specialized cooking appliances, which he projected as antecedents to the larger burned rock middens, these stained hearths were not specialized nor dominated by burned rocks. Consequently, this component is a very important to central Texas prehistory.

The moderately large lithic debitage assemblage reflects stone tool manufacturing focused on reduction of local cobble cherts towards middle and late stage biface preparation, with some finishing and resharpening processes. Evidence of stone tool manufacturing tasks is complimented evidence of meat procurement tasks, which include diverse resources, such as deer, pronghorn, jackrabbits, cottontail rabbits, canid, turtles, birds, and fish. Plants were also gathered, processed, and cooked. Identified plants include grass (probably wildrye) and cheno-am seeds, wild buffalo gourds, and true lily bulbs. The multiple plant and animal resources were directly associated with three small fire hearths for heating and or cooking. All resources were probably cooked through stone boiling.

The horizontal artifact patterning documented in the Martindale component (see Figure 11-7) clearly reveals an intact and distinct single use episode. Like the Bell/Andice component, association between smashed bone fragments, burned rocks, and cooking features indicate stone boiling probably for bone grease extraction with refuse piles next to the cooking feature. Binford (1978:159) provides information to support this association in a discussion of debris resulting from bone grease extraction. He states:

"The archeological remains of
such an operation are
unmistakable. There is a large pile
of pulverized bone approaching
the appearance of bone meal. This
is generally a dump to one side of
a substantial hearth containing
large quantities of ash."

The discard of material was patterned, wellpreserved, and remained intact. Two of the features were identical and probably represented the same cooking method and discard activity performed the same way. These two similar activity areas probably represent two separate individuals from different families who processed their own food following the established routine set by the group.

If gender roles documented in the ethnographic literature hold true prehistorically, then the combined data reflects a group of males and females, which probably represented a population comprised of multiple families. Multiple families are inferred through spatial arrangement of the three hearths dispersed across the excavation areas. The similarity in processing plants and small game through the same cooking strategy dispersed over space is supported by the ethnographic statement above by Opler (1965:355) in that women "keep their finds separately." This mobile hunter-gatherer group pursued a broad diet breath during their seasonal movements and best reflects a foraging strategy (Binford 1980).

\subsubsection{Component Summary}

The two middle Holocene components in Blocks B and $\mathrm{D}$ are among best defined and well-preserved components in central Texas and adjacent regions. They also represent a very poorly known and understood time interval during the later Altithermal 
period. The excellent context and high integrity combined with preserved vertebrate faunal assemblages provide ideal conditions and a unique opportunity to investigate how groups of huntergatherers operated during this middle Holocene period. Specific human behaviors were identified through distinct activity areas reflected by the distribution of cultural features and artifacts in both occupations. The microfossil and macrobotanical remains combined also indicate that the season of occupation for each component was during the fall. Seasons of occupation, particularly fall events, are rarely documented in Texas.

The current understanding of the environmental conditions during Altithermal period relies on proxy data and considerable speculation (see Chapter 2.0). Technical analyses following the excavation of 41TV2161 was directed towards different proxy datasets to address questions concerning the paleoenvironment (as alluded to in the research design and Hypothesis 2); unfortunately, only limited data was obtained as a result of poor microfossil preservation. The results indicate that between roughly 5200 and 5500 B.P., the local environment was dominated by prairie grassland conditions that most likely contained scattered low brush and grassy areas, which bordered a localized riparian zone along the flowing Onion Creek.

\subsection{DISCUSSIONS}

In Texas, human populations potentially present during the middle Holocene period have not been well-defined, documented, or extensively studied (see Chapter 3.0). This fact reflects few excavated sites or components that clearly date to this period. Components and sites that have yielded projectile point types and radiocarbon dates attributed to the middle Holocene period are typically palimpsest deposits of mostly mixed assemblages in shallow, deflated context, or amid thick stratigraphic zones with multiple point types represented (Table 13-2). Although a few projectile point types are attributed to the Middle Holocene, specific charcoal radiocarbon dates from good context in direct association with a single point type are lacking. Collins (2004:109) stated, "Many sites in Central Texas are deficient or totally lacking in suitable organic materials for conventional radiocarbon dating." The two middle Holocene components documented at the Big Hole site also lacked sizable charcoal fragments and clearly defined charcoal lenses; however, eight radiocarbon dates on charcoal flecks and a single date on burned seeds from multiple cultural features in each component provide high quality radiocarbon dates to document the specific age of the three recovered point types. Multiple organic materials (i.e., animal bones and teeth, Rabdotus snail shells, and carbon enriched sediment from well-defined features) were also dated and compared to the charcoal results. Noncharcoal results provide less precise time frames, rather than a specific age for cultural events. The difference in results between charcoal and other dated material follows the findings from many previously dated contexts (e.g., Abbott 1994; Collins 1994b; Frederick 2011).

\subsubsection{Cultural and Environmental History}

Nine radiocarbon dates obtained from charcoal and a carbonized seed sample, coupled with three projectile point types recovered (Bell, Big Sandy, and Martindale), indicate the deposits occurred over a maximum 230 radiocarbon year period from about 5280 to 5510 B.P. From an environmental perspective, this is an important and useful interval of time to study adaptation to changing conditions as this period has been considered near the end of the warm and possibly dry Altithermal period (see Chapter 2.0). With few exceptions, sites or components throughout Texas do not specifically document this time interval. Research in the western United States and portions of the Great Plains region have identified and studied this warm period (see Chapter 2.0). Implications of the Altithermal are the effects of warm conditions on plant and animal populations, and subsequent consequences for human populations that relied on those resources. 
Table 13-2. Component Integrity, Point Types, and Radiocarbon Dates from Selected Excavated Sites in Texas for the General Martindale and Bell/Andice Middle Holocene Period.

\begin{tabular}{|c|c|c|c|c|c|c|}
\hline $\begin{array}{l}\text { Site Name } \\
\text { and } \\
\text { Number }\end{array}$ & Location & $\begin{array}{l}\text { Component } \\
\text { Integrity }\end{array}$ & Point Types Present & $\begin{array}{l}\text { Radiocarbon } \\
\text { Dates (B.P.) } \\
\text { (Material) }\end{array}$ & $\begin{array}{c}\text { Comments on } \\
\text { Date }\end{array}$ & References \\
\hline $\begin{array}{l}\text { Arenosa } \\
\text { Shelter, } \\
\text { 41VV99 }\end{array}$ & Lower Pecos & Fair & 1 Early Barb/Martindale & $\begin{array}{l}5360 \pm 170 \\
\text { (Charcoal) }\end{array}$ & $\begin{array}{c}\text { Date from } \\
\text { multiple } \\
\text { charcoal } \\
\text { concentrations }\end{array}$ & $\begin{array}{c}\text { Dibble 1997; } \\
\text { Valastro et } \\
\text { al. } 1967\end{array}$ \\
\hline $\begin{array}{l}\text { Bering } \\
\text { Sinkhole, } \\
\text { 41KR241 }\end{array}$ & $\begin{array}{l}\text { Eastern } \\
\text { Edwards } \\
\text { Plateau }\end{array}$ & Good & 2 Martindale, 1 Uvalde & $\begin{array}{c}6860 \pm 170 \\
6660 \pm 110 \\
5840 \pm 190 \\
\text { (Human bones) }\end{array}$ & $\begin{array}{c}\text { Dates on } \\
\text { human petrous } \\
\text { bones }\end{array}$ & $\begin{array}{c}\text { Bement } \\
1994\end{array}$ \\
\hline $\begin{array}{l}\text { Camp Pearl } \\
\text { Wheat, } \\
41 \mathrm{KR} 243\end{array}$ & $\begin{array}{l}\text { Central } \\
\text { Edwards } \\
\text { Plateau }\end{array}$ & Fair & $\begin{array}{c}3 \text { Martindale, } 1 \text { Uvalde, } \\
1 \text { Uvalde-like, } 1 \text { Bell }\end{array}$ & None & NA & $\begin{array}{l}\text { Collins et al. } \\
1990\end{array}$ \\
\hline $\begin{array}{l}\text { Gatlin, } \\
\text { 41KR621, } \\
\text { Occupation } \\
\text { Zone 2, }\end{array}$ & $\begin{array}{l}\text { Central } \\
\text { Edwards } \\
\text { Plateau }\end{array}$ & Poor & $\begin{array}{c}88 \text { points; } 64 \text { Early } \\
\text { Archaic, } 34 \text { Martindale, } \\
21 \text { Gower, } 10 \text { Early } \\
\text { Triangular, } 5 \text { Bandy, } 2 \\
\text { Bell, } 1 \text { Andice, } 1 \text { Baker, } \\
1 \text { Marcos }\end{array}$ & $\begin{array}{c}13 \text { dates }= \\
6100 \text { to } 4600 \\
\text { (Charcoal) }\end{array}$ & $\begin{array}{l}13 \text { Dates on } \\
\text { wood charcoal }\end{array}$ & $\begin{array}{l}\text { Houk et al. } \\
2008\end{array}$ \\
\hline $\begin{array}{c}\text { Cibolo } \\
\text { Crossing, } \\
41 \mathrm{BX} 377, \\
\text { Martindale } \\
\text { Component }\end{array}$ & $\begin{array}{l}\text { Southeastern } \\
\text { Edwards } \\
\text { Plateau }\end{array}$ & Good & $\begin{array}{l}2 \text { Martindale and } 1 \\
\text { Bandy }\end{array}$ & $\begin{array}{c}790 \pm 40^{*} \\
820 \pm 50^{*} \\
2110 \pm 40^{*} \\
\text { Mean A/l } \\
\text { values }=5953 \\
\& 6290 \\
\text { (Rabdotus) }\end{array}$ & $\begin{array}{l}\text { Three charcoal } \\
\text { dates intrusive. } \\
\text { A/l values on } \\
\text { Rabdotus } \\
\text { shells OK }\end{array}$ & $\begin{array}{l}\text { Kibler and } \\
\text { Scott } 2000\end{array}$ \\
\hline $\begin{array}{l}\text { Cibolo } \\
\text { Crossing, } \\
\text { 41BX377, } \\
\text { Bell/Andice } \\
\text { Component }\end{array}$ & $\begin{array}{l}\text { Southeastern } \\
\text { Edwards } \\
\text { Plateau }\end{array}$ & Fair & $\begin{array}{c}9 \text { Bell,1 Andice, } 2 \text { Baird } \\
\text { triangular }\end{array}$ & $\begin{array}{c}800 \pm 40^{*} \\
780 \pm 50^{*}, \\
2110 \pm 40^{*} \\
4420 \pm 50 \\
4400 \pm 60 \\
4370 \pm 80 \\
\text { (Charcoal) } \\
3550 \pm 70^{*} \\
3510 \pm 80^{*} \\
3060 \pm 70^{*} \\
\text { (Sediment) }\end{array}$ & $\begin{array}{l}\text { Two charcoal } \\
\text { dates intrusive. } \\
\text { Sediment } \\
\text { dates to young, } \\
\text { three charcoal } \\
\text { dates accepted } \\
\text { by author }\end{array}$ & $\begin{array}{l}\text { Kibler and } \\
\text { Scott } 2000\end{array}$ \\
\hline $\begin{array}{c}\text { Clear Creek } \\
\text { Golf Course, } \\
41 \mathrm{CV} 413, \\
\text { Lower } \\
\text { Component }\end{array}$ & $\begin{array}{l}\text { Eastern } \\
\text { Edwards } \\
\text { Plateau }\end{array}$ & Poor & $\begin{array}{l}42 \text { points: } 22 \text { Early } \\
\text { Archaic: } 8 \text { Yarbrough, } 5 \\
\text { Martindale, } 4 \text { Wells, } 4 \\
\text { Gower, } 1 \text { Merrell }\end{array}$ & $\begin{array}{l}4320 \pm 40 \\
4580 \pm 40 \\
4280 \pm 40 \\
\text { (Charcoal) }\end{array}$ & $\begin{array}{l}3 \text { dates on } \\
\text { charcoal }\end{array}$ & $\begin{array}{l}\text { Quigg et al. } \\
\quad 2011\end{array}$ \\
\hline $\begin{array}{c}\text { Eckols, } \\
\text { 41TV528, } \\
\text { Analytic Unit } \\
2\end{array}$ & $\begin{array}{l}\text { Edwards } \\
\text { Plateau }\end{array}$ & Mixed & $\begin{array}{l}1 \text { Pedernales, } 1 \text { Early } \\
\text { Triangular, } 3 \\
\text { Martindale. } 2 \\
\text { Martindale-like, } 2 \text { Bell- } \\
\text { like }\end{array}$ & $\begin{array}{l}5199 \pm 124 \\
\text { (Charcoal) }\end{array}$ & $\begin{array}{l}\text { Charcoal date } \\
\text { from zone }\end{array}$ & $\begin{array}{l}\text { Karbula } \\
2000\end{array}$ \\
\hline
\end{tabular}




\section{Table 13-2. Component Integrity, Point Types, and Radiocarbon Dates from Selected Excavated Sites in Texas for the General Martindale and Bell/Andice Middle Holocene Period (continued).}

\begin{tabular}{|c|c|c|c|c|c|c|}
\hline $\begin{array}{l}\text { Site Name } \\
\text { and } \\
\text { Number }\end{array}$ & Location & $\begin{array}{l}\text { Component } \\
\text { Integrity }\end{array}$ & Point Types Present & $\begin{array}{c}\text { Radiocarbon } \\
\text { Dates (B.P.) } \\
\text { (Material) }\end{array}$ & $\begin{array}{c}\text { Comments on } \\
\text { Date }\end{array}$ & References \\
\hline $\begin{array}{l}\text { Halls Cave, } \\
\text { 41KR474 }\end{array}$ & $\begin{array}{l}\text { Central } \\
\text { Edwards } \\
\text { Plateau }\end{array}$ & Good & $\begin{array}{c}1 \text { Uvalde, } 1 \text { probable } \\
\text { Uvalde, } 1 \text { Martindale at } \\
102 \mathrm{cmbs}\end{array}$ & $\begin{array}{l}4850 \pm 130 \\
\text { (Charcoal) }\end{array}$ & $\begin{array}{l}\text { Charcoal date } \\
\text { from hearth at } \\
100 \mathrm{cmbs}\end{array}$ & $\begin{array}{l}\text { Toomey } \\
1993\end{array}$ \\
\hline $\begin{array}{l}\text { Haws, } \\
\text { 41WM56, } \\
\text { Area C }\end{array}$ & $\begin{array}{l}\text { Eastern } \\
\text { Edwards } \\
\text { Plateau }\end{array}$ & Poor & $\begin{array}{c}1 \text { Martindale above } 1 \\
\text { Nolan }\end{array}$ & None & NA & $\begin{array}{l}\text { Peter et al. } \\
\quad 1982\end{array}$ \\
\hline $\begin{array}{l}\text { Higgins, } \\
\text { 41BX184, } \\
\text { Hearth-Field, } \\
\text { Slice 1 }\end{array}$ & $\begin{array}{l}\text { Southeastern } \\
\text { Edwards } \\
\text { Plateau }\end{array}$ & Poor & $\begin{array}{c}3 \text { Martindale, } 2 \text { Early } \\
\text { Triangular, } 1 \text { Bell, } 1 \\
\text { Early Stemmed, } 3 \\
\text { unnamed types }\end{array}$ & None & NA & $\begin{array}{l}\text { Black et al. } \\
\quad 1998\end{array}$ \\
\hline $\begin{array}{l}\text { Landslide, } \\
\text { 41BL85, } \\
\text { Stratum V }\end{array}$ & $\begin{array}{l}\text { Eastern } \\
\text { Edwards } \\
\text { Plateau }\end{array}$ & Fair & $\begin{array}{l}1 \text { Bell, } 4 \text { Gower, } 4 \\
\text { Gower-like, } 6 \\
\text { Martindale }\end{array}$ & None & NA & $\begin{array}{l}\text { Sorrow et al. } \\
\quad 1967\end{array}$ \\
\hline $\begin{array}{l}\text { Panther } \\
\text { Springs, } \\
\text { 41BX228, } \\
\text { Area M, }\end{array}$ & $\begin{array}{l}\text { Southeastern } \\
\text { Edwards } \\
\text { Plateau }\end{array}$ & Poor & $\begin{array}{l}5 \text { Martindale, } 7 \text { Early } \\
\text { corner-notch, } 7 \\
\text { Expanding stem, } 2 \text { Bell, } \\
3 \text { Nolan, } 1 \text { Travis }\end{array}$ & $\begin{array}{l}4720 \pm 170 \\
\text { from Area I }\end{array}$ & $\begin{array}{l}\text { Charcoal from } \\
\text { Area I at 98.60, } \\
84 \text { cmbs, } \\
\text { "transition" } \\
\text { zone, Local } \\
\text { Period } 5\end{array}$ & $\begin{array}{l}\text { Black and } \\
\text { McGraw } \\
1985\end{array}$ \\
\hline $\begin{array}{l}\text { Richard } \\
\text { Beene, } \\
\text { 41BX831, } \\
\text { Lower } \\
\text { Medina } \\
\text { Component, } \\
\text { Block G }\end{array}$ & $\begin{array}{l}\text { Northern } \\
\text { Coastal Plain }\end{array}$ & Poor & $\begin{array}{l}\text { Stemmed corner- } \\
\text { notched, straight based } \\
\text {, Martindale/Bandy-like, } \\
\text { stemmed corner- } \\
\text { notched, Baker, } \\
\text { Pandale-like }\end{array}$ & $\begin{array}{l}6700 \pm 110 \\
6985 \pm 65 \\
6900 \pm 70 \\
7000 \pm 70 \\
6930 \pm 65 \\
\text { (Charcoal) }\end{array}$ & $\begin{array}{c}\text { Dates on } \\
\text { charcoal from } \\
\text { Features } 30 \text {, } \\
43, \& 44,1 \text { on } \\
\text { burned tree }\end{array}$ & $\begin{array}{l}\text { Thoms and } \\
\text { Mandel } 2007\end{array}$ \\
\hline $\begin{array}{l}\text { Seminole } \\
\text { Sink, } \\
\text { 41VV620 }\end{array}$ & $\begin{array}{l}\text { Southwestern } \\
\text { Edwards } \\
\text { Plateau }\end{array}$ & Good & $\begin{array}{l}1 \text { Early Corner-notched } \\
\text { (not Martindale) }\end{array}$ & $\begin{array}{c}3 \text { dates, } 4671 \\
\text { to } 5750 \\
\text { (Human bones) }\end{array}$ & $\begin{array}{c}\text { Dates on } \\
\text { human bones }\end{array}$ & Turpin 1988 \\
\hline $\begin{array}{c}\text { Skyline } \\
\text { Shelter, } \\
\text { 41VV930, } \\
\text { Early } \\
\text { Archaic }\end{array}$ & Lower Pecos & Poor & $\begin{array}{c}14 \text { devils Triangular, } 7 \\
\text { triangular preforms, } 6 \\
\text { Martindale/Bandy, } 4 \\
\text { Baker/Uvalde, } 3 \text { Gower, } \\
\text { 1 Early-barbed, } 2 \\
\text { Golondrina }\end{array}$ & $\begin{array}{l}5920 \pm 70 \\
\text { (Charcoal) }\end{array}$ & $\begin{array}{l}\text { Wood charcoal } \\
\text { from central } \\
\text { pit/hearth }\end{array}$ & $\begin{array}{l}\text { Turpin and } \\
\text { Bement } \\
1992\end{array}$ \\
\hline $\begin{array}{l}\text { Sleeper, } \\
\text { 41BC65 } \\
\text { Early } \\
\text { Archaic }\end{array}$ & $\begin{array}{l}\text { Central } \\
\text { Edwards } \\
\text { Plateau }\end{array}$ & Poor & $\begin{array}{c}6 \text { Uvalde, } 4 \text { Gower, } 1 \\
\text { Bell, } 1 \text { Martindale, } 4 \\
\text { Unclassified }\end{array}$ & None & No charcoal & $\begin{array}{l}\text { Johnson } \\
1991\end{array}$ \\
\hline
\end{tabular}


Table 13-2. Component Integrity, Point Types, and Radiocarbon Dates from Selected Excavated Sites in Texas for the General Martindale and Bell/Andice Middle Holocene Period (continued).

\begin{tabular}{|c|c|c|c|c|c|c|}
\hline $\begin{array}{l}\text { Site Name } \\
\text { and } \\
\text { Number }\end{array}$ & Location & $\begin{array}{l}\text { Component } \\
\text { Integrity }\end{array}$ & Point Types Present & $\begin{array}{c}\text { Radiocarbon } \\
\text { Dates (B.P.) } \\
\text { (Material) }\end{array}$ & $\begin{array}{c}\text { Comments on } \\
\text { Date }\end{array}$ & References \\
\hline $\begin{array}{l}\text { Varga, } \\
\text { 41ED28, } \\
\text { Early } \\
\text { Archaic } \\
\text { Component }\end{array}$ & $\begin{array}{l}\text { Southwestern } \\
\text { Edwards } \\
\text { Plateau }\end{array}$ & Poor & $\begin{array}{c}179 \text { points: } 32 \text { Gower, } \\
22 \text { Martindale, } 21 \\
\text { Bandy, } 15 \text { Group 1, } 12 \\
\text { Baker, } 12 \text { Merrell, } 7 \\
\text { Group 2, } 3 \text { Carrizo, } 1 \\
\text { Pandale }\end{array}$ & 6280 to 5200 & $\begin{array}{c}17 \text { Dates; } 2 \\
\text { sediment, } 3 \\
\text { bones, } 4 \\
\text { charcoal, } 5 \\
\text { walnut shells, } 1 \\
\text { Rabdotus, } 2 \\
\text { charred seeds }\end{array}$ & $\begin{array}{l}\text { Quigg et al. } \\
2008\end{array}$ \\
\hline $\begin{array}{l}\text { Wilson- } \\
\text { Leonard, } \\
\text { 41WM235, } \\
\text { Early } \\
\text { Archaic } \\
\text { Period }\end{array}$ & $\begin{array}{l}\text { Eastern } \\
\text { Edwards } \\
\text { Plateau }\end{array}$ & Poor & $\begin{array}{c}266 \text { points, } 20 \\
\text { Angostura, } 30 \text { bifurcate } \\
\text { stem, } 17 \text { Hoxie, } 16 \\
\text { thrall, } 11 \text { Gower, } 30 \\
\text { lanceolate, } 10 \\
\text { Martindale, \& others }\end{array}$ & $\begin{array}{l}8700 \text { to } 6000 \\
\text { (Geophytes) }\end{array}$ & $\begin{array}{l}\text { Charcoal and } \\
\text { charred } \\
\text { geophytes }\end{array}$ & $\begin{array}{c}\text { Collins et al. } \\
1998\end{array}$ \\
\hline $\begin{array}{l}\text { Woodrow } \\
\text { Heard, } \\
\text { 41UV88, } \\
\text { Geological } \\
\text { Unit lla }\end{array}$ & $\begin{array}{l}\text { Southwestern } \\
\text { Edwards } \\
\text { Plateau }\end{array}$ & Poor & $\begin{array}{c}\text { Baker, Bell, Early } \\
\text { Triangular, Martindale, } \\
\text { Uvalde }\end{array}$ & $\begin{array}{l}15 \text { dates, } 6500 \\
\text { to } 4400 \\
\text { (Charcoal) }\end{array}$ & $\begin{array}{l}\text { Charcoal } \\
\text { dates, not } \\
\text { directly } \\
\text { associated }\end{array}$ & $\begin{array}{l}\text { Decker et al. } \\
2000\end{array}$ \\
\hline
\end{tabular}

The Altithermal is most often viewed as a xeric interval of relatively dry and or warm conditions that lasted approximately 3,000 years from ca. 8000 to 5000 B.P. Effects are often stream incision and erosion, which created stable alluvial terrace surfaces in which soils formed. Those paleoenvironmental conditions had major impacts on the archeological record. One effect is the limited number of sites identified across the open Plains, with most recorded sites located towards the margins of the Plains or along major water courses. The limited number of recorded Altithermal period sites may simply reflect the lack of excavation in deep, alluvial terraces along the streams and rivers.

Walker (1980) revealed many middle Holocene sites across the Central and Northern Plains that have yielded various side-notched projectile point forms. The side-notched form in the Southern Plains and eastward is generally referred to as Big Sandy (Bell 1960:8), while similar side-notched forms across the Central and Northern Plains have a variety of names including Early Side-notched, Bitterroot Side-Notched, Northern Side-Notched, Gowen Side-Notched, Hawken Side-Notched, Mummy Cave Side-notched, Mount Albion SideNotched, and Blackwater Side-Notched (Walker 1980). Turner and colleagues (2011:66) discuss the Big Sandy point type as Late Paleoindian in association and suggest that it has only been recently recognized in Texas (and was not described by Suhm and Jelks 1962). Revised chronologies for central Texas have not included early side-notched forms (e.g., Bousman et al. 2004; Collins 1995, 2004; Prewitt 1981, 1985).

The early side-notched point was originally suggested as a type at the Big Sandy site (25HY18) in Henry County, Tennessee. This point type was described by Kneberg (1956:25a) (quoted in Bell 1960:8) as a side-notched dart point of medium size with incurved base, thin cross-section, and wellretouched by pressure flaking. It occurred across Kentucky and northern Alabama (Bell 1960:8). A 
pure "Early Side-Notched" component was defined and radiocarbon dated at Dust Cave (1LU496) in Alabama with initial occupations around 10,000 to 9700 B.P. (Sherwood et al. 2004). Sherwood and colleagues (2004) also state the "Early SideNotched" period in the Midsouth region has remained enigmatic. This point type occurs in contexts spanning a broad time range across an extensive region of the central United States, and it is not well-represented or well-dated in Texas. A recycled Big Sandy point from mixed context was from the Gatlin site (Oksanen 2008:4-5), one was from the Wilson-Leonard site (Dial et al. 1998: Figure 13-102), as well as a few other sites scattered across seven Texas counties (Figure 13-1) (Prewitt 1995:93).

The Big Sandy point identified in the Bell/Andice component at the Big Hole site was documented in good context, in situ within an isolated component, and in association with six charcoal dates that average ca. 5333 B.P., which is much later than the presumed age of the enigmatic point time. Possibly, this single Big Sandy point was a trade item or represents an individual or group unrelated

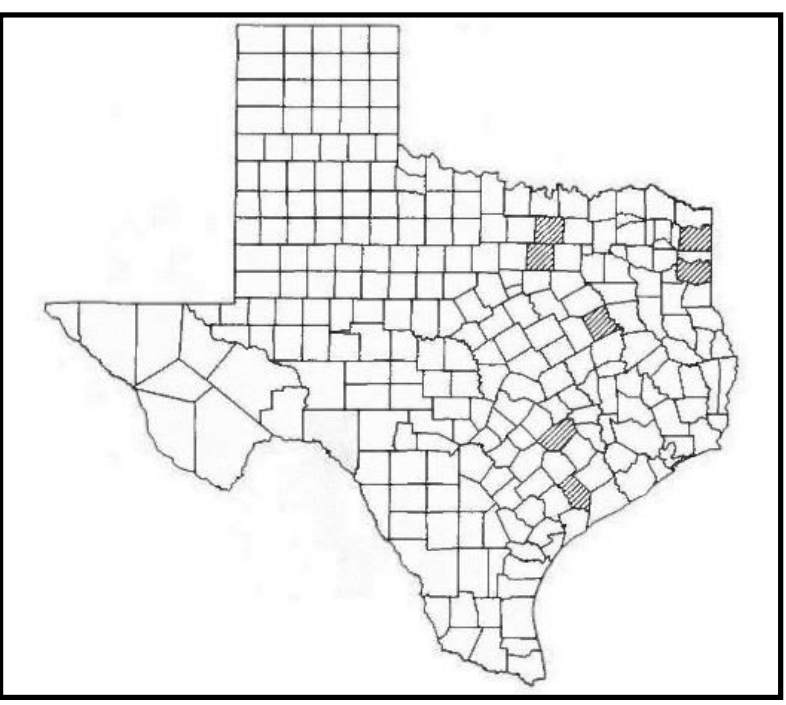

Figure 13-1. As of the early 1990s, Big Sandy point distribution depicting counties with 1 to 11 points (Prewitt 1995:93, Figure 7). to the population that employed the Bell/Andice points. The complete and unmodified Big Sandy point recovered from the same component as Bell/Andice points indicates that multiple point types were employed during that time.

Although early side-notched points are not wellknown, not often documented, or dated in Texas, sites north of Texas have yielded early side-notched forms and a number are radiocarbon dated (e.g., Agogino and Frankforter 1960; Anderson and Shutler 1978; Carlson et al. 1999; Frison et al. 1976; Hoard et al. 2004; Kivett 1962a, 1962b; McCracken 1978; Nicholson and Playford 2009; Reeves 1978; Smith and Creasman 1988). In central Kansas, a human burial associated with a large side-notched point, a drill and deer bone, yielded a radiocarbon date on an associated deer bone to $6160 \pm 35$ B.P. (Hoard et al. 2004). In northeastern Wyoming the Hawken site (48CK303), a communally operated, arroyo bison trap dated to 6400 B.P., yielded large, wellmanufactured, side-notched projectile points (Frison et al. 1976). The earliest side-notched type known from northwestern Plains was associated with a bison kill containing small-sized Bison occidentalis reflecting the dwarfing of the species during the middle Holocene (Frison 1978; Frison et al. 1976). In southwestern Wyoming at the Taliaferro site (48LN1468), Component I yielded several early side-notched points associated with a radiocarbon date on charcoal to $5290 \pm 190$ B.P. (Smith and Creasman 1987). In southwestern Nebraska, the Sidney Burial (25CN55) yielded a large side-notched point associated with human bones was radiocarbon dated to $3910 \pm 60$ B.P. (Carlson et al. 1999). The Logan Creek phase in Nebraska, in the northern Flint Hills of Kansas into Iowa, contains early side-notched forms between 6300 and 5200 B.P. (Hofman 1989). These examples and others reveal early side-notched forms occurred north of Texas and generally from the early to middle Holocene. Currently, no early side-notched points were recovered from 
Paleoindian context indicating Big Sandy points occurred later in time than often assumed.

Populations employing different projectile point styles appear during the middle Holocene across broad regions and date to the same or similar times. Consequently, the different populations in a region created opportunities for interactions and trading. Groups that employed Early Triangular point styles (e.g., Taylor points) generally occupied southern Texas at similar or slightly later times as those groups with Bell/Andice and Martindale points (Collins 2004). Johnson and Goode (1994) indicated Early Triangular points were approximately the same age as Calf Creek/Bell points. More recently Collins (1995, 2004) suggested this triangular style post-dated the Bell/Andice interval. Turner et al. (2011:88) indicate Early Triangular points range from 5800 to 4700 B.P. with the possibility this type persisted to about 3900 B.P. at sites across the broader Texas region.

The Holt site (41HY341), just $42 \mathrm{~km}$ south of the Big Hole site in a similar Blackland environmental setting in Hays County yielded four stratified cultural deposits that included Paleoindian through Middle Archaic period materials (Brownlow 2004). The second youngest cultural zone (III) was radiocarbon dated on charcoal to $4740 \pm 40$ B.P. from burned rock Feature 4 and yielded a projectile point assemblage of three Early Triangular points and one displaced Gower point (Brownlow 2004). Other radiocarbon dates associated with Early Triangular points during this Middle Archaic period are also not associated with Bell/Andice or Martindale/corner-notched points of any type (e.g., Black and McGraw 1985; Mahoney et al. 2003). A single date for an Early Triangular component (Local Period 5) at the Panther Springs Creek site (41BX228) in southern Texas indicates this type is dated to about $5330 \pm 170$ B.P. to support populations employing different point types were in the same region during the Middle Archaic (Black and McGraw 1985).
The Royal Coachman site (41CM111), further south in Comal County, yielded three mixed components with multiple point types, including 16 Early Triangular, two Bell, one Andice-like, three Nolan, two Pandale, and two Nolan/Pandale point types dated from a range of ca. 4770 to 5000 B.P. from the radiocarbon results of five charcoal samples (Mahoney et al. 2003). These point types lack a clear temporal separation between the types and the radiocarbon dates indicate a range of some 1,000 years between 4820 and 5880 B.P. The Bell/Andice component at the Cibolo Crossing site (41BX377) in Bexar County also yielded two Baird (Early Triangular) points, unfortunately this component is not well-dated with reliable charcoal dates (Kibler and Scott 2000). At the Pavo Real site (41BX52) also in Bexar County, Feature 10 dated by wood charcoal to $4780 \pm 40$ B.P. was in associations with Early Triangular points (Black 2003:209). At least six Early Triangular points were from an isolated Middle Archaic component radiocarbon dated by two charcoal samples to a period between 4200 and 4800 B.P. at the Varga site (41ED28) in Edwards County (Quigg et al. 2008). At present, no sites with good context have yielded Early Triangular points as old as the Bell/Andice points at the Big Hole component. From a technological aspect these Early Triangular points, which apparently proceeded the highly crafted and technologically complex Bell/Andice points, were probably not a direct antecedent from the latter. This indicates no cultural continuity in the technology. Shaffer and Tomka (2003:86) suggest Early Triangular points probably have numerous and significant adaptive advantages for highly mobile hunter-gatherers.

If groups that employed the Early Triangular point styles (e.g., Taylor points) were present at the same time as groups that employed the Bell/Andice and possibly the Martindale points, then exchanges and interactions would have been possible. The same is true for groups to the north, such as those that used the Big Sandy points and others such as lanceolate 
points in the Munkers Creek phase. As additional components or sites in good context are excavated and provide multiple charcoal dates, the beginning and ending dates of various intervals will become clearer and associations can be addressed.

\subsubsection{Subsistence Practices}

Since the two Big Hole components each yielded a rarely preserved vertebrate faunal assemblage and limited but significant microfossil plant data, it is appropriate to discuss how the studied components contribute understanding of subsistence practices during the middle Holocene. Across Texas, a few excavated Early Archaic components reveal human populations exploited a broad spectrum of resources that included plants (lily bulbs, gourds, and grasses, and cheno-am seeds), big and small game, fish, and birds. These early sites document that populations were highly successful foragers, knowledgeable of resource habitats, and sought diverse resources (Table 13-3) (Dering 2007; Thoms 2007b:354). The two Big Hole components clearly document a continuation of broad spectrum resource exploitation and a broad diet breath during ca. 300 years between 5200 to 5500 B.P. of the Middle Archaic period. This included continued reliance on diverse plant resources, such as grass seeds, cheno-am seeds, gourds, and geophytes; in combination with pursuit of small mammals, such as cottontail rabbits, jackrabbits, deer, and other resources such as fish and birds.

Evidence continues to mount that broad spectrum subsistence strategies were well-established prior to the Early and Middle Archaic periods, and Altithermal drying event (Dering 2007:191). Paleoindian sites in Texas that reflect more broad diet breadth include the Wilson-Leonard site (Baker 1998), the Levi site (Alexander 1963), the Gault site (Collins 2002), and others (e.g., Bousman et al. 2004; Dering 2007; Hester 1982; Johnson 1987a; Turpin 1991; Yates and Lundelius 2001). To elaborate, the Paleoindian components at the Wilson-Leonard site (41WM235) yielded turtles, snakes, birds, rodents, cottontail rabbits, jackrabbits, canid, deer, bison, and horse (Baker 1998). The Clovis component at the Gault site yielded small mammals, birds, frogs along with horse, bison, and mammoth (Collins 2002). A Late Paleoindian basin-shaped hearth radiocarbon dated to $9020 \pm 150$ B.P. at Baker Cave (41VV213) in Val Verde County yielded diverse array of faunal and floral remains (Hester 1982). Multiple technical analyses revealed evidence of sunflower, grasses, Mormon tea, buckhorn, cattail, prickly pear, Mexican buckeye, mescal beans, grape, hackberry, oak, Texas black walnut, persimmon, and mesquite, among others. Identified fauna include at least 12 species of mammals, 23 species of reptiles, and six species of fish (Hester 1982).

Moreover, subsistence data from the Bell/Andice component at the Big Hole site does not support assumptions by Johnson and Goode (1994) and others that Bell/Andice populations during the Middle Archaic in Texas were specialized bison hunters. Wyckoff $(1994,1995)$ also linked the Oklahoma Calf Creek horizon to bison exploitation, although the number of bison directly associated with the Calf Creek horizon is extremely limited and no actual bison kill sites have been identified in Texas or Oklahoma (see Chapter 3.0). The revised view that middle Holocene populations, including those using Bell/Andice points, practiced broad spectrum foraging and exploited a wide-ranging variety of resources is supported by the data recovered at the Big Hole site and elsewhere.

\section{Animal Subsistence Discussion}

According to optimum foraging theory, bison is a highly valued, sought after resource; however, it appears as though these large heard animals were not plentiful or readily available across the entire region during all seasons. Interannual and seasonal variability in bison populations have been documented for the historic period in Texas (e.g., Mauldin and Thompson 2012; Wade 1998, 2002), and this pattern probably occurred during the 
Table 13-3. Selected Early Sites and Their Subsistence Data.

\begin{tabular}{|c|c|c|c|c|c|c|c|c|c|c|c|c|}
\hline $\begin{array}{c}\text { Site Name, No. } \\
\text { \& Phase }\end{array}$ & $\begin{array}{l}\text { Large } \\
\text { Mammal }\end{array}$ & $\begin{array}{l}\text { Small } \\
\text { Mammal }\end{array}$ & $\begin{array}{l}\text { Canis/ } \\
\text { Carnivore }\end{array}$ & Rodent & Turtle & Mussels & Geophytes & Seeds & Snakes & Bird & Fish & Reference \\
\hline $\begin{array}{l}\text { Cervenka, } \\
\text { 41WM267, San } \\
\text { Geronimo }\end{array}$ & $\begin{array}{l}\text { deer, } \\
\text { pronghorn } \\
\text { bison }\end{array}$ & $\begin{array}{c}\text { cottontail } \\
\text { jackrabbit } \\
\text { beaver }\end{array}$ & yes & $\begin{array}{l}\text { gopher } \\
\text { mouse } \\
\text { cotton rat }\end{array}$ & multiple & yes & no & hackberry & yes & $\begin{array}{c}\text { bobwhite, } \\
\text { turkey, } \\
\text { prairie } \\
\text { chicken }\end{array}$ & $\begin{array}{l}\text { gar, } \\
\text { catfish, } \\
\text { perch }\end{array}$ & Yates 1982 \\
\hline $\begin{array}{c}\text { Wilson-Leonard } \\
\text { 41WM235 Early } \\
\text { Archaic }\end{array}$ & $\begin{array}{l}\text { pronghorn, } \\
\text { deer }^{1}\end{array}$ & $\begin{array}{l}\text { cottontail, } \\
\text { jackrabbit }{ }^{1} \\
\text { Squirrel }\end{array}$ & yes $^{1}$ & yes, rats & yes $^{1}$ & yes & bulbs & no & yes & yes, small & yes, 5 & Baker 1998 \\
\hline $\begin{array}{l}\text { 41WM57 San } \\
\text { Geronimo }\end{array}$ & no & no & no & no & yes $^{1}$ & no & no & no & no & no & catfish $^{1}$ & Yates 1982 \\
\hline $\begin{array}{l}\text { Hawes } \\
\text { 41WM56 San } \\
\text { Geronimo }\end{array}$ & $\begin{array}{l}\text { pronghorn }{ }^{1} \\
\text { deer }^{1}\end{array}$ & $\begin{array}{l}\text { rabbit } \\
\text { beaver }\end{array}$ & no & $\begin{array}{l}\text { gopher }^{1} \\
\text { wood rat }\end{array}$ & no & no & no & acorns & no & turkey ${ }^{1}$ & $\begin{array}{l}\text { gar, } \\
\text { catfish }\end{array}$ & Yates 1982 \\
\hline $\begin{array}{l}\text { Landslide } \\
\text { 41BL85 Strata } \\
\text { III, IV, V }\end{array}$ & bison & no & no & no & no & yes & no & no & no & no & no & $\begin{array}{c}\text { Sorrow et al. } \\
\quad 1967\end{array}$ \\
\hline $\begin{array}{c}\text { Berdoll } \\
\text { 41TV2125 Early } \\
\text { Archaic }\end{array}$ & $\begin{array}{l}\text { deer, } \\
\text { bison }\end{array}$ & $\begin{array}{l}\text { jackrabbit, } \\
\text { cottontail }\end{array}$ & no & yes & yes & yes & bulbs & no & yes & no & yes & $\begin{array}{c}\text { Karbula et al. } \\
2011\end{array}$ \\
\hline $\begin{array}{l}\text { Camp Pearl } \\
\text { Wheat } \\
41 \mathrm{KR} 243\end{array}$ & no & no & no & no & no & yes 1 & no & no & no & no & no & $\begin{array}{c}\text { Collins et al. } \\
1990\end{array}$ \\
\hline $\begin{array}{c}\text { Cibolo Crossing } \\
\text { 41BX377, } \\
\text { Martindale }\end{array}$ & no & no & no & no & no & yes 1 & no & no & no & no & no & $\begin{array}{l}\text { Kibler \& Scott } \\
\quad 2000\end{array}$ \\
\hline Holt, 41HY342 & no & no & no & no & no & yes 2 & bulbs & no & no & no & no & $\begin{array}{c}\text { Brownlow } \\
2004\end{array}$ \\
\hline
\end{tabular}


Table 13-3. Selected Early Sites and Their Subsistence Data (continued).

\begin{tabular}{|c|c|c|c|c|c|c|c|c|c|c|c|c|}
\hline $\begin{array}{l}\text { Site Name, No. } \\
\text { \& Phase }\end{array}$ & $\begin{array}{l}\text { Large } \\
\text { Mammal }\end{array}$ & $\begin{array}{c}\text { Small } \\
\text { Mammal }\end{array}$ & $\begin{array}{l}\text { Canis/ } \\
\text { Carnivore }\end{array}$ & Rodent & Turtle & Mussels & Geophytes & Seeds & Snakes & Bird & Fish & Reference \\
\hline $\begin{array}{l}\text { Armstrong, } \\
\text { 41CW54 Early } \\
\text { Archaic }\end{array}$ & $\begin{array}{c}\text { bison }^{2}, \\
\text { deer, } \\
\text { pronghorn }\end{array}$ & no & no & yes & no & yes 12 & bulbs & hackberry & no & no & no & $\begin{array}{l}\text { Schroeder } \\
2011\end{array}$ \\
\hline $\begin{array}{c}\text { Eckols } \\
\text { 41TV528 } \\
\text { AU-3 }\end{array}$ & $\begin{array}{l}\text { deer, } \\
\text { bison }\end{array}$ & rabbit & no & no & yes & no & no & no & no & no & no & Karbula 2000 \\
\hline $\begin{array}{c}\text { Varga, 41ED28, } \\
\text { Early Archaic }\end{array}$ & $\begin{array}{l}\text { deer, } \\
\text { bison }\end{array}$ & yes & no & no & no & yes 7 & no & $\begin{array}{l}\text { prickly } \\
\text { pear, } \\
\text { juniper }\end{array}$ & no & no & no & $\begin{array}{l}\text { Quigg et al. } \\
\qquad 2008\end{array}$ \\
\hline $\begin{array}{c}\text { 41TV540 } \\
\text { Middle Archaic }\end{array}$ & deer & $\begin{array}{l}\text { cottontail } \\
\text { jackrabbit }\end{array}$ & yes & yes & yes & yes & bulbs & no & yes & $\begin{array}{l}\text { ducks, } \\
\text { turkey } \\
\text { size, }\end{array}$ & no & $\begin{array}{l}\text { Figueroa et } \\
\text { al. 2011; } \\
\text { Meissner } \\
\text { 2011b }\end{array}$ \\
\hline $\begin{array}{l}\text { Richard Beene } \\
\text { 41BX831, lower } \\
\text { Medina, } \\
\text { Block G }\end{array}$ & $\begin{array}{c}\text { deer, } \\
\text { pronghorn }\end{array}$ & $\begin{array}{l}\text { rabbits, } \\
\text { squirrel, } \\
\text { porcupine }\end{array}$ & yes & yes & yes & yes & no & no & yes & no & yes & $\begin{array}{l}\text { Thoms and } \\
\text { Mandel } 2007\end{array}$ \\
\hline
\end{tabular}


middle Holocene also. Periods of bison scarcity were observed elsewhere on the Plains. For example, bison are absent in the archeological record for the entire 3,500 year period between ca. 7500 and 4000 B.P. in the Plains of eastern Colorado (Butler 1992, 1997). Further north in Wyoming, Frison (1978) noticed that evidence of bison was limited and generally restricted to niche habitats like the Black Hills and along the mountains. The numbers of bison also reduced through time across much of the Plains, and especially in the Southern Plains, probably related to climate stress, which significantly reduced human reliance on bison (Wolverton 2005).

Bison and bison hunters hold an important place in Texas prehistory. Researchers (e.g., Collins 2004; Johnson and Goode 1994; Lohse et al. 2013; Wyckoff 1995) argue that human populations employing Bell/Andice projectiles arrived in Texas from the north in pursuit of bison. During the middle Holocene an expanded grassland across the region should have provided plenty of nutritional food resources to bison populations (and consequently human populations) from approximately 6200 to 5000 B.P., if the Boriak bog pollen data are correct (see Chapter 2.0) (Bousman 1998). Bison remains were identified at minimally six Early Archaic sites (Table 13-3), and at least four sites attributed to the Middle Archaic and contemporaneous with the two components at the Big Hole site.

A bison tooth from the Gatlin site (41KR621) in the central Edwards Plateau was radiocarbon dated to $5320 \pm 40$ B.P. (Smith and Oksanen 2008:7-8). At $41 \mathrm{ME} 147$ on the southern edge of the Edwards Plateau, bison bone was dated to $5205 \pm 20$ B.P. (Lohse et al. 2014a, 2014b). Also nine bison bones were directly radiocarbon dated to between 5065 and 5290 B.P. from the Spring Lake site (41HY160) along the eastern edge of the Edwards Plateau (Lohse 2013; Lohse et al. 2014a, 2014b). Bison were also present on the Blackland Prairie at $5135 \pm 20$ B.P. on the basis of a bison bone date from the Cervenka site (Lohse et al. 2014a, 2014b). The radiocarbon dates on bison bones document bison presence in the region at roughly the time of the Big Hole components (ca. 5000 to 5500 B.P.); however, no bison remains were recovered from the Big Hole site.

The Altithermal period is also the period that witnessed the change in size of the bison species and this change potentially altered how these animals were hunted. Most previous references to bison only addressed bison in general terms and not to a specific species. So which species was represented in this middle Holocene in Texas is unclear. Bison antiquus was identified in Feature 5 at the Armstrong site (41CW54) in the Terminal Paleoindian (8490 \pm 40 B.P.) and Early Archaic components (7960 $\pm 60,8070 \pm 60$ B.P.; Schroeder 2001). Therefore, this fossil bison species was still present and hunted as a food resource close to 8000 B.P. just south of the Big Hole site. A juvenile Bison antiquus occidentalis skull from the Arkansas River near Tulsa, Oklahoma was embedded with the broken Calf Creek point and radiocarbon dated to $5120 \pm 25$ B.P. (Bement et al. 2004, 2005; Bement and Carlson 2014). This latter identification is surprising and calls into question what bison species were present in central Texas during the two Big Hole components. Wilson $(1975,1978)$ in studying the Holocene bison record in Wyoming concluded and or advanced the concept of chronoclinal variation in bison through the Holocene on the Northern Plains. Through the Holocene, bison became progressively smaller and possibly at a more or less constant rate. Wilson (1975:234, 1978:19) placed the boundary between B. bison occidentalis and B. bison bison at ca. 5000 B.P. McDonald (1981) also saw the shift in size to modern B. bison bison on the Southern Plains sometime around 5000 B.P. An alternate view of the timing of body size reduction in bison is presented by Lewis and colleagues (2007), which argue for transition to smaller modern bison between 8000 and 6500 B.P. across the southern 
Plains. They correlate the morphological change to the spread and nutritional properties of $\mathrm{C}_{4}$ grasses. This latter argument, however, is contrary to the dated juvenile $B$. antiquus occidentalis skull from the Arkansas River. Consequently, exactly what bison species was in the above referenced assemblages and in central Texas at roughly 5300 B.P., if only one, needs research and clarification. Regardless which species was present, bison were generally present in the region during the period ca. 5200 to 5500 B.P. of the two Big Hole components and probably available, if only seasonally. If bison were not available for whatever reason, the populations were sufficiently adapt and knowledgeable to pursue and procure multiple other plant and animal food resources in time of need as demonstrated here.

In central Texas, pronghorn are documented in minimally four Early Archaic components (Table 13-3), in addition to the pronghorn remains identified in the Martindale component at the Big Hole site. Sites with identified pronghorn material are typically located along the existing Blackland Prairie ecological region. Pronghorn remains have also been identified in the archeological record at ca. 6900 B.P. and in the late Pleistocene component from the Richard Beene site (41BX831) south of San Antonio (Baker and Steel 2007; Thoms 2007a), and as far south as Victoria, Texas in Late Paleoindian, Late Archaic, and Late Prehistoric context (Scott 2012).

Pronghorn prefer open range but also take advantage of riparian zones and consume a variety of grasses, forbs, and shrubs, within a range of 5 to $6.5 \mathrm{~km}$ (Giest 2001). They are often referred to as an animal of the plains (Davis 1974:248), or the quintessential prairie animal (Pastor et al. 2000:1). For much of the year adult males are solitary, with females forming groups, with a significant commingling of sexes over winter (Giest 2001). Their presence in sites in central Texas indicates a difference in past vegetation across this region in prehistory, which reflects an open grassland community in the immediate vicinity. The data indicate pronghorn range extended into south Texas during much of the Holocene and was a constant resource through time, potentially in small numbers. Beyond Texas borders, pronghorn were hunted for over 10,000 years across the Great Plains with ethnographic records and multiple archeological sites to testify to their procurement (Pastor and Lubinski 2000).

Deer are the most often identified game animal in prehistoric sites, which testifies to their long presence in the region and hunting them was a common throughout prehistoric lifeways. Deer habitat is dominated by brushy or wooded country, which probably was in the immediate vicinity in the riparian zone along Onion Creek and or in nearby Edwards Plateau. However, 67 percent of their total feeding time was spent grazing on forbs and grasses (Martin 1974:258). White-tail deer are territory-minded with restricted home ranges of 1.6 to $2.0 \mathrm{~km}$ (Davis 1974:257). Deer would probably have been available in relatively high numbers in the Onion Creek vicinity at the time of the Big Hole components. Just the opposite is reflected in the Big Hole components as deer are represented by very few animals.

Exploitation of rabbits in early sites appears quite consistent as at least seven of the ten components with faunal remains contain rabbits (see Table 133). Earlier examples included Paleoindian sites such as the Aubry Clovis site in north-central Texas and Paleoindian components at the Wilson-Leonard site in central Texas yielded rabbit bones along with other small game (Baker 1998; Ferring 2001).

Rabbits apparently were a consistent resource and should have been available on a regular, year round basis across nearly all of Texas. These small animals are easily trapped with small snares or nets, or can be driven and captured during rabbit drives (e.g., Shaffer and Gardner 1995). Jackrabbits prefer hot, dry, desert scrubland and even sometimes in coniferous forest (Davis 1974). They 
consume a variety of plants that include cactus, sagebrush, mesquite, and many grasses and herbs (Davis 1974). Eastern cottontails prefer brushland, marginal areas, and coastal prairies. Their feeding habits vary seasonally and they consume green grasses and herbs, and sometimes bark and twigs of shrubs and trees (Davis 1974).

Exploitation of turtles is nearly as frequent as deer (see Table 13-3). They are slow and easily caught and probably a resource young children could have captured and contributed to the greater good. Not only was the meat a food resource, their shells potentially served as utensils or other functions. One example of use, are four modified turtle shells in the Paleoindian double burial in Sub-Stratum 5G, Upper member in Horn Shelter 2 (Redder 1985:43). Although probably not a subsistence resource in that context, it does document a very early procurement of turtles in the region. The infrequent recovery of turtle remains indicates this resource was not a major importance to groups or plentiful in this setting.

Fish bones were from minimally five other early central Texas sites (see Table 13-3). Fish bones and otoliths have also been recovered further west in Tom Green County at site 41TG307, in 8200 B.P. deposits (Quigg et al. 1996) and south of San Antonio at ca. 6900 B.P. from the Richard Beene site (Thoms 2007a). Gar and catfish are most common fish identified and probably the easiest to acquire because of their size and habits. Multiple fish hooks and hook manufacturing debris $(N=33)$ from Stratum 10, in an Archaic midden in the south unit at Horn Shelter No. 2 (41BQ46) in Bosque County of central Texas document one procurement strategy (Redder 1985:60). The midden also yielded a suite of projectile points, mostly Pedernales $(N=17)$ and Gary $(N=8)$, plus five other types. Redder (1985:60) mentions at least nine other sites and or shelters that have yielded fishhook materials from across the greater central Texas region. Collins (1998a) suggested that a
Waco Sinker potentially served as equipment for fish procurement. Fish bones in a Paleoindian hearth in the Baker Cave site documents an earlier procurement (Hester 1982). Both gar and catfish species mentioned here are documented and were resources employed at roughly 5300 to 5500 B.P. at the Big Hole components. Exploitation of fish resources does not appear unusual where faunal resources are preserved, and apparently where procured over a long period of time.

Bird bones were identified at minimally three early sites in the region (see Table 13-3), plus a large quantity of bird bones $(N=157)$ from a terminal Early Archaic component at site 41TV540 (Mauldin et al. 2011a:134). At the latter site, 36 percent were from a single amorphous lens of burned rocks, bones, Rabdotus shells, and charcoal, over a small hearth-like Feature 27/29. The bird bones consist of 99 percent duck or duck-size birds. Another 35 percent were from an amorphous scatter (Feature 13; Mauldin et al. 2011a). Also, 11 bird bones were from the Ice House site (41HY161) in Hays County in San Marcos. One bird bone is the size of a probable prairie chicken (Tympanuchus sp.) (Meissner 2011a). Bird egg shells are represented in two early sites (i.e., Wilson-Leonard [Decker 1998] and Horn Shelter No. 2 [Redder 1985:61]). Multiple discoveries document bird exploitation in general, probably for meat and feathers, and possibly for other purposes. Their presence also indicates mammals were not the sole targeted meat resource by early and middle Holocene populations. Not only have bird bones been recovered, highpowered use-wear studies documented a feather barbule attached to an edge-modified flake in the ca. 5400 B.P. Martindale component at the Big Hole site. This documents actual bird processing. The latter is not the only bird processing example as two edgemodified flakes from a Late Prehistoric occupation at site 41LM50 in Lampasas County also exhibited feather barbules (Hardy 2014). Obviously, birds were hunted and processed by multiple groups over a long time, which began during the early Holocene and continued through modern settlement. 


\section{Plant Subsistence Discussion}

Plants, and more specifically geophytes are represented in increasing numbers of early sites (see Table 13-3). Examples include the WilsonLeonard site (Dering 1998) where they are radiocarbon dated between $7890 \pm 70$ B.P. and $8130 \pm 70$ B.P. from Feature 181, and $8250 \pm 80$ B.P. from Feature 8 (Collins et al. 1998; Stafford 1998:1054); the Berdoll site from Feature 11 dated approximately 7854 to 8103 B.P. (Karbula et al. 2011). Their use continued into the middle Holocene at the Big Hole site, Features 3 and 27/29 at 41 TV540 (Dering 2011), the Armstrong site Feature 2 dated to $6780 \pm 50$ B.P. (Schroeder 2002, 2011), and in the Lower Pecos such as Lens 13 in Area B at the Hinds Cave site dated to ca. 6000 B.P. (Dering 2007; Williams-Dean 1978). Acuna (2006) documented geophyte occurrences in archeological sites across central Texas and demonstrated their employment from Early Archaic sites on. Geophytes such as wild hyacinth (Camassia scillordes) grow in locally dense stands in the Blackland Prairie along the eastern Edwards Plateau (Scooter Cheatham personal communication 1995 cited in Dering 1998; Boyd et al. 2004b). These and other geophyte bulbs such as wild onion (Allium canadense), winecup (Callirhoe involucrate), dog-tooth violet (Erythronium mesochoreum), rain lily (Cooperia pedunculata), Prairie celestial (Nemastylis geminiflora), wood sorrel (Oxalis drummondii), crow poison (Nothoscordum bivalve [L.]) and many other roots and tubers are all present across this same region. Many geophytes and other plants are documented as used by Native Americans (Moerman 1998). Geophytes such as wild onions and related bulbs are said to have been boiled (Moerman 1998). Their value and habitats were obviously know to prehistoric populations and therefore, utilized early on with no apparent reason to cease their exploitation.

The lily starch from the two Big Hole components is that of a true lily (Lilium) and definitely not from wild onion, camas, or rain lily as they contain no starches. True lilies, Lilium do not occur in modern Texas flora, although potentially present some 5,000 years ago. This specific starch was in Features 24 and 29 in the Bell/Andice component and on an edge-modified flake from the Martindale component. A possible candidate for true Lilium starch would be Catesby's or pine lily (Lilium catesbaei) that currently grows across the coastal prairie region from Louisiana to Florida. This coastal plant grows in wet savannas, damp areas, requires hot conditions, and acidic sandy loam or clay loam soils. It blooms in late summer through fall and has a small scaly bulb, or rhizome, 1 to 3 $\mathrm{cm}$ in diameter and produces a $60 \mathrm{~cm}$ tall stalk. These lilies grow in open sites with limited leaf litter and extensive light (Sommers et al. 2011). They have edible flower buds, shoots, and roots that are often baked or boiled and taste like potato (The Flower Expert 2015). The lily identification is rare and unusual, but the presence of a geophyte is in keeping with other recent discoveries of plant remains at younger and older sites.

The gourd phytoliths from the two Big Hole components are not the first identified in Texas as one was also recovered from a 4800 to 5100 B.P. burned rock Feature 1 in a Andice component at the Slippery Slope site (41MS69) in Mason County (Quigg et al. 2015; Sudbury 2015). Gourds continued to play a role in later periods with gourd phytoliths identified during the Late Archaic component at site 41LM51 and the Late Prehistoric period at site 41LM50, both in Lampasas County (Sudbury 2014). In Texas, gourd seeds were in prehistoric context at the Hinds Cave site (41VV456) (Dering 1979, 1999). Apparently gourd use began quite early, and played a role throughout the late Holocene. Cabeza de Vaca mentions Natives employed gourds for water and cooking (Krieger 2002). It is not clear exactly how gourds were employed as food, utensils, floats for fishing nets, or other functions. Considerable historical evidence exist for native use of buffalo 
gourds including "seeds that can be ground to mush, and the bitter roots processed for their starch and protein" (Jones and Cushman 2004:145), as well as medical applications (Foster and Hobbs 2002). Gourd use has a long history in the United States as a bottle gourd (Lagenaria siceraria) in association with a human burial at the Windover site (8RB246) in Florida was directly radiocarbon dated to $7290 \pm 120$ B.P. (Doran et al. 1990). Cucurbits were in the American Southwest, and recent research indicates members of the genus were among the first domesticated plants in the New World, even predating the domestication of maize (Smith 1997). Southward in Tamaulipas Mexico cucurbit materials from three cave sites represent roughly the 9,000 years of prehistory (Whitaker et al. 1957). Wild gourds are wide spread in open, disturbed places all across the southern plains environment. Therefore, it should be expected that wild cucurbits were present and or used in some capacity 5,400 years ago in central Texas.

The two Big Hole components yielded evidence of multiple grasses. Multiple unidentified taxa of charred grass seeds from a Middle Archaic context were reported from the Wilson-Leonard site (Dering 1998, 2007). As Dering (1998:1626) indicates, no central Texas historic or ethnographic references exist for grass consumption, although abundant such references exist for Native Americans in the arid southwest (e.g., Castetter 1935; Castetter and Bell 1951; Doebley 1984; Yanovsky 1936). Yanovsky (1936:7-9) lists minimally 40 grasses, beside maize, used by Native Americans across the United States. This indicates grasses were very important to Native populations. Unfortunately, very limited archeological evidence exists for their use. At the Hinds Cave site, grass seeds were also recovered from 40 percent of the coprolites radiocarbon dated between 7490 B.P. and 6750 B.P. (Dering 2007), to support the early prehistoric diet included grass seeds.
One particular grass species, wildrye (Figure 13-2), probably present in the Big Hole components, has been identified through starch grain analysis (Perry and Quigg 2011) and is listed by Yanovsky (1936:8) as one taxa utilized by early populations. Wildrye grass starch has been identified at numerous prehistoric components and or sites across different regions and periods in Texas where starch grain analysis has been conducted. Examples include a ca. 2500 B.P. Late Archaic, fall bison processing camp (41PT185 - the Pipeline site) in Potter County in the Texas Panhandle. There wildrye starch was on 34 percent of the analyzed burned rocks, all eight metate fragments analyzed, and also on a chopper and large side scraper (Quigg et al. 2010; Perry and Quigg 2011a, 2011b). Also, wildrye was identified on three small burned rock features (Features 2, 4, and 7) radiocarbon dated by organic residues from the same burned rocks to between ca. 900 and 1500 B.P. at site $41 \mathrm{MI} 96$ in Mills County of central Texas. At the same site and next to the dated features, three edge-modified flakes yielded 13 lenticular grass starches, probably wildrye (Quigg et al. 2013a; Perry 2013). A Late Archaic camp radiocarbon dated between 1100 and 1500 B.P. dominated by mussel shells in Young County of north-central Texas also yielded wildrye grass starch from five burned rock features (Features 1, 5, 7, 14 and 15), plus one ground stone tool (Quigg et al. 2011a). Although the actual number of individual wildrye starch grains is never very high, their consistent appearance in small burned rock features and on stone tools at multiple prehistoric sites in different regions provide strong support for this specific grass to have been consistently collected by prehistoric populations for its seeds. Results from the Big Hole components push the cultural use of probable wildrye seeds back to at least ca. 5400 B.P.

Dering (Appendix B) states the recovery of chenoam seeds in the Big Hole site in central Texas is quite unusual, especially for an open air site. 
However, this is not the first reported instance of these seeds, which are identified in several much younger prehistoric sites. Cheno-ams have a long history of use across many parts of the United States especially in the Great Basin region (e.g., Cummings 2004; Kindscher 1987). The few identified seeds in the Martindale component push back the use of these related plants in central Texas. Evidence continues to mount that small-grain use was not energetically efficient in most settings. Consequently, efficiency probably is a poor measure of utility for prehistoric peoples in general or when populations were facing periods of food shortages (e.g., Gremillion 2004). Seasonal availability of specific food resources, such as seeds, will prove significant to seed harvesting and use as detective measures increase. Seasonality played a major role in the profitability of low ranked resources such as seeds and geophytes (see Winterhalder 1981). Therefore, the optimal diet shifts seasonally, especially if one or more highly ranked resources such as bison were scarce. As Gremillion (2004) discusses small seeds offered low rates of return (measured as energy per unit time spent), even so, peoples gathered and used this resource. Although the collection and processing

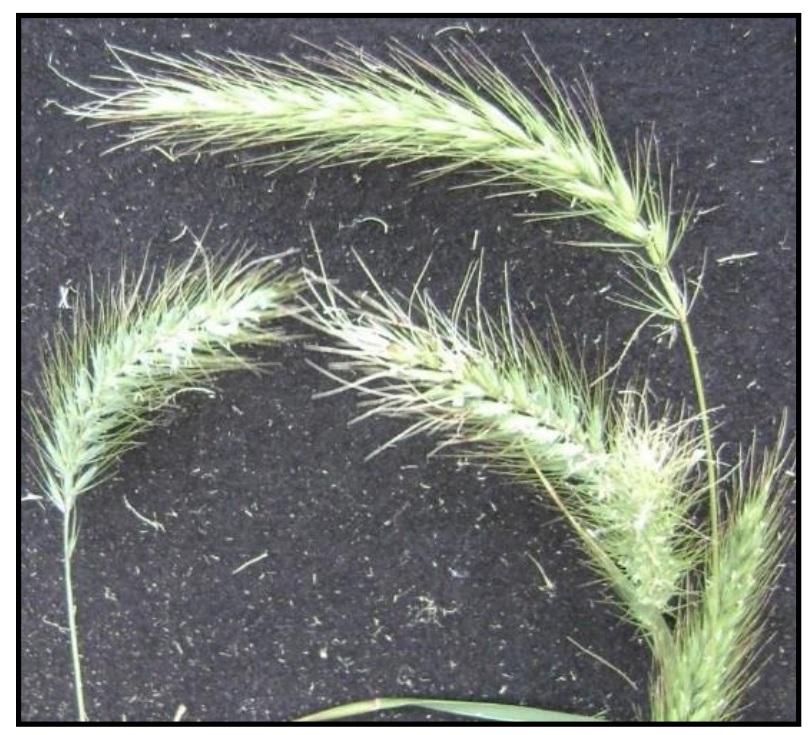

Figure 13-2. Close-up of wildrye (Elymus canadensis) grass seed heads (photograph by M. Quigg). of small seeds was labor intensive, this effort may have been necessitated by the lack of more readily available resources at this specific location and or season. It may also indicate the existing local environment had an abundance of seed resources, and or this was an adaptive response on the part of the regional human population to the relative dry climate. It definitely documents the use of small seed plant resources at this fall event.

Mussel shells were present in very limited numbers in both Big Hole components. No mussel shell concentrations or features were recognized. Although mussel meat was a potential food resource, animals and birds were potentially responsible for their sparse presence in apparent association with the components. If mussels were a culturally targeted food resource here, then mussel meat played a very minor role in the overall subsistence pattern. Their low frequency in the Big Hole components potentially reflects their limited availability in the lower Onion Creek valley during the occupation periods. Mussel shell low frequency is also documented at adjacent sites 41TV410 and 41TV540 (Figueroa et al. 2011), and the Berdoll site (41TV2125) (Karbula et al. 2011), in the immediate vicinity of the Big Hole site.

\section{Summary of Subsistence}

Dering's (2007) detailed examination of Early Holocene plant and animal assemblages from sites on the southern Plains periphery clearly demonstrated the broad diet breath nature of the subsistence patterns and the complexity unfolding. Generalized early hunter-gatherers utilized diverse resources to continually have food and sought multiple food options depending on local, seasonal, and regional conditions. Obviously with poor preservation more the norm in Paleoindian, Early and Middle Archaic sites across Texas (e.g., Dering 1998, 2011a; Dering and Bryant 2007) and the positive results from microfossil analyses clearly demonstrated here, a greater need exists to employ these types of analyses in all early sites. Continued 
use of multiple microfossil analyses will continue to document and expand upon plant resources utilized by early hunter-gatherer populations.

\subsubsection{Seasonality Discussion}

Seasonality has only been identified or even postulated for few early and middle Holocene sites. Lack of identification of seasonality severely limits our understanding concerning settlement patterns and seasonal camp and or subsistence variations across the landscape. Our multidisciplinary approach explored diverse lines of evidence and combined with the preserved faunal and macrobotanical remains allowed seasonality to be identified for both the Big Hole components.

For the Martindale component, the first molar of a deer in the erupting stage was identified and reflects a 3 to 6 month old fawn. This age animal indicates a fall death in roughly October through November time frame. The identified charred cheno-am and grass seeds in feature fill, plus charred hackberry nutlets from Feature 26 are most abundant and mature in fall. Also, the identification of lenticular grass starch (probably wildrye), on an expedient tool and one burned rock from Feature 26 support intentional collection and processing grass seeds, which mature in the fall. The presence of buffalo gourd phytoliths from Features 15 and 33 also supports a fall encampment. The combined evidence clearly reflects a fall use for this Martindale occupation.

For the Bell/Andice component at Big Hole, charred hackberry nutlets were present in Feature 32. Hackberry nutlets are most prominent and abundant in the fall. Ground and or damaged grass starch grains from three manos provide excellent evidence grass seeds were processed. True lily and lenticular grass (probably wildrye) starch grains were on three burned rocks from Feature 24 and Feature 29. Wildrye and other grass seeds would have been most abundant and ripe in the fall with true lily bulbs a possibility in the fall. Combined with buffalo gourd phytoliths in Features 24 and 30, which are again most available and ripe in the fall, the evidence again supports a fall event for the Bell/Andice.

\subsubsection{Technology Issues}

As data concerning subsistence practices continues to accumulate and expand, it is paramount we also strive towards a greater understanding of cooking technologies employed by the early populations (e.g., Black and Creel 1997; Black and Thoms 2014; Black et al. 1997; Collins 2011; Ellis 1997; Hester 1991; Jackson 1998; Quigg et al. 2001, 2002, 2010; Stark 1997, 2002; Thoms 2003, 2008, 2009; Thoms and Mandel 2007). As Driver and Massey (1957) state, the ethnographic records indicate patterned uses of cooking facilities to process different food resources. Burned rocks, the most obvious and abundant cultural debris at hunter-gatherer sites across Texas, reflect cooking practices. These rocks combined with identified features should reflect the diverse cooking practices employed. Many researchers have postulated various types of cooking strategies such as stone boiling, pit steaming, griddle cooking, earth-ovens, and others through a variety of means (e.g., Black and Thoms 2014; Collins 2011; Ellis 1997; Quigg et al. 2001, 2002, 2010; Thoms 2003, 2008, 2009). With different plant and animal resources identified at the two Big Hole components, the question arises as to what cooking technology or technologies were employed and are they specific to this time, and or to a specific food?

The eight burned rock features in the Bell/Andice component were small (less than $1.2 \mathrm{~m}$ in diameter) and appeared in both formal and informal rock patterns, some in shallow (less than $10 \mathrm{~cm}$ deep) basins, with less than 100 rocks per feature. Previously in this document we argued these feature characteristics, combined with plant and animal preparation data and technical results (highly fragments bones, carbonized seeds, lipid residues documenting both plant and animals, and 
gelatinized starch grains) clearly document diverse foods were cooked through a stone boiling technology. Lowie (1954:26) states some Plains nomads practiced stone boiling and describes it as "they lined a pit with a hide, filled it with water, and dropped red-hot rocks into it. The same effect was obtained by suspending a hide or paunch from four sticks driven into the ground". The above ground method depicted in Figure 13-3 is also supported by Wissler (1910). Collins (2011) provides multiple detailed ethnographic accounts that describe episodes of stone boiling to support this cooking technology as a common and often performed practice. Many accounts document boiling fish, meat, pulverized bone, and acorns (Collins 2011). Driver and Massey (1957) document stone boiling to cook multiple types of plant and animal food resources. Those same authors also document stone pottery was absent and populations were highly mobile. Wandsnider (1997) also documents plants high in starch and very lean or dried meat would be prepared by boiling. Grinnell (1961:106) indicates meat was usually boiled only long enough to change the color. Reid (1989) reported boiling was important for cooking starchy cereal grains and roots, which do not completely gelatinize below a temperature of $93^{\circ} \mathrm{C}$. Therefore, the ethnohistoric and ethnographic evidence of stone boiling supports the findings at the Bell/Andice component at the Big Hole site.
The boiling technology contrasts with earth oven technology employed by some foraging groups across the adjacent Edwards Plateau (Black and Thoms 2014). Earth ovens require large quantities of rock (ca $250 \mathrm{~kg}$ ), generally concentrated over a $2 \mathrm{~m}$ area, in 50 to $100 \mathrm{~cm}$ deep pits, with closely spaced and relatively large rocks in a circular pattern (e.g., Black and Creel 1997; Black and Thoms 2014; Collins 1991, 1998; Dering 1999). Fires in the large, deep pits create intense heat (ca. 500 to $900^{\circ} \mathrm{C}$ ) that caused charring of plant remains, abundant ash, carbon-stained and thermally oxidized sediment, and fragmented burned rocks (Black and Thoms 2014:215). Most large earth ovens are considered specialized plant-processing facilities for long-term heating and or cooking of plants that require break down (hydrolysis) of specific chemicals in inedible or minimally edible plants such as sotol, lechuguilla, or camas, to allow human digestion (Black and Thoms 2014; Dering 1999; Wandsnider 1997). The earth oven characteristics and or foods most often targeted for long-term oven cooking were not documented in the two Big Hole components.

Earth ovens may not only be specialized cooking facilities for specific foods, they potentially were limited to certain seasons along the eastern Plateau when specific resources were available. Dering (2007:188) states the full expression of the Early Archaic is characterized as a shift to bulk processing

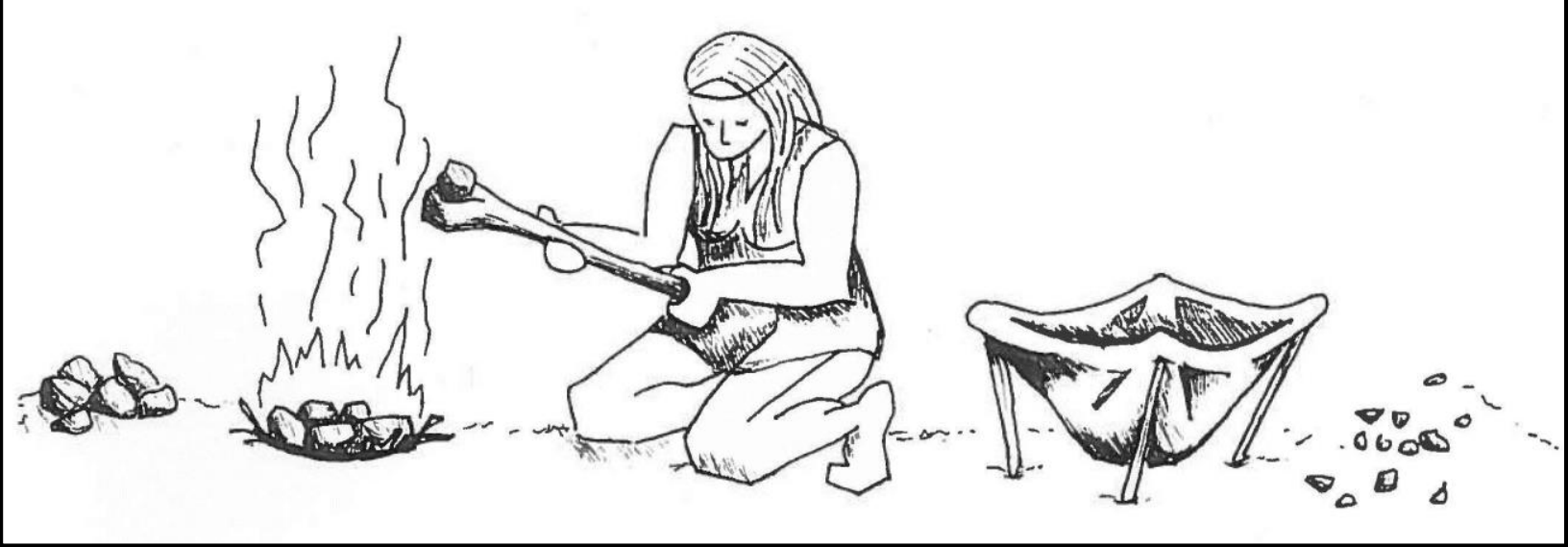

Figure 13-3. Schematic drawing of the stone boiling process and burned rock patterning (drawing by and with permission of $\mathrm{C}$. Lintz). 
of geophytes in earth ovens around 8000 B.P. The absence of earth ovens from the Big Hole components may potentially reflect their non-employment during the fall season. Plants cooked in earth ovens (e.g., sotol, lechuguilla, or camas) are generally not procured during the fall season. Foods generally not oven processed include; high sugar foods, starch-rich seeds, and lean meats (Wandsnider 1997:13). Consequently, different food resources procured at different season were probably cooked by different strategies.

Fossil Exogyra shells were observed in some burned rock features at the Big Hole site. Although an unusual material, other burned rock features in the immediate area and or region have also revealed fossil Exogyra use. Examples include Feature 13 in the San Geronimo phase at the Cervenka site (Peter et al. 1982), and multiple Early Archaic features at the adjacent Berdoll site (Karbula et al. 2011). It is highly unlikely these thick, heavy fossilized shells were specifically selected. Probably they were randomly collected with other rocks. This reflects a nonbiased or random rock selection for employment in the heat transferring process. It may also reflect the individuals collecting rocks were not familiar with different rock types and shapes, which would imply young children probably conducted the collection.

The highly fragmented bones in both Big Hole components document intentionally targeting bone grease (Figure 13-4). Zierhut (1967:35) states:

"...The process of making grease is as follows; all bone fragments are gathered and placed in a large pot containing water. The bones are boiled very slowly, and the fat which floats to the top is collected and eventually eaten."

Small, highly fragmented pieces are often connected to pemmican processing, as the extraction of bone grease through boiling is one of two principal ingredients, along with meat, as identified in ethnohistoric literature and archeological sites (e.g., Binford 1978; Brackenridge 1904; Brink et al. 1985;
Leechman 1951; Lowie 1954, 1963; Mandelbaum 1940; Mason and Holderby 1994; Newcomb 1993; Quigg and Peck 1995; Quigg 1997; Schilz 1983; Vehik 1977; Wissler 1910; Zierhut 1967). The Plains Cree performed similar operations in 1860-70 as Mandelbaum (1940:193 cited in Dyck 1977:264) states:

"Large bones were split open and pounded with a maul. The crushed splinters were placed in boiling water; the grease that rose to the surface was skimmed off with shell spoons and stored in buffalo pouches. It was called oskanpimi (bone grease)."

The production of pemmican as described by Driver (1972:93):

"The dried meat was first softened by holding it over a fire, then pulverized, mixed with melted fat and marrow and finally with berry or fruit paste to give it the desired taste and texture. The whole mess was then packed in a folded rawhide container called a parflech."

Lowie (1954:27) in describing pemmican production by Plains Indians in general states:

"Sun-dried slices of meat, pounded fine with a maul, were mixed with melted fat, marrow, and the dry paste from wild cherries that had been crushed, pits and all."

In describing the Tonkawa, Schilz (1983:31) states:

"Pemmican, a staple of the diet, was made by shredding dried buffalo meat, pounding it with a small mallet, and flavoring it with pecans or berries. This concoction was then mixed with hot grease and stored in rawhide bags. Pemmican would keep for several months." 


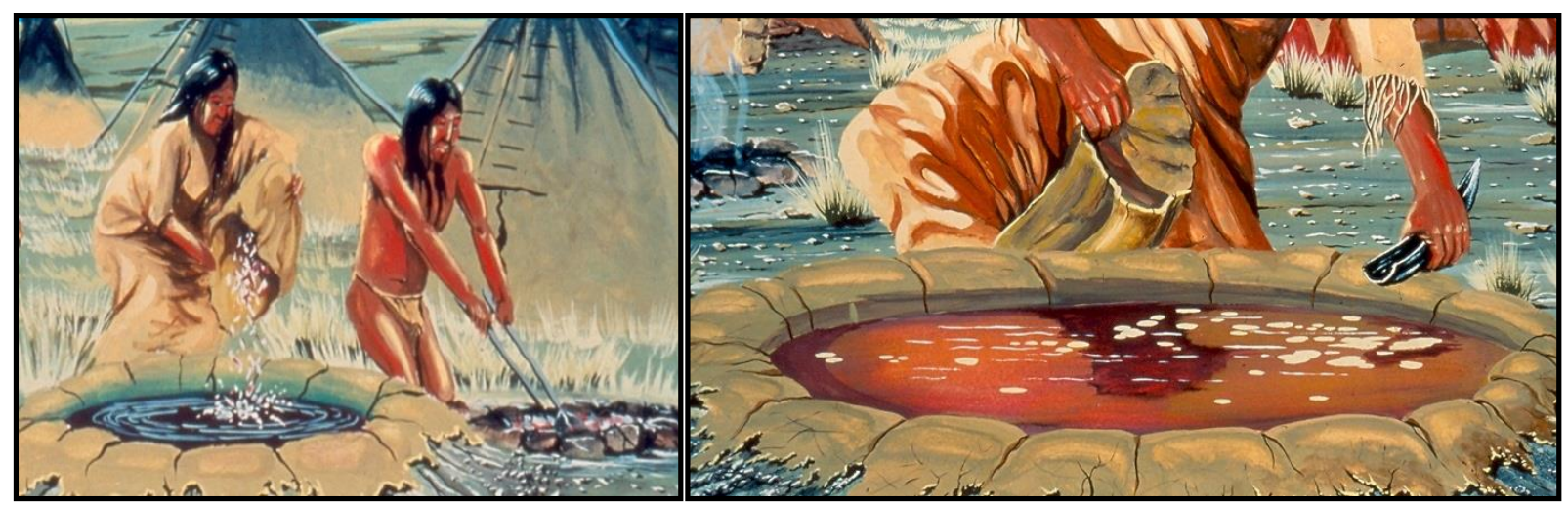

Figure 13-4. Impressions of bone grease extraction process (left) and scooping bone grease floating on surface of water (right) (paintings by and with permission of Shayne Tolman).

The two smashed bone assemblages from the Big Hole components are rare instances for bone preservation in Middle or even many Late Archaic components. Their recovery allows documentation of subsistence resources and also reflects specific human processing behaviors. Most early sites across Texas have not yielded vertebrate assemblages to allow researchers to address subsistence or related processing tasks.

Mason and Holderby (1994) discuss Late Prehistoric Toyah groups as probably producing pemmican, specifically for trading purposes. They state this time was potentially a "critical transition point in the evolutions of production in the Southern Plains, setting the Toyah strategy apart from the Archaic components which proceeded it" (Mason and Holderby 1994:478). If bone grease was processed for pemmican production in the two middle Holocene components at the Big Hole site, then it extends back in time this grease extraction and potential pemmican process, specifically in Texas. In the northern Plains, Reeves (1990:168194) documented pemmican production back to 4800 B.P., although it reached its peak during the "classic' bison hunting tradition of the Late Prehistoric period. Even if these smashed bones and the grease extracted from them were not part of pemmican production at the Big Hole components, the Late Prehistoric Toyah groups were not the only ones intensively processing bones for grease, as it occurred in these two middle Holocene components and elsewhere at other times across Texas (e.g., Quigg and Peck 1995; Quigg et al. 2001, 2002, 2010). The importance of fats in the prehistoric diet has been well-documented by Speth (1983). With relatively few animals per species represented in the two Big Hole components, apparently the desire for bone grease or potentially pemmican production, was not solely dependent upon large communal kills or large single prey species. When bone grease was desired, it was apparently extracted from whatever animal resource was procured, regardless the quantity or species. Bone grease extraction in the fall was potentially for preparation of what was envisioned concerning food supplies during the winter months ahead.

Lithic technology and game procurement is minimally represented in the two Big Hole components. Johnson and Goode (1994) stated Bell/Andice point using populations employed a specialized bison hunting and processing tool kit to reflect a shift in lithic technology from the previous period. Those authors did not specify what was specialized about those tool kits, except to indicate their projectiles were very broad. As Speer and colleagues (2014), and others such as Weber and Collins (1994), before them point out, the production of deep, narrow basal-notches in the Bell/Andice/Calf Creek projectile points and the skill in the production of large bifacial forms is not 
only unique, it requires a highly specific tool and technique for indirect percussion (Collins 1994a; Weber and Collins 1994). Although the large, broad projectile points with their complex and precise notching were probably manufactured by specialists, many different projectile point types were successful in killing bison. Therefore, it was not the processing tool kit or the killing of bison that was specialized, but the tremendous skill and craftsmanship in the production of the point used to kill the animals. In support of the skill employed in the technology to produce projectile points is the extensive use of heat treatment of raw material throughout the multiple stages in preparing and reducing large, thin bifaces into the final projectile for Calf Creek points across Oklahoma (e.g., Bartlett 1994; Duncan 2014; Duncan and Wyckoff 1994; Sullivan 1995; Wyckoff et al. 1994). Notching, adding the long, deep, narrow notches to the carefully crafted thin biface, was the final and critical step in projectile point manufacturing. To obtain the necessary notching skill, this very technical procedure was practiced on flakes and other chert pieces prior to attempting notching the thin finished biface. The notching pattern and the flakes from notching are so distinctive Collins (1994a) was able to identify a Bell/Andice knapping feature at the Barton site by the presence of multiple notching flakes alone. Ayala's (2014a, 2014b) research into notching patterns of Bell, Andice, and Calf Creek points is exposing new technological data. He sees, perhaps, a "typological series", which separates the technological traditions north and south of the Red River.

The high value placed on these finally crafted Bell/Andice projectiles is demonstrated in the fact many dulled, damaged, and or even broken points were often resharpened along blade edges or extensively reworked to extend their use life. Often resharpening was so extensive the original form was nearly obliterated or significantly reduced in size (Collins 1994a; Weber 2000). Broken points were often reworked into other tool forms.
Refurbishing was not due to the absence or scarcity of raw material as raw material resources were and are present across all the regions where these populations are known to occur. Given the tremendous skill and effort expended into the original production of finely crafted points, it is reasonable the craftsmen placed high value on each artifact and sought to maintain that artifact as long as possible.

Newly manufactured Bell/Andice points exhibit larger blade widths than most dart points to potentially indicate a broader shaft was necessary. Using only the four point examples from the two Big Hole components, the generally smaller and narrower Martindale point has a neck width roughly $1.0 \mathrm{~mm}$ wider than the two Bell points. This indicates a larger shaft was probably not employed with the broader Bell/Andice points. The Big Sandy point has the largest neck width at $21.1 \mathrm{~mm}$, which is significantly wider than the neck widths above. Consequently, the Big Sandy point was possibly hafted to a wider shaft, potentially implying a different delivery system.

Unmixed Bell/Andice tool assemblages are rare and limited in Texas as indicated by the sparse formal tool assemblage from the Big Hole component. Consequently, the full range of associated tools and technologies for their production is not known. The Hunter site (34TG6) in Oklahoma has yielded one of the few assemblages with scrapers. Those scarpers were manufactured on thick flakes (mean thickness of nearly $13 \mathrm{~mm}$ ), 4 to $6 \mathrm{~cm}$ long, removed from tabular nodules by a soft-hammer technique (Brooks 1995). Brooks (1995) also noted intentional removal of striking platforms and bulbs of percussion. Thirty-two percent demonstrate heat treatment to improve knapping (Brooks 1995). The presence of scrapers at some sites rather than others, implies seasonal variation in the tool kits, and probably not just in scrapers.

Martindale points, which clearly preceded the Bell/Andice forms, are much smaller, not as wide, 
corner-notched and exhibit a manufacturing technology unlike Bell/Andice forms. These attributes support the Bell/Andice forms did not originate directly from the Martindale technology. Functional analysis on 21 Martindale points from the Gatlin site clearly demonstrated all, except one, served as projectile points (Root et al. 2008). Nearly 30 percent exhibit evidence of being resharpened and reused, which continued the life span of a projectile into multifunctional tools. A potentially distinctive subtype and or substyle of Martindale points was observed in 37 points from the Woodrow Heard site (Decker et al. 2000). Sixteen percent exhibit alternate basal edgebeveling of the fishtail-shaped ears. Another 46 percent have unifacially beveled bases (see Figure 3-24). Beveling potentially reflects a localized characteristic and may imply regional variations. However, beveling and resharpening of multiple projectile point types were also detected in the lower Medina assemblage (ca, 6900 B.P.) at the Richard Beene site (Dockall and Pevny 2007:203). Since limited research has been conducted into the Martindale point style, and only a single complete point was from the Big Hole component, much remains to be learned concerning this early technology. From the few identified Martindale assemblages, the broader tool classes appear similar to many other Archaic assemblages (see Chapter $3.0)$.

The common hunter-gatherer chipped stone tools; points, bifaces, and edge-modified flakes were the only chipped stone tool classes recovered in the two Big Hole components. No unusual or unique formal stone tools (e.g., gouges, Guadalupe tools, or pieces esquillees, etc.) were from either Big Hole component. Absence of specialized tools indicate these huntergatherers had not significantly altered their hunting and processing tool kits to combat apparently difficult environmental conditions. Both components yielded manos and metates, artifacts which should be expected as they functioned for processing plants and potentially other subsistent resources.
As stated in Chapter 3.0, the stone tool technology at Middle Archaic sites is not well-defined as most lithic debitage and core assemblages have not been intensively scrutinized, even though lithic debris is frequent at many Early and Middle Archaic sites and or components. Lithic technology information derived from the two Big Hole components is also somewhat limited due to few formal chipped stone tools combined with the low frequency of lithic debitage. Formal tools only include the usual points and bifaces, which occur in most huntergatherer camps in the region and support the acquisition and processing of large game resources. The only potential unusual nature of the two Big Hole assemblages was the absence of scraping tools. It is not clear if their absence reflects the tasks performed in each component, seasonal characteristic, or if this is a broader trend for these groups. The low frequency of formal chipped and ground stone tools probably reflects the short-term nature of both components.

An in-depth lithic debitage analysis from the Big Hole Bell/Andice component reveals nearly all lithic reduction originated from cobble reduction towards production of large flakes probably for immediate and general tasks. Reduction was primarily by a hard-hammer percussion technique. In contrast to a high percent of heat treated materials in Calf Creek assemblages in Oklahoma, a high percentage (ca. 73 percent) of complete flakes in the Big Hole assemblage were not heat treated. This strategy to improve the knapping process was not extensively performed during this Bell/Andice occupation. This probably reflects the fact knapping only targeted general flake production, which apparently did not warrant time consuming, and controlled heating of targeted nodules. It may also reflect the local Edwards cherts were of such high quality, heat treatment was not always required. In support of the lack of heat treating Edwards chert, only 1 of 13 finished bifaces made from Edwards chert at the Primrose site (34MR65) in Oklahoma was heat treated in 
contrast to all local materials having been heat treated (Wyckoff et al. 1994). What is also noticeably absent in the Big Hole Bell/Andice component are "practice pieces", late stage biface production debris, and notching flakes. The absent of these latter items indicates projectile point manufacturing was not conducted here and potentially implies skilled artisans were not present or not actively producing at the Big Hole component. In broad terms, the limited Bell/Andice debitage assemblage represents a very basic unskilled, unstructured core-flake reduction sequence from local Edwards chert cobbles to create flakes for use as expedient tools for immediate on-site use.

The Big Hole Martindale component yielded a larger lithic debitage assemblage. It was also oriented towards reduction of small Edwards chert cobbles, plus the production of formal bifacial tools. Following the initial core reduction, much of the middle to late stage biface reduction was conducted through soft-hammer percussion technique. In contrast to the Bell/Andice assemblage, 70 percent of the complete flakes were heat treated to enhance the knapping process (see Crabtree and Butler 1964). Heat treatment was applied to stages in the biface reduction process. The higher frequency of lithic debitage, the application of heat treatment, and the production of formal bifaces reflect a greater commitment to formal tool production, probably by skilled craftsmen. If heat treatment occurred on site, no investigated features can be attributed to this process. This time consuming task would have probably extended the stay, or perhaps in this instance was performed prior to this occupation.

Although the two Big Hole lithic debitage assemblages are relatively limited, especially in the Bell/Andice component, the general reduction techniques of reducing chert cobbles and cores with hard-hammers, then switching to soft-hammers in preparing and reducing bifaces were similar. Additionally, relatively small cobbles of medium quality Edwards chert were reduced in both components. As demonstrated by INAA, these cherts were locally derived from the adjacent hilltop gravel source.

Bone technology is nonexistent in the Big Hole components and at other early sites, as most middle Holocene sites and or components have yielded little or no vertebrate faunal remains. This includes the absence of bone tools such as; awls, weaving needles, knapping tools, tool handles, rasps, bone beads, bone ornaments, tubes, etc. These tool types were probably employed, although poor site preservation or site functions have contributed to the absence of such artifacts.

Shell technology is also absent from most early and middle Holocene investigated inland sites in Texas and here in the Big Hole components. Mussel meat, a probable food source at many sites (e.g., the Richard Beene site [Thoms and Mandel 2007]), would have provided the necessary raw material from which a variety of shell tools and or ornaments could have been fashioned. The absence of shell may reflect a sampling problem, but more importantly reflect the nature of the individual occupations and or the populations themselves. The few excavated sites in the immediate vicinity of the Big Hole site, the Berdoll site (Karbula et al. 2011), sites 41TV410, and 41TV540 (Figueroa et al. 2011), also yielded meager mussel shell assemblages and no mussel shell features. Their availability in the lower Onion Creek valley potentially accounts for their limited presence, or a limited desire on behalf of those early populations.

The two ground stone tool assemblages of manos and metates from the Big Hole components are nearly identical to each other. They are also similar in type and style to those identified at other huntergatherer camps in the region, where ground stone tools were recovered. No unusual aspects concerning these two assemblages were detected. The metates are relatively thin slabs of sandstone characterized by shallow ill-defined use-wear to 
document limited grinding efforts. Manos are small one handed types with limited evidence of grinding. No specialize technology was needed to produce these ground stone tools. With the easy in which these tools can be manufactured and the need for grinding evident, it seems unusual more ground stone has not been recovered. As an example, the massive $233 \mathrm{~m}^{2}$ excavated Block $\mathrm{G}$ at the Richard Beene site (41BX831) yielded nearly 7,000 cultural items, and only one ground stone was identified (Thoms 2007a). Ground stone tools are much heavier and bulker than chipped stone tools and more often than not, left at the actual plant processing locations, rather than transported from camp to camp. Their absence from other components probably reflects the nature of tasks performed at other camps, which potentially lacked plant and or seed processing. It also might reflect the small excavated areas were not adequate to yield adequate samples from sites and or components.

Archeologically, many collecting and processing technologies are invisible but are known to exist. Poor preservation of perishable goods is the reason for our limited understanding. Basketry, wooden bowls and mortars, and other utensils employed in collecting and processing of grass and cheno-ams seeds are known from very few sites. Researchers in Texas have documented several wooden utensils (i.e., Collins and Hester 1968; Prewitt 1981; Waters et al. 2011). These perishable items were definitely part of prehistoric hunter-gatherer societies, although not preserved, except in rare circumstances in open air sites. Perishable objects played a major role in everyday tasks and are assumed to have been present at the Big Hole components. As stone metates were present, it is unlikely that wooden mortars were employed at these components. It is assumed basketry was the primary equipment for collecting small seeds; although skin bags potentially severed in a similar capacity (e.g., Geib and Jolie 2008). High-powered use-wear did reveal informal chert tools were one procurement instrument for harvesting seeds.
Other than projectile points employed to tip their darts, other procurement and or hunting technologies are not visible as they probably involved perishable objects. Baskets, nets, rabbit sticks, digging sticks, wooden traps, etc. from caves and rock shelters sites in the Lower Pecos provide evidence of perishable materials employed for resource procurement through time. Procurement of small game such as rabbits and rodents is documented without direct evidence as to the means employed. Arid regions of the west and southwest provide data in which to interpret procurement methods as rabbit drives that employed nets and sticks were an important procurement method. These methods were even depicted on pottery vessels (Shaffer and Gardner 1995).

\subsubsection{Environmental Characteristics}

Diverse datasets provide spotty information concerning past environments. Wood, seeds and plant identifications from early and middle Holocene archeological components and or sites across central Texas have yielded information concerning past vegetation. Live oak, juniper, pecan, walnut, and arboreal legume (e.g., mesquite, acacia, or Honey locus) have been identified at the Wilson-Leonard site in adjacent Williamson County in the Edwards Plateau since Paleoindian times (Dering 1998). Therefore, it stands to reason by 5500 B.P. minimally these same tree species were in the general vicinity and probably along major river and stream valleys. Tree species identified at adjacent site 41 TV540 that date to 5240 to 5300 B.P. include hackberry, persimmon, black walnut, oak, cherry or plum-type, cottonwood/willow-type, and elm, with oak the highest frequency identified (Dering 2011a). These species typify a riparian zone in a Blackland Prairie setting. In other Blackland Prairie settings, oak, mesquite, hackberry, elm, and walnut were identified from 6780 B.P. at the Armstrong site in Caldwell County (Schroeder 2011; Schroeder and Oksanen 2002). 
Burned trees were identified and radiocarbon dated at several prehistoric sites in, along, and adjacent to the Balcones Escarpment (e.g., the Wilson-Leonard site, the Richard Beene site). Those from the WilsonLeonard site date to the mid 5000 B.P., specifically at $5560 \pm 60$ B.P. and $5520 \pm 80$ B.P. (Collins et al. 1998a). These dates are similar to three burned trees (Features 19, 21, and 23) at the Big Hole site that date between 5800 and 6400 B.P. The burned trees potentially indicate natural fires during dry periods, or intentionally set fires to maintain open grasslands in this general area. Prehistoric populations intentionally burned areas as a means to attract bison to a specific area in the spring time (Lewis 1973:55; Stewart 1954; Wedel 1957). Bison select recent burned areas over unburned or old burned areas (Coppedge and Shaw 1998).

Three types of charred grass seeds, panic grass (Panicum), paspalum (Paspalum), and bristlegrass (Setaria) were identified from Feature 209, a Middle Archaic feature at the Wilson-Leonard site (Dering 1998, 2007). Dering (1998:1626) suggests these grasses flourished in open, well-drained soils of moderate depth and are indicative of an open canopy parkland for south-central Williamson County. The probable presence of wildrye grass in the two Big Hole components indicates shaded and moist areas were probably present in the riparian zone along Onion Creek. In the immediate vicinity of the Big Hole site, Bozarth and Woodburn (2011) document a dynamic but younger vegetation history and paleoenvironment over the last 3820 B.P. Their data came from a $7 \mathrm{~m}$ vertical section through depositional Unit Qa4 in Area 3 at 41TV410. In the vicinity of the Big Hole site, vegetation from ca. 3820 B.P. was dominated by warm season Chloridoid phytoliths, which reflect short $\mathrm{C}_{4}$ grasses adapted to arid environments. The upper $3 \mathrm{~m}$ of the section, younger than 2870 B.P. based on bulk soil dates, also reflect charred phytoliths and particulate charcoal to document burning the local environment. These phytolith results support a general short-grass prairie for the Big Hole site environment and across the region.
The presence of pronghorn and grouse or prairie chickens during 5200 to 5400 B.P. in the Big Hole Martindale component faunal assemblages indirectly supports an open prairie environment. Pronghorns require access to nutritious forbs, browse, grasses, and sedges and also eat grease wood, rabbit bush, and sagebrush, all in an arid environment. Pronghorn do not do well in true deserts, but take advantage of riparian zones (Geist 2001). Pronghorn remains have also been identified in similar settings in Early Archaic components at the Berdoll site (Karbula et al. 2011), in Late Paleoindian times at the Armstrong site (Schroeder 2011; Schroeder and Oksanen 2002), in Early Archaic components at the Cervenka site (Yates 1982), the Hawes site (Yates 1982), in the adjacent Edwards Plateau at during the Early Archaic at the Wilson-Leonard site (Baker 1998), and even in the Gulf Coastal Plain in Early Archaic (ca. 6900 B.P.) assemblages (lower Medina) at the Richard Beene site (Baker and Steele 2007; Thoms and Clabaugh 2011). If the presence of pronghorn implies an open prairie region during these early periods, it apparently extended over a long period clear to the Texas coast.

The Attwater Prairie-Chicken is a medium sized grouse heavily barred with brown, black and buff feathers. Although now nearly extinct in Texas, their historic range is shown in Figure 13-5 (Silvy et al. 2004). This species was once a resident of the tall grass prairie ecosystem all across the coastal prairie habitat of southern Texas. The birds prefer open prairies without any woody cover, and avoid areas with greater than 25 percent cover of shrubs. Modern and historic range data indicate the Attwater Prairie Chicken as the most probable candidate for those prairie chicken elements identified as belonging to grouse family in the Big Hole Martindale component. The general expansion of prairie taxa has also been demonstrated in western Missouri during the warm and dry middle Holocene (Wolverton 2002). 


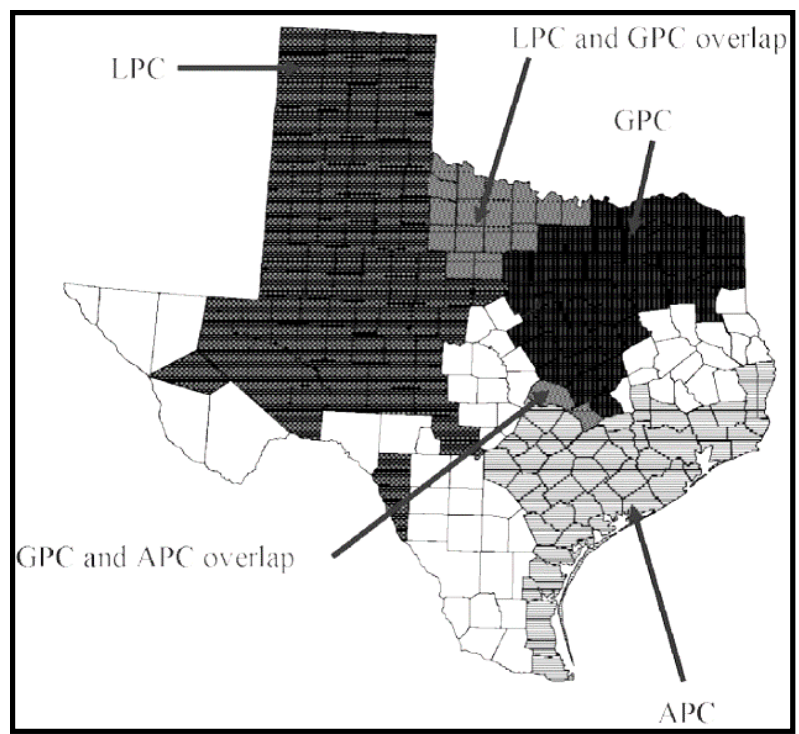

Figure 13-5. Historic range for lesser prairiechicken (LPC), Greater Prairie-Chicken (GPC) and Attwater's Prairie-Chicken (APC) (from Silvy et al. 2003:18).

Prewitt's (1985) San Geronimo phase (ca. 6700 to 6200 B.P.) component at the Cervenka site, also in the Blackland Prairie setting just north in Williamson County, yielded unburned bird elements specifically identified as prairie chicken elements $(N=6)$, as did two later phases in Area D at the same site (Yates 1982:15-193). This prairie species was apparently present in the immediate environment and hunted as a resource by multiple groups during minimally the middle Holocene.

Onion Creek was definitely more than seasonally flowing as evidenced by the presence of minimally three fish species (i.e., catfish, gar, and possibly bass or perch) in the Big Hole Martindale component. This constant water source would have provided an excellent attraction for human populations and other resources such as fish and a diverse plant community. The Yellow Sandshell mussel identified from the Martindale component does not tolerate dewatering or droughts (Howells et al. 1996). This mussel combined with viable fish populations document Onion Creek was flowing during these fall occupations and probably throughout much of its history. This constantly flowing water is in contrast to multiple streams across and near the Llano Estacado in northwestern Texas where above ground water disappeared and prehistoric populations hand-dug wells to capture water (e.g., Meltzer 1991, 1995, 1999; Meltzer and Collins 1987; Quigg et al. 1994) during the Altithermal period.

Noticeably absent from the two Big Hole botanical assemblages are nut shells of any type, such as walnut, hickory or oak acorns. If the two Big Hole components were occupied in the fall as the evidence supports, this often sought nut resource would probably have been procured during the occupations, if nut producing trees were in the area. Nut producing trees are more noted in the Post Oak Savanna along the western edge of the deciduous and or coniferous forest. The absence of nuts implies these tree species were probably not in immediate site environments. Their absence also supports this locality was an open prairie grassland environment during these occupations.

During phytolith analyses of the Big Hole sediments, soil texture data from a stratigraphic column in noncultural deposits clearly document a significant increase in sand content at the expense of silt between 300 and $330 \mathrm{cmbs}$ (Appendix E). Sand is generally deposited in wetter periods or floods, and support an interpretation of a wet event along Onion Creek just below or prior to the Martindale component dated to ca. 5400 B.P. Prior to that potential wet event, the silt deposition rate was higher at $340 \mathrm{cmbs}$, which potentially reflects eolian deposition during a relatively dry interval. Following the potential wet event and the Martindale component between 260 and $290 \mathrm{cmbs}$, the silt component again increased to offset the decrease in sand deposition. This silt deposit potentially implies another drying interval. It is significant that an apparent wet event or flood is documented by texture data between two dry periods. Texture changes in the deposits may lend support for a two part Altithermal or minimally, more complex wet and dry periods, which created 
geomorphic changes to the landscape as discussed in Chapter 2.0. Lohse et al. (2014) present stable carbon and nitrogen isotope data from bison remains in central Texas to indicate the "Calf Creek" period was characterized by cool and dry conditions. The rare preservation of vertebrate faunal assemblages and well-preserved activity areas in both Big Hole components reflects rapid burial of cultural deposits by late fall or early spring floods.

\subsubsection{Settlement Patterns and Interaction}

In terms of settlement, the two Big Hole components were occupied during the fall season, adjacent flowing waters of Onion Creek. The immediate area obviously provided necessary life sustaining resources hunter-gatherer populations relied upon; water, wood, rocks, plant, and animal resources. Repeated use of this lower Onion Creek valley location (e.g., sites 41TV410, 41TV540, 41TV2115, and 41TV2161) and adjacent alluvial deposits was influenced by multiple ecotones along the Balcones Escarpment with good quality water flowing from there and access to multiple needed resources. This setting allowed multiple huntergatherer groups over time to access the diverse resources in various niche habitats. The availability of multiple resources in one location created an ideal area and an obvious choice for camping over time, and specifically reliable for fall resources. One specific plant resource, geophytes were potentially the main attraction to the lower Onion Creek valley for multiple populations. These were potentially available during one or possibly two seasons, and were identified in minimally three other sites in this immediate area (i.e., sites 41TV410, 41TV540, and 41TV2115). No chipped stone tool caches, in ground storage pits, or elaborate and costly cooking facilities were detected to support intentional food storage or stock piling raw materials in anticipation of returning this exact spot. The absence of such features reflects the mobility of these groups and the short-term nature of the occupations. The short-term nature and apparent small group sizes reflected by the two Big Hole components and adjacent sites document residential mobility remained high, and group sizes small, during this late Altithermal period in central Texas.

The settlement pattern detected at the two Big Hole components may not appear as a specific adaptive strategy for this warm and or dry period. However a similar site settlement pattern was detected for 115 middle Holocene sites across the Northern Plains (Walker 1980, 1992). Walker (1992:128129) states virtually all middle Holocene sites are very close to reliable water sources, and a second characteristic is a tendency for habitation sites to be of limited extent with sparse cultural assemblages. The two Big Hole components are excellent examples of this same settlement pattern detected for the Northern Plains. Benedict (1979) also sees population movements from the Plains proper and more intensive settlement in the mountains during this period. Back in 1981 McKinney's (1981) observed most known Early Archaic sites in Texas are concentrated along the southern Edwards Plateau. This latter settlement pattern is further supported by data from the Big Hole site and the adjacent early sites of 41TV410, 41TV540, and 41TV2115. This pattern of short-term occupation by small groups in similar environmental settings again indicates mobile populations probably followed a scheduled exploitation of seasonally available resources.

In terms of regional interactions, evidence is lacking from the two Big Hole components, at least on the basis of the absence of recognizable nonlocal lithic materials or exotic goods. Local interactions with adjacent groups potentially occurred with perishable materials not preserved in the archeological record. However, the absence of nonlocal lithic materials, especially from Oklahoma, where both abundant lithic resources are present and Calf Creek horizon materials are well-represented is surprising. Although Wyckoff 
(1995) postulates distinct territories for Calf Creek horizon populations in Oklahoma on the basis of use of local raw materials in different regions of the state, those assemblages also reveal minor amounts of Edwards chert and other nonlocal materials. Edwards cherts in Oklahoma indicates exchange and or trading of lithic resources did occur during this period, some over great distances, and between these regionally localized populations. Apparently, broader social networks were in operation between Texas and Oklahoma, especially during the Calf Creek horizon time, although they were not discernible in the two Big Hole components. The use of local Edwards chert combined with the absence of Oklahoma cherts may indicate a population regionally specific to central Texas.

About this same time in the middle Mississippi and lower Ohio Rivers, carved and engraved bone pins were shown as objects that document regional-scale social interactions among increasingly more sedentary late Middle Archaic (6000 to 5000 B.P.) hunter-gatherer groups (Jefferies 1997). The latter example documents regional-scale interaction networks had developed between some eastern groups by 6000 B.P. Therefore, it would not be surprising similar networks were operational across Texas and Oklahoma. The apparent absence at the Big Hole components potentially reflects existing climatic conditions hindered interactions, the dispersed nature of the human populations, or even the abundance of local, high quality lithic resources immediately available to local groups. The latter would have limited their need and or desire to exchange lithic goods or interact. Until additional middle Holocene sites are excavated in Texas, exchange, movements, and interactions between populations across Texas and Oklahoma will remain unresolved.

Hofman (1989) provided a region-wide synthesis of hunter-gatherer cultural history followed by five assumptions he thought characteristic of Archaic groups across the Southern Plains. In general terms, each of those five assumptions appear validated and or supported by the two Big Hole middle Holocene components. Those assumptions include the following: 1) seasonally varied economies, 2) flexible group structure with periods of aggregation and dispersal, 3) seasonally variable needs such as fuel, shelter, and clothing, 4) a variety of site types which result from diverse economic, social, and maintenance activities, and 5) a variety of alternative strategies for coping with seasonal, or yearly, economic shortfalls or windfalls (Hofman 1989). Meltzer (1999), in discussing specific conditions during the Altithermal, also projected a widening of the diet breath to incorporate highercost, lower-return seed and plant resources, which are documented at the two Big Hole components. The two Big Hole components fit nicely into the previously stated expectations and clearly document these behaviors.

\subsection{ASSESSING AND CRITIQUING TECHNICAL ANALYSES}

Twelve different technical analyses were implemented to extract as much information as possible from these rarely explored and important occupations. Each technical analysis implemented is individually addressed below.

\subsubsection{Radiocarbon Dating}

Radiocarbon dating (Appendix A) has long been established and accepted as the most appropriate and most reliable means to establish age of cultural and natural deposits. More than dozen radiocarbon dates from each identified component resulted in significant information, particularly in refining the time of occupations and the specific use periods for the three projectile point types recovered. Radiocarbon dating of multiple material classes has demonstrated once again that wood charcoal and charred annual seeds provide greater precision than other organic materials in determining the specific age of a cultural event. It has also been demonstrated charred annual plants (e.g., corn, beans, grass seeds, and cheno-ams) provide even 
more accurate and precise timing of an event over wood charcoal (Roper and Adair 2011). If charcoal is not present, radiocarbon results from other substances (e.g., soil humates, shells, and bones) yield ages that must be considered as "ball park" timing for the associated cultural event, rather than a specific occupation age. Continued funding of many radiocarbon dates per event or component is necessary to adequately document the age specific features or cultural events. Multiple dates also continue to refine the broader chronology of Texas. Adequate radiocarbon dating potentially leads to the synthesis of information concerning overlapping group territories and movements of human populations across space and through time. The willingness of TxDOT to fund multiple dates per component at the Big Hole site was significant and yielded very important age associations for three understudied projectile point types.

\subsubsection{Flotation and Macrobotanical Analysis}

Flotation and subsequent macrobotanical analysis (Appendix B) are more routinely applied than years previous. These analytical processes are necessary to address questions regarding the kinds of plant species and wood types utilized by past populations. Since the beginning of the "flotation revolution" in the 1960s (Chapman and Watson 1993), macrobotanical analysis has led to remarkable discoveries of plant utilization and will continue to do so. Macrobotanical identification may also provide information regarding plant communities in past environments, the species of plant material for radiocarbon dating, human selection patterns of certain plant resources, and potentially the processes and technology used to prepare or cook plants. The specifics of collecting flotation samples is not standardized (e.g., sample volume, documentation, number of samples, etc.). At minimum, samples of 10 liters should be targeted to maximize returns, sample volumes should be documented to address densities, and the flotation of 50 to 100 percent of feature fill should be the goal in most situations.
Macrobotanical identifications from the two Big Hole components were very limited as preservation of organic residues was quite poor, which is common in many sites and areas across Texas especially in early to middle Holocene deposits (Dering 2007). The few charred seeds identified are quite significant and, as Dering believes, quite noteworthy (Appendix B). Although most analyzed samples yielded unidentifiable carbonized material, some very important charred grass and cheno-ams seeds, as well as wood charcoal from live oak and mesquite, remained preserved for identification. The results from the excavation of the Big Hole components exemplify the need to routinely collect samples for flotation and macrobotanical analysis with specific focus on cultural features to determine which plant resources were potentially in use at a particular feature, event, or camp. Flotation and macrobotanical analysis are necessary procedures to evaluate long-held assumptions regarding plant exploitation and subsistence. In regions of poor macrobotanical preservation, flotation and macrobotanical analyses could be applied more liberally to ensure that important and significant subsistence information is detected within every feature and archaeological component.

\subsubsection{Diatom Analysis}

Diatom analysis (Appendix C) was anticipated to yield data necessary to address paleowater conditions and potentially support water use in features during the cooking processes. However, diatoms were not well-preserved with only about half the analyzed samples providing any results whatsoever. Only 45 diatom taxa were identified plus 12 unidentified diatom fragments, for a total of 2,137 cells. One sample extracted from calcium carbonate adhering to a deer bone contained 81 percent of the total. The best preserved and most abundant diatoms were those trapped in small calcium carbonate nodules attached to deer bones, a late discovery in the process of collecting samples for diatom analysis; such accumulations in 
association with cultural materials should be targeted when sampling calcium carbonate rich deposits.

Diatom types identified allow interpretations of water characteristics that supplemented other datasets. The analysis of the poorly preserved samples were only partially helpful in determining the presence of water in features and whether or not water was used in cooking processes. The unexpected and unusual discovery of marine diatoms in this setting contributes to a small and growing body of similar discoveries. These chance discoveries may increase understanding of their presence in or near prehistoric components (see Chapter 10.0 for more discussion). Diatom analysis should be employed as needed to address specific research questions, but even at that, chance discoveries and identification such as the marine diatoms will go undiscovered, if this analysis is not pursued on a regular basis.

\subsubsection{High-Powered Use-Wear Analysis}

High-powered use-wear (Appendix D) analysis was implemented to identify specific tool functions and potentially materials the tools came in contact with. Use-wear was very informative, especially on the edge-modified flakes, which most often were employed to conduct an array of tasks on diverse materials. This technique is also useful if multiple tools of one specific type are recovered to clearly document their function (e.g., Quigg et al. 2008). The added bonus in this work was the ability of the analyst to identify microfossils on tools, which often provides the specific materials the tool came in contact with, rather than an unspecific polish type documenting use. The identification of a fish scale and a feather particle during use-wear analysis was extremely informative as to the specific function of that tool, and definitely adds strong support for specific tasks undertaken by the inhabitants, and specific resources processed by the occupants.
Implementation of high-power use-wear is important, although use-wear can only be interpreted to a limited extent (e.g., Bamforth et al. 1990; Barrett 2012; Grace 1990; Hudler 2003a; Root et al. 2008). Use-wear combined with additional identification of microfossils helps identify specific contact materials. It is this author's opinion that more high-powered use-wear combined with residue and or microfossil identification will yield significant information concerning specific human behaviors and specific tasks conducted at individual occupations.

\subsubsection{Phytolith Analysis}

Phytolith analysis (Appendices E and K) was conducted to document the site's immediate environment and conditions these groups operated within, and to contribute to the identification of potential foods and or food groups utilized by the occupants. An initial phytolith assessment was conducted and positive results indicated continued analysis would yield useful information and contribute to establishing the composition of the grassland environment at the time of these occupations. Subsequent in-depth analysis on additional samples documented short cell grass phytoliths, important for reconstructing the local grassland environment, were not well-preserved and a major disappointment. Even with generally poor presentation of short grass cells, various other phytoliths were present to indicate possible food resources (e.g., wild gourd), while other identified phytoliths document the presence of wood employed as fuel for the fires. Although one primary goal was not successful here, other important phytolith information made contributions. It is important to continue pursuing this technique, as in most circumstances, phytoliths should yield information towards a greater understanding of past environments and use of potential food products. At other projects and or sites in the region, such as at Ivie Reservoir (ScottCummings 1993) and the Wilson-Leonard site, phytolith analyses documented regional and local 
changes in vegetation. These analyses also showed close correlation with other independent records to validate the usefulness of phytoliths for paleoenvironmental reconstruction (Fredlund 1998). Fredlund (1998) states those positive site results warrant continued use of this method. Interestingly, phytolith analysis at the adjacent 41TV410 site yielded well-preserved and interpretable assemblages (Bozarth and Woodburn 2011). Another important aspect of phytolith analysis is many economic plants such as maize, squash, wild gourds, sunflowers, and common beans produce distinctive phytoliths to contribute to subsistence aspects at sites (Bozarth 1986, 1987a, 1987b, 1993; Bozarth and Woodburn 2010, 2011).

\subsubsection{Starch Analysis}

Starch grain analysis (Appendix F) was projected to identify specific plant foods employed, and food preparation techniques by each human population, as macrobotanical remains were anticipated to yield minimal data concerning specific foods employed because of preservation problems. Carbonized plant remains are rare in most Texas sites, especially in early sites, and starch grain analysis provides a very promising means to identify specific plants employed, plus processing techniques applied to that resource (e.g., Fullagar et al. 1998; Perry 2001, 2010, 2013, 2014; Piperno and Holst 1998; Piperno et al. 2000; Zarrillo and Kooyman 2006). Starch grain preservation was poor for the first time in multiple studies across Texas. Even so, starch analysis yielded very significant information concerning plant use and food preparation techniques, which otherwise would have remained undetected. When starches are preserved, often they can be identified to specific plants (e.g., Perry and Quigg 2011b). Not only is the specific plant identification important, the condition of individual starch grains (ground, parched, crushed, or gelatinized) can inform towards processing and cooking techniques plants were subjected to. Ground and damage grains can be identified and document one plant processing strategy, as identified gelatinized grains document cooking with heat and water such as stone boiling strategies (e.g., Perry and Quigg 2011a). These processing and cooking strategies are very significant in understanding human behaviors concerning a particular feature and or occupation. This author believes strongly in this technique, and it should be more widely employed when dealing with burned rock features and various chipped and ground stone tools to broaden our understanding of feature and tool functions towards targeted plant resources. Gathering plants was a major and significant activity of mobile hunter-gatherer societies and is often overlooked or understated as the archeological visibility is often low. As one example, this technique has allowed significant discovery and identification of maize starch on multiple burned rocks from multiple Late Archaic components in central Texas (Perry 2010, 2013, 2014). It is this type of discovery that will advance our understanding of specific subsistence and processing practices of prehistoric populations across Texas.

\subsubsection{Lipid Residue Analysis}

Lipid residue analysis (Appendix G) is a chemical means of identifying general food groups - meats and or plants cooked by burned rocks, and was also applied to ground stone tools. Lipid residue analysis has not gained wide acceptance and is not pursued as a standard analytical practice. Understanding what food groups were cooked by burned rocks is very significant and informative towards how different groups and or populations treated, processed, and cooked various plant and animals resources. If lipid residues can document rocks from one type of burned rock feature were employed to cook only plants and another feature type was employed to cook both plants and animal products, that is important information that otherwise would have gone undetected. Without that information, researches are prevented from obtaining knowledge on how different features functioned and the purpose behind the cooking 
process. Eventually, researchers may determine certain types of features were employed to cook certain types of resources. The new, additional and more sophisticated techniques currently employed in Malainey's lipid analysis, which include incorporation of high-temperature gas chromatography (HT-GC) and gas chromatography with mass spectrometry (GC-MS, fully implemented in 2009) to detect plant and animal products through specific lipid biomarkers (in use since 2007) has greatly enhanced lipid results and strengthened interpretations. This new and improved approach was employed here and in the Mills County analysis (Malainey and Figol 2013).

High temperature gas chromatography was employed to separate and assess a wide range of lipid components, including fatty acids, long chain alcohols and hydrocarbons, sterols, waxes, terpenoids and triacylglycerols (Evershed et al. 2001). The molecular structure of separated components is elucidated by mass spectrometry (Evershed 2000).

Triacylglycerols, diacylglycerols and sterols can be employed to distinguish animal derived residues, which contain cholesterol and significant levels of both triacylglycerols, from plant derived residues, indicated by plant sterols, such as $\beta$-sitosterol, stigmasterol and campersterol, and only traces of triacylglycerols (Dudd and Evershed 1998; Evershed 1993; Evershed et al. 1997a). Waxes, which are long-chain fatty acids and long-chain alcohols that form protective coatings on skin, fur, feathers, leaves and fruit, also resist decay. Evershed et al. (1991) discovered epicuticular leaf waxes from plants of the genus Brassica in vessel residues from a Late Saxon or Medieval settlement. Cooking experiments later confirmed the utility of nonacosane, nonacosan-15-one and nonacosan-15ol to indicate the preparation of leafy vegetables, such as turnip or cabbage (Charters et al. 1997). Reber et al. (2004) suggested n-dotriacontanol could serve as an effective biomarker for maize in vessel residues from sites located in Midwestern and Eastern North America. Beeswax can be identified by the presence and distribution of $n$ alkanes with carbon chains 23 to 33 atoms in length and palmitic acid wax esters with chains between 40 and 52 carbons in length (Evershed et al. 1997b; Heron et al. 1994).

Terpenoid compounds, or terpenes, are long chain alkenes that occur in the tars and pitches of higher plants. For example, the use of GC and GC-MS to detect the diterpenoid, dehydroabietic acid, from conifer products in archeological residues extends over a span of 25 years (Heron and Pollard 1988; Shackley 1982). Lupeol, $\alpha$ - and $\beta$-amyrin and their derivatives indicate the presence of plant materials (Regert 2007). Eerkens (2002) used the predominance of the diterpenoid, $\Delta-8(9)$ isopimaric acid, in a vessel residue from the western Great Basin to argue it contained piñyon resins. Other analytical techniques have also been employed to identify terpenoid compounds. Sauter et al. (1987) detected the triterpenoid, betulin, in Iron Age tar using both ${ }^{1} \mathrm{H}$ and ${ }^{13} \mathrm{C}$ nuclear magnetic resonance spectroscopy, confirming the tar was produced from birch.

The employment of these improved methods and biomarkers allow more specific informative and useful data for interpreting what products burned rocks and ground stone came in contact with from human behaviors. This author strongly supports continued employment of lipid residue analysis with the added new techniques when sites yield burned rock features and or ground stone tools to determine what those rocks came in contact with. As a means of extracting additional and useful information from artifacts, Feder (2008), Kelly and Thomas (2013), and Thomas et al. (2009) all advocate for lipid residue analysis.

\subsubsection{Instrumental Neutron Activation Analysis}

Instrumental neutron activation analysis (Appendix H) was employed to identify lithic sources and determine possible population's movements and or 
interaction with other populations in adjacent regions. The incorporation of local natural chert samples from the adjacent hilltop into our analysis was a significant addition to the cultural chert samples from each component. This allowed a direct comparison and yielded positive results. The laboratory established a chemical signature for the local natural hilltop cherts, and in conjunction with signatures from the cultural artifacts, determined nearly all cultural artifacts analyzed from both components were manufactured from cherts collected from this local gravel source. It also revealed these two different hunter-gatherer populations were not transporting or trading lithic artifacts from distant sources, at least not in this specific instance. This means of establishing lithic and ceramic source areas continues to build a greater understanding of human population movements, trading and settlement patterns, and resource procurement activities across Texas. This is an excellent means of identifying many different internal and social aspects of prehistoric groups and can be applied to lithic and ceramic materials (e.g., Boulanger and Glascock 2012; Cogwell et al. 2004; Ferguson and Glascock 2011, 2012; Frederick et al. 1994; Hudler 2003b; Perttula 2011; Perttula et al. 2003; Quigg et al. 2011b). Although many different source areas for Edwards chert are identified, it has also been demonstrated different sources of Edwards chert can be documented (e.g., Boulanger and Glascock 2012; Hudler 2003b). This author advocates for continued implementation of this technique and to include sizable samples $(N=20$ or more for statistical purposes) of cultural cherts and ceramic artifacts from components and sites, and large samples $(N=$ 20 or more) of natural raw materials from across the region. Continued use of INAA will gradually expand the current state wide databases for lithic artifacts and pottery. This will provide necessary data to address complex social issues and interactions.

\subsubsection{X-Ray Diffraction Analysis}

XRF analysis (Appendix I) in this report was very limited and focused on identifying reddish and blackish residues on two nonfeature rocks. Red stained rocks are unusual and analysis directed towards identifying the stain was important and would ultimately add to our understanding various tasks performed, what substances were processed, and to extrapolate potential uses of that material. XRF results positively identified the stained substance as iron oxide and enlightened us as to potential tasks conducted such as grinding pigment. This author supports the use of XRF in determination of mineral residues on carefully selected objects from components and identifying obsidian composition to determine sources (e.g., Shackley 2010, 2012).

\subsubsection{Cementation Analysis}

Cementation analysis (Appendix $\mathbf{J}$ ) was conducted in a very limited way. It was directed towards two deer incisors to determine the age of the individual at the time of death and thereby contribute toward establishing the season of use for the cultural component. The two incisors did not reveal any growth lines, therefore the laboratory could not provide useful data to address the animals age. Although this technique has good potential to determine the animals actual age at death through cementation analysis, the very old age of these teeth prevented preservation of any growth lines, which could establish the animals season of death. Although this particular study was not successful with this small sample of very old teeth, sites with much younger deer incisors would probably yield a greater potential to establish the season of death, and therefore contribute towards establishing the season of occupation. I support the use of this technique as a means to address ages of deer and seasonality of cultural occupations. 


\subsubsection{Pollen Analysis}

Pollen assessment (Appendix L) was initially implemented on three sediment samples and three ground stone fragments as a feasibility study. This was to determine pollen concentrations and potential to yield information concerning the paleoenvironment and potential foods processed by the artifacts during this early period. Initial results yielded low pollen concentrations and poor preservation, so further analysis would not be beneficial and continued pollen analysis was not recommended (Bozarth, Appendix L). Pollen from open sites is generally poorly preserved across most of Texas (e.g., Bryant 2012; Dering 1995; Dering and Bryant 2007; Holloway 1993, 2008), with the exception of studies directed at individual ground stone artifacts (e.g., Cummings 1991), or in wet or previously wet environments (e.g., Albert 2012; Bozarth and Woodburn 2010; Cummings 2002). Therefore, most pollen analysts do not recommend pollen analysis of sediments from open sites for environmental reconstruction (e.g., Bryant 2006; Dering 1995; Gorham 2000).

\subsubsection{Soil Chemistry}

Soil chemistry (Appendix M) was conducted to specifically address a question of chemical contents of dark stained soil from burned rock features, and to contribute pertinent data to address soil characteristics that contained the cultural materials. Two completely different types of soil laboratories were sent split sediment samples to determine the chemical construct of dark substance in the sediment. Both laboratories determined that carbon was a major constituent. This significant result allowed TRC archeologists to pursue radiocarbon dating this carbon based sediment to determine the age of the deposits in these components, where charcoal was poorly preservation.

The other soil chemistry conducted (texture analysis, percent carbonate, percent organic carbon, percent organic matter, $\mathrm{pH}$, and total phosphate) yielded useful information towards classifying the soils and determining their chemical characteristics. Given extensive geomorphological investigations towards soil and stratigraphic characterization from deeply buried sites 41TV410 and 41TV540 of similar age immediately adjacent the Big Hole site (Frederick 2011), similar efforts were limited here. Geomorphology combined with soil chemistry has been quite useful and important in establishing context and environmental and or depositional changes at stratified sites, as demonstrated many times across Texas (e.g., Abbott 1994; Collins 2003; Frederick 1996, 2010, 2011; Frederick and Bateman 2012; Goldberg and Holliday 1998; Mandel et al. 2007; Stafford 1981). Soil chemistry analyses is recommended as a regular component of testing and data recovery projects.

\subsection{CONCLUSIONS}

Data recovery specifically targeted a 60 to $70 \mathrm{~cm}$, vertically restricted zone in a deeply stratified site. This zone yielded two stratified, well-defined, rare middle Holocene components in great context with high integrity. The rapidly aggraded strata in this zone exhibited limited postdepositional disturbances, provided excellent time resolution and great data integrity for interpreting human behaviors in the two isolated components. Despite low frequency of formal chipped stone tools, generally poor preservation of microfossils, recovered data includes a rare vertebrate faunal assemblages and subsequent interdisciplinary technical analyses, significantly broadened our understanding of two separate and distinct huntergatherer groups. The latter groups hunted with Bell/Andice and Martindale projectiles while they pursued their lifeways during the fall season in the latter part of the Altithermal climate period.

These two components reflect individual, shortterm, well-defined seasonally restricted occupations. Such circumstances are quite rare and unlike most excavated sites across Texas. More often than not sites have yielded mixed and or 
palimpsests deposits on repeatedly occupied stable or slowly aggrading surfaces (e.g., Collins et al. 1998; Collins et al. 2003; Decker et al. 2000; Houk et al. 2008; Johnson 1991, 1995, 1997; Mehalchick and Kibler 2008; see Mahoney et al. 2003 for a discussion of stable surface sites; Sorrow et al. 1967).

For one of the very few instances the Bell/Andice and Martindale projectile points are radiocarbon dated and documented to a specific time period. The earlier Martindale component is charcoal dated to only a few hundred years prior to the charcoal dated Bell/Andice component. This is not the existing perception by many Texas archeologists or what is in the current literature concerning the age of Martindale projectile points. A non-reworked and non-patinated Big Sandy point was also recovered from the Bell/Andice component to indicate these two projectile point types co-occur and were likely contemporaneous at ca. 5322 B.P. here in Texas. This is one of the few instances where a Big Sandy point in secure context is associated with a charcoal radiocarbon date.

These are some of the first insolated components to yielded well-defined cultural features for these two groups. Their presence, overall form, and subsequent technical analyses of their parts allows interpretations as to general site functions, specific cooking and heating technologies, and various human behaviors. Their horizontal distribution allows insights into camp structure.

The exceptional integrity of the two targeted components provides a rare opportunity to investigate human behaviors at isolated campsites. The clarity in the horizontal distribution of the two cultural assemblages reveals single isolated cooking features with discarded subsistence remains immediately next to them to imply individual work spaces. In some instances cooking rocks and the plant and animal remains cooked were discarded in selected dump areas. Dump areas document intentional cleaning and or maintenance of work spaces. Of interest is that these same identified human behaviors occurred in both components.

Interesting aspects concerning the recovered data include the actual preservation of faunal assemblages, and the identification of fish and birds and multiple other game in the Martindale assemblage. In support of the bird and fish faunal remains is the presence of a bird feather barbule and a fish scale attached to two separate edge-modified flakes. This fact testifies to the actual processing of these two resources and the tools used for that processing. One remarkable aspect of the faunal assemblage is the complete absence of bison bones during a time when bison are reportedly present. The very fragmented faunal assemblage is interpreted to indicate that bone grease extraction was conducted through stone boiling in both components at ca. 5300 B.P. The targeting of bone grease at this early age of ca. 5300 B.P. is significant and a new development for Texas and perhaps the Southern Plains. It is also significant that the targeted grease was extracted from bones of multiple small and diverse animal resources and not large animal bones such as bison. Bone grease extraction is a technology that might imply maximizing the resources available at a specific season. It may also indicate planning and preparation for the lean winter months ahead. The cooking of bones may also be related to preparations of stews or soups for immediate consumption or the grease may have been part of the preparation of food storage in the making of pemmican.

Documentation of grass and cheno-am seed use here at ca. 5300 B.P. is also an important discovery and quite unusual for an open air site. The charred cheno-am and grass seeds recovered is one of the earliest use of these seeds known in Texas. For one of the few times, seasonality was also identified for both components based on the faunal and floral remains recovered. The absence of bison remains combined with the presence of multiple plant 
remains documents these populations employed a broad diet-breath. Based on the recovered cultural assemblages from each component it was argued that the Bell/Andice component represented a small female specialized task oriented group focused primarily on plant gathering, processing and cooking. This latter interpretation is in general conflict to what is reported in the existing literature for the Bell/Andice populations who are seen as bison hunters. Whereas the Martindale assemblage reflected a diverse hunter-gatherer group that conducted a broad range of hunting and camping activities.

Diverse technical analyses, specifically starch grain, phytolith, and lipid residues have brought to light plant gathering aspects of these huntergatherers that so often go undetected. Overall, diverse analyses combined with excellent context and integrity have enhanced a greater understanding of intrasite human behaviors researchers strive for with each and every excavation. Seasonality was also documented for both components for one of the few times in Texas.

Radiocarbon dates of multiple material classes in support of this work, again verifies previous radiocarbon dating results from multiple sites. Results clearly document bulk sediment ages and Rabdotus snail results overestimate the true age of the cultural occupations. Consequently, if and when charcoal or charred food resources (e.g., seeds, nuts, maize kernels, etc.) are recovered, they yield a much greater precision of the timing of cultural events, compared to other classes.

Future developments would probably not propose disturbances to penetrate depths greater than $2 \mathrm{~m}$ in alluvial settings. Therefore, deeply buried cultural remains such as documented at the Big Hole site, the Berdoll site, and sites 41TV410 and 41TV540, will continue to be infrequently encountered in development zones. Artz (1996) argues that depositional processes have buried many Altithermal sites beyond detection by conventional survey methods. Upland soil erosion in response to warmer climate conditions during the middle Holocene (e.g., Nordt 2004; Toomey 1993; Toomey et al. 1993) have contributed to widespread valley filling of low-order tributaries like Onion Creek (Nordt 2004). In contrast, many stratigraphic sequences in large streams across Kansas have gaps between ca. 7000 and 5000 B.P. due to floodplain erosion (Meltzer 2006), which was also the case along the Double Mountain Fork of the Brazos in northwest Texas (Blum et al. 1992). If prehistoric occupations greater than ca. 5,000 years are of interest to researchers, then they must consider probing and or testing deposits minimally $2 \mathrm{~m}$ deep. In contrast, if proposed developments anticipate disturbances greater than $2 \mathrm{~m}$ in alluvial deposits (e.g., oil and gas pipelines, water and sewer lines, culverts, dams, etc.), then testing and or assessing those depths should be a significant and specific part that targets the older cultural resources. At those depths, cultural materials encountered will probably be old, horizontally dispersed, probably exhibit good context, and because they are rarely encountered and in good context, will tend to yield significant contributions to Texas prehistory.

Deep alluvial deposits such as encountered at the Big Hole site, provide excellent conditions to encounter cultural materials in good context, contain significant cultural resources, which allow researchers greater interpretations of human behaviors. The Big Hole components, the Berdoll site (Karbula et al. 2011), the Holt site (Brownlow 2004), the Richard Beene site (Thoms and Mandel 2007), and sites similar to 41TV410 and 41TV540 (Figueroa et al. 2011) are just a few examples with deeply buried and early cultural remains in good context. Comparing the different approaches applied to the excavation strategies employed at these sites, the more horizontal approach instituted at the Big Hole site and the Richard Beene site resulted in a greater understanding of human behaviors and internal human patterns. The horizontal approach should be applied at future 
deeply buried sites. The horizontal approach has been argued for by Collins $(1995,2004)$ as the most appropriate means of revealing human behavior patterns and viewed as a positive development in Texas Archeology. In part, intracultural material patterns identified in each component resulted from larger, continuous block excavations in contrast to multiple smaller blocks in targeting cultural remains in tight context. High sedimentation rates and frequent flooding with low energy deposits contributed to the excellent integrity in these components.

The two largest blocks, B and D, investigated with $51 \mathrm{~m}^{2}$ and $74 \mathrm{~m}^{2}$ respectively, only yielded a total of four diagnostic projectile points. With the importance placed on recovering these specific diagnostic tools, it would be advantageous for future researchers to excavate much larger, continuous blocks to obtain sizable assemblages of diagnostic projectiles from single components. From previous investigations (e.g., Figueroa et al. 2011; Karbula et al. 2011; Quigg et al. 1996) it is apparent blocks smaller than $50 \mathrm{~m}^{2}$ would probably not yield diagnostic projectile points to enable assignment to cultural affiliations, and $50 \mathrm{~m}^{2}$ may not be sufficient as the $74 \mathrm{~m}^{2}$ excavated in the Martindale component yielded a single diagnostic point.

Macrobotanical analysis following flotation of feature sediments yielded very limited subsistence data, not only at these Big Hole components, but at many other early sites (e.g., Dering 1998, 2007, 2011b; Dering and Bryant 2007; Figueroa et al. 2011; Iruegas and Brownlow 2004; Quigg et al. 1996). As an example, 51 samples that totaled 551 liters from 11 sites at Fort Hood, yielded no carbonized subsistence remains (Dering 2000b). At the Wilson-Leonard site only 3 of the 76 flotation samples examined contained identifiable carbonized plant remains (Dering 1998:1634). To overcome poor preservation or unevenly preserved nature of the subsistence economy from most sites across Texas, this author reiterates and strongly supports statements made by Collins (1991:1). He stated, researches should "escalate our application of specialized analytical techniques" and the greatest effort should be directed towards the smaller scale data through microbotanical and microfossil analyses. Microfossil results from the Big Hole components clearly document the importance of these approaches. Even with generally poor preservation they added significant understanding to the employment of multiple plant resources.

This author strongly supports the use of multiple technological analyses, such as employed at the Big Hole components and other sites. These provide means to extract more specific and detailed information to maximize data return from the cultural material recovered from features, components, and sites. These data will contribute more towards interpreting the great diversity of human behaviors at specific points in time and changes over time for these nonrenewable resources. 


\subsection{MANAGEMENT CONSIDERATIONS AND RECOMMENDATIONS}

\section{J. Michael Quigg}

Based on the completion of hand-excavated volume of $38.5 \mathrm{~m}^{3}\left(150 \mathrm{~m}^{2}\right)$, the artifact and component descriptions, discussions, and interpretations presented in the previous chapters, TRC archeologists successfully mitigated the roadway impact to part of prehistoric site 41TV2161 and fulfilled the requirements under the Antiquities Code of Texas of 1977 (revised 2013), Title 9 Chapter 191 VACS, Art. 6145-9 and for Texas Antiquities Committee Permit No. 4064 plus Section 106 of the National Historic Preservation Act, the implementing regulations of 360CRF Part 800. No further recommendations concerning this impacted part of the Big Hole site are warranted. The excellent context and high integrity of diversified materials recovered provided significant data sets to address a wide-range of questions pertaining to human behaviors during the targeted middle Holocene period and address the stated hypothesis. The recovered data has contributed significant information to understanding a narrow and rare time window between 5200 and 5400 B.P. in Texas prehistory.
Although a portion of the Big Hole site within the right-of-way was successfully mitigated, it is this author's belief that the site extends beyond the existing roadway APE. Consequently, it is important that any further land disturbing development(s), which includes any additional roadway right-of-way in the immediate vicinity of 41TV2161, be required to conduct cultural resource assessment through an interdisciplinary approach of these deep deposits to insure more cultural deposits are not in those proposed development zones.

This section of the lower Onion Creek valley contains the deep alluvial deposits, which contain multiple cultural resource sites and components significant to Texas prehistory. Sites that illustrate the importance of the Onion Creek valley for prehistoric occupations include the Berdoll site (Karbula et al. 2011), sites 41TV420 and 41TV540 (Figueroa et al. 2011), the Big Hole site, and others. Consequently, it is recommended that future proposed developments in this general area and along other parts of the Onion Creek valley, be carefully reviewed to protect this very rich nonrenewable cultural resource area from future impacts. 
This page intentionally left blank. 


\subsection{REFERENCES}

Abbott, J. T.

1994 Chapter 12: Geomorphic Context of the Barton Site (41HY202) and the Mustang Branch Site (41HY209). In Archaic and Late Prehistoric Human Ecology in the Middle Onion Creek Valley, Hays County, Texas-Volume 2: Topical Studies, by R. A. Ricklis and M. B. Collins, pp. 353-379. Studies in Archeology, No. 19. Texas Archeological Research Laboratory, The University of Texas at Austin.

Abbott, J. and W. N. Trierweiler (editors)

1995 NRHP Significance Testing of 57 Prehistoric Archaeological Sites on Fort Hood, Texas. United States Army Fort Hood, Archeological Resource Management Series, Research Report No. 34.

Acuna, L. I.

2006 The Economic Contribution of Root Foods and Other Geophytes in Prehistoric Texas. Unpublished Master's thesis, Texas State University-San Marcos.

2010 Appendix C: Plant Remains from Site 41CV389, Fort Hood, Coryell County, Texas. In Data Recovery Investigations on the Cowdog Crossing Site: A Study of the End of the Archaic, Fort Hood, Coryell County, Texas, by S. Carpenter, C. T. Hartnett, J. D. Lowe, and K. A. Miller, pp. 143-154. United States Army Fort Hood, Archeological Resource Management Series, Research Report No. 56.

Adams, L. E.

1958 Archeological Investigations of Southwestern Missouri. The Missouri Archeologist 20.
Agogino, G. A. and W. O. Frankforter

1960 A Pale-Indian Bison Kill in Northwestern Iowa. American Antiquity 25(3):414-415.

Agvise Labs

2007 Soil Organic Matter: A Choice of Methods. Electronic document, http://www.agvise.com/tech_art/om.php, accessed May 16, 2007.

Albert, B. M.

2007 Holocene Environmental Change around the Buckeye Knoll Site: The Palynological Evidence. In Archaeology and Bioarchaeology of the Buckeye Knoll Site, 41VT98, A Major Locus of HunterGatherer Occupation and an Early Archaic Cemetery Near the Lower Guadalupe River, Victoria County, Texas, R. A. Ricklis, compiler, pp. 1281-1340. Report submitted to the U.S. Army Corps of Engineers, Galveston District. Coastal Environments, Inc., Corpus Christi.

2012 Appendix B: Pollen Analysis. In Archaeology and Bioarchaeology of the Buckeye Knoll Site (41VT98), Victoria County, Texas, Volume 3 (Appendices), edited by R. A. Ricklis, R. A. Weinstein and D. G. Wells, pp. 779-822. Prepared by Coastal Environments, Inc., Corpus Christi, for U.S. Army Corps of Engineers, Galveston District.

Albert, L. E.

1981 Ferndale Bog and Natural Lake: Five Thousand Years of Environmental Change in Southeastern Oklahoma. Oklahoma Archeological Survey, Studies in Oklahoma's Past 7:iii-99, Norman.

Albert, L. E. and D. G. Wyckoff

1984 Chapter 1: Oklahoma Environments: Past and Present. In Prehistory of Oklahoma, 
edited by R. E. Bell, pp. 1-43. Academic Press, Inc., Orlando.

Alford, J. J.

1973 The American Bison: An Ice Age Survivor. Proceedings of the Association of American Geographers 5:1-6.

Anderson, D. C. and R. Shutler, Jr.

1978 The Cherokee Sewer Site (13CK405): A Summary and Assessment. Plains Anthropologist 23(82):132-139, Part 2: Memoir 14: Bison Procurement and Utilization: A Symposium, edited by L. B. Davis and M. Wilson.

Andrefsky, W., Jr.

1994 Raw Material Availability and the Organization of Technology. American Antiquity 59(1):21-34.

2005 Lithics: Macroscopic Approaches to Analysis, Second Edition. Cambridge Manuals in Archaeology. Cambridge University Press, Cambridge.

Andrews, B.

1999 Regional Variation in Calf Creek Projectile Points from Oklahoma. Bulletin of the Oklahoma Anthropological Society Volume XLVIII:113-124.

Antevs, E.

1955 Geologic-Climate Dating in the West. American Antiquity 20(4):317-335.

Arbogast, A. F. and D. R. Muhs

2000 Geochemical and Mineralogical Evidence from Eolian Sediments for Northwesterly Mid-Holocene Paleowinds from Central Kansas, USA. Quaternary International 67:107-118.
Artz, J. A.

1996 Cultural Response or Geological Process? A Comment on Sheehan. Plains Anthropologist 41(158):383-393.

Ayala, S.

2014a Technology and Typology of the Calf Creek Horizon. Paper presented at the $79^{\text {th }}$ Annual Meeting, Society of American Archaeology, Austin.

2014b Technology and Typology of the Calf Creek Horizon. Paper presented at the $72^{\text {nd }}$ Annual Meeting of the Plains Anthropological Society, Fayetteville.

Baker, B. W.

1998 Chapter 33: Vertebrate Faunal Remains from the 1/4-inch and 1/8-inch Screens. In Wilson-Leonard: An 11,000-year Archeological Record of Hunter Gatherers in Central Texas. Volume V: Special Studies, assembled and edited by M. B. Collins, pp. 1463-1509. Studies in Archeology 31, Texas Archeological Research Laboratory, The University of Texas at Austin and Archeological Studies Program, Report 10, Texas Department of Transportation, Environmental Affairs Division.

Baker, B. W. and D. Gentry Steele

2007 Chapter 11: Late Pleistocene through Late Holocene Faunal Assemblage. In Archaeological and Paleoecological Investigations at the Richard Beene Site, South-Central Texas, Volume II: Archaeological Studies, Synthesis, and Appendixes, edited by A. V. Thoms and R. D. Mandel, editors, pp. 229-239. Reports of Investigations No. 8, Center for Ecological Archaeology, Texas A\&M University, College Station. 
Baker, V. M. and M. M. Penteado-Orellana

1977 Adjustment to Quaternary Climatic Change by the Colorado River of Central Texas. Journal of Geology 85(4):395-422.

1979 Fluvial Sedimentation Conditioned by Quaternary Climatic Change in Central Texas. Journal of Sedimentary Petrology 48(2):433-451.

Balinsky, R.

1998 Chapter 35: Pleistocene to Holocene Wilson-Leonard Microvertebrate Fauna and its Paleoenvironmental Significance. In Wilson-Leonard: An 11,000-year Archeological Record of Hunter Gatherers in Central Texas, Volume V: Special Studies, assembled and edited by M. B. Collins, pp. 1515-1542. Studies in Archeology 31, Texas Archeological Research Laboratory, The University of Texas at Austin and Archeological Studies Program, Report No. 10, Texas Department of Transportation, Environmental Affairs Division.

Bamforth, D. B.

1987 Historical Documents and Bison Ecology on the Great Plains. Plains Anthropologist 32(115):1-16.

Bamforth, D. B., G. Burns, and C. Woodman 1990 Ambiguous Use Traces and Blind Test Results: New Data. Journal of Archaeological Science 17:413-430.

Banks, L. D.

1984 Chapter 3: Lithic Resources and Quarries. In Prehistory of Oklahoma, edited by R. E. Bell, pp. 65-95. Academic Press, Inc., Orlando.

1990 From Mountain Peaks to Alligator Stomachs: A Review of Lithic Sources in the Trans-Mississippi South, the Southern Plains, and Adjacent Southwest. Oklahoma Anthropological Society, Memoir \#4, Norman.

Banks, W. E. and P. E. Wigand

2005 Reassessment of Radiocarbon Age Determinations for the Munkers Creek Phase. Plains Anthropologist 50(194):173183.

Barnes, V. E.

1974 Geological Atlas of Texas, Austin Sheet. Frances Luther Whitney, Memorial edition, Bureau of Economic Geology, The University of Texas at Austin.

1981 Geological Atlas of Texas, Austin Sheet. Frances Luther Whitney, Memorial edition, Bureau of Economic Geology, The University of Texas at Austin.

Bartlett, R.

1994 The Calf Creek Component at the Stilman Pit Site (34MR71) and its Relation to Calf Creek Caching Strategy. Bulletin of the Oklahoma Anthropological Society XL:6990.

Barrett, J. W.

2012 Appendix D: Lithic Use-Wear Analysis. In Archaeology and Bioarchaeology of the Buckeye Knoll Site (41VT98), Victoria County, Texas, edited by R. A. Ricklis, R. A. Weinstein and D. G. Wells, pp. 8871059. Prepared by Coastal Environments, Inc., Corpus Christi, for U. S. Army Corps of Engineers, Galveston District.

Bell, R. E.

1960 Guide to the Identification of Certain American Indian Projectile Points. Special Bulletin No. 2 of the Oklahoma Anthropological Society. 
Bell, R. E., editor

1984 Prehistory of Oklahoma. Academic Press, Inc., Orlando.

Bement, L. C.

1994 Hunter-Gatherer Mortuary Practices during the Central Texas Archaic. University of Texas Press, Austin.

Bement, L. C. and Kristen Carlson

2014 Bison across the Holocene: What Did Calf Creek Foragers Hunt? Paper presented at the Society for American Archaeology, $79^{\text {th }}$ Annual Meeting, Austin.

Bement, L. C., E. L. Lundelius Jr. and R. A. Ketchum

2004 Get the Point? Point of No Return, Driving Home the Point, A Data Package from the Arkansas River, Pointing out the Obvious, A Pointed Comment, Point Taken, Oklahoma Archeological Survey Newsletter 23 (4):1-3.

2005 Hoax or History: A Bison Skull with Embedded Calf Creek Projectile Point. Plains Anthropologist 50(195):221-226.

Bement, L. C., B. J. Carter, R. A. Varney, L. S. Cummings, and J. B. Sudbury

2007 Paleo-environmental Reconstruction and Bio-stratigraphy, Oklahoma Panhandle, USA. Quaternary International 169170:39-50.

Bender, M. M.

1971 Variations in the C13/C12 Ratios of Plants in Relation to the Pathway of Photosynthetic Carbon Dioxide Fixation. Phytochemistry 10:1239-1244.

Benedict, J. B.

1979 Getting Away from it All: A Study of Man, Mountains, and the Two-Drought
Altithermal. Southwestern Lore 45(3):112.

Binford L. R.

1978 Nunamiut Ethnoarchaeology. Academic Press, Inc., New York.

1980 Willow Smoke and Dog's Tails: Hunter Gatherer Settlement Systems and Archaeological Site Formation Processes. American Antiquity 45(1):4-20.

Black, S. L.

1997 Chapter 6: The Honey Creek Site, 41MS32. In Hot Rock Cooking on the Greater Edwards Plateau: Four Burned Rock Midden Sites in West Central Texas, Volume 1, by S. L. Black, L. W. Ellis, D. G. Creel, and G. T. Goode, pp. 99-167. Texas Archeological Research Laboratory, The University of Texas at Austin, Studies in Archeology 22, and Texas Department of Transportation, Environmental Affairs Department, Archeology Studies Program, Report 2.

2003 Chapter 8: Archaic Palo Real. In Pavo Real (41BX52): A Paleoindian and Archaic Camp and Workshop on the Balcones Escarpment, South-Central Texas, by M. B. Collins, D. B. Hudler, and S. L. Black, pp. 191-232. Studies in Archeology 41, Texas Archeological Research Laboratory, The University of Texas at Austin and Archeology Studies Program, Report 50. Environmental Affairs Division, Texas Department of Transportation.

Black, S. L. and A. J. McGraw

1985 The Panther Springs Creek Site: Cultural Change and Continuity within the Upper Salado Creek Watershed, South-Central, Texas. Archaeological Survey Report, No. 
100, Center for Archaeological Research, The University of Texas at San Antonio.

Black, S. L. and D. Creel

1997 Chapter 11: The Central Texas Burned Rock Midden Reconsidered. In Hot Rock Cooking on the Greater Edwards Plateau: Four Burned Rock Midden Sites in West Central Texas, Volume 1, by S. L. Black, L. W. Ellis, D. G. Creel, and G. T. Goode, pp. 269-305. Texas Archeological Research Laboratory, The University of Texas at Austin, Studies in Archeology 22, and Texas Department of Transportation, Environmental Affairs Department, Archeology Studies Program, Report 2.

Black, S. L. and A. V. Thoms

2014 Hunter-gatherer Earth Ovens in the Archeological Record: Fundamental Concepts. American Antiquity 79(2):203226.

Black, S. L., L. W. Ellis, D. G. Creel, and G. L. Goode

1997 Hot Rock Cooking on the Greater Edwards Plateau: Four Burned rocks Midden Sites in West Central Texas, Volumes 1 and 2, Texas Archeological Research Laboratory, The University of Texas at Austin, Studies in Archeology 22, and Texas Department of Transportation, Environmental Affairs Department, Archeology Studies Program, Report 2.

Black, S. L., K. Jolly, C. D. Frederick, J. R. Lucas, J. W. Karbula, P. R. Takac, and D. R. Potter

1998 Archeology along the Wurzbach Parkway: Module 3, Investigations and Experimentation at the Higgins Site (41BX184), Volume I. Studies in Archeology 27, Texas Archeological Research Laboratory, The University of Texas at Austin.
Blackmar, J. M. and J. H. Hofman

2006 Chapter 4: The Paleoarchaic of Kansas. In Kansas Archaeology, edited by R. J. Hoard and W. E. Banks, pp. 46-75. University Press of Kansas, Lawrence.

Blair, W. F.

1950 The Biotic Provinces of Texas. Texas Journal of Science 2(1):93-117.

Blum, M. D.

1987 Late Quaternary Sedimentation by the Upper Pedernales River, Central Texas. Unpublished Master's thesis, The University of Texas at Austin.

1992 Modern Depositional Environments and Recent Alluvial History of the Colorado River, Gulf Coastal Plain of Texas. Unpublished Ph.D. dissertation, The University of Texas at Austin.

Blum, M. D. and C. Lintz

1993 Chapter 5.2: Late Quaternary Geology in the Reservoir Basin. In Cultural Resource Investigations in the O. H. Ivie Reservoir, Concho, Coleman, and Runnels Counties, Texas, Volume I: Project Introduction, Setting, and Methods, by C. Lintz, W. N. Trierweiler, A. C. Earls, F. M. Oglesby, M. Blum, P. L. O’Neill, J. Kuhl, R. Holloway, L. Scott-Cummings, and D. Scurlock, pp. 280-314. Mariah Associates, Inc., Technical Report No. 346-1.

Blum, M. D. and S. Valastro Jr.

1989 Response of the Pedernales River of Central Texas to Late Holocene Climate Change. Annals of the Association of American Geographers 79(3):435-456.

1992 Quaternary Stratigraphy and Geoarchaeology of the Colorado and 
Concho Rivers, West Texas. Geoarchaeology, 7(5):419-448.

1994 Late Quaternary Sedimentation, Lower Colorado River, Gulf Coastal Plain of Texas. Geological Society of America Bulletin 106(8):1002-1016.

Blum, M. D., J. T. Abbott and S. Valastro Jr.

1992 Evolution of Landscapes on the Double Mountain Fork of the Brazos River, West Texas: Implications for Preservation and Visibility of the Archaeological Record. Geoarchaeology 7(4):339-370.

Blum, M. D., R. S. Toomey III, and S. Valastro Jr. 1994 Fluvial Response to Late Quaternary Climatic and Environmental Change, Edwards Plateau, Texas. Palaeogeography, Palaeoclimatology, and Palaeoecology 108(1\&2):1-21.

Bohrer, V.

1987 The Plant Remains from La Ciudad, A Hohokam Site in Phoenix. In Specialized Studies in the Economy, Environment and Culture of La Ciudad, edited by J. A. E. Kisselburg, G. E. Rice, and B. Spears, pp. 67-202. Office of Cultural Resource Management, Department of Anthropology, Arizona State University, Tempe.

Bond, C.

1978 Three Archeological Sites at Hoxie Bridge, Williamson County, Texas. Report No. 43, Anthropology Laboratory, Texas A\&M University, College Station.

Boulanger, M. T. and M. D. Glascock

2012 Appendix N: Analysis of Edwards Formation Chert and the Llano River Gravels and 41KM69. In Archeological Testing and Data Recovery at the Flatrock
Road Site, 41KM69, Kimble County, Texas, by J. L. Thompson, R. P. Mauldin, S. A. Tomka, and E. Oksanen, pp. 487-499. Texas Department of Transportation, Environmental Affairs Division, Archeological Studies Program, Report No. 133, Austin and Center for Archaeological Research, The University of Texas at San Antonio, Archaeological Report, No. 419.

2014 Chert Sourcing for the Big Hole (41TV2161) Project, Compositional Analyses of Chert Gravels and Artifacts. Report on file with TRC Environmental Corporation, Austin.

Bousman, C. B.

1998 Paleoenvironmental Change in Central Texas: The Palynological Evidence. Plains Anthropologist 43(164):201-219.

Bousman, C. B., B. W. Baker, and A. C. Kerr 2004 Chapter 2: Paleoindian Archeology in Texas. In The Prehistory of Texas, edited by T. K. Perttula, pp. 15-97. Texas A\&M University Press, College Station.

Boyd, D. K., C. W. Ringstaff, and G. Mehalchick 2004a Chapter 8: Analysis and Interpretations of the Cultural Occupations at the Firebreak Site. In Shifting Sands and Geophytes: Geoarcheological Investigations at Paluxy Sites on Fort Hood, Texas, by G. Mehalchick, D. K. Boyd, K. W. Kibler, and C. H. Ringstaff, pp. 129-198. United States Army Fort Hood, Archeological Resource Management Series, Research Report No. 48.

Boyd, D. K., C. W. Ringstaff, and G. Mehalchick 2004b Chapter 9: Rethinking Paluxy Site Archeology. In Shifting Sands and Geophytes: Geoarcheological 
Investigations at Paluxy Sites on Fort Hood, Texas, by G. Mehalchick, D. K. Boyd, K. W. Kibler, and C. H. Ringstaff, pp. 199-224. United States Army Fort Hood, Archeological Resource Management Series, Research Report No. 48.

Bozarth, S.

1986 Morphological Distinctive Phaseolus, Cucurbita and Helianthus annuns Phytoliths. In Plant Opal Phytolith Analysis in Archeology and Paleoecology: Proceeding of the 1984 Phytolith Research Workshop, edited by I. Rover, pp. 56-66. Occasional Papers No. 1, The Phytolitharien, North Carolina State University, Raleigh.

1987a Diagnostic Opal Phytoliths from Rinds of Selected Cucurbita Species. American Antiquity 52(3):607-615.

1987b Opal Phytolith Analysis of Edible Fruits and Nuts Native to the Central Plains. Phytolitharien Newsletter 4(3):9-10.

1993 Maize (Zea mays) Cob Phytoliths from a Central Kansas Great Bend Aspect Archaeological Site. Plains Anthropologist 38(146):279-286.

1995 Fossil Biosilicates. In Stratigraphy and Paleoenvironments of Late Quaternary Valley Fills on the Southern High Plains, by V. T. Holliday, pp. 47-50. Geological Society of America, Inc., Memoir 186, Boulder.

Bozarth, S. and T. Woodburn

2010 Appendix D: Paleoenvironmental Reconstruction at West Amarillo Creek, Potter County, Texas, Based on Biosilicate Analysis and Palynology. In Landis
Property: Data Recovery at Three Prehistoric Sites (41PT185, 41PT186, and 41PT245) in Potter County, Texas, Volume II: Appendices, by J. M. Quigg, C. D. Frederick, P. M. Matchen and K. G. DuBois, pp. 695-733. TRC Technical Report 150832. Manuscript on file with TRC in Austin and Bureau of Land Management, Santa Fe.

2011 Appendix F: Paleoenvironmental Reconstruction at 41TV410 (Area-3) based on Biosilicate, Charred Phytolith, and Particulate Charcoal Analysis. In Results of Archeological Significance Testing at $41 T V 410$ and $41 T V 540$ and Associated Geomorphological Investigations on a Segment of Onion Creek in Travis County, Texas, by A. Figueroa, R. Mauldin, J. C. Fredrick, S. A. Tomka, and J. L. Thompson, pp. 211-236. Texas Department of Transportation, Environmental Affairs Division, Archeological Studies Program, Report No. 134, Austin and Center for Archaeological Research, The University of Texas at San Antonio, Archaeological Report No. 420.

Brackenridge, H. M.

1904 Journal of the Voyage Up the Missouri River Performed in Eighteen Hundred and Eleven. In Early Western Travels, 17481846, edited by R. G. Thwaites, 6:19-166. A. H. Clark, Cleveland.

Brady, R. G.

1989 Geology of the Quaternary Dune Sands in Eastern Major and Southern Alfalfa Counties, Oklahoma. Unpublished Ph.D. dissertation, Graduate College, Oklahoma State University, Stillwater. 
Brink, J., M. Wright, B. Dawe, and D. Gaum

1985 Final Report of the 1983 Season at HeadSmashed-in Buffalo Jump, Alberta. Archaeological Survey of Alberta, Manuscript Series No. 1. Alberta Culture, Historical Resources Division, Edmonton.

Brock, F., D. G. Froese, and R. G. Roberts

2010 Low Temperature (LT) Combustion of Sediments does not Necessarily Provide Accurate Radiocarbon Ages for Site Chronology. Quaternary Geochronology 5(6):625-630.

Brooks, R. L.

1995 Trying to Scrape Up Some Answers: An Analysis of Scraping Tools from a Calf Creek Assemblage at the Hunter Site, 34GT6. Bulletin of the Oklahoma Anthropological Society XLII:53-88.

Brownlow, R. K.

2004 Data Recovery Investigations at the Holt Site (41YHY341), San Marcos, Hays County, Texas. HJN 040032 AR, Prepared for Fairfield Residential, LLC, by Horizon Environmental Services, Inc., Austin.

Brune, G.

1981 Springs of Texas. Volume I. Brance-smith, Inc., Fort Worth.

Bryant, V. M., Jr.

1977 A 16,000 Year Old Pollen Record of Vegetation Change in Central Texas. Palynology 1:143-155.

2006 Appendix B: Pollen Analysis of Bastrop Soil Samples from 41BP627. In The McKinney Roughs Site (41BP627): A Stratified Late Archaic II Site on the Colorado River Terraces, Bastrop County, Texas, by S. Carpenter, M. Chavez, K. Miller, and K. Lawrence, pp. B-1 through
B-9. SWCA Cultural Resources Report No. 02-313, SWCA Environmental Consultants, Austin.

2012 Appendix I: Pollen and Phytolith Extraction from Archeological Sediments in Area 1 of Site 41KM69, Kimble County, Texas. In Archeological Testing and Data Recovery at the Flatrock Road Site, 41KM69, Kimble County, Texas, by J. L. Thompson, R. P. Mauldin, S. A. Tomka, and E. Oksanen, pp. 417-428. Texas Department of Transportation, Environmental Affairs Division, Archeological Studies Program, Report No. 133, Austin and Center for Archaeological Research, The University of Texas at San Antonio, Archaeological Report, No. 419.

Bryant, V. M., Jr. and R. G. Holloway

1985 A Late Quaternary Paleoenvironmental Record of Texas: An Overview of the Pollen evidence. In Pollen Records of Late Quaternary North America Sediments, edited by V. M. Bryant, Jr. and R. G. Holloway, pp. 36-70. American Association of Stratigraphic Palynologist Foundation, Dallas.

Bryant, V. M., Jr. and J. Schoenwetter

1987 Chapter 5: Pollen Records from Lubbock Lake. In Lubbock Lake: Late Quaternary Studies on the Southern High Plains, edited by E. Johnson, pp. 36-40. Texas A\&M University Press, College Station.

Buckner, A. P.

1980 Cultural Responses to Altithermal (Atlantic) Climate Along the Eastern Margins of the North American Grasslands 5500 to 3000 B.C. National Museum of Canada, National Museum of Man, 
Mercury Series, Archaeological Survey of Canada, Paper No. 97, Ottawa.

Bureau of Economic Geology

1974 Geologic Atlas of Texas, Austin Sheet. The Bureau of Economic Geology, The University of Texas at Austin.

Butler, W. B.

1992 Bison Presence and Absence in Colorado. Southwestern Lore 58(3):114.

1997 Cultural and Climatic Patterns in the Faunal Record from Western Plains Archeological Sites. Southwestern Lore 63(4):1-36.

Butzer, K. W.

1982 Archaeology as Human Ecology: Method and Theory for a Contextual Approach. Cambridge University Press, United Kingdom.

Byrd, C. L.

1971 Origins and History of the Uvalde Gravel of Central Texas. Waco, Texas, Baylor University, Baylor Geological Studies Bulletin No. 20.

Calame, D. Sr., C. Weber, L. Banks, and R. McReynolds

2002 Projectile Points of the Calf Creek Horizon from Frio, Medina, and Uvalde Counties, Texas. La Tierra 29(2):29-38

Callahan, E.

1979 The Basics of Biface Knapping in the Eastern Fluted Point Tradition: A Manual for Flintknappers and Lithic Analysts. Archeology of Eastern North America 7(1):1-180.
Campbell, J. A., J. W. Karbula, and M. C. Stotts

2007 Archeological Investigations of Additional Properties in Segment B of the Proposed State Highway 130, Williamson and Travis Counties, Texas. Archeology Series No. 170. Hicks \& Company, Austin.

Campbell, J. A., S. C. Caran, J. W. Karbula, and B. King

2005 Results of Archeological Field Investigations at the Berdoll SH 130 Project Specific Location Along Onion Creek, Austin, Texas, Travis County, Texas. Hicks \& Company, Austin.

Campbell, J. A., M. C. Stotts, S. C. Caran, and B. Jones

2006 Results of Survey Investigations of SH 130 Schematic Right-of-way in segment $B$, Segment 4, Section 14 Parcel 365 within Site 41TV2161. Unpublished report on file with Environmental Affairs Division, Texas Department of Transportation, Austin.

Campbell, J. A., J. W. Karbula, B. M. Jones, and M. C. Stotts

2010 The Berdoll Site, 41TV2125, A Transitional Late Paleoindian - Early Archaic Camp in the Lower Onion Creek Basin, Travis County, Texas. Archeology Series No. 186, Hicks and Company, Austin.

Campbell, T. N.

1948 The Merrell Site: Archaeological Remains Associated with Alluvial Terrace Deposits in Central Texas. Bulletin of the Texas Archeological and Paleontological Society 19:7-35.

Caran, S. C.

1998a Quaternary Paleoenvironmental and Paleoclimate Reconstruction: A Discussion and Critique, with Examples from the 
Southern High Plains. Plains Anthropologist 43(164):111-124.

1998b Chapter 3: Quaternary stratigraphy and geomorphology of the property acquisition area. In Archeological Testing for the New Austin-Bergstrom International Airport in the Lower Onion Creek Drainage of Travis County, edited by J. C. Lohse and S. Ireguas, p. 7-32. Hicks \& Company Archeology Series 50, Hicks \& Company, Austin.

1999 Quaternary Stratigraphy and Geomorphology of Town Lake Civic Center Project Area, Austin, Travis County, Texas. In Cultural Resource Survey of the Town Lake Civic Center, Austin, Texas, Travis County, Texas, by M. F. Godwin and S. C. Caran. Antiquities Planning and Consulting, Kyle.

2003 Quaternary Stratigraphy and Geomorphology of the Proposed New City Hall Project, Construction Complex, Blocks 2 and 4B, Austin, Travis County, Texas. In Boarding Houses, Bar Rooms and Brothels - Life in a Vice-District. Archeological Investigations of $A$ Changing Neighborhood in Austin, Texas, by R. Feit, J. Karbula, J. Clark, S. C. Caran, J. A. Moreman, T. Griffith, and J. Jarvis, pp. 3-22. Hicks \& Company, Archeology Series 104, Hicks \& Company, Austin.

2009 Project Environment and Geomorphology. In The Berdoll Site, A Transitional Late A Paleoindian-Early Archaic Camp in the Lower Onion Creek Basin, Travis County, Texas, by J. A. Campbell, J. W. Karbula, B. M. Jones, and M. C. Stotts, pp. 7-14. Archeology Series No. 186. Hicks \& Company, Austin.
Caran, S. C., D. G. Robinson, K. L. Scott, H. H. Eling, Jr., and D. K Utley

1992 Archeological and Geomorphological Investigations at Two LCRA River Access Sites on the Colorado River, Colorado and Fayette Counties, Texas. Texas Archeological Research Laboratory Technical Series 26, The University of Texas at Austin.

Carlson, G. F., J. R. Bozell, T. L. Steinacher, M. B. Lovvorn, and G. W. Gill

1999 The Sidney Burial: A Middle Plains Archaic Mortuary Site from Western Nebraska. Plains Anthropologist 44(168):105-119.

Cassells, E. S.

1997 The Archaeology of Colorado. Johnson Books, Boulder.

Casteel, R.

1976 Fish Remains in Archaeology and Paleoenvironmental Studies. Academic Press, Inc., New York.

Castetter, E. F.

1935 Uncultivated Native Plants Used as Sources of Food. University of New Mexico Bulletin 266. Albuquerque.

Castetter, E. and W. Bell

1951 Yuman Indian Agriculture. University of New Mexico Press. Albuquerque.

Cestaro, G. C. and M. H. Carrell

1994 The Hester/Adams Site: 34ML83, McClain County, Oklahoma. Bulletin of the Oklahoma Anthropological Society XL:107-130. 
Chandler, C. K.

1983 Notes on Some Bell Points from San Patricio County, Texas. La Tierra 10(2):710.

Chandler, C. K. and D. Kumpe

1993 Stemmed Points with Massive Barbs from Lower Rio Grande Valley. La Tierra 20(3):26-28.

Chapman J. and P. J. Watson

1993 The Archaic Period and the Flotation Revolution. In Foraging and Farming in the Eastern Woodlands, edited by C. M. Scary, pp. 27-38. University Press of Florida, Gainesville.

Charters, S., R. P. Evershed, A. Quye, P. W. Blinkhorn and V. Denham

1997 Simulation Experiments for Determining the Use of Ancient Pottery Vessels: The Behavior of Epicuticular Leaf Wax during Boiling of a Leafy Vegetable. Journal of Archaeological Science 24:1-7.

Clark, J. S., E. C. Grimm, J. J. Donovan, S. C. Fritx, D. R. Engstrom, and J. E. Almerndinger

2002 Drought Cycles and Landscape Responses to Past Aridity on Prairies of the Northern Great Plains, USA. Ecology 83(3):595601.

Cogwell, J. W., H. Neff, and M. D. Glascock

2004 Chapter XIV, Chemical Variation in Northeast Texas Ceramics. In The Oak Hill Village Site (41RK214), Ruck County, Texas, by R. Rogers and T. K. Perttula, pp. 307-321. PBS\&J, Document No. 030083, PBS\&J Job No. 15152, Austin.

Collins, M. B., Assembler and Editor

1998a Wilson-Leonard, An 11,000-year Archeological Record of Hunter-Gatherers in Central Texas,5 Volumes. Studies in
Archeology 31, Texas Archeological Research Laboratory, The University of Texas at Austin and Archeology Studies Program, Report 10, Texas Department of Transportation, Environmental Affairs Division.

Collins, M. B.

1991 Thoughts on Future Investigation of Burned Rock Middens. In The Burned Rock Middens of Texas: An Archeological Symposium, edited by T. R. Hester, pp. 124. Studies in Archeology 13, Texas Archeological Research Laboratory, The University of Texas at Austin.

1994a Chapter 5: Evidence of Early Archaic Occupation. In Archaic and Late Prehistoric Human Ecology in the Middle Onion Creek Valley, Hays County, Texas, Volume 1: Archeological Components, by R. A. Ricklis and M. B. Collins, pp. 67100. Studies in Archeology 19, Texas Archeological Research Laboratory, The University of Texas at Austin.

1994b Chapter 17: Chronometric Investigations. In Archaic and Late Prehistoric Human Ecology in the Middle Onion Creek Valley, Hays County, Texas, Volume 2, Topical Studies, by R. A. Ricklis and M. B. Collins, pp. 499-505. Studies in Archeology 19, Texas Archeological Research Laboratory, The University of Texas at Austin.

1995 Forty Years of Archeology in Central Texas. Bulletin of the Texas Archeological Society 66:361-400.

1998b Chapter 11: The Place of Wilson-Leonard in Southern Plains Prehistory. In WilsonLeonard, An 11,000-year Archeological Record of Hunter-Gatherers in Central Texas, Volume I: Introduction, 
Background, and Syntheses, assembled and edited by M. B. Collins, pp. 277-291. Studies in Archeology 31, Texas Archeological Research Laboratory, The University of Texas at Austin and Archeology Studies Program, Report 10, Texas Department of Transportation, Environmental Affairs Division.

2002 The Gault Site, Texas, and Clovis Research. Athena Review 3(2):31-41.

2003 Chapter 4: Quaternary Geology of the Pavo Real Locality. In Pavo Real (41BX52): A Paleoindian and Archaic Camp and Workshop on the Balcones Escarpment, South-Central Texas, by M. B. Collins, D. B. Hudler, and S. L. Black, pp. 39-60. Studies in Archeology 41, Texas Archeological Research Laboratory, The University of Texas at Austin and Archeology Studies Program, Report 50. Environmental Affairs Division, Texas Department of Transportation.

2004 Chapter 3: Archeology in Central Texas. In The Prehistory of Texas, edited by T. K. Perttula, pp. 101-126. Texas A\&M University Press, College Station.

2011 Heated Rocks as Evidence of Prehistoric Hunter-Gatherer Adaptations in Texas. Bulletin of the Texas Archeological Society 82:181-191.

Collins, M. B. and T. R. Hester

1968 A Wooden Mortar and Pestle from Val Verde County, Texas. Bulletin of the Texas Archeological Society 39:1-8.

Collins, M. B. and P. Headrick

1990 Fluorescent Properties of "Edwards" and Related Cherts of Central Texas. Manuscript in possession of the authors.
Collins, M. B. and R. A. Ricklis

1994 Chapter 9: Conclusions. In Archaic and Late Prehistoric Human Ecology in the Middle Onion Creek Valley, Hays County, Texas, Volume 1, Archeological Components, by R. A. Ricklis and M. B. Collins, pp. 317-328. Studies in Archeology 19, Texas Archeological Research Laboratory, The University of Texas at Austin.

Collins, M. B., B. Ellis, and C. Dodt-Ellis

1990 Excavations at the Camp Pearl Wheat Site (41KR243): An Early Archaic Campsite on Town Creek, Kerr County, Texas. Studies in Archeology 6, Texas Archeological Research Laboratory, The University of Texas at Austin.

Collins, M. B., J. Guy, and S. W. Dial

1998 Chapter 9: The Archaic Period, 8800 To 1300 B.P. In Wilson-Leonard, An 11,000year Archeological Record of HunterGatherers in Central Texas, Volume I: Introduction, Background, and Syntheses, assembled and edited by M. B. Collins, pp. 211-270. Studies in Archeology 31, Texas Archeological Research Laboratory, The University of Texas at Austin and Archeology Studies Program, Report 10, Texas Department of Transportation, Environmental Affairs Division.

Collins, M. B., D. B. Hudler, and S. L. Black

2003 Pavo Real (41BX52): A Paleoindian and Archaic Camp and Workshop on the Balcones Escarpment, South-Central Texas. Studies in Archeology 41, Texas Archeological Research Laboratory, The University of Texas at Austin and Archeology Studies Program, Report 50. Environmental Affairs Division, Texas Department of Transportation. 
Conyers, L. B.

2004 Ground-penetrating Radar for Archaeology. AltaMira, Walnut Creek, California.

Conyers, L. B. and D. Goodman

1997 Ground-penetrating Radar: An Introduction for Archaeologists. AltaMira, Walnut Creek, California.

Cook, R. L.

1975 Learn About Whitetails. Revised and updated by H. G. Gore 1987. Texas Parks and Wildlife Magazine.

Coppedge B. R. and J. H. Shaw

1998 Bison Grazing Patterns on Seasonally Burned Tallgrass Prairie. Journal of Range Management 51:258-264

Cotterell, B. and J. Kamminga

1987 The Formation of Flakes. American Antiquity 52(4):675-708.

Coupland, R. T.

1958 The Effects of Fluctuations in Weather Upon the Grasslands of the Great Plains. The Botanical Review 24(5):273-317.

Cox, K. A.

1996 Surface Collections as Indicators of Change in Coastal Human ecology. La Tierra 23(1):24-42.

Crabtree, D. E.

1987 An Introduction to Flintworking. Occasional Papers No. 28. Idaho State Museum, Pocatello.

Crabtree, D. and R. Butler

1964 Notes on Experiment in Flintknapping: 1. Heat Treatment of Silica Materials. Tebiwa 7(1):1-6.
Cummings, L. S.

1991 Appendix B: Pollen, Phytolith, Macrofloral, and Charcoal Analyses in the Jewitt Mine Project Area, Texas. In Excavations at the Bottoms, Rena Branch, and Moccasin Springs Sites, Jewitt Mine Project, Freestone and Leon Counties, Texas, by R. C. Fields, L. W. Klement, C. B. Bousman, S. A. Tomka, E. F. Gadus, and M. A. Howard, pp. 329-362. Reports of Investigations, Number 82, Prewitt and Associates, Inc., Austin.

2002 Appendix G: Pollen and Phytolith Analysis for 41CW54. In Data Recovery at the Armstrong Site (41CW54) Caldwell County, Texas, Volume II, by E. A. Schroeder, pp. 145-159. Paul Price Associates, Inc., PPA Cultural Resources Report Number 330, Austin.

2004 Chapter 6: Great Basin Paleoethnobotany. In People and Plants in Ancient Western North America, edited by P. E. Minnis, pp. 205-277. The University of Arizona Press, Tucson.

Davis, W. B.

1974 The Mammals of Texas. Bulletin No. 61 Texas Parks and Wildlife Department, Austin, Reprinted in 1978.

Decker, S.

1998 Chapter 36: Eggshell Analysis. In WilsonLeonard, An 11,000-year Archeological Record of Hunter-Gatherers in Central Texas, Volume I: Introduction, Background, and Syntheses, assembled and edited by M. B. Collins, pp. 1543-1554. Studies in Archeology 31, Texas Archeological Research Laboratory, The University of Texas at Austin and Archeology Studies Program, Report 10, 
Texas Department of Transportation, Environmental Affairs Division.

Decker, S. S., S. L. Black, and T. Gustavson

2000 The Woodrow Heard Site (41UV88): A

Holocene Terrace Site in the Western

Balcones Canyonlands of Southwestern

Texas. Studies in Archeology 33, Texas

Archeological Research Laboratory, The

University of Texas at Austin and Texas

Department of Transportation,

Environmental Affairs Department,

Archeology Studies Program, Report 14.

Deevey, E. S. and R. F. Flint

1957 Postglacial Hypsithermal Interval. Science 125(3240):182-184.

Dempsey, H. A.

2001 Blackfoot. In Handbook of North American Indians: Plains, edited by R. J. DeMallie, 13(2):604-628. Smithsonian Institution, Washington.

Denniston, R. H.

1907 The Growth and Organization of the Development of Triticeae Starch Grains. Die Starke 34:109-111.

Dering, J. P.

1979 Pollen and Plant Macrofossil Record Recovered from Hinds Cave, Val Verde County, Texas. Unpublished Master's Thesis, Department of Biology, Texas A\&M University, College Station.

1995 Appendix G: Pollen and Phytolith Assessment. In The Rush Site (41TG346): A Stratified Late Prehistoric Locale in Tom Green County, Texas, by J. M. Quigg and J. Peck, pp. G-1. Mariah Associates, Inc., Technical Report No. 816c, Austin.
1997 Appendix D: Macrobotanical Remains. In Hot Rock Cooking on the Greater Edwards Plateau: Four Burned Rock Midden Sites in West Central Texas. Volume II, by S. L. Black, L. W. Ellis, D. G. Creel, and G. T. Goode, pp. 571-600. Texas Archeological Research Laboratory, The University of Texas at Austin, Studies in Archeology 22, and Texas Department of Transportation, Environmental Affairs Department, Archeology Studies Program, Report 2.

1998 Chapter 40: Carbonized Plant Remains. In Wilson-Leonard: An 11,000-year Archeological Record of Hunter Gatherers in Central Texas, Volume V: Special Studies, assembled and edited by M. B. Collins, pp. 1609-1636. Studies in Archeology 31, Texas Archeological Research Laboratory, The University of Texas at Austin and Archeological Studies Program, Report 10, Texas Department of Transportation, Environmental Affairs Division.

2000a Appendix II: Macrobotanical Remains from Soil Flotation Samples from the Bessie Kruze Site, with Thoughts about the Blackland Prairie. In Life and Death at the Bessie Kruze Site (41WM13) on the Blackland Prairie of Williamson County, Texas, by L. Johnson, pp. 213-220. Texas Department of Transportation, Environmental Affairs Division, Archeological Studies Program, Report No. 22.

2000b Appendix E: Analysis of Flotation and Macroplant Samples. In Geoarcheological Investigations and National Register Testing of 52 Prehistoric Sites on Fort Hood, Texas: The 1997 Season, by G. Mehalchick, K. Kleinbach, D. K. Boyd, and K. W. Kibler, pp. 319-325. United 
States Army Fort Hood, Archeological Resource Management Series, Research Report No. 48.

2003 Appendix C: Plant Remains from Rice's Crossing (41WM815). In Archeological Investigations at 41WM815, A Backland Prairie Site, Williamson County, Texas, by R. K. Brownlow, pp. 113-120. Studies in Archeology 36, Texas Archeological Research Laboratory, The University of Texas at Austin and Texas Department of Transportation, Environmental Affairs Division, Archeological Studies Program, Report 23, Austin.

2004 Appendix B: Analysis of Macrobotanical Remains form Three Paluxy Sites on Fort Hood, Texas. In Shifting Sands and Geophytes: Geoarcheological Investigations at Paluxy Sites on Fort Hood, Texas, by G. Mehalchick, D. K. Boyd, K. W. Kibler, and C. H. Ringstaff, pp. 245-257. United States Army Fort Hood, Archeological Resource Management Series, Research Report No. 48.

2006 Plant Remains. In Prehistoric Encampments at the Shepherd Site: Testing and Data Recovery at 41WM1010, Williamson County, Texas, by B. Dixon and R. Rogers, pp. 153-156. Prepared for Texas Department of Transportation, by PBS\&J, Austin.

2007 Assessment of Botanical and Faunal Assemblages from Paleoindian and Early Archaic Components on the Periphery of the Southern Plains. Bulletin of the Texas Archeological Society 78:177-195.

2011a Appendix A: Plant Remains form 41TV410 and 41TV540. In Results of
Archeological Significance Testing at $41 T V 410$ and $41 T V 540$ and Associated Geomorphological Investigations on a Segment of Onion Creek in Travis County, Texas, by A. Figueroa, R. Mauldin, C. Frederick, S. A. Tomka, and J. L. Thompson, pp. 159-164. Texas Department of Transportation, Environmental Affairs Division, Archeology Studies Program, Report No. 134, Austin and Center for Archaeological Research, University of Texas at San Antonio, Archaeological Report No. 420.

2011b Appendix E: Botanical Analysis of Flotation Samples from 41BX17. In Archeological Significance Testing at 41BX17/271, the Granberg Site: A MultiComponent Site along the Salado Creek in Bexar County, Texas, by C. M. Munoz, R. P. Mauldin, J. L. Thompson, and S. C. Caran, pp. 239-244. Texas Department of Transportation, Environmental Affairs Division, Archeology Studies Program, Report No. 140 and Center for Archaeological Research, The University of Texas at San Antonio, Archaeological Report No. 393.

Dering, J. P. and V. M. Bryant, Jr.

2007 Chapter 12: Plant Remains. In Archaeological and Paleoecological Investigations at the Richard Beene Site, South-Central Texas, Volume II: Archaeological Studies, Synthesis, and Appendixes, edited by A. V. Thoms and R. D. Mandel, pp. 241-249. Reports of Investigations No. 8, Center for Ecological Archaeology, Texas A\&M University, College Station.

Dial, S. W., A. C. Kerr, and M. B. Collins

1998 Chapter 13: Projectile Points. In WilsonLeonard, An 11,000-year Archeological 
Record of Hunter-Gatherers in Central Texas, Volume II: Chipped Stone Artifacts, assembled and edited by M. B. Collins, pp. 313-445. Studies in Archeology 31, Texas Archeological Research Laboratory, The University of Texas at Austin and Texas Department of Transportation, Environmental Affairs Division, Archeology Studies Program, Report No. 10.

Dibble, D. S.

1967 Excavations at Arenosa Shelter, 1965-66. Texas Archeological Salvage Project, The University of Texas at Austin.

1997 Excavations at Arenosa Shelter, 1965-66. Reprinted by the Texas Archeological Research Laboratory, The University of Texas at Austin.

Dickens, W. A.

1993 Lithic Analysis. In Archaeological Investigations in Bull Branch: Results of the 1990 Summer Archaeological Field School, edited by D. L. Carlson, pp. 79115. United States Army Fort Hood, Archeological Resource Management Series, Research Report Number 19.

Dickson, D. R.

1968 Two Provisional Projectile Point Types. The Arkansas Amateur 7(6):5-7, Fayetteville.

Diffenbaugh, N. S., M. Ashfaq, B. Shuman, J. W. Williams, and P. J. Bartlein

2006 Summer Aridity in the United States: Response to Mid-Holocene Changes in Insolation and Sea Surface Temperature. Geophysical Research Letters 33, L22712:1029.
Dillehay, T. D.

1974 Late Quaternary Bison Population Changes on the Southern Plains. Plains Anthropologist 19(65):180-196.

Denlinger, R. P., D. R. H. O'Connell, and P. K. House

2002 Robust Determination of Stage and Discharge: An Example from an Extreme Flood on the Verde River, Arizona. In Ancient Floods, Modern Hazards: Principles and Applications of Paleoflood Hydrology, edited by P. K. House, R. H. Webb, V. R. Baker, and D. R. Levish, pp.127-146. Water Science and Application Volume 5, The American Geophysical Union, Washington D.C.

Doebley, J. F.

1984 "Seeds" of Wild Grasses: A Major Food of Southwestern Indians. Economic Botany 38 (1):52-64.

Dockall, J. and C. D. Pevny

2007 Chapter 10: Lithic Assemblages: Transitions of Holocene Technological Organization. In Archaeological and Paleoecological Investigations at the Richard Beene Site, South-Central Texas, Volume II: Archaeological Studies, Synthesis, and Appendixes, edited by A. V. Thoms, and R. D. Mandel, pp. 177-228. Reports of Investigations No. 8, Center for Ecological Archaeology, Texas A\&M University, College Station.

Doran, G. H., D. N. Dickel, and L. A. Newsom

1990 A 7,290-Year-Old Bottle Gourd from the Windover Site, Florida. American Antiquity 55(2):354-359.

Driver, H. E.

1972 Indians of North America. The University of Chicago Press, Chicago. 
Driver, H. E. and W. C. Massey

1957 Comparative Studies of North American Indians. Vol. 47. Transactions of the American Philosophical Society, Philadelphia.

Duncan, $M$.

1994 The Williams' Orchard Site: An Analysis of Lithic Procurement. Bulletin of the Oklahoma Anthropological Society XL:91106.

1995 Calf Creek Foragers: Mobility on the Southern Plains during the Altithermal. Bulletin of the Oklahoma Anthropological Society XLII:89-144.

1996 Test Excavations at the Kubik Site, 34KA354, Oklahoma Anthropological Survey Newsletter 15(4):3.

2014 Lithic Heat-Treatment Experiments on the Southern Plains. Paper presented at the $72^{\text {nd }}$ Annual Meeting of the Plains Anthropological Society, Fayetteville.

Duncan, M. and D. G. Wyckoff

1994 The McKellips Site: An Analysis of the Calf Creek Component. Bulletin of the Oklahoma Anthropological Society XL:257-276.

Dyck, I. G.

1977 The Harder Site: A Middle Period Bison Hunting's Campsite in the Northern Great Plains. National Museum of Man, Mercury Series, Archaeological Survey of Canada, Paper No. 67.

Eerkens, J. W.

2002 The Preservation and Identification of Pinon Resins by GC-MS in Pottery from the Western Great Basin. Archaeometry 44(1):95-105.
Ellis, L. W.

1997 Chapter 3: Hot Rock Technology. In Hot Rock Cooking on the Greater Edwards Plateau: Four Burned Rock Midden Sites in West Central Texas, Volume 1, by S. L. Black, L. W. Ellis, D. G. Creel, and G. T. Goode, pp. 43-81. Studies in Archeology 22, Texas Archeological Research Laboratory, University of Texas at Austin. Archeology Studies Program, Report 2, Texas Department of Transportation, Environmental Affairs Department, Austin.

Ellis, G. L. and G. A. Goodfriend

1994 Chapter 7: Chronometric and SiteFormation Studies Using Land Snail Shells: Preliminary Results. In Archeological Investigations on 571 Prehistoric Sites at Fort Hood, Bell and Coryell Counties, Texas, edited by W. N. Trierweiler, pp. 183-202. United States Army Fort Hood, Archeological Resource Management Series, Research Report No. 31.

Ellis, G. L., G. A. Goodfriend, J. T. Abbott, P. E. Hare, and D. W. Von Endt

1996 Assessment of Integrity and Geochronology of Archeological Sites Using Amino Acid Racemization in Land Snails: Examples from Central Texas. Geoarchaeology 11(3):189-213.

Evans, G. L.

1951 Prehistoric Water Wells in Eastern New Mexico. American Antiquity 17(1):1-9.

Evershed, R. P.

1993 Biomolecular Archaeology and Lipids. World Archaeology 25(1):74-93.

2000 Biomoecular Analysis by Organic Mass Spectrometry. In Modern Analytical 
Methods in Art and Archaeology, Chemical Analysis Series, Vol. 155, edited by E. Ciliberto and G. Spoto, pp. 177239. Wiley, New York.

Evershed, R. P., C. Heron and L. J. Goad

1991 Epicuticular Wax Components Preserved in Potsherds as Chemical Indicators of Leafy Vegetables in Ancient Diets. Antiquity 65:540-544.

Evershed, R. P., H. R. Mottram, S. N. Dudd, S. Charters, A. W. Stott, G. J. Lawrence, A. M. Gibson, A. Conner, P. W. Blinkhorn and V. Reeves 1997a New Criteria for the Identification of Animal Fats in Archaeological Pottery. Naturwissenschaften 84:402-406.

Evershed, R. P., S. J. Vaugh, S. N. Dudd and J. S. Soles

1997b Fuel for Thought? Beeswax in Lamps and Conical Cups from Late Minoan Crete. Antiquity 71:979-985.

Evershed, R. P., S. N. Dudd, M. J. Lockheart and S. Jim

2001 Lipids in Archaeology. In Handbook of Archaeological Sciences, edited by D. R. Brothwell and A. M. Pollard, pp. 331-350. Wiley, Chichester.

Ewers, J.

1982 The Blackfoot Raiders on the Northwestern Plains. Reprint from 1958. Oklahoma University Press, Norman.

Feder, K. L.

2008 A Brief Introduction to Archaeology. Second edition. Oxford University Press, New York.

Fenneman, N. M.

1931 Physiography of Western United States. McGraw-Hill, New York.
Ferguson, J. R. and M. D. Glascock

2011 Appendix D: Instrumental Neutron Activation Analysis of Caddo Pottery from Ten Sites in Eastern Texas. In Archeological Investigations at the Lang Pasture Site (41AN38) in the Upper Neches River Basin of East Texas, assembled and edited by T. K. Perttula, D. B. Kelley and R. A. Ricklis, pp. 591-612. Texas Department of Transportation, Archeological Studies Program, Report No. 129, Environmental Affairs Division, and TRC Environmental Corporation, Technical Report No. 174202, Austin.

2012 Appendix M: Instrumental Neutron Activation Analysis of Toyah Phase Ceramics from 41KM69, Kimble County, Texas, and Results of Scanning Electron Microscopy of Sherds from 41KM69, Kimble County, Texas. In Archeological Testing and Data Recovery at the Flatrock Road Site, 41KM69, Kimble County, Texas, by J. L. Thompson, R. P. Mauldin, S. A. Tomka, and E. Oksanen, pp. 469-483. Texas Department of Transportation, Environmental Affairs Division, Archeological Studies Program, Report No. 133, Austin and Center for Archaeological Research, The University of Texas at San Antonio, Archaeological Report, No. 419.

Ferring C. R.

2001 The Archaeology and Paleoecology of the Aubrey Clovis Site (41DN479), Denton County, Texas. Center for Environmental Archaeology, Department of Geology, University of North Texas, Denton.

Ferring C. R. and B. C. Yates

1997 Archaeological Investigations at Five Prehistoric Sites at Lewisville Lake, Denton County, Texas. Center for 
Environmental Archaeology, University of North Texas, Denton.

Figueroa, A., R. Mauldin, J. C. Fredrick, S. A. Tomka, and J. L. Thompson

2011 Results of Archeological Significance

Testing at 41TV410 and $41 T V 540$ and Associated Geomorphological Investigations on a Segment of Onion Creek in Travis County, Texas. Texas Department of Transportation, Environmental Affairs Division, Archeological Studies Program, Report No. 134, Austin and Center for Archaeological Research, The University of Texas at San Antonio, Archaeological Report No. 420.

Fisher, J. W. Jr.

1995 Bone Surface Modifications in Zooarchaeology. Journal of Archaeological Methods and Theory 2(1):7-68.

Fowler, L. and R. Flannery

2001 Gros Ventre. In Handbook of North American Indians: Plains, edited by R. J. DeMallie, 13 (2):677-694. Smithsonian Institution, Washington.

Forman, S. L., R. Oglesby and R. S. Webb

2001 Temporal and Spatial Patterns of Holocene Dune Activity on the Great Plains of North America: Megadroughts and Climate Links. Global and Planetary Change 29:129.

Foster, S. and R. C. Hobbs

2002 A Western Medicinal Plants and Herbs. The Peterson Field Guide Series, Houghton Miffton Company, Boston.
Fox, A. A. and T. R. Hester

1976 An Archaeological Survey of Coleto Creek, Victoria and Goliad Counties, Texas. The University of Texas at San Antonio, Center for Archaeological Research, Archaeological Survey Report, No. 18.

Frederick, C. D.

1987 A Paleoenvironmental Interpretation of the Austin Mastodon Site. Unpublished M.A. Thesis, The University of Texas at Austin, Austin.

1996 Chapter 5: Geomorphic Investigations. In Early Archaic Use of the Concho River Terraces: Archaeological Investigations at $41 T G 307$ and 41TG309, Tom Green County, Texas, by J. M. Quigg, J. Peck, C. Lintz, A. C. Treece, C. D. Frederick, R. Clem, G. L. Ellis, P. Schuchert, and J. T. Abbott, pp. 85-108. Technical Report Number 11058, TRC-Mariah Associates, Inc., Austin.

2006 Chapter 6: Geomorphology and Geoarchaeology. In The McKinney Roughs Site 41BP627: A Stratified Late Archaic II Site on the Colorado River Terraces, Bastrop County, Texas, by S. Carpenter, M. Chavez, K. Miller, and K. Lawrence and with contributions by $\mathrm{C}$. Frederick, C. Crawford, V. M. Bryant, R. Howells, and A. Malof, pp. 51-64. Cultural Resources Report No. 02-313. SWCA Environmental Consultants, Austin.

2010 Chapter 6.0, Stratigraphic Overview of Upper West Amarillo Creek Valley. In Landis Property: Data Recovery at Three Prehistoric Sites (41PT185, 41PT186, and 41PT245) in Potter County, Texas, Volume I, by J. M. Quigg, C. D. Frederick, P. M. Matchen, and K. DuBois, pp. 151-163. TRC Technical Report No. 150832. Report 
on file with TRC Austin and Bureau of Land Management, Santa Fe.

2011 Chapter 8: Late Quaternary Alluvial Stratigraphy of the Lower Onion Creek Valley. In Results of Archeological Significance Testing at $41 T V 410$ and $41 T V 540$ and Associated Geomorphological Investigations on a Segment of Onion Creek in Travis County, Texas, by A. Figueroa, R. Mauldin, C. Frederick, S. A. Tomka, and J. L. Thompson, pp. 69-95. Texas Department of Transportation, Environmental Affairs Division, Archeological Studies Program, Report No. 134, Austin, and Center for Archaeological Research, The University of Texas at San Antonio, Archaeological Report No. 420.

Frederick, C. D. and C. Ringstaff

1994 Chapter 6: Lithic Resources at Fort Hood: Further Investigations. In Archaeological Investigations on 571 Prehistoric Sites at Fort Hood, Bell, and Coryell Counties, Texas, edited by W. N. Trierweiler, pp. 125-181. United States Army Fort Hood, Archeological Resource Management Series, Research Report No. 31.

Frederick, C. D. and M. D. Bateman

2012 Appendix A: Geoarcheological Investigations. In Archeology and Biology of the Buckeye Knoll Site (41VT98), Victoria County, Texas, Volume 3, edited by R. A. Ricklis, R. A. Weinstein, and D. C. Wells, pp. 731-777. Coastal Environments, Inc., Corpus Christi and U.S. Corps of Engineers, Galveston District.

Frederick, C., E. Schroeder and J. M. Quigg 2007 Chapter 3.0: Geoarcheological Evaluations. In Data Recovery at
41TV2161, Travis County, Texas: Interim Report. Interim Report No. 51831, TRC Environmental Corporation, Austin, pp. 24-33. Submitted to Environmental Affairs Division, Texas Department of Transportation, Austin.

Frederick, C. D., J. L. Thompson and K. J. Córdova 2008 A Geoarcheological Survey of the Proposed Plainview Hike and Bike Trail, Hale County, Texas. Environmental Affairs Division, Texas Department of Transportation, Austin, Texas and Center for Archaeological Research, The University of Texas at San Antonio Archaeological Report No. 396.

Frederick, C. D., M. D. Glascock, H. Neff and C. M. Stevenson

1994 Evaluation of Chert Patination as a Dating Technique: A Case Study from Fort Hood, Texas. Research Report No. 32, Archaeological Resource Management Series. United States Army, Fort Hood.

Fredlund, G.

1998 Chapter 41: Phytolith Analysis. In WilsonLeonard: An 11,000-year Archeological Record of Hunter Gatherers in Central Texas, Volume V: Special Studies, assembled and edited by M. B. Collins, pp. 1637-1651. Studies in Archeology 31, Texas Archeological Research Laboratory, The University of Texas at Austin and Archeological Studies Program, Report No. 10, Texas Department of Transportation, Environmental Affairs Division.

Fredlund, G. G. and L. L. Tieszen

1998 Chapter 42: Stable Carbon Isotope Analysis of Soil Organic Matter. In Wilson-Leonard An 11,000-year Archeological Record of Hunter-Gatherers 
in Central Texas, Volumes V: Special Studies, assembled and edited M. B. Collins, pp. 1653-1656. Studies in Archeology 31, Texas Archeological Research Laboratory, The University of Texas at Austin, and Archeology Studies Program, Report No. 10, Texas Department of Transportation, Environmental Affairs Division, Austin.

Fredlund, G. G., C. B. Bousman, and D. K. Boyd 1998 The Holocene Phytolith Record from Morgan Playa in the Rolling Plains of Texas. Plains Anthropologist 43(164):187-200.

Frison, G. C.

1978 Prehistoric Hunters of the High Plains. Academic Press, Inc., New York.

1991 Prehistoric Hunters of the High Plains. $2^{\text {nd }}$ edition, Academic Press, Inc., San Diego.

Frison, G. C., M. Wilson, and D. J. Wilson

1976 Fossil Bison and Artifacts from an Early Altithermal Period Arroyo Trap in Wyoming. American Antiquity 41(1):2857.

Fullagar, R., T. Loy, and S. Cox

1998 Starch Grains, Sediments, Stone Tool Function: Evidence form Bitikara, Papua New Guinea. In: A Closer Look: Australian Studies of Stone Tools, edited by R. Fullagar, pp. 49-60. University of Sydney Archaeological Computing Laboratory, Sydney.

Fullington, R. and K. Fullington

1982 Chapter 15: Invertebrate Faunal Analysis. In Archeological Investigations at the San Gabriel Reservoir District, Central Texas, Volume 2, compiled and edited by T. R. Hays, pp. $15-33$ to $15-56$. Archaeology
Program, Institute of Applied Sciences, North Texas State University, Denton.

Gee, K. L., J. H. Holman, M. K. Causey, A. N. Rossi, and J. B. Armstrong

2002 Aging White-tail Deer by Tooth Replacement and Wear: A Critical Evaluation of a Time- honored Technique. Wildlife Society Bulletin 30:387-393.

Geib, P. R. and E. A. Jolie

2008 The Role of Basketry in Early Holocene Small Seed Exploitation: Implications of a Ca. 9,000 Year-Old Basket from Cowboy Cave, Utah. American Antiquity 73(1):83102.

Geist, V.

1996 Buffalo Nation: History and Legend of the North American Bison. Voyageur Press, Stillwater.

Geist, V. and M. Frances

2001 Antelope Country, Pronghorns: The Last Americans. Krause Publications, Iola.

Girard, J. and H. S. Carr

1991 Archeological Investigations at the Bellcow Site (34LN29), Lincoln County, Oklahoma. Oklahoma Conservation Commission, Archaeological Research Report 15. Oklahoma City.

1994 Calf Creek Bifaces and Middle Archaic Period Occupations in the Bellcow Creek Drainage, Central Oklahoma. Bulletin of the Oklahoma Anthropological Society XL:195-208.

Glascock, M. D.

1995 A Report on the INAA Characterization of Source Specimens from the Segovia Formation (Site 41HW52, Howard County, Texas) of the Edwards Group Chert. In 
Cultural Resource Investigations of Lithic

Procurement Sites (41RG39, 41RG40 and 41HW52) in Howard and Reagan Counties, Texas, by C. A. Turnbow and D. P. Staley, pp. D-1 through D-16. Prepared for Cap Rock Electric Cooperation, Inc., by Mariah Associates, Inc., Albuquerque. Confidential Report.

Goldberg, P. and V. T. Holliday

1998 Chapter 6: Geology and Stratigraphy. In Wilson-Leonard, An 11,000-year Archeological Record of Hunter-Gatherers in Central Texas, 5 Volumes, assembler and editor M. B. Collins, pp. 77-121. Studies in Archeology 31, Texas Archeological Research Laboratory, The University of Texas at Austin and Texas Department of Transportation, Environmental Affairs Division, Archeology Studies Program, Report No. 10.

Goodfriend, G. A.

1987 Radiocarbon Age Anomalies in Shell Carbonate of Land Snails from Semi-arid Areas. Radiocarbon 29(2):159-167.

Gorham, L. D.

2000 Appendix H: Pollen Results. In Data Recovery at 41ZP364: An Upland Campsite at Falcon Reservoir, Zapata County, Texas, by J. M. Quigg and C. Cordova, pp. 281-283. TRC Technical Report No. 22317, TRC Mariah Associates Inc., Austin.

Gould, F. W.

1975 Texas Plants: A Checklist and Ecological Summary. The Texas A\&M University System, Texas Agricultural Experimental Station, College Station.
Grace, R.

1990 The Limitations and Applications of UseWear Analysis. In The Interpretive Possibilities of Microwear Analysis, edited by B. Graslund, H. Knutsson, K. Knutsson, and J. Taffinder, pp. 9-14. Societas Archaeologica Upsaliensis, Uppsala, AUN 14 , Sweden.

Green, D. E.

1962 Additional Notes on Prehistoric Water Wells at the Clovis Site. American Antiquity 28(2):230-234.

Gremillion, K. J.

2004 Seed Processing and the Origins of Food Production in Eastern North America. American Antiquity 69(2):215-233.

Grinnell, G. B.

1961 Pawnee, Blackfoot and Cheyenne. Charles Scribner's Sons, New York.

1972 The Cheyenne Indians, Vol.1. University of Nebraska Press, Lincoln.

Grueger, J.

1973 Studies on the Late Quaternary Vegetation History of Northeastern Kansas. Geological Society of America Bulletin 84(1):239-250.

Hall, S. A.

1988 Environment and Archaeology of the Central Osage Plains. Plains Anthropologist 33(120):203-218.

1995 Pollen. In Stratigraphy and Paleoenvironments of Late Quaternary Valley Fills on the Southern High Plains, by V. T. Holliday, pp. 53-54. Geological Society of America, Inc., Memoir 186, Boulder. 
2001 Chapter 6: Pollen Analysis of Late-Glacial Pond and Spring deposits, Aubrey Clovis Site, Denton County, Texas. In The Archaeology and Paleoecology of the Aubrey Clovis Site (41DN479), Denton County, Texas, by C. R. Ferring, pp. 79-88. Center for Environmental Archaeology, Department of Geology, University of North Texas, Denton.

Hardy, B. L.

2014 Appendix C: Residue and Use-Wear Analysis of Stone Artifacts, Lampasas County, Texas, Sites 41LM50 and 41LM51. In Eligibility Testing at Three Prehistoric Sites at Lynch Creek, Lampasas County, Texas, by J. M. Quigg, P. M. Matchen, R. A. Ricklis, S. Gray, C. D. Frederick, and J. Barrett, pp. 303-348. Texas Department of Transportation, Environmental Affairs Division, Archeological Studies Program Report No. 161 and TRC Environmental Corporation, Technical Report Nos. 43243 (106892), 18125, and 201313, Austin.

Harlow, R. and M. DeFoor

1962 How to Age White-Tail Deer. Florida Wildlife 16:18-21

Hayden, B. and W. Hutchings

1989 Whither the Billet Flake? In Experiments in Lithic Technology, edited by D. Amick and R. Mauldin, pp. 235-258. British Archaeological Reports, British Series 528, Oxford.

Hayden, B., N. Franco, and J. Spafford

1996 Evaluating Lithic Strategies and Design Criteria. In Stone Tools: Theoretical Insights into Human Prehistory, edited by G. Odell, pp. 9-45. Plenum Press, New York and London.
Haynes, C. V.

1967 Geochronology of Late-Quaternary Alluvium. In Means of Correlation of the Quaternary Successions, edited by R. B. Morrison and H. E. Wright. Proceedings VII Congress International Association for Quaternary Research 8:591-631.

1995 Geochronology of Paleoenvironmental Change, Clovis Type Site, Blackwater Draw, New Mexico. Geoarchaeology 10(5):317-188.

Heron, C. and A. M. Pollard

1988 The Analysis of Natural Resinous Materials from Roman Amphoras. In Science and Archaeology Glasgow 1987. Proceedings of a Conference on the Application of Scientific Techniques to Archaeology, Glasgow, 1987, edited by E. A. Slater and J. O. Tate, pp. 429-447. BAR British Series 196 (ii), Oxford.

Heron, C., N. Nemcek, K. M. Bonfield, J. Dixon and B. S. Ottaway

1994 The Chemistry of Neolithic Beeswax. Naturwissenschaften 81:266-269.

Hester, T. R.

1971 Archeological Investigations at the La Jita Site, Uvalde County, Texas. Bulletin of the Texas Archeological Society 42:51-148.

1980 Digging into South Texas Prehistory: A Guide for Amateur Archaeologists. Corona Publishing, San Antonio.

1982 Late Paleo-Indian Occupations at Baker Cave, Southwestern Texas. Bulletin of the Texas Archeological Society 53:101-119.

1990 Notes on South Texas Archeology: 1990-3: Early Archaic "Eccentric" Lithic Artifacts 
in Southern and Central Texas. La Tierra 17(3):1-5.

Hester, T. R. (editor)

1991 The Burned Rock Middens of Texas: An Archeological Symposium. Studies in Archeology 13, Texas Archeological Research Laboratory, The University of Texas at Austin.

Hester, T. R. and R. L. McReynolds

2003 A Look Back at the Contributions of C. K. Chandler to the Study of South Texas Archaeology. La Tierra 30(3 \& 4):5-14.

Hill, M. E. Jr., M. G. Hill, and C. C. Widga

2008 Late Quaternary Bison Diminution on the Great Plains of North America: Evaluating the Role of Human Hunting versus Climate Change. Quaternary Science Review 27(17):1752-1771.

Hill, R. T. and T. W. Vaughan

1897 Description of the Austin Quadrangle. U.S.G.S. Atlas Folio 76, United States Geological Survey, Washington D. C.

Hoard, R. J., W. E. Banks, R. D. Mandel, M. Finnegan, and J. E. Epperson

2004 A Middle Archaic Burial from East Central Kansas. American Antiquity 69(4):717739 .

Hofman, J. L.

1989 Chapter 4: Prehistoric Cultural History Hunters and Gatherers in the Southern Great Plains. In From Clovis to Comanchero: Archaeological Overview of the Southern Great Plains, by J. L. Hofman, R. L. Brooks, J. S. Hays, D. W. Owsley, R. L. Jantz, M. K. Marks, and M. H. Manheim, pp. 25-60. Arkansas Archaeological Survey Research Series 35, Fayetteville.
Hofman, J. L., L. C. Todd, and M. B. Collins 1991 Identification of Central Edwards Chert at the Folsom and Lindenmeier Sites. Plains Anthropologist 36(137):297-308.

Holliday, V. T.

1985 Archaeological Geology of the Lubbock Lake Site, Southern High Plains of Texas. Geological Society of American Bulletin 96(12):1483-1492.

1989 Middle Holocene Drought on the Southern High Plains. Quaternary Research 31:7482.

1995a Stratigraphy and Paleoenvironments of Late Quaternary Valley Fills on the Southern High Plains. Geological Society of America, Inc., Memoir 186, Boulder.

1995b Stable Isotopes. In Stratigraphy and Paleoenvironments of Late Quaternary Valley Fills on the Southern High Plains, by V. T. Holliday, pp. 54-58. Geological Society of America, Inc., Memoir 186, Boulder.

Holliday, V. T. and C. M. Welty

1981 Lithic Tool Resources of the Eastern Llano Estacado. Bulletin of the Texas Archeological Society 52:201-214.

Holloway, R. G.

1993 Appendix E: Pollen Results. In Cultural Resource Investigations in the O. H. Ivie Reservoir, Concho, Coleman, and Runnels Counties, Volume IIB: Appendices, by C. Lintz, A. C. Treece, F. M. Oglesby, K. Kibler, P. L. O’Neill, W. N. Trierweiler, C. Frederick, J. M. Quigg, and A. J. Taylor, pp. E-1 through E-35. Mariah Associates, Inc., Technical Report No. 346-IIB, Austin. 
2008 Appendix E: Pollen and Phytolith Analyses of Samples from the Varga Site, 41ED28, Edwards County, Texas. In The Varga Site: A Multicomponent, Stratified Campsite in the Canyonlands of Edwards County, Texas, by J. M. Quigg, J. D. Owens, P. M. Matchen, G. Smith, R. A. Ricklis, M. C. Cody, and C. D. Frederick, pp. 843-883. Texas Department of Transportation, Environmental Affairs Division, Archeological Studies Program Report No. 110 and TRC Environmental Corporation, Technical Report No. 35319, Austin.

Holloway, R. G., L. M. Raab, and R. Stuckenrath 1987 Pollen Analysis of Late-Holocene Sediments from a Central Texas Bog. The Texas Journal of Science 39(1):71-79.

Honea, K.

1980 Marks Beach, Stratified Paleoindian Site, Lamb County, Texas: Preliminary Report. Bulletin of the Texas Archeological Society 51:243-269.

Houk, B. A., K. A. Miller, and E. R. Oksanen

2008 The Gatlin Site (41KR621): Investigating Archaic Lifeways on the Southern Edwards Plateau of Central Texas. Texas Department of Transportation, Environmental Affairs Division, Archeological Studies Program, Report No. 108, and SWCA Environmental Consultants, SWCA Cultural Resources Report No. 2008-149, Austin.

House, P. K., P. A. Pearthree, and J. E. Klawon 2002 Historical Flood and Paleoflood Chronology of the Lower Verde River, Arizona: Stratigraphic Evidence and Related Uncertainties. In Ancient Floods, Modern Hazards: Principles and Applications of Paleoflood Hydrology, edited by P. K. House, R. H. Webb, V. R. Baker, and D. R. Levish, pp. 267-293. Water Science and Application Volume 5, The American Geophysical Union, Washington D.C.

Howells, R. G., R. W. Neck, and H. D. Murray

1996 Freshwater Mussels of Texas. Texas Parks and Wildlife Department, Inland Fisheries Division, Austin.

Hudler, Dale B.

2000 Modeling Paleolandscapes in Central Texas. Unpublished Ph.D. dissertation. Department of Anthropology, The University of Texas at Austin.

2003a Appendix 2: Use-Wear Analysis of Selected Pavo Real Artifacts. In Pavo Real (41BX52): A Paleoindian and Archaic Camp and Workshop on the Balcones Escarpment, South-Central Texas, by M. B. Collins, D. B. Hudler, and S. L. Black, pp. 253-266. Studies in Archeology 41, Texas Archeological Research Laboratory, The University of Texas at Austin and Archeology Studies Program, Report No. 50. Environmental Affairs Division, Texas Department of Transportation.

2003b Appendix 5: Instrumental Neutron Activation Analysis Results. In Pavo Real (41BX52): A Paleoindian and Archaic Camp and Workshop on the Balcones Escarpment, South-Central Texas, by M. B. Collins, D. B. Hudler, and S. L. Black, pp. 287-290. Studies in Archeology 41, Texas Archeological Research Laboratory, The University of Texas at Austin and Archeology Studies Program, Report No. 50. Environmental Affairs Division, Texas Department of Transportation. 
Hughes, D. T.

1984 Chapter 5: The Foragers: Western Oklahoma. In Prehistory of Oklahoma, edited by R. E. Bell, pp. 109-118. Academic Press, Inc., Orlando.

Hughes, S. S.

1991 Division of Labor at a Besant Hunting Camp in Eastern Montana. In Approaches to Gender Processes on the Great Plains. Plains Anthropologist, Memoir 26, edited by M. Kornfeld, 36(134):25-50.

Humphrey, J. and C. R. Ferring

1994 Stable Isotopic Evidence for Late Pleistocene to Holocene Climatic Change, North-Central Texas. Quaternary Research 41(2):200-213.

2001 Chapter 4: Late Quaternary Stable Isotope of the Aubrey Clovis Site. In The Archaeology and Paleoecology of the Aubrey Clovis Site (41DN479), Denton County, Texas, by C. R. Ferring, pp. 55-68. Center for Environmental Archaeology, Department of Geology, University of North Texas, Denton.

Iruegas, M. T. and R. Brownlow

2004 Chapter 9.0: Plant Remains Recovered from 41HY341. In Data Recovery Investigations at the Holt Site (41YHY341), San Marcos, Hays County, Texas, by R. K. Brownlow, pp. 94-105. HJN 040032 AR, Prepared for Fairfield Residential, LLC, by Horizon Environmental Services, Inc., Austin.

Jackson, M. A.

1998 The Nature of Fire-cracked Rock: New Insights from Ethnoarchaeological and Laboratory Experiments. Unpublished Master's thesis, Texas A\&M University, College Station.
Jefferies, R.

1997 Middle Archaic Bone Pins: Evidence of Mid-Holocene Regional-Scale Social Groups in the Southern Midwest. American Antiquity 62(3):464-487.

Jensen, W.

1998 Aging Antelope: It's all in the Teeth. North Dakota Outdoors 61(2):16-20.

Jochim, M. A.

1981 Strategies for Survival: Cultural Behavior in an Ecological Context. Academic Press, Inc., New York.

Johnson, E.

1987a Chapter 7: Vertebrate Remains. In Lubbock Lake: Late Quaternary Studies on the Southern High Plains, edited by E. Johnson, pp. 49-89. Texas A\&M University Press, College Station.

1987b Chapter 8: Paleoenvironmental Overview. In Lubbock Lake: Late Quaternary Studies on the Southern High Plains, edited by E. Johnson, pp. 90-99. Texas A\&M University Press, College Station.

Johnson, E. and V. T. Holliday

1986 The Archaic Record at Lubbock Lake. Plains Anthropologist 31(114) Part 2:7-54. Memoir 21.

1995 Archeology and Late Quaternary Environments of the Southern High Plains. Bulletin of the Texas Archeological Society 66:519-540.

2004 Chapter 9: Archeology and Late Quaternary Environments of the Southern High Plains. In The Prehistory of Texas, edited by T. K. Perttula, pp. 283-295. Texas A\&M University Press, College Station. 
Johnson, L. Jr.

1964 The Devil's Mouth Site: A Stratified Campsite at Amsted Reservoir, Val Verde County, Texas. Archeology Series, Number 6, Department of Anthropology, The University of Texas, Austin.

1967 Toward a Statistical Overview of the Archaic Cultures of Central and Southwestern Texas. Bulletin of the Memorial Museum, Number 12. The University of Texas at Austin.

1991 Early Archaic Life at the Sleeper Archaeological Site (41BC65) of the Texas Hill Country, Blanco County, Texas. Texas State Department of Highways and Public Transportation, Highway Division, Publications in Archaeology, Report No. 39, Austin.

1995 Past Cultures and Climates at the Jonas Terrace Site, 41ME29 of Medina County, Texas. Office of the State Archeologist Report 40. Texas Department of Transportation and Texas Historical Commission, Austin.

1997 The Lion Creek Site (41BT105): Aboriginal Houses and Other Remains at a Prehistoric Rancheria in the Texas Hill County (Burnet County). Texas Department of Transportation, Environmental Affairs Division, Archeology Studies Program, Report 1, and Texas Historical Commission, Office of the State Archeologist, Report 41, Austin.

Johnson, L. Jr. and G. T. Goode

1994 A New Try at Dating and Characterizing Holocene Climates, as Well as Archeological Periods, on the Eastern
Edwards Plateau. Bulletin of the Texas Archeological Society 65:1-51

Johnson, W. C. and C. W. Martin

2010 Holocene Alluvial-stratigraphic Studies from Kansas and adjoining States of the East-central, Plains. Electronic document, http://www.kgs.ku.edu/Publications/Bullet ins/GB5/Johnson/index.html, accessed December 172014.

Jones, S. R. and R. C. Cushman

2004 A Field Guide to the North American Prairie. The Peterson Field Guide Series, Houghton Mifflin Company, Boston.

Karbula, J. W.

2000 Investigations of the Eckols Site (41TV528): A Stratified Prehistoric Terrace Site on Barton Creek in Travis County, Texas. Unpublished Ph.D. dissertation, The University of Texas at Austin.

Karbula, J. W., J. A. Campbell, and B. M. Jones

2011 The Berdoll Site: An Early Archaic Camp on Onion Creek, Travis County, Texas. Bulletin of the Texas Archeological Society 82:135-173.

Kay, M.

1998 Chapter 6: The Central and Southern Plains Archaic. In Archeology on the Great Plains, edited by W. R. Wood, pp. 173200. University Press of Kansas, Lawrence.

Kelly, R. L. and D. H. Thomas

2013 Archaeology. Sixth Edition. Wadsworth, Cengage Learning, Boston. 
Kenberg, M.

1956 Some Important Projectile Point Types found in the Tennessee Area. Tennessee Archaeologist, Volume XII(1):17-28.

Kenmotsu, R.

1982 Chapter 3: Environmental Setting. In Archeological Investigations at the San Gabriel Reservoir Districts, Central Texas, Volume 1, compiled and edited by T. R. Hays, pp. 3-1 to 3-23. Institute of Applied Sciences, North Texas State University, Denton.

Kerr, A. C. and S. W. Dial

1998 Chapter 14: Statistical Analysis of Unfluted Lanceolate and Early Bifurcate Stem Projectile Points. In Wilson-Leonard, An 11,000-year Archeological Record of Hunter-Gatherers in Central Texas, Volume II: Chipped Stone Artifacts, assembled and edited by M. B. Collins, pp. 447-505. Studies in Archeology 31, Texas Archeological Research Laboratory, The University of Texas at Austin and Texas Department of Transportation, Environmental Affairs Division, Archeology Studies Program, Report No. 10.

Kibler K. W. and A. M. Scott

2000 Archaic Hunters and Gatherers or the Balcones Canyon Lands: Data Recovery at the Cibolo Crossing Site (41BX377), Camp Bullis Military Reservation, Bexar County, Texas. Reports of Investigations, Number 126, Prewitt and Associates, Inc., Austin.

Kindscher, K.

1987 Edible Wild Plants of the Prairie. University Press of Kansas, Lawrence.
Kivett, M. F.

1962a Logan Creek Complex. Paper presented at the Twentieth Plains Anthropological Conference at Lincoln.

1962a Archeological Field Report: 1961. Plains Anthropologist 7(16):77.

Knox, J. C.

1983 Chapter 3: Responses of River Systems to Holocene Climates. In Late Quaternary Environments of the United States, Volume 2, The Holocene, edited by $\mathrm{H}$. E. Wright, Jr., pp. 26-41. University of Minnesota, Minneapolis.

Kooyman, B. P.

2002 Understanding Stone Tools and Archaeological Sites. University of New Mexico Press, Albuquerque.

Kraft, K. C.

1997 The Distribution of Alibates Silicified Dolomite Clasts along the Canadian River. Current Research in the Pleistocene 14:106-109.

Krieger, A. D. (editor)

2002 We Come Naked and Barefoot: The Journey of Cabeza de Vaca across North America. The University of Texas Press, Austin.

Kwiatkowski, S.

1992 The Rye Creek Flotation and Macrobotanical Analyses. In The Rye Creek Project: Archaeology in the Upper Tonto Basin. Volume 2: Artifact and Specific Analyses, pp. 325-375. Anthropological Papers No. 11. Center for Desert Archeology, Tucson. 
Laird, K. R., S. C. Fritz, E. C. Grimm, and P. G. Mueller

1996 Century-scale Paleoclimate Reconstruction from Moon Lake, A Closed-basin Lake in the Northern Great Plains. American Society of Limnology and Oceanography, Inc., 41(5):890-902.

Larson, D. A., V. M. Bryant, and T. S. Patty

1972 Pollen Analysis of a Central Texas Bog. The American Midland Naturalist 88(2):358-367.

Lee, R. B. and I. DeVore

1968 Man the Hunter. Aldine, Chicago.

Leechman, D.

1951 Bone Grease. American Antiquity 16(4):355-356.

Lewis, H. T.

1973 Patterns of Indian Burning in California: Ecology and Ethnohistory. Ballena Press, Ramona.

Lewis, P. J., E. Johnson, B. Buchanan, and S. E. Churchill

2007 The Evolution of Bison bison: A View from the Southern Plains. Bulletin of the Texas Archeological Society 78:197-204.

Lintz, C., W. N. Trierweiler, A. C. Earls, F. M. Oglesby, M. Blum, P. L. O'Neill, J. Kuhl, R. Holloway, L. Scott-Cummings, and D. Scurlock

1993 Cultural Resource Investigations in the O. H. Ivie Reservoir, Concho, Coleman, and Runnels Counties, Texas-Volume I: Project Introduction, Setting and Methods. Technical Report No. 346-I, Mariah Associates, Inc., Austin.
Lohse, J. C., B. J. Culleton, and D. J. Kennett

2014a Dating Calf Creek Bison in Texas. Paper presented at the Society of American Archeology $79^{\text {th }}$ Annual Meeting, Austin.

Lohse, J. C., S. L. Black, and L. M. Cholak

2014c Towards an Improved Archaic Radiocarbon Chronology for Central Texas. Bulletin of the Texas Archeological Society 85:243-279.

Lohse, J. C., A. E. Reid, D. M. Yelacic, and C. L. Timperley

2013 Data Recovery and Analysis at the Texas State University Ticket Kiosk Project, Located at 41HY160, Spring Lake, Hays County, Texas. Archaeological Studies Report No. 32, Center for Archaeological Studies, Texas State University-San Marcos.

Lohse, J. C., D. B. Madsen, D. J. Kennett, and B. J. Culleton

2014b Isotope Paleoecology of Episodic Mid-toLate Holocene Bison Population Expansions in the Southern Great Plains. Quaternary Science Reviews 101(2014):113, Elsevier Ltd.

Looney, R. M.

1977 Late Quaternary Geomorphic Evolution of the Colorado River, Bastrop and Fayette Counties, Texas. Unpublished M. A. Thesis, The University of Texas at Austin, Austin.

Looney, R. M. and V. M. Baker

1977 Late Quaternary Geomorphic Evolution of the Colorado River, Inner Texas Coastal Plain. Transactions of the Gulf Coast Association of Geological Societies 27:323-333. 
Lowie, R. H.

1954 Indians of the Plains. American Museum Science Books, The Natural History Press, New York.

1963 The Crow Indians. University of Nebraska Press, Lincoln.

Lubinski, P. A.

2001 Observations on Seasonality and Mortality from a Recent Catastrophic Death Assemblage. Journal of Archaeological Science 28(8):833-842.

Lundelius, E. L., Jr.,

1992 The Avenue Local Fauna: Late Pleistocene Vertebrates from Terrace Deposits at Austin, Texas. Annals Zoologici Fennici 28:329-340.

MacMasters, M. M.

1964 Microscopic Techniques for Determining Starch Granule Properties. In Methods in Carbohydrate Chemistry, edited by R. L. Whistler, pp. 233-240. Academic Press, New York.

Malainey, M. E.

2000 Appendix K: Analysis of the Fatty Acid Compositions of Burned Rock Residues from Site 41ZP364, Zapata County, Texas. In Data Recovery at 41ZP364: An Upland Campsite at Falcon Reservoir, Zapata County, Texas, by J. M. Quigg and C. Cordova, pp. 331-352. Technical Report No. 22317, TRC Mariah Associates Inc., Austin.

2003 Appendix F: Analysis of the Fatty Acid Compositions of Archeological Residues from 41MM340. In Data Recovery Excavations at 41MM340: A Late Archaic Site along Little River in Milam County, Texas, by R. B. Mahoney, S. A. Tomka, R.
P. Mauldin, H. J. Shafer, L. C. Nordt, R. D. Graves, and R. R. Galdeano, pp. 235-244. Texas Department of Transportation, Environmental Affairs Division, Archeological Studies Program, Report No. 54, Austin, and The University of Texas at San Antonio, Center for Archaeological Research, Archaeological Survey Report No. 340, San Antonio.

Malainey, M. E. and K. L. Malisza

2004 Appendix C: Analysis of Fatty Acid Compositions of Archeological Residues from 41CV595. In Shifting Sands and Geophytes: Geoarcheological Investigations at Paluxy Sites on Fort Hood, Texas, by G. Mehalchick, D. K. Boyd, K. W. Kibler, and C. H. Ringstaff, pp. 259-270. United States Army Fort Hood, Archeological Resource Management Series, Research Report No. 48.

2008 Appendix G: Analysis of the Fatty Acid Compositions of Archaeological Pottery and Rock Residues from the Varga Site (41ED28). In The Varga Site: A Multicomponent, Stratified Campsite in the Canyonlands of Edwards County, Texas Volume II, by J. M. Quigg, J. D. Owens, P. M. Matchen, G. D. Smith, R. A. Ricklis, M. C. Cody, and C. D. Frederick, pp. 951-983. Texas Department of Transportation Environmental Affairs Division, Archeological Studies Program Report No. 110 and TRC Environmental Corporation, Austin, Technical Report No. 35319.

2011 Appendix B: Analysis of the Fatty Acid Composition of Archeological Burned Rock Samples from 41CV413, Central Texas. In Cultural Resource Investigations to Offset Mechanical Impacts to the Clear Creek Golf Course Site (41CV413), Fort 
Hood, Texas, by J. M. Quigg, C. Lintz, G. Smith, D. DeMar and J. D. Owens, pp. 169183. United States Army Fort Hood, Archeological Resource Management Series, Resource Report No. 60.

Malainey, M. E. and T. Figol

2010 Appendix G: Analysis of Lipids Extracted from Archaeological Burned Rock and Pottery Residues from Sites in Potter County, Texas. In Landis Property: Data Recovery at Three Prehistoric Sites (41PT185, 41PT186, and 41PT245) in Potter County, Texas, Volume II: Appendices, by J. M. Quigg, C. D. Frederick, P. M. Matchen, and K. DuBois, pp. 791-831. TRC Technical Report No. 150832. Manuscript on file with TRC, Austin and Bureau of Land Management, Santa Fe.

2011 Appendix H: Analysis of the Lipid Compositions of Archeological Burned Rock Residues from Site 41YN452. In Root-Be-Gone (41YN452): Data Recovery of Late Archaic Components in Young County, Texas, Volume II, by J. M. Quigg, P. M. Matchen, C. D. Frederick and R. A. Ricklis, pp. 579-604. Texas Department of Transportation, Environmental Affairs Division, Archeological Studies Program Report No. 135 and TRC Environmental Corporation, Technical Report Nos. 50907 and 171219, Austin.

2013 Appendix D: Analysis of the Lipid Compositions of Archeological Burned Rock Residues from Site 41MI96. In Data Recovery at $41 M 196$ in Mills County, Texas, by J. M. Quigg, R. A. Ricklis, P. M. Matchen, and J. T. Abbott, pp. 177-194. Texas Department of Transportation, Environmental Affairs Division,
Archeological Studies Program Report No. 150 and TRC Environmental Corporation, Technical Report No. 192832, Austin.

Mandel, R. D.

1992 Chapter 2: Soils and Holocene Landscape Evolution in Central and Southern Kansas: Implications for Archaeological Research. In Soils in Archaeology: Landscape Evolution and Human Occupation, edited by V. T. Holliday, pp. 41-100. Smithsonian Institute Press, Washington, D.C.

1995 Chapter 3: Geomorphic Controls of the Archaic Record in the Central Plains of the United States. In Archaeological Geology of the Archaic Period in North America, edited by E. A. Bettis III, pp. 37-66. Special Paper 297. Geological Society of America, Boulder.

2006 Chapter 3: The Effects of Late Quaternary Landscape Evolution on the Archaeological Record of Kansas. In Kansas Archaeology, edited by R. J. Hoard and W. E. Banks, pp. 28-45. Kansas State Historical Society by the University press of Kansas, Lawrence.

Mandel, R. D., J. S. Jacob, and L. C. Nordt

2007 Chapter 3: Geomorphic Investigations at the Richard Beene Site. In Archaeological and Paleoecological Investigations at the Richard Beene Site 41BX831: SouthCentral Texas, Volume I: Paleoecological Studies, Cultural Contexts, and Excavation Strategies, edited by A. V. Thoms and R. D. Mandel, pp. 27-60. Reports of Investigations No. 8. Center for Ecological Archaeology, Texas A\&M University, College Station. 
Mandelbaum, D. G.

1940 The Plains Cree. Anthropological Papers of the American Museum of Natural History, 37 (Part II):153-316. American Museum of Natural History, New York.

Martin, P. S.

1963 The Last 10,000 Years: A Fossil Pollen Record of the American Southwest. University of Arizona Press, Tucson.

Mason, M. A. and M. W. Holderby

1994 Chapter 15: Subsistence Patterns at 41HY209 and 41HY202: An Analysis of Vertebrate Faunal Remains. In Archaic and Late Prehistoric Human Ecology in the Middle Onion Creek Valley, Hays County, Texas, Volume 2: Topical Studies, by R. A. Ricklis and M. B. Collins, pp. 403-489. Studies in Archeology 19, Texas Archeological Research Laboratory, The University of Texas at Austin.

Mauldin, R. P. and D. S. Amick

1989 Investigating Patterning in Debitage from Experimental Bifacial Core Reduction. In Experiments in Lithic Technology, edited by D. S. Amick and R. P. Maudlin, pp. 6788. BAR International Series 528. British Archaeological Reports, Oxford.

Mauldin, R. P. and J. L. Thompson

2012 Chapter 9: Modeling Adaptions in the Late Archaic and Late Prehistoric Period. In Archeological Testing and Data Recovery at the Flatrock Road Site, 41KM69, Kimble County, Texas, by J. L. Thompson, R. P. Mauldin, S. A. Tomka, and E. Oksanen, pp. 87-107. Texas Department of Transportation, Environmental Affairs Division, Archeological Studies Program, Report No. 133, Austin and Center for Archaeological Research, The University of Texas at San Antonio, Archaeological Report, No. 419.

Mauldin, R., A. Figueroa, and S. Tomka

2011a Chapter 10: Site Use and Function at 41TV540. In Results of Archeological Significance Testing at $41 T V 410$ and $41 T V 540$ and Associated Geomorphological Investigations on a Segment of Onion Creek in Travis County, Texas, by A. Figueroa, R. Mauldin, J. C. Fredrick, S. A. Tomka, and J. L. Thompson, pp. 109-135. Texas Department of Transportation, Environmental Affairs Division, Archeological Studies Program, Report No. 134, Austin and Center for Archaeological Research, The University of Texas at San Antonio, Archaeological Report, No. 420.

Mauldin, R., S. Tomka, C. Frederick, J. Thompson, and A. Figueroa

2011b Chapter 6: Archeological Investigations at 41TV540-Field and Laboratory Methods and Summary of Results. In Results of Archeological Significance Testing at $41 T V 410$ and 41TV540 and Associated Geomorphological Investigations on a Segment of Onion Creek in Travis County, Texas, by A. Figueroa, R. Mauldin, J. C. Fredrick, S. A. Tomka, and J. L. Thompson, pp. 41-68. Texas Department of Transportation, Environmental Affairs Division, Archeological Studies Program, Report No. 134, Austin and Center for Archaeological Research, The University of Texas at San Antonio, Archaeological Report No. 420.

McCracken, H., editor

1978 The Mummy Cave Project in Northwestern Wyoming. The Buffalo Bill Historical Center, Cody. 
McDonald, G.

1981 North American Bison: Their Classification and Evolution. University of California Press, Berkeley.

McKinney, W. W.

1981 Early Archaic Adaptations in Central and Southwestern Texas: The Problem of the Paleoindian-Archaic Transition. Bulletin of the Texas Archeological Society 52:91120.

McReynolds, R.

2002 Calf Creek Horizon Points from Wilson County, Texas. La Tierra 29(2):39-44.

Mear, C. E.

1998 Terrace Deposits and Late Quaternary Climate, South-Central Edwards Plateau, Texas. Bulletin of the Texas Archeological Society 69:79-88.

Mehalchick, G. D. and K. W. Kibler

2008 Hunters and Gathers of the North Bosque River Valley: Excavations at the Baylor, Britton, McMillan, and Higginbotham Sites, Waco Lake, McLennan County, Texas. Reports of Investigations, Number 156, Prewitt \& Associates, Inc., Austin. For the U.S. Army Corps of Engineers, Fort Worth District.

Mehalchick, G., D. K. Boyd, K. W. Kibler, and C. H. Ringstaff

2004 Shifting Sands and Geophytes: Geoarcheological Investigations at Paluxy Sites on Fort Hood, Texas. United States Army Fort Hood, Archeological Resource Management Series, Research Report No. 48.

Meissner, B. A.

2011a Appendix B: Vertebrate Faunal Remains from 41HY161. In Archaeological
Investigations at the Ice House Site, 41HY161: Early Archaic Technology, Subsistence, and Settlement along he Balcones Escarpment, Hays County, Texas, assembled by E. Oksanen, pp. 149151. Archaeological Studies Report No. 14, Center for Archaeological Studies, Texas State University-San Marcos.

2011b Appendix N: Faunal Material from 41TV540. In Results of Archeological Significance Testing at $41 T V 410$ and $41 T V 540$ and Associated Geomorphological Investigations on a Segment of Onion Creek in Travis County, Texas, by A. Figueroa, R. Mauldin, J. C. Fredrick, S. A. Tomka, and J. L. Thompson, pp. 357-362. Texas Department of Transportation, Environmental Affairs Division, Archeological Studies Program, Report No. 134, Austin and Center for Archaeological Research, The University of Texas at San Antonio, Archaeological Report No. 420.

Meltzer, D. J.

1991 Altithermal Archeology and Paleoecology at Mustang Springs, on the Southern High Plains of Texas. American Antiquity 56(2):236-267.

1995 Modeling the Prehistoric Response to Altithermal Climates on the Southern High Plains. In Ancient Peoples and Landscapes, edited by E. Johnson, pp. 349368. Texas Tech University Press, Lubbock.

1999 Human Responses to Middle Holocene (Altithermal) Climates on the North American Great Plains. Quaternary Research 52(3):404-416. 
Meltzer, D. J. and M. B. Collins

1987 Prehistoric Water Wells on the Southern High Plains: Clues to Altithermal Climate. Journal of Field Archaeology 14(1):9-28.

Menking, K. M. and R. Y. Anderson

2003 Contributions of La Nina and El Nino to Middle Holocene Drought and Late Holocene Moisture in the American Southwest. Geology 31(11):937-940.

Miao, X., J. Mason, R. J. Goblet, and P. R. Hanson 2005 Loess Record of Dry Climate and Aeolian Activity in the Early-to mid-Holocene, Central Great Plains, North America. The Holocene 15(3):339-346.

Miller, E. O. and E. B. Jelks

1952 Archeological Excavations at the Belton Reservoir, Coryell County, Texas. Bulletin of the Texas Archeological Society 23:168217.

Moerman, D. E.

1998 Native American Ethnobotany. Timber Press, Inc., Portland.

Munoz, C. M., R. P. Mauldin, J. L. Thompson, and S. C. Caran

2011 Archeological Significance Testing at 41BX17.271, The Granberg Site: A MultiComponent Site along the Salado Creek in Bexar County, Texas. Texas Department of Transportation, Environmental Affairs Division, Archeological Studies Program Report No. 135 and Center for Archaeological Research, The University of Texas at San Antonio, Archaeological Report No. 393, Austin.

Muir, J.

1979 My First Summer in the Sierra. Houghton Mifflin Company, Boston.
National Oceanic and Atmospheric Administration 2007 A Paleo Perspective on Global Warming: The Mid-Holocene "Warm Period". www.ncdc.noaa.gov/paleo/globalwarming /holocene.html.

Neal, L.

1994a A Calf Creek Component from the Lamar Site, 34BR8, Bryan County. Bulletin of the Oklahoma Anthropological Society XL:139-180.

1994b The Brandon Site, 34TU82, Tulsa Oklahoma. Bulletin of the Oklahoma Anthropological Society XL:209-250.

1999 Dating the Kubik Site, 34KA354. Newsletter of the Oklahoma Archeological Survey, The University of Oklahoma 19(2):1-3.

2002 Activities at the Kubik Site, 2002. Oklahoma Archeology 50(3):5-7.

Neal, W. L. and R. R. Drass

1998 Middle Holocene Archeology in Northeastern Oklahoma. Oklahoma Anthropological Society Bulletin XLVII:39-66.

Neal, W. L. and M. Duncan

1998 Mid-Holocene Radiocarbon Dates from the Kubik Site (34KA354). Oklahoma Archeological Survey Newsletter 18(1):2.

Neal, W. L., D. Morgan, B. Ross, and D. G. Wyckoff

1994 The Red Clay and Island Locations in Haskell County: Eastern Oklahoma Manifestations of the Calf Creek Horizon. Bulletin of the Oklahoma Anthropological Society XL:277-306. 
Newcomb, W. W., Jr.

1993 The Indians of Texas: From Prehistoric to Modern Times. University of Texas Press, Austin.

Newman, R. W.

1983 The Buffalo in Southeastern United States Post-Pleistocene Prehistory. In Southeastern Natives and Their Past, edited by D. G. Wyckoff and J. L. Hofman, pp. 261-180. Oklahoma Archeological Survey, Studies in Oklahoma's Past No. 11. Norman.

Nicholson, R. A.

1993 A Morphological Investigation of Burnt Animal Bone and an Evaluation of its Utility in Archeology. Journal of Archaeological Science 20(4):411-428.

Nicholson, B. A. and T. Playford

2009 The Atkinson Site-A 6,000 Year Old Gowen (Mummy Cave) Occupation near Lauder, Manitoba. Plains Anthropologist 54(209):29-48.

Nickels, D. L, C. B. Bousman, J. D. Leach, and D. A. Cargill

2001 Test Excavations at the Culebra Creek Site (41BX126), Bexar County, Texas. Archaeological Survey Report, No. 265, Center for Archaeological Research, The University of Texas at San Antonio and Archeological Studies Program, Report 3, Environmental Affairs Division, Texas Department of Transportation, Austin.

Nordt, L. C.

1992 Archeological Geology of the Fort Hood Military Reservation Ft. Hood, Texas. United States Army Fort Hood, Archeological Resource Management Series, Research Report Number 25.
1993

Additional

Geoarcheological

Investigations at the Fort Hood Military Reservation, Ft. Hood, Texas. United States Army Fort Hood, Archeological Resource Management Series, Research Report Number 28, Addendum to Research Report Number 25.

2002 Geomorphological Investigations. In Archaeological Investigations for the McKinney Roughs Resort Project, Bastrop County, Texas, by K. Lawrence, K. A. Miller, L. Nordt, and B. Young, pp. 17-21 and pp. 41-47. SWCA Cultural Resources Report No. 01-354, Austin.

Nordt, L. C., J. von Fisher, and L. Tieszen 2007 Late Quaternary Temperature Record from Buried Soils of the North American Great Plains. Geological Society of America 35(2):159-162.

Nordt, L. C., T. W. Boutton, C. T. Hallmark, and M. R. Waters

1994 Late Quaternary Vegetation and Climate Changes in Central Texas Based on The Isotopic Composition of Organic Carbon. Quaternary Research 41(1):109-120.

Nordt, L. C., T. W. Boutton, J. S. Jacob, and R. D. Mandel

$2002 \mathrm{C}_{4}$ Plant Production and Climate- $\mathrm{CO}_{2}$ Variations in South-Central Texas during the Late Quaternary. Quaternary Research 58(2):182-188.

Odell, G. H.

2004 Lithic Analysis. Manuals in Archaeological Method, Theory, and Technique. Springer Science and Business Media, New York. 
Oksanen, E. R., C. A. Weaver, E. A. Schroeder, and G. T. Goode

2002 Archaeological Testing of Four Prehistoric Sites Along the Guadalupe-Blanco River Authority's San Marcos Raw Water Pipeline, Caldwell and Hays Counties, Texas. PPA Cultural Resources Report 0262, prepared for Guadalupe-Blanco River Authority.

Oksanen, E. R., T. R. Hester, H. J. Shafer and M. Cody

2008 Appendix C: Technology. In The Gatlin Site (41KR621): Investigating Archaic Lifeways on the Southern Edwards Plateau of Central Texas, by B. A. Houk, K. A. Miller, and E. R. Oksanen, pp. 13-105. Texas Department of Transportation, Environmental Affairs Division, Archeological Studies Program, Report No. 108, and SWCA Environmental Consultants, SWCA Cultural Resources Report No. 2008-149, Austin.

Opler, M. E.

1965 An Apache Life-way: The Economic, Social, and Religious Institutions of the Chiricahua Indians. Reprinted by Cooper Square Publishers, New York.

Parker, W. and J. Mitchell

1979 Notes on Some Bell Points from a Site in Crosby County, Texas. La Tierra 6(2):2627.

Parks, D. R.

2001 Pawnee. In Handbook of North American Indians: Plains, edited by R. J, DeMallie 13(2):515-547. Smithsonian Institution, Washington.

Parry, W. J. and R. L. Kelly

1987 Expedient Core Technology and Sedentism. In The Organization of Core
Technology, edited by J. K. Johnson and C. A. Morrow, pp. 285-304. Westview Press, Boulder.

Pastor, J. V. and P. M. Lubinski (editors)

2000 Pronghorn Past and Present: Archaeology, Ethnology, and Biology. Plains Memoir 32.

Pastor, J. V., K. Thompson, and R. L. Tanner

2000 Pronghorn Past and Present. In Pronghorn Past and Present: Archaeology, Ethnology, and Biology, edited by J. V. Pastor and P. M. Lubinski, pp. 1-2. Plains Memoir 32.

Patterson, L. W. and James B. Sollberger

1978 Replication and Classification of Small Size Lithic Debitage. Plains Anthropologist 23(80):103-112.

Perino, G.

1968 Guide to the Identification of Certain American Indian Projectile Points. Special Bulletin No. 3, of the Oklahoma Anthropological Society.

Perry, L.

2001 Prehispanic Subsistence in the Middle Orinoco Basin: Starch Analyses Yield New Evidence. Unpublished Ph.D. dissertation, Southern Illinois University at Carbondale, Carbondale.

2010 Appendix F: Starch Analyses from the BLM Landis Property. In Landis Property: Data Recovery at Three Prehistoric Sites (41PT185, 41PT186, and 41PT245) in Potter County, Texas, Volume II: Appendices, by J. M. Quigg, C. D. Frederick, P. M. Matchen, and K. DuBois, pp. 767-689. TRC Technical Report No. 150832. Report on file with TRC in Austin and Bureau of Land Management, Santa Fe. 
2013 Appendix C: Starch Analysis of Artifacts from 41MI96. In Data Recovery at 41 MI96 In Mills County, Texas, by J. M. Quigg, R. A. Ricklis, P. M. Matchen, and J. T. Abbott, pp. 165-176. Texas Department of Transportation, Environmental Affairs Division, Archeological Studies Program Report No. 150 and TRC Environmental Corporation, Technical Report No. 192832, Austin.

2014 Appendix D: Starch Analysis of Sixty Samples, Lampasas County, Texas. In Eligibility Testing at Three Prehistoric Sites at Lynch Creek, Lampasas County, Texas, by J. M. Quigg, P. M. Matchen, R. A. Ricklis, S. Gray, C. D. Frederick, and J. Barrett, pp. 283-298. Texas Department of Transportation, Environmental Affairs Division, Archeological Studies Program Report No. 161 and TRC Environmental Corporation, Technical Report Nos. 43243 (106892), 18125, and 201313, Austin.

Perry, L. and J. M. Quigg

2011a Starch Remains and Stone Boiling in the Texas Panhandle, Part I: The Pipeline, Corral, and Pavilion Sites. Plains Anthropologist 56(218):95-107.

2011b Starch Remains and Stone Boiling in the Texas Panhandle, Part II: Identifying Wildrye (Elymus spp.). Plains Anthropologist 56(218):109-119.

Perry, L., D. Sandweiss, D. R. Piperno, K. Rademaker, M. Malpass, A Umire, and P. de la Vera

2006 Early Maize Agriculture and Interzonal Interaction in Southern Peru. Nature 440(2):76-78.
Perttula, T. K.

2004 Chapter 1: An Introduction to Texas Prehistoric Archeology. In The Prehistory of Texas, T. K. Perttula, editor, pp. 5-14. Texas A\&M University Press, College Station.

2011 Chapter 6: The Ceramic Artifacts from the Lang Pasture Site (41AN38) and the Place of the Site within an Upper Neches River Basin Caddo Ceramic Tradition. In Archeological Investigations at the Lang Pasture Site (41AN38) in the Upper Neches River Basin of East Texas, assembled and edited by T. K. Perttula, D. B. Kelley and R. A. Ricklis, pp. 145-320. Texas Department of Transportation, Archeological Studies Program, Report No. 129, Environmental Affairs Division, and TRC Environmental Corporation, Technical Report No. 174202, Austin.

Perttula, T. K., S. A. Iruegas, and H. Neff

2003 Caddoan Pottery in Central Texas: Geochemical Analyses of Ceramics from Fort Hood and Vicinity. United States Army Fort Hood, Archeological Resource Management Series, Research Report No. 51.

Peter, D. E., D. Prikryl, O. F. McCormick, and M. Demuynck

1982 Chapter 8: Site Excavation Reports: Primary Contract. In Archeological Investigations at the San Gabriel Reservoir District, Central Texas, Volume I, compiled and edited by T. R. Hays, pp. 829 to 8-76, 8-241 to 8-297. Archaeology Program, Institute of Applied Sciences, North Texas State University, Denton.

Pierce, H. G.

1987 Chapter 6: The Gastropods, with Notes on Other Invertebrates. In Lubbock Lake: 
Late Quaternary Studies on the Southern High Plains, edited by E. Johnson, pp. 4148. Texas A\&M University Press, College Station.

Piperno D. R. and I. Holst

1998 The Presence of Starch Grains on Prehistoric Stone Tools from the Humid Neotropics: Indications of Early Tuber Use and Agricultural in Panama. Journal of Archeological Science 25(8):765-776.

Piperno, D. R., A. J. Ranere, I. Holst, and P. Hansell 2000 Starch Grains Reveal Early Root Crop Horticulture in the Panamanian Tropical Forest. Nature 407(6806):894-897.

Poore, R. Z., M. J. Pavich, and H. D. GrissinoMayer

2005 Record of the North American Southwest Monsoon from Gulf of Mexico Sediment Cores. Geological Society of America 33(3):209-212.

Powell, V.

1995 Bifaces of the Calf Creek Horizon: A Collection from Cedar Canyon, Oklahoma. Bulletin of the Oklahoma Anthropological Society XLII:45-166.

Prewitt, E. R.

1966 Preliminary Report on the Devil's Rockshelter Site, Val Verde County, Texas. Texas Journal of Science 18(2):206-224.

1981a Cultural Chronology in Central Texas. Bulletin of the Texas Archeological Society 52:65-89.

1981b A Wooden Mortar from the Stockton Plateau of Texas. Journal of Field Archaeology 8(1):111-117.
1982 Archeological Investigations at the Loeve-

Fox, Loeve and Tombstone Bluff Sites in the Granger Lake District of Central Texas. In Archeological Investigations at the San Gabriel Reservoir Districts, Central Texas, Volume 4, compiled and edited by T. R. Hays, Institute of Applied Sciences, North Texas State University, Denton.

1983 Andice: An Early Archaic Dart Point Type. La Tierra 10(3):1-6.

1985 From Circleville to Toyah: Comments on Central Texas Archeology. Bulletin of the Texas Archeological Society 54:201-238.

1987 Observations on Seasonality of Selected Fish Remains from 41AS16. In: National Register Assessment of the Swan Lake Site, 41AS16, on Copano Bay, Aransas County, Texas, by E. R. Prewitt, S. V. Lisk, and M. A. Howard, pp. 259-268. Reports of Investigations No. 56, Prewitt and Associates, Inc., Austin.

1995 Distribution of Typed Projectile Points in Texas. Bulletin of the Texas Archeological Society 66:83-173.

Quigg, J. M.

1997 Bison Processing at the Rush Site, 41TG346, and Evidence for Pemmican Production in the Southern Plains. Plains Anthropologist 42(159):145-161, Memoir 29.

2008 Chapter 10.0 Middle Archaic Component. In The Varga Site: A Multicomponent, Stratified Campsite in the Canyonlands of Edwards County, Texas, Volumes I and II, by J. M. Quigg, J. D. Owens, P. M. Matchen, G. Smith, R. A. Ricklis, M. C. Cody, and C. D. Frederick, pp. 373-405. Texas Department of Transportation, 
Environmental Affairs Division, Archeological Studies Program Report No. 110 and TRC Environmental Corporation, Technical Report No. 35319, Austin.

2011 Use-Wear and Starch Residue Analyses on an In Situ Corner-Tang Knife from the Texas Panhandle: Towards an Understanding of Function. Plains Anthropologist 56(217):37-44.

Quigg, J. M. and G. L. Ellis

1994 Chapter 8: Burned Rock Mound Chronometric Investigations. In Archeological Investigations on 571 Prehistoric Sites at Fort Hood, Bell and Coryell Counties, Texas, edited by W. N. Trierweiler, pp. 203-274. United States Army Fort Hood, Archeological Resource Management Series, Research Report No. 31.

Quigg, J. M. and J. Peck

1995 The Rush Site (41TG346): A Stratified Late Prehistoric Locale in Tom Green County, Texas. Mariah Associates, Inc., Technical Report No. 816c, Austin.

Quigg, J. M. and C. D. Frederick

2005 Assessment of TxDOT Right-of-Way at 41MS69 in Mason County, Texas: An Interim Report. Prepared for Texas Department of Transportation, Environmental Affairs Division, by TRC Environmental Corporation, Austin.

Quigg, J. M., C. D. Frederick, and C. Lintz 1994 Sulphur Springs Draw: Geoarchaeological and Archaeological Investigations at Sulphur Draw Reservoir, Martin County, Texas. Prepared for Colorado River Municipal Water District, Big Springs, Texas. Mariah Technical
Report No. 776, Mariah Associates, Inc., Austin.

Quigg, J. M., S. Pritchard, and G. Smith

2002 The Boiler Site (41WB557): Utilization of an Upland Setting Over the Last 4200 Years, Webb County, Texas. TRC Technical Report No. 27277, TRC Environmental Corporation, Austin, Texas and Texas Department of Transportation, Environmental Affairs Division, Archeological Studies Program, Report No. 45, Austin.

Quigg, J. M., P. M. Matchen, and C. D. Frederick

2015 Eligibility Assessment of the Slippery Slope Site (41MS69) in TxDOT Right-ofWay in Mason County, Texas. Texas Department of Transportation, Environmental Affairs Division, Archeological Studies Program, Report No. 172 and TRC Technical Report Nos. 43252 (106895) and 211462, TRC Environmental Corporation, Austin.

Quigg, J. M., M. T. Boulanger, and M. Glascock

2011b Geochemical Characterization of Tecovas and Alibates Source Samples. Plains Anthropologist 56(219):259-284.

Quigg, J. M., M. Glascock, and R. J. Speakman

2006 Reconnaissance in Phase II East and Chert Collection across Phase I and II East for FPL Energy's Horse Hollow Project, Taylor County, Texas. Confidential Report. TRC Environmental Corporation, Technical Report 49150, Austin.

Quigg, J. M., C. Lintz, G. Smith, and S. Wilcox

2000 The Lino Site: A Stratified Late Archaic Campsite in a Terrace of the San Idelfonzo Creek, Webb County, Southern Texas. Technical Report No. 23756, TRC Mariah Associates Inc., and Texas Department of 
Transportation, Environmental Affairs Division, Archeological Studies Program, Report No. 20.

Quigg, J. M., M. Malainey, R. Przybylski, and G. Monks

2001 No Bones about It: Using Lipid Analysis of Burned Rock and Ground Stone Residues to Examine Late Archaic Subsistence Practices in South Texas. Plains Anthropologist 46(177):283-303.

Quigg, J. M., P. M. Matchen, C. D. Frederick, and E. Schroeder

2007 Data Recovery at 41TV2161, Travis County, Texas: Interim Report. Prepared for Texas Department of Transportation, Environmental Affairs Division by TRC Environmental Corporation, Austin,

Quigg, J. M., C. D. Frederick, P. M. Matchen, and K. DuBois

2010 Landis Property: Data Recovery at Three Prehistoric Sites (41PT185, 41PT186, and 41PT245) in Potter County, Texas, Volumes I and II. TRC Technical Report No. 150832. Report on file with TRC in Austin and Bureau of Land Management, Santa Fe.

Quigg, J. M., P. M. Matchen, C. D. Frederick, and R. A. Ricklis

2011a Root-Be-Gone (41YN452): Data Recovery of Late Archaic Components in Young County, Texas, Volumes I and II. Texas Department of Transportation, Environmental Affairs Division, Archeological Studies Program Report No. 135 and TRC Environmental Corporation, Technical Report Nos. 50907 and 171219, Austin.
Quigg, J. M., R. A. Ricklis, P. M. Matchen, and J. T. Abbott

2013a Data Recovery at 41MI96 In Mills County, Texas. Texas Department of Transportation, Environmental Affairs Division, Archeological Studies Program Report No. 150 and TRC Environmental Corporation, Technical Report No. 192832, Austin.

Quigg, J. M., C. Lintz, G. Smith, D. DeMar, and J. D. Owens

2011b Cultural Resource Investigations to Offset Mechanical Impacts to the Clear Creek Golf Course Site (41CV413), Fort Hood, Texas. United States Army Fort Hood, Archeological Resource Management Series, Resource Report No. 60.

Quigg, J. M., P. M. Matchen, C. D. Frederick, B. Gregory, and R. A. Ricklis

2014a Barrett Site (41MM382) Assessment, Milam County (CSJ: 0590-05-027), Texas. Texas Department of Transportation, Environmental Affairs Division, Archeological Studies Program Report No. 163 and TRC Environmental Corporation, TRC Technical Report Nos. 192919 and 211462, Austin.

Quigg, J. M., J. D. Owens, P. M. Matchen, G. Smith, R. A. Ricklis, M. C. Cody, and C. D. Frederick

2008 The Varga Site: A Multicomponent, Stratified Campsite in the Canyonlands of Edwards County, Texas, Volumes I and II. Texas Department of Transportation, Environmental Affairs Division, Archeological Studies Program Report No. 110 and TRC Environmental Corporation, Technical Report No. 35319, Austin. 
Quigg, J. M., P. M. Matchen, C. D. Frederick, R. A. Ricklis, B. Gregory, D. Maki, and M. Bateman

2013b Long View (41RB112): Data Recovery of Two Plains Village Components in Roberts County, Texas, Volumes I and II. Texas Department of Transportation, Environmental Affairs Division, Archeological Studies Program Report No. 147 and TRC Environmental Corporation, Technical Report Nos. 50907 and 171219, Austin.

Quigg, J. M., P. M. Matchen, R. A. Ricklis, S. Gray, C. D. Frederick, and J. Barrett

2014b Eligibility Testing at Three Prehistoric Sites at Lynch Creek, Lampasas County, Texas. Texas Department of Transportation, Environmental Affairs Division, Archeological Studies Program Report No. 161 and TRC Environmental Corporation, Technical Report Nos. 43243 (106892), 18125, and 201313, Austin.

Quigg, J. M., J. Peck, C. Lintz, A. C. Treece, C. D. Frederick, R. Clem, G. L. Ellis, P. Schuchert, and J. T. Abbott

1996 Early Archaic Use of the Concho River Terraces: Cultural Resource Investigations at $41 T G 307$ and 41TG309, Tom Green County, San Angelo, Texas. Report for the City of San Angelo, by TRC Mariah Associates, Inc., Austin. Technical Report No. 11058.

Quinn, J. H.

1957 Paired River Terraces and Pleistocene Glaciation. Journal of Geology. 65(2):149166.

Raab, L. M., R. F. Cande, and D. W. Stahle

1979 Debitage Graphs and Archaic Settlement Patterns in the Arkansas Ozarks. Midcontinental Journal of Archaeology 4(2):167-82.
Ramsey, C. B. and S. Lee

2013 Oxcal Program Version 4.2. Radiocarbon Accelerator Unit, University of Oxford, U.K.

Reber, E. A., S. N. Dudd, N. J. van der Merwe, and R. P. Evershed

2004 Direct Detection of Maize in Pottery Residue via Compound Specific Stable carbon Isotope Analysis. Antiquity 78:682691.

Redder, A. J.

1985 Horn Shelter No. 2: The South End, a Preliminary Report. Central Texas Archeologist 10:37-65.

Reeves, B. O. K.

1973 The Concept of an Altithermal Cultural Hiatus in Northern Plains Prehistory. American Anthropologist 75(5):12211253.

1978 Head-Smashed-In: 5500 Years of Bison Jumping in the Alberta Plains. Plains Anthropologist 23(82):151-174. Part 2: Memoir 14: Bison Procurement and Utilization: A Symposium, edited by L. B. Davis and M. Wilson.

1990 Chapter 10: Communal Bison Hunting of the Northern Plains. In Hunters of the Recent Past, edited by L. B. Davis and B. O. K. Reeves, pp. 168-194. Unwin Hyman Ltd., London.

Regert, M.

2007 Chapter 6: Elucidating Pottery Function using a Multi-step Analytical Methodology combining Infrared Spectroscopy, Chromatographic Procedures and Mass Spectrometry. In Theory and Practice of Archaeological Residue Analysis, edited by H. Barnard and J. W. Eerkens, pp. 61-76. 
British Archaeological Reports International Series 1650. Oxford.

Reher, C. A.

1977 Adaptive Process on the Shortgrass Plains. In For Theory Building in Archaeology, Essays On Faunal Remains, Aquatic Resources, Spatial Analysis, and Systematic Modeling, edited by L. R. Binford, pp. 13-40. Academic Press, Inc., New York.

Reichert, E. T.

1913 The Differentiation and Specificity of Starches in Relation to Genera, Species, etc. Carnegie Institute, Washington, D. C.

Reid, K. C.

1989 A Material Science Perspective on HunterGatherer Pottery. In Pottery Technology: Ideas and Approaches, edited by $\mathrm{G}$. Bronitsky, pp. 167-180. Westview, Boulder.

Reimer, P. J., M. G. L. Baillie, E. Bard, A. Bayliss, J. W. Beck, C. J. H. Bertrand, P. G. Blackwell, C. E. Buck, G. S. Burr, K. B. Cutler, P. E. Damon, R. L. Edwards, R. G. Fairbank, M. Friedrich, T. P. Guilderson, A. G. Hogg, K. A. Hughen, B. Kromer, G. McCormac, S. Manning, C. B. Ramsey, R. W. Reimer, S. Remmele, J. R. Southon, M. Stuiver, S. Talamo, F. W. Taylor, J. van der Plicht, and C. E. Weyhenmeyer,

2004 IntCal04 Terrestrial Radiocarbon Age Calibration, 0-26 CAL KYR BP. Radiocarbon 46(3):1029-1058.

Reimer, J. P., E. Bard, A. Bayliss, J. W. Beck, P. G. Blackwell, C., B. Ramsey, C. E. Buck, H. Cheng, R. L. Edwards, M. Friedrich, P. M. Grootes, T. P. Guilderson, H. Haflidason, I. Hajdas, C. Hatté, T. J. Heaton, D. L. Hoffmann, A. G. Hogg, K. A. Hughen, K. F. Kaiser, B. Kromer, S. W. Manning, M. Niu, R. W. Reimer, D. A. Richards, E. M. Scott,
J. R. Southon, R. A. Staff, C. S. M. Turney, and J. van der Plicht

2013 IntCal13 and Marine 13 Radiocarbon Age Calibration Curves 0-50,000 Years cal B.P. Radiocarbon 55(4):1869-1887

Rhoton, C.

1995 Calf Creek on the High Plains, Part II: Finds from Cimarron County, Oklahoma, and Adjacent Parts of Colorado and Texas. Bulletin of the Oklahoma Anthropological Society XLII:171-177.

Ricklis, R. A.

1988 Archeological Investigations at the McKinzie Site (41NU221), Nueces County, Texas: Description and Contextual Interpretations. Bulletin of the Texas Archeological Society 58:1-76.

1993 A Model of Holocene Environmental and Human Adaptive Change on the Central Texas Coast, Geoarchaeological Investigations at White's Point, Nueces Bay, and Surrounding Area. Coastal Archaeological Studies, Inc., Corpus Christi.

1996 The Karankawa Indians of Texas: An Ecological Study of Cultural Tradition and Change. University of Texas Press, Austin.

2011 Appendix J: Aging and Seasonality Estimations of Freshwater Drum Otoliths from 41YN452 in Young County Texas. In Root-Be-Gone (41YN452): Data Recovery of Late Archaic Components in Young County, Texas, Volume II, by J. M. Quigg, P. M. Matchen, C. D. Frederick and R. A. Ricklis, pp. 605-614. TRC Report No. 171219, TRC Environmental Corporation, Austin. 
2012a Chapter 7: Non-Mortuary Artifacts. In Archaeology and Bioarchaeology of the Buckeye Knoll Site (41VT98), Victoria County, Texas, Volume 1, edited by R. A. Ricklis, R. A. Weinstein and D. G. Wells, pp. 163-254. Prepared by Coastal Environments, Inc., Corpus Christi, for U.S. Army Corps of Engineers, Galveston District.

2012b Chapter 19: Cultural and Ecological Change. In Archaeology and Bioarchaeology of the Buckeye Knoll Site (41VT98), Victoria County, Texas, Volume 2, edited by R. A. Ricklis, R. A. Weinstein and D. G. Wells, pp. 679-700. Prepared by Coastal Environments, Inc., Corpus Christi, for U.S. Army Corps of Engineers, Galveston District.

Ricklis, R. A. and M. B. Collins

1994 Archaic and Late Prehistoric Human Ecology in the Middle Onion Creek Valley, Hays County, Texas, Volume 1: Archeological Components. Studies in Archeology 19, Texas Archeological Research Laboratory, The University of Texas at Austin.

Ricklis, R. A. and M. D. Blum

1997 The Geoarchaeological Record of Holocene Sea Level Change and Human Occupation of the Texas Gulf Coast. Geoarchaeology: An International Journal 12(4):287-314.

Ricklis, R. A., R. A. Weinstein and D. G. Wells (editors)

2012 Archaeology and Bioarchaeology of the Buckeye Knoll Site (41VT98), Victoria County, Texas. Three Volumes, Prepared by Coastal Environments, Inc., Corpus Christi, for U.S. Army Corps of Engineers, Galveston District.
Roberts, R. G.

1965 Tick Creek Cave, An Archaic Site in the Gasconade River Valley of Missouri. The Missouri Archeologist 27(2).

Robinson, D. G. and S. C. Caran

1995 Geomorphology and Quaternary Stratigraphy of Proposed Site of Travis County Correctional Center, Texas. Borderlands Archeological Research Unit Cultural Resources Report 1, The University of Texas at Austin.

Root, M. J., D. E. Ferguson, and D. R. Harro

2008 Appendix D: Functional Analysis of Selected Tools from 41KR621. In The Gatlin Site (41KR621): Investigating Archaic Lifeways on the Southern Edwards Plateau of Central Texas, by B. A. Houk, K. A. Miller, and E. R. Oksanen, pp. 28-35. Texas Department of Transportation, Environmental Affairs Division, Archeological Studies Program, Report No. 108, and SWCA Environmental Consultants, SWCA Cultural Resources Report No. 2008-149, Austin.

Roper, D. C. and M. J. Adair

2011 Interpreting AMS Radiocarbon Age Determinations from Selected Central Plains Tradition Sites Plains Anthropologist 56(217):3-22.

Sala, O. E., W. J. Parton, L. A. Joyce, and W. K. Lauenroth

1988 Primary Production of the Central Grassland Region of the United States. Ecology 69(1):40-45.

Sauter, F., E. W. H. Hayek, W. Moche and U. Jordis 1987 Betulin aus archäologischem Schwelteer. Z. für Naturforsch 42c (11-12):1151-1152. 
Schilz, T. F.

1983 People of the Cross Timbers: A History of the Tonkawa. Unpublished Ph.D. dissertation, Texas Christian University, Fort Worth.

Schmits, L. J.

1976 The Coffey Site: Environment and Cultural Adaptation at a Prairie Plains Archaic Site. Kansas University, National Park Service, Denver, Interagency Archeology Services.

1978 The Coffey Site: Environment and Cultural Adaptation at a Prairie Plains Archaic Site. Mid-Continental Journal of Archeology 3(1):69-185.

Schoeneberger, P. J., A. D. Wysocki, E. C. Benham, and W. D. Broderson (editors)

2002 Field Book for Describing and Sampling Soils. Version 2.0. Natural Resources Conservation Service, National Soil Survey Center, Lincoln.

Schroeder, E. A.

2002 Data Recovery at the Armstrong Site (41CW54) Caldwell County, Texas. Volume II. Paul Price Associates, Inc., PPA Cultural Resources Report Number 330, Austin.

2011 The Armstrong Site: A Transitional Archaic Occupation along the Eastern Balcones Escarpment. Bulletin of the Texas Archeological Society 82:117-134.

Schroeder, E. A. and E. R. Oksanen

2002 Data Recovery at the Armstrong Site (41CW54) Caldwell County, Texas. Volume I. Paul Price Associates, Inc., PPA Cultural Resources Report Number 0284, Austin.
Schuetz, M. K.

1957 A Report on Williamson County Mound Material. Bulletin of the Texas Archeological Society 28:135-168.

Scott-Cummings, L.

1993 Appendix F: Phytolith Results. In Cultural Resource Investigations in the O. H. Ivie Reservoir, Concho, Coleman, and Runnels Counties, Volume IIB: Appendices, by C. Lintz, A. C. Treece, F. M. Oglesby, K. Kibler, P. L. O’Neill, W. N. Trierweiler, C. Frederick, J. M. Quigg, and A. J. Taylor, pp. F-37 through F-17. Mariah Associates, Inc., Technical Report No. 346-IIB, Austin.

Scott, S. L.

2012 Chapter 8: Vertebrate Faunal Bones. In Archaeology and Bioarchaeology of the Buckeye Knoll Site (41VT98), Victoria County, Texas, Volume 1, edited by R. A. Ricklis, R. A. Weinstein and D. G. Wells, pp. 255-293. Prepared by Coastal Environments, Inc., Corpus Christi, for U.S. Army Corps of Engineers, Galveston District.

Seebach, J. D.

2002 Stratigraphy and Bonebed Taphonomy at Blackwater Draw Locality No. 1 during the Middle Holocene (Altithermal). Plains Anthropologist 47(183):339-358.

Shackley, M.

1982 Gas Chromatographic Identification of a Resinous Deposit from a 6th Century Storage Jar and Its Possible Identification. Journal of Archaeological Science 9:305306.

Shackley, M. S.

2010 Appendix C: Obsidian Source Analysis. In Landis Property: Data Recovery at Three 
Prehistoric Sites (41PT185, 41PT186, and 41PT245) in Potter County, Texas Volume II: Appendices, by J. M. Quigg, C. D. Frederick, P. M. Matchen, and K. DuBois, pp. 685-6693. TRC Technical Report No. 150832. Report on file with TRC in Austin and Bureau of Land Management, Santa $\mathrm{Fe}$.

2012 Appendix I: An Energy-Dispersive X-Ray Fluorescence Analysis of Obsidian Artifact from Little Paint Site (41KM226), Central Texas. In The Little Paint Site: A Classic Toyah Camp on the South Llano River, Kimble County, Texas, by S. M. Carpenter, K. A. Miller, C. D. Frederick, L. G. Cecil, M. C. Cody, and A. Peyton, pp. 393-395. SWCA Environmental Consultants, SWCA Cultural Resources Report No. 12429 ,

Shaeffer, J. B.

1958 The Alibates Flint Quarry, Texas. American Antiquity 24(2):189-191.

Shafer, H. J.

1963 Test Excavations at the Youngsport Site: A Stratified Terrace Site in Bell County, Texas. Bulletin of the Texas Archeological Society 34:57-81.

Shafer, H. J. and S. Tomka

2003 Chapter 10: Early Middle Archaic Lithic Assemblages. In Royal Coachman (41CM111), An Early Middle Archaic Site along Cordova Creek in Comal County, Texas, by R. B. Mahoney, H. J. Shafer, S. A. Tomka, L. C. Nordt, and R. P. Mauldin, pp. 83-86. Texas Department of Transportation, Environmental Affairs Division, Archeological Studies Program Report No. 49 and the Center for Archaeological Research, The University of Texas at San Antonio, Archaeological Survey Report No. 332.

Shaffer, B. S. and K. M. Gardner

1995 The Rabbit Drive Through Time: Analysis of the North American Ethnographic and Prehistoric Evidence. Utah Archaeology 8(1):13-25.

Sherwood, S. C., B. N. Driskell, A. R. Randall, and S. C. Meeks

2004 Chronology and Stratigraphy at Dust Cave, Alabama. American Antiquity 69(3):533554.

Shideler, G. L.

1970 Provenance of Johns Valley Bounders in Late Paleozoic Ouachita Fancies, Southeastern Oklahoma and Southwestern Arkansas. The American Association of Petroleum Geologists Bulletin 554(5):789806.

Silvy, N. J., M. J. Peterson, and R. R. Lopez

2004 The Cause of the Decline of Pinnated Grouse: The Texas Example. Wildlife Society Bulletin 32(1):16-21.

Shockley, D., P. Benefield, and D. G. Wyckoff 1994 Calf Creek on the Cherokee Prairie, Part II: The Woodard-Benefield Site (34MS258). Bulletin of the Oklahoma Anthropological Society, XL:329-346.

Smith, B. D.

1997 The Initial Domestication of Cucurbita pepo in the Americas 10,000 Years Ago. Science 276:932-934.

Smith, H. A.

1983 Determination of Seasonality in Archaeological Sites through Examination of Fish Otoliths. Journal of Field Archaeology 10:498-500. 
Smith, B. N. and W. V. Brown

1973 The Kranz Syndrome in the Gramineae as Indicated by Carbon Isotope Ratios. American Journal of Botany 60(6):505513.

Smith, C. S, and S. D. Creasman

1988 The Taliaferro Site: 5000 Years of Prehistory in Southwest Wyoming. For Bureau of Land Management, Wyoming, Cultural Resource Series No. 6, by Archaeological Services, Western Wyoming College, Rock Springs.

Smith, M. E. and E. R. Oksanen

2008 Chapter 7: Data Recovery Methods and Summary of Results. In The Gatlin Site (41KR621): Investigating Archaic Lifeways on the Southern Edwards Plateau of Central Texas, by B. A. Houk, K. A. Miller, and E. R. Oksanen, pp. 7-1 through 7-28. Texas Department of Transportation, Environmental Affairs Division, Archeological Studies Program, Report No. 108, and SWCA Environmental Consultants, SWCA Cultural Resources Report No. 2008-149, Austin.

Smith, C., J. Runyon, and G. Agogino

1966 A Progress Report on a Pre-Ceramic Site at Rattlesnake Draw, Eastern New Mexico. Plains Anthropologist 11(34):302-313.

Sommers, K. P., M. Elswick, G. I. Herrick, and G. A. Fox

2011 Inferring Microhabitat Preferences of Lilium catesbaei (Liliaceae). American Journal of Botany 98(5):819-28.

Sorrow, W. M., H. J. Shafer, and R. E. Ross

1967 Excavations at Stillhouse Hollow Reservoir. Papers of the Texas Archeological Salvage Project, No. 11, Austin.
Speer, C. A., M. B. Collins, and S. Ayala

2014 The Uniqueness of Calf Creek Horizon Notching Flakes. Paper presented at the $72^{\text {nd }}$ Annual Meeting of the Plains Anthropological Society, Fayetteville.

Speth, J. D.

1983 Bison Kills and Bone Counts. University of Chicago Press, Chicago.

Spivey, T., F. Freeze, and D. G. Wyckoff

1994 The Frazier Site: A Calf Creek-Bison Association in the Southern Osage Plains, South-Central Oklahoma. Bulletin of the Oklahoma Anthropological Society, XL:131-137.

Splawn, B.

1995 A Complete Calf Creek Biface from the Arkansas River, Kay County, Oklahoma. Bulletin of the Oklahoma Anthropological Society XLII:37-39.

Splawn, B. and D. G. Wyckoff

1995 Calf Creek Diagnostics from the Arkansas River, Kay County, Oklahoma. Bulletin of the Oklahoma Anthropological Society XLII:29-35.

Stafford, T., Jr.

1981 Alluvial Geology and Archaeological Potential of the Texas Southern High Plains. American Antiquity 46(3):548-565.

1998 Radiocarbon Chronostratigraphy. In Wilson-Leonard: An 11,000-year Archeological Record of Hunter-Gatherers in Central Texas, Volume IV: Archeological Features and Technical Analyses, assembled and edited by M. B. Collins, pp. 1035-1066. Studies in Archeology 31, Texas Archeological Research Laboratory, The University of Texas at Austin and Texas Department of 
Transportation, Environmental Affairs Division, Archeology Studies Program, Report 10.

Stark, R. T.

1997 Piedras Calientes: Ethnohistory, Experimental Archaeology, and Cooking with Hot Stones. Unpublished Master's thesis, The University of Texas at Austin.

2002 Fire-Cracked Rock Experiments: The Potential of Three Analytical Techniques. La Tierra 29(4):12-28.

Stewart, O. C.

1954 The Forgotten Side of Ethnogeography. In Method and Perspective in Anthropology: Papers in Honor Wilson D. Wallis, edited by R. F. Spencer, pp. 211-248. University of Minnesota Press, Minneapolis.

Stites, M. D.

2006 The Calf Creek Complex: Preliminary Research on the Lithic Technology in Kansas. Oklahoma Archeology 54(4):1042.

Story, D. A., J. A. Guy, B. A. Burnett, J. D. Freeman, J. C. Rose, D. G. Steele, B. W. Olive and K. J. Reinhard

1990 The Archeology and Bioarchaeology of the Gulf Coastal Plain, Volume 1. Research Series No. 38, Arkansas Archeological Survey, Fayetteville.

Stuiver, M. and P. J. Reimer

1993 Extended 14C Database and Revised CALIB Radiocarbon Calibration Program. Radiocarbon 35(1):215-230.

Sudbury, J. B.

2014 Appendix F: Environmental Basilica Data 41LM50 and 41LM51: Early Archaic through Late Prehistoric Periods. In
Eligibility Testing at Three Prehistoric Sites at Lynch Creek, Lampasas County, Texas, by J. M. Quigg, P. M. Matchen, R. A. Ricklis, S. Gray, C. D. Frederick, and J. Barrett, pp. 303-348. Texas Department of Transportation, Environmental Affairs Division, Archeological Studies Program Report 161 and TRC Environmental Corporation, Technical Report Nos. 43243 (106892), 18125, and 201313, Austin.

Suhm, D. A.

1962 Footbridge: A Terrace Site. In Salvage Archeology of Canyon Reservoir: The Wunderlich, Footbridge, and Oblate Sites, by L. Johnson, Jr., D. A. Suhm, and C. D. Tunnel, pp. 49-76. Texas Memorial Museum, Bulletin Number 5, The Museum of The University of Texas, Austin.

Suhm, D. A. and E. B. Jelks

1962 Handbook of Texas Archeology: Type Descriptions. The Texas Archeological Society, Special Publication Number One and The Texas Memorial Museum Bulletin Number Four, Austin.

Suhm, D. A., A. D. Krieger and E. B. Jelks

1954 An Introduction Handbook of Texas Archeology. Bulletin of the Texas Archeological Society 25.

Sullivan, M. J.

1995 Calf Creek in North-Central Oklahoma. Bulletin of the Oklahoma Anthropological Society, XLII:41-52.

Sullivan, A. P. and K. C. Rozen

1985 Debitage Analysis and Archaeological Interpretation. American Antiquity 50(4):755-779 
Texas Beyond History

2015 Buffalo Gourd. Electronic document, http://texasbeyondhistory.net/ethnobot/im ages/cucurbitabg.html, accessed April 10, 2015 .

Texas Department of Transportation (TxDOT)

2010 TxDOT Lithic Protocol Version 2.1, Chipped Stone Analytical Protocol, TxDOT Archeological Studies Program, On File with Environmental Affairs Division, Texas Department of Transportation, Austin.

Tharp, B. C.

1939 The Vegetation of Texas, Texas Academy of Science publications in Natural History, Non-Technical Series 1.

The Flower Expert

2015 Tiger Lily, http://www.the wildflowerexpert.com. Electronic document, accessed February 29, 2015.

Thomas, C., T. H. Bonner, and B. G. Whiteside 2007 Freshwater Fishes of Texas: A Field Guide. Texas A\&M University Press, College Station.

Thomas, D. H., R. L. Kelly, and P. C. Dawson 2009 Archaeology, First Canadian Edition, Nelson Education, Toronto.

Thompson, J. L.

1967 Chapter 4: Modern, Historic, and Fossil Flora. In Lubbock Lake: Late Quaternary Studies on the Southern High Plains, edited by E. Johnson, pp. 26-35. Texas A\&M University Press, College Station.

Thoms, A. V.

2007a Chapter 9: Excavation Strategies and the General Nature of Archaeological Deposits. In Archaeological and
Paleoecological Investigations at the Richard Beene Site, South-Central Texas, Volume I: Paleoecological Studies, Cultural Contexts, and Excavation Strategies, edited by A. V. Thoms and R. D. Mandel, pp. 139-176. Reports of Investigations No. 8, Center for Ecological Archaeology, Texas A\&M University, College Station.

2007b Chapter 15: Archaeological and Paleoecological Records: Summary and Synthesis. In Archaeological and Paleoecological Investigations at the Richard Beene Site, South-Central Texas, Volume II: Archaeological Studies, Synthesis, and Appendixes, edited by A. V. Thoms and R. D. Mandel, pp. 337-382. Reports of Investigations No. 8, Center for Ecological Archaeology, Texas A\&M University, College Station.

2008 The Fire Stones Carry: Ethnographic Records and Archaeological Expectations for Hot-Rock Cookery in Western North America. Journal of Anthropological Archaeology 27(4):443-460.

2009 Rocks of Ages: Propagation of Hot-Rock Cookery in Western North America. Journal of Archaeological Science 31(2):213-232.

Thoms, A. V. and R. D. Mandel (editors)

2007 Archaeological and Paleoecological Investigations at the Richard Beene Site, South-Central Texas. Volumes I and II. Reports of Investigations No. 8, Center for Ecological Archaeology, Texas A\&M University, College Station.

Thoms, A. V. and P. A. Clabough

2011 The Archaic Period at the Richard Beene Site: Six Thousand Years of Hunter- 
Gatherer Family Cookery in South-Central North America. Bulletin of the Texas Archeological Society 82:77-115.

Thurmond, J. P. and D. G. Wyckoff

1994 Some Surprising Dune Dates on the Washita. Newsletter of the Oklahoma Anthropological Society 42(6):4-5.

1999 The Calf Creek Horizon in Northwestern Oklahoma. Plains Anthropologist 44(169):231-250.

Tinkler, K. J.

2001 The Case of the Missing Flood: The Unrecorded Flood of 1935 on the James River, Mason County, Texas. Geomorphology 39(3\&4):239-250.

Tomka, S. A.

1989 Differentiating Lithic Reduction Techniques: An Experimental Approach. In Experiments in Lithic Technology, edited by D. S. Amick and R. P. Maudlin, pp. 137-162. BAR International Series 528. British Archaeological Reports, Oxford.

Toomey, R. S. III

1993 Late Pleistocene and Holocene Faunal and Environmental Changes at Hall's Cave, Kerr County, Texas. Unpublished Ph.D. dissertation, The University of Texas at Austin.

Toomey, R. S., III, M. D. Blum, and S. Valastro, Jr. 1993 Late Quaternary Climates and Environments of Edwards Plateau, Texas. Global and Planetary Change 7(4):299320.

Torrence, R. (editor)

1989 Time, Energy and Stone Tools. Cambridge University Press, New York.
Turner, E. S. and T. R. Hester

1999 A Field Guide to Stone Artifacts of Texas Indians. Gulf Publishing, Rowman \& Littlefield Publishers, Inc. London, New York, Oxford.

Turner, E. S., T. R. Hester, and R. McReynolds

2011 Stone Artifacts of Texas Indians. Completely Revised Third Edition. Taylor Trade Publishing, London, New York.

Turpin, S. A.

1991 Chapter 1: Time Out of Mind: The Radiocarbon Chronology of the Lower Pecos River Region. In Papers on Lower Pecos Prehistory, edited by S. A. Turpin, pp. 1-49. Studies in Archeology 8, Texas Archeological Research Laboratory, The University of Texas at Austin.

1998 Wroe Ranch: Small Shelter Occupancy on the Edge of the Trans-Pecos, Terrell County, Texas. Cultural Resource Report 3, Borderlands Archeological Research Unit, University of Texas at Austin.

Turpin, S. A., compiler

1988 Seminole Sink: Excavation of a Vertical Shaft Tomb, Val Verde County, Texas.

Plains Anthropologist 33(122) Part 2, Memoir 22.

Turpin, S. A. and L. C. Bement

1992 Skyline Shelter and Devils Triangular Dart Points: Evidence for A New Component of the Lower Pecos Early Archaic Sequence, Southwest Texas. Plains Anthropologist 37(138):41-57.

United States Department of Agriculture (USDA)

2015 Official Soil Series Descriptions, USDANRCS Soil Survey Division. Electronic document, https://soilseries.sc.egov.usda.gov/OSD_D 
ocs/H/HOUSTON_BLACK.html, accessed March 2015

United States Fish and Wildlife Service

2015 Attwater Prairie Chicken National Wildlife

Refuge. Electronic document, http://www.fws.gov/.../Attwater_Prairie, accessed April 10, 2015.

University of Minnesota, Department of Anthropology

2015 Simomsen, Electronic document, http://anthropology.umn.edu/labs/wlnaa/p oints/clusters/simonsen.html, accessed May 10, 2015.

Urbanec, D. A.

1963 Stream Terraces and Related Deposits in the Austin Area, Texas. Unpublished M. A. thesis, The University of Texas at Austin.

Valastro, S., F. J. Pearson, Jr. and E. M. Davis

1967 University of Texas Radiocarbon Dates. Radiocarbon 9(1):445.

Vehik, S. C.

1977 Bone Fragments and Bone Grease Manufacturing: A Review of their Archeological Use and Potential. Plains Anthropologist 21(77):169-182).

Voget, F. W.

2001 Crow. In Handbook of North American Indians: Plains, edited by R. J. DeMallie 13(2):695-717. Smithsonian Institution, Washington.

Wade, M. F.

1998 The Native Americans of the Texas Edwards Plateau and Related Areas: 1582-1799. Unpublished Ph.D. dissertation, Department of Anthropology, The University of Texas at Austin.
2002 Patterns of Buffalo Seasonality During the Protohistoric and Historic Periods in Texas. Appendix C. In The Smith Creek Bridge Site (41DW270): A Terrace Site in De Witt County, Texas, by D. Hudler, K. Prilliman, and T. Gustavson, pp. 171-186. Studies in Archeology 35, Texas Archaeological Research Laboratory, The University of Texas at Austin and Archeology Studies Program, Report No. 17, Environmental Affairs Division, Texas Department of Transportation.

Walker, E. G.

1980 The Gowen Site: An Early Archaic Site on the Northern Plains. Unpublished Ph.D. dissertation, The University of Texas at Austin.

1992 The Gowen Site: Cultural Responses to Climate Warming on the Northern Plains (7500-5000 B.P.). Archaeological Survey of Canada, Mercury Series Paper No. 145, Ottawa. Canadian Museum of Civilization, Hull, Quebec.

Walter, Richard W.

1990 A Preliminary Report of Andice Points found on the Llano Estacado. South Plains Archaeological Society Newsletter 97:1-3.

2013 The Elusive Calf Creek Horizon in the Big Bend. In Archaeological Explorations of the Eastern Trans-Pecos and Big Bend: Collected Papers, Volume 1, edited by Pat Dasch and Robert J. Mallouf, pp. 6170. Papers of the Trans-Pecos Archaeological Program, No 6. Center for Big Bend Studies, Sul Ross State University, Alpine.

Wandsnider, L.

1997 The Roasted and the Boiled: Food Composition and Heat Treatment with 
Special Emphasis on Pit-Hearth Cooking. Journal of Anthropological Archaeology 16:1-48.

Waters, M. R. and L. C. Nordt

1995 Late Quaternary Floodplain History of the Brazos River in East-Central Texas. Quaternary Research 43(3):311-319.

Waters, M. R., S. Daniel, J. Urista, and R. Bonnichsen

2001 An Archaic Wooden Bowl from the Gulf Coastal Plain of Texas. Bulletin of the Texas Archeological Society 82:175-179.

Watt, F. H.

1978 Radiocarbon Chronology of Sites in the Central Brazos Valley. Bulletin of the Texas Archeological Society 492:111-138.

Weaver, J. E. and F. W. Albertson

1956 Grasslands of the Great Plains. Johnson Publishing, Lincoln.

Webb, T. and R. A. Bryson

1972 Late and Post Glacial Climate Change in the Northern Midwest, USA: Quantitative Estimates Derived from Fossil Pollen Spectra by Multivariate Statistical Analysis. Quaternary Research 2(1):70115 .

Weber, C. D.

1986 An Analysis of Discriminant Function Values of Andice and Bell Points. $L a$ Tierra 13(3):32-38.

1991 Andice/Bell Projectile Point Notching Failures. La Tierra 18(4):23-38.

2000 Andice/Bell Resharpening Attributes. La Tierra 27(4):45-61.
2002 Andice/Bell Point Use Fractures. La Tierra 29(2):31-44.

Weber, C. D. and L. W. Patterson

1985 A Quantitative Analysis of Andice and Bell Points. La Tierra 12(2):21-27.

Weber, C. D. and M. B. Collins

1994 Chapter 24: A Replication Technique for Andice/Bell Points. In Archaic and Late Prehistoric Human Ecology in the Middle Onion Creek Valley, Hays County, Texas, Volume 2, Topical Studies, by R. A. Ricklis and M. B. Collins, pp. 629-651. Studies in Archeology 19, Texas Archeological Research Laboratory, The University of Texas at Austin.

Weber, G. E.

1968 Geology of the Fluvial Deposits of the Colorado River Valley, Central Texas. Unpublished Master's thesis, The University of Texas at Austin.

Wedel, W.

1957 The Central North American Grassland: Man-Made or Natural. In Studies in Human Ecology, pp. 39-69. Social Science Monographs III. Pan American Union, Washington, D.C.

Weeks, A. W.

1945 Quaternary Deposits of the Texas Coastal Plain between the Brazos River and the Rio Grande. Bulletin of the Association of Petroleum Geologists 29(12):1693-1720.

Weir, F. A.

1976a The Central Texas Archaic. Unpublished Ph.D. dissertation, Department of Anthropology, Washington State University, Spokane. 
1976b The Central Texas Archaic Reconsidered. In The Texas Archaic: A symposium, edited by T. R. Hester, pp. 60-66. Center for Archaeological Research, The University of Texas at San Antonio, Special Report, No. 2.

1979 Greenhaw: An Archaic Site in Central Texas. Bulletin of the Texas Archeological Society 50:5-67.

Werchan, L. E., A. C. Lowther, and R. N. Ramsey 1974 Soil Survey of Travis County, Texas. United States Department of Agriculture, Soil Conservation Service in cooperation with Texas Agricultural Experimental Station.

Wesolowsky, A. B., T. R. Hester, and D. R. Brown 1976 Archeological Investigations at the Jetta Court Site (41TV151), Travis County, Texas. Bulletin of the Texas Archeological Society 47:25-87.

White, R. W.

1995 Calf Creek on the High Plains, Part I: Some Central Oklahoma Panhandle Finds. Bulletin of the Oklahoma Anthropological Society XLII:167-169.

Whitaker, T. W., H. C. Cutler, and R. S. MacNeish 1957 Cucurbit Materials from Three Caves Near Ocampo, Tamaulipas. American Antiquity 22(4, part 1):352-358.

Whittaker, J. C.

1994 Flintknapping: Making and Understanding Stone Tools. University of Texas Press, Austin.

Wilder, M. C., J. K. McWilliams, K. W. Kibler, and M. D. Freeman

2003 Cultural Resources Survey of 4,423 Acres and National Register Testing at Five
Prehistoric Sites, Camp Bullis Military Reservation, Bexar and Comal Counties, Texas. For the U.S. Army Corps of Engineers Fort Worth District, Prewitt \& Associates, Inc., Reports of Investigations, Number 135.

Williams-Dean, G. J.

1978 Ethnobotany and Cultural Ecology of Prehistoric Man in Southwest Texas. Anthropology Research Laboratory, Texas A\&M University, College Station.

Wilson, C. A.

2002 Seasonal Occupation of the Guadalupe Bay Site Based on Fish Otoliths. In: Archaeological Investigations at the Guadalupe Bay Site (41CL2): Late Archaic through Late Prehistoric Occupation along the Channel to Victoria, Calhoun County, Texas, Richard A. Weinstein, editor, pp. 701-714. Coastal Environments, Inc., Baton Rouge.

Wilson, M.

1975 Holocene Fossil Bison from Wyoming and Adjacent Areas. Unpublished Master of Arts thesis, Department of Anthropology, University of Wyoming, Laramie.

1978 Archaeological Kill Site Populations and the Holocene Evolution of the Genus Bison. Plains Anthropologist 23(82):9-22, Part 2: Memoir 14: Bison Procurement and Utilization: A Symposium, edited by L. B. Davis and M. Wilson.

Winsborough, B. M.

1997 Diatoms. In Stratigraphy and Paleoenvironments of Late Quaternary Valley Fills on the Southern High Plains, by V. T. Holliday, pp. 67-82. Geological Society of America, Inc., Memoir 186, Boulder. 
2002a Appendix E: Microscopic Analysis of Sediments from Archaeological Site 41CW54. In Archaeological Testing of Four Prehistoric Sites Along the Guadalupe-Blanco River Authority's San Marcos Raw Water Pipeline, Caldwell and Hays Counties, Texas, by E. R. Oksanen, C. A. Weaver, E. A. Schroeder, and G. T. Goode. PPA Cultural Resources Report 0262 prepared for: Guadalupe-Blanco River Authority.

2002b Appendix F: Paleoenvironmental Analysis of Archaeological Sediments. In Archaeological Caldwell County, Texas, Volume II, by E. A. Schroeder, pp. 139143. Paul Price Associates, Inc., PPA Cultural Resources Report 0330.

2008 Appendix A: Diatom Paleoenvironmental Analysis of Sediments from Archaeological Site 41HA1, in Plainview, Texas. In A Geoarchaeological Survey of the Proposed Plainview Hike and Bike Trail, Hale County, Texas, by C. D. Frederick, J. L. Thompson and K. J. Córdova, pp. 59-68. Texas Department of Transportation, Environmental Affairs Division, Austin, Texas and Center for Archaeological Research, The University of Texas at San Antonio Archaeological Report No. 396.

2011a Appendix F: Paleoenvironmental Analysis of Diatoms and Associated Material in Sediment and Burned Rock Samples from the Root-Be-Gone Archeological Site (41YN452), Young County, Texas, U.S.A. In Root-Be-Gone (41YN452): Data Recovery of Late Archaic Components in Young County, Texas, Volume II, by J. M. Quigg, P. M. Matchen, and C. D. Frederick, pp. 489-514. Texas Department of Transportation, Environmental Affairs
Division, Archeological Studies Program Report 135 and TRC Environmental Corporation, Technical Report Nos. 50907 and 171219, Austin.

2011b Appendix C: Diatom Paleoenvironmental Analysis of Middle and Late Archaic Material from the Granberg Archaeological Site (41BX17), Bexar County, Texas. In Archaeological Significance Testing at 41BX17/271, the Granberg Site along Salado Creek in Bexar County, Texas, by C. M. Munoz, R. P. Mauldin, J. L. Thompson, and S. C. Caran, pp. 219-227. Center for Archaeological Research The University of Texas at San Antonio Archaeological Survey Report No. 393 and Texas Department of Transportation, Environmental Affairs Division, Archeological Studies Program, Report No. 140.

Winsborough, B. M. and J. M. Quigg

2012 Oldest Evidence for Salt Use in Texas and Possibly the New World. Paper presented at the Texas Archeological Society $83^{\text {rd }}$ Annual Meeting in Tyler.

Winterhalder, B.

1981 Chapter 2: Optimal Foraging Strategies and Hunter-Gatherer Research in Anthropology: Theory and Models. In Hunter Gatherer Foraging Strategies: Ethnographic and Archaeological Analyses, edited by B. Winterhalder and E. A. Smith, pp. 13-35. The University of Chicago Press, Chicago.

Wissler, C.

1910 Material Culture of the Blackfoot Indians. Reprinted 1976. AMS Press, Inc., New York. 
Witty, T. A., Jr.

1969 Notes on Flint Hills Archeology. Kansas Anthropological Association Newsletter 14(8):1-5.

1982 The Slough Creek, Two Dog, and William Young Sites, Council Grove Lake, Kansas. Kansas State Historical Society, Anthropological Series Number 10, Topeka.

Wolverton, S.

2002 Zooarchaeological Evidence of Prairie Taxa in Central Missouri during the MidHolocene. Quaternary Research 58(2):200-204.

2005 The Effects of the Hypsithermal on Prehistoric Foraging Efficiency in Missouri. American Antiquity 70(1):91106.

Wood, W. R., editor

1998 Archeology of the Great Plains. University Press of Kansas, Lawrence.

Word, J. H. and C. L. Douglas

1970 Excavations at Baker Cave, Val Verde County, Texas. Bulletin of the Texas Memorial Museum 16, Austin.

White, R. W.

1995 Calf Creek on the High Plains, Part I: Some Central Oklahoma Panhandle Finds. Bulletin of the Oklahoma Anthropological Society XLII:167-170.

Wyckoff, D. G.

1984 Chapter 6: The Foragers: Eastern Oklahoma. In Prehistory of Oklahoma, edited by R. E. Bell, pp. 119-160. Academic Press, Inc., Orlando.
1990 A Radiocarbon Date for Dewey County Dune Development. Oklahoma Archeological Survey Newsletter 10(2):1.

1993 Calf Creek: Traces of 6,000 Year Old Hunting-Gathering Societies. Oklahoma Archeological Survey Newsletter 1:1-2.

1994a Introduction to the 1991 Bulletin. Bulletin of the Oklahoma Anthropological Society XL:1-8.

1994b The Calf Creek Horizon in the Osage Savannah. Bulletin of the Oklahoma Anthropological Society XL:9-10.

1995 A Summary of the Calf Creek Horizon in Oklahoma. Bulletin of the Oklahoma Anthropological Society XLII:179-210.

2005 A Calf Creek Potpourri. Oklahoma Archeology 53(1):20-28.

Wyckoff, D. G. and L. Woodard

1988 At Last, A Radiocarbon Date for Calf Creek. Oklahoma Anthropological Society Newsletter 36(9):4-6.

Wyckoff, D. G. and D. Shockey (editors)

1994 Bulletin of the Oklahoma Anthropological Society XL.

1995 Bulletin of the Oklahoma Anthropological Society XLII.

Wyckoff, D. G., W. L. Neal, and M. Duncan

1994a The Primrose Site, 34MR65, Murray County, Oklahoma. Bulletin of the Oklahoma Anthropological Society XL:1167.

Wyckoff, D. G., D. Morgan, and L. Woodard 1994b Calf Creek on the Cherokee Prairie, Part I: The Arrowhead Ditch Site (34MS174). 
Bulletin of the Oklahoma Anthropological Society XL:307-328.

Yanovsky, E.

1936 Food Plants of the North American Indians. Miscellaneous Publications 27. United States Department of Agriculture, Washington D.C.

Yates, B. C.

1982 Chapter 15: Vertebrate Faunal Remains. In Archeological Investigations at the San Gabriel Reservoir District, Central Texas, Volume 2, compiled and edited by T. R. Hays, pp. 15-57 to 15-227. Archaeology Program, Institute of Applied Sciences, North Texas State University, Denton.
Yates, B. C. and E. L. Lundelius, Jr.

2001 Chapter 8: Vertebrate Faunal Remains from the Aubrey Clovis Site. In The Archaeology and Paleoecology of the Aubrey Clovis Site (41DN479), Denton County, Texas, by C. Reid Ferring, pp. 103119. Center for Environmental Archaeology, Department of Geology, University of North Texas, Denton.

Zarrillo, S. and B. Kooyman

2006 Evidence for Berry and Maize Processing on the Canadian Plains from Starch Grain Analysis. American Antiquity 71(3):473499.

Zierhut, N. W.

1967 Bone Breaking Activities of the Calling Lake Cree. Alberta Anthropologist 1(3):33-37. 
This page intentionally left blank. 


\subsection{GLOSSARY OF TECHNICAL TERMS}

A Horizon: The near surface horizon of a natural soil. This is a carbon rich soil horizon characterized by an accumulation of partially decomposed to decomposed organic matter and eluvial loss of constituents such as clays and carbonates, which tend to accumulate in the deeper B horizon. The A horizon represents the upper solum of a soil. Lower case letters with the upper case letter A indicate specific characteristics of that A horizon. An Ab designation indicates the A horizon is buried. An Ap designation indicates a disturbed or anthropically modified soil such as in a plow zone.

Accelerated Mass Spectrometry (AMS): A laboratory technique that separates and identifies ions based on their mass to charge ratios, to measure the relative concentrations of carbon atoms. This technique is used in radiocarbon dating tiny particles of carbon in organic remains and residues.

Acidic: A chemical term that refers to any acidforming compound, which is a compound that dissolves in water to produce a solution with a high concentration of hydrogen ions. If a material is acidic, its $\mathrm{pH}$ is less than 7.0.

Acryloid B-72: This is a conservation material used to stabilize or glue artifacts together. It is an ethyl methacrylate copolymer.

A.D.: Anno domini in Latin. "In the year of our Lord." For example, A.D. 1000 is 1,000 years After Christ (A.D.). This is generally used when a B.P. radiocarbon date is calibrated to the tree ring results with a calibration formula.

Aerophilous Habitats: This means depending on free oxygen or air; "aerobic fermentation. This can include damp soils, wet plants and rocks, marshes, and mudlands.
Agavaceae: A plant family name that refers to fiber, vascular bundle, or the central stem sections that cannot be specifically identified as agave (Agave), yucca (Yucca) or sotol (Dasylirion).

A/I Ratio: The ratio of $\mathrm{D}$-allosioleucine to $\mathrm{L}$ isoleucine (A/I) proteins contained in Rabdotus snail shells. This ratio is a measure of age. All amino acids are initially in the L-form, but over time, it converts or racemizes to the D-form. This ratio can be used to measure age unless the shells have been exposed to prolonged heat from a hearth, which will accelerate the chemical change and cause exceptionally abnormal results.

Alkalinity: A chemical term that refers to any acid forming compound that dissolves in water to produce a solution with few hydrogen atoms. If the solution is alkaline it is has a $\mathrm{pH}$ greater than 7.0 in contrast with acidic solution which has a $\mathrm{pH}$ less than 7.0.

Allostratigraphic Unit: The term is used in discussion soil stratigraphy. Allostratigraphy is a method of subdividing and correlating rocks and sediments into units on the basis of bounding unconformities.

Allounits may be lithologically diverse and may or may not be formally defined. The allostratigraphic unit is defined by 3D bounding surfaces.

Alluvium: Clastic sediments, such as sand, silt, or clay deposited by a flowing stream, either in the channel or outside the channel during overbank flooding.

Antiquities Code of Texas: This is the state law passed in 1977 to protect and preserve prehistoric and historic cultural resources on lands owned by the State and its political subdivisions (i.e., cities, towns, municipalities, and parks).

Anthropogenic: This relates to or resulting from the influence of human beings on nature. 
Applied Force: Force is applied to remove a flake from a parent object/core. This term is used when the type of force is unknown or questionable, in contrast to percussion, indirect percussion, direct pressure or natural force.

Argillans: These are clay coatings on ped- or pore surfaces.

Atlatl: This is a short stick, roughly 40 to $60 \mathrm{~cm}$ long, with a handle on one end and a groove or peg at the other end, used for throwing a dart shaft or light spear. It provides much greater leverage and force to the throw by extending the length of the arm. This was the primary instrument used to propel projectiles before the bow and arrow.

Azelaic Acid: This is a chemical biomarker in lipid residue analysis of rocks and a short chain dicarboxylic acid associated with the oxidation of unsaturated fatty acids. Its presence may indicate plant seed processing.

Barbule: A small barb or pointed projection, especially one that fringes the edges of the barbs of feathers. Feather barbules are of two types, pennaceous (making up the flight surface of the feather) and plumulaceous (insulation barbule). Plumulaceous barbules are the most useful for identification of the type of bird from which the barbule came, but the pennaceous barbules are also useful though generally not as specific as to the type of bird.

B.C.: The abbreviation for Before Christ, in contrast to After Christ (A.D.).

Benthic: This relates to or occurring at the bottom of a body of water and is used in discussing diatoms. Benthic diatoms include those attached to or crawling on aquatic plants, larger algae, rocks, pebbles, sand, mud and debris. These species are typically found in vegetated, shallow streams and ponds.
Bentonitic Clay: Bentonite is an absorbent aluminum phyllosilicate, impure clay consisting mostly of montmorillonite, also referred to as Montmorillonite. This clay has a high ionic displacement, and shrinks and swells with moisture content.

Beta ( $\beta$ )-Sitosterol: This is a sterol found in plant tissue and is a structural lipid present in cell membranes. This is one of the chemical markers used in the lipid residue analysis.

Biface or Bifacial: A stone tool that has two distinct sides or faces, both of which have been substantially worked and/or flaked. The biface may take the form of many shapes and sizes and used in diverse activities.

B Horizon: The lower solum of a natural soil. A B horizon is a mineral soil horizon characterized by an accumulation of constituents such as clays, carbonates or salts, or organic complexes that have been translocated from the A horizon. Common subordinates include lowercase letters such as $t$ as $\mathrm{Bt}$, which indicates accumulation of illuvial clays. The lowercase $\mathrm{k}(\mathrm{Bk})$ indicates accumulation of carbonate. The lower case $\mathrm{w}$ indicates structural or color changes with no significant accumulations of illuvial material.

Biogenetic: This includes such microfossils as freshwater sponge spicules, phytoliths, diatoms, and are presented in the phytolith report. These particles all have the same chemical composition and specific gravity, if present in a soil sample they are recovered together.

Biomarker: This is in lipid residue analysis, a molecular associated with a narrow range of substances, or the presence and distribution of certain types of lipids that enables a residue to be identified with a high degree of precision.

Biosilicates: This is a general term to include various tiny hard bodies that contain silicon and are 
developed in plants and include; phytoliths, diatoms, algal statospores, and sponge spicules.

Bioturbation: The churning and mixing of sediments by living organisms, including burrowing rodents, insects, worms, and plant roots.

Biplot: A biplot is a special type of graph following from principal component analysis on which both the samples and elements are displayed. Examination of a biplot from the principal component analysis of ceramic specimens often leads to identification of the analyzed elements responsible for differentiating groups of specimens from one another.

Bivariate Plot (Scatter Plot): A two-dimensional graph where the $\mathrm{x}$-axis and $\mathrm{y}$-axis symbolize a pair of measured or calculated variables. The points on a bivariate plot represent the position of individual samples. These graphs are used to recognize possible structure in a data set.

B.P.: An abbreviation for before present, which in radiocarbon dating is referenced to the standard year A.D. 1950, which is considered "present". Generally B.P. dates have not been tree ring corrected using one of the calibration formulas.

$\boldsymbol{\beta}$-sitosterol and Stigmasterol: These are sterols associated with plant products, which can be detected during lipid analysis. Its presence indicates plant residues.

Bulb of Force or Percussion: This is the rounded protrusion on the ventral/inside surface of a flake just below the point of contact caused by a conelike diffusion of force of the detaching blow.

Bulliform Cells: These are plant cells involved in tissue contraction or water storage, or of uncertain function and are a type of phytoliths. These are large keystone shaped-cells.

Burned Rock Dump: A loose cluster of previously heated rocks that exhibits no horizontal patterning to the positions of the rocks and lacks indications of in situ heating/burning, such as a prepared basin, lenses of charcoal or ash, and/or the absence of an oxidation rim. Scattered charcoal or other cultural items may be present between or around the burned rocks.

Burned Rock Midden: This is an accumulation of a large quantity of discarded burned rocks previously employed in multiple cooking activities. These accumulations were the results of long extensive cooking episodes generally in association with rock ovens.

Cache: The deliberate placement or storage of artifacts in a tight grouping such as tools or pottery purposely placed for storage, generally in the ground. Caches are thought to represent storage with the intention of subsequently returning to retrieve the stored objects.

C $_{3}$ Plants: A Calvin-Benson photosynthetic pathway that most trees and flowering bushes use to assimilate carbon dioxide into their systems. The average carbon isotope of $\mathrm{C}_{3}$ matter is $-26.5 \%$ with a range from about $-19.0 \%$ to $-34.0 \%$.

C $_{4}$ Plants: A Hatch-Slack photosynthetic pathway used by most arid (xeric) grasses and maize (corn) to assimilate carbon dioxide into their systems. The average carbon isotope of $\mathrm{C}_{4}$ matter is $-12.5 \%$ with a range of $-6 \%$ to $-19 \%$. These plants are more resistant to stress due to lack of water, but more susceptible to cold temperatures.

C Horizon: Weathered, but relatively unaltered parent material at the base of a soil profile, generally below the B horizon. This term is roughly synonymous with subsoil, although the latter term is often used to encompass the lower B horizon.

Calcareous: Rocks, minerals, or sediment containing calcium carbonates.

Calcic Horizon: This is a mineral soil horizon of secondary carbonate enrichment that is $>15 \mathrm{~cm}$ thick, has a $\mathrm{CaCO}_{3}$ equivalent of $>150 \mathrm{~g} \mathrm{~kg}^{-1}$, and 
has at least $50 \mathrm{~g} \mathrm{~kg}^{-1}$ more calcium carbonate equivalent than the underlying $\mathrm{C}$ horizon.

Calcined: To heat a substance like bone to a high temperature but below the melting or fusing point, causing loss of moisture, reduction or oxidation, and the decomposition of carbonates and other compounds. This turns a bone white color.

Calcite: These are colorless to white crystals of a carbonate mineral mainly consisting of calcium, the principal mineral of limestone and marble.

Calcium: A chemical element with the symbol ' $\mathrm{Ca}$ ' and atomic number 20. Calcium is a soft gray alkaline earth metal, and is the fifth most abundant element by mass in the Earth's crust. Calcium is also the fifth most abundant dissolved ion in seawater by both molarity and mass, after sodium, chloride, magnesium, and sulfate.

Calibration: This is used in radiocarbon dating to convert a radiocarbon age in B.P. time into calendar years.

Caliche: A more or less cemented deposit of calcium carbonate in soils of warm-temperate, subhumid to arid areas. Caliche, normally white, occurs as soft, thin layers in the soil or as hard, thick beds just beneath the solum, or it is exposed at the surface by erosion.

CAM Plants: A photosynthetic pathway for assimilating carbon dioxide into plants that can change from $\mathrm{C}_{3}$-like to $\mathrm{C}_{4}$-like pathways depending on the diurnal (day or night) cycle. Most succulent plants such as cactus have crassulacean acid metabolism (CAM) pathways. The carbon isotope values of most CAM plants in Texas such as Agave lechuguilla and Opuntia englmannii are similar to the values in $\mathrm{C}_{4}$ plants (see Eickmeier and Bender 1976).

Cambic Horizon: This is a nonsandy, mineral soil horizon that has soil structure rather than rock structure, contains some weatherable minerals and is characterized by the alteration or removal of mineral material.

Campesterol: This and stigmasterol and sitosterol are sterols found in plant tissue, which can be detected during lipid analysis. Its presence documents plants were processed.

Canonical Discriminant (CD) Analysis: This is a dimension-reduction technique related to principal component analysis and canonical correlation.

Carbonates: These are rock or mineral classes that include limestone, calcite, ooids, and bioclasts. White carbonate filaments are often observed in C horizons of soils.

Cementation: The term here is used in reference to teeth. This is the process of adding calcified substance in layers to a tooth over time. The layering is a reflection of the growth of the animal and thus potential for determining the age the tooth and thereby the animal.

Chalcedony: A cryptocrystalline variety of quartz or chert. Chalcedony is often a component of other cherts. It may be translucent or semitranslucent, has a wax-like luster, and generally is white, pale blue, gray, brown, or black in color.

Cheno-am: A term used in botanical classification that includes the plant family of Chenopodiaceae (goosefoot) and the genus Amaranthus (pigweed), with tiny seeds that are indistinguishable from each other.

Chloridoid: These are short cell phytoliths that are squat and tall saddle-shaped, and occur dominantly in $\mathrm{C}_{4}$ grasses such as grama grass (Bouteloua sp.) and buffalo grasses (Bouchloe sp.). These plants thrive in warm, arid to semiarid regions in which the available soil moisture is very low, thus thrive in the shortgrass prairies during the hot summers.

Cholesterol: This is the major sterol in animal tissue, which can be detected during lipid analysis. Its presence indicates animal residues. 
Clast: Any detrital particle of sediment created by the weathering and disintegration of a larger rock mass and transported by water, wind, or ice. Clasts also include discrete particulates created and deposited by volcanic action.

Clay: This is mineral sediment particles less than 0.002 millimeters in diameter. As a soil textural class, soil mineral that is 40 percent or more clay, less than 45 percent sand, and less than 40 percent silt.

Cluster Analysis (CA): A type of numerical classification that uses the value of attributes to cluster data. Clustering is the classification of objects into groups so that objects from the same cluster are more similar to each other than to objects from different clusters. Often similarity is defined according to a distance measure. Clustering is a common technique for statistical data analysis, which is used in many fields, including data mining, pattern recognition, image analysis and bioinformatics.

Collagen: This is the fibrous protein that forms most of the organic component of bone. This is the material generally radiocarbon dated when dating bones.

Colluvium: Soil material, rock fragments, or both, moved by creep, slide, or local wash that is deposited at the base of steep slopes.

Complex: A group of archeological sites that date to the same time period and that contain similar artifacts. This term expresses a relationship of common cultural or technological traits in assemblages within widespread geographic area.

Component: An archeological site or portion of a site that is spatially and chronologically discrete from other accumulations of artifacts. These can be horizontally or vertically differentiated.

Conifers: Any member of the order Pinales, woody plants that bear their seeds and pollen on separate, cone-shaped structures. They constitute the largest division of gymnosperms, with more than 550 species. Most are evergreen, upright trees and shrubs. They grow throughout North American and prefer temperate climate zones. Conifers include the pines (Pinus), junipers (Juniperus), spruces (Picea), hemlocks (Tsuga), firs (Abies), larches (Larix), yews (Taxus), cypresses (Cupressus), bald cypresses (Taxodium), Douglas firs (Pseudotsuga), and related groups. The trees are the source of resins, volatile oils, turpentine, tars, and pharmaceuticals detected in the lipid analysis.

Conventional Radiocarbon Age: This is the age determined by the laboratory using the Libby halflife of 5569 years, with correction for sample isotopic fractionation to a normalized or base value of -25.0 per mille relative to the ratio of carbon 12 /carbon 13 in the carbonate standard VPDB Cretaceous belemnite formation at Peedee in South Carolina, zero B.P. (Before Present) is defined as AD 1950, and the assumption that global radiocarbon levels are constant.

Context: The association and position of artifacts, materials, and cultural features that are used by archeologists to interpret space, time, and culture.

Core: This is the nucleus/mass/cobble from which flakes are driven off either to obtain the flakes or reduce the piece into a tool. The core usually has negative flake scars with either patterned flake removals (prepared core) or random scars (unprepared core).

Cortex: The natural surface or rind, on chert. This can be either smooth from being round in water or rough from it natural condition.

Crazing: These are minute surface cracks observed in chert debitage, often in cross-hatched patterns that are caused by the object having been subjected to intensive heat. 
Criterion of Abundance: If a large groups of specimens in an assemblage is represented by a single, homogenous, compositional fingerprint and the actual source is unknown, then the criterion of abundance indicates that these is a high probability the groups was produced at lone location.

Ctenoid: Fish scales that have a toothed/spiny outer edge, and are usually found on fish with spiny fin rays, such as bass, sunfish, perch, and crappie.

Cumulic Soil: A soil formed in a setting experiencing relatively slow deposition, so that freshly introduced sediment is incorporated into the A horizon, leading to overthickening of the surface horizon. Cumulic soils are common in alluvial overbank and colluvial settings.

Cucurbits: A plant of the squash family (Cucurbitaceae) and includes wild gourds (Cucurbita foetidissima or Buffalo gourd) and the melon subgroup. These are tuberous plants; require little water, well-adapted to desert environments. They contain high amounts of protein and carbohydrates and yield abundant oils. The fresh young gourd can be eaten like squash. The seeds are edible and several Native American groups used them for food. (see www.texasbeyondhistory.net/ethnobot/images/cuc urbitabg).

D-alloisoleucine: This is an amino acid used in part with L-isoleucine, another amino acid, to measure the organic content in snail shells. In modern material essentially all amino acids are in the Lform, but they gradually convert/epimerize to the D-form over time and therefore can be used to determine a relatively age.

Debitage: The waste material from stone tool manufacturing, which has little or no specific characteristics such as a bulb of force or platform.

Dehydroabietic Acid: This is a biomarker that indicates the presence of conifer products, which may have been introduced from firewood, resins or other conifer products. This acid can be detected in lipid residue analysis.

Dendrite: An oxide of manganese that has crystallized in a branching pattern as in the dark inclusions in moss agate.

Deposition: The accumulation of sediments or gravels laid down by natural agencies such as moving water, or artificial agencies such as dumping.

Diatoms: These are single-celled algae whose cellular contents are enclosed between two valves of silica that are preserved when the organism dies. Often these are preserved in ponds, streams, and important to stream ecology. These are useful in reconstructing aquatic paleoenvironments.

Downy Feathers: These are small, soft, fluffy, feathers found under the contour feathers. They are plumaceous, and have many non-interlocking barbs, lacking the barbules and hooklets seen in contour and flight feathers.

Epiphyseal: This is the end of a long bone, initially separated from the shaft (diaphysis) by a section of cartilage that eventually ossifies so that the two portions fuse together uniting with the bone through further ossification over time. When separated from the shaft or main body, the bone is considered immature and once attached the shaft it is considered mature.

Epiphyte: This is a plant that derives its moisture and nutrients from the air and rain and grows usually on another plant. This is used in the discussion of the diatoms.

Eraillure Scar: A small enigmatic flake scar formed between the bulb of force and the bulbar scar.

Erosional Unconformity: A significant break or gap in the geological or depositional record, indicative of removal of the older unit prior to renewed deposition. 
Ester: This is an organic compound that contains a carbonyl group linked to an alkyl group through an oxygen atom; organic compounds synthesized from a carboxylic acid and an alcohol in the presence of water.

Euclidean Distance: In mathematics, the distance or metric is the ordinary distance between two points that one would measure with a ruler, which can be proven by repeated application of the Pythagorean Theorem.

Eutrophic: Having waters rich in mineral and organic nutrients that promote a proliferation of plant life, especially algae, which reduces the dissolved oxygen content and often causes the extinction of other organisms. This is used in the discussion of diatoms.

Facies: A definable subdivision of a formal or informal stratigraphic unit. This term is used in discussing soil deposition.

FAMES: This is an abbreviation for fatty acid methyl esters (FAMES) and is prepared by treating the dry lipid extract with $3 \mathrm{~mL}$ of $0.5 \mathrm{~N}$ anhydrous hydrochloric acid in methanol $\left(68^{\circ} \mathrm{C} ; 60 \mathrm{~min}\right)$. This is part of the lipid residue analysis.

Fatty Acids: The major constituents of fats and oils (lipids) that occur in nature in plants and animals. They are insolubility in water and relatively abundant compared to other classes of lipids. Fatty acids may be absorbed into porous archeological materials during cooking, including heated rocks and ceramics, or ground into manos, metates, or mortar holes. Some major fatty acids are referred to as C12:0, C14:0, C15:0, C16:0, C16:1, C17:0, C18:0, C18:1w9, C18:1w11, C18:2.

Feather Termination: This is found on the distal end of a flake that terminates at the margin of the target object and leaves a sharp edge. This reflects precision in flaking an object.
Feature: In archeological terms it is used to identify a nonportable item or group of items produced by people or natural causes. These require documentation in the field, such as a house structure, a cluster of artifacts, a hearth, cluster of lithic debitage, concentration of burned rocks, a tree burn, etc.

Floodplain: A nearly level land, alluvial plain that borders a stream or river and is subject to periodic flooding during periods of high water.

Flotation: This is the process by which organic remains, primarily charred plant fragments, are recovered from archeological sediment samples using water as the separating agent. This may be performed either by hand in various containers or by mechanical equipment especially designed for this process.

Flotsam: Debris floating on the water and in this case it would have been wood, grass and other organics.

Foraminifera: These are a phylum or class of amoeboid protists, characterized by streaming granular ectoplasm that among other things is used for catching food, and commonly by an external shell, or test, made of various materials and constructed in diverse forms. All but perhaps a very few are aquatic and most are marine, the majority of which live on or within the seafloor sediment, while a smaller variety are floaters in the water column at various depths (plankton). A few are known from freshwater or brackish conditions and some soil species have been identified through molecular analysis.

Frustules: This is the hard and porous cell wall or external layer of diatoms. The frustule is composed almost purely of silica, made from silicic acid, and is coated with a layer of organic substance, which was referred to in the early literature on diatoms as pectin, a fiber most commonly found in cell walls of plants. 
Gar: This is a fish in the Lepisosteidae family and recognized by their long sharply-toothed jaws, diamond-shaped and nonoverlapping hard scales, with their dorsal and anal fins far back on the body.

Gas Chromatography (GC): This highly technical measuring instrument separates and measures the amount of elemental components of a specific sample by the measurement of light passed through gas at regulated temperatures, which allows the detection of fatty acids at the nonogram $\left(1 \mathrm{X} \quad 10^{-9} \mathrm{~g}\right)$ level. High temperature gas chromatography is used to separate and assess a wide range of lipid components, including fatty acids, long chain alcohols and hydrocarbons, sterols, waxes, terpenoids and triacylglycerols (Evershed et al. 2001). The molecular structure of separated components is elucidated by mass spectrometry (Evershed 2000).

\section{Gas Chromatography-Mass Spectrometry (GC-} MS): This is an analytical technique that enables the mass analysis and identification of components separated from a sample by gas chromatography; an analytical technique that combines gas chromatography with mass spectrometry.

Gelatinization: In regards to starch grains this is a morphological change (distortion of the original shape) in the grain caused by the exposure to heat and water when starches are cooked.

Geomorphology: That part of geography concerned with the form and development of the landscape.

Geophytes: These are plants with underground storage organ such as bulbs (i.e., onions, camas, and false garlic), tubers, roots, and rhizomes that are a reserve of carbohydrates, nutrients, and water. These storage organs can be collected, cooked, and eaten as part of the human diet. The study of these geophytes from an archeological site aids in determining the diet of the past occupants.
Glume: A plant biology term that pertains to small dry membranous tissues found at the base of a grass spikelet or each flower in a sedge or related plant.

Gorget: These are usually a polished stone, sometimes of shell or limestone, with holes drilled in it. These were presumably worn as jewelry.

Gourd: see Cucurbits

GPR: This is the abbreviation for Groundpenetrating radar, which is a geophysical method that uses radar pulses to image the subsurface. This nondestructive method uses electromagnetic radiation in the microwave band (UHF/VHF frequencies) of the radio spectrum, and detects the reflected signals from subsurface structures. GPR can have applications in a variety of media, including rock, soil, ice, fresh water, pavements and structures. In the right conditions, practitioners can use GPR to detect subsurface objects, changes in material properties, and voids and cracks.

Graticule: A device used in the microscope to measure the size of items under magnification.

Hard-Hammer: This is a striking instrument, usually a rock used to apply direct force to remove a flake from a core or target object.

Hard/High Silica Polish: This is a residue that comes from the material that a stone tool came in contact with. This type of polish is generally produced when processing plants with high silica content in the plant tissues such as grasses, wood, reeds, and potentially soil. This polish was detected during high-powered microscopic use-wear studies conducted during stone tools analysis.

Hard Polish: This is polish observed on stone tools that have come in contact with bone, antler, wood, or possibly plants with high silica.

HCL: Hydrochloric acid, which is the solution of hydrogen chloride (HCL) in water. It is a highly corrosive, strong mineral acid and has major industrial uses. 
Heat Treated: This term is used to describe lithic materials that exhibit waxy luster or traces of reddish colors and results from low, controlled heating of objects to improve the material quality and control in knapping.

Heating Element: This is an intentional, intact and localized spot were a human created a fire in an archeological site or component. This is generally evidenced by quantities of wood charcoal, prepared basin, lenses of charcoal or ash, and possibly an oxidation rim often accompanied by intentionally placed rocks, either lining the margins or directly amongst the charcoal. The function of this fire may reflect many different things, such as for heat to warm a person, to cook on, or to heat rocks for other uses. The specific contents may provide clues as to a more specific function or length of use.

Heavy Fraction: This is in reference to the heavier materials that do not float, but are collected during the flotation process. That material is collected and often sorted into materials types, counted and weight by category.

Hematite: A blackish-red to brick-red mineral, $\mathrm{Fe}_{2} \mathrm{O}_{3}$, the chief ore of iron. This mineral is found naturally in the various geological formations.

High Temperature-Gas Chromatography (HTGC): This instrument is used to separate and assess a wide range of lipid components, including fatty acids, long chain alcohols and hydrocarbons, sterols, waxes, terpenoids and triacylglycerols (Evershed et al. 2001). This is used with mass spectrometry (MS) to elucidate the molecular structure of separated components. Triacylglycerols, diacylglycerols and sterols can be used to distinguish animal-derived residues, which contain cholesterol and significant levels of both triacylglycerols, from plant-derived residues, indicated by plant sterols, such as $\beta$-sitosterol, stigmasterol and campesterol, and only traces of triacylglycerols.
Hilum: The scar on a seed, such as a bean, indicating the point of attachment to the funiculus. The nucleus of a starch grain.

Hinge Termination: This is the distal end of a flake that has not traveled through the entire target piece and results in a right angle to the longitudinal axis. This is usually a rounded or blunt end of the flake.

Holocene: Geological time period spanning roughly the last 10,000 years before present. The Holocene is roughly equivalent to the Post-glacial period, and often referred to as the "Recent" period in geology. Many investigations consider the Holocene to be an interstadial in the ongoing Pleistocene epoch.

Horizon: A discrete, relatively uniform layer in a soil profile that is typically parallel with the surface and formed as the result of pedogenic process.

Humates: These are substances formed from the biological and chemical breakdown of animal and plant life over time. Humates are made up of compounds and materials that plant life on earth absolutely needs for growth. Humates contain a mixture of organic acids, including humic acids, fulvic acids, and macromolecules of amino acids, amino sugars, and peptides. The chemistry of humate is so complex it can't really be broken down.

Humus: A dark, organic-rich substance consisting of decomposed organic material (animal or vegetable) and is found in the soil.

Hydrolysis: This is a process by which complex molecules are cleaved into smaller molecules through the uptake of a water molecule. This process in conjunction with heat facilitates digestion of complex plant tissues.

In Situ: An artifact in its original position that was placed or deposited within the landscape. 
Instrumental Neutron Activation Analyses (INAA): This is a technique used to determine the elemental composition of a sample (i.e., chert or sherd) through the analysis of characteristics gamma rays emitted by specific radioactive isotopes artificially created through neutron capture reactions in a nuclear reactor.

Integrity: This refers to the degree of intactness of archeological deposits, components, features, or artifacts.

Inulin: This is a carbohydrate, a fructan is not digestible via acid hydrolysis, the typical way we digest carbohydrates such as starch.

Isomers: These are compounds with the same molecular formula that differ with respect to how the atoms are joined. Structural isomers differ with respect to the order in which atoms are joined. Stereoisomers differ with respect to the arrangement of atoms in space but the order in which the atoms are attached is identical. These are found during the lipid analysis.

Isotope: An atom of an element. One of two or more forms of a chemical element, differentiated by the number of neutrons contained in the nucleus. Unstable isotopes are radioactive whereas stable isotopes are not.

Jasper: A dense, cryptocrystalline, opaque to slightly translucent variety of chert associated with iron oxide impurities that give the rock various colors. Most often red, but can be yellow, green, grayish-blue, brown, or black.

Knapping: A term used to describe the manufacturing of prehistoric chipped stone tools using different techniques, such as pressure and/or percussion methods, to chip/flake a target mass of material to form a useful tool.

Lamellae: This is a term used to discuss structure observed in soils. A thin plate-like structure, often one amongst many lamellae very close to one another, with open space between.

Legume: A plant that produces a bean or seedpod in various forms consisting of one cell and/or two valves. Common legume plants across Texas include such plants as; mesquite, Texas ebony, various acacia, retama, Dalea sp., mimosa, and rattlebush.

Light Fraction: This is in reference to light organic material that floats to the surface during the flotation process. That material is collected and often sorted into materials types with charred seeds and charcoal, then identified by a botanist.

Lily: These are flowers with bulbs and are different than other lilies such as rain lilies. True lilies (Lilium) as identified by the starch grains are of the family Liliaceac. These like well-drained, rich acidic $6.5 \mathrm{pH}$ soils and need both moisture and light. True lilies are not currently part of the flora of Texas.

L-isoleucine: This is an amino acid used in part with D-alloisolucine, another amino acid, to measure the organic content in snail shells. In modern material essentially all amino acids are in the L-form, but they gradually convert/epimerize to the D-form and can be used to determine the approximate age.

Lip: This term is used in describing the projection on a core or artifact, which results from the bulbar scar. A concavity caused by a slight overhang on the proximal, ventral surface of some flakes. This is generally associated with percussion using a softhammer or by pressure.

Lipids: These are hydrophobic constituents of living tissues including fatty acids, alcohols, triacylglycerols, sterols, bile acids, and waxes. Lipids are present in tissues of all living organisms in varying proportions. These are insoluble in water, relatively easy to extract, and are readily amenable to separation and characterization. 
Lithic: Means "of stone". This term is used by archeologists to refer to stone artifacts and the debris that result from the manufacture of stone artifacts.

Lithology: The scientific study and description of rocks, especially at the macroscopic level, in terms of their color, texture, and composition. The gross physical character of a rock or rock formation.

Little Barley: This is a short winter annual bunch grass with a scientific name of Hordeum pusillum in the Poaceae grass family. It has a rapid growth period with a brown seed that develops after spring and is available in the early summer. The seed head consists of flattened spikes. It is considered low in protein and is intolerant to shade. This grass has a low drought tolerance but can grow with only 10 inches of rain per year. It is considered a $\mathrm{C}_{3}$ grass (-26.7\%o; Smith and Brown 1973) adapted to fine and medium soil (http://plants.usda.gov 2011).

Loam: This is soil composed of sand, silt, and clay in relatively even concentration (about 40-40-20\% concentration respectively). Loam soils generally contain more nutrients and humus than sandy soils, have better drainage and infiltration of water and air than silty soils, and are easier to till than clay soils.

Macrobotanical: These are remains of plant tissues, such as wood, charcoal, and seeds that one can see with the naked eye.

Mahalanobis Distance: This is a statistical calculation used to determine the probability that a specimen is a member of any of the groups identified through discriminant analysis. This makes it possible to describe the separation between groups or between individual samples and groups on multiple dimensions.

Major Element: A category of seven elements that typically occur in systems at levels greater than 1 wt. percent.
Mano: This is a hand-held stone, usually sandstone or quartzite, used to grind plants such as corn, nuts, seeds, or other vegetable matter and sometimes other rocks. It is used in conjunction with a stone metate that plants are placed on to perform the grinding.

Manuport: An object, usually a rock, that was transported by humans to the place it was recovered, but its macroscopic appearance does not indicate it had been artificially altered to form a specific tool or other kind of artifact.

Mass Spectrometer (MS): This is an instrument used to produce molecular and elemental ions, sort them according to mass and detect abundances to establish the composition, determine molecular structure or measure isotopic ratios of specific elements.

Matrix: Refers to the sediments in which the artifacts at an archeological site are encased, or surrounds.

Megahertz: This is a unit of alternating current (AC) or electromagnetic (EM) wave frequency equal to one million hertz $(1,000,000 \mathrm{~Hz})$, and is abbreviated as MHz. Megahertz is commonly used to express microprocessor clock speed.

Mesic Condition: A relatively moist interval of time generally used in the context of climatic conditions.

Metate: A slab of rock in which vegetable matter is placed upon for the purpose of grinding. The natural surface becomes polished and a concave depression forms on the metate surface from continued grinding. The grinding stone used with the metate is called a mano.

Microdebitage: Any stone or lithic material from the manufacture of stone tools that is less than 4.0 $\mathrm{mm}$ in diameter. Microdebitage is often recovered in sieving or floating sediments from archeological deposits. 
Microfossils: These include a variety of very tiny microscopic residues that include such things as starch grains, diatoms, phytoliths, pollen, and organic remains that are only detectable and visible under high-powered microscopes.

Midden: This is somewhat of a catch-all term. It generally refers to an accumulation of cultural material such as a zone of burned rocks, and it is often used to refer to a thick accumulation of mixed cultural material in a vertical zone.

Migmatite: This rock is a mixture of igneous and metamorphic rock. These rocks form under extreme temperature conditions during volcanic activity, prograde metamorphism.

MNI: The minimum number of individuals represented in a given faunal or human osteological collection. This is determined by the greatest number of any particular bone element representing a given species in a sample of bones.

Mollusks: These include bivalve clams, mussels (Pelecypoda), and univalve snails, whelks, and conches (Gastropoda). They are soft bodied and unsegmented with a muscular foot, a head region, a visceral mass, and a fleshy mantle. The shell is composed of proteins and crystalline calcium carbonate. The association of mollusks in the sediments reflects water quality, salinity, and stream flow.

National Historic Preservation Act: This is the federal law passed in 1966 that establishes a program for the preservation of significant historic properties throughout the United States.

National Register of Historic Places: This is the federal list of significant historic properties maintained by the National Parks Service.

Organic: Compounds that contain carbon and are associated with living organisms. Materials or objects that contain organic carbon can be radiocarbon dated.
Overbank Deposits: The deposition of fine silts and clay particles that are left on terrace tops and banks when water in creeks exceeds the capacity of the channel and drops the suspended sediments in the lower energy environment. Overbank depositional processes usually cause minimal movement to large objects on the terrace top.

Overshot: This is a flake that goes beyond the opposite margin of the tool and bends over to the other side. This is also referred to as outrepassé in French.

Oxidation: A chemical process wherein oxygen is added to minerals or other compounds; weathering oxidizes minerals; burning wood and rusting metal are types of oxidation.

Paleoenvironment: Ancient or past environments.

Paleosol: Generally refers to a soil that developed an A horizon and was subsequently buried by younger deposits.

Palimpsest: Archeologically, refers to the inability to distinguish and separate material remains from repeated occupations by a succession of cultural events of different ages due to their deposition and intermixing over time on relatively stable surfaces. Some palimpsest assemblages are buried following a long period of exposure.

Palynology: The study of fossil pollens and spores that are produced by plants. Commonly used to reconstruct the floral communities in paleoenvironment.

Panicoid: A group of short cell grass phytoliths that result from tall $\mathrm{C}_{4}$ grasses and are taxonomically diagnostic of switch grass (Panicum), big and little bluestem (Andropogon sp.), and Indian grass (Sorghostrum). These grasses do well in warm, moist environments and are a major species in the tallgrass prairies. These include basic morphotypes that include simple lobate, panicoid-type, cross, and other Lobate forms. 
Ped: A unit of soil structure such as an aggregate, crumb, prism, block, or granule, formed by natural processes.

Pedogenesis: The dynamic process of soil formation and development, which typically leads to the formation of a darkened, organic-rich Ahorizon at or near the surface, and the downward movement of fine clays into, and/or the formation of carbonate nodules within, the underlying B horizons.

Pedoturbation: A general term used to describe soil that has been mixed.

Pee Dee Belmnite: A limestone found in Southern Carolina used as the international standard for various compositional (carbon and oxygen isotopic and elemental) analyses such as radiocarbon dating.

Percussor: An implement used for striking an object such as a soft (antler billet) or hard-hammer (rock).

pH: The standard numerical designation of acidity and alkalinity commonly used in reference to soil. A neutral $\mathrm{pH}$ value as in distilled water is 7.0. Lower and higher values are acidic, and base (alkaline, respectively.

Phase: A group of related archeological traits (e.g., artifacts, features) that contain similar cultural material and date to one relatively narrow time period within a limited region.

Phytoliths: These are microscopic, inorganic siliceous bodies/residues that form in plant cells and frequent mirror parent cell shape. They are produced in multiple shapes and sizes. After the plant dies, the silica bodies become part of the mineral component of soils. A single plant may produce many different phytolith forms. A single phytolith form may be produced by a number of plant taxa. Phytoliths may survive for thousands of years and provide evidence of past plants. Phytoliths are generally incorporated in the silt fraction of the soil (2-50 micron particle size) as are most specimens of diatoms and statospores.

Pieces Esquillees: This is a French term used to identify a chert tool created by bipolar flaking. The artifact is created by placing the target object on a hard anvil stone or bone and striking the opposite end. The force creates flake removals from both opposing ends. Flake scars tend to be paired and opposed, have heavy rippling, and edges often show crushing on opposite margins. Bipolar flaking is often directed to small pieces and also can result from pounding a piece into wood or bone in a wedge like fashion.

Planktonic Foraminifera: These are unicellular organisms with a complex cell (Eukaryotes), and genetic material within a cell nucleus.

Platform: This is the surface area that receives the force necessary to detach a flake from the target piece/core. This can be prepared through flaking or grinding, or natural.

Pleistocene: The first epoch, which along with the Holocene Epoch constitutes the Quaternary period, spanning the time between roughly 2.0 or 1.65 million years ago and 10,000 years ago. Characterized by repeated continental glaciations, the Pleistocene witnessed the evolution of modern humans.

Poaceae: These, also called Gramineae or true grasses, are a large and nearly ubiquitous family of monocotyledonous flowering plants. There are more than 10,000 domesticated and wild species, the Poaceae represent the fifth-largest plant family. The three Poaceae subfamilies include Pooids, Panicoids, and Chloridoids. These short cell phytoliths are climatic indicators.

Polyunsaturated Fatty Acids: Pertaining to longchain carbon compounds (e.g., C18:2) like fats with multiple double bonds. These fats are very unstable and degrade very rapidly over time. These are 
detected in archeological samples during lipid residue analysis.

Pooids: A group of phytoliths from mostly coolmoist $\mathrm{C}_{3}$ grasses such as fescue (Festuca sp.), Canadian wildrye (Elymus sp.), Foxtail barley (Hordeum sp.), and western wheatgrass (Agropyron sp.). These grasses often grow in shaded areas and in riparian environments. Basic morphotypes for Pooids include keeled, conical, pryramidal, and crenate forms.

Potlid: This is a flake that exhibits a plano-convex shape, which leaves a circular concave scar on a larger piece. This is the result of differential expansion and contraction caused by heat when the object is subjected to extensive heat.

Pressure Flaking: A method used to shape stone tools through the application of force applied by pushing rather than striking. This is generally part of the final stages of finishing a stone tool to remove large ridges from previous scars.

Principal Component Analysis (PCA): This is a pattern recognition technique used for reducing the dimensionality of multivariate data, similar to factor analysis. It uses all the variables measured in a sample and calculates the variation among those variables.

Profile: A cross-sectional exposure of the sequence of horizons that make up a soil or a sequence of sedimentary deposits. It can be the result of either natural erosional down cutting or an artificial excavation.

Provenience: The specific vertical and horizontal location of where an object is found.

Quaternary: The second period, which along with the Tertiary Period, make up the Cenozoic Era, encompassing the Pleistocene and Holocene epochs; roughly the last 2.0 or 1.65 million years.

Rabdotus: This is a genus of air-breathing land snails, terrestrial pulmonate gastropod mollusks.
This is a common land snail found across much of Texas, often in archeological context. These live in a habitat of semiarboreal, grass and shrubs primarily in central, south, and west Texas. These hold promise for determining subsistence strategies and recreating of past environments and can be radiocarbon dated.

Radiocarbon Dating: The process of determining the age of a sample based on the amount of radioactive carbon (carbon 14 or $\mathrm{C}^{14}$ ) retained in that object.

Raphides: Needle-shaped crystals in a plant cell, typically of calcium oxalate. These are small (30 to $500 \mu \mathrm{m})$ crystals, generally with points on the ends and of similar lengths. They are often found in plants of the Agavaceae family such as sotol, yucca, agave, and lechuguilla. They are not diagnostic of any particular plant. Bohrer (1987) and Kwiatkowski (1992) believe that only agave contain these crystals. In contrast, Dering (2003) believes raphides occur in a variety of Agavaceae including sotol, yucca, agave, and beargrass.

Rare Earth Elements (REE): One of a group of 17 elements with atomic number 57 to 71 . These are detected in the instrumental neutron activation analysis.

Retouch: A technique of chipped stone artifact manufacture in which pressure flaking is used to detach small flakes to sharpen or otherwise modify the edge of a stone tool.

Riparian Zone: The generally well-watered area along a stream course with trees, bushes, and grasses in contrast to the open prairies.

Root Etching: Thin, shallow, squiggly lines or pits that are etched into the surfaces of bones by acids associated with plant roots that grow against the bone after the bone was deposited in the ground.

Saturated Fatty Acids: Each carbon in the chain is connected to its neighboring carbon by a single 
bond, which makes them relatively stable. The most abundant saturated fatty acids have chainlengths of either, 14, 16, or 18 carbons. Mammal fats consist primarily of saturated fatty acids and are solid at room temperature. These are detected in archeological samples during lipid residue analysis.

Scar: This is the negative area following the removal of a flake from the target object/core or tool.

Seasonality: The season of death of the animals killed at a campsite. This is often determined by the presence of fetal or neonatal bones (i.e., bison or deer), linked to the birthing period or the age of the animals determined by tooth eruption and wear patterns.

Section 106 Process: This is the federal process to assess whether or not a project will have effects on historic properties. The basic steps include establishing the parameters of the development/undertaking, identifying the historic properties within the undertaking, if historic properties are affected then assess the effects, and resolve the adverse effects. The assessing is generally done in terms of evaluating/testing, whereas the resolving the adverse effects is through avoidance or data recovery/mitigation.

Sedges: These are any number of grass like plants of the family Cyperaceae, characteristically having solid three-sided stems, leaves arranged in three rows, and spikelets of inconspicuous flowers. These are moist environment indicators.

Silt: A particle size that has a range from $0.06 \mathrm{~mm}$ to $0.002 \mathrm{~mm}$. These are smaller than sand grains and larger than clay particles.

Siliceous: Pertaining to silica, as in silicon dioxide, the most common chemical constituent on earth, and the dominant component of chert and quartz.

Site Structure: The spatial distribution of features, artifacts, and debris across a single occupation (or within a component) of an archeological site that is used to reconstruct manufacturing, maintenance, processing, production, and disposal activities at specific loci, and the spatial ways prehistoric groups organized their space at a site.

Slackwater: Water that is essentially still/unstressed or with no movement either way.

Smectitic Clay: Smectite is applied as a group or family name and Montmorillonite is a mineral species name. This clay has a high ionic displacement, and shrinks and swells with moisture content. When it contains water, the molecular structure allows easy movement of ions, making it a good electrical conductor.

Soft-Hammer: This is a striking instrument, usually an antler billet or wooden object used to apply force to remove a flake from a core or target object.

Soft Polish: This is polish observed on stone tools that have come in contact with animal skins, muscle, or soft plants.

Soil Horizon: A layer of soil, approximately parallel to the surface, having distinct characteristics produced by soil-forming processes. In the identification of soil horizons an upper case letter (i.e., A, B, C, R, and O) represents the major horizons with $\mathrm{A}$ at the top. Lower case letters that follow the upper case letters represent subdivisions of the major horizons.

Sponge Spicules: These are structural elements found in most sponges (Porifera). They provide structural support and deter predators. Large spicules that are visible to the naked eye are referred to as megascleres, while smaller, microscopic ones are termed microscleres. Megascleres are large spicules measuring from 60$2000 \mu \mathrm{m}$. Spicules are found in a range of symmetry types. Sponges prefer clear water, are very versitile and can be calcareous, siliceous, or composed of organic substance called spongin. The 
composition, size, and shape of spicules are one of the largest determining factors in sponge taxonomy. Fresh water sponge spicules are quite common and were recovered in the phytolith extraction process.

Sponge Gemmoscleres: Gemmoscleres are important for sponge species survival. They are part of the sponge's reproductive mechanism. Gemmules--which are spheres with the axially aligned gemmoscleres forming the outer "wall" of the gemmule as a layer or shell protecting the sponge larvae inside--are released from adult sponges to establish new sponge colonies.

Stable Isotope: An isotope not subjected to radioactive decay, such as carbon $\left(\mathrm{C}^{13}\right)$, oxygen $\left(\mathrm{O}^{18}\right)$, or nitrogen $\left(\mathrm{N}^{15}\right)$ isotopes. This contrasts with radioactivity isotopes that decay over time.

Starch: Starch is produced by all green plants for energy storage and is a major food source for humans. Pure starch is a white, tasteless and odorless powder that is insoluble in cold water or alcohol. Starch can be used as a thickening, stiffening or gluing agent when dissolved in warm water, giving, for example, wheat paste. In photosynthesis, plants use light energy to produce glucose from carbon dioxide. The glucose is stored mainly in the form of starch granules. Toward the end of the growing season, starch accumulates in twigs of trees near the buds. Fruit, seeds, rhizomes, and tubers store starch to prepare for the next growing season.

State Antiquities Landmark (SAL): This is any archeological site on county or municipal property, according to the Antiquities Code of Texas (Texas National Resources Code of 1977, Title 9, Chapter 191 as amended). State Antiquities Landmarks with high research potential may be designated by the Texas Historical Commission.

Statospores: These are the resting phase cysts of certain algae. These are the remains after the death of the plant or organism that produced it and in a terrestrial environment is incorporated into the soil mineral fraction.

Sterols: These are structural lipids that are present in cell membranes and contain the perhydrocyclopentanophenanthrene ring system. Sterols are a special king of alcohol that serves as precursors to a wide variety of products known as steroids. The cholesterol is the major sterol in animal tissue. Campesterol, stigmasterol and sitosterol are sterols found in plant tissue.

Stigmasterol: This and sitosterol are chemical sterols found in plant tissue that can be detected in lipid analysis.

Stratigraphy: The study of layering in rocks and/or sediments, and how the layers correlate to each other.

Striae: These are tiny, thin, narrow grooves, channels, or lines, often called striations. Here, they were observed during high-powered microscopic use-wear analysis on stone tools and are an indication of the direction of the movement of the tools during their use. They were observed under high magnification in the residues left on the tools.

Taxon: In biology, a taxon (plural taxa) is a group of one or more populations of an organism or organisms seen by taxonomists to form a unit.

Terrace: In geologic terms this is an old alluvial plain that is generally flat and borders a river, stream, lake, or sea. Terraces are recognized by different elevations and generally labeled $\mathrm{T}_{0}, \mathrm{~T}_{1}$ and $\mathrm{T}_{2}$ from lowest to highest.

Thermal Damage: This is used to describe lithic materials that exhibit potlidding, crazing, cracking, etc. through exposure to extreme heat.

Trace Elements: One of many chemical elements such as zinc, manganese, and iron in soils that typically occur in all systems in extremely small amounts. 
Tracheids: These are elongated cells in the xylem of vascular plants that serve in the transport of water and mineral salts. Tracheids are one of two types of tracheary elements, vessel elements being the other.

Triacylglycerol (TAGs): This is a glycerol molecule to which three fatty acids are bounded through ester linkages. These can be detected in lipid analysis.

Triticeae: This is a tribe within the Pooideae subfamily of grasses that includes genera with many domesticated species. Major crop genera are found in this tribe including wheat, barley, and rye; crops in other genera include some for human consumption and others used for animal feed or rangeland protection. Seed storage proteins in Triticeae are implicated in various food allergies and intolerances.

Turbation: Disturbance to natural matrix deposits generally caused by biological agents (burrowing rodents, insects, worms, and plant roots) and natural (soil creep, desiccation crack displacement, frost heaving, landslides, etc.) processes. These actions tend to move/displace cultural objects in the ground.

Tuber: This is the thick, fleshy underground stem of a plant. This stem serves as the primary storage organ of nutrients that stores food over winter and produces new growth in spring.

Type: This is a group of similar items having common characteristics (e.g., ceramic sherds or projectile points) all of which are more or less the same.

Ultraviolet (UV) Light: The wave length of light above that is usually detected by the human eye and that fluoresces various kinds of minerals and emits distinctive colors. Here, a multiband light source (UV light 254/366 nm Model UVGI-58) was used to investigate the visual fluorescence of culturally modified stones to help in identifying their source and detect new/recent scars from old flake scars.

Unconformity: Stratigraphic term for a boundary or break created by a depositional hiatus. This boundary separates younger strata from older strata. An unconformity is usually caused by erosion and therefore deposits are missing.

Uniface: An artifact that is flaked only on one surface.

Unsaturated Fatty Acids: These types of fatty acids contain at least one carbon-carbon double bond or point of unsaturation. That point of unsaturation is susceptible to additional reactions. Unsaturated fatty acids are the primary constituents of plant and fish oils and tend to be in liquid-state at room temperature. Their chain-lengths vary with a minimum of 12 carbons but most common ones contain at least 18 carbons.

Use-wear: The high-powered microscopic evidence on a stone tool that was created from sustained use. The wear may appear as striations, tiny nicks, abrasive particles, polish, rounding, soluble inorganic residues, etc. The accompanying use-wear study used magnification between 100x and 500x to observe wear and edge-modification on selected artifacts. This detailed analysis contributes to our understanding of the function of tools and potentially substances that tools were used on.

Variance-covariance Matrix: This is the matrix of covariance between all pairs of measured variables in a study.

Vector: In mathematics this is used to represent physical quantities that have both magnitude and direction.

Ventral: The underside or interior surface of a flake or blade.

Waxes: These are long-chain fatty acids and longchain alcohols that form protective coatings on 
skin, fur, feathers, leaves and fruit, also resist decay. These can be detected during lipid analysis.

Wildrye (Elymus sp.): A common grass throughout the Plains of the United States, from Mexico to Canada and is all across Texas. The seeds of this genus are large and it possesses a large distinctive starch grain. This is a cool season $\mathrm{C}_{3}$ grass (ca. $-27.6 \%$, $-27.1 \%$, Bender 1971) that produces short cell phytoliths. The seeds are available during the summer and fall.

Xeric Condition: A dry or relatively arid condition often in reference to climatic conditions.
Xerophic Plants: These are plants that have adapted to survive in an environment that lacks water, such as a desert. These include cactus, sotol, yucca, agave, and lechuguilla, and others.

X-Ray Fluorescence (XRF): This is an elemental analysis technique that involves the excitation of inner shell electrons with primary X-rays and analysis of characteristics secondary, fluorescence $\mathrm{X}$-ray emitted as sample atoms return to ground state. 